\title{
A RELIMITATION AND REVISION OF THE AUSTRALASIAN GROUND SPIDER FAMILY LAMPONIDAE (ARANEAE: GNAPHOSOIDEA)
}

\author{
NORMAN I. PLATNICK \\ Peter J. Solomon Family Curator \\ Division of Invertebrate Zoology \\ American Museum of Natural History; \\ Adjunct Professor, Department of Biology \\ City College, City University of New York; \\ Adjunct Professor, Department of Entomology \\ Cornell University
}

BULLETIN OF THE AMERICAN MUSEUM OF NATURAL HISTORY

Number 245, 330 pages, 819 figures, 93 maps

Issued January 27, 2000

Price: $\$ 27.00$ a copy 


\section{CONTENTS}

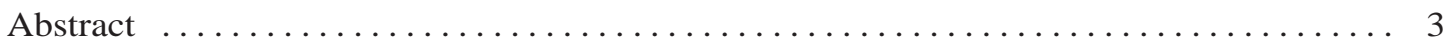

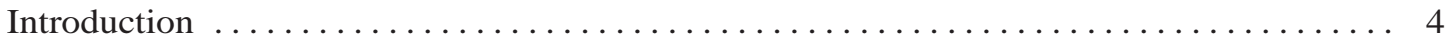

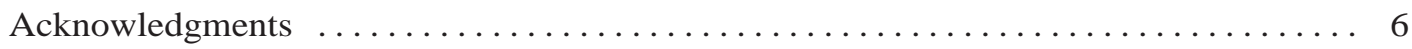

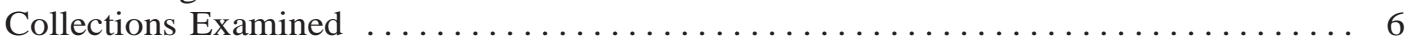

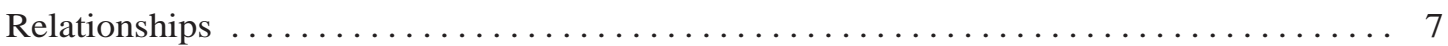

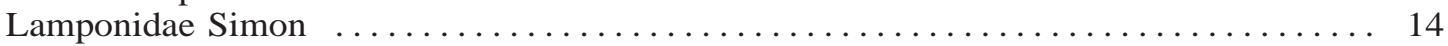

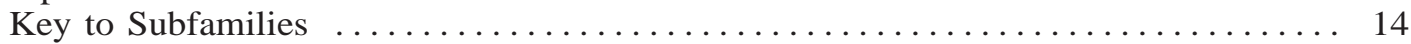

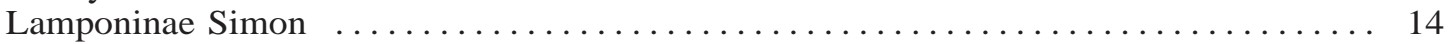

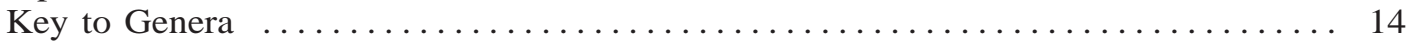

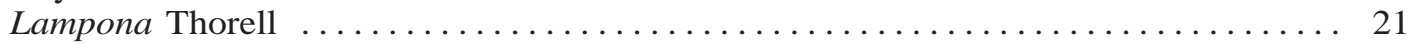

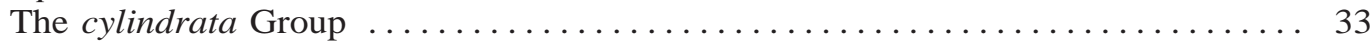

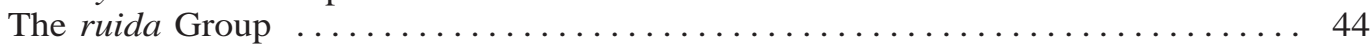

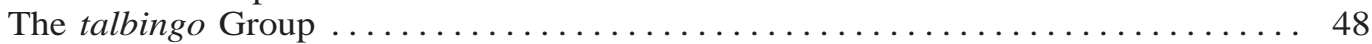

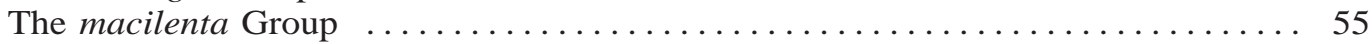

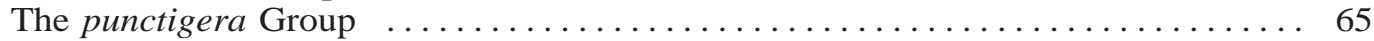

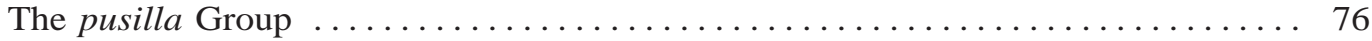

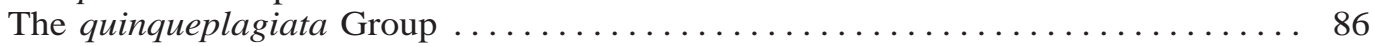

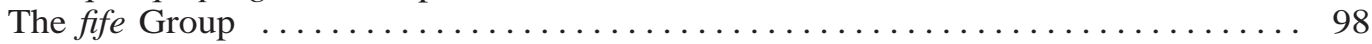

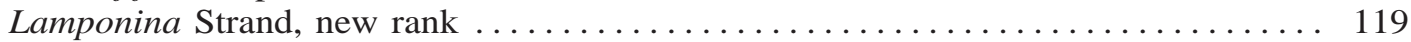

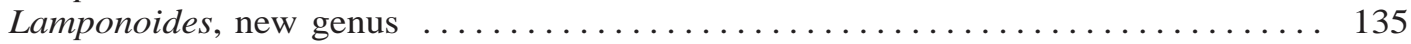

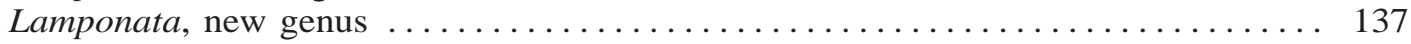

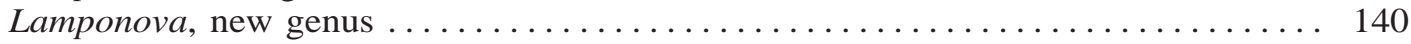

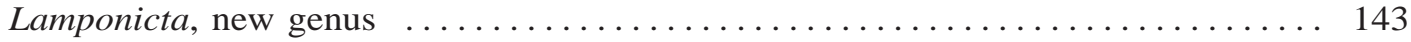

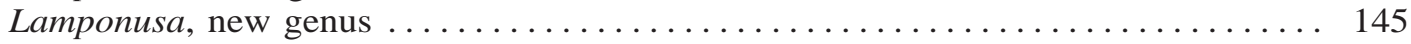

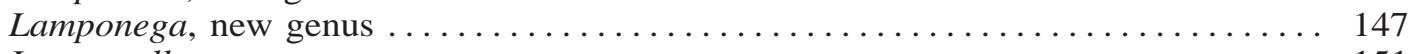

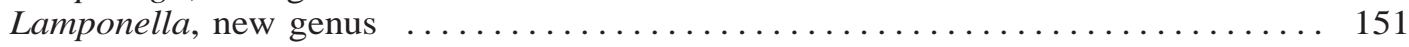

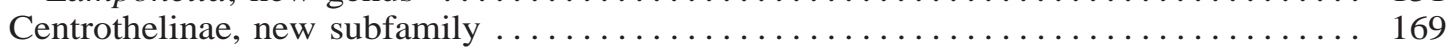

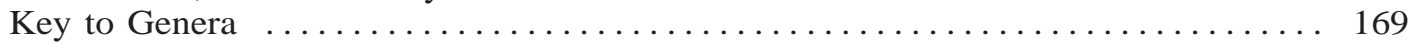

Centrothele L. Koch ................................... 173

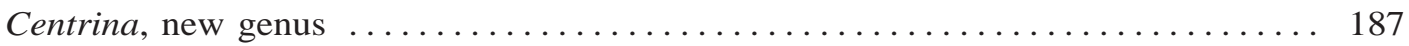

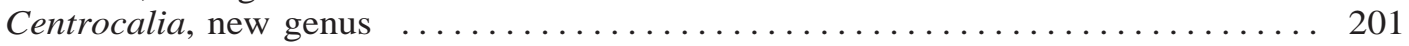

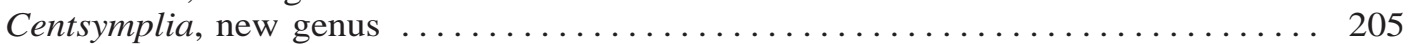

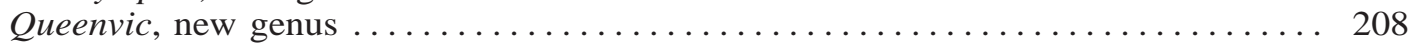

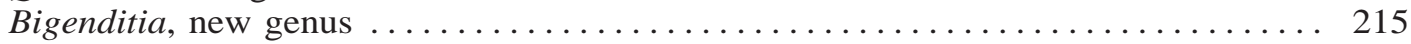

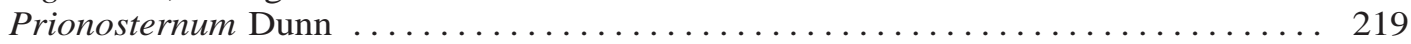

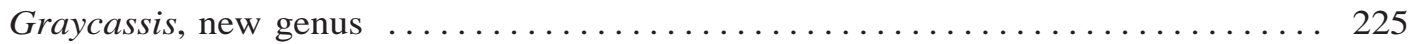

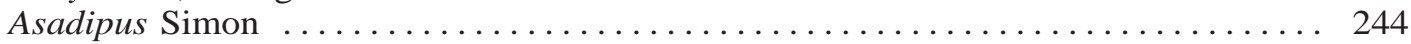

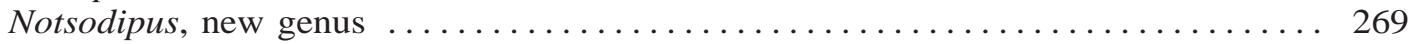

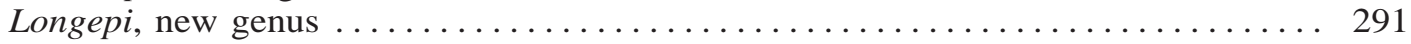

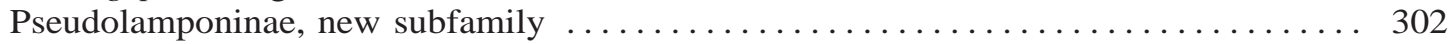

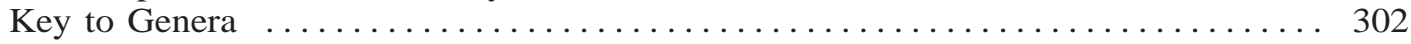

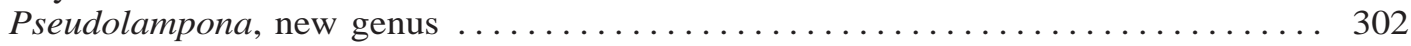

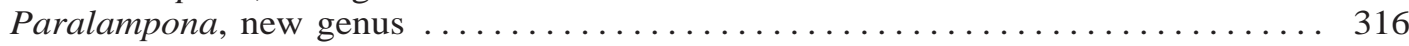

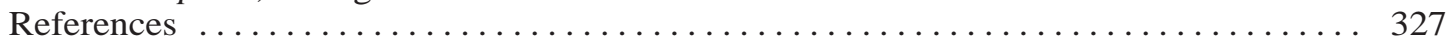

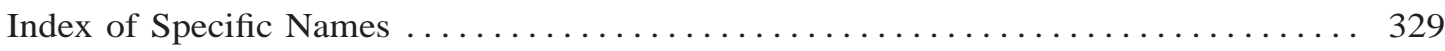




\begin{abstract}
The ground spider family Lamponidae Simon, previously construed to include only the type genus Lampona Thorell, is relimited to encompass 190 species of Australasian gnaphosoids, most of which have been either undescribed or misplaced in the families Corinnidae and Gnaphosidae. Three subfamilies are recognized: the Lamponinae Simon (including nine genera totalling 81 species), the Centrothelinae, new subfamily (type genus Centrothele L. Koch, including 11 genera, totalling 89 species), and the Pseudolamponinae, new subfamily (type genus Pseudolampona, new genus, including two genera, totalling 20 species). Putative synapomorphies for the family, as relimited, include two newly noted characters: a pair of oval, invaginated abdominal sclerites situated just behind the epigastric furrow, and a highly modified promarginal seta originating near the base of the cheliceral fang. As relimited, the lamponids are exclusively Australasian, and are hypothesized to represent the sister group of the worldwide families Gnaphosidae plus Prodidomidae.
\end{abstract}

The most commonly encountered lamponids are large, synanthropic, and occasionally medically important spiders that have generally been identified as Lampona cylindrata (L. Koch) but actually constitute a group of three closely related species: L. cylindrata, L. murina L. Koch, and $L$. papua, new species. Of these three species, only L. cylindrata has been found in Western and South Australia and in Tasmania, and that species is also common in Victoria and New South Wales, extends north into south-central Queensland, and has apparently been introduced into the South Island of New Zealand. In contrast, only L. murina has been found in eastern Queensland, and that species also occurs south to Victoria and has apparently been introduced into Lord Howe Island, Norfolk Island, the North Island of New Zealand, and the Kermadec Islands; L. papua is known only from New Guinea. Lampona pseudocylindrata Strand is newly synonymized with $L$. cylindrata; L. formicaria (Urquhart) is removed from the synonymy of L. cylindrata and placed as a junior synonym of L. murina. Three other specific names are newly synonymized within Lampona: $L$. subaquila Urquhart with $L$. ruida L. Koch, and $L$. obnubila Simon and $L$. paupercula Simon, both with $L$. brevipes L. Koch. The female of L. quiqueplagiata Simon and the males of $L$. flavipes L. Koch, L. macilenta L. Koch, L. pusilla L. Koch, L. ruida L. Koch, L. punctigera Simon, and L. foliifera Simon are described for the first time; 47 new species of Lampona are described.

The subgenus Lamponina Strand is elevated to generic level. Lampona scutata Strand and Lampona asperrima Hickman are transferred to Lamponina; the male of the former species and the female of the latter are described for the first time. Four new species of Lamponina are described. Seven new genera of lamponines are described: Lamponoides, Lamponata, Lamponova, Lamponicta, and Lamponusa, each for one new species; Lamponega, for three new species; and Lamponella, for 10 new species that are hypothesized to represent the sister group of all other lamponines.

Four genera (Aristerus Simon, Asadipus Simon, Centrothele L. Koch, and Stratius Simon) are transferred from the Corinnidae to the Centrothelinae; Prionosternum Dunn is transferred from the Gnaphosidae to the Centrothelinae. Aristerus is placed as a junior synonym of Asadipus, and 18 new species of Asadipus are described. Stratius is placed as a junior synonym of Centrothele; the males of C. lorata L. Koch and C. mutica (Simon) are described for the first time, and eight new species of Centrothele are described. Asadipus lifoui Berland is transferred to the new genus Centrocalia, its female is newly described, and two new species of Centrocalia are described; the genus appears to be endemic to New Caledonia. Asadipus nitidiceps Simon is transferred to Prionosternum and its male is newly described; males previously associated with its newly designated female lectotype belong to $P$. scutatum Dunn, the female of which is newly described; one new species of Prionosternum is described. Seven other new centrotheline genera are described: Centrina, for 11 new species; Centsymplia, for 1 new species; Longepi, for 8 new species; Queenvic, for 4 new species; Bigenditia, for 2 new species; Graycassis, for 10 new species; and Notsodipus, for 17 new species.

The Pseudolamponinae contains two new genera: Pseudolampona, described for 12 new species, and Paralampona, described for 8 new species. Pseudolamponines are hypothesized to represent the sister group of lamponines plus centrothelines. Hypothesized lamponine synapomorphies include a uniquely even endite shape and tubular pedicel sclerites; putative centrotheline synapomorphies include a highly tuberculate carapace, an anterior abdominal scutum in females, an anteriorly truncated ventral pedicel segment, a weakly sclerotized spot on the 
anterior surface of the chelicerae, and a longitudinal row of three highly modified cylindrical gland spigots on the posterior median spinnerets. Lamponines and centrothelines are united by a deep and sharply demarcated median groove on the palpal endites.

\section{INTRODUCTION}

This project started with the seemingly modest goal of reviewing the status of the spider family Lamponidae Simon. At the outset, the family was relatively small. In the process of investigating the limits and relationships of the various families of ground spiders, Platnick (1990) restricted the classical family Gnaphosidae to those taxa in which the piriform gland spigots on the anterior lateral spinnerets have greatly enlarged and widened shafts. As a result, 3 of the 115 or so genera that had previously been placed in that family had to be moved out of the group. Two of those genera had characters that allowed them to be placed in one of the other gnaphosoid families, but the Australasian genus Lampona Simon was not so cooperative and Simon's (1893) group Lamponeae was therefore elevated to the family level. At the start of the present project, then, the family Lamponidae contained a single genus and 17 described species, known only from Australia and New Zealand.

Of those species, only one, Lampona cylindrata (L. Koch), is at all well known. That animal is commonly called the "white-tailed spider" because of the conspicuous white spot found at the back of the abdomen, just above the spinnerets. It is among the most frequently collected spiders in Australia, not only because of its size (it is easily the largest species of Lampona) but also because, even though it does live in native forests, it is also synanthropic. These spiders are commonly found in and around buildings, where they fearlessly invade the webs of other spiders (including those of the large species of Badumna Thorell that are frequently found around houses as well). As a result of their synanthropic habits, the species is occasionally found in odd places (such as the specimen recorded below from "inside a plasticwrapped head of lettuce").

In recent years, Lampona cylindrata has received a considerable amount of publicity, especially in the Australian popular press.
Because of its synanthropic habits, there are occasional cases of bites in humans, and some toxicologists and physicians, such as Sutherland (1987, 1991) and Gray (1989), have suggested that the species is responsible for cases where putative bites have led to severe necrosis. There seems to be little evidence for this suggestion; the cases involving severe necrotic lesions have not been accompanied by captured specimens of the spiders involved, and in the bite cases where Lampona specimens have been recovered, the symptoms of the bites have not been severe (White et al., 1989; Raven and Harvey, 1991). Harvey and Raven (1991) suggested that the cases of severe necrotic lesions might have been caused by mycobacteria, perhaps carried on spider mouthparts, rather than by spider venom per se.

In addition, laboratory study of Lampona venom (Atkinson and Wright, 1991) did not find any severe reactions. Unfortunately, that particular study has turned out to be less than definitive. As indicated below, what has commonly been referred to as Lampona $c y$ lindrata is actually a complex of three closely related species. One (the true L. cylindra$t a$ ) is common throughout southern Australia; a second species (Lampona murina L. Koch) occurs only on the east coast, being most common in Queensland and becoming progressively less common at higher latitudes in New South Wales and into Victoria, and a third (Lampona papua, new species) is known only from New Guinea. As it happens, the spiders whose venoms were examined and found to be relatively harmless by Atkinson and Wright (1991) were from the Toowoomba, Queensland, area, and thus were almost certainly members of $L$. murina rather than L. cylindrata. Because it is possible that the increased size of $L$. cylindrata has been accompanied by an increase in the virulence of its venom, toxicological studies of southern specimens would be welcome. A recent report of sexual dimorphism in $L$. $c y$ - 
lindrata venom activity (Rash et al., 1998), presumably based on specimens from Victoria, may well have been compromised by mixed samples of L. cylindrata and L. murina.

The process of investigating the diversity of $L$. cylindrata and its relatives turned out to be a much larger project than could have been predicted. As argued below, the Lamponidae are actually a very diverse family, members of which have frequently been misplaced not only in the Gnaphosidae but even in some non-gnaphosoid families, such as the Clubionidae or Corinnidae. Thanks to numerous newly available collections, the family is relimited here to include not just Lampona, but a total of three subfamilies (two of them new), 22 genera (17 of them new), and 190 species (171 of them new).

Dealing with this unexpected diversity has been challenging, particularly as some taxa (such as Lampona itself) have relatively simple genitalia, impeding both the discrimination of species and the matching of males and females. Arachnologists historically have taken various approaches to the latter problem. When working with relatively small samples, it is frequently the case that males and females have never been taken together. Faced with such difficulties, some workers have routinely described the sexes separately, so that the resulting species names are frequently synonymized when further collecting allows likely matches to be made. However, that approach can result in significant overestimates of the actual diversity of various groups within particular regions. With the increased public awareness of the importance of biodiversity, and the vast amount of information on habitat diversity potentially offered by megadiverse groups like spiders, it seems inappropriate to bias diversity estimates in that manner.

Instead, I have adopted the viewpoint that the relevant null hypothesis, within any group being examined, is that all specimens belong to a single species, and that additional evidence is required to support each increase in the number of species hypothesized to exist. As a result, it is likely that some of the samples here hypothesized to be conspecific will subsequently be found to be composites of more than one taxon. For example, con- sider the new genus described below as Lamponoides. At present, this curious taxon is known from only two specimens, a male taken in New South Wales and a female taken in Queensland. This is obviously unsatisfactory, and my colleagues and I have therefore attempted to collect additional specimens at both of the original localities. In both cases, other quite unusual lamponids were collected, but no additional specimens of Lamponoides have been forthcoming. The two specimens seem to have congruent relationships; the male seems to represent the sister group of the males assigned below to the genus Lamponina Strand, new rank, and the female seems to represent the sister group of the females assigned below to Lamponina. It therefore seems reasonable to suggest that the two isolated specimens are congeneric. Given the distance and ecological differences between the localities at which they were collected, it is certainly possible, perhaps even likely, that they represent two different species of Lamponoides. However, at present there is no direct evidence to suggest that they are not conspecific as well as congeneric, and the null hypothesis that they are conspecific is therefore accepted.

To help restrict the results to a manageable size, species descriptions have been kept as short as possible, with most of the important information appearing in the figures, diagnoses, keys, lists of material examined, and maps. Measurements have been made only of total body length of single specimens, and reported only to the nearest $0.1 \mathrm{~mm}$, as they provide little information beyond an indication that one is in the right "ball park" when trying to identify a species not seen before.

Complete data are provided on all specimens examined, except in the case of the three most commonly collected species. In those cases, only a short version of the locality name, the geographic coordinates, the depository, and a registration number (if available) are provided, for a single specimen from each locality sampled. Full data for those three species will be made available, subsequently, in the Internet version of this monograph (at http://research.amnh.org/entomology). 


\section{ACKNOWLEDGMENTS}

A project of this magnitude clearly requires the involvement and assistance of many colleagues. First and foremost, I must thank the National Science Foundation's PEET (Partnerships for Enhancing Expertise in Taxonomy) program for providing the funding that has enabled two postdoctoral fellows, Drs. Vladimir Ovtsharenko and Kefyn Catley, to join with me in tackling the enormous task of revising the Australasian ground spider fauna. Without that funding and the multiple personnel involved, provided through grant DEB-9521631, we would never even have considered trying to tackle so large a project. We also thank the Australian Biological Resources Study for additional funding that has greatly enhanced our flexibility in dealing with the unpredicted challenges of handling a group that has proved to be far more diverse than we, or anyone else, suspected. In addition, the American Museum of Natural History has provided significant cost-sharing, enabling us to use the artistic talents of Dr. Mohammad Shadab as well as additional technical assistance.

All three investigators involved with the PEET project are enormously indebted to several Australian colleagues who have gone far above and beyond the call of duty in helping make specimens available for study. We are grateful to the curators of each of the 38 collections (listed below) that have supplied lamponid material for this study, but several colleagues cannot be thanked enough. In Brisbane, Drs. Robert Raven and Val Davies, and Mr. Phil Lawless, of the Queensland Museum have provided a virtual "home away from home" during our visits; Dr. Raven has worked especially closely with us, in the context of his surveys of Australian clubionoid spiders, to ensure that no taxa are "falling between the cracks" of our respective studies. In Sydney, Drs. Mike Gray and Gerry Cassis, and Mr. Graham Milledge, of the Australian Museum have made available to us the magnificent collections made in their recent and ongoing surveys of montane habitats in New South Wales. Without their efforts, entire groups (such as the speciose new genus called Graycassis below) would have been represented by only a handful of enigmatic specimens. In Adelaide, Mr. David Hirst of the South Australian Museum has allowed us access to important material even before it has gone through the normal cataloging process, and shared with us his discoveries about the vibration collecting technique. In Perth, Dr. Mark Harvey and Ms. Julianne Waldock of the Western Australian Museum have kept up a continual supply line of fascinating and puzzling new material, and provided invaluable assistance in the field. And in Darwin, Dr. Tracey Churchill has been the most gracious of hosts, supplying logistic support as well as collections from many habitats never before sampled for ground spiders. The efforts of Drs. Gray, Harvey, and Raven in reviewing the manuscript are enormously appreciated. To all these colleagues and the others listed below, a hearty "Goodonya!"

\section{COLLECTIONS EXAMINED}

AMNH American Museum of Natural History

AMNZ Auckland Museum Entomology Collections, Auckland, J. Early

AMS Australian Museum, Sydney, M. Gray

BMNH Natural History Museum, London, P. Hillyard

BMM Burke Memorial Museum, University of Washington, R. Crawford

CAS California Academy of Sciences, San Francisco, C. Griswold

CBB B. Baehr, München

CBM B. Y. Main, Perth

CCD C. Deeleman, Ossendrecht, Netherlands

CMNH Carnegie Museum of Natural History, Pittsburgh, J. Rawlins

CSID CSIRO, Darwin, T. Churchill

CVIC Central Victoria Regional Insect Collection, La Trobe University, Bendigo, Victoria, J. Shield

FCTH Tasmanian Forest Invertebrate Collection, Hobart, R. Bashford

FMNH Field Museum of Natural History, Chicago, P. Sierwald

FSCA Florida State Collection of Arthropods, Gainesville, G. Edwards

HNHM Hungarian Natural History Museum, Budapest, S. Mahunka, J. Balogh

JAM J. A. Murphy, Hampton, England

LUNZ Lincoln University, Canterbury, C. Vink

MCZ Museum of Comparative Zoology, 
Harvard University, H. Levi, L. Leibensperger

MNHN Muséum National d'Histoire Naturelle, Paris, C. Rollard

MNT Museum and Art Gallery of the Northern Territory, Darwin, G. R. Brown, G. Dally

MONZ Museum of New Zealand, P. Sirvid

NMS Natur-Museum Senckenberg, Frankfurt, M. Grasshoff

NMV Museum of Victoria, Abbotsford, G. Milledge

NMVS Museum of Victoria survey collection, A. Yen

NMW Naturhistorisches Museum Wien, J. Gruber

NRS Naturhistoriska Riksmuseet, Stockholm, T. Kronestedt

NZAC New Zealand Arthropod Collection, Auckland, G. Hall
OMD Otago Museum, Dunedin, J. Darby

QMB Queensland Museum, Brisbane, R. Raven, including material formerly in the Australian National Insect Collection, Canberra, lent by R. Halliday

QVM Queen Victoria Museum, Launceston, L. McGowan

SAM South Australian Museum, Adelaide, D. Hirst

TMH Tasmanian Museum, Hobart, E. Turner USNM National Museum of Natural History, Smithsonian Institution, Washington, J. Coddington, S. Larcher

WAM Western Australian Museum, Perth, M. Harvey, J. Waldock

ZMB Museum für Naturkunde, Berlin, M. Moritz

ZMH Zoologisches Museum, Hamburg, H. Dastych

\section{RELATIONSHIPS}

Platnick (1990) allocated those gnaphosoids with unmodified piriform gland spigots to five families (the Cithaeronidae, Gallieniellidae, Trochanteriidae, Ammoxenidae, and Lamponidae), and suggested that the other two gnaphosoid families (the Gnaphosidae and Prodidomidae) might be sister groups, united by the loss of a sclerotized ring on the anterior lateral spinnerets. The sclerotized ring apparently represents the remnants of a plesiomorphically present distal article on those spinnerets. Such a ring is found in the various "clubionoid" families that presumably include the closest relatives of gnaphosoids. For that 1990 study, only a few specimens of L. cylindrata were available for study, and none of those specimens had wellextended spinnerets. Subsequent investigation has shown that L. cylindrata and the other taxa assigned below to the Lamponidae actually differ, on one hand, from the Gnaphosidae and Prodidomidae, and on the other, from the remaining gnaphosoid families as well.

In the Gnaphosidae and Prodidomidae, the sclerotized ring is represented at most by a tiny crescent of sclerotized, seta-bearing cuticle found only around the base of the major ampullate gland spigots; the piriform gland spigots are surrounded only by soft cuticle, so that the anterior lateral spinnerets have a single article (i.e., are "unisegmented"). In the Cithaeronidae, Gallieniellidae, Trochanteriidae, and Ammoxenidae, the distal article is clearly marked by a complete ring of sclerotized, seta-bearing cuticle, so that the anterior lateral spinnerets are "bisegmented." In contrast, L. cylindrata and its relatives actually have an intermediate condition; the sclerotized ring is present but incomplete, being interrupted along its posterior edge by soft, unsclerotized cuticle that connects the area bearing the piriform gland spigots with the similarly unsclerotized areas proximal to the sclerotized ring. Hence, lamponid anterior lateral spinnerets are actually "unisegmented."

Discovery of this intermediate condition suggested that the Lamponidae, as here relimited, might constitute the sister group of the Gnaphosidae plus Prodidomidae. To test that hypothesis, a data matrix was compiled that includes 30 taxa: the 22 genera assigned below to the Lamponidae, plus eight outgroup taxa. Two genera each represent the families Gnaphosidae (Gnaphosa Latreille and Zelotes Gistel) and Prodidomidae (Prodidomus Hentz and Lygromma Simon). The putatively more plesiomorphic gnaphosoid families are represented by two genera of the Ammoxenidae (Ammoxenus Simon and Rastellus Platnick and Griffin). Because some of 


\begin{tabular}{|c|c|c|c|c|c|c|}
\hline Genus & & 5 & $\begin{array}{l}1 \\
0\end{array}$ & $\begin{array}{l}\text { er N } \\
1 \\
5\end{array}$ & $\begin{array}{c}\text { nber } \\
2 \\
0\end{array}$ & $\begin{array}{l}2 \\
5\end{array}$ \\
\hline Corinna & 00000 & 00000 & 00001 & 00000 & 00000 & 00000 \\
\hline Trachelas & 00000 & 00000 & 00011 & 00001 & 00000 & 00001 \\
\hline Ammoxenus & 00000 & 00001 & 10001 & 00000 & $0002-$ & -1000 \\
\hline Rastellus & 00000 & 00001 & 10001 & 00000 & $0002-$ & -1000 \\
\hline Gnaphosa & 00000 & 00011 & 10000 & 10000 & 00011 & 00000 \\
\hline Zelotes & 00000 & 00011 & 10000 & 10100 & 00011 & 00000 \\
\hline Prodidomus & 00000 & 00011 & 10001 & 10000 & 00010 & 10000 \\
\hline Lygromma & 00000 & 00011 & 10000 & 10000 & 00010 & 000 \\
\hline Pseudol ampona & 00000 & 00011 & 11010 & 10000 & 00000 & 00001 \\
\hline Paralampona & 00000 & 00011 & 11011 & 10000 & 00000 & 00001 \\
\hline Centrothele & 00 & 20011 & 11010 & 11111 & 11 & \\
\hline Centrina & 00000 & 20011 & 11010 & 11111 & 11000 & 00 \\
\hline Centrocalia & 00001 & 20011 & 11010 & 11111 & 11000 & 00000 \\
\hline Centsymplia & 00000 & 20011 & 11010 & 11101 & 11000 & 00000 \\
\hline Longepi & 00 & 11 & 11 & 11101 & 00 & \\
\hline dipus & 00000 & 20011 & 11001 & 11101 & 11100 & 10 \\
\hline Queenvic & 00000 & 20011 & 11010 & 11101 & 11000 & 00000 \\
\hline Bigenditia & 01 & 11 & 11010 & 11101 & 11000 & 00000 \\
\hline Prionostern & 00000 & 20011 & 11000 & 11101 & 11000 & 00 \\
\hline Graycassis & 00000 & 20011 & 11010 & 11101 & 11000 & 00000 \\
\hline Asadipus & 00000 & 20011 & 11010 & 11101 & 11100 & 00000 \\
\hline Lampona & 01110 & 01011 & 11011 & 10000 & 01000 & 00001 \\
\hline Lamponoides & 01110 & 11111 & 11011 & 10000 & 01000 & 00001 \\
\hline Lamponina & 01111 & 11111 & 11011 & 10000 & 01000 & 00001 \\
\hline Lamponata & 01100 & 01011 & 11011 & 10000 & 01000 & 00001 \\
\hline Lamponova & 01100 & 01011 & $1101 ?$ & 10000 & 01000 & 00001 \\
\hline Lamponel Ia & 01000 & 01001 & 11210 & 10000 & 01000 & 00001 \\
\hline Lamponega & 01001 & 01011 & 11101 & 10000 & 01000 & 00001 \\
\hline Lamponusa & 01001 & 11011 & 11011 & 10000 & 01000 & 00001 \\
\hline Lamponicta & 01001 & 11011 & 11111 & 10000 & 01000 & 00001 \\
\hline
\end{tabular}

Fig. 1. Character matrix for 30 genera; see text for character descriptions.

the taxa here placed in the Lamponidae have been considered members of the Corinnidae, two genera of that family are used to root the tree and test lamponid monophyly: the type genus Corinna C. L. Koch and the tracheline genus Trachelas L. Koch (chosen because the Australian taxa that have been misplaced as corinnids were originally described as members or close relatives of Trachelas). Both Corinna and Trachelas are currently heterogeneous groups, and comparisons in each case have been made with their type species (and not, for example, with the New
World taxa currently misplaced in Trachelas).

An extensive search was made for new characters that might be relevant to resolving the relationships of these taxa, and several have been found. The data matrix (fig. 1) comprises 29 characters (plus an initial allzero dummy character, no. 0 ).

Spinneret Characters

As indicated above, the anterior lateral spinnerets consist of either one or two arti- 
cles; in character 15 , those taxa with a complete sclerotized ring surrounding the spigots of the anterior lateral spinnerets, and with that sclerotized ring separated from the proximal article by unsclerotized cuticle, are coded as having two articles (state 0). Those taxa in which the sclerotized ring is incomplete (i.e., is itself interrupted by unsclerotized cuticle) are coded as having a single article (state 1). Among the latter group of taxa, the gnaphosids and prodidomids differ from the lamponids in having the sclerotized ring reduced to a tiny crescent of cuticle, but that additional difference was not coded in the matrix, so that other characters would have a maximal chance of disputing the relationships initially suggested by these differences.

When L. Koch (1873) described the genus Centrothele, he was struck (as his choice of name indicates) by the peculiar "center" or posterior median spinnerets of these spiders; Koch even illustrated the three large and peculiar spigots found on each of those spinnerets! Unfortunately, Centrothele has remained an obscure taxon; for Simon (1897a: 186), it was merely a "genus invisum," and Simon overlooked the peculiar spigots in his own specimens of the genus, which he therefore redescribed under a different name. Koch's character is actually quite distinctive, consisting of a longitudinal row of three cylindrical gland spigots, each of which has an enormously widened base and greatly shortened shaft (figs. 410, 411). These striking spigots (character 16) are found in females of 11 of the genera included in the matrix.

Three characters in the matrix refer to modifications of the piriform gland spigots found in various gnaphosoid families. In most spiders, the piriform gland spigots are quite small, and are smaller than the major ampullate gland spigots that are also present on the anterior lateral spinnerets. However, in some gnaphosoids these spigots can be much larger than the major ampullate gland spigots (state 1 of character 23); in others, the piriform gland spigots can be lost instead (state 2 of character 23). In true gnaphosids, the piriform gland spigots have wide shafts, with broad openings (character 24), whereas in prodidomids, the piriform gland spigots have greatly elongated bases accompanied by plumose setae (character 25).
Also, in the ammoxenids, the posterior median spinnerets have moved anteriorly, so that they separate the anterior lateral spinnerets (character 26), a feature paralleled only in distantly related groups such as the Caponiidae.

\section{Cephalothoracic Characters}

Gnaphosoids are often most easily recognized by their modified posterior median eyes. In most spiders those eyes have normal, rounded lenses; seen in profile, for example, the lenses of the posterior median eyes have the same shape as do those of the posterior lateral eyes. In gnaphosoids, however, the posterior median eyes are flattened and are often irregularly shaped; these modified lenses (character 9) presumably do not function in normal vision.

Another feature often used to diagnoses gnaphosoids is the presence of an oblique depression on the palpal endites (character 10), although the character does occur sporadically in some "clubionoids." Some of the taxa included in the matrix have additional endite modifications as well. Members of the genus Lampona and its close relatives have distinctively shaped endites, with a rather evenly rectangular outline (character 1; fig. 15). Those taxa and others also have a deep and sharply demarcated longitudinal groove along the median edge of the endites, opposite the labium (character 21; fig. 15).

In some of the genera, the carapace is heavily coated with large tubercles (character 20); similar tubercles are often found on the sternum, but their presence may be correlated with the carapace tubercles and they are therefore not coded as an independent character.

At the front of the carapace, there is usually a small, triangular sclerite (the chilum) situated between the anterior edge of the carapace and the proximal margins of the chelicerae. In some cases, the chilum may be fused to the carapace, producing a medially prolonged carapace outline (state 1 of character 12), or the chilum may instead be lost entirely (state 2 of character 12).

Some of the taxa analyzed here have a distinctive cheliceral seta that seems not to have been noted before. The promargin of the che- 
liceral fang furrow is generally bordered by an entire series of setae that normally parallel each other in size, shape, and orientation. However, the seta which originates closest to the base can be quite modified; in the taxa showing character 13 , that seta is greatly elongated, bent at a right angle just beyond its origin, extends across the other promarginal cheliceral setae toward the midline, and is distally plumose (figs. 9-11). The modified seta is found in most of the taxa here considered lamponids, but also in some outgroup taxa as well.

Centrothele and its relatives have an additional cheliceral modification, consisting of an oval area situated on the anterior surface of the chelicerae, not far dorsal of the promarginal setae. This oval area is relatively weakly sclerotized and much paler than the remainder of the paturon. This feature (character 17) also occurs in some of outgroup taxa (such as Zelotes), where it also seems not to have been noted before.

Several features of the sternum and the adjacent epimeric sclerites, which are situated between the lateral edge of the carapace and the coxae, may also be informative. In some lamponids, the sternal surface is notably rugose (character 3), rather than smooth. In some, the lateral edges of the sternum are fused to the epimeric sclerites (character 4). The epimeric sclerites can also fuse, producing a single long sclerite that extends above all of the leg coxae (character 8). The latter fusion can happen regardless of whether the epimeric sclerites also fuse to the sternum, but when both characters co-occur, the coxae each open from a circular foramen (i.e., the coxae are each completely encircled by sclerotized cuticle).

Some lamponids have a distinctive divided scopula, composed of sharply bent hairs (figs. 31, 32), on the ventral surface of metatarsi and tarsi I and II (character 2). All these genera have fewer leg spines than do typical ground spiders, but in some genera, the leg spination is dramatically reduced (character 29); in these cases, there is at most a single proventral spine at the distal tip of tibiae III and IV, with an occasional dorsal spine on femur IV, or spines may be lacking entirely.

\section{Other Abdominal Characters}

The pedicel is often highly modified in these spiders. In some genera, the pedicel sclerites form a tube (character 6), which either bears an anterior extension on its ventral surface or has the entire tube extended anteriorly. In other genera, the anterior edge of the pedicel becomes truncated ventrally (character 19), fitting across the breadth of the posterior margin of the sternum with only a narrow sliver of unsclerotized cuticle separating the pedicel and sternum, or (in extreme cases) even fusing with the posterior margin of the sternum.

Males of these groups commonly have a dorsal abdominal scutum, but in some genera the females may also have a scutum, which may be a dorsal scutum much like that of the male (only smaller; state 1 of character 5), or instead may be an anterior scutum, situated on the anterior face of the abdomen, just above the pedicel (state 2 of character 5).

One of the oddest features of Lampona and its relatives seems not to have been noted before. The ventral surface of the abdomen bears, just behind the epigastric furrow, a pair of oval, invaginated sclerites, one behind each booklung cover (figs. 20, 37, 38; character 11). These invaginated sclerites have a distinctive anterior rim, which is often retracted under the epigastric sclerite itself (at least in preserved specimens). The function of these peculiar sclerites is unknown; scanning electron microscopy has revealed no pores or sensillae on their surface, and there seem to be no anatomical connections to either the respiratory or reproductive organs. In other spiders, similar invaginated sclerites occur in females and are used by males as holdfasts during mating, but in these animals the sclerites are identical in both sexes. They are usually very conspicuous, and although some corinnids and liocranids have flat sclerites, of various shapes, in analogous positions on the abdomen, these invaginated sclerites have not yet been noticed in other taxa. In two new genera that contain only very small species (Pseudolampona and Paralampona), the sclerites are much less conspicuous and were initially thought to be absent. Compound microscopic examination of the relevant parts of the cu- 
ticle indicates that they are present, although they are not heavily sclerotized and are relatively much smaller than in the remaining genera that bear them. These two genera are therefore coded as having the character, but since its small size might represent either an early stage in their evolution or a subsequent reduction correlated with a reduced body size, the relative size is not coded as an additional feature.

\section{Genitalic Characters}

In many genera considered here, both the male and female genitalia are relatively simple, offering few clues about higher-level relationships. Nevertheless, a few characters can be coded as representing likely synapomorphies. In some cases the epigynal area forms part of an elongate sclerite, with edges parallel to the lateral edges of the booklung covers and separated from the rest of the epigastric sclerite by only a narrow strip of unsclerotized cuticle on each side; in these cases (character 7), the epigynal sclerite reaches all the way to the pedicel.

In these genera, a median apophysis may be present on the male palp, as in most hunting spiders, or reduced (character 14), being represented at most by a slight sclerotization situated within a membranous conductor, or lost entirely. In some cases, the male embolus bears a distinctive apophysis at its base (character 18); in others, the embolus may be greatly elongated and twice recurved (character 22). The male tegulum may have a distinctive bulge near its anteromedial corner (character 28), and the female epigynum may have a pair of blind ducts (character 27).

\section{Analysis}

Although phylogenetic analyses are still most commonly carried out with equally weighted characters, it seems clear that this is usually inappropriate. If analysis of a data set indicates that some of the characters are homoplasious, then the analysis itself provides evidence that not all the characters deserve equal weight. At least two reasonable methods of analysis are available that allow the total suite of characters to determine the weights of any individual character, and both are used here.
Successive approximations weighting (Farris, 1969) was applied to the character matrix using Hennig86, version 1.5 (Farris, 1988). The states of the few multistate characters were presumed to be independent modifications and were therefore considered unordered. Four runs using the $\mathrm{h}, \mathrm{h} * \mathrm{~m}$, and $\mathrm{m}^{*}$ tree-generating algorithms, with the results then subjected to the $b^{*}$ branch swapper, each produced a total of 780 cladograms (length 51, consistency index 0.62 , retention index 0.87). Three rounds of successive approximations weighting produced a stable solution (24 cladograms of length 278 , consistency index 0.87, retention index 0.96).

One of the 24 resulting cladograms was chosen as the preferred solution (fig. 2), for the following reasons. In 16 out of the 24 cladograms, the new genera Pseudolampona and Paralampona do not appear as sister taxa, falling either as unresolved at the basal lamponid node (six cases) or as paraphyletic (with either of these genera treated as closer to other lamponids than to each other; 10 cases). While the evidence is not overwhelming, the similarities between these two genera in size and carapace shape (not coded in the matrix), as well as their joint loss of all leg spines, suggest that they do indeed form a monophyletic group.

In two of the remaining eight cladograms, the basal lamponine dichotomy between the new genus Lamponella and the other eight lamponine genera was not recovered, producing a trichotomy at that level. Although most of the many odd features of Lamponella may be autapomorphic, the loss of the median apophysis in the remaining genera suggests that they do indeed form a monophyletic group.

The remaining six cladograms differ only with regard to relationships within the centrothelines. All six include two components: they each group Centrothele with the new genera Centrina and Centrocalia, and they each place the two new genera Notsodipus and Longepi as sister taxa. One cladogram (fig. 2) shows no further resolution. Three resolve the first group, with Centrothele and Centrina shown as sister taxa, relative to Centrocalia. This result was contrary to my expectations, and seems to be produced by associating the new genus Bigenditia with 


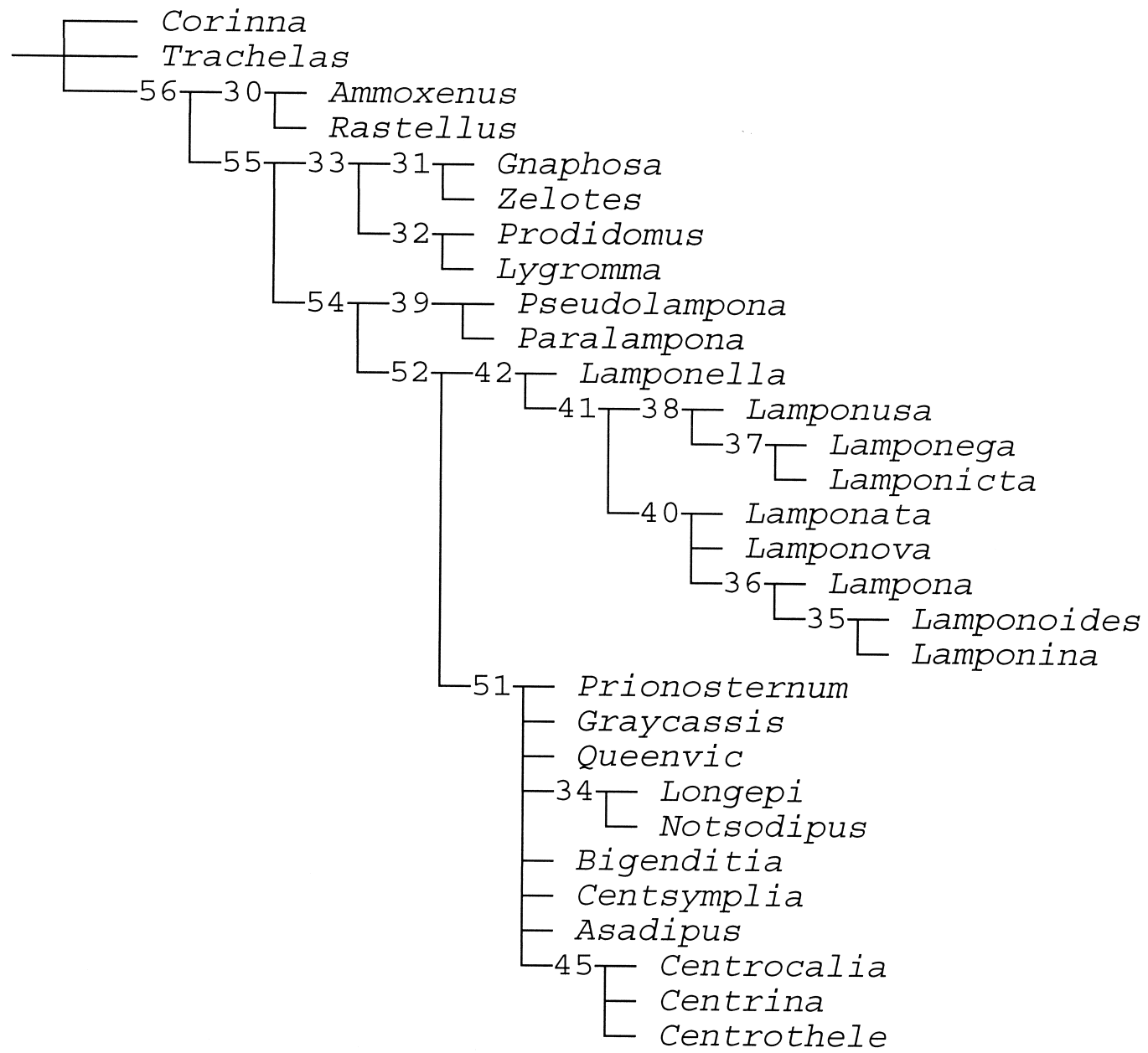

Fig. 2. Preferred cladogram for 30 genera; see text for discussion.

this component. Bigenditia and Centrocalia are both extremely heavily sclerotized, which is reflected in their both having the sternum fused to the epimeric sclerites (the only coded character in which Centrocalia differs from Centrothele and Centrina). This association allows the program to consider the fusion to evolve in the group of all four genera and then become lost in Centrothele and Centrina. Fusion of the sternum to the epimeric sclerites seems to have occurred independently twice within the lamponines as well (once in the clade including the new genera Lamponicta, Lamponega, and Lamponusa, and a second time in Lamponoides), and a fourth origin, correlated with increased sclerotization, therefore seems more likely than the reversal required by these three cladograms.

Of the remaining three cladograms, one places the Notsodipus plus Longepi clade as the sister group of Prionosternum Dunn, whereas one places the Notsodipus plus Longepi clade as the sister group of all other centrothelines. Neither of these contradictory hypotheses seems well supported, and the preferred solution (fig. 2) therefore shows just the two components, within the centrothelines, that are indisputably supported by the data.

Implied weighting (Goloboff, 1993) was also applied to the matrix, using Pew-Wee, version 2.6 (Goloboff, 1997). This program downweights homoplasious characters using 
a concave function of the homoplasy; the range of available settings for the concavity function (1-6) was used, in conjunction with the mult*20 command, which randomizes the order of the taxa, creates a weighted cladogram, submits it to tree-bisection and reconnection, and repeats the process 20 times using a different random number seed. At each concavity, the program found the same three cladograms (with, of course, different total weights of $233.1,247.0,255.4,260.9$, 264.6, and 267.6 for concavities 1-6, respectively).

One of these three cladograms is identical to the preferred solution (fig. 2) except that the clade including Pseudolampona plus Paralampona is once again not recovered, producing a trichotomy among these two genera and a group including all other lamponids. The second cladogram also fails to resolve Lamponella as the sister group of the other eight lamponine genera, and the third also resolves a clade including all nine centrotheline genera other than Longepi and Notsodipus. For the reasons cited above, none of these three solutions is seen as an improvement, although the basal dichotomy within the centrothelines resolved in the third cladogram remains a possibility for future testing.

\section{Conclusions}

The superfamily Gnaphosoidea is resolved at node 56, supported by flattened posterior median eyes and obliquely depressed endites; the genera Centrothele and Asadipus Simon cluster as gnaphosoids rather than in their current position as tracheline corinnids. The Ammoxenidae cluster at node 30, united by anteriorly advanced posterior median spinnerets and the loss of piriform gland spigots. The true Gnaphosidae cluster at node 31, based on their widened piriform gland spigot shafts; the Prodidomidae cluster at node 32, based on the elongated bases of their piriform gland spigots; and the gnaphosids and prodidomids cluster at node 33 , supported by having the piriform gland spigots larger than the major ampullate gland spigots.

The Lamponidae, as here relimited, cluster at node 54, supported by the pair of oval, invaginated abdominal sclerites and the mod- ified first seta on the cheliceral promargin (despite homoplasy, in the latter character, both inside and outside the group). The lamponids are placed as the sister group of the Gnaphosidae plus Prodidomidae, based on their joint loss of a distal article on the anterior lateral spinnerets (i.e., the interruption of the sclerotized ring around the spigots on these spinnerets). All these higher level relationships will be reexamined, of course, when the substantial Australian faunas of the more plesiomorphic gnaphosoid families (such as the Gallieniellidae and Trochanteriidae) can be added to the matrix. It will be interesting to see whether character 8 (fusion of the epimeric sclerites above the leg coxae) continues to support a separation between the more plesiomorphic gnaphosoid families (such as the Ammoxenidae) and the Lamponidae, Prodidomidae, and Gnaphosidae.

Within the Lamponidae, the new (albeit weakly supported) subfamily Pseudolamponinae clusters at node 39 (supported by the complete loss of leg spines and an uncoded carapace shape character) and appears to represent the sister group of the other two subfamilies recognized, the Lamponinae (node 42) and Centrothelinae (node 51). The latter two subfamilies are united at node 52 by the deep, sharply demarcated longitudinal groove occupying the median side of the endites.

The Lamponinae cluster at node 42, supported by their peculiar endite shape and tubular pedicel sclerites. A rugose sternal surface unites Lampona with the genera Lamponina and Lamponoides at node 36, with the latter two genera clustering at node 35 (supported by their long epigynal sclerite and by the presence of a dorsal abdominal scutum in females, paralleled elsewhere). The new genera Lamponata and Lamponova join that clade at node 40 , supported by the presence of divided scopulae on the anterior tarsi and metatarsi.

The three new genera Lamponusa, Lamponega, and Lamponicta cluster at node 38 (sternum fused with the epimeric sclerites, paralleled in Lamponina), with the latter two genera being resolved as sister taxa at node 37, based on their fused chilum. Lamponella appears to represent the sister group of all other lamponines (node 41), retaining a me- 
dian apophysis on the male palp that is lost in the other genera.

The members of the new subfamily Centrothelinae cluster at node 51, well supported by their highly tuberculate carapace (and sternum), an anterior (rather than dorsal) abdominal scutum in females, an anteriorly truncated ventral pedicel sclerite, a weakly sclerotized spot on the anterior surface of the chelicerae, and, of course, the row of three highly modified cylindrical gland spigots found on the posterior median spinnerets of females. Genitalic characters support the two centrotheline clades recognized (an embolar apophysis at node 45, and blind epigynal ducts and a male tegular bulge at node 34 ).

\section{LAMPONIDAE SIMON}

Lamponeae Simon, 1893: 349, 375 (type genus Lampona Thorell).

Lamponidae: Platnick, 1990: 40.

DiagnOSIS: Lamponids differ from ammoxenids, cithaeronids, gallieniellids, and trochanteriids in having anterior lateral spinnerets consisting of only a single article, and from gnaphosids and prodidomids in having an incomplete ring of sclerotized cuticle (rather than just a tiny crescent) that represents the remains of the base of a distal article. Most lamponids are easily recognized by the pair of large, oval, invaginated sclerites situated just behind the epigastric furrow in both sexes (figs. 20, 37, 38), but these sclerites are inconspicuous in the small species of the subfamily Pseudolamponinae.

Distribution: Native to Australia (including Tasmania), New Guinea, and New Caledonia; apparently introduced into Lord Howe and Norfolk Islands as well as the Kermadec, North, and South Islands of New Zealand.
INCLUDED SUBFAMILIES: Lamponinae Simon; Centrothelinae, new subfamily; and Pseudolamponinae, new subfamily.

\section{KEY TO SUBFAMILIES}

1. Endites with deep, sharply demarcated groove extending along their medial margins, adjacent to the labium (figs. 15, 43) . . . . 2

- Endites without a deep groove ........ ............. Pseudolamponinae

2. Endites with an evenly rectangular outline (figs. 15, 43); pedicel often with an anterior projection at the front of its ventral sclerite (fig. 43); females with dorsal or no abdominal scutum, without enlarged cylindrical gland spigots ......... Lamponinae

- Endites with an uneven outline; pedicel with anterior margin of its ventral sclerite truncated or fused with the sternum; females with anterior abdominal scutum, their posterior median spinnerets each with longitudinal row of three enormously enlarged cylindrical gland spigots (fig. 410) ....... ............... Centrothelinae

\section{LAMPONINAE SIMON}

DIAGNOSIS: Lamponines are most easily recognized by their distinctive endite shape (figs. 15, 43), not shared with any other gnaphosoids. The pedicel sclerites form a tube, which either has an anterior projection on the anterior surface of its ventral sclerite (fig. 43) or forms part of a greatly elongated tube (fig. 274). The sternum usually has steeply sloping margins (fig. 18) and is usually expanded anteriorly, just behind its anterior margin (fig. 43).

Distribution: Native to Australia (including Tasmania) and New Guinea; apparently introduced into Lord Howe and Norfolk Is- lands as well as the Kermadec, North, and South Islands of New Zealand.

InCluded Genera: Lampona Thorell; Lamponina Strand, new rank; and seven new genera: Lamponoides, Lamponata, Lamponova, Lamponicta, Lamponusa, Lamponega, and Lamponella.

\section{KEY TO GENERA OF LAMPONINAE}

1. Ventral surface of metatarsi and tarsi I and II with divided scopula composed of distally bent setae (figs. 31, 32; N.B.: males of Lamponata sometimes have relatively few scop- 

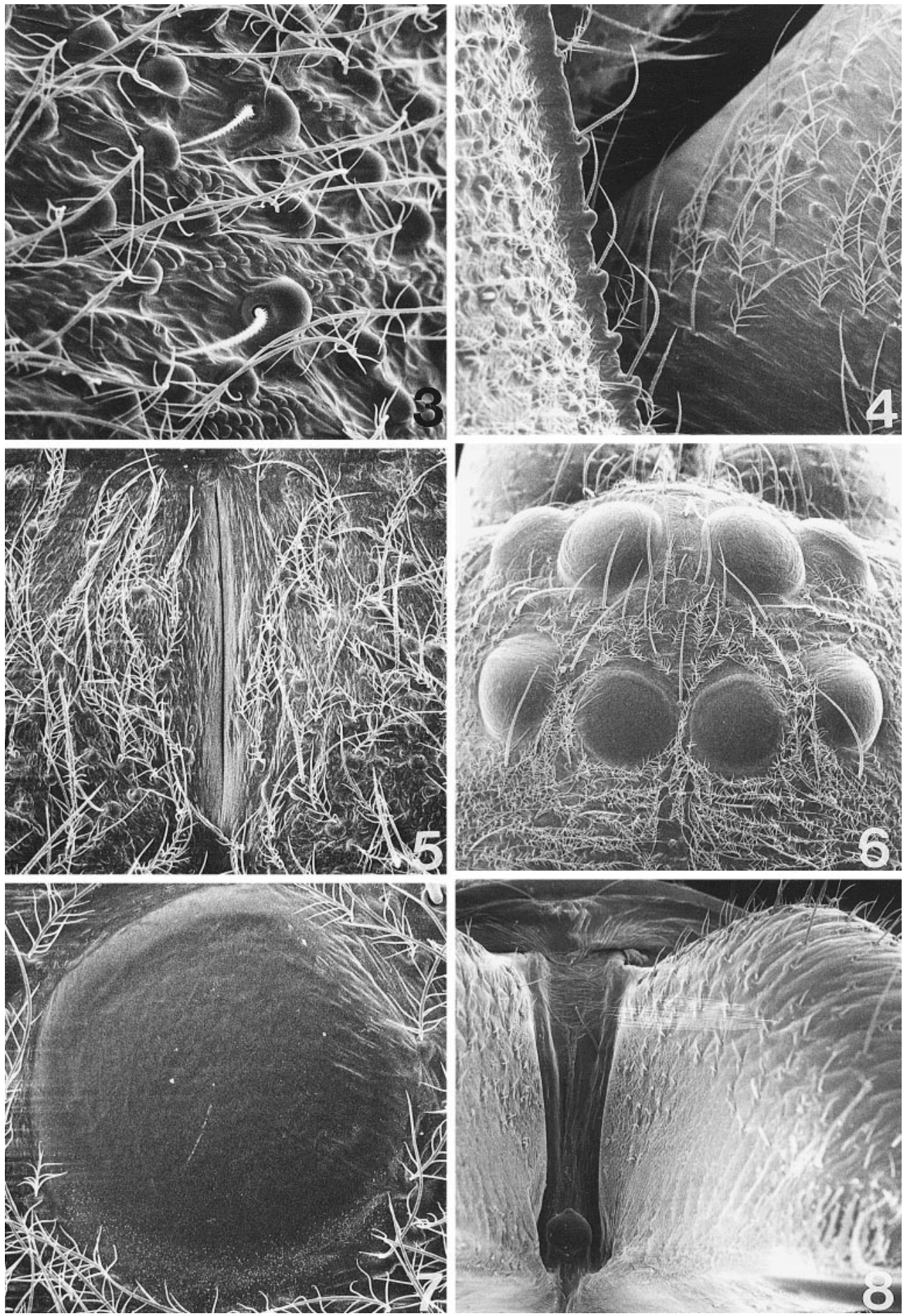

Figs. 3-8. Lampona cylindrata (L. Koch), male. 3. Feathery and hairy setae of carapace, dorsal view. 4. Lateral margin of carapace and coxa II, dorsal view. 5. Thoracic groove, dorsal view. 6. Eyes, dorsal view. 7. Posterior median eye, dorsal view. 8. Posterior chilum, a narrow sclerite situated between the bases of the chelicerae, ventral view. 

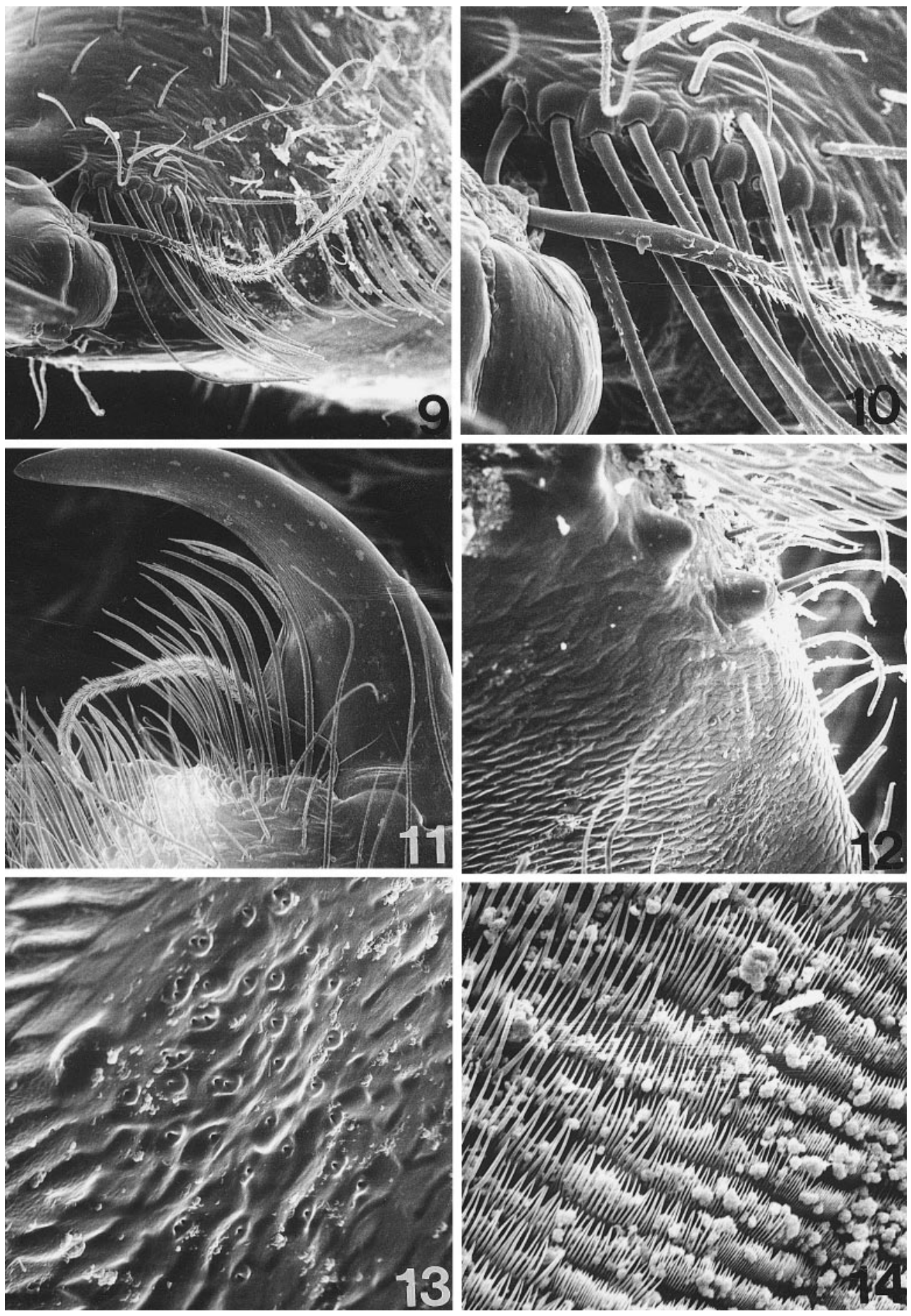

Figs. 9-14. Lampona cylindrata (L. Koch). 9. Promarginal cheliceral setae of male, ventral view. 10. Modified first promarginal chelicerae seta of male, ventral view. 11. Same, female, posterior view. 12. Promarginal cheliceral teeth of male, posterior view. 13. Cheliceral gland openings of male, posterior view. 14. Straining teeth of labrum, female, posterior view. 

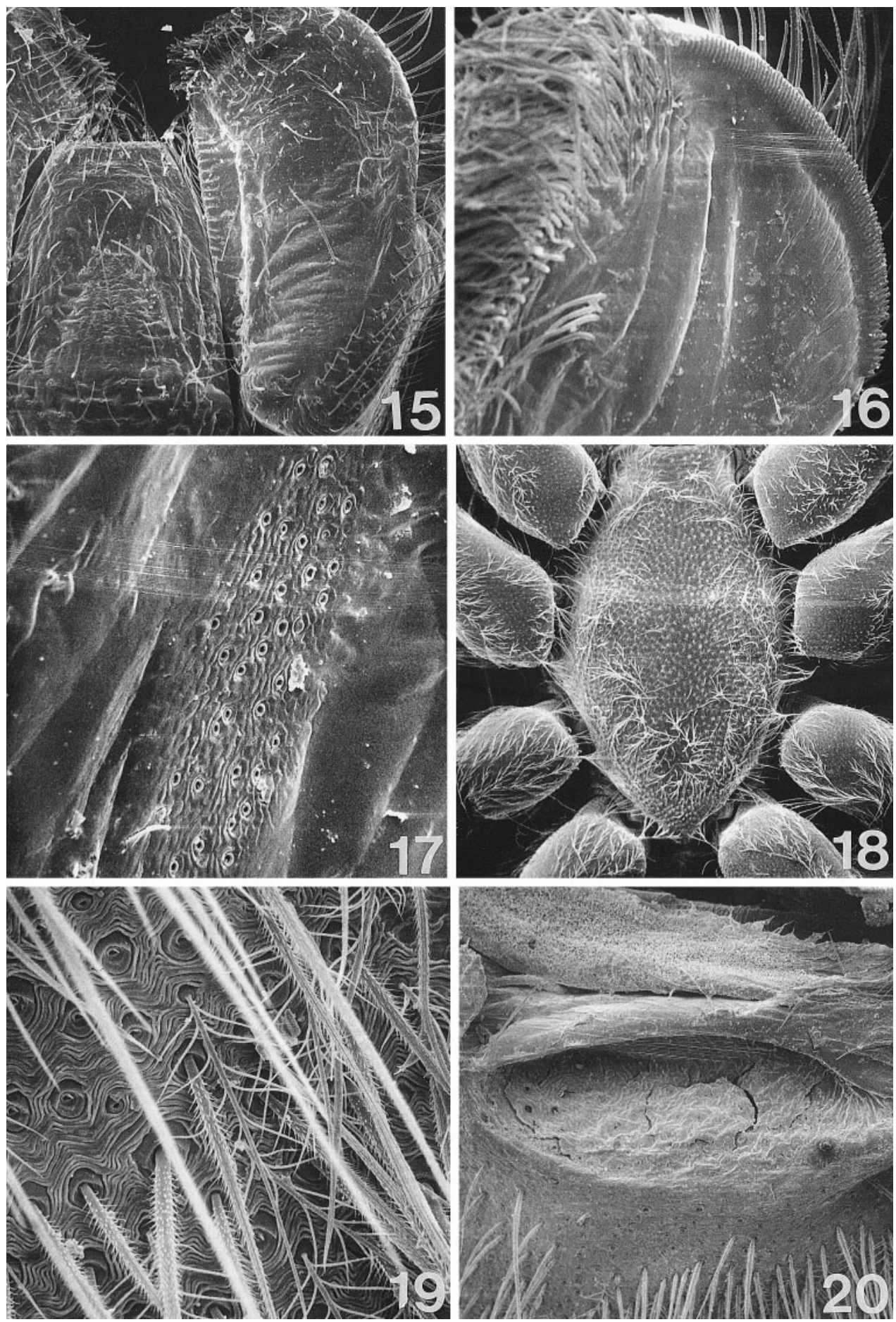

Figs. 15-20. Lampona cylindrata (L. Koch). 15. Labium and endite, male, ventral view; note conspicuous groove along median margin of endite. 16. Endite, female, anterior view, showing serrula. 17. Anterior surface of endite, female, showing sieve plate. 18. Sternum, female, ventral view; note steep sternal margins. 19. Cuticle of abdominal venter, female, dorsal view, showing feathery and hairy setae. 20. One of the paired, postepigastric invaginated sclerites, ventral view; note elevated anterior rim. 

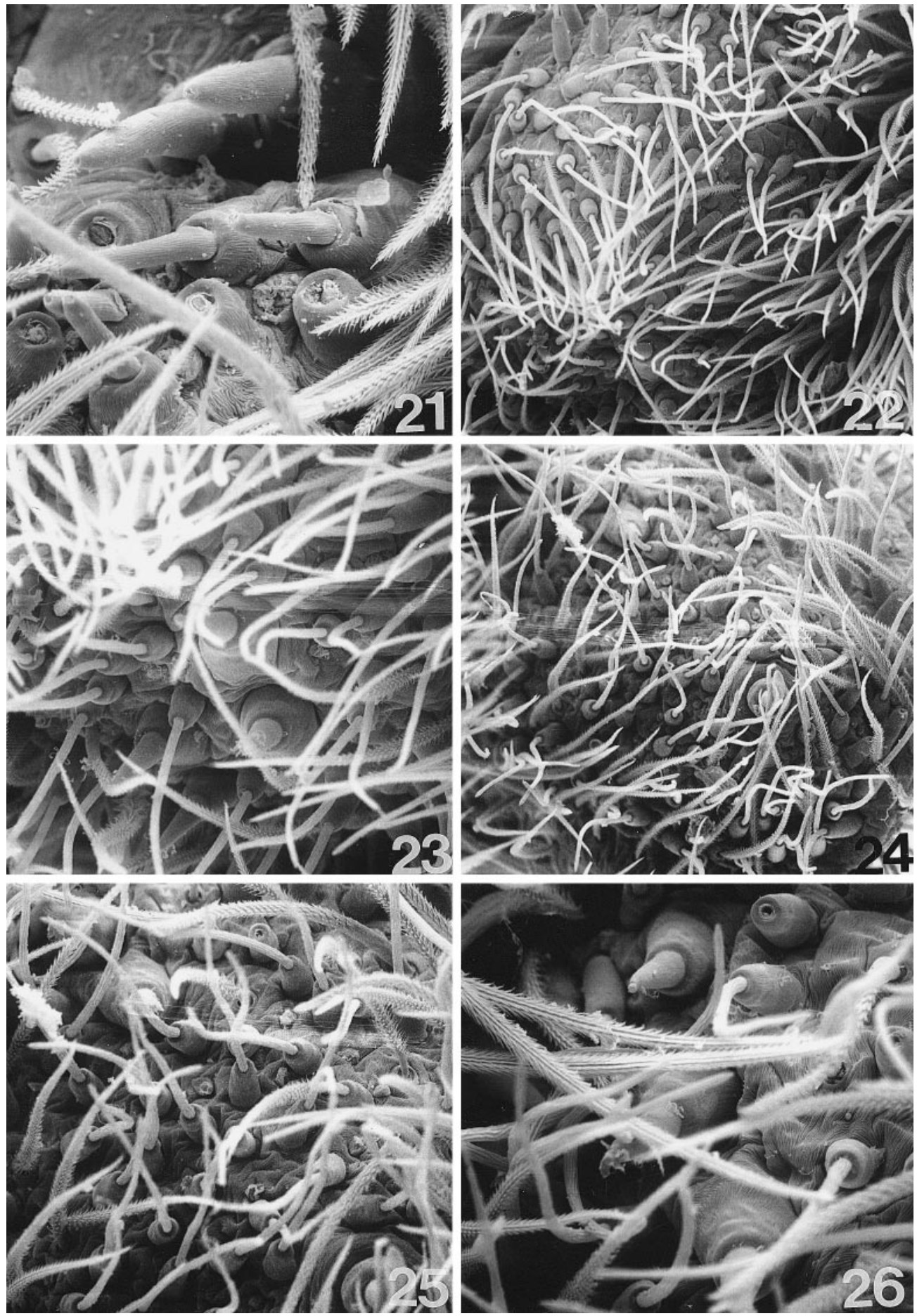

Figs. 21-26. Lampona cylindrata (L. Koch), female, spinneret spigots, posterior views. 21. Anterior lateral spinneret. 22, 23. Posterior median spinneret. 24-26. Posterior lateral spinneret. 

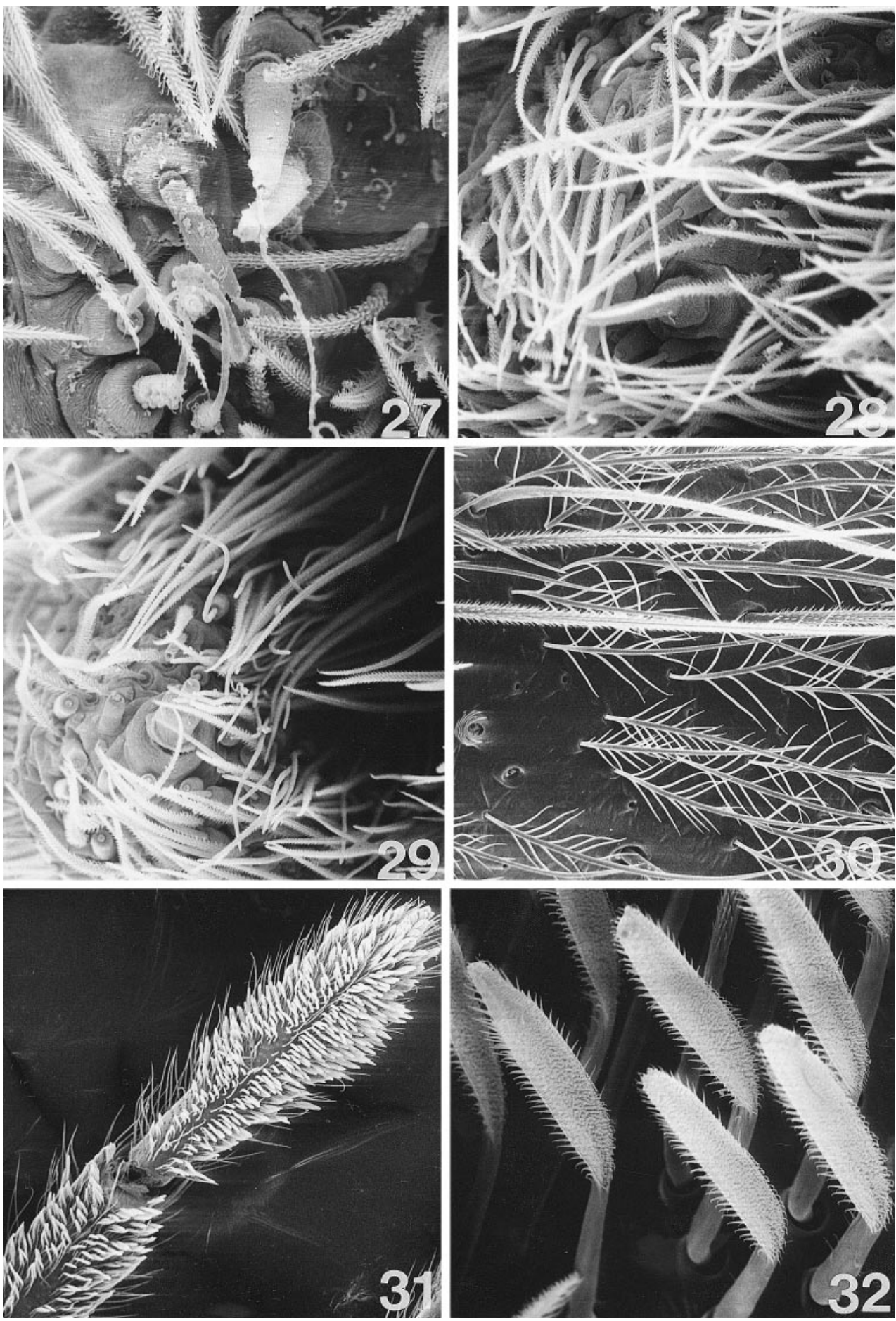

Figs. 27-32. Lampona cylindrata (L. Koch), male. 27-29. spinneret spigots, posterior views. 27. Anterior lateral spinneret. 28. Posterior median spinneret. 29. Posterior lateral spinneret. 30. Metatarsus IV, dorsal view, showing feathery and hairy setae. 31. Metatarsus and tarsus I, ventral view, showing divided scopula. 32. Scopular setae of leg I, ventral view. 

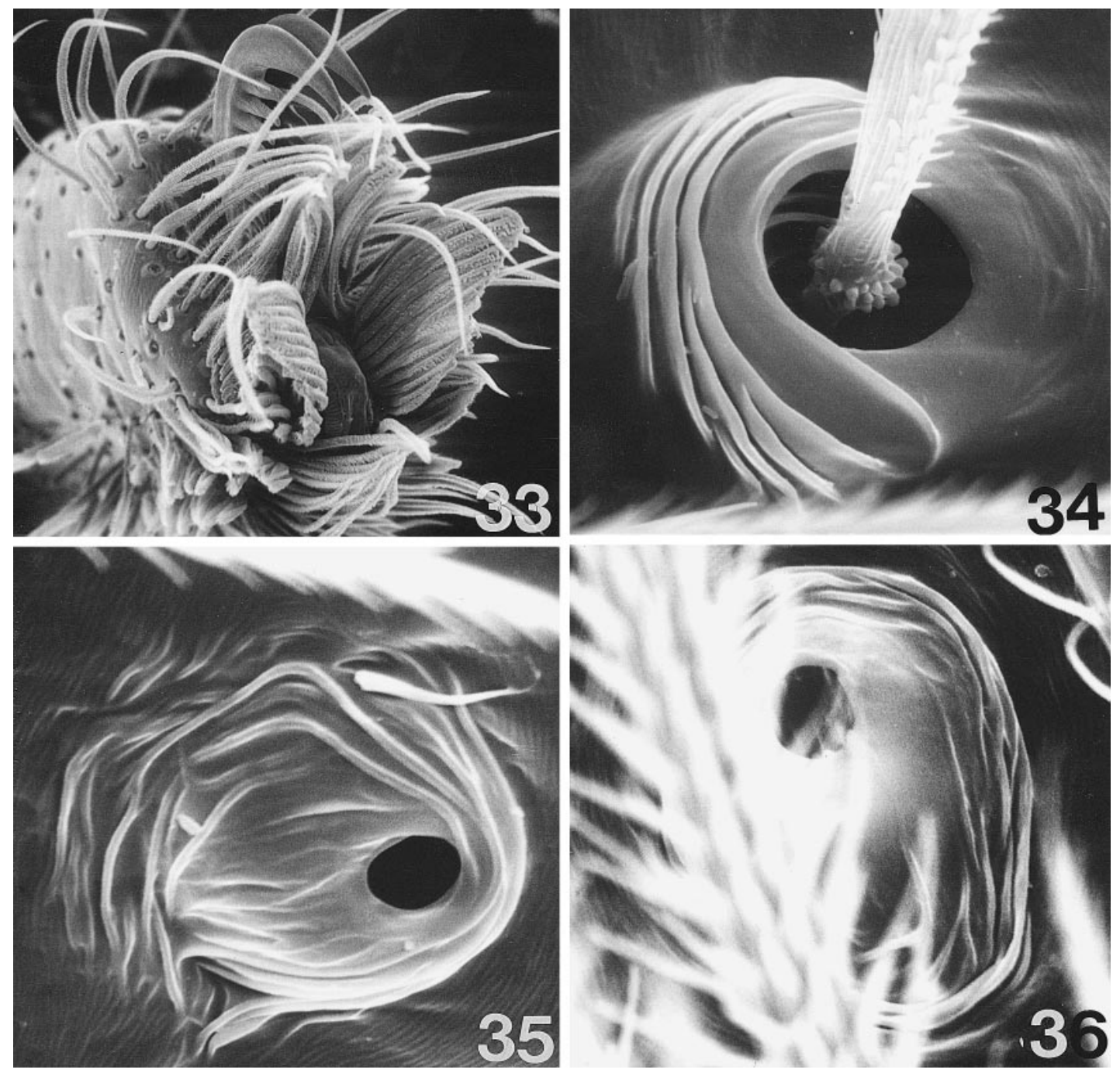

Figs. 33-36. Lampona cylindrata (L. Koch), male. 33. Tarsal claws and claw tufts of leg IV, distal view. 34. Trichobothrial base on tarsus IV, dorsal view. 35. Tarsal organ on tarsus IV, dorsal view. 36. Tarsal organ on palpal tarsus, dorsal view.

ula hairs, but those males have a distinctively long tibial apophysis, fig. 320) ..... 2

- Ventral surface of metatarsi and tarsi I and II without distinct, divided scopula . . . . . 6

2. Surface of sternum rugose (fig. 18) . . . 3

- Surface of sternum with circular punctations separated by smooth cuticle (fig. 325) . . 5

3. Epimeric sclerites fused with sternum, so that coxae originate from circular openings fully surrounded by sclerotized cuticle (fig. 274) . . . . . . . . . . . . . . . Lamponina

- Epimeric sclerites not fused with sternum . . . 4

4. Female with dorsal abdominal scutum; male palp with embolus situated medially (fig. 315) . . . . . . . . . . . Lamponoides

- Female without dorsal abdominal scutum; male palp with embolus situated prolaterally

Lampona

5. Female with anterior epigynal hood (fig. 321); male with long retrolateral tibial apophysis (fig. 320) .............. Lamponata

- Female without anterior epigynal hood (fig. 323); male unknown ....... Lamponova

6. Epimeric sclerites fused with sternum, so that coxae originate from circular openings fully 

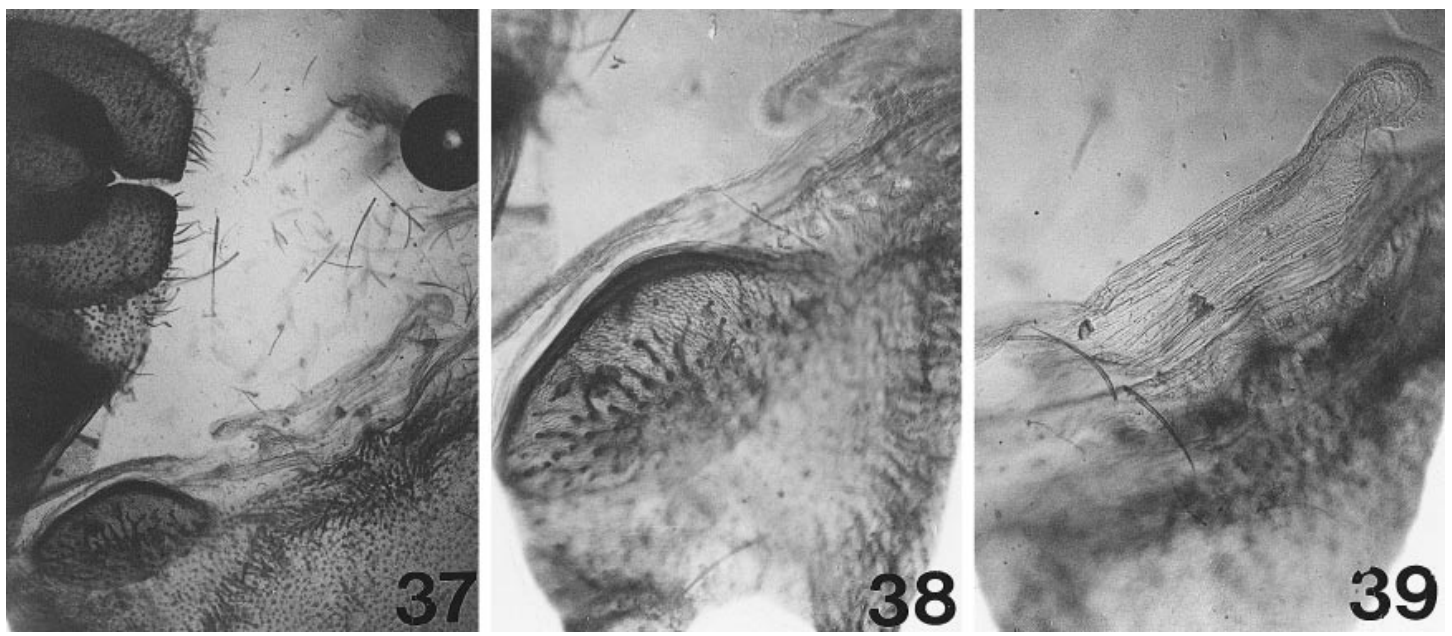

Figs. 37-39. Lampona cylindrata (L. Koch), female. 37, 38. One of the paired, postepigastric invaginated sclerites, and the membranous lobe situated between the pair of postepigastric sclerites. 39. Detail of that membranous lobe.

surrounded by sclerotized cuticle (as in fig. 274) .................... 7

- Epimeric sclerites not fused with sternum ... .................. Lamponella

7. Sternum greatly elevated (figs. 338,341 ); cheliceral promargin without a modified seta .................. Lamponega

- Sternum normal; cheliceral promargin with a typical lamponine modified seta (figs. 9-11)

8. Carapace with white scales; male palp with small retrolateral tibial apophysis (fig. 331); female with distal spine on tibiae III and IV

Lamponusa

- Carapace without white scales; male palp without retrolateral apophysis, with distal excavation instead (fig. 327); female without leg spines .............. Lamponicta

\section{LAMPONA THORELL}

Latona L. Koch, 1866: 3 (type species by monotypy Latona cylindrata L. Koch). Preoccupied in the Mollusca, Crustacea, and Coleoptera.

Lampona Thorell, 1869: 37 (replacement name for Latona L. Koch).

DiagnOSIS: Specimens resemble those of Lamponina and Lamponoides in having a divided scopula on metatarsi and tarsi I and II, and in having the sternal surface rugose (rather than smooth with circular pits). They can be separated from those of Lamponina by having the sternum and epimeric sclerites unfused, and from those of Lamponoides by the absence of a dorsal abdominal scutum in females and by having the male embolus situated prolaterally rather than medially.

DESCRIPTION: Medium to large spiders, total length of males 4.3-12.3, of females 4.617.0. Carapace dark red, with low tubercles, coated with both feathery and hairy setae originating from bases of tubercles (fig. 3), tubercles protruding from lateral margin (fig. 4); thoracic groove long, longitudinal (fig. 5). Eight eyes in two rows, anterior medians largest, circular, dark, other eyes subequal in size, light, laterals oval, posterior medians irregularly oval, flattened (figs. 6, 7); from above, both eye rows slightly procurved, from front, both rows strongly procurved; anterior medians separated by at least their radius, by their radius or less from anterior laterals; posterior medians varying from almost contiguous to separated by more than their diameter, separated by at least their radius from posterior laterals; anterior and posterior laterals separated by at least their diameter; median ocular quadrangle wider in front than in back, usually slightly longer than wide. Chelicerae, sternum, and mouthparts dark red; chilum small, triangular, accompanied by second, elongated posterior chilum (extremely narrow sclerite separating bases of chelicerae posteriorly, fig. 8); chelicerae with distinct lateral boss, promargin 

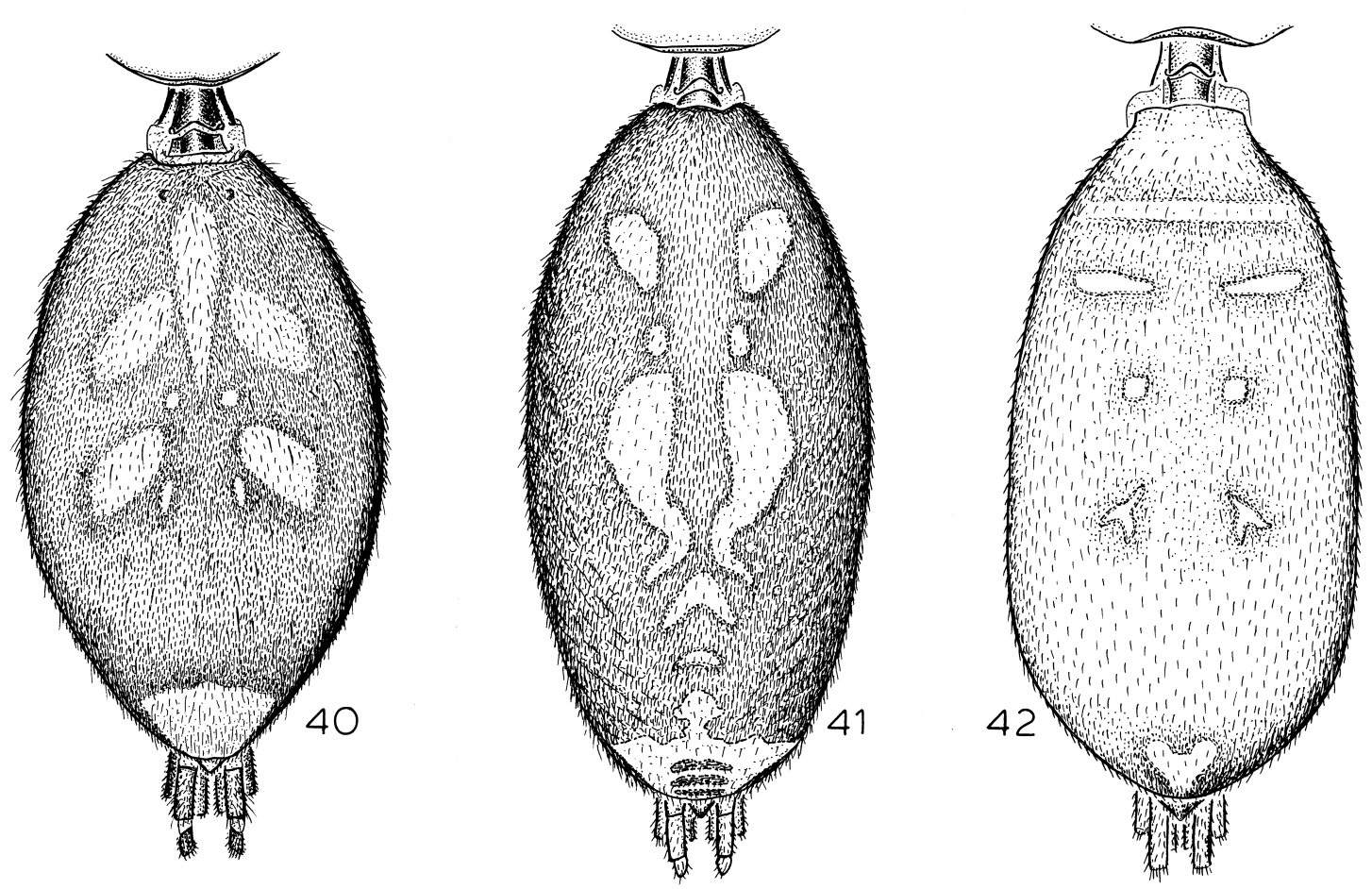

Figs. 40-42. Abdominal color patterns of females, dorsal views. 40. Lampona cylindrata (L. Koch). 41. L. ruida L. Koch. 42. L. braemar, new species.

with series of short setae originating in line along base of fang plus one long, thick seta originating closest to fang (fig. 9), immediately bent at $90^{\circ}$ angle (fig. 10), extending to median line (fig. 11); promargin with three or four teeth, retromargin with none (fig. 12); cheliceral gland openings in almost unsclerotized, slight depression situated posteroproximally of teeth (fig. 13). Labium truncate anteriorly and posteriorly, distinctly depressed medially; posterior surface of labrum with rows of long straining teeth, teeth shorter on median surface than on sides (fig. 14). Endites obliquely depressed, with sharply demarcated, deep groove along margin near labium (figs. 15, 43); serrula long, with single row of basally fused teeth (fig. 16); anterior surface with distinct sieve plate of maxillary gland openings (fig. 17). Sternum elevated, with steep lateral margins (fig. 18), expanded anteriorly to sides beyond heel at posterolateral corner of endites, with extensions to and between coxae; surface rugose. Two epimeric sclerites on each side, one opposite palpal endites, one surrounding all coxae, not reaching sternal triangles, not fused to carapace. Pedicel composed of two small, flat dorsal sclerites and rounded sclerite covering venter and sides, rounded sclerite with median longitudinal keel prolonged anteriorly into distinct protrusion extending toward sternum.

Anterior edge of abdomen of male with almost complete sclerotic ring formed by epigastric scutum plus dorsal abdominal scutum reaching to at least one-third of abdominal length, females without dorsal scutum, usually with pattern of white spots (figs. 40$42,44)$; cuticle with feathery and hairy setae (fig. 19); epigastric scutum accompanied posterolaterally by pair of oval, deeply invaginated sclerites bearing clearly elevated anterior rim (fig. 20); sclerites separated by membranous lobe (in at least some females, lobe bears distinct tubular extension on each side, figs. 37-39); anterior edge of oval sclerites often fitting under epigastric scutum; colulus represented by setae; tiny semicircular to u-shaped sclerite sometimes present, well 

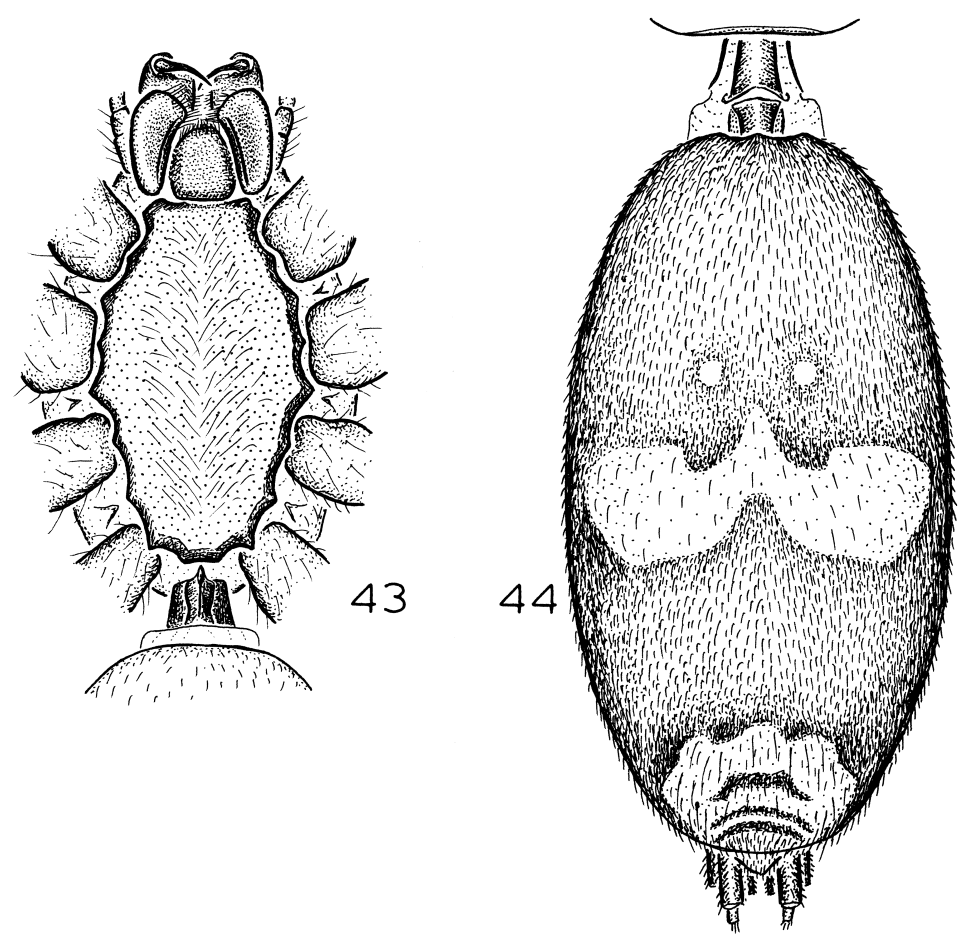

Figs. 43, 44. 43. Lampona cylindrata (L. Koch), female cephalothorax, ventral view, showing medial groove on endites, anteriorly expanded sternum (not fused to epimeric sclerites), and tubular pedicel sclerite. 44. L. cumberland, new species, abdominal color pattern of female, dorsal view.

removed from spinnerets, marking position of small posterior spiracle leading to four extremely narrow tracheal tubes. Anterior lateral spinnerets tubular, separated by more than their diameter, with two thick major ampullate gland spigots plus 8-10 small, unmodified piriform gland spigots (figs. 21, 27 , cuticle representing distal, second spinneret segment restricted to semicircle surrounding major ampullate gland spigots (piriform gland spigots surrounded only by soft cuticle); posterior median spinnerets large, tubular, without anteriorly expanded tips, with numerous long aciniform gland spigots with short bases covering most of spinneret, posterior tip with two minor ampullate gland spigots with triangular shafts (fig. 28) plus, in females, two cylindrical gland spigots with wide bases (figs. 22, 23); posterior lateral spinnerets two-segmented, with spigots as on posterior medians (figs. 24-26, 29).

Legs almost spineless, usually with single proventral spine at distal tip of tibiae III, IV; most surfaces with both feathery and hairy setae (fig. 30); in males, all coxae and posterior trochanters dorsally tuberculate; anterior coxae with protuberant posterolateral corners; trochanters unnotched; anterior metatarsi and tarsi with divided scopulae (fig. 31 ), composed of flattened, distally bent setae (fig. 32), scopulae sometimes entire on distal tip of tarsi; posterior metatarsi with thick, dark, distal preening brushes; posterior tarsi with entire scopulae; tarsi with two dentate claws, claw tufts composed of lateral pads of closely appressed setae (fig. 33); trichobothria present on tibiae, metatarsi, and tarsi, in 2-3 irregular rows, bases ridged (fig. $34)$; tarsal organ long, longitudinally ridged on proximal two-thirds, opening situated distally (fig. 35), similar in structure on palpal tarsus (fig. 36). Female palpal tibia and tarsus with several long, thin spines; female palpal tarsus with dentate claw.

Male palp with at least one retrolateral tibial apophysis (figs. 45, 48, 49); cymbial sur- 

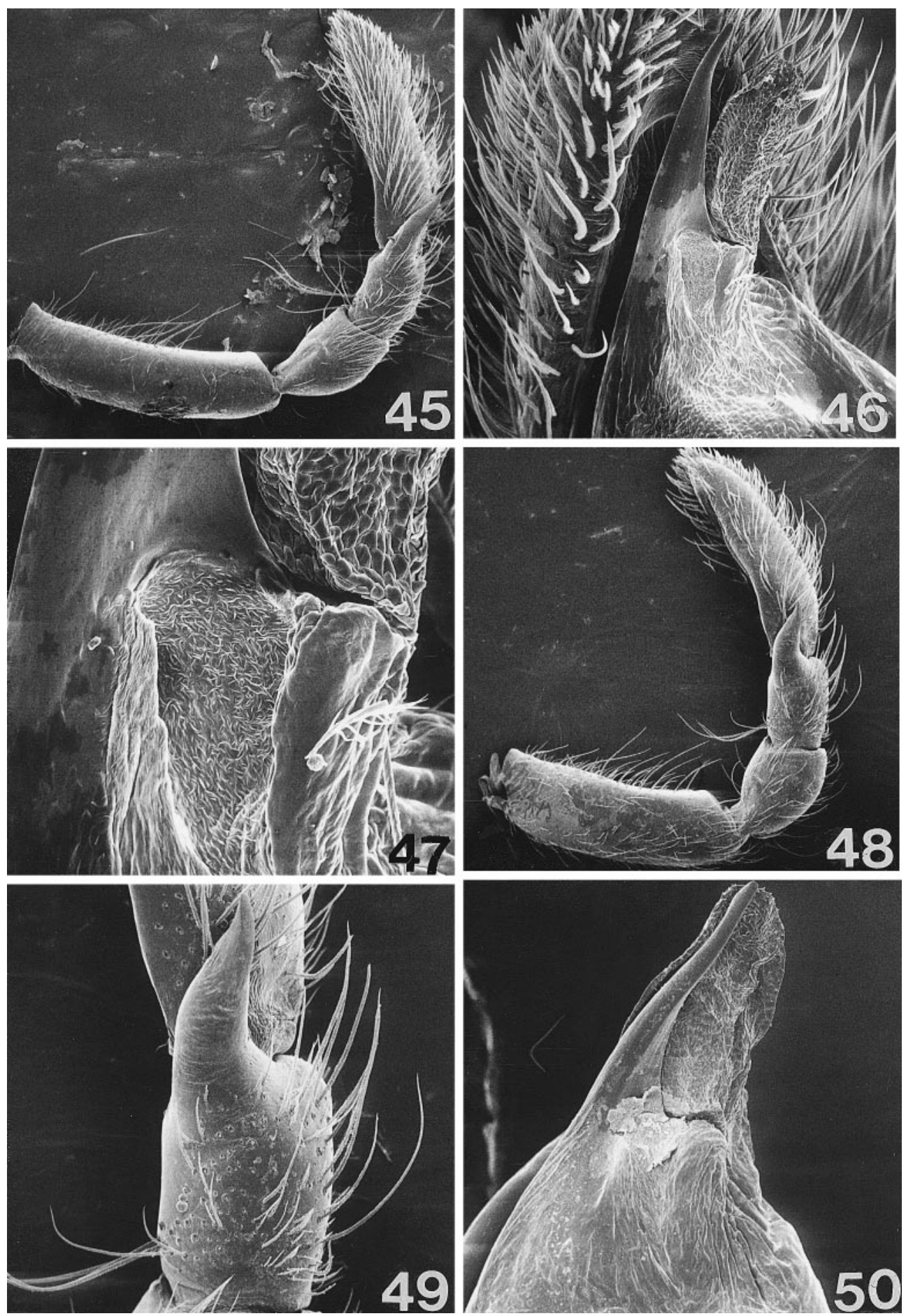

Figs. 45-50. Left male palp. 45-47. Lampona cylindrata (L. Koch). 48-50. L. murina L. Koch. 45, 48, 49. Lateral view, showing retrolateral tibial apophysis. 46, 47, 50. Ventral view, showing palpal embolus and conductor. 
face usually excavated opposite tibial apophysis; tegulum expanded (often greatly), extending beyond posterior rim of tarsus; embolus prolaterally situated, elongated, typically fused basally to prolateral portion of membranous conductor (retrolateral portion of conductor extending to tip of embolus, figs. 46, 47, 50); median apophysis absent, represented at most by slight sclerotization within conductor. Epigynum variable in shape, usually with plug of solidified secretions; pre-epigynum present at least two instars before maturity.

Misplaced SPECIES: Lampona scutata Strand and Lampona asperrima Hickman are transferred below to Lamponina.

Nomina DUBIA: Three names are here considered nomina dubia. The types of Lampona quadrimaculata L. Koch (1872: 365, pl. 28, fig. 4) from Bowen, Queensland (holotype, $\mathrm{ZMH}$, examined), of Lampona fasciata L. Koch (1872: 366, pl. 28, fig. 5) from Port Mackay, Queensland (two syntypes, ZMH, examined), and of Lampona sordida L. Koch (1873: 372, pl. 29, fig. 3) from "Neuholland" (holotype, NRS, examined) are unidentifiable juveniles.

IDENTIFICATION: Because the genus is so large, and the genitalic differences among species are often small, geographically limited keys seem more useful (and more likely to lead efficiently to accurate results) than would a single key to all the species. Separate keys are presented to the species known from (1) Western Australia, (2) the Northern Territory, (3) South Australia, (4) northern Queensland (north of $20^{\circ} \mathrm{S}$ ), (5) southern Queensland (south of $20^{\circ} \mathrm{S}$ ), (6) southeastern Australia (New South Wales, Victoria, and the Australian Capital Territory), and (7) Tasmania.

For the same reasons, interrelationships among the many species of this genus are often obscure, and for a few species, identifying any close relatives is quite difficult. With far too few characters available to attempt a cladistic analysis, species have been placed here in eight informal species groups, which should at least serve as a partial guide to future investigations of the cladistic structure of the genus.

\section{KEY TO SPECIES OF LAMPONA \\ KNOWN FROM WeSTERn AUSTRALIA}

1. Males (those of L. torbay unknown) . . . 2

- Females (those of L. barrow unknown) . 13

2. Palpal femur with distinct subbasal excavation or bulge ............. 3

- Palpal femur unmodified (as in fig. 52) . . 9

3. Palpal femur with distinct subbasal excavation (fig. 95) .......... macilenta

- Palpal femur with distinct subbasal projection ................ 4

4. Two retrolateral tibial apophyses (fig. 91) .. ............... whaleback

- Only a single retrolateral tibial apophysis .. $\ldots \ldots \ldots \ldots \ldots \ldots \ldots \ldots \ldots \ldots \ldots \ldots$

5. Retrolateral tibial apophysis with dorsal expansion (fig. 99) ............ barrow

- Retrolateral tibial apophysis without dorsal expansion ..............6

6. Subbasal projection on palpal femur relatively long, retrolateral tibial apophysis with ventral ledge (fig. 187) . . quinqueplagiata

- Subbasal projection on palpal femur relatively short, retrolateral tibial apophysis without ventral ledge (figs. 123, 159, 192) . . 7

7. Subbasal projection on palpal femur rounded, retrolateral tibial apophysis relatively broad (fig. 123) ............ punctigera

- Subbasal projection on palpal femur distally abrupt, retrolateral tibial apophysis relatively narrow (figs. 159, 192) . . . . . 8

8. Retrolateral tibial apophysis relatively short (fig. 192) ............ dwellingup

- Retrolateral tibial apophysis relatively long (fig. 159) ............. yanchep

9. Retrolateral tibial apophysis relatively long (figs. 51, 71, 111) ......... 10

- Retrolateral tibial apophysis relatively short (figs. 87, 195) . . . . . . . . . 12

10. Retrolateral tibial apophysis expanded at tip (fig. 71) ...............foliffera

- Retrolateral tibial apophysis narrowed at tip

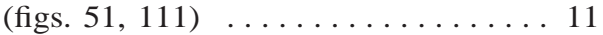

11. Palpal bulb expanded ventrally, extending half length of palpal tibia (fig. 51) ..... .................... cylindrata

- Palpal bulb not expanded, extending only to anterior edge of tibia (fig. 111) ... walsh

12. Retrolateral tibial apophysis directed dorsally (fig. 87) . . . . . . . . . . brevipes

- Retrolateral tibial apophysis directed ventrally (fig. 195) . . . . . . . . a ampeinna

13. Epigynum with long, wide, deep median excavation, spermathecae restricted to posterior one-third of epigynum (figs. 53-56) 
- Epigynum without large median excavation, spermathecae larger ........... 14

14. Epigynum with bulbous, nose-shaped median projection (fig. 88) ......... brevipes

- Epigynum without bulbous median projection $\ldots \ldots \ldots \ldots \ldots \ldots \ldots$

15. Epigynum with arrow-shaped midpiece (figs. $188,192,196)$; spermathecal bulbs rounded, on long, narrow stalks (figs. 189, 193,

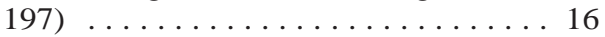

- Epigynum without arrow-shaped midpiece, spermathecae otherwise ......... 18

16. Epigynal midpiece relatively wide (fig. 196) ................. ampeinna

- Epigynal midpiece relatively narrow (figs. $188,192) \ldots \ldots \ldots \ldots \ldots \ldots \ldots 17$

17. Anterior epigynal margin joined to epigynal midpiece (fig. 188) ... quinqueplagiata

- Anterior epigynal margin not joined to epigynal midpiece (fig. 192) . . . dwellingup

18. Epigynum with anterior excavation (figs. 72, 160) .................. 19

- Epigynum without anterior excavation . 20

19. Anterior epigynal excavation relatively narrow (fig. 72), spermathecae bipartite (fig. 73) .............. foliifera

- Anterior epigynal excavation relatively wide (fig. 160), spermathecae tripartite (fig. 161) .................. yanchep

20. Epigynum with triangular, posterior excavation (fig. 112), spermathecae extending far anterior of excavation (fig. 113) .. walsh

- Epigynum without triangular, posterior excavation ................ 21

21. Epigynum with relatively wide midpiece (figs. 92, 100) $\ldots \ldots \ldots \ldots \ldots \ldots 22$

- Epigynum with relatively narrow midpiece (figs. 96, 124) .............. 23

22. Posterior portion of spermathecae relatively long, narrow (fig. 93) ..... whaleback

- Posterior portion of spermathecae relatively short, wide (fig. 101) . . . . . . . torbay

23. Spermathecae globular (fig. 125) ...... $\ldots \ldots \ldots \ldots \ldots$ punctigera

- Spermathecae elongate (fig. 97) macilenta

\section{Key to SPeCIES OF LAMPONA KNOWN FROM THE NORTHERN TERRITORY}

1. Males (those of L. olga unknown) .... 2

- Females .................. 7

2. Retrolateral tibial apophysis expanded at tip (figs. 71, 75, 107) .......... 3

- Retrolateral tibial apophysis narrowed at tip (figs. 115, 171, 195) .......... 5

3. Palpal femur with subbasal projection (figs. $75,107) \ldots \ldots \ldots \ldots \ldots \ldots \ldots$
- Palpal femur without subbasal projection (fig. 71) ................ foliifera

4. Palpal bulb short, triangular (fig. 106) .... .................. flavipes

- Palpal bulb long, oval (figs. 74, 75) . f finke

5. Retrolateral tibial apophysis relatively long (fig. 115) .............. kapalga

- Retrolateral tibial apophysis relatively short (figs. 171, 195) …........6 6

6. Palpal femur with subbasal expansion (fig. 171) .............. danggali

- Palpal femur unmodified (fig. 195) ...... ................. ampeinna

7. Posterior portions of spermathecae much narrower than anterior portions (figs. 109, 117, $157,197) \ldots \ldots \ldots \ldots \ldots \ldots$

- Posterior portions of spermathecae about as wide as anterior portions (figs. 73, 77, 173)

8. Anterior epigynal margin situated in posterior half of epigynum (figs. 116, 196) ....9

- Anterior epigynal margin situated in anterior half of epigynum (figs. 108, 156) . . . 10

9. Epigynum with transverse anterior margin (fig. 116) ............. kapalga

- Epigynum without transverse anterior margin (fig. 196) ............ ampeinna

10. Epigynal midpiece relatively wide (fig. 108) $\ldots \ldots \ldots \ldots \ldots \ldots$ flavipes

- Epigynal midpiece relatively narrow (fig. 156) .................. olga

11. Spermathecae extending far anterior of anterior epigynal margin (fig. 172) .... .................. danggali

- Spermathecae not extending far anterior of anterior epigynal margin (figs. 72, 76) ...

12. Epigynal midpiece relatively small situated behind anterior excavation (fig. 72) ... ................. foliifera

- Epigynal midpiece relatively large, extending full length of epigynum (fig. 76) . . finke

\section{Key to SPECIES OF LAMPONA KnOwn From South Australia}

1. Males ................... 2

- Females ................... 19

2. Palpal femur with subbasal excavation (fig. 95) ............. macilenta

- Palpal femur without subbasal excavation ..

3. Palpa. fe..................... figs. $67,123,127,203) \ldots \ldots \ldots \ldots$

- Palpal femur unmodified (as in fig. 52) 14

4. Palpal femur with distinct projection near its base (figs. 75, 107) . . . . . . . . 5

- Palpal femur with projection or expansion 
farther from base (as in figs. 127, 207) . . $\ldots \ldots \ldots \ldots \ldots 6$

5. Palpal bulb triangular (fig. 106) ... flavipes

- Palpal bulb oval (fig. 74) ........ finke

6. Retrolateral tibial apophysis with ventral ledge or tubercle (figs. 183, 199, 203, 207) $\ldots \ldots \ldots \ldots \ldots \ldots \ldots \ldots$

- Retrolateral tibial apophysis without ventral prominence (figs. 67, 123, 127, 131, 171)

alpal femur with long, low swelling at base

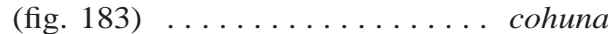

- Palpal femur with larger expansion (figs. 199, 203, 207) ............. 8

8. Retrolateral tibial apophysis relatively short (fig. 203) ................. gilles

- Retrolateral tibial apophysis relatively long (figs. 199, 207) ..............99

9. Palpal femur with subbasal bulge (fig. 199) .................. ooldea

- Palpal femur with subbasal lobe (fig. 207) . . hirsti

10. Retrolateral tibial apophysis apically hooked (fig. 171) ............. danggali

- Retrolateral tibial apophysis not apically hooked ................11

11. Retrolateral tibial apophysis relatively long, narrow (figs. 67, 131) . . . . . . . . 12

- Retrolateral tibial apophysis relatively short, wide (figs. 123, 127) . . . . . . . 13

12. Palpal femur with subbasal point (fig. 67) . . $\ldots \ldots \ldots \ldots \ldots$ ruida

- Palpal femur with subbasal swelling only (fig. 131) ............. moorilyanna

13. Palpal femur with subbasal swelling steep distally, retrolateral tibial apophysis relatively large (fig. 123) ...... punctigera

- Palpal femur with subbasal swelling shallow distally, retrolateral tibial apophysis relatively small (fig. 127) ....... braemar

14. Retrolateral tibial apophysis relatively long (figs. 52, 71) . . . . . . . . . 15

- Retrolateral tibial apophysis relatively short (figs. 79, 119, 195, 211) . . . . . . 16

15. Retrolateral tibial apophysis distally expanded (fig. 71) ............... foliifera

- Retrolateral tibial apophysis narrowed distally (fig. 52) . . . . . . . . . cylindrata

16. Two retrolateral tibial apophyses present (fig. 79) ................ talbingo

- Only one retrolateral tibial apophysis present

17. Retrolateral tibial apophysis very narrow di rected dorsally (fig. 119) ........ ewens

- Retrolateral tibial apophysis wider, directed ventrally (figs. 195, 211) . . . . . . 18

18. Retrolateral tibial apophysis with ventral ledge (fig. 211) . . . . . . . . . . eba
- Retrolateral tibial apophysis without ventral ledge, short (fig. 195) . . . . . ampeinna

19. Epigynum with long, wide, deep median excavation, spermathecae restricted to posterior one-third of epigynum (figs. 53-56) ................. cylindrata

- Epigynum without large median excavation, spermathecae larger . . . . . . . . . 14

20. Epigynum with two paramedian flanges (fig. 184), spermathecae triangular (fig. 185) . . .........................

- Epigynum otherwise, spermathecae not triangular ................ 21

21. Spermathecae on long stalks that are much narrower than spermathecal bulbs (figs. 97, $109,121,125,197,201,205) \ldots 22$

- More posterior portions of spermathecae not much narrower than spermathecal bulbs (figs. 69, 73, 77, 81, 129, 133, 173, 209, 213) .............. 28

22. Epigynal midpiece abruptly narrowed (fig.

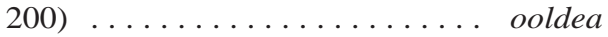

- Epigynum otherwise ........... 23

23. Epigynal midpiece or atrium broadly triangular (figs. 108, 120, 196) . . . . . . . 24

- Epigynal midpiece much narrower (figs. 96,

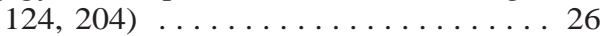

24. Epigynum with triangular midpiece, bordered at sides by lateral margins (fig. 196) .... ................. ampeinna

- Epigynum without separate lateral margins (figs. 108, 120) . . . . . . . . . 25

25. Spermathecal stalks relatively long (fig. 108) ................... flavipes

- Spermathecal stalks relatively short (fig. 120)

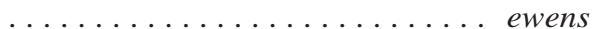

26. Spermathecae extending far anterior of epigynal midpiece (figs. 97, 204) . . . . 27

- Spermathecae not extending far anterior of epigynal midpiece (fig. 124) ... punctigera

27. Epigynum with arrow-shaped midpiece (fig. 204), spermathecae globose anteriorly (fig. 205) ................. gilles

- Epigynum with diamond-shaped midpiece (fig. 96), spermathecae not globose anteriorly (fig. 97) .......... macilenta

28. Epigynum with deep anterior excavation (figs. 68, 72) . . . . . . . . . . . 29

- Epigynum without deep anterior excavation ................ 30

29. Epigynal excavation relatively wide (fig. 68), spermathecae kidney-shaped (fig. 69) .... ................. ruida

- Epigynal excavation relatively narrow (fig. 72), spermathecae almost straight (fig. 73) ................foliffera

30. Anterior portion of spermathecae much 
smaller than median portion (figs. 81, 173, 209, 213) .............. 31

- Anterior portion of spermathecae as large as median portion (figs. 77, 129, 133) . . 34

31. Epigynum with triangular midpiece and distinct lateral margins (fig. 172) .. danggali

- Epigynum otherwise . . . . . . . . . 32

32. Epigynum with tongue-shaped midpiece (fig. 208), spermathecae relatively short (fig. 209) .................. hirsti

- Epigynum with diamond-shaped midpiece (figs. 78, 212), spermathecae relatively long (figs. 79, 213) .......... 33

33. Anterior portion of spermathecae less than one-third as long as median portion (fig. 79) ............... talbingo

- Anterior portion of spermathecae more than one-third as long as median portion (fig. 213) .............eba

34. Epigynal midpiece and spermathecae massive (figs. 76, 77) ............ finke

- Epigynal midpiece and spermathecae smaller (figs. 128, 132) . . . . . . . . . 32

35. Epigynal midpiece relatively short (fig. 132) ............... moorilyanna

- Epigynal midpiece relatively long (fig. 128) ............... braemar

\section{KEY TO SPECIES OF LAMPONA KNOWN FROM NORTHERN QUEENSLAND}

1. Males ................... 2

- Females ................... 15

2. Retrolateral tibial apophysis almost as long as tibia (figs. 60, 107, 115) . . . . . . . 3

- Retrolateral tibial apophysis much shorter than tibia ............... 5

3. Palpal femur with subbasal knob (fig. 107), palpal bulb triangular (fig. 106) flavipes

- Palpal femur unmodified (figs. 60, 115) . . 4

4. Palpal bulb expanded ventrally, reaching to most of tibial length (figs. 59, 60) ......

$\ldots \ldots \ldots \ldots \ldots$ murina alpal bulb almost circular, reaching only to half of tibial length (figs. 114, 115) .....

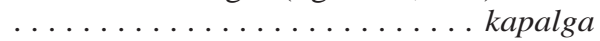

5. Palpal femur expanded at base (figs. 239,

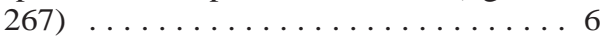

- Palpal femur unmodified ........... 7

6. Retrolateral tibial apophysis a narrow spike (fig. 267) ............... molloy

- Retrolateral tibial apophysis wide at base, hooked distally (fig. 239) . . . . kirrama

7. Retrolateral tibial apophysis relatively massive (figs. 135, 175) . . . . . . . . 8

- Retrolateral tibial apophysis relatively weak (figs. 139, 147, 243, 247, 255, 259, 271)
8. Retrolateral tibial apophysis distally truncated (fig. 135) ................ spec

- Retrolateral tibial apophysis distally sinuous (fig. 175) .............. garnet

9. Dorsal margin of retrolateral tibial apophysis distinctly narrowed below tip, sinuous (figs. 147, 243, 255) . . . . . . . 10

- Dorsal margin of retrolateral tibial apophysis not distinctly narrowed below tip (figs. 139, $247,259,271) \ldots \ldots \ldots 12$

10. Palpal bulb relatively short (fig. 242), tip of retrolateral tibial apophysis relatively short (fig. 243) ............. monteithi

- Palpal bulb relatively long (figs. 146, 254), tip of retrolateral tibial apophysis relatively long (figs. 147, 255) . . . . . . . . 11

11. Tip of retrolateral tibial apophysis bent at almost a right angle (fig. 147) .... terrors

- Tip of retrolateral tibial apophysis bent at much smaller angle (fig. 255) ... carlisle

12. Palpal bulb relatively short, not extending past distal margin of tibia (fig. 259) . . . . . $\ldots \ldots \ldots \ldots$ davies

- Palpal bulb longer, extending past distal margin of tibia (figs. 139, 247, 271) . . . 13

13. Palpal bulb narrowed proximally (fig. 138) ............... russell

- Palpal bulb not narrowed proximally (figs. 246, 270) .............. 14

14. Palpal bulb relatively wide (fig. 270), tip of retrolateral tibial apophysis relatively long (fig. 271) ................. tully

- Palpal bulb relatively narrow (fig. 246), tip of retrolateral tibial apophysis relatively short (fig. 247) ............. finnigan

15. Epigynum with long, deep, narrow excavation (fig. 61), spermathecae short, with distinct shoulders behind their distal tips (figs. $57,58,62) \ldots \ldots \ldots \ldots$ murina

- Epigynum without long excavation, spermathecae much larger ............ 16

16. Spermathecae on long, narrow stalks (figs.

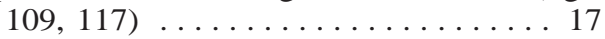

- Spermathecae not on long, narrow stalks ... ................. 18

17. Epigynal atrium relatively large (fig. 108), anterior portion of spermathecae relatively small (fig. 109) ............. flavipes

- Epigynal atrium relatively small (fig. 116), anterior portion of spermathecae relatively large (fig. 117) . . . . . . . . . kapalga

18. Spermathecae bipartite (figs. 141, 149) . . . 19

- Spermathecae tripartite, at least as seen in ventral view (as in figs. 136, 244, 268) ..

19. Anterior portion of spermathecae very small (fig. 141) ............... russell 
- Anterior portion of spermathecae much larger (fig. 149) $\ldots \ldots \ldots \ldots \ldots$ terrors

20. Median portion of spermathecae squeezed toward epigynal plate, scarcely visible in dorsal view (figs. 136, 137) ....... spec

- Median portion of spermathecae easily visible in dorsal view .............. 21

21. Spermathecae widely separated anteriorly (figs. 176, 177) ........... garnet

- Spermathecae approximate anteriorly ...22

22. Posterior portion of spermathecae much larger than median and anterior portions (figs. 257, 273) ............. 23

- Posterior portion of spermathecae not much larger than median and anterior portions (figs. 241, 245, 249, 261, 269) . . . . 24

23. Spermathecae greatly widened (fig. 273) ... .................. tully

- Spermathecae not greatly widened (fig. 257)

24. Epigynum with long lateral margins forming anterior arch (fig. 244), spermathecae almost bullet-shaped (fig. 245) . . monteithi

- Epigynum with at most a weak anterior margin (figs. 240, 248, 260, 268), spermathecae not bullet-shaped (figs. 241, 249, 261, 269) ................. 25

25. Median portion of spermathecae larger than anterior or posterior portions (figs. 261, 269) ................. 26

- Median portion of spermathecae not larger than anterior or posterior portions (figs. $241,249) \ldots \ldots \ldots \ldots \ldots \ldots \ldots 27$

26. Median portion of spermathecae rounded (fig. 261) ................ davies

- Median portion of spermathecae elongated (fig. 269) .............. molloy

27. Anterior portion of spermathecae rounded, larger than median portion (fig. 249) .... ................. finnigan

- Anterior portion of spermathecae truncated posteriorly, smaller than median portion (fig. 241) ............. kirrama

\section{Key to SPECIES OF LAMPONA KNOWN FROM SOUTHERN QUEENSLAND}

1. Males ....................... 2

- Females ..................24 24

2. Palpal femur with distinct subbasal expansion (figs. 67, 107, 123, 131, 163, 171, 267) . . $\ldots \ldots \ldots \ldots \ldots \ldots \ldots \ldots$

- Palpal femur without subbasal expansion ... ...................... 9

3. Retrolateral tibial apophysis a wide triangle (fig. 123), palpal femur with long subbasal ledge (fig. 123) ........ punctigera
- Retrolateral tibial apophysis relatively narrow (figs. 67, 107, 131, 163, 171, 267) ... 4

4. Palpal femur with distinct subbasal protuberance (figs. 67, 107, 163) . . . . . . 5

- Palpal femur with subbasal expansion not in form of distinct protuberance (figs. 131, 171, 267) $\ldots \ldots \ldots \ldots \ldots \ldots \ldots 7$

5. Retrolateral tibial apophysis expanded at tip (fig. 107) ................ flavipes

- Retrolateral tibial apophysis narrowed at tip (figs. 67, 163)

6. Retrolateral tibial apophys tip of embolus relatively small (fig. 66) (fig. 66) ...

- Retrolateral tibial apophysis angled (fig. 163), tip of embolus relatively large (fig. 162) . .

....................... taroom

7. Retrolateral tibial apophysis very narrow (fig. 267) .................. molloy

- Retrolateral tibial apophysis wider (figs. 131,

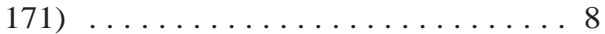

8. Retrolateral tibial apophysis with distal hook (fig. 171) ............ danggali

- Retrolateral tibial apophysis without distal hook (fig. 131) . ........ moorilyanna

9. Retrolateral tibial apophysis relatively massive (figs. 52, 60, 103) . . . . . . 10

- Retrolateral tibial apophysis relatively slight (figs. 79, 127, 143, 147, 151, 195, 223, 227, $231,251,255,263,271) \ldots \ldots \ldots 12$

10. Retrolateral tibial apophysis directed ventrally (fig. 103) ............... chalmers

- Retrolateral tibial apophysis directed dorsally (figs. 52, 60) .............. 11

11. Palpal femur relatively long (fig. 45), retrolateral tibial apophysis relatively long, sinuous (fig. 52) . . . . . . . cylindrata

- Palpal femur relatively short (fig. 48), retrolateral tibial apophysis relatively short, straight (fig. 60) ........... murina

12. Two retrolateral tibial apophyses (fig. 79) ..

.................... talbingo

- Only a single retrolateral tibial apophysis present ................ 13

13. Retrolateral tibial apophysis directed ventrally (fig. 195) . . . . . . . . ampeinna

- Retrolateral tibial apophysis directed dorsally .................... 14

14. Retrolateral tibial apophysis with long, greatly narrowed tip (figs. 143, 147, 223, 227, $255,271) \ldots \ldots \ldots \ldots \ldots \ldots \ldots 15$

- Retrolateral tibial apophysis with shorter, wider tip (figs. 127, 151, 231, 251, 263) $\ldots$. ....................20

15. Retrolateral tibial apophysis abruptly expanded below tip (figs. 147, 255) . . . . . 16

- Retrolateral tibial apophysis not abruptly ex- 
panded below tip (figs. 143, 223, 227, 271)

.................. 17

16. Palpal bulb with anteromedial expansion (fig. 146), tip of embolus hidden behind tegulum (fig. 146) ............. terrors

- Palpal bulb without anteromedial expansion (fig. 254), tip of embolus protruding past tegulum (fig. 254) ........... carlisle

17. Subdistal portion of retrolateral tibial apophysis directed distally (figs. 143, 271) . . . 18

- Subdistal portion of retrolateral tibial apophysis directed dorsally (figs. 223, 227) . . . . ..................... 19

18. Palpal bulb relatively narrow (fig. 142) ... .................... airlie

_ Palpal bulb relatively wide (fig. 270) . . tully

19. Posterior portion of palpal bulb relatively short (fig. 222) . . . . . . . . . . bunya

- Posterior portion of palpal bulb relatively long (fig. 226) ............. cudgen

20. Retrolateral tibial apophysis of subequal width throughout most of its length (fig.

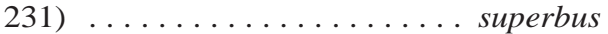

- Retrolateral tibial apophysis narrowed toward tip (figs. 127, 151, 251, 263) . . . . 21

21. Retrolateral tibial apophysis gradually narrowed toward tip (figs. 127, 151) . . . 22

- Retrolateral tibial apophysis abruptly narrowed near tip (figs. 251, 263) ..... 23

22. Retrolateral tibial apophysis situated near dorsal margin of tibia (fig. 151) . . pusilla

- Retrolateral tibial apophysis situated near middle of lateral surface of tibia (fig. 127)

braemar

23. Posterior portion of palpal bulb narrow, angled (fig. 250) . . . . . . . . lamington

- Posterior portion of palpal bulb rounded (fig.

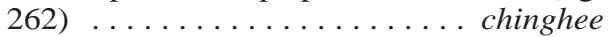

24. Epigynum with long, deep depression (sometimes filled with post-copulatory plug) occupying most of epigynal length (figs. 53,

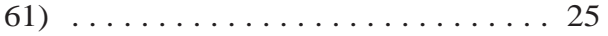

- Epigynum without long depression ... 26

25. Epigynal depression relatively wide (fig. 53), anterior lobes of spermathecae without dorsal demarcation (figs. 54-56)

$\ldots \ldots \ldots \ldots$ cylindrata

- Epigynal depression relatively narrow (fig. $61)$, anterior lobes of spermathecae dorsally demarcated by distinct ridge (figs. 57, 58, 62) .............. murina

26. Spermathecae on long, narrow stalks at least as long as anterior portion of spermathecae (figs. 105, 109, 197) . . . . . . . . 27

- Spermathecae on short stalks (as in fig. 265) .................... 29

27. Epigynum with wide, v-shaped atrium (fig. 108) ................. flavipes
- Epigynum without distinct atrium, with distinct midpiece (figs. 104, 196) . . . . 28

28. Anterior portion of spermathecae globose (fig. 197) ............. ampeinna

- Anterior portion of spermathecae tubular (fig. 105) .................. chalmers

29. Spermathecae bipartite (figs. 69, 81, 145, 149, 173), those two parts sometimes on narrow stalks (figs. 125, 133) . . . . . . . 30

- Spermathecae clearly tripartite ...... 36

30. Anterior and median portions of spermathecae subequal in size (figs. $69,125,133$ ) . .

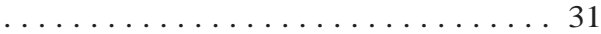

- Anterior portion of spermathecae smaller than median portion (figs. 81, 145, 149, 173) pigynum with deep anterior excavation (fig. 68) ................ ruida

- Epigynum without anterior excavation . . 32

32. Anterior portions of spermathecae approximate (fig. 133) .......... moorilyanna

- Anterior portions of spermathecae divergent (fig. 125) ........... punctigera

33. Epigynal midpiece relatively wide (fig. 172) .............. danggali

- Epigynal midpiece relatively narrow (figs. 80, 144, 148) .............. 34

34. Anterior portion of spermathecae rounded (fig. 145) ................ airlie

- Anterior portion of spermathecae not rounded (figs. 81, 149) ............. . 35

35. Median portion of spermathecae greatly elongated (fig. 81) ........... talbingo

- Median portion of spermathecae shorter (fig. 149) ................ terrors

36. Median portion of spermathecae much larger than anterior and posterior portions (figs. $153,165,269) \ldots \ldots \ldots \ldots 37$

- Median portion of spermathecae not much larger than anterior and posterior portions

37. Anterior portion of spermathecae reduced to round knob (figs. 153, 165) . . . . . . 38

- Anterior portion of spermathecae not reduced (fig. 269) . . . . . . . . . . . . molloy

38. Epigynum with anterior excavation (fig. 164) .................... taroom

- Epigynum without anterior excavation (fig. 152) ............... pusilla

39. Epigynal midpiece relatively narrow (figs. 128, 224) . . . . . . . . . . 40

- Epigynal midpiece relatively wide (figs. 228, $232,252,256,264,272) \ldots \ldots 41$

40. Anterior portion of spermathecae relatively large (fig. 129) ............ braemar

- Anterior portion of spermathecae relatively small (fig. 225) . . . . . . . . . bunya 
41. Epigynum with distinct anterior margin (figs. $232,252,256,272) \ldots \ldots \ldots 4$

- Epigynum without distinct anterior margin (figs. 228, 264) . . . . . . . . . 45

42. Epigynum with anterior margin medially incised (figs. 252, 256, 272) . . . . . . 43

- Epigynum with anterior margin not medially incised (fig. 232) ........... superbus

43. Spermathecae relatively wide (fig. 273) .... .................... tully

- Spermathecae relatively narrow (figs. 253, 257) . . . . . . . . . . . 44

44. Epigynal midpiece relatively short (fig. 252) ............... lamington

- Epigynal midpiece relatively long (fig. 256) $\ldots \ldots \ldots \ldots$. . . . . . . . .

45. Anterior portion of spermathecae relatively small (fig. 229) . . . . . . . . . . . cudgen

- Anterior portion of spermathecae relatively large (fig. 265) ............. chinghee

\section{Key to SPECIES OF LAMPONA KNOWN FROM VICTORIA, New South WaLes,}

\section{AND the Australian Capital Territory}

1. Males ..................2

- Females .................... 22

2. Retrolateral tibial apophysis relatively long, reaching to about one-fifth of cymbial length (as in figs. 52, 67) . . . . . . 3

- Retrolateral tibial apophysis relatively short, not reaching to about one-fifth of cymbial length (as in fig. 79) ...........8

3. Palpal femur with subbasal protuberance or excavation (figs. 67, 83, 95, 107) . . . 4

- Palpal femur unmodified (figs. 52, 60) . . 7

4. Palpal femur with subbasal excavation (fig. 95) ............. macilenta

- Palpal femur with subbasal protuberance (figs. 67, 83, 107) . . . . . . . . 5

5. Palpal bulb widened proximally (fig. 106) . . ................. flavipes

- Palpal bulb not widened proximally (figs. 66, 82) ...............6

6. Palp with one retrolateral tibial apophysis (fig. 67) ................ ruida

- Palp with two retrolateral tibial apophyses (fig. 83) ............... lomond

7. Palpal femur relatively long (fig. 45), retrolateral tibial apophysis relatively long, sinuous (fig. 52) ............ . cylindrata

- Palpal femur relatively short (fig. 48), retrolateral tibial apophysis relatively short, straight (fig. 60) ........... murina

8. Palp with two retrolateral tibial apophyses (fig. 79) .............. talbingo
- Palp with one retrolateral tibial apophysis .. .................... 9

9. Palpal femur with basal or subbasal expansion (as in fig. 123) ........... 10

- Palpal femur without expansion ...... 13

10. Retrolateral tibial apophysis covered with stiff setae, gradually narrowed toward tip (fig. 215) ................ fife

- Retrolateral tibial apophysis without stiff setae (sometimes with soft setae; if so, abruptly narrowed below tip) ...... 11

11. Retrolateral tibial apophysis with subdistal denticle (fig. 199) ........... ooldea

- Retrolateral tibial apophysis without subdistal denticle ............... 12

12. Retrolateral tibial apophysis distally hooked (fig. 171) ............. danggali

- Retrolateral tibial apophysis not distally hooked (figs. 123, 127) . . . . . . . 13

13. Retrolateral tibial apophysis relatively wide near base (fig. 123) ....... punctigera

- Retrolateral tibial apophysis relatively narrow near base (fig. 127) . . . . . . braemar

14. Retrolateral tibial apophysis with very narrow tip (fig. 219), body of apophysis almost obscured by numerous soft setae ...................... cumberland

- Retrolateral tibial apophysis less abruptly narrowed at tip ............. 15

15. Retrolateral tibial apophysis with subdistal denticle on ventral surface (fig. 183) ....

............................

- Retrolateral tibial apophysis without denticle

16. Tip of retrolateral tibial apophysis directed ventrally (fig. 195) ......... ampeinna

- Tip of retrolateral tibial apophysis directed dorsally or retrolaterally ........ 17

17. Palpal tibia relatively short (figs. 151, 167) .................. 18

- Palpal tibia relatively long (figs. 179, 227, $235,263) \ldots \ldots \ldots \ldots \ldots \ldots$

18. Palpal bulb relatively large (fig. 150) . . . . . .................. pusilla

- Palpal bulb relatively small (fig. 166) .... $\ldots \ldots \ldots \ldots$ allyn

19. Tip of retrolateral tibial apophysis relatively long (fig. 227) ............. cudgen

- Tip of tibial apophysis relatively short (figs. $179,235,263) \ldots \ldots \ldots 20$

20. Palpal femur dorsally expanded, darkened

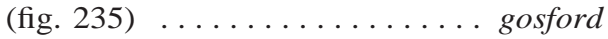

- Palpal femur unmodified .......... 21

21. Retrolateral tibial apophysis short, stubby (fig. 179) ............. mildura

- Retrolateral tibial apophysis longer, not stubby (fig. 263) .............. chinghee

22. Epigynum with long, deep depression (some- 
times filled with post-copulatory plug) occupying most of epigynal length (figs. 53, 61) .................. 23

- Epigynum without long depression ... . 24

23. Epigynal depression relatively wide (fig. 53), anterior lobes of spermathecae without dorsal demarcation (figs. 54-56) . . . cylindrata

- Epigynal depression relatively narrow (fig. $61)$, anterior lobes of spermathecae dorsally demarcated by distinct ridge $(57,58,62)$

............... murina

24. Epigynum relatively wide (figs. 167, 184); spermathecae roughly triangular (figs. 168, 185) ................ 25

- Epigynum not as wide; spermathecae longitudinal ............... 26

25. Epigynum with midpiece (fig. 167) .. allyn

- Epigynum without midpiece (fig. 184) . . . . . ...........................

26. Spermathecae consisting of narrow posterior stalk and two subdivisions (as in fig. 69)

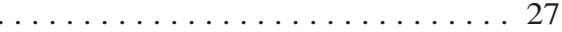

- Spermathecae consisting of stalk and at least three subdivisions (as in fig. 221) . . . 32

27. Spermathecal subdivisions roughly equal in size (figs. 69, 125) . . . . . . . . . . 28

- Posterior spermathecal subdivision longer than anterior (figs. 81, 85, 173, 201) 29

28. Epigynum with deep anterior depression (fig. 68), spermathecae bean-shaped (fig. 69) ..

$\ldots \ldots \ldots \ldots \ldots$. . . . . . . . .

Epigynum with shallow anterior depression (fig. 124), spermathecae relatively short (fig. 125) ........... punctigera

29. Anterior portion of spermathecae at least half as long as posterior portion (fig. 173, 201)

- Anterior portion of spermathecae much shorter than posterior portion (figs. 81,85 ) ... $\ldots \ldots \ldots \ldots \ldots \ldots \ldots \ldots \ldots$

30. Posterior portion of spermathecae about as wide as anterior portion (fig. 173) .... $\ldots \ldots \ldots$ danggali

- Posterior portion of spermathecae much narrower than anterior portion (fig. 201) ....

31. Spermathecae relatively short (fig 85 ooldea .

- Spermathecae relatively long (fig 81) ... $\ldots \ldots \ldots \ldots$ talbingo

32. Epigynum with wide, distinct anterior margin

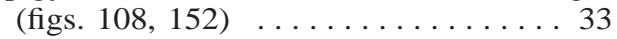

- Epigynum without distinct anterior margin ..................... 34

33. Spermathecae widest anteriorly (fig. 109) .. .................. flavipes

- Spermathecae widest medially (fig. 153) ...
34. Anterior lobes of spermathecae much narrower than posterior lobes (figs. 181, 229) ...

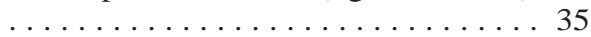

- Anterior lobes of spermathecae roughly as wide as posterior lobes, or wider ... 36

35. Median and anterior lobes of spermathecae displaced laterally (fig. 181) ... . mildura

- All spermathecal lobes longitudinally arrayed (fig. 229) ................. cudgen

36. Anterior lobes of spermathecae much wider than posterior lobes (fig. 197) . . ampeinna

- Anterior lobes of spermathecae not wider than posterior lobes .......... 37

37. Posterior spermathecal lobe subdivided or crenulated (figs. 97, 129, 237) . . . . 38

- Posterior spermathecal lobe entire (figs. 217, 221, 265) ............. . 40

38. Epigynal midpiece relatively wide (fig. 96) ............ macilenta

- Epigynal midpiece relatively narrow (figs. 128, 236) . . . . . . . . . . . 39

39. Epigynal midpiece relatively long (fig. 128) ................ braemar

- Epigynal midpiece relatively short (fig. 236) ............... gosford

40. Epigynal midpiece relatively wide (fig. 264)

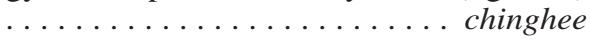

- Epigynal midpiece relatively narrow (figs. 220, 216) .............44

41. Epigynal midpiece relatively long (fig. 220)

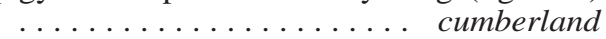

- Epigynal midpiece relatively short (fig. 216) $\ldots \ldots \ldots \ldots \ldots$ fife

\section{KEY TO SPECIES OF LAMPONA KNOWN FROM TASMANIA}

1. Males (those of L. hickmani unknown) . . 2

- Females ................6 6

2. Retrolateral tibial apophysis relatively long

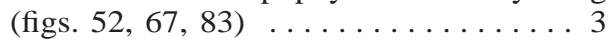

- Retrolateral tibial apophysis relatively short (figs. 127, 119) . . . . . . . . . 5

3. Two retrolateral tibial apophyses present (fig. 83) ................. lomond

- Only one retrolateral tibial apophysis (figs. $52,67) \ldots \ldots \ldots \ldots \ldots \ldots \ldots \ldots \ldots \ldots$

4. Retrolateral tibial apophysis narrowed at tip (fig. 52) .............. cylindrata

- Retrolateral tibial apophysis expanded at tip (fig. 67) ................. ruida

5. Retrolateral tibial apophysis relatively wide (fig. 127) ............. braemar

- Retrolateral tibial apophysis relatively narrow (fig. 119) ................ ewens

6. Epigynum with wide, deep anterior excavation (figs. 53, 68) . . . . . . . . 7

- Epigynum without anterior excavation ... 8 
7. Epigynal excavation relatively long (fig. 53) .................. cylindrata

- Epigynal excavation relatively short (fig. 68) ................. ruida

8. Epigynum with anteromedian projection (figs. $84,128) \ldots \ldots \ldots \ldots \ldots \ldots \ldots$. 9

- Epigynum without anteromedian projection (figs. 120, 154) $\ldots \ldots \ldots \ldots \ldots \ldots$

9. Anterior portion of spermathecae relatively small (fig. 85) ............ lomond

- Anterior portion of spermathecae relatively large (fig. 129) . . . . . . . . braemar

10. Epigynum with distinct anterior margin (fig. 120) ............... ewens

- Epigynum without distinct anterior margin (fig. 154) .............. hickmani

\section{THE CYLINDRATA GROUP}

This group includes three closely related species, united by having a deep excavation (that extends for most of the length of the epigynum) and relatively small spermathecae. As in many other lamponids, the epigynal excavation is often filled with a presumptive mating plug that can be difficult to remove.

\section{Lampona cylindrata (L. Koch)} Figures 3-40, 43, 45-47, 51-56; Map 1

Latona cylindrata L. Koch, 1866: 3, pl. 1, figs. 2, 3 (male lectotype, in effect designated by $\mathrm{L}$. Koch, 1873: 373-375, who removed the female paralectotype from this species by using it as the holotype of $L$. ruida, from Australia [as "Neuholland"], no specific locality, in BMNH, examined).

Lampona cylindrata: L. Koch, 1873: 373, pl. 29, figs. 4, 4a, 4b. -Main, 1964: 80, figs. A-D. -Hickman, 1967: 90, figs. 157, 158.

Lampona obscoena L. Koch, 1873: 376, pl. 29, figs. 6, 6a (female syntype from Australia [as "Neuholland"], no specific locality, in ZMH, examined). First synonymized with $L$. cylindrata by Dalmas, 1917: 350 .

Lampona cylindrata herculanea Dalmas, 1917: 351 (female holotype from Nelson, South Island, New Zealand, in MNHN, examined). First synonymized with $L$. cylindrata by Forster, 1979: 71.

Lampona pseudocylindrata Strand, 1922: 145 (name established for unspecified males illustrated as L. cylindrata by Simon, 1893: 376, fig. 334, and described as L. cylindrata by Simon, 1908: 401). NEW SYNONYMY.

Diagnosis: This species is very similar to
L. murina and has often been confused with it in the past. Females are relatively easy to distinguish by spermathecal structure; the narrow, tubular, bulging spermathecae of $L$. cylindrata (figs. 54-56) are quite different from the basally widened spermathecae with distinctly offset tips that are characteristic of L. murina (figs. 57, 58, 62). The epigynal fossa of L. cylindrata (fig. 53) is also typically wider and more rounded than that of $L$. murina (fig. 61), with the outline of the spermathecae typically presenting a pair of circular spots just behind the posterior rim of the fossa. Differences in the male palpi are subtle; in L. cylindrata, the palpal femur is relatively long and narrow, and the retrolateral tibial apophysis is relatively long, relatively narrow at its base, and almost straight (figs. 45, 52), whereas in L. murina the palpal femur is relatively short and thick, and the retrolateral tibial apophysis is relatively short, widened at its base, and distally curved (figs. 48, 60). The medial portion of the membranous palpal conductor also differs between the two species, barely extending over the lateral portion of the conductor in L. cylindrata (figs. 46, 47, 51), but produced into a wide, rounded lobe covering the base of the lateral portion of the conductor in $L$. murina (figs. 50, 59).

MALE: Total length 12.3. Abdomen dark gray, dorsum with two pairs of anterior transverse white spots plus fifth spot medially at posterior tip; venter with large, anteromedian light spot surrounding two paramedian, longitudinal rows of small sclerites. Legs with coxae, trochanters, and femora dark orange, other segments orange, without distinct dark bands. Palpal femur relatively long, thin; retrolateral tibial apophysis relatively long, narrow, as long as tibia itself, incised on ventral side at tip (fig. 52); tegulum globose, extending proximally almost to base of tibia; embolus dorsoventrally flattened, membranous conductor bipartite, medial part short ridge covering base of embolus (but only edge of lateral part of conductor) in ventral view, lateral part extending to embolar tip (fig. 51).

FEMALE: Total length 17.0. Coloration as in male. Epigynum separated from booklung covers by soft cuticle, consisting of elongate, excavate oval fossa (usually filled with plug 

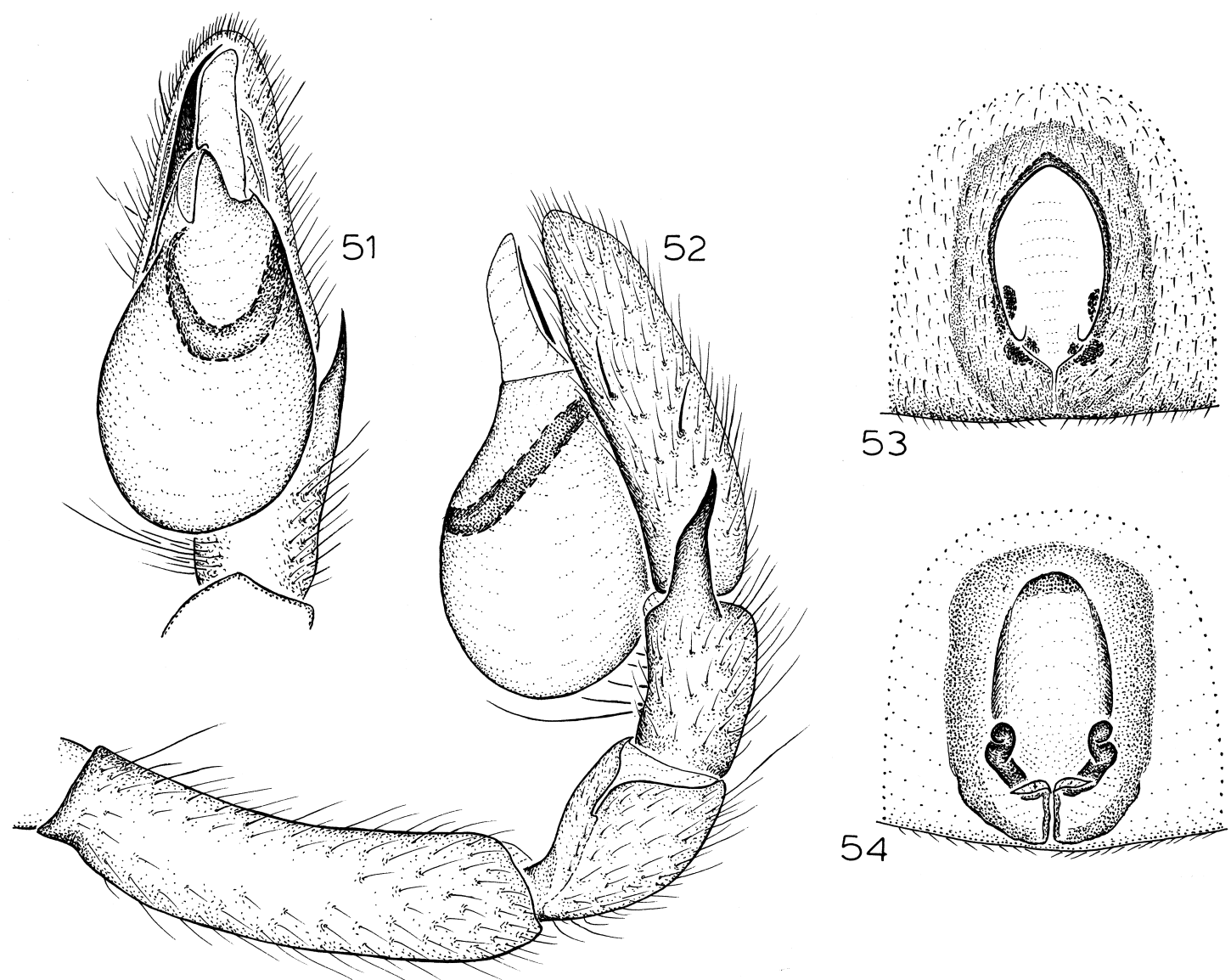

Figs. 51-54. Lampona cylindrata (L. Koch). 51. Left male palp, ventral view. 52. Same, retrolateral view. 53. Epigynum, ventral view. 54. Same, dorsal view.

of sticky secretion), anterior rim arched, elevated, openings at posterior edges of lateral rims, separated by pair of (rarely by three) slightly to distinctly raised longitudinal ridges (fig. 53); spermathecae narrow, tubular, usually with three bulging portions, amount and details of bulges highly variable, even between right and left sides of single specimen (fig. 54).

SElected Records: AUSTRALIA: Australian Capital Territory: Black Mountain, $35^{\circ} 16^{\prime} \mathrm{S}, 149^{\circ} 06^{\prime} \mathrm{E}, \mathrm{QMB}$ S30161; Blundells Creek, $35^{\circ} 22^{\prime} \mathrm{S}, 148^{\circ} 50^{\prime} \mathrm{E}$, QMB; Campbell, $35^{\circ} 18^{\prime} \mathrm{S}, \quad 149^{\circ} 09^{\prime} \mathrm{E}$, QMB; Canberra, $35^{\circ} 20^{\prime} \mathrm{S}, 149^{\circ} 10^{\prime} \mathrm{E}$, WAM 95/926; Cotter River Dam, $35^{\circ} 20^{\prime} \mathrm{S}, 148^{\circ} 54^{\prime} \mathrm{E}, \mathrm{CAS}$; Downer, $35^{\circ} 15^{\prime} \mathrm{S}, 149^{\circ} 09^{\prime} \mathrm{E}$, QMB; Kingston, $35^{\circ} 19^{\prime} \mathrm{S}, 149^{\circ} 08^{\prime} \mathrm{E}, \mathrm{QMB}$; Piccadilly Circus, $35^{\circ} 22^{\prime} \mathrm{S}, 148^{\circ} 48^{\prime} \mathrm{E}, \mathrm{QMB}$; Red Hill, $35^{\circ} 20^{\prime} \mathrm{S}$, $149^{\circ} 08^{\prime} \mathrm{E}, \mathrm{QMB}$; Turner, $35^{\circ} 16^{\prime} \mathrm{S}, 149^{\circ} 07^{\prime} \mathrm{E}$, QMB; Yarralumla, 35 $18^{\circ} \mathrm{S}, 149^{\circ} 04^{\prime} \mathrm{E}, \mathrm{QMB}$. New South Wales: Albury, $36^{\circ} 05^{\prime} \mathrm{S}$, $146^{\circ} 55^{\prime} \mathrm{E}, \mathrm{CAS}$; Balladoran, $31^{\circ} 52^{\prime} \mathrm{S}$, $148^{\circ} 40^{\prime} \mathrm{E}$, QMB; Baulkam Hills, $33^{\circ} 48^{\prime} \mathrm{S}$, $151^{\circ} 00^{\prime} \mathrm{E}$, AMS KS31928; Broken Hill, $31^{\circ} 58^{\prime} \mathrm{S}, 141^{\circ} 27^{\prime} \mathrm{E}$, SAM N1996/153; Coolah Valley, $31^{\circ} 50^{\prime} \mathrm{S}, 149^{\circ} 43^{\prime} \mathrm{E}$, AMS KS31919; Cootamundra, $34^{\circ} 38^{\prime} \mathrm{S}, 148^{\circ} 02^{\prime} \mathrm{E}$, AMS KS31916; Dubbo, $32^{\circ} 15^{\prime} \mathrm{S}, 148^{\circ} 37^{\prime} \mathrm{E}$, AMS KS31844; Frying Pan, $36^{\circ} 09^{\prime} \mathrm{S}$, $148^{\circ} 50^{\prime} \mathrm{E}$, AMS KS55265; Guthega, $36^{\circ} 21^{\prime} \mathrm{S}, 148^{\circ} 25^{\prime} \mathrm{E}$, AMS KS55260; Jerilderie, $35^{\circ} 22^{\prime} \mathrm{S}, 1^{\circ} 45^{\circ} 43^{\prime} \mathrm{E}$, AMS KS20167; Kooringal, $35^{\circ} 07^{\prime} \mathrm{S}, 147^{\circ} 22^{\prime} \mathrm{E}$, WAM 95/ 887; Merriwa, $32^{\circ} 09^{\prime} \mathrm{S}, 150^{\circ} 21^{\prime} \mathrm{E}, \mathrm{CBB}$; Parkes, $33^{\circ} 08^{\prime} \mathrm{S}, 148^{\circ} 11^{\prime} \mathrm{E}$, AMS KS42363; 

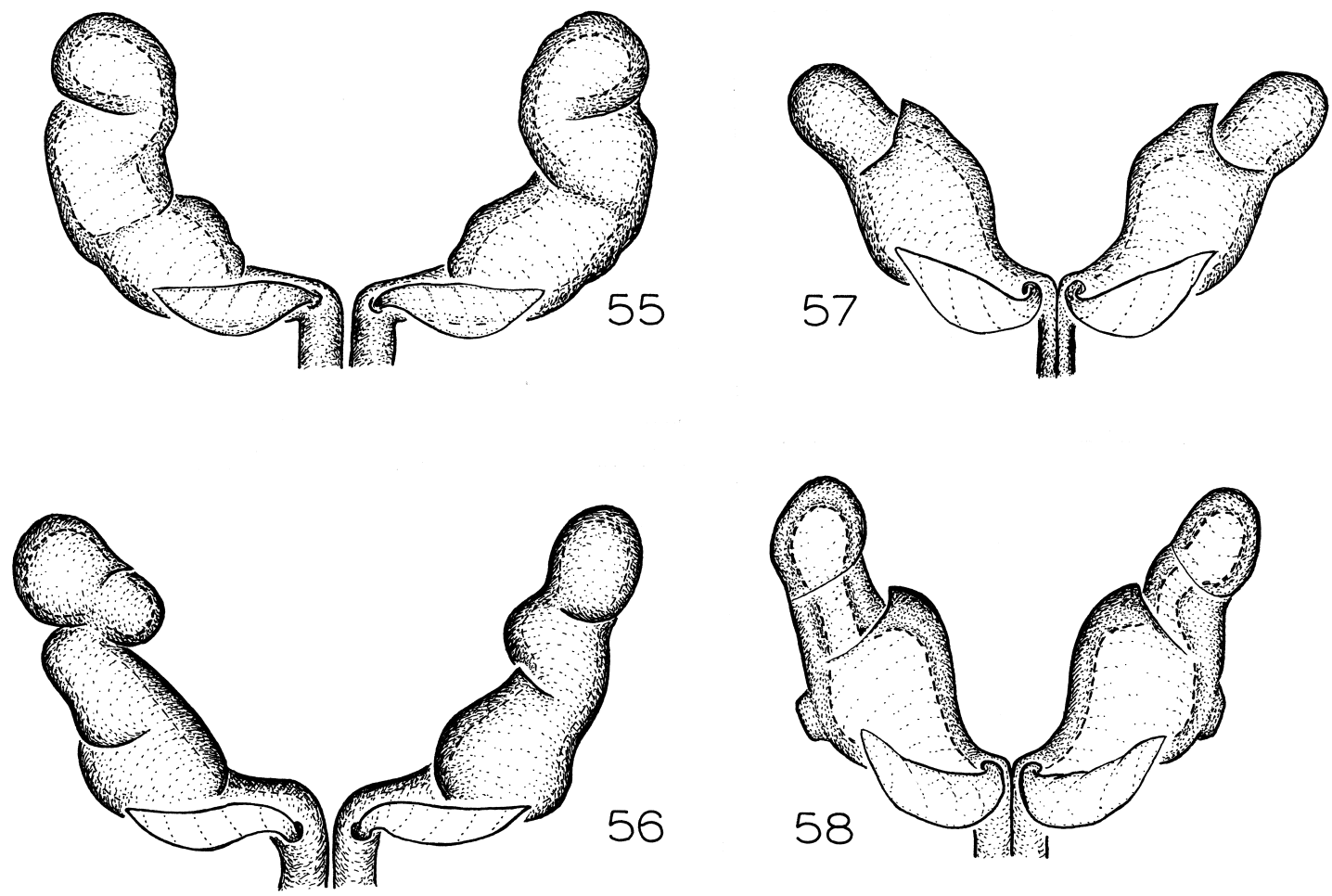

Figs. 55-58. Female spermathecae, dorsal views. 55, 56. Lampona cylindrata (L. Koch). 57, 58. $L$. murina L. Koch.

South Yathong National Park, $32^{\circ} 43^{\prime} \mathrm{S}$, $145^{\circ} 32^{\prime} \mathrm{E}$, NMV K2990; Sydney, 335' $3^{\prime}$, $151^{\circ} 13^{\prime} \mathrm{E}$, SAM N1996/156; Wee Jasper, $35^{\circ} 09^{\prime} \mathrm{S}, 148^{\circ} 42^{\prime} \mathrm{E}$, QMB; Yanco Creek, $35^{\circ} 02^{\prime} \mathrm{S}, 146^{\circ} 13^{\prime} \mathrm{E}$, WAM 95/894. Queensland: Blackall, $24^{\circ} 25^{\prime} \mathrm{S}, 145^{\circ} 28^{\prime} \mathrm{E}, \mathrm{QMB}$ S26124; Endfield Station, $27^{\circ} 55^{\prime} \mathrm{S}, 149^{\circ} 43^{\prime} \mathrm{E}$, QMB S28021; Roma, $26^{\circ} 35^{\prime} \mathrm{S}, 148^{\circ} 47^{\prime} \mathrm{E}$, QMB S15587. South Australia: Adelaide, $34^{\circ} 56^{\prime} \mathrm{S}, 138^{\circ} 36^{\prime} \mathrm{E}$, SAM N1996/5; Aldgate, $35^{\circ} 01^{\prime} \mathrm{S}, 138^{\circ} 44^{\prime} \mathrm{E}$, SAM N1996/73; Baird Bay, $33^{\circ} 09^{\prime} \mathrm{S}, 134^{\circ} 22^{\prime} \mathrm{E}$, WAM 95/877; Belair National Park, $35^{\circ} 01^{\prime} \mathrm{S}, 138^{\circ} 39^{\prime} \mathrm{E}$, SAM N1996/79; Billiat Conservation Park, $34^{\circ} 59^{\prime} \mathrm{S}, 140^{\circ} 28^{\prime} \mathrm{E}$, SAM N1997/129; Black Hill Conservation Park, $34^{\circ} 53^{\prime} \mathrm{S}, 138^{\circ} 43^{\prime} \mathrm{E}$, SAM N1996/25; Blackwood, 3501'S, $138^{\circ} 37^{\prime} \mathrm{E}$, SAM N1996/62; Brady Creek, $33^{\circ} 59^{\prime} \mathrm{S}, \quad 1^{\circ} 09^{\circ} \mathrm{E}, \quad \mathrm{SAM}$ N1996/126; Bridgewater, $35^{\circ} 01^{\prime} \mathrm{S}, 138^{\circ} 45^{\prime} \mathrm{E}, \quad \mathrm{SAM}$ N1996/74; Cape Ellen, 36 $00^{\prime} \mathrm{S}, 137^{\circ} 11^{\prime} \mathrm{E}$, SAM N1996/103; Ceduna, 32 08'S, $133^{\circ} 41^{\prime} \mathrm{E}$, SAM N1996/145; Clare, 3350'S, $138^{\circ} 37^{\prime}$ E, SAM N1996/127; Coffin Bay, $34^{\circ} 38^{\prime} \mathrm{S}, 135^{\circ} 28^{\prime} \mathrm{E}, \mathrm{SAM}$ N1996/140; Coomandook, $35^{\circ} 28^{\prime} \mathrm{S}, 139^{\circ} 42^{\prime} \mathrm{E}$, SAM N1996/ 111; Cortina Station, $36^{\circ} 20^{\prime} \mathrm{S}, 139^{\circ} 45^{\prime} \mathrm{E}$, SAM N1996/113; Dingley Dell National Pleasure Resort, $38^{\circ} 02^{\prime} \mathrm{S}, 140^{\circ} 41^{\prime} \mathrm{E}$, SAM N1996/121; Dudley Conservation Park, $35^{\circ} 48^{\prime} \mathrm{S}, 137^{\circ} 52^{\prime} \mathrm{E}$, SAM N1996/102; Elizabeth Field, $34^{\circ} 43^{\prime} \mathrm{S}, 138^{\circ} 41^{\prime} \mathrm{E}$, SAM N1996/ 67; Flinders Monument, 34 $46^{\prime} \mathrm{S}, 135^{\circ} 56^{\prime} \mathrm{E}$, SAM N1996/139; Goat Island, 35³0'S, 138 57'E, SAM N1996/93; Goolwa, $35^{\circ} 30^{\prime} \mathrm{S}, 138^{\circ} 47^{\prime} \mathrm{E}$, SAM N1996/91; Greenock, $34^{\circ} 28^{\prime} \mathrm{S}, 138^{\circ} 56^{\prime} \mathrm{E}$, SAM N1996/68; Gum Lagoon Conservation Park, 36 37'S, $140^{\circ} 02^{\prime}$ E, SAM N1996/114; Honan's Scrub, $37^{\circ} 44^{\prime} \mathrm{S}, 140^{\circ} 38^{\prime} \mathrm{E}$, SAM N1996/117; Jimmys Well, $35^{\circ} 51^{\prime} \mathrm{S}, 140^{\circ} 18^{\prime} \mathrm{E}, \mathrm{SAM}$ N1996/ 115; Kingscote, $35^{\circ} 39^{\prime} \mathrm{S}, 137^{\circ} 38^{\prime} \mathrm{E}$, SAM N1996/101; Kolay Outstation, $32^{\circ} 33^{\prime}$ S, $135^{\circ} 36^{\prime}$ E, SAM N1996/150; Kulliparu Conservation Park, $33^{\circ} 08^{\prime} \mathrm{S}, 134^{\circ} 49^{\prime} \mathrm{E}$, SAM N1996/142; Langhorne Creek, $35^{\circ} 18^{\prime} \mathrm{S}$, $139^{\circ} 02^{\prime}$ E, SAM N1996/89; Loftia Recreation Reserve, $35^{\circ} 02^{\prime} \mathrm{S}, 138^{\circ} 42^{\prime} \mathrm{E}$, SAM N1996/ 
66; Long Point, $35^{\circ} 42^{\prime} \mathrm{S}, 139^{\circ} 10^{\prime} \mathrm{E}$, SAM N1996/112; Marion Bay, $35^{\circ} 14^{\prime} \mathrm{S}, 137^{\circ} 02^{\prime} \mathrm{E}$, SAM N1996/128; Meadows, 35 ${ }^{\circ} 11^{\prime} \mathrm{S}$, 138 45'E, SAM N1996/76; Melrose, $32^{\circ} 50^{\prime} \mathrm{S}, 138^{\circ} 11^{\prime} \mathrm{E}$, SAM N1996/136; Millaton, 34 $46^{\prime} \mathrm{S}, 137^{\circ} 36^{\prime} \mathrm{E}$, SAM N1996/129; Moonta Bay, $34^{\circ} 03^{\prime} \mathrm{S}, 137^{\circ} 34^{\prime} \mathrm{E}$, WAM 95/ 890; Moorak, $37^{\circ} 50^{\prime} \mathrm{S}, 140^{\circ} 47^{\prime} \mathrm{E}$, SAM N1996/122; Morgan, $34^{\circ} 02^{\prime} \mathrm{S}, 139^{\circ} 40^{\prime} \mathrm{E}$,

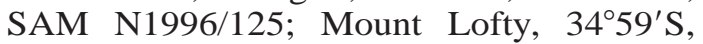
$138^{\circ} 43^{\prime}$ E, SAM N1996/65; Mount Magnificent, $35^{\circ} 19^{\prime} \mathrm{S}, 138^{\circ} 41^{\prime} \mathrm{E}$, SAM N1996/84; Murray River banks, $34^{\circ} 58^{\prime} \mathrm{S}, 139^{\circ} 16^{\prime} \mathrm{E}$,

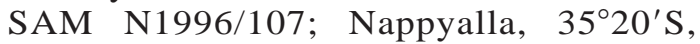
$139^{\circ} 07^{\prime} \mathrm{E}, \mathrm{SAM}$ N1996/77; Nepean Bay, $35^{\circ} 42^{\prime} \mathrm{S}, 137^{\circ} 37^{\prime} \mathrm{E}$, SAM N1996/100; Nullarbor, $31^{\circ} 19^{\prime} \mathrm{S}, 131^{\circ} 11^{\prime} \mathrm{E}$, SAM N1996/292; Nuriootpa, $34^{\circ} 29^{\prime} \mathrm{S}, 139^{\circ} 00^{\prime} \mathrm{E}$, WAM 95/ 961; Orrorroo, $32^{\circ} 44^{\prime} \mathrm{S}, 138^{\circ} 37^{\prime} \mathrm{E}, \mathrm{SAM}$ N1996/134; Penneshaw Township, 3543'S, $137^{\circ} 56^{\prime} \mathrm{E}, \mathrm{SAM}$ N1996/99; Pond Roadside Reserve, $37^{\circ} 32^{\prime} \mathrm{S}, 140^{\circ} 49^{\prime} \mathrm{E}$, SAM N1996/ 120; Ravine de Casoars, $35^{\circ} 48^{\prime} \mathrm{S}, 136^{\circ} 05^{\prime} \mathrm{E}$, SAM N1996/104; Reevesby Island, $34^{\circ} 32^{\prime} \mathrm{S}$, $136^{\circ} 16^{\prime} \mathrm{E}$, NMV K48; Rothe, $34^{\circ} 34^{\prime} \mathrm{S}$, $139^{\circ} 18^{\prime} \mathrm{E}, \mathrm{SAM}$ N1996/124; Roxby Downs, $30^{\circ} 42^{\prime} \mathrm{S}, 136^{\circ} 46^{\prime} \mathrm{E}$, SAM N1996/146; Second Valley, $35^{\circ} 32^{\prime} \mathrm{S}, 138^{\circ} 14^{\prime} \mathrm{E}$, WAM 95/ 990; Serpentine Lakes, $28^{\circ} 30^{\prime} \mathrm{S}, 129^{\circ} 00^{\prime} \mathrm{E}$, SAM N1996/291; Sleaford Bay, 3454'S, $135^{\circ} 47^{\prime}$ E, SAM N1996/138; Snowgum Reserve, $37^{\circ} 56^{\prime} \mathrm{S}, 140^{\circ} 56^{\prime} \mathrm{E}$, SAM N1996/118;

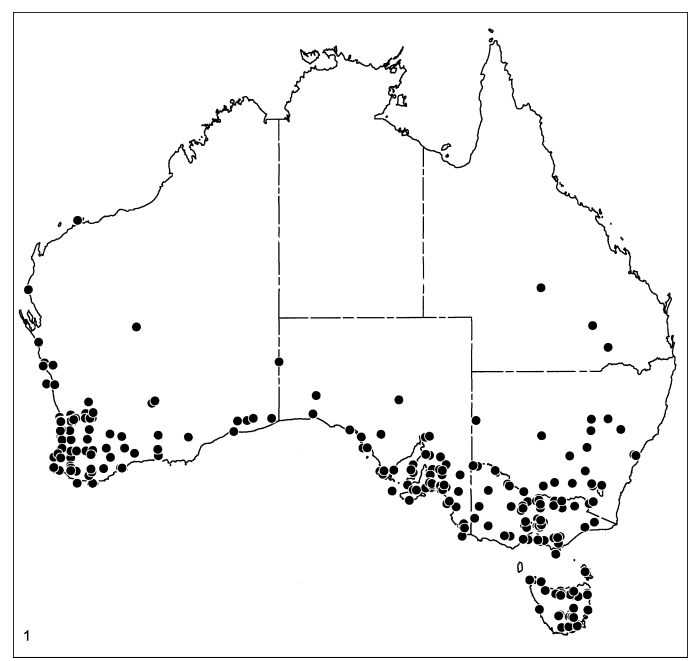

Map 1. Australian records of Lampona cylindrata (L. Koch).
Snowtown, $33^{\circ} 45^{\prime} \mathrm{S}, 138^{\circ} 13^{\prime} \mathrm{E}$, SAM N1996/ 131; Stirling, $35^{\circ} 00^{\prime} \mathrm{S}, 138^{\circ} 43^{\prime} \mathrm{E}$, SAM N1996/71; Stoneleigh Park, 35 41's, $139^{\circ} 20^{\prime} \mathrm{E}, \mathrm{SAM}$ N1996/108; Strathalbyn, $35^{\circ} 16^{\prime} \mathrm{S}, 138^{\circ} 54^{\prime} \mathrm{E}$, SAM N1996/85; Streaky Bay, $32^{\circ} 48^{\prime} \mathrm{S}, 134^{\circ} 13^{\prime} \mathrm{E}$, SAM N1996/143; Sturt Road, $35^{\circ} 02^{\prime} \mathrm{S}, 138^{\circ} 38^{\prime} \mathrm{E}$, QMB S26754; Tailem Bend, $35^{\circ} 15^{\prime} \mathrm{S}, 139^{\circ} 29^{\prime} \mathrm{E}$, SAM N1996/106; Tod River mouth, 343'ㅇ' 135 54'E, SAM N1996/137; Victor Harbour, $35^{\circ} 33^{\prime} \mathrm{S}, 138^{\circ} 37^{\prime} \mathrm{E}$, SAM N1996/90; Wardang Island, $34^{\circ} 30^{\prime} \mathrm{S}, 137^{\circ} 22^{\prime} \mathrm{E}$, SAM N1996/130; Wistow, $35^{\circ} 07^{\prime} \mathrm{S}, 138^{\circ} 53^{\prime} \mathrm{E}$, SAM N1996/69; Yarle Lakes, 30¹7'S, $131^{\circ} 31^{\prime} \mathrm{E}, \mathrm{SAM}$ N1996/151. Tasmania: Bagdad, $42^{\circ} 38^{\prime} \mathrm{S}, 147^{\circ} 14^{\prime} \mathrm{E}$, TMH J207; Bellerive, $42^{\circ} 52^{\prime} \mathrm{S}, 147^{\circ} 22^{\prime} \mathrm{E}$, TMH J2702; Blackmans Bay, $43^{\circ} 01^{\prime} \mathrm{S}, 147^{\circ} 19^{\prime} \mathrm{E}, \mathrm{TMH}$ J208; Broadmarsh, $42^{\circ} 39^{\prime} \mathrm{S}, 147^{\circ} 07^{\prime} \mathrm{E}, \mathrm{TMH}$ J3069; Bruny Island, $43^{\circ} 17^{\prime} \mathrm{S}, 147^{\circ} 18^{\prime} \mathrm{E}$, TMH J1535; Burnie, $41^{\circ} 04^{\prime} \mathrm{S}, 145^{\circ} 55^{\prime} \mathrm{E}$, TMH J2923; Charlton, $42^{\circ} 02^{\prime} \mathrm{S}, 147^{\circ} 30^{\prime} \mathrm{E}$, TMH J1085; Claremont, $42^{\circ} 47^{\prime} \mathrm{S}, 147^{\circ} 15^{\prime} \mathrm{E}$,

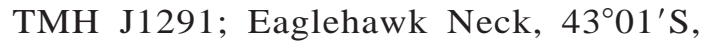
$147^{\circ} 52^{\prime} \mathrm{E}, \mathrm{QMB}$ S11617; Evandale, $41^{\circ} 35^{\prime} \mathrm{S}$, $147^{\circ} 14^{\prime} \mathrm{E}, \mathrm{QVM}$ 13/21200; Fentonbury, $42^{\circ} 39^{\prime} \mathrm{S}, 146^{\circ} 46^{\prime} \mathrm{E}, \mathrm{TMH}$ J3097; Fingal, $41^{\circ} 38^{\prime} \mathrm{S}, 147^{\circ} 58^{\prime} \mathrm{E}, \mathrm{BMNH}$ 1931.7.30.25; Flinders Island, $40^{\circ} 00^{\prime} \mathrm{S}, 148^{\circ} 00^{\prime} \mathrm{E}, \mathrm{QMB}$; Franklin, $43^{\circ} 05^{\prime} \mathrm{S}, 147^{\circ} 00^{\prime} \mathrm{E}, \mathrm{TMH} \mathrm{J} 770$; Freycinet Peninsula, $42^{\circ} 13^{\prime} \mathrm{S}, 148^{\circ} 18^{\prime} \mathrm{E}$, CBB; Geeveston, $43^{\circ} 09^{\prime} \mathrm{S}, 146^{\circ} 55^{\prime} \mathrm{E}, \mathrm{TMH}$ J1263; Georgetown, $41^{\circ} 06^{\prime} \mathrm{S}, 146^{\circ} 50^{\prime} \mathrm{E}$, QVM 13/21030; Hadspen, $41^{\circ} 30^{\prime} \mathrm{S}$, $147^{\circ} 05^{\prime} \mathrm{E}, \mathrm{QVM} 13 / 21194$; Hobart, $42^{\circ} 55^{\prime} \mathrm{S}$, $147^{\circ} 20^{\prime} \mathrm{E}, \mathrm{MNHN}$ AR3086; Howrah, $42^{\circ} 54^{\prime} \mathrm{S}, 147^{\circ} 24^{\prime} \mathrm{E}$, TMH J1307; Kingston, $42^{\circ} 58^{\prime} \mathrm{S}, 147^{\circ} 19^{\prime} \mathrm{E}$, TMH J2842; Lachlan, $42^{\circ} 50^{\prime} \mathrm{S}, 147^{\circ} 03^{\prime} \mathrm{E}$, TMH J2016; Lauderdale, $42^{\circ} 54^{\prime} \mathrm{S}, 147^{\circ} 29^{\prime} \mathrm{E}$, TMH J989; Launceston, $41^{\circ} 25^{\prime} \mathrm{S}, 147^{\circ} 08^{\prime} \mathrm{E}$, QVM 13/21032; Maydena, $42^{\circ} 45^{\prime} \mathrm{S}, 146^{\circ} 37^{\prime} \mathrm{E}$, TMH J3068; Newnham, $41^{\circ} 27^{\prime} \mathrm{S}, 147^{\circ} 10^{\prime} \mathrm{E}$, QVM 13/ 10408; New Town, $42^{\circ} 51^{\prime} \mathrm{S}, 147^{\circ} 19^{\prime} \mathrm{E}, \mathrm{TMH}$ J130; North Hobart, $42^{\circ} 55^{\prime} \mathrm{S}, 147^{\circ} 20^{\prime} \mathrm{E}$, TMH J3125; Notley Gorge, $41^{\circ} 21^{\prime} \mathrm{S}$, $146^{\circ} 55^{\prime} \mathrm{E}$, WAM 95/997; Queenstown, $42^{\circ} 05^{\prime} \mathrm{S}, 145^{\circ} 33^{\prime} \mathrm{E}, \mathrm{TMH} \mathrm{J} 204$; Risdon, $42^{\circ} 48^{\prime} \mathrm{S}, 147^{\circ} 20^{\prime} \mathrm{E}, \mathrm{AMNH}$; Rocky Cape, $40^{\circ} 55^{\prime} \mathrm{S}, 145^{\circ} 29^{\prime} \mathrm{E}, \mathrm{QVM}$ 13/21188; Rokeby, $42^{\circ} 54^{\prime} \mathrm{S}, 147^{\circ} 26^{\prime} \mathrm{E}$, FCTH 29; Rosetta, $42^{\circ} 50^{\prime} \mathrm{S}, 147^{\circ} 17^{\prime} \mathrm{E}, \mathrm{TMH}$ J131; Saint Helens, $41^{\circ} 19^{\prime} \mathrm{S}, 148^{\circ} 15^{\prime} \mathrm{E}$, TMH J1222; Scamander, 
$41^{\circ} 28^{\prime} \mathrm{S}, 148^{\circ} 16^{\prime} \mathrm{E}$, QVM 13/7030; Sheffield, $41^{\circ} 23^{\prime} \mathrm{S}, 146^{\circ} 20^{\prime} \mathrm{E}$, TMH J1938; Stony Point, $40^{\circ} 45^{\prime} \mathrm{S}, 144^{\circ} 58^{\prime} \mathrm{E}$, WAM 95/906; Strahan, $42^{\circ} 09^{\prime} \mathrm{S}, 145^{\circ} 19^{\prime} \mathrm{E}, \mathrm{TMH}$; Summerleas, $42^{\circ} 58^{\prime} \mathrm{S}, 147^{\circ} 19^{\prime} \mathrm{E}, \mathrm{TMH}$ unregistered; Targa, $41^{\circ} 19^{\prime} \mathrm{S}, 147^{\circ} 22^{\prime} \mathrm{E}, \mathrm{QVM} 13 / 21189$; Westbury, $41^{\circ} 31^{\prime} \mathrm{S}, 146^{\circ} 51^{\prime} \mathrm{E}$, QVM 13/ 21199; West Moonah, 42 $51^{\prime} \mathrm{S}, 147^{\circ} 18^{\prime} \mathrm{E}$, TMH J3115; West Scottsdale, $41^{\circ} 09^{\prime} \mathrm{S}$, $147^{\circ} 31^{\prime} \mathrm{E}, \mathrm{QVM}$ 13/21203; Windermere, $41^{\circ} 46^{\prime} \mathrm{S}, 145^{\circ} 57^{\prime} \mathrm{E}$, QVM 13/21207. Victoria: Anderson, $38^{\circ} 32^{\prime} \mathrm{S}, 145^{\circ} 27^{\prime} \mathrm{E}$, NMV K70; Ashburton, $37^{\circ} 52^{\prime} \mathrm{S}, 145^{\circ} 05^{\prime} \mathrm{E}$, WAM 95/835; Balwyn, $37^{\circ} 49^{\prime} \mathrm{S}, 145^{\circ} 04^{\prime} \mathrm{E}$, NMV K2959; Bendigo, $36^{\circ} 46^{\prime} \mathrm{S}, 144^{\circ} 16^{\prime} \mathrm{E}$, NMV K2971; Black Rock, $37^{\circ} 59^{\prime} \mathrm{S}, 145^{\circ} 01^{\prime} \mathrm{E}$, NMV K85; Brighton, $36^{\circ} 45^{\prime} \mathrm{S}, 146^{\circ} 58^{\prime} \mathrm{E}$, NMV K2957; Broken Creek, 36 06' S, $145^{\circ} 16^{\prime} \mathrm{E}$, NMVS; Brooms Road, $36^{\circ} 06^{\prime} \mathrm{S}$, $145^{\circ} 05^{\prime} \mathrm{E}, \mathrm{NMVS}$; Bundoora, $37^{\circ} 42^{\prime} \mathrm{S}$, $145^{\circ} 03^{\prime} \mathrm{E}$, NMV K2968; Buxton, $37^{\circ} 25^{\prime} \mathrm{S}$, $145^{\circ} 43^{\prime} \mathrm{E}$, NMV K2997; Canterbury, $37^{\circ} 50^{\prime} \mathrm{S}, 145^{\circ} 05^{\prime} \mathrm{E}$, NMV K44; Carnegie, $37^{\circ} 53^{\prime} \mathrm{S}, 145^{\circ} 04^{\prime} \mathrm{E}$, NMV K47; Cheniston, $37^{\circ} 25^{\prime} \mathrm{S}, 144^{\circ} 34^{\prime} \mathrm{E}, \mathrm{BMNH}$ 1924.3.95; Churchill, $38^{\circ} 18^{\prime} \mathrm{S}, 146^{\circ} 25^{\prime} \mathrm{E}$, SAM N1996/166; Clayton, $37^{\circ} 55^{\prime} \mathrm{S}, 145^{\circ} 07^{\prime} \mathrm{E}$, NMV K80; Cobon South Coupe, $37^{\circ} 25^{\prime} \mathrm{S}, 148^{\circ} 57^{\prime} \mathrm{E}$, AMNH; Cressy, $38^{\circ} 02^{\prime} \mathrm{S}, 143^{\circ} 38^{\prime} \mathrm{E}$, NMV K88; Cullulleraine, $34^{\circ} 12^{\prime} \mathrm{S}, 141^{\circ} 35^{\prime} \mathrm{E}$, NMVS; Darlington, $38^{\circ} 00^{\prime} \mathrm{S}, 143^{\circ} 03^{\prime} \mathrm{E}$, NMV K2980; Dartmouth, $36^{\circ} 34^{\prime} \mathrm{S}$, $147^{\circ} 35^{\prime} \mathrm{E}, \mathrm{NMV}$ K3407; Deep Creek, $36^{\circ} 05^{\prime} \mathrm{S}, 139^{\circ} 59^{\prime} \mathrm{E}$, NMVS; Drysdale HS, $36^{\circ} 45^{\prime} \mathrm{S}, 145^{\circ} 24^{\prime} \mathrm{E}$, NMVS; Echuca, $36^{\circ} 08^{\prime} \mathrm{S}$, $144^{\circ} 45^{\prime} \mathrm{E}$, NMV K2998; Edenhope, $37^{\circ} 02^{\prime} \mathrm{S}$, $141^{\circ} 17^{\prime} \mathrm{E}, \quad$ SAM N1996/161; Eltham, $37^{\circ} 44^{\prime} \mathrm{S}, 145^{\circ} 09^{\prime} \mathrm{E}$, NMV K3000; Essendon, $37^{\circ} 46^{\prime} \mathrm{S}$, $144^{\circ} 55^{\prime} \mathrm{E}$, NMV K63; Fern Tree Gully, $37^{\circ} 53^{\prime} \mathrm{S}, 145^{\circ} 18^{\prime} \mathrm{E}$, SAM N1996/162; Gardenvale, $37^{\circ} 54^{\prime} \mathrm{S}, 145^{\circ} 00^{\prime} \mathrm{E}$, NMV K2951; Glen Iris, $37^{\circ} 51^{\prime} \mathrm{S}$, $145^{\circ} 03^{\prime} \mathrm{E}$, WAM 95/933; Glenmaggie, $38^{\circ} 05^{\prime} \mathrm{S}, 146^{\circ} 47^{\prime} \mathrm{E}$, SAM N1996/165; Gunbower Island State Forest, $35^{\circ} 53^{\prime} \mathrm{S}, 144^{\circ} 23^{\prime} \mathrm{E}$, NMVS; Hamilton, $37^{\circ} 45^{\prime} \mathrm{S}, 142^{\circ} 02^{\prime} \mathrm{E}$, NMV K2962; Harrietville, $33^{\circ} 55^{\prime} \mathrm{S}, 147^{\circ} 04^{\prime} \mathrm{E}$, NMV K2973; Hawthorn, $37^{\circ} 50^{\prime} \mathrm{S}, 145^{\circ} 02^{\prime} \mathrm{E}$, NMV K2975; Ivanhoe, $37^{\circ} 46^{\prime} \mathrm{S}, 145^{\circ} 03^{\prime} \mathrm{E}$, NMV K46; Kaarimba, $36^{\circ} 10^{\prime} \mathrm{S}, 145^{\circ} 14^{\prime} \mathrm{E}$, NMVS; Kew, $37^{\circ} 49^{\prime} \mathrm{S}, 145^{\circ} 03^{\prime} \mathrm{E}$, WAM 95/923; Kilsyth, $37^{\circ} 48^{\prime} \mathrm{S}, 145^{\circ} 19^{\prime} \mathrm{E}, \mathrm{NMV}$ K72; Korumburra, $38^{\circ} 26^{\prime} \mathrm{S}, 145^{\circ} 49^{\prime} \mathrm{E}$, NMV K2963; Kotupna-
Barmah Road, $36^{\circ} 05^{\prime} \mathrm{S}, 145^{\circ} 03^{\prime} \mathrm{E}$, NMVS; Lake Albacutya Park, $35^{\circ} 46^{\prime} \mathrm{S}, 142^{\circ} 03^{\prime} \mathrm{E}$, NMV K76; Lakes Entrance, 37 53'S, $148^{\circ} 00^{\prime} \mathrm{E}, \mathrm{QMB}$; Lindsay River, $34^{\circ} 08^{\prime} \mathrm{S}$, $141^{\circ} 08^{\prime} \mathrm{E}$, NMVS; Little Desert, $36^{\circ} 35^{\prime} \mathrm{S}$, $141^{\circ} 45^{\prime} \mathrm{E}$, NMV K2960; Longforest Road, $37^{\circ} 41^{\prime} \mathrm{S}, 144^{\circ} 27^{\prime} \mathrm{E}$, NMV K20; Lower Moira, $36^{\circ} 04^{\prime} \mathrm{S}, 145^{\circ} 00^{\prime} \mathrm{E}$, NMVS; Maldon, $36^{\circ} 59^{\prime} \mathrm{S}, 144^{\circ} 05^{\prime} \mathrm{E}$, NMV K2983; Malvern, $37^{\circ} 52^{\prime} \mathrm{S}, 145^{\circ} 02^{\prime} \mathrm{E}$, NMV K2966; Melbourne, $37^{\circ} 50^{\prime} \mathrm{S}, 145^{\circ} 00^{\prime} \mathrm{E}$, MNHN AR3084; Melville Caves, $36^{\circ} 36^{\prime} \mathrm{S}, 143^{\circ} 43^{\prime} \mathrm{E}$, SAM N1996/294; Montmorency, 37 43'S, $145^{\circ} 07^{\prime} \mathrm{E}$, NMV K2974; Morwell National Park, $38^{\circ} 22^{\prime} \mathrm{S}, 146^{\circ} 24^{\prime} \mathrm{E}$, SAM N1996/167; North Carlton, $37^{\circ} 47^{\prime} \mathrm{S}$, $144^{\circ} 58^{\prime} \mathrm{E}$, NMV K89; Northcote, $37^{\circ} 46^{\prime} \mathrm{S}, 145^{\circ} 00^{\prime} \mathrm{E}$, NMV K2978; North Melbourne, $37^{\circ} 48^{\prime} \mathrm{S}$, $144^{\circ} 58^{\prime} \mathrm{E}$, NMV K62; Nyah, $35^{\circ} 10^{\prime} \mathrm{S}$, $143^{\circ} 23^{\prime} \mathrm{E}$, NMV K2953; Oakleigh, $37^{\circ} 54^{\prime} \mathrm{S}$, $145^{\circ} 06^{\prime} \mathrm{E}$, NMV K75; Ovens River, $36^{\circ} 05^{\prime} \mathrm{S}$, $146^{\circ} 11^{\prime} \mathrm{E}, \mathrm{SAM}$ N1996/164; Pascoe Vale, $37^{\circ} 44^{\prime} \mathrm{S}, 144^{\circ} 56^{\prime} \mathrm{E}$, NMV K60; Pomfrets Road, $36^{\circ} 00^{\prime} \mathrm{S}, 145^{\circ} 14^{\prime} \mathrm{E}$, NMVS; Rathbones Road, $36^{\circ} 09^{\prime} \mathrm{S}, 145^{\circ} 13^{\prime} \mathrm{E}$, NMVS; Raywood, $36^{\circ} 32^{\prime} \mathrm{S}, 144^{\circ} 13^{\prime} \mathrm{E}$, NMV K2958; Ringwood, $37^{\circ} 49^{\prime} \mathrm{S}, 145^{\circ} 14^{\prime} \mathrm{E}$, NMV K3406; Robinvale, $34^{\circ} 35^{\prime} \mathrm{S}, 142^{\circ} 46^{\prime} \mathrm{E}$, WAM 95/855; Rosanna, $37^{\circ} 45^{\prime} \mathrm{S}, 145^{\circ} 04^{\prime} \mathrm{E}$, NMV K2976; Rowville, $37^{\circ} 56^{\prime} \mathrm{S}, 145^{\circ} 14^{\prime} \mathrm{E}$, QMB S26760; Rye, $38^{\circ} 22^{\prime} \mathrm{S}, 144^{\circ} 49^{\prime} \mathrm{E}$, NMV K34; Sassafras, $37^{\circ} 52^{\prime} \mathrm{S}, 145^{\circ} 21^{\prime} \mathrm{E}$, NMV K81; Shepparton/ Bendigo, $36^{\circ} 23^{\prime} \mathrm{S}, 145^{\circ} 24^{\prime} \mathrm{E}$, K2996; Sherbrook Forest, $37^{\circ} 53^{\prime} \mathrm{S}, 145^{\circ} 21^{\prime} \mathrm{E}$, HNHM; Snake Gully, $37^{\circ} 55^{\prime} \mathrm{S}, 145^{\circ} 07^{\prime} \mathrm{E}$, QMB S26748; Somers, $38^{\circ} 24^{\prime} \mathrm{S}, 145^{\circ} 10^{\prime} \mathrm{E}$, NMV K2985; Spring Creek, $38^{\circ} 20^{\prime} \mathrm{S}$, $144^{\circ} 19^{\prime} \mathrm{E}$, QMB; Sunday Island, $38^{\circ} 43^{\prime} \mathrm{S}, 146^{\circ} 37^{\prime} \mathrm{E}$, NMV K2967; Surrey Hills, 37 49's, $145^{\circ} 06^{\prime} \mathrm{E}$, NMV K87; Swan Hill, $35^{\circ} 21^{\prime} \mathrm{S}$, $143^{\circ} 34^{\prime} \mathrm{E}$, NMV K2972; Tallarook, 3706'S, $145^{\circ} 06^{\prime} \mathrm{E}, \mathrm{MNV}$ K61; Trafalgar, $38^{\circ} 12^{\prime} \mathrm{S}$, $146^{\circ} 09^{\prime} \mathrm{E}$, NMV K2970; Traralgon, $38^{\circ} 11^{\prime} \mathrm{S}$, $146^{\circ} 32^{\prime} \mathrm{E}$, QMB S4538; Upper Lurg, $36^{\circ} 35^{\prime} \mathrm{S}, 146^{\circ} 11^{\prime} \mathrm{E}$, CVIC 629; Wangaratta, $36^{\circ} 22^{\prime} \mathrm{S}, 146^{\circ} 20^{\prime} \mathrm{E}, \mathrm{NMV}$ K83; Watts River, $37^{\circ} 39^{\prime} \mathrm{S}, 145^{\circ} 32^{\prime} \mathrm{E}$, QMB S26763; White Hills, $36^{\circ} 46^{\prime} \mathrm{S}, 144^{\circ} 16^{\prime} \mathrm{E}$, NMV K2956; Werribee, $37^{\circ} 54^{\prime} \mathrm{S}, 144^{\circ} 40^{\prime} \mathrm{E}$, NMV K2977; Wilson's Promontory, $39^{\circ} 02^{\prime} \mathrm{S}, 146^{\circ} 19^{\prime} \mathrm{E}, \mathrm{NMV}$ K19; Windsor, $37^{\circ} 52^{\prime} \mathrm{S}, 145^{\circ} 00^{\prime} \mathrm{E}$, NMV K2995; Winton, $36^{\circ} 31^{\prime} \mathrm{S}, 146^{\circ} 05^{\prime} \mathrm{E}$, NMV K67; Wonga Park, $37^{\circ} 44^{\prime}$ S, $145^{\circ} 16^{\prime} \mathrm{E}$, NMV 
K2991; Woodend, $37^{\circ} 21^{\prime} \mathrm{S}, 144^{\circ} 32^{\prime} \mathrm{E}$, NMV K59; Yea, $37^{\circ} 13^{\prime} \mathrm{S}, 145^{\circ} 26^{\prime} \mathrm{E}$, QMB S26758. Western Australia: Albany, $35^{\circ} 02^{\prime} \mathrm{S}$, $117^{\circ} 53^{\prime} \mathrm{E}$, WAM 95/946; Alfred Cove, $31^{\circ} 57^{\prime} \mathrm{S}, 115^{\circ} 52^{\prime} \mathrm{E}$, SAM N1996/160; Amelia Hights, $31^{\circ} 53^{\prime} \mathrm{S}, 115^{\circ} 49^{\prime} \mathrm{E}$, WAM 84/193; Applecross, $31^{\circ} 59^{\prime} \mathrm{S}, 115^{\circ} 56^{\prime} \mathrm{E}$, WAM 84/ 192; Armadale, $32^{\circ} 10^{\prime} \mathrm{S}, 116^{\circ} 01^{\prime} \mathrm{E}$, WAM 95/974; Arthur River, $33^{\circ} 21^{\prime} \mathrm{S}, 117^{\circ} 02^{\prime} \mathrm{E}$, WAM 95/780; Augusta, $34^{\circ} 19^{\prime} \mathrm{S}, 115^{\circ} 10^{\prime} \mathrm{E}$, MCZ; Australind, $33^{\circ} 16^{\prime} \mathrm{S}, 115^{\circ} 44^{\prime} \mathrm{E}$, WAM 95/951; Baskerville, $31^{\circ} 54^{\prime} \mathrm{S}, 116^{\circ} 01^{\prime} \mathrm{E}$, WAM 95/955; Bayswater, $31^{\circ} 55^{\prime} \mathrm{S}$, $115^{\circ} 54^{\prime} \mathrm{E}$, WAM 84/195; Black Bay, $33^{\circ} 03^{\prime} \mathrm{S}, 115^{\circ} 56^{\prime} \mathrm{E}$, AMNH; Booanya, $32^{\circ} 46^{\prime} \mathrm{S}, 123^{\circ} 36^{\prime} \mathrm{E}$, NMV K74; Boolathana Station, $24^{\circ} 25^{\prime} \mathrm{S}, 113^{\circ} 45^{\prime} \mathrm{E}$, WAM 96/1444; Boranup, $34^{\circ} 09^{\prime} \mathrm{S}, 115^{\circ} 02^{\prime} \mathrm{E}$, WAM 95/882; Boulder, 30 $47^{\prime} \mathrm{S}, 121^{\circ} 29^{\prime} \mathrm{E}$, WAM 95/938; Bridgetown, $33^{\circ} 58^{\prime} \mathrm{S}, 116^{\circ} 08^{\prime} \mathrm{E}$, WAM 95/ 862; Bull Creek, $32^{\circ} 03^{\prime} \mathrm{S}, 115^{\circ} 52^{\prime} \mathrm{E}$, WAM 95/888; Bunbury, $33^{\circ} 19^{\prime} \mathrm{S}, 115^{\circ} 38^{\prime} \mathrm{E}$, WAM 84/196; Bunnings Treefarms, $34^{\circ} 15^{\prime} \mathrm{S}$, $116^{\circ} 09^{\prime} \mathrm{E}$, WAM 95/1077; Busselton, $33^{\circ} 39^{\prime} \mathrm{S}, 115^{\circ} 20^{\prime} \mathrm{E}$, WAM 84/198; Carter Road, 33 $55^{\prime} \mathrm{S}, 115^{\circ} 03^{\prime} \mathrm{E}$, WAM 95/957; Claremont, $31^{\circ} 59^{\prime} \mathrm{S}, 115^{\circ} 47^{\prime} \mathrm{E}$, WAM 95/ 917; Collie, $33^{\circ} 22^{\prime} \mathrm{S}, 116^{\circ} 09^{\prime} \mathrm{E}$, CBB; Cottesloe, $32^{\circ} 00^{\prime} \mathrm{S}, 115^{\circ} 46^{\prime} \mathrm{E}$, WAM 95/908; Como, $32^{\circ} 00^{\prime} \mathrm{S}, 115^{\circ} 52^{\prime} \mathrm{E}$, WAM 95/988; Coolgardie, $30^{\circ} 57^{\prime} \mathrm{S}, 121^{\circ} 10^{\prime} \mathrm{E}$, WAM 95/ 807; Cottesloe, $31^{\circ} 59^{\prime} \mathrm{S}, 115^{\circ} 45^{\prime} \mathrm{E}$, WAM 95/ 981; Craigie, $31^{\circ} 47^{\prime} \mathrm{S}, 115^{\circ} 46^{\prime} \mathrm{E}$, WAM 96/ 1422; Crawley, $31^{\circ} 59^{\prime} \mathrm{S}, 115^{\circ} 49^{\prime} \mathrm{E}$, WAM 95/787; Darkan, $33^{\circ} 21^{\prime} \mathrm{S}, 116^{\circ} 44^{\prime} \mathrm{E}$, WAM 95/931; Darlington, $31^{\circ} 55^{\prime} \mathrm{S}, 116^{\circ} 04^{\prime} \mathrm{E}$, WAM 95/922; Denmark, $34^{\circ} 58^{\prime} \mathrm{S}, 117^{\circ} 21^{\prime} \mathrm{E}$, WAM 95/838; D'Entrecasteaux National Park, $34^{\circ} 28^{\prime} \mathrm{S}, 115^{\circ} 39^{\prime} \mathrm{E}, \mathrm{CCD}$; Dianella, $31^{\circ} 54^{\prime} \mathrm{S}, 115^{\circ} 53^{\prime} \mathrm{E}$, WAM 84/201; Dowerin, $31^{\circ} 12^{\prime} \mathrm{S}, 117^{\circ} 02^{\prime} \mathrm{E}$, WAM 95/911; Dumbleyung, $33^{\circ} 19^{\prime} \mathrm{S}, 117^{\circ} 44^{\prime} \mathrm{E}$, WAM 95/945; East Chapman, $28^{\circ} 30^{\prime} \mathrm{S}, 114^{\circ} 47^{\prime} \mathrm{E}$, WAM 32/ 2303; East Freemantle, $32^{\circ} 02^{\prime} \mathrm{S}, 115^{\circ} 46^{\prime} \mathrm{E}$, WAM 95/978; East Hyden Siding, 32 $27^{\prime} \mathrm{S}$, $118^{\circ} 52^{\prime} \mathrm{E}$, WAM 95/861; East Perth, $31^{\circ} 57^{\prime} \mathrm{S}, 115^{\circ} 52^{\prime} \mathrm{E}$, WAM 95/983; East Victoria Park, $31^{\circ} 59^{\prime} \mathrm{S}, 115^{\circ} 54^{\prime} \mathrm{E}$, WAM 95/875; Eneabba, $29^{\circ} 50^{\prime} \mathrm{S}, 115^{\circ} 15^{\prime} \mathrm{E}$, WAM 96/1420; Esperance, $33^{\circ} 52^{\prime} \mathrm{S}, 121^{\circ} 53^{\prime} \mathrm{E}$, WAM 95/ 958; Eucla, $31^{\circ} 43^{\prime} \mathrm{S}, 128^{\circ} 54^{\prime} \mathrm{E}$, WAM 96/ 1427; Eyre Bird Observatory, $32^{\circ} 15^{\prime} \mathrm{S}$, $126^{\circ} 18^{\prime} \mathrm{E}$, WAM 95/796; Fairfields, $31^{\circ} 39^{\prime} \mathrm{S}$, $117^{\circ} 35^{\prime} \mathrm{E}$, WAM 95/854; Ferndale, 32 $59^{\prime} \mathrm{S}$, $115^{\circ} 55^{\prime} \mathrm{E}$, WAM 95/841; Fremantle, $32^{\circ} 07^{\prime} \mathrm{S}, \quad 115^{\circ} 45^{\prime} \mathrm{E}, \mathrm{AMNH}$; Gelorup, $33^{\circ} 23^{\prime} \mathrm{S}, 115^{\circ} 39^{\prime} \mathrm{E}$, WAM 95/818; Geraldton, $28^{\circ} 46^{\prime} \mathrm{S}, 114^{\circ} 37^{\prime} \mathrm{E}$, MCZ; Gidgegannup, $31^{\circ} 20^{\prime} \mathrm{S}, 116^{\circ} 09^{\prime} \mathrm{E}$, WAM 95/844; Glenburnie farm, $31^{\circ} 48^{\prime} \mathrm{S}, 116^{\circ} 19^{\prime} \mathrm{E}$, WAM 84/247; Glen Forrest, $31^{\circ} 55^{\prime} \mathrm{S}, 116^{\circ} 06^{\prime} \mathrm{E}$, WAM 84/ 1134; Grasmere, $35^{\circ} 01^{\prime} \mathrm{S}, 117^{\circ} 45^{\prime} \mathrm{E}$, WAM 95/987; Grasspatch, $33^{\circ} 14^{\prime} \mathrm{S}, 121^{\circ} 43^{\prime} \mathrm{E}$, WAM 84/725; Great Southern Highway, $31^{\circ} 54^{\prime} \mathrm{S}, 116^{\circ} 28^{\prime} \mathrm{E}$, WAM 95/1089; Greenmount, $31^{\circ} 51^{\prime} \mathrm{S}, 116^{\circ} 03^{\prime} \mathrm{E}$, WAM 95/930; Gum Tree Bay, $29^{\circ} 47^{\prime} \mathrm{S}, 114^{\circ} 58^{\prime} \mathrm{E}$, WAM 95/811; Hamersley, $31^{\circ} 51^{\prime} \mathrm{S}, 115^{\circ} 47^{\prime} \mathrm{E}$, WAM 84/205; Harvey, $33^{\circ} 05^{\prime} \mathrm{S}, 115^{\circ} 54^{\prime} \mathrm{E}$, WAM 96/1423; Heathridge, $31^{\circ} 46^{\prime} \mathrm{S}$, $115^{\circ} 46^{\prime} \mathrm{E}$, WAM 95/819; Helena Valley, $31^{\circ} 56^{\prime} \mathrm{S}, 116^{\circ} 04^{\prime} \mathrm{E}$, WAM 95/837; Holt Rock, 32 $41^{\prime} \mathrm{S}, 119^{\circ} 24^{\prime} \mathrm{E}$, WAM 84/1141; Huntingdale, $32^{\circ} 05^{\prime} \mathrm{S}, 115^{\circ} 58^{\prime} \mathrm{E}$, WAM 95/ 954; Hyden, $32^{\circ} 27^{\prime} \mathrm{S}, 118^{\circ} 52^{\prime} \mathrm{E}$, WAM 95/ 919; Inglewood, $31^{\circ} 55^{\prime} \mathrm{S}, 115^{\circ} 53^{\prime} \mathrm{E}$, WAM 84/1143; Jandakot Airport, $32^{\circ} 06^{\prime} \mathrm{S}$, $115^{\circ} 53^{\prime} \mathrm{E}$, WAM 95/1088; Jerramungup, $33^{\circ} 57^{\prime} \mathrm{S}, 118^{\circ} 54^{\prime} \mathrm{E}, \mathrm{CAS}$; Joondanna, $31^{\circ} 54^{\prime} \mathrm{S}, 115^{\circ} 50^{\prime} \mathrm{E}$, WAM 95/829; Kalgoorlie, $30^{\circ} 44^{\prime} \mathrm{S}, 121^{\circ} 28^{\prime} \mathrm{E}$, WAM 84/208; Kallaroo, $31^{\circ} 47^{\prime} \mathrm{S}, 115^{\circ} 45^{\prime} \mathrm{E}$, WAM 95/1086; Karratha, $20^{\circ} 44^{\prime} \mathrm{S}, 116^{\circ} 51^{\prime} \mathrm{E}$, WAM 84/209; Karridale, $34^{\circ} 12^{\prime} \mathrm{S}, 115^{\circ} 04^{\prime} \mathrm{E}$, WAM 95/813; Karrinyup, $31^{\circ} 52^{\prime} \mathrm{S}, 115^{\circ} 46^{\prime} \mathrm{E}$, WAM 95/ 789; Katanning, $33^{\circ} 42^{\prime} \mathrm{S}, 117^{\circ} 33^{\prime} \mathrm{E}$, WAM 95/918; Kellerberrin, $31^{\circ} 38^{\prime} \mathrm{S}, 117^{\circ} 43^{\prime} \mathrm{E}$, WAM 95/859; Kelmscott, $32^{\circ} 07^{\prime} \mathrm{S}$, $116^{\circ} 01^{\prime} \mathrm{E}$, WAM 95/785; Kensington, $31^{\circ} 59^{\prime} \mathrm{S}, 115^{\circ} 53^{\prime} \mathrm{E}$, WAM 84/1155; Kewdale, $31^{\circ} 59^{\prime} \mathrm{S}, 115^{\circ} 57^{\prime} \mathrm{E}$, WAM 84/211; Lake Dundas, 32 $34^{\prime} \mathrm{S}, 121^{\circ} 50^{\prime} \mathrm{E}, \mathrm{SAM}$ N1997/ 125; Lake Grace, $33^{\circ} 06^{\prime} \mathrm{S}, 118^{\circ} 28^{\prime} \mathrm{E}$, WAM 95/846; Lake Indoon, $29^{\circ} 52^{\prime} \mathrm{S}, 115^{\circ} 09^{\prime} \mathrm{E}$, WAM 95/834; Lake King, $33^{\circ} 06^{\prime} \mathrm{S}$, $119^{\circ} 41^{\prime} \mathrm{E}$, WAM 95/786; Lake Poorginup, $34^{\circ} 33^{\prime} \mathrm{S}, 116^{\circ} 44^{\prime} \mathrm{E}$, WAM 95/832; Langford, $32^{\circ} 03^{\prime} \mathrm{S}, 115^{\circ} 56^{\prime} \mathrm{E}$, WAM 95/817; Leda, $32^{\circ} 16^{\prime} \mathrm{S}, 115^{\circ} 49^{\prime} \mathrm{E}$, WAM 95/992; Leederville, $31^{\circ} 56^{\prime} \mathrm{S}, 115^{\circ} 50^{\prime} \mathrm{E}$, WAM $84 / 212$; Ludlow, 3336' $\mathrm{S}, 115^{\circ} 29^{\prime} \mathrm{E}$, WAM 95/848; Maddington, $32^{\circ} 04^{\prime} \mathrm{S}, 115^{\circ} 58^{\prime} \mathrm{E}$, WAM 95/852; Madura, $31^{\circ} 57^{\prime} \mathrm{S}, 126^{\circ} 42^{\prime} \mathrm{E}$, WAM 95/866; Mandurah, $32^{\circ} 32^{\prime} \mathrm{S}$, $115^{\circ} 43^{\prime} \mathrm{E}$, WAM 95/ 912; Maylands, $31^{\circ} 56^{\prime} \mathrm{S}, 115^{\circ} 54^{\prime} \mathrm{E}$, WAM 84/219; Meckering, $31^{\circ} 38^{\prime} \mathrm{S}, \quad 117^{\circ} 00^{\prime} \mathrm{E}$, 
WAM 84/220; Meltham, $31^{\circ} 55^{\prime} \mathrm{S}, 115^{\circ} 54^{\prime} \mathrm{E}$, WAM 54/3085; Mirrabooka, $31^{\circ} 52^{\prime} \mathrm{S}$, $115^{\circ} 52^{\prime} \mathrm{E}$, WAM 96/1416; Mockerdillup Road, 34 $04^{\prime} \mathrm{S}, 116^{\circ} 06^{\prime} \mathrm{E}$, WAM 95/839; Monjinup, $33^{\circ} 49^{\prime} \mathrm{S}, 121^{\circ} 50^{\prime} \mathrm{E}, \mathrm{CBB}$; Moons Crossing, $34^{\circ} 30^{\prime} \mathrm{S}, 116^{\circ} 09^{\prime} \mathrm{E}$, WAM 95/967; Mosman Park, $32^{\circ} 01^{\prime} \mathrm{S}, 115^{\circ} 46^{\prime} \mathrm{E}$, WAM 95/ 927; Mount Barker, $34^{\circ} 38^{\prime} \mathrm{S}, 117^{\circ} 40^{\prime} \mathrm{E}$, WAM 95/867; Mount Claremont, 31 ${ }^{\circ} 58^{\prime} \mathrm{S}$, $115^{\circ} 46^{\prime} \mathrm{E}$, WAM 95/1087; Mount Hawthorn, $31^{\circ} 55^{\prime} \mathrm{S}, 115^{\circ} 50^{\prime} \mathrm{E}$, WAM 96/1414; Mount Helena, $31^{\circ} 52^{\prime} \mathrm{S}, 116^{\circ} 12^{\prime} \mathrm{E}$, WAM 95/831; Mount Henry, $32^{\circ} 02^{\prime} \mathrm{S}, 115^{\circ} 52^{\prime} \mathrm{E}$, WAM 95/ 1081; Mount Lawley, $31^{\circ} 56^{\prime} \mathrm{S}, 115^{\circ} 53^{\prime} \mathrm{E}$, WAM 84/222; Mount Pleasant, $32^{\circ} 02^{\prime} \mathrm{S}$, $115^{\circ} 51^{\prime} \mathrm{E}$, WAM 95/878; Mount Yokine, $31^{\circ} 54^{\prime} \mathrm{S}, 115^{\circ} 51^{\prime} \mathrm{E}$, WAM, 95/952; Mullaloo, $31^{\circ} 47^{\prime} \mathrm{S}, 115^{\circ} 44^{\prime} \mathrm{E}$, WAM 95/956; Mundaring, $31^{\circ} 54^{\prime} \mathrm{S}, 116^{\circ} 10^{\prime} \mathrm{E}$, WAM 84/224; Murray $\operatorname{Road}, 34^{\circ} 14^{\prime} \mathrm{S}, 119^{\circ} 17^{\prime} \mathrm{E}$, WAM 95/901; Nannup, 33 $59^{\prime} \mathrm{S}, 115^{\circ} 46^{\prime} \mathrm{E}$, WAM 84/225; Narrogin, $32^{\circ} 56^{\prime} \mathrm{S}, 117^{\circ} 11^{\prime} \mathrm{E}$, WAM 95/989; Nedlands, $31^{\circ} 59^{\prime} \mathrm{S}, 115^{\circ} 48^{\prime} \mathrm{E}$, WAM 95/915; Neerabup, $31^{\circ} 42^{\prime} \mathrm{S}, 115^{\circ} 47^{\prime} \mathrm{E}$, WAM 96/ 1424; Nornalup, $35^{\circ} 00^{\prime} \mathrm{S}, 116^{\circ} 49^{\prime} \mathrm{E}, \mathrm{AMNH}$; Northam, $31^{\circ} 39^{\prime} \mathrm{S}, 116^{\circ} 40^{\prime} \mathrm{E}$, WAM 95/893; Palmyra, $32^{\circ} 03^{\prime} \mathrm{S}, 115^{\circ} 47^{\prime} \mathrm{E}$, WAM 84/230; Parmelia, $32^{\circ} 15^{\prime} \mathrm{S}, 115^{\circ} 47^{\prime} \mathrm{E}$, WAM 95/892; Peppermint Grove, $32^{\circ} 00^{\prime} \mathrm{S}, 115^{\circ} 46^{\prime} \mathrm{E}$, WAM 95/822; Perth, $31^{\circ} 57^{\prime} \mathrm{S}, 115^{\circ} 52^{\prime} \mathrm{E}$, WAM 84/231; Quaalup Homestead, $34^{\circ} 17^{\prime} \mathrm{S}$, $119^{\circ} 26^{\prime} \mathrm{E}$, WAM 95/889; Quairading, $32^{\circ} 01^{\prime} \mathrm{S}, 117^{\circ} 24^{\prime} \mathrm{W}$, WAM 84/237; Quinns Rock, $31^{\circ} 41^{\prime} \mathrm{S}, 115^{\circ} 41^{\prime} \mathrm{W}$, WAM 96/1433; Rangeview, $28^{\circ} 46^{\prime} \mathrm{S}, 114^{\circ} 37 \mathrm{E}$, WAM 95/ 809; Ravensthorpe, $33^{\circ} 35^{\prime} \mathrm{S}, 120^{\circ} 02^{\prime} \mathrm{E}, \mathrm{CAS}$; Riverton, $32^{\circ} 02^{\prime} \mathrm{S}, 115^{\circ} 54^{\prime} \mathrm{E}$, WAM 95/791; Rivervale, $31^{\circ} 58^{\prime} \mathrm{S}, 115^{\circ} 55^{\prime} \mathrm{E}$, WAM 84/238; Rockingham, $32^{\circ} 17^{\prime} \mathrm{S}, 115^{\circ} 43^{\prime} \mathrm{E}$, WAM 95/ 840; Roleystone, $32^{\circ} 07^{\prime} \mathrm{S}, 116^{\circ} 04^{\prime} \mathrm{E}$, WAM 84/239; Rossmoyne, $32^{\circ} 02^{\prime} \mathrm{S}, 115^{\circ} 46^{\prime} \mathrm{E}$, WAM 84/1214; Savage Highway Quarry, $31^{\circ} 54^{\prime} \mathrm{S}, 127^{\circ} 01^{\prime} \mathrm{E}$, AMS KS17130; Shenton Park Bush, $31^{\circ} 58^{\prime} \mathrm{S}, 115^{\circ} 48^{\prime} \mathrm{E}$, WAM 96/ 1580; Sieda, $33^{\circ} 14^{\prime} \mathrm{S}, 121^{\circ} 46^{\prime} \mathrm{E}$, WAM 96/ 1578; Shenton Park, $31^{\circ} 58^{\prime} \mathrm{S}, 115^{\circ} 48^{\prime} \mathrm{E}$, WAM 84/241; South Perth, $31^{\circ} 59^{\prime} \mathrm{S}$, $115^{\circ} 52^{\prime}$ E, WAM 84/1216; South Tammin, $31^{\circ} 39^{\prime} \mathrm{S}, 117^{\circ} 29^{\prime} \mathrm{E}$, WAM 95/932; Spearwood, $32^{\circ} 06^{\prime} \mathrm{S}, 115^{\circ} 47^{\prime} \mathrm{E}$, WAM 84/681; Stirling Range Caravan Park, $34^{\circ} 19^{\prime} \mathrm{S}$, $118^{\circ} 12^{\prime} \mathrm{E}$, WAM 95/984; Subiaco, $31^{\circ} 57^{\prime} \mathrm{S}$, $115^{\circ} 50^{\prime} \mathrm{E}, \mathrm{ZMH}$; Sunset Beach, $28^{\circ} 46^{\prime} \mathrm{S}$, $114^{\circ} 37^{\prime} \mathrm{E}$, WAM 96/1431; Tammin, $31^{\circ} 39^{\prime} \mathrm{S}$, $117^{\circ} 29^{\prime} \mathrm{E}$, WAM 95/1085; Tenilba Park, $31^{\circ} 46^{\prime} \mathrm{S}, 116^{\circ} 28^{\prime} \mathrm{E}$, WAM 95/815; Tenterden, $34^{\circ} 22^{\prime} \mathrm{S}, 117^{\circ} 34^{\prime} \mathrm{E}$, AMS KS31852; Thampanna Cave, $31^{\circ} 42^{\prime} \mathrm{S}, 127^{\circ} 40^{\prime} \mathrm{E}$, WAM 95/ 794; Trayning, $31^{\circ} 07^{\prime} \mathrm{S}, 117^{\circ} 48^{\prime} \mathrm{E}$, WAM 95/ 828; Tuart Hill, $31^{\circ} 53^{\prime} \mathrm{S}, 115^{\circ} 52^{\prime} \mathrm{E}$, WAM 95/1076; Tumblo Block, $32^{\circ} 58^{\prime} \mathrm{S}, 116^{\circ} 10^{\prime} \mathrm{E}$, WAM 95/1158; University of Western Australia Research Park, $31^{\circ} 57^{\prime} \mathrm{S}, 115^{\circ} 48^{\prime} \mathrm{E}$,

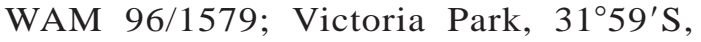
$115^{\circ} 53^{\prime} \mathrm{E}$, WAM 95/814; Walk Walkin, $30^{\circ} 49^{\prime} \mathrm{S}, 117^{\circ} 19^{\prime} \mathrm{E}$, WAM 95/879; West Cape Howe National Park, $35^{\circ} 05^{\prime} \mathrm{S}, 117^{\circ} 38^{\prime} \mathrm{E}$, WAM 95/902; Wicherina Dam, $28^{\circ} 43^{\prime} \mathrm{S}$, $115^{\circ} 00^{\prime} \mathrm{E}, \mathrm{AMNH}$; Willetton, $32^{\circ} 03^{\prime} \mathrm{S}$, $115^{\circ} 53^{\prime} \mathrm{E}$, WAM 84/246; Wiluna, $26^{\circ} 35^{\prime} \mathrm{S}$, $120^{\circ} 14^{\prime} \mathrm{E}$, WAM 84/245; Woodman Point, $32^{\circ} 08^{\prime} \mathrm{S}, 115^{\circ} 45^{\prime} \mathrm{E}$, WAM 95/1090; Woorree, $28^{\circ} 47^{\prime} \mathrm{S}, 114^{\circ} 39^{\prime} \mathrm{E}$, WAM 95/804; Yanchep, $31^{\circ} 33^{\prime} \mathrm{S}, 115^{\circ} 41^{\prime} \mathrm{E}$, WAM 95/788; Yokine, $31^{\circ} 54^{\prime} \mathrm{S}, 115^{\circ} 45^{\prime} \mathrm{E}$, WAM 95/792; Zuytdorp, $27^{\circ} 16^{\prime} \mathrm{S}, 114^{\circ} 09^{\prime} \mathrm{E}$, WAM 96/1513. NEW ZEALAND: South Island: Beckenham, LUNZ; Canterbury, LUNZ; Christchurch, FSCA; Halswell, AMNH; Lincoln, AMNH; McCormicks Bay, AMNH; Nelson, OMD; Richmond, OMD; Rolleston, LUNZ; Russley, AMNH; Springston, AMNH; Woolston, AMNH.

Distribution: Southern Australia, but rare along east coast (map 1). Apparently introduced into the South Island of New Zealand, perhaps more than once. The earliest record is the Dalmas holotype, collected in Nelson in 1913; there are additional records from the Nelson area in 1948 and 1966, but the remainder of the records are all subsequent to 1988.

NATURAl History: Adults have been collected year-round, most abundantly in summer (November through February), least commonly in winter (May through August). Females have been found with egg cases from September through April. Specimens have been taken at elevations up to $1240 \mathrm{~m}$, in gardens, heath, meadows, and dry sclerophyll forests; under casuarina, eucalypt, karri, mallee, redgum, and wandoo bark; in stumps, drier parts of the rocky littoral zone, and the twilight zone within $20 \mathrm{~m}$ of a cave entrance; in drift fence, flight intercept, and wet and dry pitfall traps, at lights, and by 
vibration; under bricks, logs, and wood; in houses, sheds, swimming pools, beds, parcels, luggage, cupboards, drawers of clothes, handbags, sinks, bathtubs, museum exhibition cases, lawn mowers, a photo album, a cow skull, and a plastic-wrapped head of lettuce. The favored prey seems to be other spiders, and specimens have been found in the webs of (and eating) mygalomorphs (Cethegus Thorell, Dipluridae), pholcids, redbacks (Latrodectus hasselti Thorell, Theridiidae), and desids, especially Badumna insignis (L. Koch) and Badumna longinqua (L. Koch).

Bite records from authenticated specimens include the following: bit patient on neck, producing local muscle spasms, swelling, and local reaction; bit woman on arm, arm sore for 4 days; bit girl on neck while in bath, causing swelling that went down by next morning; bit 4-year-old child on chest, child developed itchy weal, no further problems; bites produced large weals that remained sore for 3-4 weeks.

SyNONYMY: The name Lampona pseudocylindrata was provided by Strand (1922: 143) for specimens he considered to have been misidentified as L. cylindrata by Simon (1893, 1908). Strand was correct in arguing that Simon's males of L. cylindrata are not conspecific with the male from Cape York, Queensland, studied by Strand and correctly identified by him as the first described male of L. murina. However, Strand's argument that Simon's specimens are also not conspecific with the lectotype male of $L$. cylindrata was based only on published illustrations and is incorrect.

\section{Lampona murina $\mathrm{L}$. Koch \\ Figures 48-50, 57-62; Map 2}

Lampona murina L. Koch, 1873: 378, pl. 29, figs. 7, 7a (four female syntypes: one from Port Mackay, Queensland, in ZMH, examined; one from same locality, in NMW, examined; one from same locality, in BMNH, examined; one from Australia [as "Neuholland”], no specific locality, in NRS, examined).

Drassus formicarius Urquhart, 1887: 109 (juvenile male and adult female syntypes from Waiwera and Drury, North Island, New Zealand, lost). NEW SYNONYMY.

Herpyllus formicarius: Urquhart, 1892: 221.

Lampona cylindrata (misidentification): Forster,
1979: 71, figs. 282-285 (North Island records and genitalic illustrations).

DiAgnosis: This species has often been confused with $L$. cylindrata but can be distinguished by the characters discussed in the diagnosis of that species.

MALE: Total length 9.2. Coloration as in $L$. cylindrata. Palpal femur relatively short, thick; retrolateral tibial apophysis relatively short, greatly widened at base, shorter than tibia itself, greatly narrowed at tip (fig. 60); tegulum globose, extending proximally to about half length of tibia; embolus dorsoventrally flattened, membranous conductor bipartite, medial part forming expanded, anteriorly rounded lobe covering base of lateral part, which extends to embolar tip (fig. 59).

Female: Total length 12.6. Coloration as in L. cylindrata. Epigynum separated from booklung covers by soft cuticle, consisting of narrow, elongate, excavate oval fossa (usually filled with plug of sticky secretion), variable in width, anterior rim arched, elevated (sometimes with median extension to base of excavation), openings at posterior edges of lateral rims, separated by pair of slightly to distinctly raised longitudinal ridges (fig. 61); spermathecae widened posteriorly, with distinctly offset anterior tips (fig. 62).

Selected Records: AUSTRALIA: Australian Capital Territory: Canberra, $35^{\circ} 20^{\prime} \mathrm{S}$, $149^{\circ} 10^{\prime} \mathrm{E}$, HNHM. Lord Howe Island: Salmon Beach, $31^{\circ} 33^{\prime} \mathrm{S}, 159^{\circ} 05^{\prime} \mathrm{E}$, AMS KS31867. New South Wales: Bankstown, $33^{\circ} 55^{\prime} \mathrm{S}, 151^{\circ} 03^{\prime} \mathrm{E}$, AMS KS31875; Bawley Point, $35^{\circ} 30^{\prime} \mathrm{S}, 150^{\circ} 24^{\prime} \mathrm{E}, \mathrm{QMB}$ S28542; Beecroft, $33^{\circ} 45^{\prime} \mathrm{S}, \quad 151^{\circ} 04^{\prime} \mathrm{E}, \quad$ AMS KS55261; Bexley, $33^{\circ} 56^{\prime} \mathrm{S}, 151^{\circ} 05^{\prime} \mathrm{E}$, AMS KS31927; Blacktown, $33^{\circ} 46^{\prime} \mathrm{S}, 150^{\circ} 55^{\prime} \mathrm{E}$,

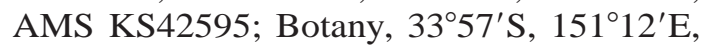
AMS KS31869; Brooklana, $30^{\circ} 16^{\prime} \mathrm{S}$, $152^{\circ} 53^{\prime} \mathrm{E}$, AMS KS31874; Carlingford, $33^{\circ} 48^{\prime} \mathrm{S}, 151^{\circ} 02^{\prime} \mathrm{E}$, AMS KS31923; Chatswood, $33^{\circ} 48^{\prime} \mathrm{S}, 151^{\circ} 11^{\prime} \mathrm{E}$, AMS KS31855; Collaroy, $\quad 33^{\circ} 45^{\prime} \mathrm{S}, \quad 151^{\circ} 15^{\prime} \mathrm{E}, \quad \mathrm{AMS}$ KS31925; Condong Falls, $28^{\circ} 37^{\prime} \mathrm{S}$, $153^{\circ} 23^{\prime} \mathrm{E}$, QMB; Corunna Lake, $36^{\circ} 17^{\prime} \mathrm{S}$, $150^{\circ} 06^{\prime} \mathrm{E}, \mathrm{AMNH}$; Croydon, $33^{\circ} 53^{\prime} \mathrm{S}$, $151^{\circ} 07^{\prime} \mathrm{E}$, AMS KS40979; Croydon Park, $33^{\circ} 54^{\prime} \mathrm{S}, 151^{\circ} 08^{\prime} \mathrm{E}$, AMS KS31884; Cundletown, $31^{\circ} 54^{\prime} \mathrm{S}, 152^{\circ} 33^{\prime} \mathrm{E}$, AMS KS55264; Dubbo, $32^{\circ} 15^{\prime} \mathrm{S}, 148^{\circ} 37^{\prime} \mathrm{E}$, AMS KS31935; 

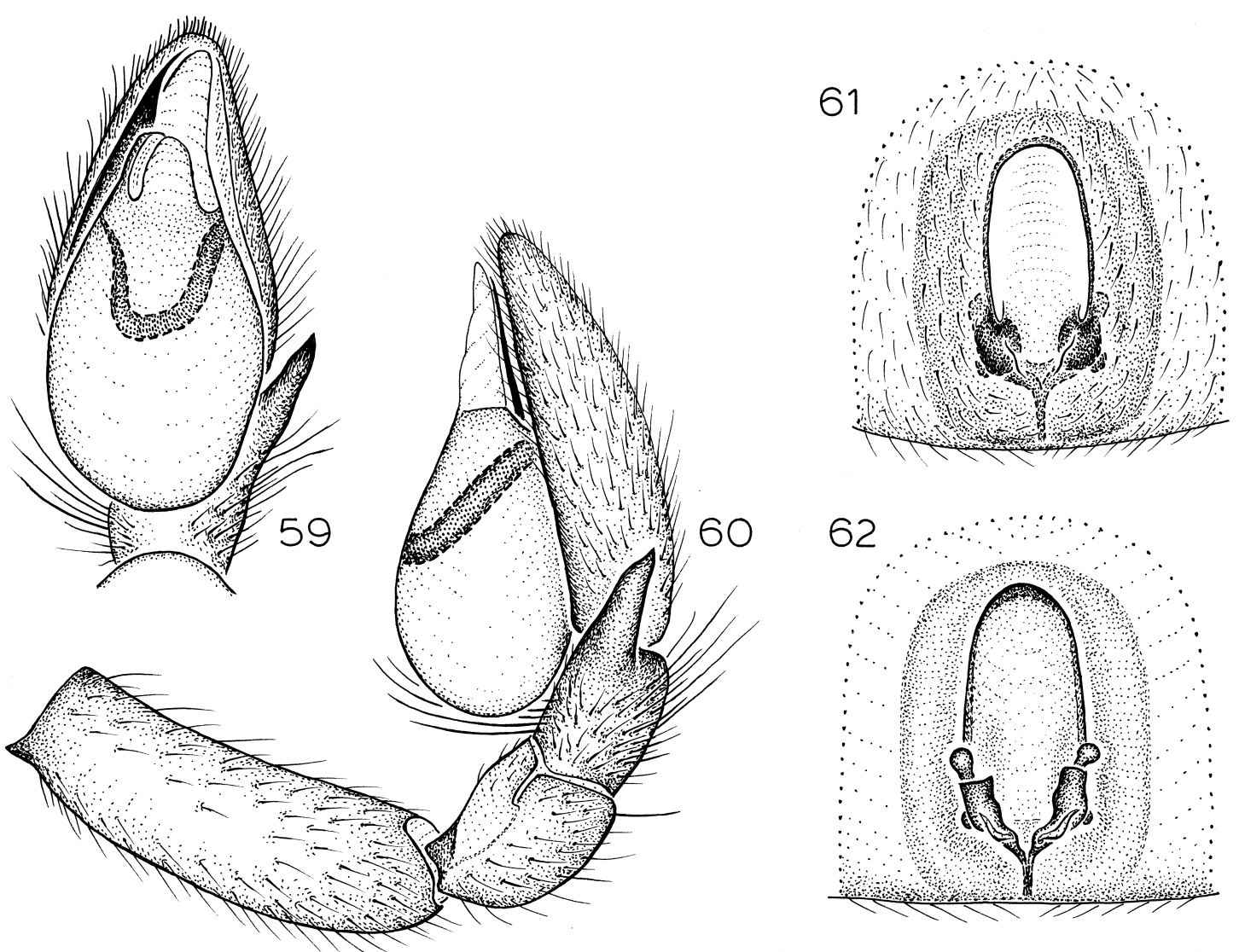

Figs. 59-62. Lampona murina L. Koch. 59. Left male palp, ventral view. 60. Same, retrolateral view. 61. Epigynum, ventral view. 62. Same, dorsal view.

East Kurrajong, $33^{\circ} 31^{\prime} \mathrm{S}, 150^{\circ} 47^{\prime} \mathrm{E}$, AMS KS31840; Five Dock, $33^{\circ} 40^{\prime} \mathrm{S}, 151^{\circ} 06^{\prime} \mathrm{E}$, AMS KS31873; Gerroa, $34^{\circ} 46^{\prime} \mathrm{S}, 150^{\circ} 49^{\prime} \mathrm{E}$, AMS KS30242; Gladesville, $33^{\circ} 50^{\prime} \mathrm{S}$, $151^{\circ} 08^{\prime} \mathrm{E}, \mathrm{OMD}$; Gwydir Road, $29^{\circ} 56^{\prime} \mathrm{S}$, $150^{\circ} 35^{\prime} \mathrm{E}, \mathrm{AMS} \mathrm{KS} 29926$; Heathcote, $34^{\circ} 05^{\prime} \mathrm{S}, 151^{\circ} 02^{\prime} \mathrm{E}$, AMS KS31924; Kensington, $33^{\circ} 55^{\prime} \mathrm{S}, 151^{\circ} 15^{\prime} \mathrm{S}$, AMS KS31929; Kyogle, $28^{\circ} 37^{\prime} \mathrm{S}, 153^{\circ} 00^{\prime} \mathrm{E}, \mathrm{QMB}$ S21628; Lane Cove, $33^{\circ} 53^{\prime} \mathrm{S}, 151^{\circ} 12^{\prime} \mathrm{E}$, SAM N1996/ 296; Lawson, $33^{\circ} 43^{\prime} \mathrm{S}, 150^{\circ} 26^{\prime} \mathrm{E}$, AMS KS16336; Malabar, $33^{\circ} 58^{\prime} \mathrm{S}, 151^{\circ} 14^{\prime} \mathrm{E}$, AMS KS31921; Maroubra, $33^{\circ} 57^{\prime} \mathrm{S}, 151^{\circ} 15^{\prime} \mathrm{E}$, AMS KS42372; Minnamurra Falls, $34^{\circ} 28^{\prime} \mathrm{S}$, 150 51'E, AMS KS31938; Mosman, $33^{\circ} 50^{\prime} \mathrm{S}, 151^{\circ} 15^{\prime} \mathrm{E}$, AMS KS5188; Mount Tindal Road, $29^{\circ} 42^{\prime} \mathrm{S}, 152^{\circ} 37^{\prime} \mathrm{E}$, AMS KS40495; Mudgee, $32^{\circ} 36^{\prime} \mathrm{S}, 149^{\circ} 35^{\prime} \mathrm{E}$, AMS KS42636; Murwillumbah, $28^{\circ} 20^{\prime} \mathrm{S}$, $153^{\circ} 24^{\prime} \mathrm{E}, \mathrm{QMB}$ S28071; Nadgee Nature Re- serve, $37^{\circ} 27^{\prime} \mathrm{S}, 149^{\circ} 59^{\prime} \mathrm{E}$, AMS KS2268; Naremburn, $33^{\circ} 53^{\prime} \mathrm{S}, 151^{\circ} 12^{\prime} \mathrm{E}$, MCZ; Neutral Bay, $33^{\circ} 49^{\prime} \mathrm{S}, 151^{\circ} 14^{\prime} \mathrm{E}$, AMS KS31930; Pacific Palms, $32^{\circ} 21^{\prime} \mathrm{S}, 152^{\circ} 31^{\prime} \mathrm{E}$, AMS KS55268; Randwick, $33^{\circ} 55^{\prime} \mathrm{S}, 151^{\circ} 15^{\prime} \mathrm{E}$, AMS KS19897; Rockdale, 33 ${ }^{\circ} 53^{\prime} \mathrm{S}$, $151^{\circ} 13^{\prime} \mathrm{E}$, AMS KS31915; Royal National Park, $34^{\circ} 08^{\prime} \mathrm{S}, 151^{\circ} 04^{\prime} \mathrm{E}$, CAS; Rydalmere, $33^{\circ} 47^{\prime} \mathrm{S}, 151^{\circ} 03^{\prime} \mathrm{E}$, AMS KS31876; Scalloway, $34^{\circ} 45^{\prime} \mathrm{S}, 150^{\circ} 50^{\prime} \mathrm{E}$, AMS KS17424; South Camden, $34^{\circ} 03^{\prime} \mathrm{S}, 151^{\circ} 42^{\prime} \mathrm{E}$, AMS KS31917; Springwood, $33^{\circ} 42^{\prime} \mathrm{S}, 150^{\circ} 34^{\prime} \mathrm{E}$, AMS KS31931; Surry Hills, 3351'S, $151^{\circ} 13^{\prime} \mathrm{E}$, AMS KS31926; Strathfield, $33^{\circ} 52^{\prime} \mathrm{S}, 151^{\circ} 06^{\prime} \mathrm{E}$, AMS KS31918; Sydney, $33^{\circ} 53^{\prime} \mathrm{S}, 151^{\circ} 13^{\prime} \mathrm{E}$, AMS KS31957; Thredbo Camping Ground, $36^{\circ} 30^{\prime} \mathrm{S}, 148^{\circ} 19^{\prime} \mathrm{E}$, SAM N1996/155; Tugalow Creek, 31 ${ }^{\circ} 54^{\prime} \mathrm{S}$, $151^{\circ} 26^{\prime} \mathrm{E}$, AMS KS40494; Wallacia, $33^{\circ} 52^{\prime} \mathrm{S}, 150^{\circ} 39^{\prime} \mathrm{E}$, AMS KS31936; Woom- 
bah, $29^{\circ} 22^{\prime} \mathrm{S}, 153^{\circ} 17^{\prime} \mathrm{E}$, QMB S30858. Norfolk Island: Anson Bay Reserve, $29^{\circ} 01^{\prime} \mathrm{S}$, $167^{\circ} 55^{\prime} \mathrm{E}, \mathrm{QMB}$; near Highlands Guesthouse, $29^{\circ} 02^{\prime} \mathrm{S}, 167^{\circ} 57^{\prime} \mathrm{E}$, QMB. Queensland: Albany Creek, $27^{\circ} 21^{\prime} \mathrm{S}, 152^{\circ} 58^{\prime} \mathrm{E}$, QMB S15661; Ashmore, $28^{\circ} 00^{\prime} \mathrm{S}, 153^{\circ} 22^{\prime} \mathrm{E}$, QMB S21554; Aspley, $27^{\circ} 22^{\prime} \mathrm{S}, 153^{\circ} 01^{\prime} \mathrm{E}$, QMB S25986; Bahr's Scrub, 27²5'S, $153^{\circ} 10^{\prime} \mathrm{E}$, QMB S28066; Bald Hills, $27^{\circ} 19^{\prime} \mathrm{S}, 153^{\circ} 01^{\prime} \mathrm{E}$, QMB S3638; Ballandean, $28^{\circ} 48^{\prime} \mathrm{S}, 151^{\circ} 50^{\prime} \mathrm{E}, \mathrm{QMB}$ S28064; Beenleigh, $27^{\circ} 43^{\prime} \mathrm{S}, \quad 153^{\circ} 12^{\prime} \mathrm{E}, \quad \mathrm{QMB}$ S28069; Beerburrum, $26^{\circ} 58^{\prime} \mathrm{S}, 152^{\circ} 58^{\prime} \mathrm{E}$, QMB S28068; Beerwah, 26 $51^{\circ} \mathrm{S}, 152^{\circ} 58^{\prime} \mathrm{E}$, QMB S4541; Bellthorpe, 26 $6^{\circ} 1^{\prime} \mathrm{S}, 152^{\circ} 43^{\prime} \mathrm{E}$, QMB S21522; Benowa, $28^{\circ} 01^{\prime} \mathrm{S}, 153^{\circ} 23^{\prime} \mathrm{E}$, QMB S28070; Binjour Plateau, $25^{\circ} 33^{\prime} \mathrm{S}$, $151^{\circ} 28^{\prime} \mathrm{E}$, QMB S44222; Birkdale, $27^{\circ} 29^{\prime} \mathrm{S}$, $153^{\circ} 13^{\prime} \mathrm{E}$, QMB S21275; Boggomoss, $25^{\circ} 26^{\prime} \mathrm{S}, 150^{\circ} 01^{\prime} \mathrm{E}$, QMB S36291; Bottle Tree Bluff, $26^{\circ} 50^{\prime} \mathrm{S}, 151^{\circ} 33^{\prime} \mathrm{E}$, QMB S28024; Brisbane, $27^{\circ} 30^{\prime} \mathrm{S}, 153^{\circ} 01^{\prime} \mathrm{E}, \mathrm{QMB}$ S25967; Broken River Camp, 20 55'S, $148^{\circ} 30^{\prime} \mathrm{E}$, CAS; Bunya Mountains, $26^{\circ} 50^{\prime} \mathrm{S}$, $151^{\circ} 33^{\prime} \mathrm{E}, \mathrm{CBB}$; Burleigh Heads, $28^{\circ} 06^{\prime} \mathrm{S}$, $153^{\circ} 27^{\prime} \mathrm{E}, \mathrm{QMB}$ S25995; Caboolture, $27^{\circ} 05^{\prime} \mathrm{S}, 152^{\circ} 57^{\prime} \mathrm{E}$, QMB S25992; Caloundra, $26^{\circ} 48^{\prime} \mathrm{S}, 153^{\circ} 08^{\prime} \mathrm{E}$, QMB S25996; Canungra, $28^{\circ} 02^{\prime} \mathrm{S}, 153^{\circ} 10^{\prime} \mathrm{E}, \mathrm{QMB} \mathrm{S} 28054$; Cherwell River, $25^{\circ} 15^{\prime} \mathrm{S}, 152^{\circ} 16^{\prime} \mathrm{E}$, QMB; Cooloola, $26^{\circ} 12^{\prime} \mathrm{S}, 153^{\circ} 03^{\prime} \mathrm{E}$, QMB S26888; Coolum, $26^{\circ} 33^{\prime} \mathrm{S}, 153^{\circ} 05^{\prime} \mathrm{E}$, NMV K2989;

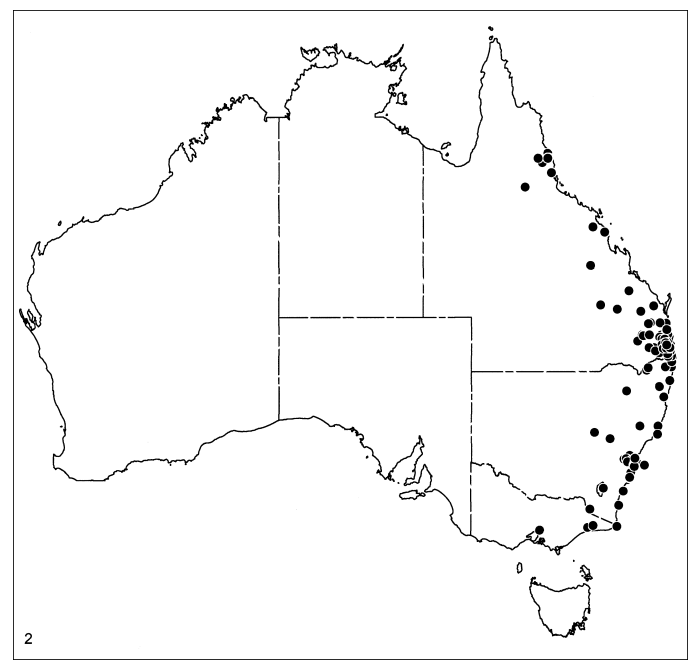

Map 2. Australian records of Lampona murina L. Koch.
Cunningham National Park, $27^{\circ} 50^{\prime} \mathrm{S}$, $152^{\circ} 37^{\prime} \mathrm{E}, \mathrm{QMB}$; Danbulla State Forest, $17^{\circ} 09^{\prime} \mathrm{S}, 145^{\circ} 37^{\prime} \mathrm{E}$, JAM 21340; Einasleigh River, $18^{\circ} 43^{\prime} \mathrm{S}, 144^{\circ} 20^{\prime} \mathrm{E}, \mathrm{CBB}$; Expedition Range National Park, $25^{\circ} 12^{\prime} \mathrm{S}, 148^{\circ} 59^{\prime} \mathrm{E}$, QMB S42439; Ferny Grove, $27^{\circ} 24^{\prime} \mathrm{S}$, $152^{\circ} 56^{\prime} \mathrm{E}, \mathrm{QMB}$ S7001; Freshwater, $16^{\circ} 53^{\prime} \mathrm{S}$, $145^{\circ} 43^{\prime} \mathrm{E}$, FSCA; Gatton, $27^{\circ} 33^{\prime} \mathrm{S}, 152^{\circ} 17^{\prime} \mathrm{E}$, QMB S30157; Goomeri, $26^{\circ} 11^{\prime} \mathrm{S}, 152^{\circ} 04^{\prime} \mathrm{E}$, QMB S17689; Gordonvale, $17^{\circ} 08^{\prime} \mathrm{S}$, $145^{\circ} 45^{\prime} \mathrm{E}, \quad \mathrm{AMNH}$; Gympie, $26^{\circ} 11^{\prime} \mathrm{S}$, $152^{\circ} 40^{\prime} \mathrm{E}, \mathrm{QMB}$ S30165; Herberton, $17^{\circ} 23^{\prime} \mathrm{S}, 145^{\circ} 23^{\prime} \mathrm{E}$, AMS KS31939; Kroombit Tops, $24^{\circ} 26^{\prime} \mathrm{S}, 150^{\circ} 43^{\prime} \mathrm{E}$, QMB S28007; Lake Broadwater, $27^{\circ} 11^{\prime} \mathrm{S}, 151^{\circ} 16^{\prime} \mathrm{E}$, QMB S28029; Lamington National Park, $27^{\circ} 27^{\prime} \mathrm{S}$, $153^{\circ} 02^{\prime} \mathrm{E}, \mathrm{QMB}$ S17674; Little Mulgrave, $17^{\circ} 09^{\prime} \mathrm{S}, \quad 145^{\circ} 43^{\prime} \mathrm{E}$, FSCA; Loganlea, $27^{\circ} 40^{\prime} \mathrm{S}, 1^{\circ} 03^{\circ} 08^{\prime} \mathrm{E}$, QMB S28056; Logan River, $27^{\circ} 43^{\prime} \mathrm{S}, 153^{\circ} 18^{\prime} \mathrm{E}, \mathrm{CBB}$; Margate, $27^{\circ} 15^{\prime} \mathrm{S}, 153^{\circ} 07^{\prime} \mathrm{E}$, QMB S30156; Marlaybrook, 26 $53^{\prime} \mathrm{S}, 151^{\circ} 37^{\prime} \mathrm{E}, \mathrm{QMB}$ S28028; Morningside, $27^{\circ} 29^{\prime} \mathrm{S}, 153^{\circ} 08^{\prime} \mathrm{E}$, QMB S3622; Mount Archer, $26^{\circ} 59^{\prime} \mathrm{S}, 152^{\circ} 38^{\prime} \mathrm{E}$, QMB S28059; Mount Cotton, $27^{\circ} 36^{\prime} \mathrm{S}$, $153^{\circ} 13^{\prime} \mathrm{E}, \mathrm{QMB}$ S42481; Mount Mee, $27^{\circ} 05^{\prime} \mathrm{S}, 152^{\circ} 46^{\prime} \mathrm{E}$, QMB S15648; Mount Nebo, $27^{\circ} 23^{\prime} \mathrm{S}, 152^{\circ} 47^{\prime} \mathrm{E}, \mathrm{QMB}$ S28048; Mulgowie, $27^{\circ} 44^{\prime} \mathrm{S}, 152^{\circ} 22^{\prime} \mathrm{E}$, QMB S25972; Murgon, $26^{\circ} 14^{\prime} \mathrm{S}, 151^{\circ} 57^{\prime} \mathrm{E}$, QMB S25968; Murrarie, Brisbane, $27^{\circ} 30^{\prime} \mathrm{S}$, $153^{\circ} 01^{\prime} \mathrm{E}, \mathrm{QMB}$ S30159; Petrie, $27^{\circ} 16^{\prime} \mathrm{S}$, $152^{\circ} 59^{\prime} \mathrm{E}, \mathrm{QMB}$ S20313; Port Mackay, $21^{\circ} 12^{\prime} \mathrm{S}, 149^{\circ} 13^{\prime} \mathrm{E}, \mathrm{BMNH}$ 1915.3.5.5751; Riverview, $27^{\circ} 36^{\prime} \mathrm{S}, 152^{\circ} 51^{\prime} \mathrm{E}, \mathrm{QMB}$ S21115; Rochedale, $27^{\circ} 34^{\prime} \mathrm{S}, 153^{\circ} 08^{\prime} \mathrm{E}$, QMB S28034; South Brisbane, $27^{\circ} 29^{\prime} \mathrm{S}$, $153^{\circ} 01^{\prime} \mathrm{E}, \mathrm{QMB}$ S30164; Stafford, $27^{\circ} 24^{\prime} \mathrm{S}$, $153^{\circ} 01^{\prime} \mathrm{E}, \mathrm{QMB}$ S28032; Stanthorpe, $28^{\circ} 40^{\prime} \mathrm{S}, 151^{\circ} 56^{\prime} \mathrm{E}$, QMB S30170; Strathpine, $27^{\circ} 17^{\prime} \mathrm{S}, 153^{\circ} 00^{\prime} \mathrm{E}$, QMB S30163; Sunnybank, $27^{\circ} 35^{\prime} \mathrm{S}, 153^{\circ} 03^{\prime} \mathrm{E}, \mathrm{QMB}$ S28012; Tambourine, $27^{\circ} 53^{\prime} \mathrm{S}, 153^{\circ} 08^{\prime} \mathrm{E}, \mathrm{CBB}$; Thornlands, $27^{\circ} 33^{\prime} \mathrm{S}, 153^{\circ} 16^{\prime} \mathrm{E}$, QMB S26030; Tieri, $23^{\circ} 02^{\prime} \mathrm{S}, 148^{\circ} 22^{\prime} \mathrm{E}$, QMB S30208; Toowoomba, $27^{\circ} 33^{\prime} \mathrm{S}, 151^{\circ} 58^{\prime} \mathrm{E}$, QMB S28020; Tully, $17^{\circ} 56^{\prime} \mathrm{S}, 145^{\circ} 56^{\prime} \mathrm{E}$, SAM N1996/293; Villeneuve, 26 $6^{\circ} 58^{\prime} \mathrm{S}$, $152^{\circ} 38^{\prime} \mathrm{E}, \mathrm{QMB}$ S28055; Walsh River, $17^{\circ} 09^{\prime} \mathrm{S}, 145^{\circ} 07^{\prime} \mathrm{E}, \mathrm{CBB}$; West Chermside, $27^{\circ} 24^{\prime} \mathrm{S}, 153^{\circ} 04^{\prime} \mathrm{E}, \mathrm{QMB}$ S28017; West End, $27^{\circ} 29^{\prime} \mathrm{S}, 153^{\circ} 00^{\prime} \mathrm{E}, \mathrm{QMB}$ S22434; Yarraman, $26^{\circ} 50^{\prime} \mathrm{S}, 151^{\circ} 59^{\prime} \mathrm{E}$, QMB S28025. Victoria: 
Buchan, $37^{\circ} 30^{\prime} \mathrm{S}, 148^{\circ} 11^{\prime} \mathrm{E}$, NMV K2965; Hurstbridge, $37^{\circ} 39^{\prime} \mathrm{S}, 145^{\circ} 13^{\prime} \mathrm{S}$, NMV K2992; Sardine Coupe, $37^{\circ} 24^{\prime} \mathrm{S}, 148^{\circ} 31^{\prime} \mathrm{E}$, NMV K3562. NEW ZEALAND: Kermadec Islands: SE Denham Bay, NZAC; Raoul Island, MONZ. North Island: Aramaho, OMD; Auckland, NZAC; Avondale, NZAC; Beechlands, OMD; Berhamphore, MONZ; Birkenhead, MONZ; Castlecliff, OMD; Chelsea Refinery, OMD; Cuvier Island, OMD; $10 \mathrm{mi} \mathrm{N}$ Dargaville, OMD; Epsom, NZAC; Foxton, OMD; Glen Eden, NZAC; Glenfield, AMNZ 6017; Grafton, AMNZ 6021; Greenhithe, OMD; Helensville, MONZ; Howick, NZAC; Island Bay, MONZ; Jacks Bay, AMNZ 6015; Judgeford, MONZ; Kamo, AMNZ 6019; Kawa, MONZ; Kawakawa, OMD; Levin, MONZ; Lower Hutt, MONZ; Maarama Crescent, MONZ; Mairangi Bay, AMNZ 6024; Manaia, MONZ; Manawahe, QMB; Mangere, NZAC; Mangonui, OMD; Manurewa, NZAC; Masterton, OMD; Matamata, OMD; Mercury Island, NZAC; Millstream Falls, QMB S34404; Mount Albert, NZAC; Mount Eden, AMNZ 6027; Mount Holdsworth, OMD; Mount Roskill, NZAC; Muriwai Beach, MONZ; Napier, OMD; New Lynn, NZAC; New Plymouth, OMD; Newtown, MONZ; Ngapuhi Road, NZAC; Otaihanga, MONZ; Otorohanga, OMD; Owairaka, NZAC; Paihia, OMD; Pakuranga, NZAC; Palmerston North, MONZ; Parnell, AMNZ 6014; Pukekohe, QMB S30174; Remuera, AMNZ 6036; Riverbend Road, QMB; Rotorua, MNHN AR3088; Ruawai, OMD; Sandringham, AMNZ 6034; Stokes Valley, MONZ; Sunnyvale, AMNZ 6016; Taita, MONZ; Takapuna, AMNZ 6023; Taradale, OMD; Taranaki, MONZ; Titirangi, AMS KS31610; Unuwhau, NZAC; Waiheke Island, MONZ; Waipoua State Forest Lookout, NZAC; Waitarere, MONZ; Wellington, OMD; Westmere, OMD; Whangarei, NZAC.

Distribution: Eastern Australia (map 2). Apparently introduced into Lord Howe Island, Norfolk Island, the Kermadec Islands, and the North Island of New Zealand (where it has been taken repeatedly for more than a century).

NATURAl History: Adults have been collected year round, most abundantly in summer (December through March), least com- monly in fall and winter (April through June). Specimens have been taken at elevations up to $1270 \mathrm{~m}$, in gardens, vine scrub, and open, dry, and rain forests, under logs and eucalypt, lagunaria, yellow box, and river red gum bark, in houses, sheds, swimming pools, and pitfall and intercept traps.

There is a bite record involving an authenticated specimen (from New Plymouth, North Island, New Zealand, in CLF): bit man on front of upper leg, bite felt, spider captured, area of bite swelled up like a boil, massive bright red erythema over much of front of leg above knee.

SYNONYMY: Lampona murina was placed as a junior synonym of L. cylindrata by Dalmas (1917), but that synonymy was correctly rejected by Strand (1922), who first described the male of L. murina. Drassus formicarius was also placed as a junior synonym of L. cylindrata by Dalmas (1917), but the type locality, from the northern end of the North Island of New Zealand, suggests that Urquhart had this species rather than $L$. cylindrata.

\section{Lampona papua, new species} Figures 63-65

TYPE: Female holotype taken under bark of Araucaria cunninghami (middle stem, tree no. 7) at Beenleigh Lake, Wau, Morobe District, Papua New Guinea, $7^{\circ} 20^{\prime} \mathrm{S}, 146^{\circ} 40^{\prime} \mathrm{E}$ (Sept. 22, 1970; B. Gray), deposited in AMS (KS13258).

ETYMOLOGY: The specific name is a noun in apposition taken from the type locality.

Diagnosis: This species resembles $L$. $c y$ lindrata and $L$. murina but can be easily distinguished by the shape of the spermathecae, which have laterally directed tips (figs. 64, 65).

MALE: Unknown.

Female: Total length 8.8. Coloration as in L. cylindrata except abdomen uniformly brownish gray. Epigynum separated from booklung covers by soft cuticle, consisting of narrow, elongate, excavate oval fossa, slightly wider anteriorly than posteriorly, anterior rim arched, elevated, openings at posterior edges of lateral rims, without distinct longitudinal ridges (fig. 63); spermathecae with laterally directed tips (figs. 64, 65). 

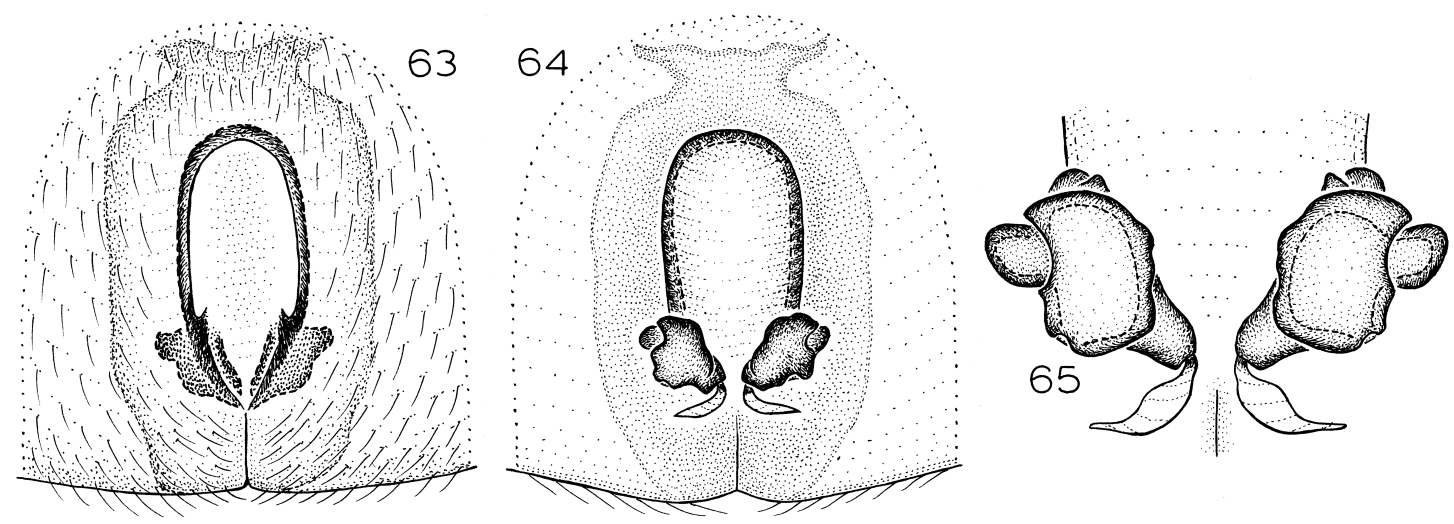

Figs. 63-65. Lampona papua, new species. 63. Epigynum, ventral view. 64. Same, dorsal view. 65. Spermathecae, dorsal view.

Other Material ExAmined: One female taken with the holotype (AMS), and one female also taken under bark of Araucaria cunninghami (lower stem, tree no. 2) at Stony Lake, Bulolo, Morobe District, Papua New Guinea, 6 ${ }^{\circ} 45^{\prime} \mathrm{S}, 146^{\circ} 30^{\prime} \mathrm{E}$ (Oct. 18, 1970; B. Webber, AMS KS13216).

DistRIBUTION: Known only from Papua New Guinea.

\section{THE RUIDA GROUP}

This group includes three species in which the male retrolateral apophysis is relatively long, distally widened, and distally flattened; the palpal bulb is relatively long. The spermathecae are large, long, and bipartite.

\section{Lampona ruida $\mathrm{L}$. Koch \\ Figures 41, 66-69; Map 3}

Latona cylindrata (misidentification): L. Koch, 1866: 3, pl. 1, fig. 1 (female only, misidentified).

Lampona ruida L. Koch, 1873: 375, pl. 29, figs. 5, 5a (female holotype from Australia [as "Neuholland"], no specific locality, in BMNH, examined).

Lampona sub-aquila Urquhart, 1893: 96 (female holotype from Launceston, Tasmania, depository unknown, lost). NEW SYNONYMY.

Lampona subaquila: Roewer, 1955: 473.

Diagnosis: This taxon seems to be eastern sister species of $L$. foliifera Simon, which it resembles in having a dorsoventrally flattened tip of the retrolateral tibial apophysis in males and a deeply depressed epigynal fossa and greatly enlarged, bipartite spermathecae in females. Males can be distinguished from those of L. foliifera by the subbasal knob on the palpal femur (fig. 67), females by the much wider epigynal fossa (fig. 68).

MALE: Total length 7.1. Abdomen dark gray, posterior one-fourth of dorsum with light chevrons (as in fig. 41). Legs orange, leg I darkest, without dark annulations. Palpal femur with subbasal knob protruding from ventral surface; retrolateral tibial apophysis relatively long, straight, dorsoventrally flattened at tip (fig. 67); tegulum globose, extending to half length of tibia; embolus long, originating on proximal half of bulb, tip greatly narrowed; conductor bifid, with distinctly sclerotized prolateral rim of prolateral portion, which extends to embolar tip, retrolateral portion extending even further anteriorly (fig. 66).

Female: Total length 9.9. Coloration as in male. Epigynum with deeply excavated, almost circular anterior fossa with bipartite anterior rim (fig. 68); spermathecae huge, almost bean-shaped, with sharply demarcated anterior and posterior portions (fig. 69).

Material Examined: AUSTRALIA: no specific locality (BMNH 1915.3.5.5752), 1 우 (holotype), (Kaufmann, FSCA), 19 . New South Wales: Bimbadeen Lookout, SW Cessnock, $32^{\circ} 50^{\prime} \mathrm{S}, 151^{\circ} 21^{\prime} \mathrm{E}$, Apr. 21, 1990 (D. Hirst, SAM N1996/329), 1ठ; Flaggy Creek, Wallaroo State Forest, $0.95 \mathrm{~km} \mathrm{~S}$ on Ripley's Trail from Clarence Town Road, 

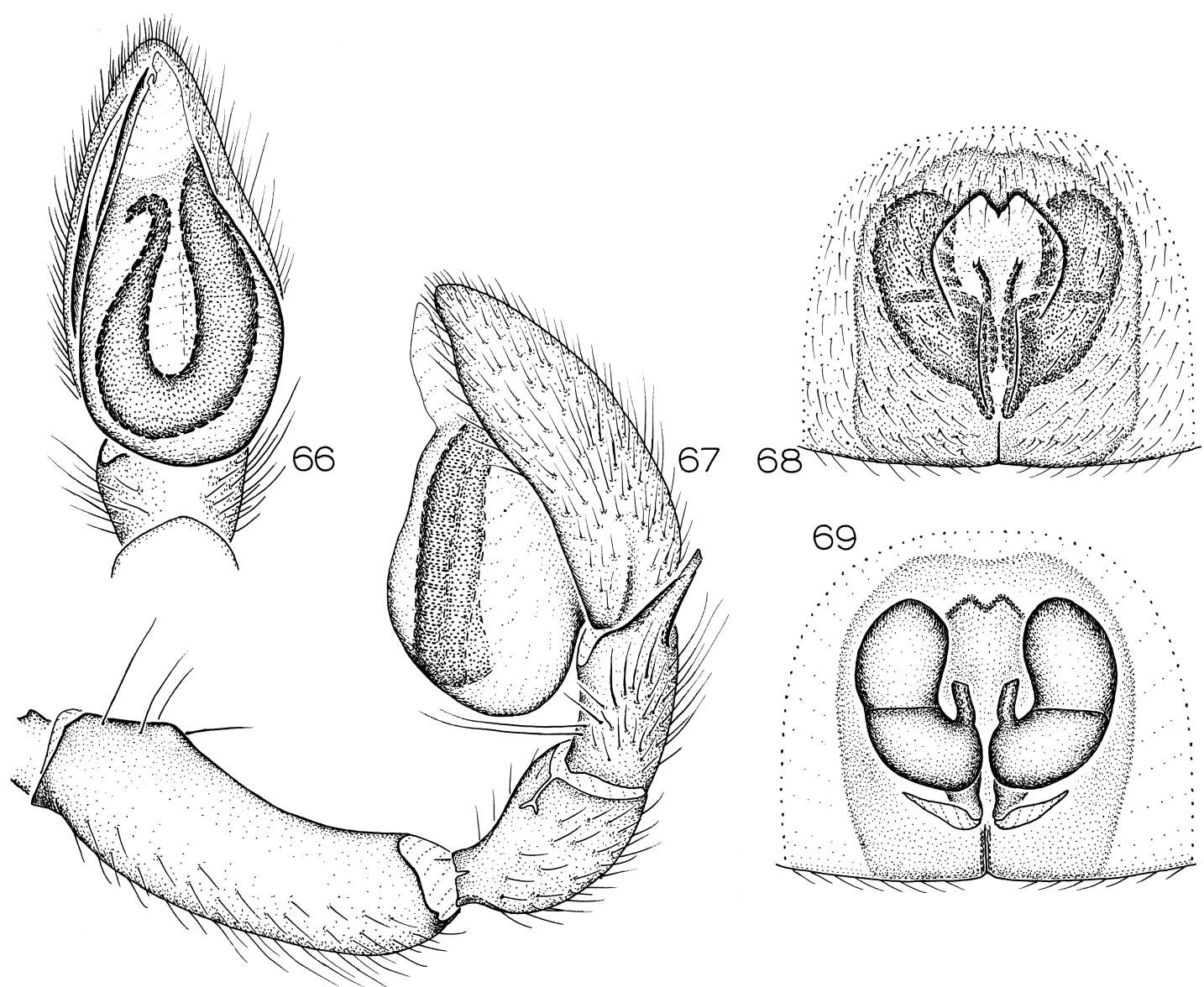

Figs. 66-69. Lampona ruida L. Koch. 66. Left male palp, ventral view. 67. Same, retrolateral view. 68. Epigynum, ventral view. 69. Same, dorsal view.

$32^{\circ} 36^{\prime} \mathrm{S}, 151^{\circ} 48^{\prime} \mathrm{E}$, Feb. 4-Apr. 9, 1993, pitfall, elev. $20 \mathrm{~m}$ (M. Gray, G. Cassis, AMS KS40481), 1 $q$; above Gibraltar Falls, $30^{\circ} 11^{\prime} \mathrm{S}, 151^{\circ} 11^{\prime} \mathrm{E}$, Dec. 11,1977 (M. Gray, AMS KS48944), 1 \% ; Malabar, $33^{\circ} 58^{\prime} \mathrm{S}$, $151^{\circ} 14^{\prime} \mathrm{E}$, June 16, 1966 (R. Mascord, AMS KS31845), 1 우 $\quad$ Scheyville, $33^{\circ} 36^{\prime} \mathrm{S}$, $150^{\circ} 53^{\prime} \mathrm{E}$, Feb. 2-5, 1987, pyrethrum knockdown (H. Recher, AMS KS55267), 1 \% . Queensland: base of Kenniffs Lookout, Mount Moffat National Park, $25^{\circ} 01^{\prime} \mathrm{S}$, $147^{\circ} 57^{\prime} \mathrm{E}$, Dec. 13, 1987, open woodland (J. Gallon, QMB S14559), $1 \%$; Lake Broadwater, SW Dalby, $27^{\circ} 11^{\prime} \mathrm{S}, 151^{\circ} 16^{\prime} \mathrm{E}$, Jan. 26Feb. 18, 1985, pitfall (QMB S30487), 10, Aug. 1986 (E. Zillman, QMB S26777), 1 \%; Tallarenha Creek, W Alpha on highway to Longreach, $23^{\circ} 38^{\prime} \mathrm{S}, 146^{\circ} 28^{\prime} \mathrm{E}$, July 9, 1995 , eucalypt beside dry creek bed (J. Thompson, M. Tio, S. Cowan, AMS KS44182), 1 ㅇ․ South Australia: Jimmys Well, Mount Rescue Conservation Park, $35^{\circ} 51^{\prime} \mathrm{S}, 140^{\circ} 18^{\prime} \mathrm{E}$, Mar. 17, 1992, vibration (D. Hirst, SAM N1996/173), 1 đ; Jip Jip Conservation Park, $36^{\circ} 29^{\prime} \mathrm{S}, 140^{\circ} 11^{\prime} \mathrm{E}$, Mar. 3, 1992, vibration (J. Forrest, D. Hirst, SAM N1996/174), 1 \% ; Loftia Recreation Park, Mount Lofty Ranges, $35^{\circ} 02^{\prime} \mathrm{S}, 138^{\circ} 42^{\prime} \mathrm{E}$, Mar. 20-27, 1990, pitfall (D. Hirst, SAM N1996/195), $19 ; 4.7$ km NE Mount Gow, $26^{\circ} 31^{\prime} \mathrm{S}, 140^{\circ} 44^{\prime} \mathrm{E}$, Nov. 4, 1994, pitfall (R. Brandle, SAM N1996/449), 1 \% ; Parndana Conservation Park, Kangaroo Island, $35^{\circ} 45^{\prime} \mathrm{S}, 137^{\circ} 18^{\prime} \mathrm{E}$, Nov. 3, 1987 (D. Hirst, SAM N1996/172), 19 ; track to Waterfall Creek, Western River Conservation Park, Kangaroo Island, $35^{\circ} 42^{\prime} \mathrm{S}, 136^{\circ} 54^{\prime} \mathrm{E}$, 
Nov. 3-4, 1987 (D. Hirst, SAM N1996/194), 1ㅇ․ Tasmania: King Island, Bass Strait, $40^{\circ} 00^{\prime} \mathrm{S}, 144^{\circ} 00^{\prime} \mathrm{E}$, Feb. 12, 1975 (G. Cann, AMS KS 31868), 10; Knocklofy Reserve, Hobart, $42^{\circ} 53^{\prime} \mathrm{S}, 147^{\circ} 18^{\prime} \mathrm{E}$, Nov. 29, 1997, under rock, open eucalypt forest, elev. 200 $\mathrm{m}$ (L. Boutin, TMH), $1 \widehat{o}^{\text {\% }}$; Launceston, $41^{\circ} 25^{\prime} \mathrm{S}, 147^{\circ} 08^{\prime} \mathrm{E}$, Aug. 30, 1929, under loose eucalypt bark (V. Hickman, AMS KS31858), $2 q$, May 27, 1930, in dry grass tussocks (V. Hickman, AMS KS31860), 1 , $1+$, May 25, 1931, under loose eucalypt bark (V. Hickman, AMS KS31865, 31900), 2; Pats River, Flinders Island, $40^{\circ} 00^{\prime} \mathrm{S}$, $148^{\circ} 00^{\prime} \mathrm{E}$, Feb. 1974 (J. Whinray, TMH J925), $10^{\top}$; Trevallyn, $41^{\circ} 27^{\prime} \mathrm{S}, 147^{\circ} 10^{\prime} \mathrm{E}$, Sept. 4, 1929, under gum tree bark (V. Hickman, AMS KS29260), 2q, May 28, 1931, under loose eucalypt bark (V. Hickman, AMS KS31862), 1 0 , 2q. Victoria: $3 \mathrm{~km} \mathrm{E}$ Anglers Rest, $37^{\circ} 00^{\prime} \mathrm{S}, 147^{\circ} 29^{\prime} \mathrm{E}$, Mar. 3, 1993 (G. Milledge, NMV K3668), 1 đo; Bendigo area, 36 $46^{\prime} \mathrm{S}, 144^{\circ} 16^{\prime} \mathrm{E}$, Feb. 1991 (B. Harvey, NMV K3664), 1 o $^{\text {; }}$ Coranderrk Reserve, Healesville, $37^{\circ} 39^{\prime} \mathrm{S}, 145^{\circ} 31^{\prime} \mathrm{E}$, Mar. 5-9, 1979, under bark (M. Harvey, NMV K3408), $1 \%$; $23 \mathrm{~km}$ SE Halls Gap, Ararat Road, $37^{\circ} 18^{\prime} \mathrm{S}, 142^{\circ} 46^{\prime} \mathrm{E}$, June 20 , 1989, under redgum bark (D. Hirst, SAM N1996/ 298), 1\%; Mandurang South, 36 $51^{\prime} \mathrm{S}$, $144^{\circ} 17^{\prime} \mathrm{E}$, May 11, 1997 (F. Johnstone, CVIV 471), 1 연 Sherbrook Forest, $37^{\circ} 53^{\prime} \mathrm{S}$,

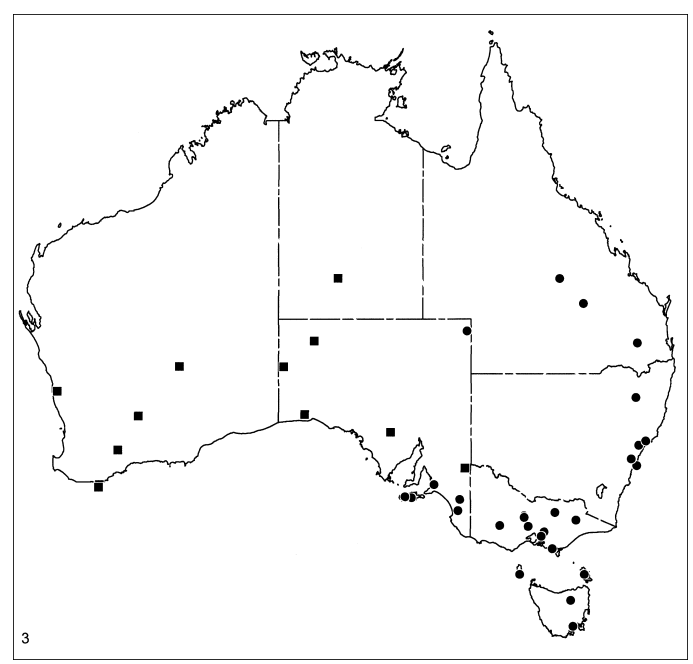

Map 3. Records of Lampona ruida L. Koch (circles) and L. foliifera Simon (squares). $145^{\circ} 21^{\prime} \mathrm{E}$, July 24,1968 , under bark (J. Balogh, HNHM), $2 q$; Stony Creek, South Gippsland, $38^{\circ} 35^{\prime} \mathrm{S}, 146^{\circ} 02^{\prime} \mathrm{E}$, Jan. 1978 (K. Bell, NMV K3434), 10; Upper Lurg, $36^{\circ} 35^{\prime} \mathrm{S}, 146^{\circ} 11^{\prime} \mathrm{E}$, Dec. 24, 1994, house (J. Strudwick, CVIC 633), 1 , , Feb. 13-May 9, 1996-1997, garden (J. Strudwick, CVIC 631, 641), $20^{\top}$; Woodend, $37^{\circ} 21^{\prime} \mathrm{S}, 144^{\circ} 32^{\prime} \mathrm{E}$, Nov. 1903 (M. Walcott, NMV K3428), 1 ․

Distribution: Eastern Australia (map 3).

SYNONYMY: Urquhart's measurements and his descriptions of the color pattern and epigynum (with "a large, deep, subcircular fovea") indicate that he had this species rather than any of the others now known from Tasmania.

\section{Lampona foliifera Simon}

Figures 70-73; Map 3

Lampona foliifera Simon, 1908: 400 (female holotype from Boorabbin, Western Australia, in ZMB, examined).

Diagnosis: This species seems to be the western sister species of $L$. ruida (see above under that name), but can be distinguished by the absence of a subbasal knob on the male palpal femur (fig. 71) and the much narrower female epigynal fossa (fig. 72).

MALE: Total length 6.1. Abdomen dark gray, posterior half of dorsum with light chevrons. Legs light brown, anterior coxae and basal third of femur I darkest, posterior femora, tibiae, metatarsi slightly darkened distally. Palpal femur thickened basally but without distinct subbasal knob protruding from ventral surface; retrolateral tibial apophysis relatively long, straight, dorsoventrally flattened at tip (fig. 71); tegulum globose, extending to half length of tibia, with distinct midventral dilation; embolus long, originating on proximal half of bulb, tip greatly narrowed; conductor bifid, with distinctly sclerotized prolateral rim of prolateral portion, which extends to embolar tip, retrolateral portion extending even further anteriorly (fig. 70).

FEMALE: Total length 6.5. Coloration as in male. Epigynum with deeply excavated, narrow anterior fossa with bipartite anterior rim (fig. 72); spermathecae huge, with sharply demarcated anterior and posterior portions (fig. 73). 

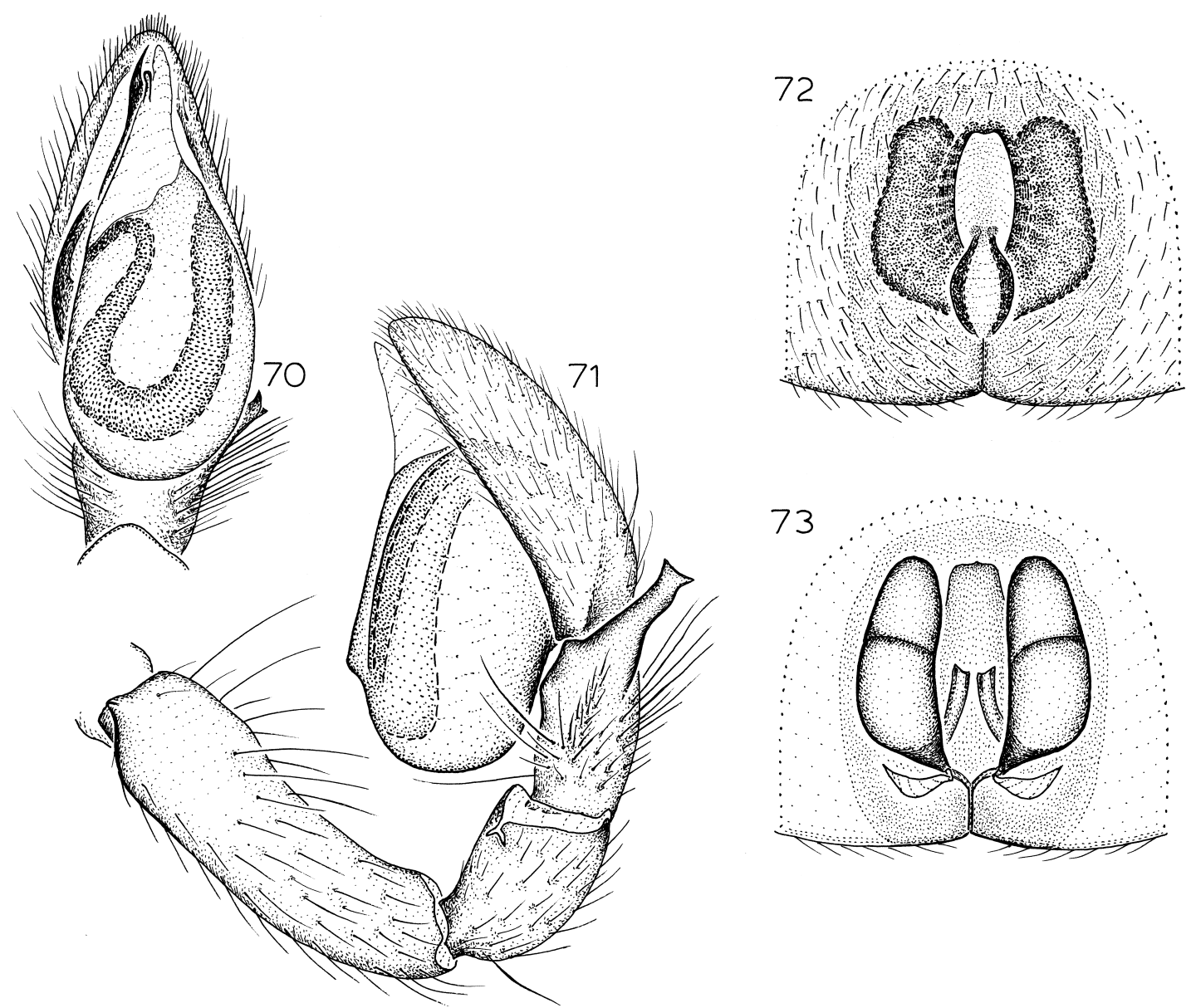

Figs. 70-73. Lampona foliifera Simon. 70. Left male palp, ventral view. 71. Same, retrolateral view. 72. Epigynum, ventral view. 73. Same, dorsal view.

Material Examined: Northern Territory: Ormiston Gorge, $23^{\circ} 37^{\prime} \mathrm{S}, 132^{\circ} 42^{\prime} \mathrm{E}$, Oct. 1994 (T. Churchill, QMB S34288), 1 ㅇ․ South Australia: $10.5 \mathrm{~km} \mathrm{E} \mathrm{Ampeinna}$ Hills, $27^{\circ} 05^{\prime} \mathrm{S}, 131^{\circ} 13^{\prime} \mathrm{E}$, Mar. 19-23, 1995, pitfall (SAM N1997/133), 10; Kudna Rockhole, $31^{\circ} 07^{\prime} \mathrm{S}, 130^{\circ} 38^{\prime} \mathrm{E}$, Sept. 30, 1988 (D. Hirst, SAM N1996/218), 1 \% ; Pinkawillinie Conservation Park, $32^{\circ} 07^{\prime} \mathrm{S}, 136^{\circ} 00^{\prime} \mathrm{E}$, Nov. 29, 1995, vibration (D. Hirst, SAM N1997/ 134), 1 \% $14 \mathrm{~km}$ WNW Renmark, $34^{\circ} 07^{\prime} \mathrm{S}$, $140^{\circ} 37^{\prime} \mathrm{E}$, Nov. 7-Dec. 13, 1995, intercept trap (K. Pullen, ex QMB S32529), $10^{\star}$; Serpentine Lakes, $28^{\circ} 30^{\prime} \mathrm{S}, 129^{\circ} 20^{\prime} \mathrm{E}$, Apr. 18, 1994, under rock on lake (D. Hirst, SAM N1996/314), 1․ Western Australia:
Boorabbin, $31^{\circ} 12^{\prime} \mathrm{S}, 120^{\circ} 18^{\prime} \mathrm{E}$, July 3,1905 (W. Michaelsen, R. Hartmeyer, ZMB 24592), 1 ㅇ (holotype); Eneabba, $29^{\circ} 49^{\prime} \mathrm{S}$, $115^{\circ} 16^{\prime}$ E, Sept. 4-5, 1992, leaf litter (K. Aplin, WAM 95/995), 19 ; 39 km E Laverton, $28^{\circ} 28^{\prime} \mathrm{S}, 122^{\circ} 50^{\prime} \mathrm{E}$, Nov. 12-28, 1989-1990, pitfall (E. Pianka, WAM 96/ 236, 237), 20; Mount Clarence, Albany, $35^{\circ} 01^{\prime} \mathrm{S}, 117^{\circ} 54^{\prime} \mathrm{E}$, Oct. 30, 1981, on ground at night (D. Hirst, SAM N1996/ 337), 1 \% ; $20 \mathrm{~km}$ NE Newdegate, $33^{\circ} 06^{\prime} \mathrm{S}$, $119^{\circ} 01^{\prime} \mathrm{E}$, Apr. 8, 1993, mallee (G. Harold, WAM 95/994), $10^{\hat{*}}$; Torndirrup National Park, $9 \mathrm{~km} \mathrm{~S}$ Albany, $35^{\circ} 10^{\prime} \mathrm{S}, 117^{\circ} 50^{\prime} \mathrm{E}$, May 25-Nov. 9, 1983, pitfall (P. Dyer, J. Lyon, WAM 95/996), 1 \%. 
Distribution: Western and central Australia (map 3).

\section{Lampona finke, new species Figures 74-77; Map 4}

TYPE: Female holotype from Finke River, $25^{\circ} 51^{\prime} \mathrm{S}, 135^{\circ} 03^{\prime} \mathrm{E}$, Northern Territory (Oct. 1994; R. Raven), deposited in QMB (S34284).

ETYMOLOGY: The specific name is a noun in apposition taken from the type locality.

DiAGNOSIS: Males resemble those of $L$. foliifera but have a more pronounced ventral protuberance on the base of the palpal femur (fig. 75); females also resemble those of $L$. foliifera but have a large epigynal midpiece rather than a deep anterior excavation (fig. 76).

MALE: Total length 5.5. Abdominal dorsum with anterior pair of white spots almost fused, median pair fully fused into transverse stripe; legs yellow, undarkened, unmarked. Palpal femur with small, pimple-shaped ventral protuberance; retrolateral tibial apophysis expanded laterally at both sides of tip (fig. 75); tegulum massively expanded, reaching almost to tip of tibia; embolus long, wide, with short, arched tip (fig. 75).

FEMALE: Total length 6.8. Coloration as in male. Epigynal midpiece large, wide, reaching beyond tips of spermathecae (fig. 76), spermathecae bipartite, widely separated (fig. 77).

Other Material Examined: South Australia: Serpentine Lakes, $28^{\circ} 30^{\prime} \mathrm{S}, 129^{\circ} 00^{\prime} \mathrm{E}$, Apr. 16, 1994, under bark (D. Hirst, SAM N1996/313), 1 \%, Apr. 17, 1994, under eucalypt bark (J. Forrest, SAM N1996/225), 10; E side, Serpentine Lakes, $28^{\circ} 30^{\prime} \mathrm{S}$, $129^{\circ} 02^{\prime}$ E, Apr. 18, 1994, under bark (P. Hudson, SAM N1996/312), 1 ㅇ.

Distribution: Known only from the Northern Territory and South Australia (map 4).

\section{THE TALBINGO GROUP}

This group includes four species in which the retrolateral tibial apophysis of males is accompanied by a second, small apophysis situated on the dorsal margin of the tibia.
Lampona talbingo, new species

Figures 78-81; Map 4

TyPES: Female holotype and male allotype from Talbingo, $35^{\circ} 34^{\prime} \mathrm{S}, 148^{\circ} 18^{\prime} \mathrm{E}$, New South Wales (J. Wiburd), deposited in AMS (KS31885).

ETYMOLOGY: The specific name is a noun in apposition taken from the type locality.

DiagnOSIS: This species resembles L. lomond in having two tibial apophyses on the male palp (fig. 79) and an elongated epigynum with an anteromedian projection (fig. 80) but can be distinguished by the shorter tibial apophyses of males (figs. 78, 79) and the longer spermathecae of females (figs. 80, 81).

MALE: Total length 7.2. Coloration as in $L$. cylindrata. Palpal femur slightly sinuous, darkened for most of length, with subbasal projection; tibia with two retrolateral tibial apophyses, ventral one wide basally, followed dorsally by smaller hooklike apophysis (fig. 79); tegulum globose, extending to half length of tibia, embolus prolaterally arched, basally widened (fig. 78).

Female: Total length 8.6. Coloration as in L. cylindrata. Epigynum elongated, with rounded anterior rim, openings at anteromedian elevation (fig. 80); spermathecae greatly elongated, with small anterior portion (fig. 81).

Other Material Examined: Australian Capital Territory: Canberra, $35^{\circ} 20^{\prime} \mathrm{S}$, $149^{\circ} 10^{\prime}$ E, Aug. 4, 1968 (J. Balogh, HNHM), $1 \%$, May 24, 1992 (G. Milledge, NMV K3646), $1 \%$; $10 \mathrm{~km}$ NW Cotter River, $35^{\circ} 19^{\prime} \mathrm{S}, 148^{\circ} 57^{\prime} \mathrm{E}$, Dec. 10, 1987, elev. 700 m (B., M. Baehr, CBB), 1 ․ New South Wales: Corunna Lake, $36^{\circ} 17^{\prime} \mathrm{S}, 150^{\circ} 06^{\prime} \mathrm{E}$, Nov. 25, 1996, elev. 160 m (N. Platnick, R. Raven, V. Ovtsharenko, K. Catley, AMNH), $10^{\circ} ; 6 \mathrm{~km} \mathrm{SW}$ Ebor, $30^{\circ} 24^{\prime} \mathrm{S}, 152^{\circ} 21^{\prime} \mathrm{E}$, Oct. 21, 1980, montane woodland, elev. $1200 \mathrm{~m}$ (D. Bickel, AMNH), 1 \% ; Enfield, 33⒌' $151^{\circ} 06^{\prime}$ E, Aug. 16, 1904 (E. Ramsay, AMS KS31914), 1 \% ; Frying Pan, Cooma, 36 $09^{\prime} \mathrm{S}$, $148^{\circ} 50^{\prime} \mathrm{E}$, Dec. 3, 1992 (J. Noble, AMS KS55266), 1 đ̊; $0.65 \mathrm{~km} \mathrm{~N}$ along Oakwood Fire Trail from London Bridge Fire Trail, Oakwood State Forest, $29^{\circ} 53^{\prime} \mathrm{S}, 152^{\circ} 05^{\prime} \mathrm{E}$, Feb. 4-Apr. 9, 1993, pitfall, elev. $1060 \mathrm{~m}$ (M. Gray, G. Cassis, AMS KS37089), 1 ๙ Tamworth, $31^{\circ} 06^{\prime} \mathrm{S}, 150^{\circ} 56^{\prime} \mathrm{E}$, July 4, 1970 

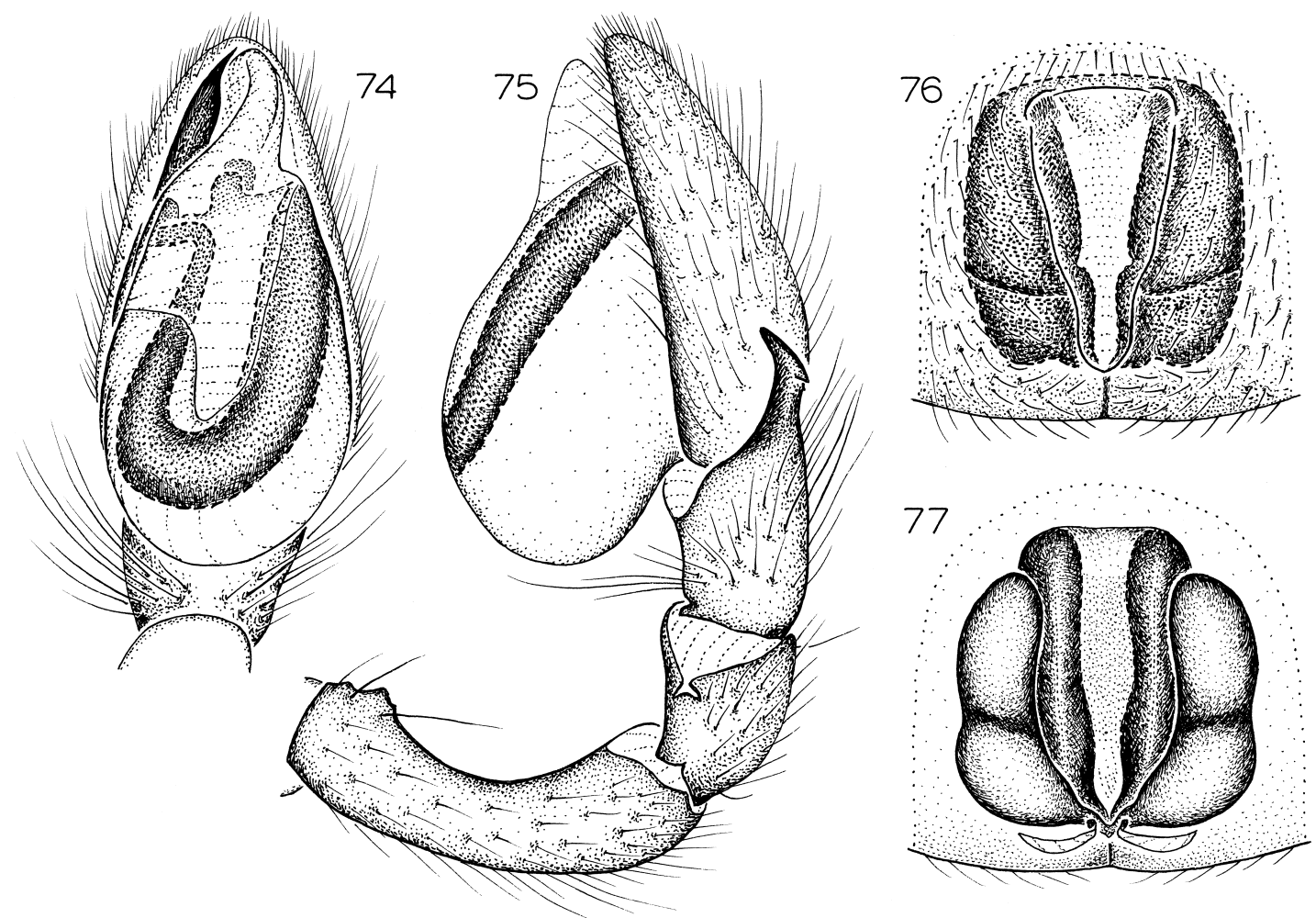

Figs. 74-77. Lampona finke, new species. 74. Left male palp, ventral view. 75. Same, retrolateral view. 76. Epigynum, ventral view. 77. Same, dorsal view.

(L., R. Forster, OMD), 1 ; Wahroonga, Frazer Reserve, $33^{\circ} 43^{\prime} \mathrm{S}, 151^{\circ} 07^{\prime} \mathrm{E}$, July 10 , 1993 (J. Noble, AMS KS66262), 1 오. Queensland: Rochedale State Forest, $27^{\circ} 34^{\prime} \mathrm{S}, 153^{\circ} 08^{\prime} \mathrm{E}$, Jan. 31, 1980, under bark (V. Davies, R. Raven, QMB S26913), 1 ; 10 $\mathrm{km}$ SE Stanthorpe, $28^{\circ} 40^{\prime} \mathrm{S}, 151^{\circ} 57^{\prime} \mathrm{E}$, May 8, 1983 (A. Rozefelds, QMB S25985), 1 \% ; Tarana, $26^{\circ} 57^{\prime} \mathrm{S}, 151^{\circ} 10^{\prime} \mathrm{E}$, Apr. 24, 1966 (R. Mascord, AMS KS31848), 19 . South Australia: Ewens Ponds Reserve, $38^{\circ} 02^{\prime} \mathrm{S}$, $140^{\circ} 47^{\prime} \mathrm{E}$, Apr. 21, 1979 (D. Lee, SAM N1996/224), 10; Mannum, 34⒌' S, $139^{\circ} 18^{\prime} \mathrm{E}$, Apr. 14, 1989, under bark of Eucalyptus camaldulensis (G. Gross, SAM N1996/193), 19; Murray River banks opposite Caloote Landing, $34^{\circ} 58^{\prime} \mathrm{S}, 139^{\circ} 16^{\prime} \mathrm{E}$, Jan. 7, 1971, under bark (J. Szent-Ivany, J. Lakatos, SAM N1996/192), 1 \%, Jan. 30, 1971, under bark (J. Szent-Ivany, SAM N1996/190, 191), 1 o , 1 ㅇ. Victoria: no specific locality (MNHN AR1606), 1 \% ; Abbotsford, $37^{\circ} 48^{\prime} \mathrm{S}, 145^{\circ} 00^{\prime} \mathrm{E}$, July 2, 1992 (G.

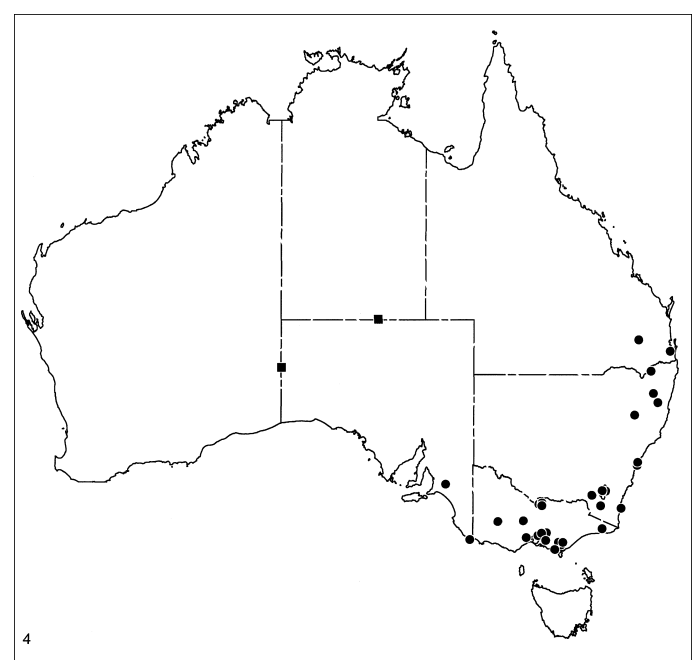

Map 4. Records of Lampona finke, new species (squares) and L. talbingo, new species (circles). 

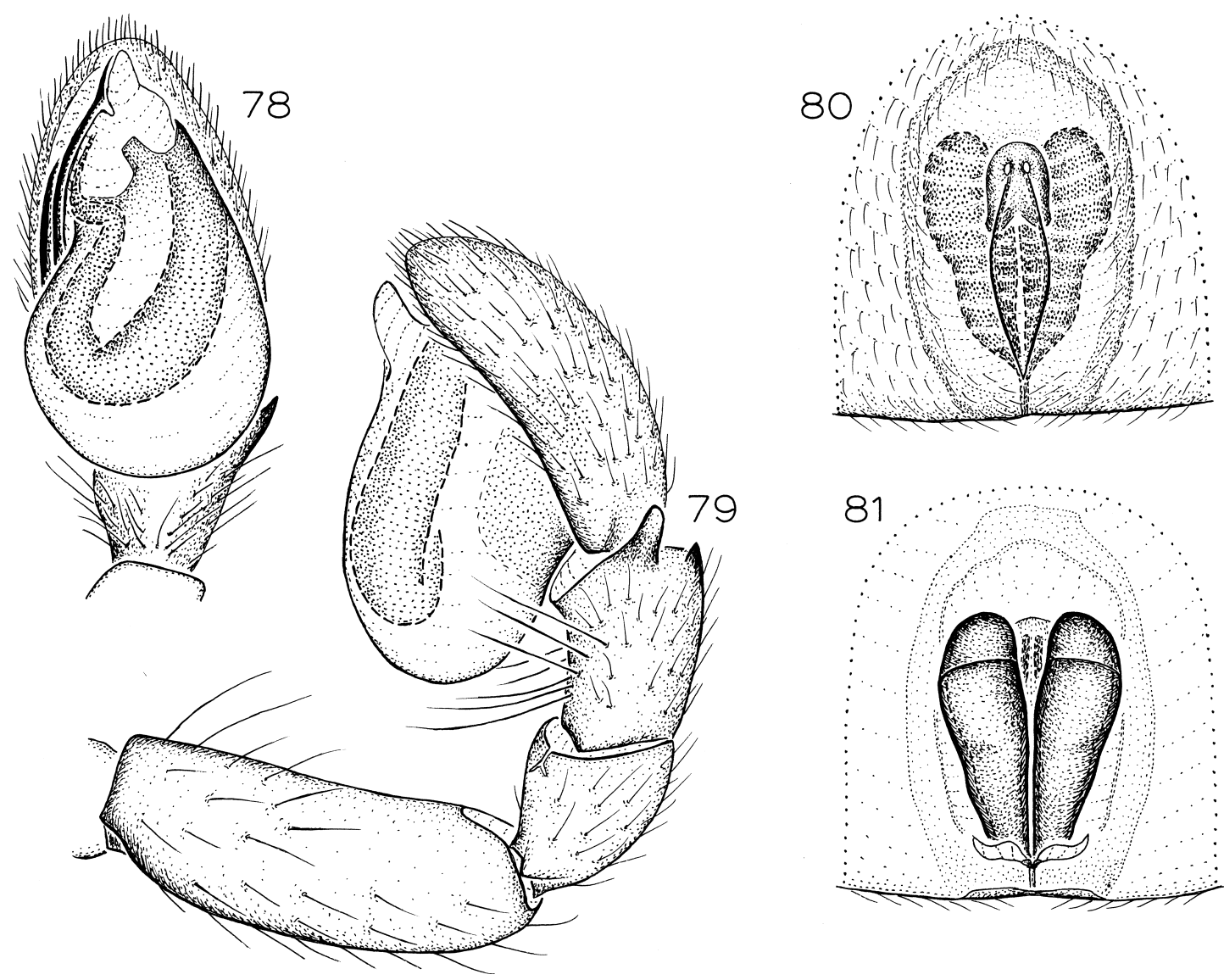

Figs. 78-81. Lampona talbingo, new species. 78. Left male palp, ventral view. 79. Same, retrolateral view. 80. Epigynum, ventral view. 81. Same, dorsal view.

Milledge, NMV K3635), 1 \%; Brisbane Ranges, $1 \mathrm{~km}$ NW Anakie Junction, $37^{\circ} 55^{\prime} \mathrm{S}$, $144^{\circ} 16^{\prime} \mathrm{E}$, June 3, 1989, under bark (D. Hirst, SAM N1996/307), 19; Brooms Road, 7.5 $\mathrm{km}$ NE Yambuna, $36^{\circ} 06^{\prime} \mathrm{S}, 145^{\circ} 05^{\prime} \mathrm{E}$, Apr. 10-17, 1994 (P. Lillywhite, G. Milledge, NMVS), 19 ; Churchill, $38^{\circ} 19^{\prime} \mathrm{S}, 146^{\circ} 25^{\prime} \mathrm{E}$, Aug. 28, 1972 (QMB S26724), 19; Cobon South Coupe, $37^{\circ} 25^{\prime} \mathrm{S}, 148^{\circ} 57^{\prime} \mathrm{E}$, Nov. 2728, 1996, elev. 700 m (N. Platnick, R. Raven, V. Ovtsharenko, K. Catley, AMNH), 20; Coranderrk Reserve, Healesville, $37^{\circ} 39^{\prime} \mathrm{S}$, $145^{\circ} 31^{\prime} \mathrm{E}$, Mar. 6, 1980, under bark of $\mathrm{Eu}$ calyptus viminalis (M. Harvey, QMB), 1 ; ; Grampians, near Mt. William, $37^{\circ} 02^{\prime} \mathrm{S}$, $142^{\circ} 30^{\prime} \mathrm{E}$, Nov. 7, 1932 (NMV K3419), 1 우 $5 \mathrm{~km}$ ESE Kaarimba, $36^{\circ} 10^{\prime} \mathrm{S}, 145^{\circ} 14^{\prime} \mathrm{E}$, Jan. 17-22, 1994 (G. Milledge, P. Lillywhite, NMVS), 1 ㅇ, July 18-23, 1994 (G. Milledge,
P. Lillywhite, NMVS), $1 \delta^{\star}$; Kangaroo Ground, $37^{\circ} 41^{\prime} \mathrm{S}, 145^{\circ} 13^{\prime} \mathrm{E}$, winter 1974 , under bark of Eucalyptus rubida (J. Wainer, QMB S26716), 1 9 ; Kotupna Barmah Road, $0.5 \mathrm{~km}$ NE Ellingtons Bridge, $36^{\circ} 05^{\prime} \mathrm{S}$, 14503'E, Apr. 10-17, 1994 (P. Lillywhite, G. Milledge, NMVS), 1 t ; 5 km SE Maldon, 36 $59^{\prime} \mathrm{S}, 144^{\circ} 05^{\prime} \mathrm{E}$, May 29, 1988 (P. Meyer, R. Seymour, NMV K3418), 10; McDonalds Road, 1.8 km S Shepparton-Barmah Road, $36^{\circ} 04^{\prime} \mathrm{S}, 145^{\circ} 02^{\prime} \mathrm{E}$, Jan. 17-22, 1994 (G. Milledge, P. Lillywhite, NMVS), $10^{\star}, 1$, Apr. 10-17, 1994 (P. Lillywhite, G. Milledge, NMVS), 10; Moe, Latrobe River, $38^{\circ} 10^{\prime} \mathrm{S}, 146^{\circ} 16^{\prime} \mathrm{E}$, Oct. 1973 (NMV K3425), 1 \% ; Murray Valley Highway, 0.3 km NNW Walshs Bridge Road, 36 $06^{\prime} \mathrm{S}$, $145^{\circ} 12^{\prime}$ E, July 18-23, 1994 (G. Milledge, P. Lillywhite, NMVS), 1 \% ; Pakenham, 

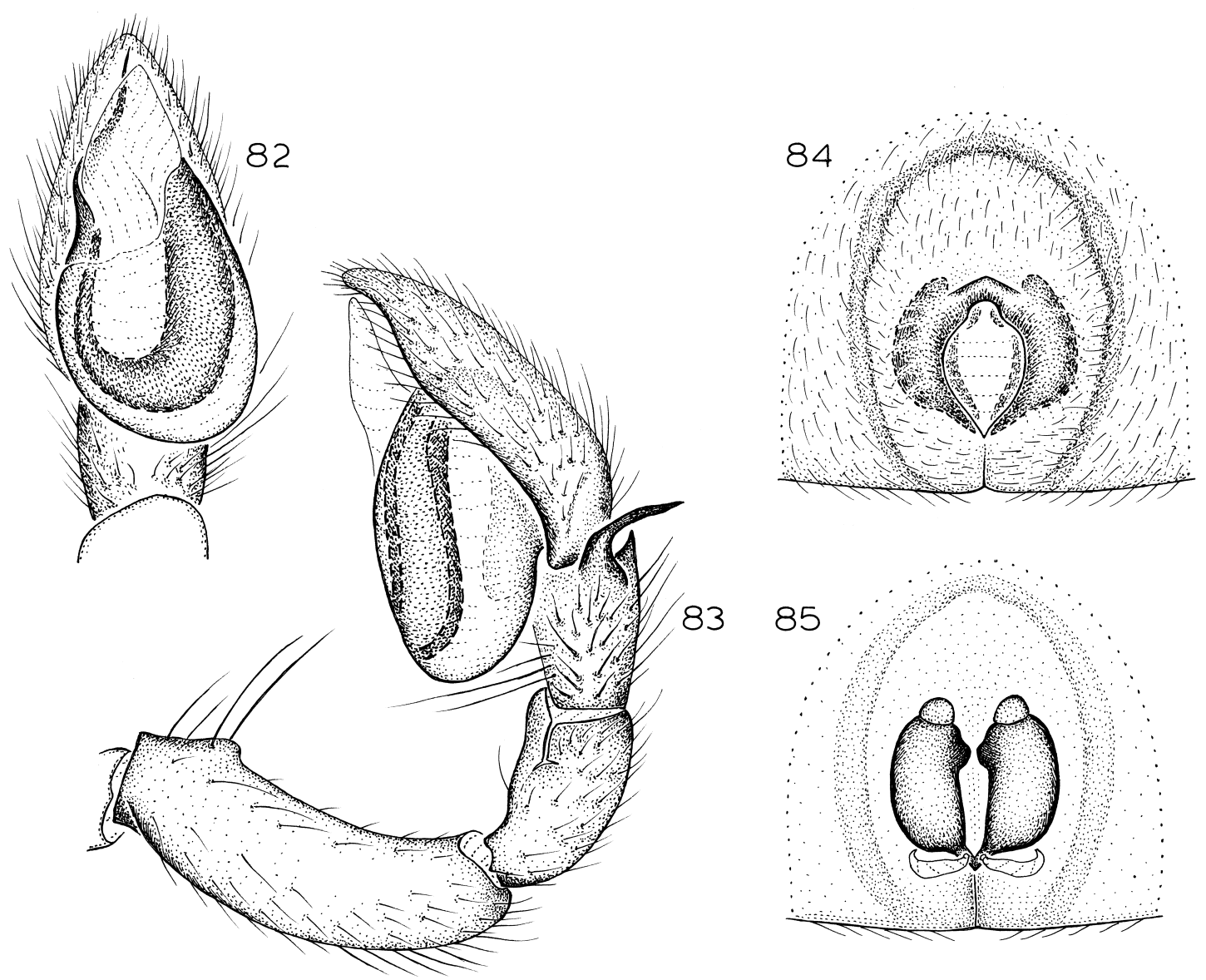

Figs. 82-85. Lampona lomond, new species. 82. Left male palp, ventral view. 83. Same, retrolateral view. 84. Epigynum, ventral view. 85. Same, dorsal view.

$38^{\circ} 04^{\prime} \mathrm{S}, 145^{\circ} 29^{\prime} \mathrm{E}$, Oct. 23, 1976, manna gum bark (V. Salanitri, QMB S26755), 1 ; ; Pomfrets Road, $0.6 \mathrm{~km}$ S Picola-Katunga Road, $36^{\circ} 00^{\prime} \mathrm{S}, 145^{\circ} 14^{\prime} \mathrm{E}$, Apr. 10-17, 1994 (P. Lillywhite, G. Milledge, NMVS), 1 , July 18-23, 1994 (G. Milledge, P. Lillywhite, NMVS), 10, 1 ㅇ, Jan. 26-30, 1995 (J. Evans, S. Hinkley, J. Wainer, NMVS), 10 , 2 \%; Rathbones Road, $3 \mathrm{~km}$ E Booths Road, $36^{\circ} 09^{\prime} \mathrm{S}, 145^{\circ} 13^{\prime} \mathrm{E}$, Jan. 27-30, 1995 (J. Evans, S. Hinkley, J. Wainer, NMVS), 19 ; Stoney Creek, South Gippsland, 38 $35^{\prime} \mathrm{S}$, $146^{\circ} 02^{\prime}$ E, Jan. 1978 (K. Bell, NMV K3430), 1 ㅇ (penultimate but with full epigynum); Traralgon, Latrobe River, $38^{\circ} 11^{\prime} \mathrm{S}, 146^{\circ} 32^{\prime} \mathrm{E}$, Oct. 24, 1973 (NMV K3436), $1 \delta^{\dagger}$. 4).
Lampona lomond, new species Figures 82-85; Map 5

TyPES: Female holotype and male allotype taken in open forest at an elevation of 1000 $\mathrm{m}$ in Ben Lomond National Park, $41^{\circ} 30^{\prime} \mathrm{S}$, $147^{\circ} 38^{\prime} \mathrm{E}$, Tasmania (Feb. 5, 1987; J. Gallon), deposited in QMB (S11633).

ETYMOLOGY: The specific name is a noun in apposition taken from the type locality.

Diagnosis: This species resembles L. talbingo (see above) but can be distinguished by the longer tibial apophyses of males (figs. 82,83 ) and the shorter spermathecae of females (figs. 84, 85).

MALE: Total length 5.6. Abdominal coloration as in L. cylindrata. Anterior legs with darkened femora and tibiae; posterior legs 
with distal three-fourths of femora, patella, tibiae, distal one-fourth of metatarsi darkened. Palpal femur with subbasal knob protruding from ventral surface; tibia with two retrolateral tibial apophyses, ventral one wide basally, bent distally at right angle, followed dorsally by smaller, long apophysis (fig. 83); tegulum globose, extending to half length of tibia, embolus recessed behind conductor (fig. 82).

Female: Total length 8.2. Coloration as in male. Epigynum elongated, with rounded anterior and lateral rims surrounding shallowly depressed fossa bearing elevated ridge at about half its length (fig. 84); spermathecae with tiny anterior portion (fig. 85).

Other Material ExAmined: New South Wales: Mount Kosciusko, $36^{\circ} 27^{\prime} \mathrm{S}$, 148 ${ }^{\circ} 6^{\prime}$ E, Dec. 1931, elev. 5000-6000 ft (P. Darlington, MCZ), 1 \% ; Perisher Valley, $36^{\circ} 25^{\prime} \mathrm{S}, 148^{\circ} 25^{\prime} \mathrm{E}$, Jan. 20, 1975, under stones (V. Salanitri, QMB S26768), 19 . Tasmania: Ben Lomond National Park, $41^{\circ} 30^{\prime} \mathrm{S}$, $147^{\circ} 38^{\prime} \mathrm{E}$, Feb. 5, 1987, open forest, elev. $1000 \mathrm{~m}$ (J. Gallon, QMB S11633, 11636), 2 \%; Derwent Bridge, $42^{\circ} 08^{\prime} \mathrm{S}, 146^{\circ} 14^{\prime} \mathrm{E}$, Dec. 22, 1997. eucalypt bark, open eucalypt forest (L. Boutin, TMH), $1 \%$; Dry's Bluff, $41^{\circ} 42^{\prime} \mathrm{S}, 146^{\circ} 49^{\prime} \mathrm{E}$, Dec. 31, 1929, in silk nest under stone on summit, elev. $4125 \mathrm{ft}$ (V. Hickman, AMS KS28932), 1 ; Forth Falls,

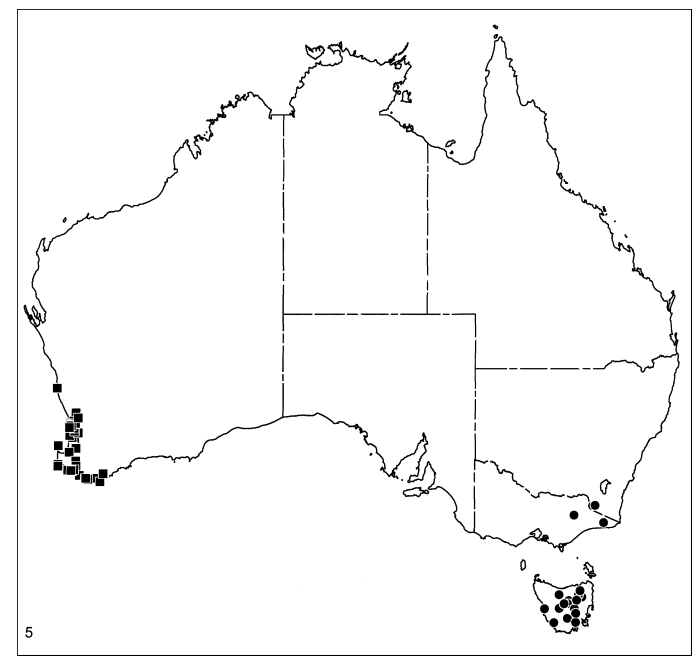

Map 5. Records of Lampona lomond, new species (circles) and L. brevipes L. Koch (squares). $41^{\circ} 23^{\prime} \mathrm{S}, 146^{\circ} 13^{\prime} \mathrm{E}$, Dec. 28, 1926 (V. Hickman, AMS KS29317), 1 9 ; Great Lake, $41^{\circ} 52^{\prime} \mathrm{S}, 146^{\circ} 45^{\prime} \mathrm{E}$, Mar. 19, 1930, under stone (V. Hickman, AMS KS31859), 1, Jan. 22, 1940, under log (V. Hickman, AMS KS28959), 1 , Nov. 20, 1972 (M. Baehr, $\mathrm{CBB}), 19$; Interlaken, $42^{\circ} 09^{\prime} \mathrm{S}, 147^{\circ} 10^{\prime} \mathrm{E}$, Mar. 1938, under lichens (V. Hickman, AMS KS31864), 1 \% ; Lake Augusta, 41 ${ }^{\circ} 52^{\prime} \mathrm{S}$, $146^{\circ} 32^{\prime} \mathrm{E}$, Nov. 21, 1972 (M. Baehr, CBB), 3 ㅇ ; Lenah Valley, $42^{\circ} 52^{\prime} \mathrm{S}, 147^{\circ} 17^{\prime} \mathrm{E}$, July 28, 1951, under stone (V. Hickman, AMS KS44051), $1 \%$; Mount Barrow, summit, E Launceston, $41^{\circ} 22^{\prime} \mathrm{S}, 147^{\circ} 25^{\prime} \mathrm{E}$, Feb. 5, 1987, heath plain, elev. 1300 m (R. Raven, T. Churchill, QMB S11679), 2\%; Mount Field National Park, 42 $41^{\prime}$ S, $146^{\circ} 49^{\prime} \mathrm{E}$, Jan. 1, 1998, under rocks (L. Boutin, TMH), 1 ㅇ (penultimate but with formed epigynum); near Mount Field National Park, $42^{\circ} 41^{\prime}$ S, $146^{\circ} 43^{\prime} \mathrm{E}$, May 1996, under bark, elev. 160 m (L. Boutin, TMH), 1 \% ; Mount Wellington, $42^{\circ} 54^{\prime} \mathrm{S}, 147^{\circ} 14^{\prime} \mathrm{E}$, Dec. 1956, high (P. Darlington, MCZ), 19 , Dec. 10, 1963, summit, with eggsac under stone (J. Hickman, AMS KS31863), 1 ; ; Nive River, 15 km N Bronte Park, 41 ${ }^{\circ} 40^{\prime} \mathrm{S}, 147^{\circ} 18^{\prime} \mathrm{E}$, Nov. 24, 1972 (M. Baehr, CBB), $2 \%$; southwestern Tasmania, $42^{\circ} 55^{\prime} \mathrm{S}, 145^{\circ} 53^{\prime} \mathrm{E}$, Feb. 15, 1978, hand collecting, heathland transect (L. Hill, AMS KS27058), 19 ; Spring Hill, $42^{\circ} 24^{\prime} \mathrm{S}$, $147^{\circ} 15^{\prime} \mathrm{E}$, Sept. 6, 1947, in grass tussocks (V. Hickman, AMS KS44041), 1 $\%$ Strahan, $42^{\circ} 09^{\prime} \mathrm{S}, 145^{\circ} 19^{\prime} \mathrm{E}$, Nov. 14-Dec. 1, 1997 , eucalypt bark (L. Boutin, TMH), $2 \%$; Tomahawk River, $40 \mathrm{~km}$ NE Scottsdale, $41^{\circ} 09^{\prime} \mathrm{S}$, 147³1'E, Nov. 3, 1972 (M. Baehr, CBB), 1․ Victoria: Cobon North Coupe, site 512.08, Vaus experimental study, $37^{\circ} 22^{\prime} \mathrm{S}$, $148^{\circ} 56^{\prime}$ E, Apr. 6-10, 1992, upslope pitfalls (R. Coy, NMV K3556), 1 đ; Mount Hotham, $36^{\circ} 58^{\prime} \mathrm{S}, 147^{\circ} 07^{\prime} \mathrm{E}$, Dec. 13 , 1962, elev. 1700 m (E. Ross, D. Cavagnaro, CAS), 2 q .

DisTRIBUTION: Southeastern Australia, including Tasmania (map 5).

\section{Lampona brevipes L. Koch Figures 86-89; Map 5}

Lampona brevipes L. Koch, 1872: 363, pl. 28, figs. 3, 3a (female holotype from Swan River, Western Australia, may be in Hope Department, Oxford, not examined).

Lampona obnubila Simon, 1908: 400 (male ho- 

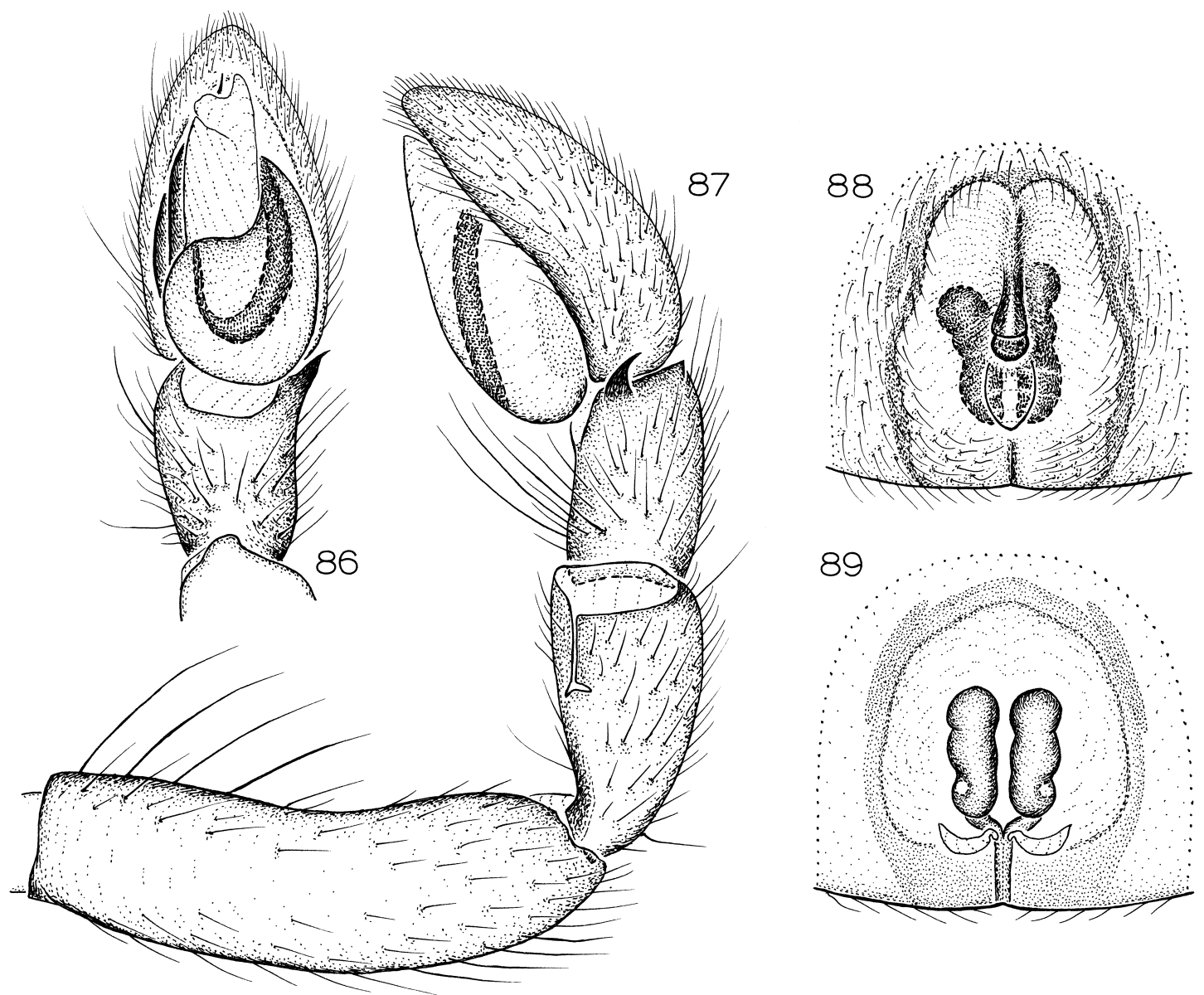

Figs. 86-89. Lampona brevipes L. Koch. 86. Left male palp, ventral view. 87. Same, retrolateral view. 88. Epigynum, ventral view. 89. Same, dorsal view.

lotype from Boyanup, Western Australia, in ZMB, examined). NEW SYNONYMY.

Lampona paupercula Simon, 1908: 402 (male syntype, presumably from Boyanup, Western Australia, in MNHN, examined). NEW SYNONYMY.

Diagnosis: Males resemble those of L. lomond in having a dorsally directed retrolateral tibial apophysis accompanied by a smaller, more dorsal tibial apophysis, but both apophyses are smaller than in that species (fig. 87); females can be easily recognized by the very large protuberance projecting from the middle of the epigynum (fig. 88).

MALE: Total length 8.3. Coloration as in $L$. cylindrata except patellae lighter than other leg segments. Palpal femur with only vague indication of ventral enlargement; tibia with two small retrolateral tibial apophyses, ventral one bent distally at right angle, followed dorsally by very short apophysis (fig. 87); tegulum globose, extending to only about one-fifth length of tibia, embolus situated beside obliquely divided conductor (fig. 86).

FEMALE: Total length 9.0. Coloration as in male. Epigynum with proboscis-shaped protuberance (fig. 88); spermathecae small, tripartite, situated on greatly invaginated plate (fig. 89).

Other Material Examined: Western Australia: Alcoa minesite and forests, $\mathrm{N}$ and NW Jarrahdale, $32^{\circ} 20^{\prime} \mathrm{S}, 1^{\circ} 6^{\circ} 04^{\prime} \mathrm{E}$, Mar.Aug. 1993 (S. Simmonds, WAM 95/1034), 
1 9 ; Bedfordale, $32^{\circ} 10^{\prime} \mathrm{S}, 116^{\circ} 03^{\prime} \mathrm{E}$, Mar. 3,

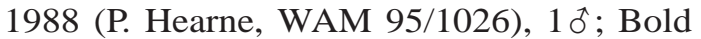
Park, $31^{\circ} 57^{\prime} \mathrm{S}, 115^{\circ} 46^{\prime} \mathrm{E}$, Sept. 24-Nov. 18, 1993, pitfall (J. Waldock, WAM 95/1102), 19 , Nov. 19, 1993-Jan. 6, 1994, pitfall (J. Waldock, WAM 95/1094), 1 \%, Jan. 6-Mar. 18, 1994, pitfalls (M. Harvey, J. Waldock, WAM 95/1091-1093), 10, 2오, Mar. 18May 19, 1994, pitfalls (M. Harvey, J. Waldock, WAM 95/1103, 1104), 20; Boranup, $10 \mathrm{mi} \mathrm{S}$ Lake Cave on Caves Road, $34^{\circ} 09^{\prime} \mathrm{S}$, $115^{\circ} 02^{\prime} \mathrm{E}$, Nov. 21, 1976, at light (FMNH), $10^{\circ}$; Boranup Forest, $34^{\circ} 15^{\prime} \mathrm{S}, 115^{\circ} 02^{\prime} \mathrm{E}$, Aug. 18, 1977, tree pitfall (S. Curry, WAM

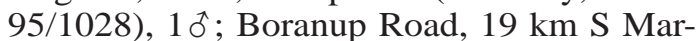
garet River, $33^{\circ} 07^{\prime} \mathrm{S}, 115^{\circ} 03^{\prime} \mathrm{E}$, Feb. 15, 1990 (M. Harvey, M. Blosfelds, WAM 95/ 1043), 1 \% ; Boyanup, $33^{\circ} 29^{\prime} \mathrm{S}, 115^{\circ} 43^{\prime} \mathrm{E}$ (V. Michaelsen, R. Hartmeyer, ZMB 24593), 1 đ (holotype), (MNHN AR1610), 1 †े (presumed syntype, labelled only Western Australia; syntype of $L$. paupercula in ZMB from this locality is juvenile), Nov. 13, 1998, marri litter (V. Ovtsharenko, K. Catley, AMNH), 10 , 1 ; Brockman Highway/ Mockerdillup Road corner, ca. $5 \mathrm{~km} \mathrm{~W}$ Bridgetown, $33^{\circ} 58^{\prime} \mathrm{S}, 116^{\circ} 08^{\prime} \mathrm{E}$, Apr. 18 , 1987 (J. Waldock, WAM 95/1048), 1 우 ; Circular Pool on Franklin River, $7 \mathrm{~km}$ NNE Walpole, 345' $\mathrm{S}, 116^{\circ} 47^{\prime} \mathrm{E}$, Aug. 30, 1987 (M. Harvey, J. Blyth, WAM 95/1037), 1 \%; Cottesloe, $31^{\circ} 59^{\prime} \mathrm{S}, 115^{\circ} 45^{\prime} \mathrm{E}$, Aug. 1986 (R. McMillan, WAM 95/1025), $10^{\circ}$; Crowea, $34^{\circ} 28^{\prime} \mathrm{S}, 116^{\circ} 10^{\prime} \mathrm{E}$, Dec. 12, 1980, pitfall, open (cleared) forest (S. Curry, WAM 95/ 1023), $10^{\top}$; Dalkeith, $32^{\circ} 00^{\prime} \mathrm{S}, 115^{\circ} 48^{\prime} \mathrm{E}$, June 15, 1969 (V. Davies, WAM 95/1038), 1 ; ; Darlington, $31^{\circ} 55^{\prime} \mathrm{S}, 116^{\circ} 05^{\prime} \mathrm{E}$, Sept. 5, 1962, elev. $450 \mathrm{ft}$ (E. Ross, D. Cavagnaro, CAS), $10^{\hat{t}}, 3$ \% ; D'Entrecasteaux National Park, $34^{\circ} 28^{\prime} \mathrm{S}, 115^{\circ} 39^{\prime} \mathrm{E}$, Dec. 19, 1994, in house (C. Deeleman, CCD), 19 , Dec. 19, 1994-Jan. 8, 1995, leaf litter (C. Deeleman, CCD), 1o; Dog Pool, Shannon National Park, $34^{\circ} 46^{\prime} \mathrm{S}, 116^{\circ} 22^{\prime} \mathrm{E}$, Mar. 2, 1989, under bark of Eucalyptus diversicolor (M. Harvey, M. Blosfelds, WAM 95/1049), 1 ㅇ, Mar. 23, 1993 (M. Harvey, J. Waldock, WAM 95/ 1028), 10; Dwellingup, $32^{\circ} 43^{\prime} \mathrm{S}, 116^{\circ} 04^{\prime} \mathrm{E}$, May 5, 1976, pitfall (S. Curry, WAM 95/ 1033), 1 \% , Oct. 1, 1976 (J. Majer, WAM 95/ 1022), 10, Nov. 11, 1976, unburned plot (J. Majer, WAM 95/1036), 1 q , Nov. 11, 1976, hot autumn burn plot (J. Majer, WAM 95/ 1042), 19; 16 km E Dwellingup along Williams Road, $32^{\circ} 40^{\prime} \mathrm{S}, 115^{\circ} 55^{\prime} \mathrm{E}$, Feb. 15, 1979, in litter (M. Gray, AMS KS14869), $10^{\dagger}$; Furnissdale, 6 km E Mandurah, 32 $34^{\prime} \mathrm{S}$, $115^{\circ} 46^{\prime}$ E, May 19, 1985 (F. Baker, WAM 95/ 1020), $10^{\circ} ; 6 \mathrm{~km}$ SW Gidgegannup, $31^{\circ} 20^{\prime} \mathrm{S}$, $116^{\circ} 09^{\prime} \mathrm{E}$, May 6, 1989 (WAM arachnology group, WAM 95/1041), 1 ; ; Harris River, 10 $\mathrm{km}$ N Collie, 3317'S, $116^{\circ} 09^{\prime} \mathrm{E}$, Aug. 26, 1987 (M. Harvey, J. Blyth, WAM 95/1047), 1 9 ; 2 km NE Lake Yeagarup, Ritter Road, $34^{\circ} 31^{\prime} \mathrm{S}, 115^{\circ} 53^{\prime} \mathrm{E}$, May 2, 1990, under marri bark (M. Harvey, J. Waldock, WAM 95/

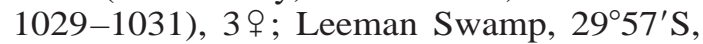
$114^{\circ} 58^{\prime}$ E, Aug. 29, 1982 (R. McMillan, WAM 95/1040), 1; Mahogany Creek, $31^{\circ} 54^{\prime} \mathrm{S}, 116^{\circ} 08^{\prime} \mathrm{E}$, Jan. 19, 1995 , bit adult male on upper thigh, producing $2-\mathrm{cm}$ welt, no other problems (T. Niven, WAM 95/ 1099), $10^{\top}$; Manjimup, $34^{\circ} 15^{\prime} \mathrm{S}, 116^{\circ} 09^{\prime} \mathrm{E}$, Apr. 26, 1978, pitfall (S. Curry, WAM 95/ 1024), $10^{\dagger} ; 12 \mathrm{mi} \mathrm{W}$ Manjimup, $34^{\circ} 15^{\prime} \mathrm{S}$, $116^{\circ} 09^{\prime} \mathrm{E}$, Mar. 11, 1971 (H. Butler, WAM 84/214-218), 20, 2ㅇ; S end, Millinup Pass, Porongurup National Park, $34^{\circ} 42^{\prime} \mathrm{S}$, $117^{\circ} 54^{\prime} \mathrm{E}$, Mar. 30, 1993, under karri bark (M. Harvey, J. Waldock, WAM 95/1017), 10 , in rotting logs (M. Harvey, J. Waldock, WAM 95/1045), 1 \% , Sept. 2-Dec. 16, 1996, pitfall (M. Harvey, J. Waldock, WAM 96/ 1581), 1 우 ; Mount Claremont, $31^{\circ} 58^{\prime} \mathrm{S}$, $115^{\circ} 46^{\prime} \mathrm{E}$, Jan. 19-Mar. 21, 1995, pitfall (M. Harvey, J. Waldock, WAM 95/1100), 1 , May 4-July 6, 1995, pitfall (J. Waldock, M. Harvey, WAM 95/1097, 96/1514), 20; Mount Cooke, $32^{\circ} 25^{\prime} \mathrm{S}, 116^{\circ} 18^{\prime} \mathrm{E}$, Nov. 28, 1991-Feb. 25, 1992, pitfall (M. Harvey, J. Waldock, WAM 95/1027), 10, Feb. 25, 1992, hand collected (M. Harvey, J. Waldock, WAM 95/1039), 1 \%; Mount Henry, $32^{\circ} 02^{\prime} \mathrm{S}, 115^{\circ} 52^{\prime} \mathrm{E}$, Mar. 21-May 4, 1995, pitfall (J. Waldock, M. Harvey, WAM 95/

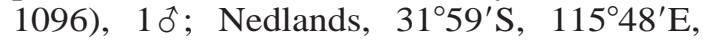
Apr. 28, 1991 (A. Baynes, WAM 95/1021), 10 , Mar. 7, 1995, in house (A. Baynes, WAM 95/1095), 19 ; Nornalup, $35^{\circ} 00^{\prime} \mathrm{S}$, $116^{\circ} 49^{\prime} \mathrm{E}$, Aug., under bark (J. Wunderlich, AMNH), $3 q$; Nyts Wilderness, $10 \mathrm{~km} \mathrm{SW}$ Walpole, 34 $57^{\prime} \mathrm{S}, 116^{\circ} 44^{\prime} \mathrm{E}$, Dec. 1, 1987 (B., M. Baehr, CBB), $1 \%$; Pemberton, $34^{\circ} 27^{\prime} \mathrm{S}, 116^{\circ} 02^{\prime} \mathrm{E}$, Aug. 21, 1956 (B. Main, WAM 95/1035), 1 \%, Nov. 16, 1978, tree trap 
(S. Curry, WAM 95/1044), 1 \%; Salter Point, $32^{\circ} 02^{\prime} \mathrm{S}, 115^{\circ} 52^{\prime} \mathrm{E}$, Mar. 14, 1986 (L. Koch, WAM 95/1032), 1 \% , Sept. 21, 1986, found dead indoors near open window (L. Koch, WAM 95/1019), 10; $7 \mathrm{~km}$ N South Coast Highway, $34^{\circ} 56^{\prime} \mathrm{S}, 117^{\circ} 22^{\prime} \mathrm{E}$, Apr. 26, 1990, under bark of Eucalyptus diversicolor (M. Harvey, J. Waldock, WAM 95/1018), 1 ; ; Swan River near Upper Swan, $31^{\circ} 46^{\prime} \mathrm{S}$, $116^{\circ} 01^{\prime} \mathrm{E}$, Nov. 18, 1987 (M. Baehr, CBB), $10 ; 25 \mathrm{~km}$ W Toodyay, Avon Valley National Park, $31^{\circ} 36^{\prime} \mathrm{S}, 116^{\circ} 18^{\prime} \mathrm{E}$, Nov. 19-21,

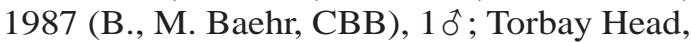
West Cape Howe, $35^{\circ} 08^{\prime} \mathrm{S}, 117^{\circ} 39^{\prime} \mathrm{E}$, Mar. 3-19, 1983, pitfall (B. Main, CBM), 1 đ̊; S Torbay Hill, near South Road, West Cape Howe National Park, $35^{\circ} 05^{\prime} \mathrm{S}, 117^{\circ} 38^{\prime} \mathrm{E}$, Mar. 27, 1993, under karri bark (M. Harvey, J. Waldock, WAM 95/1013-1016), 2 ô, 2 웅 Tree in the Rock, Porongurup National Park, 34 $41^{\prime}$ S, $117^{\circ} 50^{\prime} \mathrm{E}$, Mar. 30, 1993 (M. Harvey, J. Waldock, WAM 95/1046), 1 ; ; Trigg Dune Bush, $31^{\circ} 53^{\prime} \mathrm{S}, 115^{\circ} 46^{\prime} \mathrm{E}$, Nov. 28, 1995-Jan. 29, 1996, pitfall (M. Harvey, J. Waldock, WAM 96/1515), 10; Valley of the Giants, 34 $59^{\prime} \mathrm{S}, 116^{\circ} 54^{\prime} \mathrm{E}$, Sept. 24, 1962, elev. 150 m (E. Ross, D. Cavagnaro, CAS), 10 , 1 \% ; Walpole-Nornalup National Park, $140 \mathrm{~km}$ W Albany, 3456' S, 116 $46^{\prime} \mathrm{E}$, Feb. 3, 1994 (B. Baehr, CBB), 19 (penultimate but with full epigynum); W of road to Warren National Park, $34^{\circ} 30^{\prime} \mathrm{S}, 115^{\circ} 50^{\prime} \mathrm{E}$, Nov. 13, 1986 (B. Main, WAM 96/1516), 1; Woodman Point, $32^{\circ} 08^{\prime} \mathrm{S}, 115^{\circ} 45^{\prime} \mathrm{E}$, Jan. 19-Mar. 21, 1995, pitfall (M. Harvey, J. Waldock, WAM 95/1098), 19, May 4-July 6, 1995, pitfall (J. Waldock, M. Harvey, WAM 95/1101), 10 .

Distribution: Southwestern Australia (map 5).

SYNONYMY: Simultaneous collections of both sexes indicate that $L$. obnubila is the male of L. brevipes. Simon separated L. paupercula from $L$. obnubila by differences in the eye arrangement that are not corroborated by direct comparison of the two adult specimens.

Lampona whaleback, new species Figures 90-93; Map 6

TYPE: Female holotype taken in dry pitfall trap on Mount Whaleback, $23^{\circ} 21^{\prime} \mathrm{S}$,
119 39'E, Western Australia (Aug. 7-12, 1997; D. Knowles), deposited in WAM (96/ 1525).

ETYMOLOGY: The specific name is a noun in apposition taken from the type locality.

DiagnOSIS: This species resembles $L$. lomond in having a subbasal knob on the palpal femur, but differs in having a shorter retrolateral tibial apophysis in males (fig. 91) and anterolateral epigynal invaginations in females (fig. 92).

MALE: Total length 7.0. Abdominal dorsum with only posterior white spot clearly marked, with only vague indications of anterior white spots, venter palp gray, without markings; legs with posterior tibia distolaterally darkened. Palpal femur with distinct subbasal knob, retrolateral tibial apophysis situated near ventral edge of segment, accompanied by short, more dorsal apophysis (fig. 91); embolus with long, retrolaterally curved tip (fig. 90).

FEMALE: Total length 8.8. Abdominal dorsum with four anterior white spots each almost rectangular, leg coloration as in male. Epigynum with $\mathrm{v}$-shaped median septum separating anterolateral depressions marked by strong ridges (fig. 92); spermathecae with conical basal portion, rounded distal portion, situated between lateral invaginations (fig. 93).

Other Material Examined: The single male here associated with the type was taken in a pitfall trap at Bush Bay, $25^{\circ} 07^{\prime} \mathrm{S}$, $113^{\circ} 48^{\prime} \mathrm{E}$, Western Australia, Jan. 16-May 23, 1995 (P. West, WAM 96/1524).

Distribution: Western Australia (map 6).

\section{THE MACILENTA GROUP}

This group includes seven species in which the posterior portions of the spermathecae are narrowed and crenulated. In some cases the retrolateral tibial apophyses are distally elaborated; in others the apophysis is almost straight. The female epigyna vary, but in four of the six species known from females, the epigynal midpiece is an inverted triangle, and the anterior portions of the spermathecae are globose and directed toward the midline. 

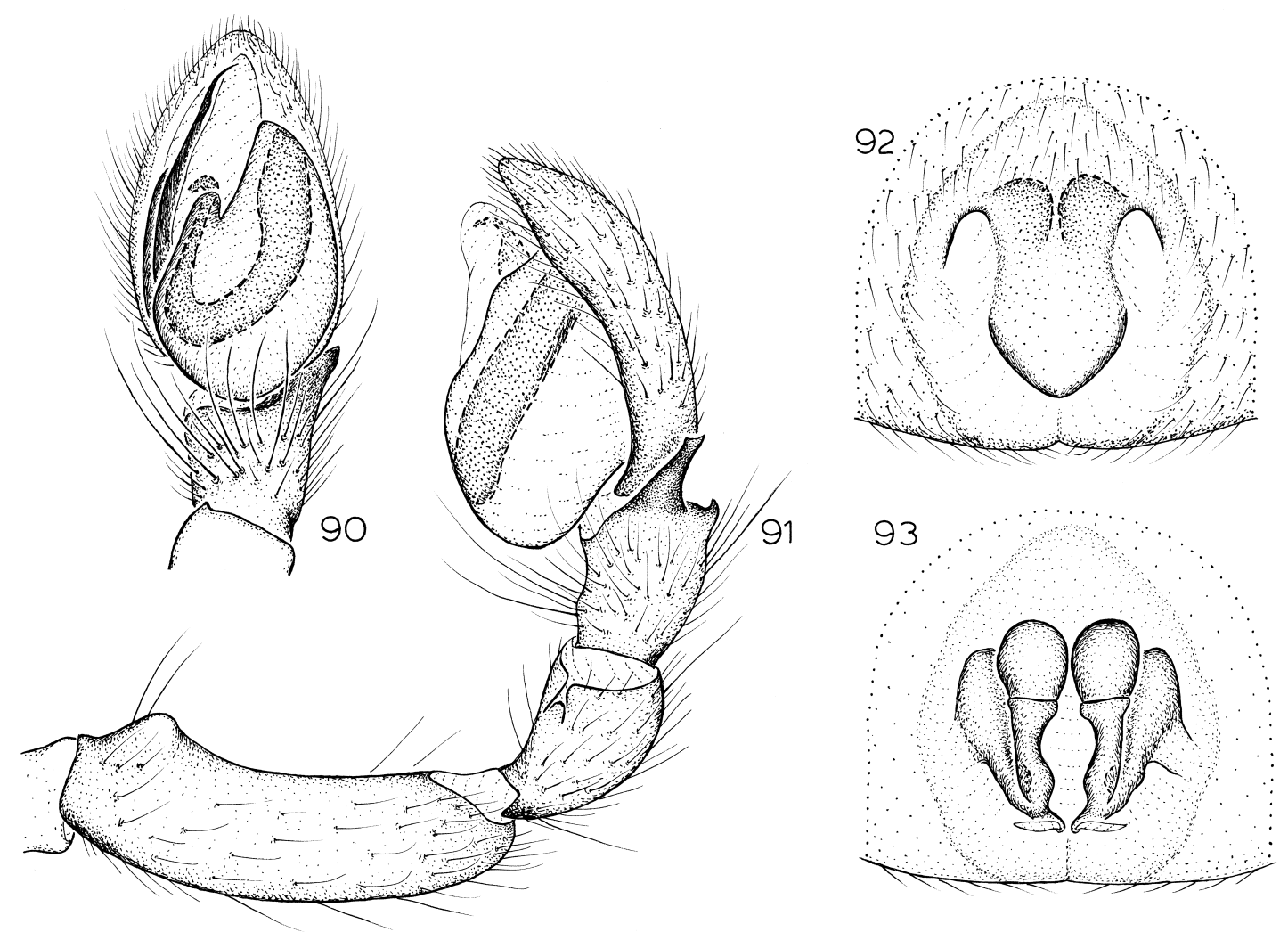

Figs. 90-93. Lampona whaleback, new species. 90. Left male palp, ventral view. 91. Same, retrolateral view. 92. Epigynum, ventral view. 93. Same, dorsal view.

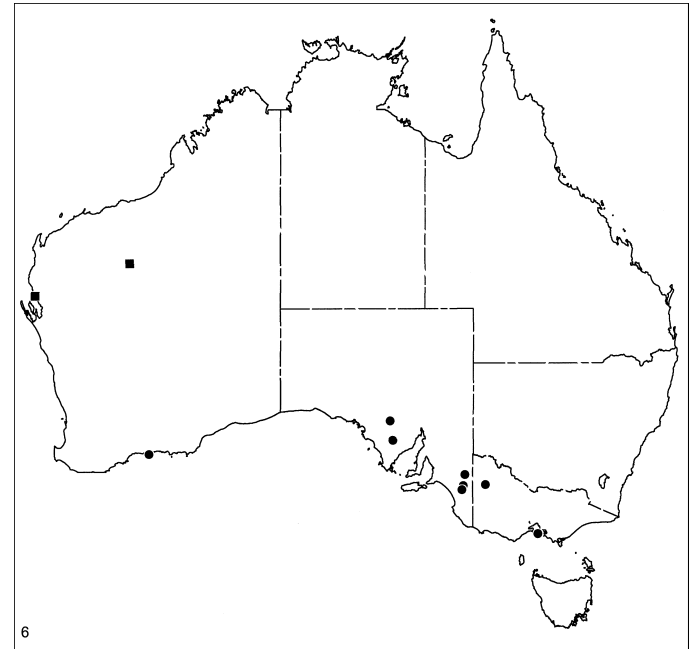

Map 6. Records of Lampona whaleback, new species (squares) and L. macilenta L. Koch (circles).

\section{Lampona macilenta L. Koch} Figures 94-97; Map 6

Lampona macilenta L. Koch, 1873: 369, pl. 28, figs. 7, 7a (female holotype from Australia [as "Neuholland"], no specific locality, in NRS, examined).

DiAgnosis: This species can easily be recognized by the saddle-shaped depression on the palpal femur and the elongated embolus, extending behind and over the palpal conductor of males (figs. 94, 95), and by the elongated basal portion of the female spermathecae (fig. 97).

MALE: Total length 9.2. Abdominal dorsum gray, lightest behind scutum, without distinct pattern; venter with paired, oval sclerites fused to epigastric scutum posteriorly and laterally. Legs dark reddish brown, with basal halves of femora lightest. Base of palpal femur with ventral, saddle-shaped depression; retrolateral tibial apophysis wide, 

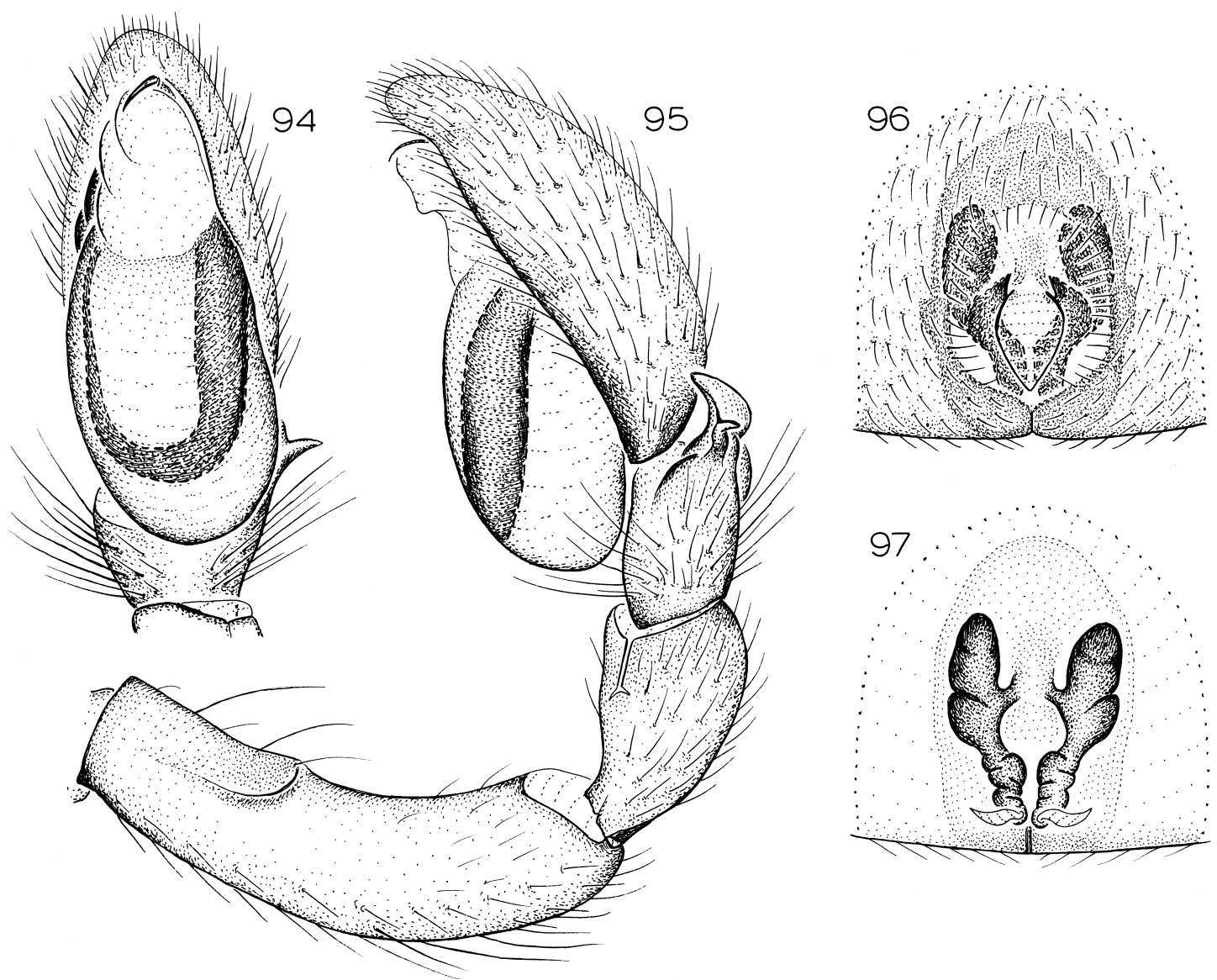

Figs. 94-97. Lampona macilenta L. Koch. 94. Left male palp, ventral view. 95. Same, retrolateral view. 96. Epigynum, ventral view. 97. Same, dorsal view.

flattened, bifid, with truncate flange extending anteriorly and sharply pointed flange extending laterally (fig. 95); tegulum globose, reaching beyond half length of tibia, embolus curling behind palpal conductor, tip directed ventrally across tip of conductor (fig. 94).

FEMALE: Total length 9.2. Coloration as in male; abdominal dorsum with small, triangular sclerotization representing rudimentary scutum; venter with paired, oval sclerites not fused to epigastric sclerite. Epigynum long, with median depression flanked by paired openings closer to anterior rim than to epigastric furrow (fig. 96); basal portion of spermathecae elongated, distinctly crenulated (fig. 97).

Material ExAmINed: AUSTRALIA: no specific locality (NRS), 19 (holotype).
South Australia: near trig point, Billiatt Conservation Park, $35^{\circ} 01^{\prime} \mathrm{S}, 140^{\circ} 29^{\prime} \mathrm{E}$, Nov. 28, 1996, vibration (D. Hirst, SAM N1997/ 135, 136), 10, 1\%; $4 \mathrm{~km} \mathrm{~S}$ Box Flat, $35^{\circ} 38^{\prime} \mathrm{S}, 140^{\circ} 23^{\prime} \mathrm{E}$, Mar. 19, 1992, mallee/red sand (D. Hirst, SAM N1996/175), 1 \% ; NE Jimmys Well, $35^{\circ} 51^{\prime} \mathrm{S}, 140^{\circ} 18^{\prime} \mathrm{E}$, Mar. 20, 1992, vibration (D. Hirst, SAM N1996/264), 10; Pinkawillinie Conservation Park, $32^{\circ} 03^{\prime} \mathrm{S}, 135^{\circ} 50^{\prime} \mathrm{E}$, Nov. 24, 1995 (D. Hirst, SAM N1997/137), 1 \%; Pinkawillinie Conservation Park, $33^{\circ} 07^{\prime} \mathrm{S}, 136^{\circ} 00^{\prime} \mathrm{E}$, May $19-$ 20, 1990, mallee trunk (D. Hirst, SAM N1996/217), 1 . . Victoria: near junction of Freeway and Everard tracks, Wyperfeld National Park, $35^{\circ} 34^{\prime} \mathrm{S}, 141^{\circ} 45^{\prime} \mathrm{E}$, July 4, 1982, under bark of Eucalyptus incrassata (M. Harvey, B. Roberts, NMV K3420), 1 \%; 2 km 

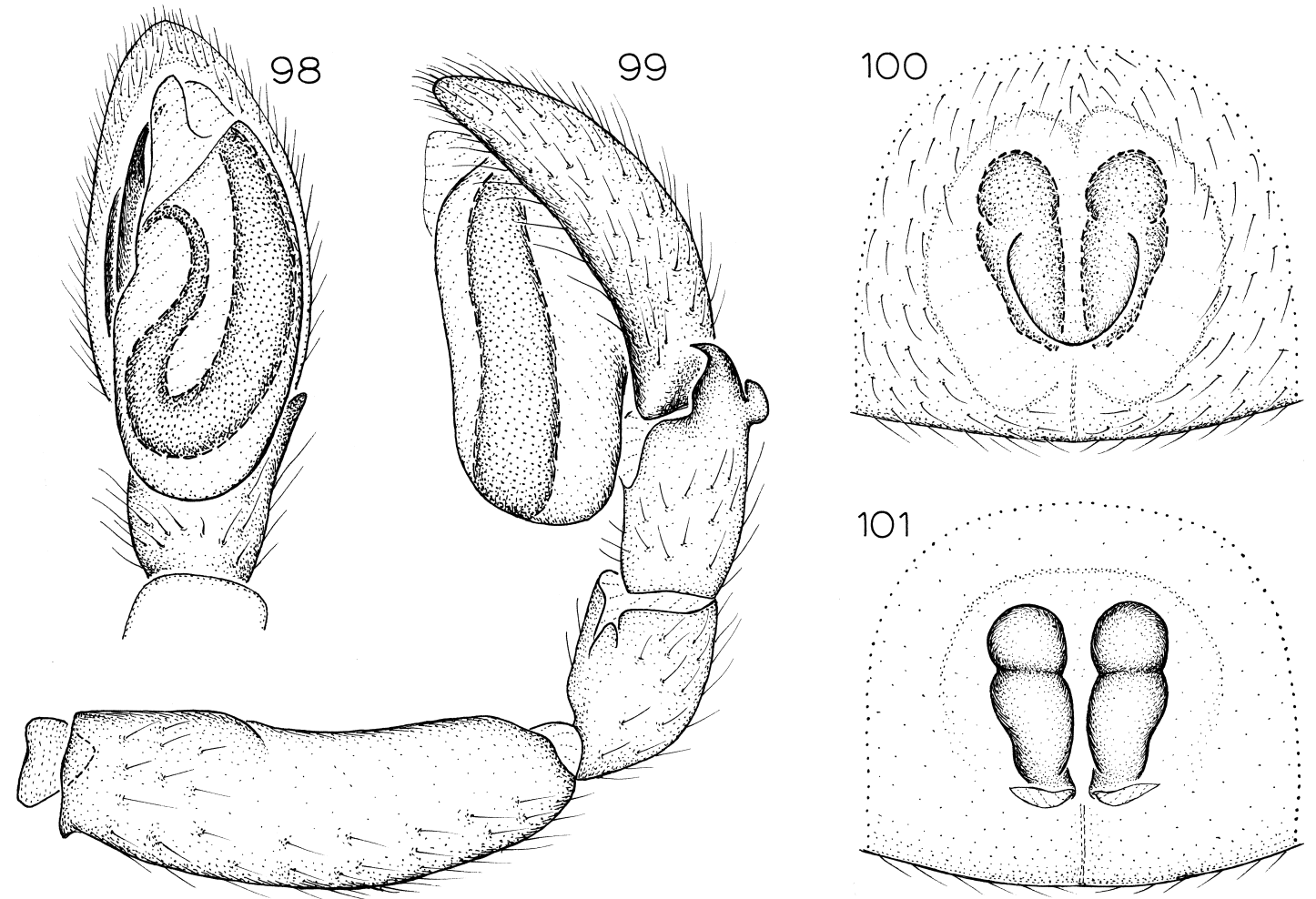

Figs. 98-101. 98, 99. Lampona barrow, new species. 100, 101. L. torbay, new species. 98. Left male palp, ventral view. 99. Same, retrolateral view. 100. Epigynum, ventral view. 101. Same, dorsal view.

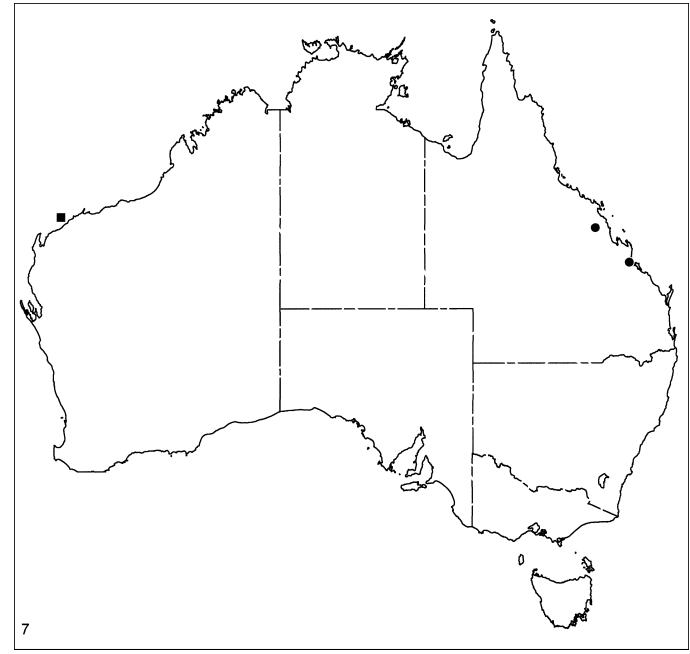

Map 7. Records of Lampona barrow, new species (square) and L. chalmers, new species (circles).
N McKenzie Junction, E Mount Martha Rest Area, $38^{\circ} 18^{\prime} \mathrm{S}, 145^{\circ} 01^{\prime} \mathrm{E}$, June 18,1989 (D. Hirst, SAM N1996/299), 1 ; ; Mount Martha, $38^{\circ} 18^{\prime} \mathrm{S}, 145^{\circ} 00^{\prime} \mathrm{E}$, June 18, 1989 (D. Hirst, SAM N1996/300), 1 ㅇ. Western Australia:

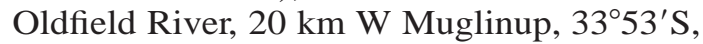
$120^{\circ} 51^{\prime} \mathrm{E}$, Nov. 12-13, 1987 (M. Baehr, CBB), 1 하. 1 ㅇ․

DistRIBUTION: Southern Australia (map 6).

Lampona barrow, new species Figures 98, 99; Map 7

TyPE: Male holotype from Barrow Island, $20^{\circ} 48^{\prime} \mathrm{S}, 115^{\circ} 26^{\prime} \mathrm{E}$, Western Australia (Feb. 1997; H. Heatwole), deposited in QMB (S34403).

ETYMOLOGY: The specific name is a noun in apposition taken from the type locality.

DiAGNOSIS: Males of this species can easily be recognized by their oddly shaped, distally bifid retrolateral tibial apophysis (fig. 99). 

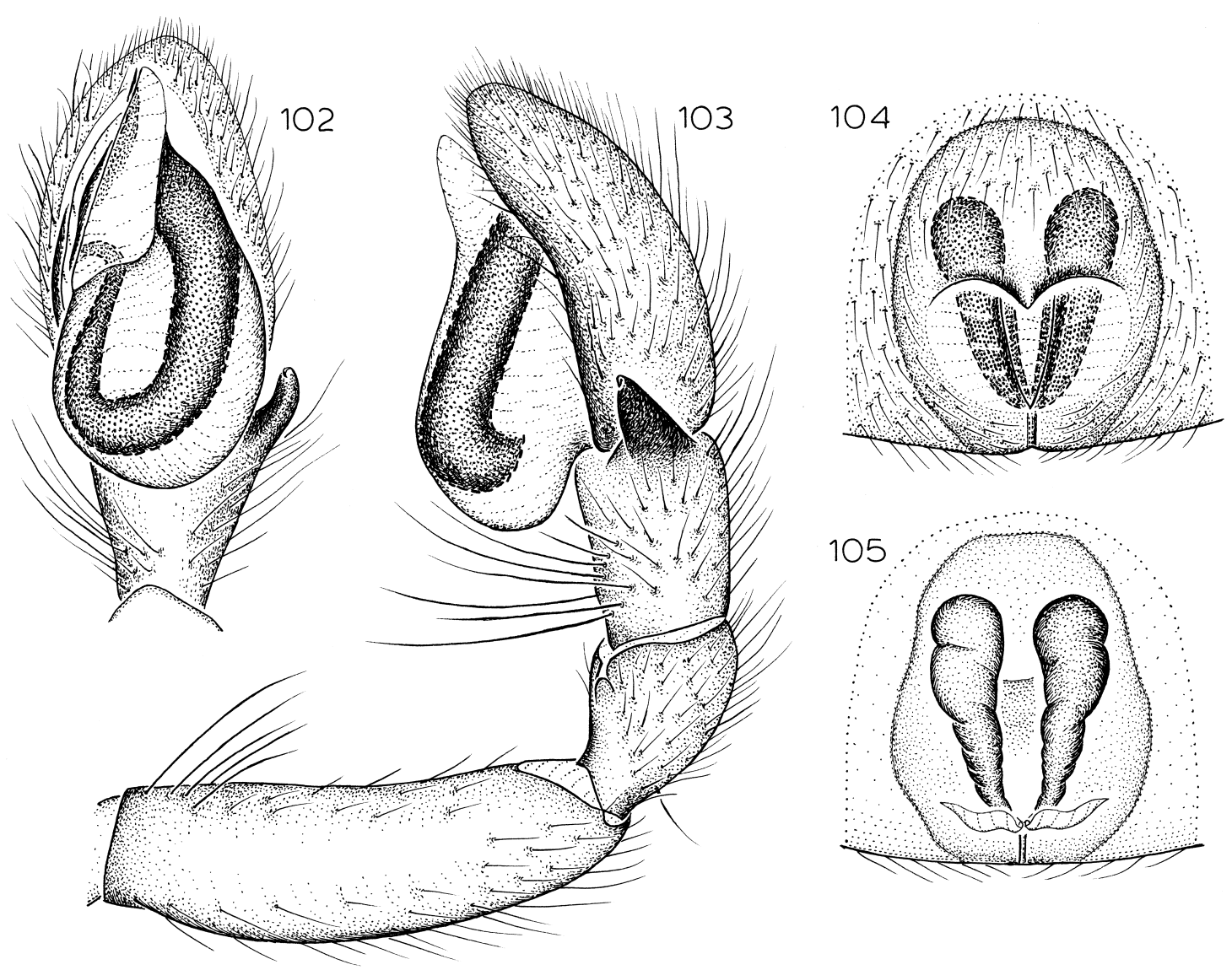

Figs. 102-105. Lampona chalmers, new species. 102. Left male palp, ventral view. 103. Same, retrolateral view. 104. Epigynum, ventral view. 105. Same, dorsal view.

MALE: Total length 5.5. Holotype badly faded, few details of original coloration preserved. Palpal femur darkened, expanded proximally, retrolateral tibial apophysis bifid, with square subdistal lobe (fig. 99); embolus relatively short, distal edge of tegulum heavily sclerotized (fig. 98).

FEMALE: Unknown.

Other Material ExAmined: None.

DisTRIBUTION: Known only from Barrow Island, Western Australia (map 7).

Lampona chalmers, new species Figures 102-105; Map 7

TYPE: Female holotype taken in a pitfall trap at C. Hollins on Mount Chalmers, $23^{\circ} 18^{\prime} \mathrm{S}, 150^{\circ} 38^{\prime} \mathrm{E}$, Queensland (Mar. 16, 1993; D. Wallace), deposited in QMB (S22184).
ETYMOLOGY: The specific name is a noun in apposition taken from the type locality.

DIAGNOSIS: Both sexes can be recognized by their distinctively dark coloration, males by the prolaterally hooked tip of the retrolateral tibial apophysis (figs. 102, 103), and females by their heart-shaped epigynal atrium (fig. 104).

MALE: Total length 5.0. Abdominal dorsum almost black, with five white spots reduced to narrow transverse bands; distal portions of femora, all of patellae, all of anterior tibiae, and distal portions of posterior tibiae greatly darkened. Palpal femur darkened throughout its length but otherwise unmodified; tip of retrolateral tibial apophysis hooked prolaterally (fig. 103); tegulum expanded, reaching to about one-third of tibial length, embolus hidden in ventral view by 

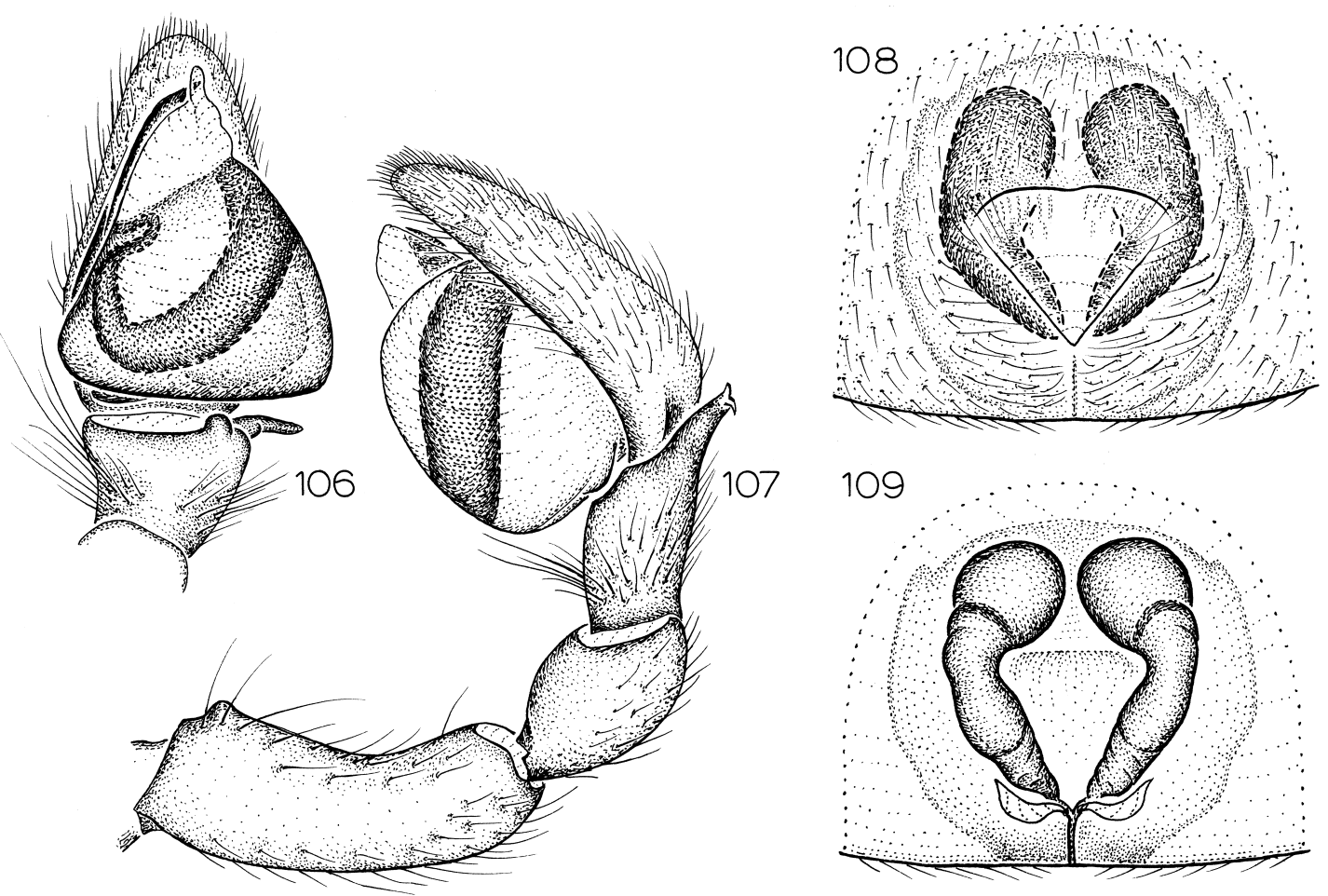

Figs. 106-109. Lampona flavipes L. Koch. 106. Left male palp, ventral view. 107. Same, retrolateral view. 108. Epigynum, ventral view. 109. Same, dorsal view.

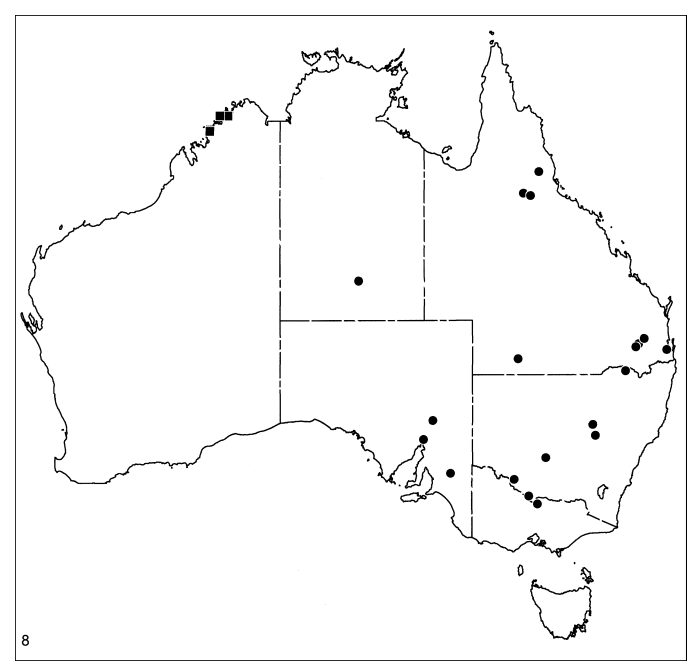

Map 8. Records of Lampona flavipes L. Koch (circles) and L. walsh, new species (squares). sclerotized prolateral rim of conductor (fig. 102).

FEMALE: Total length 5.4. Coloration as in male. Epigynum with large, posteriorly situated, heart-shaped atrium (fig. 104); spermathecae tripartite, posterior portions long, narrow (fig. 105).

Other MATERIAL EXAMINED: The male associated with the holotype was taken in litter at Homevale, $21^{\circ} 24^{\prime} \mathrm{S}, 148^{\circ} 33^{\prime} \mathrm{E}$, April 1-7, 1975 (V. Davies, R. Kohout, QMB S25979).

DistRIBUTION: Known only from mid-eastern Queensland (map 7).

\section{Lampona flavipes L. Koch \\ Figures 106-109; Map 8}

Lampona flavipes L. Koch, 1872: 368, pl. 28, figs. 6, 6a (female syntype from Rockhampton or Port Mackay, Queensland, in ZMH, examined).

Diagnosis: This distinctive species can easily be recognized by the posteriorly widened tegulum of males (figs. 106, 107) and 
the widened epigynal atrium (fig. 108) of females.

MALE: Total length 9.0. Abdominal coloration as in L. cylindrata; venter with paired, oval sclerites free of epigastric scutum. Legs with only coxae, trochanters, and bases of posterior femora orange, remainder yellow. Base of palpal femur with sharply angled protrusion; retrolateral tibial apophysis wide, flattened, bifid, with truncate flange extending anteriorly and sharply pointed flange extending laterally (fig. 107); tegulum globose, reaching beyond half length of tibia, widened posteriorly, embolus broad, with long tip (fig. 106).

FEMALE: Total length 14.3. Coloration as in male; abdominal dorsum without small, triangular sclerotization, venter with paired, oval sclerites free of epigastric sclerite. Epigynum with broad median atrium occupying most of spermathecal width (fig. 108); basal portion of spermathecae short, distinctly crenulated, distal portion bulbous, on narrowed neck (fig. 109).

Material Examined: New South Wales: Berida Station, $22 \mathrm{~km}$ W Gilgandra, 3139'S, $148^{\circ} 27^{\prime} \mathrm{E}$, Mar. 27, 1982 (W. Bell, AMS KS8859), $10^{\star}$; Boggabilla, 28 $40^{\prime} \mathrm{S}, 150^{\circ} 29^{\prime} \mathrm{E}$ (R. Mascord, AMS KS43652), 1 ㅇ ; Caldwell, 35 38'S, 14430'E, Jan. 1953 (V. Robb, NMV K3423), $10^{\top}$; Dubbo, $32^{\circ} 15^{\prime} \mathrm{S}$, $148^{\circ} 37^{\prime} \mathrm{E}$, July 31, 1966 (R. Mascord, AMS KS31846), 1 \% ; Hillston, $33^{\circ} 29^{\prime} \mathrm{S}, 145^{\circ} 32^{\prime} \mathrm{E}$, Sept. 9, 1959 (F. Gay, QMB), 1 \% Yanga Creek, Balranald, $34^{\circ} 42^{\prime} \mathrm{S}, 143^{\circ} 35^{\prime} \mathrm{E}$, Apr. 8, 1990 (D. Hirst, SAM N1996/297), 1 ㅇ․

Northern Territory: environs of Alice Springs, $23^{\circ} 42^{\prime} \mathrm{S}, 133^{\circ} 53^{\prime} \mathrm{E}$, May 9, 1965 (J. Balogh, HNHM), $10^{\star}$. Queensland: uncertain locality (Port Mackay or Rockhampton) (ZMH), 1 \% (syntype); Bowen district, Apr. 17, 1971 (R. Monroe, QMB S28431), 3; Camira, Brisbane, $27^{\circ} 30^{\prime} \mathrm{S}, 153^{\circ} 01^{\prime} \mathrm{E}$, Sept. 1986 (R. Raven, extracted from QMB S30168), 1 ㅇ, Jan. 1-Aug. 1, 1987 (R., J., D. Raven, QMB S30173), 1 $\%$; 14 km N Kidston, $18^{\circ} 52^{\prime} \mathrm{S}, 144^{\circ} 10^{\prime} \mathrm{E}$, June 12, 1993 (B., M. Baehr, CBB), 3 ; ; Lake Broadwater, SW Dalby, $27^{\circ} 11^{\prime} \mathrm{S}, 151^{\circ} 16^{\prime} \mathrm{E}$, Apr. 22-June 12, 1986, pitfall (M. Bennie, QMB S28599), 1 \% ; Lake Broadwater, track to $\mathrm{N}$ end (lagoon), $27^{\circ} 21^{\prime} \mathrm{S}, 1^{\circ} 1^{\circ} 06^{\prime} \mathrm{E}$, Jan. 25, 1985 (V. Davies, J. Gallon, QMB S34396), 1 đ̊; Marlaybrook,
Bunya Mountains National Park, 26 53'S, $151^{\circ} 37^{\prime} \mathrm{E}$, Mar. 6, 1976, under bark (R. Raven, V. Davies, extracted from QMB S28028), 10; McKinnons Creek, 9 km SSW Lynd junction, $19^{\circ} 00^{\prime} \mathrm{S}, 144^{\circ} 37^{\prime} \mathrm{E}$, June 13, 1993 (B., M. Baehr, CBB), 2q; 30 km SW Mount Garnet, $17^{\circ} 41^{\prime} \mathrm{S}, 145^{\circ} 07^{\prime} \mathrm{E}$, June 14, 1993 (B., M. Baehr, CBB), 1 \% ; Thargomindah, $28^{\circ} 00^{\prime} \mathrm{S}, 143^{\circ} 49^{\prime} \mathrm{E}$, Dec. 25-30, 1974, under bark (G. Ingram, QMB S28026), 1 ㅇ. South Australia: near Blanchetown, $34^{\circ} 21^{\prime} \mathrm{S}, 139^{\circ} 37^{\prime} \mathrm{E}$, Mar. 1966, under eucalypt bark (B. Edwards, SAM N1996/171), 1 ; ; Bunyeroo Creek, ABC Range, $31^{\circ} 25^{\prime} \mathrm{S}$, $138^{\circ} 34^{\prime} \mathrm{E}$, May 14-15, 1990, under bark (J. Forrest, SAM N1996/169), 1 $q$; Bunyeroo Gorge, Heysen Range, $31^{\circ} 25^{\prime} \mathrm{S}, 138^{\circ} 32^{\prime} \mathrm{E}$, May 16, 1990 (D. Hirst, J. Forrest, SAM N1996/168), 1 ठ; Quorn Road, 20 km NE Pt. Augusta, $32^{\circ} 28^{\prime} \mathrm{S}, 137^{\circ} 57^{\prime} \mathrm{E}$, Apr. 26, 1987, in creek bed (D. Hirst, SAM N1996/170), 1 ㅇ. Victoria: McDonalds Road, $1.8 \mathrm{~km} \mathrm{~S}$ Shepparton-Barmah Road, $36^{\circ} 04^{\prime} \mathrm{S}$, $145^{\circ} 02^{\prime} \mathrm{E}$, Jan. 17-22, 1994 (G. Milledge, P. Lillywhite, NMVS), 1 ㅇ.

DisTRIBUTION: Widespread in central and eastern Australia (map 8).

\section{Lampona walsh, new species Figures 110-113; Map 8}

TYPE: Female holotype (penultimate but with fully developed epigynum) taken in a Berlese sample at Walsh Point, Admiralty Gulf, $14^{\circ} 34^{\prime} \mathrm{S}, 125^{\circ} 15^{\prime} \mathrm{E}$, Western Australia (May 16, 1983; J. Balderson), deposited in QMB.

ETYMOLOGY: The specific name is a noun in apposition taken from the type locality.

DIAGNOSIS: Males resemble those of $L . k a$ palga but have a smaller retrolateral tibial apophysis (fig. 111); females also resemble those of L. kapalga but have a straight anterior epigynal margin (fig. 112) and smaller anterior portions of the spermathecae (fig. 113).

MALE: Total length 4.5. Coloration as in $L$. cylindrata, with anterior pair of transverse light stripes more pronounced than median pair. Palpal femur unmodified; retrolateral tibial apophysis about half as long as tibia, triangular (fig. 111); tegulum globose but extending only to about one-fifth of tibial 

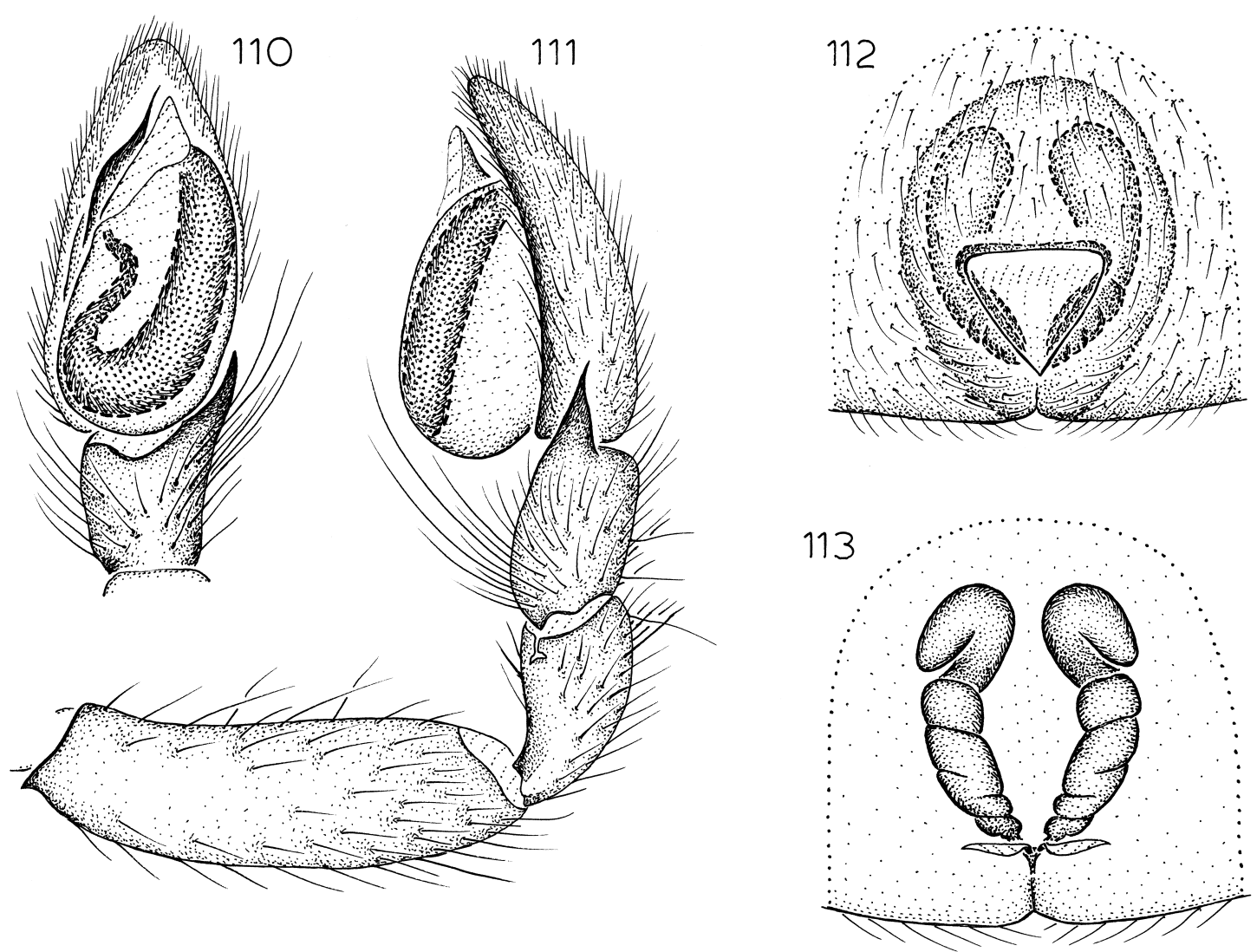

Figs. 110-113. Lampona walsh, new species. 110. Left male palp, ventral view. 111. Same, retrolateral view. 112. Epigynum, ventral view. 113. Same, dorsal view.

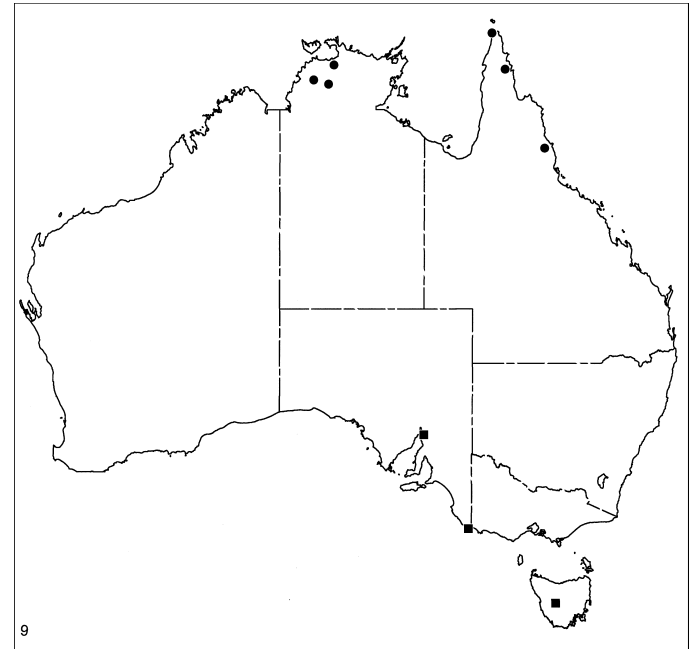

Map 9. Records of Lampona kapalga, new species (circles) and L. ewens, new species (squares). length; embolus narrow, gradually narrowed toward tip; palpal conductor small, with very small prolateral lobe (fig. 110).

FEMALE: Total length 6.0. Coloration as in male. Epigynum triangular (fig. 112); spermathecae elongate, anteriorly bulbous (fig. 113).

Other Material Examined: Western Australia: Augustus Island, CALM site 26/ $1,15^{\circ} 25^{\prime} \mathrm{S}, 124^{\circ} 38^{\prime} \mathrm{E}$, June $11-16,1988$, yellow pan trap in open forest (I. Naumann, QMB S25998), 10; Lone Dingo, Mitchell Plateau, $14^{\circ} 35^{\prime} \mathrm{S}, 125^{\circ} 45^{\prime} \mathrm{E}$, May 9-19, 1983, pan trap (I. Naumann, Cardale, QMB), $1 \delta^{\star}$.

Distribution: Northwestern Australia (map 8).

\section{Lampona kapalga, new species}

Figures 114-117; Map 9

TyPE: Female holotype from North Point, Kapalga, $12^{\circ} 25^{\prime} \mathrm{S}, 132^{\circ} 22^{\prime} \mathrm{E}$, Northern Ter- 

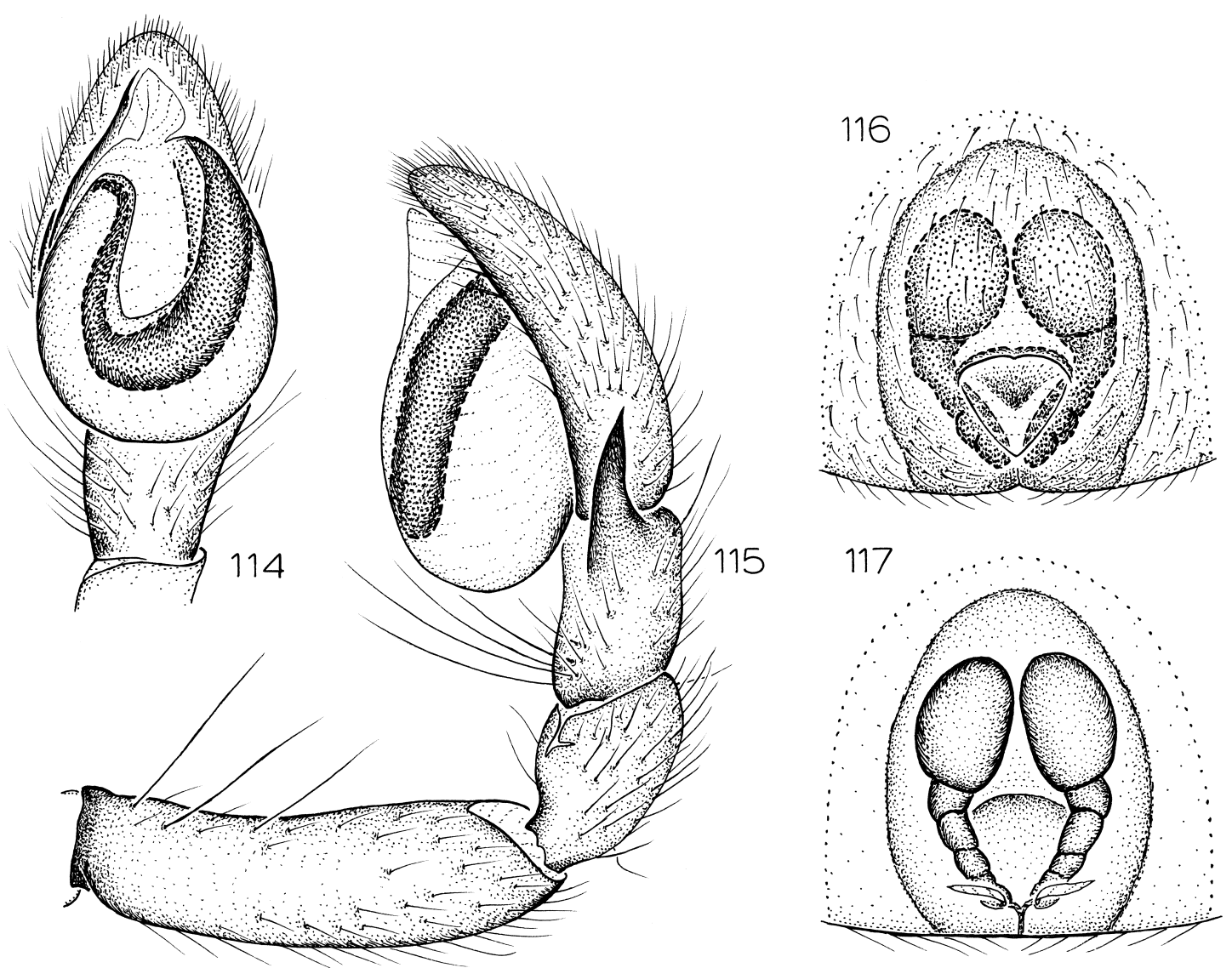

Figs. 114-117. Lampona kapalga, new species. 114. Left male palp, ventral view. 115. Same, retrolateral view. 116. Epigynum, ventral view. 117. Same, dorsal view.

ritory (Nov. 11, 1979; R. Raven), deposited in QMB (S34369).

ETYMOLOGY: The specific name is a noun in apposition taken from the type locality.

DiAGNOSIS: Males resemble those of $L$. walsh but can be recognized by the larger, longer retrolateral tibial apophysis and more globose tegulum (fig. 115); females also resemble those of $L$. walsh but have much larger anterior bulbs on the spermathecae (fig. 117).

MALE: Total length 4.9. Abdominal dorsum with anterior and median pairs of white spots fused into narrow paramedian longitudinal stripes; anterior femora, patellae, and tibiae darkened throughout their length, posterior femora darkened distally, patellae darkened laterally, tibiae darkened distally. Palpal femur unmarked, unmodified; retro- lateral tibial apophysis long, gradually narrowed distally (fig. 115); tegulum globose, extending to almost half of tibial length, embolus sharply narrowed below tip (fig. 114).

FEMALE: Total length 5.7. Abdominal dorsum with only median pair and posterior white spots, anterior pair obsolete; sides of femora, patellae, and tibiae darkened distally. Epigynum triangular, median plate invaginated on insides of lateral margins (fig. 116); anterior portion of spermathecae enlarged, bulbous (fig. 117).

OTHER MATERIAL EXAMINED: Northern Territory: Adelaide River, $70 \mathrm{mi} S$ Darwin, $13^{\circ} 15^{\prime} \mathrm{S}, 131^{\circ} 06^{\prime} \mathrm{E}$, Mar. 20-27, 1945 (B. Malkin, AMNH), 1 ; ; Mary River 2, 115 km E Darwin, $13^{\circ} 29^{\prime} \mathrm{S}, 132^{\circ} 01^{\prime} \mathrm{E}$, July 29-30, 1995, eucalypts (M., U. Baehr, CBB), 2 ㅇ․ Queensland: Mareeba, $17^{\circ} 00^{\prime} \mathrm{S}, 145^{\circ} 26^{\prime} \mathrm{E}$, 

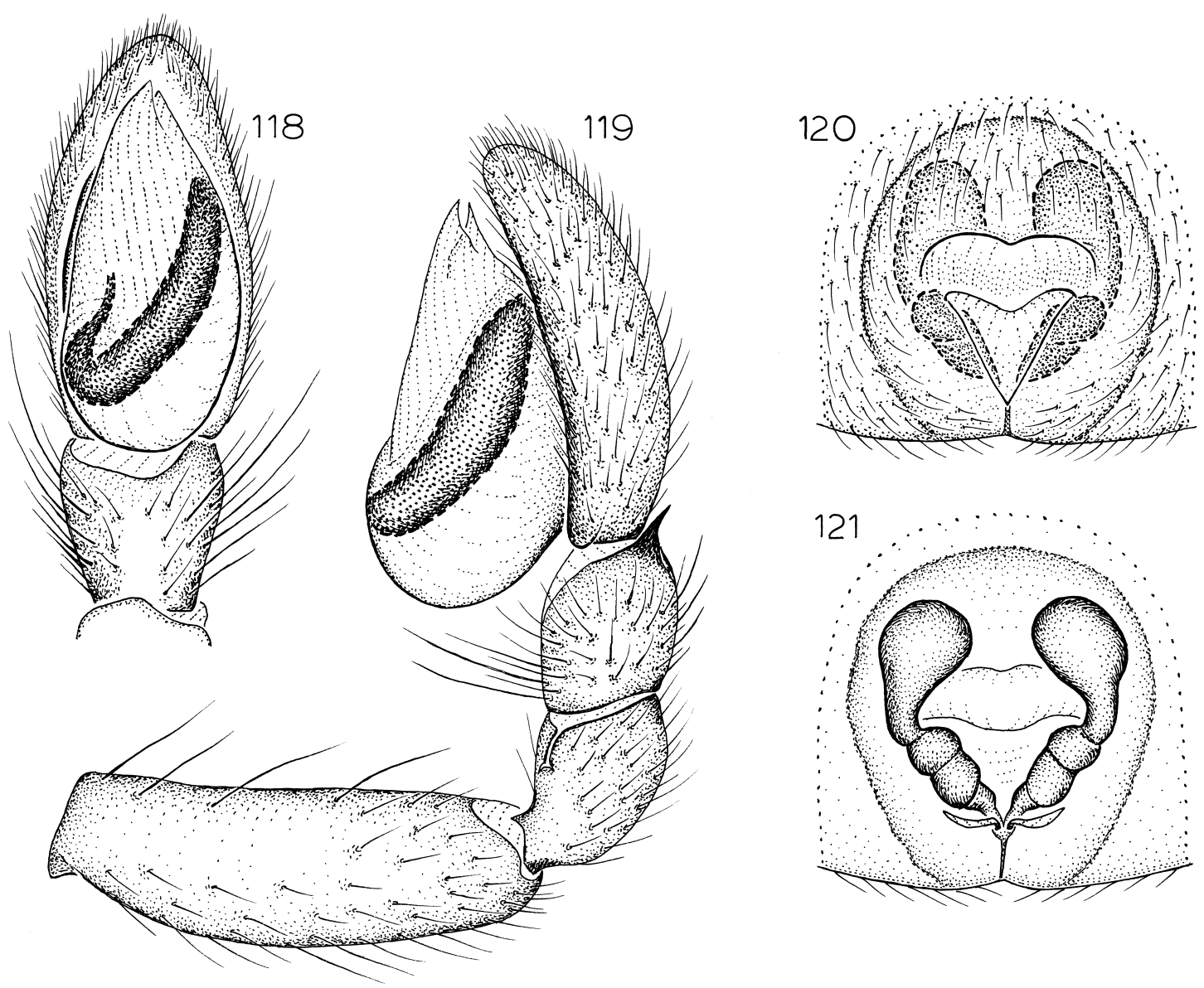

Figs. 118-121. Lampona ewens, new species. 118. Left male palp, ventral view. 119. Same, retrolateral view. 120. Epigynum, ventral view. 121. Same, dorsal view.

Feb. 12, 1972 (N. Coleman, AMS KS31866), $10^{\top}$; Prince of Wales Island, Torres Strait, $10^{\circ} 40^{\prime} \mathrm{S}, 142^{\circ} 10^{\prime} \mathrm{E}$, Sept. 12-14, 1913 (H. Clark, MCZ), $1 \% ; 13 \mathrm{~km} \mathrm{E}$ by $\mathrm{S}$ Weipa, $12^{\circ} 40^{\prime} \mathrm{S}, 143^{\circ} 00^{\prime} \mathrm{E}$, Apr, 20-May 24, 1994, intercept trap (P. Zborowski, QMB), 10,1 ㅇ․

Distribution: Northern Australia (map 9).

Lampona ewens, new species Figures 118-121; Map 9

TYPE: Female holotype taken under bark or stone at Ewens Pond Reserve, $38^{\circ} 02^{\prime} \mathrm{S}$, $140^{\circ} 47^{\prime} \mathrm{E}$, South Australia (Apr. 21, 1979; D. Lee), deposited in SAM (26).

ETYMOLOGY: The specific name is a noun in apposition taken from the type locality.

Diagnosis: Males resemble those of $L$. walsh but have a much smaller, shorter retrolateral tibial apophysis (fig. 119); females can be recognized by the bulbous, laterally displaced anterior spermathecal bulbs (fig. 121).

MALE: Total length 4.3. Abdominal dorsum with white markings expanded to cover most of dorsum except for dark chevrons in front of posterior white spot; legs unmarked. Palpal femur unmodified; retrolateral tibial apophysis short, sharply pointed (fig. 119); tegulum small, not posteriorly expanded, reaching only to about one-fourth of tibial length; embolar base long, tip narrow (fig. 118).

Female: Total length 6.5. Coloration as in male except abdominal dorsum also with 

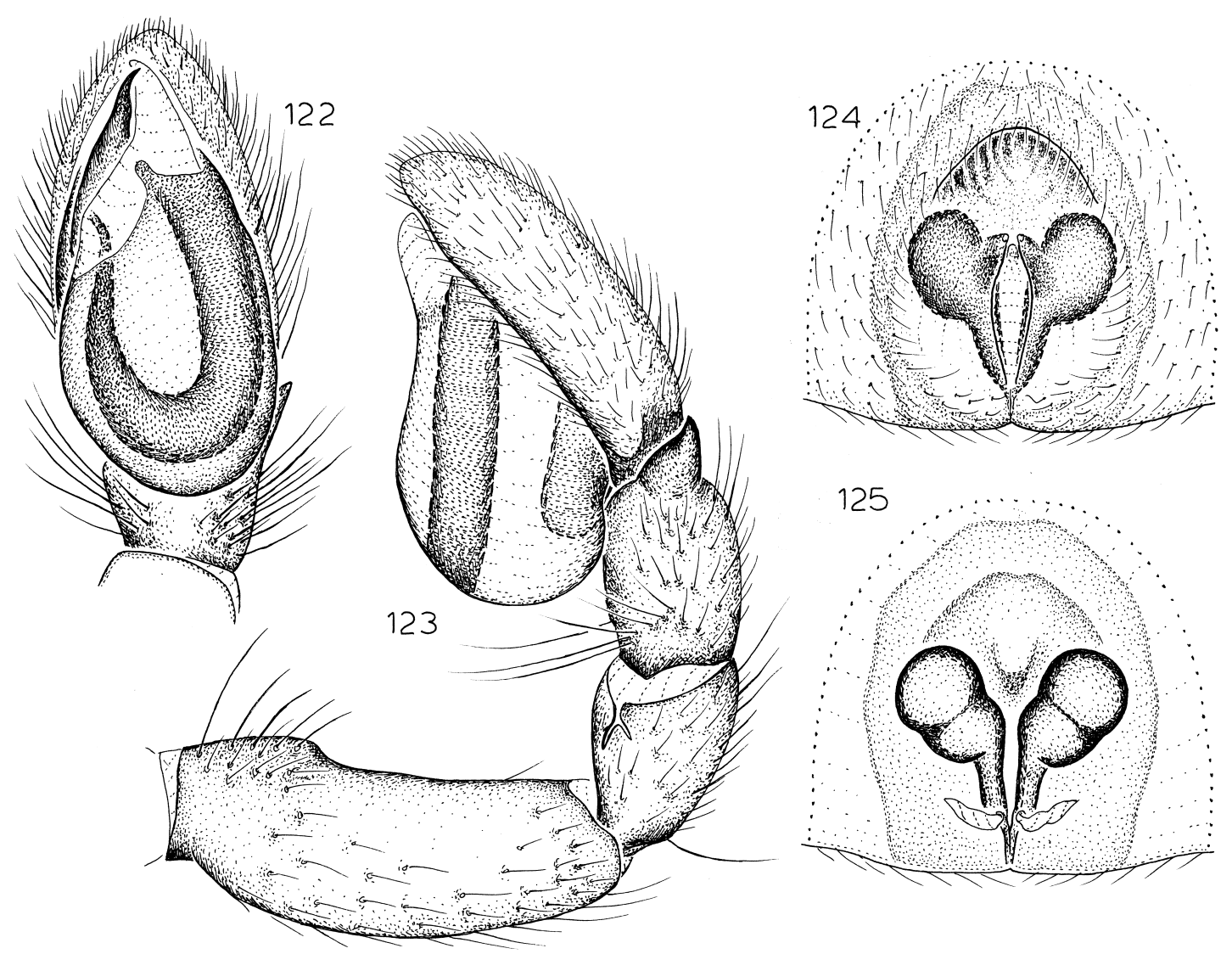

Figs. 122-125. Lampona punctigera Simon. 122. Left male palp, ventral view. 123. Same, retrolateral view. 124. Epigynum, ventral view. 125. Same, dorsal view.

dark cardiac mark and anterolateral dark spots. Epigynal midpiece triangular, widely expanded anteriorly, anterior margin weak (fig. 120); anterior portion of spermathecae laterally displaced, bulbous (fig. 121).

Other Material Examined: South Australia: Mambray Creek, $32^{\circ} 50^{\prime} \mathrm{S}, 137^{\circ} 59^{\prime} \mathrm{E}$, Mar. 11, 1979, under rock (M. Gray, AMS KS15008), 1 t. Tasmania: Lake St. Clair, $42^{\circ} 08^{\prime} \mathrm{S}, 146^{\circ} 10^{\prime} \mathrm{E}$, Jan. 29 , 1987, under logs and rocks, lake edge, elev. $700 \mathrm{~m}$ (R. Raven, J. Gallon, QMB S5637), 1 .

DisTRIBUTION: Known only from South Australia and Tasmania (map 9).

\section{THE PUNCTIGERA GROUP}

This group includes eight species in which the spermathecae tend to be relatively short and basically bipartite, although there may be a long posterior stalk, and the anterior portions of the spermathecae are often reduced in size. In two species, L. punctigera and $L$. braemar, the retrolateral tibial apophysis is triangular and the epigynal midpiece is long. In the remaining species, the epigynal midpiece tends to be diamond-shaped; in two ( $L$. moorilyanna, L. spec) the retrolateral tibial apophysis is long and wide; in three (L. russell, L. airlie, L. terrors) it is small and curved.

\section{Lampona punctigera Simon Figures 122-125; Map 10}

Lampona punctigera Simon, 1908: 399 (female syntypes from Midland, Western Australia, in $\mathrm{ZMH}$, examined, and from Western Australia, no specific locality, in MNHN, examined).

Diagnosis: This species resembles $L$. 
braemar in having a triangular retrolateral tibial apophysis and in the shape of the spermathecae, but can be distinguished in males by the presence of a ledgelike ventral protrusion near the base of the palpal femur, the more dorsally directed tip of the retrolateral tibial apophysis, and the widened, triangular embolus (figs. 122, 123), and in females by the distinct anterior depression of the epigynum and the short, bean-shaped spermathecae (figs. 124, 125).

MALE: Total length 7.3. Coloration as in $L$. cylindrata. Palpal femur with ledgelike ventral protrusion near base; retrolateral tibial apophysis greatly widened at base, triangular, skewed dorsally (fig. 123), tegulum globose, extending to half length of tibia, with short, wide embolus (fig. 122).

Female: Total length 9.8. Coloration as in male. Epigynum elongate, with semicircular anterior depression situated just anterior of paired openings (fig. 124); spermathecae short, bean-shaped, with anterior portion larger than posterior (fig. 125).

MAterial ExAmined: Queensland: Braemar State Forest, $27^{\circ} 13^{\prime} \mathrm{S}, 150^{\circ} 50^{\prime} \mathrm{E}$, Oct. 15-19, 1979, under rocks and bark, cypress and brigalow (R. Raven, QMB S25975), $10^{\star}$; Lake Broadwater, SW Dalby, $27^{\circ} 11^{\prime} \mathrm{S}$, $151^{\circ} 16^{\prime}$ E, Feb. 22-25, 1986 (G. Monteith, G. Thompson, QMB S26778), 1 ㅇ. South Australia: Avenue Range, $36^{\circ} 47^{\prime} \mathrm{S}, 140^{\circ} 14^{\prime} \mathrm{E}$,

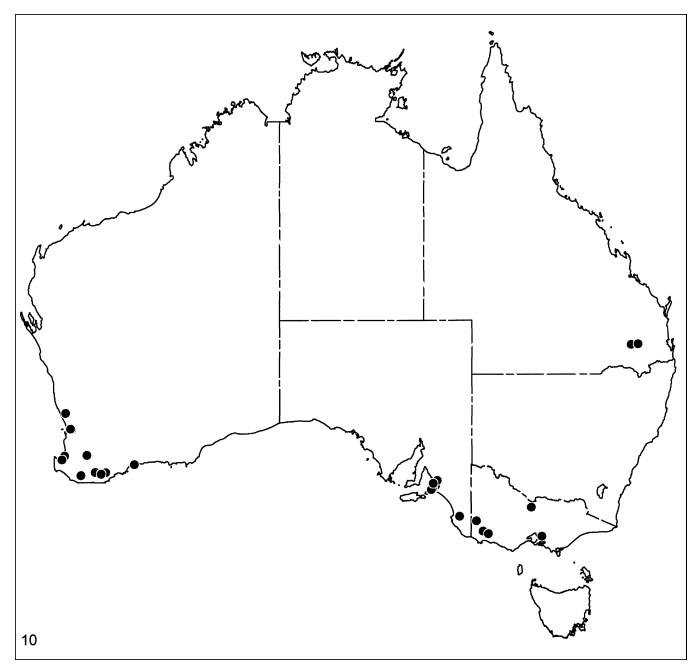

Map 10. Records of Lampona punctigera Simon.
Mar. 1908 (SAM N1996/187), 1 \%; Belair National Park, Mount Lofty Ranges, $35^{\circ} 01^{\prime} \mathrm{S}, 138^{\circ} 39^{\prime} \mathrm{E}$, Jan. 1936 (H. Womersley, SAM N1996/182, 183), 2 ; ; Brown Hill Creek, Adelaide foothills, $35^{\circ} 00^{\prime} \mathrm{S}, 138^{\circ} 38^{\prime} \mathrm{E}$, Feb. 23, 1936 (H. Womersley, SAM N1996/ 184), 1 \%; Loftia Recreation Park, Mount Lofty Ranges, $35^{\circ} 02^{\prime} \mathrm{S}, 138^{\circ} 42^{\prime} \mathrm{E}$, Mar. 20, 1990 (D. Hirst, SAM N1996/180), 1 \%; Mount Lofty, $34^{\circ} 59^{\prime} \mathrm{S}, 138^{\circ} 42^{\prime} \mathrm{E}$, Aug. 31, 1931, elev. $1000 \mathrm{ft}$ (P. Darlington, W. Wheeler, MCZ), 2 \% Mylor, Mount Lofty Ranges, $35^{\circ} 03^{\prime} \mathrm{S}, 138^{\circ} 46^{\prime} \mathrm{E}$, Sept. 1935 (H. Womersley, SAM N1996/181), $19 ; 5$ km S Mylor, $35^{\circ} 03^{\prime} \mathrm{S}, 138^{\circ} 45^{\prime} \mathrm{E}$, Dec. 14, 1980 (A. Austin, AMS KS10536), 3 ; ; Pedlars Creek, 35 ${ }^{\circ} 13^{\prime} \mathrm{S}, 138^{\circ} 34^{\prime} \mathrm{E}$, Sept. 23, 1954 (G. Gross, SAM N1996/179), 19; Roachdale Nature Trail, Kersbrook, $34^{\circ} 47^{\prime}$ S, $138^{\circ} 51^{\prime}$ E, Apr. 17 , 1980 (J., M. Szent-Ivany, SAM N1996/186), $1 \%$; Sellicks, Aldinga Scrub, $35^{\circ} 17^{\prime} \mathrm{S}$, $138^{\circ} 29^{\prime}$ E, Mar. 25, 1987 (E. Matthews, J. Forrest, SAM N1996/177), 1 ô, June 16, 1987 (E. Matthews, J. Forrest, SAM N1996/ 176), 10, June 26, 1987, under bark (D. Hirst, SAM N1996/178), 1 ; Tea Tree Gully, Adelaide, 345'' S, $138^{\circ} 36^{\prime}$ E (SAM N1996/ 185), $1 \delta^{t}$. Victoria: Campaspe River, $15 \mathrm{~km}$ S Echuca, $36^{\circ} 16^{\prime} \mathrm{S}, 144^{\circ} 41^{\prime} \mathrm{E}$, May 23,1988 (D. Hirst, SAM N1996/310, 311), 10,1 \% Coleraine, $37^{\circ} 36^{\prime} \mathrm{S}, \quad 141^{\circ} 42^{\prime} \mathrm{E}$ (NMV $\mathrm{K} 3438), 1$; ; Edenhope, $37^{\circ} 02^{\prime} \mathrm{S}, 141^{\circ} 17^{\prime} \mathrm{E}$, July 31, 1913 (SAM N1996/188, 189), 2 \% ; Hamilton, $37^{\circ} 45^{\prime} \mathrm{S}, 142^{\circ} 02^{\prime} \mathrm{E}$, Aug. 14, 1948 (G. Stephens, NMV K3437), 1 \%, Oct. 10, 1948 (NMV K3435), 1 \%, Nov. 15, 1948 (G. Stephens, NMV K3422), 1 ; Sherbrook Forest, $37^{\circ} 53^{\prime} \mathrm{S}, 145^{\circ} 21^{\prime} \mathrm{E}$, July 24 , 1968 , under bark (J. Balogh, HNHM), 2 ㅇ. Western Australia: no specific locality (MNHN AR1612), 19 (syntype); Arthur River, $33^{\circ} 21^{\prime} \mathrm{S}, 117^{\circ} 02^{\prime} \mathrm{E}$, Aug. 15,1992 , under eucalypt bark (C. Car, WAM 95/1003, 1004), 2\%; W Cranbrook, $1 \mathrm{~km} \mathrm{~N}$ junction Boyup Brook and Franklin roads, $34^{\circ} 18^{\prime} \mathrm{S}$, $117^{\circ} 33^{\prime} \mathrm{E}$, Nov. 18, 1992, under $\log$ (A. Longbottom, WAM 95/998), 1 \%; Darlington, $31^{\circ} 55^{\prime} \mathrm{S}, 116^{\circ} 05^{\prime} \mathrm{E}$, Sept. 5, 1962, elev. 450 ft (E. Ross, D. Cavagnaro, CAS), 1 ; Fitzgerald River National Park, $9.4 \mathrm{~km}$ WSW Annie Peak, $33^{\circ} 52^{\prime} \mathrm{S}, 119^{\circ} 58^{\prime} \mathrm{E}$, Nov. 1996, pitfall (A. Sanders, WAM 96/1519, 1520), 10; Gelorup Rise, $33^{\circ} 23^{\prime} \mathrm{S}, 115^{\circ} 39^{\prime} \mathrm{E}$, Feb. 

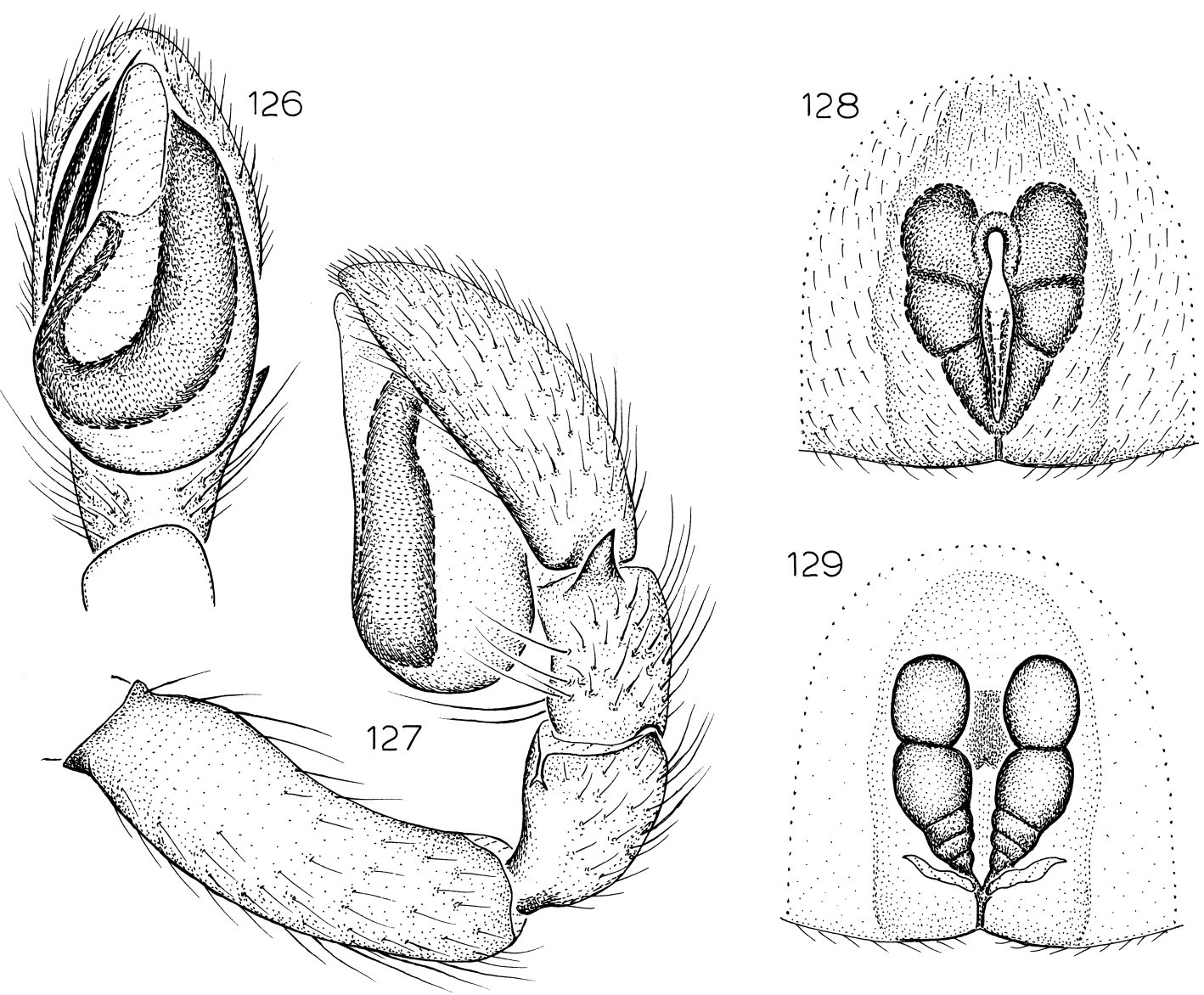

Figs. 126-129. Lampona braemar, new species. 126. Left male palp, ventral view. 127. Same, retrolateral view. 128. Epigynum, ventral view. 129. Same, dorsal view.

1997, in house (K. Longbottom, WAM 96/ 1517), $10^{\top}$; Ludlow, $33^{\circ} 36^{\prime} \mathrm{S}, 115^{\circ} 29^{\prime} \mathrm{E}$, Apr. 5, 1979 (WAM 95/1001), 1\%; Midland, $31^{\circ} 52^{\prime} \mathrm{S}, 116^{\circ} 01^{\prime} \mathrm{E}$, Aug. 27, 1905 (W. Michaelsen, R. Hartmeyer, ZMH), 1 ㅇ (syntype); Moore River, Brand Highway, $47 \mathrm{~km}$ $\mathrm{N}$ Gin Gin, $31^{\circ} 00^{\prime} \mathrm{S}, 115^{\circ} 42^{\prime} \mathrm{E}$, Feb. 24, 1979, under bark (M. Gray, AMS KS14951), 10; near Nthn Ranger's residence, Stirling Range National Park, $34^{\circ} 19^{\prime} \mathrm{S}, 118^{\circ} 12^{\prime} \mathrm{E}$, June 10, 1993, under wandoo bark (J. Waldock, A. Sampey, WAM 95/1002), 1 \% ; Red Lake b., Lake Muir, $34^{\circ} 28^{\prime} \mathrm{S}, 116^{\circ} 40^{\prime} \mathrm{E}$, Nov. 24-25, 1987 (B., M. Baehr, CBB), 1 \% S Stirling Range Caravan Park, $34^{\circ} 19^{\prime} \mathrm{S}, 118^{\circ} 12^{\prime} \mathrm{E}$, Apr. 1, 1993, active at night (M. Harvey, J. Waldock, WAM 95/1005), $10^{\text {; }}$; White Gum Flat, Stirling Range National Park, $34^{\circ} 24^{\prime} \mathrm{S}$, $117^{\circ} 55^{\prime} \mathrm{E}$, Apr. 1, 1993, under wandoo bark (M. Harvey, J. Waldock, WAM 95/999, 1000), 2 q .

Distribution: Southern Australia (map 10).

\section{Lampona braemar, new species} Figures 42, 126-129; Map 11

TYPES: Female holotype and male allotype taken in pitfall traps in cypress and brigalow litter in Braemar State Forest, $27^{\circ} 13^{\prime} \mathrm{S}$, $150^{\circ} 50^{\prime} \mathrm{E}$, Queensland (Oct. 15-19, 1979; R. Raven), deposited in QMB (S25974).

ETYMOLOGY: The specific name is a noun in apposition taken from the type locality.

Diagnosis: This species resembles $L$. punctigera (see above) but can be distin- 
guished by its smaller size, the reduced pattern on the abdominal dorsum (fig. 42), the short, thickened, darkened, setose tibia I, in males by the very slight ventral protrusion near the base of the palpal femur, the more distally directed tip of the retrolateral tibial apophysis, and the narrower embolus (figs. $126,127)$, and in females by the anteriorly flat epigynum and the longer spermathecae (figs. 128, 129).

MALE: Total length 5.6. Abdominal dorsum with two anterior pairs of white stripes reduced to narrow bands (fig. 42); sides of carapace with scattered white, scalelike setae. Distal halves of femora, entire surface of anterior patellae and tibiae, distal halves of posterior tibiae and patellae darkened; tibia I incrassate, much wider than metatarsus, highly setose. Palpal femur with only slight ventral expansion near base; retrolateral tibial apophysis widened at base, triangular, tip directed distally (fig. 127), tegulum globose, extending to half length of tibia, with narrow embolus (fig. 126).

FEMALE: Total length 8.0. Coloration as in male. Epigynum elongate, anterior edge without distinct rim, without anterior depression (fig. 128); spermathecae elongate, quadripartite (fig. 129).

Other Material Examined: New South Wales: Blood Filly Creek near Jenolan Caves, Kanangra-Boyd National Park,

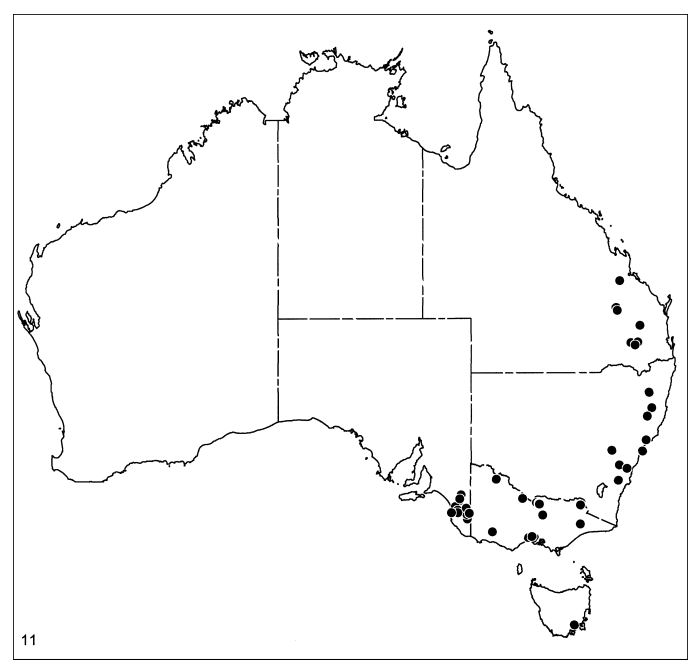

Map 11. Records of Lampona braemar, new species. $33^{\circ} 59^{\prime} \mathrm{S}, 150^{\circ} 08^{\prime} \mathrm{E}$, Mar. 27, 1976, eucalypt litter (M. Gray, G. Hunt, J. McDougall, AMS KS29877), 10 ; Bungonia Caves area, near Information Centre, $34^{\circ} 50^{\prime} \mathrm{S}, 150^{\circ} 04^{\prime} \mathrm{E}$, Nov. 1989, pitfall (G. Hunt, AMS KS22564), 1 o; Flaggy Creek, $0.95 \mathrm{~km} \mathrm{~S}$ along Ripleys Trail from Clarence Town Road, Wallaroo State Forest, $32^{\circ} 36^{\prime} \mathrm{S}, 151^{\circ} 48^{\prime} \mathrm{E}$, Feb. 4-Apr. 9, 1993, pitfall, elev. 20 m (M. Gray, G. Cassis, AMS KS40480), 1 đ; NE facing slope above Kunderang Station Creek, $30^{\circ} 48^{\prime} \mathrm{S}$, $152^{\circ} 06^{\prime}$ E, Feb. 4-Apr. 9, 1993, pitfall, elev. 410 m (M. Gray, G. Cassis, AMS KS40478), $10^{\dagger}$; Kunderang Trail, $1.2 \mathrm{~km}$ from homestead, $30^{\circ} 49^{\prime} \mathrm{S}, 152^{\circ} 08^{\prime} \mathrm{E}$, Feb. 4-Apr. 9, 1993, pitfall, elev. 210 m (M. Gray, G. Cassis, AMS KS40479), 19; Lake Munmorah State Recreation Reserve, Geebung, 33 ${ }^{\circ} 13^{\prime} \mathrm{S}$, $151^{\circ} 34^{\prime} \mathrm{E}$, Oct. 30, 1980, litter (M. Gray, AMS KS17799), 10; Moggs Swamp Creek, Moggs Swamp Fire Trail, Warra State Forest,

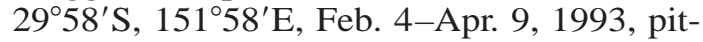
fall, elev. 1080 m (M. Gray, G. Cassis, AMS KS37090), $10^{\text {to }}$; Mount Wiagdon, $33^{\circ} 11^{\prime} \mathrm{S}$, 149 41'E, Dec. 23, 1962, elev. 900 m (E. Ross, D. Cavagnaro, CAS), $1 \%$; Mummel Forest Road, $7.6 \mathrm{~km} \mathrm{~N}$ junction with Enfield Forest Road, Enfield State Forest, $31^{\circ} 17^{\prime} \mathrm{S}$, $151^{\circ} 51^{\prime} \mathrm{E}$, Feb. 4-Apr. 9, 1993, pitfall, elev. $1340 \mathrm{~m}$ (M. Gray, G. Cassis, AMS KS40477), 10; The Razorback, near Picton, $34^{\circ} 11^{\prime} \mathrm{S}, 150^{\circ} 36^{\prime} \mathrm{E}$, Dec. 19, 1962, elev. 230 $\mathrm{m}$ (E. Ross, D. Cavagnaro, CAS), 1 ㅇ․ Queensland: Boggomoss, via Taroom, $25^{\circ} 25^{\prime} \mathrm{S}, 150^{\circ} 01^{\prime} \mathrm{E}$, Nov. 11, 1996-Jan. 1997, pitfall (D. Cook, G. Monteith, QMB S36213), 1 \% ; Braemar State Forest, $27^{\circ} 13^{\prime} \mathrm{S}$, $150^{\circ} 50^{\prime} \mathrm{E}$, Oct. $15-19,1979$, cypress and brigalow litter, pitfalls (R. Raven, QMB 25983), 3 ơ $^{\text {; }}$ Brigooda, $26^{\circ} 16^{\prime} \mathrm{S}, 151^{\circ} 25^{\prime} \mathrm{E}$, Jan. 26Apr. 20, 1995, pitfall, vine scrub (G. Mon-

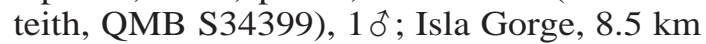
SSW lookout, $25^{\circ} 16^{\prime} \mathrm{S}, 149^{\circ} 56^{\prime} \mathrm{E}$, Dec. 19, 1997-Mar. 3, 1998, pitfall, vine scrub, elev. $360 \mathrm{~m}$ (G. Monteith, D. Cook, QMB S44205), 2 9 ; Lake Broadwater, SW Dalby, $27^{\circ} 11^{\prime} \mathrm{S}, 151^{\circ} 16^{\prime} \mathrm{E}$, Jan. 26-Feb. 19, 1985, pitfalls (M. Bennie, QMB S34386, 34387), 20, 1 ㅇ, Feb. 19-Mar. 26, 1985, pitfalls (M. Bennie, QMB S34372, 34373), 1 o, 1 \% , Mar. 26-May 17, 1985, pitfall (M. Bennie, QMB

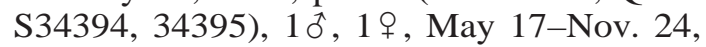
1985, pitfalls (M. Bennie, QMB S34371, 
34375-34377), 20, 5 오, Nov. 24, 1985-Jan. 3, 1986, pitfalls (M. Bennie, QMB S34393, 34405), 3 ㅈ, 1 으, Jan. 3-Feb. 25, 1986, pitfall (M. Bennie, QMB S34388, 34389, 34391, 34392), 60, 3 오 , Feb. 25-Apr. 22, 1986, pitfalls (M. Bennie, QMB S28618, 3437934383), 7o, 3 ㅇ, Apr. 22-June 12, 1986, pitfall (M. Bennie, QMB S28647, 34384, 34385), 80, 1 우 ; Lake Broadwater, via Dalby, $27^{\circ} 21^{\prime} \mathrm{S}, 151^{\circ} 06^{\prime} \mathrm{E}$, May $16-$ Nov. 23, 1985, river gums, pitfall (G. Thompson, QMB S26832), 1 ơ; Pheasant Creek, Wowan, $23^{\circ} 46^{\prime} \mathrm{S}, 150^{\circ} 09^{\prime} \mathrm{E}$, June 9, 1994, pitfall (F. Armstrong, D. Wallace, QMB S34420), 1 o $^{\text {; }}$; Taroom District, Boggomoss, $25^{\circ} 26^{\prime} \mathrm{S}$, $150^{\circ} 01^{\prime} \mathrm{E}$, Sept. 9-Nov. 11, 1996, pitfall (P. Lawless, QMB S34408, 34411, 34412), 40ิ, 2 ㅇ, Nov. 26, 1996-Jan. 12,1997, pitfall (D. Cook, G. Monteith, QMB S36328, 36364, 36633), 20, 1 ․ South Australia: 4 km S Box Flat, $35^{\circ} 38^{\prime} \mathrm{S}, 140^{\circ} 23^{\prime} \mathrm{E}$, Mar. 19, 1992, mallee/red sand (D. Hirst, SAM N1996/208, 209), $1 \widehat{0}, 19$; hill N Bucks Camp Well, $35^{\circ} 56^{\prime} \mathrm{S}, 140^{\circ} 18^{\prime} \mathrm{E}$, Mar. 18, 1992, vibration (D. Hirst, SAM N1996/196, 197), 1 ㅎ, 1 \% ; $22 \mathrm{~km}$ W Frances, 36 $43^{\prime} \mathrm{S}, 140^{\circ} 43^{\prime} \mathrm{E}$, Jan. 31-Feb. 4, 1994, Eucalyptus camaldulensis woodland (T. Croft, G. Carpenter, SAM N1996/214), 19 ; Gum Lagoon Conservation Park, $36^{\circ} 17^{\prime} \mathrm{S}, 140^{\circ} 02^{\prime} \mathrm{E}$, Mar. 26, 1992, vibration, lagoon edge (D. Hirst, SAM N1996/ 210), 1 đo; Jimmys Well, Mount Rescue Conservation Park, $35^{\circ} 51^{\prime} \mathrm{S}, 140^{\circ} 18^{\prime} \mathrm{E}$, Mar. 1620, 1992, pitfall (D. Hirst, SAM N1996/198, 199), 20 , Mar. 17, 20, 1992, vibration (D. Hirst, SAM N1996/200-207), 20ิ, 6우 Jip Jip Conservation Park, $36^{\circ} 29^{\prime} \mathrm{S}, 140^{\circ} 11^{\prime} \mathrm{E}$, Mar. 23, 1992, vibration (J. Forrest, D. Hirst, SAM N1996/211), 10; 13 km N Keilira Station, $36^{\circ} 37^{\prime} \mathrm{S}, 140^{\circ} 10^{\prime} \mathrm{E}$, Mar. 23, 1992, vibration, pitfall (D. Hirst, SAM N1996/212, 1997/139), 20; $0.4 \mathrm{~km}$ SE Mundulla, $36^{\circ} 22^{\prime} \mathrm{S}, 140^{\circ} 42^{\prime} \mathrm{E}$, Dec. 13-15, 1995 (SAM N1997/138), 10; Naracoorte, 3658'S, $140^{\circ} 45^{\prime} \mathrm{E}$, received Aug. 2, 1994 (K. Alcock, SAM N1996/213), 10َ; 3.5 km N Saint Aubins, $36^{\circ} 39^{\prime} \mathrm{S}, 140^{\circ} 52^{\prime} \mathrm{E}$, Dec. 4-8, 1995, pitfall (SAM N1997/140), 10; 8 km S Western Flat, $36^{\circ} 37^{\prime} \mathrm{S}, 139^{\circ} 46^{\prime} \mathrm{E}$, Feb. 28-Mar. 4, 1994, pitfall, Eucalyptus arenacea, E. baxteri low woodland on sand (T. Croft, G. Carpenter, J. Matthew, SAM N1996/241), 10 . Tasmania: East Risdon, $42^{\circ} 50^{\prime} \mathrm{S}, 147^{\circ} 21^{\prime} \mathrm{E}$, Apr.
19, 1966, from dry Xeroter (V. Hickman, AMS KS31861), 1 ․ Victoria: Barr Creek, Cohuna, $35^{\circ} 50^{\prime} \mathrm{S}, 144^{\circ} 11^{\prime} \mathrm{E}$, Dec. 1995-Jan. 1996, pitfalls (J. Hooper, CVIC 503), $1 \delta^{\hat{\sigma}}$, 3 ; Booths Road, 0.2 km S Murray Valley Highway, $36^{\circ} 08^{\prime} \mathrm{S}, 145^{\circ} 11^{\prime} \mathrm{E}$, Jan. 17-22, 1994, pitfall (G. Milledge, NMVS), 1 9 ; Brooms Road, $7.5 \mathrm{~km}$ NE Yambuna, $36^{\circ} 06^{\prime} \mathrm{S}, 145^{\circ} 05^{\prime} \mathrm{E}$, Apr. 10-17, 1994, pitfall (G. Milledge, NMVS), $1 \delta^{\Uparrow}$; Burrowa, Pine Mountain National Park, N Cudgewa, $36^{\circ} 12^{\prime} \mathrm{S}, 147^{\circ} 45^{\prime} \mathrm{E}$, Dec. 13, 1980 (B., M. Baehr, CCB), $10^{\text {to }}$; Flora Reserve, DunkeldPenshurst Road, $5.5 \mathrm{~km}$ SW Dunkeld, $37^{\circ} 41^{\prime} \mathrm{S}, 142^{\circ} 18^{\prime} \mathrm{E}$, Feb. 17-21, 1994, pitfall (A. Kobelt, NMVS), 19 ; Kotupna Barmah Road, $0.5 \mathrm{~km}$ NE Ellingtons Bridge, $36^{\circ} 05^{\prime} \mathrm{S}$, $145^{\circ} 03^{\prime} \mathrm{E}$, Apr. 10-17, 1994, pitfall (G. Milledge, NMVS), $1 \delta$; Longwood-Pranjip Road, $2.5 \mathrm{~km}$ S Pranjip Road, 36 $45^{\prime} \mathrm{S}$, $145^{\circ} 24^{\prime} \mathrm{E}$, Jan. 1993, pitfall (A. Bennett, NMVS), $1 \%$; McDonalds Road, $1.8 \mathrm{~km} \mathrm{~S}$ Shepparton-Barmah Road, $36^{\circ} 04^{\prime} \mathrm{S}$, $145^{\circ} 02^{\prime} \mathrm{E}$, Jan. 17-22, 1994, pitfall (G. Milledge, NMVS), 1 , , Apr. 10-17, 1994, pitfall (G. Milledge, NMVS), 1 ô, Jan. 26-30, 1995, pitfall (J. Evans, S. Hinkley, J. Wainer, NMVS), 10َ; McLellands Road, $0.1 \mathrm{~km} \mathrm{~N}$ Rathbones Road, $36^{\circ} 09^{\prime} \mathrm{S}, 145^{\circ} 14^{\prime} \mathrm{E}$, Dec. $2-$ 7, 1994, pitfall (J. Evans, M. Griffiths, S. Hinkley, NMVS), $1 \%$; Metropolitan Farm, Werribee, Murtcain Reserve, 28 Mile Road, $38^{\circ} 00^{\prime} \mathrm{S}, 144^{\circ} 32^{\prime} \mathrm{E}$, Jan. 23-30, 1992, pitfall, salt bush flood plain (B. Van Praagh, P. Lillywhite, C. Knight, NMVS), 10; Murray Valley Highway, Skeleton Creek Crossing, $36^{\circ} 07^{\prime} \mathrm{S}, 145^{\circ} 11^{\prime} \mathrm{E}$, Dec. $2-7$, 1994, pitfall (J. Evans, M. Griffiths, S. Hinkley, NMVS), 1 \% ; Murray Valley Highway, $0.3 \mathrm{~km}$ NNW Walshs Bridge Road, $36^{\circ} 06^{\prime} \mathrm{S}, 145^{\circ} 12^{\prime} \mathrm{E}$, Apr. 10-17, 1994, pitfall (G. Milledge, NMVS), 1 ð, Jan. 26-30, 1995 (J. Evans, S. Hinkley, J. Wainer, NMVS), 1 ; 7.9 km NW junction Murray Valley Highway and Annuello Road, $34^{\circ} 46^{\prime} \mathrm{S}, 142^{\circ} 33^{\prime} \mathrm{E}$, drift fence pitfall (A. Yen, NMVS), $10^{\text {t }}$; Point Cook, opposite Car Park One, $37^{\circ} 56^{\prime} \mathrm{S}, 144^{\circ} 45^{\prime} \mathrm{E}$, Jan. 23-30, 1992, pitfall, chenopod herbfield (B. Van Praagh, P. Lillywhite, C. Knight, NMVS), 10, 1 ; ; Rathbones Road, $3 \mathrm{~km} \mathrm{E}$ Booths Road, $36^{\circ} 09^{\prime} \mathrm{S}, 145^{\circ} 13^{\prime} \mathrm{E}$, Apr. 1017, 1994 (G. Milledge, P. Lillywhite, NMVS), 20, $1 \% ; 3 \mathrm{mi} \mathrm{N}$ Swifts Creek, 

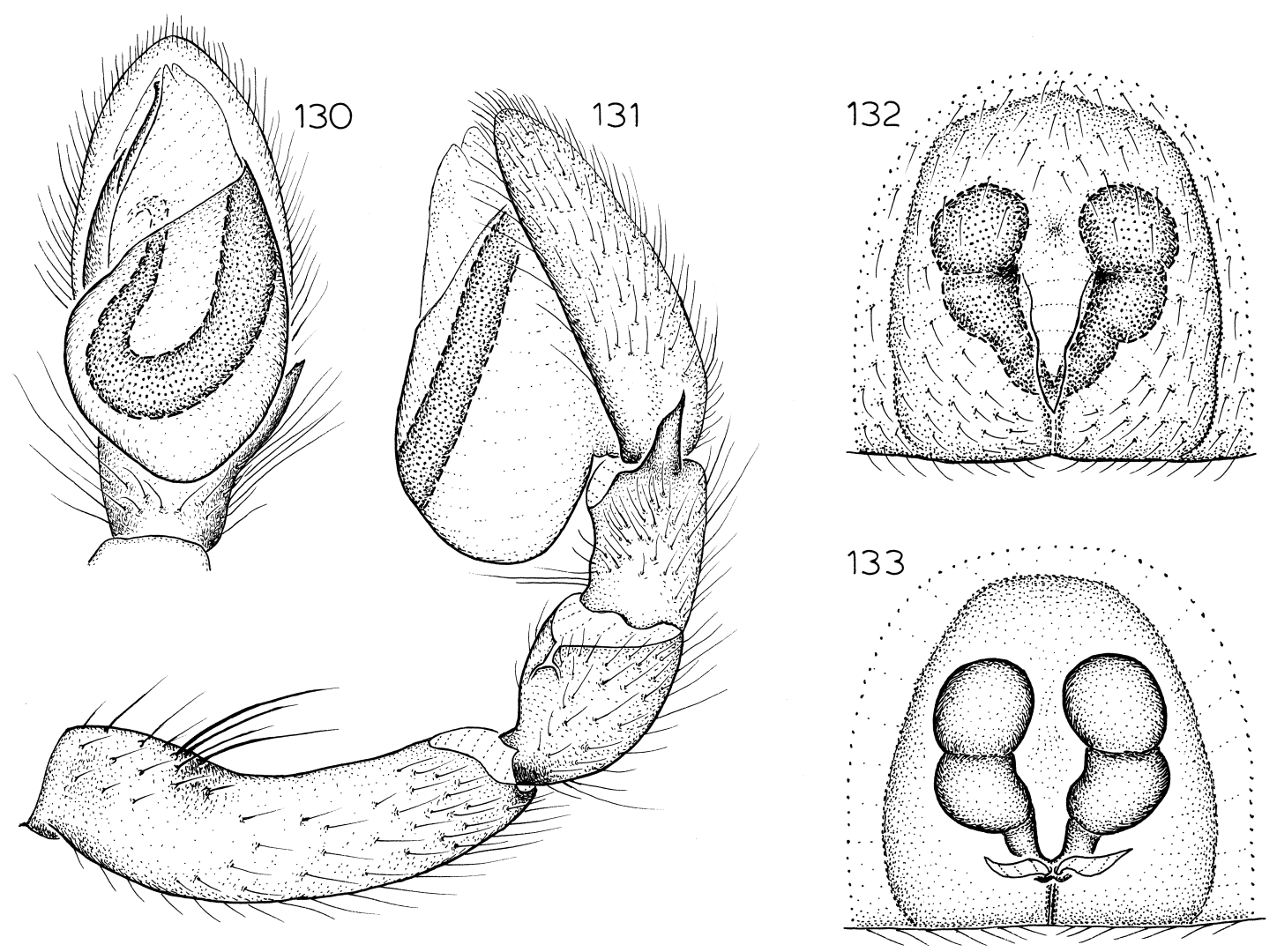

Figs. 130-133. Lampona moorilyanna, new species. 130. Left male palp, ventral view. 131. Same, retrolateral view. 132. Epigynum, ventral view. 133. Same, dorsal view.

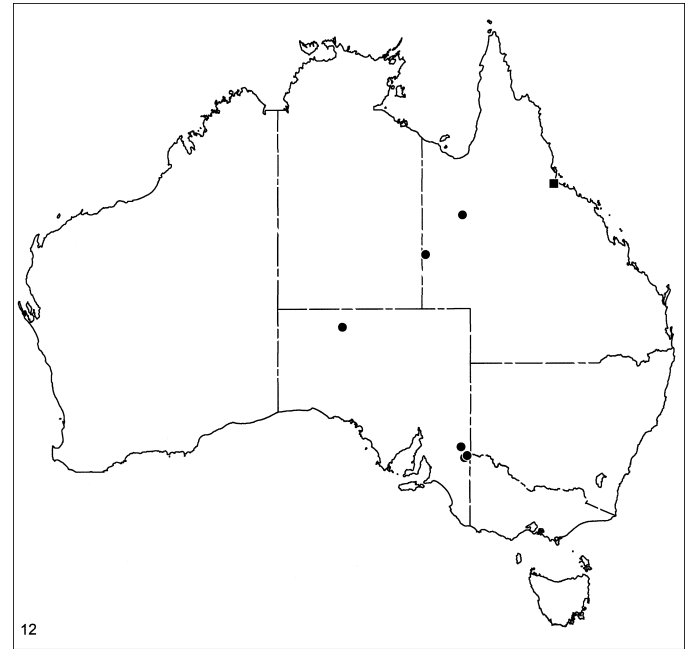

Map 12. Records of Lampona moorilyanna, new species (circles) and L. spec, new species (square). $37^{\circ} 15^{\prime} \mathrm{S}, 147^{\circ} 43^{\prime} \mathrm{E}$, Dec. 13,1962 , elev. 300 m (E. Ross, D. Cavagnaro, CAS), 1 \% .

DisTRIBUTION: Eastern Australia, including Tasmania (map 11).

\section{Lampona moorilyanna, new species}

Figures 130-133; Map 12

TYPE: Female holotype from Moorilyanna Native Well, $26^{\circ} 52^{\prime} \mathrm{S}, 133^{\circ} 01^{\prime} \mathrm{E}$, South Australia (July 2, 1914), deposited in SAM (38).

ETYMOLOGY: The specific name is a noun in apposition taken from the type locality.

DiAgnosis: This species resembles $L$. spec in having a moderately long, wide retrolateral tibial apophysis; males can be distinguished by the distally pointed (rather than truncated) retrolateral tibial apophysis (fig. 131), females by the narrow epigynal midpiece and long posterior spermathecal stalks (figs. 132, 133). 

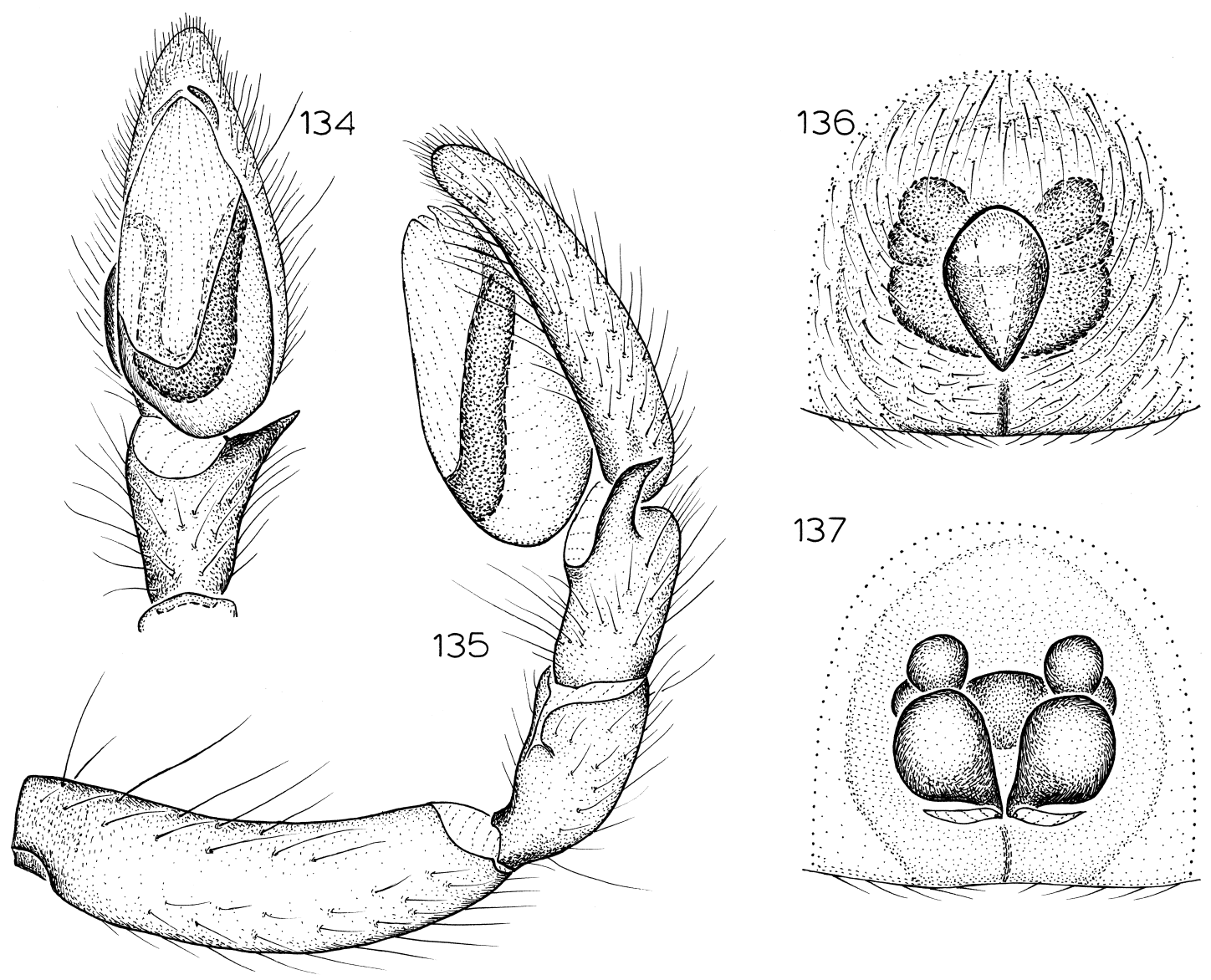

Figs. 134-137. Lampona spec, new species. 134. Left male palp, ventral view. 135. Same, retrolateral view. 136. Epigynum, ventral view. 137. Same, dorsal view.

MALE: Total length 7.2. Abdominal dorsum with indistinct pair of median white spots and more distinct posterior white spot over spinnerets; legs with femoral bases slightly darkened. Palpal femur with slightly enlarged base bearing elongated setae; retrolateral tibial apophysis relatively long, straight, abruptly narrowed distally (fig. 131); palpal bulb enlarged, reaching to about half of tibial length, embolus narrow, distally hooked (fig. 130).

Female: Total length 6.4. Coloration in $L$. cylindrata except paired white stripes on abdominal dorsum indistinct. Epigynum simple, with narrow midpiece (fig. 132); spermathecae tripartite, kidney-shaped (fig. 133).
Other MATERIAL ExAMINED: Queensland: Cloncurry, $20^{\circ} 42^{\prime} \mathrm{S}, 140^{\circ} 30^{\prime} \mathrm{E}$, Sept. 10 , 1972 (M. Baehr, CBB), 1; 1.5 m SE Poodyea Point, Glenormiston Station, $22^{\circ} 52^{\prime} \mathrm{S}$, $138^{\circ} 13^{\prime} \mathrm{E}$, June 16,1974 , under stone in dry creek bed (A. Elliot, QMB S34414), 1 ㅇ. South Australia: $14 \mathrm{~km}$ WNW Renmark, $34^{\circ} 07^{\prime} \mathrm{S}, 140^{\circ} 37^{\prime} \mathrm{E}$, July 4-24, 1995, pitfall, mallee on dune (A. Lambie, QMB S32548), $10^{\top} ; 19 \mathrm{~km} \mathrm{~N}$ Renmark, $34^{\circ} 00^{\prime} \mathrm{S}, 140^{\circ} 47^{\prime} \mathrm{E}$, June 6-July 6, 1995, intercept trap (K. Pullen, QMB), 1ठ; 79 km NNW Renmark, $33^{\circ} 31^{\prime} \mathrm{S}, 140^{\circ} 24^{\prime} \mathrm{E}$, June 6-July 6, 1995, intercept trap (K. Pullen, QMB), $1 \delta$.

DisTRIBUTION: Known only from Queensland and South Australia (map 12). 

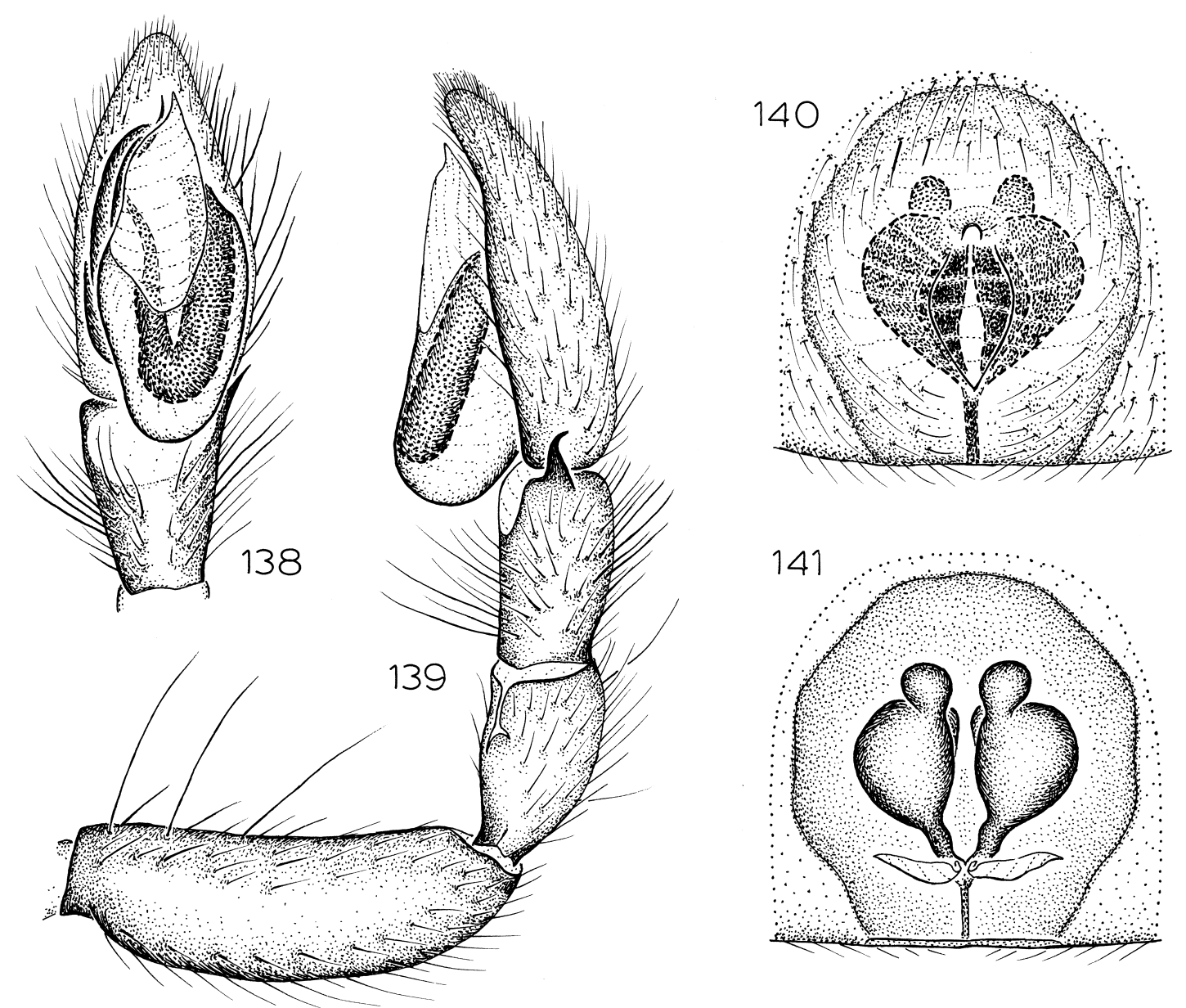

Figs. 138-141. Lampona russell, new species. 138. Left male palp, ventral view. 139. Same, retrolateral view. 140. Epigynum, ventral view. 141. Same, dorsal view.

\section{Lampona spec, new species}

Figures 134-137; Map 12

TYPE: Male holotype taken in a Malaise trap at an elevation of $875 \mathrm{~m}$ on Mount Spec, $19^{\circ} 00^{\prime} \mathrm{S}, 146^{\circ} 11^{\prime} \mathrm{E}$, Queensland (May 8-June 5, 1995; M. Cermak), deposited in QMB.

ETYMOLOGY: The specific name is a noun in apposition taken from the type locality.

DiAGNOSIS: This odd species resembles $L$. moorilyanna but has a distally truncated retrolateral tibial apophysis (fig. 135); females are unique in having tripartite spermathecae in which the median portions have been squeezed behind the anterior and posterior portions, so that they are readily visible only in ventral view (figs. 136, 137).
MALE: Total length 6.2. Abdominal dorsum with large anterior and smaller posterior white spots occupying almost full width of abdomen, venter unmarked, posterior femora and tibiae with subdistal dark rings. Palpal femur with very long setae but otherwise unmodified; retrolateral tibial apophysis wide, almost straight, with dorsally directed projection at tip (fig. 135); palpal bulb elongate, embolus narrow, recessed behind translucent membranes (fig. 134).

Female: Total length 7.9. Coloration as in male. Epigynum with diamond-shaped septum, not extending as far anteriorly as spermathecae (fig. 136); spermathecae tripartite but with median portion squeezed into dorsal 
position, scarcely visible in ventral view (fig. 137).

Other MATERIAL ExAmined: Queensland: Mount Spec, $19^{\circ} 00^{\prime} \mathrm{S}, 146^{\circ} 11^{\prime} \mathrm{E}$, Feb. 6-Mar. 9, 1995, intercept trap, elev. 875 m (M. Cermak, QMB), 1 . .

DISTRIBUTION: Known only from northern Queensland (map 12).

Lampona russell, new species Figures 138-141; Map 13

TYPE: Female holotype taken in pitfall trap in Russell River National Park, $17^{\circ} 18^{\prime} \mathrm{S}$, $145^{\circ} 58^{\prime} \mathrm{E}$, Queensland (Oct. 30, 1991-July 24, 1992; P. Lawless, R. Raven, M. Shaw), deposited in QMB (S19912).

ETYMOLOGY: The specific name is a noun in apposition taken from the type locality.

DiAgNosis: This species seems to be the northern sister species of L. airlie; males have a shorter, wider tip on the retrolateral tibial apophysis (fig. 139); females have a diamond-shaped epigynal midpiece (fig. 140) and widened spermathecae with very small anterior portions (fig. 141).

MALE: Total length 5.1. Abdominal dorsum with anterior pair of white spots small, median pair forming $\mathrm{W}$-shaped stripe, not connected to posterior spot; anterior femora darkened, femora with distal, tibiae with proximal and distal dark rings. Palpal femur darkened throughout its length; retrolateral tibial apophysis basally triangular, tip long, directed dorsally (fig. 139); tegulum produced posteroventrally, extending to about one-third of tibial length; embolus narrow throughout its length (fig. 138).

FEMALE: Total length 7.2. Coloration as in male. Epigynal midpiece short, diamondshaped (fig. 140); spermathecae bipartite, posterior portion wide, globose, anterior portion small, bulbous, situated near midline (fig. 141).

Other MATERial ExAmined: Queensland: Broadwater Park, $35 \mathrm{~km}$ NW Ingham, $18^{\circ} 22^{\prime} \mathrm{S}, 145^{\circ} 57^{\prime} \mathrm{E}$, Dec. 16, 1986, rainforest, elev. $60 \mathrm{~m}$ (G. Monteith, G. Thompson, S. Hamlet, QMB S15633), 1 ; ;ission Beach, $17^{\circ} 50^{\prime} \mathrm{S}, 146^{\circ} 06^{\prime} \mathrm{E}$, Jan. 29-Mar. 4, 1996, pitfall, elev. $10 \mathrm{~m}$ (M. Cermak, QMB), 1 \%, July 29-Sept. 2, 1996, pitfall, elev. 10 m (M. Cermak, QMB), 1, (M. Cermak, QMB

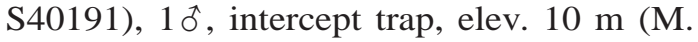
Cermak, QMB S40183), 10 ; Mission Beach, $17^{\circ} 52^{\prime} \mathrm{S}, 146^{\circ} 04^{\prime} \mathrm{E}$, July 29-Sept. 2, 1996, intercept trap, elev. $40 \mathrm{~m}$ (M. Cermak, QMB

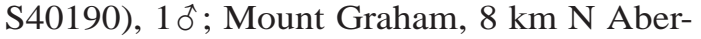
gowrie, $18^{\circ} 22^{\prime} \mathrm{S}, 145^{\circ} 52^{\prime} \mathrm{E}$, Dec. 29, 1986, rainforest litter (S. Hamlet, QMB S34282), 1 ; ca. $5 \mathrm{~km} \mathrm{~W}$ Paluma, $19^{\circ} 00^{\prime} \mathrm{S}, 146^{\circ} 12^{\prime} \mathrm{E}$, July 12, 1971, Berlese, rainforest, elev. ca. 950 m (Taylor, Feehan, QMB), $1 \%$; Russell River National Park, $17^{\circ} 18^{\prime} \mathrm{S}, 145^{\circ} 58^{\prime} \mathrm{E}$, July 24-Nov. 25, 1992, pitfall (R. Raven, P., E. Lawless, M. Shaw, QMB S21743), 10,1 우 (missing epigynum).

DisTRIBUTION: Known only from northern Queensland (map 13).

\section{Lampona airlie, new species}

Figures 142-145; Map 13

TyPES: Female holotype and male allotype taken in rainforest litter at Airlie Beach, $20^{\circ} 16^{\prime} \mathrm{S}, 148^{\circ} 43^{\prime} \mathrm{E}$, Queensland (Feb. 16, 1986; R. Raven, J. Gallon), deposited in QMB (S8069).

ETYMOLOGY: The specific name is a noun in apposition taken from the type locality.

Diagnosis: This species seems to be the southern sister species of L. russell; males have a longer, narrower tip on the retrolateral tibial apophysis (fig. 143); females have a

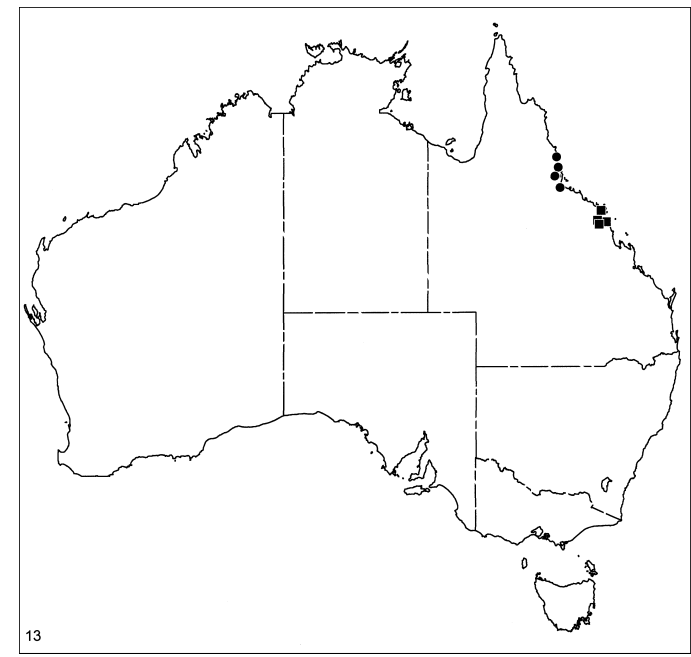

Map 13. Records of Lampona russell, new species (circles) and L. airlie, new species (squares). 

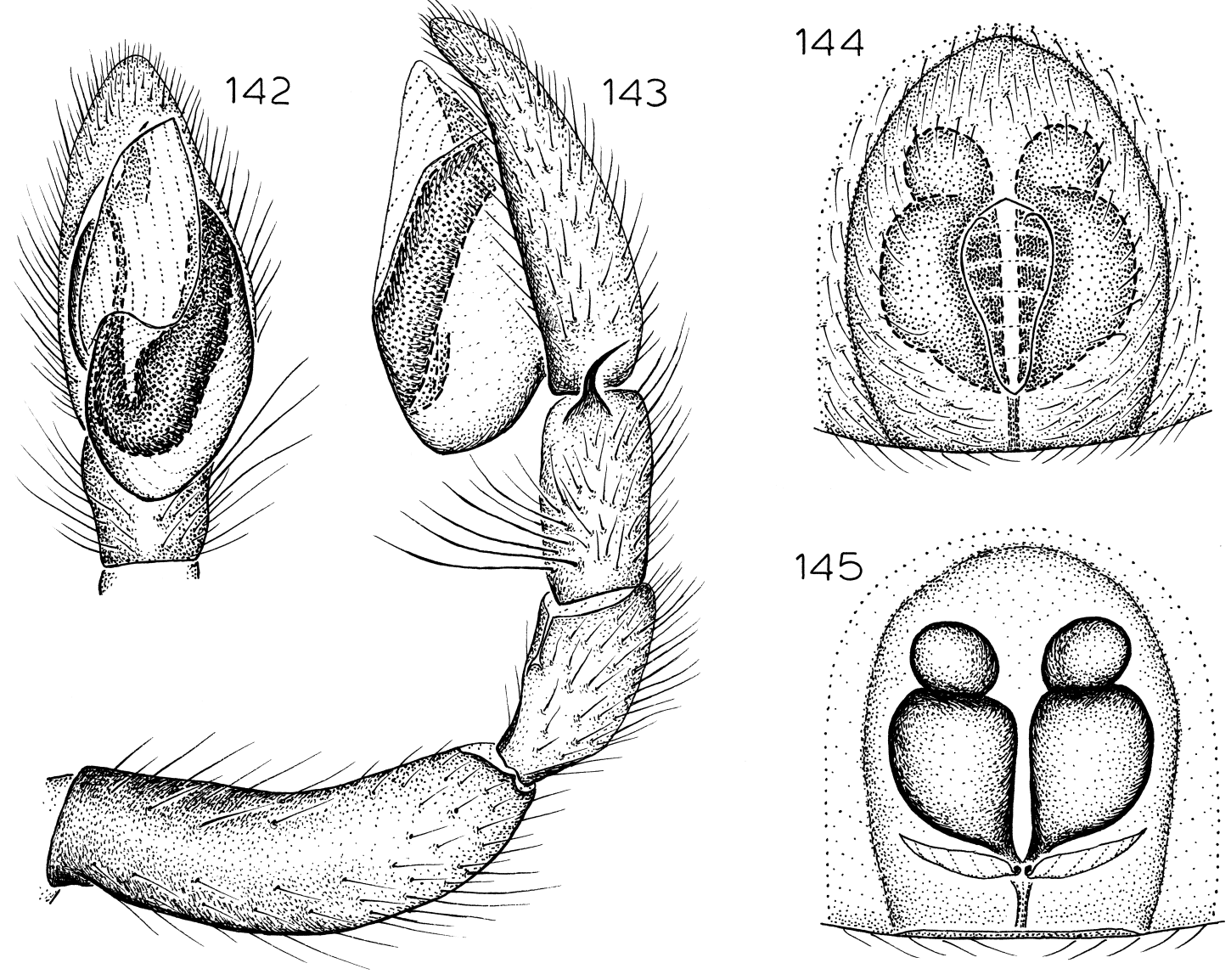

Figs. 142-145. Lampona airlie, new species. 142. Left male palp, ventral view. 143. Same, retrolateral view. 144. Epigynum, ventral view. 145. Same, dorsal view.

more rectangular epigynal midpiece and larger anterior portions of the spermathecae (figs. 144, 145).

MALE: Total length 4.5. Abdominal dorsum with anterior and median pairs of white spots united transversely into two transverse stripes; femora with weak subdistal, tibiae with weak proximal and subdistal dark rings. Palpal femur short, darkened basally, otherwise unmodified; retrolateral tibial apophysis with long, narrow tip (fig. 143); tegulum greatly expanded, reaching to about half of tibial length; embolus obscured by conductor in ventral view (fig. 142).

FEMALE: Total length 7.1. Coloration as in male except abdominal dorsum with anterior and median pairs of white spots united transversely as well as longitudinally. Epigynal midpiece widest anteriorly (fig. 144); spermathecae resembling those of $L$. russell but with larger anterior portions (fig. 145).

OTHER MATERIAL EXAMINED: Queensland: Airlie Beach, $20^{\circ} 16^{\prime} \mathrm{S}, 148^{\circ} 43^{\prime} \mathrm{E}$, Feb. 16, 1986, rainforest litter (R. Raven, J. Gallon, QMB S8069), 1\%; Cape Hillsborough National Park, $20^{\circ} 54^{\prime} \mathrm{S}, 149^{\circ} 03^{\prime} \mathrm{E}$, pitfall, rainforest, Nov. 24, 1992-Apr. 22, 1993 (R., J., S. Raven, P., E. Lawless, QMB S24475), 1 ; $5 \mathrm{~km} \mathrm{NW}$ Mount Macartney, 20 49'S, $148^{\circ} 30^{\prime} \mathrm{E}$, Apr. 21,1989 , Berlese, sieved litter, open forest, elev. $480 \mathrm{~m}$ (G. Monteith, QMB S28085), 1 $q$; Mount William, Eungella National Park, $21^{\circ} 02^{\prime} \mathrm{S}, 148^{\circ} 36^{\prime} \mathrm{E}$, Apr. 19, 1979, Berlese, moss on trees, rainforest, elev. 1100 m (G. Monteith, QMB S34419), 1 ㅇ. 

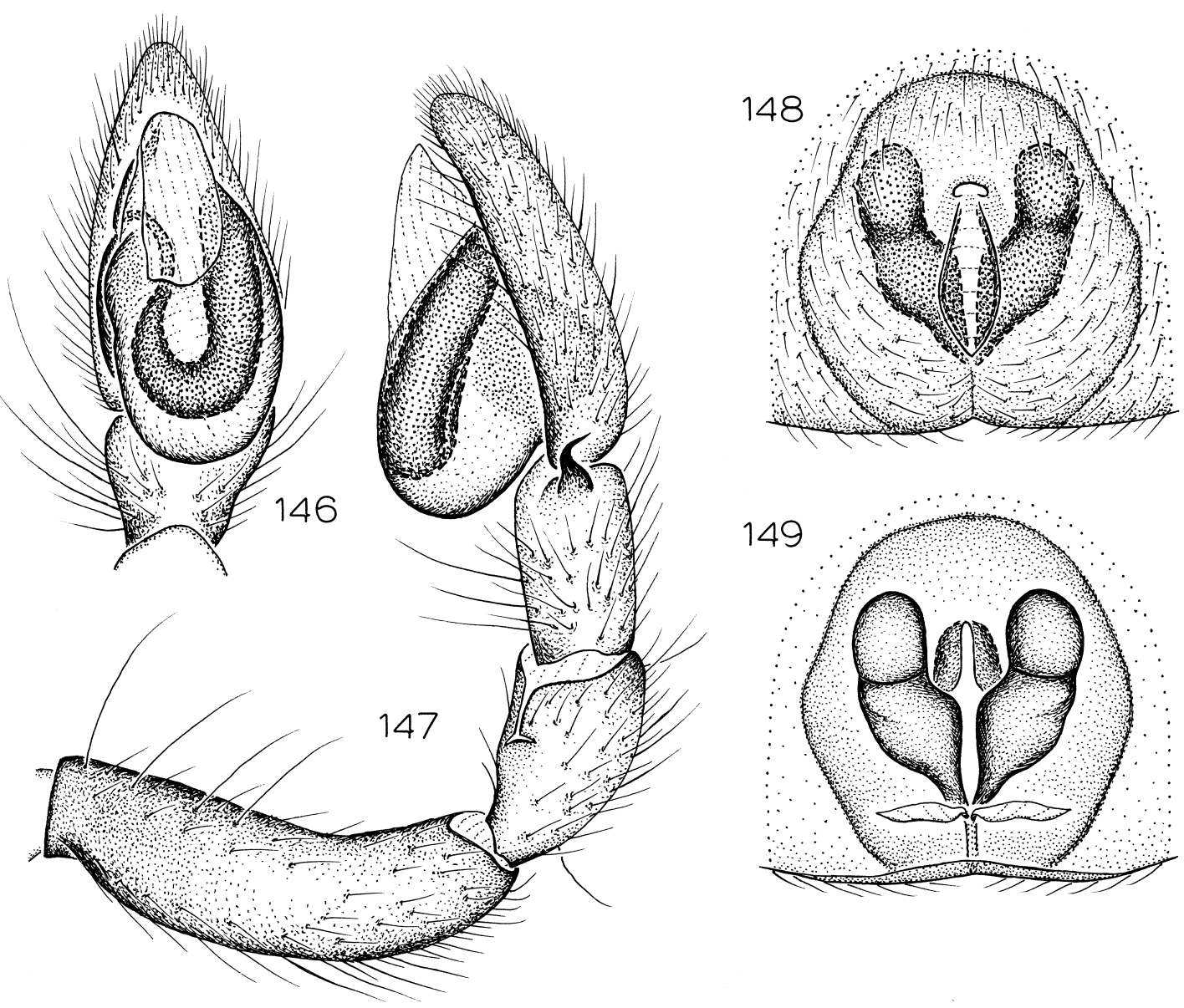

Figs. 146-149. Lampona terrors, new species. 146. Left male palp, ventral view. 147. Same, retrolateral view. 148. Epigynum, ventral view. 149. Same, dorsal view.

DistRIBUTION: Known only from mid-eastern Queensland (map 13).

\section{Lampona terrors, new species}

Figures 146-149; Map 14

TYPE: Female holotype from Terrors Creek, $27^{\circ} 13^{\prime} \mathrm{S}, 152^{\circ} 50^{\prime} \mathrm{E}$, Queensland (Nov. 1908; J. Lamb), deposited in QMB (S28118).

EтYMOLOGY: The specific name is a noun in apposition taken from the type locality.

Diagnosis: Males resemble those of $L$. russell and $L$. airlie but have the retrolateral tibial apophysis more abruptly narrowed posterior to the tip (fig. 147); females have larger anterior portions of the spermathecae that are displaced laterally, to the side of the epigynal midpiece (figs. 148, 149).
MALE: Total length 5.3. Abdominal dorsum apparently with median white spots fused to each other and to anterior pair; anterior femora darkened, posterior femora with subdistal, posterior tibiae with proximal and distal dark rings. Palpal femur darkened throughout most of its length but otherwise unmodified; retrolateral tibial apophysis distinctly expanded posterior of tip (fig. 147); tegulum expanded, reaching to half of tibial length; embolar base rounded prolaterally (fig. 146).

Female: Total length 7.9. Coloration as in male except abdominal dorsum with only vague indications of anterior and median white spots. Epigynal midpiece long, narrow (fig. 148); anterior portions of spermathecae displaced to sides of epigynum (fig. 149). 
Other MAterial ExAmined: Queensland: Clarke Creek, Sarina Road, 20 35'S, $148^{\circ} 15^{\prime} \mathrm{E}$, Dec. 1979 , in colony of Badumna candida (L. Koch) (R. Jackson, QMB S25984), 1 9 ; Conway National Park, $20^{\circ} 17^{\prime} \mathrm{S}, 148^{\circ} 46^{\prime} \mathrm{E}$, July $28-$ Dec. 3, 1992, pitfall, eucalypt woodland (R. Raven, P., E. Lawless, M. Shaw, QMB S24993), 10; Douglas Creek Road, Kirrama State Forest, $18^{\circ} 12^{\prime} \mathrm{S}, 145^{\circ} 45^{\prime} \mathrm{E}$, Dec. 10, 1986-Jan. 11, 1987, rainforest, elev. $800 \mathrm{~m}$, intercept trap (G. Monteith, G. Thompson, Hamlet, QMB

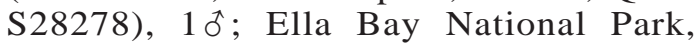
$17^{\circ} 29^{\prime} \mathrm{S}, 146^{\circ} 04^{\prime} \mathrm{E}$, July 24-Nov. 25, 1992, pitfall (R. Raven, P., E. Lawless, M. Shaw, QMB S24921), 10; Mount Cleveland, $19^{\circ} 16^{\prime} \mathrm{S}, 147^{\circ} 03^{\prime} \mathrm{E}$, Mar. 22-24, 1991, dung, pitfall, open forest, elev. $550 \mathrm{~m}$ (G. Monteith, D. Cook, QMB S43880), 1 t ; Rockhampton, $23^{\circ} 23^{\prime} \mathrm{S}, 150^{\circ} 30^{\prime} \mathrm{E}$, Mar. 10-11, 1965 (J. Balogh, HNHM), 1 9 ; Rockhampton District, $23^{\circ} 22^{\prime} \mathrm{S}, 150^{\circ} 32^{\prime} \mathrm{E}, 1991$, pitfall, open forest (R. Raven, QMB S30349), 20 . 14).

DisTRIBUTION: Eastern Queensland (map

Lampona torbay, new species Figures 100, 101; Map 14

TyPE: Female holotype from Torbay Inlet, $35^{\circ} 01^{\prime} \mathrm{S}, 117^{\circ} 41^{\prime} \mathrm{E}$, Western Australia (Jan. 2,

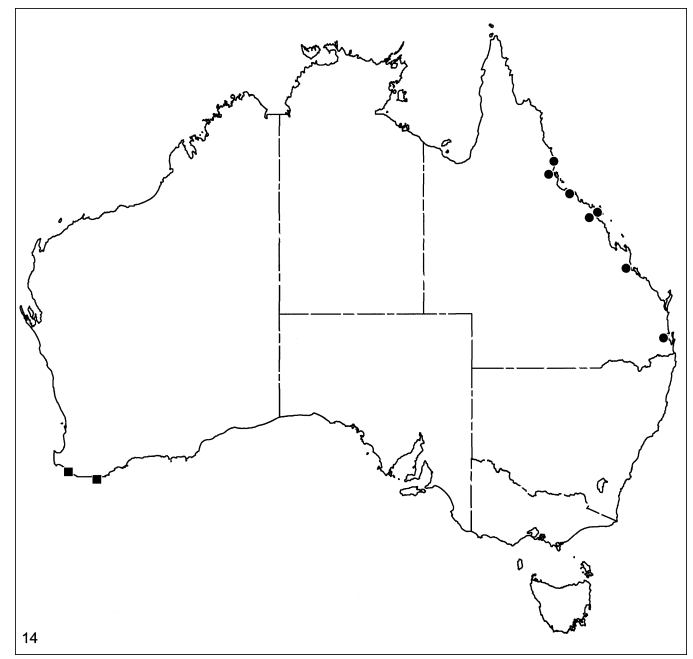

Map 14. Records of Lampona terrors, new species (circles) and L. torbay, new species (squares).
1978; B. Main), deposited in WAM (96/ 1526) courtesy of Dr. Barbara York Main.

ETYMology: The specific name is a noun in apposition taken from the type locality.

DiAGNOSIS: Only females are known, and it is difficult to identify their closest relatives; the epigynal midpiece is wide (fig. 100) and the spermathecae are bipartite, with the posterior portion long and the anterior portion rounded (fig. 101).

MALE: Unknown.

Female: Total length 9.2. Abdominal dorsum with anterior pairs of white spots fused, leaving median, longitudinal dark stripes on cardiac area and most of posterior half of abdomen; venter unmarked except where lateral dark markings approach spinnerets. Epigynal septum v-shaped, wide posteriorly, without distinct anterior margin although surface is depressed across top of septum (fig. 100); basal portion of spermathecae short, wide, distal portion rounded (fig. 101).

Other Material Examined: Western Australia: Callcup, Warren River, $34^{\circ} 36^{\prime} \mathrm{S}$, $115^{\circ} 54^{\prime} \mathrm{E}$, Oct. 30-Nov. 5, 1997, bush, elev. $100 \mathrm{~m}$ (J. Murphy, JAM), 1 ; Torbay Inlet, $35^{\circ} 01^{\prime} \mathrm{S}, 117^{\circ} 41^{\prime} \mathrm{E}$, Jan. 5, 1979 (B. Main, CBM), 1 ㅇ․

Distribution: Southwestern Australia (map 14).

\section{THE PUSILLA GROUP}

This group includes 10 species in which females usually have a triangular epigynal midpiece and reduced anterior spermathecal lobes, and males usually have an angular retrolateral tibial apophysis (often shifted to the dorsal edge of the tibia) that is directed obliquely and dorsally.

\section{Lampona pusilla L. Koch Figures 150-153; Map 15}

Lampona pusilla L. Koch, 1873: 371, pl. 28, figs. 8, 8a (female holotype from Australia [as "Neuholland"], no specific locality, in NRS, examined).

DiAgnosis: Males resemble those of $L$. yanchep in having a relatively long, narrow retrolateral tibial apophysis, but lack a distinct subbasal protuberance on the palpal femur (fig. 151); females resemble those of $L$. hickmani but differ in having a distinct an- 

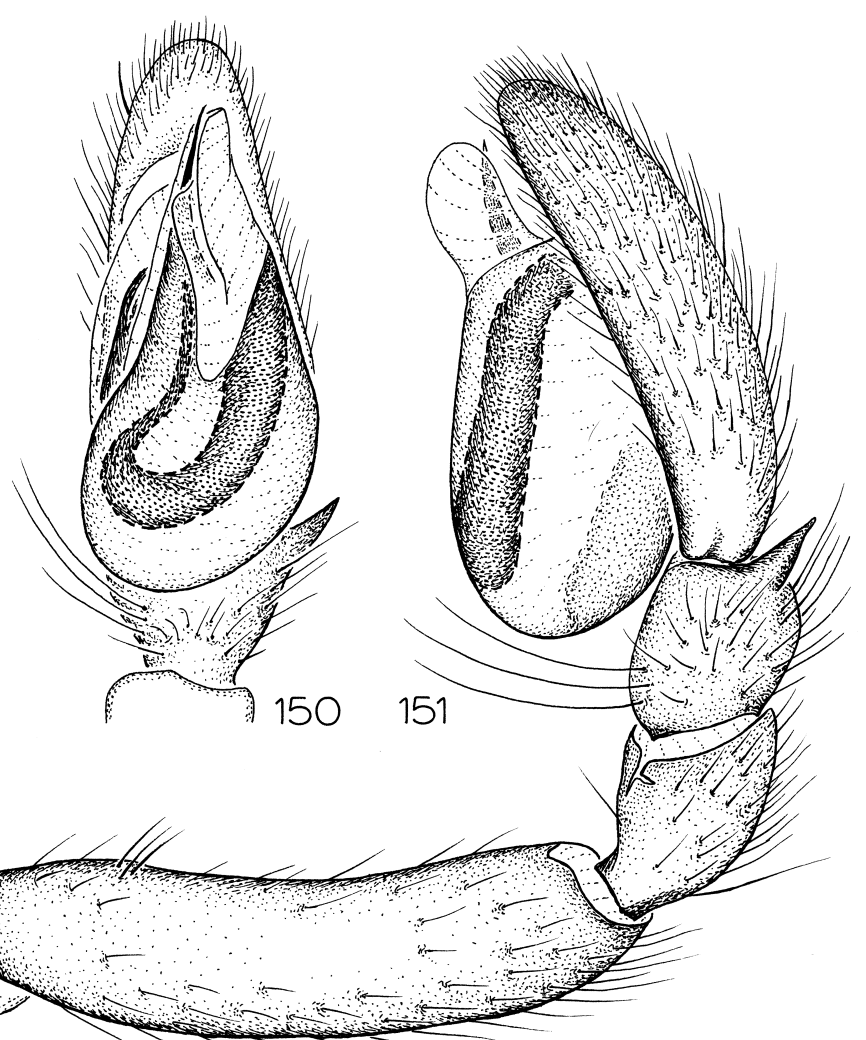
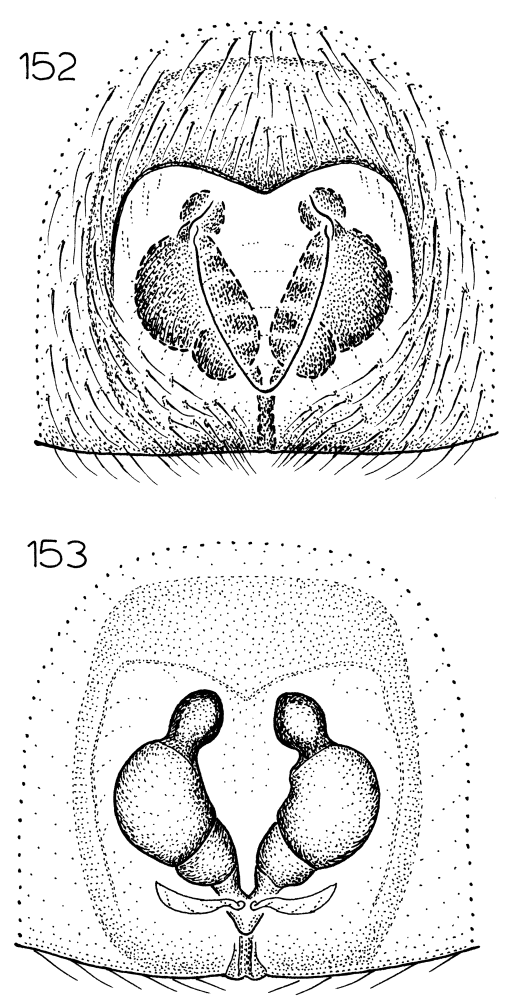

Figs. 150-153. Lampona pusilla L. Koch. 150. Left male palp, ventral view. 151. Same, retrolateral view. 152. Epigynum, ventral view. 153. Same, dorsal view.

terior epigynal margin and a larger, bulbous, anterior portion of the spermathecae (figs. $152,153)$.

MALE: Total length 5.2. Abdomen light, dorsal dark markings reduced to three incomplete transverse stripes; legs unmarked, patellae lighter than tibiae. Palpal femur with only slightly elevated ventral protrusion bearing stiff setae; retrolateral tibial apophysis relatively long, narrow throughout most of length, directed almost dorsally (fig. 151); tegulum globose, expanded posteriorly, extending to about one-half of tibial length; embolus narrow throughout its length, base of conductor narrow (fig. 150).

FEMALE: Total length 6.5. Coloration as in male. Epigynum with long, almost m-shaped anterior and lateral margins, large $\mathrm{v}$-shaped midpiece with openings situated anteriorly (fig. 152); spermathecae tripartite, distal portion small, bulbous (fig. 153).

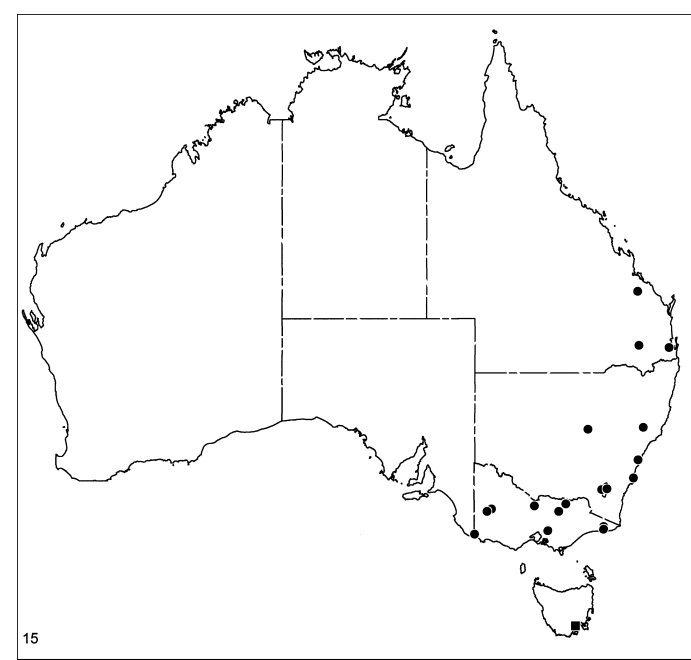

Map 15. Records of Lampona pusilla L. Koch (circles) and L. hickmani, new species (square). 
Material ExAmined: AUSTRALIA: no specific locality (NRS), 1 (holotype). Australian Capital Territory: Blundells Creek, $3 \mathrm{~km}$ E Piccadilly Circus, $35^{\circ} 22^{\prime} \mathrm{S}, 148^{\circ} 50^{\prime} \mathrm{E}$, Jan.-Feb., 1984-1985, elev. $850 \mathrm{~m}$ (Weir, Lawrence, Johnson, QMB), 7\$, 2q; Canberra, $35^{\circ} 20^{\prime} \mathrm{S}, 149^{\circ} 10^{\prime} \mathrm{E}$, May. 23, 1965 (J. Balogh, HNHM), $2 q$. New South Wales: Pennant Hills, $33^{\circ} 44^{\prime} \mathrm{S}, 151^{\circ} 04^{\prime} \mathrm{E}$, Feb. 7, 1997 (J. Noble, AMS KS55275), 1 9 ; Trangie, $32^{\circ} 02^{\prime} \mathrm{S}, 147^{\circ} 59^{\prime} \mathrm{E}$, Mar. 8, 1968, Berlese, dry sclerophyll leaf litter (L. Mound, QMB), 20, 2\%; junction, Tubrabucca and Omadale Brook Roads, Stewarts Brook State Forest, $31^{\circ} 55^{\prime} \mathrm{S}, 151^{\circ} 24^{\prime} \mathrm{E}$, Nov. 22-23, 1996, elev. 1360 m (N. Platnick, R. Raven, V. Ovtsharenko, K. Catley, AMNH), 1 \%; Willowvale, near Gerringong, 344ㄴ $\mathrm{S}$, $150^{\circ} 48^{\prime} \mathrm{E}$, Nov.-Dec. 1979, pitfall (W. Wishart, AMS KS4313), 10 . Queensland: Kroombit Tops, $24^{\circ} 22^{\prime} \mathrm{S}, 151^{\circ} 01^{\prime} \mathrm{E}$, Feb. 25, 1982, open forest (R. Raven, QMB S28095), $1 \%$; Lake Broadwater, via Dalby, $27^{\circ} 21^{\prime} \mathrm{S}$, $151^{\circ} 06^{\prime} \mathrm{E}$, May 17-Nov. 24, 1985, pitfall (M. Bennie, QMB S34397), 10 ; Mount Coot-tha, Brisbane, $27^{\circ} 28^{\prime} \mathrm{S}, 152^{\circ} 58^{\prime} \mathrm{E}$, Dec. 14, 1996, elev. $140 \mathrm{~m}$ (N. Platnick, R. Raven, V. Ovtsharenko, K. Catley, AMNH), 10,1 ㅇ․ Victoria: Campaspe River, $15 \mathrm{~km}$ S Echuca, $36^{\circ} 16^{\prime} \mathrm{S}, 144^{\circ} 41^{\prime} \mathrm{E}$, May 23, 1988 (D. Hirst, SAM N1996/308, 309), 10, 1 웅 Chiltern Forest, $36^{\circ} 10^{\prime} \mathrm{S}, 146^{\circ} 37^{\prime} \mathrm{E}$, Feb. 1967, Berlese, leaf litter, Eucalyptus sideroxylon (R. McInnes, QMB), 10, 3q; Cobon South Coupe, $37^{\circ} 25^{\prime} \mathrm{S}, 148^{\circ} 57^{\prime} \mathrm{E}$, Nov. 27-28, 1996, elev. 700 m (N. Platnick, R. Raven, V. Ovtsharenko, K. Catley, AMNH), 40, 1 ; Coranderrk Reserve, Healesville, $37^{\circ} 39^{\prime} \mathrm{S}$, $145^{\circ} 31^{\prime} \mathrm{E}$, Mar. 20-21, 1982, under bark of Eucalyptus viminalis (M. Harvey, QMB), $10^{\top}$; Dimboola, $36^{\circ} 27^{\prime} \mathrm{S}, 142^{\circ} 02^{\prime} \mathrm{E}$, May 25 , 1988 (D. Hirst, SAM N1996/301-306), 2 , 4 ; Dingo Creek, Lind National Park, $37^{\circ} 34^{\prime} \mathrm{S}, 148^{\circ} 57^{\prime} \mathrm{E}$, June 18 , 1987, under eucalypt bark (M. Harvey, WAM 95/1012), 10; Horseshoe Bend, Little Desert National Park, $36^{\circ} 35^{\prime} \mathrm{S}, 141^{\circ} 45^{\prime} \mathrm{E}$, July 6, 1982, under bark of Eucalyptus camaldulensis (M. Harvey, B. Roberts, NMV), 1 \% ; 4 km S Horseshoe Bend, Little Desert National Park, $36^{\circ} 35^{\prime} \mathrm{S}, 141^{\circ} 45^{\prime} \mathrm{E}$, July 6 , 1982 , under bark of Eucalyptus camaldulensis (M. Harvey, B. Roberts, NMV), $1 \%$; 15 km E Mount Gam- bier, $37^{\circ} 50^{\prime} \mathrm{S}, 141^{\circ} 00^{\prime} \mathrm{E}$, Oct. 7, 1972 (B., M. Baehr, CBB), 19 ; Upper Lurg, $36^{\circ} 35^{\prime} \mathrm{S}$, $146^{\circ} 11^{\prime}$ E, Jan. 20-May 24, 1995, house (J. Strudwick, CVIC 636, 637), 2 9, Nov. 29Apr. 9, 1995-1997, house (J. Strudwick, CVIC 634, 635, 638, 640, 644), 5 万े.

Distribution: Southeastern Australia (map 15).

\section{Lampona hickmani, new species}

Figures 154, 155; Map 15

TYPE: Female holotype taken under bark on Mount Wellington, $42^{\circ} 54^{\prime} \mathrm{S}, 147^{\circ} 14^{\prime} \mathrm{E}$, Tasmania (Aug. 19, 1924; V. Hickman), deposited in AMS (KS29253).

ETYMOLOGY: The specific name is a patronym in honor of the collector, the late Dr. V. Hickman.

Diagnosis: Females resemble those of $L$. pusilla but have longer lateral margins of the epigynal midpiece (fig. 154) and almost obsolete anterior portions of the spermathecae (fig. 155).

MALE: Unknown.

Female: Total length 8.5. Abdomen brownish gray, without distinct pattern; legs unmarked. Epigynum with indistinct anterior margin, with $\mathrm{v}$-shaped midpiece greatly widened anteriorly (fig. 154); spermathecae tripartite, anterior portion tiny, almost obsolete (fig. 155).

OTHER MATERIAL EXAMINED: Four females taken with the holotype.

DistRIBUTION: Known only from Tasmania (map 15).

\section{Lampona yanchep, new species}

Figures 158-161; Map 16

TYPE: Male holotype taken in pitfall trap at Yanchep, $31^{\circ} 33^{\prime} \mathrm{S}, 115^{\circ} 41^{\prime} \mathrm{E}$, Western Australia (Feb. 2, 1978; S. Curry), deposited in WAM (95/1106).

ETYMOLOGY: The specific name is a noun in apposition taken from the type locality.

DiAGNOSIS: Males resemble those of L. $t a-$ room in having a subbasal knob protruding from the ventral surface of the palpal femur, but have a shorter retrolateral tibial apophysis (fig. 159); females also resemble those of L. taroom in having an anterior epigynal excavation, but have the excavation wider and less clearly demarcated posteriorly (fig. 160). 

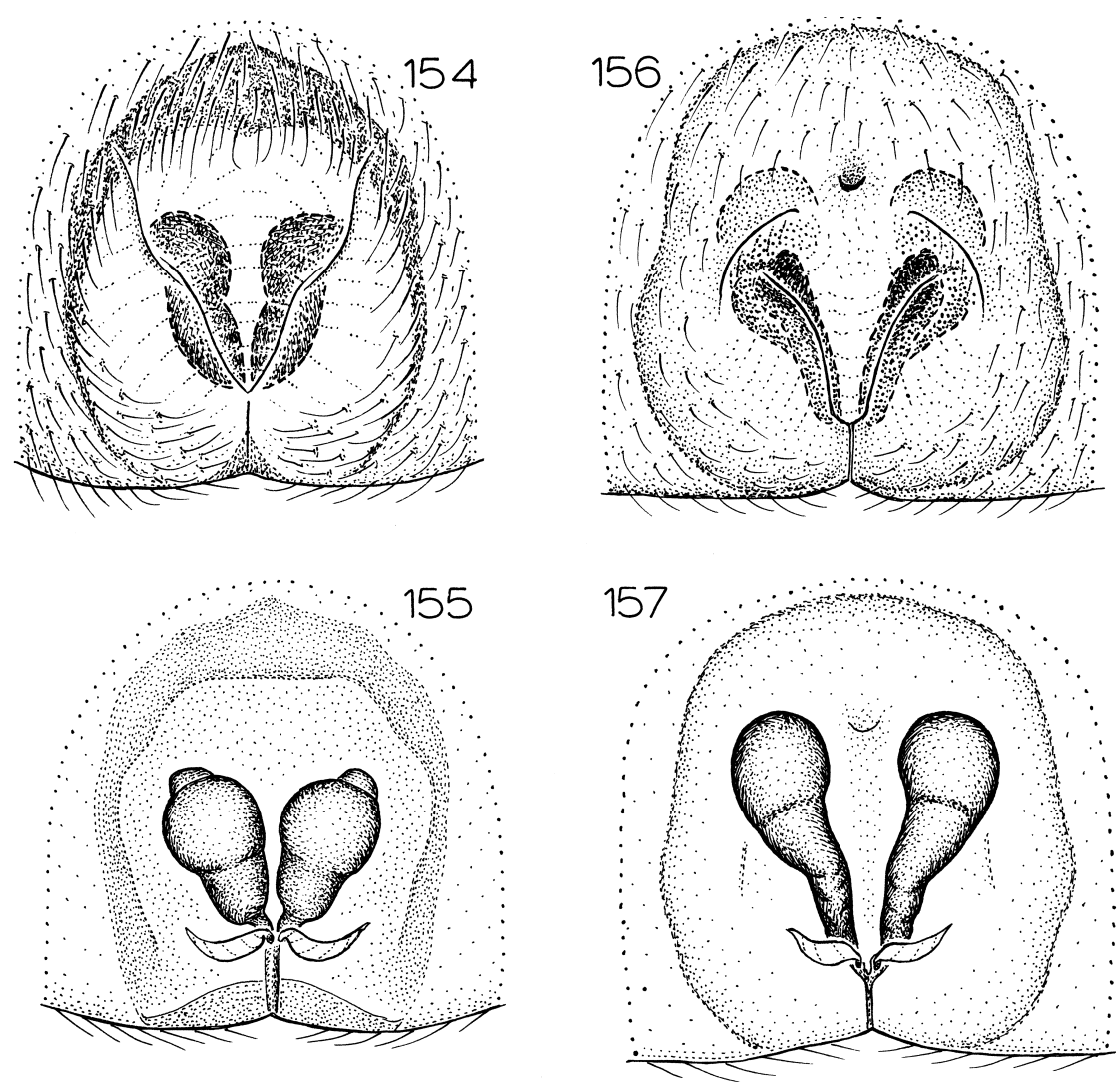

Figs. 154-157. 154, 155. Lampona hickmani, new species. 156, 157. L. olga, new species. 154, 156. Epigynum, ventral view. 155, 157. Same, dorsal view.

MALE: Total length 7.9. Coloration as in $L$. cylindrata except white markings on abdominal dorsum connected laterally. Palpal femur with subbasal knob protruding from ventral surface; retrolateral tibial apophysis relatively long, triangular (fig. 159); tegulum globose, extending to one-half length of tibia, embolar base long, gradually narrowed toward tip, prolateral lobe of conductor extending behind embolus (fig. 158).

FEMALE: Total length 6.1. Coloration as in male except posterior portion of abdominal dorsum also with white chevrons. Epigynal atrium almost conical, depressed anteriorly (fig. 160); spermathecae short, tripartite (fig. 161).

Other Material Examined: Western Australia: Mount Cooke, $32^{\circ} 25^{\prime} \mathrm{S}$, $116^{\circ} 18^{\prime} \mathrm{E}$, Feb. 17, 1991, along gully at base of mountain, under log (M. Harvey, WAM 95/1108), 1 \%; Wexcombe, W side Talbot
Road, outside NE corner of reserve, $31^{\circ} 52^{\prime} \mathrm{S}$, $116^{\circ} 03^{\prime} \mathrm{E}$, May 10-June 24, 1993, pitfall (M. Harvey, J. Waldock, WAM 95/1107), 1 to.

Distribution: Southwestern Australia (map 16).

\section{Lampona taroom, new species}

Figures 162-165; Map 16

TYPE: Male holotype taken in baited flight intercept trap at Boggomoss, via Taroom, $25^{\circ} 26^{\prime} \mathrm{S}, 150^{\circ} 01^{\prime} \mathrm{E}$, Queensland (Nov. 12, 1996-Jan. 1997; D. Cook, G. Monteith), deposited in QMB (S36315).

ETYMOLOGY: The specific name is a noun in apposition taken from the type locality.

DiagnOsIs: This species seems closest to L. yanchep, sharing with it a subbasal knob on the male palpal femur and a deeply excavated epigynal atrium; males differ in having a longer, more curved tip on the retro- 

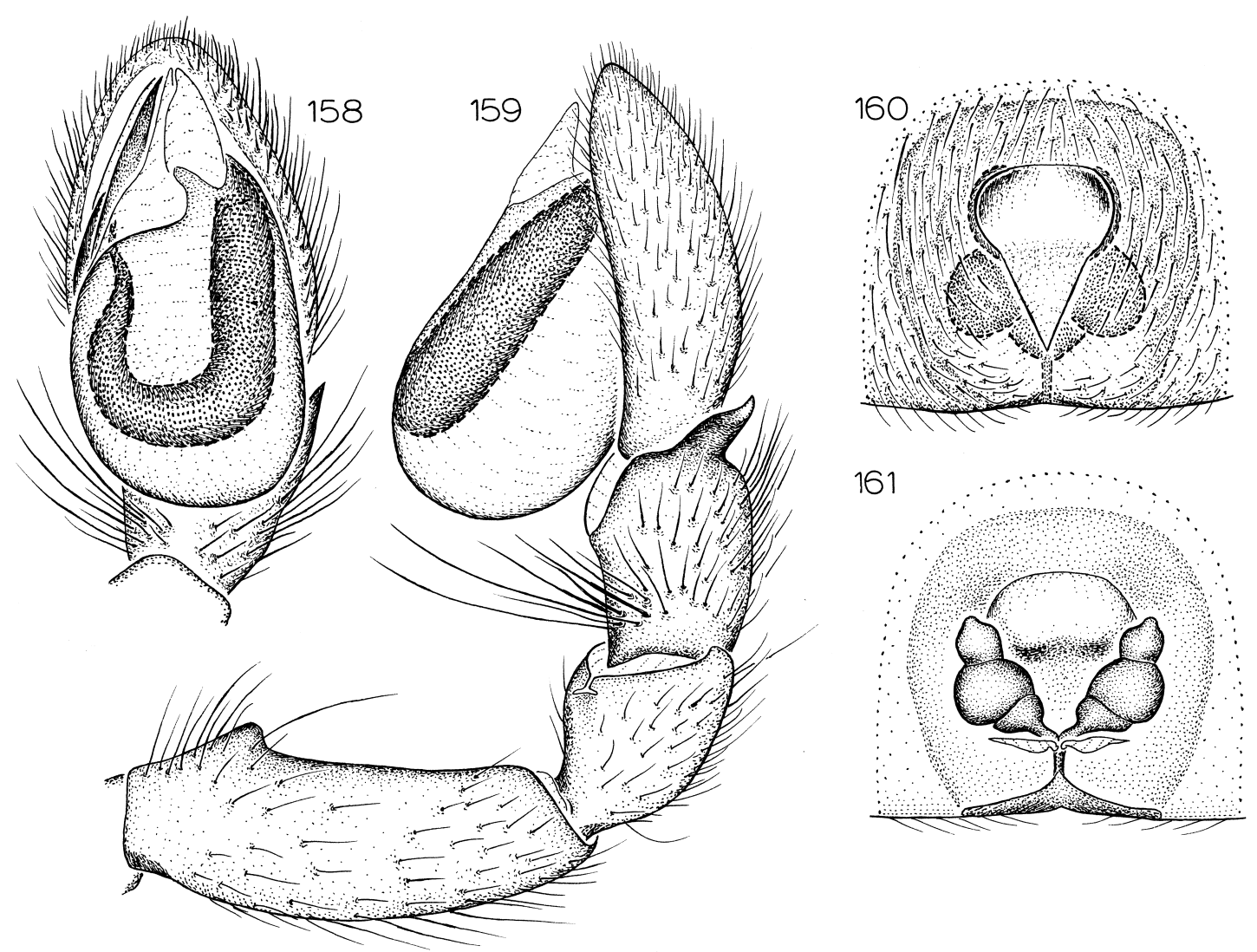

Figs. 158-161. Lampona yanchep, new species. 158. Left male palp, ventral view. 159. Same, retrolateral view. 160. Epigynum, ventral view. 161. Same, dorsal view.

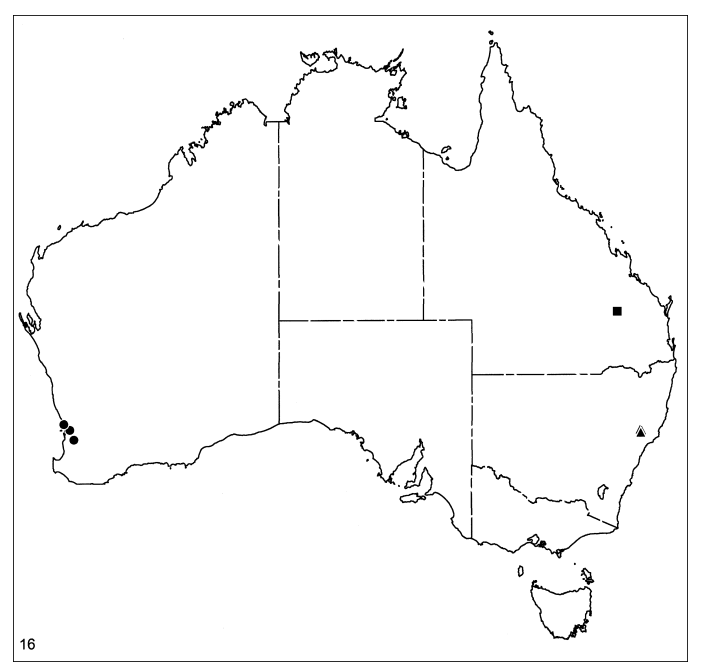

Map 16. Records of Lampona yanchep, new species (circles), L. taroom, new species (square), and L. allyn, new species (triangles). lateral tibial apophysis (fig. 163), females in having an anteriorly narrower epigynal atrium (fig. 164).

MALE: Total length 7.2. Abdominal dorsum shrunken from cuticle, markings indistinct, apparently with paramedian white markings, venter and legs unmarked. Palpal femur with distinct subbasal knob; retrolateral tibial apophysis long, with recurved tip (fig. 163); palpal bulb elongate, embolus with long, strong tip (fig. 162).

Female: Total length 7.0. Abdominal dorsum with markings indistinct but apparently with posterior white chevrons, venter unmarked, tibiae IV with subdistal, slightly darkened rings. Epigynum with longitudinal atrium, deeply excavated anteriorly (fig. 164); spermathecae tripartite, median portions much longer than anterior or posterior portions (fig. 165). 

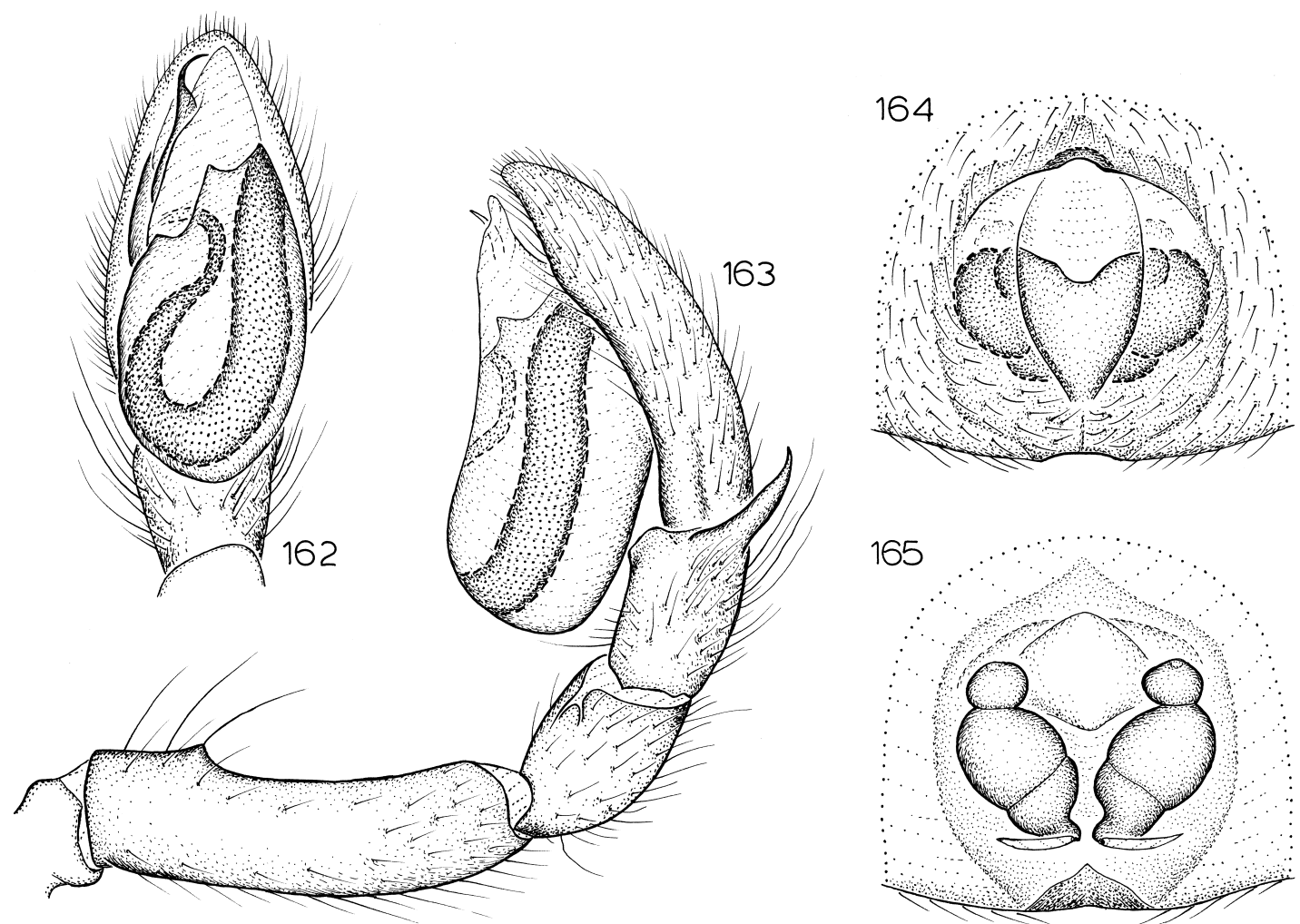

Figs. 162-165. Lampona taroom, new species. 162. Left male palp, ventral view. 163. Same, retrolateral view. 164. Epigynum, ventral view. 165. Same, dorsal view.

Other MAterial ExAmined: Queensland: Taroom District, $25^{\circ} 25^{\prime} \mathrm{S}, 149^{\circ} 58^{\prime} \mathrm{E}$, Sept. 10-Nov. 12, 1996, pitfall, vine thicket on hill (P. Lawless, QMB S34406), 1 ㅇ.

Distribution: Known only from southeastern Queensland (map 16).

\section{Lampona allyn, new species}

Figures 166-169; Map 16

TYPE: Female holotype taken in pitfall trap at an elevation of $970 \mathrm{~m}$ along Mount Allyn Road, adjacent to Mount Allyn Forest Park, $900 \mathrm{~m}$ from junction of Mount Allyn Lookout Road, Chichester State Forest, $32^{\circ} 07^{\prime} \mathrm{S}$, $151^{\circ} 25^{\prime}$ E, New South Wales (Feb. 4-Apr. 9, 1993, M. Gray, G. Cassis), deposited in AMS (KS40486).

ETYMOLOGY: The specific name is a noun in apposition taken from the type locality.

DiAgnOsis: This species resembles other members of the pusilla group in the shape of the male retrolateral tibial apophysis and epi- gynum, but can be distinguished in males by the more dorsally situated and directed retrolateral tibial apophysis (fig. 167) and in females by the broad posterior portion of the spermathecae (fig. 169).

MALE: Total length 5.2. Abdominal dorsum with median row of dark markings separated by white areas from dark rows of markings on side of abdomen; legs with patellae lighter than other segments, posterior tibiae with proximal and distal dark rings. Palpal femur with long, low ventral protuberance bearing long, stiff setae; retrolateral tibial apophysis long, narrow, straight, situated near dorsal side of segment (fig. 167); tegulum relatively small, scarcely protruding past base of tarsus; embolar base almost fused to tegulum (fig. 166).

FEMALE: Total length 6.5. Coloration as in male except abdominal dorsum with few white paramedian markings. Epigynum excavate, v-shaped median plate with elevated 

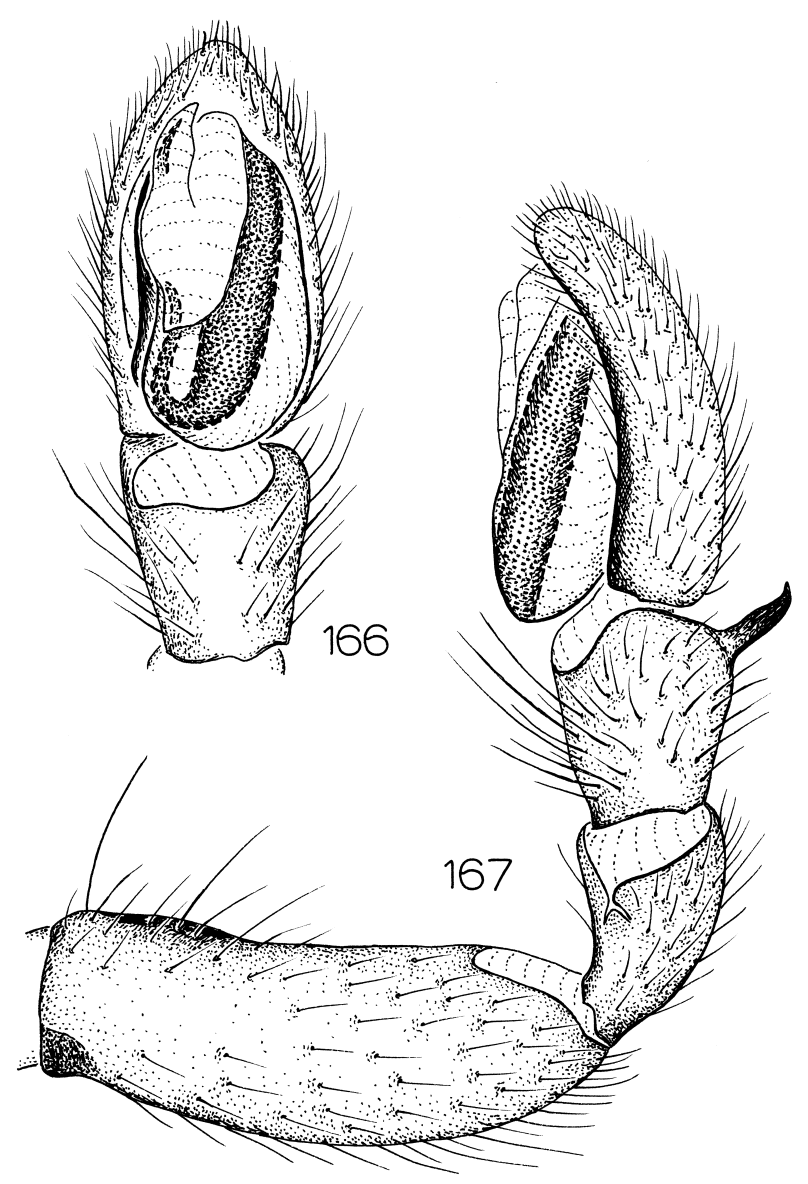

Figs. 166-169. Lampona allyn, new species. 166. Left male palp, ventral view. 167. Same, retrolateral view. 168. Epigynum, ventral view. 169. Same, dorsal view.
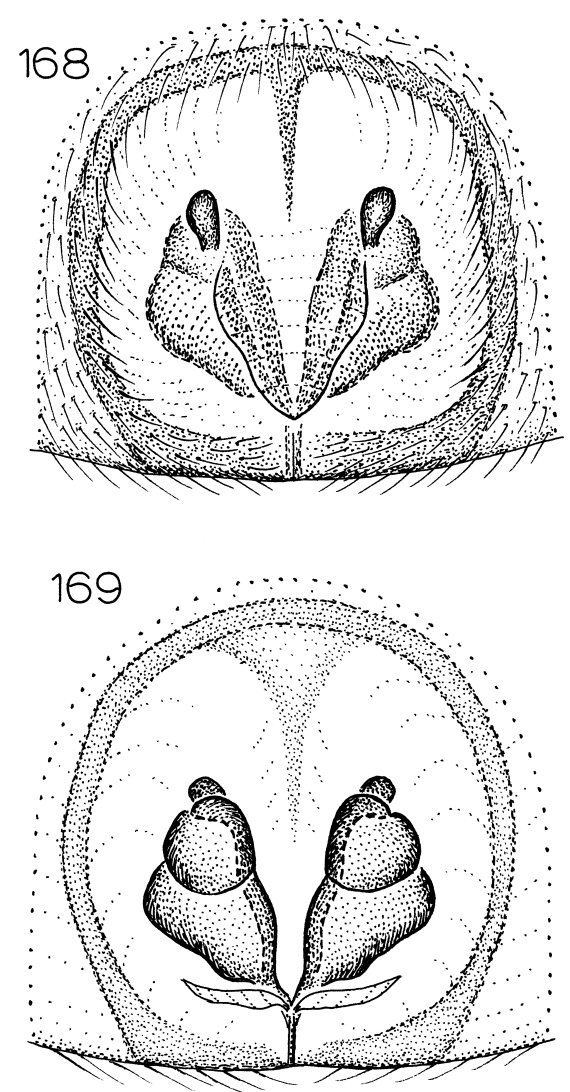

anterior margins (fig. 168); posterior portion of spermathecae wide, distal portion reduced to tiny lobe (fig. 169).

Other MAterial ExAmined: New South Wales: Bull Gully near junction of Bull Gully and Bull Ridge Roads, Stewarts Brook State Forest, $31^{\circ} 57^{\prime} \mathrm{S}, 151^{\circ} 22^{\prime} \mathrm{E}$, Feb. 4-Apr. 9, 1993, pitfall, elev. 1290 m (M. Gray, G. Cassis, AMS KS40483), $10^{\text {t} ; ~ C a r e y ' s ~ P a r k, ~}$ Barrington Tops, $32^{\circ} 00^{\prime} \mathrm{S}, 151^{\circ} 28^{\prime} \mathrm{E}$, Nov. 1 , 1957, moss, elev. 5064 ft (F. Perkins, OMD), 19; Mount Allyn, near Barrington Tops, $32^{\circ} 06^{\prime} \mathrm{S}, 151^{\circ} 26^{\prime} \mathrm{E}$, Sept. 1993, bark scraped from Nothofagus trunks, temperate rainforest (G. Hunt, AMS KS44099), 20.

Distribution: Known only from New South Wales (map 16).

\section{Lampona danggali, new species} Figures 170-173; Map 17

TYPE: Female holotype taken by vibration $2.4 \mathrm{~km}$ N Sandford Dam, Danggali Conservation Park, $33^{\circ} 19^{\prime} \mathrm{S}, 140^{\circ} 55^{\prime} \mathrm{E}$, South Australia (Nov. 22, 1996; D. Hirst, J. Forrest), deposited in SAM (N1997/149).

ETYMOLOGY: The specific name is a noun in apposition taken from the type locality.

DiAgnosis: This species shares with $L$. olga a triangular epigynal midpiece that almost meets a pair of curved, anterolateral epigynal margins; males of L. olga are unknown but males of this species have a long, low basal expansion on the palpal femur and an apically hooked retrolateral tibial apoph- 

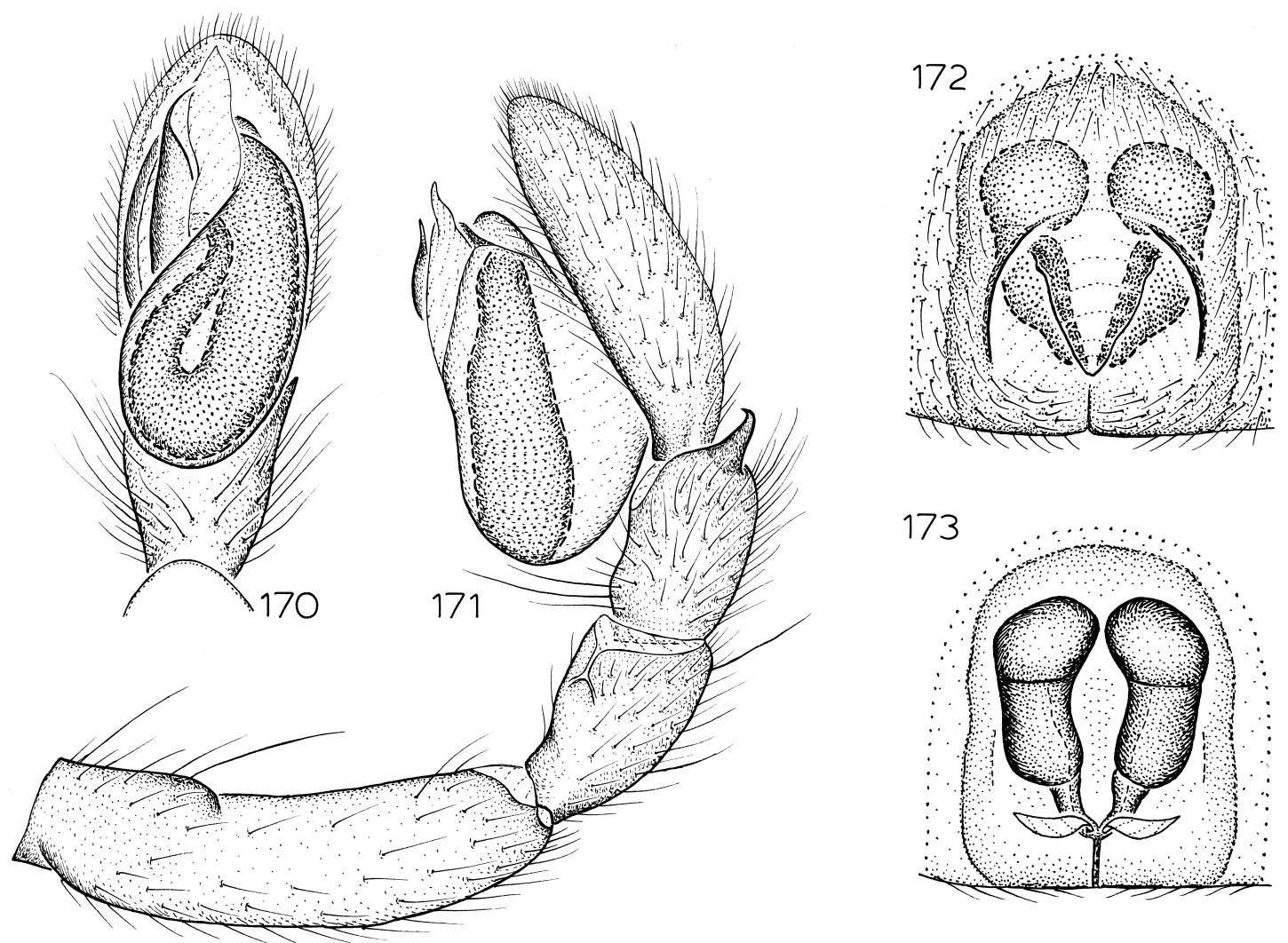

Figs. 170-173. Lampona danggali, new species. 170. Left male palp, ventral view. 171. Same, retrolateral view. 172. Epigynum, ventral view. 173. Same, dorsal view.

ysis (fig. 171), and females have a much wider epigynal midpiece (fig. 172).

MALE: Total length 5.6. Abdominal coloration as in L. cylindrata; anterior femora darkened except for light oval occupying basal two-thirds of prolateral surface, tibiae IV slightly darkened on distal prolateral surface. Palpal femur with very slight basal expansion; retrolateral tibial apophysis short, triangular (fig. 171); tegulum only moderately expanded, reaching to about one-fourth of tibial length; embolus wide at base, abruptly narrowed below tip (fig. 170).

Female: Total length 7.6. Coloration as in male. Epigynum with $\mathrm{m}$-shaped appearance produced by $\mathrm{v}$-shaped midpiece and almost angular lateral margins (fig. 172); middle portion of spermathecae broad (fig. 173).

Other Material Examined: New South Wales: Waukeroo, near Broken Hill, $33^{\circ} 31^{\prime} \mathrm{S}, 142^{\circ} 01^{\prime} \mathrm{E}$, July 19, 1970 (R. Mew,

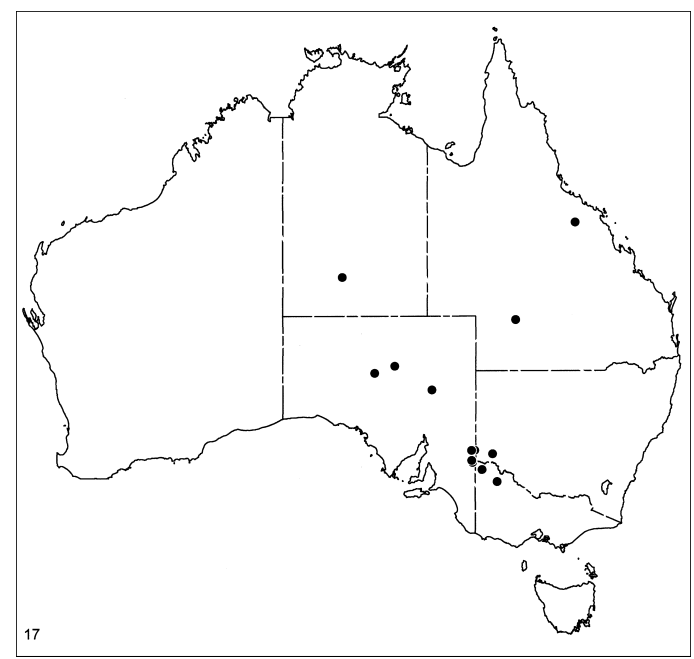

Map 17. Records of Lampona danggali, new species. 
AMS KS31854), 19 . Northern Territory: Ormiston, $23^{\circ} 43^{\prime} \mathrm{S}, 132^{\circ} 42^{\prime} \mathrm{E}$, Oct. 1994 (R. Raven, QMB S34287), 1\%. Queensland: Coopers Creek, $20^{\circ} 41^{\prime} \mathrm{S}, 147^{\circ} 08^{\prime} \mathrm{E}$, Aug. 28, 1978, under bark (M. Jahnke, QMB S34409), 1 ; Thylungra, $26^{\circ} 04^{\prime} \mathrm{S}, 143^{\circ} 28^{\prime} \mathrm{E}$, Oct. 1995, pitfall, mulga (T. Churchill, CSID), 10. South Australia: $85 \mathrm{~km}$ NE Coober Pedy on Stuart Highway, $29^{\circ} 01^{\prime} \mathrm{S}, 134^{\circ} 43^{\prime} \mathrm{E}$, Aug. 9, 1980, under rock (M. Harvey, QMB), 1 ; 5.5 km ESE Patsy Dam, 28 $8^{\circ} 7^{\prime} \mathrm{S}$, $135^{\circ} 58^{\prime} \mathrm{E}$, Oct. $26-31$, 1995, pitfall (SAM N1996/339), 1 đ; 19 km N Renmark, $34^{\circ} 00^{\prime} \mathrm{S}, 140^{\circ} 47^{\prime} \mathrm{E}$, Aug. 10-Sept. 7, 1995, intercept trap (K. Pullen, QMB), 1 , Nov. 8-22, 1995, pitfall (A. Lambie, K. Pullen QMB S34370), 1, Dec. 14-21, 1995, pitfall, saltbush (S. Dominelli, K. Pullen, QMB), 10, Dec. 14, 1995-Jan. 25, 1996, intercept trap (K. Pullen, QMB ex S32501), 10, Jan. 24-Feb. 20, 1996, intercept trap (K. Pullen, QMB), 20; $32 \mathrm{~km} \mathrm{~N}$ Renmark, $33^{\circ} 53^{\prime} \mathrm{S}, 140^{\circ} 44^{\prime} \mathrm{E}$, Nov. 9-Dec. 12, 1995, intercept trap (K. Pullen, QMB ex S32525), $1 \%$; near Tomahawk Dam, Danggali Conservation Park, $33^{\circ} 20^{\prime} \mathrm{S}, 140^{\circ} 43^{\prime} \mathrm{E}$, Nov. 25, 1996, vibration (D. Hirst, J. Forrest, SAM N1997/150), 1 đ̊; 7 km WNW Wilpoorinna High School, 29 $56^{\prime} \mathrm{S}, 138^{\circ} 16^{\prime} \mathrm{E}$, Oct. 31Nov. 5, 1994, pitfall (SAM N1997/148), 1 . Victoria: $3.2 \mathrm{~km}$ ESE Meringur, $34^{\circ} 24^{\prime} \mathrm{S}$, $141^{\circ} 22^{\prime} \mathrm{E}, \mathrm{Feb} .1986$, drift fence pitfall (A. Yen, NMVS), 1 \% ; Ouyen, $35^{\circ} 04^{\prime} \mathrm{S}$, $142^{\circ} 19^{\prime}$ E, Nov. 23, 1914, mallee (C. French, NMV K3433), 1 ㅇ․

DistRIBUTION: Widespread in the drier parts of central and eastern Australia (map 17).

\section{Lampona olga, new species}

Figures 156, 157; Map 18

TYPE: Female holotype from the summit of the second highest peak on Mount Olga, $25^{\circ} 19^{\prime} \mathrm{S}, 130^{\circ} 46^{\prime} \mathrm{E}$, Northern Territory (Aug. 19, 1969; D. Giuliani), deposited in WAM (84/722).

ETYMOLOGY: The specific name is a noun in apposition taken from the type locality.

DiAGNOSIS: This distinctive species resembles $L$. danggali in external epigynal structure, but can easily be distinguished by the narrower, more divergent spermathecae (fig. 157).

MALE: Unknown.

Female: Total length 14.7. Abdominal coloration uncertain (specimen worn); legs unmarked. Epigynum with arched, weak anterior and lateral margins; midpiece greatly widened anteriorly (fig. 156); spermathecae tripartite, middle portion elongate, sinuous (fig. 157).

Other Material ExAmined: None.

DistRIBUTION: Known only from the type locality (map 18).

\section{Lampona garnet, new species} Figures 174-177; Map 18

TYPE: Female holotype from Forty Mile Scrub, SW Mount Garnet, $18^{\circ} 05^{\prime} \mathrm{S}$, 14451'E, Queensland (Apr. 10-14, 1978; R. Raven, V. Davies), deposited in QMB (S28096).

ETYMology: The specific name is a noun in apposition taken from the type locality.

DiAGNOSIS: Males resemble those of $L$. mildura in having a basally widened retrolateral tibial apophysis, but that apophysis is longer and bent below its tip (fig. 175); females also resemble those of $L$. mildura but have a more distinct anterior epigynal margin and more evenly triangular epigynal midpiece (fig. 176) as well as smaller median portions of the spermathecae (fig. 177).

MALE: Total length 5.8. Abdominal dorsum with anterior and median white spots fused into longitudinal stripes; all femora and tibiae darkened distally. Palpal femur unmarked, unmodified; retrolateral tibial apophysis with sinuous tip (fig. 175); tegulum globose, extending to about half of tibial length; embolus twisted, with tip extending dorsally before returning to ventral side of bulb (fig. 174).

Female: Total length 6.8. Abdominal dorsum light anteriorly, with median white markings posteriorly; legs as in male. Epigynum with semicircular anterior margin (fig. 176); spermathecae short, tripartite, with anterior portion on twisted neck (fig. 177).

Other MATERIAL ExAMined: The male tentatively associated with the holotype was taken at Trovers (coordinates unknown, possibly Travers Island, Torres Strait, $10^{\circ} 22^{\prime} \mathrm{S}$, 

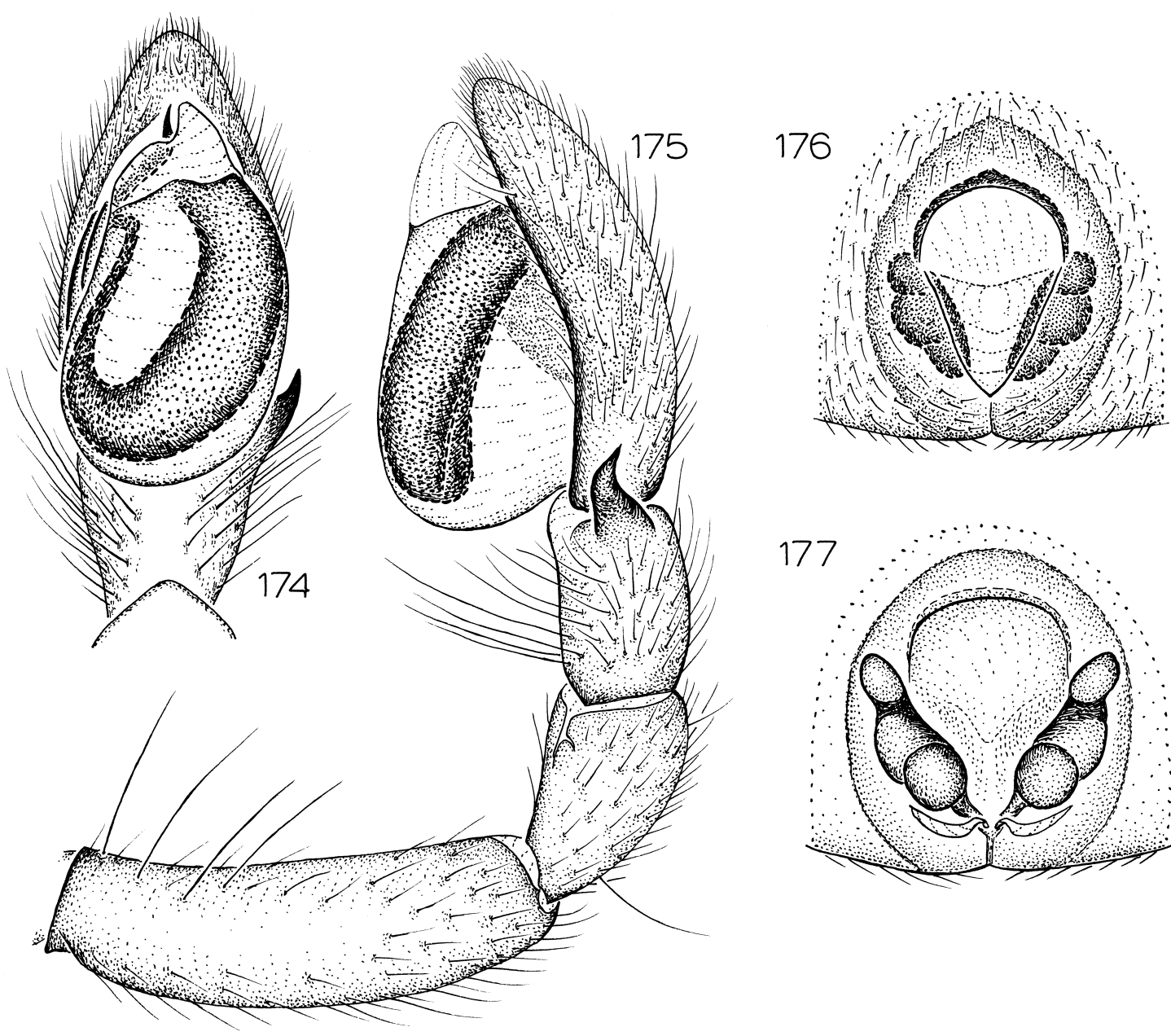

Figs. 174-177. Lampona garnet, new species. 174. Left male palp, ventral view. 175. Same, retrolateral view. 176. Epigynum, ventral view. 177. Same, dorsal view.

$\left.142^{\circ} 22^{\prime} \mathrm{E}\right)$ in northeastern Queensland on Apr. 6, 1986 (J. Turner, QMB S30189).

DisTRIBUTION: Known only from northeastern Queensland (map 18).

\section{Lampona mildura, new species}

Figures 178-181; Map 18

TYPE: Female holotype taken under bark of Eucalyptus camaldulensis $5 \mathrm{~km}$ W Mildura, Victoria, $34^{\circ} 10^{\prime} \mathrm{S}, 142^{\circ} 06^{\prime} \mathrm{E}$ (Sept. 21, 1985; M. Harvey, B. Scott, L. Hoare), deposited in AMS (KS31888).

ETYMOLOGY: The specific name is a noun in apposition taken from the type locality.

DiAgNOSIS: Females resemble those of $L$. yanchep and $L$. garnet but can be distin- guished by the almost pentagonal epigynal midpiece (fig. 180). The male tentatively matched with the holotype resembles that specimen in coloration, sternal sculpturing, and femoral shape, and can easily be recognized by the odd shape of the retrolateral tibial apophysis (fig. 179).

MALE: Total length 5.2. Abdominal dorsum light anteriorly, with dark chevrons posteriorly; anterior femora slightly darkened, posterior femora with subdistal, tibiae with proximal and distal dark rings. Palpal femur darkened throughout its length but otherwise unmodified; retrolateral tibial apophysis lobe-shaped, with narrow tip (fig. 179); tegulum short; embolus short, narrow, almost 
obscured by conductor in ventral view (fig. 178).

FEMALE: Total length 4.6. Coloration as in male, except posterior leg markings not evident (specimen probably collected soon after molt, before fully sclerotized). Epigynum with v-shaped lateral margins (fig. 180); spermathecae with anterior portion rotund (fig. 181).

Other Material ExAmined: New South Wales: Barren Grounds, $34^{\circ} 42^{\prime} \mathrm{S}, 150^{\circ} 43^{\prime} \mathrm{E}$, Sept. 3, 1996 (AMS KS49695), 10; Mount Keira, $34^{\circ} 24^{\prime} \mathrm{S}, 150^{\circ} 51^{\prime} \mathrm{E}$, no date, Berlese, rotting $\log$, rainforest, elev. ca. $1000 \mathrm{ft}$ (E. Britton, QMB), 1 స.

DisTRIBUTION: Known only from northwestern Victoria and southern New South Wales (map 18).

\section{Lampona cohuna, new species}

Figures 182-185; Map 19

TyPES: Male holotype and female allotype collected during watering at Barr Creek, Cohuna, $35^{\circ} 50^{\prime} \mathrm{S}, 144^{\circ} 11^{\prime} \mathrm{E}$, Victoria (Apr. 18, 1998; J. Hooper, CVIC 506), deposited in NMV courtesy of J. Shield.

ETYMOLOGY: The specific name is a noun in apposition taken from the type locality.

DIAGNOSIS: This distinctive species is unlikely to be confused with any other member

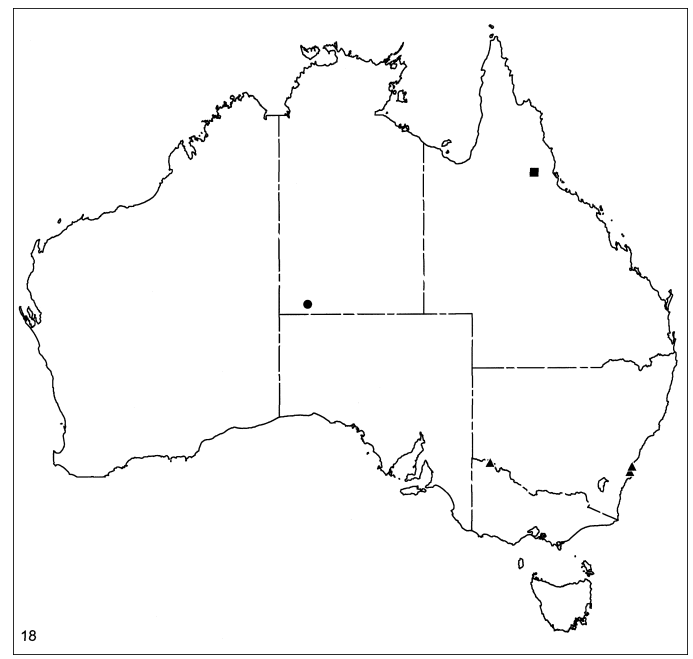

Map 18. Records of Lampona olga, new species (circle), L. garnet, new species (square), and L. mildura, new species (triangles). of the genus; males have a long, dorsally situated and obliquely directed retrolateral tibial apophysis with its ventral surface abruptly narrowed below the tip (fig. 183), and females have a peculiar epigynum bearing a pair of hooded pockets posteriorly (fig. 184).

MALE: Total length 6.7. Abdominal dorsum with markings subdued gray rather than white, anterior femora darkened proximally. Palpal femur darkened on proximal half, distal half narrowed, twisted relative to proximal half; retrolateral tibial apophysis shifted dorsally, expanded behind tip (fig. 183); embolar tip long, strong, arched (fig. 182).

FEMALE: Total length 8.8. Coloration as in male. Epigynum with pair of posteriorly situated pockets bearing anterior hoods (fig. 184); spermathecae situated on internal projections of pockets (fig. 185).

Other Material Examined: South Australia: $14 \mathrm{~km}$ SSW Beresford Bore, Stuart Creek Station, $29^{\circ} 22^{\prime} \mathrm{S}, 136^{\circ} 37^{\prime} \mathrm{E}$, Sept. 2530, 1995, pitfall (SAM N1997/142), 10 ; $9.95 \mathrm{~km}$ NNE Coongie, $27^{\circ} 05^{\prime} \mathrm{S}, 140^{\circ} 09^{\prime} \mathrm{E}$, Feb. 6, 1987, pitfall (J. Reid, SAM N1996/ 288), $10^{\top}$; Lake Arthur, $29^{\circ} 32^{\prime} \mathrm{S}, 138^{\circ} 46^{\prime} \mathrm{E}$, Oct. 31-Nov. 5, 1994, pitfall (SAM N1997/ 143), 10; Memory Bore, $26^{\circ} 41^{\prime} \mathrm{S}, 135^{\circ} 40^{\prime} \mathrm{E}$, Nov. 23, 1995, pitfall (SAM N1996/340), 10; Stuart Creek Station, $7.5 \mathrm{~km}$ E Sheep Camp WH, $29^{\circ} 45^{\prime} \mathrm{S}, 137^{\circ} 00^{\prime} \mathrm{E}$, Sept. 27-30, 1995, pitfall (SAM N1997/141), $10^{\uparrow}$. Victoria: Barr Creek, Cohuna, $35^{\circ} 50^{\prime} \mathrm{S}, 144^{\circ} 11^{\prime} \mathrm{E}$, Apr. 18, 1998, collected during watering (J. Hooper, CVIC 507), $19 ; 5 \mathrm{~km}$ W Mildura, $34^{\circ} 10^{\prime} \mathrm{S}, 142^{\circ} 06^{\prime} \mathrm{E}$, Sept. 21, 1985 , under bark of Eucalyptus camaldulensis (M. Harvey, B. Scott, L. Hoare, QMB), 19.

Distribution: Known only from South Australia and Victoria (map 19).

\section{THE QUINQUEPLAGIATA GROUP}

This group includes seven species that share an arrow-shaped epigynal midpiece. Males have a short triangular retrolateral tibial apophysis, and often have a highly modified palpal femur. The spermathecae are often on greatly elongated posterior stalks.

\section{Lampona quinqueplagiata Simon Figures 186-189; Map 19}

Lampona quinqueplagiata Simon, 1908: 403 (male syntype from Dirk Hartog, Western Australia, in $\mathrm{ZMB}$, examined). 

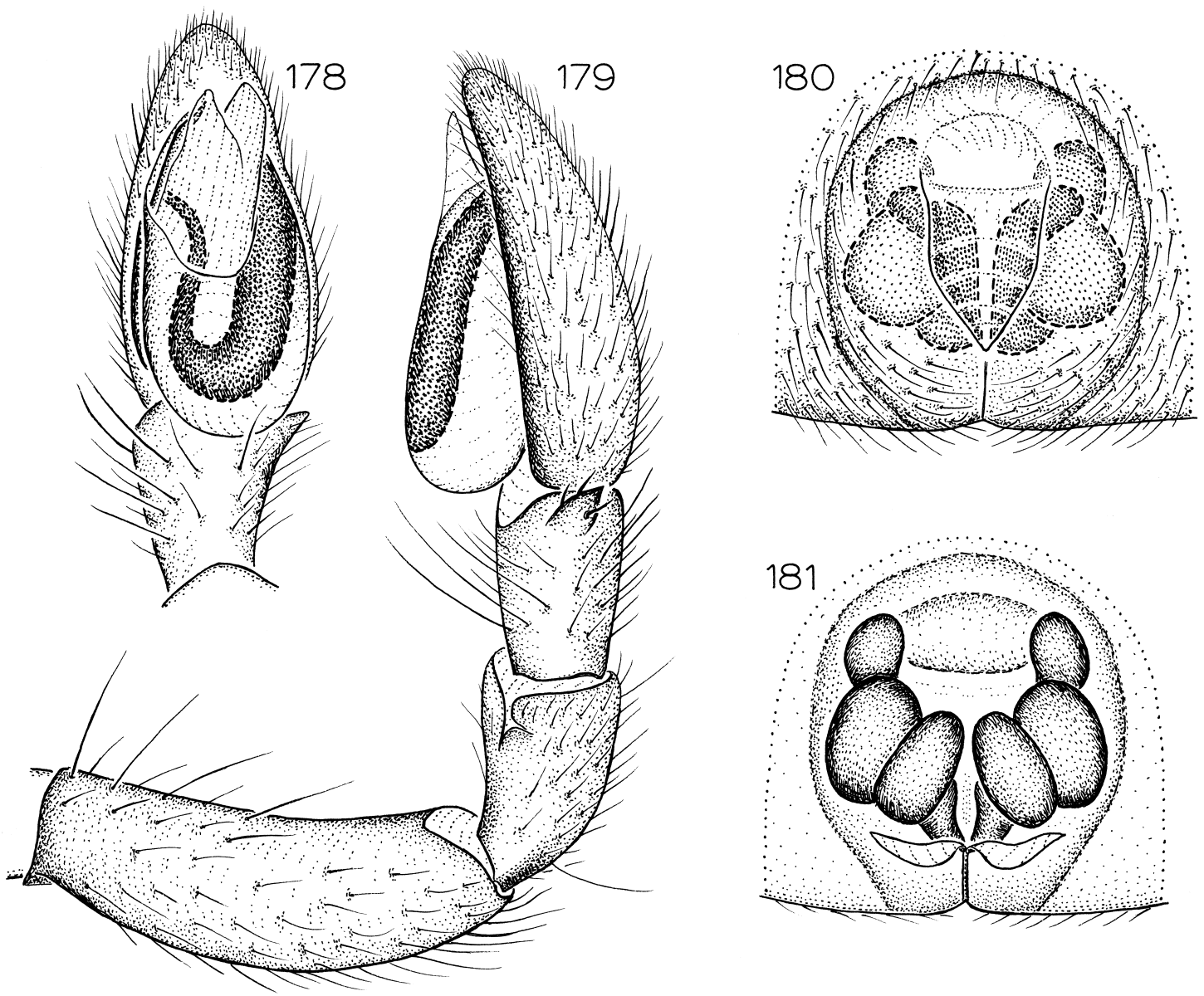

Figs. 178-181. Lampona mildura, new species. 178. Left male palp, ventral view. 179. Same, retrolateral view. 180. Epigynum, ventral view. 181. Same, dorsal view.

Diagnosis: This species resembles $L$. dwellingup in having a highly elaborated protrusion on the male palpal femur, but can be distinguished by the smaller protrusion and embolus in males (figs. 186, 187) and the longer epigynal midpiece in females (fig. 188).

MALE: Total length 5.1. Abdominal dorsum as in L. cylindrata; coxae and anterior femora orange, other leg segments yellow, unmarked. Palpal femur relatively small, with elaborate, pointed ventral protrusion; retrolateral tibial apophysis triangular, excavated, situated on dorsal edge of segment (fig. 187); tegulum globose, extending to only about one-fifth length of tibia, embolar base short, wide, triangular, tegulum extending behind embolus and conductor (fig. 186).
FEMALE: Total length 6.7. Coloration as in male. Epigynum with relatively narrow, flat, dagger-shaped midpiece, lateral margins almost semicircular (fig. 188); spermathecal bulbs round, situated on extremely long, medially situated ducts (fig. 189).

Material Examined: Western Australia: Barlee Range Nature Reserve, $23^{\circ} 05^{\prime} \mathrm{S}$, $115^{\circ} 47^{\prime} \mathrm{E}$, June 15-18, 1994, dry pitfall (P., G. Kendrick, WAM 95/1056, 1059), 2 \% ;

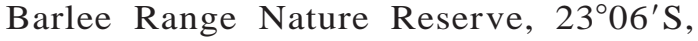
$116^{\circ} 00^{\prime} \mathrm{E}$, Aug. 1993 (S. van Leeuwen, B.

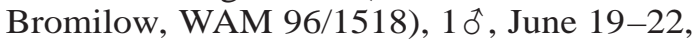
1994, dry pitfall (P., G. Kendrick, WAM 95/ 1055), 19 ; Barlee Range Nature Reserve, $23^{\circ} 23^{\prime} \mathrm{S}, 115^{\circ} 53^{\prime} \mathrm{E}$, June 11-14, 1994, dry pitfall (P., G. Kendrick, WAM 95/1051), 10; Barlee Range Nature Reserve, $23^{\circ} 25^{\prime} \mathrm{S}$, 

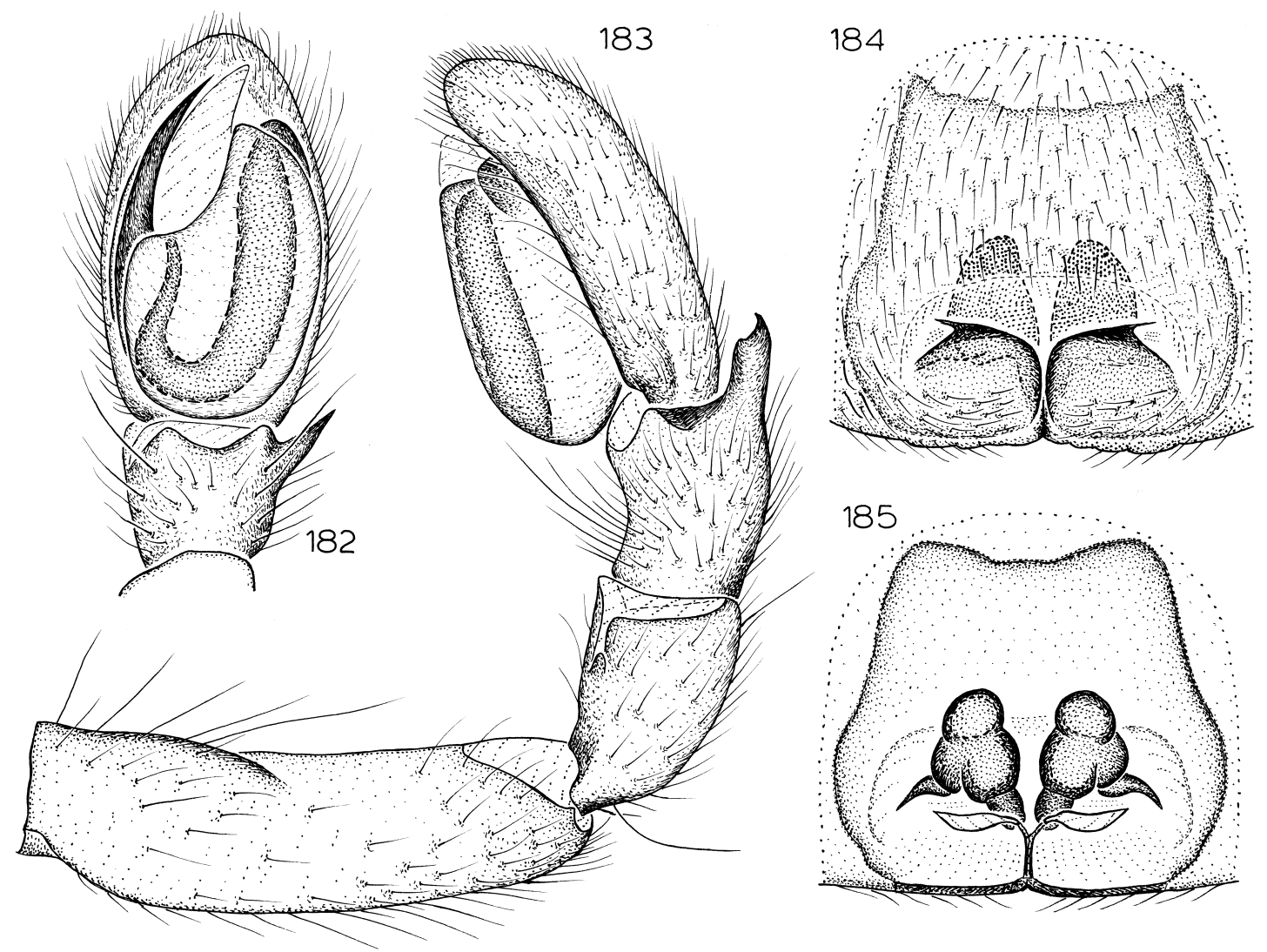

Figs. 182-185. Lampona cohuna, new species. 182. Left male palp, ventral view. 183. Same, retrolateral view. 184. Epigynum, ventral view. 185. Same, dorsal view.

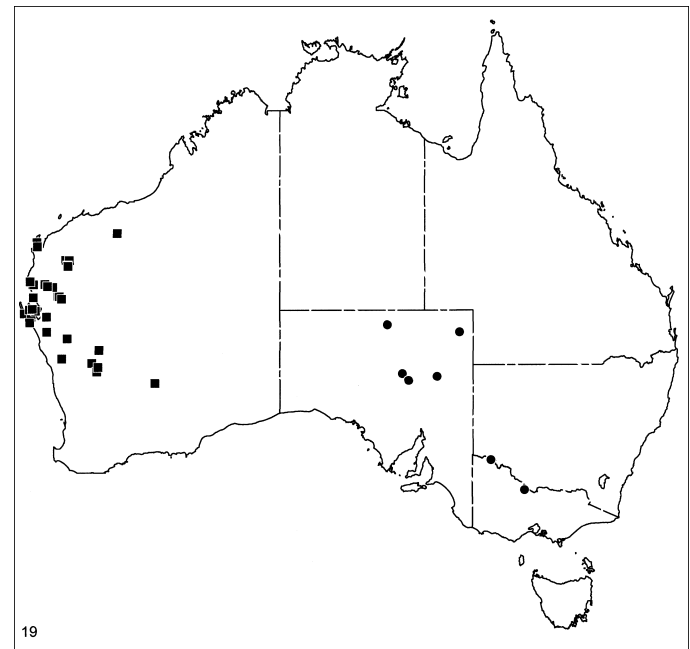

Map 19. Records of Lampona cohuna, new species (circles) and L. quinqueplagiata Simon (squares). $115^{\circ} 54^{\prime} \mathrm{E}$, June $11-14,1994$, dry pitfall (P., G. Kendrick, WAM 95/1060), 1 \% ; Bidgemia Station, Gasgoyne Junction, $25^{\circ} 03^{\prime} \mathrm{S}$, $115^{\circ} 18^{\prime}$ E, Aug. 17-Oct. 5, 1994, pitfall (A. Sampey, WAM 96/1437), 1 ô, Oct. 5, 1994Jan. 13, 1995, pitfall (N. McKenzie, J. Rolfe, WAM 96/1436), 1 ô, June 6-Aug. 20, 1995, pitfall (N. Hall, WAM 96/1434), $1 \delta^{\hat{\alpha}}$; Bidgemia Station, Gasgoyne Junction, $25^{\circ} 05^{\prime} \mathrm{S}$, $115^{\circ} 23^{\prime} \mathrm{E}$, Jan. 13-June 6, 1995, pitfall (J. Waldock, WAM 96/1439), 1; Bidgemia Station, Gasgoyne Junction, $25^{\circ} 13^{\prime} \mathrm{S}$, $115^{\circ} 31^{\prime}$ E, Aug. 17-Oct. 4, 1994, pitfall (A. Sampey, WAM 96/1435), 10, Jan. 13-June 5, 1995, pitfall (J. Waldock, WAM 96/1438), 19 ; Billabong Roadhouse, $27^{\circ} 25^{\prime} \mathrm{S}$, $115^{\circ} 50^{\prime}$ E, July 9, 1991 (D. Elford, K. Brimmell, N. Schroder, WAM 95/1058), 1 \%; Boolathana Station, $24^{\circ} 25^{\prime} \mathrm{S}, 113^{\circ} 40^{\prime} \mathrm{E}$, May 28-June 2, 1995, pitfall (J. Waldock, WAM 

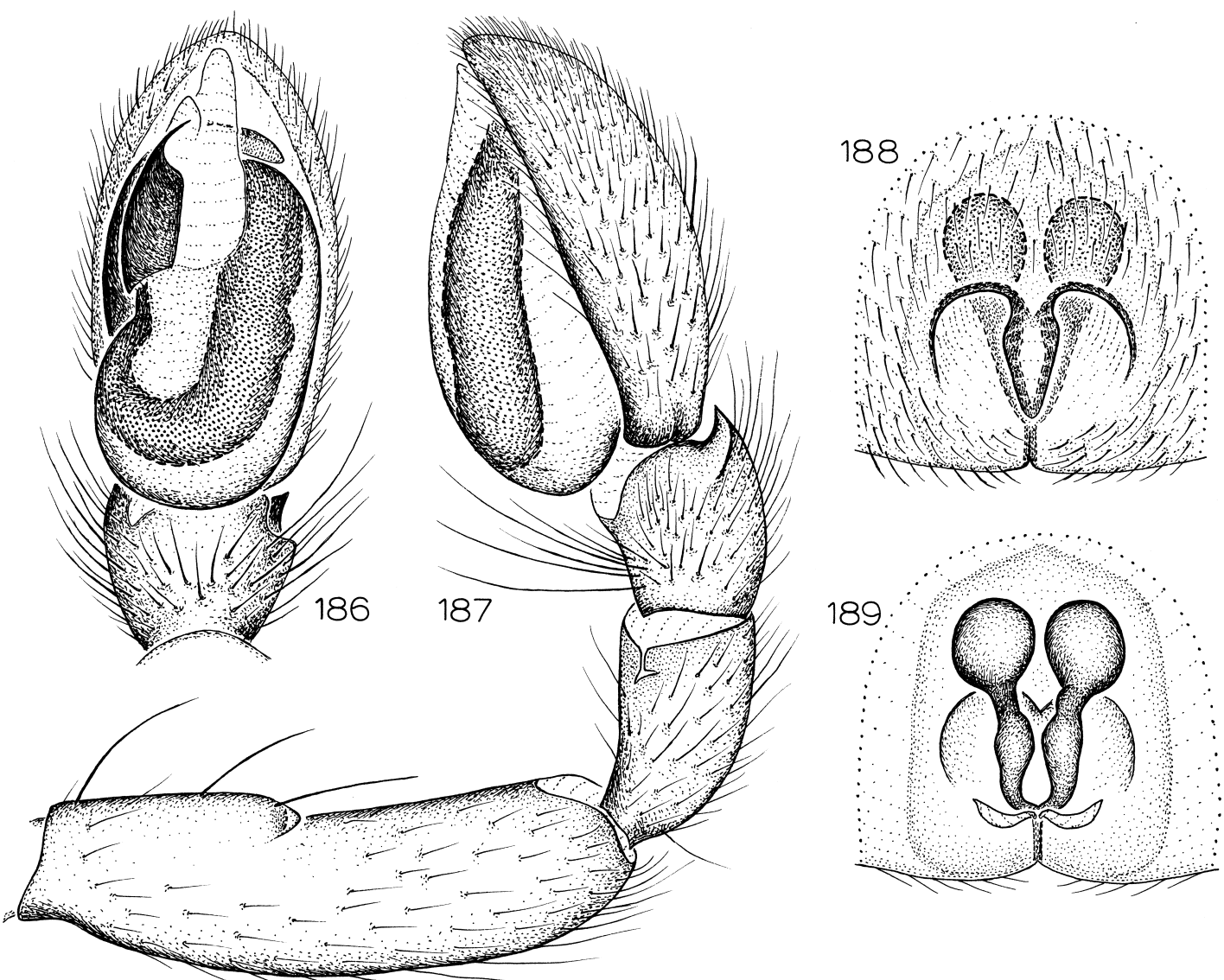

Figs. 186-189. Lampona quinqueplagiata Simon. 186. Left male palp, ventral view. 187. Same, retrolateral view. 188. Epigynum, ventral view. 189. Same, dorsal view.

96/1442), 10; Boolathana Station, $24^{\circ} 25^{\prime} \mathrm{S}$, $113^{\circ} 41^{\prime}$ E, Sept. 30, 1994-Jan. 15, 1995, pitfall (N. McKenzie, J. Rolfe, WAM 96/1443, 1445), $10^{\star}, 1$; ; Boolathana Station, $24^{\circ} 25^{\prime} \mathrm{S}$, $113^{\circ} 42^{\prime}$ E, Sept. 30, 1994-Jan. 15, 1995, pitfall (N. McKenzie, J. Rolfe, WAM 96/1440), $10^{\circ}$; Boolathana Station, $24^{\circ} 25^{\prime} \mathrm{S}, 113^{\circ} 45^{\prime} \mathrm{E}$, Jan. 15-May 31, 1995, pitfall (J. Waldock, WAM 96/1441), 1\%; Boolathana Station, $24^{\circ} 25^{\prime} \mathrm{S}, 113^{\circ} 46^{\prime} \mathrm{E}$, Aug. 20-Sept. 30, 1994, pitfall (A. Sampey, WAM 98/1950), $1 \%$; Burnabinmah, SE Yalgoo, 28 47'S, $117^{\circ} 22^{\prime}$ E, Aug. 1976 (N. Witherow, WAM 84/197), 1 \% ; Bush Bay, $25^{\circ} 05^{\prime} \mathrm{S}, 113^{\circ} 43^{\prime} \mathrm{E}$, Aug. 16-Sept. 30, 1994, pitfalls (M. Harvey, WAM 96/1490-1494), 30ิ, 2 ㅇ, Sept. 27Oct. 2, 1994, dry pitfall (M. Harvey, WAM 96/1447), 1 ㅇ, Sept. 30, 1994-Jan. 16, 1995 , pitfall (N. McKenzie, J. Rolfe, WAM 96/
1448, 1149), 20; Bush Bay, $25^{\circ} 08^{\prime} \mathrm{S}$, $113^{\circ} 46^{\prime} \mathrm{E}$, Aug. 16-Sept. 28, 1994, pitfall (M. Harvey, WAM 98/1947), 1 đ, Sept. 28, 1994-Jan. 16, 1995, pitfall (N. McKenzie, J. Rolfe, WAM 96/1446, 1948, 1949), $20^{\text {o, }} 1$ 우; Cape Cuvier, Quobba Station, $24^{\circ} 15^{\prime} \mathrm{S}$, $113^{\circ} 33^{\prime}$ E, Sept. 29, 1994-Jan. 15, 1995, pitfall (N. McKenzie, J. Rolfe, WAM 96/1495), $1 \%$; Cape Range, NW Cape Peninsula camp, $22^{\circ} 15^{\prime} \mathrm{S}, 114^{\circ} 03^{\prime} \mathrm{E}$, June 5, 1990 (J. Waldock, WAM 96/1521), 1 ; ; Cape Range, near Cape Range No. 2 well, $22^{\circ} 06^{\prime} \mathrm{S}, 114^{\circ} 00^{\prime} \mathrm{E}$, May 1965 (G. Kendrick, B. Hitchin, WAM 95/ 1110), 1 $\delta^{\text {* }}$; Cape Range, Area $C$, head of well 3, 22 $20^{\prime} \mathrm{S}, 114^{\circ} 02^{\prime} \mathrm{E}$, May 18, 1993 (R. Brooks, W. Humphreys, WAM 95/1105), 1 \% ; Central Peron Peninsula, 26 $06^{\prime} \mathrm{S}$, $113^{\circ} 38^{\prime} \mathrm{E}$, Nov. 15-19, 1989 (G. Harold, WAM 95/1057), 19 ; Dirk Hartog, $26^{\circ} 02^{\prime} \mathrm{S}$, 

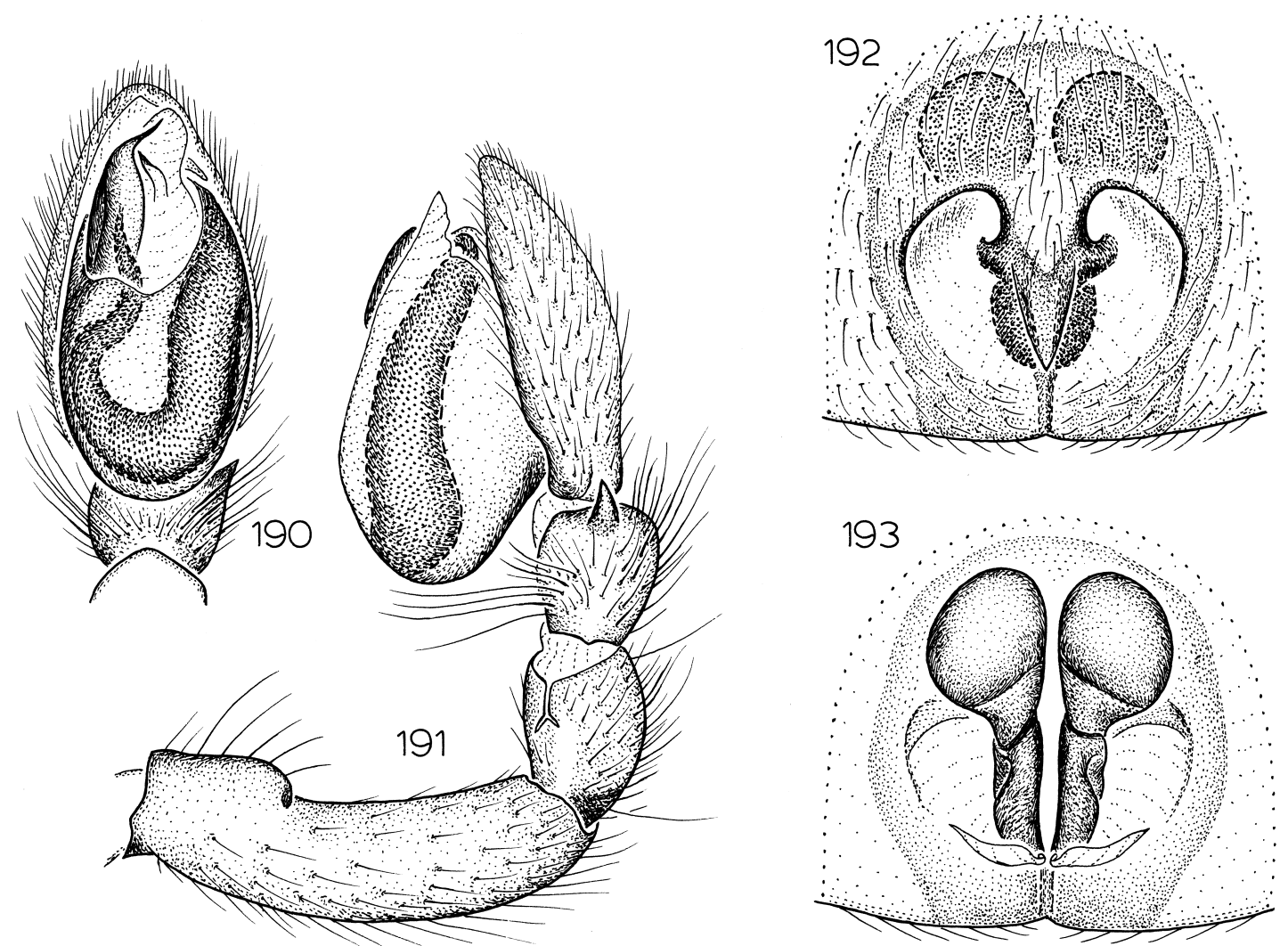

Figs. 190-193. Lampona dwellingup, new species. 190. Left male palp, ventral view. 191. Same, retrolateral view. 192. Epigynum, ventral view. 193. Same, dorsal view.

$113^{\circ} 12^{\prime} \mathrm{E}$ (V. Michaelsen, R. Hartmeyer, ZMB 24599), $10^{\star}$ (syntype); Edel Land, $26^{\circ} 32^{\prime} \mathrm{S}, 113^{\circ} 32^{\prime} \mathrm{E}$, Jan. 17-May 9, 1995, pitfall (A. Sampey, WAM 96/1496), 19 ; Faure Island, $25^{\circ} 53^{\prime} \mathrm{S}, 113^{\circ} 54^{\prime} \mathrm{E}$, Nov. 22-27, 1989 (G. Harold, WAM 95/1050), $1 \delta^{\hat{\alpha}}, 1$ \% ; Francois Peron National Park, $25^{\circ} 49^{\prime} \mathrm{S}, 113^{\circ} 32^{\prime} \mathrm{E}$, Aug. 24-Oct. 11, 1994, pitfall (A. Sampey, WAM 96/1497, 1498), 20 ; Kennedy Range National Park, $24^{\circ} 30^{\prime} \mathrm{S}, 115^{\circ} 01^{\prime} \mathrm{E}$, Oct. 3-8, 1994, dry pitfall (M. Harvey, WAM 96/ 1505), 1 0 ; Kennedy Range National Park, $24^{\circ} 30^{\prime} \mathrm{S}, 115^{\circ} 02^{\prime} \mathrm{E}$, Oct. 3-8, 1994, dry pit-

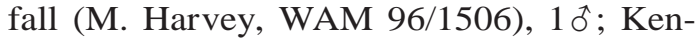
nedy Range National Park, 24\%31'S, $114^{\circ} 58^{\prime} \mathrm{E}$, Oct. 3-8, 1994, dry pitfall (M. Harvey, WAM 96/1501, 1502), 20, May 29Aug. 28, 1995, pitfall (N. Hall, WAM 96/ 1503), 19 ; Kennedy Range National Park, $24^{\circ} 33^{\prime} \mathrm{S}, 114^{\circ} 58^{\prime} \mathrm{E}$, May 29-Aug. 28, 1995,

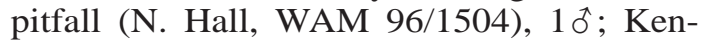

nedy Range National Park, 243' $\mathrm{S}$, $114^{\circ} 57^{\prime} \mathrm{E}$, Oct. 3-8, 1994, dry pitfall (M. Harvey, WAM 96/1500), 10, May 29-Aug. 28, 1995, pitfall (N. Hall, WAM 96/1499), 1 \% ; Mardathuna Station, $24^{\circ} 24^{\prime} \mathrm{S}, 114^{\circ} 28^{\prime} \mathrm{E}$, May 23-28, 1995, dry pitfall (A. Sampey, WAM 96/1507), 1 \%; Mardathuna Station, $24^{\circ} 31^{\prime} \mathrm{S}, 114^{\circ} 38^{\prime} \mathrm{E}$, May 26-Aug. 26, 1995, pitfall (N. Hall, WAM 96/1508), 10; Monkey Mia, Peron Peninsula, $25^{\circ} 47^{\prime} \mathrm{S}$, $113^{\circ} 42^{\prime} \mathrm{E}$, Nov. 7, 1998 (V. Ovtsharenko, $\mathrm{AMNH}), 10^{\star}, 2{ }^{\circ}$; Mount Magnet, $28^{\circ} 04^{\prime} \mathrm{S}$, $117^{\circ} 49^{\prime} \mathrm{E}$, Oct. 4, 1962, elev. 410 m (E. Ross, D. Cavagnaro, CAS), $2+$ (1 penultimate but with full epigynum); Mullewa, $28^{\circ} 32^{\prime} \mathrm{S}$, $115^{\circ} 31^{\prime}$ E, Sept. 18, 1931 (W. Wheeler, MCZ), 1 ; ; Nerren Nerren Station, $27^{\circ} 03^{\prime} \mathrm{S}$, $114^{\circ} 34^{\prime} \mathrm{E}$, Jan. 11-May 11, 1995, pitfall (P. West, WAM 96/1509), 1 \%; granite rock, 8 mi from Paynes Find on Yalgoo Road, $29^{\circ} 15^{\prime} \mathrm{S}, 117^{\circ} 41^{\prime} \mathrm{E}$, Sept. 3, 1967 (B. Main, 
WAM 95/1054), $1 \%$; 17 mi N Paynes Find, $29^{\circ} 00^{\prime} \mathrm{S}, 117^{\circ} 45^{\prime} \mathrm{E}$, Oct. 3, 1962, elev. $400 \mathrm{~m}$ (E. Ross, D. Cavagnaro, CAS), $1+$; White Quartz Dam, Goongarrie, $29^{\circ} 54^{\prime} \mathrm{S}, 121^{\circ} 15^{\prime} \mathrm{E}$, Oct. 6-11, 1980 (W. Humphreys, WAM 95/ 1052), $10^{\dagger}$; Woodleigh Station, $26^{\circ} 12^{\prime} \mathrm{S}$, $114^{\circ} 32^{\prime} \mathrm{E}$, Oct. 9-14, 1994, dry pitfall (M. Harvey, WAM 96/1510), 10\%; Woodleigh Station, $26^{\circ} 13^{\prime} \mathrm{S}, 114^{\circ} 35^{\prime} \mathrm{E}$, Oct. 9-14, 1994 , dry pitfall (M. Harvey, WAM 96/1511), 1 đo; $^{\text {; }}$ Woodstock (Abydos) Station, 21 $37^{\circ} \mathrm{S}$, $118^{\circ} 57^{\prime} \mathrm{E}$, June 7, 1951, from stomach of adult $q$ frog Uperolia russelli (E. Ealey, WAM 95/1053), 10 .

Distribution: Known only from Western Australia (map 19).

\section{Lampona dwellingup, new species}

Figures 190-193; Map 20

TYPE: Female holotype taken in pitfall trap at Dwellingup, $32^{\circ} 43^{\prime} \mathrm{S}, 116^{\circ} 04^{\prime} \mathrm{E}$, Western Australia (Mar. 9, 1978; S. Curry), deposited in WAM (95/1065).

ETYMOLOGY: The specific name is a noun in apposition taken from the type locality.

DIAGNOSIS: This species resembles L. quinqueplagiata (see above), but can be distinguished by the larger palpal femur bearing a smaller ventral protrusion in males (fig. 191) and the posteriorly depressed epigynal midpiece in females (fig. 192).

MALE: Total length 8.2. Abdominal dorsum with only posterior spot pronounced, anterior pair obscure, median pair obsolete; coxae orange, other leg segments yellow, unmarked. Palpal femur relatively large, with small, elaborate, pointed ventral protrusion; retrolateral tibial apophysis triangular, slightly excavated, situated on side of segment (fig. 191); tegulum globose, extending to half length of tibia, embolar base long, wide, tegulum extending behind embolus and conductor (fig. 190).

Female: Total length 9.5. Coloration as in male. Epigynum with relatively narrow midpiece high anteriorly, greatly depressed posteriorly, lateral margins rounded (fig. 192); spermathecal bulbs oval, situated on extremely long, medially situated, anteriorly expanded ducts (fig. 193).

Other Material Examined: Western Australia: Dwellingup, $32^{\circ} 43^{\prime} \mathrm{S}, 116^{\circ} 04^{\prime} \mathrm{E}$,
Jan. 12, 1978, pitfalls (S. Curry, WAM 95/ 1061-1063), 1o, 2오. Apr. 15, 1978 (J. Majer, WAM 95/1066), 1 o, May 5, 1978, pitfall (S. Curry, WAM 95/1064), 1 ô; Kings Park, Perth, $31^{\circ} 57^{\prime} \mathrm{S}, 115^{\circ} 52^{\prime} \mathrm{E}$, Sept. 5, 1962, elev. $100 \mathrm{ft}$ (E. Ross, D. Cavagnaro, CAS), 1 ㅇ.

Distribution: Southwestern Australia (map 20).

Lampona ampeinna, new species Figures 194-197; Map 20

TYPE: Female holotype taken in pitfall trap

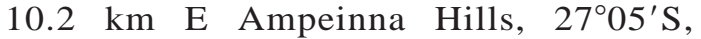
$131^{\circ} 14^{\prime} \mathrm{E}$, South Australia (Mar. 19-23, 1995), deposited in SAM (N1996/448).

ETYMOLOGY: The specific name is a noun in apposition taken from the type locality.

DIAGNOSIs: This species seems to represent a northeastern vicar of $L$. quinqueplagiata and L. dwellingup; males lack the pronounced protrusion on the palpal femur and have a recurved tip on the retrolateral tibial apophysis (fig. 195); females have an anteriorly widened epigynal midpiece and shorter, more angular lateral epigynal margins (fig. 196).

MALE: Total length 5.7. Abdominal dorsum with only posterior spot pronounced, anterior and median stripes reduced to small, elongate ovals; coxae and anterior femora

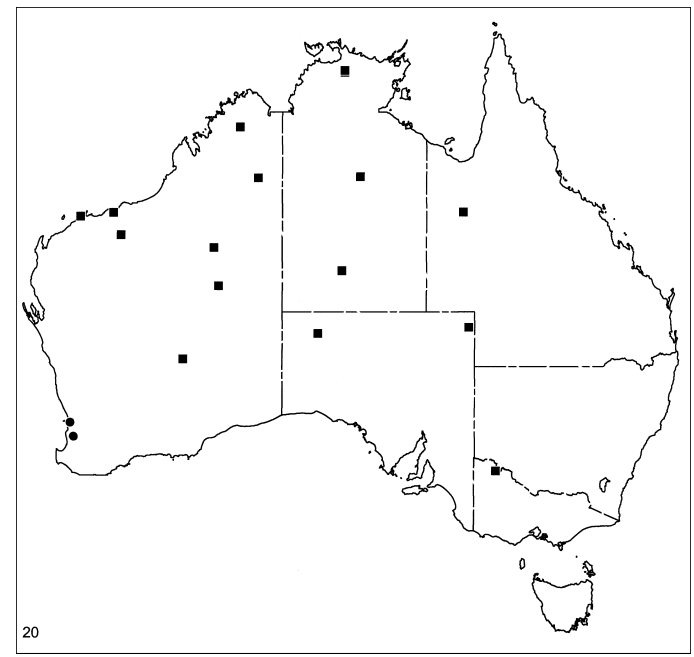

Map 20. Records of Lampona dwellingup, new species (circles) and L. ampeinna, new species (squares). 

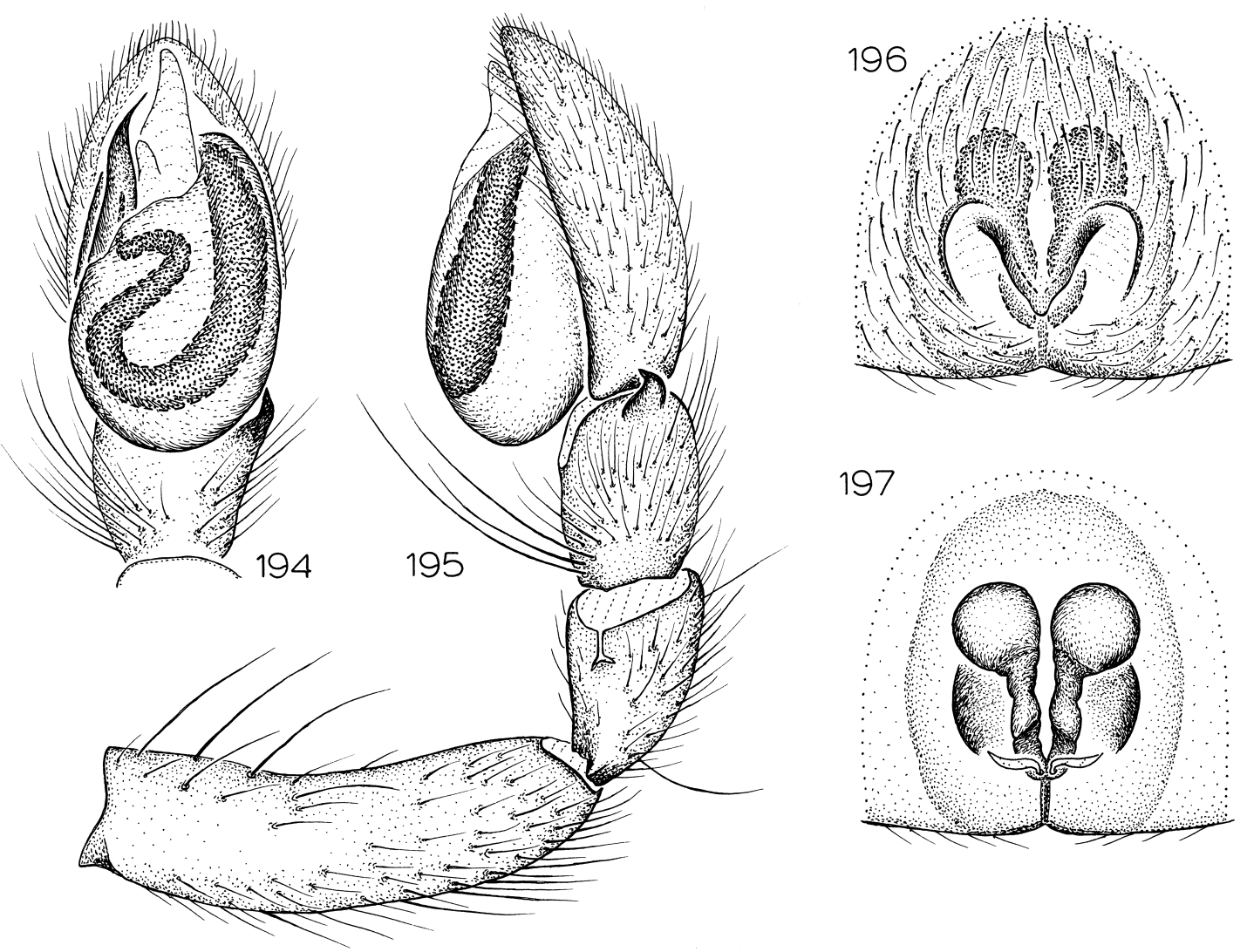

Figs. 194-197. Lampona ampeinna, new species. 194. Left male palp, ventral view. 195. Same, retrolateral view. 196. Epigynum, ventral view. 197. Same, dorsal view.

red, other leg segments orange, unmarked. Palpal femur relatively small, with only slight ventral expansion; retrolateral tibial apophysis triangular, with recurved tip (fig. 195); tegulum globose, extending to onethird length of tibia, embolar base short, wide, triangular, tegulum extending behind embolus and conductor (fig. 194).

FEMALE: Total length 9.5. Coloration as in male, with anterior and median abdominal stripes sometimes connected longitudinally. Epigynum with anteriorly widened, v-shaped midpiece, lateral margins short, angular (fig. 196); spermathecal bulbs round, situated on extremely long, medially situated, anteriorly expanded ducts (fig. 197).

Other MAterial ExAmined: Northern Territory: Helen Springs Station, Barkly Tableland, $18^{\circ} 26^{\prime} \mathrm{S}, 133^{\circ} 52^{\prime} \mathrm{E}$, Mar. 1996, pit-

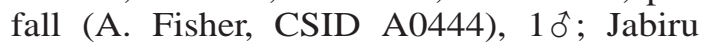

Residency, $12^{\circ} 41^{\prime} \mathrm{S}, 132^{\circ} 53^{\prime} \mathrm{E}$, May 28, 1992, head torch at night (M. Harvey, J. Waldock, WAM 95/1074), 10; Mudginberri, $12^{\circ} 35^{\prime} \mathrm{S}, 132^{\circ} 53^{\prime} \mathrm{E}$, Oct. 11,1977 (R. Pengilley, NTM T025), 1ठ; Ormiston Gorge, $23^{\circ} 37^{\prime} \mathrm{S}, 132^{\circ} 43^{\prime} \mathrm{E}$, Oct. 1994 (T. Churchill, ex QMB 34288), 1 t. Queensland: Quamby, $20^{\circ} 22^{\prime} \mathrm{S}, 140^{\circ} 17^{\prime} \mathrm{E}$ (J. Williams, AMS KS17484), 19 . South Australia: $3.8 \mathrm{~km}$ SSE Cordillo Downs, $26^{\circ} 44^{\prime} \mathrm{S}, 140^{\circ} 38^{\prime} \mathrm{E}$, Nov. 5, 1994, pitfall (R. Brandle, SAM N1996/450), 10 . Victoria: 4.8 km E HattahKulkyne National Park, $34^{\circ} 38^{\prime} \mathrm{S}, 142^{\circ} 20^{\prime} \mathrm{E}$, Feb. 6, 1970, Berlese, roadside mallee (R. Taylor, QMB), 1 ㅇ. Western Australia: Boodarie Hill area, c. $15 \mathrm{~km}$ SW Port Hedland, $20^{\circ} 24^{\prime} \mathrm{S}, 118^{\circ} 31^{\prime} \mathrm{E}$, Oct. 11-17, 1994, dry pitfall (G. Harold, J. Dell, WAM 95/ 1070), 1 ; ; Canning Stock Route, Well 33, $22^{\circ} 20^{\prime} \mathrm{S}, 124^{\circ} 45^{\prime} \mathrm{E}$, Aug. 4, 1987 (A. De 

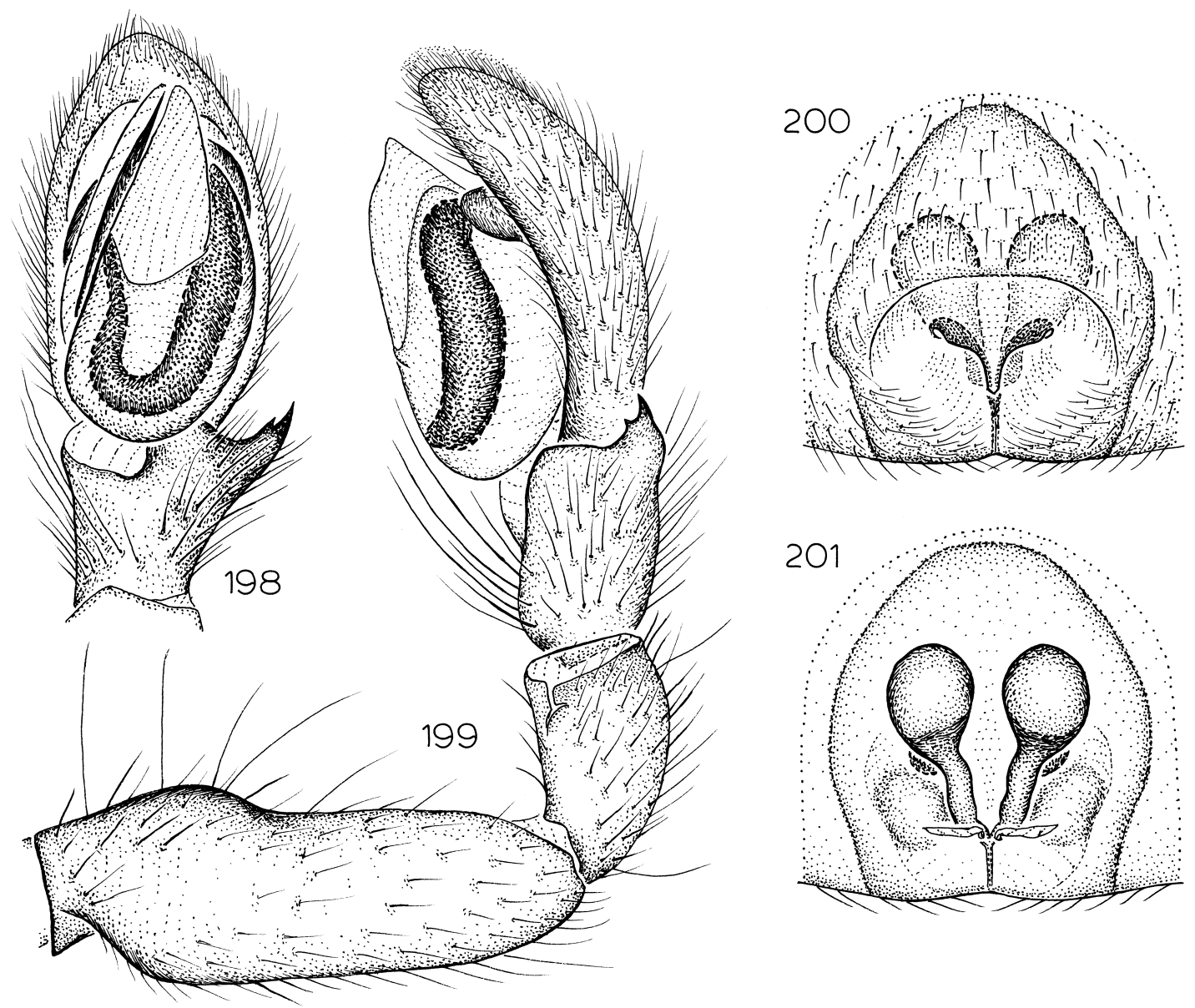

Figs. 198-201. Lampona ooldea, new species. 198. Left male palp, ventral view. 199. Same, retrolateral view. 200. Epigynum, ventral view. 201. Same, dorsal view.

Jong, WAM 95/1071), 1 \%; Drysdale River Station, $15^{\circ} 42^{\prime} \mathrm{S}, 126^{\circ} 23^{\prime} \mathrm{E}$, Mar. 10, 1994, under airfield runway marker (A. Longbottom, WAM 95/1073), $1 \delta^{\text {t }}$; Enderby Island, $20^{\circ} 36^{\prime} \mathrm{S}, 116^{\circ} 29^{\prime} \mathrm{E}$, Aug. 18, 1985 (R. McMillan, WAM 95/1072), $19 ; 2.5$ km S Great Northern Highway on Tanami Track, $18^{\circ} 30^{\prime} \mathrm{S}, 127^{\circ} 30^{\prime} \mathrm{E}$, May 10,1994 , under tire in gravel pit (A. Longbottom, WAM 96/238), 1 ; ; Lake Cohen, $24^{\circ} 27^{\prime} \mathrm{S}, 125^{\circ} 02^{\prime} \mathrm{E}$, July 18 , 1982 running on sparse mulga litter (B. Muir, WAM 95/1069), 1\%; $39 \mathrm{~km} \mathrm{E} \mathrm{Laverton,}$ $28^{\circ} 28^{\prime} \mathrm{S}, 122^{\circ} 50^{\prime} \mathrm{E}$, Nov. 22-24, 1990, pitfall (E. Pianka, WAM 96/239), 1 ; ; Woodstock Station, $21^{\circ} 37^{\prime} \mathrm{S}, 118^{\circ} 57^{\prime} \mathrm{E}$, Oct. 25, 1990, under spinifex (M. Harvey, WAM 95/1068), 1 ㅇ ; Woodstock Station, $21^{\circ} 37^{\prime} \mathrm{S}, 118^{\circ} 59^{\prime} \mathrm{E}$,
Oct. 31, 1990, pitfall (M. Harvey, WAM 95/ 1067), 1 ㅊ․

DisTRIBUTION: Widespread in western and central Australia (map 20).

\section{Lampona ooldea, new species} Figures 198-201; Map 21

TyPE: Female holotype from Ooldea, $30^{\circ} 27^{\prime} \mathrm{S}, 131^{\circ} 50^{\prime} \mathrm{E}$, South Australia (July 1921; J. Rushaw), deposited in NMV (K3431).

ETYMOLOGY: The specific name is a noun in apposition taken from the type locality.

DiAGNOSIS: Males and females have not been taken together and may be mismatched, but both resemble the preceding three spe- 
cies; males differ in having a low, rounded subbasal protuberance on the palpal femur (fig. 199), females in having a much shorter epigynal midpiece (fig. 200).

MALE: Total length 7.6. Coloration as in $L$. cylindrata but with paired white abdominal stripes reduced to spots. Palpal femur with sinuously bulging ventral protuberance; retrolateral tibial apophysis dorsally angular, bearing ventral tooth (fig. 199); tegulum globose, extending to about one-third of tibial length; embolar tip long, abruptly narrowed, prolaterally situated (fig. 198).

FEMALE: Total length 8.0. Coloration as in male. Epigynal midpiece very short, greatly widened anteriorly, lateral margins very short, rounded (fig. 200); anterior portion of spermathecae bulbous (fig. 201).

Other MATERIAl ExAmined: Victoria: McLellands Road, $0.1 \mathrm{~km} \mathrm{~N}$ Rathbones Road, $36^{\circ} 09^{\prime} \mathrm{S}, 145^{\circ} 14^{\prime} \mathrm{E}$, July 18-23, 1994, pitfall (S. Hinkley, P. Lillywhite, G. Milledge, NMVS), 10 .

DisTRIBUTION: Known only from southern South Australia and Victoria (map 21).

\section{Lampona gilles, new species} Figures 202-205; Map 21

TyPE: Female holotype from Lake Gilles Conservation Park, $33^{\circ} 04^{\prime} \mathrm{S}, 136^{\circ} 41^{\prime} \mathrm{E}$, South

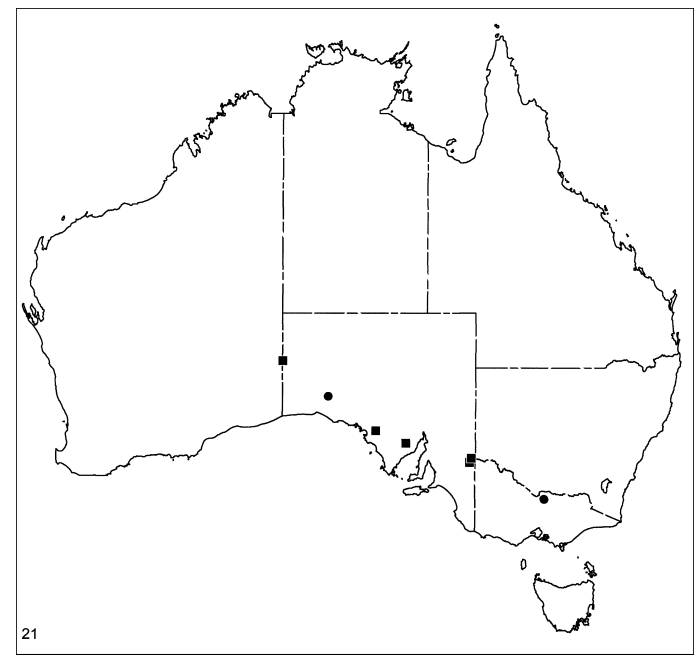

Map 21. Records of Lampona ooldea, new species (circles) and L. gilles, new species (squares).
Australia (Apr. 2, 1987; D. Lee, D. Hirst), deposited in SAM (N1996/216).

ETYMOLOGY: The specific name is a noun in apposition taken from the type locality.

DiagnOSIS: Males resemble those of $L$. quinqueplagiata and L. dwellingup in having a large protrusion on the palpal femur, but have the tip of the retrolateral tibial apophysis apically incised and bifid (fig. 203); females also have an arrow-shaped epigynal midpiece similar to that found in those species, but have longer epigynal margins that are less heavily sclerotized anteriorly, and the epigynal midpiece is greatly elevated (fig. 204).

MALE: Total length 8.1. Abdominal dorsum with four indistinct anterior white spots and very distinct posterior white spot situated just over spinnerets; legs unmarked. Palpal femur with large protrusion at base; retrolateral tibial apophysis short, apically incised, producing bifid tip (fig. 203); tegulum greatly expanded, reaching to about fourfifths of tibial length; embolus long, with strong, gently curved tip (fig. 202).

FEMALE: Total length 10.6. Coloration as in male except that all segments of leg I are deeper red than are legs II-IV. Epigynum with narrow, triangular, elevated midpiece and lightly sclerotized, m-shaped margins (fig. 204); spermathecae bulbous, on long, twisted stalks (fig. 205).

Other Material Examined: South Australia: Conservation Park NE Wirulla, $32^{\circ} 23^{\prime} \mathrm{S}, 134^{\circ} 49^{\prime} \mathrm{E}$, Nov. 25-26, 1995, pitfall (D. Hirst, SAM N1997/147), 10; $14 \mathrm{~km}$ WNW Renmark, 34 $07^{\circ}$ S, $140^{\circ} 37^{\prime}$ E, Sept. 5Oct. 12, 1995, Malaise trap, mallee on dune (K. Pullen, QMB S32455), 1\%; 32 km N Renmark, $33^{\circ} 53^{\prime} \mathrm{S}, 140^{\circ} 44^{\prime} \mathrm{E}$, Nov. 9-22, 1995, pitfall, mallee (A. Lambie, K. Pullen, QMB S32537), 10; E side, Serpentine Lakes, 28 $30^{\prime} \mathrm{S}, 129^{\circ} 01^{\prime} \mathrm{E}$, Apr. 18, 1994 (P. Hudson, SAM N1996/289), 1 ㅇ.

Distribution: Known only from South Australia (map 21).

Lampona hirsti, new species Figures 206-209; Map 22

TYPE: Female holotype taken in pitfall trap $6.8 \mathrm{~km} \mathrm{~S}$ Binda Boudna Hill, 29 27'S, 

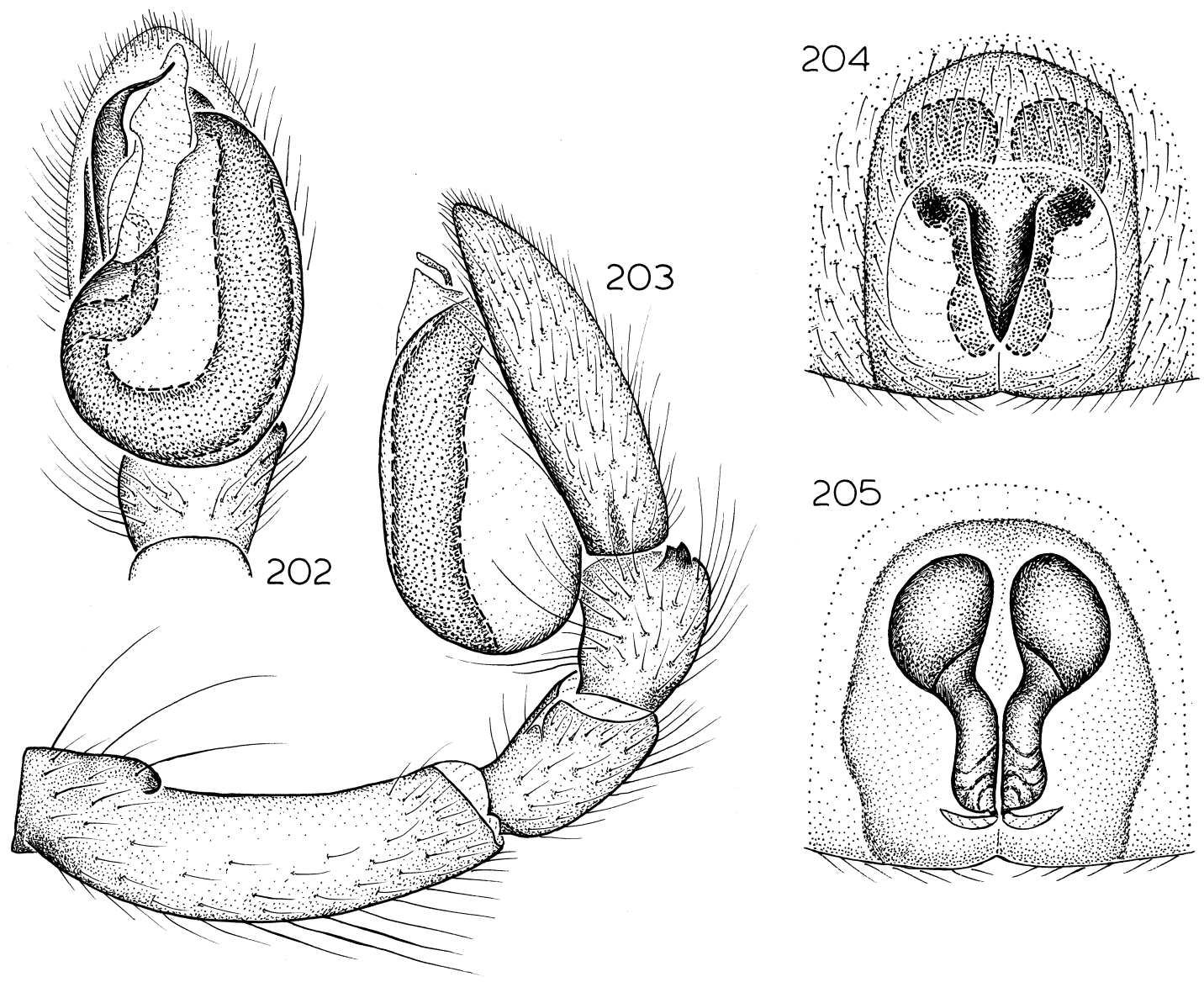

Figs. 202-205. Lampona gilles, new species. 202. Left male palp, ventral view. 203. Same, retrolateral view. 204. Epigynum, ventral view. 205. Same, dorsal view.

$136^{\circ} 13^{\prime}$ E, South Australia (Oct. 7, 1995); deposited in SAM (N1997/144).

ETYMOLOGY: The specific name is a patronym in honor of Mr. David Hirst of the South Australian Museum, who first suggested the pairing of these males and females.

DiAgnosis: Males resemble those of L. eba but have a distinct basal protrusion on the ventral surface of the palpal femur (fig. 207); females also resemble those of $L$. eba but have an anteriorly widened epigynal midpiece and relatively shorter posterior portions of the spermathecae (figs. 208, 209).

MALE: Total length 6.8. Abdominal dorsum as in L. cylindrata; anterior femora darkened distally, anterior tibiae darkened laterally, posterior patellae and tibiae dark- ened laterally. Palpal femur with small, rectangular basal protrusion on ventral surface; retrolateral tibial apophysis with retrolaterally directed protrusion below tip (fig. 207); tegulum moderately expanded, reaching to about one-third of tibial length; embolar base short, wide, tip arched (fig. 206).

FEMALE: Total length 6.9. Coloration as in male. Epigynum with tongue-shaped midpiece extending full length of spermathecae, midpiece elevated anteriorly, level with remainder of cuticle posteriorly (fig. 208); spermathecae bipartite, with large anterior portions (fig. 209).

Other Material Examined: South Australia: Coober Pedy, $29^{\circ} 01^{\prime} \mathrm{S}, 134^{\circ} 45^{\prime} \mathrm{E}$, May 1969 (B. Evans, WAM 84/1223), 10 \%; $5.4 \mathrm{~km}$ E Emerald Springs, $29^{\circ} 22^{\prime} \mathrm{S}$, 

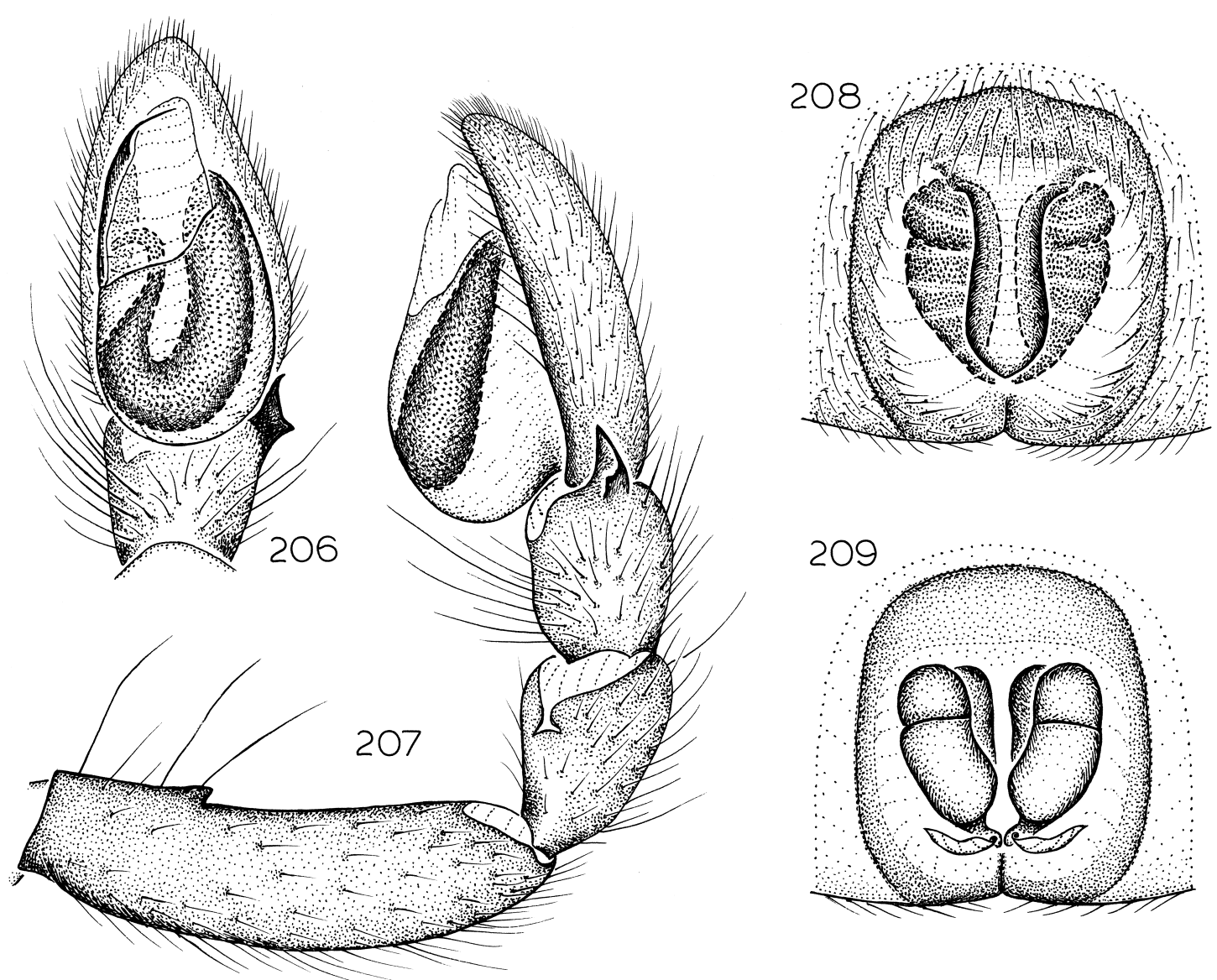

Figs. 206-209. Lampona hirsti, new species. 206. Left male palp, ventral view. 207. Same, retrolateral view. 208. Epigynum, ventral view. 209. Same, dorsal view.

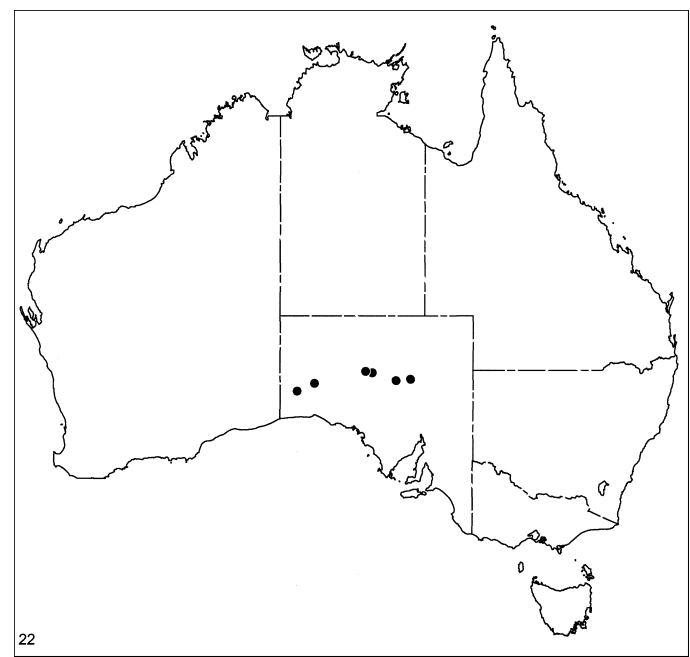

Map 22. Records of Lampona hirsti, new species. $137^{\circ} 07^{\prime} \mathrm{E}$, Apr. 15-19, 1996, pitfall, canegrass/bluebush (D. Hirst, SAM N1997/145, 146), 20; Mabel Creek, $28^{\circ} 56^{\prime} \mathrm{S}, 134^{\circ} 20^{\prime} \mathrm{E}$, Oct. 1984, pitfall (SAM N1996/222), 10; Muckera, Nullarbor Nth., $30^{\circ} 02^{\prime} \mathrm{S}, 130^{\circ} 03^{\prime} \mathrm{E}$, Sept. 1984, pitfall (L. Jansen, SAM N1985/ 119), 19; 47 KM N Muckera Rockhole, $29^{\circ} 36^{\prime} \mathrm{S}, 131^{\circ} 08^{\prime} \mathrm{E}$, Oct. $16-21,1987$, pitfall, interdune, black oak-acacia woodland with cassia (SAM N1996/221), 1 \% ; Muckera Waterhole, $30^{\circ} 02^{\prime} \mathrm{S}, 130^{\circ} 03^{\prime} \mathrm{E}$, Sept. 1984 , pitfall (B. Guerin, SAM N1996/220), 1 ㅇ.

DistRIBUTION: Known only from South Australia (map 22).

Lampona eba, new species Figures 210-213; Map 23

TyPE: Female holotype from Mount Eba Station, $30^{\circ} 11^{\prime} \mathrm{S}, 135^{\circ} 40^{\prime} \mathrm{E}$, South Australia 

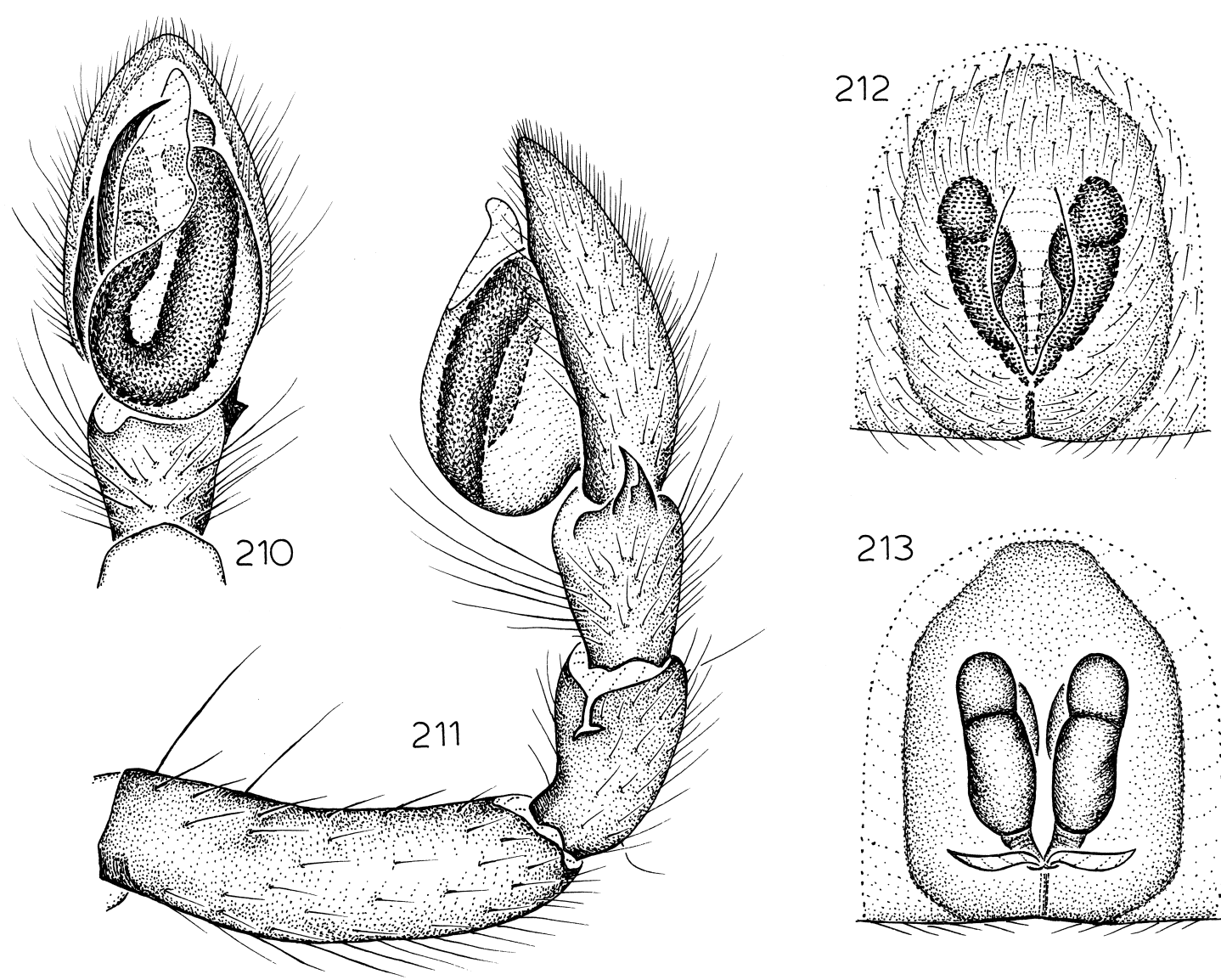

Figs. 210-213. Lampona eba, new species. 210. Left male palp, ventral view. 211. Same, retrolateral view. 212. Epigynum, ventral view. 213. Same, dorsal view.

(May 25-26, 1956; G. Gross), deposited in SAM (N1996/223).

ETYMOLOGY: The specific name is a noun in apposition taken from the type locality.

DiAgNosis: Males resemble those of L. hir$s t i$ in having a lateral protrusion on the retrolateral tibial apophysis, but lack the distinct protuberance on the palpal femur found in that species (fig. 211); female also resemble those of $L$. hirsti but have an anteriorly narrowed epigynal midpiece and relatively longer posterior portions of the spermathecae (fig. 213).

MALE: Total length 4.6. Abdominal dorsum as in L. cylindrata; anterior femora darkened distally, anterior tibiae darkened laterally, posterior patellae and tibiae darkened laterally. Palpal femur without basal protrusion on ventral surface; retrolateral tib-

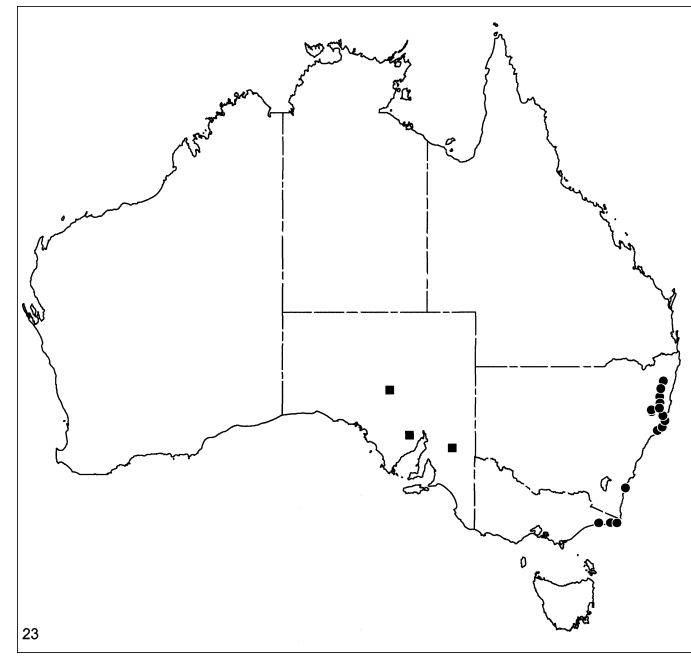

Map 23. Records of Lampona eba, new species (squares) and L. fife, new species (circles). 
ial apophysis with retrolaterally directed protrusion below tip (fig. 211); tegulum slightly expanded, reaching to about one-fifth of tibial length; embolar base short, wide, tip arched (fig. 210).

FEMALE: Total length 8.3. Coloration as in male. Epigynal midpiece not extending as far anteriorly as spermathecae, narrowed anteriorly, otherwise as in L. hirsti (fig. 212); spermathecae bipartite, posterior portions longer than in that species (fig. 213).

Other Material Examined: South Australia: $2 \mathrm{~km}$ ENE The Coffins Dam, $33^{\circ} 24^{\prime} \mathrm{S}$, $139^{\circ} 34^{\prime} \mathrm{E}$, Oct. 21, 1992, pitfall (SAM N1996/ 315), 10; Lake Gilles, $32^{\circ} 41^{\prime} \mathrm{S}, 136^{\circ} 55^{\prime} \mathrm{E}$, Feb. 1993, under plank at lake edge (P. Hudson, SAM N1996/215), 1 ㅇ.

DisTRIBUTION: Known only from South Australia (map 23).

\section{THE FIFE GROUP}

This group comprises the remaining 15 species of the genus, all of which have tripartite spermathecae. Males generally have a small, apically narrowed retrolateral tibial apophysis. The group seems to be confined to eastern Australia, and is most speciose in Queensland.

\section{Lampona fife, new species Figures 214-217; Map 23}

TYPES: Female holotype and male allotype taken in pitfall trap at an elevation of $350 \mathrm{~m}$ at Branch Creek tributary, $250 \mathrm{~m}$ downslope from Fife Fire Trail at point about $2.5 \mathrm{~km}$ NE Fife's Knob, 30 $54^{\prime} \mathrm{S}, 152^{\circ} 23^{\prime} \mathrm{E}$, New South Wales (Feb. 4-Apr. 9, 1993; M. Gray, G. Cassis), deposited in AMS (KS40492).

ETYMOLOGY: The specific name is a noun in apposition taken from the type locality.

DiAGNOSIS: Males share with L. cumberland the presence of numerous stiff setae situated at about two-thirds of the length of the retrolateral tibial apophysis (fig. 215), but the apophysis is smoothly narrowed toward its tip; females have almost circular median epigynal openings and rounded anterior portions of the spermathecae (figs. 216, 217).

MALE: Total length 5.6. Coloration as in $L$. cylindrata, except markings on abdominal dorsum less distinct, tibiae IV with distal dark ring. Palpal femur darkened for about two-thirds its length, ventral surface produced at base into rounded knob bearing long setae; retrolateral tibial apophysis long, narrow, bearing stiff setae at about two-thirds its length (fig. 215); tegulum globose, extending to only about one-third of tibial length but greatly projecting ventrally; conductor extending to prolateral side of embolus (fig. 214).

Female: Total length 8.5. Coloration as in male. Epigynum with relatively wide median septum leading to almost circular, laterally facing openings (fig. 216); spermathecae tripartite, basal portion small, both distal portions large, globose (fig. 217).

Other Material Examined: New South Wales: $11 \mathrm{~km}$ NE Bulahdelah, O'Sullivan's Gap Reserve, $32^{\circ} 25^{\prime} \mathrm{S}, 152^{\circ} 13^{\prime} \mathrm{E}$, June $11-$ Aug. 27, 1982, intercept trap, wet sclerophyll forest (S., J. Peck, AMNH), 30 ; Chaelundi Road, 2.9 km S Big Bull Road, Mount Hyland Nature Reserve, $30^{\circ} 09^{\prime} \mathrm{S}, 152^{\circ} 27^{\prime} \mathrm{E}$, Feb. 4-Apr. 9, 1993, pitfall, elev. $1080 \mathrm{~m}$ (M. Gray, G. Cassis, AMS KS35319), 20; bottom end, Cliffs Trail, $1.3 \mathrm{~km}$ from Oxley Road, Styx River State Forest, 30 33'S, $152^{\circ} 20^{\prime}$ E, Feb. 4-Apr. 9, 1993, pitfall, elev. 1080 m (M. Gray, G. Cassis, AMS KS35452), 1 đ; $^{\text {; }}$ bottom end, Cliffs Trail, 2.8 $\mathrm{km}$ from Oxley Road, Styx River State Forest, $30^{\circ} 33^{\prime} \mathrm{S}, 152^{\circ} 21^{\prime} \mathrm{E}$, Feb. 4-Apr. 9, 1993, pitfall, elev. $1130 \mathrm{~m}$ (M. Gray, G. Cassis, AMS KS35453), 1 ơ; Daisy Patch Fire Trail, $1.9 \mathrm{~km}$ S Enfield Road, Enfield State Forest, $31^{\circ} 20^{\prime} \mathrm{S}, 151^{\circ} 54^{\prime} \mathrm{E}$, Feb. 4-Apr. 9, 1993, pitfall, elev. 1130 m (M. Gray, G. Cassis, AMS KS40489), 1 đ; Dodd's Fire Trail, 2 km from Enfield Road on Scrubby Creek, Enfield State Forest, $31^{\circ} 23^{\prime} \mathrm{S}, 151^{\circ} 52^{\prime} \mathrm{E}$, Feb. 4-Apr. 9, 1993, pitfall, elev. 930 m (M. Gray, G. Cassis, AMS KS40490), 10; Fife's Knob Road, about $4 \mathrm{~km}$ from Fifes Fire Trail, Carrai State Forest, $30^{\circ} 54^{\prime} \mathrm{S}, 152^{\circ} 22^{\prime} \mathrm{E}$, Feb. 4Apr. 9, 1993, pitfall, elev. 740 m (M. Gray, G. Cassis, AMS KS40491), 19 ; Hallidays Point (Black Head), SE Taree, $32^{\circ} 14^{\prime} \mathrm{S}$, $152^{\circ} 32^{\prime} \mathrm{E}$, Jan. 22, 1991, litter on loan, headland type rainforest (G., B. Williams, AMS KS29637), 1 + ; 3-5 km NE Harrington, $31^{\circ} 52^{\prime} \mathrm{S}, 152^{\circ} 42^{\prime} \mathrm{E}$, Aug. 18, 1990, litter on sandy soil, littoral rainforest (G. Williams, AMS KS23422), 2 ; Kerewong State Forest, near Lorne, $31^{\circ} 36^{\prime} \mathrm{S}, 152^{\circ} 34^{\prime} \mathrm{E}$, Nov. 7-Dec. 

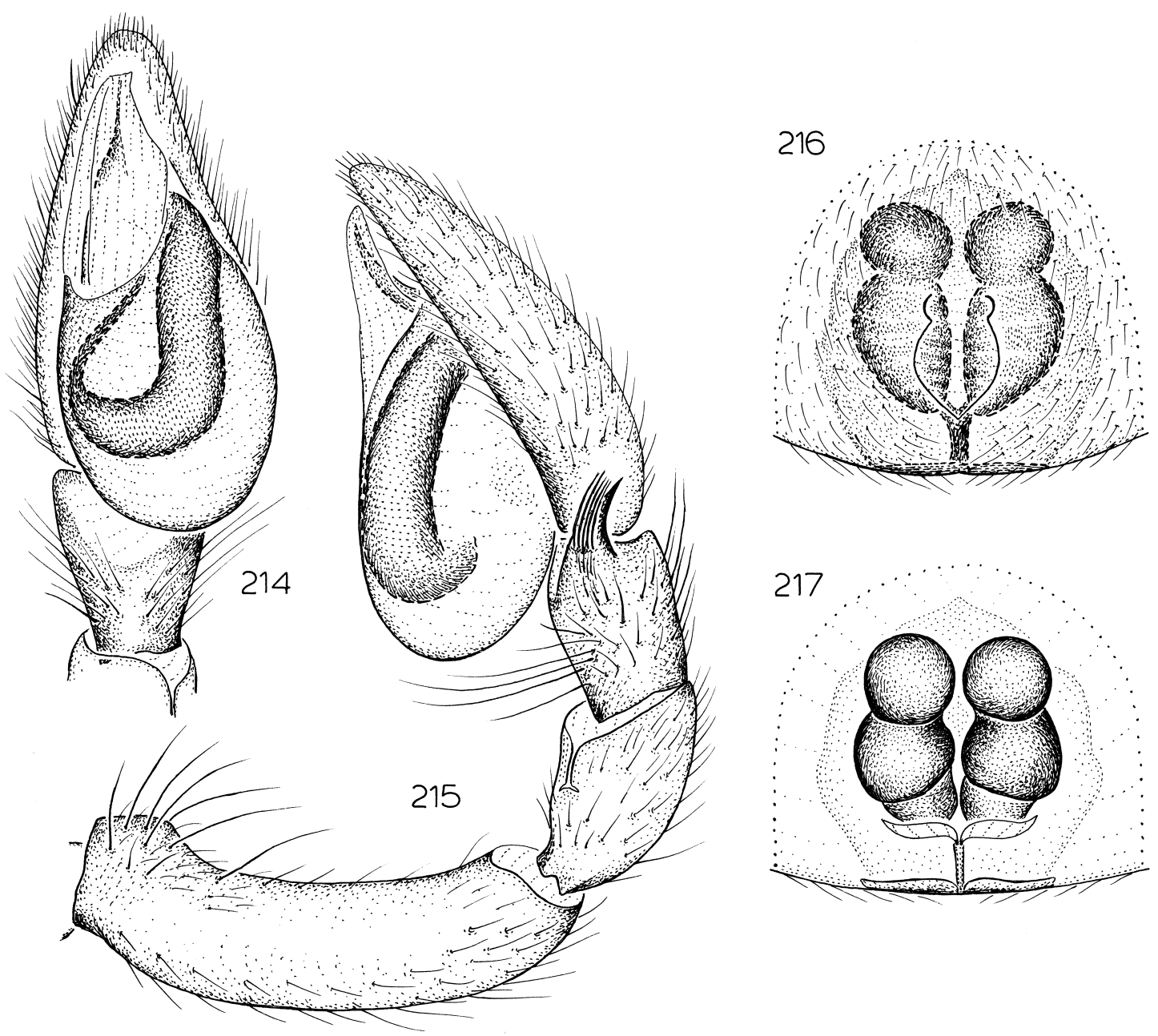

Figs. 214-217. Lampona fife, new species. 214. Left male palp, ventral view. 215. Same, retrolateral view. 216. Epigynum, ventral view. 217. Same, dorsal view.

10, 1978, pitfall, litter (D. Milledge, AMS KS16176), 19 ; Kiola State Forest Drive, 15 $\mathrm{km}$ N Batemans Bay, $35^{\circ} 37^{\prime} \mathrm{S}, 150^{\circ} 16^{\prime} \mathrm{E}$, Nov. 2, 1978 (C. Horseman, AMS KS2271), 19; Mount Tindal Road, Ramornie State Forest, $29^{\circ} 41^{\prime} \mathrm{S}, 152^{\circ} 35^{\prime} \mathrm{E}$, Feb. 4-Apr. 9, 1993, pitfall, elev. 490 m (M. Gray, G. Cassis, AMS KS40484), 10; Mummel Forest Road, $6.1 \mathrm{~km} \mathrm{~N}$ junction with Enfield Road, Enfield State Forest, $31^{\circ} 17^{\prime} \mathrm{S}, 151^{\circ} 51^{\prime} \mathrm{E}$, Feb. 4-Apr. 9, 1993, pitfall, elev. 1330 m (M. Gray, G. Cassis, AMS KS40488), 10 ; Mummel Forest Road, 7.6 km N junction with Enfield Road, Enfield State Forest, $31^{\circ} 17^{\prime} \mathrm{S}$, $151^{\circ} 51^{\prime} \mathrm{E}$, Feb. 4-Apr. 9, 1993, pitfall, elev.
$1340 \mathrm{~m}$ (M. Gray, G. Cassis, AMS KS40487), $20^{\text {; }}$; Oppossum Creek, upstream of Foamy Creek Road, Marengo State Forest, $30^{\circ} 06^{\prime} \mathrm{S}, 152^{\circ} 25^{\prime} \mathrm{E}$, Feb. 4-Apr. 9, 1993, pitfall, elev. $830 \mathrm{~m}$ (M. Gray, G. Cassis, AMS KS35440), 20; Rimau Road, 11.2 km E Cockerawombeeba Road, Mount Boss State Forest, $31^{\circ} 11^{\prime} \mathrm{S}, 152^{\circ} 21^{\prime} \mathrm{E}$, Feb. 4-Apr. 9, 1993, pitfall, elev. 690 m (M. Gray, G. Cassis, AMS KS40485), 10 . Victoria: Alfred National Park, $37^{\circ} 33^{\prime} \mathrm{S}, 149^{\circ} 21^{\prime} \mathrm{E}$, May 21, 1978, Berlese, bark and rotten logs, temperate rain forest, elev. $200 \mathrm{~m}$ (S., J. Peck,

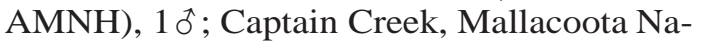
tional Park, $37^{\circ} 34^{\prime} \mathrm{S}, 149^{\circ} 45^{\prime} \mathrm{E}$, May 22, 

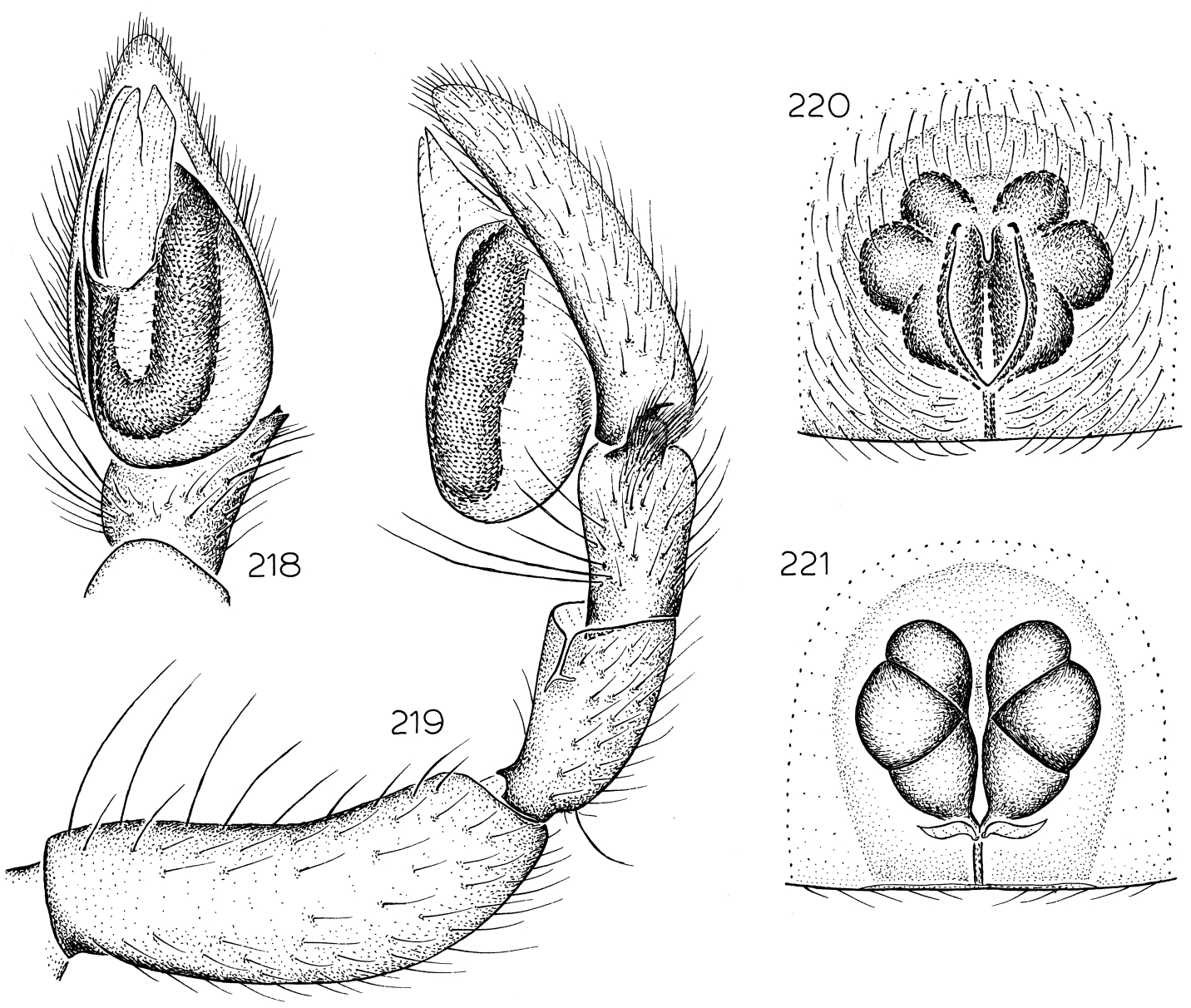

Figs. 218-221. Lampona cumberland, new species. 218. Left male palp, ventral view. 219. Same, retrolateral view. 220. Epigynum, ventral view. 221. Same, dorsal view.

1978, Berlese, eucalypt, banksia litter, bark, fungi, elev. $10 \mathrm{~m}$ (S., J. Peck, AMNH), 1\%; Vaus Experimental Study site 510.08, RichMurrungowar Coupe, $37^{\circ} 34^{\prime} \mathrm{S}, 148^{\circ} 38^{\prime} \mathrm{E}$, Mar. 18-25, 1992, pitfall, midslope (R. Coy, NMV K3525), 10; Vaus Experimental Study site 515.05, Rich-Murrungowar Coupe, $37^{\circ} 34^{\prime} \mathrm{S}, 148^{\circ} 38^{\prime} \mathrm{E}$, May 7-13, 1992, pitfall, gully (R. Coy, NMV K3542), 1 đ.

Distribution: Known only from New South Wales and Victoria (map 23).

\section{Lampona cumberland, new species}

Figures 44, 218-221; Map 24

TYPES: Female holotype and male allotype taken under Eucalyptus regnans bark at Cumberland Falls, $37^{\circ} 34^{\prime} \mathrm{S}, 145^{\circ} 53^{\prime} \mathrm{E}$, Vic- toria (May 27, 1991; M. Harvey, M. Blosfelds), deposited in WAM (95/1006, 1007).

ETYMOLOGY: The specific name is a noun in apposition taken from the type locality.

DiAgnosis: Males of this species resemble those of L. fife in having many setae on the retrolateral tibial apophysis (largely omitted from fig. 219 so that the apophysis shape can be seen) but can be distinguished by the abrupt subdistal narrowing of that apophysis (fig. 219); females can be recognized by the pronounced lateral epigynal margins and triangular median portions of the spermathecae (figs. 220, 221).

MALE: Total length 6.9. Dorsum of abdomen with one pair of anteromedian and three pairs of posteromedian light chevrons (as in 
fig. 44); posterior femora, tibiae, and metatarsi with distal dark rings, tibiae with slightly darkened proximal ring as well. Palpal femur darkened, sinuous, but without distinct ventral protrusion; retrolateral tibial apophysis with wide rectangular base and hooklike tip, dorsally with stiff setae (fig. 219); tegulum globose, extending to about one-half of tibial length; conductor extending to prolateral side of embolus (fig. 218).

FEMALE: Total length 7.9. Coloration as in male, except anteromedian abdominal light chevron larger. Epigynum with distinct lateral margins, with median depression between anterior ends of lateral margins (fig. 220); spermathecae tripartite, median part largest (fig. 221).

Other MATERial ExAmined: Victoria: 0.7 km N Acheron Gap, 7 km NE Mount Donna Buang, $37^{\circ} 40^{\prime} \mathrm{S}, 145^{\circ} 44^{\prime} \mathrm{E}$, Oct. 26-Dec. 28, 1995, pitfall, eucalypt forest (G. Milledge, NMV K4660), 19 ; The Big Culvert, $2.5 \mathrm{~km}$ ENE Mount Observation, $37^{\circ} 34^{\prime} \mathrm{S}, 145^{\circ} 52^{\prime} \mathrm{E}$, Feb. 19, 1996, beech forest (G. Milledge,

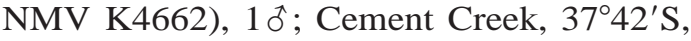
$145^{\circ} 44^{\prime} \mathrm{E}$, Sept. 8, 1990, under eucalypt bark (M. Harvey, M. Blosfelds, WAM 95/1011), 10 ; Cobb Hill, 14 km SE Bonang, Goonmirk Range, $37^{\circ} 12^{\prime} \mathrm{S}, 148^{\circ} 45^{\prime} \mathrm{E}$, Nov. 24, 1985 , Berlese, litter under tree ferns (J., N. Lawrence, QMB), 10; near Churchill, $38^{\circ} 18^{\prime} \mathrm{S}, 146^{\circ} 27^{\prime} \mathrm{E}$, Dec. 23, 1992 (R. de Souza-Daw, SAM N1996/219), 1 \%; Cumberland Falls, $37^{\circ} 34^{\prime} \mathrm{S}, 145^{\circ} 53^{\prime} \mathrm{E}$, May 27, 1991, under Eucalyptus regnans bark (M. Harvey, M. Blosfelds, WAM 95/1008), 1 ; Jeeralang West Road, $0.1 \mathrm{~km}$ N Binns Hill Junction, Strzelecki Ranges, $38^{\circ} 27^{\prime} \mathrm{S}$, $146^{\circ} 29^{\prime} \mathrm{E}$, Mar. 4, 1996, eucalypt forest (G. Milledge, NMV K4663), 1 ; Mount Macedon, $37^{\circ} 25^{\prime} \mathrm{S}, 144^{\circ} 35^{\prime} \mathrm{E}$, Nov. 30, 1996, elev. 700 m (N. Platnick, R. Raven, V. Ovtsharenko, K. Catley, AMNH), $3 \sigma^{\text {; }}$, Mount Saint Leonard, $37^{\circ} 34^{\prime} \mathrm{S}, 145^{\circ} 32^{\prime} \mathrm{E}$, Apr. 6, 1991, under eucalypt bark (M. Harvey, M. Blosfelds, WAM 95/1009, 1010), $10^{\star}, 1$ \% ; Sherbrook Forest, $37^{\circ} 53^{\prime} \mathrm{S}, 145^{\circ} 21^{\prime} \mathrm{E}$, Sept. 1974 (NMV K3655), $1 \%$; Warburton, $37^{\circ} 45^{\prime} \mathrm{S}$, $145^{\circ} 41^{\prime}$ E, Jan. 14, 1968 (R. Kelly, NMV K3417), 1 ㅇ․

DisTRIBUTION: Known only from Victoria (map 24).
Lampona bunya, new species

Figures 222-225; Map 24

TYPES: Male holotype and female allotype taken under $\log$ at Dandabah, Bunya Mountains National Park, 26 $6^{\circ} 3^{\prime} \mathrm{S}, 151^{\circ} 37^{\prime} \mathrm{E}$, Queensland (Feb. 29, 1976), deposited in QMB (S28076).

ETYMOLOGY: The specific name is a noun in apposition taken from the type locality.

DiAgNOSIS: This species seems closest to L. cudgen, based on the sharply angled retrolateral tibial apophysis of males and the elongated spermathecae of females; males of $L$. bunya can be distinguished by the longer palpal femur, the shorter retrolateral tibial apophysis, and the retrolaterally directed embolar tip (figs. 222, 223), females by the relatively large anterior portion of the spermathecae (fig. 225).

MALE: Total length 8.0. Abdominal dorsum with pattern indistinct, posterior white stripes clearer than anterior; posterior tibiae with slightly darkened proximal and distal dark bands. Palpal femur long, without distinct ventral protrusion; retrolateral tibial apophysis acutely bent at about half its length, distal portion relatively short (fig. 223); tegulum elongate, reaching to about one-third of tibial length; embolus recessed

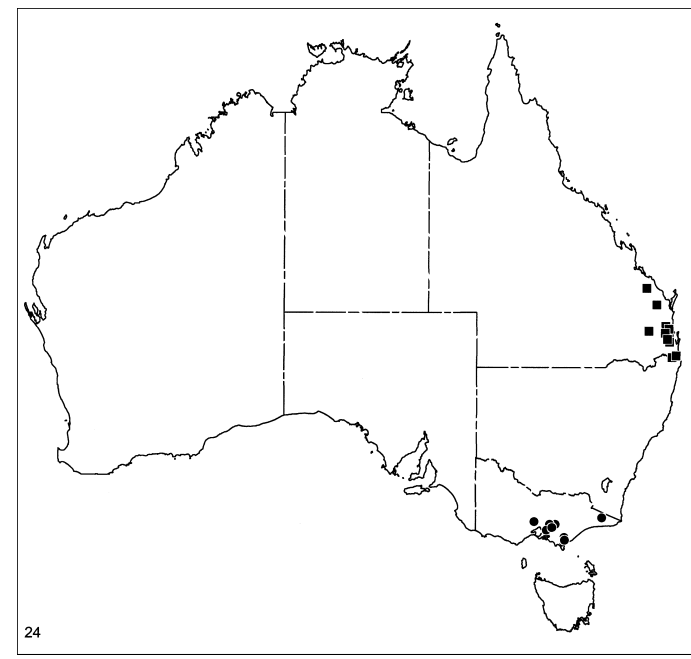

Map 24. Records of Lampona cumberland, new species (circles) and L. bunya, new species (squares). 

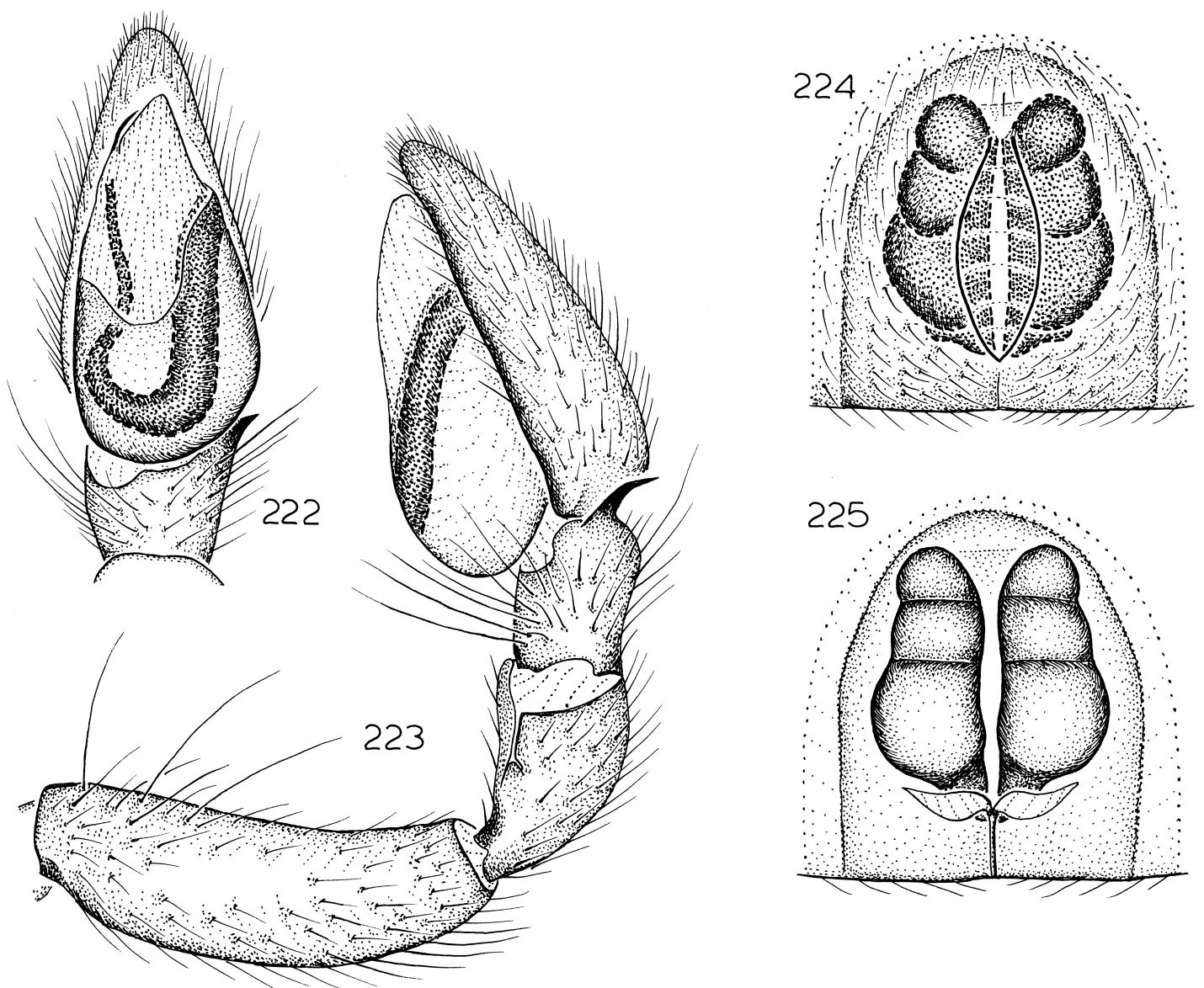

Figs. 222-225. Lampona bunya, new species. 222. Left male palp, ventral view. 223. Same, retrolateral view. 224. Epigynum, ventral view. 225. Same, dorsal view.

behind striated conductor, with long, retrolaterally directed tip (fig. 222).

FEMALE: Total length 8.5. Coloration as in male. Epigynal midpiece long, leading to anteriorly situated, closely spaced openings (fig. 224); spermathecae tripartite, posterior portion largest, anterior portion almost as large as median portion (fig. 225).

OTHER MATERIAL ExAMINED: Queensland: Booloumba Creek, Conondale Range, $26^{\circ} 37^{\prime} \mathrm{S}, 152^{\circ} 41^{\prime} \mathrm{E}$, Apr. 13-18, 1976, leaf litter (R. Raven, QMB S30172), 2 \% ; Brookfield, $27^{\circ} 29^{\prime} \mathrm{S}, 152^{\circ} 54^{\prime} \mathrm{E}$, Nov. 9, 1975-Feb. 27, 1976, rainforest pitfall, elev. $110 \mathrm{~m}$ (G., S. Monteith, QMB S28082), 19 ; Brookfield, $27^{\circ} 29^{\prime} \mathrm{S}, 152^{\circ} 55^{\prime} \mathrm{E}$, Nov. 27, 1975-May 20, 1976, rainforest (G., S. Monteith, QMB
S34400), 19; Bulburin Forestry Nursery, NW Bundaberg, $24^{\circ} 31^{\prime} \mathrm{S}, 151^{\circ} 29^{\prime} \mathrm{E}$, Mar. 1975, on ground, elev. $580 \mathrm{~m}$ (M. Gray, C. Horseman, AMS KS6785), 20; Bulburin State Forest, Mar. 25-28, 1977 (R. Raven, V. Davies, QMB S28093), 1 đ; Bunya Mountains National Park, 26 $53^{\prime} \mathrm{S}, 151^{\circ} 37^{\prime} \mathrm{E}$, Feb. 28, 1976, litter (R. Raven, V. Davies, QMB S28078), 1 o $^{\text {; }}$ Gold Creek, Brookfield, $27^{\circ} 29^{\prime} \mathrm{S}, 152^{\circ} 54^{\prime} \mathrm{E}$, July 3, 1980, eating mygalomorph in its tube (R. Raven, QMB S28089), 19 ; Lamington Plateau, $28^{\circ} 19^{\prime} \mathrm{S}$, $153^{\circ} 04^{\prime} \mathrm{E}$, Apr. 2, 1975, rainforest (R. Raven, QMB S34417), 10; Mary Cairncross Park, 7 km SE Maleny, $26^{\circ} 46^{\prime} \mathrm{S}, 152^{\circ} 52^{\prime} \mathrm{E}$, June 18 Aug. 15, 1982, rainforest intercept trap, elev. c. 900 m (S., J. Peck, AMNH), 10, Jan. 8- 

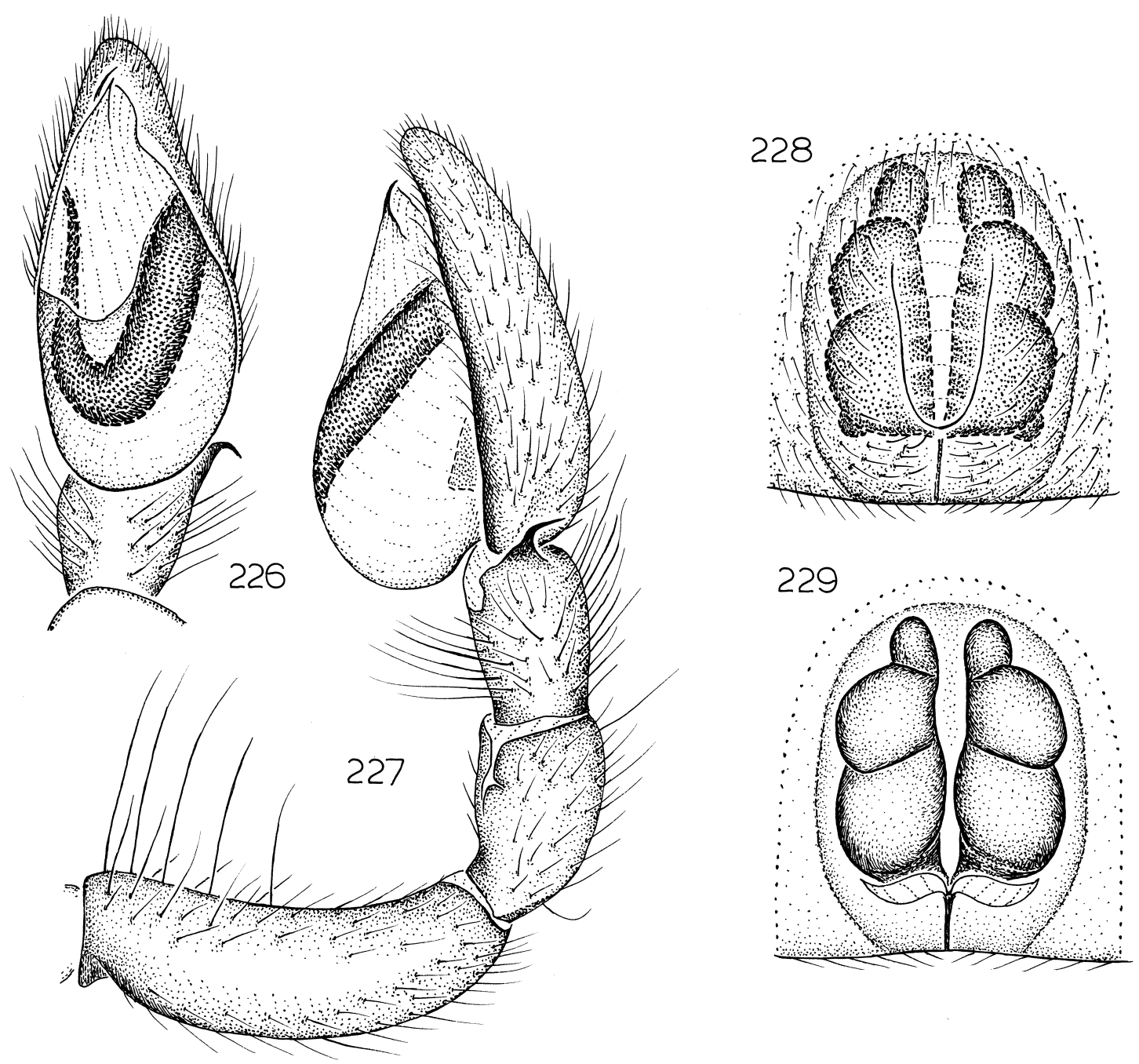

Figs. 226-229. Lampona cudgen, new species. 226. Left male palp, ventral view. 227. Same, retrolateral view. 228. Epigynum, ventral view. 229. Same, dorsal view.

Mar. 3, 1992, rainforest intercept trap, elev. 500 m (D. Cook, QMB S25015), 1 \% ; Mount Archer, Kilcoy, $26^{\circ} 59^{\prime} \mathrm{S}, 152^{\circ} 38^{\prime} \mathrm{E}$, Apr. 29, 1985 (J. Gallon, QMB S28099), 1 ơ $^{\text {; Mount }}$ Glorious State Forest, $27^{\circ} 20^{\prime} \mathrm{S}, 152^{\circ} 46^{\prime} \mathrm{E}$, Feb. 5, 1988, subtropical rainforest, Argyrodendron actinophyllum (Y. Basset, QMB S5171), 10; Mount Goonaneman, near Childers, $25^{\circ} 26^{\prime} \mathrm{S}, 152^{\circ} 07^{\prime} \mathrm{E}$, Nov. 3-7, 1980, litter, elev. 670 m (V. Davies, R. Raven, QMB S28092), 3o, 2 ; ; Tallebudgera Valley, falls area, $28^{\circ} 14^{\prime} \mathrm{S}, 153^{\circ} 19^{\prime} \mathrm{E}$, Nov. 11, 1997-Jan. 28, 1998, pitfall (D. Cook, QMB S42482), 20 .
DISTRIBUTION: Known only from southeastern Queensland (map 24).

\section{Lampona cudgen, new species}

Figures 226-229; Map 25

TYPE: Female holotype taken under melaleuca bark at Cudgen, $28^{\circ} 16^{\prime} \mathrm{S}, 153^{\circ} 33^{\prime} \mathrm{E}$, New South Wales (Nov. 20, 1978; R. Raven), deposited in QMB (S28103).

ETYMOLOGY: The specific name is a noun in apposition taken from the type locality.

Diagnosis: This species seems closest to L. bunya (see above); males of L. cudgen can 
be distinguished by the shorter palpal femur, the longer retrolateral tibial apophysis, and the more distally directed embolar tip (figs. $226,227)$, females by the relatively small anterior portion of the spermathecae (fig. 229).

MALE: Total length 5.2. Abdominal dorsum with anterior and median white stripes fused at sides, posterior white spot large, including small dark markings; posterior femora with distal, posterior tibiae with proximal and distal dark rings. Palpal femur short, without distinct ventral protrusion; retrolateral tibial apophysis acutely bent at about half its length, distal portion relatively long (fig. 227); tegulum elongate, reaching to about one-half of tibial length; most of embolus not recessed behind conductor, with long, almost distally directed tip (fig. 226).

Female: Total length 7.2. Coloration as in male except leg markings less pronounced. Epigynal midpiece long, leading to anteriorly situated, widely spaced openings (fig. 228); spermathecae tripartite, posterior portion largest, anterior portion smaller than median portion (fig. 229).

Other Material Examined: New South Wales: Brown Mountain Flora Reserve, 0.5 km SSW Cochrane Dam, 36 35' S, 149 $27^{\circ} \mathrm{E}$, Feb. 8, 1993, pyrethrum fogging, tree, cool temperate rainforest, elev. $940 \mathrm{~m}$ (A. Newton, M. Thayer, FMNH), $1 \%$; Cudgen, $28^{\circ} 16^{\prime} \mathrm{S}, 153^{\circ} 33^{\prime} \mathrm{E}$, Nov. 20, 1978, under

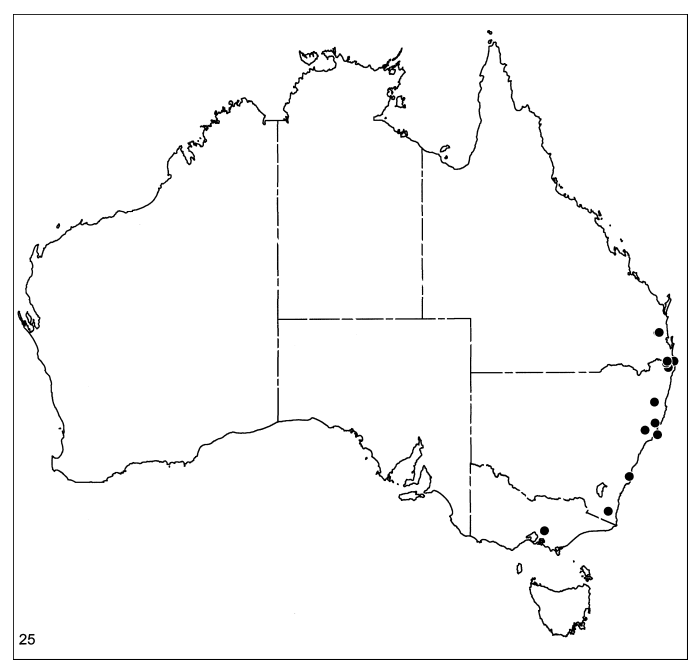

Map 25. Records of Lampona cudgen, new species. melaleuca bark (R. Raven, QMB S28103), 1 9 ; off Cunnawarra Trail c. 2 km N Cunnawarra Creek, Styx River State Forest, $30^{\circ} 32^{\prime} \mathrm{S}, 152^{\circ} 20^{\prime} \mathrm{E}$, Feb. 4-Apr. 9, 1993, pitfall, elev. 1070 m (M. Gray, G. Cassis, AMS KS35455), 1 đ; Jamberoo Mountains, $34^{\circ} 39^{\prime} \mathrm{S}, 150^{\circ} 46^{\prime} \mathrm{E}$, Aug. 27, 1966 (R. Mascord, AMS KS31837), 1 ; Nimbin Rocks, near Nimbin, $28^{\circ} 37^{\prime} \mathrm{S}, 153^{\circ} 12^{\prime} \mathrm{E}$, Nov. 20, 1983 (D. Rentz, M. Harvey, QMB), 1 đo; Pacific Palms, $32^{\circ} 21^{\prime}$ S, $152^{\circ} 31^{\prime}$ E, May 9, 1991 (C. Peterson, AMS KS55269), 1\%; $100 \mathrm{~m}$ $\mathrm{N}$ trig tower, Berrico Road, Chichester State Forest, $32^{\circ} 06^{\prime} \mathrm{S}, 151^{\circ} 45^{\prime} \mathrm{E}$, Feb. 4-Apr. 9, 1993, pitfall, elev. 940 m (M. Gray, G. Cassis, AMS KS40482), $10^{\widehat{*}}$; Wiangaree State Forest, $28^{\circ} 22^{\prime} \mathrm{S}, 153^{\circ} 05^{\prime} \mathrm{E}$, Feb. 10-12, 1983, elev. $1050 \mathrm{~m}$ (T. Weir, A. Calder, QMB), 10; Wingham Brush, $31^{\circ} 42^{\prime} \mathrm{S}$, $152^{\circ} 22^{\prime} \mathrm{E}$, Oct. 12, 1993 (J. Noble, AMS KS55263), 10 . Queensland: Binna Burra, Lamington Plateau, $28^{\circ} 16^{\prime} \mathrm{S}, 153^{\circ} 08^{\prime} \mathrm{E}$, Mar. 27-30, 1976, night collecting (R. Raven, V. Davies, QMB S28088), 1 đ̊; Lamington Plateau, $28^{\circ} 16^{\prime} \mathrm{S}, 153^{\circ} 08^{\prime} \mathrm{E}$, Apr. 2, 1975 (R. Raven, QMB S28097), 10َ; Sunday Creek Road, junction with Gigher Creek Road, Kenilworth State Forest, $26^{\circ} 42^{\prime} \mathrm{S}, 152^{\circ} 33^{\prime} \mathrm{E}$, May 7, 1998, wet sclerophyll forest (G. Milledge, AMS KS55270), 1 ; ; Sunday Creek Road, 9.8 km W Charlie Moreland Park, Kenilworth State Forest, $26^{\circ} 40^{\prime} \mathrm{S}, 152^{\circ} 37^{\prime} \mathrm{E}$, May 7, 1998, wet sclerophyll forest (G. Milledge, AMS KS55271), 1 \%. Victoria: Watts River, Healesville, $37^{\circ} 39^{\prime} \mathrm{S}, 145^{\circ} 32^{\prime} \mathrm{E}$, Sept. 4, 1974, under eucalypt bark (QMB S26764), 1 ㅇ.

Distribution: Southeastern Queensland south to Victoria (map 25).

\section{Lampona superbus, new species}

Figures 230-233; Map 26

TYPE: Female holotype taken at night in rainforest at an elevation of $850 \mathrm{~m}$ on Teviot Brook Track, Mount Superbus, $28^{\circ} 14^{\prime} \mathrm{S}$, $152^{\circ} 29^{\prime}$ E, Queensland (Mar. 8, 1990; T. Churchill), deposited in QMB (S15902).

ETYMology: The specific name is a noun in apposition taken from the type locality.

DiAgnosis: Males resemble those of $L$. bunya and L. cudgen but have a larger tip on 

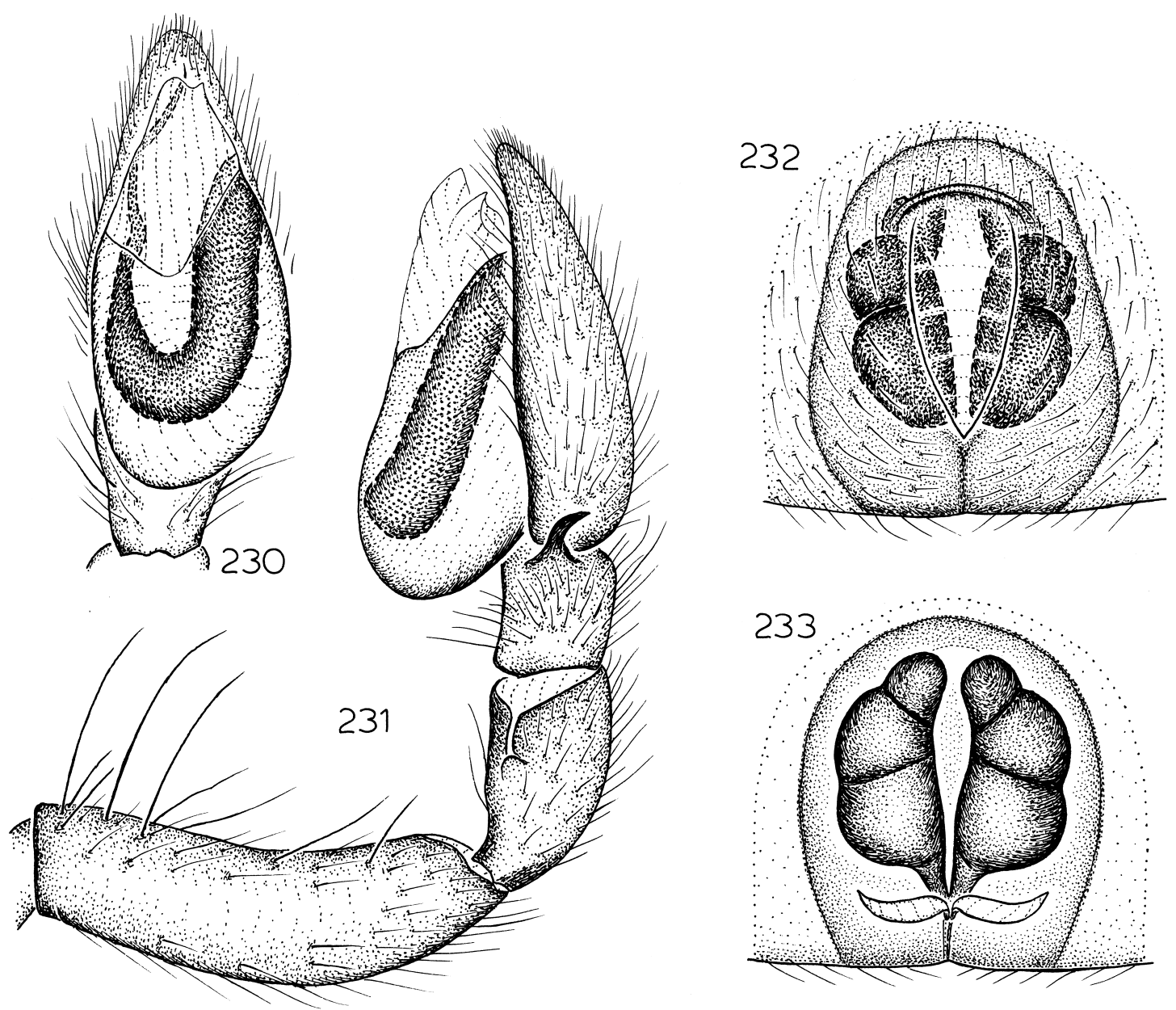

Figs. 230-233. Lampona superbus, new species. 230. Left male palp, ventral view. 231. Same, retrolateral view. 232. Epigynum, ventral view. 233. Same, dorsal view.

the retrolateral tibial apophysis and a retrolateral protrusion on the membranous conductor (figs. 230, 231); females also resemble those of L. bunya and L. cudgen but can easily be recognized by the arched anterior epigynal margin (fig. 232).

MALE: Total length 5.3. Abdominal dorsum with anterior and median paired white spots fused into w-shaped pattern; anterior femora darkened, posterior femora with subdistal, tibiae with proximal and distal dark rings. Palpal femur darkened throughout its length but otherwise unmodified; retrolateral tibial apophysis abruptly bent, transverse portion relatively large (fig. 231); both palps of single available specimen expanded, but tegulum probably reaching to at least onethird of tibial length; embolus abruptly narrowed prolaterally below tip, retrolaterally at tip, membranous conductor with distinct retrolateral protrusion (fig. 230).

FEMALE: Total length 9.5. Coloration as in male. Epigynum elongate, with distinct, arched anterior margin (fig. 232); spermathecae tripartite, anterior portions small, rounded (fig. 233).

Other MAterial Examined: Queensland: Little Yabba Creek, $26^{\circ} 37^{\prime} \mathrm{S}, 152^{\circ} 41^{\prime} \mathrm{E}$, Mar. 2-Apr. 12, 1992, pitfall, rainforest, elev. 150 m (D. Cook, QMB S30322), 10َ; Swimming Pool Track, near Binaburra Lodge, Lamington National Park, $28^{\circ} 11^{\prime} \mathrm{S}, 153^{\circ} 11^{\prime} \mathrm{E}$, July 1 , 
1986 (M. Harvey, P. Vaughan, WAM 95/ 1109), 1 우.

DisTRIBUTION: Known only from southeastern Queensland (map 26).

\section{Lampona gosford, new species}

Figures 234-237; Map 26

TYPE: Female holotype from Gosford, $33^{\circ} 26^{\prime} \mathrm{S}, 151^{\circ} 20^{\prime} \mathrm{E}$, New South Wales (Oct. 12, 1977; R. Mascord), deposited in AMS (KS31932).

ETYMOLOGY: The specific name is a noun in apposition taken from the type locality.

DiAGNOSIS: The tentatively associated males resemble those of $L$. fife in having a long, sinuous palpal femur and a triangular, setose retrolateral tibial apophysis, but differ in having that apophysis and the embolar tip shorter (figs. 234, 235). Females can easily be recognized by the elongate epigynum with a distinct anterior margin and club-shaped spermathecae (figs. 236, 237).

MALE: Total length 7.6. Abdominal dorsum with median pair of white stripes fused medially into w-shaped markings; basal portion of anterior femora greatly darkened. Palpal femur long, sinuous, darkened; retrolateral tibial apophysis triangular, setose, relatively small (fig. 235); tegulum globose, extending to half of tibial length; conductor not

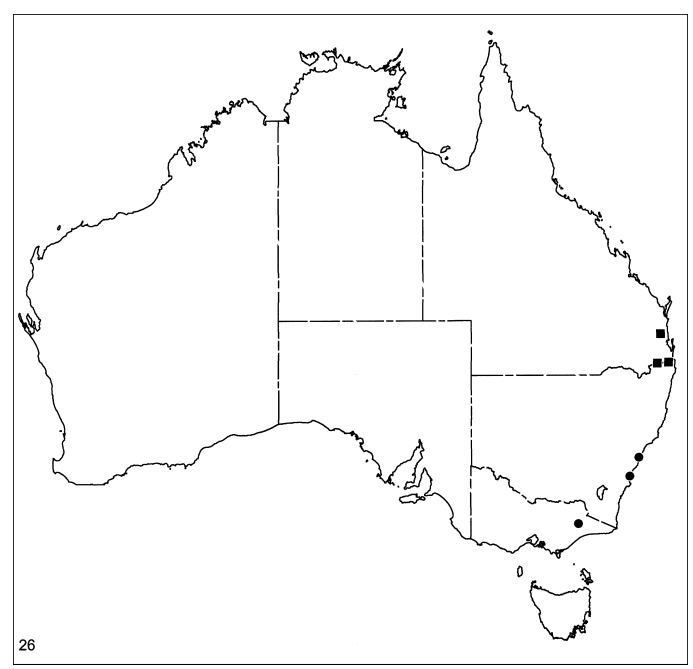

Map 26. Records of Lampona superbus, new species (squares) and L. gosford, new species (circles). quite covering prolateral side of embolus (fig. 234).

FEMALE: Total length 11.5. Abdominal dorsum with median pair of white stripes not quite fused medially; anterior femora completely darkened. Epigynum long, with distinct anterior margin, openings at about onethird of length (fig. 236); spermathecae tripartite, club-shaped (fig. 237).

Other Material Examined: New South Wales: Wongawilli, $34^{\circ} 29^{\prime} \mathrm{S}, 150^{\circ} 46^{\prime} \mathrm{E}$, Aug. 13, 1966 (R. Mascord, AMS KS31933), 3 ‥ Victoria: Mitta Mitta River, $5 \mathrm{~km}$ N Omeo, $37^{\circ} 06^{\prime} \mathrm{S}, 147^{\circ} 36^{\prime} \mathrm{E}$, Feb. 28, 1993 (G. Milledge, NMV K3671), 1 స.

Distribution: Known only from New South Wales and Victoria (map 26).

\section{Lampona kirrama, new species}

Figures 238-241; Map 27

TYPE: Female holotype taken under a long in Kirrama Forest, $18^{\circ} 09^{\prime} \mathrm{S}, 145^{\circ} 37^{\prime} \mathrm{E}$, Queensland (1981), deposited in QMB (S34407).

ETYMology: The specific name is a noun in apposition taken from the type locality.

DIAGNOSIS: Males have a basally expanded palpal femur and a sinuous retrolateral tibial apophysis with a tip directed at about a $30^{\circ}$ angle (fig. 239); females have a tongueshaped epigynal midpiece with laterally directed openings situated at about one-third the length of the spermathecae (fig. 240).

MALE: Total length 5.2. Abdominal dorsum with anterior pair of longitudinal white stripes connected to median transverse white band, band with posterolateral white extensions almost reaching transverse posterior white band; femora with distal, tibiae with proximal and distal dark rings. Palpal femur basally expanded, especially along dorsal edge; retrolateral tibial apophysis sinuous, with tip directly distodorsally (fig. 239); tegulum enlarged, extending to about one-third length of palpal tibia; embolar base truncated, tip directed retrolaterally (fig. 238).

Female: Total length 6.0. Coloration as in male. Epigynum with tongue-shaped midpiece, openings directed laterally, situated near anterior edge of median portion of spermathecae (fig. 240); spermathecae tripartite, 

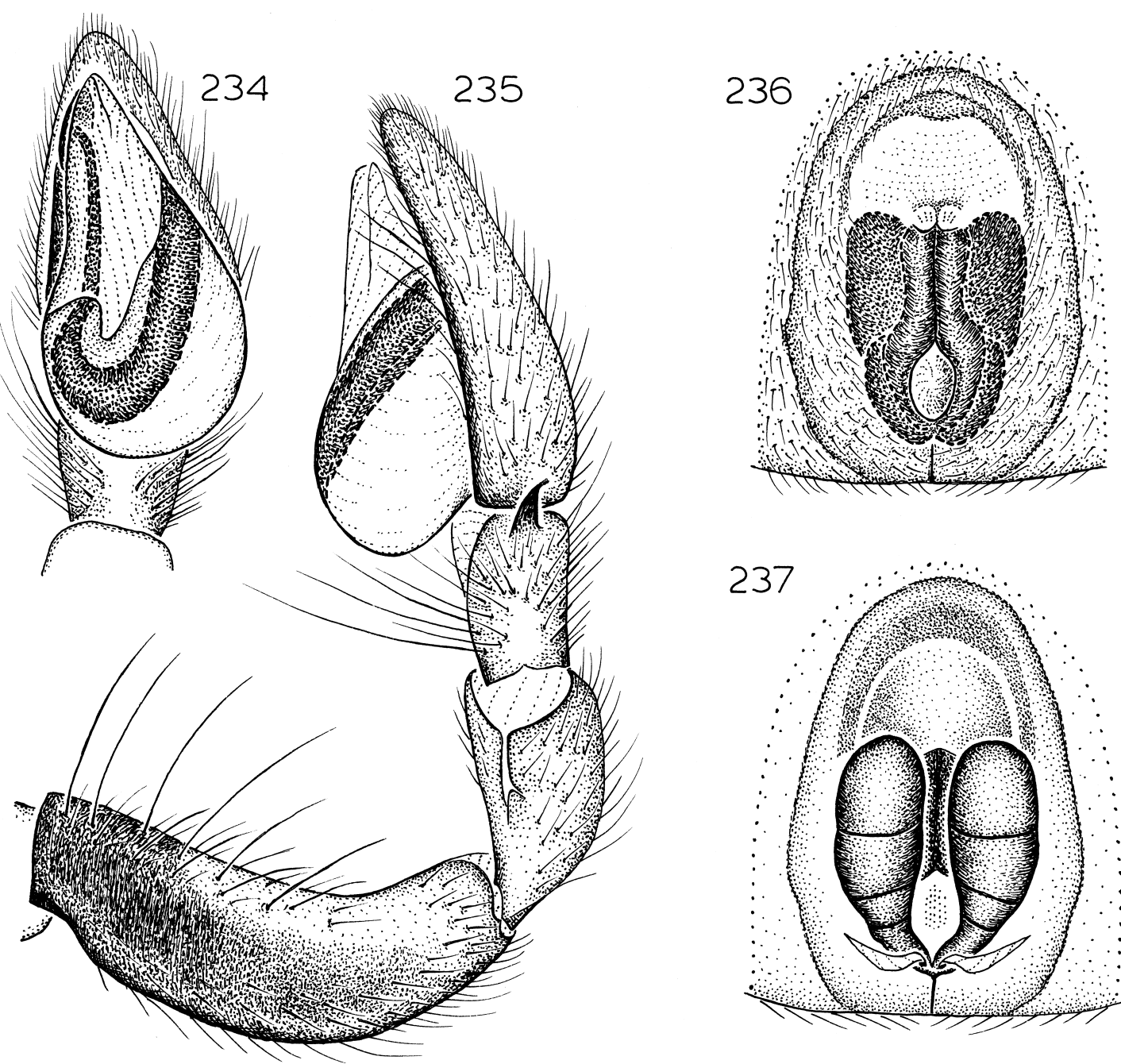

Figs. 234-237. Lampona gosford, new species. 234. Left male palp, ventral view. 235. Same, retrolateral view. 236. Epigynum, ventral view. 237. Same, dorsal view.

posterior portion longest, median portion widest, anterior portion rounded (fig. 241).

Other MATERIAl ExAmined: Queensland: Cable tower 3, Bellenden Ker Range, $17^{\circ} 16^{\prime} \mathrm{S}, 145^{\circ} 51^{\prime} \mathrm{E}$, Oct. $17-24,1981$, pyrethrum knockdown, tree trunks, elev. $1054 \mathrm{~m}$ (QMB S26250), 19; Cableway base station, Bellenden Ker Range, $17^{\circ} 16^{\prime} \mathrm{S}, 145^{\circ} 54^{\prime} \mathrm{E}$, Oct. 25-31, 1981 (QMB S27668), 1 \% ; Crystal Caverns, June 10, 1987, nest on rock ledge (FSCA), $10^{\text {t }}$; Edge Hill, Botanic Garden, Cairns, $16^{\circ} 55^{\prime} \mathrm{S}, 145^{\circ} 46^{\prime} \mathrm{E}$, beaten from bushes, rainforest, Aug. 9, 1968 (J. Balogh, HNHM), 2q; Freshwater Creek, Cairns, $16^{\circ} 55^{\prime} \mathrm{S}, 145^{\circ} 46^{\prime} \mathrm{E}$, Aug. 11, 1968, beating in eucalypt forest (J. Balogh, HNHM), $10^{\text {t}}$; Gayundah Creek, Hinchinbrook Island, $18^{\circ} 22^{\prime} \mathrm{S}, 146^{\circ} 13^{\prime} \mathrm{E}$, Nov. 7-15, 1984 (D. Cook, G. Thompson, QMB S34285), 10; Kirrama State Forest, $32 \mathrm{~km}$ NW Cardwell, $18^{\circ} 16^{\prime} \mathrm{S}, 146^{\circ} 02^{\prime} \mathrm{E}$, June 23-Aug. 8, 1982, intercept trap, rainforest, elev. $800 \mathrm{~m}$ (S., J. Peck, AMNH), $1 \delta^{\circ}$; Kuranda, $16^{\circ} 30^{\prime} \mathrm{S}$, $145^{\circ} 30^{\prime} \mathrm{E}$ (T. Henry, K. Henderson, S. Weller, USNM), $10^{\circ}$; Kuranda, $16^{\circ} 49^{\prime} \mathrm{S}, 145^{\circ} 39^{\prime} \mathrm{E}$, Aug. 17, 1969 (N. Coleman, OMD), 1 \% Kuranda Road, $16^{\circ} 49^{\prime} \mathrm{S}, 145^{\circ} 39^{\prime} \mathrm{E}$, May 13 , 1987, in rolled leaf in pisaurid (Inola sp.) 

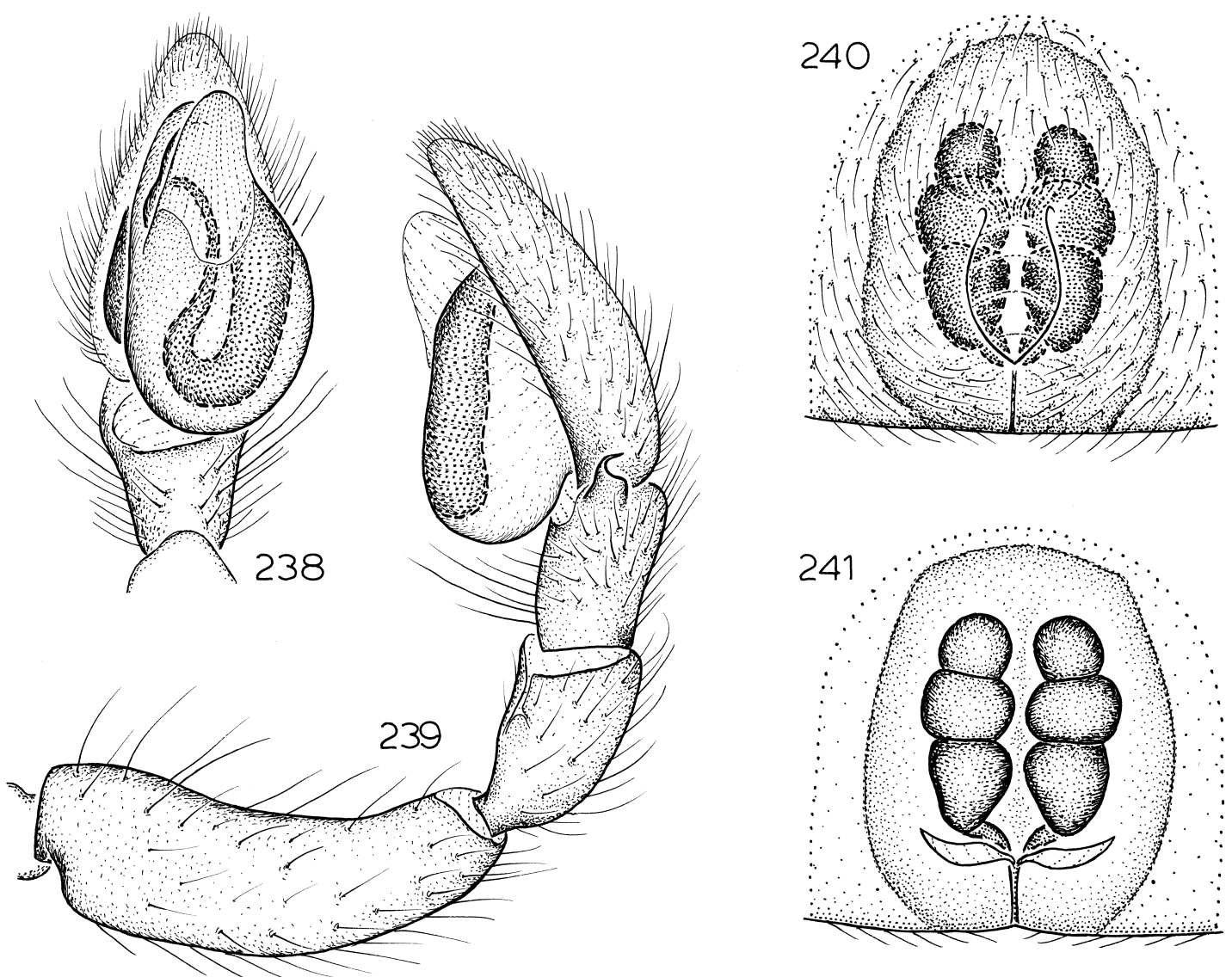

Figs. 238-241. Lampona kirrama, new species. 238. Left male palp, ventral view. 239. Same, retrolateral view. 240. Epigynum, ventral view. 241. Same, dorsal view.

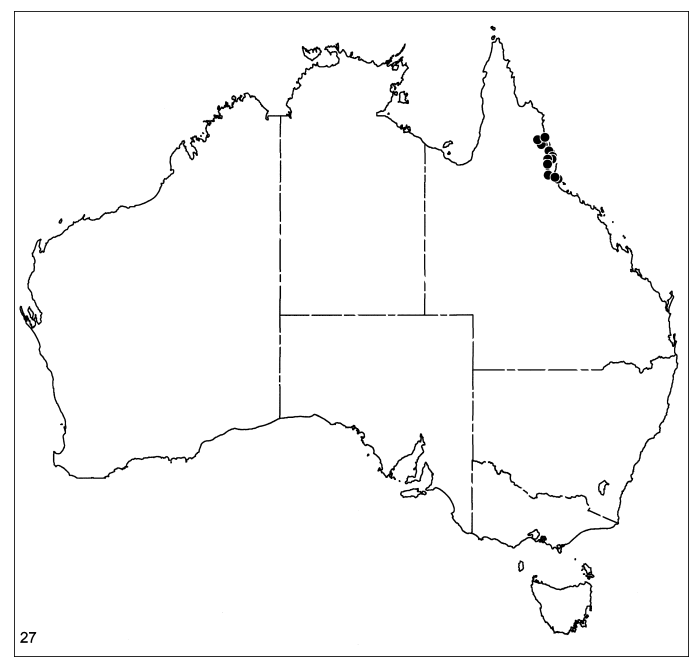

Map 27. Records of Lampona kirrama, new species. web (R. Jackson, FSCA), 19 ; Kuranda State Forest, $3 \mathrm{~km} \mathrm{~N}$ Kuranda, $16^{\circ} 49^{\prime} \mathrm{S}, 145^{\circ} 39^{\prime} \mathrm{E}$, June 25-Aug. 3, 1982, intercept trap, rainforest, elev. 360 m (S., J. Peck, AMNH), 10; Massey Creek, $17^{\circ} 37^{\prime} \mathrm{S}, 145^{\circ} 34^{\prime} \mathrm{E}$, May 30July 1, 1996, Malaise trap, elev. 1000 m (P.

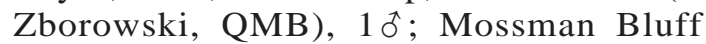
Track, 5-10 km W Mossman, $16^{\circ} 28^{\prime} \mathrm{S}$, $145^{\circ} 22^{\prime} \mathrm{E}$, Dec. 16-30, 1988, intercept trap, elev. $480 \mathrm{~m}$ (G. Monteith, G. Thompson, QMB S32409), 19; Mossman Gorge, Daintree National Park, $16^{\circ} 29^{\prime} \mathrm{S}, 145^{\circ} 20^{\prime} \mathrm{E}$, Apr. 29, 1998 (G. Milledge, AMS KS55272), 1 \% ; Mount Demi summit, $16^{\circ} 30^{\prime} \mathrm{S}, 145^{\circ} 19^{\prime} \mathrm{E}$, Dec. 17, 1995-Jan. 25, 1996, intercept trap, elev. $1100 \mathrm{~m}$ (G. Monteith, G. Thompson, Ford, QMB S34410), 1\%; Mount Fisher, $17^{\circ} 33^{\prime} \mathrm{S}, 145^{\circ} 32^{\prime} \mathrm{E}$, Mar. 26-May 2, 1996, in- 

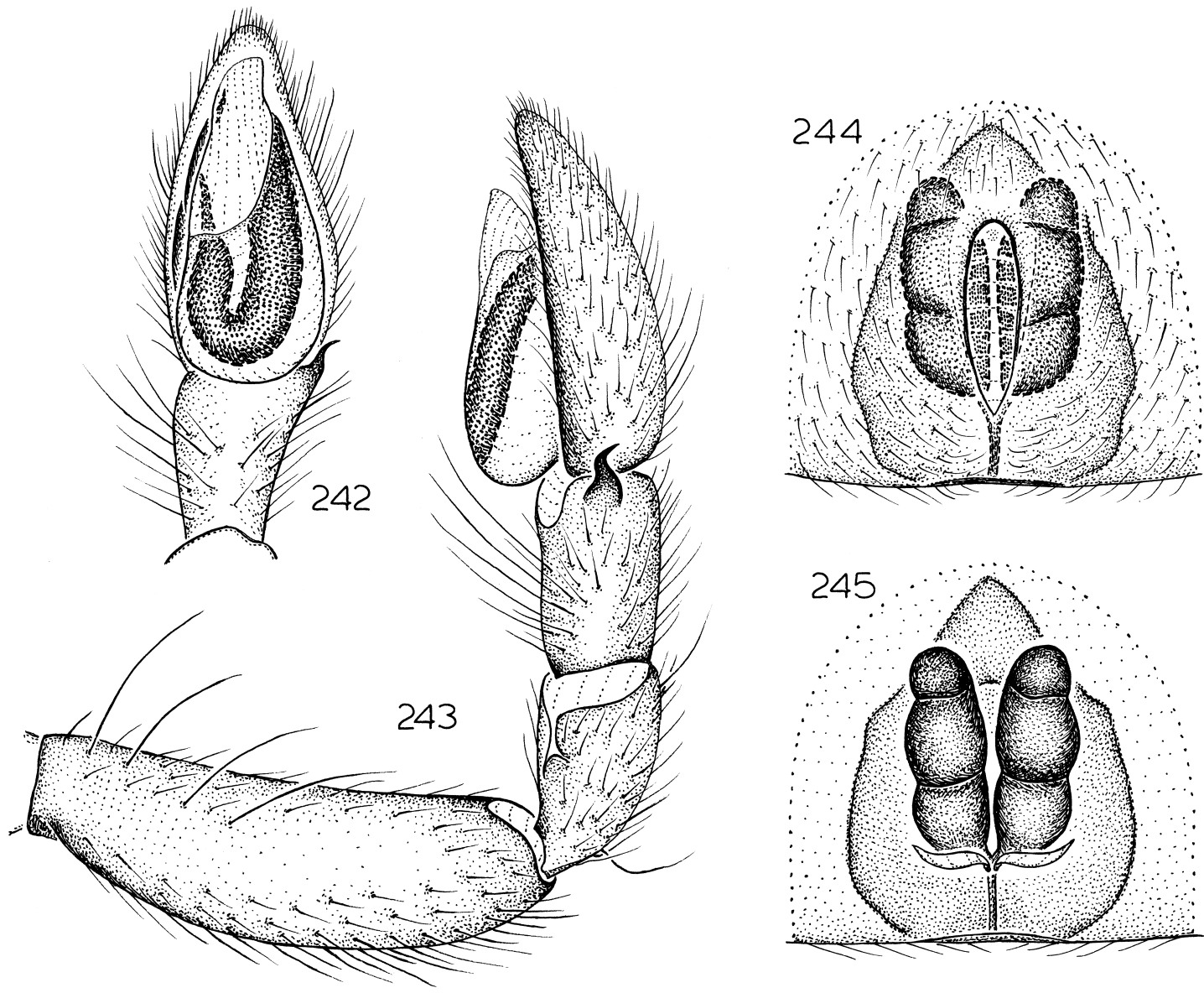

Figs. 242-245. Lampona monteithi, new species. 242. Left male palp, ventral view. 243. Same, retrolateral view. 244. Epigynum, ventral view. 245. Same, dorsal view.

tercept trap, elev. $1150 \mathrm{~m}$ (P. Zborowski, QMB), 1 \% ; Mount Fisher, 7 km SW Millaa Millaa, Whiteing Road, $17^{\circ} 33^{\prime} \mathrm{S}, 145^{\circ} 34^{\prime} \mathrm{E}$, May 5, 1983, pyrethrum knockdown, rainforest (G. Monteith, D. Yeates, QMB S28084), 1 \%; Mount Spurgeon, $16^{\circ} 28^{\prime} \mathrm{S}$, $145^{\circ} 12^{\prime} \mathrm{E}$, Nov. 20, 1997-Feb. 8, 1998, pitfall, elev. $1140 \mathrm{~m}$ (G. Monteith, D. Cook, QMB S43936), 1 \%; Mount Windsor Tableland, Whypalla State Forest, $16^{\circ} 12^{\prime} \mathrm{S}$, $144^{\circ} 58^{\prime} \mathrm{E}$, summer, 1992-1993, pitfall (S. Barnett, QMB S30453), 1\%; North Bell Peak, Malbon Thompson Range, $17^{\circ} 06^{\prime} \mathrm{S}$,

Map 28. Records of Lampona monteithi, new species.

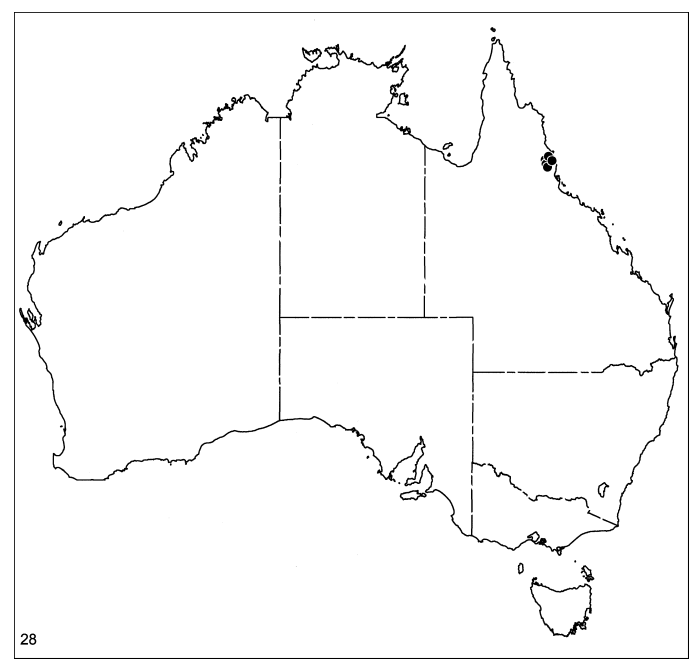



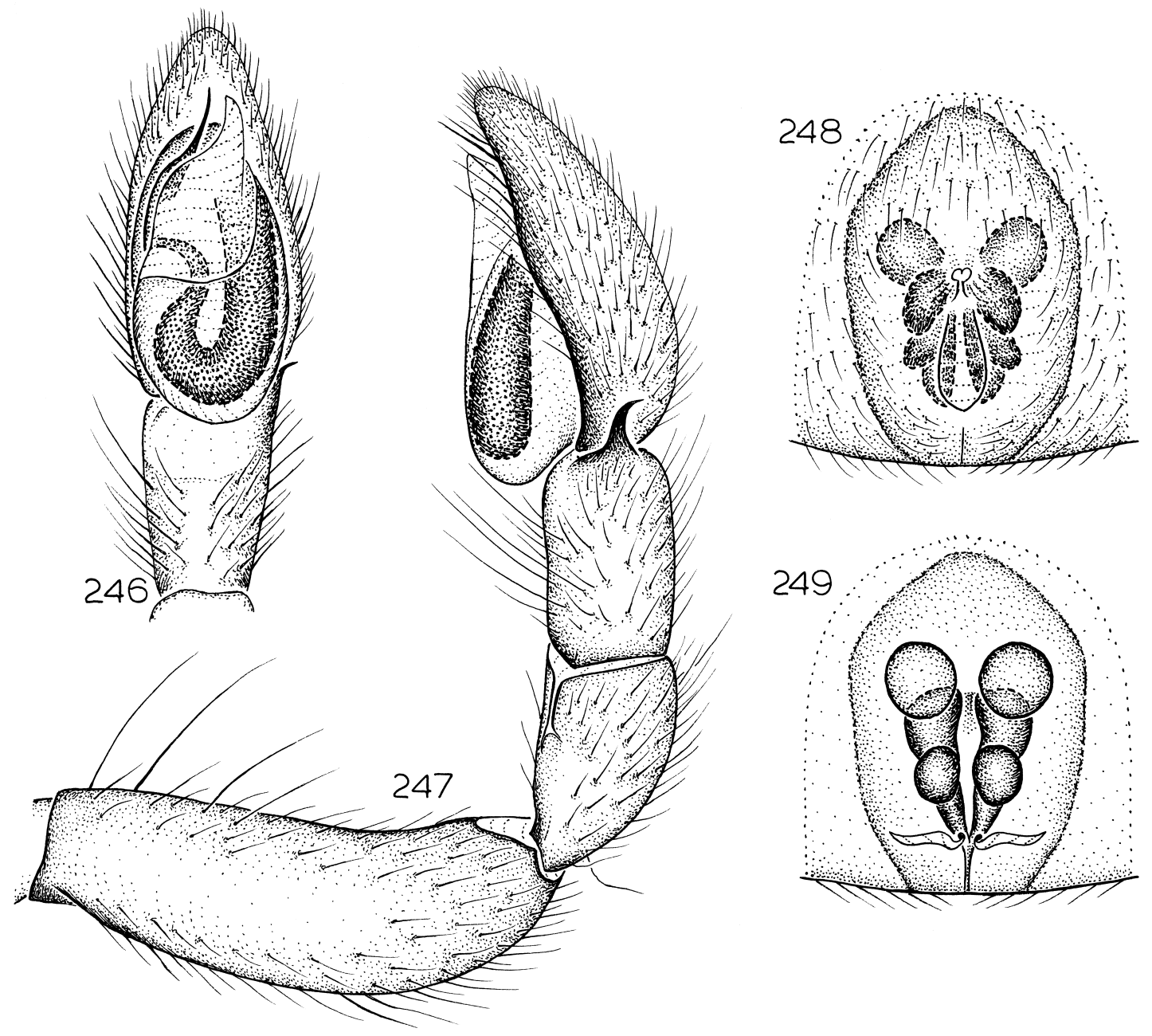

Figs. 246-249. Lampona finnigan, new species. 246. Left male palp, ventral view. 247. Same, retrolateral view. 248. Epigynum, ventral view. 249. Same, dorsal view.

$145^{\circ} 53^{\prime} \mathrm{E}$, Nov. 20, 1990, pyrethrum knock down, trees and $\operatorname{logs}$ (G. Monteith, H. Janetzki, QMB S34418), 2\%; Roaring Meg Valley, $16^{\circ} 04^{\prime} \mathrm{S}, 145^{\circ} 25^{\prime} \mathrm{E}$, Dec. 9, 1993 , pyrethrum, trees and logs, elev. $680 \mathrm{~m}$ (G. Monteith, QMB S34401), 1 ; ; Saddle Mountain, $16^{\circ} 49^{\prime} \mathrm{S}, 145^{\circ} 40^{\prime} \mathrm{E}$, Nov. 21,1994 , Berlese, sieved litter from summit, elev. $650 \mathrm{~m}$ (G. Monteith, QMB S27957), 10 ; South Bell Peak, Malbon Thompson Range, $17^{\circ} 08^{\prime} \mathrm{S}$, 1455'ㄹ, Nov. 20-21, 1990, elev. 900 m (G. Monteith, G. Thompson, QMB S34283), 1 \%; Summit TV station, Bellenden Ker Range, $17^{\circ} 16^{\prime} \mathrm{S}, 145^{\circ} 51^{\prime} \mathrm{E}$, Oct. $25-31$, 1981, palm leaf bracts, elev. $1560 \mathrm{~m}$ (QMB S27858), 19 , Nov. 1-7, 1981, pyrethrum knockdown on $\operatorname{logs}$, stones, and tree trunks, elev. 1560 m (QMB S28087), 1 \%; Windsor Tableland, $28 \mathrm{~km}$ NNW Mount Carbine, $16^{\circ} 16^{\prime} \mathrm{S}$, $145^{\circ} 08^{\prime} \mathrm{E}$, Apr. $15-18,1982$, pyrethrum knockdown, rainforest (G. Monteith, D. Yeates, D. Cook, QMB S28079), 1 ; 16 km NE Yungaburra on Gillies Road, NE Atherton, $17^{\circ} 17^{\prime} \mathrm{S}, 145^{\circ} 35^{\prime} \mathrm{S}$, June 24-Aug. 3, 1982, intercept trap, rainforest (S., J. Peck, AMNH), 10 ..

DISTRIBUTION: Known only from northern Queensland (map 27). 
Lampona monteithi, new species

Figures 242-245; Map 28

TYPE: Female holotype taken at an elevation of $1100 \mathrm{~m}$ at a site $3 \mathrm{~km} \mathrm{~W}$ Bones Knob, $17^{\circ} 13^{\prime} \mathrm{S}, 145^{\circ} 25^{\prime} \mathrm{E}$, Queensland (Dec. 10, 1995; G. Monteith, D. Cook, G. Thompson), deposited in QMB (S28538).

ETYMOLOGY: The specific name is a patronym in honor of Dr. Geoff Monteith of the Queensland Museum, whose indefatigable collecting has vastly improved our knowledge of the Queensland lamponid fauna.

Diagnosis: Males resemble those of L. kirrama but have an unmodified palpal femur (fig. 243); females have a distinctively arched and elevated anterior epigynal margin (fig. 244).

MALE: Total length 5.8. Abdominal dorsum with anterior pair of longitudinal white stripes connected to median transverse white band, band with posterolateral white extensions almost reaching transverse posterior white band; femora with distal, tibiae with proximal and distal slightly darkened rings. Palpal femur short, unmodified; retrolateral tibial apophysis sinuous, with tip directly distodorsally (fig. 243); tegulum not enlarged, extending posteriorly only slightly past base of tarsus; embolus small, recesses behind conductor (fig. 242).

Female: Total length 9.2. Abdominal dorsum with median pair of white spots fused medially, extended anteriorly along midline; leg coloration as in male. Epigynum with arched, elevated anterior margin (fig. 244); spermathecae tripartite, middle portion largest (fig. 245).

Other MATERial ExAmined: Queensland: Hugh Nelson Range, $17^{\circ} 27^{\prime} \mathrm{S}, 145^{\circ} 29^{\prime}$ E, July 3-Aug. 1, 1995, intercept trap, elev. $1150 \mathrm{~m}$ (P. Zborowski, QMB), 10; Lambs Head, 10 $\mathrm{km}$ W Edmonton, $17^{\circ} 02^{\prime} \mathrm{S}, 145^{\circ} 38^{\prime} \mathrm{E}$, Dec. 4, 1988, rainforest (G. Monteith, G. Thompson, QMB S34281), 10; Massey Creek, $17^{\circ} 37^{\prime} \mathrm{S}, 145^{\circ} 34^{\prime} \mathrm{E}$, Jan. 3-Feb. 4, 1995, pitfall, elev. 1000 m (P. Zborowski, QMB), 1 ô; Summit TV station, Bellenden Ker Range, $17^{\circ} 16^{\prime} \mathrm{S}, 145^{\circ} 51^{\prime} \mathrm{E}$, Nov. 1-7, 1981, pyrethrum knockdown on logs, stones, and tree trunks, elev. $1560 \mathrm{~m}$ (ex QMB S28087), $10^{\star}$.

DISTRIBUTION: Known only from northern Queensland (map 28).
Lampona finnigan, new species

Figures 246-249; Map 29

TYPE: Female holotype taken by pyrethrum knockdown in rainforest at an elevation of $1100 \mathrm{~m}$ on the summit of Mount Finnigan, via Helenvale, $15^{\circ} 49^{\prime} \mathrm{S}, 145^{\circ} 17^{\prime} \mathrm{E}$, Queensland (Nov. 28, 1985; G. Monteith, D. Cook, L. Roberts), deposited in QMB (S30369).

ETYMOLOGY: The specific name is a noun in apposition taken from the type locality.

DiAGNOSIS: Males can be recognized by the almost c-shaped retrolateral tibial apophysis (fig. 247, females by the very small epigynum with anteriorly situated openings (fig. 248).

MALE: Total length 5.3. Coloration as in $L$. kirrama. Palpal femur darkened but unmodified; retrolateral tibial apophysis almost cshaped (fig. 247); tegulum only slightly expanded, reaching only to about one-sixth of tibial length; in ventral view, conductor covering middle portion of embolus (fig. 246).

FEMALE: Total length 5.6. Coloration as in male. Epigynal midpiece long, reaching entire length of spermathecae, openings situated at anterior edge (fig. 248); spermathecae tripartite, anterior portion expanded laterally (fig. 249).

Other MAterial Examined: Queensland:

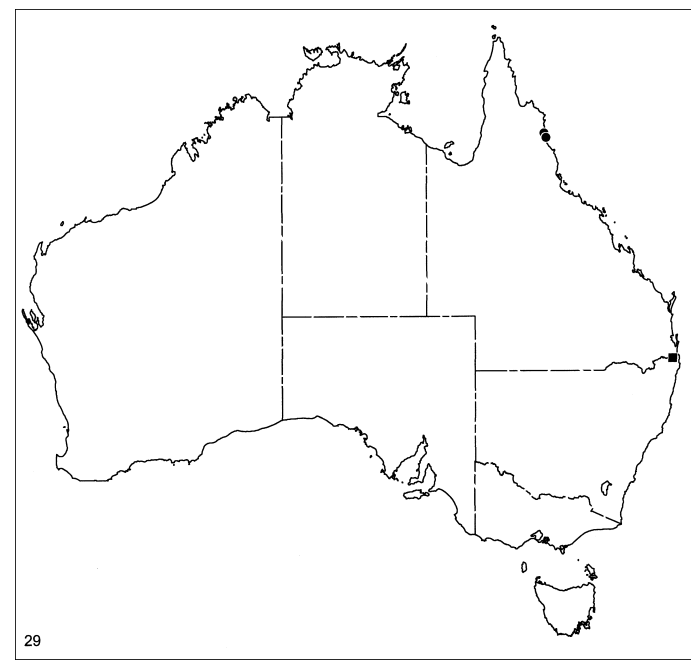

Map 29. Records of Lampona finnigan, new species (circles) and L. lamington, new species (square). 

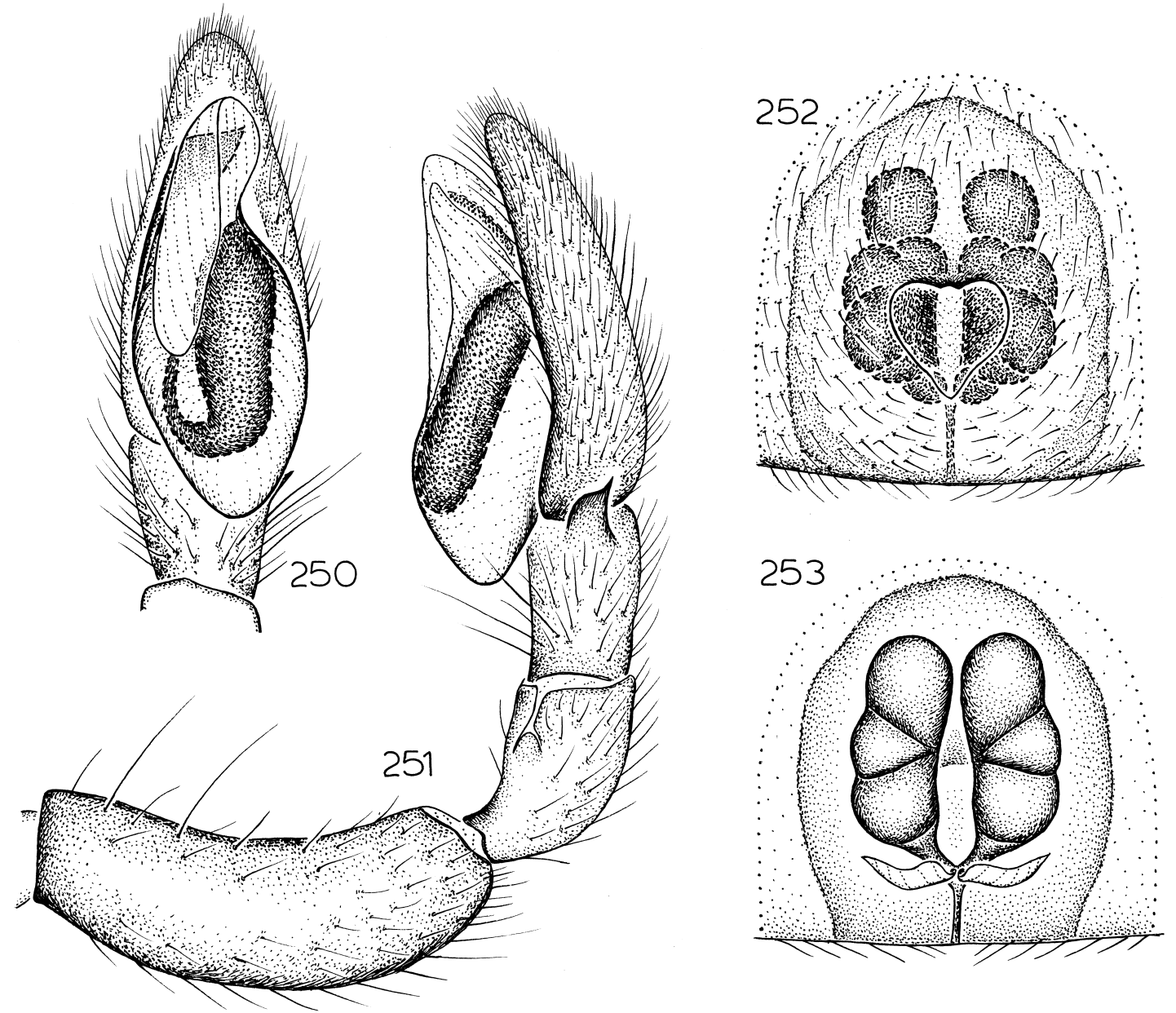

Figs. 250-253. Lampona lamington, new species. 250. Left male palp, ventral view. 251. Same, retrolateral view. 252. Epigynum, ventral view. 253. Same, dorsal view.

4.5-5.0 km W Cape Tribulation (top camp), $16^{\circ} 05^{\prime} \mathrm{S}, 145^{\circ} 26^{\prime} \mathrm{E}$, Sept. 27-Oct. 7, 1982, rainforest, elev. 760-780 m (G. Monteith, D. Yeates, G. Thompson, QMB S28086), 10; Mount Finnigan, $37 \mathrm{~km} \mathrm{~S}$ Cooktown, $15^{\circ} 49^{\prime} \mathrm{S}, 145^{\circ} 17^{\prime} \mathrm{E}$, Apr. 19-22, 1982, rainforest (G. Monteith, D. Yeates, D. Cook, QMB S28081), 10, Dec. 20, 1982, pyrethrum knockdown, elev. 1100 m (L. Roberts, QMB S28075), 10; Mount Finnigan, summit, via Helenvale, $15^{\circ} 49^{\prime} \mathrm{S}, 145^{\circ} 17^{\prime} \mathrm{E}$, Nov. 28-30, 1985, rainforest, elev. $1100 \mathrm{~m}$ (G. Monteith, D. Cook, L. Roberts, QMB S30171), 20ิ, Dec. 3-5, 1990, pyrethrum knockdown, elev. 1050 m (G. Monteith, R. Sheridan, L. Roberts, G. Thompson, QMB
S30338), 20; Mount Pieter Botte, $16^{\circ} 04^{\prime} \mathrm{S}$, $145^{\circ} 24^{\prime} \mathrm{E}$, Nov. 21, 1993, pyrethrum, trees, logs, rocks (G. Monteith, H. Jantezki, QMB S34415), 10 .

DistRIBUTION: Known only from northern Queensland (map 29).

\section{Lampona lamington, new species}

Figures 250-253; Map 29

TYPES: Female holotype and male allotype taken in rainforest at Lamington National Park, $28^{\circ} 12^{\prime} \mathrm{S}, 153^{\circ} 10^{\prime} \mathrm{E}$, Queensland (Aug. 9-10, 1977; R. Raven), deposited in QMB (S28094).

ETYMOLOGY: The specific name is a noun in apposition taken from the type locality. 

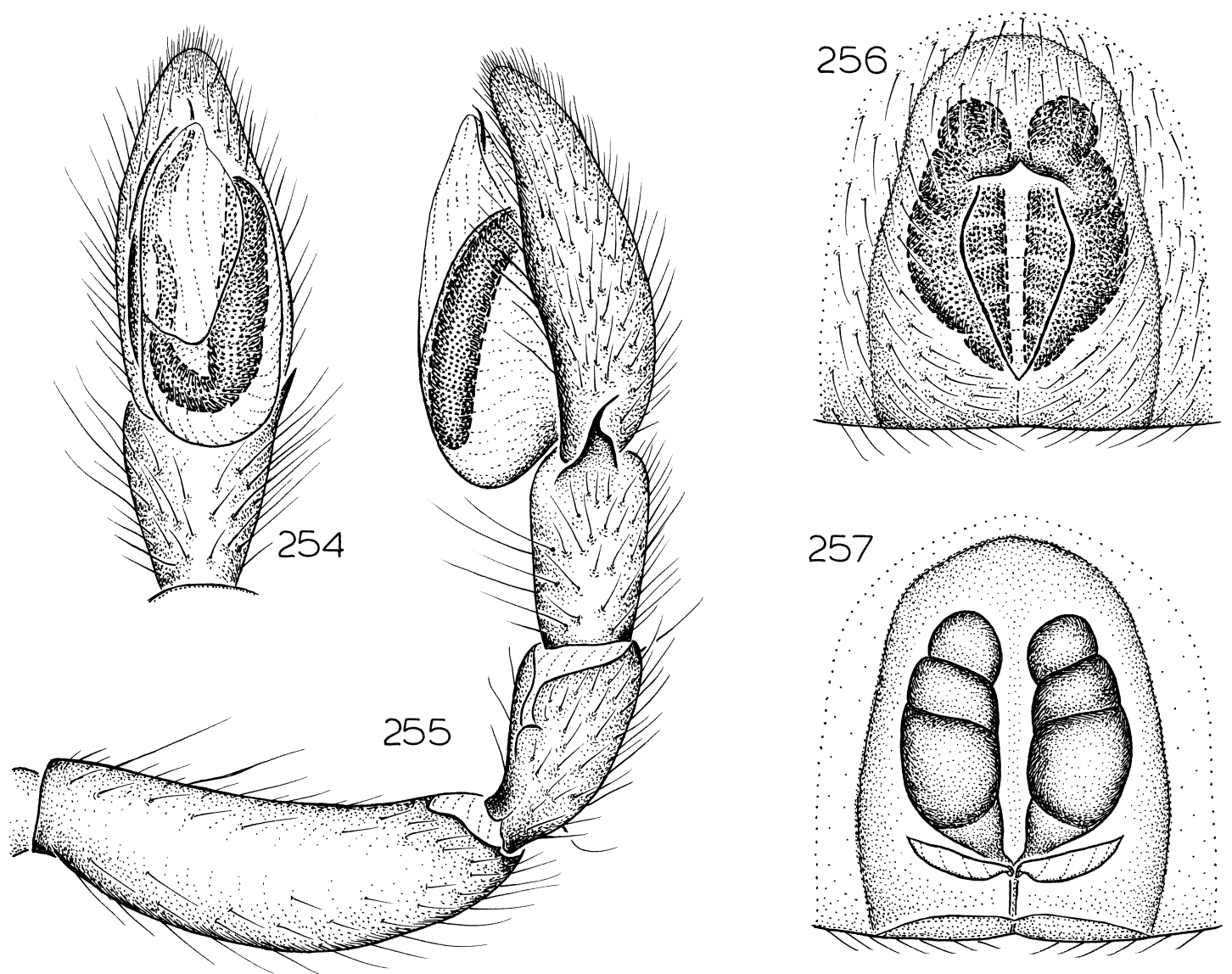

Figs. 254-257. Lampona carlisle, new species. 254. Left male palp, ventral view. 255. Same, retrolateral view. 256. Epigynum, ventral view. 257. Same, dorsal view.

DiAgNosis: Males have a broadly truncated retrolateral tibial apophysis and a strongly protuberant tegulum (fig. 251); females have distinctive, anteriorly truncated epigynal midpiece (fig. 252).

MALE: Total length 5.0. Abdominal dorsum without distinct pattern of white spots; anterior femora darkened, posterior femora with subdistal, tibiae with proximal and distal dark rings. Palpal femur darkened throughout most of its length, otherwise unmodified; retrolateral tibial apophysis distally truncated (fig. 251); tegulum strongly protuberant basally, extending to half length of tibia; embolus very wide, distally arched (fig. 250).

Female: Total length 6.5. Coloration as in male. Epigynal midpiece anteriorly truncated (fig. 252); spermathecae tripartite, anterior portions narrowed (fig. 253).

Other MATERIAL EXAMINED: None.

DisTRIBUTION: Known only from southeastern Queensland (map 29).

\section{Lampona carlisle, new species}

Figures 254-257; Map 30

TYPE: Female holotype taken at night in a Melaleuca forest on Carlisle Island, Whitsunday Islands, $20^{\circ} 47^{\prime} \mathrm{S}, 149^{\circ} 17^{\prime} \mathrm{E}$, Queensland (Dec. 16, 1986; D. Reeves), deposited in QMB (S30175).

ETYMOLOGY: The specific name is a noun in apposition taken from the type locality.

DiAGNOSIS: Males can be recognized by the prong-shaped tip of the retrolateral tibial 

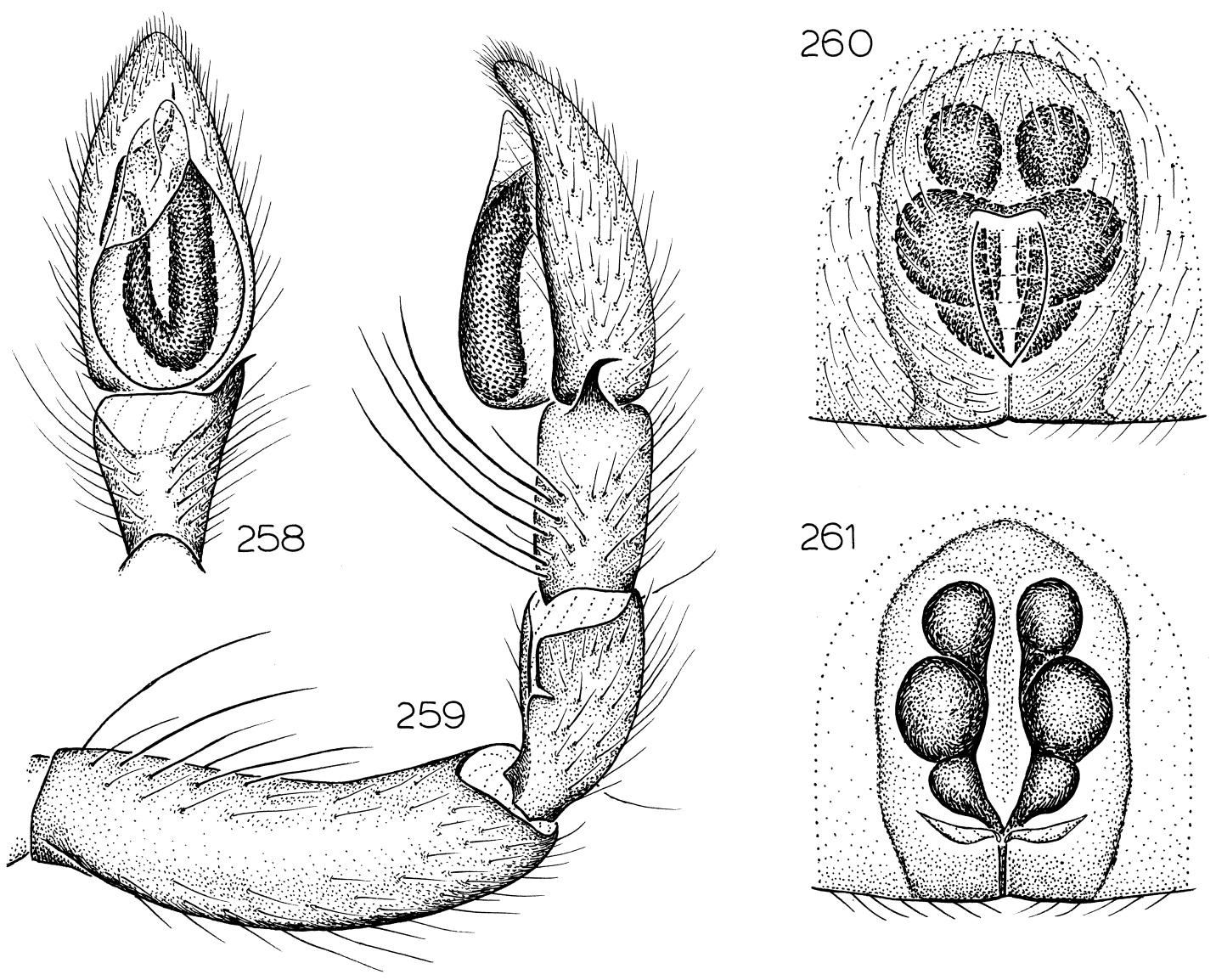

Figs. 258-261. Lampona davies, new species. 258. Left male palp, ventral view. 259. Same, retrolateral view. 260. Epigynum, ventral view. 261. Same, dorsal view.

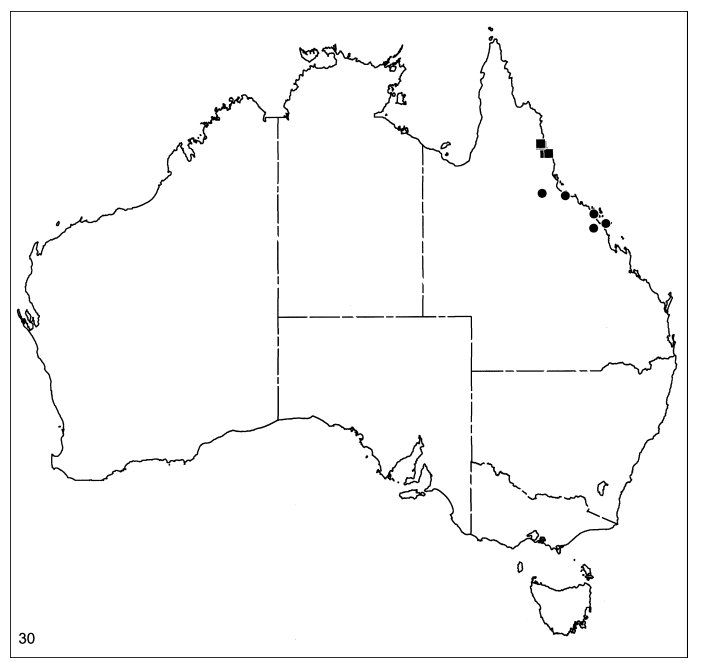

apophysis (fig. 255), females by the large, diamond-shaped epigynal midpiece and the enlarged posterior portions of the spermathecae (figs. 256, 257).

MALE: Total length 6.0. Abdominal dorsum too faded to distinguish white spots; anterior femora scarcely darkened, posterior femora with subdistal, posterior tibiae with proximal and distal dark rings. Palpal femur darkened throughout its length but otherwise unmodified; retrolateral tibial apophysis wide basally, with long distal prong (fig. 255); tegulum slightly expanded, reaching to about

$\leftarrow$

Map 30. Records of Lampona carlisle, new species (circles) and L. davies, new species (squares). 

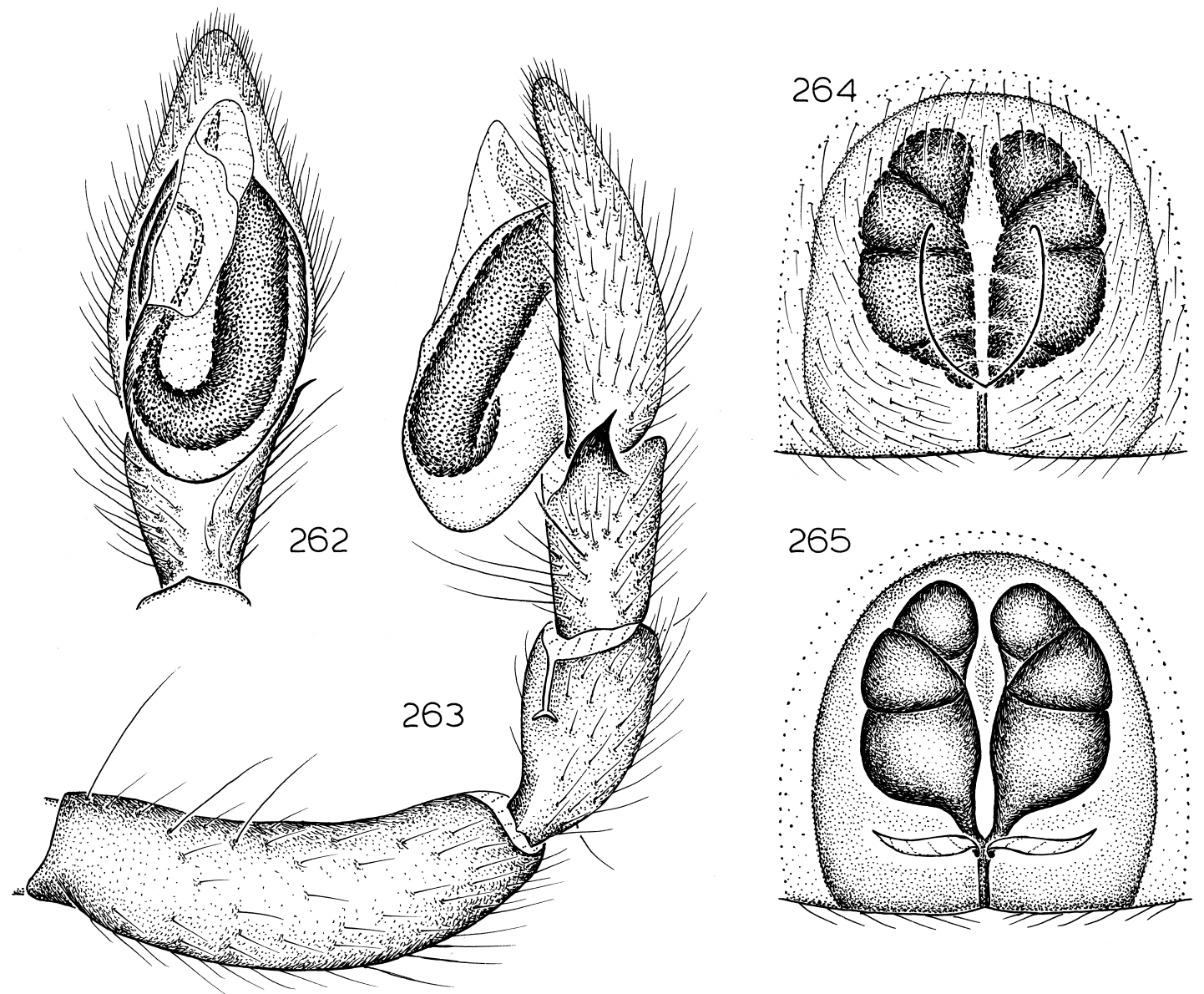

Figs. 262-265. Lampona chinghee, new species. 262. Left male palp, ventral view. 263. Same, retrolateral view. 264. Epigynum, ventral view. 265. Same, dorsal view.

one-fourth of tibial length; embolus almost entirely hidden by conductor in ventral view (fig. 254).

FEMALE: Total length 6.8. Abdominal dorsum with anterior and median pairs of white spots fused into w-shaped mark; leg coloration as in male. Epigynum long, diamondshaped (fig. 256); spermathecae tripartite, posterior portions greatly enlarged (fig. 257).

Other MAterial ExAMINED: Queensland: Eungella area, Snake Road, $8.1 \mathrm{~km}$ NW Dalrymple Road, $21^{\circ} 02^{\prime} \mathrm{S}, 148^{\circ} 32^{\prime} \mathrm{E}$, eucalypt forest (G. Milledge, AMS KS52295), 1 \% ; Mount Dryander, $20^{\circ} 15^{\prime} \mathrm{S}, 148^{\circ} 33^{\prime} \mathrm{E}$, Apr. 24, 1979, Berlese, sieved rainforest litter (G. Monteith, QMB S28091). 10; SE ridge, Mount Halifax, $19^{\circ} 07^{\prime} \mathrm{S}, 145^{\circ} 23^{\prime} \mathrm{E}$, Mar. 19-
21, 1991, rainforest pitfall, elev. $950 \mathrm{~m}$ (G. Monteith, D. Cook, QMB S34279), $10^{\text {t。 }}$ Townsville, $19^{\circ} 15^{\prime} \mathrm{S}, 146^{\circ} 48^{\prime} \mathrm{E}$, in nest of Badumna candida (M. Downes, QMB S32444), 1 ㅇ․

DisTRIBUTION: Known only from eastern Queensland (map 30).

\section{Lampona davies, new species} Figures 258-261; Map 30

TyPE: Female holotype from Davies Creek, $16^{\circ} 55^{\prime} \mathrm{S}, 145^{\circ} 32^{\prime} \mathrm{E}$, Queensland (Mar. 12, 1972; N. Coleman), deposited in QMB (28102).

ETYMOLOGY: The specific name is a noun in apposition taken from the type locality. 
DiAGNOSIS: Males can be recognized by the almost straight retrolateral edge of those parts of the retrolateral tibial apophysis posterior to the angled tip (fig. 259), females by the greatly widened median portions of the spermathecae, which flank the narrow epigynal midpiece (figs. 260, 261).

MALE: Total length 4.9. Abdominal dorsum with vague indications of fused anterior and median white spots; anterior femora darkened, posterior femora with subdistal, posterior tibiae with proximal and distal dark rings. Palpal femur darkened, basal portion flattened laterally, otherwise unmodified; re trolateral tibial apophysis almost straight below angled tip (fig. 259); tegulum slightly expanded, reaching to about one-fourth of tibial length; embolus with long, narrow tip recessed far behind conductor (fig. 258).

FEMALE: Total length 5.2. Coloration as in male. Epigynal midpiece long, narrow, flanked by enlarged median portions of spermathecae (figs. 260, 261).

Other MATERIAl ExAMINED: Queensland: Davies Creek, $16^{\circ} 55^{\prime} \mathrm{S}, 145^{\circ} 32^{\prime} \mathrm{E}$, Mar. 12, 1972 (N. Coleman, QMB 28102), 1 우 (plus holotype); Mount Lewis, 20 km SW Mossman, $16^{\circ} 28^{\prime} \mathrm{S}, 145^{\circ} 22^{\prime} \mathrm{E}$, June 26-Aug. 1, 1982, intercept trap, rainforest, elev. $1000 \mathrm{~m}$ (S., J. Peck, AMNH), 10; North Bell Peak, $20 \mathrm{~km} \mathrm{~S}$ Cairns, $16^{\circ} 55^{\prime} \mathrm{S}, 145^{\circ} 46^{\prime} \mathrm{E}$, Sept.

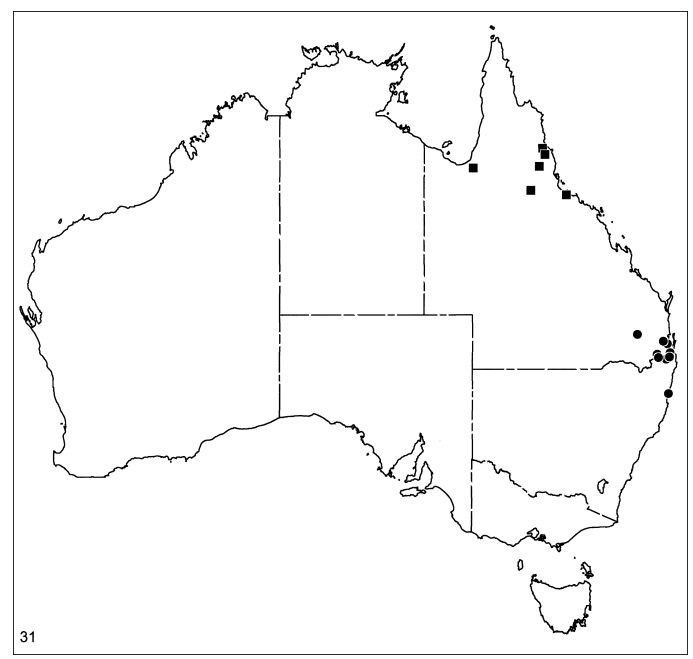

Map 31. Records of Lampona chinghee, new species (circles) and L. molloy, new species (squares).
15-16, 1981, pyrethrum knockdown, elev. 900-1000 m (G. Monteith, D. Cook, QMB S28080), 10; Pauls Luck, Carbine Tableland, $16^{\circ} 27^{\prime} \mathrm{S}, 145^{\circ} 16^{\prime} \mathrm{E}$, Nov. 29, 1990 (G. Monteith, H. Janetzki, QMB S34402), $10^{\star}$; Upper Whyanbeel Creek, $16^{\circ} 23^{\prime} \mathrm{S}, 145^{\circ} 17^{\prime} \mathrm{E}$, Sept. 5, 1992, pyrethrum, mossy rocks, elev. 1150 m (G. Monteith, QMB S40185), 10 .

DistRIBUTION: Known only from northern Queensland (map 30).

Lampona chinghee, new species Figures 262-265; Map 31

TYPE: Female holotype taken in a Berlese sample of sieved rainforest litter from an elevation of $720 \mathrm{~m}$ on Mount Chinghee, $12 \mathrm{~km}$ S Rathdowney, $28^{\circ} 19^{\prime} \mathrm{S}, 152^{\circ} 58^{\prime} \mathrm{E}$, Queensland (Dec. 17, 1982; G. Monteith, D. Yeates, G. Thompson), deposited in QMB (S28077).

ETYMOLOGY: The specific name is a noun in apposition taken from the type locality.

DiAGNOSIS: This species can be distinguished by the almost triangular retrolateral tibial apophysis and narrow embolus of males (figs. 262, 263) and the large, wide epigynal midpiece and large posterior portions of the spermathecae of females (figs. 264, 265).

MALE: Total length 5.7. Abdominal dorsum with white spots united into lateral longitudinal stripes, median dark stripe interrupted by chevrons; anterior femora darkened, posterior femora with subdistal, posterior tibiae with proximal and distal dark rings. Palpal femur darkened throughout most of its length, otherwise unmodified; retrolateral tibial apophysis triangular, setose (fig. 263); tegulum globose, extending to almost half length of tibia; embolus relatively narrow, even at base (fig. 262).

Female: Total length 7.2. Coloration as in male except white stripes much less extensive. Epigynum with wide, tongue-shaped midpiece (fig. 264); spermathecae tripartite, posterior portions largest (fig. 265).

Other Material Examined: New South Wales: Bruxner Park via Coffs Harbour, $30^{\circ} 14^{\prime} \mathrm{S}, 153^{\circ} 06^{\prime} \mathrm{E}$, Nov. 12, 1980 (R. Raven, G. Monteith, QMB S28100), 10. Queensland: $15 \mathrm{mi} \mathrm{W}$ Brisbane, $27^{\circ} 28^{\prime} \mathrm{S}, 153^{\circ} 01^{\prime} \mathrm{E}$, Nov. 24, 1962, elev. 400 m (E. Ross, D. Cavagnaro, CAS), 1\%; Cunningham's Gap, 

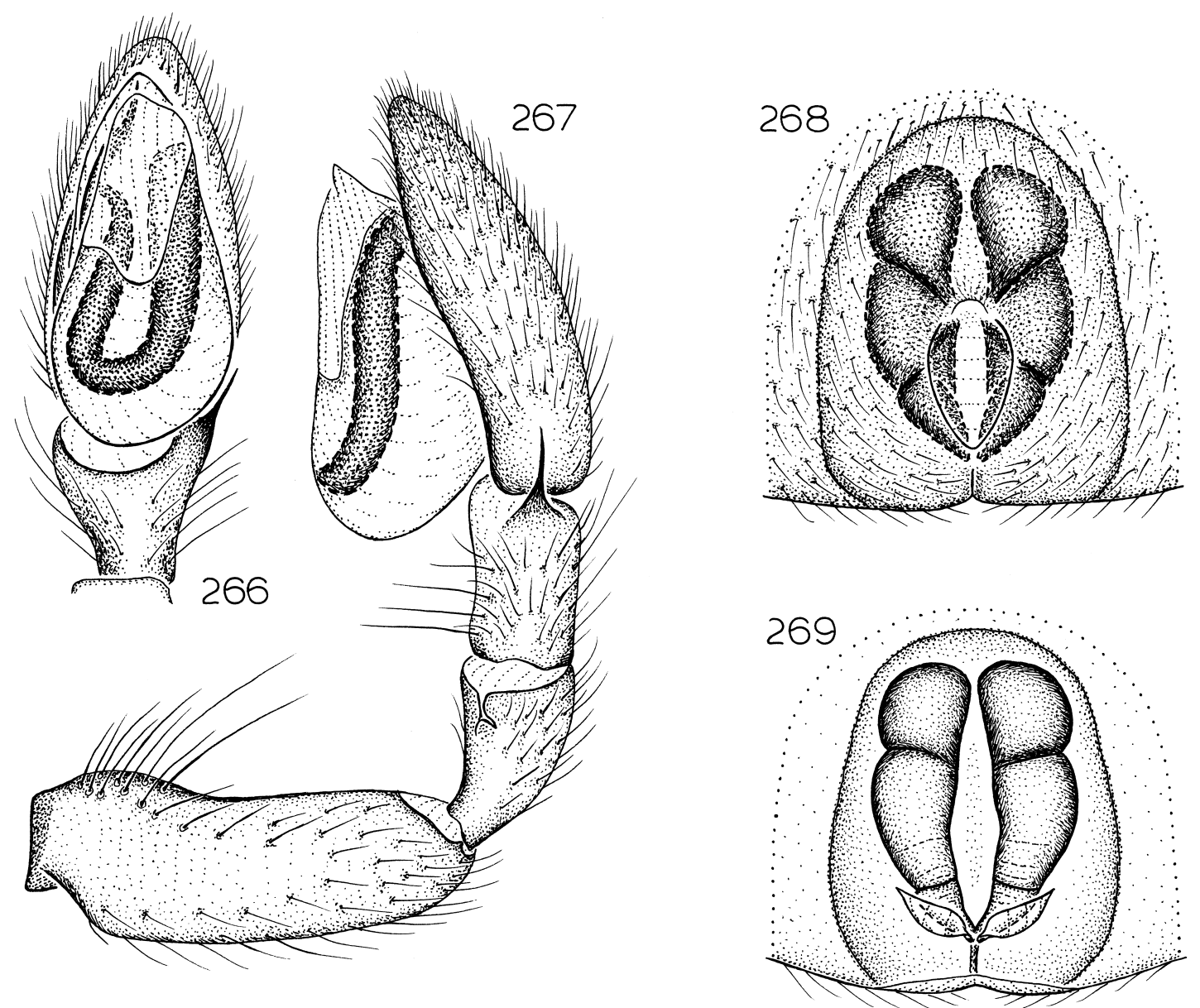

Figs. 266-269. Lampona molloy, new species. 266. Left male palp, ventral view. 267. Same, retrolateral view. 268. Epigynum, ventral view. 269. Same, dorsal view.

$28^{\circ} 03^{\prime} \mathrm{S}, 152^{\circ} 24^{\prime} \mathrm{E}$, Jan. 6-Mar. 1, 1992, pitfall, rainforest, elev. $790 \mathrm{~m}$ (D. Cook, QMB S25044), 19; Mount Chinghee, $12 \mathrm{~km} \mathrm{~S}$ Rathdowney, $28^{\circ} 19^{\prime} \mathrm{S}, 152^{\circ} 58^{\prime} \mathrm{E}$, Dec. 17 , 1982, Berlese, sieved litter, rainforest, elev. 720 m (G. Monteith, D. Yeates, G. Thompson, QMB S28077), $1 \%$; Mount Glorious, $27^{\circ} 20^{\prime} \mathrm{S}, 152^{\circ} 46^{\prime} \mathrm{E}$, Sept. 1978, Malaise trap (A. Hiller, QMB S30162), 1 , Jan. 10-31, 1981, Malaise trap (J. Grimshaw, QMB S30213), 19; Mount Glorious State Forest, $27^{\circ} 20^{\prime} \mathrm{S}, 152^{\circ} 46^{\prime} \mathrm{E}$, Feb. 17-24, 1987, subtropical rainforest (Y. Besart, QMB S5353), 1 웅 Mount Superbus, $28^{\circ} 14^{\prime} \mathrm{S}, 152^{\circ} 29^{\prime} \mathrm{E}$, Feb. 8, 1990, rainforest, under logs, night (T. Churchill, QMB S15813), 10; Mount Tamborine, $27^{\circ} 58^{\prime} \mathrm{S}, 153^{\circ} 12^{\prime} \mathrm{E}$, Oct. $12-18,1978$,
Malaise trap (J. Grimshaw, QMB S30215), 1 ${ }^{\top}$; Nagarigoon, Lamington National Park, $28^{\circ} 12^{\prime} \mathrm{S}, 153^{\circ} 10^{\prime} \mathrm{E}$, Apr. 1-8, 1976, rainforest (R. Raven, V. Davies, QMB S28098), $10^{\text {to; }}$ Tarana, $26^{\circ} 57^{\prime} \mathrm{S}, 151^{\circ} 10^{\prime} \mathrm{E}$, Apr. 23, 1966 (R. Mascord, AMS KS35560), 1 ㅇ.

DisTRIBUTION: Southeastern Queensland and northern New South Wales (map 31).

\section{Lampona molloy, new species} Figures 266-269; Map 31

TyPE: Female holotype from Molloy Road, Mount Molloy, $16^{\circ} 41^{\prime} \mathrm{S}, 145^{\circ} 20^{\prime} \mathrm{E}$, Queensland (Feb. 18, 1972; N. Coleman), deposited in QMB (S25982).

ETYMOLOGY: The specific name is a noun in apposition taken from the type locality. 
Diagnosis: The sexes have not been collected together, but the male here associated matches the females well in abdominal color pattern. Males can easily be recognized by the long, narrow retrolateral tibial apophysis (fig. 267), females by the elongate spermathecae with small posterior portions (fig. 269).

MALE: Total length 4.3. Abdominal dorsum with anterior and median pairs of white spots united into lateral longitudinal stripes; anterior femora darkened, posterior femora with subdistal, posterior tibiae with proximal and distal dark rings. Palpal femur with low, setose ventral protuberance at base; retrolateral tibial apophysis with long, needleshaped dorsal spur (fig. 267); tegulum moderately expanded, probably reaching to about one-third of tibial length (uncertain, as only available palp is expanded); embolar tip recessed behind conductor (fig. 266).

FEMALE: Total length 7.3. Coloration as in male except abdominal stripes wider. Epigynum with relatively wide, short, midpiece reaching to only half of spermathecal length (fig. 268); spermathecae tripartite but posterior portions very small (fig. 269).

OTHER MATERIAl ExAMINED: Queensland: Atherton Tablelands, near Mareeba, $17^{\circ} 00^{\prime} \mathrm{S}$, $145^{\circ} 26^{\prime} \mathrm{E}$, Dec. 1982 , in Badumna web (R. Jackson, QMB S25966), 3 ; Bowen district, Apr. 17, 1971 (R. Monroe, ex QMB S28431), 1 \% ; McKinnons Creek, 9 km SSW Lynd Junction, $19^{\circ} 00^{\prime} \mathrm{S}, 144^{\circ} 37^{\prime} \mathrm{E}$, June 13, 1993 (B., M. Baehr, CBB), 1 \% ; Mount Garnet, $17^{\circ} 41^{\prime} \mathrm{S}, 145^{\circ} 07^{\prime} \mathrm{E}$, Feb. 24, 1972 (N. Coleman, QMB S34421), 1\%; 5 km S Normanton, $17^{\circ} 46^{\prime} \mathrm{S}, 141^{\circ} 02^{\prime} \mathrm{E}$, July 12,1995 (J. Thompson, M. Tio, S. Cowan, AMS KS44176), 19; Tinaroo Creek Road, $17^{\circ} 02^{\prime} \mathrm{S}, 145^{\circ} 28^{\prime} \mathrm{E}$, Jan. 8, 1974 (J. Thomson, BMM), $10^{\top}$; Townsville, $19^{\circ} 15^{\prime} \mathrm{S}, 146^{\circ} 48^{\prime} \mathrm{E}$, Feb. 1, 1988, in nest of Badumna candida (M. Downes, QMB S32443), 1§, 1989 (M. Downes, QMB S18768), 1 ㅇ.

DisTRIBUTION: Known only from northern Queensland (map 31).

\section{Lampona tully, new species}

Figures 270-273; Map 32

TYPE: Female holotype taken by pyrethrum spraying of $\operatorname{logs}$ and trees at an ele- vation of $1000 \mathrm{~m}$ at Upper Boulder Creek, $11 \mathrm{~km}$ NNW Tully, $17^{\circ} 50^{\prime} \mathrm{S}, 145^{\circ} 54^{\prime} \mathrm{E}$, Queensland (Dec. 5, 1989; G. Monteith, G. Thompson, H. Janetzki), deposited in QMB (S34413).

ETYMOLOGY: The specific name is a noun in apposition taken from the type locality.

DiAGNOSIS: Both sexes are darkly colored, with only a small pair of white spots on the abdominal dorsum; males can easily be recognized by the triangular base and long shaft of the retrolateral tibial apophysis (fig. 271); females have a distinctive epigynum, with the basal portion of the spermathecae much larger than either the median or distal portions (fig. 273).

Male. Total length 8.0. Abdominal dorsum with only median pair of white spots conspicuous, venter pale gray; anterior femora darkened, posterior femora with subdistal, posterior tibiae with proximal and distal dark rings. Palpal femur enlarged, darkened throughout its length, but otherwise unmodified; retrolateral tibial apophysis with triangular base and long, narrow, angled shaft (fig. 271); tegulum globose, reaching almost to half length of tibia; only prolateral edge of embolus visible in ventral view (fig. 270).

Female: Total length 8.7. Coloration as in male. Epigynum with small anterior hood, invaginated at tip (fig. 272); basal portion of spermathecae greatly enlarged, median and distal portions small (fig. 273).

Other Material Examined: Queensland: Douglas Creek Road, Kirrama State Forest, $18^{\circ} 12^{\prime} \mathrm{S}, 145^{\circ} 45^{\prime} \mathrm{E}$, Dec. $10,1986-$ Jan. 11, 1987, rainforest, elev. 800 m, intercept trap (G. Monteith, G. Thompson, Hamlet, QMB S28278), 10; Massey Creek, $17^{\circ} 37^{\prime} \mathrm{S}, 145^{\circ} 34^{\prime} \mathrm{E}$, Jan. 3-31, 1996, intercept trap, elev. 1000 m (L. Umback, QMB), 1 o, Jan. 3-Feb. 4, 1995, pitfall, elevation 1000 m (P. Zborowski, QMB), 1 ㅇ, Feb. 4Mar. 6, 1995, pitfall, elev. 1000 m (P. Zborowski, QMB), 1ठ; Upper Boulder Creek, $11 \mathrm{~km}$ NNW Tully, $17^{\circ} 50^{\prime} \mathrm{S}, 145^{\circ} 54^{\prime} \mathrm{E}$, Dec. 5-7, 1989, pitfall, rainforest, elev. $900 \mathrm{~m}$ (G. Monteith, G. Thompson, H. Janetzki, QMB S30468, 1 î.

Distribution: Known only from northern Queensland (map 32). 

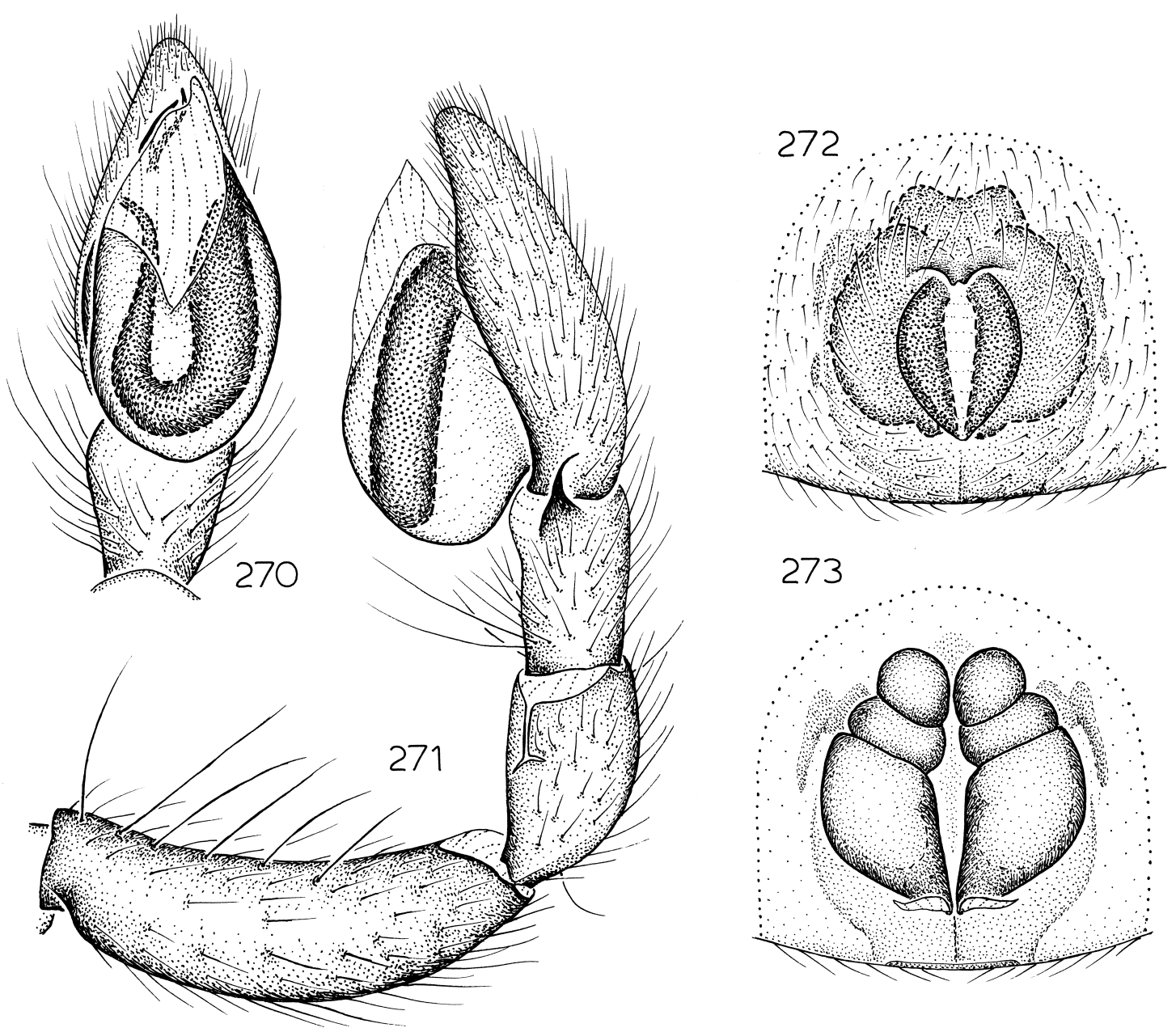

Figs. 270-273. Lampona tully, new species. 270. Left male palp, ventral view. 271. Same, retrolateral view. 272. Epigynum, ventral view. 273. Same, dorsal view.

\section{Lamponina Strand, new rank}

Lamponina Strand, 1913: 606 (type species by original designation Lampona scutata Strand; described as a subgenus of Lampona Thorell).

Note: The status of Strand's name Lamponina has been ambiguous. Roewer's (1955) catalog, for example, doesn't even cite the name. Strand (1913: 606) merely appended a paragraph to his description of Lampona scutata, translated as follows:

Because of the divergent posterior eye row, the abdominal scuta, and the spinnerets, a special subgenus [Untergattung] ought to be set up for this species (eventually Lamponina mihi).

Petrunkevitch (1928: 152) erroneously listed
Lamponina as a genus (rather than a subgenus), and that error was perpetuated in Bonnet's catalog (1957: 2343). Because there is a clear indication of a type species and a putative diagnosis, there seems no doubt that Lamponina is an available name, despite Strand's noxious habit of providing provisional names for taxa (usually new subspecies) in case they might prove distinct at a later date. As Strand's species belongs to a group that is clearly monophyletic and distinct from Lampona, his name is here accepted and explicitly elevated to generic rank.

DiAgNOSIS: The fusion of the sternum with the epimeric sclerites separates specimens 
from those of all lamponine genera other than Lamponega, Lamponusa, and Lamponicta. The presence of a divided scopula on metatarsi and tarsi I and II, a rugose sternal surface, and the typical form of the retrolateral tibial apophysis (figs. 292, 312) and epigynum (figs. 293, 313) all separate Lamponina specimens from those of the other three genera with fused sterna and epimeric sclerites.

DESCRIPTION: Medium-sized spiders, total length 6.1-10.5. Carapace dark reddishblack, elongate, surface coated with setabearing tubercles (extending to lateral margins, which therefore appear serrate), with median longitudinal row of thick white scales (fig. 278), row wide on posterior half of pars cephalica, narrow on pars thoracica; thoracic groove long, longitudinal, inconspicuous. From above, anterior eye row procurved, posterior row straight (fig. 277); from front, anterior row very strongly procurved, with laterals almost forming third row, posterior row procurved; AME separated by their radius, closer to ALE; PME almost contiguous, slightly farther from PLE; MOQ slightly longer than wide, wider in back than in front; clypeus at midline almost twice AME diameter. Chelicerae reddishblack proximally, lightened to dark orange on distal one-fifth; sternum and mouthparts

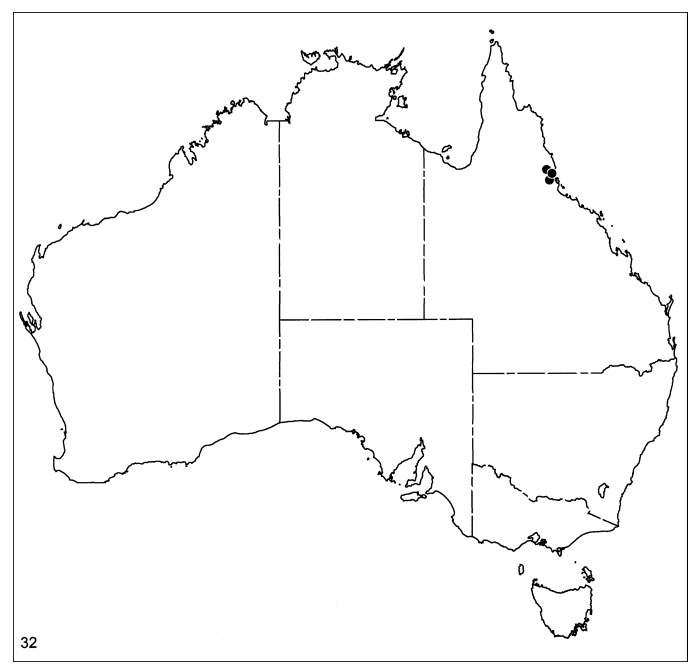

Map 32. Records of Lampona tully, new species. dark red. Anterior chilum small, almost diamond-shaped, protuberant; posterior chilum (extremely narrow sclerite between chelicerae posteriorly at base) present. Each cheliceral promargin with long, thick seta originating near insertion of fang, immediately bent at 90 degree angle, extending to median line (fig. 282); chelicerae with three promarginal teeth, no retromarginals, with distinct lateral boss. Epimeric sclerites completely fused with sternum on each side, so that each leg coxae appears set in foramen completely surrounded by sclerotized cuticle; epimeric sclerites also completely fused with carapace from level of endites back to rear of coxae II foramina (from there back to pedicel, separated from carapace by extremely narrow line of unsclerotized cuticle). Anterior margin of labium slightly incised at middle, posterior margin rounded at sides; labium depressed medially. Endites obliquely depressed, inner margins with sharply demarcated, deep groove extending from level of scopula back to over half length of labium; serrula teeth may be fused into long ridge, sieve plate small (fig. 281). Sternum elevated, with deep grooves between coxal foramina; surface rugose, with elevated ridges connecting seta-bearing tubercles. Pedicel elongate (figs. 274, 279), dorsally incised, antlike in appearance, composed of two dorsal sclerites (anterior sclerite elongate; posterior sclerite normal in length, composed of five triangular subsclerites) and rounded sclerite covering venter and sides, separated from elongate dorsal sclerite only by narrow strip of unsclerotized cuticle; ventral sclerite without median longitudinal keel prolonged anteriorly into distinct protrusion extending toward sternum.

Abdomen dark gray, dorsum usually with white markings and scales; venter usually brownish-gray, without longitudinal rows of small sclerites. Anterior edge of abdomen of both sexes with almost complete sclerotic ring formed by epigastric scutum and long, narrow, reddish dorsal abdominal scutum reaching to almost half of abdominal length in males, to about one-fourth of abdominal length in females, dorsal scutum with white, scalelike setae; epigastric scutum dark orange, deepened to dark red and highly setose 
over booklungs. Pair of oval sclerites bearing elevated anterior ridge, situated just behind epigastric scutum; anterior edges of those sclerites fitting under epigastric scutum. Anterior lateral spinnerets with two major ampullate gland spigots and unenlarged piriform gland spigots (figs. 287, 288); posterior median spinnerets of males with at least one minor ampullate gland spigot and many aciniform gland spigots (fig. 289), females with at least one cylindrical gland spigot as well (fig. 290); posterior lateral spinnerets twosegmented, distal segment short; colulus represented by setae; oval sclerite just in front of spinnerets presumably marks posterior spiracle.

Legs often with some dark markings; coxae elongate, coxae IV with few dorsal setabearing tubercles; trochanters short, unnotched; anterior metatarsi and tarsi with divided scopulae (fig. 283); posterior metatarsi with distal preening brushes; posterior tarsi with entire scopulae; claw tufts with relatively few setae (fig. 285); legs almost spineless, single proventral spine present at distal end of tibiae III, IV; tarsal organ flat (fig. 284), trichobothrial bases ridged (fig. 286). Female palpal tibia and tarsus with several long, thin spines; female palpal tarsus with dentate claw.

Male palp with retrolateral tibial apophysis short, wide, distally serrate; bulb with posterior protrusion on retrolateral side, with long, prolaterally directed embolus and membranous conductor wrapping around embolus dorsally (fig. 280), without median apophysis; sperm duct s-shaped in retrolateral view. Epigynum on distinct sclerite reaching to pedicel, separated from sclerites bearing booklung covers by narrow longitudinal strips of unsclerotized cuticle; epigynum with small, posteriorly situated median plate with openings at anterolateral corners; spermathecal bulbs on elaborate, mshaped ducts.

Penultimate males and females with epimeric sclerites not fused to carapace, not fused posteriorly to sternum between coxae IV, ventral sclerite of pedicel composed of two separate halves divided along midline by narrow strip of unsclerotized cuticle; penultimate females with substantial pre-epigynum.

\section{Key to SPeCIES OF LAMPONINA}

1. Males ..................... 2

- Females ................... 7

2. Distal half of palpal femur widened (fig. 304) ...................... L. kakadu

- Distal half of palpal femur not widened . 3

3. Palpal femur with small but distinct narrowing at about half its length (as in fig. 292) $\ldots \ldots \ldots \ldots \ldots \ldots \ldots \ldots$

- Palpal femur not narrowed at about half its length ............... 6

4. Femur IV narrowed, elongate, yellow; anterior half of abdomen white . L. elongata

- Femur IV not narrowed, dark orange or darker; anterior half of abdomen dark gray 5

5. Retrolateral tibial apophysis with produced ventral edge, unexpanded dorsal edge (fig. 292) ................. L. scutata

- Retrolateral tibial apophysis with unproduced ventral edge, expanded and pointed dorsal edge (fig. 312) ............. L. isa

6. Abdominal venter with longitudinal dark stripe along midline ......... L. loftia

- Abdominal venter without longitudinal dark stripe .............. L. asperrima

7. Abdominal venter with longitudinal dark stripe along midline ......... L. loftia

- Abdominal venter without longitudinal dark stripe ............... 8

8. Femur IV narrowed, elongate, yellow; anterior half of abdomen white . L. elongata

- Femur IV not narrowed, dark orange or darker; anterior half of abdomen dark gray .. 9

9. Spermathecae relatively large, extending to about one-third of length of epigynal sclerite (figs. 293, 313) . ............ 10

- Spermathecae relatively small, extending only to about one-fourth of length of epigynal sclerite (figs. 297, 305) . . . . . 11

10. Spermathecal tips relatively small (fig. 294) .................... L. scutata

- Spermathecal tips relatively large (fig. 314) $\ldots \ldots \ldots \ldots \ldots \ldots \ldots$. . . . isa

11. Epigynal midpiece relatively large (fig. 297) ............... L. asperrima

- Epigynal midpiece relatively small (fig. 305) .................... L. kakadu

\section{Lamponina scutata (Strand)}

Figures 274, 277-294; Map 33

Lampona scutata Strand, 1913: 606 (female holotype from central Australia, no specific locality, in NMS, examined).

Lamponina scutata: Bonnet, 1957: 2343.

Diagnosis: Males have a distinct, oblique constriction at roughly half the length of the 

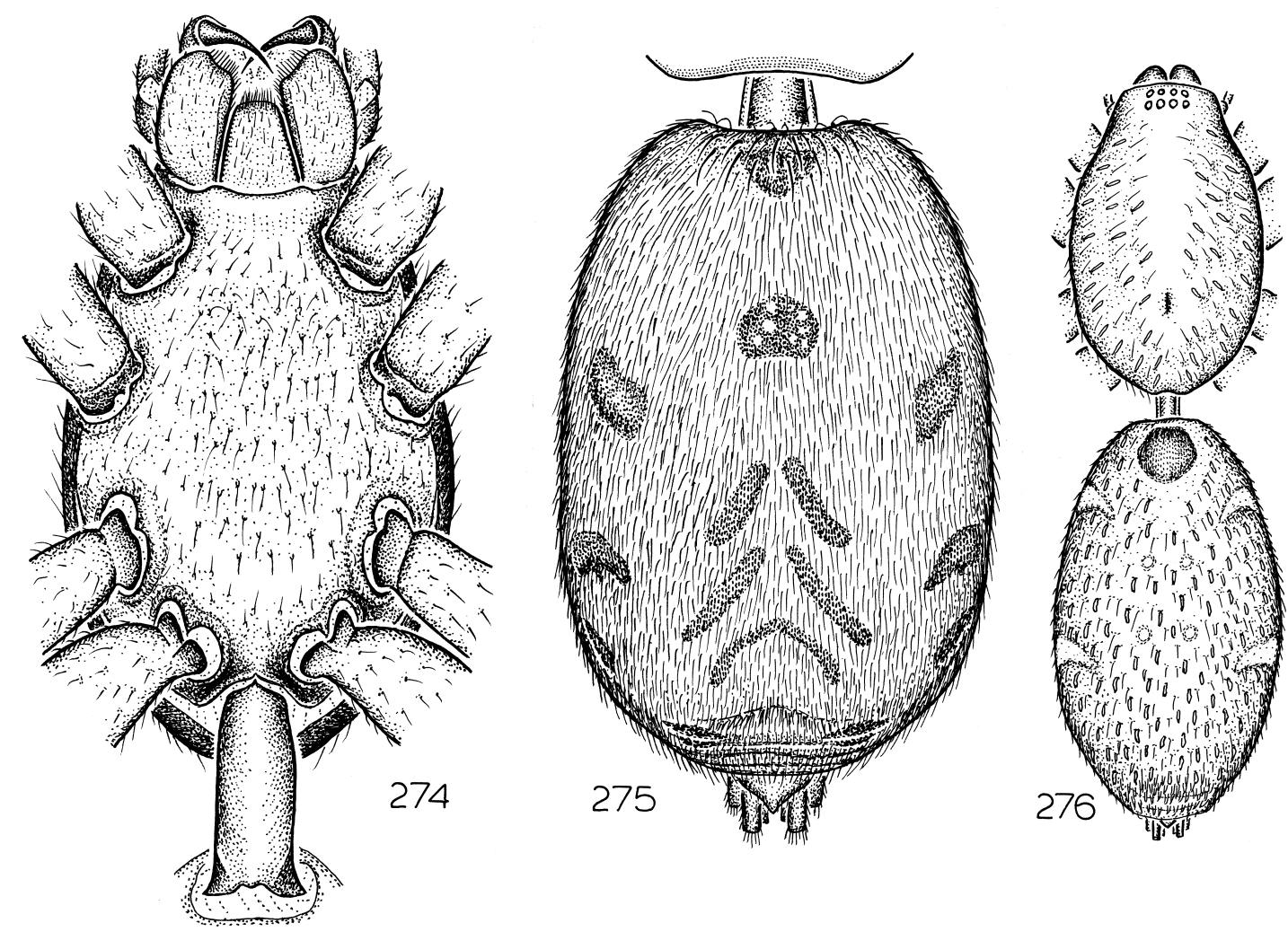

Figs. 274-276. 274. Lamponina scutata (Strand), female cephalothorx and pedicel, ventral view. 275. Lamponata daviesae, new species, female abdomen, dorsal view. 276. Lamponusa gleneagle, new species, female, dorsal view.

ventral surface of the palpal femur and a protruding distoventral edge on the retrolateral tibial apophysis (fig. 292), as well as an elongated conductor reaching beyond the tip of the palpal bulb (fig. 291). Females have relatively large spermathecae, with medially directed ducts budding off relatively long stalks (fig. 294).

MALE: Total length 10.0. Abdominal dorsum with median transverse white stripe with anteromedian longitudinal extension, situated at about half of abdominal length, narrowest at middle, extended posteriorly on sides of abdomen (stripe often reduced to single median and pair of lateral white spots); additional white spot above spinnerets; coxae and trochanters dark orange, anteriors darkest; femur I and proximal half of patella I dark reddish-black, femur II and proximal end of patella II dark reddish-black, remainder of anterior legs yellow; posterior femora orange, remainder of posterior legs yellow. Palpal femur with slight ventral invagination at about half its length; tibia globose, with distally directed, thumb-shaped retrolateral apophysis, ventral edge of apophysis with enlarged setal bases (fig. 292); tegulum with pronounced posterolateral corner, embolus long, arched (fig. 291).

Female: Total length 10.5. Coloration as in male. Epigynum with small, arched, median openings (fig. 293); spermathecae on long stalks, with medially directed ducts (fig. 294).

SELECTED RECORDS: AUSTRALIA: central Australia, no specific locality (v. Leonhardt, NMS 2863), 1 i (holotype). New South Wales: Morton Plains Station, $29^{\circ} 01^{\prime} \mathrm{S}$, $146^{\circ} 13^{\prime} \mathrm{E}$, AMS KS32552. Northern Territory: E bank, Finke River, $24^{\circ} 03^{\prime} \mathrm{S}, 132^{\circ} 45^{\prime} \mathrm{E}$, NMV K4674; Finke River, $25^{\circ} 51^{\prime} \mathrm{S}, 135^{\circ} 03^{\prime} \mathrm{E}$, QMB S34284; Frewena, $18^{\circ} 57^{\prime} \mathrm{S}, 135^{\circ} 10^{\prime} \mathrm{E}$, 

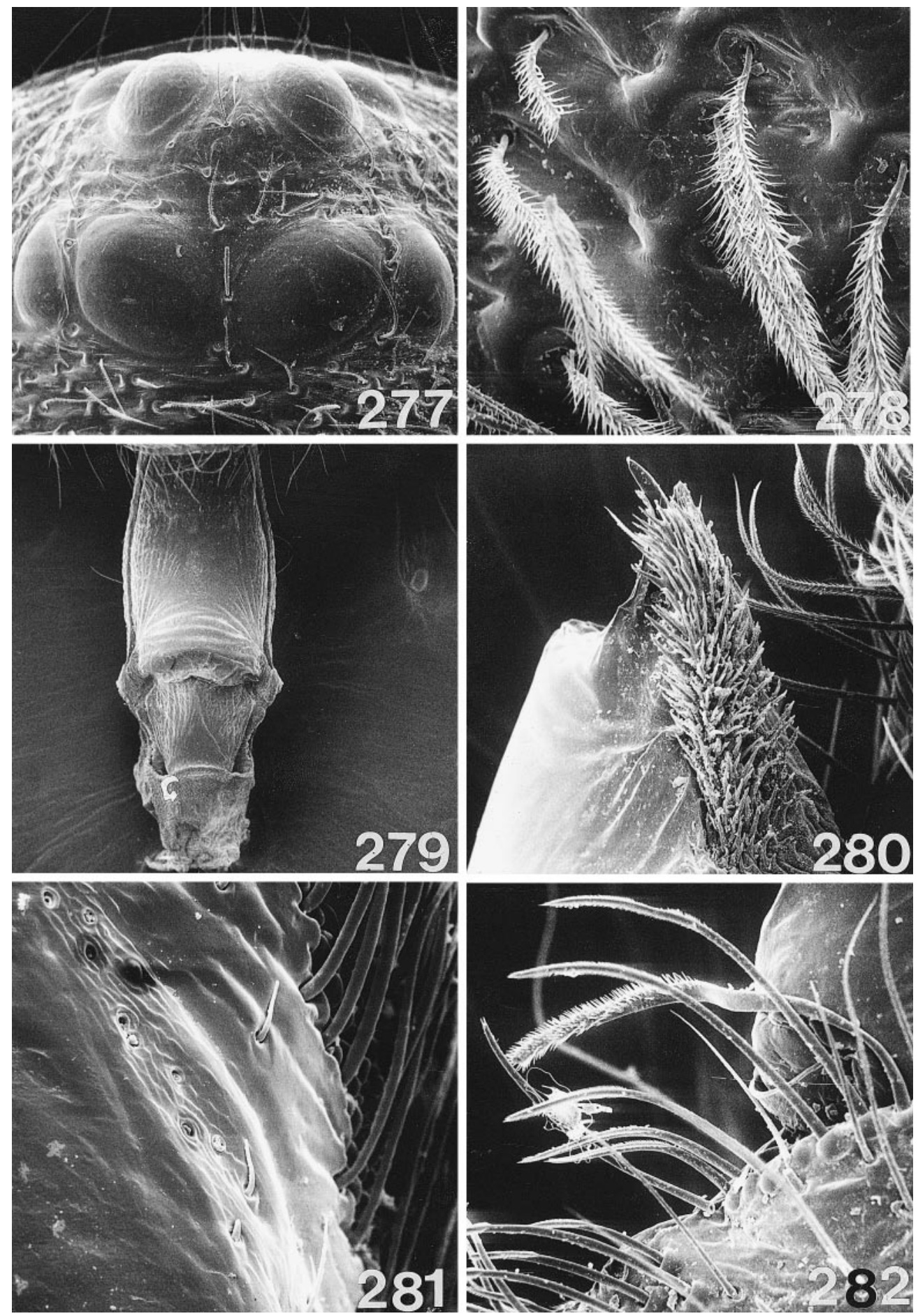

Figs. 277-282. Lamponina scutata (Strand), male. 277. Eyes, dorsal view. 278. Scales from side of carapace, dorsal view. 279. Pedicel, dorsal view. 280. Right palp, prolateral view. 281. Endite, anterior view, showing sieve plate. 282. Modified first promargin seta of chelicera, anterior view. 

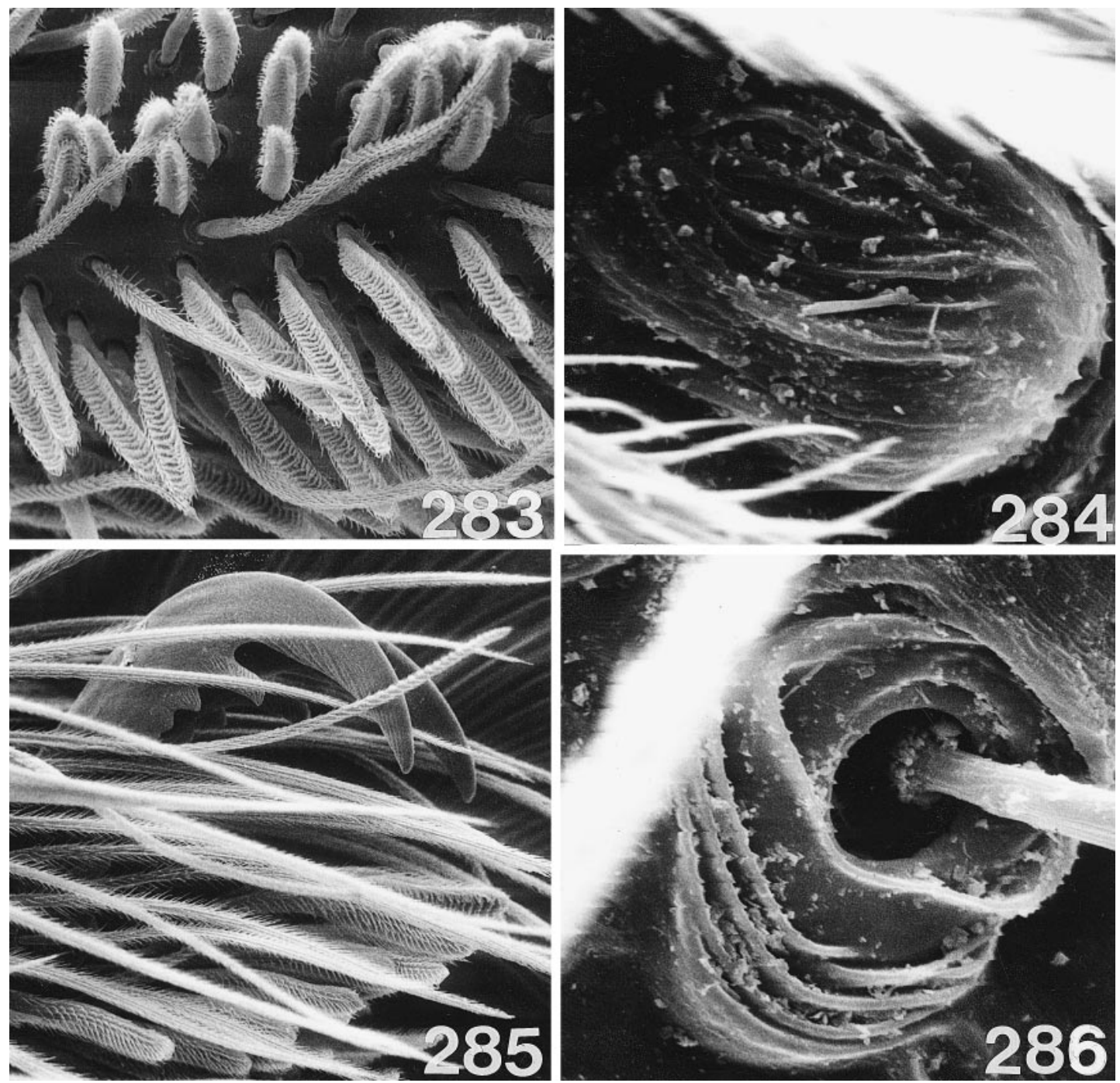

Figs. 283-286. Lamponina scutata (Strand), male. 283. Setae of divided scopula, leg II, ventral view. 284. Tarsal organ from leg IV, dorsal view. 285. Tarsal claws and claw tufts, leg III, lateral view. 286. Trichobothrial base from tarsus IV, dorsal view.

MNT; Harts Range, $23^{\circ} 06^{\prime} \mathrm{S}, 134^{\circ} 55^{\prime} \mathrm{E}$, WAM 96/229; Illamurta Spring, $24^{\circ} 18^{\prime} \mathrm{S}, 132^{\circ} 41^{\prime} \mathrm{E}$, SAM N1996/332; Kathleen Springs Track, $24^{\circ} 21^{\prime} \mathrm{S}, 131^{\circ} 41^{\prime} \mathrm{E}$, NMV K4671; Kidman Springs Station, $16^{\circ} 06^{\prime} \mathrm{S}, 130^{\circ} 53^{\prime} \mathrm{E}$, CSID A0250; Ormiston Gorge, $23^{\circ} 37^{\prime} \mathrm{S}, 132^{\circ} 42^{\prime} \mathrm{E}$, QMB S34300; Palm Plains, $24^{\circ} 04^{\prime} \mathrm{S}$, $132^{\circ} 39^{\prime} \mathrm{E}$, NMV K4667; Sherwin Creek, $14^{\circ} 39^{\prime} \mathrm{S}, 134^{\circ} 21^{\prime} \mathrm{E}, \mathrm{CSID}$ A0260; Uluru-Kata Tjuta National Park, $25^{\circ} 21^{\prime} \mathrm{S}, 131^{\circ} 22^{\prime} \mathrm{E}$, NMV K4668; Waite Creek, $22^{\circ} 29^{\prime} \mathrm{S}, 134^{\circ} 30^{\prime} \mathrm{E}$, MNT. Queensland: Amber Station, $17^{\circ} 45^{\prime} \mathrm{S}$,
144²1'E, QMB S34304; Muncoonie Lakes, $25^{\circ} 12^{\prime} \mathrm{S}, 138^{\circ} 41^{\prime} \mathrm{E}$, QMB S26459; Sandringham Station, $24^{\circ} 03^{\prime} \mathrm{S}, 139^{\circ} 04^{\prime} \mathrm{E}$, QMB S34303; Thylungra, $26^{\circ} 04^{\prime} \mathrm{S}, 143^{\circ} 28^{\prime} \mathrm{E}$, CSID. South Australia: Alinga Bore, $26^{\circ} 13^{\prime} \mathrm{S}$, $135^{\circ} 41^{\prime} \mathrm{E}$, SAM N1997/64; Ampeinna Hills, $27^{\circ} 09^{\prime} \mathrm{S}, 131^{\circ} 08^{\prime} \mathrm{E}$, SAM N1996/455; Anvil Hole Native Well, $26^{\circ} 17^{\prime} \mathrm{S}, 135^{\circ} 42^{\prime} \mathrm{E}$, SAM N1997/72; Burnside, $34^{\circ} 56^{\prime}$ S, $138^{\circ} 38^{\prime}$ E, SAM N1996/263; Coffins Dam, 33⒉ $4^{\prime}$ S, $139^{\circ} 34^{\prime} \mathrm{E}$, SAM N1996/323; Danggali Conservation Park, 33¹9'S, 14055'E, SAM N1997/67; Er- 

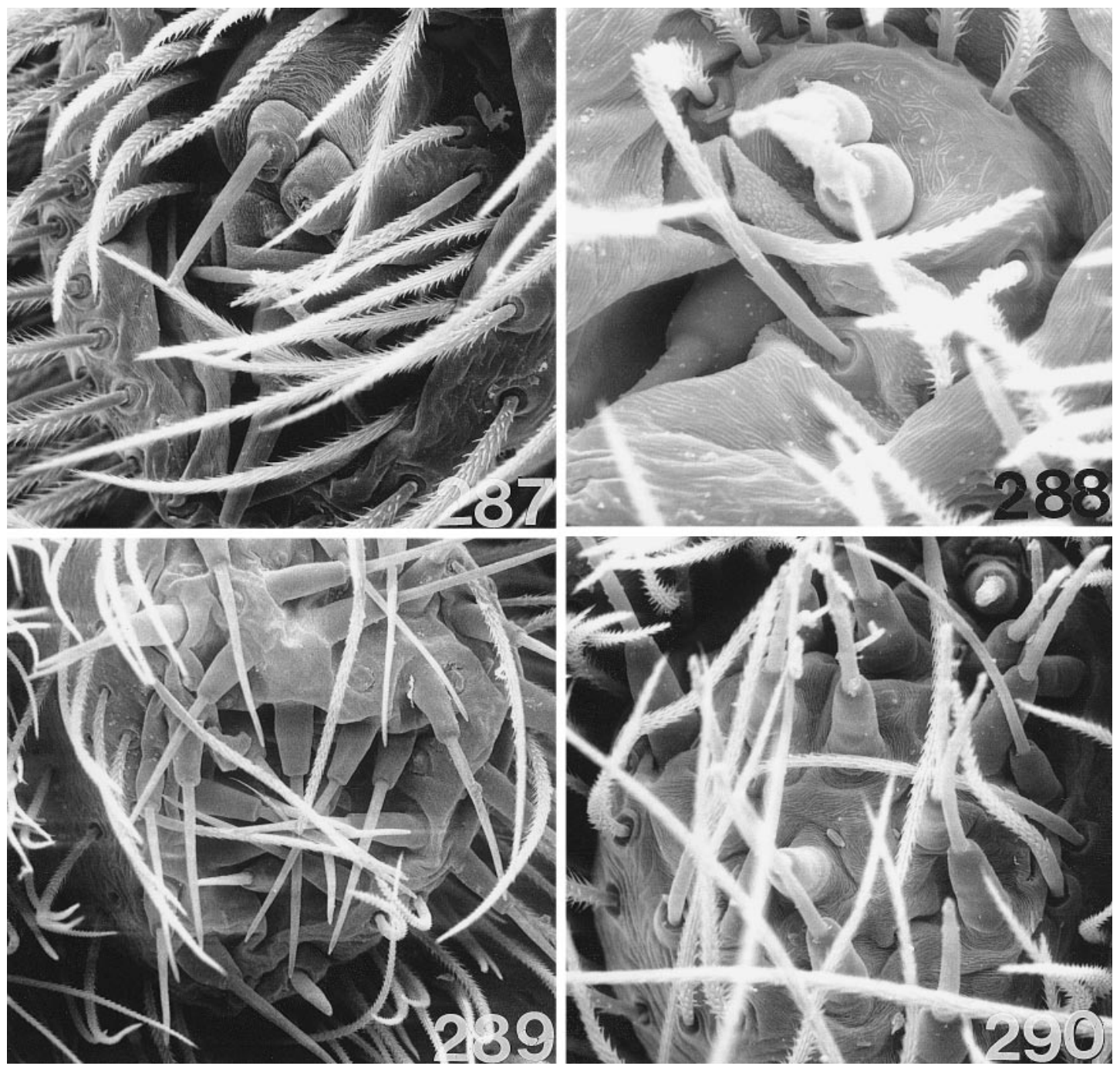

Figs. 287-290. Lamponina scutata (Strand), spinnerets, posterior views. 287. Anterior lateral spinneret of male. 288. Same, female. 289. Posterior median spinneret of male. 290. Same, female.

inga Station, $26^{\circ} 34^{\prime} \mathrm{S}, 135^{\circ} 28^{\prime} \mathrm{E}$, SAM N1997/ 63; Grainger Lagoon, $35^{\circ} 54^{\prime} \mathrm{S}, 136^{\circ} 53^{\prime} \mathrm{E}$, SAM N1996/321; Granite Downs, 26 $56^{\prime} \mathrm{S}$, $133^{\circ} 30^{\prime} \mathrm{E}$, SAM N1996/280; Halifax Hill, $29^{\circ} 41^{\prime} \mathrm{S}, 135^{\circ} 49^{\prime} \mathrm{E}$, SAM N1996/451; Hambridge Conservation Park, $33^{\circ} 25^{\prime} \mathrm{S}, 135^{\circ} 55^{\prime} \mathrm{E}$, SAM N1985/118; Jump Off Road, 35 44'S, 136² 45'E, SAM N1996/232; Maryinna Hill, $27^{\circ} 02^{\prime} \mathrm{S}, 131^{\circ} 19^{\prime} \mathrm{E}$, SAM N199766; Muckera Rockhole, $29^{\circ} 34^{\prime} \mathrm{S}, 130^{\circ} 08^{\prime} \mathrm{E}$, SAM N1996/ 284; Mundulla, $36^{\circ} 25^{\prime} \mathrm{S}, 140^{\circ} 41^{\prime} \mathrm{E}$, SAM N1997/65; Oolarinna E Bore, $27^{\circ} 40^{\prime} \mathrm{S}$, $132^{\circ} 55^{\prime}$ E, SAM N1997/75; Pinkawillinie Con- servation Park, $32^{\circ} 07^{\prime} \mathrm{S}, 136^{\circ} 00^{\prime} \mathrm{E}$, SAM N1997/62; Renmark, 3400'S, $140^{\circ} 47^{\prime} \mathrm{E}$, QMB S32501; Stony Creek, 32 $41^{\prime} \mathrm{S}, 138^{\circ} 06^{\prime} \mathrm{E}$, SAM N1996/338; Teatrick, 36 24' S, $140^{\circ} 53^{\prime} \mathrm{E}$, SAM N1997/74; Wolseley, 36²1'S, $140^{\circ} 58^{\prime} \mathrm{E}$, SAM N1997/61. Victoria: Cullulleraine, $34^{\circ} 15^{\prime} \mathrm{S}, 141^{\circ} 35^{\prime} \mathrm{E}$, NMVS; Hattah, $34^{\circ} 47^{\prime} \mathrm{S}$, $142^{\circ} 20^{\prime} \mathrm{E}$, NMVS; Lindsay River, $34^{\circ} 07^{\prime} \mathrm{S}$, $141^{\circ} 05^{\prime} \mathrm{E}$, NMVS; Mount Hope, $36^{\circ} 00^{\prime} \mathrm{S}$, $144^{\circ} 12^{\prime} \mathrm{E}$, CVIC 627; Point Cook, 37 $56^{\prime} \mathrm{S}$, $144^{\circ} 45^{\prime} \mathrm{E}$, NMVS; Terrick Terrick National Park, $36^{\circ} 08^{\prime} \mathrm{S}, 144^{\circ} 15^{\prime} \mathrm{E}$, CVIC 531; Wychitella, $36^{\circ} 21^{\prime} \mathrm{S}, 143^{\circ} 36^{\prime} \mathrm{E}$, NMV K4665. West- 

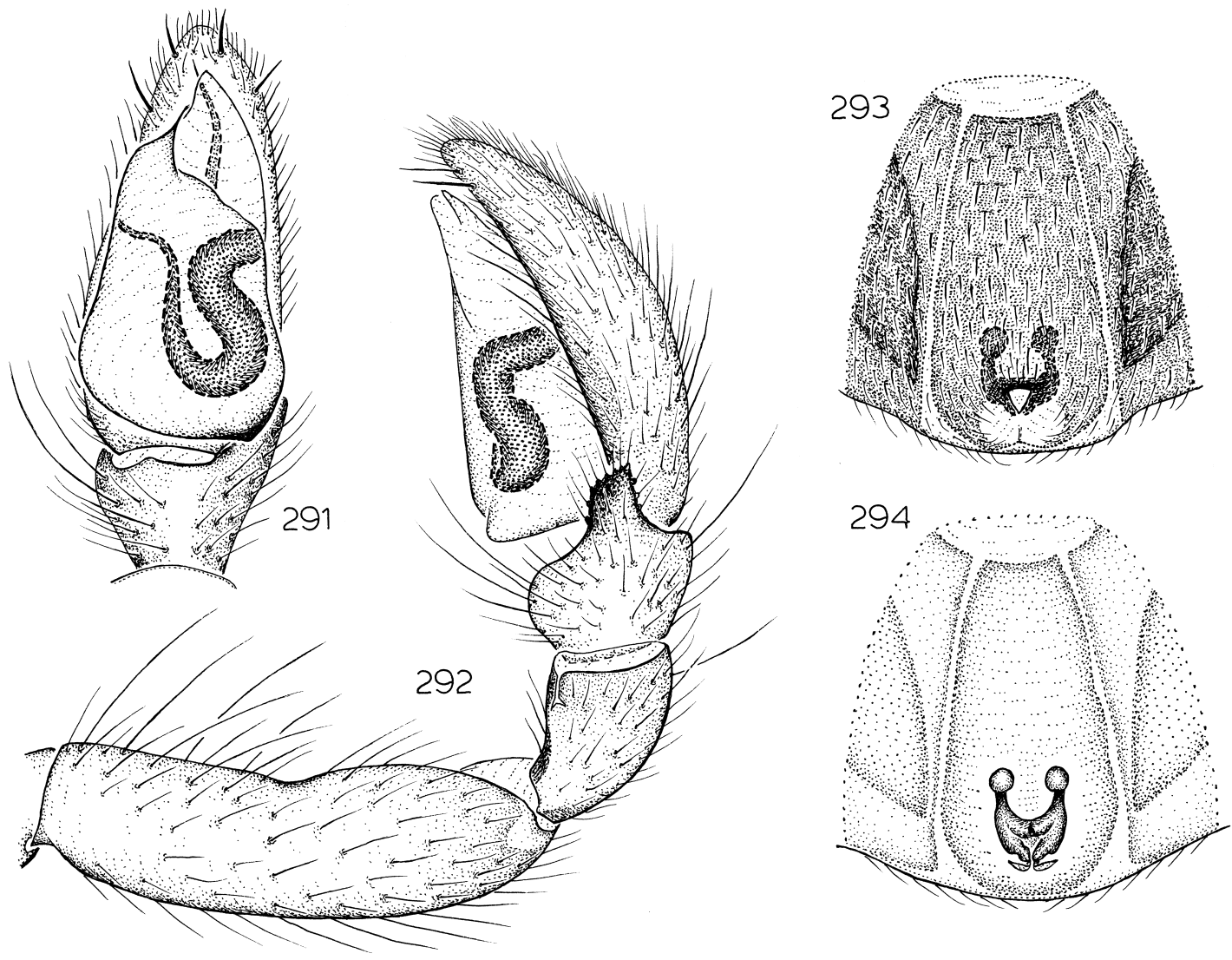

Figs. 291-294. Lamponina scutata (Strand). 291. Left male palp, ventral view. 292. Same, retrolateral view. 293. Epigynum, ventral view. 294. Same, dorsal view.

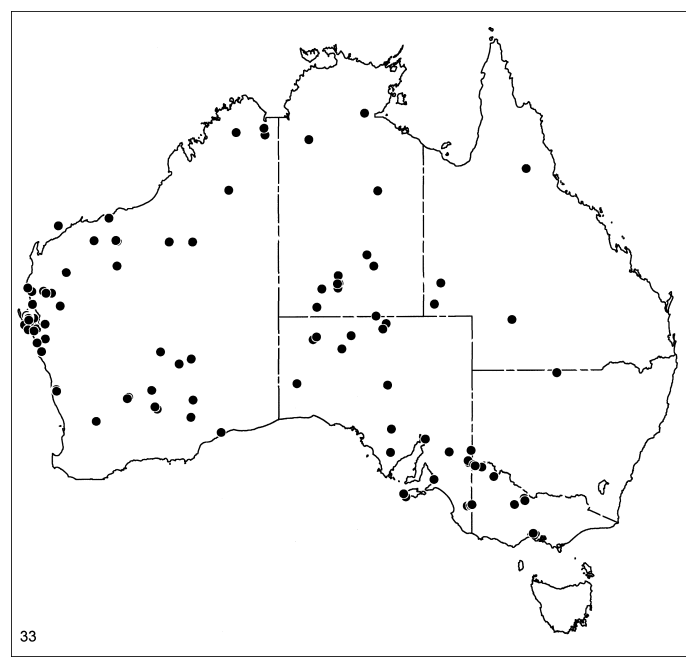

Map 33. Records of Lamponina scutata (Strand). ern Australia: Banjiwarn, $27^{\circ} 48^{\prime} \mathrm{S}, 121^{\circ} 41^{\prime} \mathrm{E}$, WAM 95/1135; Barlee Range Nature Reserve, $23^{\circ} 23^{\prime} \mathrm{S}, 115^{\circ} 53^{\prime} \mathrm{E}$, WAM 96/215; Barrow Island, $20^{\circ} 48^{\prime} \mathrm{S}, 115^{\circ} 24^{\prime} \mathrm{E}$, WAM 90/577; Bidgemia Station, $25^{\circ} 11^{\prime} \mathrm{S}, 115^{\circ} 29^{\prime} \mathrm{E}$, WAM 96/ 1219; Big Lagoon, $25^{\circ} 42^{\prime} \mathrm{S}, 113^{\circ} 27^{\prime} \mathrm{E}$, WAM 95/1148; Boodarie Hill, $20^{\circ} 24^{\prime} \mathrm{S}, 118^{\circ} 31^{\prime} \mathrm{E}$, WAM 95/1112; Boolathana Station, $24^{\circ} 25^{\prime} \mathrm{S}$, $113^{\circ} 41^{\prime} \mathrm{E}$, WAM 96/1141; Bungalbin Hill, $30^{\circ} 18^{\prime} \mathrm{S}, 119^{\circ} 43^{\prime} \mathrm{E}$, WAM 95/1153; Buningonia Spring, $31^{\circ} 27^{\prime} \mathrm{S}, 123^{\circ} 31^{\prime} \mathrm{E}$, WAM 95/1151; Bush Bay, $25^{\circ} 07^{\prime} \mathrm{S}, 113^{\circ} 48^{\prime} \mathrm{E}$, WAM 96/1215; Caiguna Tank, $32^{\circ} 17^{\prime} \mathrm{S}, 125^{\circ} 25^{\prime} \mathrm{E}$, WAM 56/ A77; Cape Cuvier, $24^{\circ} 12^{\prime} \mathrm{S}, 113^{\circ} 27^{\prime} \mathrm{E}$, WAM 96/1195; Cape Lesueur, $25^{\circ} 43^{\prime} \mathrm{S}, 113^{\circ} 25^{\prime} \mathrm{E}$, WAM 95/1132; Central Peron Peninsula, 26응' $\mathrm{S}, 113^{\circ} 38^{\prime} \mathrm{E}$, WAM 95/1150; Christmas Creek Station, $18^{\circ} 53^{\prime} \mathrm{S}, 125^{\circ} 55^{\prime} \mathrm{E}$, WAM 95/ 1130; Drysdale River Station, $15^{\circ} 42^{\prime} \mathrm{S}$, $126^{\circ} 23^{\prime} \mathrm{E}$, WAM 96/2210; Edel Land, $26^{\circ} 15^{\prime} \mathrm{S}$, 
$113^{\circ} 18^{\prime} \mathrm{E}$, WAM 95/1144; Emma Gorge, $15^{\circ} 50^{\prime} \mathrm{S}, 128^{\circ} 10^{\prime} \mathrm{E}$, WAM 95/1149; Eneabba, $29^{\circ} 50^{\prime} \mathrm{S}, 115^{\circ} 15^{\prime} \mathrm{E}$, WAM 96/746; Faure Island, $25^{\circ} 53^{\prime} \mathrm{S}, 113^{\circ} 53^{\prime} \mathrm{E}$, WAM 95/1136; Francois Peron National Park, $25^{\circ} 49^{\prime} \mathrm{S}, 113^{\circ} 32^{\prime} \mathrm{E}$, WAM 96/1207; Goongarrie, 29 $54^{\prime} \mathrm{S}$, $121^{\circ} 10^{\prime} \mathrm{E}$, WAM 95/1138; Helena-Aurora Ranges, $30^{\circ} 23^{\prime} \mathrm{S}, 119^{\circ} 38^{\prime} \mathrm{E}$, WAM 96/1151; Hope Downs, $23^{\circ} 02^{\prime} \mathrm{S}, 119^{\circ} 01^{\prime} \mathrm{E}$, WAM 96/ 1576; Kalbarri National Park, $27^{\circ} 45^{\prime} \mathrm{S}$, $114^{\circ} 21^{\prime} \mathrm{E}$, WAM 96/230; Kalgoorlie, $30^{\circ} 58^{\prime} \mathrm{S}$, $121^{\circ} 28^{\prime} \mathrm{E}$, WAM 94/1505; Kellerberrin, $31^{\circ} 38^{\prime} \mathrm{S}, 117^{\circ} 43^{\prime} \mathrm{E}$, WAM 96/219; Kennedy Range National Park, $24^{\circ} 30^{\prime} \mathrm{S}, 115^{\circ} 02^{\prime} \mathrm{E}$, WAM 96/1188; Kurrawang Reserve, $30^{\circ} 50^{\prime} \mathrm{S}$, $121^{\circ} 20^{\prime} \mathrm{E}$, WAM 96/226; Lake Auld, $21^{\circ} 44^{\prime} \mathrm{S}$, $123^{\circ} 40^{\prime} \mathrm{E}$, WAM 95/1147; Laverton, $28^{\circ} 28^{\prime} \mathrm{S}$, $122^{\circ} 50^{\prime} \mathrm{E}$, WAM 95/1163; Mardathuna Station, $24^{\circ} 24^{\prime} \mathrm{S}, 114^{\circ} 27^{\prime} \mathrm{E}$, WAM 96/1222; Nanga Station, $26^{\circ} 29^{\prime} \mathrm{S}, 114^{\circ} 03^{\prime} \mathrm{E}$, WAM 96/1138; Nerren Nerren Station, $27^{\circ} 00^{\prime} \mathrm{S}, 114^{\circ} 32^{\prime} \mathrm{E}$, WAM 96/1220; Point Salvation, $28^{\circ} 12^{\prime} \mathrm{S}$, $123^{\circ} 36^{\prime}$ E, WAM 96/1572; Red Sands, $28^{\circ} 12^{\prime} \mathrm{S}, 123^{\circ} 35^{\prime} \mathrm{E}$, WAM 96/223; Queen Victoria Spring, $30^{\circ} 28^{\prime} \mathrm{S}, 123^{\circ} 41^{\prime} \mathrm{E}$, WAM 95/ 1154; Tambrey Station, $21^{\circ} 38^{\prime} \mathrm{S}, 117^{\circ} 36^{\prime} \mathrm{E}$, WAM 95/1124; Telfer, $21^{\circ} 43^{\prime} \mathrm{S}, 122^{\circ} 14^{\prime} \mathrm{E}$, WAM 96/231; Woodleigh Station, 26 $12^{\prime}$ S, $114^{\circ} 31^{\prime}$ E, WAM 96/1179; Woodstock Station, $21^{\circ} 37^{\prime} \mathrm{S}, 118^{\circ} 58^{\prime} \mathrm{E}$, WAM 95/1141; Wyndham, $15^{\circ} 28^{\prime} \mathrm{S}, 128^{\circ} 06^{\prime} \mathrm{E}, \mathrm{CAS}$; Zuytdorp, $27^{\circ} 16^{\prime} \mathrm{S}$, $114^{\circ} 04^{\prime} \mathrm{E}$, WAM 96/1214.

Distribution: Western, central, and drier parts of eastern Australia (map 33).

NATURAL History: Adult males have been collected year round, females in all months except June and July, both sexes most commonly in November. Specimens have been taken in open forest and in acacia, brigalow, casuarina, eucalypt, mallee, mulga, and triodia vegetation, on the ground and sand (including dunes), under an airfield runway marker, from a straight, webbed burrow in a sandy creek bed, by wet and dry pitfall, drift fence, and intercept traps, and by vibration.

\section{Lamponina asperrima (Hickman), new combination \\ Figures 295-298; Map 34}

Lampona asperrima Hickman, 1950: 11, f. 11 (male and juvenile female syntypes from Reevesby Island, South Australia, in NMV, examined).
DiagnOsis: This species closely resembles L. scutata, but males lack the oblique constriction at roughly half the length of the ventral surface of the palpal femur, have a less protuberant distoventral edge on the retrolateral tibial apophysis (fig. 296), and have a shorter conductor (fig. 295); females have relatively small spermathecae, with medially directed ducts budding off relatively long stalks (fig. 298).

MALE: Total length 7.7. Abdominal dorsum with median transverse white stripe with anteromedian longitudinal extension, situated at about half of abdominal length, narrowest near middle, extended anteriorly and posteriorly on sides of abdomen (stripe often reduced to single median and pair of lateral white spots); additional white spot above spinnerets; coxae and trochanters dark orange, anteriors darkest; anterior femora I, II dark reddish-black, posterior femora orange, remainder of legs yellow. Palpal femur without ventral invagination; tibia cup-shaped, with distally directed, sloping, thumb-shaped retrolateral apophysis, ventral edge of apophysis with enlarged setal bases (fig. 296); tegulum with pronounced posterolateral corner, embolus short, situated medially, conductor short (fig. 295).

Female: Total length 8.5. Coloration as in male. Epigynal midpiece anteriorly arched (fig. 297); spermathecae small, on short bases (fig. 298).

Material Examined: South Australia: Arcoona Creek, 2 km NE Owieandana, Gammon Ranges National Park, $30^{\circ} 26^{\prime} \mathrm{S}$, $138^{\circ} 58^{\prime} \mathrm{E}$, May 6, 1989, pitfall (J. Forrest, SAM N1996/278), 10; Eyre Peninsula, no specific locality, Sept. (J. Sutton, SAM N1996/268), 19 ; Kolay Hut, 32 33'S, $135^{\circ} 36^{\prime}$ E, Dec. $8-11,1989$, pitfall (D. Hirst, SAM N1996/324-327), 40; Lake Gilles Conservation Park, $33^{\circ} 05^{\prime} \mathrm{S}, 136^{\circ} 39^{\prime} \mathrm{E}$, Nov. 20-22, 1995, pitfall (D. Hirst, J. Forrest, SAM N1997/55), 10; 22.5 km SW Loxton, $34^{\circ} 33^{\prime} \mathrm{S}, 140^{\circ} 22^{\prime} \mathrm{E}$, May 9, 1991, pitfall (A. McArthur, SAM N1996/266), 1 , Sept. 1991, pitfall (A. McArthur, SAM N1996/ 265), 1 ㅇ $3 \mathrm{~km}$ SSW Mongolata, $33^{\circ} 37^{\prime} \mathrm{S}$, $139^{\circ} 05^{\prime} \mathrm{E}$, Sept. 28-Oct. 31, 1992, pitfall (South Olary Plain Survey, SAM N1996/ 322), 10; N Mount Woodroffe, $26^{\circ} 16^{\prime} \mathrm{S}$, $131^{\circ} 45^{\prime} \mathrm{E}$, Oct. 21,1994 , Eucalyptus socialis 

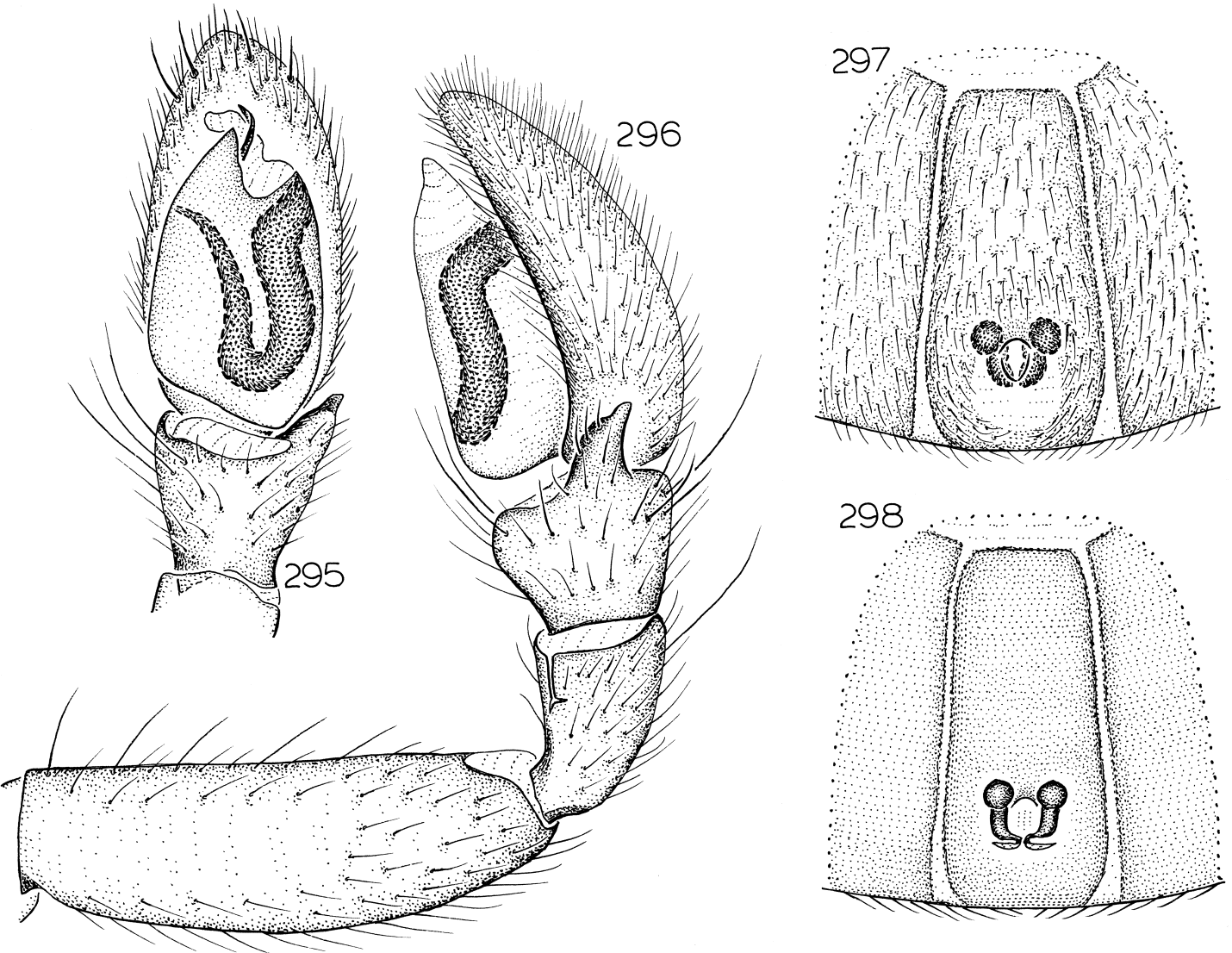

Figs. 295-298. Lamponina asperrima (Hickman). 295. Left male palp, ventral view. 296. Same, retrolateral view. 297. Epigynum, ventral view. 298. Same, dorsal view.

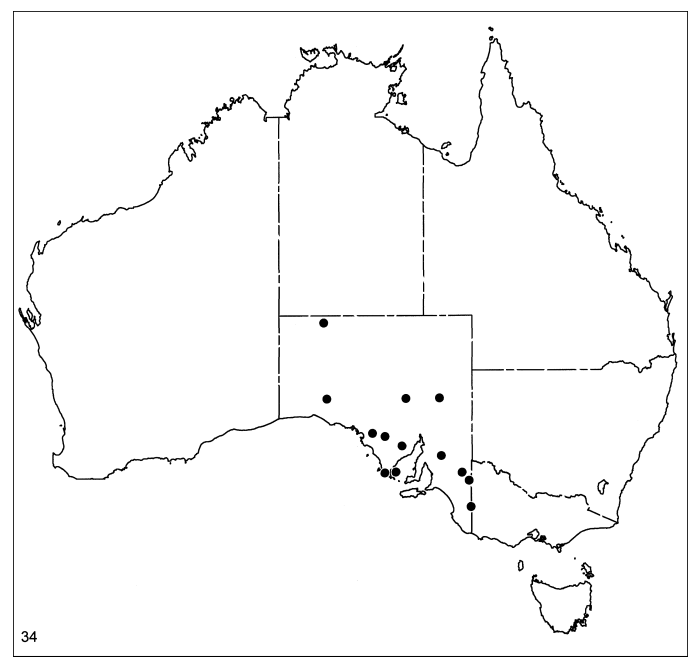

Map 34. Records of Lamponina asperrima (Hickman). area, vehicle vibration (D. Hirst, SAM N1996/269), $10^{\text {` }} 7$ km NE Mount Woodroffe, $26^{\circ} 17^{\prime} \mathrm{S}, 131^{\circ} 48^{\prime} \mathrm{E}$, Oct. $13-17$, 1994 , pitfall (J. Forrest, SAM N1996/270), $19 ; 8$ km NNE Mount Woodroffe, $26^{\circ} 16^{\prime} \mathrm{S}, 131^{\circ} 47^{\prime} \mathrm{E}$, Oct. 13-17, 1994, pitfall (J. Forrest, SAM N1996/271), 10ิ; Murrunata Conservation Park, $34^{\circ} 35^{\prime} \mathrm{S}, 135^{\circ} 35^{\prime} \mathrm{E}$, Mar. 29, 1987, leaf litter, roadside vegetation (D. Lee, D. Hirst, SAM N1996/262), 10; Olympic Dam site, Roxby Downs, $30^{\circ} 27^{\prime} \mathrm{S}, 136^{\circ} 53^{\prime} \mathrm{E}$, Mar.June 1987, pitfall (A. Smith, SAM N1996/ 272), 1 ô, Nov. 3-5, 1987 (E. Matthews, C. Watts, SAM N1996/273-276), 40; 13 km E Ooldea, $30^{\circ} 29^{\prime} \mathrm{S}, 131^{\circ} 58^{\prime} \mathrm{E}$, Sept. 30, 1988 (D. Hirst, SAM N1996/277), 1 đ̊; Peebinga, $34^{\circ} 59^{\prime} \mathrm{S}, 140^{\circ} 49^{\prime} \mathrm{E}$, Oct. 6-8, 1990 (Strathalbyn Field Naturalists, SAM N1996/267), 1 \% ; $3.7 \mathrm{~km}$ NE Pooginagoric, $36^{\circ} 27^{\prime} \mathrm{S}, 140^{\circ} 55^{\prime} \mathrm{E}$, 

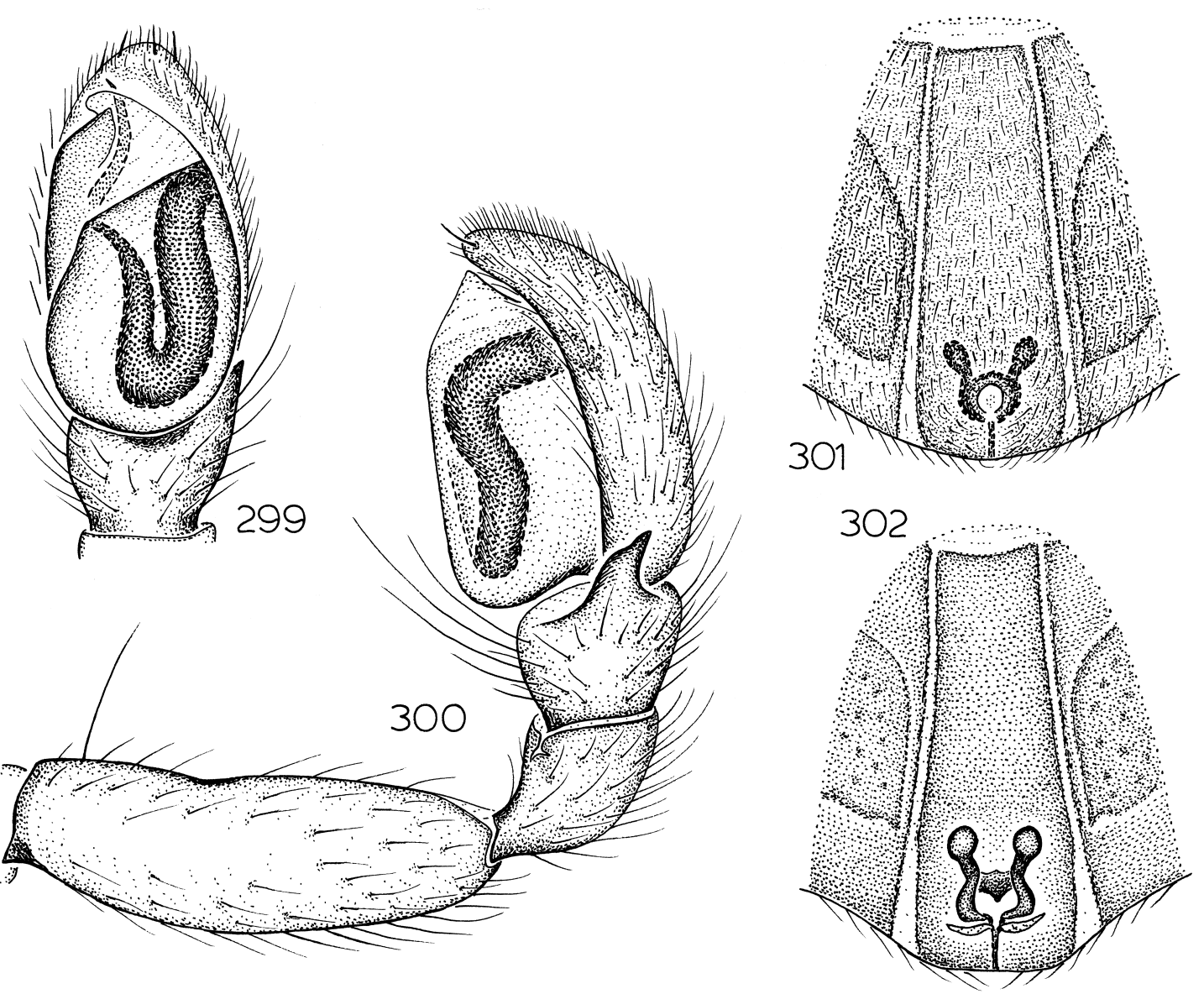

Figs. 299-302. Lamponina elongata, new species. 299. Left male palp, ventral view. 300. Same, retrolateral view. 301. Epigynum, ventral view. 302. Same, dorsal view.

Dec. 5-8, 1995, pitfall (SAM 1997/56), 1 웅 Reevesby Island, $34^{\circ} 32^{\prime} \mathrm{S}, 136^{\circ} 16^{\prime} \mathrm{E}$, Dec. 1936 (J. Clark, NMV K149), 1 đ̊ (syntype); Conservation Park NE Wirrulla, $32^{\circ} 23^{\prime} \mathrm{S}$, $134^{\circ} 49^{\prime}$ E, Nov. 25-26, 1995, pitfall (D. Hirst, SAM N1997/57-60), 40.

DistRIBUTION: Known only from South Australia (map 34).

\section{Lamponina elongata, new species} Figures 299-302; Map 35

TYPE: Female holotype taken in pitfall trap in Francois Peron National Park, $25^{\circ} 50^{\prime} \mathrm{S}$, $113^{\circ} 36^{\prime}$ E, Western Australia (Oct. 12, 1994Jan. 18, 1995; N. McKenzie, J. Rolfe), deposited in WAM (96/1254).

ETYMOLOGY: The specific name refers to the elongated pedicel.
Diagnosis: Specimens can easily be distinguished from those of the other known Lamponina species by their relatively long pedicel, their relatively narrow, elongated fourth femora, and (usually) their relatively pale coloration (the legs are usually yellow, and the anterior half of the abdomen is usually white). Males resemble those of $L$. scutata in having an oblique constriction at roughly half the length of the ventral surface of the palpal femur, but have a ventrally less protuberant retrolateral tibial apophysis (figs. 299, 300). The female spermathecae are relatively long, and are often anteriorly divergent (figs. 301, 302).

NotE: The males available from Victoria and the Northern Territory have the oval sclerites behind the epigastric furrow greatly 
enlarged, equalling in length about twothirds the length of the epigastric scutum; the palps, however, seems normal for the species, and one male from South Australia (Yarna Station) has an intermediate condition, with moderately enlarged sclerites.

MALE: Total length 8.3. Dorsum of abdomen white, with transverse gray stripe at about two-thirds its length, stripe extended posteriorly around posterior white spot, remainder of sides and venter white; coxae orange, other leg segments yellow. Palpal femur with slight ventral invagination at about half its length; tibia globose, with dorsodistally directed, thumb-shaped retrolateral apophysis, ventral edge of apophysis receding rather than protuberant (fig. 299); tegulum with pronounced posterolateral corner, embolus relatively short, evenly arched (fig. 298).

Female: Total length 9.4. Coloration as in male. Epigynal midpiece relatively wide (fig. 300); spermathecae small, on long bases (fig. $301)$.

Other MAterial ExAmined: Northern Territory: Kathleen Springs Track, Watarrka National Park, $24^{\circ} 21^{\prime} \mathrm{S}, 131^{\circ} 41^{\prime} \mathrm{E}$, Oct. 1994 , pitfalls, casuarina (NMV K4672), 7o; Watarrka National Park, sand dunes W workshops, $24^{\circ} 19^{\prime} \mathrm{S}, 131^{\circ} 32^{\prime} \mathrm{E}$, pitfalls (NMV K4670), 30 . South Australia: Anna Creek

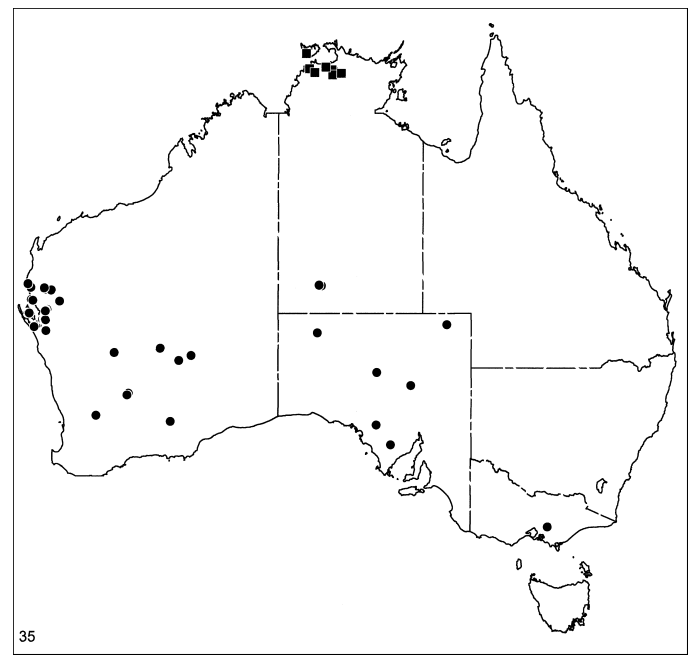

Map 35. Records of Lamponina elongata, new species (circles) and L. kakadu, new species (squares).
Station, $18.1 \mathrm{~km}$ W Backadinna Dam, $29^{\circ} 07^{\prime} \mathrm{S}, 135^{\circ} 09^{\prime} \mathrm{E}$, pitfall (SAM N1997/54), $10^{\top}$; Clifton Hills Outstation, $26^{\circ} 30^{\prime} \mathrm{S}$, $139^{\circ} 29^{\prime} \mathrm{E}$, Nov. 18-20, 1993, pitfall, sand dune with triodia (J. Forrest, D. Hirst, SAM N1996/282), 1 9 ; S shore, Lake Eyre, $29^{\circ} 50^{\prime} \mathrm{S}, 137^{\circ} 15^{\prime} \mathrm{E}$, May 1953 (K. PeakeJones, SAM N1996/283), 19; $21.5 \mathrm{~km} \mathrm{E}$ Maryinna Hill, $26^{\circ} 57^{\prime} \mathrm{S}, 131^{\circ} 26^{\prime} \mathrm{E}$, Mar. $14-$ 18, 1995, pitfall (SAM N1996/447), 1 웅 Pickawillinie Conservation Park, $33^{\circ} 07^{\prime} \mathrm{S}$, $136^{\circ} 00^{\prime}$ E, May 21, 1990 (D. Hirst, SAM N1996/281), 19; $1 \mathrm{~km} \mathrm{~N} \mathrm{Yarna} \mathrm{Station,}$ $32^{\circ} 02^{\prime} \mathrm{S}, 135^{\circ} 07^{\prime} \mathrm{E}$, Sept. 26, 1988 (D. Hirst, SAM 1996/328), 1 o. Victoria: $0.7 \mathrm{~km} \mathrm{~N}$ Acheron Gap, $7 \mathrm{~km}$ NE Mount Donna Buang, $37^{\circ} 40^{\prime} \mathrm{S}, 145^{\circ} 44^{\prime} \mathrm{E}$, Oct. 26-Dec. 28, 1995, pitfall, eucalypt forest (G. Milledge, NMV K4661), 10. Western Australia: 11 $\mathrm{km}$ ENE Anketell Homestead, $28^{\circ} 02^{\prime} \mathrm{S}$, $118^{\circ} 51^{\prime} \mathrm{E}$, Sept. 4-6, 1981, on ground at night (T. Houston, WAM 96/245), $1 \delta^{\star}$; Banjiwarn, $27^{\circ} 48^{\prime} \mathrm{S}, 121^{\circ} 41^{\prime} \mathrm{E}$, May 26-31, 1979, mulga, eremophila, bunchgrass (R. How, WAM 96/244), 1 \% ; Bidgemia Station, Gasgoyne Junction, $25^{\circ} 11^{\prime} \mathrm{S}, 115^{\circ} 29^{\prime} \mathrm{E}$, Aug. 17-Oct. 4, 1994, pitfall (A. Sampey, WAM 96/1258), 1 ㅇ, June 4-Aug. 20, 1995, pitfall (N. Hall, WAM 96/1259), $10^{\text {đ; }}$ Boolathana Station, $24^{\circ} 25^{\prime} \mathrm{S}, 113^{\circ} 40^{\prime} \mathrm{E}$, Sept. 30, $1994-$ Jan. 15, 1995, pitfalls (N. McKenzie, J. Rolfe, WAM 96/1261, 1262), 20; Boolathana Station, $24^{\circ} 25^{\prime} \mathrm{S}, 113^{\circ} 41^{\prime} \mathrm{E}$, Aug. 20Sept. 30, 1994, pitfalls (A. Sampey, WAM 96/1295-1303), 8ㅊ, 1ㅇ, Sept. 27-Oct. 2, 1994, dry pitfall (A. Sampey, WAM 96/ 1142-1145), 3o, 1 ㅇ, Sept. 30, 1994-Jan. 15, 1995, pitfalls (N. McKenzie, J. Rolfe, WAM 96/1263-1269), 60ิ, 1 ㅇ, Jan. 15-May 30, 1995, pitfalls (J. Waldock, WAM 96/ 1270-1272), 3ơ; Bungalbin Hill, 30 $17^{\prime} \mathrm{S}$, $119^{\circ} 43^{\prime} \mathrm{E}$, Dec. 1981 , pitfall, tree mallee ecotone (W. Humphreys, et al., WAM 96/248), 1 ; ; Bush Bay, $25^{\circ} 05^{\prime} \mathrm{S}, 113^{\circ} 43^{\prime} \mathrm{E}$, Sept. $27-$ Oct. 2, 1994, dry pitfalls (M. Harvey, WAM

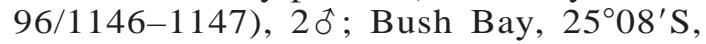
$113^{\circ} 49^{\prime}$ E, Sept. 27-Oct. 2, 1994, dry pitfalls (M. Harvey, WAM 96/1149), 1 to; $^{-}$Cape Cuvier, Quobba Station, $24^{\circ} 13^{\prime} \mathrm{S}, 113^{\circ} 30^{\prime} \mathrm{E}$, Aug. 21-Sept. 28, 1994, pitfall (P. West, WAM 96/1160), 19 ; Durokoppin Nature Reserve, $31^{\circ} 30^{\prime} \mathrm{S}, 117^{\circ} 44^{\prime} \mathrm{E}$, Nov. 1-13, 1987 (D. Mitchell et al., WAM 96/249), 1 ơ; Fran- 
cois Peron National Park, $25^{\circ} 49^{\prime} \mathrm{S}, 113^{\circ} 32^{\prime} \mathrm{E}$, Aug. 24-Oct. 11, 1994, pitfalls (A. Sampey, WAM 96/1238-1240), 30 , Oct. 11, 1994Jan. 18, 1995, pitfalls (N. McKenzie, J. Rolfe, WAM 96/1242-1244), 20, 1 \%, Jan. 18-May 24, 1995, pitfalls (M. Harvey, WAM 96/1250-1253), 40; Helena-Aurora Ranges, $30^{\circ} 23^{\prime} \mathrm{S}, 119^{\circ} 38^{\prime} \mathrm{E}$, Sept. 26, 1995, pitfall (R. McMillan, WAM 96/247), 10; Kennedy Range National Park, $24^{\circ} 30^{\prime} \mathrm{S}$, $115^{\circ} 02^{\prime} \mathrm{E}$, Aug. 16-Oct. 6, 1994, pitfall (M. Harvey, WAM 96/1241), 1 ô, Oct. 3-8, 1994, dry pitfall (M. Harvey, WAM 96/ 1260), $1 \delta^{t}$; Kennedy Range National Park, $24^{\circ} 31^{\prime} \mathrm{S}, 114^{\circ} 58^{\prime} \mathrm{E}$, Oct. 3-8, 1994, dry pitfalls (M. Harvey, WAM 96/1157, 1158), $20^{\star}$; Kennedy Range National Park, 24 $33^{\prime} \mathrm{S}$, $114^{\circ} 58^{\prime} \mathrm{E}$, Oct. 3-8, 1994, dry pitfalls (M. Harvey, WAM 96/1134-1136), 30, Oct. 4, 1994-Jan. 14, 1995, pitfalls (N. McKenzie, J. Rolfe, WAM 96/1255-1257), 2 ๙ , 1 ㅇ , Jan. 14-May 29, 1995, pitfall (P. West, WAM 96/ 1245), 1 \%; Kennedy Range National Park, $24^{\circ} 33^{\prime} \mathrm{S}, 114^{\circ} 58^{\prime} \mathrm{E}$, Aug. 18-Oct. 4, 1994, pitfalls (M. Harvey, WAM 96/1163, 1164), $20^{\top}$; Kennedy Range National Park, 24 34'S, $114^{\circ} 57^{\prime} \mathrm{E}$, Oct. 3-8, 1994, dry pitfalls (M. Harvey, WAM 96/1246-1249), 40, May 29-Aug. 28, 1995, pitfall (N. Hall, WAM 96/ 1189), $19 ; 39 \mathrm{~km}$ E Laverton, $28^{\circ} 28^{\prime} \mathrm{S}$, $122^{\circ} 50^{\prime} \mathrm{E}$, Dec. 22-Jan. 15, 1990-1991, pitfalls (E. Pianka, WAM 96/253-256), 40; Mardathuna Station, $24^{\circ} 24^{\prime} \mathrm{S}, 114^{\circ} 27^{\prime} \mathrm{E}$, Aug. 19-Oct. 5, 1994, pitfall (P. West, WAM

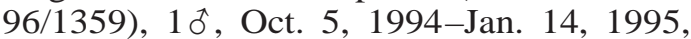
pitfalls (N. McKenzie, J. Rolfe, WAM 96/ 1360, 1361), 2 ô, Apr. 6-May 24, 1995, pitfall (A. Sampey, WAM 96/1349), $1 \delta^{\hat{\sigma}}$, May 24-Aug. 28, 1995, pitfalls (N. Hall, WAM 96/1362-1364), 3 \% ; Mardathuna Station, $24^{\circ} 24^{\prime} \mathrm{S}, 114^{\circ} 28^{\prime} \mathrm{E}$, Aug. 19-Oct. 5, 1994, pitfall (P. West, WAM 96/1345), 1 o $^{\uparrow}$, Oct. 38, 1994, dry pitfalls (P. West, WAM 96/ 1346-1348), 3 oे, Oct. 5, 1994-Jan. 14, 1995, pitfalls (N. McKenzie, J. Rolfe, WAM 96/ 1371, 1372), 1 ऽ, 1오, Jan. 14-May 24, 1995, pitfalls (A. Sampey, WAM 96/1365-1368), $20^{\circ}, 2+$; Mardathuna Station, $24^{\circ} 26^{\prime} \mathrm{S}$, $114^{\circ} 31^{\prime} \mathrm{E}$, Aug. 19-Oct. 5, 1994, pitfalls (P. West, WAM 96/1373-1375), 30े, Oct. 5, 1994-Jan. 14, 1995, pitfall (N. McKenzie, J. Rolfe, WAM 96/1377), 10, May 24-Aug. 26, 1995, pitfalls (N. Hall, WAM 96/1369,
1370), $1 \delta^{\dagger}, 1$ q ; Mardathuna Station, $24^{\circ} 27^{\prime} \mathrm{S}$, $114^{\circ} 31^{\prime} \mathrm{E}$, Oct. 3-8, 1994, dry pitfall (P. West, WAM 96/1376), 1 ô, Oct. 5, 1994-Jan. 14, 1995, pitfall (N. McKenzie, J. Rolfe, WAM 96/1350), 10̃; Mardathuna Station, $24^{\circ} 31^{\prime}$ S, $114^{\circ} 38^{\prime} \mathrm{E}$, Aug. 19-Oct. 5, 1994, pitfalls (P. West, WAM 96/1181-1187), 60, 1 , Oct. 3-8, 1994, dry pitfalls (P. West, WAM 96/1165-1178), 13 \%, 1 우 ; Meedo Station, $25^{\circ} 37^{\prime} \mathrm{S}, 114^{\circ} 42^{\prime} \mathrm{E}$, Oct. 9-14, 1994, dry pitfall (P. West, WAM 96/1159), 1 ; ; Meedo Station, $25^{\circ} 38^{\prime} \mathrm{S}, 14^{\circ} 42^{\prime} \mathrm{E}$, Oct. 11 , 1994-Jan. 12, 1995, pitfall (N. McKenzie, J. Rolfe, WAM 96/1190), 19 ; Meedo Station, $25^{\circ} 41^{\prime} \mathrm{S}, 114^{\circ} 37^{\prime} \mathrm{E}$, Jan. 12-May 18, 1995, pitfalls (A. Sampey, WAM 96/1328, 1329), $10^{\circ}, 1$ \% ; Meedo Station, $25^{\circ} 43^{\prime} \mathrm{S}, 114^{\circ} 36^{\prime} \mathrm{E}$, Oct. 9-14, 1994, dry pitfalls (P. West, WAM 96/1330-1336), 7ð, Oct. 11, 1994-Jan. 12, 1995, pitfalls (N. McKenzie, J. Rolfe, WAM 96/1351-1358), 5ô, 3 q , Jan. 12-May 19, 1995, pitfalls (A. Sampey, WAM 96/1237, 1339-1143), 4o, 2으. May 19-Aug. 22, 1995, pitfalls (N. Hall, WAM 96/13181322), 4ô, 1 으, Aug. 22-Oct. 11, 1995, pitfalls (P. West, WAM 96/1323-1327), 40ิ, 1 ; Nanga Station, $26^{\circ} 29^{\prime} \mathrm{S}, 114^{\circ} 05^{\prime} \mathrm{E}$, May 11-15, 1995, dry pitfall (A. Sampey, WAM 96/1274), $10^{\top}$; Nanga Station, $26^{\circ} 33^{\prime} \mathrm{S}$, $113^{\circ} 58^{\prime} \mathrm{E}$, Oct, 16, 1994-Jan. 19, 1995, pitfall (N. McKenzie, J. Rolfe, WAM 96/1275), 10; Nanga Station, $26^{\circ} 36^{\prime} \mathrm{S}, 113^{\circ} 53^{\prime} \mathrm{E}$, Jan. 19-May 11, 1995, pitfalls (A. Sampey, WAM 96/1276-1278), 10, 2ㅇ, May 11Aug. 30, 1995, pitfall (N. Hall, WAM 96/ 1273), 1 $\delta^{\dagger} ; 7-8 \mathrm{~km}$ WNW Point Salvation, $28^{\circ} 12^{\prime} \mathrm{S}, 123^{\circ} 36^{\prime} \mathrm{E}$, Nov. 8-10, 1990, pitfall (E. Pianka, WAM 96/252), 1 9, Feb. 25-Mar. 5, 1991, pitfalls (E. Pianka, WAM 96/250, 251), $20^{\circ} ; 13 \mathrm{~km} \mathrm{~S}$ Wannoo, $26^{\circ} 49^{\prime} \mathrm{S}$, $114^{\circ} 37^{\prime} \mathrm{E}$, Aug. 24-28, 1984, pitfall, interdune swale (T. Houston, B. Hanich, WAM 96/243), 10; Woodleigh Station, $26^{\circ} 12^{\prime} \mathrm{S}$, $114^{\circ} 25^{\prime} \mathrm{E}$, Oct. 9-14, 1994, dry pitfalls (M. Harvey, WAM 96/1312, 1313), 20; Woodleigh Station, $26^{\circ} 12^{\prime} \mathrm{S}, 114^{\circ} 31^{\prime} \mathrm{E}$, Oct. 10, 1994-Jan. 12, 1995, pitfalls (N. McKenzie, J. Rolfe, WAM 96/1287, 1288), 20, Jan. 12May 17, 1995, pitfalls (P. West, WAM 96/ 1316, 1317), 10े, 1 , May 17-Aug. 21, 1995, pitfalls (N. Hall, WAM 96/1307, 1344), $20^{\star}$; Woodleigh Station, $26^{\circ} 12^{\prime} \mathrm{S}$, $114^{\circ} 32^{\prime} \mathrm{E}$, Aug. 22-Oct. 9, 1994, pitfalls (M. 
Harvey, WAM 96/1308-1311), 40ิ, Oct. 914, 1994, dry pitfall (M. Harvey, WAM 96/ 1315), 10, May 17-Aug. 21, 1995, pitfalls (N. Hall, WAM 96/1289-1294), 30, 3 \%; Woodleigh Station, $26^{\circ} 13^{\prime} \mathrm{S}, 114^{\circ} 35^{\prime} \mathrm{E}$, Oct. 9-14, 1994, dry pitfalls (M. Harvey, WAM 96/1304-1306), 20, 1 9 , May 17-Aug. 21, 1995, pitfall (N. Hall, WAM 96/1314), 1 ơ; Woodleigh Station, $26^{\circ} 13^{\prime} \mathrm{S}, 114^{\circ} 36^{\prime} \mathrm{E}$, Aug. 22-Oct. 11, 1994, pitfalls (M. Harvey, WAM 96/1337, 1338), 2 đે, Oct. 11, 1994-Jan. 12, 1995, pitfalls (N. McKenzie, J. Rolfe, WAM 96/1280-1286), 3ô, 4, May 17-Aug. 21, 1995, pitfall (N. Hall, WAM 96/1279), 1 đ; Woodline, $31^{\circ} 50^{\prime} \mathrm{S}, 122^{\circ} 19^{\prime} \mathrm{E}$, Aug. 1980, mallee, triodia (W. Humphreys et al., WAM 96/246), 1 ㅇ․ $35)$.

Distribution: Southern Australia (map

\section{Lamponina kakadu, new species}

Figures 303-306; Map 35

TyPES: Male holotype from Kapalga, Kakadu National Park, Northern Territory, $12^{\circ} 36^{\prime} \mathrm{S}, 132^{\circ} 25^{\prime} \mathrm{E}$ (Apr. 28, 1992; A. Andersen et al.), deposited in WAM (96/257), and female allotype from Kapalga, Kakadu National Park, Northern Territory, $12^{\circ} 29^{\prime} \mathrm{S}$, $132^{\circ} 19^{\prime} \mathrm{E}$ (Dec. 17, 1986; A. Andersen et al.), deposited in WAM (96/258).

ETYMOLOGY: The specific name is a noun in apposition taken from the type locality.

DiAGNOSIS: Males can easily be separated from those of all other known Lamponina species by the large, ventral expansion on the palpal femur (fig. 304); females resemble those of L. asperrima in having a squared epigynal midpiece, but have that midpiece proportionately much smaller (fig. 305).

MALE: Total length 7.2. Dorsum of abdomen gray, with transverse anterior and median white bands and posterior white spot; legs orange, femora I darkened. Palpal femur with slight ventral invagination at about half its length, invagination immediately behind distinct ventral expansion; tibia globose, with dorsodistally directed, sharply curved retrolateral apophysis, ventral edge of apophysis receding rather than protuberant (fig. 304); tegulum with greatly pronounced posterolateral corner, embolus relatively short, tip almost straight (fig. 303).
Female: Total length 8.9. Coloration as in male except dorsal abdominal white stripes reduced to paired spots. Epigynal midpiece small, squared (fig. 305); spermathecae small, on posteriorly expanded stalks (fig. 306).

Other MATERIAl EXAMined: Northern Territory: Berrimah, Darwin, $12^{\circ} 25^{\prime} \mathrm{S}$, $130^{\circ} 55^{\prime} \mathrm{E}$, May 7-Oct. 8, 1996, pitfall, lawn (T. Churchill, CSID, A0043, A0077, A0184, A0191, A0251-A0253, A0255, A0256, A0258), 90, 2 우 ; E Flying Fox Creek, Arnhem Highway, $12^{\circ} 45^{\prime} \mathrm{S}, 132^{\circ} 22^{\prime} \mathrm{E}$, July 4-8, 1977 (R. Pengilley, MNT T020), 1; Georgetown Billabong, $12^{\circ} 40^{\prime} \mathrm{S}, 132^{\circ} 55^{\prime} \mathrm{E}$, Aug. 8, 1975 (R. Pengilley, MNT T021), 1 o; Humpty Doo, $12^{\circ} 38^{\prime} \mathrm{S}, 131^{\circ} 15^{\prime} \mathrm{E}$, May 3, 1992 (Burns, MNT), $1 \%$; Jabiru, 12 40'S, $132^{\circ} 54^{\prime}$ E, July 5-9, 1977 (R. Pengilley, MNT T022), 10; Taracumbi Falls, near Melville Island, $11^{\circ} 36^{\prime} \mathrm{S}, 130^{\circ} 43^{\prime} \mathrm{E}$, Oct. 4-7, 1996 (P. Horner, MNT T024), $20^{\star}$; Wildman River, $12^{\circ} 20^{\prime} \mathrm{S}, 131^{\circ} 56^{\prime} \mathrm{E}$, Feb. 8-9, 1989, cashew plantation, pit light (M. Malipatil, W. Houston, MNT T026), 1 .

DisTRIBUTION: Known only from far northern Australia (map 35).

\section{Lamponina loftia, new species}

Figures 307-310; Map 36

TYPE: Male holotype taken in a pitfall trap in Loftia Recreation Park, Mount Lofty Ranges, South Australia, $35^{\circ} 02^{\prime} \mathrm{S}, 138^{\circ} 42^{\prime} \mathrm{E}$ (Mar. 20-27, 1990; D. Hirst), deposited in SAM (N1996/240).

ETYMOLOGY: The specific name is a noun in apposition taken from the type locality.

DiAGNOSIS: Members of this species can easily be separated from those of the other known Lamponina species by the presence of a distinctive dark, longitudinal stripe extending along the midline of the abdominal venter, from the epigastric furrow to the spinnerets. Males have an unmodified palpal femur and a low retrolateral tibial apophysis (fig. 308); females have the median epigynal ducts forming an m-shaped pattern (fig. 310).

MALE: Total length 8.4. Abdominal dorsum dark gray, with white markings usually restricted to tiny posterior white spot no wider than spinneret field; venter mottled gray, with dark, longitudinal stripe extending 

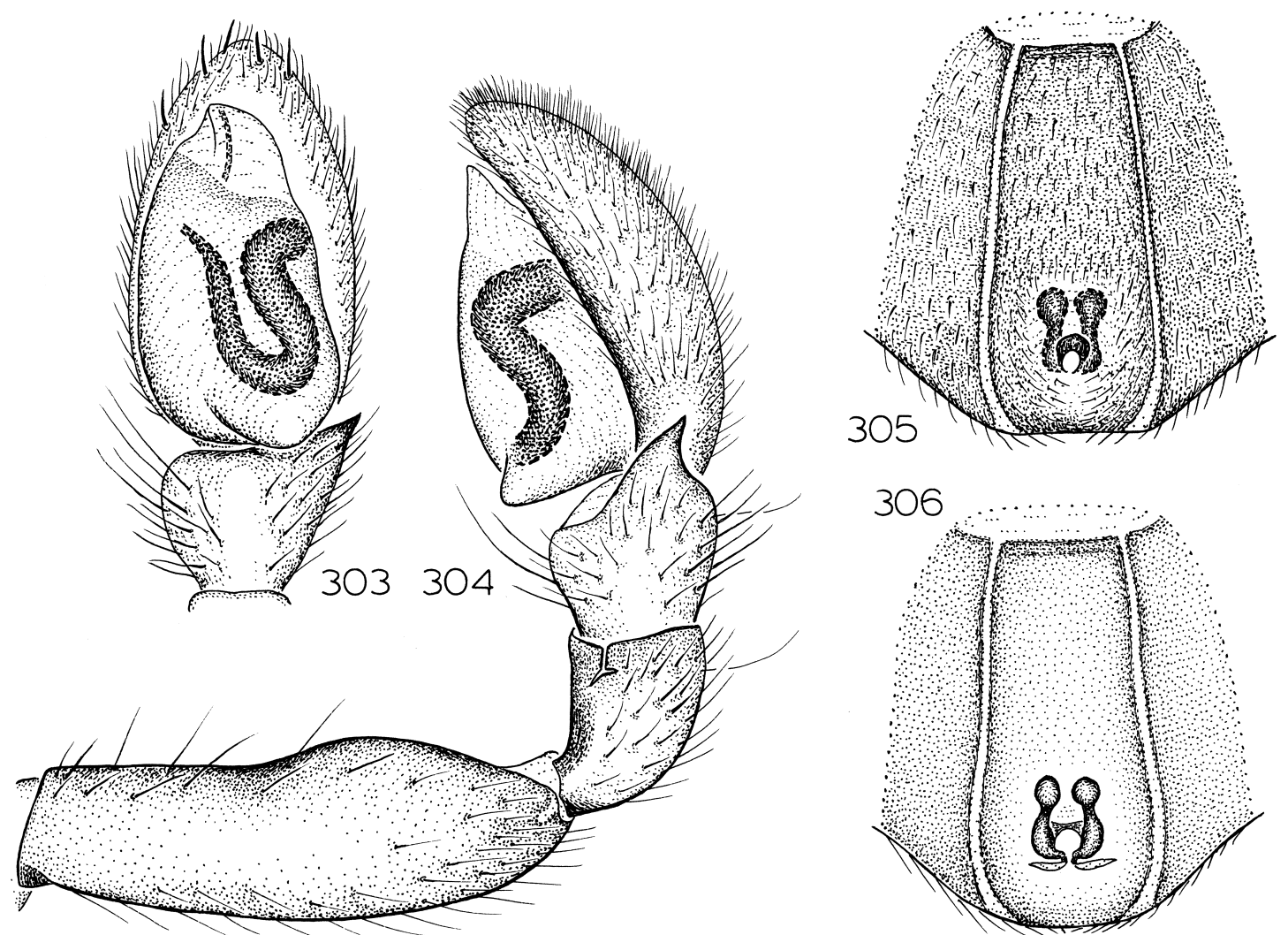

Figs. 303-306. Lamponina kakadu, new species. 303. Left male palp, ventral view. 304. Same, retrolateral view. 305. Epigynum, ventral view. 306. Same, dorsal view.

along midline from epigastric furrow to spinnerets; legs reddish-brown, with femora and sides of tibiae darkest. Palpal femur without ventral invagination; retrolateral tibial apophysis short, not protuberant ventrally (fig. 308); tegulum with pronounced posterolateral corner, embolus relatively short, tip abruptly bent at base (fig. 307).

Female: Total length 6.1. Coloration as in male. Epigynal midpiece shield-shaped (fig. 309); spermathecae small, on long, narrow stalks, median ducts forming $\mathrm{m}$-shaped pattern (fig. 310).

Other Material Examined: South Australia: SE Big Desolation, Gum Lagoon, $36^{\circ} 16^{\prime} \mathrm{S}, 139^{\circ} 59^{\prime} \mathrm{E}$, Mar. 25, 1992, vibration on stony ground (D. Hirst, SAM N1996/245247), $10^{*}, 29$; Bretag Scrub, Monarto Zoological Park, $35^{\circ} 07^{\prime} \mathrm{S}, 139^{\circ} 10^{\prime} \mathrm{E}$, Oct. $11-15$, 1993 (T. Morley, P. Goonan, SAM N1996/

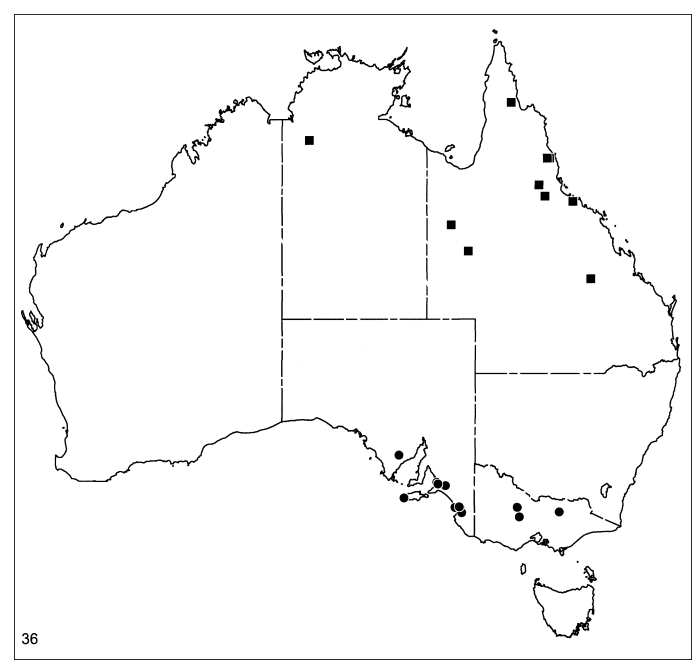

Map 36. Records of Lamponina loftia, new species (circles) and L. isa, new species (squares). 

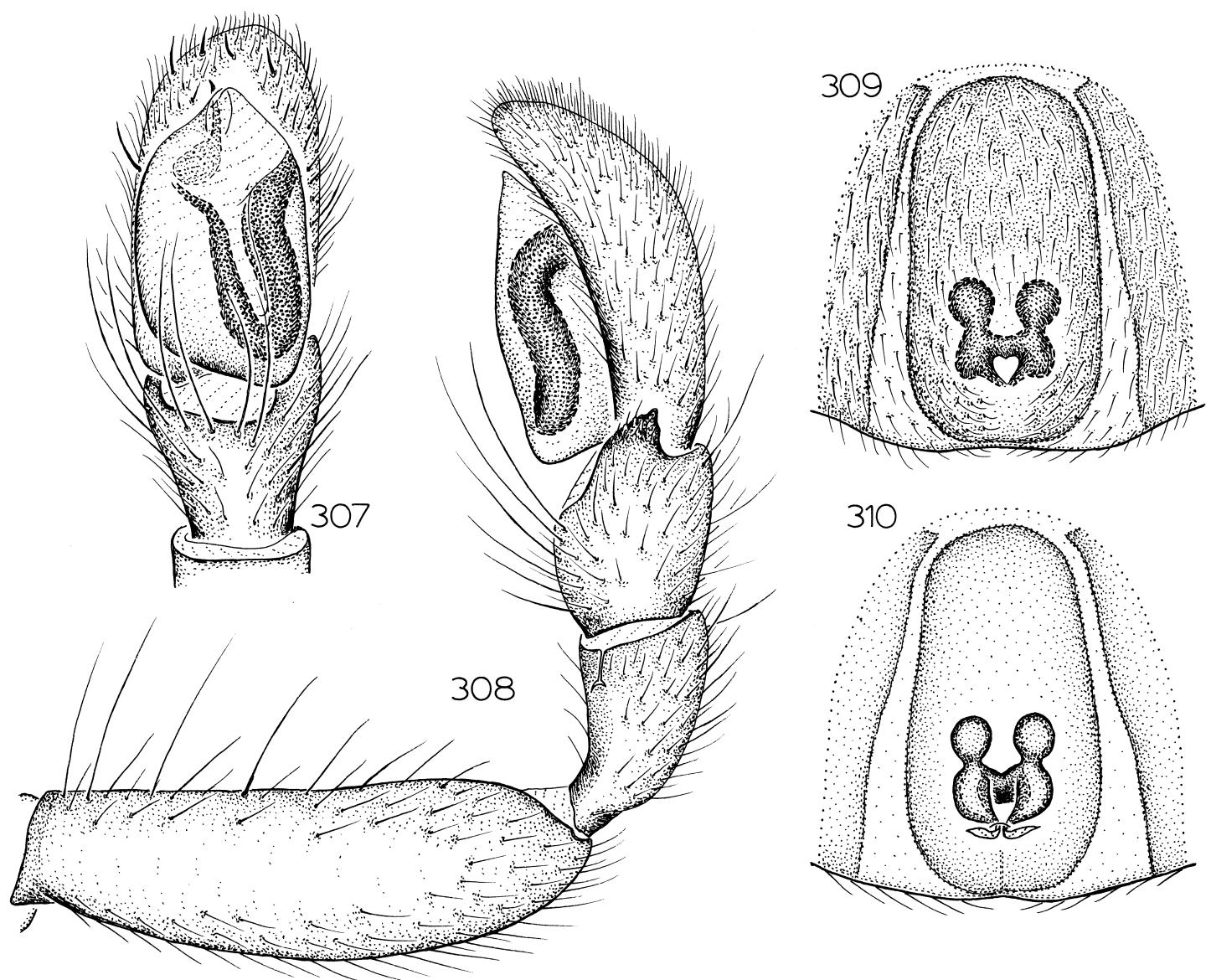

Figs. 307-310. Lamponina loftia, new species. 307. Left male palp, ventral view. 308. Same, retrolateral view. 309. Epigynum, ventral view. 310. Same, dorsal view.

236), 19 ; Burnside, Adelaide, $34^{\circ} 56^{\prime} \mathrm{S}$, $138^{\circ} 38^{\prime} \mathrm{E}$, Jan. 6, 1990 (D. Hirst, SAM N1996/235), 1 đ; Carrappee Hill Conservation Park, $33^{\circ} 25^{\prime} \mathrm{S}, 136^{\circ} 16^{\prime} \mathrm{E}$, Apr. 2, 1987 , under rock (D. Lee, D. Hirst, SAM N1996/ 261), 1\%; Cortina Station, Coorong, via Kingston, $36^{\circ} 20^{\prime} \mathrm{S}, 139^{\circ} 45^{\prime} \mathrm{E}$, Jan. 20, 1968 (A. Forbes, SAM N1996/242), 1 \%; Gum Lagoon Conservation Park, $36^{\circ} 17^{\prime} \mathrm{S}, 140^{\circ} 02^{\prime} \mathrm{E}$, Mar. 16-26, 1992, vibration, lagoon edge (D. Hirst, SAM N1996/248-252), 10 , 4 ㅇ, pitfall (D. Hirst, J. Forrest, SAM N1996/243, 244), $10^{\star}, 1$; ; Jip Jip Conservation Park, $36^{\circ} 29^{\prime} \mathrm{S}$, $140^{\circ} 11^{\prime} \mathrm{E}$, Mar. 23, 1992, vibration (J. Forrest, D. Hirst, SAM N1996/256), 1 ठ̊̊; N Keilira, $36^{\circ} 35^{\prime} \mathrm{S}, 140^{\circ} 10^{\prime} \mathrm{E}$, Mar. 23, 1992, pitfall (D. Hirst, SAM N1996/259, 260), 20 ; 13 km N Keilira Station, $36^{\circ} 37^{\prime} \mathrm{S}, 140^{\circ} 10^{\prime} \mathrm{E}$, Mar.
23, 1992, vibration (D. Hirst, SAM N1996/ 257, 258), 2 \%; Loftia Recreation Park, Mount Lofty Ranges, $35^{\circ} 02^{\prime} \mathrm{S}, 138^{\circ} 42^{\prime} \mathrm{E}$, Mar. 25, 1984, Berlese sample, litter and soil under Eucalyptus obliqua (R. Southcott, SAM N1996/237), 10, Sept. 14, 1989, in litter with moss (D. Hirst, SAM N1996/238), 1 9 , Mar. 20-27, 1990, pitfall (D. Hirst, SAM N1996/240), $10^{\text {t }}$, in litter (D. Hirst, SAM N1996/239), 1 \%; northern lagoon, Gum Lagoon Conservation Park, $36^{\circ} 18^{\prime} \mathrm{S}$, $140^{\circ} 01^{\prime} \mathrm{E}$, Mar. 25, 1992, vibration (J. Forrest, D. Hirst, SAM N1996/253-255), 3 우 Ravine de Casoars, Kangaroo Island, $35^{\circ} 48^{\prime} \mathrm{S}, 136^{\circ} 36^{\prime} \mathrm{E}$, Nov. 5-6, 1987 (D. Hirst, SAM N1996/234), 1 ㅇ. Victoria: $5 \mathrm{~km} \mathrm{E}$ Dunolly, $36^{\circ} 51^{\prime} \mathrm{S}, 143^{\circ} 44^{\prime} \mathrm{E}$, Aug. 4, 1983 , leaf litter (R. Moran, NMV K3439), $1 \%$; 4.5 
km S Wychitella, 36 $19^{\prime} \mathrm{S}, 143^{\circ} 36^{\prime} \mathrm{E}$, Mar. 26-29, 1990, mallee (A. Coventry, NMV K4666), $10^{\top}$; Upper Lurg, $36^{\circ} 35^{\prime} \mathrm{S}, 146^{\circ} 11^{\prime} \mathrm{E}$, Feb. 18, 1995, house at night (J. Strudwick, CVIC 632), 10 .

Distribution: South Australia and Victoria (map 36).

\section{Lamponina isa, new species}

Figures 311-314; Map 36

TYPES: Male holotype and female allotype from spinifex at an elevation of $310 \mathrm{~m}$ on Lake Moondarra Road, Mount Isa, Queensland, $20^{\circ} 41^{\prime} \mathrm{S}, 139^{\circ} 30^{\prime} \mathrm{E}$ (Apr. 18, 1996; R. Raven), deposited in QMB (S31252).

ETYMOLOGY: The specific name is a noun in apposition taken from the type locality.

DiAgNOSIS: Males resemble those of $L$. scutata but have a much lower retrolateral tibial apophysis that has a much more pronounced dorsal tip (fig. 312); the palpal bulb is shorter, with the anterior prolateral ledge much less abruptly separated from the translucent distal portion of the bulb (fig. 311). Females have a distinctive heart-shaped epigynal midpiece (fig. 313) and large, ovoid spermathecae on anteriorly narrowed stalks (fig. 314). In both sexes, the lateral white abdominal markings are small, square patches, much narrower than the transverse stripes typical of L. scutata.

MALE: Total length 8.4. Abdominal dorsum gray, with lateral white spots square, narrow, connected median pair of white spots forming inverted $\mathrm{v}$-shaped mark, and small posterior white spot over spinnerets, venter gray; legs reddish orange, femora and sides of tibiae darkest. Palpal femur long, with ventral invagination at about half its length; retrolateral tibial apophysis short, with prolonged dorsal tip (fig. 312); tegulum with pronounced posterolateral corner, embolus relatively short, tip relatively straight (fig. 311).

FEMALE: Total length 9.4. Coloration as in male, except median white spots widely separated. Epigynal midpiece heart-shaped, openings at anterolateral arches of heart (fig. $313)$; spermathecae ovoid, situated on anteriorly narrowed stalks (fig. 314).

Other MATERIAL EXAMINED: Northern Territory: Jasper Gorge, $16^{\circ} 01^{\prime} \mathrm{S}, 130^{\circ} 42^{\prime} \mathrm{E}$,
June 12, 1978, under rock (H. Cogger, E. Cameron, AMS KS18827), 1․ Queensland: Coen, Cape York, $13^{\circ} 56^{\prime} \mathrm{S}, 143^{\circ} 12^{\prime} \mathrm{E}$,

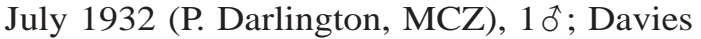
Creek National Park, $17^{\circ} 00^{\prime} \mathrm{S}, 145^{\circ} 34^{\prime} \mathrm{E}$, July 23-Nov. 26, 1992, pitfall (R. Raven, P., E. Lawless, M. Shaw, QMB S19859), 2 \% ; Fairbairn Dam, S Emerald, $23^{\circ} 40^{\prime} \mathrm{S}, 148^{\circ} 06^{\prime} \mathrm{E}$, Apr. 21, 1995 (K. McDonald, J. Covacevich, P. Couper, QMB S26328), $10^{\pi}$; Killymoon Creek, S Townsville, $19^{\circ} 24^{\prime} \mathrm{S}, 147^{\circ} 01^{\prime} \mathrm{E}$, Oct. 26, 1991-July 27, 1992, pitfall (P. Lawless, R. Raven, M. Shaw, QMB S19952), 2\%; Lake Moondarra Road, Mount Isa, $20^{\circ} 41^{\prime} \mathrm{S}$, $139^{\circ} 30^{\prime} \mathrm{E}$, Apr. 18, 1996, spinifex, elev. 310 m (R. Raven, QMB S31252), 10; Mareeba, $17^{\circ} 00^{\prime} \mathrm{S}, 145^{\circ} 26^{\prime} \mathrm{E}$, Feb. 8, 1970 (N. Coleman, AMS KS31843), 1 \% ; Osbourne Mine site, SSE Mount Isa, $22^{\circ} 07^{\prime} \mathrm{S}, 140^{\circ} 34^{\prime} \mathrm{E}$, Dec. 1996 (A. Nicholson, QMB S34302), 1\%; Thatch Creek, $19^{\circ} 06^{\prime} \mathrm{S}, 145^{\circ} 18^{\prime} \mathrm{E}$, July $26-$ Dec. 1, 1992, pitfall, dry eucalypt woodland (R. Raven, P., E. Lawless, M. Shaw, QMB S25272), 1 9 ; Wyandotte Creek, $18^{\circ} 29^{\prime} \mathrm{S}$, $144^{\circ} 55^{\prime}$ E. Dec. 1, 1992-Apr. 14, 1993, pitfall, dry eucalypt woodland (R., J., S. Raven, P., E. Lawless, QMB S24163, 24170), 2 ㅇ.

Distribution: Northern Territory and Queensland (map 36).

\section{Lamponoides, new genus}

TyPE SPECIES: Lamponoides coottha, new species.

ETYMOLOGY: The generic name is an arbitrary combination of letters, considered feminine in gender.

DiAgnosis: Members of the genus resemble those of Lampona and Lamponina in having a rugose (rather than smooth) sternal surface. They differ from Lampona species in having a dorsal abdominal scutum in females and lacking a protuberant retrolateral tibial apophysis in males, and from Lamponina species in having the epimeric sclerites free (rather than fused with the sternum).

DESCRIPTION: Medium-sized spiders, total length 4-8. Carapace dark red, tuberculate, coated with long setae originating from bases of tubercles, tubercles protruding from lateral margin; thoracic groove long, longitudinal. Eight eyes in two rows, anterior medians largest, circular, dark, other eyes subequal in 

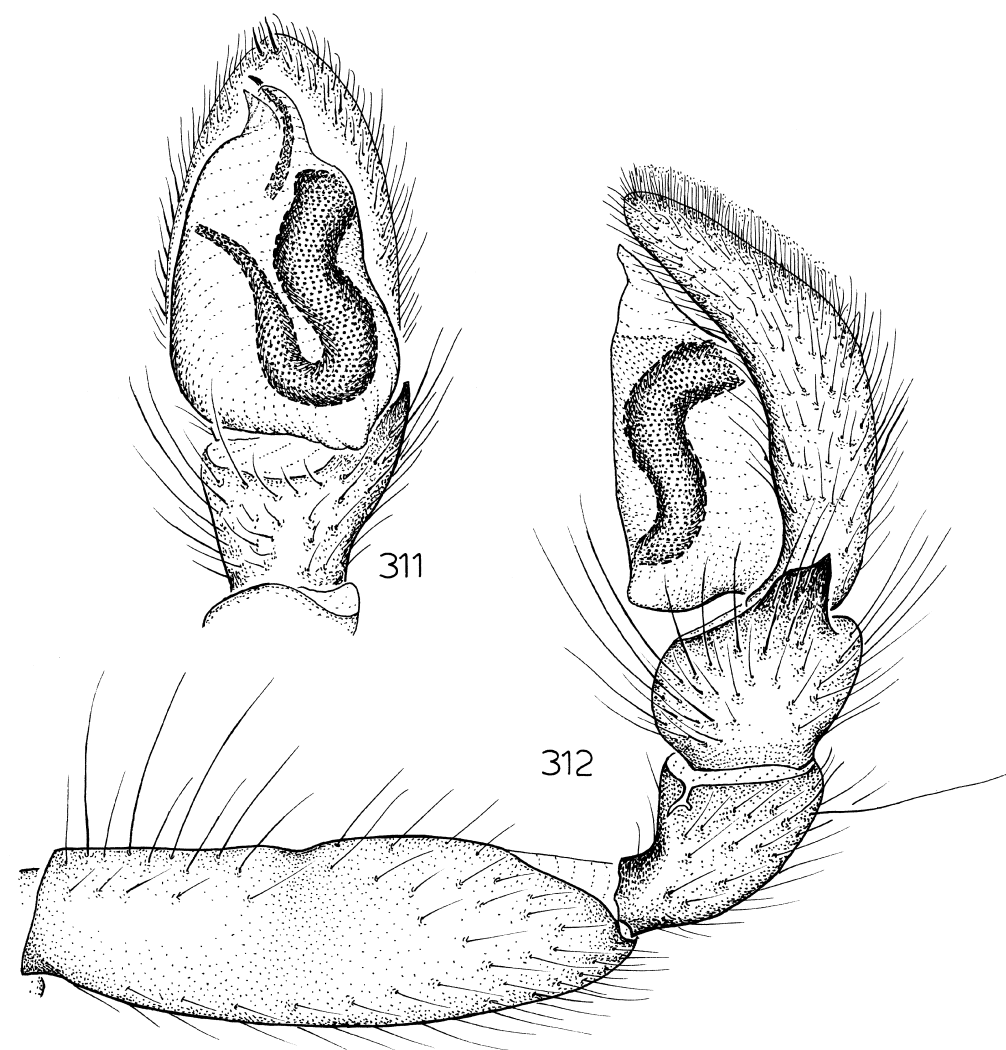

Figs. 311-314. Lamponina isa, new species. 311. Left male palp, ventral view. 312. Same, retrolateral view. 313. Epigynum, ventral view. 314. Same, dorsal view.

size, light, laterals oval, posterior medians irregularly oval, flattened; from above, both eye rows slightly procurved, from front, both rows strongly procurved; anterior medians separated by at least their radius, closer to anterior laterals; posterior medians separated by their diameter or more from each other and from posterior laterals; anterior and posterior laterals separated by at least their diameter; median ocular quadrangle wider in front than in back, slightly longer than wide. Chelicerae, sternum, and mouthparts dark red; chilum small, triangular, accompanied by second, elongated, posterior chilum (extremely narrow sclerite separating bases of chelicerae posteriorly); chelicerae with distinct lateral boss, promargin with series of short setae originating in line along base of fang plus one long, thick seta originating closest to fang, immediately bent at $90^{\circ}$ angle, extending to median line; promargin with three teeth, retromargin with none; cheliceral gland openings not scanned. Labium truncate anteriorly and posteriorly, distinctly depressed medially; anterior surface not scanned. Endites obliquely depressed, with sharply demarcated, deep groove along margin near labium; serrula long, with single row of teeth; anterior surface not scanned. Sternum elevated, with steep lateral margins, slightly expanded anteriorly at sides, reaching only to heel at posterolateral corner of endites, with extensions to and between coxae; surface rugose. Two epimeric sclerites on each side, one opposite palpal endites, one above all coxae, not reaching sternal triangles, not fused to carapace. Pedicel composed of two small, flat dorsal sclerites and rounded sclerite covering venter and sides, rounded sclerite with median longitudinal keel prolonged anteriorly into triangular protrusion extending toward sternum. 
Anterior edge of abdomen of male with almost complete sclerotic ring formed by epigastric scutum plus dorsal abdominal scutum reaching to about one-fourth of abdominal length, females with distinct, rounded scutum on anterior surface or dorsum; cuticle with long light and dark setae; epigastric scutum accompanied posterolaterally by pair of oval, deeply invaginated sclerites bearing clearly elevated anterior rim; sclerites separated by membranous lobe without extensions; anterior edge of oval sclerites fitting under epigastric scutum; colulus represented by setae; tiny transverse sclerite present, well removed from spinnerets, presumably marking position of small posterior spiracle. Anterior lateral spinnerets tubular, separated by roughly their diameter, cuticle representing distal, second spinneret segment restricted to semicircle surrounding major ampullate gland spigots (piriform gland spigots surrounded only by soft cuticle); posterior median spinnerets large, tubular, without anteriorly expanded tips; posterior lateral spinnerets two-segmented, spigots unscanned.

Legs almost spineless, with single proventral spine at distal tip of tibiae III, IV and (in females) single short spine at midlength of dorsum of femur IV; most surfaces with long, dark setae; in males, all coxae and posterior trochanters dorsally tuberculate; anterior coxae with protuberant posterolateral corners; trochanters unnotched; anterior metatarsi and tarsi with divided scopulae, composed of flattened, distally bent setae; posterior metatarsi with thick, dark, distal preening brushes; posterior tarsi with weak, entire scopulae; tarsi with two dentate claws, claw tufts composed of lateral pads of closely appressed setae; trichobothria present on tibiae, metatarsi, and tarsi, in 2-3 irregular rows, bases unscanned; tarsal organ unscanned. Female palpal tibia and tarsus with several long, thin spines; female palpal tarsus with long, dentate claw.

Male palp with retrolateral tibial apophysis reduced to low ridge not extending past distal rim of segment; cymbial surface retrobasally excavated; tegulum not expanded, not extending beyond posterior rim of tarsus; embolus medially situated, elongated, inset into membranous conductor; median apophysis absent, represented at most by slight sclero- tization within conductor. Epigynum elongated, reaching almost to pedicel, with four spermathecae; pre-epigynum unknown.

Lamponoides coottha, new species

Figures 315-318; Map 37

TYPE: Female holotype taken in a pitfall trap in open forest on Mount Coot-tha, Brisbane, Queensland, $27^{\circ} 29^{\prime} \mathrm{S}, 152^{\circ} 57^{\prime} \mathrm{E}$ (Dec. 22-Jan. 17, 1980; R. Raven), deposited in QMB (S28101).

ETYMOLOGY: The specific name is a noun in apposition taken from the type locality.

DiAGNOSIS: Males can easily be recognized by the lack of a protuberant retrolateral tibial apophysis combined with a medially situated embolus (figs. 315, 316), females by the enormously elongated epigynum combined with the additional pair of posterior spermathecae (figs. 317, 318).

MALE: Total length 4.1. Abdominal dorsum with alternating series of dark and light transverse bands (thick dark anterior, mostly under scutum, followed posteriorly by thick light, thin dark, thin light, thin dark, and thick light); tibiae with incomplete dark rings at about two-thirds their length. Palpal femur thick, unmodified; tibia without protuberant retrolateral tibial apophysis; embolus situated medially, distally hooked (figs. 315, 316).

FEMALE: Total length 7.7. Coloration as in male except anterior abdominal dark markings reduced, tibia IV with additional basal dark ring. Epigynum heavily sclerotized, elongated, paralleling sclerotized sides of booklung covers, with four spermathecae (figs. 317, 318).

Other Material Examined: A single male taken in a pitfall trap set at an elevation of $1290 \mathrm{~m}$ at a site $0.25 \mathrm{~km} \mathrm{~S}$ of the junction of Tubrabucca and Omadale Brook Roads in Stewarts Brook State Forest, New South Wales, $31^{\circ} 54^{\prime} \mathrm{S}, 151^{\circ} 23^{\prime} \mathrm{E}$ (Feb. 4-Apr. 9, 1993; M. Gray, G. Cassis), deposited in AMS (KS40493).

DISTRIBUTION: Known only from southeastern Queensland and New South Wales (map 37).

Lamponata, new genus

TyPES SPECIES: Lamponata daviesae, new species. 

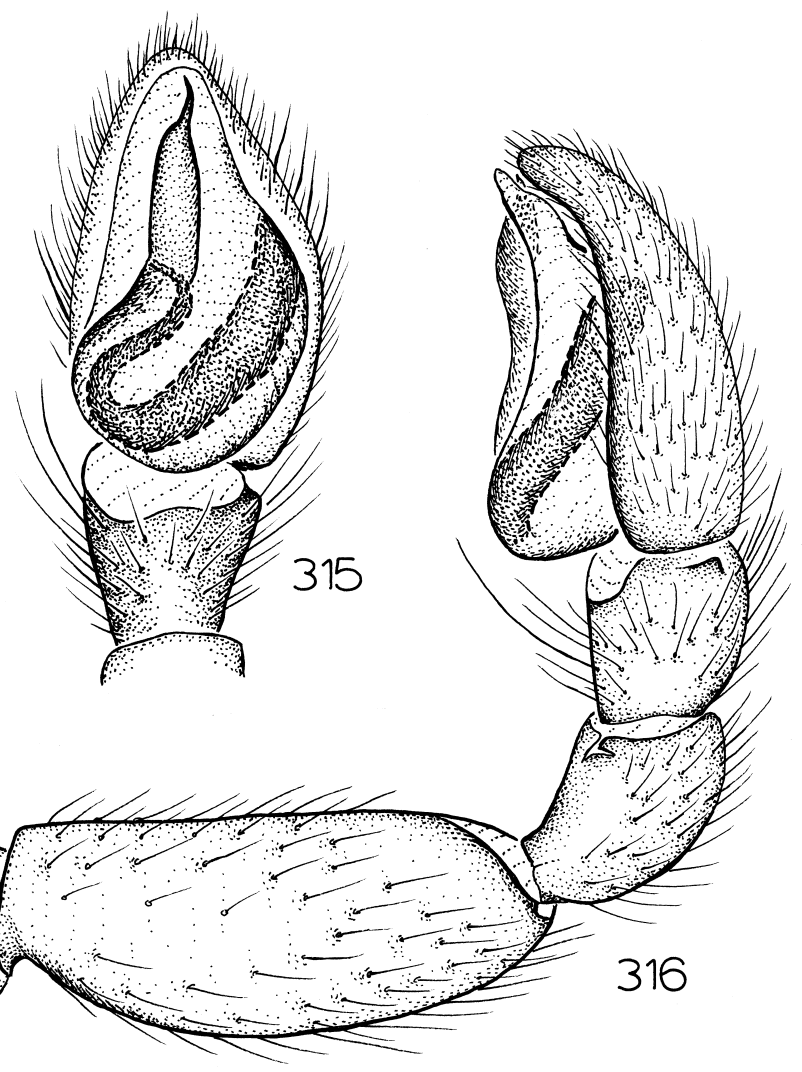

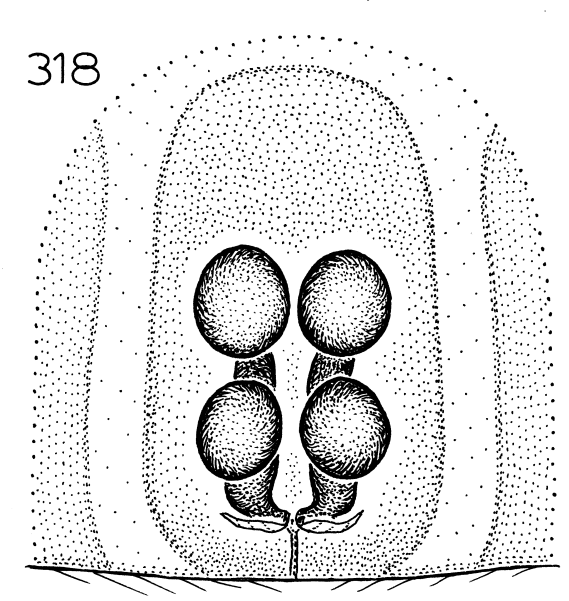

Figs. 315-318. Lamponoides coottha, new species. 315. Left male palp, ventral view. 316. Same, retrolateral view. 317. Epigynum, ventral view. 318. Same, dorsal view.

ETYMOLOGY: The generic name is an arbitrary combination of letters, considered feminine in gender.

DiagnOSIS: Members of this genus resemble those of Lampona, Lamponina, and Lamponoides in having a divided scopula on the anterior metatarsi and tarsi (although the scopula is weak and sometimes represented by relatively few hairs in males), but differ in having the sternal surface smooth with rounded pits rather than rugose. Males have a distinctive, long retrolateral tibial apophysis (fig. 320), apparently correlated with the presence in females of an anterior epigynal hood situated far anterior of the spermathecae (fig. 321).

DESCRIPTION: Medium-sized spiders, total length 2.7-3.5. Carapace dark red, tubercu- late, coated with long setae originating from bases of tubercles, tubercles protruding from lateral margin; thoracic groove long, longitudinal. Eight eyes in two rows, posterior medians largest, posterior laterals larger than anterior eyes; anterior medians circular, dark, other eyes light, laterals oval, posterior medians irregularly oval, flattened; from above, both eye rows slightly procurved, from front, both rows strongly procurved; anterior medians separated by their radius, closer to anterior laterals; posterior medians separated by less than their radius, farther from posterior laterals; anterior and posterior laterals separated by their radius; median ocular quadrangle slightly wider in back than in front, slightly longer than wide. Chelicerae, sternum, and mouthparts dark red; chilum very 
small, triangular, accompanied by second, elongated, posterior chilum (extremely narrow sclerite separating bases of chelicerae posteriorly); chelicerae with distinct lateral boss, promargin with series of short setae originating in line along base of fang plus one long, thick seta originating closest to fang, immediately bent at $90^{\circ}$ angle, extending to median line; promargin with three teeth, almost obsolete, retromargin with none; cheliceral gland openings not scanned. Labium truncate anteriorly and posteriorly, distinctly depressed medially; anterior surface not scanned. Endites obliquely depressed, with sharply demarcated, deep groove along margin near labium; serrula long, with single row of teeth; anterior surface not scanned. Sternum only slightly elevated, with low lateral margins, slightly expanded anteriorly at sides, reaching only to heel at posterolateral corner of endites, with extensions to and between coxae; surface smooth, with circular punctations. Two epimeric sclerites on each side, one opposite palpal endites, one above all coxae, not reaching sternal triangles, not fused to carapace. Pedicel composed of two small, flat dorsal sclerites and rounded sclerite covering venter and sides, rounded sclerite with median longitudinal keel prolonged anteriorly into triangular protrusion extending toward sternum.

Anterior edge of abdomen of male with almost complete sclerotic ring formed by epigastric scutum plus dorsal abdominal scutum reaching to about one-fourth of abdominal length, females without dorsal scutum; cuticle with long light and dark setae; epigastric scutum accompanied posterolaterally by pair of oval, deeply invaginated sclerites bearing clearly elevated anterior rim; sclerites separated by membranous lobe without extensions; anterior edge of oval sclerites fitting under epigastric scutum; colulus represented by setae; tiny transverse sclerite present, well removed from spinnerets, presumably marking position of small posterior spiracle. Anterior lateral spinnerets tubular, separated by more than their diameter, cuticle representing distal, second spinneret segment restricted to semicircle surrounding major ampullate gland spigots (piriform gland spigots surrounded only by soft cuticle); poste- rior median spinnerets small, tubular, without anteriorly expanded tips; posterior lateral spinnerets two-segmented, spigots unscanned.

Legs spineless; most surfaces with long, dark setae; in males, all coxae and posterior trochanters dorsally tuberculate; anterior coxae with protuberant posterolateral corners; trochanters unnotched; anterior metatarsi and tarsi with divided scopulae (weak in males), composed of flattened, distally bent setae; posterior metatarsi with thick, dark, distal preening brushes; posterior tarsi with weak, entire scopulae; tarsi with two dentate claws, claw tufts composed of lateral pads of closely appressed setae; trichobothria present on tibiae, metatarsi, and tarsi, in 2-3 irregular rows, bases unscanned; tarsal organ unscanned. Female palpal tibia and tarsus without spines; tarsus with tiny claw.

Male palp with long retrolateral tibial apophysis, reaching to about half of cymbial length; cymbial surface retrobasally excavated; tegulum not expanded, not extending beyond posterior rim of tarsus; embolus prolaterally situated, hidden in ventral view by bipartite membranous conductor; median apophysis absent, represented at most by slight sclerotization within conductor. Epigynum with anterior hood far removed from spermathecae.

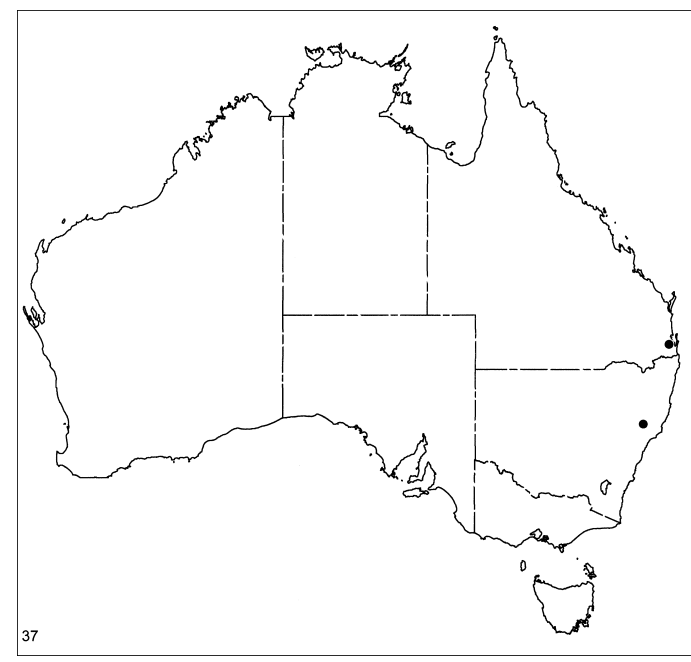

Map 37. Records of Lamponoides coottha, new species. 
Lamponata daviesae, new species Figures 275, 319-322; Map 38

TYPE: Male holotype taken in pitfall trap at Lake Broadwater, SW Dalby, $27^{\circ} 11^{\prime} \mathrm{S}$, $151^{\circ} 16^{\prime} \mathrm{E}$, Queensland (May 17-Nov. 24, 1985; M. Bennie), deposited in QMB (S34374).

ETYMOLOGY: The specific name is a patronym in honor of Dr. Valerie Davies of the Queensland Museum, whose extensive sorting of the Lake Broadwater material has produced numerous unusual lamponids, including the best series of this species.

DiAGNOSIS: With the characters of the genus and genitalia as in figures 319-322. The only taxa that could be confused with this species are males of the Lampona cylindrata group, which have a similarly long retrolateral tibial apophysis but have a basally protruding palpal bulb.

MALE: Total length 2.7. Dorsum of abdomen light gray with five broken transverse rows of dark spots (fig. 275), venter with vshaped dark marking; legs unmarked. Palpal femur sharply bent at about one-fifth its length, enlarged distally; retrolateral tibial apophysis long, distally flattened; cymbium produced ventrally (figs. 319, 320).

FEMALE: Total length 3.5. Coloration as in male except ventral abdominal dark mark absent. Epigynum with small, semicircular anterior hood far removed from midpiece (fig. 321 ); spermathecae compact, anterior portion only slightly expanded (fig. 322).

OTHER MATERIAL EXAMINED: Northern Territory: cliff $\mathrm{N}$ of old ranger station, 11.3 $\mathrm{km}$ S Hermannsburg, $24^{\circ} 03^{\prime} \mathrm{S}, 132^{\circ} 46^{\prime} \mathrm{E}$, Oct. 1994, pitfall (NMV K4669), 19 ; Todd River, Alice Springs, $23^{\circ} 42^{\prime} \mathrm{S}, 133^{\circ} 53^{\prime} \mathrm{E}$, July 1, 1969, sifted material from bark at trunk of large eucalypt trees (J. Balogh, HNMH), 1 ㅇ․ Queensland: Bushley Station, $23^{\circ} 32^{\prime} \mathrm{S}$, $150^{\circ} 15^{\prime} \mathrm{E}$, Mar. 21-Oct. 22, 1990, pitfall, open forest (D. Wallace, R. Raven, K. Williams, QMB S30410), 1 ; 33 mi. S Blackall, $24^{\circ} 25^{\prime} \mathrm{S}, 145^{\circ} 28^{\prime} \mathrm{E}$, May 31,1956 , dry leaf litter, brigalow, wilga (F. Perkins, OMD), $1 \%$; Lake Broadwater, SW Dalby, $27^{\circ} 11^{\prime} \mathrm{S}$, $151^{\circ} 16^{\prime} \mathrm{E}$, Jan. 26-Feb. 19, 1985, pitfall (M. Bennie, QMB S28651), 1 9 , May 17-Nov. 24, 1985, pitfalls (M. Bennie, QMB S28639), 1 \%, 1 ㅇ, Nov. 24, 1985-Jan. 3,
1986, pitfall (M. Bennie, QMB S28653), 10, Jan. 3-Feb. 25, 1986, pitfall (M. Bennie, QMB S28644), 10, Apr. 22-June 12, 1986, pitfall (M. Bennie, QMB S28640), $10^{\top}$; Taroom district, Boggomoss No. 3, 25 $26^{\prime} \mathrm{S}$, $150^{\circ} 01^{\prime}$ E, Nov. 12, 1996-Jan. 1997, pitfall (G. Monteith, D. Cook, QMB S36335), 10; Windermere, near Glenmorgan, $27^{\circ} 17^{\prime} \mathrm{S}$, $149^{\circ} 45^{\prime}$ E, Dec. 1990-Sept. 1991 (R. Raven, B. Smyth, QMB ex S30363), 10. South Australia: Jimmys Well, Mount Rescue Conservation Park, $35^{\circ} 51^{\prime} \mathrm{S}, 140^{\circ} 18^{\prime} \mathrm{E}$, in litter (D. Hirst, SAM N1996/316, 317), $20^{\star} ; 3$ km S Mount Sturt, 32 46'S, $135^{\circ} 24^{\prime}$ E, Dec. 13-17, 1989, pitfall (D. Hirst, SAM N1997/

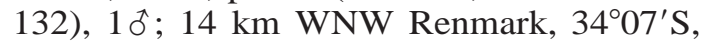
$140^{\circ} 37^{\prime}$ E, Aug. 8-Sept. 7, 1995, intercept trap (K. Pullen, QMB S34289), 10; $79 \mathrm{~km}$ NNW Renmark, $33^{\circ} 31^{\prime} \mathrm{S}, 140^{\circ} 24^{\prime} \mathrm{E}$, Aug. 9Sept. 7, 1995, intercept trap (K. Pullen, QMB), 10; W ranger station, Rocky River,

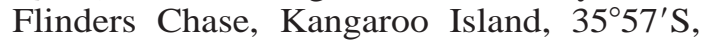
$136^{\circ} 43^{\prime} \mathrm{E}$, Nov. 7-9, 1987, pitfall (D. Hirst, SAM N1997/131), 10 . Western Australia: Tamala Station, $26^{\circ} 40^{\prime} \mathrm{S}, 113^{\circ} 40^{\prime} \mathrm{E}$, Nov. 8, 1998 (V. Ovtsharenko, AMNH), 1 \% ; Woodstock Station, $21^{\circ} 37^{\prime} \mathrm{S}, 118^{\circ} 57^{\prime} \mathrm{E}$, Feb. 1017, 1989, pitfall (J. Waldock, R. How, J. Dell, WAM 96/752), 1 ㅇ.

Distribution: Widespread in drier parts of Australia (map 38).

\section{Lamponova, new genus}

Type Species: Lamponova wau, new species.

ETYMOLOGY: The generic name is an arbitrary combination of letters, considered feminine in gender.

DiaGNOSIS: Members of this genus resemble those of Lampona, Lamponina, and Lamponoides in having a divided scopula on the anterior metatarsi and tarsi, but (like Lamponata) they differ in having the sternal surface smooth with rounded pits rather than rugose. Males of the genus are unknown, but females can easily be distinguished from all other lamponines by the median epigynal depression separating bipartite spermathecae on long stalks (figs. 323, 324).

DESCRIPTION: Medium-sized spiders, total length 3.5-4.0. Carapace dark red, tuberculate, coated with short setae originating from 

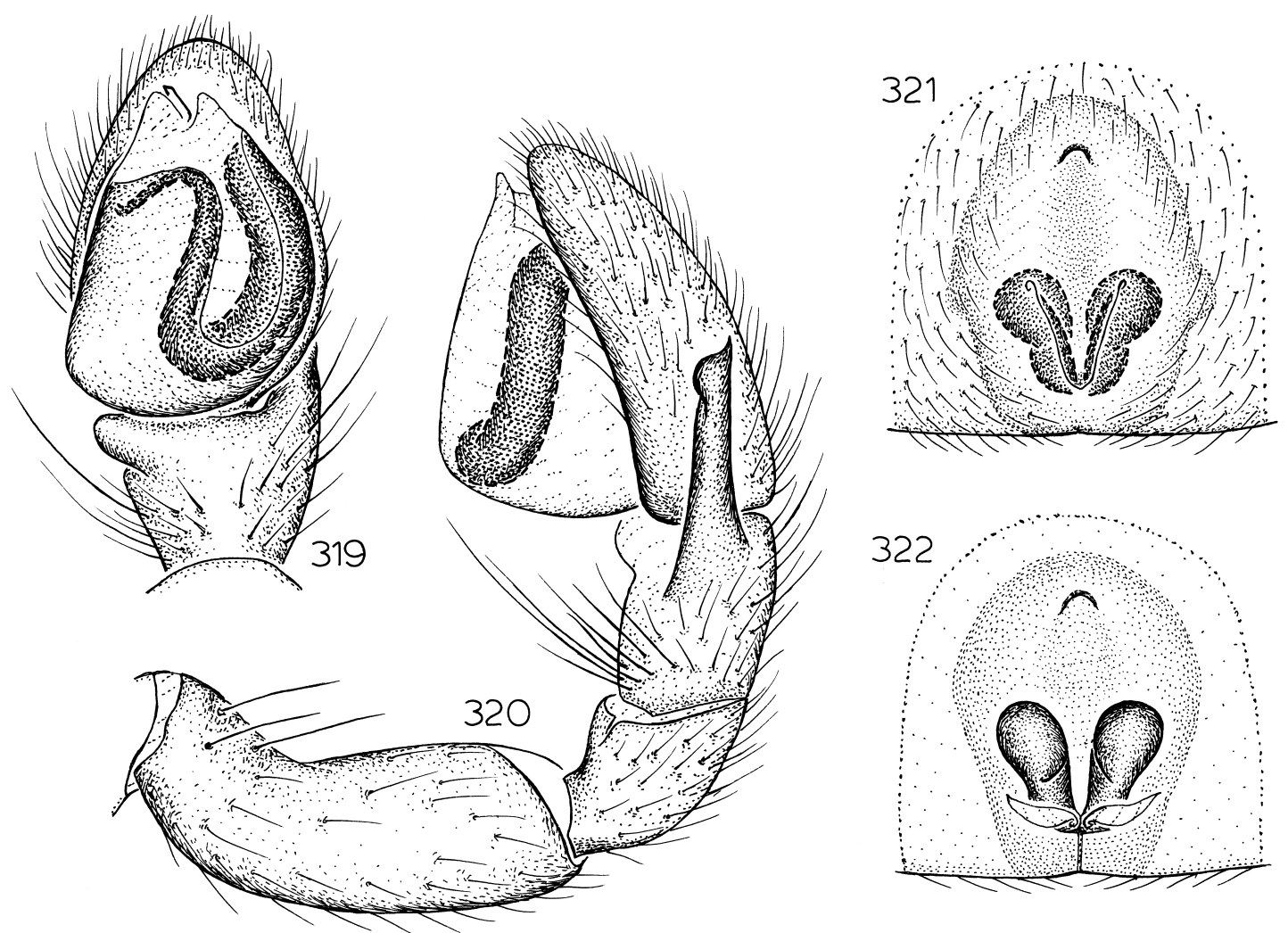

Figs. 319-322. Lamponata daviesae, new species. 319. Left male palp, ventral view. 320. Same, retrolateral view. 321. Epigynum, ventral view. 322. Same, dorsal view.

bases of low tubercles, tubercles slightly protruding from lateral margin; thoracic groove long, longitudinal, almost bipartite (deeper anteriorly than posteriorly). Eight eyes in two rows, posterior medians largest, posterior medians smaller than other subequal eyes; anterior medians circular, dark, other eyes light, laterals oval, posterior medians irregularly oval, flattened; from above, both eye rows slightly procurved, from front, both rows strongly procurved; anterior medians separated by more than their radius, closer to anterior laterals; posterior medians separated by their diameter, closer to posterior laterals; anterior and posterior laterals separated by their diameter; median ocular quadrangle slightly wider in back than in front, slightly wider than long. Chelicerae, sternum, and mouthparts dark red; chilum small, triangular, accompanied by second, short, posterior chilum (extremely narrow sclerite separating

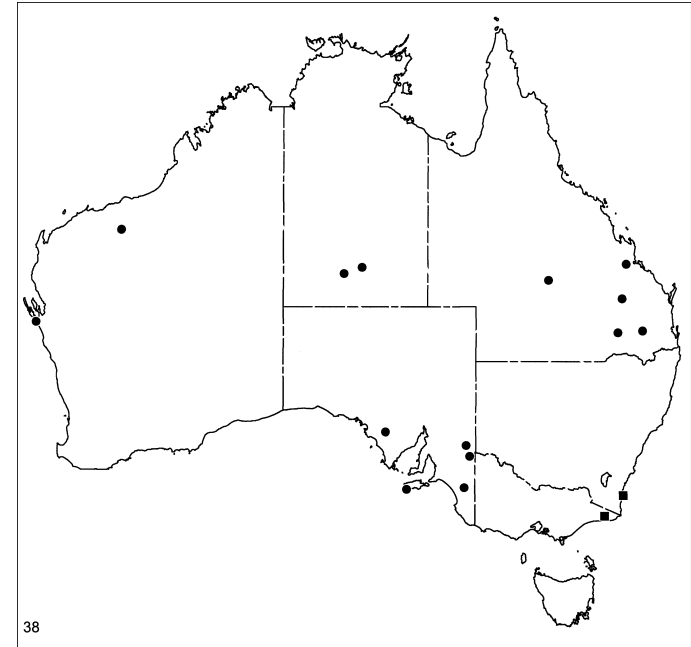

Map 38. Records of Lamponata daviesae, new species (circles) and Lamponova wau, new species (squares, Australian records only). 
bases of chelicerae posteriorly); chelicerae with distinct lateral boss, promargin with series of short setae originating in line along base of fang plus one long, thick seta originating closest to fang, immediately bent at $90^{\circ}$ angle, extending to median line; promargin with three teeth, retromargin with none; cheliceral gland openings not scanned. Labium truncate anteriorly and posteriorly, distinctly depressed medially; anterior surface not scanned. Endites obliquely depressed, with sharply demarcated, deep groove along margin near labium; serrula long, with single row of teeth; anterior surface not scanned. Sternum only slightly elevated, with low lateral margins, slightly expanded anteriorly at sides, reaching only to heel at posterolateral corner of endites, with extensions to and between coxae; surface smooth, with circular punctations. Two epimeric sclerites on each side, one opposite palpal endites, one above all coxae, not reaching sternal triangles, not fused to carapace. Pedicel composed of two small, flat dorsal sclerites and rounded sclerite covering venter and sides, rounded sclerite with median longitudinal keel prolonged anteriorly into triangular protrusion extending toward sternum.

Anterior edge of abdomen of female without dorsal scutum; cuticle with long light and dark setae; epigastric scutum accompanied posterolaterally by pair of oval, deeply invaginated sclerites bearing clearly elevated anterior rim; sclerites separated by membranous lobe without extensions; anterior edge of oval sclerites fitting under epigastric scutum; colulus represented by setae; tiny transverse sclerite present, well removed from spinnerets, presumably marking position of small posterior spiracle. Anterior lateral spinnerets tubular, separated by less than their diameter, cuticle representing distal, second spinneret segment restricted to semicircle surrounding major ampullate gland spigots (piriform gland spigots surrounded only by soft cuticle); posterior median spinnerets small, tubular, without anteriorly expanded tips; posterior lateral spinnerets two-segmented, spigots unscanned.

Legs spineless; most surfaces with long, dark setae; anterior coxae with protuberant posterolateral corners; trochanters unnotched; anterior metatarsi and tarsi with di- vided scopulae, composed of flattened, distally bent setae; posterior metatarsi with thick, dark, distal preening brushes; posterior tarsi with weak, entire scopulae; tarsi with two dentate claws, claw tufts composed of lateral pads of closely appressed setae; trichobothria present on tibiae, metatarsi, and tarsi, in 2-3 irregular rows, bases unscanned; tarsal organ unscanned. Female palpal tibia and tarsus with few weak spines; tarsus with tiny claw.

Males unknown. Epigynum with bipartite spermathecae on long stalks, separated by median depression.

\section{Lamponova wau, new species} Figures 323, 324; Map 38

TyPE: Female holotype taken under bark of Araucaria cunninghami (upper stem, tree \#6) at Beenleigh Lake, Wau, Morobe District, Papua New Guinea, $7^{\circ} 20^{\prime} \mathrm{S}, 146^{\circ} 40^{\prime} \mathrm{E}$ (Sept. 22, 1970; B. Gray), deposited in AMS (KS13251).

ETYMOLOGY: The specific name is a noun in apposition taken from the type locality.

DIAGNOSIS: Females of this small species can easily be distinguished from all other lamponines by the tiny median depression on the epigynum, which is apparent both dorsally and ventrally (figs. 323, 324).

MALE: Unknown.

Female: Total length 3.9. Abdominal dorsum mostly light anteriorly, darkened in front of posterior white spot; legs unmarked. Epigynum with small, semicircular depression situated between spermathecae (fig. 323); spermathecae with twisted anterior portion, on long stalked ducts (fig. 324).

OTHER MATERIAL EXAMINED: AUSTRALIA: New South Wales: Corunna Lake, $36^{\circ} 17^{\prime} \mathrm{S}$, $150^{\circ} 07^{\prime} \mathrm{E}$, June 2,1989 , pitfall, dry sclerophyll forest (G. Cassis, AMS KS21697), 1 ㅇ․ Victoria: Cobon South Coupe, $37^{\circ} 25^{\prime} \mathrm{S}$, $148^{\circ} 58^{\prime} \mathrm{E}$, Mar. 5-12, 1992, upslope pitfall (R. Coy, NMV K3609), 19. PAPUA NEW GUINEA: Morobe District: Beenleigh Lake, Wau, $7^{\circ} 20^{\prime} \mathrm{S}, 146^{\circ} 40^{\prime} \mathrm{E}$, Sept. 22, 1970, under bark of Araucaria cunninghami, upper stem, tree \#7 (B. Gray, AMS KS13260), 1 ; Stony Lake, Bulolo, 6 $45^{\prime} \mathrm{S}, 146^{\circ} 30^{\prime} \mathrm{E}$, Aug. 18, 1970, under bark of Araucaria cunninghami, upper stem, tree \#1 (B. Gray, AMS 

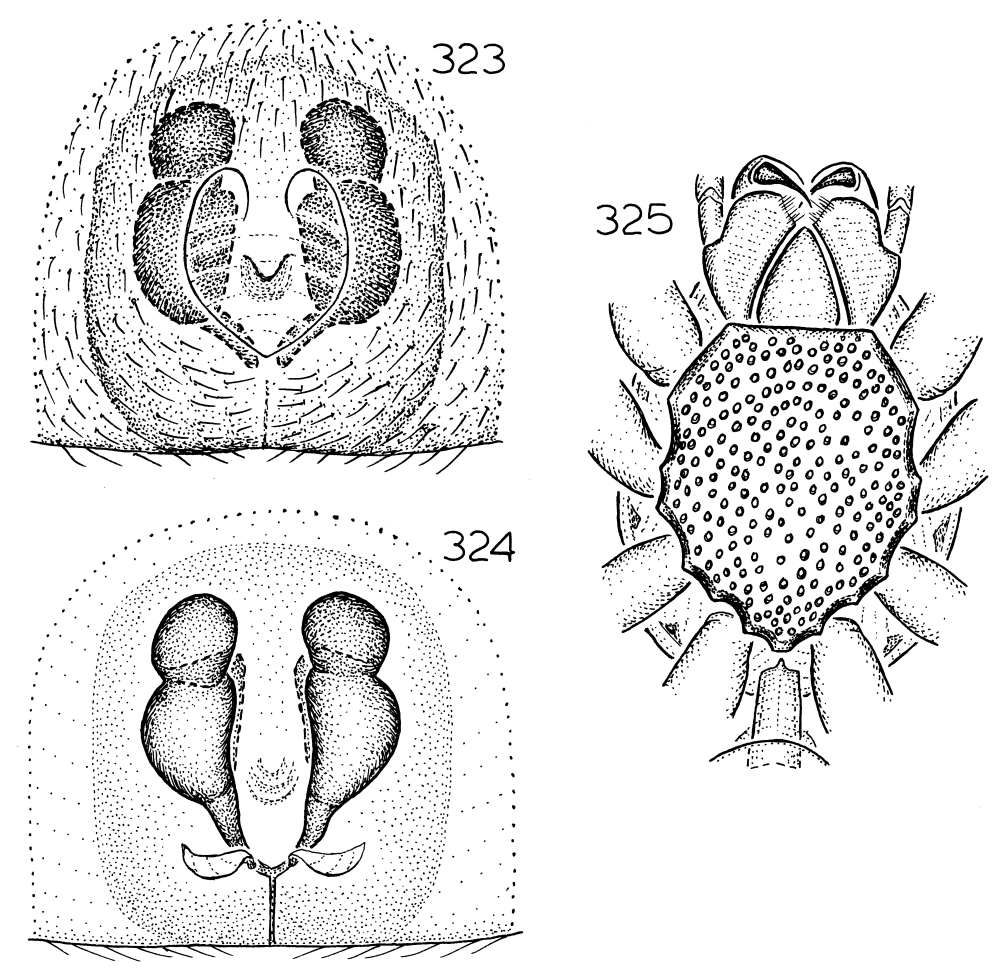

Figs. 323-325. 323, 324. Lamponova wau, new species. 323. Epigynum, ventral view. 324. Same, dorsal view. 325. Lamponella ainslie, new species, female cephalothorax, ventral view.

KS13211), 1 (penultimate but with full epigynum).

DisTRIBUTION: Known only from southeastern Australia (map 38), and from New Guinea. It is possible that the species actually occurs from New Guinea to Victoria but has not yet been collected in Queensland; alternatively, the northern or southern records could represent introductions.

\section{Lamponicta, new genus}

TyPE SPECIES: Lamponicta cobon, new species.

ETYMOLOGY: The generic name is an arbitrary combination of letters, considered feminine in gender.

DiagNosis: Specimens can be separated from those of Lampona, Lamponoides, Lamponata, Lamponova, and Lamponella by having the epimeric sclerites fused with the sternum, from Lamponina by lacking a divided scopula on the anterior metatarsi and tarsi, from Lamponega by having the ster- num normal rather than greatly elevated, and from Lamponusa by lacking white scales on the carapace. Males lack a retrolateral tibial apophysis (fig. 327), females have two spermathecal bulbs on each side (fig. 329).

DESCRIPTION: Medium to large spiders, total length 6.4-7.7. Carapace dark red, tuberculate, coated with long setae originating from bases of tubercles, tubercles protruding from lateral margin; thoracic groove short, almost obsolete. Eight eyes in two rows, eyes subequal in size; anterior medians circular, dark, other eyes light, laterals oval, posterior medians irregularly oval, flattened; from above, both eye rows very slightly procurved, from front, both rows strongly procurved; anterior medians separated by their radius, closer to anterior laterals; posterior medians separated by less than their radius, farther from posterior laterals; anterior and posterior laterals separated by their diameter; median ocular quadrangle slightly wider in front than in back, slightly wider than long. 
Chelicerae, sternum, and mouthparts dark red; chilum apparently fused to carapace, which is prolonged at midline, second, short, posterior chilum (extremely narrow sclerite separating bases of chelicerae posteriorly) present; chelicerae with distinct lateral boss, promargin with series of short setae originating in line along base of fang plus one long, thick seta originating closest to fang, immediately bent at $90^{\circ}$ angle, extending to median line; promargin with teeth obsolete, represented by slight projections on fused ridge, retromargin without teeth; cheliceral gland openings not scanned. Labium truncate anteriorly and posteriorly, distinctly depressed medially; anterior surface not scanned. Endites obliquely depressed, with sharply demarcated, deep groove along margin near labium; serrula long, with single row of teeth; anterior surface not scanned. Sternum only slightly elevated, fused to epimeric sclerites so that coxae originate from foramina completely enclosed by sclerotized cuticle; surface smooth, with circular punctations. Epimeric sclerites not fused to carapace. Pedicel elongated, composed of two small, flat dorsal sclerites and rounded sclerite covering venter and sides, rounded sclerite without median longitudinal keel or anterior protrusion extending toward sternum.

Anterior edge of abdomen of males with narrow dorsal scutum extending to about one-third of abdominal length, scutum well separated from epigastric scutum, females with small dorsal scutum bear anterior edge of dorsum; cuticle with long dark setae; epigastric scutum accompanied posterolaterally by pair of oval, deeply invaginated sclerites bearing clearly elevated anterior rim; anterior edge of oval sclerites fitting under epigastric scutum; colulus represented by setae; tiny transverse sclerite present, well removed from spinnerets, presumably marking position of small posterior spiracle. Anterior lateral spinnerets tubular, separated by less than their diameter, cuticle representing distal, second spinneret segment restricted to semicircle surrounding major ampullate gland spigots (piriform gland spigots surrounded only by soft cuticle); posterior median spinnerets small, tubular, without anteriorly expanded tips; posterior lateral spinnerets twosegmented, spigots unscanned.
Legs spineless; most surfaces with long, dark setae; anterior coxae with protuberant posterolateral corners; males with coxae tuberculate; trochanters unnotched; anterior metatarsi and tarsi without distinct, divided scopulae; posterior metatarsi with thick, dark, distal preening brushes; posterior tarsi with weak, entire scopulae; tarsi with two dentate claws, claw tufts composed of lateral pads of closely appressed setae; trichobothria present on tibiae, metatarsi, and tarsi, in 2-3 irregular rows, bases unscanned; tarsal organ unscanned.

Male palp without retrolateral tibial apophysis, position of apophysis marked by distinct excavation; cymbial surface without retrobasal excavation; tegulum not expanded, not extending beyond posterior rim of tarsus; embolus laterally situated, only tip protruding beyond tegulum, inset into membranous conductor; median apophysis represented only by distinct sclerotization on prolateral side of conductor; sperm duct greatly narrowed near midline. Epigynum with vshaped median septum, spermathecae each with two bulbous lobes.

\section{Lamponicta cobon, new species}

Figures 326-329; Map 39

TYPE: Male holotype taken in a midslope pitfall trap at Cobon South Coupe, Victoria, $37^{\circ} 25^{\prime} \mathrm{S}, 1^{\circ} 8^{\circ} 56^{\prime} \mathrm{E}$ (Apr. 6-14, 1992; R. Coy), deposited in NMV (K3598).

ETYMOLOGY: The specific name is a noun in apposition taken from the type locality.

DiAGNOSIS: With the characters of the genus, palp as in figures 326 and 327, and epigynum as in figures 328 and 329 .

MALE: Total length 6.4. Abdominal dorsum dark gray, with transverse white band at about half its length, band wider at sides than at middle; anterior legs darkened but without distinct markings. Palpal femur short, thick, sinuous when viewed dorsoventrally; tibia without retrolateral apophysis, with distal excavation (fig. 327); tegulum oval, sperm duct confined to distal half, greatly narrowed near midline (fig. 326).

Female: Total length 7.7. Abdominal dorsum without distinct transverse white band, with small, roughly pentagonal orange scu- 

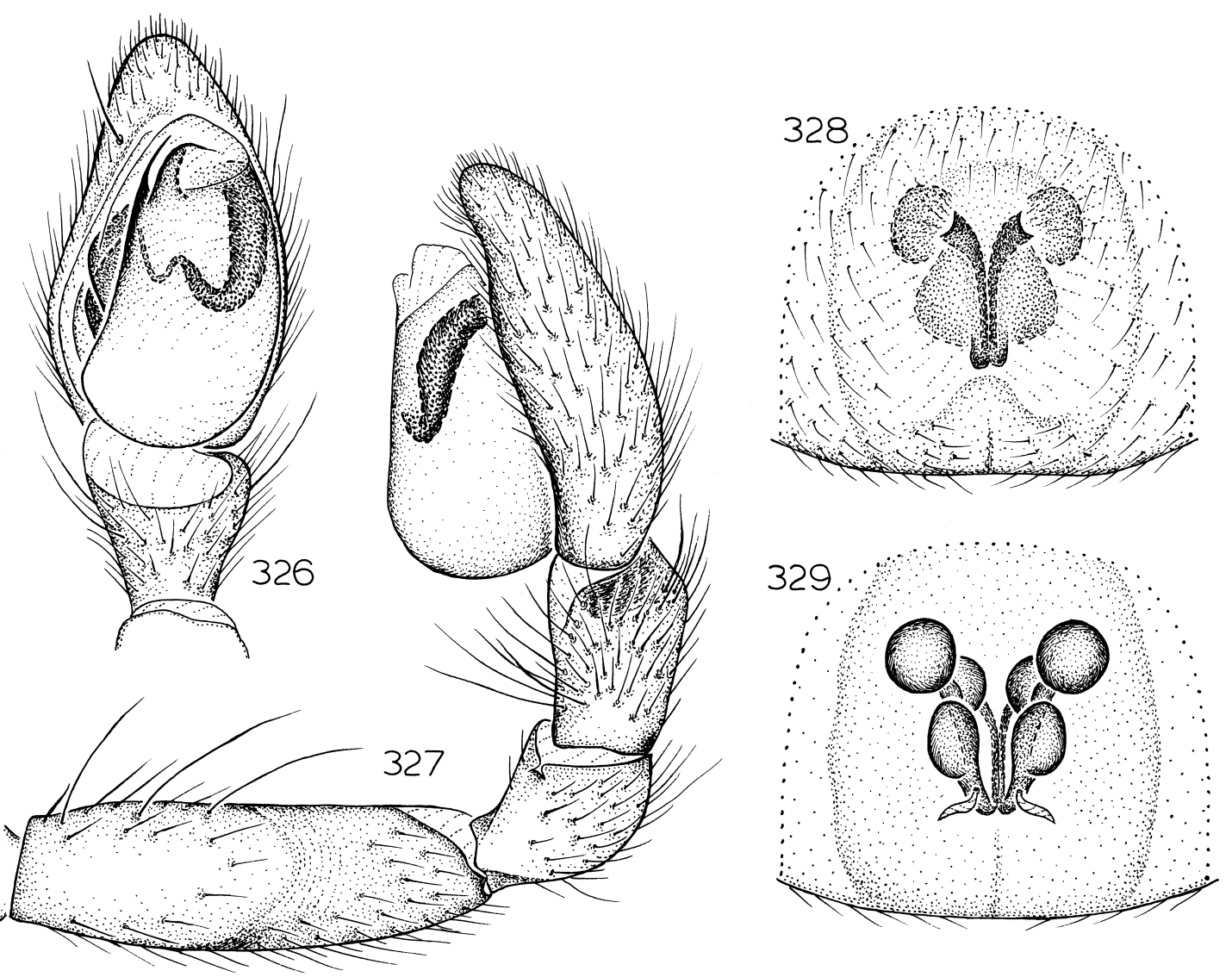

Figs. 326-329. Lamponicta cobon, new species. 326. Left male palp, ventral view. 327. Same, retrolateral view. 328. Epigynum, ventral view. 329. Same, dorsal view.

tum near anterior edge. Epigynum with long, v-shaped posteromedian septum (fig. 328); spermathecae each with two bulbous lobes (fig. 329).

Other Material Examined: New South Wales: junction, Tubrabucca and Omadale Brook Roads, Stewarts Brook State Forest, $31^{\circ} 55^{\prime} \mathrm{S}, 151^{\circ} 24^{\prime} \mathrm{E}$, Nov. 22-23, 1996, elev. 1360 m (N. Platnick, R. Raven, V. Ovtsharenko, K. Catley, AMNH), 10. Victoria: Blackwood Loam Creek, $37^{\circ} 29^{\prime} \mathrm{S}, 144^{\circ} 19^{\prime} \mathrm{E}$, Sept. 10, 1977, under bark (Parnaby, AMS KS44475), 19 ; Cobon South Coupe, $37^{\circ} 25^{\prime} \mathrm{S}, 148^{\circ} 57^{\prime} \mathrm{E}$, Nov. 27-28, 1996, elev. 700 m (N. Platnick, R. Raven, V. Ovtsharenko, K. Catley, AMNH), 1 ㅇ.

Distribution: Known only from New South Wales and Victoria (map 39).

\section{Lamponusa, new genus}

Type SPECIES: Lamponusa gleneagle, new species.

ETYMOLOGY: The generic name is an arbitrary combination of letters, considered feminine in gender.

DiagnOSIS: The presence of thick white scales scattered on both the carapace and abdomen, combined with the lack of a divided scopula on the anterior metatarsi and tarsi, will separate members of this genus from all other lamponines.

DESCRIPTION: Small spiders, total length 3.5-4.7. Carapace dark red, tuberculate, coated with long setae originating from bases of tubercles, tubercles protruding from lateral margin; sides of pars cephalica and pars thor- 
acica with scattered thick, white, scales; thoracic groove short, longitudinal. Eight eyes in two rows, eyes small, subequal in size; anterior medians circular, dark, other eyes light, laterals oval, posterior medians irregularly oval, flattened; from above, both eye rows very slightly procurved, from front, both rows strongly procurved; anterior medians separated by almost their diameter, closer to anterior laterals; posterior medians separated by almost their diameter, closer to posterior laterals; anterior and posterior laterals separated by more than their diameter; median ocular quadrangle wider in back than in front, slightly wider in back than long. Chelicerae, sternum, and mouthparts dark red; chilum small, triangular; second, short, posterior chilum (extremely narrow sclerite separating bases of chelicerae posteriorly) present; chelicerae with distinct lateral boss, promargin with series of short setae originating in line along base of fang plus one long, thick seta originating closest to fang, immediately bent at $90^{\circ}$ angle, extending to median line; promargin with teeth almost obsolete, represented by slight projections on fused ridge, retromargin without teeth; cheliceral gland openings not scanned. Labium truncate anteriorly and posteriorly, distinctly depressed medially; anterior surface not scanned. Endites obliquely depressed, with

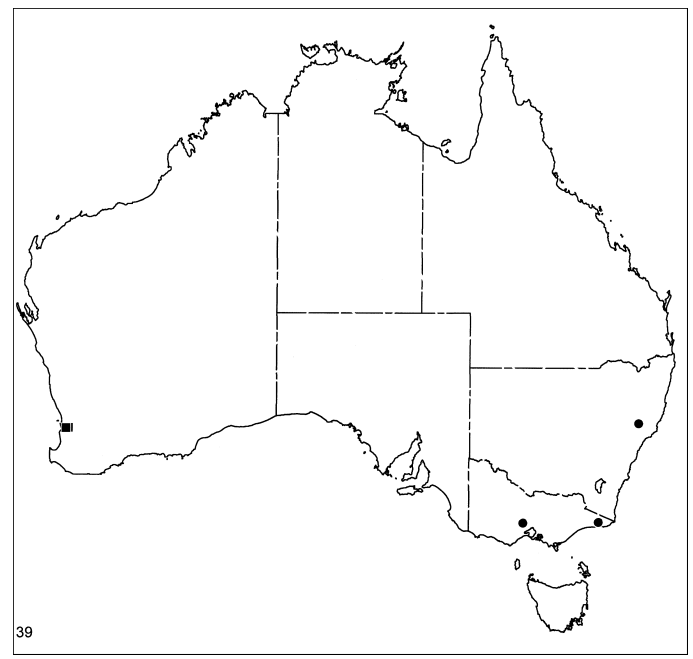

Map 39. Records of Lamponicta cobon, new species (circles) and Lamponusa gleneagle, new species (squares). sharply demarcated, deep groove along margin near labium; serrula long, with single row of teeth; anterior surface not scanned. Sternum only slightly elevated, fused to epimeric sclerites so that coxae originate from foramina completely enclosed by sclerotized cuticle; surface smooth, with circular punctations. Epimeric sclerites also completely fused with carapace from level of endites back to rear of coxae II foramina; from there back, sclerites separated from carapace by extremely narrow line of unsclerotized cuticle. Pedicel elongated, composed of two small, flat dorsal sclerites and rounded sclerite covering venter and sides, rounded sclerite without median longitudinal keel or anterior protrusion extending toward sternum.

Anterior edge of abdomen in males with oval dorsal scutum extending to about onefourth of abdominal length; dorsal scutum well separated from epigastric scutum except at anterior edge of abdomen, where both scuta join narrow sclerotized ring around pedicel opening; females with small but distinct, circular, dorsal scutum mostly confined to anterior surface of abdomen; cuticle with long dark setae and scattered thick white scales; epigastric scutum accompanied posterolaterally by pair of oval, deeply invaginated sclerites bearing clearly elevated anterior rim; anterior edge of oval sclerites fitting under epigastric scutum; colulus represented by setae; tiny transverse sclerite present, well removed from spinnerets, presumably marking position of small posterior spiracle. Anterior lateral spinnerets tubular, separated by roughly their diameter, cuticle representing distal, second spinneret segment restricted to semicircle surrounding major ampullate gland spigots (piriform gland spigots surrounded only by soft cuticle); posterior median spinnerets small, tubular, without anteriorly expanded tips; posterior lateral spinnerets twosegmented, spigots unscanned.

Legs almost spineless, single proventral spine at distal tibia of tibiae III, IV; most surfaces with long, dark setae; anterior coxae with protuberant posterolateral corners; males with coxae tuberculate; trochanters unnotched; anterior metatarsi and tarsi without distinct, divided scopulae; posterior metatarsi with thick, dark, distal preening brushes; posterior tarsi with weak, entire scopulae; 
tarsi with two dentate claws, claw tufts composed of lateral pads of closely appressed setae; trichobothria present on tibiae, metatarsi, and tarsi, in 2-3 irregular rows, bases unscanned; tarsal organ unscanned. Female palpal tarsus with few long, thin spines and dentate claw

Male palp with small, sharply pointed retrolateral tibial apophysis, cymbial surface without retrobasal excavation; distal prolateral edge of tibia with rounded, distally protuberant lobe; tegulum not expanded, not extending beyond posterior rim of tarsus; embolus laterally situated, inset into membranous conductor; median apophysis represented only by distinct sclerotization on prolateral side of conductor. Epigynum with rectangular median plate; spermathecae with anterior extensions.

\section{Lamponusa gleneagle, new species} Figures 276, 330-333; Map 39

TyPES: Female holotype and male allotype taken in a pitfall trap set in litter at Gleneagle State Forest, $32^{\circ} 15^{\prime} \mathrm{S}, 116^{\circ} 10^{\prime} \mathrm{E}$, Western Australia (Jan. 24-Mar. 3, 1979; M. Gray), deposited in AMS (KS15057).

ETYMOLOGY: The specific name is a noun in apposition taken from the type locality.

DIAGNOSIS: This species can easily be separated from all other lamponines by the white cuticular scales on both the carapace and abdomen (fig. 276); the tiny, sharply pointed retrolateral tibial apophysis of males (fig. 331 ) and the rectangular epigynal midpiece of females (fig. 332) are also diagnostic.

MALE: Total length 3.5. Dorsum of abdomen gray, with anterior and median pairs of narrow, transverse, lateral white markings and posteromedian white spot; femora, patellae, and tibiae with dusky sides. Palpal femur short, thick, with slight ventral invagination at about one-third its length; retrolateral tibial apophysis small, short, sharply pointed (fig. 331); embolar base long, basally wide (fig. 330).

FEMALE: Total length 4.7. Coloration as in male. Epigynal midpiece rectangular (fig. 332); spermathecae almost triangular, with small anterior extensions (fig. 333).

Other Material Examined: Western Australia: Alcoa minesite, Jarrahdale, $32^{\circ} 17^{\prime} \mathrm{S}, 116^{\circ} 06^{\prime} \mathrm{E}$, Apr. 1998, pitfall (L. Ashby, WAM 96/753), $10^{\star}$; Gleneagle State Forest, $32^{\circ} 15^{\prime} \mathrm{S}, 116^{\circ} 10^{\prime} \mathrm{E}$, Jan. 24-Mar. 3, 1979, pitfall, litter (M. Gray, AMS KS15057), 20 ; Norman Road, Cardup, $32^{\circ} 16^{\prime} \mathrm{S}, 116^{\circ} 01^{\prime} \mathrm{E}$, Apr. 16-June 17, 1996, pitfall (J. Waldock, P. West, A. Longbottom, WAM 96/754), 1 ๙.

DisTRIBUTION: Known only from southwestern Western Australia (map 39).

\section{Lamponega, new genus}

TyPe SPeCIES: Lamponega arcoona, new species.

ETYMOLOGY: The generic name is an arbitrary combination of letters, considered feminine in gender.

DiAGNOSIS: Specimens of this genus can easily be separated from all other lamponines by their greatly elevated sternum, with steeply sloping sides (figs. 338, 341), and by the lack of a modified promarginal cheliceral seta.

DESCRIPTION: Small spiders, total length 3.6-6.2. Carapace dark red, tuberculate, coated with long setae originating from bases of tubercles, tubercles protruding from lateral margin; thoracic groove short, almost obsolete. Eight eyes in two rows, eyes large, subequal in size; anterior medians circular, dark, other eyes light, laterals oval, posterior medians irregularly oval, flattened; from above, anterior eye row slightly recurved, posterior row slightly procurved, from front, both rows strongly procurved; anterior medians separated by less than their diameter, almost touching anterior laterals; posterior medians almost touching, separated by less than their radius from posterior laterals; anterior and posterior laterals separated by less than their radius; median ocular quadrangle slightly wider in front than in back, slightly longer than wide. Chelicerae, sternum, and mouthparts dark red; chilum absent, apparently fused to carapace, which is prolonged at midline; second, short, posterior chilum (extremely narrow sclerite separating bases of chelicerae posteriorly) present; chelicerae with distinct lateral boss, promargin with series of short setae originating in line along base of fang, without long, thick, modified seta originating closest to fang; promargin 

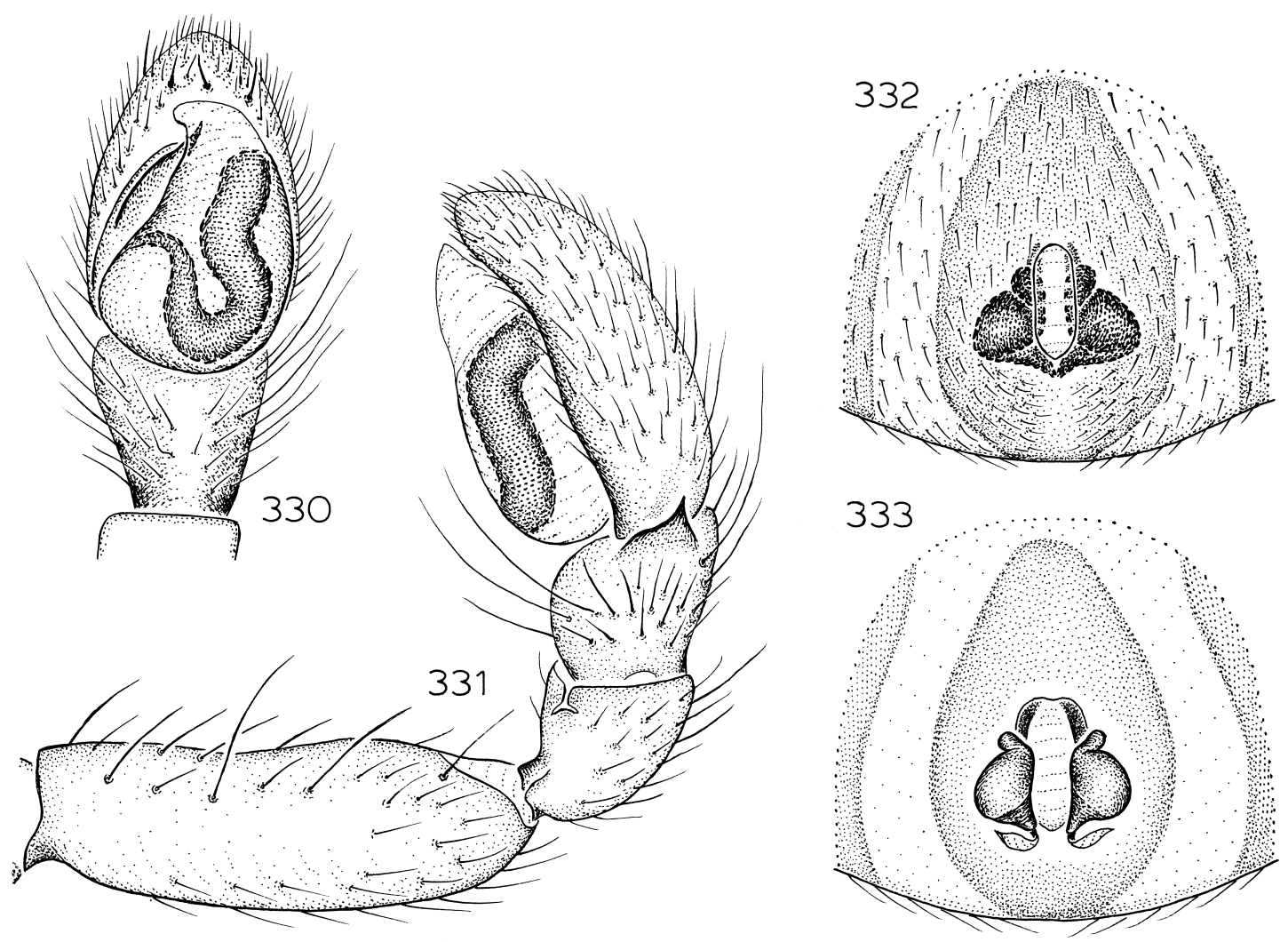

Figs. 330-333. Lamponusa gleneagle, new species. 330. Left male palp, ventral view. 331. Same, retrolateral view. 332. Epigynum, ventral view. 333. Same, dorsal view.

with teeth obsolete, represented by fused ridge, retromargin without teeth; cheliceral gland openings not scanned. Labium truncate anteriorly and posteriorly, distinctly depressed medially; anterior surface not scanned. Endites obliquely depressed, with sharply demarcated, deep groove along margin near labium; serrula long, with single row of teeth; anterior surface not scanned. Sternum greatly elevated, with steeply sloping, pinched-in sides, fused to epimeric sclerites so that coxae originate from foramina completely enclosed by sclerotized cuticle; surface smooth, with circular punctations. Epimeric sclerites not fused with carapace. Pedicel elongated, composed of two small, flat dorsal sclerites and rounded sclerite covering venter and sides, rounded sclerite without median longitudinal keel or anterior protrusion extending toward sternum.

Anterior edge of abdomen in males with oval dorsal scutum extending to about onethird of abdominal length; dorsal scutum well separated from epigastric scutum; females without dorsal scutum; cuticle with long dark setae; epigastric scutum accompanied posterolaterally by pair of oval, deeply invaginated sclerites bearing clearly elevated anterior rim; anterior edge of oval sclerites fitting under epigastric scutum; colulus represented by setae; tiny transverse sclerite present, well removed from spinnerets, presumably marking position of small posterior spiracle. Anterior lateral spinnerets tubular, separated by roughly their diameter, cuticle representing distal, second spinneret segment restricted to semicircle surrounding major ampullate gland spigots (piriform gland spigots surrounded only by soft cuticle); posterior median spinnerets small, tubular, without anteriorly expanded tips; posterior lateral 
spinnerets two-segmented, spigots unscanned.

Legs spineless; most surfaces with long, dark setae; anterior coxae with protuberant posterolateral corners; males with coxae tuberculate; trochanters unnotched; anterior metatarsi and tarsi without distinct, divided scopulae; posterior metatarsi with thick, dark, distal preening brushes; posterior tarsi with weak, entire scopulae; tarsi with two dentate claws, claw tufts composed of lateral pads of closely appressed setae; trichobothria present on tibiae, metatarsi, and tarsi, in 2-3 irregular rows, bases unscanned; tarsal organ unscanned. Female palpal tarsus with few long, thin spines and dentate claw

Male palp without retrolateral tibial apophysis, position of apophysis marked by distinct excavation, cymbial surface without retrobasal excavation; tegulum not expanded, not extending beyond posterior rim of tarsus; embolus laterally situated, inset into membranous conductor; median apophysis absent. Epigynum with long, narrow, median ridge extending posteriorly from anterior rim; spermathecae bipartite.

\section{Key to SPECIES OF LAMPONEGA}

1. Females (only those of L. arcoona known); epigynal midpiece extremely long, narrow (fig. 336) .............. L. arcoona

- Males ........... ............ 2

2. Sternum greatly elevated (fig. 338) . . . . 3

- Sternum less elevated (fig. 341) . . L. arcoona

3. Embolar base directed distally (fig. 342) .... ................... serpentine

- Embolar base directed more to side (fig. 339) ................... forceps

\section{Lamponega arcoona, new species}

Figures 334-337, 341; map 40

TyPE: Male holotype from Arcoona Creek, 2 km NE Owieandana HS, Gammon Ranges National Park, $30^{\circ} 26^{\prime} \mathrm{S}, 138^{\circ} 58^{\prime} \mathrm{E}$, South Australia (May 6, 1989; J. Forrest), deposited in SAM (N1996/279).

ETYMOLOGY: The specific name is a noun in apposition taken from the type locality.

DiAgNOSIS: Males and females have not been collected together, but are matched here because of their similar sternal modifications. Males have a less dramatically elevated sternum than do those of $L$. forceps or $L$. ser- pentine (fig. 341); females have a presumably distinctive epigynal midpiece (fig. 336).

MALE: Total length 3.6. Abdominal dorsum gray, with white markings restricted to tiny spot above spinnerets, four circular muscle impressions, and lateral lines; legs brown, unmarked. Palpal femur short, thick, unmodified; tibia without retrolateral apophysis, with slight retrodistal excavation (fig. 335); cymbium not basally excavated; tegulum not extending beyond tip of tibia, but with pronounced bulge on prolateral side; embolus with long, arched tip (fig. 334).

Female: Total length 4.2. Coloration as in male. Epigynum with narrow, scapelike median plate extending from anterior margin for about two-thirds of epigynal length (fig. 336); spermathecae bipartite, posterior portions curled (fig. 337).

Other Material Examined: South Australia: $31 \mathrm{~km}$ NW Renmark, 3359'S, $140^{\circ} 30^{\prime} \mathrm{E}$, May 2-June 8, 1995, intercept trap (K. Pullen, QMB), 10; Yardea Station, 29 km S Moonarie, $32^{\circ} 14^{\prime} \mathrm{S}, 135^{\circ} 49^{\prime} \mathrm{E}$, Oct. 3, 1988, pitfall (D. Hirst, SAM N1997/121), 19 . Western Australia: Monkey Mia, Peron Peninsula, 25 $47^{\prime} \mathrm{S}, 113^{\circ} 41^{\prime} \mathrm{E}$, Nov. 8, 1998 (V. Ovtsharenko, AMNH), 1 ; ; Peak Charles, $32^{\circ} 53^{\prime} \mathrm{S}, 121^{\circ} 10^{\prime} \mathrm{E}$, Aug. 2, 1992, salmon gum litter (M. Cowan, WAM 96/240), 1 ; York, $31^{\circ} 53^{\prime} \mathrm{S}, 116^{\circ} 43^{\prime} \mathrm{S}$, Nov. 2, 1991, pow-

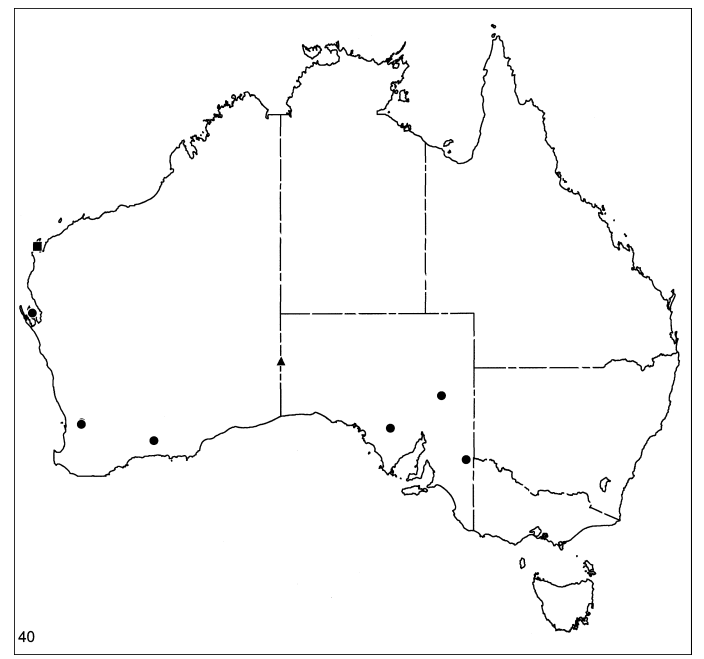

Map 40. Records of Lamponega arcoona, new species (circles), L. forceps, new species (square), and L. serpentine, new species (triangle). 

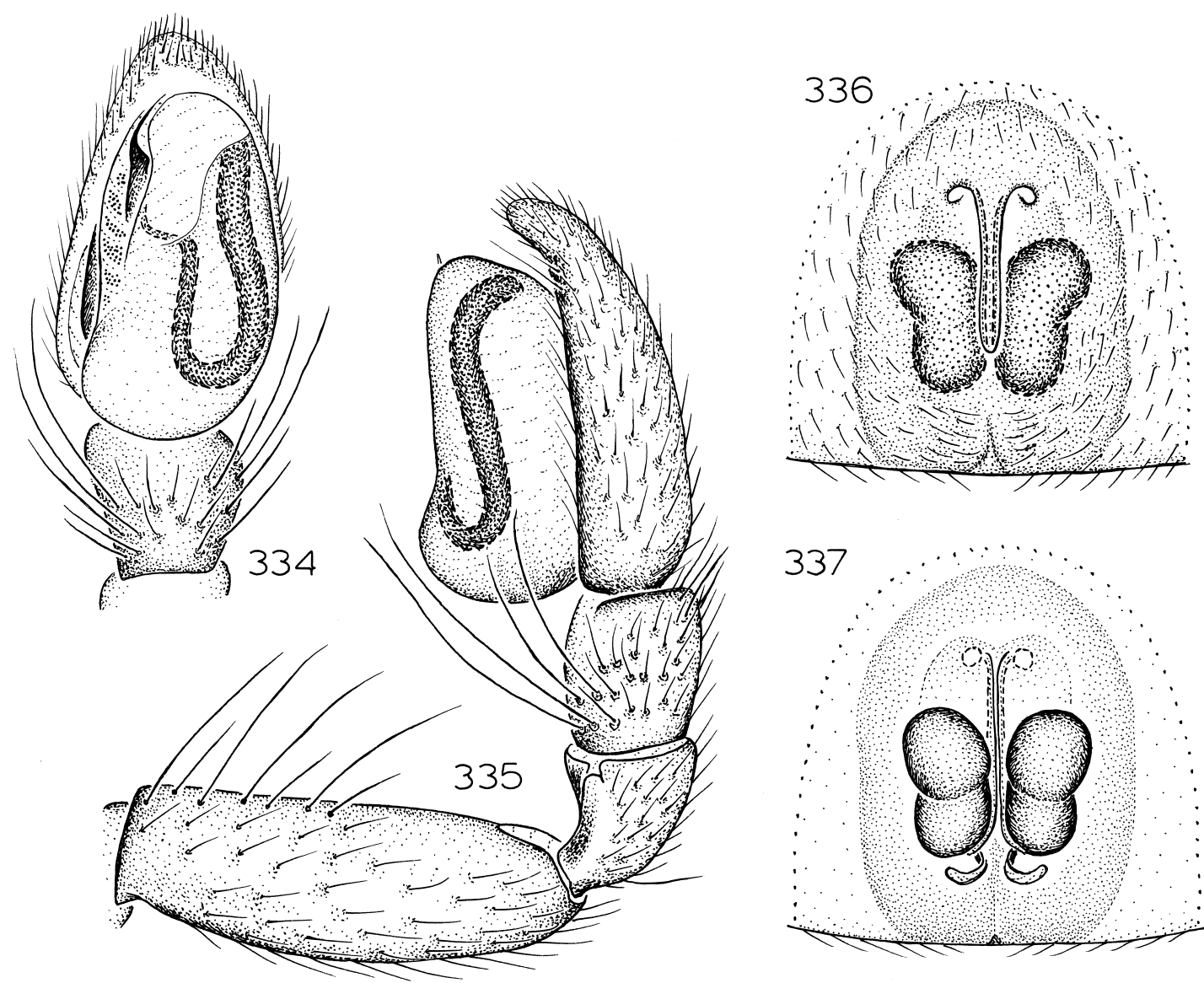

Figs. 334-337. Lamponega arcoona, new species. 334. Left male palp, ventral view. 335. Same, retrolateral view. 336. Epigynum, ventral view. 337. Same, dorsal view.

der bark/mallet leaf litter (J. Bannister, WAM 96/241), 1 \% ; $10 \mathrm{~km} \mathrm{SW}$ York, 31 $58^{\circ} \mathrm{S}$, $116^{\circ} 42^{\prime}$ S, Dec. 5, 1996, elev. 430 m (N. Platnick, R. Raven, V. Ovtsharenko, K. Catley, AMNH), 1 ㅇ.

Distribution: Southern Australia (map 40).

\section{Lamponega forceps, new species} Figures 338-340; Map 40

TyPE: Male holotype taken near Cape Range No. 2 well, Cape Range, $22^{\circ} 06^{\prime} \mathrm{S}$, $114^{\circ} 00^{\prime} \mathrm{E}$, Western Australia (May 1965; G. Kendrick, B. Hitchin), deposited in WAM (96/242).

ETYMOLOGY: The specific name is a noun in apposition, referring to the remarkable appearance of the sternum, which looks like it has been squeezed in a pair of forceps (fig. 338).

DiAGNOSIS: The bizarre sternal modifications resemble those of $L$. serpentine but males differ in having the base of the embolus directed more to the side (fig. 339).

MALE: Total length 6.1. Coloration as in $L$. arcoona, except longitudinal white lines on abdominal dorsum wider, more pronounced; legs orange. Palpal femur short, thick, unmodified; tibia without retrolateral apophysis, with slight retrodistal excavation (fig. 340); cymbium not basally excavated; tegulum not extending beyond tip of tibia, but with pronounced bulge on prolateral side; embolus with base prolaterally directed, tip relatively short (fig. 339).

FEMALE: Unknown. 

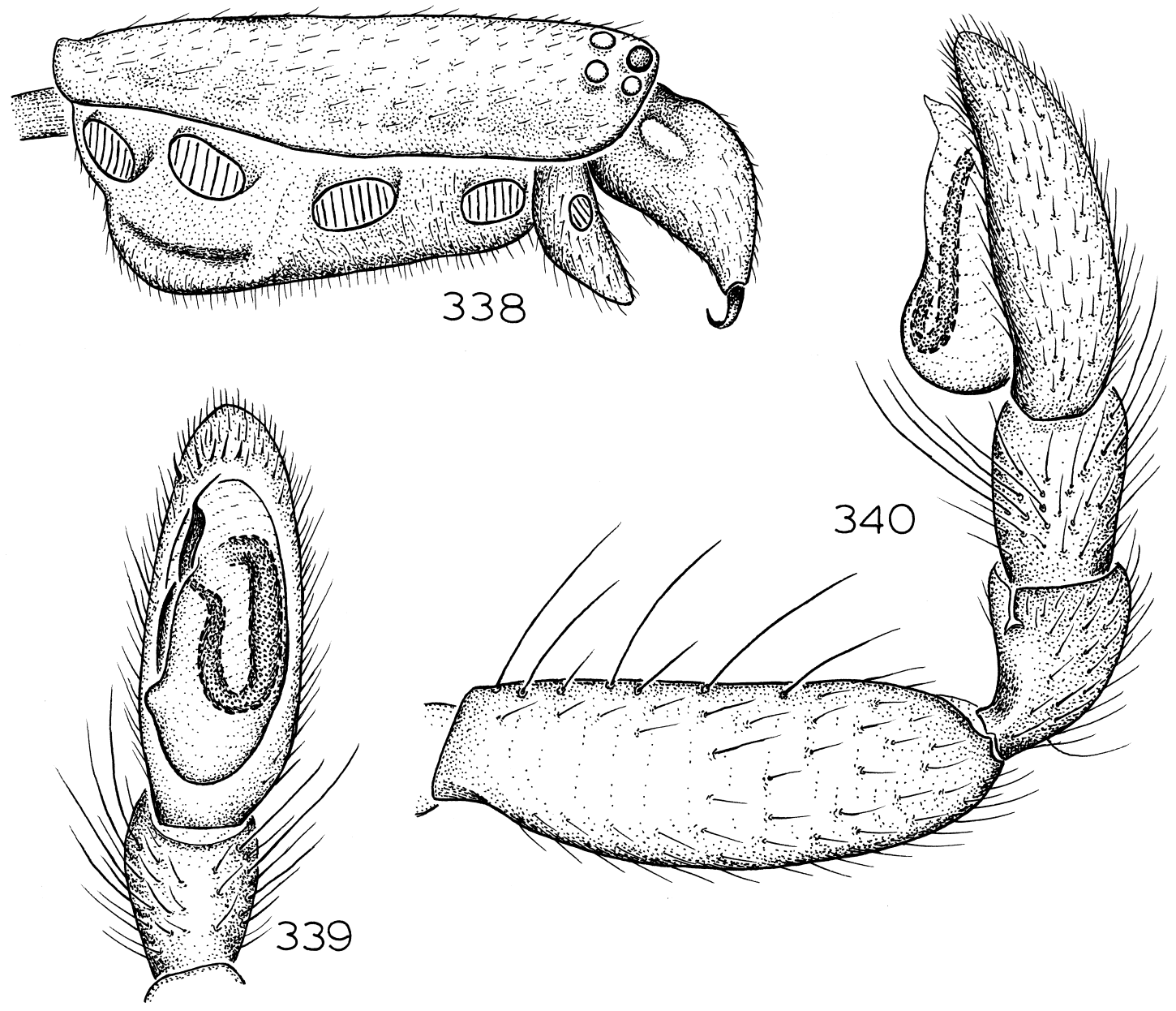

Figs. 338-340. Lamponega forceps, new species. 338. Cephalothorax, lateral view. 339. Left male palp, ventral view. 340. Same, retrolateral view.

Other MATERIAL EXAMined: None.

DisTRIBUTION: Known only from the Cape Range, Western Australia (map 40).

\section{Lamponega serpentine, new species \\ Figures 342, 343; Map 40}

TYPE: Male holotype taken under bark on the $\mathrm{E}$ side of Serpentine Lakes, $28^{\circ} 30^{\prime} \mathrm{S}$, $129^{\circ} 02^{\prime}$ E, South Australia (Apr. 18, 1994; P. Hudson), deposited in SAM (N1996/320).

ETYMOLOGY: The specific name is a noun in apposition taken from the type locality.

DIAGNOSIS: Males resemble those of $L$. forceps in sternal modifications, but have the embolar base directed more distally (fig. 342).
MALE: Total length 4.7. Coloration as in $L$. forceps. Palp as in $L$. forceps but tegulum proportionately shorter, embolar base without pronounced constriction, directed distally (figs. 342, 343).

FEMALE: Unknown.

Other MATERIAL ExAmined: None.

DISTRIBUTION: Known only from the western border of South Australia (map 40).

\section{Lamponella, new genus}

TyPE SPECIES: Lamponella ainslie, new species.

ETYMOLOGY: The generic name is an arbitrary combination of letters, considered feminine in gender. 


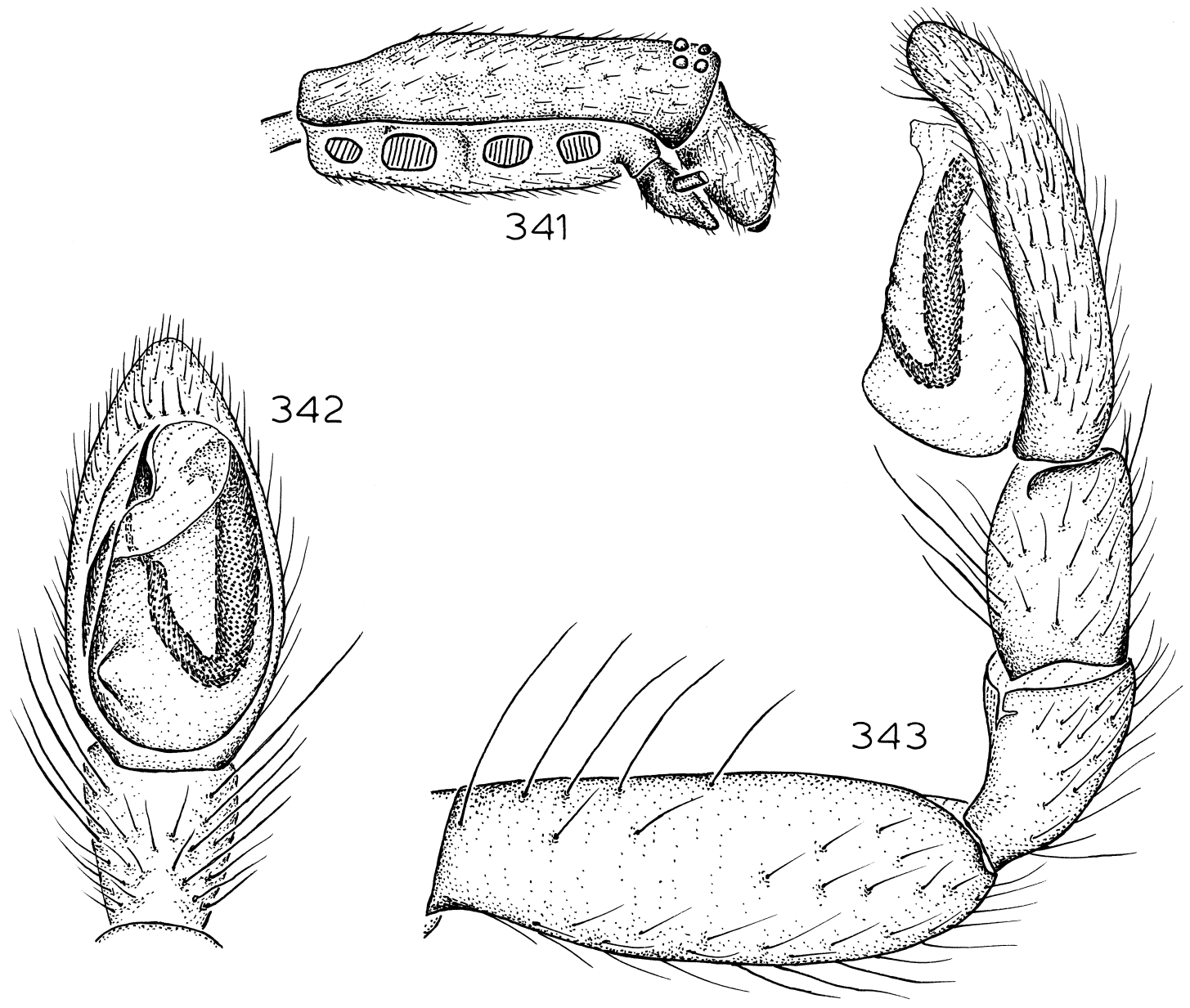

Figs. 341-343. 341. Lamponega arcoona, new species, cephalothorax, lateral view. 342. L. serpentine, new species, left male palp, ventral view. 343. Same, retrolateral view.

DiAGNOSIS: Members of this genus can easily be recognized by their deeply punctate sternum (figs. 325, 350) combined with a female palpal tarsus that is shortened, basally thickened, and has a dorsal scopula (fig. 349) or a male palp that retains a median apophysis (as in fig. 364). Other unusual features are the absence of a chilum, the anteriorly narrowed and medially invaginated labium, the presence of four unfused, triangular, epimeric sclerites above the left and right coxae, and the spineless female palpal tibia and tarsus.

DESCRIPTION: Small spiders, total length of males 2.2-3.6, of females 3.4-5.2. Carapace orange to red, tuberculate, with scattered non-feathery setae originating from bases of slight tubercles, tubercles protruding from lateral margin; thoracic groove long, longitudinal, very shallow, almost obsolete. Eight eyes in two rows, posterior medians largest, other eyes subequal in size; anterior medians circular, dark, laterals oval, light, posterior medians irregularly oval, flattened (fig. 344); from above, anterior eye row slightly procurved, posterior row strongly procurved, from front, both rows strongly procurved; anterior medians separated by at least their radius, by their radius or less from anterior laterals; posterior medians separated by less than their radius, separated by almost their radius from posterior laterals; anterior and posterior laterals separated by almost their diameter; median ocular quadrangle wider in 
front than in back, slightly longer than wide. Chelicerae, sternum, and mouthparts light orange to red; chilum absent, posterior chilum present as extremely narrow sclerite separating bases of chelicerae posteriorly (fig. 348); chelicerae with distinct lateral boss, promargin with series of short setae originating in line along base of fang plus one long, thick seta originating closest to fang, immediately bent at $90^{\circ}$ angle, extending to median line (fig. 345); promargin with about five tiny teeth on common carina, retromargin without teeth (fig. 346); cheliceral gland openings in almost unsclerotized, slight depression situated posteroproximally of teeth (fig. 347). Labium narrow, medially invaginated anteriorly, truncated posteriorly, distinctly depressed medially. Endites obliquely depressed, with sharply demarcated, deep groove along margin near labium (in ventral view, much of groove obscured by folded groove margin, fig. 351); serrula long, with single row of long teeth. Sternum slightly elevated, not expanded anteriorly to sides beyond posterolateral corner of endites, with extensions to and between coxae; surface deeply punctate (fig. 350). Four triangular epimeric sclerites on each side, between pairs of palpal and leg coxae, not reaching sternal triangles, not fused to carapace or to each other. Pedicel composed of two small, flat dorsal sclerites and rounded sclerite covering venter and sides, rounded sclerite with median longitudinal keel prolonged anteriorly into distinct protrusion extending toward sternum.

Anterior edge of abdomen of male with dorsal abdominal scutum reaching to at least one-third of abdominal length, not fused to epigastric scutum, females without dorsal scutum; cuticle with scattered short, nonfeathery setae; epigastric scutum accompanied posterolaterally by pair of oval, deeply invaginated sclerites bearing clearly elevated anterior rim; anterior edge of oval sclerites often fitting under epigastric scutum; colulus represented by setae, apparently situated just behind tracheal spiracle. Anterior lateral spinnerets short, tubular, almost contiguous at base, separated by almost their diameter at tip, with two thick major ampullate gland spigots (median one larger than lateral) plus small, unmodified piriform gland spigots (figs. 354, 355), cuticle representing distal, second spinneret segment restricted to semicircle surrounding major ampullate gland spigots (piriform gland spigots surrounded only by soft cuticle); posterior median spinnerets small, tubular, without anteriorly expanded tips, females with three spigot types (one with extremely long shaft, one with shorter shaft and narrow base, one with shorter shaft and wide base), third type lacking in males and thus presumably serving cylindrical glands (figs. 356, 357); posterior lateral spinnerets two-segmented, with spigots as on posterior medians (figs. 358, 359).

Legs spineless, with scattered non-feathery setae; in males, all coxae and posterior trochanters dorsally tuberculate; anterior coxae with slightly protuberant posterolateral corners; trochanters unnotched; anterior metatarsi and tarsi without distinct scopulae; posterior metatarsi with thick, distal preening brushes; posterior tarsi with long setae but without distinct scopulae; tarsi with two dentate claws, claw tufts composed of closely appressed setae; trichobothria present on tibiae, metatarsi, and tarsi, in single row, bases ridged (fig. 353); tarsal organ long, opening situated distally (fig. 352). Female palpal tibia and tarsus spineless; female palpal tarsus shortened, basally thickened, with short claw and dorsodistal scopula (fig. 349).

Male palp with retrolateral tibial apophysis often reduced to low lobe (figs. 361, 365, 369); embolus elongate, prolaterally situated, broadly fused basally to tegulum or free, accompanied by small, hooked median apophysis as well as membranous conductor (figs. $360,364,368$ ). Epigynum variable in shape, without plug of solidified secretions.

\section{Key to Species of LAMPONELLA}

1. Males (those of L. kroombit unknown) . 2

- Females (those of L. wombat unknown) ... ................. 10

2. Retrolateral tibial apophysis distinct (as in fig. 361) .................. 3

- Retrolateral tibial apophysis obsolete (fig. 365) ............... beaury

3. Median apophysis relatively small (figs. 360, 376) ................. 4

- Median apophysis relatively large (figs. 368, $372,380,384,388,392) \ldots \ldots \ldots .5$ 
4. Retrolateral tibial apophysis relatively short (fig. 361) ............... ainslie

- Retrolateral tibial apophysis relatively long (fig. 377) ........... . wombat

5. Median apophysis relatively short (figs. 368, 380) .................6 6

- Median apophysis relatively long (figs. 372, $384,388,392) \ldots \ldots \ldots \ldots \ldots$

6. Retrolateral tibial apophysis relatively long (fig. 381) ............. brookfield

- Retrolateral tibial apophysis relatively short (fig. 369) ............. kanangra

7. Median apophysis sinuous (fig. 388) .... ...................... . . .

- Median apophysis not sinuous ...... 8

8. Retrolateral tibial apophysis rounded (fig. 385) ........... homevale

- Retrolateral tibial apophysis triangular (figs. $373,393) \ldots \ldots \ldots \ldots \ldots$

9. Retrolateral tibial apophysis relatively wide (fig. 393) ............... taroom

- Retrolateral tibial apophysis relatively narrow (fig. 373) ............ wyandotte

10. Spermathecae widely separated (figs. 366, 367) .............. beaury

- Spermathecae approximate (as in figs. 361, 362) ............... 11

11. Anterior portion of spermathecae relatively long (figs. 375, 379, 387, 395) . . . . 12

- Anterior portion of spermathecae relatively short (figs. 363, 371, 383, 391) . . . . 15

12. Median portion of spermathecae bulging (fig. 395) ................ taroom

- Median portion of spermathecae not bulging (figs. 375, 379. 387) . . . . . . . . 13

13. Epigynal atrium oval (fig. 378) . . kroombit

- Epigynal atrium triangular or heart-shaped (figs. 374, 386) . . . . . . . . . 14

14. Anterior portion of spermathecae pointed posteriorly (fig. 387) ....... homevale

- Anterior portion of spermathecae rounded posteriorly (fig. 375) ....... wyandotte

15. Anterior portion of spermathecae pea-shaped (figs. 363, 391) ........... 16

- Anterior portion of spermathecae rounded (figs. 373, 383) . . . . . . . . 17

16. Outline of spermathecae producing diamondshaped pattern within epigynal midpiece (fig. 362) ................ ainslie

- Outline of spermathecae not producing diamond-shaped pattern (fig. 390) ... kimba

17. Anterior portion of spermathecae relatively narrow (fig. 383) .......... brookfield

- Anterior portion of spermathecae relatively wide (fig. 371) . . . . . . . . kanangra
Lamponella ainslie, new species

Figures 325, 344-363; Map 41

TyPES: Female holotype and male allotype taken in grass tussocks at Queens Domain, Hobart, $42^{\circ} 52^{\prime} \mathrm{S}, 147^{\circ} 19^{\prime} \mathrm{E}$, Tasmania (Mar. 23, 1968; V. Hickman), deposited in AMS (KS29339).

ETYMOLOGY: The specific name is a noun in apposition taken from Mount Ainslie, one of the localities at which the species has been collected.

DiAGNOSIS: Males can be recognized by the tiny median apophysis situated at the tip of the embolus (fig. 360), females by the distinctive diamond-shaped light area formed within the epigynal midpiece by the outlines of the spermathecae showing through the epigynal cuticle (fig. 362).

MALE: Total length 2.6. Abdominal dorsum dark gray, with three pairs of small white cardiac spots (anterior and part of median pair obscured by scutum) and series of posterior white hairline chevrons; legs unmarked. Palpal femur arched but unmodified; retrolateral tibial apophysis tiny, triangular (fig. 361); cymbium with dorsodistal scopula; tegulum globose, reaching to about onethird of tibial length; embolus broadly fused basally to tegulum, tip short, twisted prolaterally; median apophysis short, hook-shaped, distally situated (fig. 360).

Female: Total length 3.8. Coloration as in male. Palpal tarsus shortened, thickened at about half its length, with dorsodistal scopula. Epigynum with pair of semicircular lateral margins (fig. 362); spermathecae bipartite, anterior portion on twisted duct (fig. 363).

Other Material Examined: Australian Capital Territory: Black Mountain, $35^{\circ} 16^{\prime} \mathrm{S}, 149^{\circ} 06^{\prime} \mathrm{E}$, Jan. 18, 1975 (QMB S26715), 1 ㅇ, Oct.-Nov. 1983, pitfall (QMB), 19 , Feb. 11, 1985, leaf litter (R. Moran, QMB), 1 ; ; S slope, Black Mountain, $35^{\circ} 16^{\prime} \mathrm{S}, 149^{\circ} 06^{\prime} \mathrm{E}$, June 3, 1970, Berlese, dry sclerophyll, elev. $600 \mathrm{~m}$ (I. Taplin, QMB), 1 \%; W face, Mount Ainslie, $35^{\circ} 16^{\prime} \mathrm{S}$, $149^{\circ} 10^{\prime} \mathrm{E}$, Nov. 25,1989 , Berlese, dry sclerophyll leaf litter, elev. $820 \mathrm{~m}$ (C. Brooks, QMB), 1 . New South Wales: Blood Filly Creek near Jenolan Caves, Kanangra-Boyd National Park, $33^{\circ} 59^{\prime} \mathrm{S}, 150^{\circ} 08^{\prime} \mathrm{E}$, Mar. 27, 

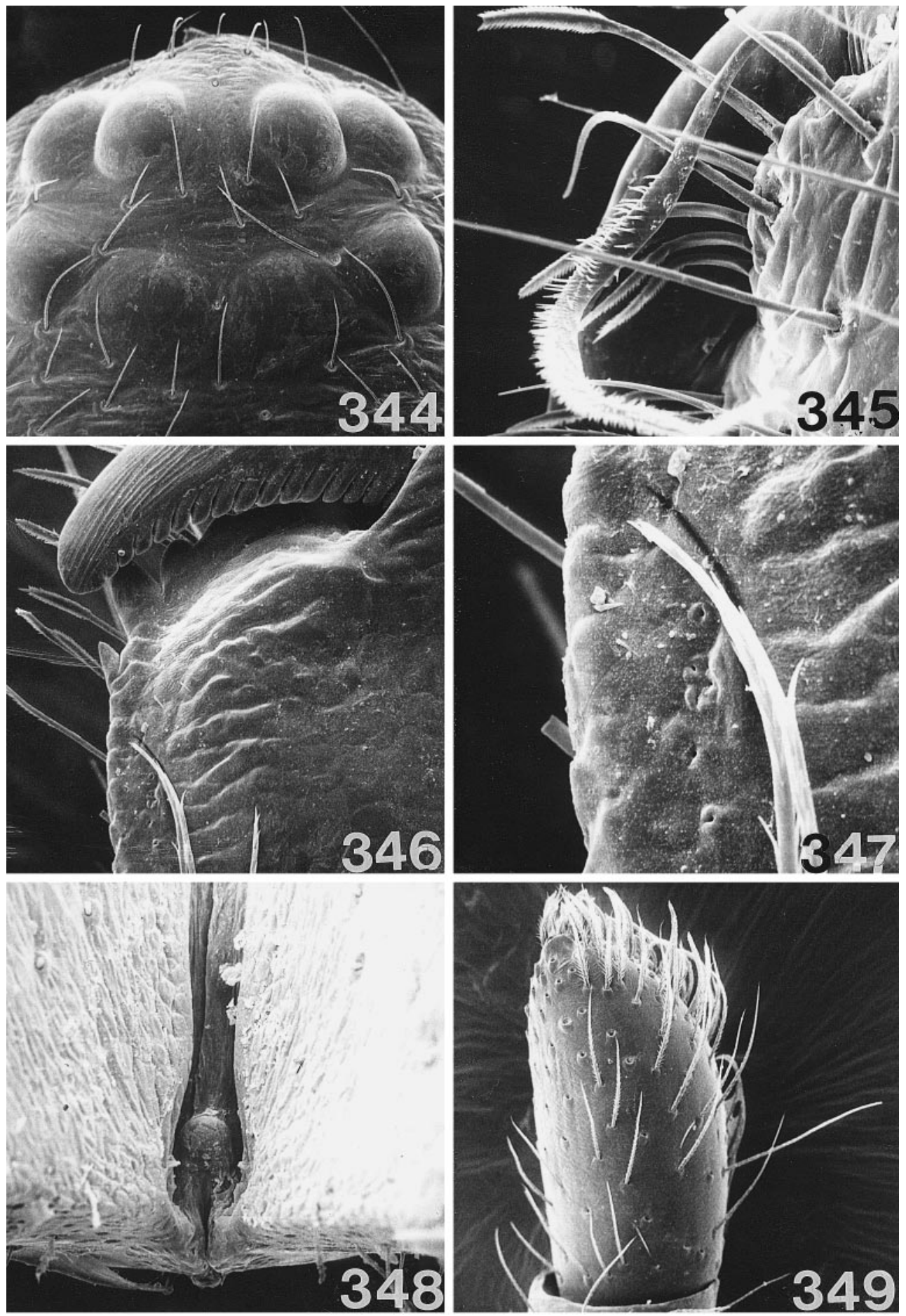

Figs. 344-349. Lamponella ainslie, new species, female. 344. Eyes, dorsal view. 345. Modified first promarginal seta of chelicera, anterior view. 346. Chelicera, posterior view. 347. Cheliceral gland openings, posterior view. 348. Posterior chilum between chelicerae, ventral view. 349. Palpal tarsus, lateral view. 

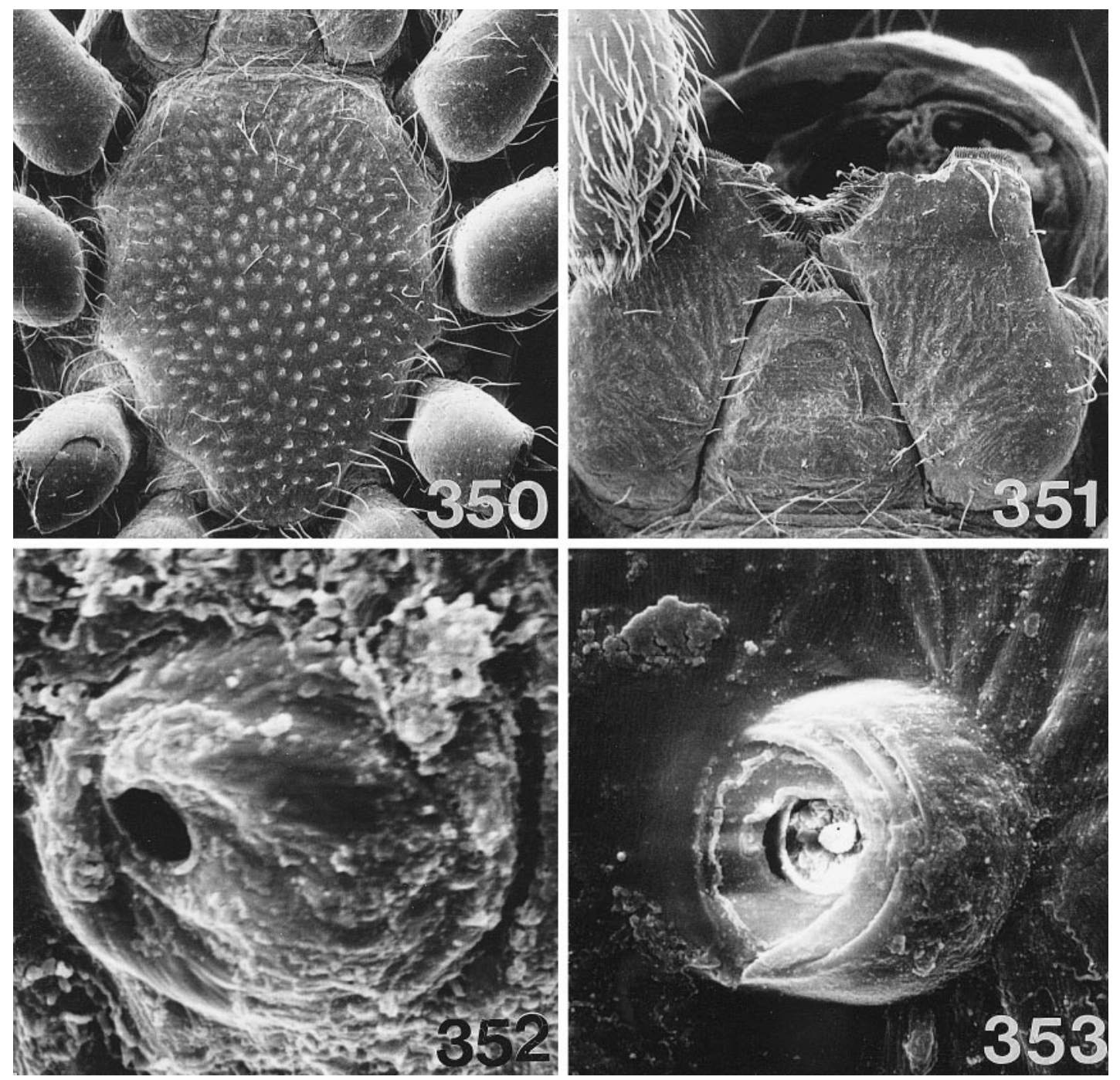

Figs. 350-353. Lamponella ainslie, new species, female. 350. Sternum, ventral view. 351. Labium and endites, ventral view; note that the lateral edges of the labium protrude into the median grooves of the endites. 352. Tarsal organ from leg IV, dorsal view. 353. Trichobothrial base from tarsus IV, dorsal view.

1976, Eucalyptus fastigata-vimnalis association (M. Gray, G. Hunt, J. McDougall, AMS KS29868), 1 ơ; Bungonia Caves, area near Information Centre, $34^{\circ} 50^{\prime} \mathrm{S}, 150^{\circ} 04^{\prime} \mathrm{E}$, mid Sept.-mid Oct. 1989, pitfall, surface (G. Hunt, AMS KS22594), 1 ㅇ ; 5 mi N Maroota, $33^{\circ} 29^{\prime} \mathrm{S}, 150^{\circ} 57^{\prime} \mathrm{E}$, Apr. 4, 1968, Berlese (E. Britton, L. Mound, QMB), $1 \%$; Trangie, $32^{\circ} 02^{\prime} \mathrm{S}, 147^{\circ} 59^{\prime} \mathrm{E}$, Mar. 3, 1968, Berlese, dry sclerophyll leaf litter (L. Mound, QMB), 1 . . South Australia: $1.2 \mathrm{~km} \mathrm{~S}$ Custon, $36^{\circ} 27^{\prime} \mathrm{S}, 140^{\circ} 55^{\prime} \mathrm{E}$, Dec. 4-8, 1995, pitfall (SAM N1997/124), 1 ơ; Gum Lagoon Conservation Park, $36^{\circ} 17^{\prime} \mathrm{S}, 140^{\circ} 02^{\prime} \mathrm{E}$, Mar. 26, 1992, vibration, lagoon edge (D. Hirst, SAM N1996/229-231), 1 đ̋, 1 우 N Jimmys Well, $35^{\circ} 51^{\prime} \mathrm{S}, 140^{\circ} 18^{\prime} \mathrm{E}$, Mar. 20, 1992, vibration (D. Hirst, SAM N1996/441-446), 20, 2 ㅇ ; 1 km E Jimmys Well, $35^{\circ} 51^{\prime} \mathrm{S}, 140^{\circ} 18^{\prime} \mathrm{E}$, Mar. 18, 1992, vibration (D. Hirst, SAM N1997/ 123), 1 우 Jip Jip Conservation Park, $36^{\circ} 29^{\prime} \mathrm{S}, 140^{\circ} 11^{\prime} \mathrm{E}$, Mar. 23, 1992, vibration 

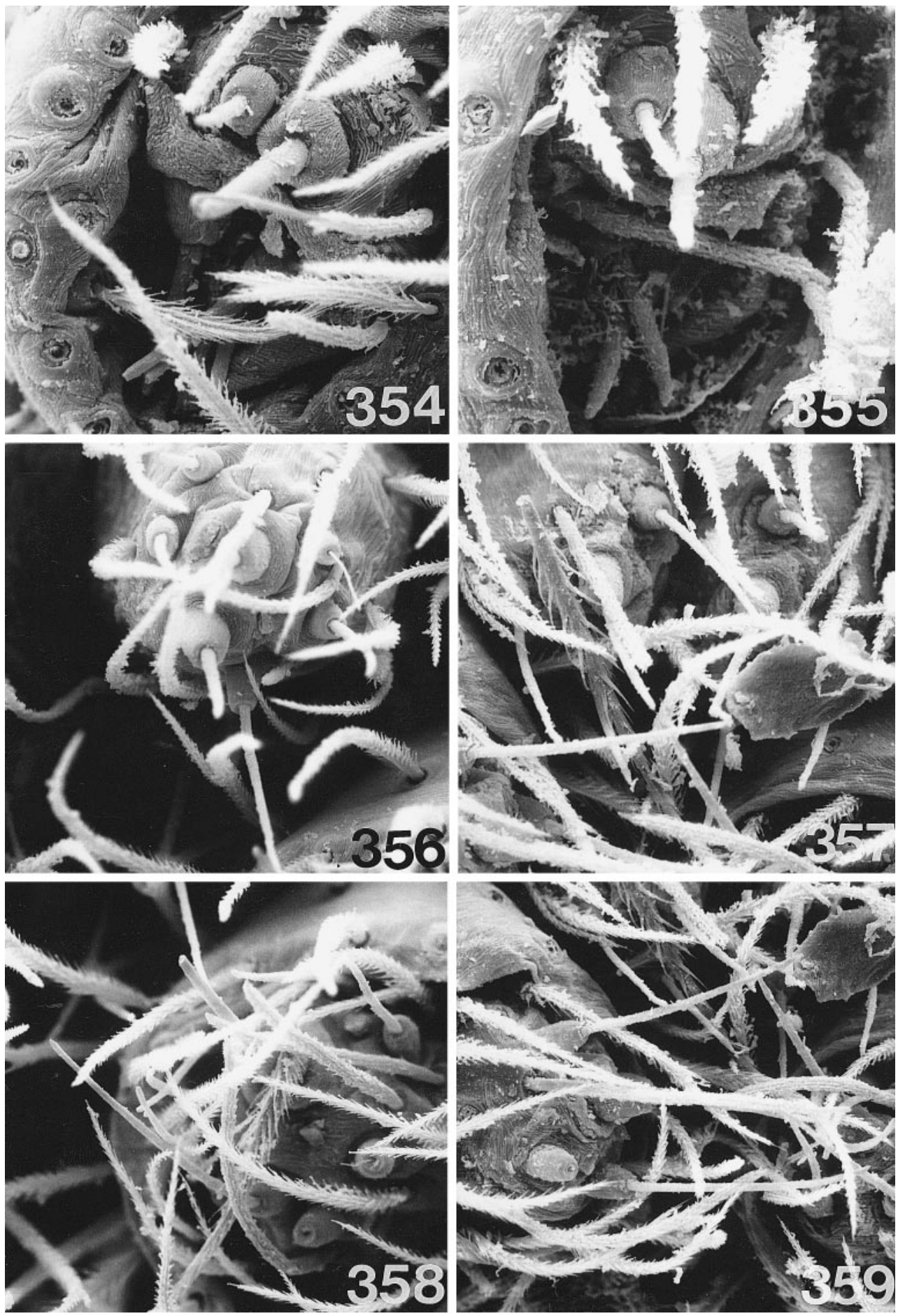

Figs. 354-359. Lamponella ainslie, new species, spinnerets of female (left) and male (right), posterior views. 354, 355. Anterior lateral spinnerets. 356, 357. Posterior median spinnerets. $358,359$. Posterior lateral spinnerets. 

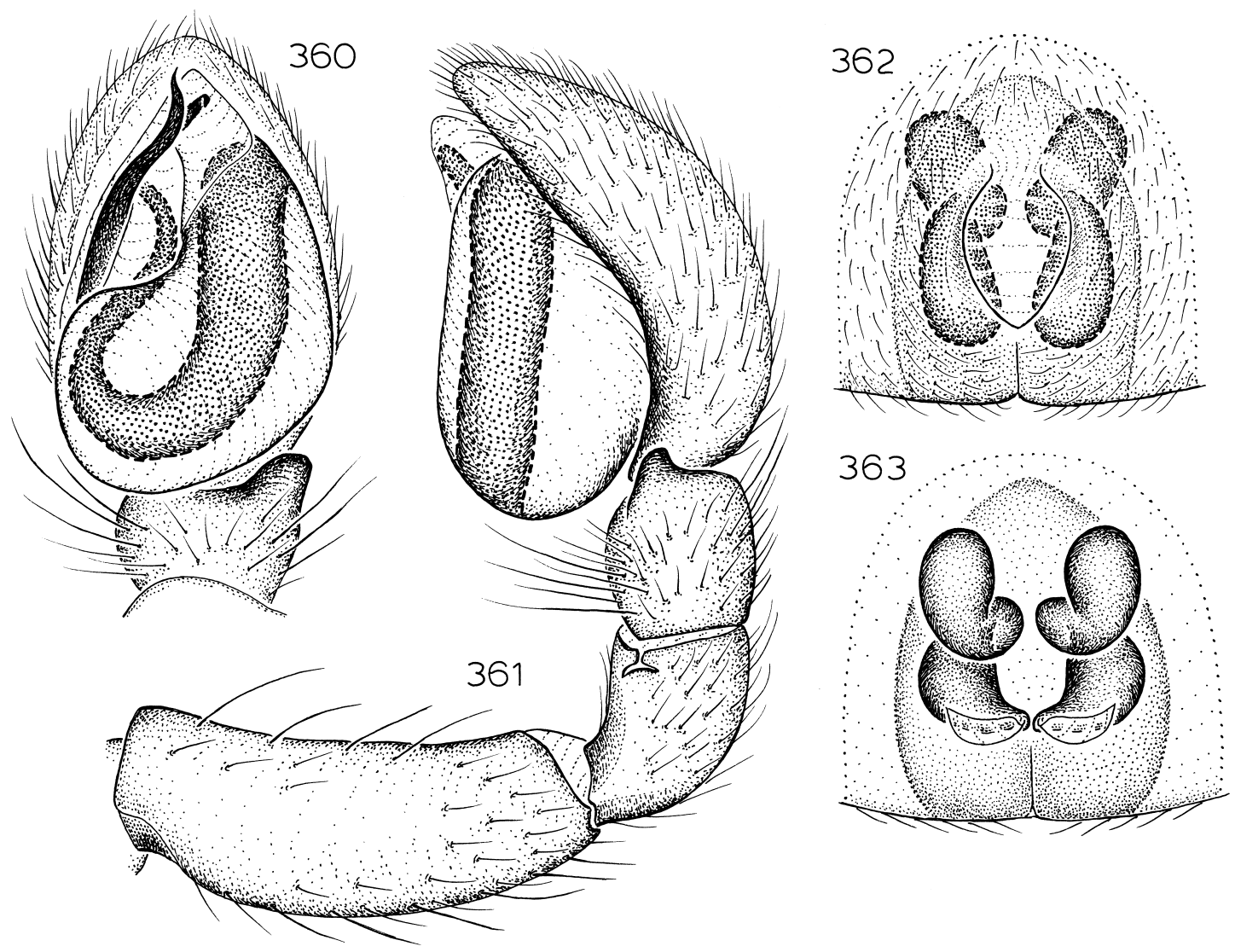

Figs. 360-363. Lamponella ainslie, new species. 360. Left male palp, ventral view. 361. Same, retrolateral view. 362. Epigynum, ventral view. 363. Same, dorsal view.

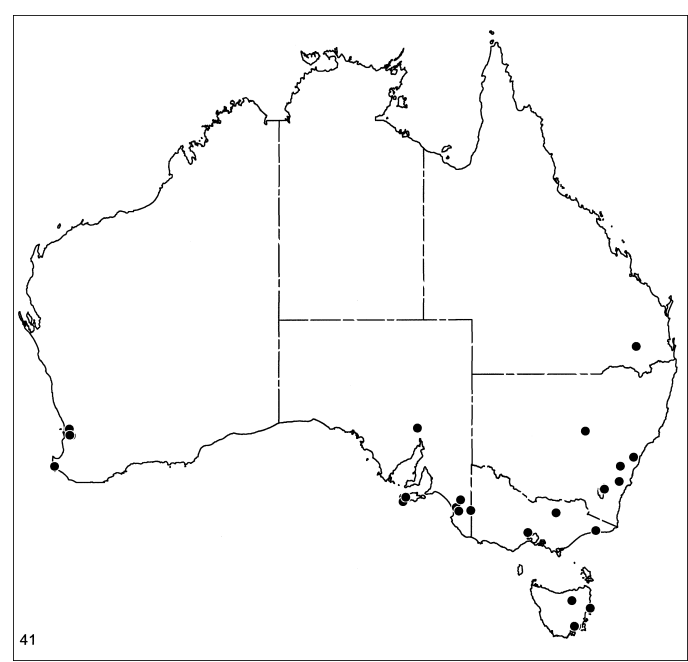

Map 41. Records of Lamponella ainslie, new species.
(D. Hirst, J. Forrest, SAM N1997/122), 1 đ; end Jump Off Road, Cape Torrens Conservation Park, Kangaroo Island, $35^{\circ} 44^{\prime} \mathrm{S}$, $136^{\circ} 45^{\prime} \mathrm{E}$, Nov. 4, 1987 (D. Hirst, SAM N1996/227), 1 \% ; Rocky River crossing, W Ranger Station, Flinders Chase, Kangaroo Island, 35 $57^{\prime} \mathrm{S}, 136^{\circ} 44^{\prime} \mathrm{E}$, Nov. 7-9, 1987 (D. Hirst, SAM N1996/228), 1 \% ; South Gap Station, Beda Hill, $31^{\circ} 51^{\prime} \mathrm{S}, 137^{\circ} 37^{\prime} \mathrm{E}$, Dec. $4-$ 6, 1989, pitfall (D. Hirst, SAM N1996/318), 19 ; track to Waterfall Creek, Western River Conservation Park, Kangaroo Island, $35^{\circ} 42^{\prime} \mathrm{S}, 136^{\circ} 54^{\prime} \mathrm{E}$, Nov. 3, 1987 (D. Hirst, SAM N1996/226), $1 \delta^{\text {t. }}$ Queensland: Lake Broadwater, via Dalby, $27^{\circ} 21^{\prime} \mathrm{S}, 151^{\circ} 06^{\prime} \mathrm{E}$, Mar. 26-May 17, 1985, pitfall (M. Bennie, QMB S34355), 10, Feb. 25-Apr. 22, 1986, pitfall (M. Bennie, QMB S34354), 1 ․ Tasmania: Bicheno lookouts, $41^{\circ} 52^{\prime} \mathrm{S}$, $148^{\circ} 18^{\prime} \mathrm{E}$, Jan. 31 , 1988, litter, dry eucalypt 
forest on granite (L. Boutin, TMH), 10; Glen Dhu, near Launceston, $41^{\circ} 27^{\prime} \mathrm{S}$, $147^{\circ} 10^{\prime} \mathrm{E}$, Nov. 8,1929 , in saclike next under stone (V. Hickman, AMS KS28962), 1, May 28, 1930, under stone (V. Hickman, AMS KS29248), 1 ; ; Mount Rumney, $42^{\circ} 52^{\prime} \mathrm{S}, 147^{\circ} 27^{\prime} \mathrm{E}$, Aug. 6, 1963, grass tussocks (J., V. Hickman, AMS KS29296), 50, 3 \% ; Risdon, $42^{\circ} 49^{\prime} \mathrm{S}, 147^{\circ} 21^{\prime} \mathrm{E}$, June 1947 , grass tussock (V. Hickman, AMS KS29301), 19 ; Queens Domain, Hobart, $42^{\circ} 52^{\prime} \mathrm{S}$, $147^{\circ} 19^{\prime}$ E, Sept. 1, 1934, grass tussocks (V. Hickman, AMS KS29342), 10, 3; Nov. 1939, under stones (V. Hickman, AMS KS29244), 3 \% , Dec. 1941-Feb. 1942 (V. Hickman, AMS KS29351), 7ㅇ, Aug.-Sept. 1942, grass tussocks (V. Hickman, AMS KS29245), 40, 7 으, May 1948, grass tussocks (V. Hickman, AMS KS28958, 29327), 10 ๙ , 18 오, June 5, 1948 (V. Hickman, AMS KS29334), 1 , Mar. 1968, grass tussock (V. Hickman, AMS KS29348), 10; Trevallyn, $41^{\circ} 27^{\prime} \mathrm{S}, 147^{\circ} 10^{\prime} \mathrm{E}$, Sept. 4, 1929, under bark of gum tree (V. Hickman, AMS KS28967), 1 ․ Victoria: Lerderderg Gorge, $9 \mathrm{~km}$ NNW Bacchus Marsh, $37^{\circ} 41^{\prime} \mathrm{S}, 144^{\circ} 27^{\prime} \mathrm{E}$, Aug. 12, 1981, debris under rock (M. Harvey, QMB), 1 \% ; Rich-Murrungowar Coupe, $37^{\circ} 34^{\prime} \mathrm{S}$, $148^{\circ} 38^{\prime} \mathrm{E}$, Mar. 18-25, 1992, upslope pitfall (R. Coy, NMV K3528), 10; Upper Lurg, $36^{\circ} 35^{\prime} \mathrm{S}, 146^{\circ} 11^{\prime} \mathrm{E}$, Aug. 29, 1996, garden (J. Strudwick, CVIC 645), 10, Jan. 14, 1997, leaf litter (J. Strudwick, CVIC 639, 646), 2 ㅇ. Western Australia: Gleneagle State Forest, $32^{\circ} 15^{\prime} \mathrm{S}, 116^{\circ} 10^{\prime} \mathrm{E}$, Jan. 24-Mar. 3, 1979, pitfall in litter (M. Gray, AMS KS15057), $19 ; 4.75 \mathrm{~km}$ ESE Margaret River, $33^{\circ} 58^{\prime} \mathrm{S}$, $115^{\circ} 07^{\prime} \mathrm{E}$, Feb. $8-14$, 1992, leaf litter, marri forest with Agonis flexuosa (M. Peterson, WAM 1995/1111), 19; Talbot Road Reserve, $31^{\circ} 52^{\prime} \mathrm{S}, 116^{\circ} 03^{\prime} \mathrm{E}$, Sept. 24-Nov. 18, 1993, pitfall (J. Waldock, WAM 96/1523), 10; Wongong Dam, Jarrahdale Mine, $32^{\circ} 13^{\prime} \mathrm{S}, 116^{\circ} 04^{\prime} \mathrm{E}$, Sept. 12-19, 1997 (K. Brennan, WAM 96/755), 1 \%.

Distribution: Southern Australia, including Tasmania (map 41).

\section{Lamponella beaury, new species}

Figures 364-367; Map 42

TYPES: Female holotype and male allotype taken in pitfall traps at an elevation of 630 $\mathrm{m}$ at Rocky Waterhole, Beaury State Forest, $28^{\circ} 33^{\prime} \mathrm{S}, 152^{\circ} 19^{\prime} \mathrm{E}$, New South Wales (Feb. 4-Apr. 9, 1993; M. Gray, G. Cassis), deposited in AMS (KS36103).

ETYMOLOGY: The specific name is a noun in apposition taken from the type locality.

DiAGNOSIS: Males can be recognized by the basally free, broadly triangular embolus and large median apophysis (figs. 364, 365), females by the heart-shaped epigynal midpiece and widely separated spermathecae (figs. 366, 367).

MALE: Total length 3.6. Abdominal dorsum dark gray, with pale anterior patch (mostly obscured by scutum), two pairs of small white cardiac spots (anterior and part of median pair obscured by scutum) and series of posterior white hairline chevrons; legs unmarked, anteriors darker than posteriors. Palpal femur unmodified; retrolateral tibial apophysis represented only by rounded lobe (fig. 365); cymbium with dorsodistal scopula; tegulum globose, reaching to about onefifth of tibial length; embolus not fused basally to tegulum, broadly triangular, tip straight; median apophysis wide, hookshaped, situated opposite base of embolus (fig. 364).

FEMALE: Total length 5.2. Coloration as in male. Epigynal midpiece heart-shaped, depressed anteriorly (fig. 366); spermathecae widely separated, medially constricted (fig. 367).

Other Material Examined: New South Wales: Bulls Ground State Forest, near Wauchope, $31^{\circ} 27^{\prime} \mathrm{S}, 152^{\circ} 44^{\prime} \mathrm{E}$, Feb. 10, 1991 (A. York, AMS KS43285), 1 ; $0.3 \mathrm{~km} \mathrm{~S}$ along Coxs Fence Trail from Boundary Road, Nerong State Forest, $31^{\circ} 38^{\prime} \mathrm{S}$, $152^{\circ} 09^{\prime}$ E, Feb. 4-Apr. 9, 1993, pitfall, elev. 70 m (M. Gray, G. Cassis, AMS KS39607), 1 $;$; 0.95 km W Henry Road, London Bridge Road, S side of road, London Bridge State Forest, near W boundary of London Bridge Flora Reserve, $29^{\circ} 50^{\prime} \mathrm{S}, 152^{\circ} 12^{\prime} \mathrm{E}$, Feb. 4Apr. 9, 1993, pitfall, elev. 630 m (M. Gray, G. Cassis, AMS KS36389), $10^{\Uparrow}$; Richmond Range State Forest, $28^{\circ} 45^{\prime} \mathrm{S}, 152^{\circ} 45^{\prime} \mathrm{E}$, Apr. 17-18, 1976, open forest (R. Raven, QMB S26518), 19 . Queensland: Beerwah Forestry Reserve, $26^{\circ} 51^{\prime} \mathrm{S}, 152^{\circ} 57^{\prime} \mathrm{E}$, Apr. 24, 1991, pitfall, heath (M. Glover, QMB S32345), 10 ; Ewan Maddock Dam, 264ㅇ' 

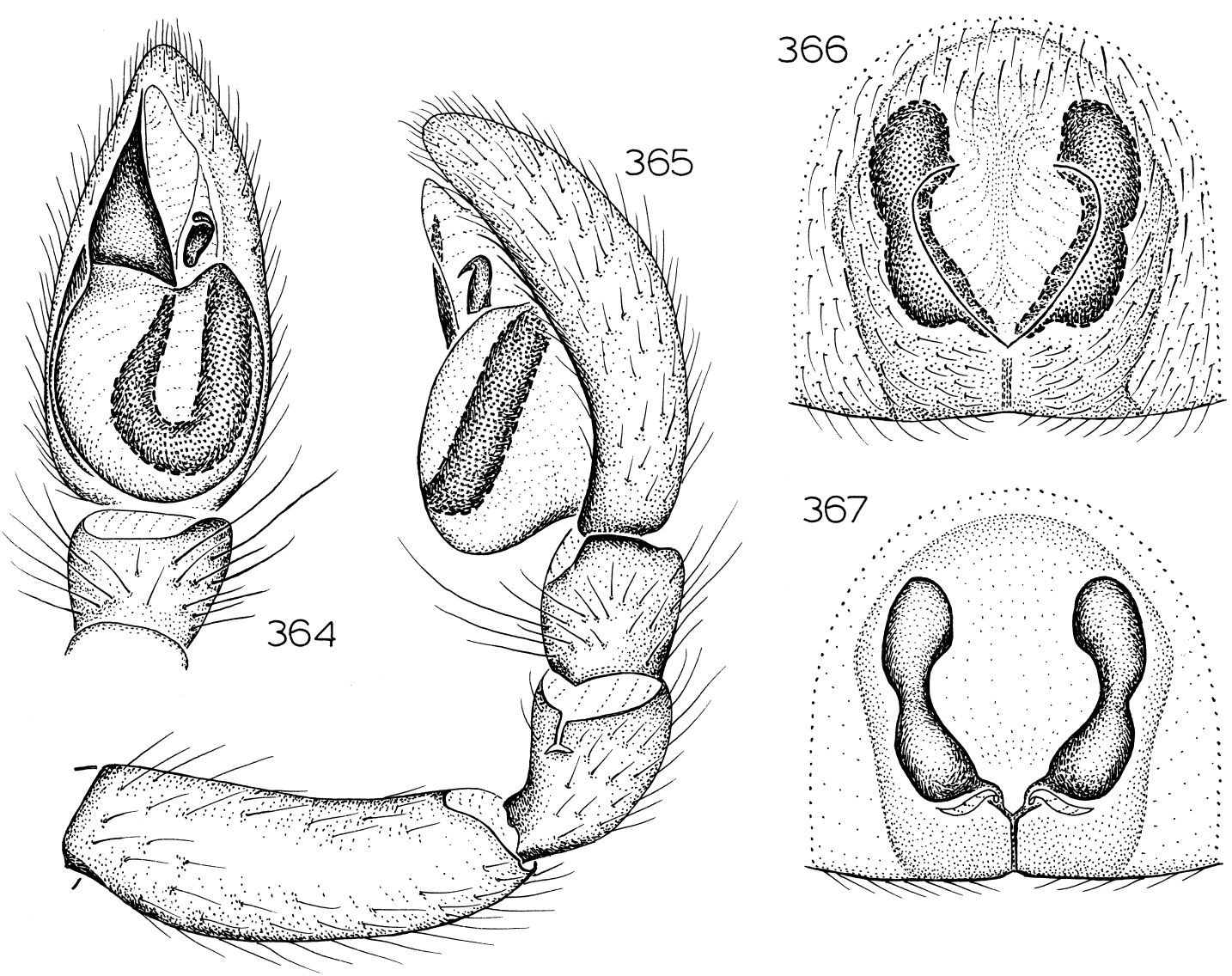

Figs. 364-367. Lamponella beaury, new species. 364. Left male palp, ventral view. 365. Same, retrolateral view. 366. Epigynum, ventral view. 367. Same, dorsal view.

$152^{\circ} 59^{\prime}$ E, Aug. 22, 1993-Mar. 22, 1994, pitfall, open forest (M. Glover, QMB S30391), 10; Rochedale State Forest, Brisbane, $27^{\circ} 37^{\prime} \mathrm{S}, 153^{\circ} 09^{\prime} \mathrm{E}$, Dec. 20, 1979-Jan. 2, 1980, litter (V. Davies, R. Raven, QMB S34361), 20; Windermere, near Glenmorgan, $27^{\circ} 17^{\prime} \mathrm{S}, 149^{\circ} 45^{\prime} \mathrm{E}$, Dec. $1990-$ Sept. 1991, pitfall (R. Raven, B. Smyth, QMB S30363), 1 ㅇ․

DistRIBUTION: Known only from southeastern Queensland and New South Wales (map 42).

\section{Lamponella kanangra, new species Figures 368-371; Map 42}

TYPE: Female holotype taken in litter in a Eucalyptus radiata-dalrympleana association at Blood Filly Creek, near Jenolan Caves, Kanangra-Boyd National Park, 335' S, $150^{\circ} 04^{\prime} \mathrm{E}$, New South Wales (Mar. 27, 1976; M. Gray, G. Hunt, J. McDougall), deposited in AMS (KS29968).

ETYMology: The specific name is a noun in apposition taken from the type locality.

DiAGNOSIS: Males can easily be recognized by the thumb-shaped retrolateral tibial apophysis and protuberant tegulum (figs. 368,369 ), females by the extremely narrow epigynal midpiece (fig. 370) and anteriorly expanded spermathecae (fig. 371).

MALE: Total length 3.2. Abdominal dorsum gray, with only vague indications of pair of anterior pale spots; legs unmarked. Palpal femur short, unmodified; retrolateral tibial apophysis thumb-shaped (fig. 369); tegulum moderately expanded, reaching to about onefourth of tibial length, with distinct protuberance on retrolateral side; embolus rela- 

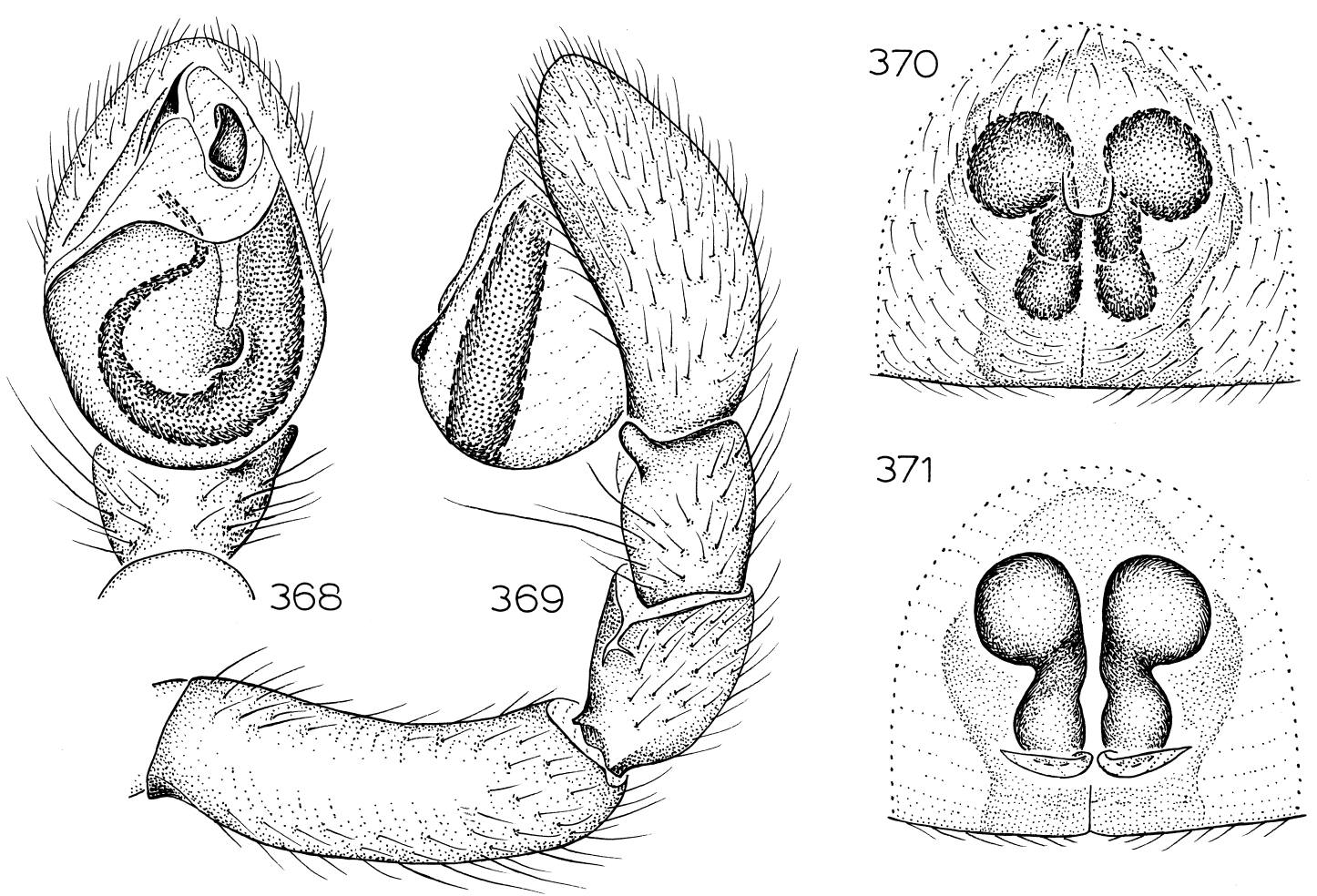

Figs. 368-371. Lamponella kanangra, new species. 368. Left male palp, ventral view. 369. Same, retrolateral view. 370. Epigynum, ventral view. 371. Same, dorsal view.

tively wide, short, median apophysis relatively large (fig. 368).

FEMALE: Total length 4.1. Coloration as in male. Epigynal midpiece narrow, situated posterior of depressed anteromedian openings (fig. 370); spermathecae expanded, bulbous anteriorly (fig. 371).

Other MATERIAl ExAmined: New South Wales: Kanangra-Boyd National Park, $34^{\circ} 01^{\prime} \mathrm{S}, 150^{\circ} 05^{\prime} \mathrm{E}$, Mar. 20, 1982, Berlese, closed forest litter and moss (L. Hill, QMB), 10 .

Distribution: Known only from New South Wales (map 42).

Lamponella wyandotte, new species Figures 372-375; Map 42

TYPE: Female holotype taken in a pitfall trap set in dry eucalypt woodland at Wyandotte Creek, $18^{\circ} 29^{\prime} \mathrm{S}, 144^{\circ} 55^{\prime} \mathrm{E}$, Queensland (July 26-Dec. 1, 1992; R. Raven, P., E. Lawless, M. Shaw), deposited in QMB (S24335).

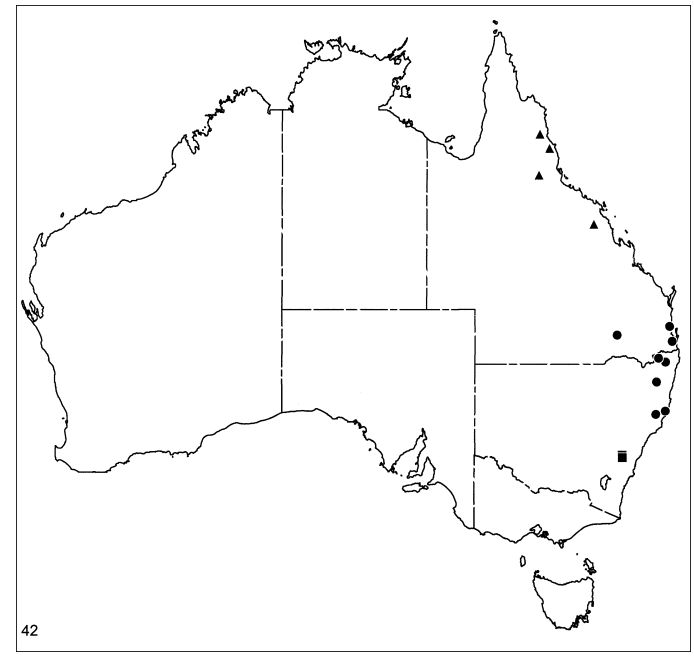

Map 42. Records of Lamponella beaury, new species (circles), L. kanangra, new species (squares), and L. wyandotte, new species (triangles). 

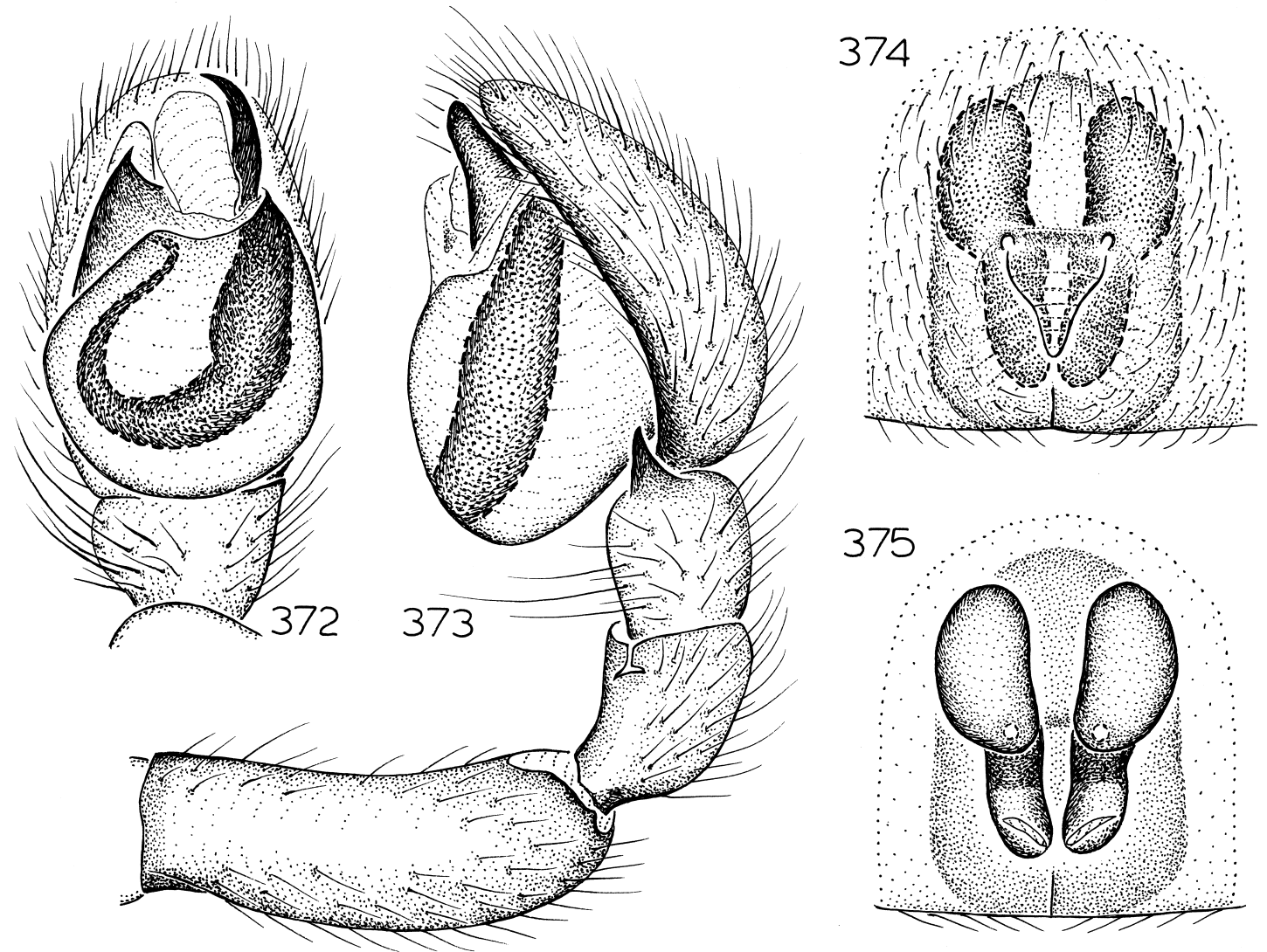

Figs. 372-375. Lamponella wyandotte, new species. 372. Left male palp, ventral view. 373. Same, retrolateral view. 374. Epigynum, ventral view. 375. Same, dorsal view.

ETYMOLOGY: The specific name is a noun in apposition taken from the type locality.

DIAGNOSIS: Males can easily be recognized by the long, prolaterally directed median apophysis, which occupies the distal onethird of the retrolateral surface of the palpal bulb (fig. 372), females by the sharply folded anterior tips of the epigynal margins (fig. 374).

MALE: Total length 3.2. Abdominal dorsum gray, with only vague indications of pair of anterior pale spots; legs unmarked. Palpal femur short, unmodified; retrolateral tibial apophysis short, triangular, directed ventrally (fig. 373); tegulum moderately expanded, reaching to about one-third of tibial length, greatly protuberant ventrally; embolus relatively narrow, widely separated from long median apophysis occupying distal third of prolateral surface of palpal bulb (fig. 372).
Female: Total length 3.8. Coloration as in male. Epigynal midpiece anteriorly expanded, margins sharply deflected at openings (fig. 374); anterior portion of spermathecae elongate, kidney-shaped (fig. 375).

Other MAterial ExAMINED: Queensland: Davies Creek National Park, $17^{\circ} 00^{\prime} \mathrm{S}$, $145^{\circ} 34^{\prime}$ E, Oct. 29 , 1991-July 23, 1992, pitfall (P. Lawless, R. Raven, M. Shaw, QMB S22741), 1 ơ; $20 \mathrm{~km}$ WSW Eungella, $21^{\circ} 12^{\prime} \mathrm{S}, 148^{\circ} 18^{\prime} \mathrm{E}$, Nov. 18, 1981, Berlese, Eucalyptus browni woodland, elev. $350 \mathrm{~m}$ (A. Gillison, QMB), 1 \% ; Mount Windsor Tableland, Whypalla State Forest, $16^{\circ} 13^{\prime} \mathrm{S}$, $144^{\circ} 59^{\prime} \mathrm{E}$, summer 1992-1993, pitfall, elev. 1120 m (S. Barnett, QMB S22667), 1 ㅇ (penultimate but with formed epigynum); Wyandotte Creek, $18^{\circ} 29^{\prime} \mathrm{S}, 144^{\circ} 55^{\prime} \mathrm{E}$, Nov. 6, 1991-July 26, 1992, pitfall, dry eucalypt woodland (P. Lawless, R. Raven, M. Shaw, 

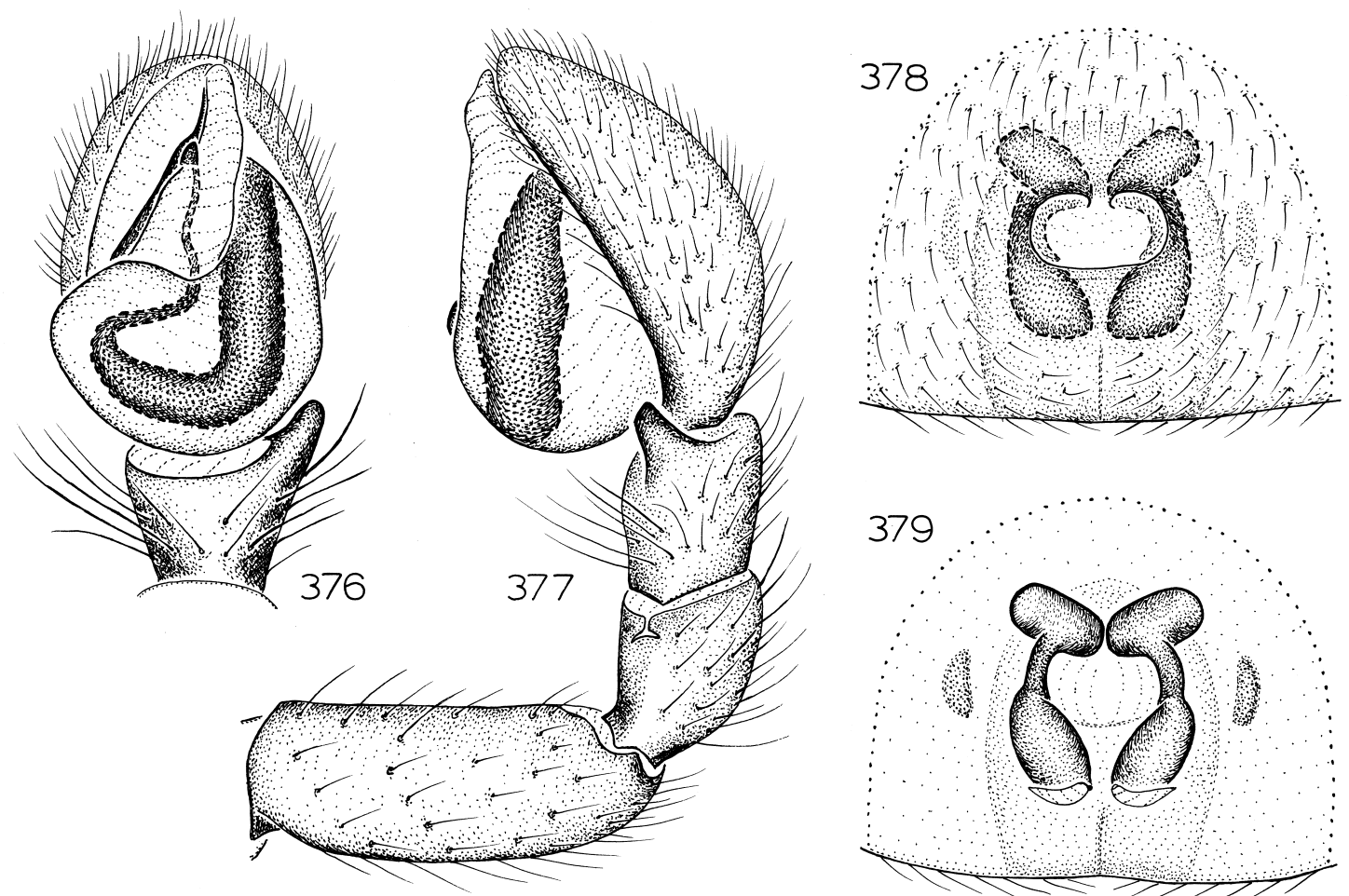

Figs. 376-379. 376, 377. Lamponella wombat, new species. 378, 379. Lamponella kroombit, new species. 376. Left male palp, ventral view. 377. Same, retrolateral view. 378. Epigynum, ventral view. 379. Same, dorsal view.

QMB S21784), 3 ㅇ, Dec. 1, 1992-Apr. 14, 1993, pitfall, dry eucalypt woodland (R., J., S. Raven, P., E. Lawless, QMB S24188), 2 ㅇ. DISTRIBUTION: Known only from northern Queensland (map 42).

\section{Lamponella wombat, new species Figures 376, 377; Map 43}

TYPE: Male holotype taken at an elevation of $750 \mathrm{~m}$ at Wombat Creek, $6 \mathrm{~km}$ NE Piccadilly Circus, $35^{\circ} 19^{\prime} \mathrm{S}, 148^{\circ} 51^{\prime} \mathrm{E}$, Australian Capital Territory (June 1985; T. Weir, J. Lawrence, M. Johnson), deposited in QMB.

ETYMOLOGY: The specific name is a noun in apposition taken from the type locality.

DiAGNOSIS: Males can be recognized by the small median apophysis almost superimposed on the embolus (fig. 376).

MALE: Total length 3.1. Abdominal dorsum gray, with only vague indications of pair of anterior pale spots; legs unmarked. Palpal femur short, straight, unmodified; retrolateral

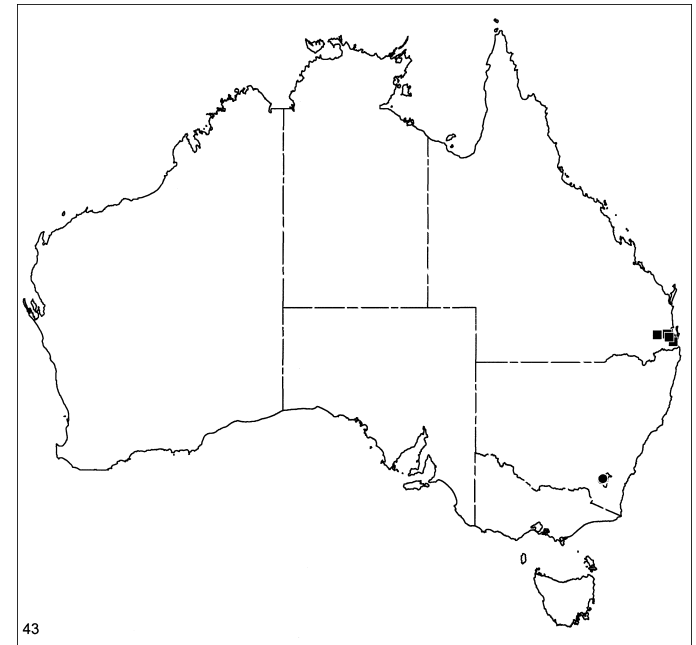

Map 43. Records of Lamponella wombat, new species (circles) and L. brookfield, new species (squares). 

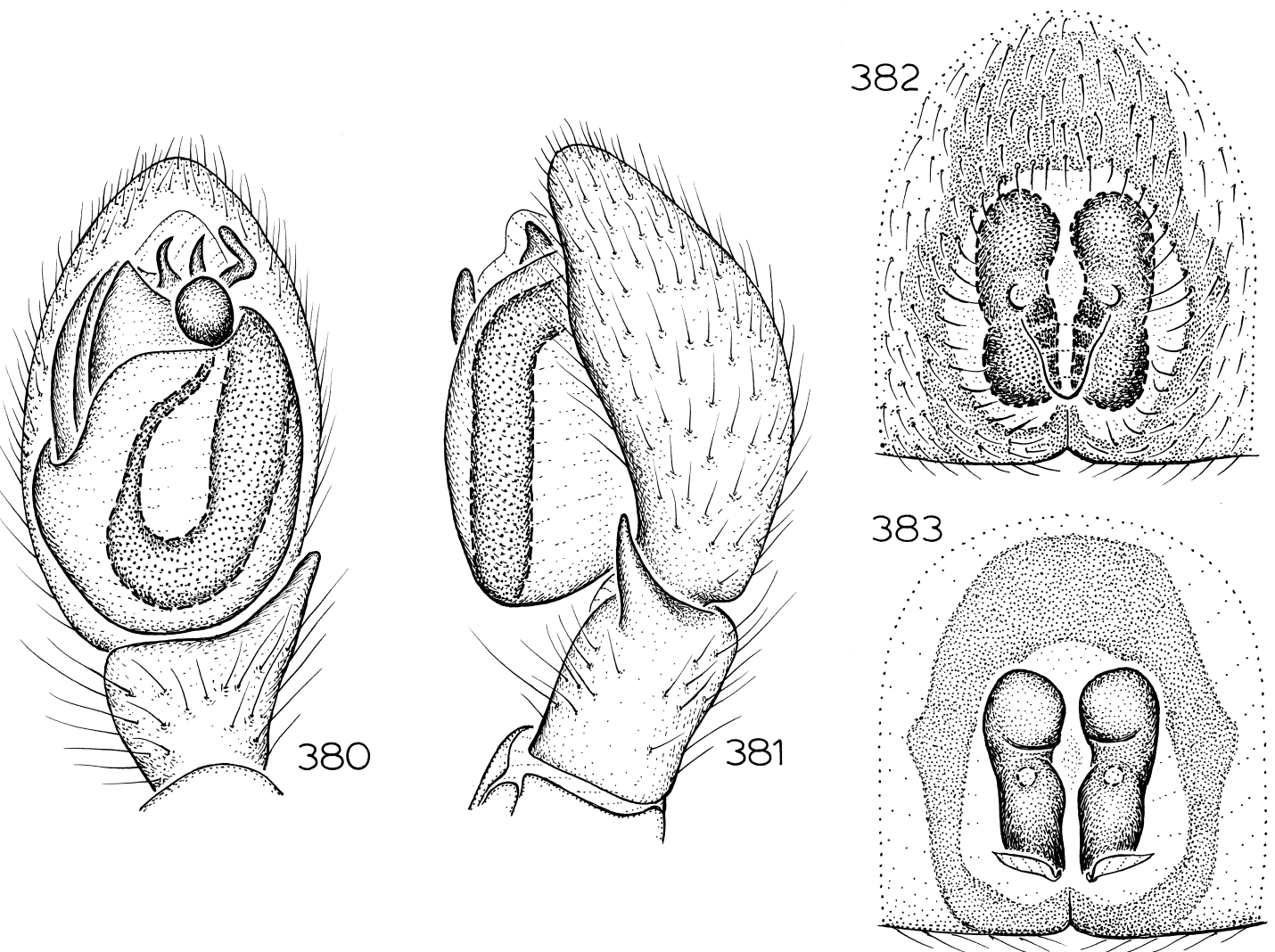

Figs. 380-383. Lamponella brookfield, new species. 380. Left male palp, ventral view. 381. Same, retrolateral view. 382. Epigynum, ventral view. 383. Same, dorsal view.

tibial apophysis short, thumb-shaped (fig. 377); tegulum moderately expanded, reaching to about one-fourth of tibial length; tip of embolus situated medially, overlapped in ventral view by small median apophysis (fig. 376).

FEMALE: Unknown.

Other Material Examined: Australian Capital Territory: Blundells Creek, $3 \mathrm{~km} \mathrm{E}$ Piccadilly Circus, $35^{\circ} 22^{\prime} \mathrm{S}, 148^{\circ} 50^{\prime} \mathrm{E}$, Jan. 1985, elev. 850 m (T. Weir, J. Lawrence, M. Johnson, QMB), 1 ơ; Piccadilly Circus, $35^{\circ} 22^{\prime} \mathrm{S}, 148^{\circ} 48^{\prime} \mathrm{E}$, Feb. 1984, elev. $1240 \mathrm{~m}$ (J. Lawrence, T. Weir, M. Johnson, QMB), 10 ; Wombat Creek, 6 km NE Piccadilly Circus, $35^{\circ} 19^{\prime} \mathrm{S}, 148^{\circ} 51^{\prime} \mathrm{E}$, Mar. 1984-1985, elev. $750 \mathrm{~m}$ (T. Weir, J. Lawrence, M. Johnson, QMB), 20 , Aug.-Sept. 1985, elev. 750 m (T. Weir, J. Lawrence, M. Johnson, QMB), 20 .
Distribution: Known only from the Australian Capital Territory (map 43).

\section{Lamponella brookfield, new species} Figures 380-383; Map 43

TYPE: Female holotype taken in litter at Gold Creek Reservoir, Brookfield, $27^{\circ} 30^{\prime} \mathrm{S}$, $152^{\circ} 55^{\prime} \mathrm{E}$, Queensland (Aug. 14, 1981; R. Raven, V. Davies), deposited in QMB (S34357).

ETYMOLOGY: The specific name is a noun in apposition taken from the type locality.

DiAGNOSIS: Males can easily be recognized by the rounded, lobe-like median apophysis (fig. 380); females resemble those of L. wyandotte but have simpler anterior tips of the epigynal midpiece and smaller anterior portions of the spermathecae (figs. 382, 383).

MALE: Total length 3.1. Abdominal dor- 

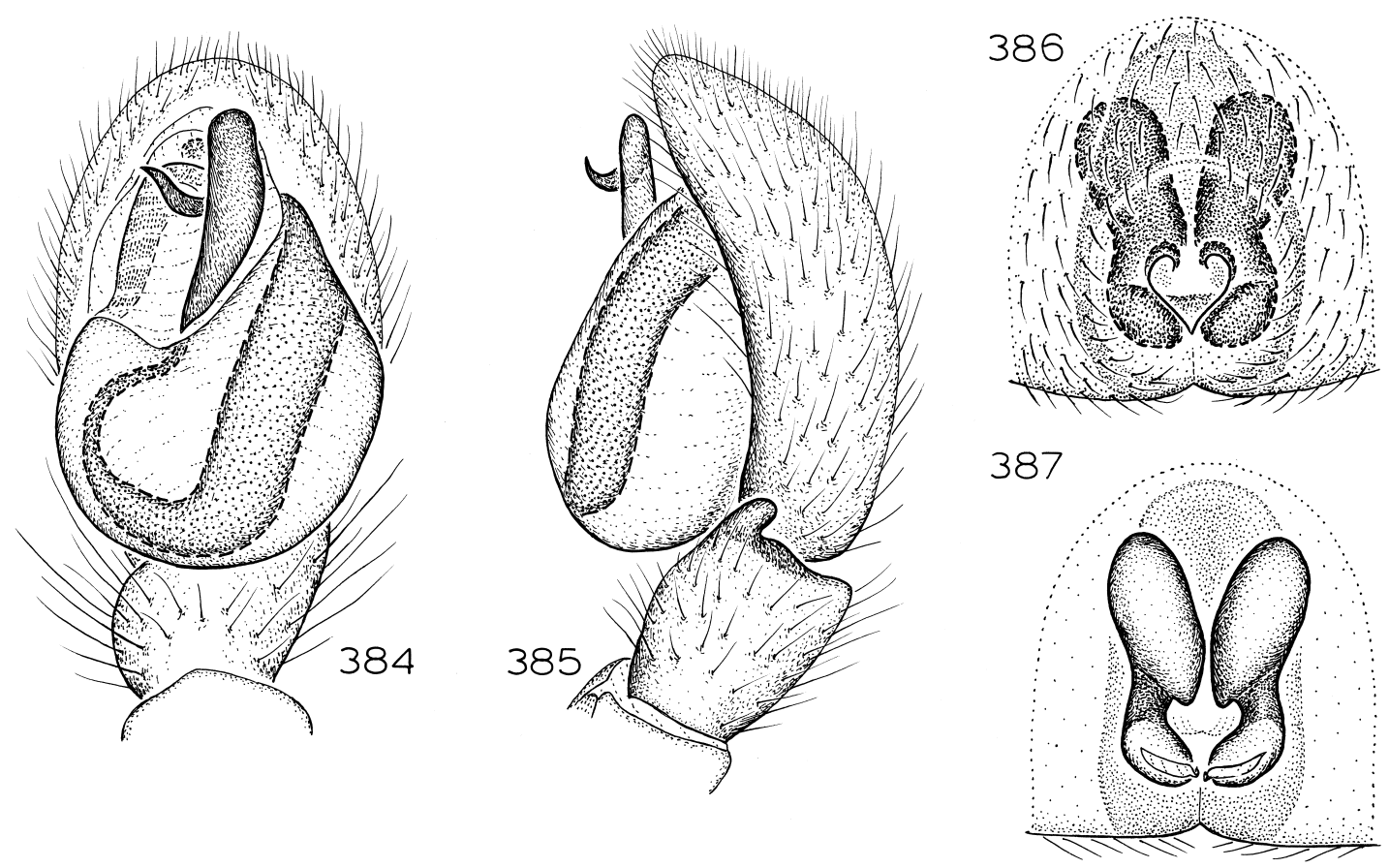

Figs. 384-387. Lamponella homevale, new species. 384. Left male palp, ventral view. 385. Same, retrolateral view. 386. Epigynum, ventral view. 387. Same, dorsal view.

sum gray, with only vague indications of fused median anterior pale spot; legs unmarked. Retrolateral tibial apophysis relatively long, narrow, sharply pointed (fig. 381); median apophysis represented by rounded lobe (fig. 380).

FEMALE: Total length 4.1. Coloration as in male. Epigynal midpiece anteriorly expanded, margins passing simple openings, continuing anteriorly to vaguely outline anterior epigynal depression (fig. 382), anterior portion of spermathecae relatively short, kidneyshaped (fig. 383).

OTHER MATERIAL ExAMINED: Queensland: Bahr's Scrub, $27^{\circ} 45^{\prime} \mathrm{S}, 153^{\circ} 10^{\prime} \mathrm{E}$, Apr. 30, 1980, dry litter (R. Raven, QMB S34360), 10े, Dec. 10, 1991-Jan. 21, 1992, pitfall, rainforest, elev. $100 \mathrm{~m}$ (D. Cook, QMB S25031), 1 q, Jan. 21-Mar. 9, 1992, pitfall, rainforest, elev. $100 \mathrm{~m}$ (D. Cook, QMB S30328), 1 \% ; Gold Creek Reservoir, Brookfield, $27^{\circ} 30^{\prime} \mathrm{S}, 152^{\circ} 55^{\prime} \mathrm{E}$, Oct. 1-30, 1980, closed forest litter (V. Davies, R. Raven, QMB S34356, 34358), 20; Ravensbourne National Park, $27^{\circ} 22^{\prime} \mathrm{S}, 152^{\circ} 11^{\prime} \mathrm{E}$, Dec. 1 ,
1991-Jan. 7, 1992, rainforest pitfall (D. Cook, QMB S34366), 10; Stony Creek, via Samford, $27^{\circ} 20^{\prime} \mathrm{S}, 152^{\circ} 48^{\prime} \mathrm{E}$, Feb. 2-Apr. 8, 1995, rainforest pitfall (G. Monteith, H. Janetzki, QMB S34368), 1\%; Upper Brookfield, $27^{\circ} 30^{\prime} \mathrm{S}, 152^{\circ} 55^{\prime} \mathrm{E}$, Dec. 11,1980 , araucaria notophyll vineforest, under $\operatorname{logs}$, rocks, in litter (V. Davies, R. Raven, QMB S34362), 1 ô.

DistRIBUTION: Known only from southeastern Queensland (map 43).

\section{Lamponella homevale, new species Figures 384-387; Map 44}

TYPE: Male holotype taken in pitfall trap at Homevale, $21^{\circ} 24^{\prime} \mathrm{S}, 148^{\circ} 33^{\prime} \mathrm{E}$, Queensland (Apr. 1-7, 1975; V. Davies, R. Kohout), deposited in QMB (S34367).

ETYMOLOGY: The specific name is a noun in apposition taken from the type locality.

DiAGNOSIS: The male and female were not collected together and may be mismatched, but are paired here on the basis of their genitalic similarities to L. taroom and $l$. kroom- 

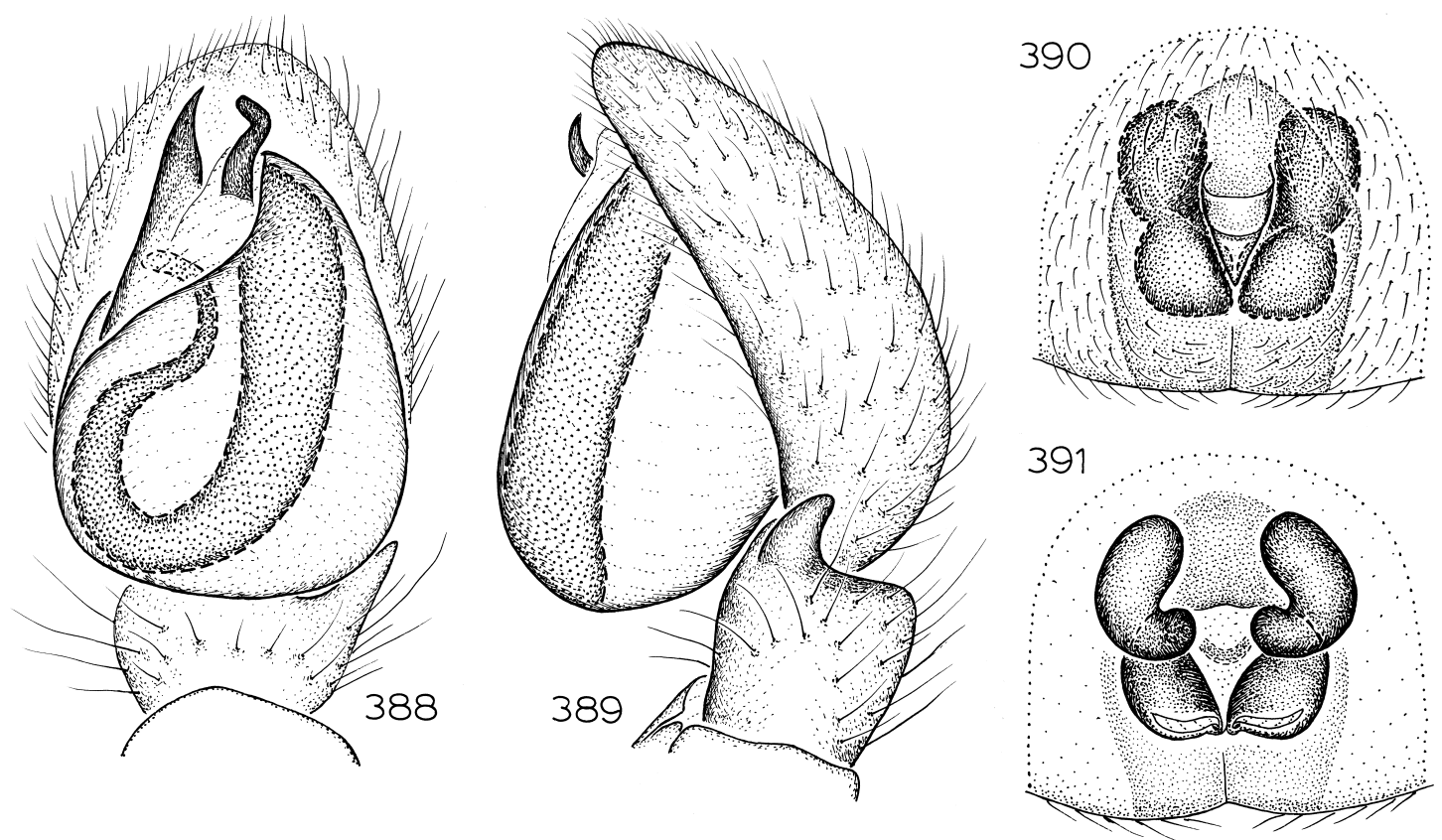

Figs. 388-391. Lamponella kimba, new species. 388. Left male palp, ventral view. 389. Same, retrolateral view. 390. Epigynum, ventral view. 391. Same, dorsal view.

bit. Males can be recognized by the recurved, prolaterally directed tip of the embolus (fig. 384), females by the short, heart-shaped, anteriorly depressed epigynal midpiece (fig. 386).

MALE: Total length 3.2. Abdominal dorsum gray, with only vague indications of fused median anterior pale spot, with few pale posterior hairline chevrons; legs unmarked. Retrolateral tibial apophysis small, rounded (fig. 385); embolar tip long, recurved, directed prolaterally (fig. 384).

FEMALE: Total length 4.1. Coloration as in male. Epigynal midpiece short, anteriorly expanded, depressed (fig. 386); anterior portion of spermathecae long, kidney-shaped (fig. 387).

Other Material ExAmined: One female taken in a pitfall trap in open forest at an elevation of $550 \mathrm{~m}$ on the summit of Mount Cleveland, $19^{\circ} 15^{\prime} \mathrm{S}, 147^{\circ} 01^{\prime} \mathrm{E}$, northeastern Queensland (Jan. 20-Mar. 12, 1991; A. Graham), deposited in QMB (S17913).

DisTRIBUTION: Known only from northand mid-eastern Queensland (map 44).

\section{Lamponella kimba, new species}

Figures 388-391; Map 44

TYPE: Female holotype taken in a Berlese sample of litter from the base of mallee 25 $\mathrm{km} \mathrm{E}$ by $\mathrm{N}$ of Kimba, $33^{\circ} 05^{\prime} \mathrm{S}, 136^{\circ} 41^{\prime} \mathrm{E}$, South Australia (Sept. 4, 1981; A. Calder), deposited in QMB.

ETYMOLOGY: The specific name is a noun in apposition taken from the type locality.

DiAGNOSIS: Males can be recognized by the sinuous median apophysis (fig. 388) and moderately long, triangular retrolateral tibial apophysis (fig. 389), females by the combined presence of an anteriorly depressed epigynum and laterally deflected anterior portions of the spermathecae (figs. 390, 391).

MALE: Total length 2.2. Abdominal dorsum light gray, without indications of anterior white spots but with pale posterior hairline chevrons. Retrolateral tibial apophysis almost triangular (fig. 389); embolus bent at tip (fig. 388).

Female: Total length 3.4. Coloration as in male. Epigynal midpiece long, anterior two- 
thirds depressed (fig. 390); anterior portions of spermathecae deflected to sides (fig. 391).

Other Material Examined: South Australia: $14 \mathrm{~km}$ WNW Renmark, $34^{\circ} 07^{\prime} \mathrm{S}$, $140^{\circ} 37^{\prime} \mathrm{E}$, July 6, 1995, intercept trap (K. Pullen, QMB), $10^{\circ}$. Western Australia: Bold Park, $31^{\circ} 57^{\prime} \mathrm{S}, 115^{\circ} 46^{\prime} \mathrm{E}$, Mar. 18-May 19, 1994, pitfall (M. Harvey, J. Waldock, WAM 96/759), 10; Boolathana Station, $24^{\circ} 25^{\prime} \mathrm{S}, 113^{\circ} 42^{\prime} \mathrm{E}$, May 29-Aug. 25, 1995, pitfall (N. Hall, WAM 96/1411), $1 \delta^{\star}$; Bush Bay, $25^{\circ} 07^{\prime} \mathrm{S}, 113^{\circ} 44^{\prime} \mathrm{E}$, Aug. 16-Sept. 28, 1994, pitfall (M. Harvey, WAM 96/760), $10^{\text {t; }}$; Hepburn Heights, $31^{\circ} 49^{\prime} \mathrm{S}$, $115^{\circ} 46^{\prime} \mathrm{E}$, July 13-Sept. 25, 1995, pitfall (M. Harvey, J. Waldock, WAM 96/756), 1 \% ; Hepburn Heights, $31^{\circ} 49^{\prime} \mathrm{S}, 115^{\circ} 47^{\prime} \mathrm{E}$, Nov. 28 , 1995 Jan. 29, 1996, pitfall (M. Harvey, J. Waldock, WAM 96/757), 1 \%; Trigg, E side old West Coast Highway, $31^{\circ} 53^{\prime} \mathrm{S}, 115^{\circ} 45^{\prime} \mathrm{E}$, Aug. 29, 1993, litter (J. Waldock, WAM 95/

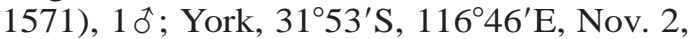
1991, powder bark/mallet litter (J. Bannister, WAM 96/758), 1 ㅇ.

Distribution: Known only from Western and South Australia (map 44).

\section{Lamponella taroom, new species Figures 392-395; Map 45}

TYPE: Male holotype taken in a pitfall trap in the Taroom District at Boggomoss No 8, $25^{\circ} 27^{\prime} \mathrm{S}, 150^{\circ} 02^{\prime} \mathrm{E}$, Queensland (Sept. 10Nov. 11, 1996; P. Lawless), deposited in QMB (S34364).

ETYMOLOGY: The specific name is a noun in apposition taken from the type locality.

DIAGNOSIS: Males can easily be recognized by the triangular embolus (fig. 392), females by the arched anterior epigynal margin and anteriorly expanded spermathecae (figs. 394, 395).

MALE: Total length 2.7. Abdominal dorsum dark gray, with scattered, small, rounded white markings and pale posterior hairline chevrons; legs unmarked. Retrolateral tibial apophysis small, triangular (fig. 393); palpal bulb posteriorly truncated, with flat, triangular embolus (fig. 392).

Female: Total length 4.5. Abdominal dorsum light gray, with markings as in male. Epigynum with arched anterior margin situated at about half of epigynal length (fig.
394); spermathecae expanded, globose anteriorly (fig. 395).

Other Material Examined: Queensland: Taroom District, Boggomoss No. 1, 25 $26^{\prime} \mathrm{S}$, $150^{\circ} 02^{\prime} \mathrm{E}$, Sept. 9-Nov. 12, 1996, pitfall (P. Lawless, QMB ex S34412), $10^{\top}$; Taroom District, Boggomoss No. 8, 25 $27^{\prime} \mathrm{S}, 150^{\circ} 02^{\prime} \mathrm{E}$, Nov. 12, 1996, Berlese, sieved open forest litter, elev. $180 \mathrm{~m}$ (G. Monteith, QMB S34363), 1 ; ; Taroom District, $7 \mathrm{~km} \mathrm{~N} \mathrm{Oo-}$ line Scrub, $25^{\circ} 36^{\prime} \mathrm{S}, 149^{\circ} 46^{\prime} \mathrm{E}$, Sept. 11-Nov. 12, 1996, road verge, pitfall (P. Lawless, QMB S34365), 1 9 ; Taroom District, $9 \mathrm{~km}$ $\mathrm{N}$ of Ooline Scrub, $25^{\circ} 35^{\prime} \mathrm{S}, 149^{\circ} 46^{\prime} \mathrm{E}$, Nov. 10, 1996-Jan. 12, 1997, road verge, pitfall (P. Lawless, QMB S36622), 1 ㅇ.

Distribution: Known only from the Taroom District, southeastern Queensland (map 45).

\section{Lamponella kroombit, new species} Figures 378, 379; Map 45

TYPE: Female holotype taken in Berlese sample of sieved open forest litter from Kroombit Tops, $24^{\circ} 23^{\prime} \mathrm{S}, 151^{\circ} 02^{\prime} \mathrm{E}$, Queensland (Dec. 14, 1983; G. Monteith, V. Davies, J. Gallon, G. Thompson), deposited in QMB (S34359).

ETYMOLOGY: The specific name is a noun in apposition taken from the type locality.

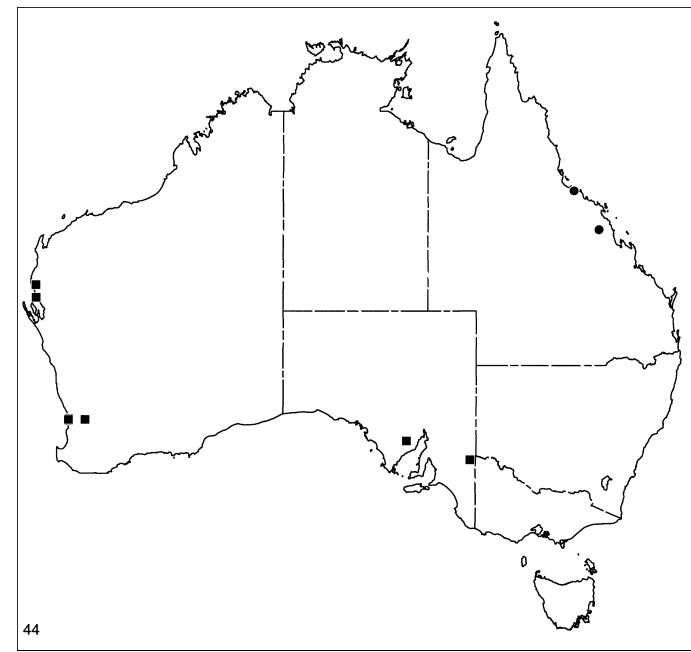

Map 44. Records of Lamponella homevale, new species (circles) and L. kimba, new species (squares). 

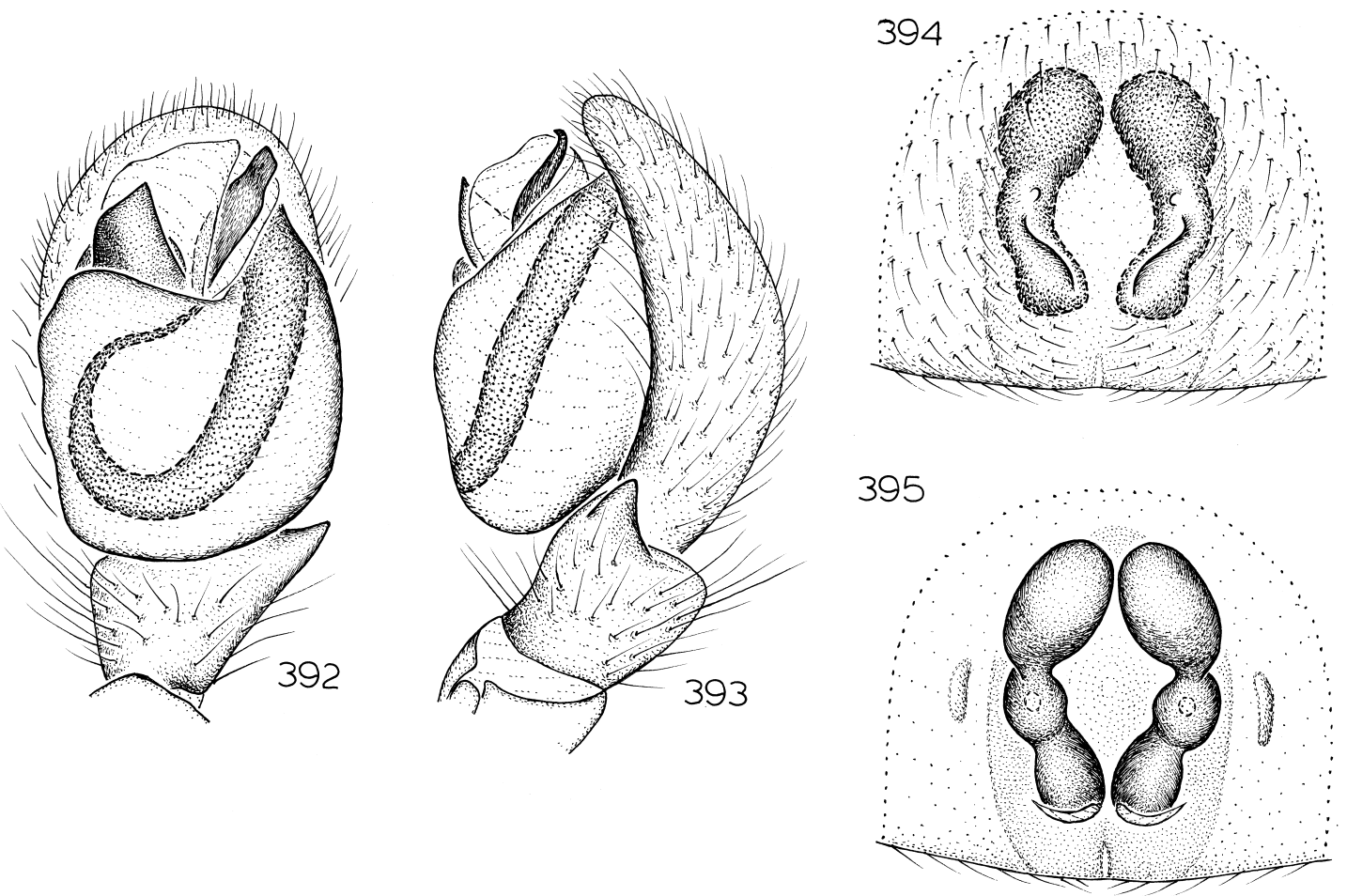

Figs. 392-395. Lamponella taroom, new species. 392. Left male palp, ventral view. 393. Same, retrolateral view. 394. Epigynum, ventral view. 395. Same, dorsal view.

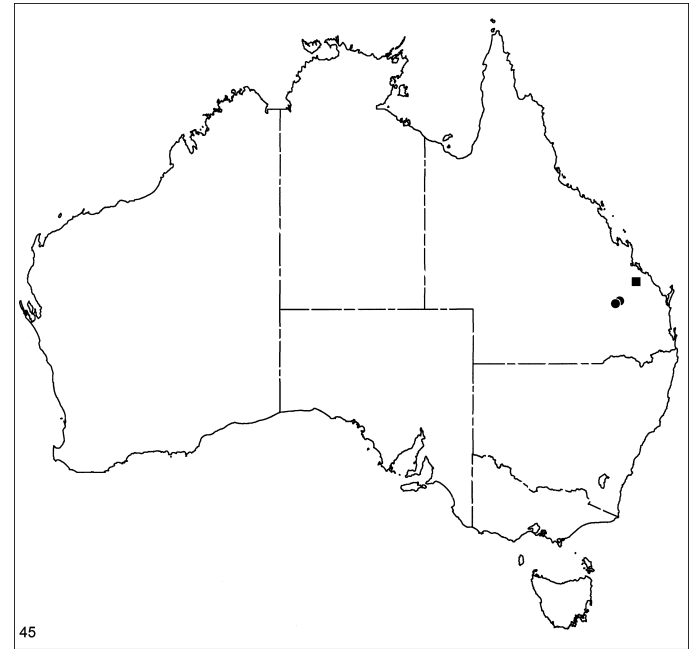

Map 45. Records of Lamponella taroom, new species (circles) and L. kroombit, new species (square).
DiAGNOSIs: Males are unknown; females have a distinctive, rounded epigynal atrium with anterior openings (fig. 378) and medially constricted spermathecae (fig. 379). Although it is conceivable that this species represents the female of $L$. wombat, epigynal similarities with the female of $L$. homevale suggest that the male of L. kroombit should be close to that of $L$. homevale, whereas the $L$. wombat appears to be closer to the less oddly modified males of $L$. ainslie and $L$. kanangra.

MALE: Unknown.

Female: Total length 4.2 Abdominal dorsum dirty white, palest in cardiac area, legs unmarked. Epigynal atrium large, rounded, with openings situated anteriorly (fig. 378); spermathecae constricted medially, anterior lobes directed laterally (fig. 379).

Other MATERIAL ExAMINED: None.

Distribution: Known only from the type locality in southeastern Queensland (map 45). 


\section{CENTROTHELINAE, NEW SUBFAMILY}

Type Genus: Centrothele L. Koch.

Diagnosis: Centrothelines share with lamponines a deep groove extending along the median margin of the endites (fig. 396) but lack the odd endite shape of lamponines. Females are easy to recognize, because of their anterior abdominal scutum (usually restricted to the anterior surface of the abdomen) and especially the row of three highly modified, greatly enlarged cylindrical gland spigots found on the posterior portion of each posterior median spinneret (figs. 410, 411); those spigots are easily visible under light microscopy. Males (like females) lack the anteriorly expanded ventral pedicel sclerite of lamponines; their ventral pedicel sclerite is anteriorly truncated, broadly meeting the posterior margin of the sternum, or even fusing with it.

Distribution: Australia (including Tasmania), New Guinea, and New Caledonia.

InCluded Genera: Centrothele L. Koch, Prionosternum Dunn, Asadipus Simon, and the new genera Centrina, Centrocalia, Centsymplia, Queenvic, Bigenditia, Graycassis, Notsodipus, and Longepi.

\section{Key to Genera of Centrothelinae}

1. Posterior margin of sternum fused to ventral pedicel sclerite .............. 2

- Posterior margin of sternum separated from anterior margin of pedicel by narrow sliver of unsclerotized cuticle ......... 3

2. Males with large projections at anterolateral corners of endites and large projections on either side of cheliceral fang base; female epigynal atria heavily rebordered posteriorly (figs. 528, 532); Australia ......

Bigenditia

- Males without projections on endites and chelicerae; female epigynal atria not heavily rebordered posteriorly (figs. 500, 504); New Caledonia ......... Centrocalia

3. Males ................. 4

- Females .................. 12

4. Embolus with apophysis at base (as in figs. $397,414) \ldots \ldots \ldots \ldots \ldots \ldots \ldots$

- Embolus without apophysis .......6 6

5. Embolus relatively long, narrow (as in fig. 414) ............. Centrothele

- Embolus relatively short, wide (as in fig. 454) Centrina

6. Most of embolus hidden, in ventral view, be- hind greatly enlarged terminal apophysis situated prolaterally on palpal bulb (as in figs. 714, 730) .......... Longepi

- Palp otherwise ............... 7

7. Embolus conspicuously recurved at anterior end of palpal bulb and again before its tip (as in figs. 582, 650) .......... 8

- Embolus not recurved twice ......... 9

8. Retrolateral tibial apophysis deeply incised (as in figs. 583, 627) ........ Asadipus

- Retrolateral tibial apophysis not incised (as in figs. 651, 663) .......... Notsodipus

9. Median apophysis greatly enlarged, highly modified (as in figs. 542, 566), retrolateral tibial apophysis lobed, ledge-shaped (as in figs. 543, 567) ......... Graycassis

- Median apophysis and retrolateral tibial apophysis otherwise .......... 10

10. Retrolateral tibial apophysis relatively short (figs. 471, 535, 539) .... Prionosternum

- Retrolateral tibial apophysis relatively long (as in figs. 507, 511) .......... 11

11. Median apophysis relatively large (as in figs. $510,514) \ldots \ldots \ldots \ldots \ldots$ Queenvic

- Median apophysis very small (fig. 506) .... ............... Centsymplia

12. Epigynum triangular, with ducts restricted to posterior portion of epigynum (figs. 508, 509) . . . . . . . . . Centsymplia

- Epigynum not as in figures 508, 509 . . 13

13. Epigynum with paired anterior hoods (figs. 536,540 ) and bipartite spermathecae (figs. $537,541) \ldots \ldots \ldots \ldots$. Prionosternum

- Epigynum otherwise ............ 14

14. Epigynum with pair of lateral pockets and medially situated, recurved ducts (figs. 512, $516,520,524) \ldots \ldots \ldots \ldots$ Queenvic

- Epigynum otherwise ............ 15

15. Epigynum internally with pair of blind ducts (as in figs. 653, 716) ......... 16

- Epigynum without blind ducts . . . . . 17

16. Epigynum with elaborate anterior margin and anteriorly recurved ducts (as in figs. 714, 715) ................ Longepi

- Epigynum otherwise, usually with anterior atrium and posteriorly coiled ducts (as in figs. 652, 653) ......... Notsodipus

17. Epigynum a flattened plate, with highly looping ducts visible through cuticle (as in figs. $588,596,628) \ldots \ldots \ldots \ldots$. Asadipus

- Epigynum otherwise . . .......... 18

18. Epigynum internally with largely parallel median and lateral ducts (as in figs. 429, 445) Centrothele

- Epigynum otherwise ............ 19

19. Posterior epigynal ducts forming a y-shaped 

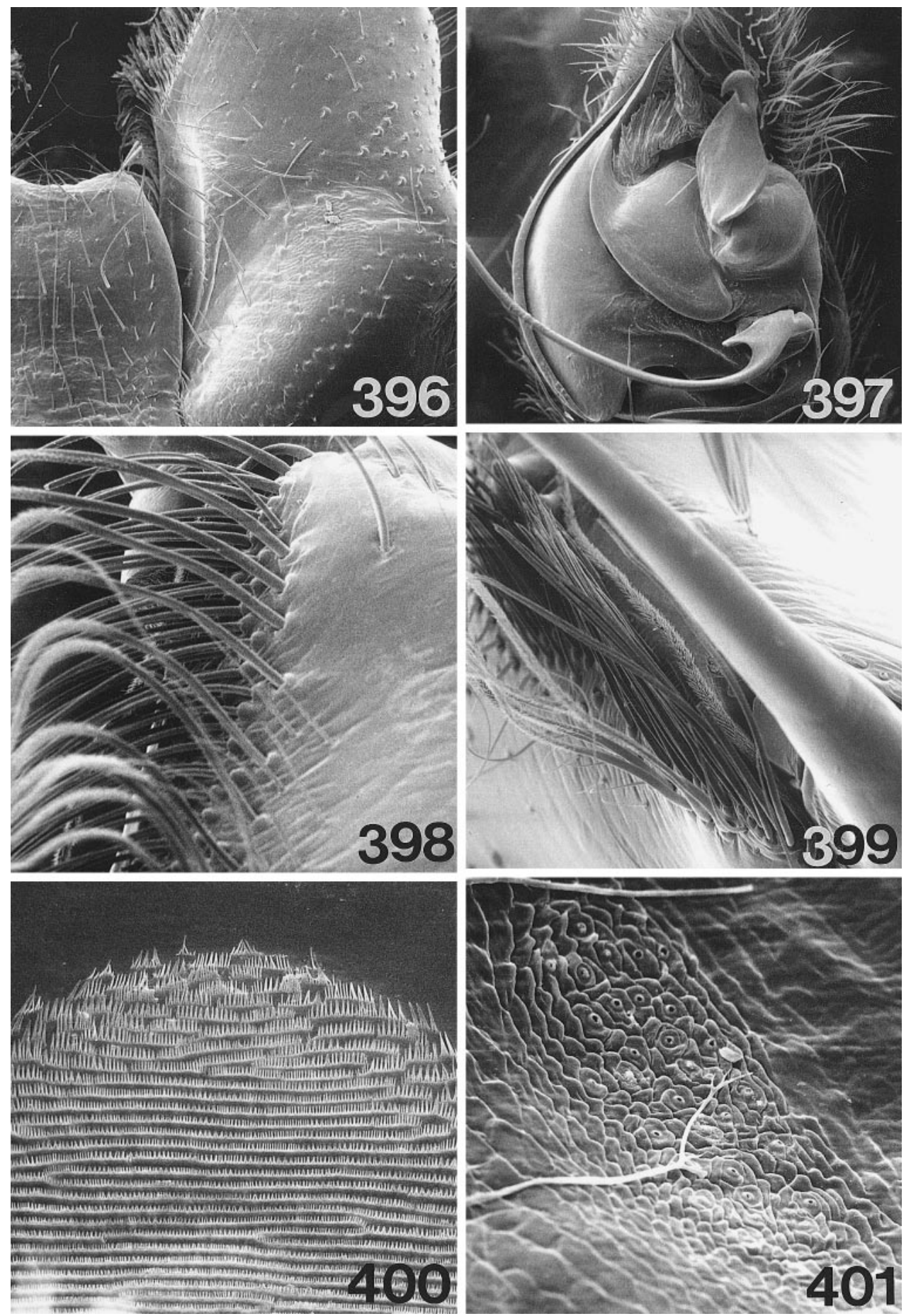

Figs. 396-401. Centrothele gordon, new species, male. 396. Labium and endite, ventral view, showing median groove on endite. 397. Left palp, ventral view, showing apophysis at base of embolus. 398. Chelicera, anterior view; modified first promarginal seta extends behind other promarginal setae. 399. Same, distal view, showing plumose shaft of modified first promarginal seta. 400. Labral teeth, posterior view. 401. Sieve plate on endite, anterior view. 

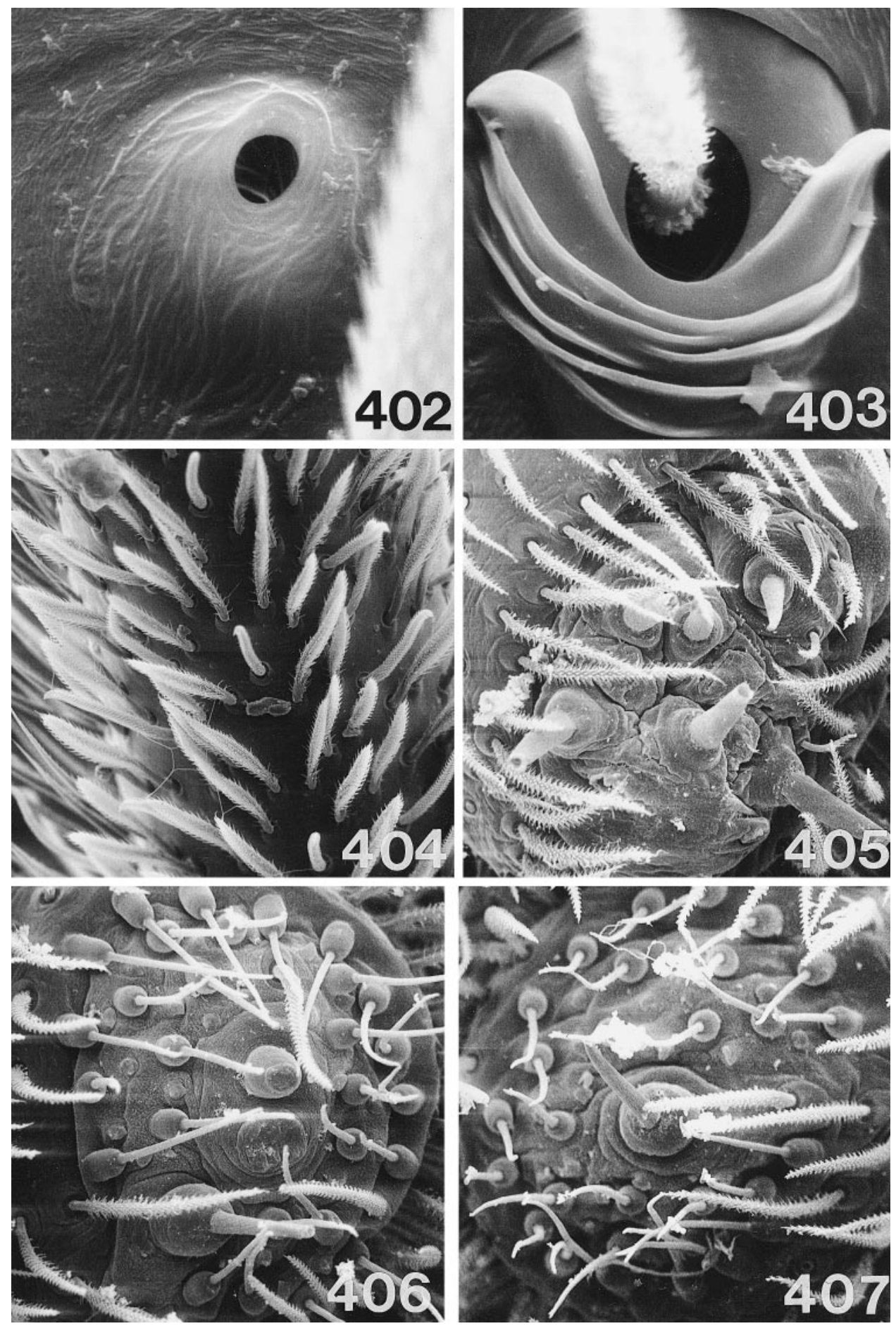

Figs. 402-407. Centrothele gordon, new species, male. 402. Tarsal organ, leg II, dorsal view. 403. Trichobothrial base, tarsus II, dorsal view. 404. Scopular setae of tarsus I, ventral view. 405. Anterior lateral spinneret, posterior view. 406. Posterior median spinneret, posterior view. 407. Posterior lateral spinneret, posterior view. 

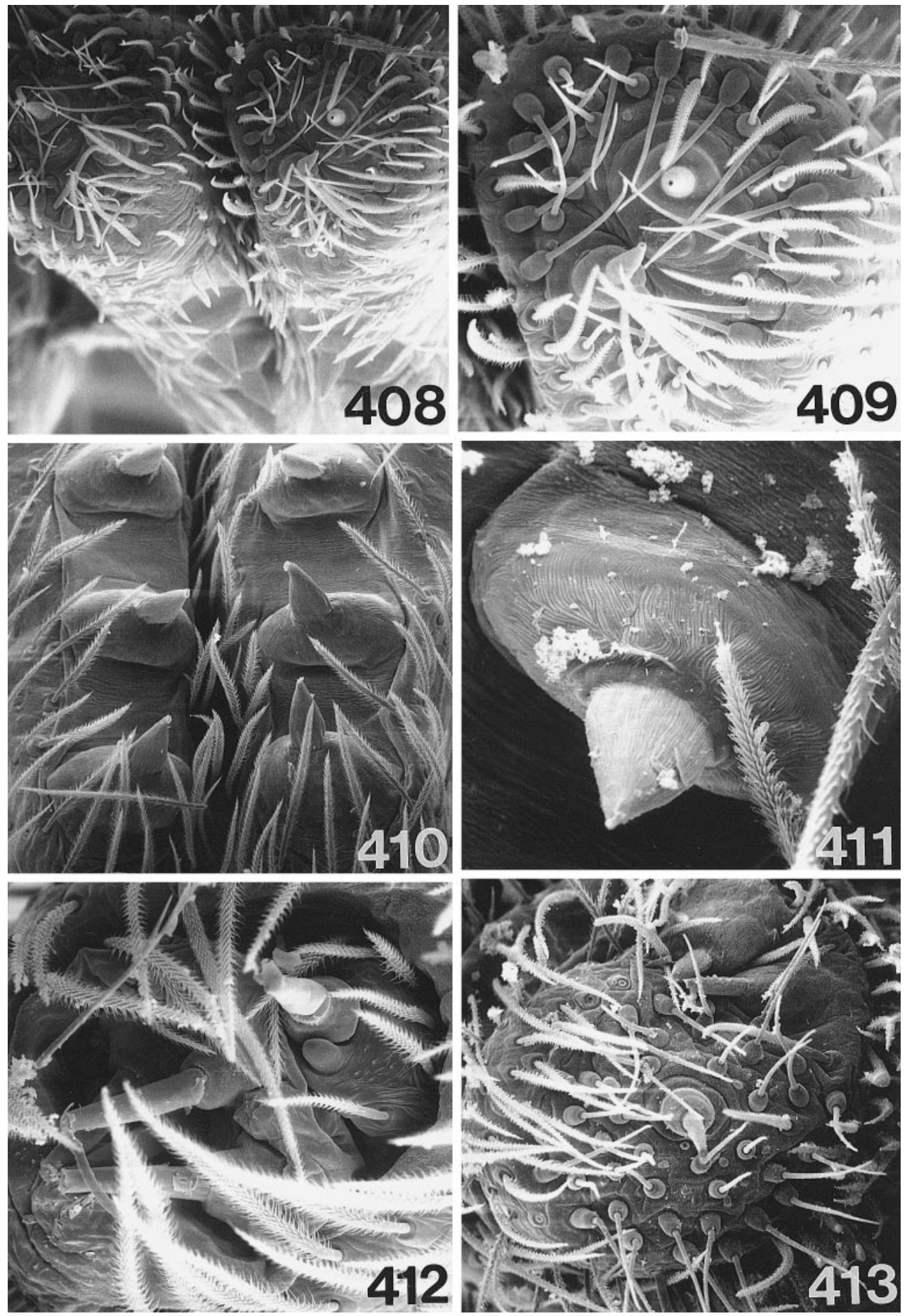

Figs. 408-413. Centrothele gordon, new species, female spinnerets, posterior views. 408. Posterior median spinnerets. 409. Anterior portion of posterior median spinneret. 410. Posterior portion of posterior median spinneret. 411. Cylindrical gland spigot from posterior portion of posterior median spinneret. 412. Anterior lateral spinneret. 413. Posterior lateral spinneret. 
structure (with the bottom stroke of the $y$ doubled, as in figs. $545,549,557) \ldots .$.

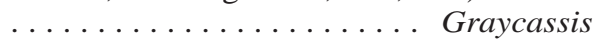

- Posterior epigynal ducts not forming yshaped structure (as in figs. 461, 469, 477)

.................. Centrina

\section{Centrothele L. Koch}

Centrothele L. Koch, 1873: 414 (type species by monotypy Centrothele lorata L. Koch).

Stratius Simon, 1898: 217 (type species by original designation Asadipus muticus Simon). NEW SYNONYMY.

Diagnosis: Males resemble those of Centrina and Centrocalia in having an apophysis at the base of the embolus, but have a relatively longer and narrower embolus (as in figs. 414, 415). Females also resemble those of Centrina and Centrocalia but have a characteristic pattern of largely parallel median and lateral epigynal ducts (figs. 429, 445) not found in those genera.

DESCRIPTION: Medium to large spiders, total length of males 7.1-10.4, of females 6.711.0. Carapace dark red, granulate, coated with short, white setae, margins with long, dark setae; thoracic groove long, longitudinal. Eight eyes in two rows, anterior medians largest, circular, dark, laterals subequal in size, oval, light, posterior medians smallest, irregularly oval, flattened, light; from above, anterior eye row slightly recurved, posterior row slightly procurved, from front, anterior row slightly procurved, posterior row strongly procurved; anterior medians separated by more than their radius, closer to anterior laterals; posterior medians separated by more than their diameter, farther from posterior laterals; anterior and posterior laterals separated by less than their diameter; median ocular quadrangle wider in front than in back, wider in front than long. Chelicerae, sternum, and mouthparts dark orange red; chilum wide, triangular, accompanied by second, inverted $\mathrm{T}$ shaped, posterior chilum (extremely narrow sclerite separating bases of chelicerae posteriorly) with bulbous anterior tip; chelicerae with distinct lateral boss outlined by rows of tubercles bearing short, stiff setae, anterior surface with depressed, relatively unsclerotized oval area near promargin; promargin with two rows of long setae originating in line along base of fang, seta closest to fang bent near base at almost $90^{\circ}$ angle, extending behind other promarginal setae to near midline (figs. 398, 399); promargin with three teeth, median one largest, proximal one smallest; retromargin with two large, widely separated teeth. Labium elongate, base narrowed at about one-third of labial length, posterior margin truncate, anterior margin medially invaginated, surface not depressed medially; labral teeth evenly spaced (fig. 400). Endites obliquely depressed, with sharply demarcated, deep groove along margin near labium, groove wider anteriorly than posteriorly (fig. 396); serrula long, with single row of teeth; anterior surface with distinct sieve plate set in oval of unsclerotized cuticle (fig. 401). Sternum not elevated, with flat lateral margins, not expanded anteriorly, with triangular extensions to and between coxae; surface tuberculate, with distinct elevations opposite, and depressions between, coxae. One epimeric sclerite on each side, above each coxa, reaching sternal triangles only by narrow strips of weakly sclerotized cuticle (strongest strip between coxae II and III), not fused to carapace. Pedicel consisting of small, diamond-shaped sclerite bearing short dorsal and ventral triangular ledges; anteroventral edge of sclerite reaching posterior tip of sternum.

Anterior edge of abdomen of male with complete sclerotic ring formed by strong epigastric scutum plus weaker dorsal abdominal scutum covering entire front edge of abdomen, reaching to almost two-thirds of abdominal length, females with dorsal scutum represented only by small semicircular plate above pedicel, restricted to lower half of anterior surface of abdomen; cuticle with long, erect and recumbent setae; epigastric scutum accompanied posterolaterally by pair of oval, deeply invaginated sclerites bearing clearly elevated anterior rim; sclerites separated by membranous lobe, anterior rim of sclerites fitting under epigastric scutum; colulus represented only by setae; tiny transverse sclerite, well removed from spinnerets, marking position of small posterior spiracle. Anterior lateral spinnerets tubular, separated by more than their diameter, with one major ampullate and few piriform gland spigots (figs. 405, 412), cuticle representing distal, second spinneret segment restricted to semicircle sur- 
rounding major ampullate gland spigot (piriform gland spigots surrounded only by soft cuticle); posterior median spinnerets of males large, tubular, with one large minor ampullate and several small aciniform gland spigots (fig. 406), of females with anteriorly expanded tips, bases occupied by three enormously widened cylindrical gland spigots (figs. 408-411); posterior lateral spinnerets two-segmented, those of males with one large minor ampullate and several small aciniform gland spigots (fig. 407), those of females with two additional greatly widened cylindrical gland spigots (fig. 413).

Leg spination reduced only on anterior legs; typical leg spination pattern (only surfaces with spines listed): femora I-IV d1-00 ; tibiae: III p0-1-1, v0-2-2, r0-0-1; IV p0-01, v2-2-2, r0-1-1; metatarsi III, IV p0-1-1, v2-2-1p, r0-1-1. Most leg surfaces with both short and long setae; males with all coxae and trochanters dorsally tuberculate; anterior coxae with slightly protuberant posterolateral corners; trochanter I unnotched, II-IV slightly notched; anterior metatarsi and tarsi with divided scopulae, composed of laterally directed setae (fig. 404); posterior metatarsi with thick, dark, distal preening brushes; tarsi III with divided, tarsi IV with entire scopulae; tarsi with two dentate claws, claw tufts composed of lateral pads of closely appressed setae; trichobothrial bases heavily ridged (fig. 403); tarsal organ capsulate (fig. 402). Female palpal femur, patella, tibia, and tarsus with long, thin spines; female palpal tarsus with long, basally dentate claw.

Male palp with strong retrolateral tibial apophysis; cymbial surface excavated opposite tibial apophysis, produced into distinct lobe on ventral side of tibial apophysis; tegulum expanded (often greatly), bearing large median apophysis opposing large terminal apophysis; embolus originating ventrally, long, looping to prolateral side of bulb, accompanied distally by long, partially membranous conductor; embolar base bearing distinct, prolaterally-directed apophysis (fig. 397). Epigynum with anterior hood and posterior atrium; spermathecae covered dorsally by two layers of semicircular ducts; anterior portion of epigynum frequently filled with hard plug.

SyNONYMY: For Simon (1897a: 186), Cen- trothele was a "genus invisum" and its redescription as Stratius can only be explained by Simon's failure to note, on his female, the enlarged cylindrical gland spigots that were well illustrated by L. Koch.

\section{Key to Species of Centrothele}

1. Males (those of C. mossman unknown) . . 2

- Females .................. 10

2. Embolus with small spur on distal side of base, in addition to large apophysis on proximal side of base (figs. 397, 422) ... $\ldots \ldots \ldots \ldots \ldots$ gordon

- Embolus with apophysis on proximal side of base only ............... 3

3. Median apophysis with proximally directed lobe below tip (figs. 418, 430) . . . . 4 4

- Median apophysis without distinct lobe below tip ................. 5

4. Retrolateral tibial apophysis very long, narrow (fig. 419) ............. coalston

- Retrolateral tibial apophysis shorter, triangular (fig. 431) . . . . . . . . . mutica

5. Median apophysis very long, narrow (fig. 414) ................ lorata

- Median apophysis shorter .........6 6

6. Retrolateral tibial apophysis relatively long, narrow at base (fig. 427) . . . . . nardi

- Retrolateral tibial apophysis shorter, wider at base ............... 7

7. Prolateral edge of terminal apophysis rounded (figs. 434, 446) …........ 8

- Prolateral edge of terminal apophysis almost straight (figs. 438, 442) . . . . . . . . 9

8. Palpal bulb relatively wide (fig. 446), retrolateral tibial apophysis bulging just beyond base (fig. 447) ............ kuranda

- Palpal bulb relatively narrow (fig. 434), retrolateral tibial apophysis not bulging beyond base (fig. 447) ............. cardell

9. Palpal bulb relatively wide (fig. 438) .... .................. fisher

- Palpal bulb relatively narrow (fig. 442) ... . ....................... spurgeon

10. Anterior epigynal margin narrow, beakshaped (figs. 424, 428, 444) . . . . . 11

- Anterior epigynal margin wider (as in figs. $416,432) \ldots \ldots \ldots \ldots \ldots$

11. Median epigynal ducts relatively short (figs. 425, 429) . . . . . . . . . . 12

- Median epigynal ducts relatively long (fig.

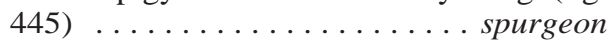

12. Epigynal midpiece relatively wide (fig. 428) $\ldots \ldots \ldots \ldots \ldots$ nardi

- Epigynal midpiece relatively narrow (fig. 424) ............... gordon 

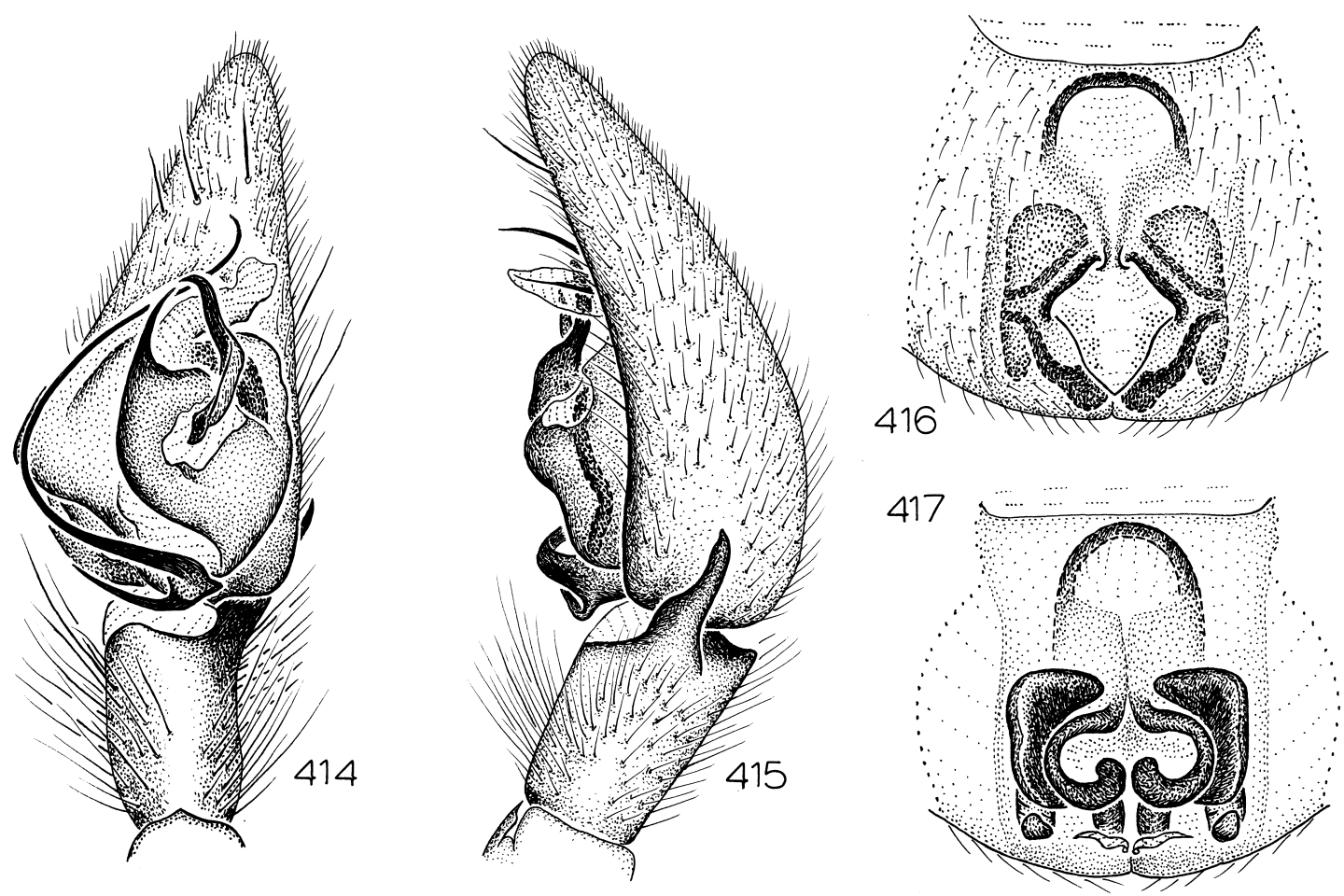

Figs. 414-417. Centrothele lorata L. Koch. 414. Left male palp, ventral view. 415. Same, retrolateral view. 416. Epigynum, ventral view. 417. Same, dorsal view.

13. Epigynal atrium situated medially (fig. 448) or anteriorly (fig. 440) .......... 14

- Epigynal atrium situated posteriorly (as in figs. 432, 436) $\ldots \ldots \ldots \ldots \ldots \ldots \ldots 15$

14. Epigynal atrium situated medially (fig. 448) ..................kuranda

- Epigynal atrium situated anteriorly (fig. 440) ............... fisher

15. Anterior epigynal margin narrow, almost straight (fig. 432); epigynal ducts n-shaped (fig. 433) .............. mutica

- Anterior epigynal ducts longer, forming arch (as in figs. 416, 436) . ........ 16

16. Lateral epigynal ducts squared laterally (figs. $417,421) \ldots \ldots \ldots \ldots \ldots \ldots 17$

- Lateral epigynal ducts rounded laterally (figs. $437,453) \ldots \ldots \ldots \ldots \ldots \ldots 18$

17. Epigynal atrium diamond-shaped (fig. 416) ................. lorata

- Epigynal atrium triangular (fig. 420) ...

18. Lateral epigynal ducts relatively narrow (fig. 453) ................. mossman

- Lateral epigynal ducts relatively wide (fig. 437) ............... cardell

\section{Centrothele lorata $\mathrm{L}$. Koch \\ Figures 414-417; Map 46}

Centrothele lorata L. Koch, 1873: 414, pl. 32, figs. 7, 7a-f (female holotype from Port Mackay, Queensland, Australia, in ZMH, examined).

DiAgnosis: Males can be recognized by the narrow median apophysis and extremely narrow apical projection on the tegular apophysis (fig. 414), females by the angular margins of the epigynal atrium (fig. 416).

MALE: Total length 7.6. Abdominal dorsum gray, with pair of narrow transverse white stripes at about half its length, soft portions of cuticle mottled with anastomosing white spots, venter pale white; legs unmarked. Leg spination: tibiae: III v2-2-2, r01-1; IV p0-1-1; metatarsi III, IV r0-1-0. Retrolateral tibial apophysis short (fig. 415); embolar apophysis long; tegular apophysis with extremely narrow apical projection; median apophysis narrow, flattened (fig. 414).

FEMALE: Total length 10.5. Coloration as 
in male. Leg spination: tibiae: III v2-2-2, r01-1; IV p0-1-2, r0-2-1; metatarsi: IV r1-0-0. Margins of epigynal atrium angular, forming almost m-shaped figure (fig. 416); dorsalmost ducts forming wide semicircle (fig. 417).

MATERIAL ExAmined: Queensland: Crediton, $21^{\circ} 12^{\prime} \mathrm{S}, 148^{\circ} 32^{\prime} \mathrm{E}$, Apr. 14-21, 1975, vagrant (V. Davies, R. Kohout, QMB S28303), $10^{\star}$; lower slopes, Mount William, Dalrymple Heights, near Eungella, $21^{\circ} 01^{\prime} \mathrm{S}$, $148^{\circ} 36^{\prime} \mathrm{E}$, Apr. 1975, pitfall, rainforest, elev. $1120 \mathrm{~m}$ (M. Gray, C. Horseman, AMS KS6727), 20; Port Mackay, $21^{\circ} 12^{\prime} \mathrm{S}$, $149^{\circ} 13^{\prime} \mathrm{E}(\mathrm{ZMH}), 1$ ㅇ (holotype).

DisTRIBUTION: Known only from mid-eastern Queensland (map 46).

\section{Centrothele coalston, new species}

Figures 418-421; Map 46

TYPES: Female holotype and male allotype taken in pitfall traps in rainforest at Coalston Lakes, $26^{\circ} 11^{\prime} \mathrm{S}, 152^{\circ} 04^{\prime} \mathrm{E}$, Queensland (Mar. 26-Sept. 5, 1977; G., S. Monteith), deposited in QMB S26400.

ETYMOLOGY: The specific name is a noun in apposition taken from the type locality.

DiAGNOSIS: Males can be recognized by the extremely long retrolateral tibial apophysis (fig. 419), females by the heart-shaped epigynal atrium (fig. 420).

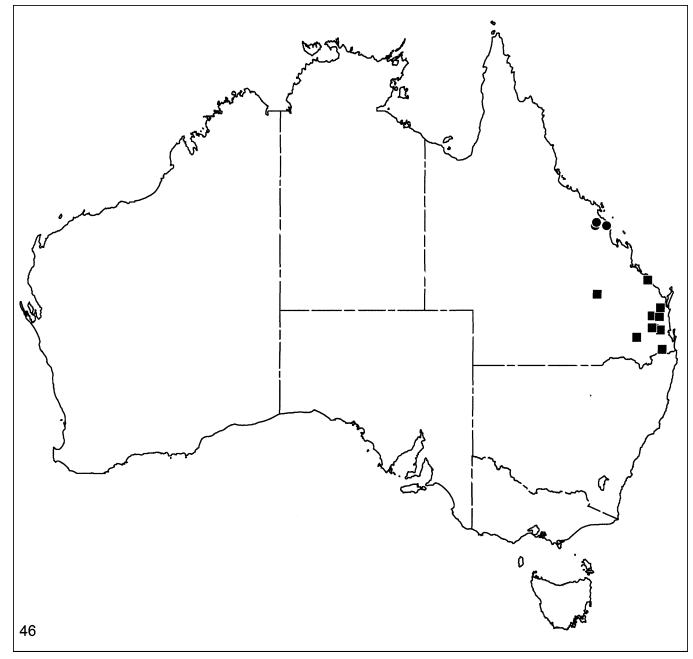

Map 46. Records of Centrothele lorata L. Koch (circles) and C. coalston, new species (squares).
MALE: Total length 11.2. Abdominal dorsum gray, with pair of narrow transverse white stripes at about half its length, soft portions of cuticle mottled with small white spots except within broad, anteriorly widened longitudinal band along midline of venter; legs unmarked. Leg spination typical for genus. Retrolateral tibial apophysis extremely long, as long as segment itself (fig. 419); embolar apophysis long; tegular apophysis with wide apical projection; median apophysis variable in shape, with or without dorsal and ventral extensions (fig. 418).

FEMALE: Total length 11.1. Coloration as in male. Leg spination typical for genus. Epigynal hood large, semicircular, atrium heartshaped, filled by midpiece (fig. 420); dorsalmost ducts c-shaped, median ducts folded, leading to crenulate spermathecae (fig. 421).

OTHER MATERIAL EXAMINED: Queensland: top of Blackbutt Range, via Benarkin, $26^{\circ} 52^{\prime} \mathrm{S}, 152^{\circ} 11^{\prime} \mathrm{E}$, Mar. 29-June 1, 1975, rainforest, pitfall (G., S. Monteith, QMB S28157), $10^{\prime}$; Boonah, $28^{\circ} 00^{\prime} \mathrm{S}, 152^{\circ} 41^{\prime} \mathrm{E}$, Apr. 21, 1973, under log (G. May, QMB S28381), 10; : Eurimbula Creek, Round Hill Head, $24^{\circ} 12^{\prime} \mathrm{S}, 151^{\circ} 47^{\prime} \mathrm{E}$, pitfall (G., S. Monteith, QMB S26397, S26401), 10 , 2 ; Lake Broadwater, via Dalby, $27^{\circ} 21^{\prime} \mathrm{S}, 151^{\circ} 06^{\prime} \mathrm{E}$, Mar. 25-May 16, 1985, pitfall (QMB S28587), 1 đo, May 16-Nov. 23, 1985, pitfalls (QMB S30493, S34351), 5ô, 3 9 , Apr. 22June 12, 1986, pitfalls (M. Bennie, QMB S28661, S30489, S34347), 30 5 , 9 ; foot of Mount Glastonbury, NW Gympie, 26 $6^{\circ} 13^{\prime} \mathrm{S}$, $152^{\circ} 31^{\prime}$ E, June 8-10, 1985 (QMB S26516), $1 \delta^{\star}$; Lake Nuga Nuga, $24^{\circ} 59^{\prime} \mathrm{S}, 148^{\circ} 40^{\prime} \mathrm{E}$, May 11, 1978 (K. McDonald, QMB S26399), $1 \%$; c. $8 \mathrm{mi} \mathrm{W}$ Tiaro on Mungar Road, $25^{\circ} 44^{\prime} \mathrm{S}, 152^{\circ} 35^{\prime} \mathrm{E}$, July 1 , 1984, in silk sac under rotting log (J. Gallon, QMB S26402), 1\%; Villeneure, via Kilcoy, $26^{\circ} 57^{\prime} \mathrm{S}, 152^{\circ} 34^{\prime} \mathrm{E}$, May 13 , 1985 , night collecting (J. Gallon, QMB S28385), $10^{\top} ; 3 \mathrm{~km}$ E Yarraman, $26^{\circ} 50^{\prime} \mathrm{S}, 152^{\circ} 03^{\prime} \mathrm{E}$, June 1 -Aug. 17, 1975, rainforest, pitfall (G., S. Monteith, QMB S26854), 10 .

DistRIBUTION: Known only from southeastern Queensland (map 46).

\section{Centrothele gordon, new species}

Figures 396-413, 422-425; Map 47

TyPES: Female holotype and male allotype taken in pitfall traps in litter at Gordon, 

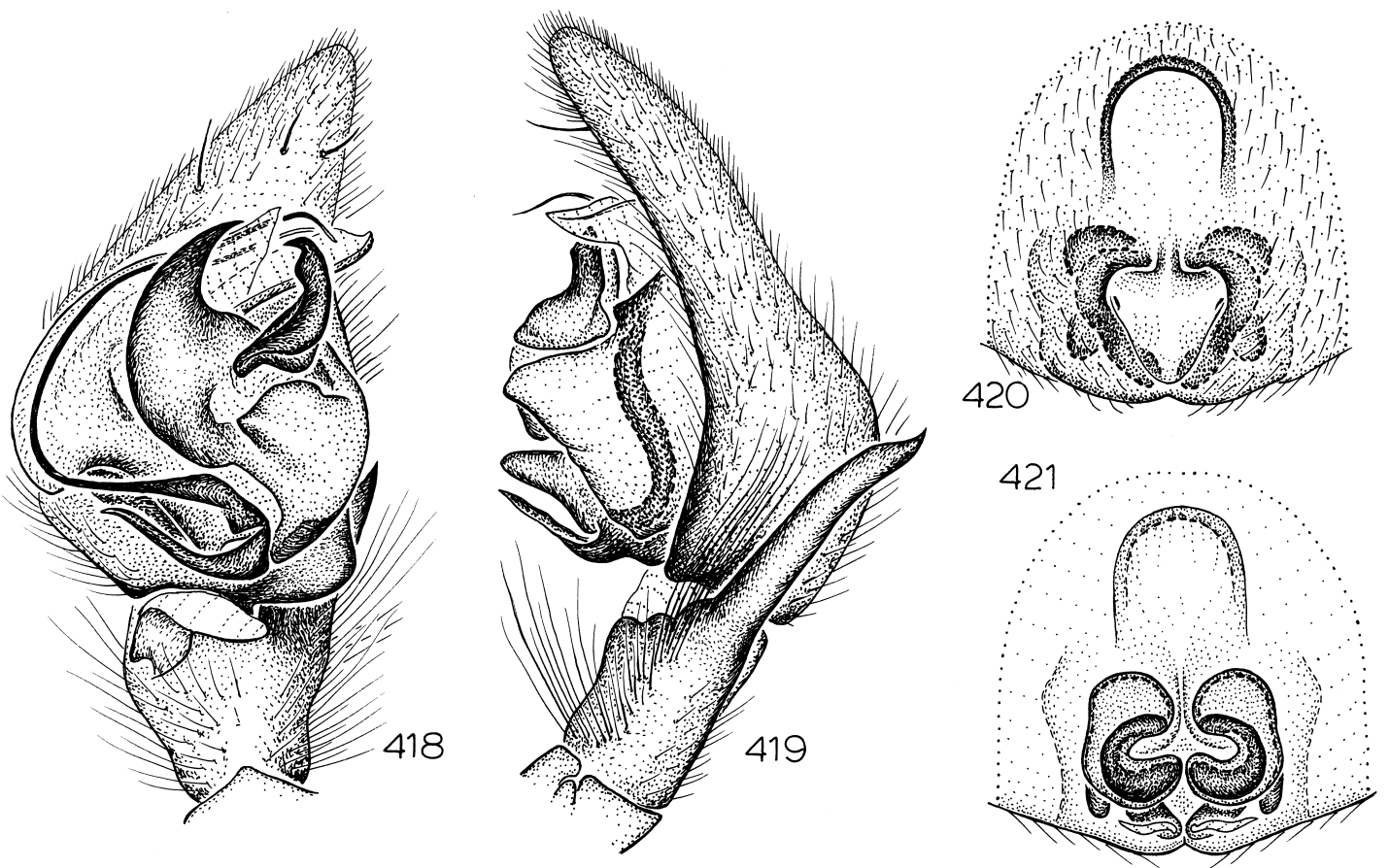

Figs. 418-421. Centrothele coalston, new species. 418. Left male palp, ventral view. 419. Same, retrolateral view. 420. Epigynum, ventral view. 421. Same, dorsal view.

$33^{\circ} 44^{\prime} \mathrm{S}, 151^{\circ} 09^{\prime} \mathrm{E}$, New South Wales (July 8-29, 1982; C. Horseman), deposited in AMS (KS9743).

ETYMOLOGY: The specific name is a noun in apposition taken from the type locality.

DiAGNOSIS: Males can be recognized by the spur on the distal side of the embolar base (fig. 422), females by the narrow, beakshaped anterior epigynal hood that is far removed from the posteriorly situated, diamond-shaped atrium (fig. 424).

MALE: Total length 10.0. Abdominal dorsum gray, without pair of transverse white stripes, soft portions of cuticle mottled with white spots, venter pale white, with four longitudinal rows of tiny orange sclerotizations; legs unmarked. Leg spination: femora I-III p0-0-1; tibiae: III v2-2-2, r0-1-1; IV p0-1-1. Retrolateral tibial apophysis relatively short (fig. 423); base of embolus with distinct distal apophysis, embolar apophysis long, tegular apophysis long, median apophysis flattened (fig. 422).

Female: Total length 10.2. Coloration as in male. Leg spination: femora I-III p0-0-1; tibiae: III v2-2-2, r0-1-1; IV p0-1-1. Epigynal hood narrow, beak-shaped (fig. 424); dorsalmost ducts greatly widened posteriorly (fig. 425).

Other Material Examined: New South Wales: Blue Gum Hut, via Mallanganee, $28^{\circ} 47^{\prime} \mathrm{S}, 152^{\circ} 43^{\prime} \mathrm{E}$, May 27-Oct. 2, 1978, pitfall, rainforest (G., S. Monteith, QMB S26480), 1 ; ; near Carrai Bat Cave, Carrai, $31^{\circ} 01^{\prime} \mathrm{S}, 152^{\circ} 30^{\prime} \mathrm{E}$, Apr. 26, 1974, rainforest litter (M. Gray, AMS KS44156), 1 \% ; Carrai area, W Kempsey, $31^{\circ} 01^{\prime} \mathrm{S}, 152^{\circ} 30^{\prime} \mathrm{E}$, July 18,1971 , in logs, subtropical rain forest (M. Gray, AMS KS52277), 2 \% Dodds Fire Trail, $1 \mathrm{~km}$ from Enfield Road, Enfield State Forest, $31^{\circ} 23^{\prime} \mathrm{S}, 151^{\circ} 53^{\prime} \mathrm{E}$, Feb. 4-Apr. 9, 1993, pitfall, elev. $1050 \mathrm{~m}$ (M. Gray, G. Cassis, AMS KS37376), 30; Gordon, 33⒋' S, $151^{\circ} 09^{\prime} \mathrm{E}$, July $8-29$, 1982, pitfall, litter (C. Horseman, AMS KS9743, 40ิ, 2 ㅇ, July 29Aug. 26, 1982, pitfall, litter (C. Horseman, AMS KS9784, KS9799), 80, 5 ㅇ, Aug. 26Oct. 6, 1982, pitfall, litter (C. Horseman, AMS KS10115, 10128), 30, 4웅. Oct. 6Nov. 4, 1982, pitfall, litter (C. Horseman, 

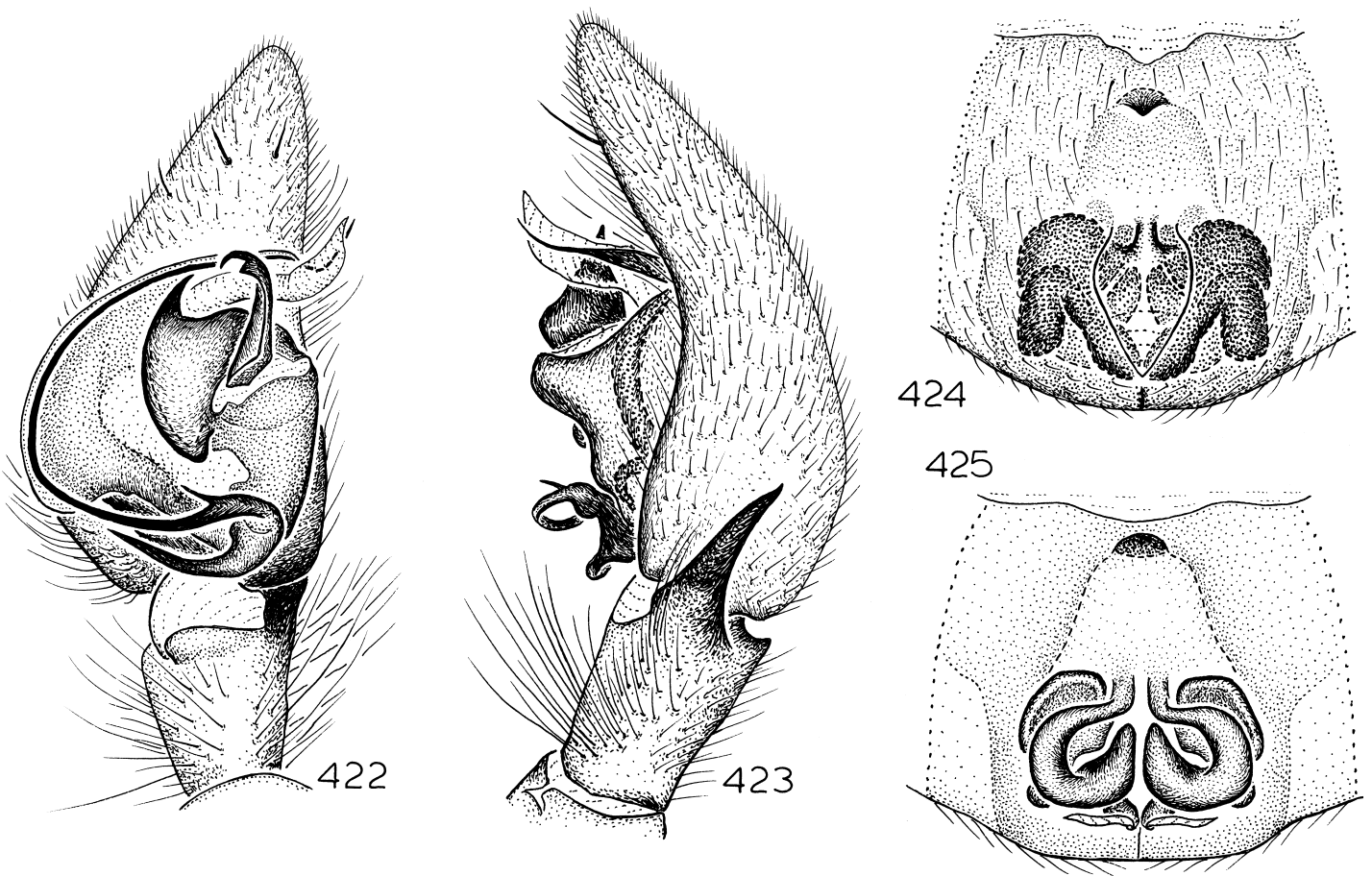

Figs. 422-425. Centrothele gordon, new species. 422. Left male palp, ventral view. 423. Same, retrolateral view. 424. Epigynum, ventral view. 425. Same, dorsal view.

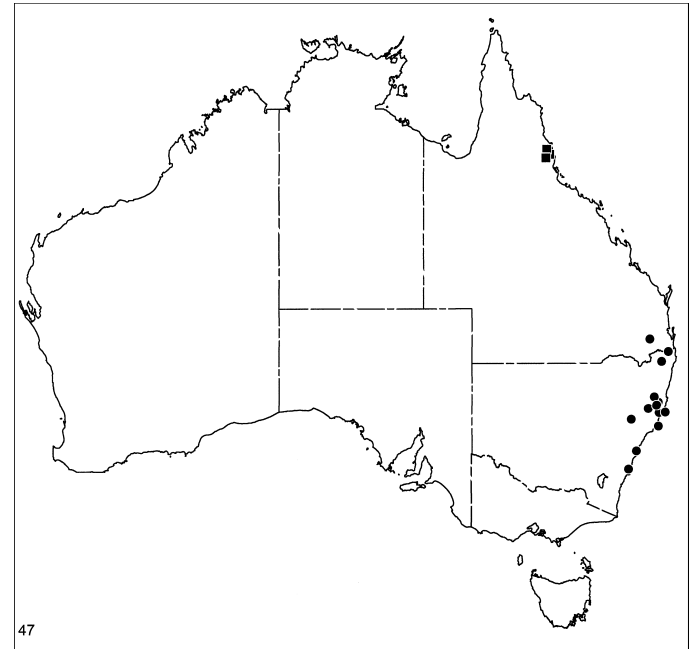

Map 47. Records of Centrothele gordon, new speices (circles) and $C$. fisher, new species (squares).
AMS KS10265, KS10275, KS10463), 1 ๙, 2ㅇ, May 12-June 9, 1983, pitfall, litter (C. Horseman, AMS KS12398), 60, 2우, June 9-July 7, 1983, pitfall, litter (C. Horseman, AMS KS12421, KS12412), 60, 5ㅇ, July 7Aug. 4, 1983, pitfall, litter (C. Horseman, AMS KS12638, KS12647), 40, 2 \% , Aug. 4Sept. 9, 1983, pitfall, litter (C. Horseman, AMS KS13285), 3ㅊ, 4오, Sept. 9-29, 1983, pitfall, litter (C. Horseman, AMS KS13311), 1 ㅇ, Sept. 29-Oct. 27, 1983, pitfall, litter (C. Horseman, AMS KS13319, KS13329), 3 ㅇ, May 9-June 7, 1984, pitfall, litter (C. Horseman, AMS KS14745, KS14754), 2 ㅇ K Kerewong State Forest, $31^{\circ} 36^{\prime} \mathrm{S}, 152^{\circ} 34^{\prime} \mathrm{E}$, May 30-Sept. 1, 1979, pitfall, litter (D. Milledge, AMS KS5445, KS5470), 2 ; Kiwarrak State Forest, near Taree, $31^{\circ} 58^{\prime} \mathrm{S}, 150^{\circ} 50^{\prime} \mathrm{E}$, Aug. 6-Sept. 19, 1979 (W. King, AMS KS3955), 1 ㅇ L Lorne State Forest, $31^{\circ} 35^{\prime} \mathrm{S}, 152^{\circ} 57^{\prime} \mathrm{E}$, May 30-Sept. 1, 1979, pitfall, litter (D. Milledge, AMS KS5380), 1 ơ; Lower Creek, 90 $\mathrm{km}$ N Kempsey, $30^{\circ} 44^{\prime} \mathrm{S}, 152^{\circ} 15^{\prime} \mathrm{E}$, July 16, 1975 , remnant rainforest, under $\log (\mathrm{R}$. Raven, QMB S34350), 1ठ; Mount Boss State 

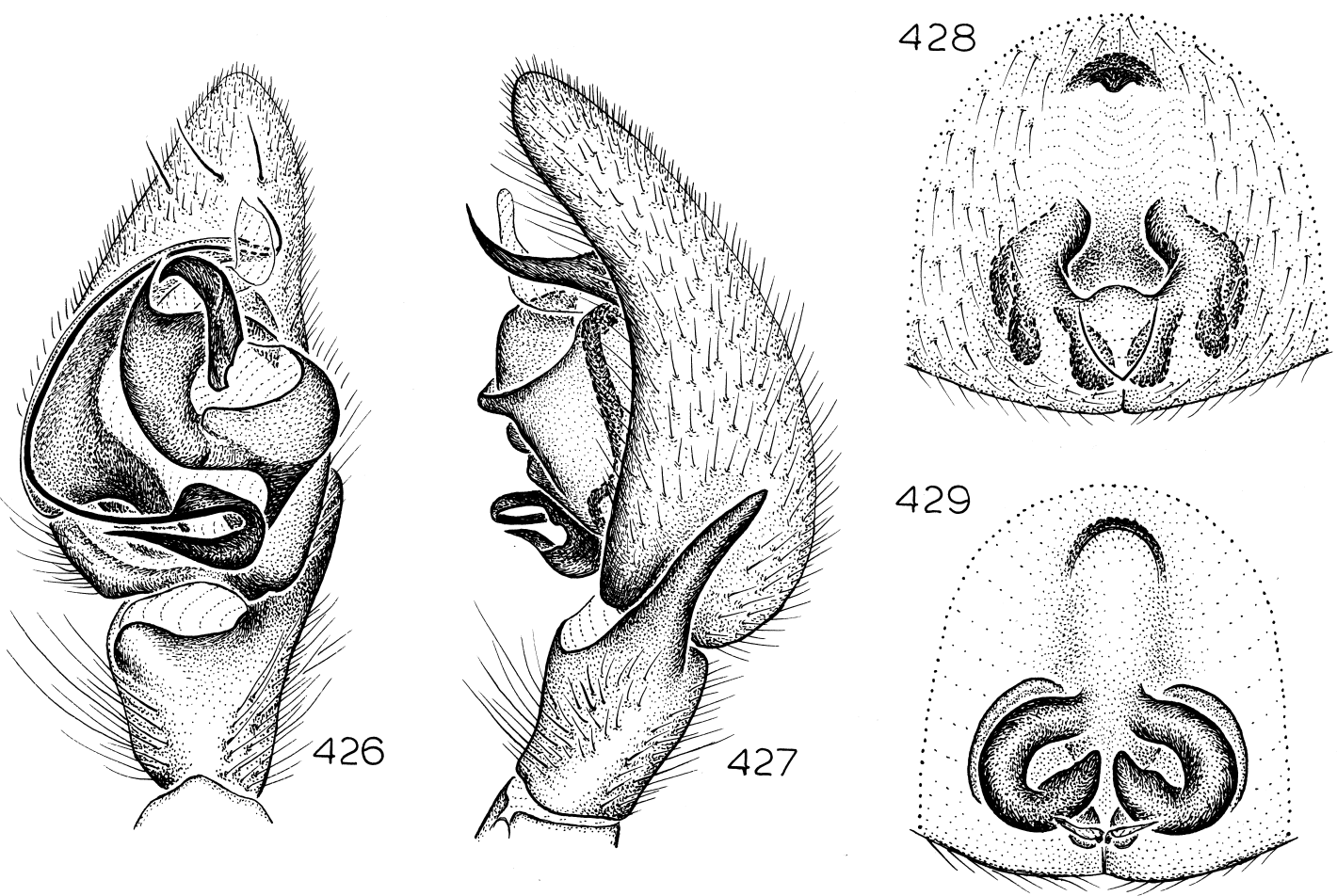

Figs. 426-429. Centrothele nardi, new species. 426. Left male palp, ventral view. 427. Same, retrolateral view. 428. Epigynum, ventral view. 429. Same, dorsal view.

Forest, South Plateau, $31^{\circ} 12^{\prime} \mathrm{S}, 152^{\circ} 24^{\prime} \mathrm{E}$, Oct. 1980 (AMS KS42884), 1 \% ; Pacific Palms, 32 $21^{\prime} \mathrm{S}, 152^{\circ} 31^{\prime} \mathrm{E}$, May 9, 1991 (C. Peterson, AMS KS55277), 1 ; ; Woodhill, W Berry, $34^{\circ} 45^{\prime} \mathrm{S}, 150^{\circ} 41^{\prime} \mathrm{E}$, Sept. 22, 1993 (J. Noble, AMS KS55328), $1 \hat{\sigma}^{\hat{\sigma}}$. Queensland: Lamington National Park, O'Reillys, $28^{\circ} 14^{\prime} \mathrm{S}, 153^{\circ} 08^{\prime} \mathrm{E}$, Jan. 1990, indoors (R. Green, QMB S28536), 1 9 ; Redwood Park, E Toowoomba, $27^{\circ} 33^{\prime} \mathrm{S}, 151^{\circ} 58^{\prime} \mathrm{E}$, July 1985-Jan. 7, 1986 (G. Monteith, G. Thompson, QMB S26837), 10 .

Distribution: Southeastern Queensland and eastern New South Wales (map 47).

\section{Centrothele nardi, new species \\ Figures 426-429; Map 48}

TYPES: Female holotype and male allotype taken in pitfall traps in rainforest at an elevation of $762 \mathrm{~m}$ on Mount Nardi, via Nimbin, $28^{\circ} 32^{\prime} \mathrm{S}, 153^{\circ} 17^{\prime} \mathrm{E}$, New South Wales (Mar. 23-Aug. 2, 1975; G., S. Monteith), deposited in QMB (S28129).
ETYMOLOGY: The specific name is a noun in apposition taken from the type locality.

DiAGNOSIS: Males can be recognized by the basally narrow embolus (fig. 426), females by the long, deeply depressed epigynal atrium (fig. 428).

MALE: Total length 8.0. Abdominal dorsum gray, without pair of transverse white stripes, soft portions of cuticle with interrupted narrow lines of white spots, venter pale white, unmarked; legs unmarked. Leg spination: tibiae: III v2-2-2, r0-1-1; IV p0-11; metatarsi: III p1-1-1; IV p1-1-1, r1-1-1. Retrolateral tibial apophysis relatively short, laterally convex (fig. 427); embolar base narrow, embolar apophysis long, straight, tegular apophysis long, median apophysis laterally flattened, situated opposite bump on tegulum (fig. 426).

FEMALE: Total length 8.2. Coloration and leg spination as in male. Epigynal hood wide, beak-shaped, situated above depression continuous with epigynal atrium (fig. 428); dor- 
salmost ducts almost as long as median ones (fig. 429).

Other Material Examined: New South Wales: Mount Nardi, via Nimbin, $28^{\circ} 32^{\prime} \mathrm{S}$, $153^{\circ} 17^{\prime} \mathrm{E}$, Mar. 23-Aug. 2, 1975, rainforest pitfall, elev. $762 \mathrm{~m}$ (G., S. Monteith, QMB S28328), 20, 1 i (penultimate). Queensland: Binna Burra, Lamington National Park, 28 $8^{\circ} 2^{\prime}$ S, $153^{\circ} 11^{\prime} \mathrm{E}$, Mar. 27, 1976 (R. Raven, V. Davies, QMB S26501), 1 đ̊; Booloumba Creek, via Conondale, $26^{\circ} 39^{\prime} \mathrm{S}$, $152^{\circ} 39^{\prime}$ E, Feb. 22-Apr. 19, 1975, rainforest pitfall, elev. 518-550 m (G., S. Monteith, QMB S12166, S28136, S28141, S28147),

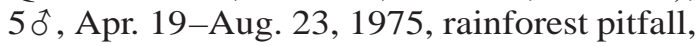
elev. 518 m (G., S. Monteith, QMB S28140), 10; Cooran Plateau, via Traveston, $26^{\circ} 21^{\prime} \mathrm{S}$, $152^{\circ} 47^{\prime} \mathrm{E}$, Mar. 28-Aug. 13, 1975, rainforest pitfall, elev. $366 \mathrm{~m}$ (G., S. Monteith, QMB S28182), 10; Dingo Creek, via Traveston, $26^{\circ} 20^{\prime} \mathrm{S}, 151^{\circ} 52^{\prime} \mathrm{E}$, Aug. 10-Nov. 9, 1974, rainforest pitfall, elev. $30 \mathrm{~m}$ (G., S. Monteith, QMB S28191), 30; Mount Glorious, Hiller property, $27^{\circ} 20^{\prime} \mathrm{S}, 152^{\circ} 46^{\prime} \mathrm{E}$, Jan. 20-June 26, 1978, rainforest pitfall (G., S. Monteith, QMB S26482), 20; Mount Glorious barracks, $27^{\circ} 18^{\prime} \mathrm{S}, 152^{\circ} 45^{\prime} \mathrm{E}$, Mar, 6-May 29, 1992, rainforest pitfall, elev. $750 \mathrm{~m}$ (G. Monteith, QMB S30482, S32474), 20, 5 ; Mount Cabinet, via Jimna, $26^{\circ} 45^{\prime} \mathrm{S}$, $152^{\circ} 27^{\prime} \mathrm{E}$, June 16-Aug. 23, 1975, rainforest

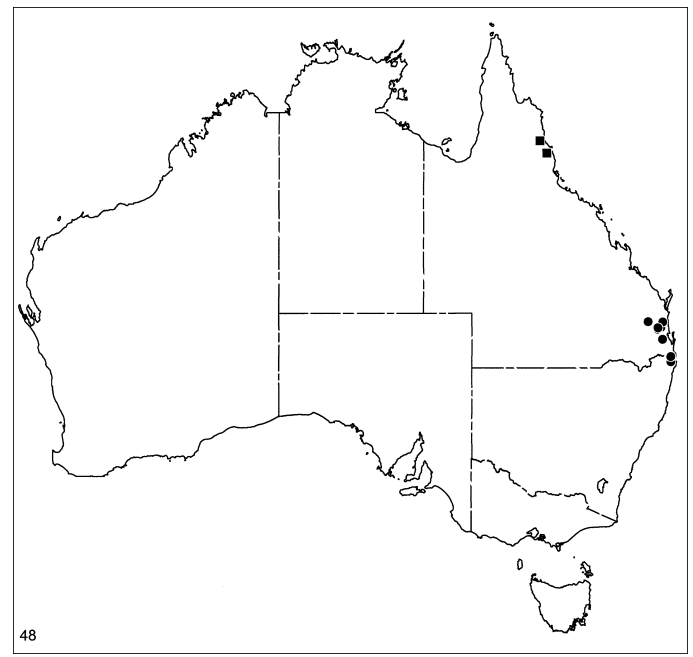

Map 48. Records of Centrothele nardi, new species (circles) and $C$. spurgeon, new species (squares). pitfall, elev. 792 m (G., S. Monteith, QMB S28330), 1 \% ; Numinbah Arch, Nerang Valley, $28^{\circ} 12^{\prime} \mathrm{S}, 153^{\circ} 13^{\prime} \mathrm{E}$, Oct. 26-Dec. 14, 1974, rainforest pitfall, elev. $305 \mathrm{~m}$ (G., S. Monteith, QMB S28353), 1 \% , Mar. 22-May 25, 1975, rainforest pitfall, elev. $305 \mathrm{~m}$ (G., S. Monteith, QMB S12198, S28371), 30 , 4 , May 25-Oct. 13, 1975, rainforest pitfall, elev. 305 m (G., S. Monteith, QMB S28339), 5 웅 Tungi Creek, via Jimna, $26^{\circ} 40^{\prime} \mathrm{S}$, $152^{\circ} 28^{\prime} \mathrm{E}$, Mar. 29-June 16, 1975, rainforest pitfall, elev. $55 \mathrm{~m}$ (G., S. Monteith, QMB S28349), $20^{\text {* }}$; Upper Tallebudgera Valley, $28^{\circ} 15^{\prime} \mathrm{S}, 153^{\circ} 16^{\prime} \mathrm{E}$, Mar.-July 1985 , rainforest, elev. 530 m (D. Cook, QMB S28393), 10 .

Distribution: Southeastern Queensland and adjacent New South Wales (map 48).

\section{Centrothele mutica (Simon), new combination \\ Figures 430-433; Map 49}

Asadipus muticus Simon, 1897b: 15 (female holotype from "Nova Hollandia orient." in MNHN, examined).

Stratius muticus: Simon, 1898: 217.

DiAGNOSIS: Males can easily be recognized by the broadened retrolateral tibial apophysis and enlarged median apophysis (figs. 430, 431), females by the posteriorly expanded epigynal midpiece (fig. 432).

MALE: Total length 9.9. Abdominal dorsum dark gray, almost completely covered by orange scutum, without pair of transverse white stripes, soft portions of cuticle with interrupted narrow lines of white spots, venter pale white, with four longitudinal rows of tiny orange sclerotizations; legs unmarked. Leg spination: tibiae: III r0-1-1; IV p0-1-1, v1p-2-2; metatarsi: III v0-2-1p. Retrolateral tibial apophysis greatly widened basally (fig. 431); embolar base shifted retrolaterally, embolar apophysis long, spikelike, tegular apophysis long, flat, median apophysis greatly enlarged, elongated (fig. 430).

FEMALE: Total length 9.3. Coloration as in male. Leg spination as in male except metatarsus III v2-2-1p. Epigynal midpiece posteriorly widened, with steeply sloping sides (fig. 432); dorsalmost ducts directed longitudinally (fig. 433). 

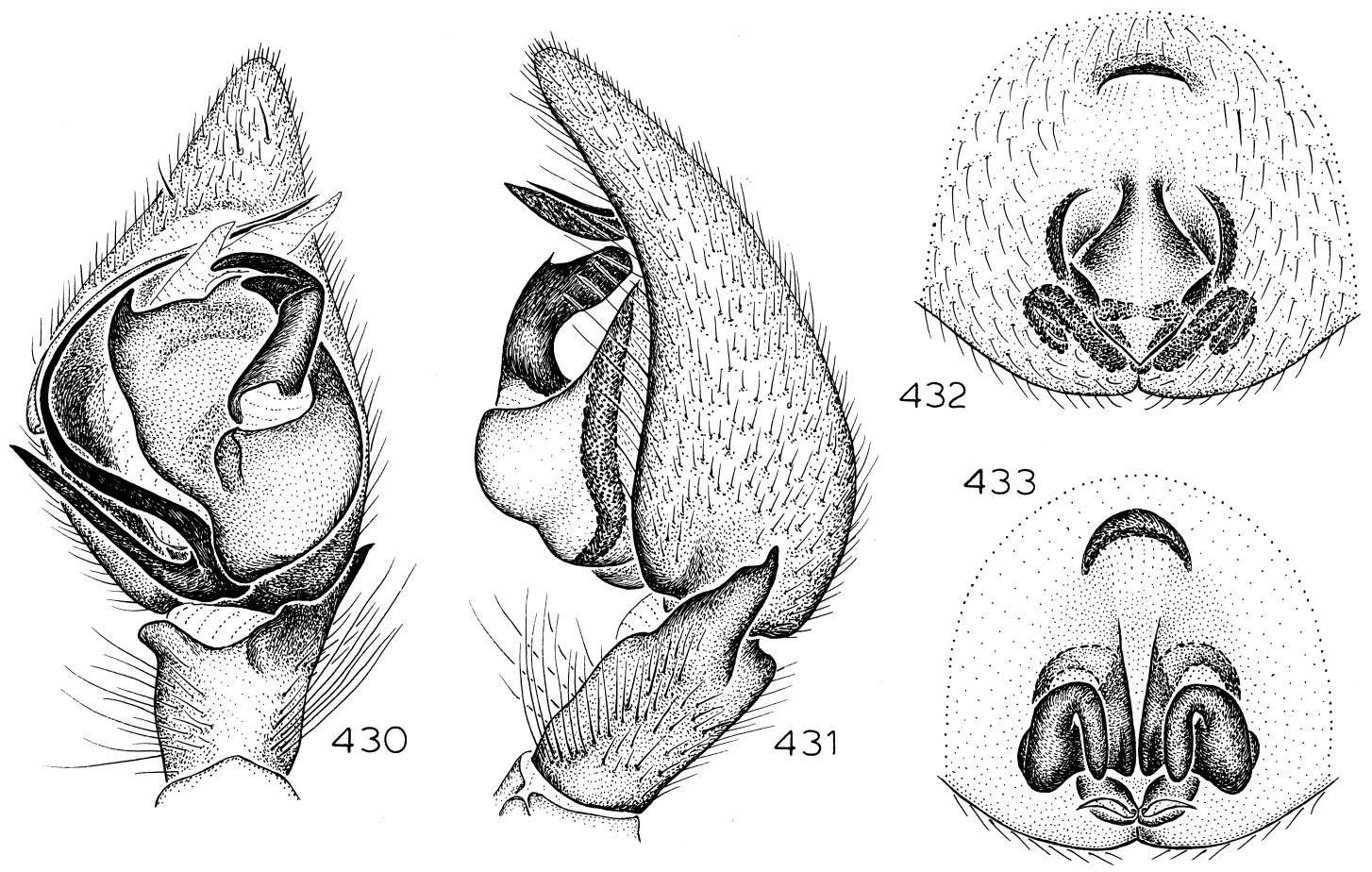

Figs. 430-433. Centrothele mutica (Simon). 430. Left male palp, ventral view. 431. Same, retrolateral view. 432. Epigynum, ventral view. 433. Same, dorsal view.

Material Examined: AUStralia: New

South Wales: no specific locality (MNHN 18090), 1 ㅇ (holotype); Gorge Creek Road, Richmond Range State Forest, $28^{\circ} 55^{\prime} \mathrm{S}$, $152^{\circ} 45^{\prime} \mathrm{E}$, Apr. 18, 1976, beating scrub (R. Raven, QMB S34352), 19 ; Lane Cove River Park, Sydney, $33^{\circ} 50^{\prime}$ S, $151^{\circ} 11^{\prime} \mathrm{E}$, May $17-$ 18, 1988 (D. Hirst, SAM N1997/78), 1 \% ; Victoria Park, via Alstonville, $28^{\circ} 53^{\prime} \mathrm{S}$, $153^{\circ} 23^{\prime} \mathrm{E}$, Mar. 23-Aug. 3, 1975, rainforest pitfall (G., S. Monteith, QMB S28329), $2 \hat{0}$. Queensland: Brookfield, $27^{\circ} 30^{\prime} \mathrm{S}, 152^{\circ} 55^{\prime} \mathrm{E}$, Feb. 27-May 20, 1976, rainforest pitfall (G., S. Monteith, QMB S34349), 1 ; Burleigh Headland, $28^{\circ} 10^{\prime} \mathrm{S}, 153^{\circ} 33^{\prime} \mathrm{E}$, May 25-Oct. 13, 1975, rainforest pitfall, elev. $10 \mathrm{~m}$ (G., S. Monteith, QMB S26850), 1 \%; Burnett Range, 15 km NE Tansey, Mar. 26-Sept. 5, 1977, rainforest pitfall, elev. $400 \mathrm{~m}$ (G., S. Monteith, QMB S26485), $1 \%$; Conondale Range, $26^{\circ} 45^{\prime} \mathrm{S}, 152^{\circ} 37^{\prime} \mathrm{E}$, May $1-3,1976$, rainforest (R. Raven, QMB S29322), 1 ㅇ, May 8-9, 1976, pine forest (R. Raven, QMB S29334), 1\%; Deer Reserve, via Kilcoy,

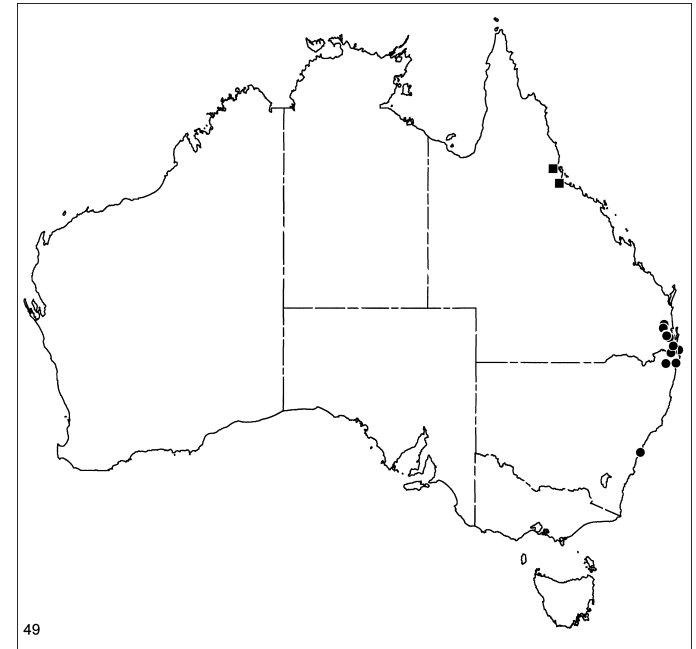

Map 49. Records of Centrothele mutica (Simon, Australian records only) (circles) and $C$. cardell, new species (squares). 

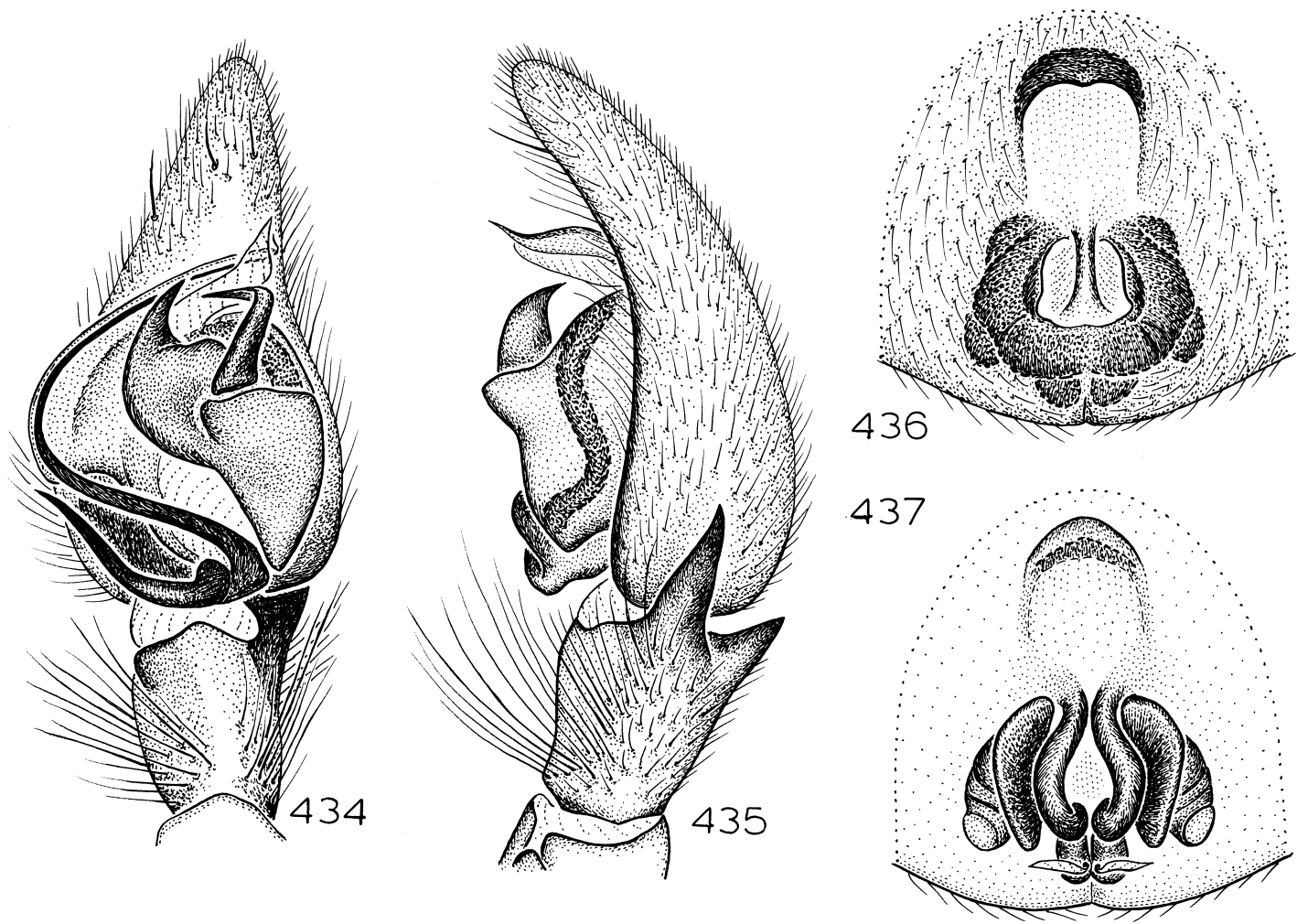

Figs. 434-437. Centrina cardell, new species. 454. Left male palp, ventral view. 455. Same, retrolateral view. 456. Epigynum, ventral view. 457. Same, dorsal view.

$26^{\circ} 57^{\prime} \mathrm{S}, 152^{\circ} 34^{\prime} \mathrm{E}$, Mar. 29-June 1, 1975, rainforest pitfall, elev. $457 \mathrm{~m}$ (G., S. Monteith, QMB S26851), 19 ; Lamington National Park, Binna Burra, $28^{\circ} 12^{\prime} \mathrm{S}, 153^{\circ} 11^{\prime} \mathrm{E}$, Apr. 5, 1995, night (R. Raven, QMB S27529), 1 $\delta^{\star}$; Lamington National Park, Daves Creek Country, $28^{\circ} 19^{\prime} \mathrm{S}, 153^{\circ} 05^{\prime} \mathrm{E}$, Apr. 3, 1976, rainforest (R. Raven, QMB S26497), 4 \% ; Lamington National Park, O'Reillys, $28^{\circ} 14^{\prime} \mathrm{S}, 153^{\circ} 08^{\prime} \mathrm{E}$, Sept. 25-26, 1986, rainforest (R. Raven, J. Gallon, QMB S26861), 1 ; Lamington National Park, Plateau, $28^{\circ} 16^{\prime} \mathrm{S}, 153^{\circ} 10^{\prime} \mathrm{E}$, June 22, 1973 (R. Raven, QMB S26495), 10; Lamington Plateau, $28^{\circ} 19^{\prime} \mathrm{S}, 153^{\circ} 04^{\prime} \mathrm{E}$, Apr. 2, 1975, rainforest (R. Raven, QMB S34348), 19 ; Mount Coot-tha, Brisbane, $27^{\circ} 29^{\prime} \mathrm{S}, 152^{\circ} 57^{\prime} \mathrm{E}$, Nov. 1973 (G. Czechura, QMB S26533), 1 9 ; Mount Glorious, $27^{\circ} 20^{\prime} \mathrm{S}, 152^{\circ} 46^{\prime} \mathrm{E}$, Feb.June 1978, malaise trap (A. Hiller, QMB S26461), 1 ${ }^{\star}$, Mar. 25-May 26, 1983, rain- forest, malaise trap (A. Hiller, QMB S26511, S26493), 20, May 10, 1988, in house (K. Hiller, QMB S6302), 10; Mount Nebo, $27^{\circ} 23^{\prime} \mathrm{S}, 152^{\circ} 47^{\prime} \mathrm{E}$, Oct. 16,1978 , mixed sclerophyll pitfall (A. Rozefelds, QMB S26498), 19 ; Mount Tamborine, $27^{\circ} 56^{\prime} \mathrm{S}$, $153^{\circ} 12^{\prime} \mathrm{E}$, July 10,1974 , beating (V. Davies, R. Raven, QMB S26503), 2 \%. PAPUA NEW GUINEA: Misima Island, Louisiade Archipelago (D. Rentoul, QMB), 1 ㅇ․

Distribution: Southeastern Queensland and New South Wales (map 49); possibly introduced into New Guinea.

\section{Centrothele cardell, new species}

Figures 434-437; Map 49

TYPE: Female holotype taken in rainforest at Mount Spec State Forest, $19^{\circ} 00^{\prime} \mathrm{S}$, $146^{\circ} 09^{\prime} \mathrm{E}$, Queensland (Sept. 2, 1988; J. Gallon), deposited in QMB (S14024). 
ETYMOLOGY: The specific name is an arbitrary combination of letters.

DiAGNOSIS: Males and females have not been collected together and are only tentatively matched here. The male has a relatively small retrolateral tibial apophysis and a distinctive, pointed dorsal tibial apophysis (figs. 434, 435); the female has a very narrow epigynal midpiece and narrow epigynal ducts (figs. 436, 437).

MALE: Carapace length 3.2 (abdomen missing). Abdominal coloration unknown, legs unmarked. Leg spination (legs IV missing): tibia III v-2-2-2, r0-1-1; metatarsus III r0-1-0. Retrolateral tibial apophysis very small, not much larger than distinctly pointed dorsal apophysis (fig. 435); embolus relatively short, not enlarged at base, embolar apophysis distally sinuous, tegular apophysis long, tip of median apophysis long, narrow (fig. 434).

Female: Total length 10.8. Abdominal dorsum gray, with faint trace of transverse white stripes, sides and venter with reticulate pattern of gray spots surrounded by paler areas; legs unmarked. Leg spination: tibiae: III v2-2-2, r0-1-1; IV p0-1-1. Epigynal hood wide, semicircular, epigynal midpiece long, narrow (fig. 436); dorsalmost ducts almost longitudinal, situated along midline, bordered at sides by narrow second pair of ducts, exposing twisted spermathecae (fig. 437).

Other MAterial ExAmined: Queensland: Kirrama State Forest, $32 \mathrm{~km}$ N Cardwell, $18^{\circ} 12^{\prime} \mathrm{S}, 145^{\circ} 45^{\prime} \mathrm{E}$, June 23-Aug. 8, 1982, rainforest, intercept trap, elev. $800 \mathrm{~m}$ (S., J. Peck, AMNH), 1 ô.

DisTRIBUTION: Known only from northern Queensland (map 49).

\section{Centrothele fisher, new species}

Figures 438-441; Map 47

TYPE: Female holotype taken in pitfall trap at an elevation of $1150 \mathrm{~m}$ on Mount Fisher, $17^{\circ} 33^{\prime} \mathrm{S}, 145^{\circ} 32^{\prime} \mathrm{E}$, Queensland (Aug. 2Sept. 4, 1995; L. Umback), deposited in QMB.

ETYMOLOGY: The specific name is a noun in apposition taken from the type locality.

DiAGNOSIS: Males can be recognized by the small dorsal tibial apophysis (fig. 439) and thin, long, embolus originating on the retrolateral edge of the bulb (fig. 438), females by the anteriorly advanced epigynal midpiece (fig. 440).

MALE: Total length 7.1. Abdominal dorsum gray, with pair of narrow transverse white stripes at about half its length, sides with interrupted narrow lines of white spots, venter pale white; legs unmarked. Leg spination: tibiae: III v2-2-2, r0-1-1; IV p0-1-1; metatarsi III, IV r0-1-0. Retrolateral tibial apophysis short, expanded basally on both dorsal and ventral sides, tibia also with small dorsal apophysis (fig. 439); embolus long, narrow, originating on retrolateral edge of bulb, embolus apophysis evenly arched, tegular apophysis almost rectangular, median apophysis laterally flattened (fig. 438).

FEMALE: Total length 6.8. Coloration as in male except abdominal venter with few orange sclerotizations. Leg spination: tibiae: III v2-2-2, r0-1-1; IV p0-1-1. Epigynal atrium and midpiece anteriorly advanced, situated near hood (fig. 440); all ducts greatly elongated (fig. 441).

Other Material Examined: Queensland: Malaan State Forest, $17^{\circ} 35^{\prime} \mathrm{S}, 145^{\circ} 35^{\prime} \mathrm{E}$, Apr. 20-24, 1978, rainforest (R. Raven, V. Davies, QMB S26515), 10; Massey Creek, W Millaa Millaa, $17^{\circ} 36^{\prime} \mathrm{S}, 145^{\circ} 33^{\prime} \mathrm{E}$, Dec. 1, 1993-Feb. 25, 1994, rainforest pitfall, elev. 1000 m (J. Hasenpusch, QMB S22932), 1 ㅇ (penultimate but with fully developed epigynum); Massey Creek, $17^{\circ} 37^{\prime} \mathrm{S}, 145^{\circ} 34^{\prime} \mathrm{E}$, July 4-Aug. 2, 1995, pitfall, elev. 1000 m (P. Zborowski, QMB), 1 t ; Mount Bartle-Frere, NW Peak summit, $17^{\circ} 23^{\prime} \mathrm{S}, 145^{\circ} 48^{\prime} \mathrm{E}$, Oct. 7, 1980, rainforest, elev. $1440 \mathrm{~m}$ (G., S. Monteith, QMB S34345), 1 \% Mount Edith, Lamb Range, $17^{\circ} 06^{\prime} \mathrm{S}, 145^{\circ} 37^{\prime} \mathrm{E}$, Oct. 12, 1982, elev. $1050 \mathrm{~m}$ (G. Monteith, D. Yeates, Thompson, QMB S28004), 1 \%, May 31June 30, 1995, pitfall, elev. $1050 \mathrm{~m}$ (P. Zborowski, QMB), $1 \%$; Mount Fisher, $17^{\circ} 33^{\prime} \mathrm{S}$, $145^{\circ} 32^{\prime}$ E, Nov. 30, 1995-Jan. 3, 1996, pitfall, elev. $1150 \mathrm{~m}$ (L. Umback, QMB), 1ठ; Mount Fisher, $7 \mathrm{~km}$ SW Millaa Millaa, $17^{\circ} 34^{\prime} \mathrm{S}, 145^{\circ} 34^{\prime} \mathrm{E}$, Apr. 27-29, 1982, rainforest, elev. 1050-1100 m (G. Monteith, D. Yeates, Cook, QMB S26510), 1 \%.

Distribution: Known only from northern Queensland (map 47). 

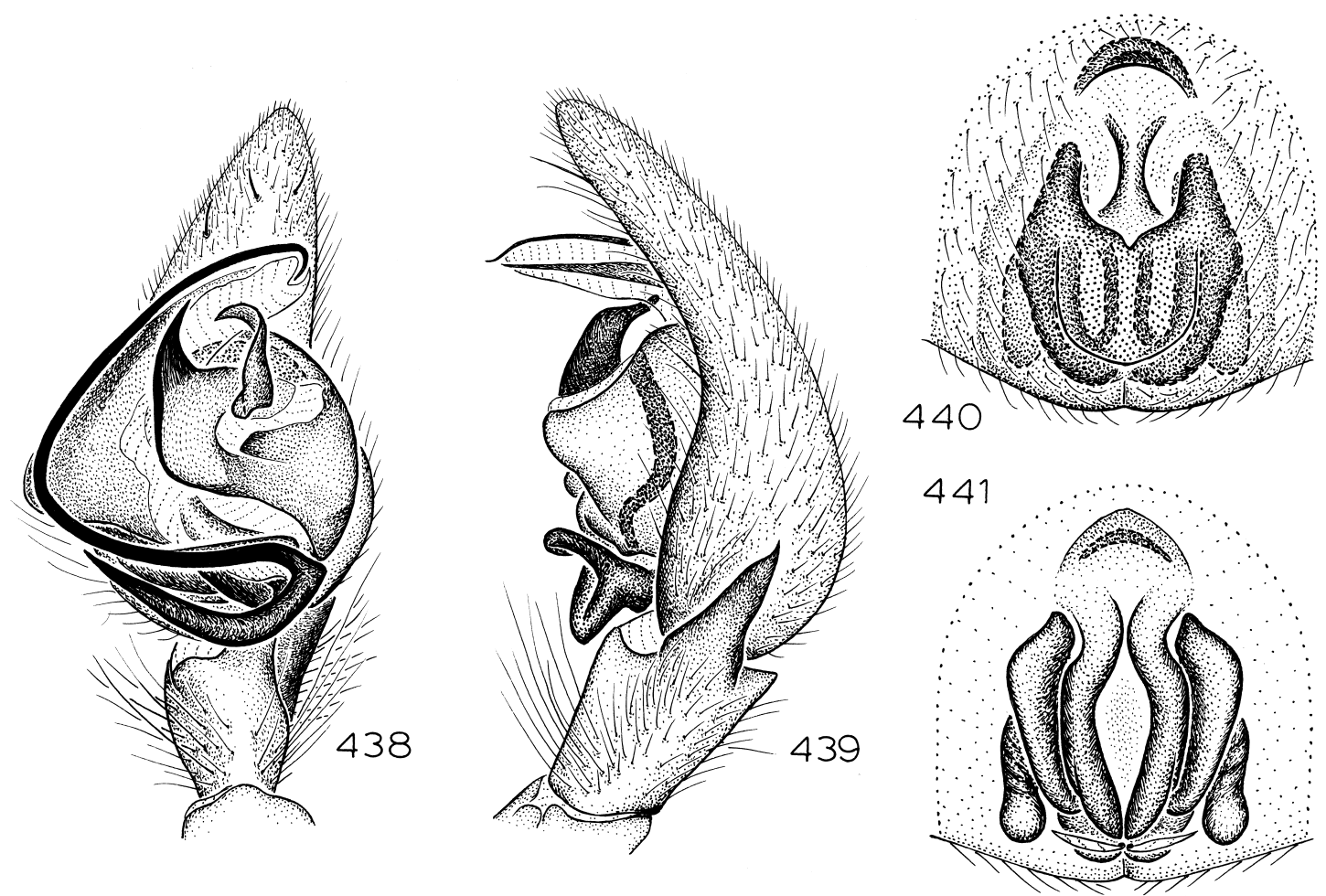

Figs. 438-441. Centrothele fisher, new species. 438. Left male palp, ventral view. 439. Same, retrolateral view. 440. Epigynum, ventral view. 441. Same, dorsal view.

\section{Centrothele spurgeon, new species} Figures 442-445; Map 48

TYPE: Female holotype taken in eucalypts in Mount Spurgeon area, $16^{\circ} 26^{\prime} \mathrm{S}, 145^{\circ} 12^{\prime} \mathrm{E}$, Queensland (Apr. 19, 1994; J. Thompson, M. Moulds, F. MacKillop), deposited in AMS (KS45911).

ETYMOLOGY: The specific name is a noun in apposition taken from the type locality.

DiAGNOSIS: Males and females have not been taken together, but males have a short, sinuous retrolateral tibial apophysis, stubby dorsal tibial apophysis, and a relatively short embolus with a thickened base (figs. 442, 443); females resemble those of $C$. fisher but have a wider epigynal midpiece (fig. 444).

MALE: Total length 10.3. Abdominal dorsum gray, with pair of narrow transverse white stripes at about half its length, stripes extending around sides, venter pale white with four longitudinal rows of small orange sclerotizations; anterior coxae, trochanters, and femoral bases darkened, almost red. Leg spination: tibiae: III r0-1-1; IV p0-1-1, r0-12. Retrolateral tibial apophysis short, sinuous, tibia also with stubby dorsal apophysis (fig. 443); embolus short, basally thickened, originating near midline of bulb, embolar apophysis very slightly invaginated on proximal edge, tegular apophysis highest medially, median apophysis with hooked tip (fig. 442).

Female: Total length 11.0 (penultimate but with fully formed epigynum). Abdominal dorsum with white stripes fused medially to form single stripe, extending around sides; coloration otherwise difficult to discern because of unshed cuticle. Leg spination: tibiae: III r0-1-1; IV p0-1-1; metatarsi III, IV p0-1-0, r0-1-0. Epigynal midpiece relatively long, wide, bulging at about half its length (fig. 444); lateral epigynal ducts with edges folded over (fig. 445).

OTHER MATERIAL EXAMINED: Queensland: Mount Edith, Lamb Range, $17^{\circ} 06^{\prime} \mathrm{S}$, $145^{\circ} 37^{\prime}$ E, Oct. 11, 1982, elev. 1140 m (G. 

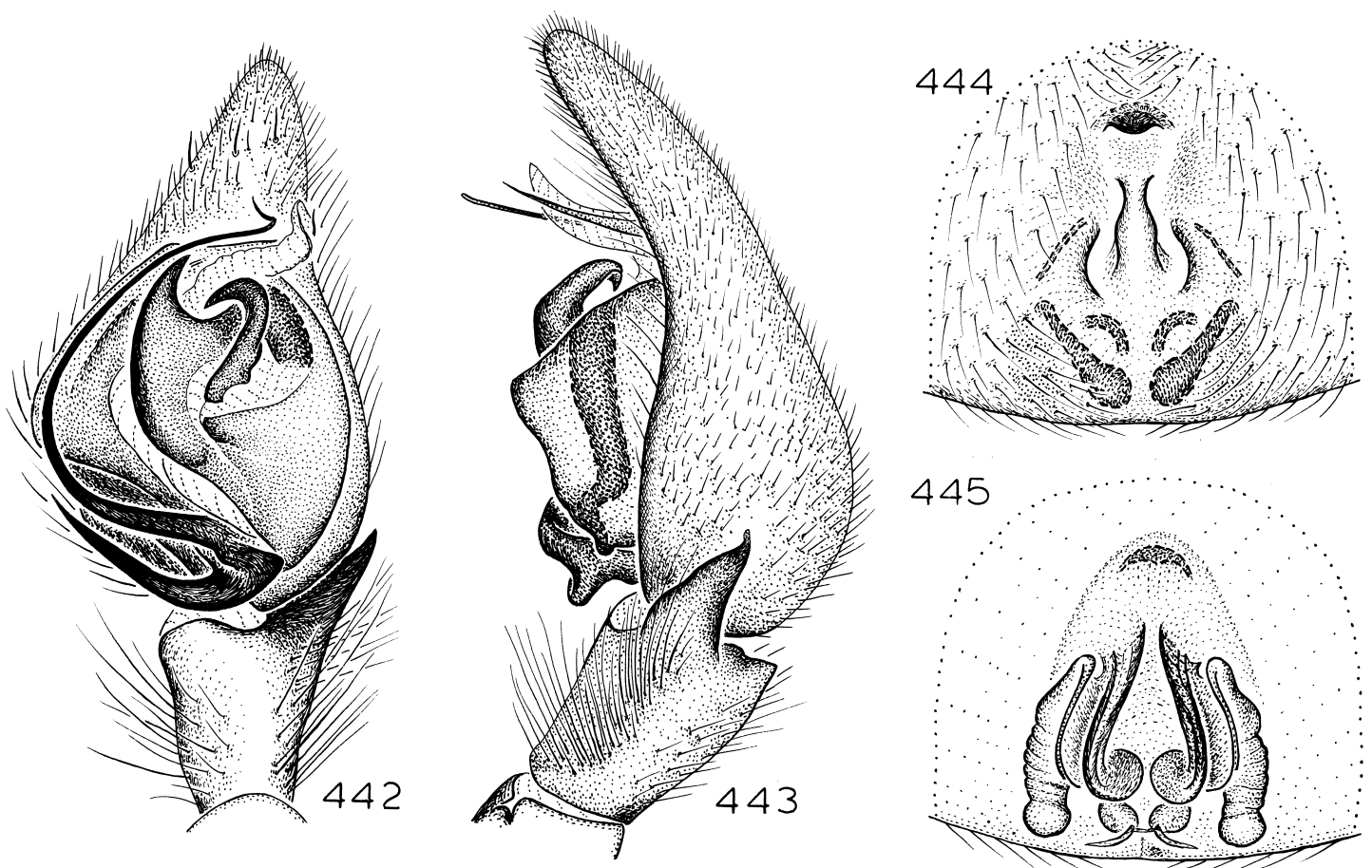

Figs. 442-445. Centrothele spurgeon, new species. 442. Left male palp, ventral view. 443. Same, retrolateral view. 444. Epigynum, ventral view. 445. Same, dorsal view.

Monteith, D. Yeates, Thompson, QMB S26479), 1 ô.

DisTRIBUTION: Known only from northern Queensland (map 48).

\section{Centrothele kuranda, new species}

Figures 446-449; Map 50

TYPES: Female holotype and male allotype taken in rainforest on Black Mountain Road, Kuranda, $16^{\circ} 49^{\prime} \mathrm{S}, 145^{\circ} 38^{\prime} \mathrm{E}$, Queensland (July 1976; P. Filewood), deposited in QMB (S26505).

ETYMOLOGY: The specific name is a noun in apposition taken from the type locality.

DIAGNOSIS: Males have a scarcely evident dorsal tibial apophysis (fig. 447) and a long embolus that is narrow even at its base (fig. 446); females have a long, parallel-sided epigynal atrium and long, narrow epigynal midpiece (fig. 448).

MaLE: Total length 10.4. Abdominal dorsum gray, with pair of narrow transverse white stripes at about half its length, stripes extending around sides, venter gray with scattered small orange sclerotizations; anterior coxae, trochanters, and femoral bases darkened, almost red. Leg spination: tibiae: III p0-1-1, v1p-2-2, r0-1-1; IV v1p-2-2, r00-1; metatarsus IV r0-1-0. Retrolateral tibial apophysis moderate in length, expanded at base on both dorsal and ventral sides, dorsal apophysis scarcely evident (fig. 447); embolus originating at retrolateral side of bulb, narrow throughout its length; embolar apophysis almost straight; tegular apophysis bulging; median apophysis almost triangular (fig. 446).

FEMALE: Total length 11.0. Coloration as in male. Leg spination: tibiae: III r0-1-1; IV p0-1-1; metatarsus IV r0-1-0. Epigynal hood wide, semicircular; epigynal atrium long, with parallel sides, midpiece long, narrow (fig. 448); epigynal ducts enlarged, dorsal most ducts making almost full circle (fig. 449).

Other Material Examined: Queensland: Davies Creek, Copperfield Road, near Cairns, $16^{\circ} 55^{\prime} \mathrm{S}, 145^{\circ} 32^{\prime} \mathrm{E}$, May 13,1984 , 

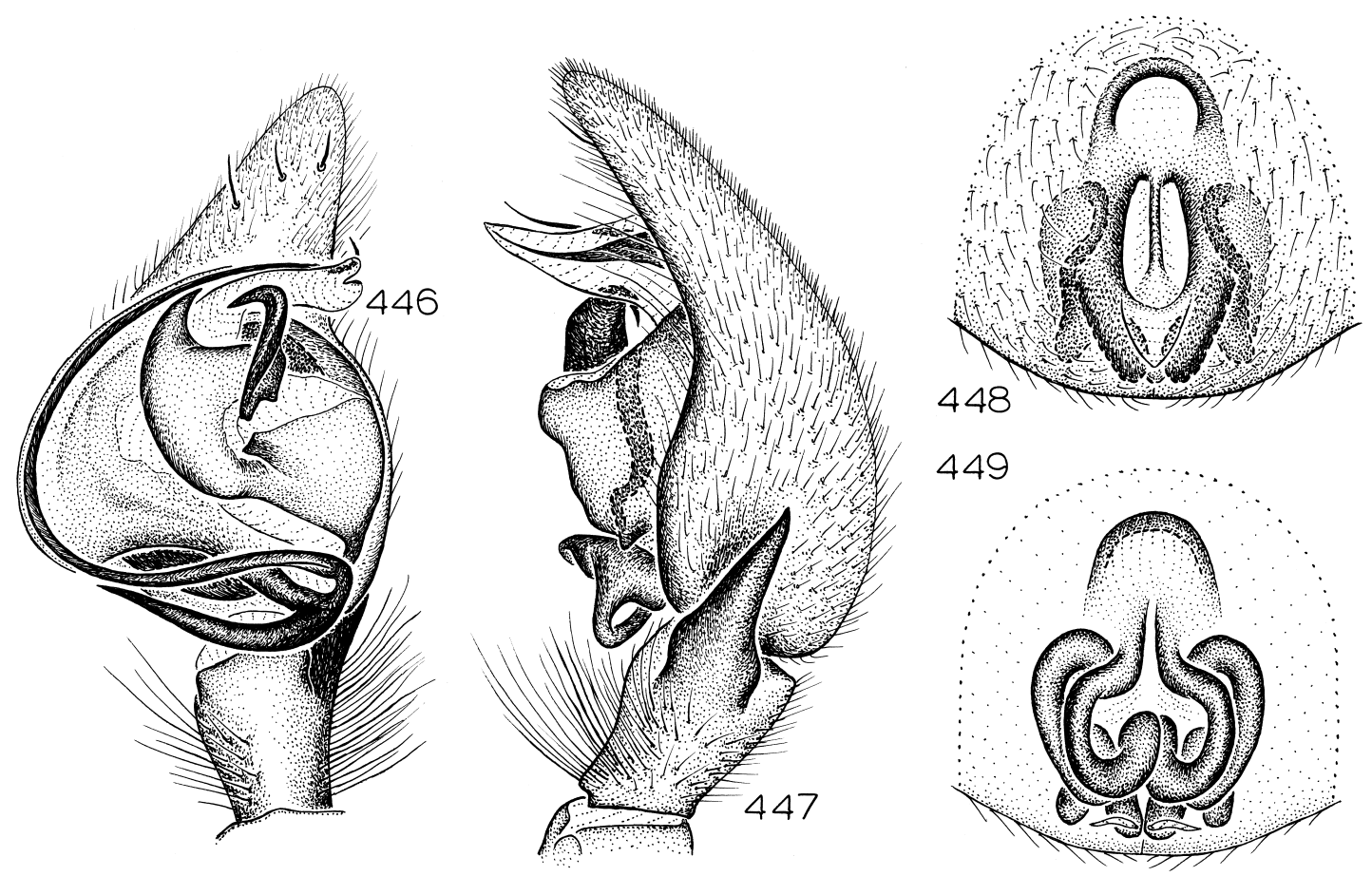

Figs. 446-449. Centrothele kuranda, new species. 446. Left male palp, ventral view. 447. Same, retrolateral view. 448. Epigynum, ventral view. 449. Same, dorsal view.

under rocks in webbed burrow (A. Dennis, QMB), 1 \% ; Kirrama Forest, $18^{\circ} 09^{\prime} \mathrm{S}$, $145^{\circ} 37^{\prime} \mathrm{E}$, May 1, 1981, rainforest (B. Ramsay, QMB S34346), 1 के.

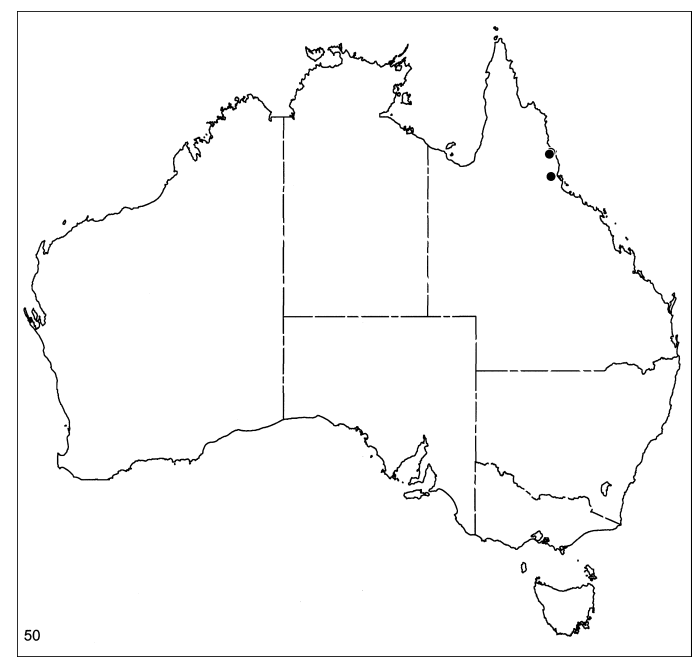

Map 50. Records of Centrothele kuranda, new species.
DistRiBUTiOn: Known only from northern Queensland (map 50).

\section{Centrothele mossman, new species} Figures 452, 453; Map 51

TYPE: Female holotype taken at an elevation of $1000-1180 \mathrm{~m}$ in the Devils Thumb area, $10 \mathrm{~km} \mathrm{NW}$ Mossman, Queensland, $16^{\circ} 23^{\prime} \mathrm{S}, 145^{\circ} 17^{\prime} \mathrm{E}$ (Oct. 9-10, 1982; G. Monteith, D. Yeates, Thompson), deposited in QMB (S26489).

ETYMOLOGY: The specific name is a noun in apposition taken from the type locality.

DiAGNOSIS: Females can easily be recognized by the shape of the epigynal atrium (fig. 452) and the widely separated dorsalmost epigynal ducts (fig. 453).

MALE: Unknown.

Female: Total length 10.4. Abdominal dorsum gray, with pair of transverse white stripes at about half its length, sides with interrupted narrow lines of white spots, venter pale white, with four sparse longitudinal rows of small orange sclerotizations; legs un- 

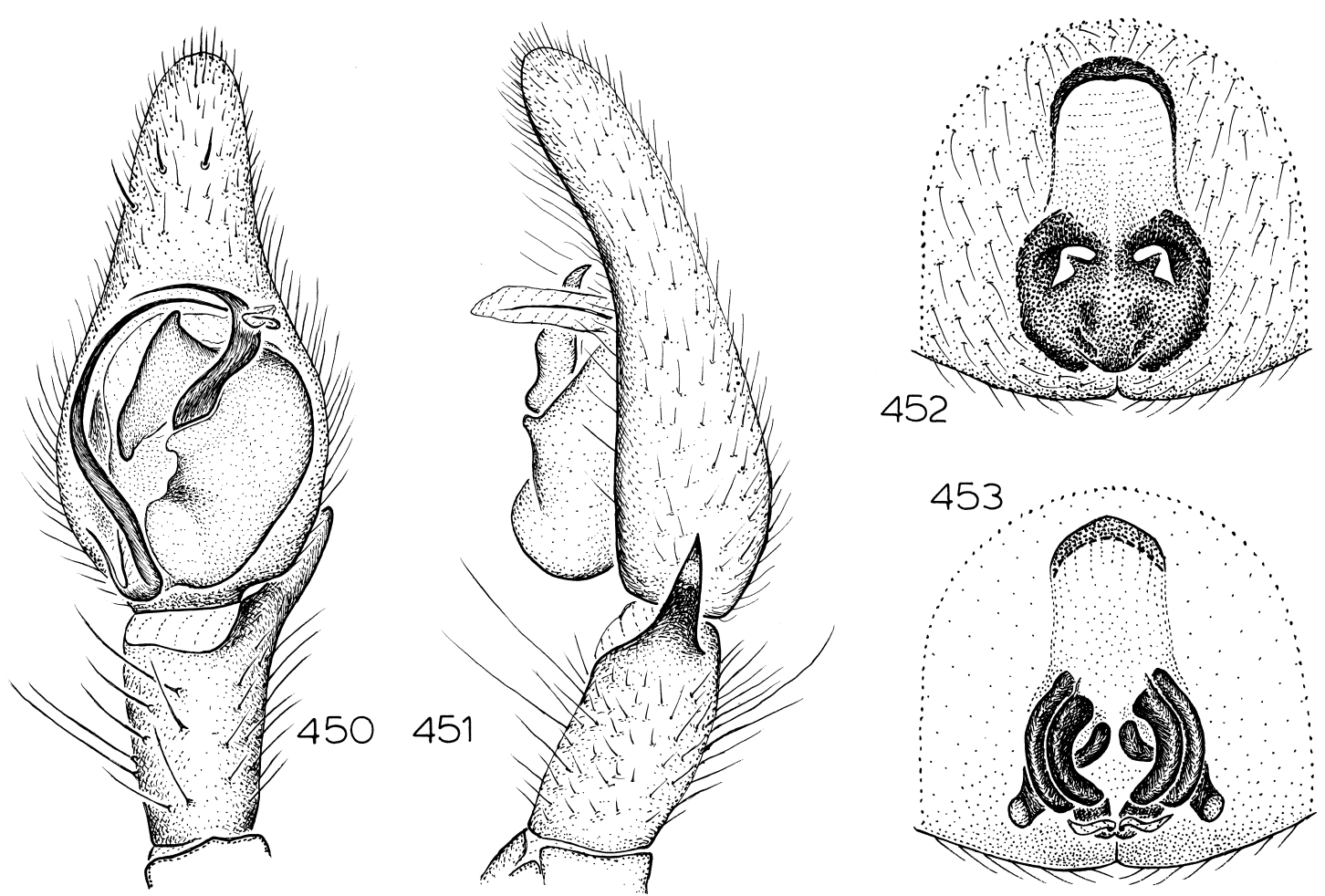

Figs. 450-453. 450, 451. Centrocalia chazeaui, new species. 452, 453. Centrothele mossman, new species. 450. Left male palp, ventral view. 451. Same, retrolateral view. 452. Left male palp, ventral view. 453. Same, retrolateral view.

marked. Leg spination: tibiae: III p0-1-1, v22-2, r0-1-1; IV p0-1-1; metatarsi III, IV r01-0. Epigynal atrium reduced to sinuous lateral depressions (fig. 452); dorsal most epigynal ducts widely separated (fig. 453).

OTHER MATERIAL EXAMINED: None.

DisTRIBUTION: Known only from northeastern Queensland (map 51).

\section{Centrina, new genus}

Type SPECIES: Centrina keira, new species. ETYMOLOGY: The generic name is an arbitrary combination of letters, considered feminine in gender.

Diagnosis: Males resemble those of Centrothele and Centrocalia in having an apophysis at the base of the embolus, but have the apophysis more distally directed and the embolus wider than in those of Centrothele. Females lack the parallel median and lateral epigynal ducts of Centrothele. Both sexes, unlike those of Centrocalia, do not have the posterior margin of the sternum fused to the pedicel.

DESCRIPTION: Medium to large spiders, total length of males 5.4-8.5, of females 4.912.8. Carapace brown, pars cephalica lightest, pars thoracica with rows of dark maculations radiating from thoracic groove, coated with long, dark setae; thoracic groove long, longitudinal. Eight eyes in two rows, anterior medians largest, circular, dark, laterals subequal in size, oval, light, posterior medians smallest, irregularly oval, flattened, light; from above, anterior eye row slightly recurved, posterior row slightly procurved, from front, anterior row slightly procurved, posterior row strongly procurved; anterior medians separated by more than their radius, closer to anterior laterals; posterior medians separated by more than their diameter, farther from posterior laterals; anterior and posterior laterals separated by less than their diameter; median ocular quadrangle wider in front than 
in back, wider in front than long. Chelicerae, sternum, and mouthparts brown; chilum wide, triangular, setose, accompanied by second, dumbbell-shaped, posterior chilum (extremely narrow sclerite separating bases of chelicerae posteriorly); chelicerae with distinct, orange lateral boss, anterior surface with depressed, relatively unsclerotized oval area near promargin; promargin with two rows of long setae originating in line along base of fang, seta closest to fang bent near base at almost $90^{\circ}$ angle, extending behind other promarginal setae to near midline; promargin with three teeth, median one largest, proximal one smallest; retromargin with two large, widely separated teeth. Labium elongate, base narrowed at about one-third of labial length, posterior margin truncate, anterior margin medially invaginated, surface not depressed medially. Endites obliquely depressed, with sharply demarcated, deep, wide groove along margin near labium, groove wider anteriorly than posteriorly; serrula long, with single row of teeth. Sternum not elevated, with flat, rebordered lateral margins, not expanded anteriorly, with triangular extensions to and between coxae; surface not tuberculate, with slight elevations opposite, and depressions between, coxae. One epimeric sclerite on each side, above each coxa, reaching sternal triangles only by narrow

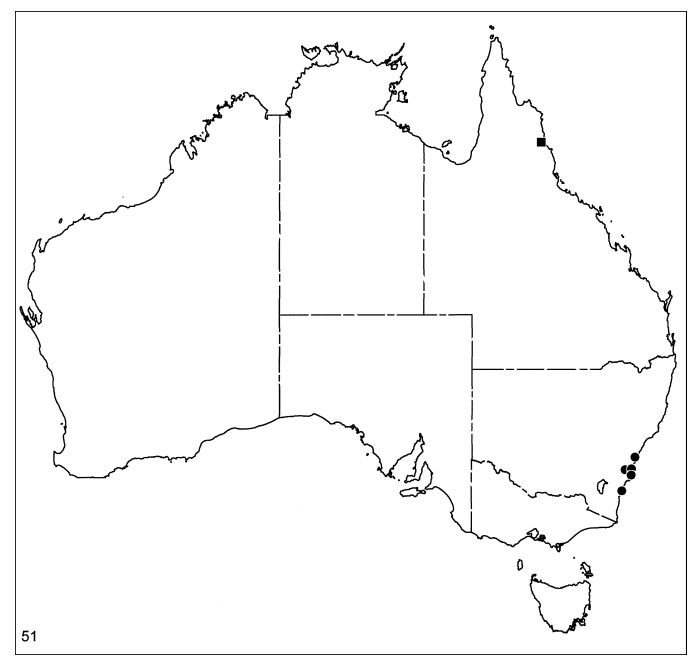

Map 51. Records of Centrothele mossman, new species (square) and Centrina keira, new species (circles). strips of weakly sclerotized cuticle (strongest strip between coxae II and III), not fused to carapace. Pedicel consisting of small, diamond-shaped sclerite bearing short dorsal and ventral triangular ledges; anteroventral edge of sclerite reaching posterior tip of sternum.

Anterior edge of abdomen of male with complete sclerotic ring formed by strong epigastric scutum plus weaker dorsal abdominal scutum covering entire front edge of abdomen, reaching to almost half of abdominal length, females with dorsal scutum represented only by small semicircular plate above pedicel, restricted to lower half of anterior surface of abdomen; cuticle with long, erect and recumbent setae; epigastric scutum accompanied posterolaterally by pair of oval, deeply invaginated sclerites bearing clearly elevated anterior rim; sclerites separated by membranous lobe, anterior rim of sclerites fitting under epigastric scutum; colulus represented only by setae on slight sclerotization; tiny transverse sclerite, well removed from spinnerets, marking position of small posterior spiracle. Anterior lateral spinnerets tubular, separated by more than their diameter, cuticle representing distal, second spinneret segment restricted to semicircle surrounding major ampullate gland spigots (piriform gland spigots surrounded only by soft cuticle); posterior median spinnerets of males large, tubular, of females with anteriorly expanded tips, bases occupied by three enormously widened cylindrical gland spigots; posterior lateral spinnerets two-segmented.

Leg spination reduced only on anterior legs; typical leg spination pattern (only surfaces with spines listed): femora I, II d1-0-0, p0-0-1; III d1-0-0, p0-0-1, r0-0-1, IV d1-0$0, \mathrm{r} 0-0-1$; tibiae III, IV p1-1-1, v2-2-2, r1-11; metatarsi III, IV p1-1-1, v2-2-1p, r1-1-1. Most leg surfaces with both short and long setae; males with all coxae and trochanters dorsally tuberculate; anterior coxae with slightly protuberant posterolateral corners; trochanters slightly notched; anterior metatarsi and tarsi with divided scopulae, composed of laterally directed setae; posterior metatarsi with thick, dark, distal preening brushes; tarsi III, IV with entire scopulae; tarsi with two dentate claws, claw tufts composed of lateral pads of closely appressed se- 
tae; trichobothria in two rows on metatarsi and tarsi. Female palpal femur, patella, tibia, and tarsus with spines; female palpal tarsus with long, basally dentate claw.

Male palp with short, strong retrolateral tibial apophysis; cymbial surface excavated opposite tibial apophysis, produced into distinct lobe on ventral side of tibial apophysis; tegulum expanded ventrally, bearing large median apophysis opposing large terminal apophysis; embolus originating prolaterally, short, wide, accompanied distally by long, partially membranous conductor; embolar base bearing distinct, distally-directed apophysis. Epigynum with anterior hood and deep atrium; spermathecae accompanied laterally by two large, translucent ducts.

\section{Key to Species of CENTRINA}

1. Males (those of $C$. whian unknown) ... 2

- Females .................... 11

2. Median apophysis relatively small (figs. 486, 490) .................. 3

- Median apophysis relatively large (as in figs. 454, 458) . . . . . . . . . . . . 4

3. Retrolateral tibial apophysis greatly widened at base (fig. 487) ... . . . . . . . macedon

- Retrolateral tibial apophysis not greatly widened at base (fig. 491) .......... . lewis

4. Embolar apophysis relatively short, wide (figs. 466, 482, 494) ..........5

- Embolar apophysis relatively long, narrow (figs. 454, 458, 462, 474, 478) .....7

5. Median apophysis with subdistal projection (fig. 494) ................. enfield

- Median apophysis without subdistal projection (figs. 466, 482) . . . . . . . . . . 6

6. Median apophysis relatively wide (fig. 466) ................. blundells

- Median apophysis relatively narrow (fig. 482) ............... sherbrook

7. Tip of median apophysis greatly narrowed, elongated (fig. 478) . . . . . . . . . bondi

- Tip of median apophysis not narrowed, elongated ................ 8

8. Median apophysis and embolar apophysis relatively short (fig. 474) ........ sawpit

- Median apophysis and embolar apophysis longer ................. 9

9. Retrolateral tibial apophysis incised at tip (figs. 455, 463) .............. 10

- Retrolateral tibial apophysis not incised at tip (fig. 459) ............... dorrigo

10. Median apophysis relatively narrow (fig. 454) ..................... keira
- Median apophysis relatively wide (fig. 462) .................. kota

11. Epigynum with rounded atrium (figs. 472,

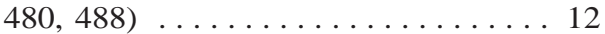

- Epigynal atrium otherwise ... . . . . . 14

12. Epigynal ducts relatively wide (fig. 488) ... ................ macedon

- Epigynal ducts relatively narrow (figs. 473, 481) . . . . . . . . . . . 13

13. Epigynal ducts situated medially (fig. 473) .. $\ldots \ldots \ldots \ldots \ldots$ whian

- Epigynal ducts situated laterally (fig. 481) .. .................. bondi

14. Epigynal atrium a broad triangle (figs. 460, $468,476) \ldots \ldots \ldots \ldots \ldots \ldots$

- Epigynal atrium otherwise ... . . . . 17

15. Epigynal midpiece relatively narrow (fig. 460) . . . . . . . . . . dorrigo

- Epigynal midpiece relatively wide (figs. 468,

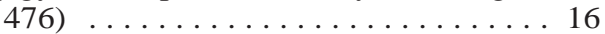

16. Epigynal midpiece relatively long (fig. 468)

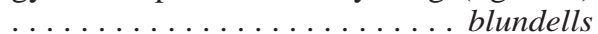

- Epigynal midpiece relatively short (fig. 476) $\ldots \ldots \ldots \ldots \ldots \ldots$ sawpit

17. Epigynum relatively short, wide (figs. 456,

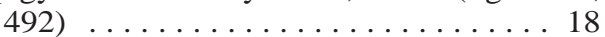

- Epigynum relatively long, narrow (figs. 464, 484, 496) . . . . . . . . . . . 19

18. Epigynal hood relatively narrow (fig. 456) ... .................keira

- Epigynal hood relatively wide (fig. 492) ... .................... lewis

19. Epigynum with anterior hood (figs. 464, 484)

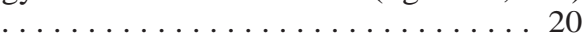

- Epigynum without anterior hood (fig. 496) . ................. enfield

20. Epigynum an inverted triangle (fig. 464) ... $\ldots \ldots \ldots \ldots \ldots \ldots$ kota

- Epigynum with hood far removed from remainder of structure (fig. 484)

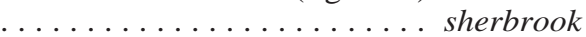

\section{Centrina keira, new species}

Figures 454-457; Map 51

TYPES: Female holotype and male allotype taken in a pitfall trap in litter on Mount Keira Fauna Reserve Scout Camp, 34 $24^{\prime}$ S, $150^{\circ} 51^{\prime} \mathrm{E}$, New South Wales (Dec. 20, 1978Jan. 17, 1979; C. Horseman), deposited in AMS (KS2370).

ETYMOLOGY: The specific name is a noun in apposition taken from the type locality.

DIAGNOSIS: Males can be recognized by the bent retrolateral tibial apophysis (fig. 455) and the long apical projection on the tegular apophysis (fig. 454), females by the 

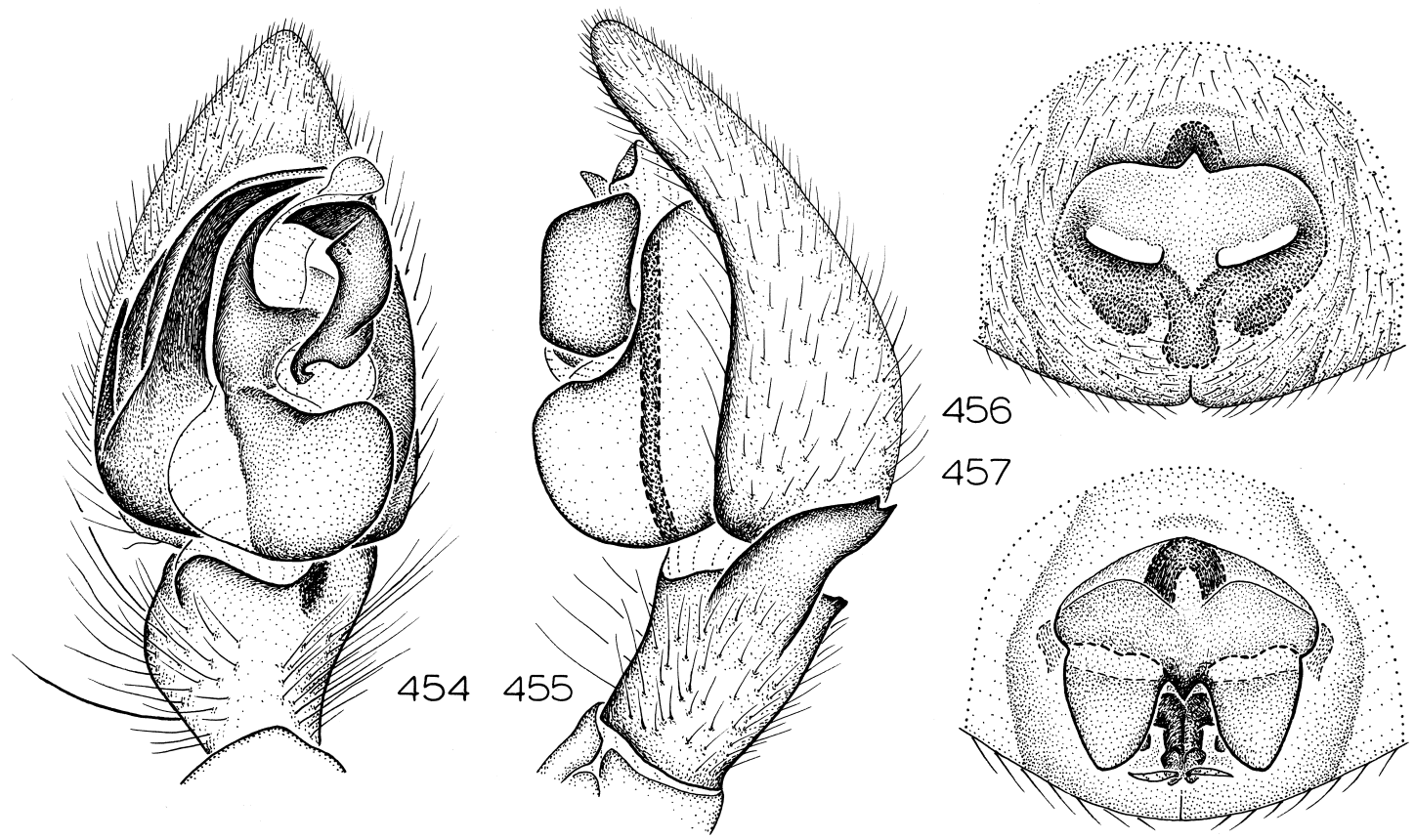

Figs. 454-457. Centrina keira, new species. 454. Left male palp, ventral view. 455. Same, retrolateral view. 456. Epigynum, ventral view. 457. Same, dorsal view.

deeply excavated lateral pockets of the epigynal atrium (fig. 456).

MALE: Total length 7.7. Abdominal dorsum gray, with narrow transverse white stripe at about half its length, sides mottled with gray, venter pale white, with two paramedian rows of small orange sclerites; legs unmarked. Leg spination: tibia III r0-1-1; metatarsus III p0-1-1. Retrolateral tibial apophysis bent, bifid at tip (fig. 455); embolar apophysis long, straight; tegular apophysis with narrow apical projection; median apophysis deeply excavated prolaterally (fig. 454).

FEMALE: Total length 10.3. Coloration as in male. Leg spination: femur III d1-1-0, p01-1. Epigynal atrium wide, almost rectangular, with posterolateral openings (fig. 456); median ducts narrow, anteriorly bent (fig. 457).

Other Material Examined: New South Wales: Beecroft, $33^{\circ} 45^{\prime} \mathrm{S}, 151^{\circ} 04^{\prime} \mathrm{E}$, Aug. 31, 1992 (J. Noble, AMS KS55329), 10 $0^{\text {; }}$ Forest Drive, Kiola State Forest, 16-18 km $\mathrm{N}$ Bateman's Bay, $35^{\circ} 37^{\prime} \mathrm{S}, 150^{\circ} 16^{\prime} \mathrm{E}$, Oct. 5-Nov. 2, 1978, litter pitfall (C. Horseman,
AMS KS2020), 10 , Nov. 2-30, 1978, litter pitfall (C. Horseman, AMS KS2114), 10 , Nov. 30, 1978-Jan. 4, 1979, litter pitfall (C. Horseman, AMS KS2297), 1 , June 14Sept. 12, 1979, litter pitfall (C. Horseman, AMS KS3839), 10, Sept. 7-Oct. 4, 1979, pitfall (C. Horseman, AMS KS3931), 10; The Gibb, Mittagong, $34^{\circ} 27^{\prime} \mathrm{S}, 150^{\circ} 27^{\prime} \mathrm{E}$, Oct. 24, 1987, under logs and rocks (G. Hunt, AMS KS18104), 10; Minnamurra Falls, 34 $38^{\prime} \mathrm{S}, 150^{\circ} 51^{\prime} \mathrm{E}$, Oct. 9-11, 1987, pitfall (AMS KS29709), 20, 1\%; Mount Keira Fauna Reserve Scout Camp, $34^{\circ} 24^{\prime}$ S, $150^{\circ} 51^{\prime}$ E, Dec. 20, 1978-Jan. 17, 1979, litter pitfall (C. Horseman, AMS KS2414), 10, Feb. 14-Mar. 14, 1979, litter pitfall (C. Horseman, AMS KS2792), $1 \%$; Scalloway, Willowvale, near Gerringong, $34^{\circ} 45^{\prime} \mathrm{S}$, $150^{\circ} 50^{\prime} \mathrm{E}$, Nov.-Dec., 1979, litter pitfall (G. Wishart, AMS KS4306), 10 , 5 ㅇ, Oct. 7Nov. 5, 1986 (G. Wishart, AMS KS17404, 17405, 17423), 3 o, Dec. 5, 1986, in pool (G. Wishart, AMS KS31716), 1 ㅇ․

Distribution: Coastal New South Wales (map. 51). 

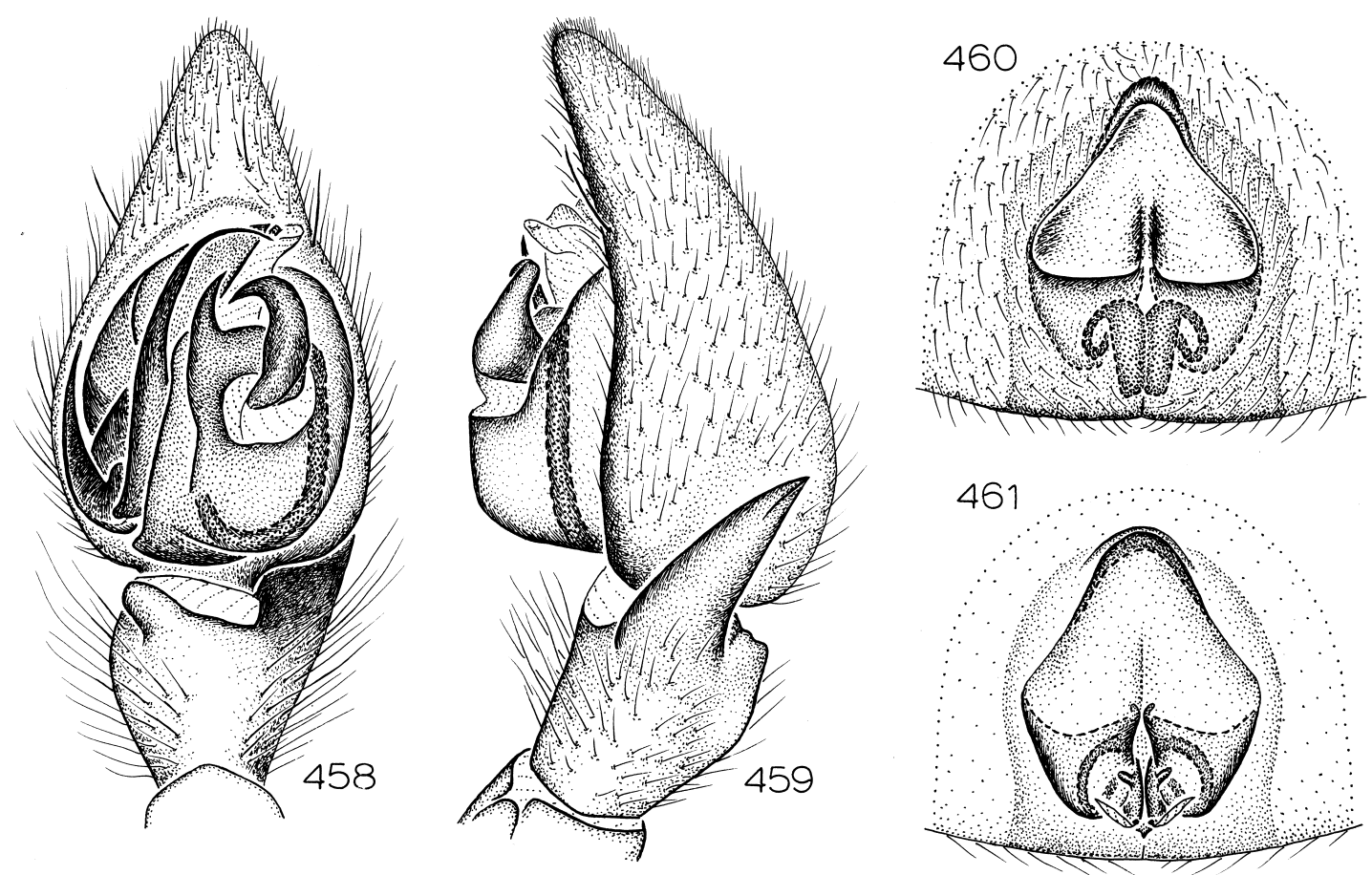

Figs. 458-461. Centrina dorrigo, new species. 458. Left male palp, ventral view. 459. Same, retrolateral view. 460. Epigynum, ventral view. 461. Same, dorsal view.

Centrina dorrigo, new species

Figures 458-461; Map 52

TYPE: Male holotype taken in pitfall trap in rainforest at an elevation of $800 \mathrm{~m}$ at "The Glade," Dorrigo, $30^{\circ} 24^{\prime} \mathrm{S}, 152^{\circ} 44^{\prime} \mathrm{E}$, New South Wales (Mar. 22-Nov. 12, 1980; G., S. Monteith), deposited in QMB (S26845).

ETYMOLOGY: The specific name is a noun in apposition taken from the type locality.

DiAGNOSIS: Males and females have not been taken together, but are paired here because of their respective resemblances to $C$. keira. Males differ from those $C$. keira in having a straight retrolateral tibial apophysis (fig. 459) and larger embolar apophysis (fig. 458), females in having a more triangular epigynum (fig. 460).

MALE: Total length 6.7. Coloration as in C. keira except abdominal venter with only about three small sclerites in each paramedian row. Leg spination: femora: II p0-1-1; III p0-1-1, r0-1-1; IV r0-1-1; tibiae III, IV p0-1-1; metatarsi III, IV p0-1-1, v2-1p-1p, r0-1-1. Retrolateral tibial apophysis not bent,

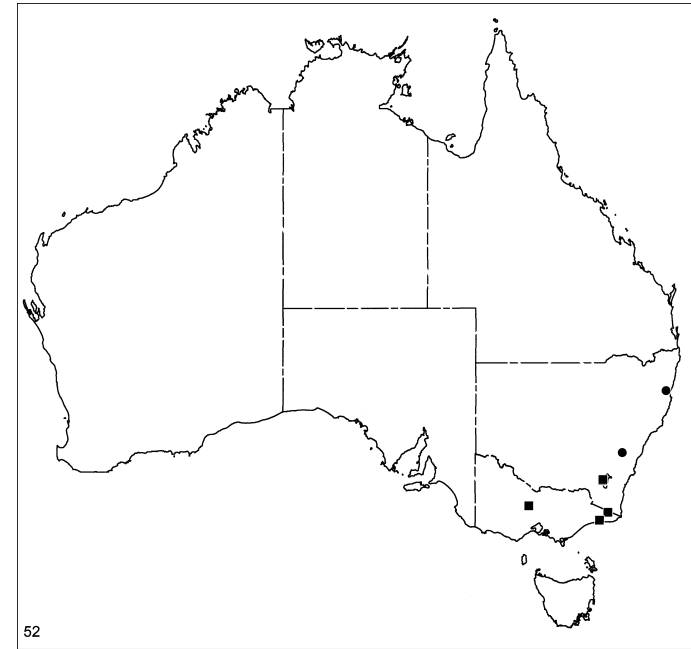

Map 52. Records of Centrina dorrigo, new species (circles) and $C$. bondi, new species (squares). 

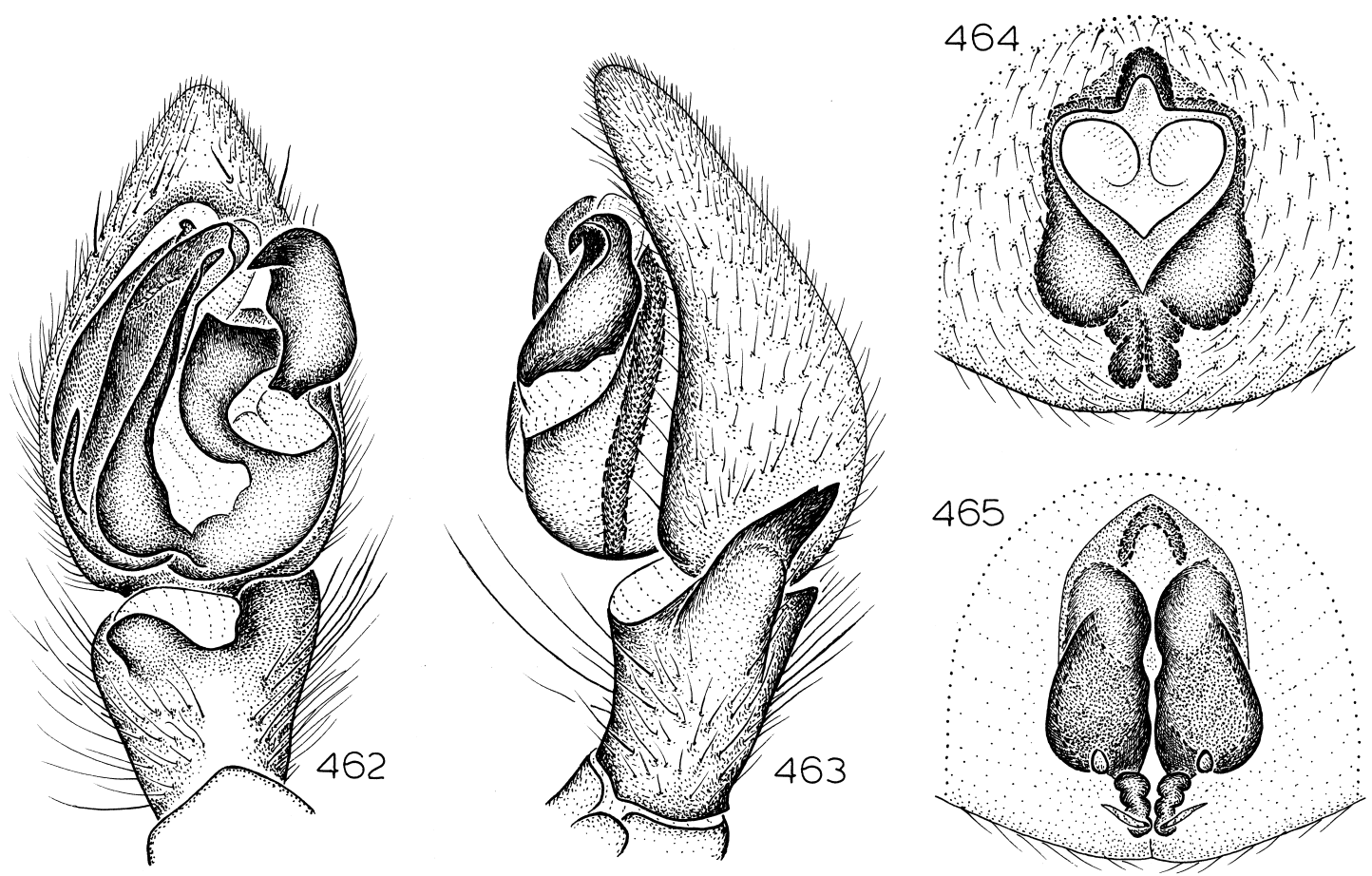

Figs. 462-465. Centrina kota, new species. 462. Left male palp, ventral view. 463. Same, retrolateral view. 464. Epigynum, ventral view. 465. Same, dorsal view.

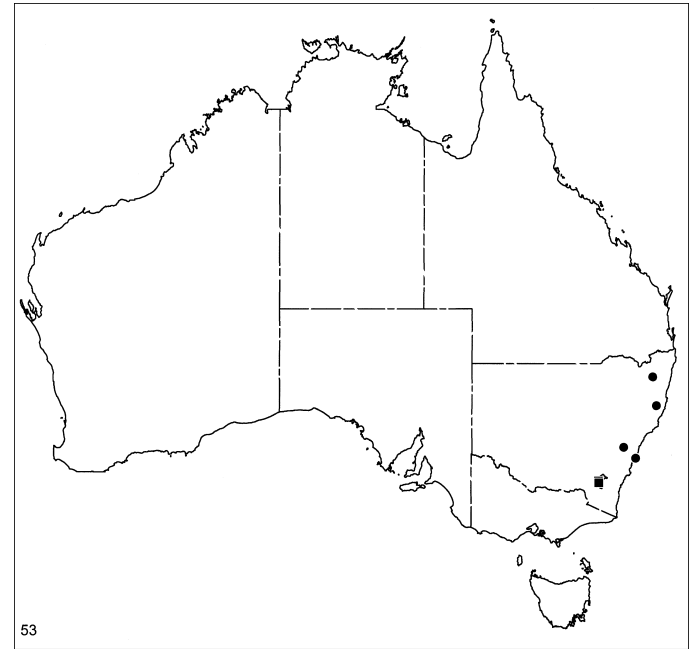

Map 53. Records of Centrina kota, new species (circles) and $C$. blundells, new species (squares). dorsoventrally flattened at tip (fig. 459); embolar apophysis large, protuberant, tegular apophysis with extremely narrow apical projection, median apophysis dorsoventrally flattened (fig. 458).

Female: Total length 10.2. Coloration as in C. keira. Leg spination: femora: I, II p00-0; III, IV r0-0-0; tibiae: III p0-1-1, r0-1-1; IV p0-0-1; metatarsi III, IV p0-1-1, r0-1-1. Epigynum triangular, with deeply excavated posterolateral pockets (fig. 460); median ducts small, dwarfed by translucent extensions to pockets (fig. 461).

Other Material Examined: New South Wales: Terrace Creek, near Jenolan, $33^{\circ} 49^{\prime} \mathrm{S}$, $150^{\circ} 02^{\prime} \mathrm{E}$, Dec. 1979 , under rocks (A. Austin, AMS KS10011), 2 ㅇ․

Distribution: Known only from New South Wales (map 52).

\section{Centrina kota, new species}

Figures 462-465; Map 53

TyPE: Female holotype from Kota, Mount Boss State Forest, $31^{\circ} 12^{\prime} \mathrm{S}, 152^{\circ} 24^{\prime} \mathrm{E}$, New 

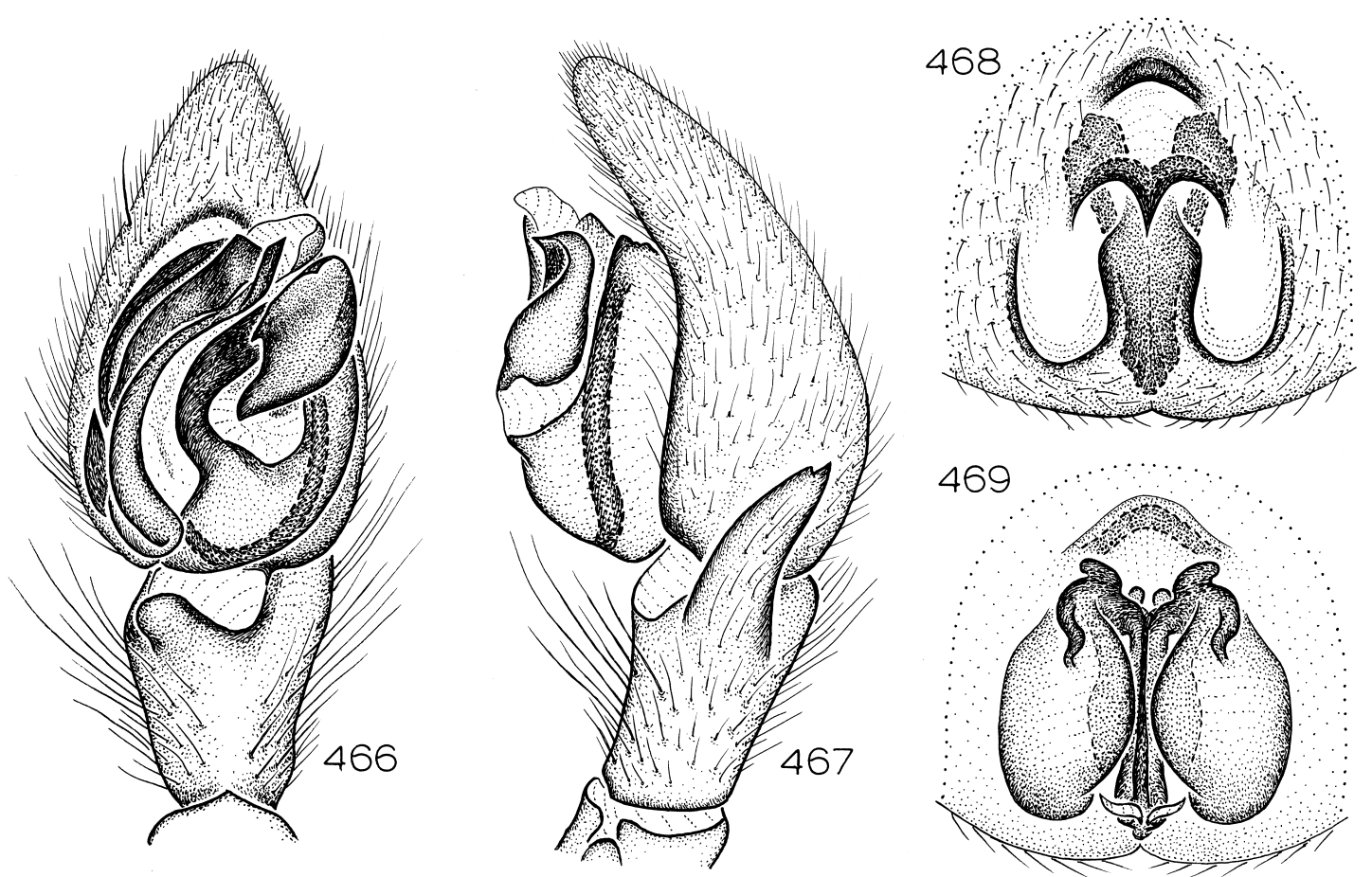

Figs. 466-469. Centrina blundells, new species. 466. Left male palp, ventral view. 467. Same, retrolateral view. 468. Epigynum, ventral view. 469. Same, dorsal view.

South Wales (Oct. 1980), deposited in AMS (KS43568).

ETYMOLOGY: The specific name is a noun in apposition taken from the type locality.

DiAGNOSIS: Males can be recognized by the blunt tip of the tegular apophysis and greatly widened conductor (fig. 462), females by the heart-shaped atrium in a rectangular epigynum (fig. 464).

MALE: Total length 6.8. Coloration as in C. keira. Leg spination: femora: I, II p0-0-0; III p0-0-0, r0-0-0; IV r0-0-0; tibiae: III p01-1, r0-1-1; IV p0-0-0, r0-1-1; metatarsi: III p0-1-1, v2-1p-1p, r0-1-1; IV p0-1-1, r0-1-1. Retrolateral tibial apophysis arched, bifid at tip (fig. 463); embolar apophysis short, straight, tegular apophysis with blunt tip, without distinct apical projection, median apophysis beak-shaped (fig. 462).

FEMALE: Total length 6.1. Coloration as in C. keira. Leg spination: femora: II p0-0-0; III p0-0-0, r0-0-0; IV r0-0-0; tibiae III, IV p0-1-1, v2-1p-2, r0-1-1; metatarsi III, IV p01-1, v2-1p-1p, r0-1-1. Epigynum rectangular, with heart-shaped, anterior situated atrium (fig. 464); median ducts situated posterior of translucent ducts (fig. 465).

Other Material Examined: New South Wales: Bonnie Vale Camp, Bundeena, Royal National Park, $34^{\circ} 06^{\prime} \mathrm{S}, 151^{\circ} 07^{\prime} \mathrm{E}$, Aug. 1113, 1990, open coastal sclerophyll (C. Griswold, T. Meikle, USNM), 29 ; Cathedral of Ferns area, Mount Wilson, $33^{\circ} 30^{\prime} \mathrm{S}$, $150^{\circ} 23^{\prime} \mathrm{E}$, Nov. $15-$ Dec. 12, 1978, litter pitfall (C. Horseman, AMS KS2144), $10^{\top}$; Gibraltar Range, $29^{\circ} 36^{\prime} \mathrm{S}, 152^{\circ} 11^{\prime} \mathrm{E}$, Nov. 10, 1980, open forest (R. Raven, QMB S28258), 2 ㅇ ; Waterfall Picnic Area Trail, Mount Wilson, $33^{\circ} 30^{\prime} \mathrm{S}, 150^{\circ} 23^{\prime} \mathrm{E}$, Nov. 15-Dec. 14, 1979, litter pitfall (C. Horseman, AMS KS4324), 10 .

Distribution: New South Wales (map 53).

Centrina blundells, new species Figures 466-469; Map 53

TYPE: Male holotype taken in pitfall trap at Lees and Blundells Creeks, Brindabella Ranges, $35^{\circ} 22^{\prime} \mathrm{S}, 148^{\circ} 50^{\prime} \mathrm{E}$, Australian Cap- 

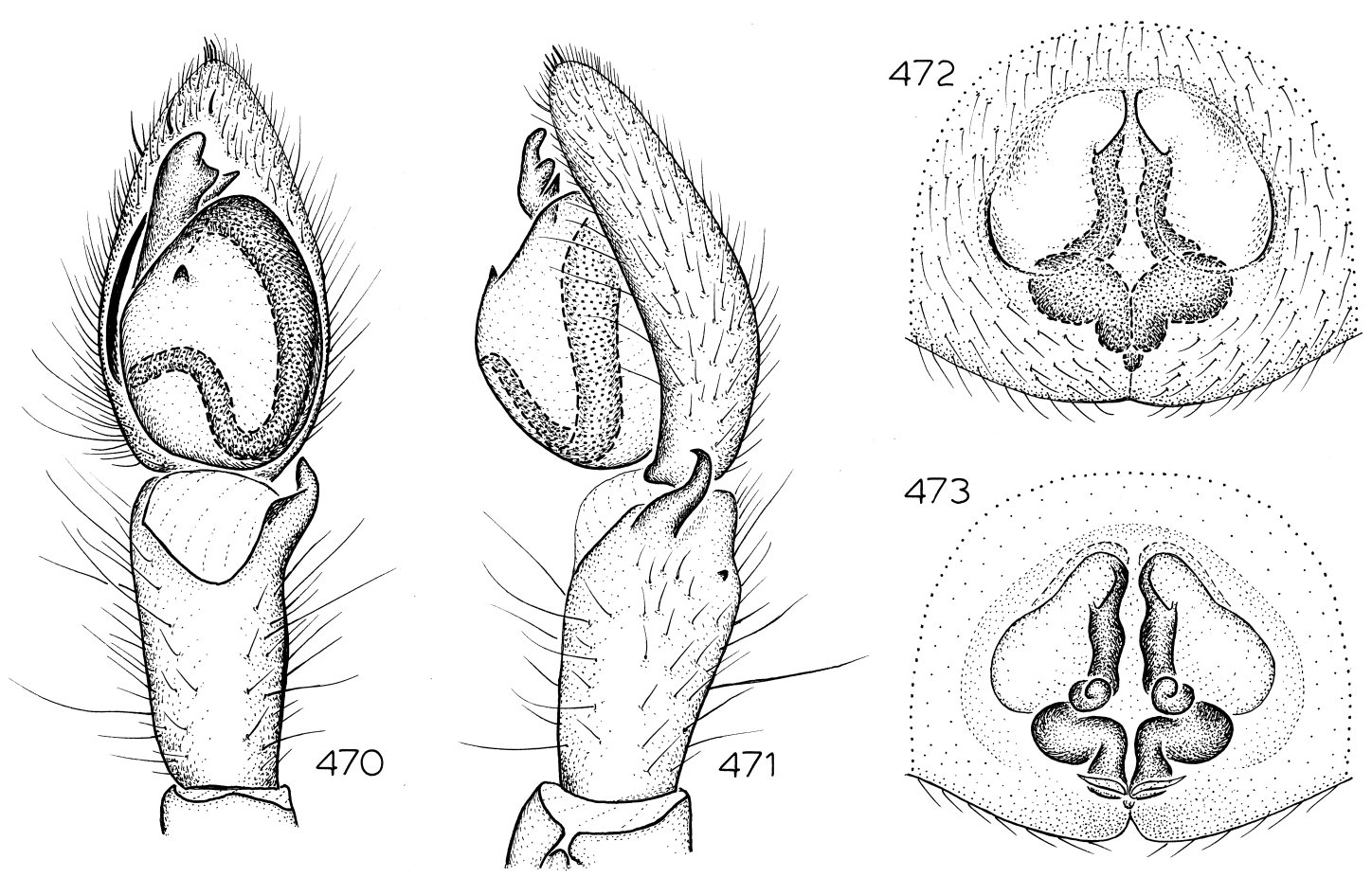

Figs. 470-473. 470, 471. Prionosternum porongurup, new species. 472, 473. Centrina whian, new species. 470. Left male palp, ventral view. 471. Same, retrolateral view. 472. Left male palp, ventral view. 473. Same, retrolateral view.

ital Territory (Oct. 1981; C. Dickman), deposited in QMB.

ETYMOLOGY: The specific name is a noun in apposition taken from the type locality.

DiAGNOSIS: Males resemble those of $C$. kota but can be distinguished by the more sinuous embolus and wider embolar apophysis (fig. 466); females can easily be recognized by the sinuous epigynal septum (fig. 468) and long median ducts (fig. 469).

MALE: Total length 8.1. Coloration as in C. keira. Leg spination: femora: III p0-1-1, r0-1-1; IV r0-0-0; tibiae III, IV p0-1-1; metatarsi III, IV p0-1-1, r0-1-1. Retrolateral tibial apophysis arched, bifid at tip (fig. 467); embolar apophysis wide, tegular apophysis with blunt tip, without distinct apical projection, median apophysis widened posteriorly (fig. 466).

Female: Total length 7.1. Coloration as in C. keira. Leg spination: femora: I, II p0-0-0; III p0-0-0, r0-0-0; IV r0-0-0; tibiae: III p01-1, r0-1-1; IV p0-0-0, r0-1-1; metatarsi III, IV p0-1-1, r0-1-1. Epigynum with long sep- tum, septum wider at middle than at front or back (fig. 468); median ducts long, extending almost full length of epigynum (fig. 469).

Other Material Examined: Australian Capital Territory: Blundells Creek Road, $35^{\circ} 22^{\prime} \mathrm{S}, 148^{\circ} 50^{\prime} \mathrm{E}$, Dec. 18, 1988, bell/pit trap $(\mathrm{CMNH}), 1 \hat{0}, 3 q$; Tidbinbilla Nature Reserve, $35^{\circ} 28^{\prime} \mathrm{S}, 148^{\circ} 52^{\prime} \mathrm{E}$, Mar. 9, 1978, pitfalls (P. Ormay, AMS KS13826, 13867), 5 ô, 14 ㅇ.

DistRibution: Known only from the Australian Capital Territory (map 53).

Centrina whian, new species Figures 472, 473; Map 54

TyPE: Female holotype taken in a rainforest pitfall trap at an elevation of $213 \mathrm{~m}$ in Whian Whian State Forest, via Dunoon, $28^{\circ} 38^{\prime} \mathrm{S}, 153^{\circ} 19^{\prime} \mathrm{E}$, New South Wales (Aug. 19-Nov. 16, 1974; G., S. Monteith), deposited in QMB (S28131).

ETYMOLOGY: The specific name is a noun in apposition taken from the type locality. 

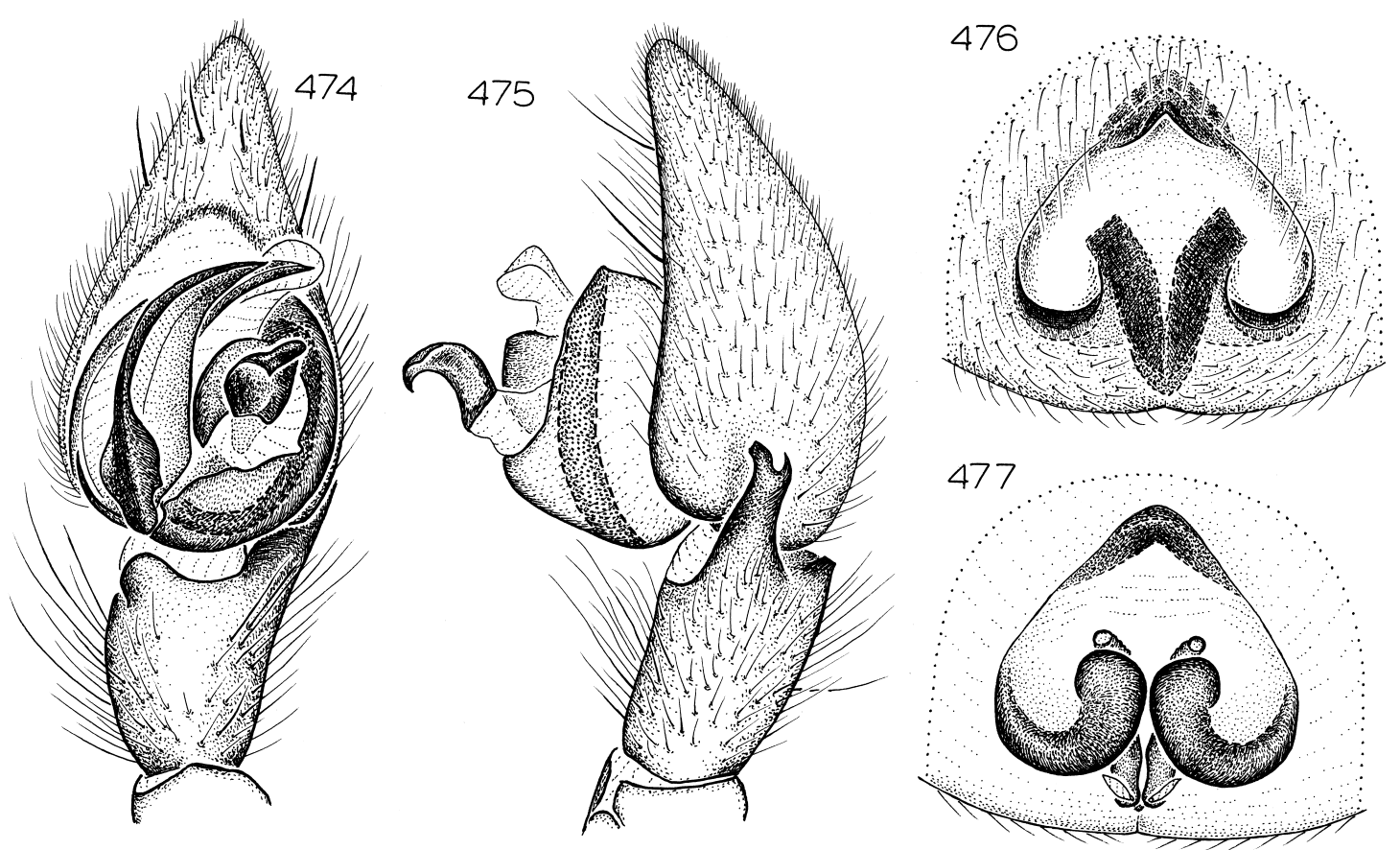

Figs. 474-477. Centrina sawpit, new species. 474. Left male palp, ventral view. 475. Same, retrolateral view. 476. Epigynum, ventral view. 477. Same, dorsal view.

Diagnosis: Females resemble those of $C$. blundells but can be distinguished by the short, narrow, anteriorly situated epigynal septum (fig. 472) and posteriorly widened spermathecae (fig. 473).

MALE: Unknown.

FEMALE: Total length 4.9. Coloration as in $C$. keira except abdominal venter gray, with two paramedian longitudinal and two lateral oblique white stripes, distal ends of femora, patellae, and proximal ends of tibiae darkened. Leg spination: femora: I, II p0-0-0, III p0-0-0, r0-0-0; IV r0-0-0; tibiae: III p1-0-1, v0-1p-2, r0-0-1; IV p0-1-1, v0-1p-2, r0-1-1; metatarsi: III p0-1-0, v0-1p-0, r0-1-0; IV p01-0, v2-1p-0, r0-1-0. Epigynum with lateral depressions and short, narrow, anteriorly situated median septum (fig. 472); spermathecae widened posteriorly, extending full length of epigynum (fig. 473).

Other Material Examined: New South Wales: Whian Whian State Forest, via Dunoon, $28^{\circ} 38^{\prime} \mathrm{S}, 153^{\circ} 19^{\prime} \mathrm{E}$, Nov. 16-Dec. 26, 1974, rainforest pitfall, elev. 213 m (G., S. Monteith, QMB S28196), 1 ㅇ.
Distribution: Known only from northern New South Wales (map 54).

\section{Centrina sawpit, new species Figures 474-477; Map 54}

TyPE: Female holotype from Sawpit Creek, Mount Kosciusko, $36^{\circ} 27^{\prime} \mathrm{S}, 148^{\circ} 16^{\prime} \mathrm{E}$, New South Wales (Jan. 1929; A. Musgrave), deposited in AMS (KS50410).

ETYMOLOGY: The specific name is a noun in apposition taken from the type locality.

DiAgNOSIS: Males resemble those of $C$. bondi in having a straight retrolateral tibial apophysis (fig. 475), but have a short embolar apophysis and a small median apophysis (fig. 474); females can easily be recognized by the wide epigynal septum (fig. 476).

MaLE: Total length 7.7. Coloration as in C. keira. Leg spination: tibiae: III p0-0-1, r00-1; IV p0-1-1; metatarsi: III r0-1-1. Retrolateral tibial apophysis straight, bifid at tip (fig. 475); embolar apophysis short, hardly protruding from bulb, tegular apophysis without apical projection, median apophysis short, with folded tip (fig. 474). 
Female: Total length 8.0. Coloration as in C. keira except abdominal dorsum without transverse white stripe. Leg spination: femora: II p0-0-0; III p0-1-1, r0-0-0; IV r0-0-0; tibiae: III p0-1-1, r0-1-1; IV p0-0-1; metatarsi III, IV p0-1-1, r0-1-1. Epigynum triangular, posterior half with wide median septum (fig. 476); spermathecae extending anterior of origin of translucent ducts (fig. 477).

Other Material Examined: Victoria: Cobon North Coupe, $37^{\circ} 23^{\prime} \mathrm{S}, 148^{\circ} 56^{\prime} \mathrm{E}$, Jan. 16-22, 1992, pitfall, midslope (R. Coy, NMV K3617), $1 \%$; Cobon South Coupe, $37^{\circ} 25^{\prime} \mathrm{S}, 148^{\circ} 56^{\prime} \mathrm{E}$, Apr. 6-14, 1992, pitfall, upslope (R. Coy, NMV K3547), $10^{\text {t }, ~ m i d-~}$ slope (R. Coy, NMV K3597), 1 웅 Cobon South Coupe, $37^{\circ} 25^{\prime} \mathrm{S}, 148^{\circ} 57^{\prime} \mathrm{E}$, Nov. 2728, 1996 (N. Platnick, R. Raven, V. Ovtsharenko, K. Catley, AMNH), 1 ; ; Cobon South Coupe, $37^{\circ} 25^{\prime} \mathrm{S}, 148^{\circ} 58^{\prime} \mathrm{E}$, Mar. 5-12, 1992, pitfall, upslope (R. Coy, NMV K4659), 1 \% , Mar. 12-18, 1992, pitfall, gully (R. Coy, NMV K3604), 1 ; Sardine Coupe, $37^{\circ} 24^{\prime} \mathrm{S}, 148^{\circ} 31^{\prime} \mathrm{E}$, Jan. 21-30, 1992, pitfall, upslope (R. Coy, NMV K3545), 1 , , Jan. 23-30, 1992, pitfall, upslope (R. Coy, NMV K3558), 10, Jan. 24-30, 1992, pitfall, midslope (R. Coy, NMV K3564), 3q; Sardine Coupe, $37^{\circ} 25^{\prime} \mathrm{S}, 148^{\circ} 31^{\prime} \mathrm{E}$, Feb. 18-25, 1992, pitfall, midslope (R. Coy, NMV $\mathrm{K} 3584), 1$ ㅇ.

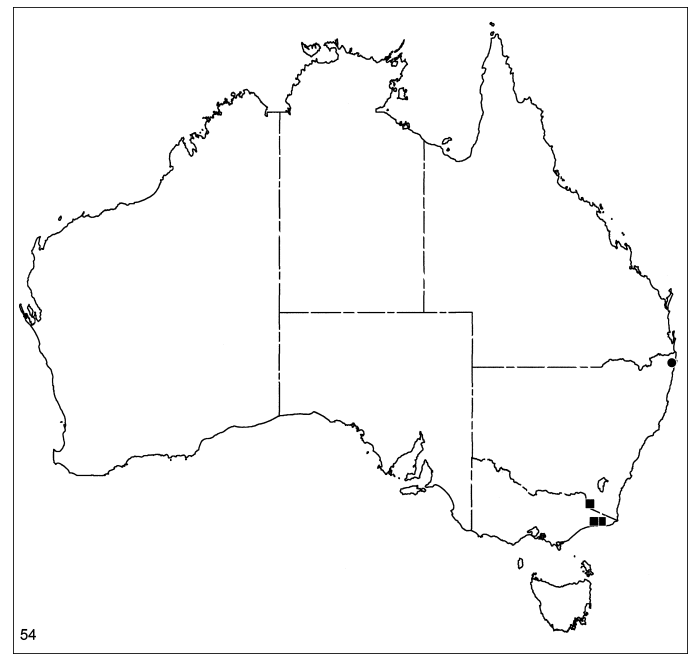

Map 54. Records of Centrina whian, new species (circle) and C. sawpit, new species (squares).
Distribution: Southern New South Wales and Victoria (map 54).

\section{Centrina bondi, new species}

Figures 478-481; Map 52

TYPE: Female holotype taken in open eucalypt forest litter in Bondi State Forest, S Bombala, $37^{\circ} 08^{\prime} \mathrm{S}, 149^{\circ} 09^{\prime} \mathrm{E}$, New South Wales (Jan. 26, 1981; G. Gowing), deposited in AMS (KS12105).

ETYMOLOGY: The specific name is a noun in apposition taken from the type locality.

DiAGNOSIS: Males and females have not been taken together, but are tentatively matched on the basis of geography; males can easily be recognized by the semicircular tip of the median apophysis (fig. 478), females by the spermathecae with distinctive compressions at about half their length (fig. 481).

MALE: Total length 5.4. Coloration as in C. keira except abdominal dorsum without transverse white stripe, scutum extending about two-thirds of abdominal length. Leg spination: femora: II p0-1-1; III p0-1-1, r01-1; IV p0-0-1. Retrolateral tibial apophysis straight, bifid at tip (fig. 479); embolar apophysis long, originating at proximal tip of bulb; tegular apophysis with long apical projection; median apophysis with semicircular, recurved tip (fig. 478).

Female: Total length 8.1. Coloration as in C. keira. Leg spination: tibiae III p0-1-1, r01-1. Epigynal atrium rounded, anterior margin bipartite, septum narrow anteriorly (fig. 480); spermathecae laterally compressed at about half their length (fig. 481).

Other Material Examined: Australian Capital Territory: Blundells Creek, $35^{\circ} 22^{\prime} \mathrm{S}, 148^{\circ} 50^{\prime} \mathrm{E}$, Mar. 1984, elev. $850 \mathrm{~m}$ (Weir, QMB), 10; Wombat Creek, $6 \mathrm{~km} \mathrm{NE}$ Piccadilly Circus, $35^{\circ} 19^{\prime} \mathrm{S}, 148^{\circ} 51^{\prime} \mathrm{E}, \mathrm{Feb} .-$ Mar. 1984, elev. $750 \mathrm{~m}$ (Weir, Lawrence, Johnson, QMB), $20^{\star}$. Victoria: Bendigo

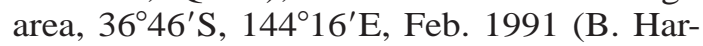
vey, NMV K3662), $10^{\star}$; Rich-Murrungowar Coupe, $37^{\circ} 34^{\prime} \mathrm{S}, 148^{\circ} 38^{\prime} \mathrm{E}$, Mar. 18-25, 1992, pitfall, midslope (R. Coy, NMV K3526), 1 ô.

Distribution: Southeastern Australia (map 52). 

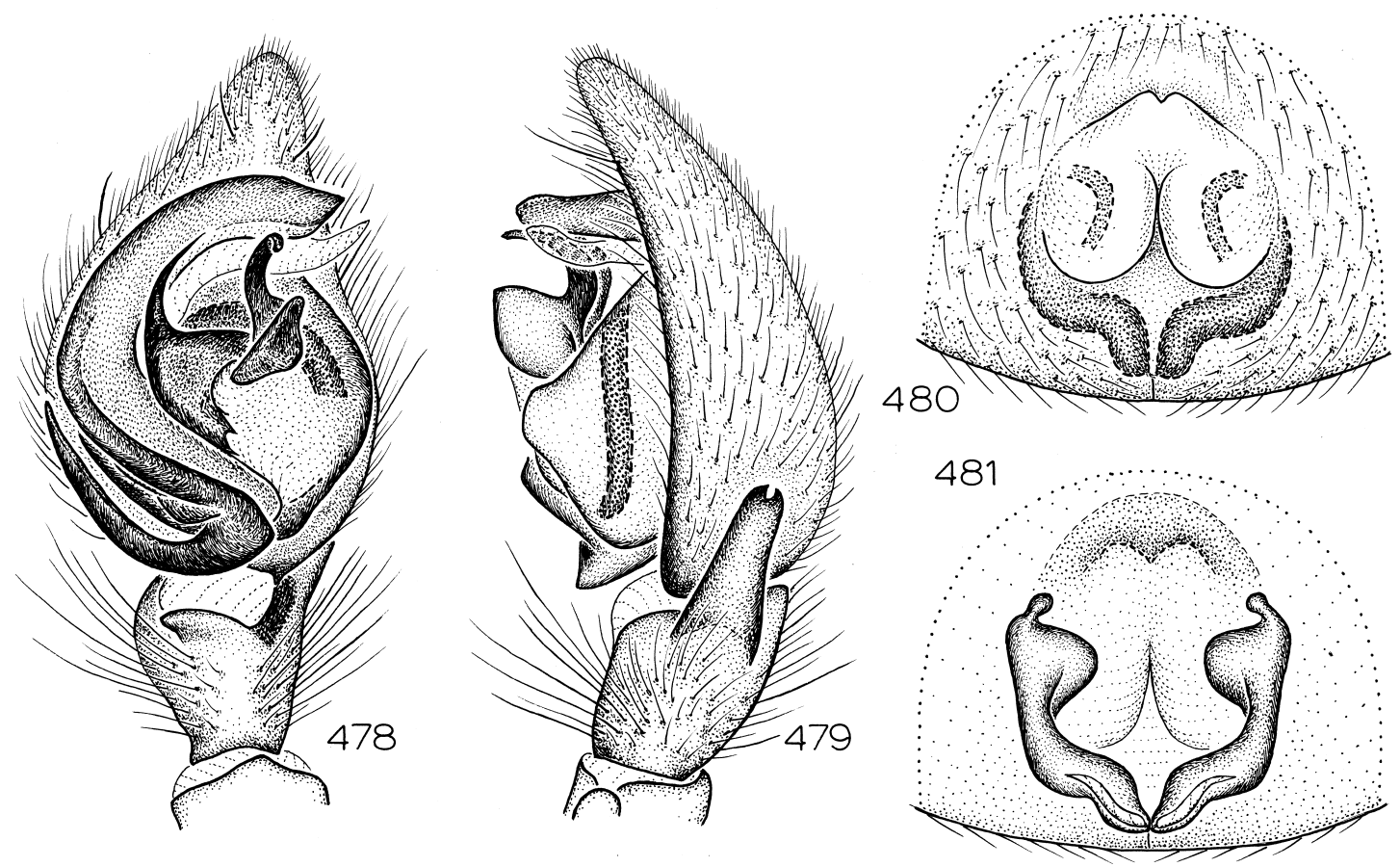

Figs. 478-481. Centrina bondi, new species. 478. Left male palp, ventral view. 479. Same, retrolateral view. 480. Epigynum, ventral view. 481. Same, dorsal view.

Centrina sherbrook, new species Figures 482-485; Map 55

TYPE: Female holotype taken in pitfall trap in Sherbrook Forest Reserve, 37 $53^{\prime} \mathrm{S}$, $145^{\circ} 21^{\prime} \mathrm{E}$, Victoria (Apr. 10, 1978; M. Gray), deposited in AMS (KS45345).

ETYMOLOGY: The specific name is a noun in apposition taken from the type locality.

DIAGNOSIS: Males and females have not been collected together but are tentatively matched here on the basis of similarities in size and coloration. Males can easily be recognized by their triangular retrolateral tibial apophysis (fig. 483) and sharply pointed conductor (fig. 482), females by the short, weak lateral epigynal margins (fig. 484).

MALE: Total length 5.8. Coloration as in C. keira except abdominal dorsum with hint of anterior transverse white stripe and leg femora darkened. Leg spination: femur III p0-1-1; tibia IV p0-1-1. Retrolateral tibial apophysis expanded at base, triangular, bifid at tip (fig. 483); embolar apophysis short, small, tegular apophysis with narrow apical projection, median apophysis sharply angled (fig. 482).

FEMALE: Total length 6.2. Coloration as in male. Leg spination: femur III p0-1-0, r0-10; tibiae: III r0-1-1; IV p0-1-1; metatarsi: III p0-1-1, r0-1-1; IV p0-1-1, v2-0-1p, r0-1-1. Epigynum with tiny anterior hood, lateral margins restricted to posterior half of epigynum (fig. 484); ducts tightly coiled posteriorly (fig. 485).

Other Material Examined: Victoria: Cobon North Coupe, $37^{\circ} 22^{\prime} \mathrm{S}, 148^{\circ} 56^{\prime} \mathrm{E}$, Apr. 6-10, 1992, pitfall, midslope (R. Coy, NMV ex K3554), 1ð, Apr. 6-14, 1992, pitfall, gully (R. Coy, NMV K3553), 10 ; 10 $\mathrm{km}$ N Healesville, $37^{\circ} 35^{\prime} \mathrm{S}, 145^{\circ} 31^{\prime} \mathrm{E}$, Apr. 6, 1991, under eucalypt bark (M. Harvey, M. Blosfelds, WAM 96/201), 1 9 ; 11.4 km ENE McMahons Creek, 37 $41^{\prime} \mathrm{S}, 145^{\circ} 57^{\prime} \mathrm{E}$, Nov. 14-24, 1988 (L. Lumsden, NMV K3631), $1 \%$; Mount Baw Baw, $40 \mathrm{~km}$ E Noojee, $37^{\circ} 50^{\prime} \mathrm{S}, 146^{\circ} 17^{\prime} \mathrm{E}$, Jan. 5, 1973 (M. Baehr, CBB), 1 우.

DISTRIBUTION: Known only from eastern Victoria (map 55). 

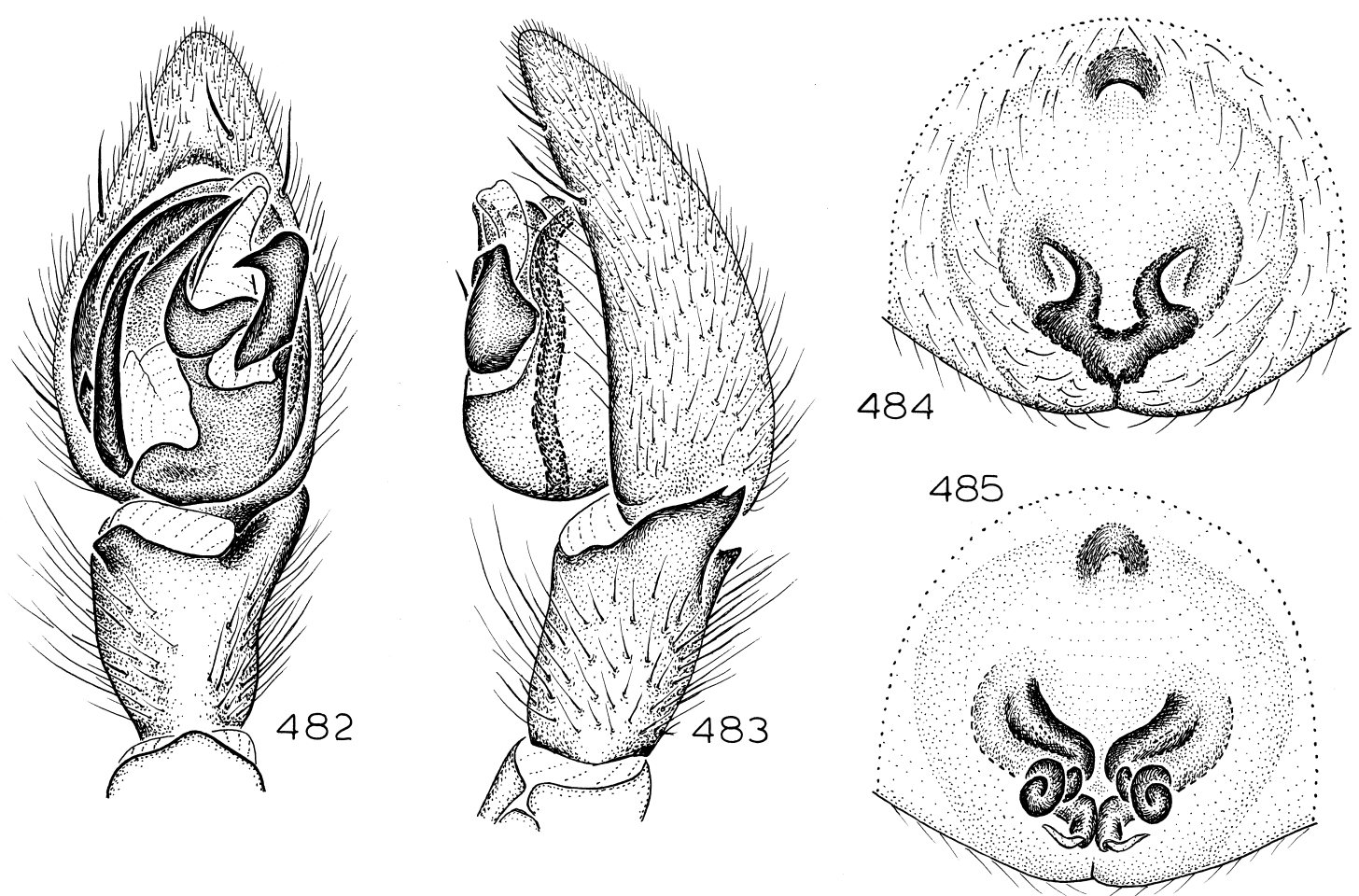

Figs. 482-485. Centrina sherbrook, new species. 482. Left male palp, ventral view. 483. Same, retrolateral view. 484. Epigynum, ventral view. 485. Same, dorsal view.

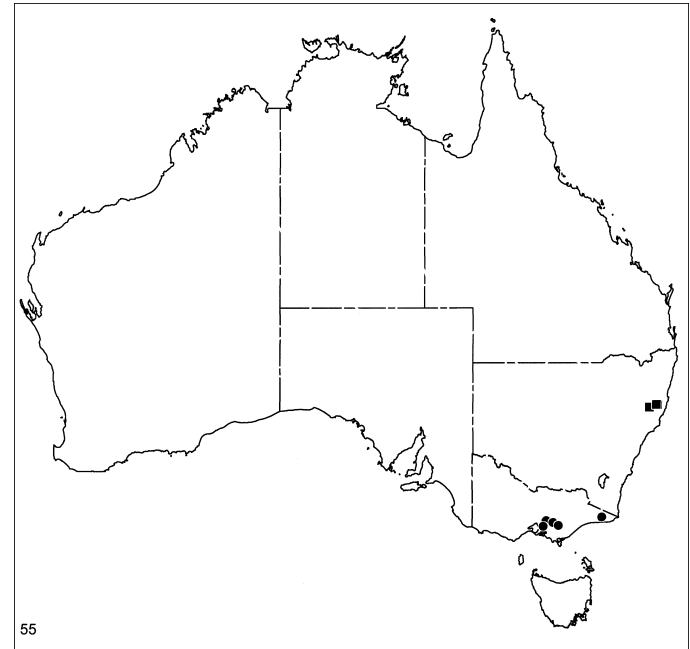

Map 55. Records of Centrina sherbrook, new species (circles) and $C$. enfield, new species (squares).
Centrina macedon, new species

Figures 486-489; Map 56

TYPE: Female holotype taken at an elevation of $700 \mathrm{~m}$ on Mount Macedon, $37^{\circ} 25^{\prime} \mathrm{S}$, $144^{\circ} 35^{\prime} \mathrm{E}$, Victoria (Nov. 30, 1996; N. Platnick, R. Raven, V. Ovtsharenko, K. Catley), deposited in QMB.

ETYMOLOGY: The specific name is a noun in apposition taken from the type locality.

DiAGNOSIS: Males and females have not been collected together but are tentatively matched here on the basis of similarities in size and coloration. Males can easily be recognized by the sinuous and basally expanded retrolateral tibial apophysis (fig. 487), females by the circular epigynal margins (fig. 488).

Male: Total length 5.7. Dorsum of abdomen with transverse white stripe covered by extensive scutum extending back to about two-thirds of abdominal length; coloration otherwise as in C. keira. Leg spination: femora: II p0-0-0; III p0-0-0, r0-0-0; IV r0-0-0; 

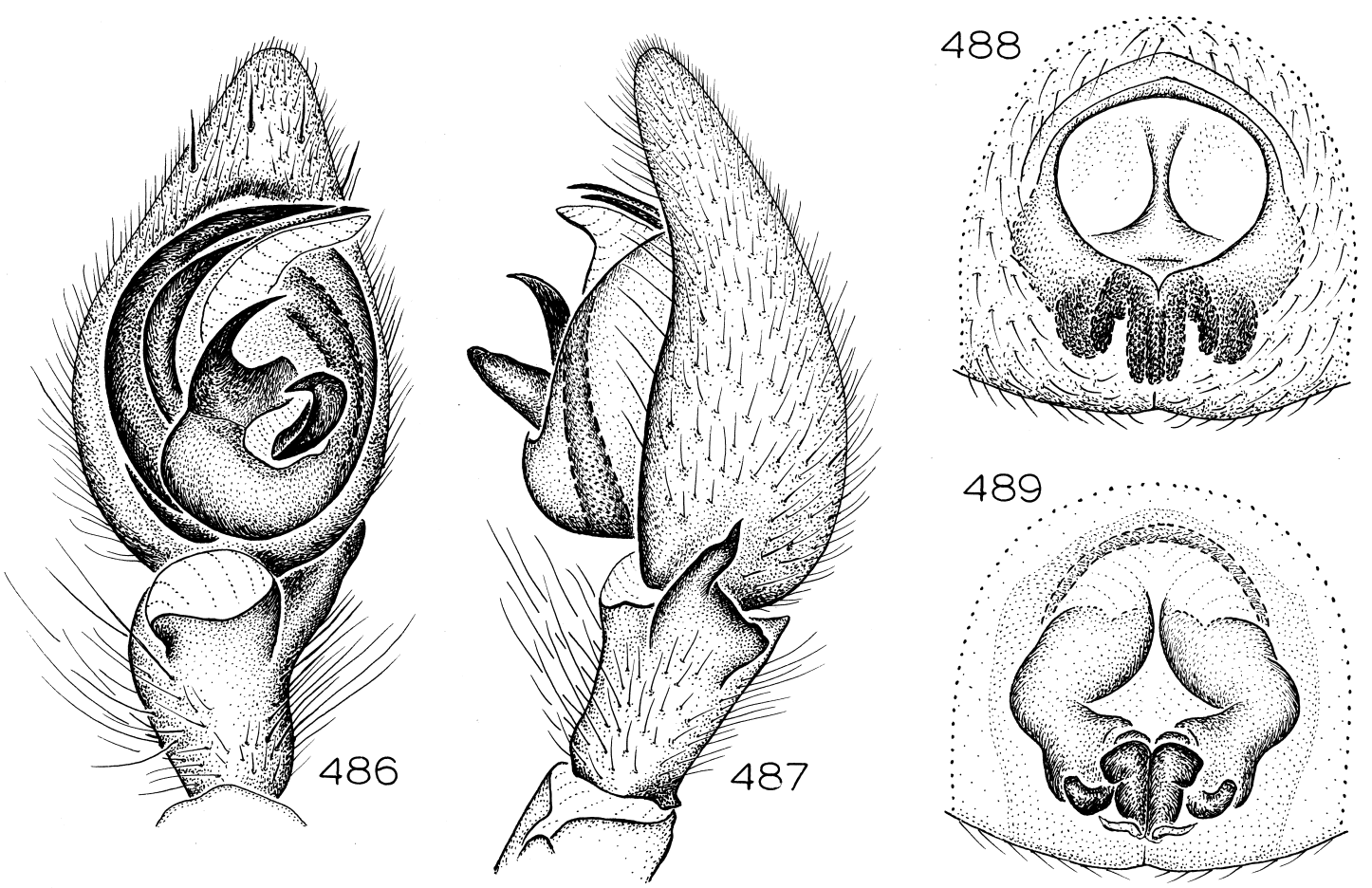

Figs. 486-489. Centrina macedon, new species. 486. Left male palp, ventral view. 487. Same, retrolateral view. 488. Epigynum, ventral view. 489. Same, dorsal view.

tibiae III, IV p0-1-1, v2-1p-2, r0-1-1; metatarsi III, IV p0-1-1, v2-1p-1p, r0-1-1. Retrolateral tibial apophysis almost bipartite, dorsal portion short, with rounded ledge, ventral portion long, sinuous (fig. 487); embolar apophysis short, small, tegular apophysis with long, rounded apical projection, median apophysis relatively small, simple, hookshaped (fig. 486).

FEMALE: Total length 6.9. Abdominal dorsum without scutum, coloration otherwise as in male. Leg spination: femora: I, II p0-0-0; III p0-0-0, r0-0-0; IV r0-0-0; tibiae: III p01-1, r0-1-1; IV p0-0-0, r0-1-1; metatarsi III, IV $\mathrm{p} 0-1-1, \mathrm{r} 0-1-1$. Epigynal margins forming full circle, atrium with narrow median septum (fig. 488); lateral ducts enlarged anteriorly (fig. 489).

Other Material Examined: New South Wales: $0.7 \mathrm{~km} \mathrm{~W}$ Omadale Brook Road along unnamed logging track, Stewarts Brook State Forest, 31 ${ }^{\circ} 54^{\prime} \mathrm{S}, 151^{\circ} 23^{\prime} \mathrm{E}$, Feb. 4-Apr. 9, 1993, pitfall, elev. $1250 \mathrm{~m}$ (M. Gray, G. Cassis, AMS KS37393), $10^{t}$. Victoria: Cobon North Coupe, $37^{\circ} 22^{\prime} \mathrm{S}$, $148^{\circ} 56^{\prime}$ E, Apr. 6-10, 1992, pitfall, midslope (R. Coy, NMV K3554), $1 \delta^{\star}$; Cobon South Coupe, $37^{\circ} 25^{\prime} \mathrm{S}, 148^{\circ} 56^{\prime} \mathrm{E}$, May 6-11, 1992, pitfall, gully (R. Coy, NMV K3548), $10^{\text {to; }}$ Rich-Murrungowar Coupe, $37^{\circ} 34^{\prime} \mathrm{S}$, $148^{\circ} 38^{\prime} \mathrm{E}$, Mar. 16-24, 1992, pitfall, midslope (R. Coy, NMV K3541), 1 ơ; Separation Creek, 38 38'S, $143^{\circ} 54^{\prime} \mathrm{E}$, Sept. 19-21, 1989, under eucalypt bark (M. Harvey, M. Blosfelds, WAM 1996/202), 1 ㅇ․

Distribution: New South Wales and Victoria (map 56).

\section{Centrina lewis, new species}

Figures 490-493; Map 56

TYPE: Female holotype taken in a pitfall trap in rainforest on Mount Lewis, $16^{\circ} 35^{\prime} \mathrm{S}$, $145^{\circ} 17^{\prime} \mathrm{E}$, Queensland (Jan.-Mar. 1988; G. Wood), deposited in QMB (S15634).

ETYMOLOGY: The specific name is a noun in apposition taken from the type locality.

DiAGNOSIS: Males can easily be recognized by the very small median apophysis (fig. 
490), females by the wide epigynal hood (fig. 492).

MALE: Total length 8.5. Abdominal dorsum without transverse white strip, scutum reaching to about two-thirds of abdominal length, venter with four longitudinal rows of small sclerotizations; legs unmarked. Leg spination: femora III, IV r0-0-0; tibiae: III p0-1-1, v0-1p-2, r0-1-1; IV p0-0-0, v1p-1p2, r0-1-1; metatarsi III, IV p0-1-0, v2-1p-0, r0-1-0. Retrolateral tibial apophysis small, longer dorsally than ventrally (fig. 491); embolar apophysis protuberant, stronger than embolus, tegular apophysis without apical projection, median apophysis small, short (fig. 490).

FEMALE: Total length 12.8. Coloration and leg spination as in male. Epigynum with distinctively wide anterior hood, openings small, situated posteriorly near midline (fig. 492); spermathecae not obscured by translucent ducts (fig. 493).

Other MATERIAl ExAmined: Queensland: Devils Thumb, $10 \mathrm{~km}$ NW Mossman, $16^{\circ} 23^{\prime} \mathrm{S}, 145^{\circ} 17^{\prime} \mathrm{E}$, Oct. 12 , 1982 , rainforest, pyrethrum knockdown (G. Monteith, D. Yeates, Thompson, QMB S26496), 1 क; 7 km N Mount Spurgeon, $16^{\circ} 26^{\prime} \mathrm{S}, 145^{\circ} 12^{\prime} \mathrm{E}$, Oct. 17-19, 1991, pitfall, elev. 1200-1250 m (G. Monteith, H. Janetzki, D. Cook, L. Roberts, QMB S30336), 10ิ, 1\%; head Roots Creek,

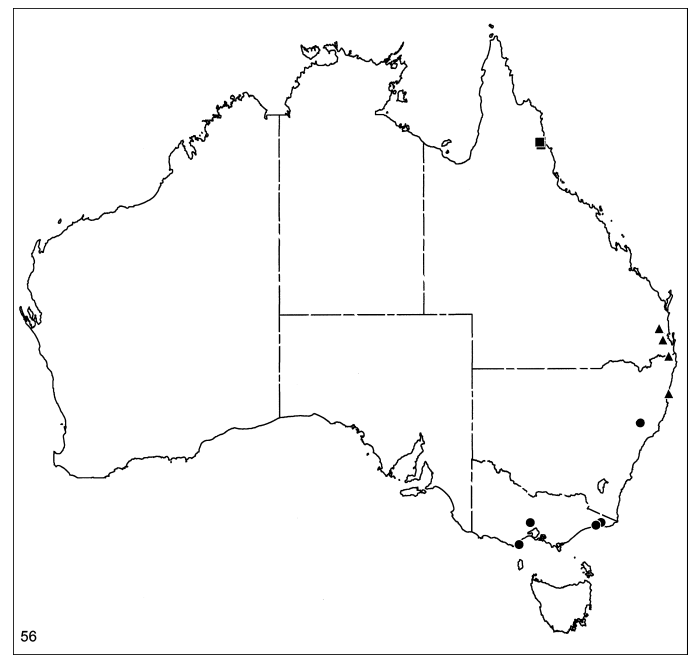

Map 56. Records of Centrina macedon, new species (circles), C. lewis, new species (squares), and Centsymplia glorious, new species (triangles).
$12 \mathrm{~km}$ WNW Mossman, $16^{\circ} 26^{\prime} \mathrm{S}, 145^{\circ} 12^{\prime} \mathrm{E}$, Dec. 28-29, 1990, elev. $1200 \mathrm{~m}$ (QMB S32414), 1 ㅇ․

DisTRIBUTION: Known only from northern Queensland (map 56).

\section{Centrina enfield, new species}

Figures 494-497; Map 55

TYPE: Male holotype taken in pitfall trap at an elevation of $1080 \mathrm{~m}$ on Double Dumps Fire Trail, 700 m off Daisy Patch Road, Enfield State Forest, $31^{\circ} 20^{\prime} \mathrm{S}, 151^{\circ} 54^{\prime} \mathrm{E}$, New South Wales (Feb. 4-Apr. 9, 1993; M. Gray, G. Cassis), deposited in AMS (KS44088).

ETYMOLOGY: The specific name is a noun in apposition taken from the type locality.

DIAGNOSIS: Males can easily be recognized by the distinctive, lobate shape of the retrolateral tibial apophysis (fig. 495), females by the long epigynum with narrow lateral margins (fig. 496).

MALE: Total length 6.2. Coloration as in C. keira. Leg spination: femora: II p0-0-0; III p0-0-0, r0-0-0; IV r0-0-0; tibiae: III p01-1, r0-1-1; IV p0-0-1, r0-1-1; metatarsi: III p0-1-0, v2-1p-0, r0-1-0; IV p0-1-0, v2-2-1p, r0-1-0. Retrolateral tibial apophysis long, with lobate ventral projection and sharp dorsal projection (fig. 495); median apophysis with beak-shaped tip bearing projection (fig. 494).

Female: Total length 7.8. Coloration as in C. keira. Leg spination: femora: I, II p0-0-0; III p0-0-0, r0-0-0; IV r0-0-0; tibiae: III p01-1, r0-1-1; IV p0-1-1, r1-0-1; metatarsi: III p0-1-0, v2-2-0, r0-1-0; IV p1-0-1, v2-2-0, r10-0. Epigynal openings situated posteriorly, between long, narrow lateral epigynal margins (fig. 496); spermathecae elongate, anteriorly twisted (fig. 497).

Other Material Examined: New South Wales: Banda Road, ca. $4.5 \mathrm{~km}$ E Hastings Forest Highway, Mount Boss State Forest, $31^{\circ} 09^{\prime} \mathrm{S}, 152^{\circ} 25^{\prime} \mathrm{E}$, Feb. 4-Apr. 9, 1993, pitfall, elev. 1100 m (M. Gray, G. Cassis, AMS KS39629), 30 ; Daisy Patch Fire Trail, 1.9 $\mathrm{km} \mathrm{S}$ Enfield Road, Enfield State Forest, $31^{\circ} 20^{\prime} \mathrm{S}, 151^{\circ} 54^{\prime} \mathrm{E}$, Feb. 4-Apr. 9, 1993, pitfall, elev. 1130 m (M. Gray, G. Cassis, AMS KS44086), $30^{\star}$; Mount Boss State Forest, $31^{\circ} 12^{\prime} \mathrm{S}, 152^{\circ} 24^{\prime} \mathrm{E}$, Oct. 1980 (G. Webb, AMS KS43519, 43529), 10, 2q; N Plateau 

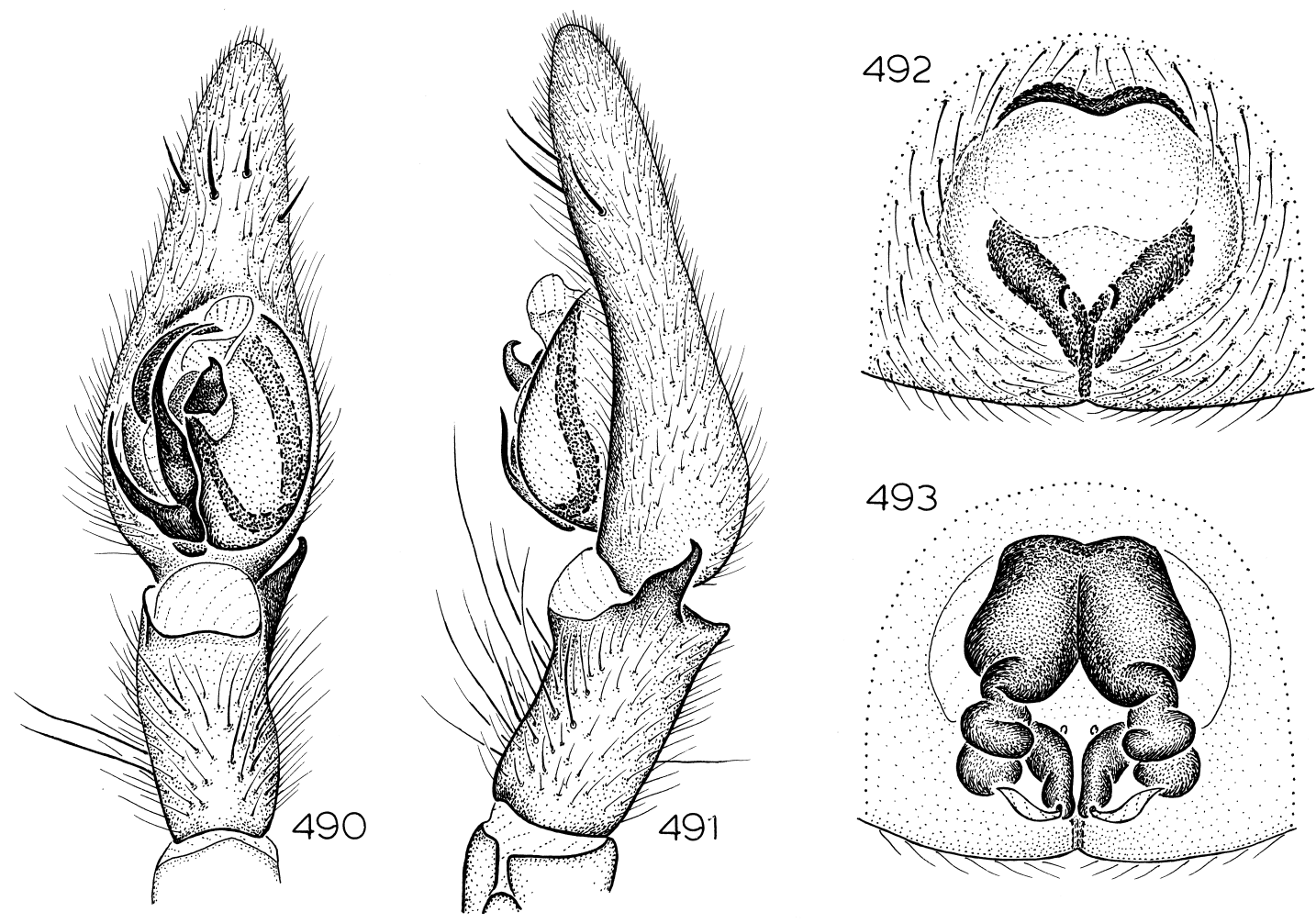

Figs. 490-493. Centrina lewis, new species. 490. Left male palp, ventral view. 491. Same, retrolateral view. 492. Epigynum, ventral view. 493. Same, dorsal view.

Road, ca. $1.5 \mathrm{~km}$ from Plateau Beach Picnic Area, Mount Boss State Forest, $31^{\circ} 10^{\prime} \mathrm{S}$, $152^{\circ} 19^{\prime}$ E, Feb. 4-Apr. 9, 1993, pitfall, elev. $1120 \mathrm{~m}$ (M. Gray, G. Cassis, AMS KS39628), 1 đ; N Plateau Road, ca. $3.5 \mathrm{~km}$ from Plateau Beach Picnic Area, Mount Boss State Forest, $31^{\circ} 11^{\prime} \mathrm{S}, 152^{\circ} 20^{\prime} \mathrm{E}$, Feb. 4-Apr. 9, 1993, pitfall, elev. 1120 m (M. Gray, G. Cassis, AMS KS39630), 20 .

DISTRIBUTION: Known only from northern New South Wales (map 55).

\section{Centrocalia, new genus}

Type Species: Asadipus lifoui Berland.

ETYMOLOGY: The generic name is an arbitrary combination of letters, considered feminine in gender.

Diagnosis: Members of this genus resemble those of Bigenditia in having the pedicel fused to the posterior rim of the sternum; males differ in lacking expanded endites and having an embolar apophysis (similar to that of Centrothele and Centrina), females in having relatively smaller epigynal atria.

DESCRIPTION: Medium-sized spiders, total length 4.0-5.7. Carapace brown, pars cephalica darkest, pars thoracica without maculations, all surfaces coated with tubercles bearing white setae; thoracic groove long, longitudinal. Eight eyes in two rows, anterior medians largest, circular, dark, laterals subequal in size, oval, light, posterior medians subequal to laterals, irregularly oval, flattened, light; from above, anterior eye row almost straight, posterior row slightly procurved, from front, anterior row slightly procurved, posterior row strongly procurved; anterior medians separated by less than their radius, slightly closer to anterior laterals; posterior medians separated by more than their diameter, about as far from posterior laterals; anterior and posterior laterals separated by less than their diameter; median ocular quadrangle wider in front than in back, wider 

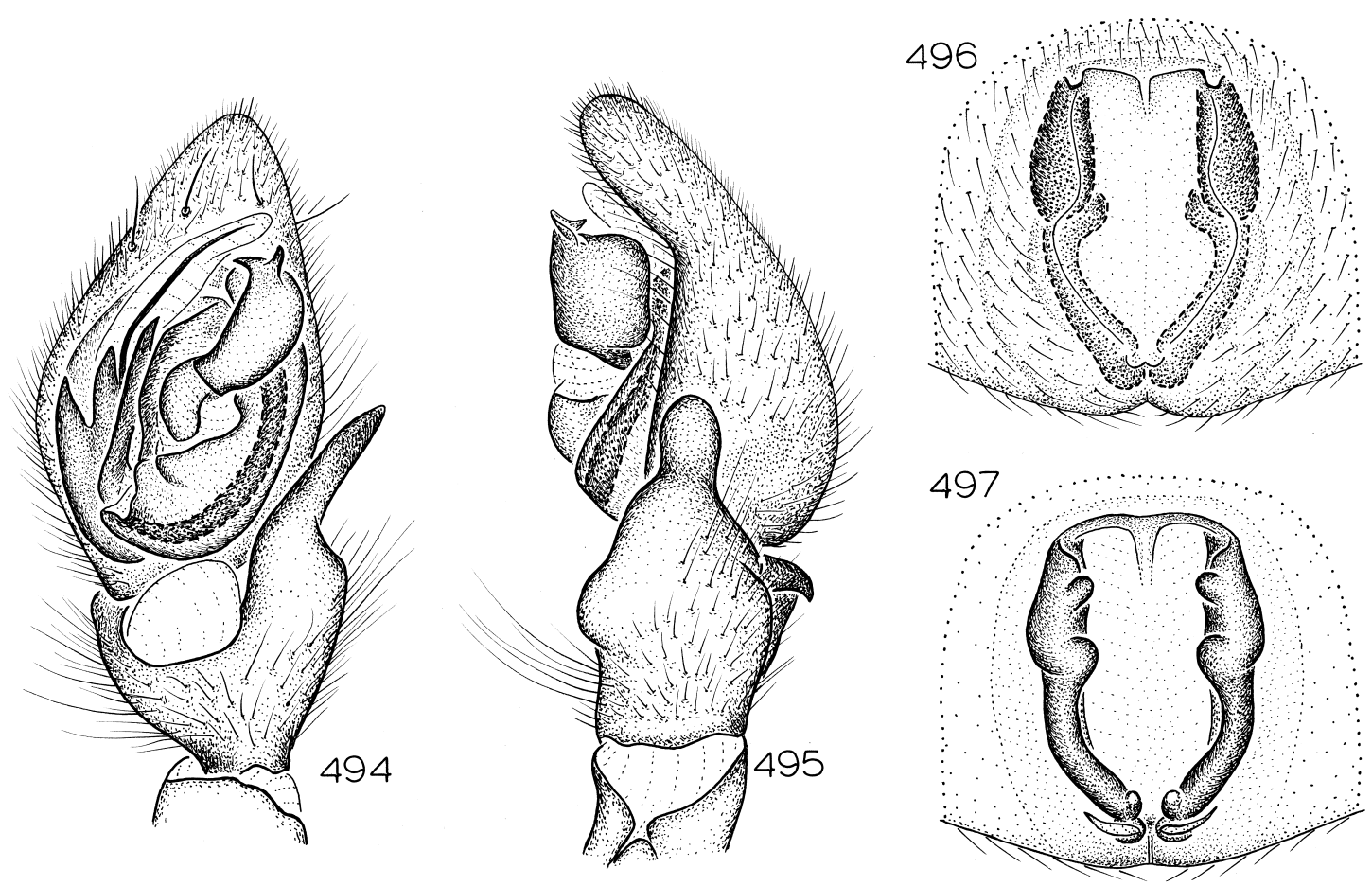

Figs. 494-497. Centrina enfield, new species. 494. Left male palp, ventral view. 495. Same, retrolateral view. 496. Epigynum, ventral view. 497. Same, dorsal view.

in front than long. Chelicerae and mouthparts reddish-brown, sternum brown; chilum wide, triangular, with seta-bearing tubercles, accompanied by second, dumbbell-shaped, posterior chilum (extremely narrow sclerite separating bases of chelicerae posteriorly); chelicerae with distinct, shiny lateral boss, anterior surface with depressed, relatively unsclerotized oval area near promargin; promargin with row of long setae originating in line along base of fang, seta closest to fang bent near base at almost $90^{\circ}$ angle, extending behind other promarginal setae to near midline; promargin with three teeth, median one largest, proximal one smallest; retromargin with two large, widely separated teeth. Labium elongate, base narrowed at about onefifth of labial length, posterior margin truncate, anterior margin medially invaginated, surface not depressed medially. Endites obliquely depressed, with sharply demarcated, deep, wide groove along margin near labium, groove wider anteriorly than posteriorly; serrula long, with single row of teeth. Sternum not elevated, with flat, rebordered lateral margins, not expanded anteriorly, with triangular extensions to and between coxae; surface with seta-bearing tubercles, without elevations or depressions opposite coxae. One continuous epimeric sclerite situated above coxae, fused to sternal triangles, not fused to carapace. Pedicel fused to epimeric sclerite and to posterior margin of sternum, that margin demarcated by distinct ridge.

Anterior edge of abdomen of male with complete sclerotic ring formed by strong epigastric scutum plus weaker dorsal abdominal scutum covering entire front edge of abdomen, reaching to almost half of abdominal length, females with dorsal scutum represented by wide semicircular plate above pedicel, restricted to lower half of anterior surface of abdomen; cuticle with long, erect and recumbent setae; epigastric scutum accompanied posterolaterally by pair of oval, deeply invaginated sclerites bearing clearly elevated anterior rim; sclerites separated by membranous lobe, anterior rim of sclerites fitting under epigastric scutum; colulus represented only by setae on slight sclerotiza- 
tion; tiny transverse sclerite, well removed from spinnerets, marking position of small posterior spiracle. Anterior lateral spinnerets tubular, separated by almost their diameter, cuticle representing distal, second spinneret segment restricted to semicircle surrounding major ampullate gland spigots (piriform gland spigots surrounded only by soft cuticle); posterior median spinnerets of males large, tubular, of females with anteriorly expanded tips, bases occupied by three enormously widened cylindrical gland spigots; posterior lateral spinnerets two-segmented.

Leg spination reduced only on anterior legs; typical leg spination pattern (only surfaces with spines listed): femora I-IV d1-00; tibiae III, IV p0-0-1, v0-2-2, r0-1-0; metatarsi III, IV p1-0-0, v0-1p-0, r1-0-0. Most leg surfaces with both short and long setae; males with all coxae and trochanters dorsally tuberculate; anterior coxae with slightly protuberant posterolateral corners; trochanters slightly notched; anterior metatarsi and tarsi with entire scopulae, composed laterally of laterally directed setae, medially of distally directed setae; posterior metatarsi with weak, distal preening brushes; tarsi III, IV with entire scopulae; tarsi with two dentate claws, claw tufts composed of lateral pads of closely appressed setae; trichobothria in two rows on metatarsi and tarsi. Female palpal femur, patella, tibia, and tarsus with spines; female palpal tarsus with long, basally dentate claw.

Male palp with short, strong retrolateral tibial apophysis; cymbial surface excavated opposite tibial apophysis, produced into distinct lobe on ventral side of tibial apophysis; tegulum expanded ventrally, bearing large median apophysis; embolus originating prolaterally, wide, accompanied distally by long, partially membranous conductor; embolar base bearing distinct, distally-directed apophysis. Epigynum with anterior hood and deep atrium (often filled with hardened secretions); spermathecal ducts expanded anteriorly into translucent chambers.

\section{Key to Species of Centrocalia}

1. Males ................... 2

- Females (those of chazeaui unknown) ... 4

2. Embolar apophysis relatively wide (fig. 498)
- Embolar apophysis narrow (figs. 450, 502)

.................... 3

3. Embolar apophysis relatively short (fig. 450)

chazeaui

- Embolar apophysis relatively long (fig. 502) ................ ningua

4. Epigynal atrium relatively long (fig. 500) .. .................... lifoui

- Epigynal atrium relatively short (fig. 504) . . $\ldots \ldots \ldots \ldots \ldots \ldots \ldots \ldots$ ningua

\section{Centrocalia lifoui (Berland), new combination \\ Figures 498-501}

Asadipus lifoui Berland, 1929: 394, figs. 5-10 (male holotype from Lifou, Loyalty Islands, New Caledonia, in MNHN, examined).

DiAGNOSIS: In both sexes, the carapace bears a diagnostic pair of ridges near the pedicel, composed of heavy tubercles and possibly stridulatory in function. The median apophysis is distinctly divided into narrow distal and wider proximal portions (fig. 498); the epigynal atrium is relatively long and divided by a septum (fig. 500).

MALE: Total length 4.0. Coloration typical for genus. Leg spination: tibiae: III p0-1-0. Retrolateral tibial apophysis short, widened and serrated distally (fig. 499); embolar apophysis wider at base than embolus, median apophysis with long, narrow tip (fig. 498).

FEMALE: Total length 4.1. Coloration typical for genus. Leg spination: tibiae: III p01-1, v0-1p-2; IV p0-1-0; metatarsi: III p0-10, r0-1-0. Epigynum with long anterior margins; atrium with median septum expanded posteriorly into plate (fig. 500); spermathecal ducts j-shaped (fig. 501).

Material Examined: New Caledonia: Lifou, Loyalty Islands, 1928 (Mme. Pruvot, MNHN), 1 ơ (holotype); Monts Koghis, May 22-24, 1987, montane forest, elev. $500 \mathrm{~m}$ (N. Platnick, R. Raven, AMNH), 10,2 , Oct. 28, 1988, humid forest at night (T. Churchill, QMB S13736), 10; Monts Koghis, Auberge, July 26-Aug. 13, 1978, Berlese, debris under bark, rainforest, elev. $500 \mathrm{~m}$ (S., J. Peck, AMNH), 10 ..

Distribution: Known only from Monts Koghis, near Nouméa on New Caledonia, and from the adjacent Loyalty Island, Lifou. 

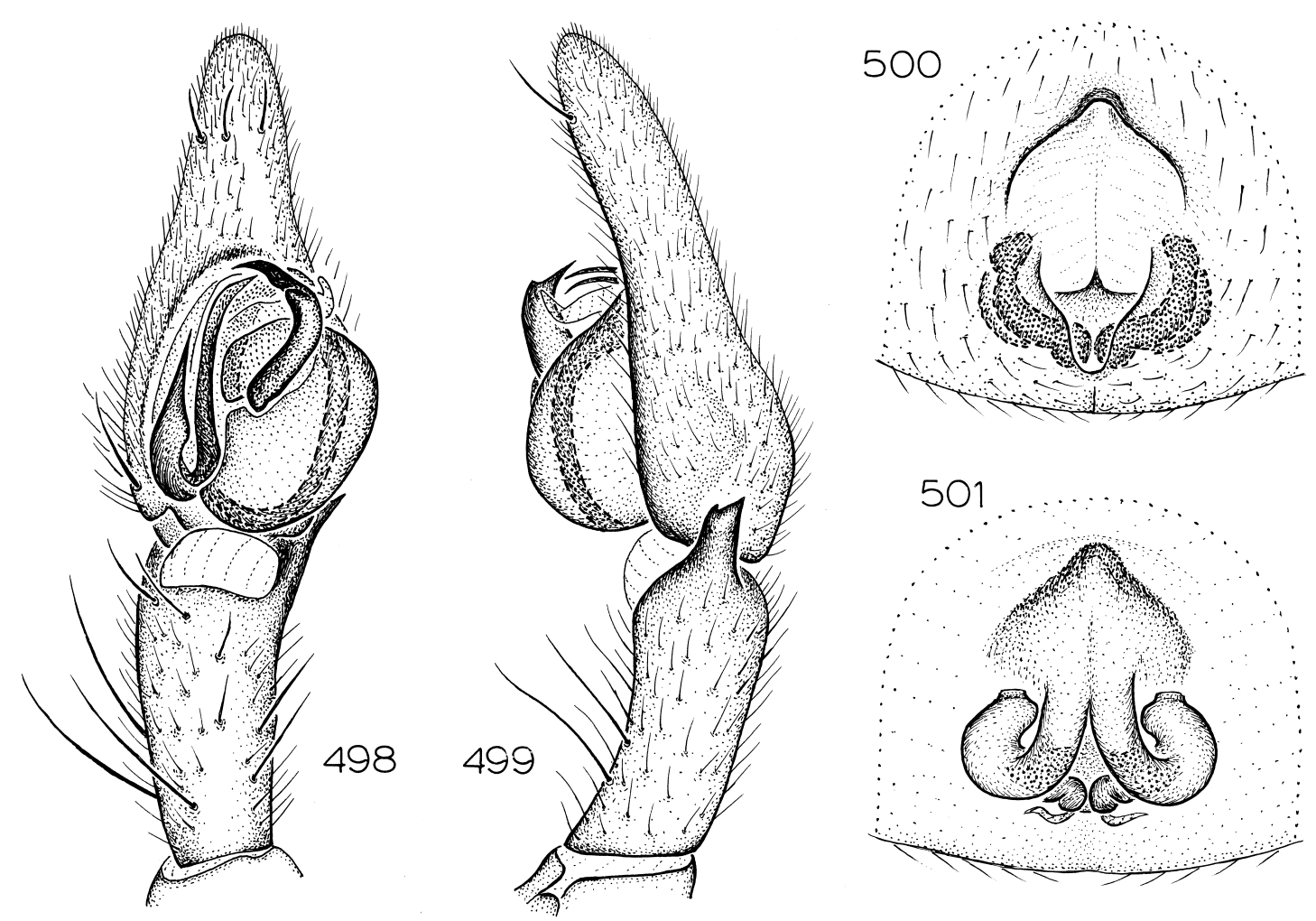

Figs. 498-501. Centrocalia lifoui (Berland). 498. Left male palp, ventral view. 499. Same, retrolateral view. 500. Epigynum, ventral view. 501. Same, dorsal view.

\section{Centrocalia chazeaui, new species} Figures 450, 451

TYPE: Male holotype taken by canopy fogging in dense forest at Rivière Bleue, New Caledonia (Jan. 20, 1993; J. Chazeau, E. Guilbert, L. Bonnet de Larbogne), deposited in MNHN.

ETYMOLOGY: The specific name is a patronym in honor of Dr. Jean Chazeau, one of the collectors and distinguished student of New Caledonian beetles.

DiaGNOSIS: Males lack the ridges found posteriorly on the carapace in $C$. lifoui but have much larger tubercles on both the carapace and sternum. The median apophysis is long and arched, the embolar apophysis short and narrow (fig. 450).

MALE: Total length 5.0. Carapace dark chestnut brown, abdomen with almost complete transverse white stripe at about twothirds of dorsal scutum length; legs orange, coxae darkest. Leg spination: femora I, II d0-
0-0; tibiae: III v0-0-1p, r0-0-0; IV p0-0-0, v0-0-1p, r0-0-0; metatarsi III, IV p0-0-0, v00-0, r0-0-0. Retrolateral tibial apophysis relatively narrow, incised at tip (fig. 451); embolar apophysis narrow, median apophysis long, arched (fig. 450).

FEMALE: Unknown.

Other MATERIAl EXAMined: None.

Distribution: Known only from Rivière Bleue, south of Nouméa, on New Caledonia.

Centrocalia ningua, new species Figures 502-505

TYPES: Male holotype taken in a pitfall trap at an elevation of $1350 \mathrm{~m}$ in virgin montane forest on Pic Ningua, New Caledonia (July 11, 1993; G. Hunt), and female allotype from the same site (Nov. 13, 1994; G. Hunt), deposited in MONZ.

ETYMOLOGY: The specific name is a noun in apposition taken from the type locality.

DiAgnosis: Males resembles those of $C$. 

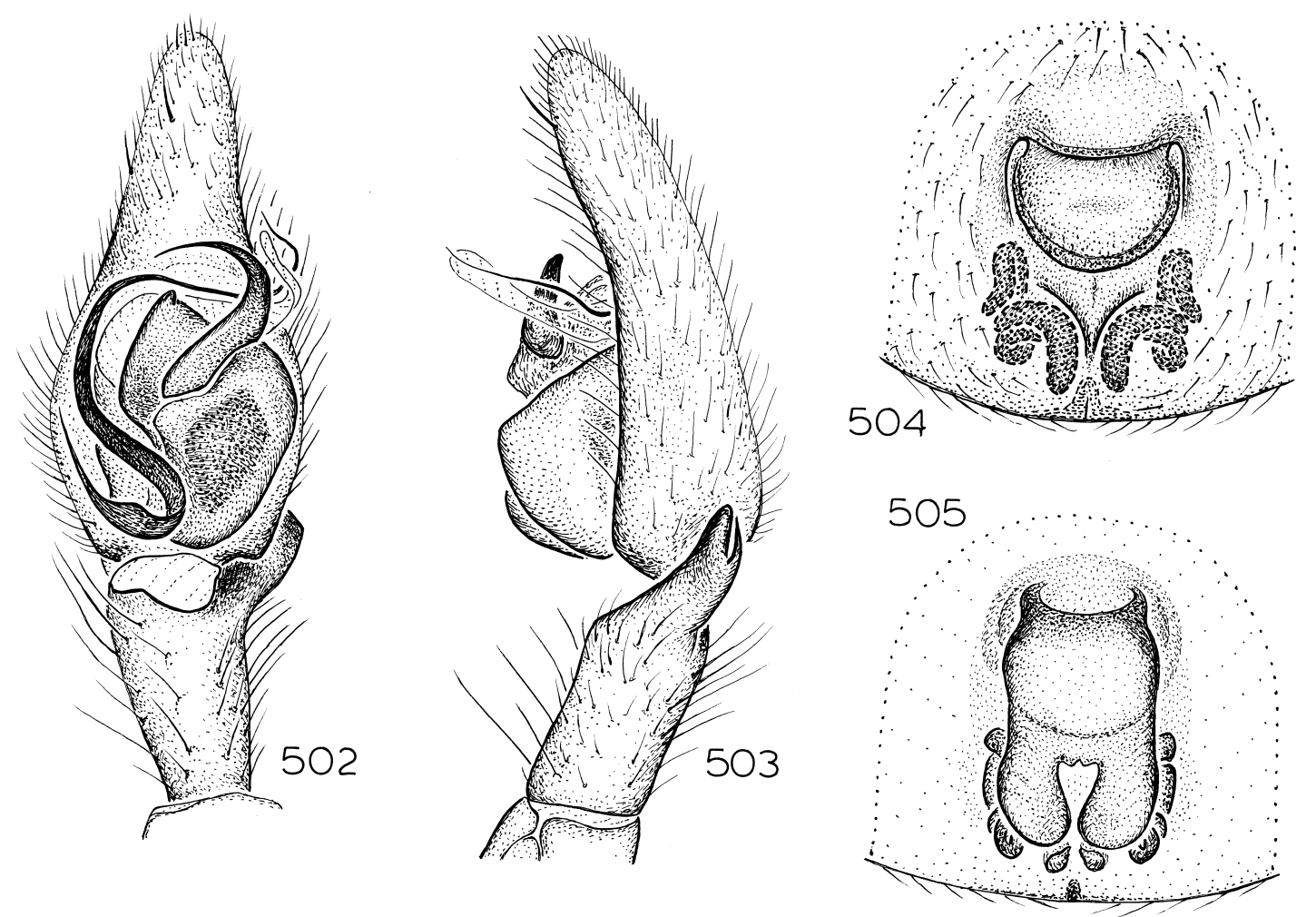

Figs. 502-505. Centrocalia ningua, new species. 502. Left male palp, ventral view. 503. Same, retrolateral view. 504. Epigynum, ventral view. 5055. Same, dorsal view.

chazeaui but have a much wider retrolateral tibial apophysis and a much longer embolar apophysis (figs. 502, 503); females have a much shorter epigynal atrium that is confined to the anterior half of the epigynal length (fig. 504).

MALE: Total length 5.5. Carapace dark chestnut brown, abdomen pale white anteriorly and posteriorly, dark gray medially; legs orange, coxae darkest. Leg spination: femora I, II d0-0-0; tibiae, metatarsi III, IV p0-0-0, v0-0-0, r0-0-0. Retrolateral tibial apophysis distally thickened, flattened (fig. 503); embolar apophysis long, reaching about half length of palpal bulb (fig. 502).

FEMALE: Total length 5.7. Coloration as in male. Leg spination: femora I-III d0-0-0; tibiae, metatarsi III, IV p0-0-0, v0-0-0, r0-0-0. Epigynal atrium restricted to anterior half of epigynum (fig. 504); spermathecal ducts fused anteriorly (fig. 505).

Other Material Examined: None.
Distribution: Known only from a montane forest on Pic Ningua, north of Nouméa on New Caledonia.

\section{Centsymplia, new genus}

TyPE SPECIES: Centsymplia glorious, new species.

ETYMOLOGY: The generic name is an arbitrary combination of letters, considered feminine in gender.

DiAGNOSIS: Members of this genus are most easily recognized by their odd genitalic characters: males have a tiny median apophysis (fig. 506) and long, twisted retrolateral tibial apophysis (fig. 507); females have a long, triangular epigynum (fig. 508) with the ducts restricted to its posterior portion (fig. 509).

DESCRIPTION: Medium spiders, total length 4.6-6.1. Carapace brown, pars cephalica lightest, pars thoracica with few rows of 
slightly darkened maculations radiating from thoracic groove, with setae largely restricted to ocular area; thoracic groove long, longitudinal. Eight eyes in two rows, anterior medians largest, circular, dark, laterals subequal in size, oval, light, posterior medians smallest, irregularly oval, flattened, light; from above, anterior eye row slightly recurved, posterior row slightly procurved, from front, anterior row slightly procurved, posterior row strongly procurved; anterior medians separated by more than their radius, closer to anterior laterals; posterior medians separated by more than their diameter, farther from posterior laterals; anterior and posterior laterals separated by less than their radius; median ocular quadrangle slightly wider in back than in front, wider in front than long. Chelicerae, sternum, and mouthparts brown; chilum wide, triangular, accompanied by second, dumbbell-shaped posterior chilum (extremely narrow sclerite separating bases of chelicerae posteriorly); chelicerae with distinct, orange lateral boss, anterior surface with depressed, relatively unsclerotized oval area near promargin; promargin with row of long setae originating in line along base of fang, seta closest to fang bent near base at almost $90^{\circ}$ angle, extending behind other promarginal setae to near midline; promargin with three teeth, median one largest, proximal one smallest; retromargin with two large, widely separated teeth. Labium elongate, base narrowed at about one-third of labial length, posterior margin truncate, anterior margin medially invaginated, surface not depressed medially. Endites obliquely depressed, with poorly demarcated, deep, wide groove along margin near labium, groove wider anteriorly than posteriorly; serrula long, with single row of teeth. Sternum not elevated, with flat, rebordered lateral margins, not expanded anteriorly, with triangular extensions to and between coxae; surface not tuberculate, with slight elevations opposite, and depressions between, coxae. One epimeric sclerite on each side, above each coxa, reaching sternal triangles only by narrow strips of weakly sclerotized cuticle (strongest strip between coxae II and III), not fused to carapace. Pedicel consisting of small, diamond-shaped sclerite bearing short dorsal and ventral tri- angular ledges; anteroventral edge of sclerite reaching posterior tip of sternum.

Anterior edge of abdomen of male with complete sclerotic ring formed by strong epigastric scutum plus weaker dorsal abdominal scutum covering entire front edge of abdomen, reaching to about seven-eighths of abdominal length, females with dorsal scutum represented only by small semicircular plate above pedicel, restricted to anterior surface of abdomen; cuticle with short, recumbent setae; epigastric scutum accompanied posterolaterally by pair of oval, deeply invaginated sclerites bearing clearly elevated anterior rim; sclerites separated by membranous lobe, anterior rim of sclerites fitting under epigastric scutum; colulus represented only by setae on slight sclerotization; tiny transverse sclerite, well removed from spinnerets, marking position of small posterior spiracle. Anterior lateral spinnerets tubular, separated by almost their diameter, cuticle representing distal, second spinneret segment restricted to semicircle surrounding major ampullate gland spigots (piriform gland spigots surrounded only by soft cuticle); posterior median spinnerets of males large, tubular, of females with anteriorly expanded tips, bases occupied by three enormously widened cylindrical gland spigots; posterior lateral spinnerets two-segmented, those of female with one enormously widened cylindrical gland spigot.

Leg spination reduced only on anterior legs; typical leg spination pattern (only surfaces with spines listed): femora I, II d1-0-0, p0-0-1; III, IV d1-0-0; tibiae III, IV p0-1-1, v2-1p-2, r0-1-1; metatarsi III, IV p1-0-0, v21p-0, r1-0-0. Most leg surfaces with coated with short setae; males with all coxae and trochanters dorsally tuberculate; anterior coxae with slightly protuberant posterolateral corners; trochanters slightly notched; anterior metatarsi and tarsi with divided scopulae, composed of laterally directed setae; posterior metatarsi with thick, dark, distal preening brushes; tarsi III, IV with entire scopulae; tarsi with two dentate claws, claw tufts composed of few lateral pads of closely appressed setae; trichobothria in two rows on metatarsi and tarsi. Female palpal femur, patella, tibia, and tarsus with strong prolateral 

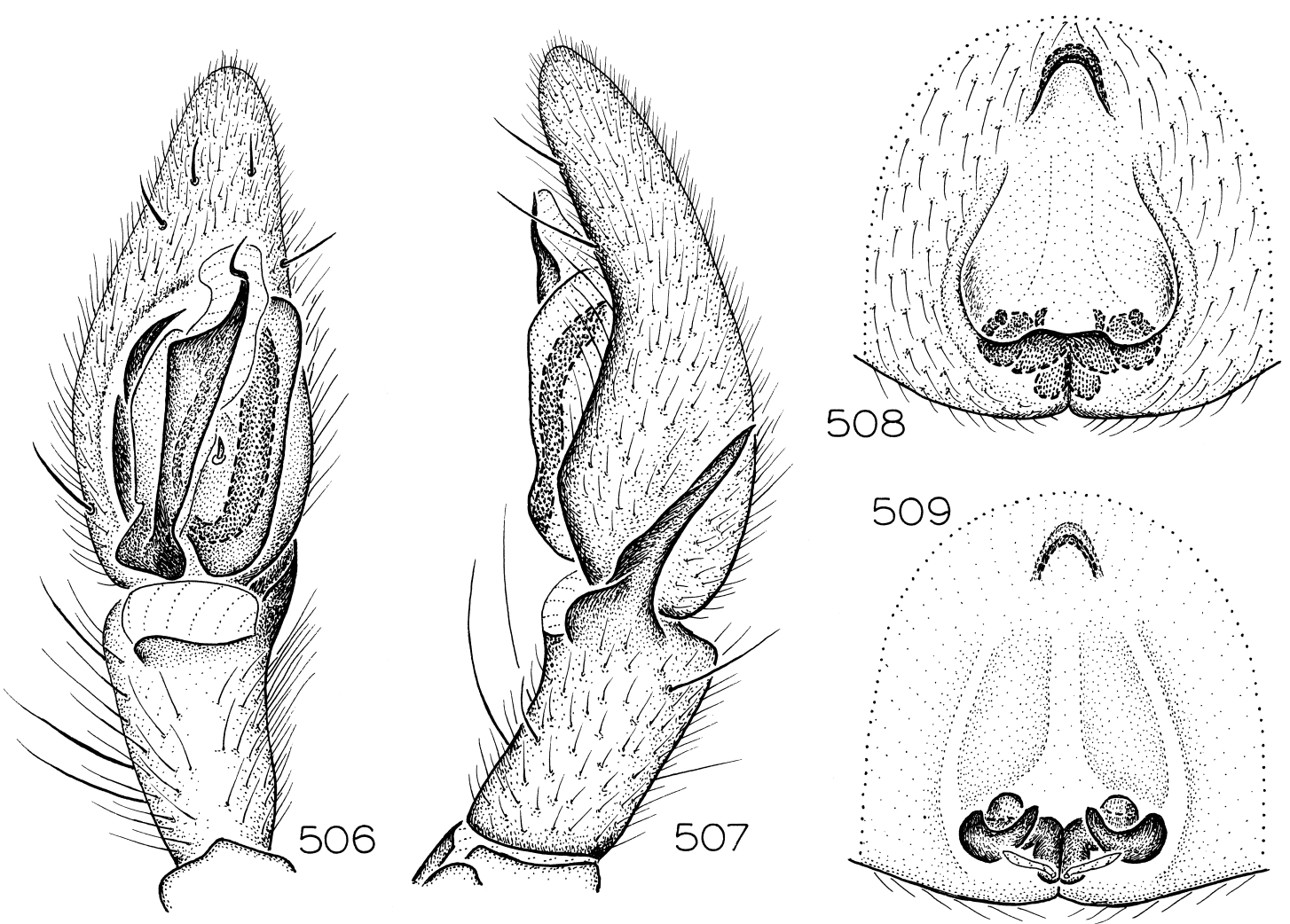

Figs. 506-509. Centsymplia glorious, new species. 506. Left male palp, ventral view. 507. Same, retrolateral view. 508. Epigynum, ventral view. 509. Same, dorsal view.

spines; female palpal tarsus with long, basally dentate claw.

Male palp with long retrolateral tibial apophysis; cymbial surface excavated opposite tibial apophysis; tegulum expanded at proventral corner, bearing tiny, vestigial median apophysis opposing small terminal apophysis; embolus originating prolaterally, long, wide, accompanied distally by membranous conductor. Epigynum with anterior hood and shallow atrium; spermathecae accompanied medially by pair of longitudinal ducts, all restricted to posterior portion of epigynum.

\section{Centsymplia glorious, new species} Figures 506-509; Map 56

TYPE: Female holotype taken under bark on Mount Glorious, $27^{\circ} 20^{\prime} \mathrm{S}, 152^{\circ} 46^{\prime} \mathrm{E}$, Queensland (July 19, 1974; V. Davies, R. Raven), deposited in QMB (S26507).
ETyMology: The specific name is a noun in apposition taken from the type locality.

DiAGNOSIS: The medially expanded retrolateral tibial apophysis (fig. 507) and apically recurved embolus (fig. 506) of males, and the u-shaped posterior epigynal ducts (fig. 509) of females, are diagnostic.

MALE: Total length 4.6. Dorsum of abdomen with transverse white stripes situated at about half of abdominal length, visible through scutum; sides gray, interrupted by narrow longitudinal pale white lines; venter pale white with two longitudinal, paramedian rows of small orange sclerotizations; legs unmarked. Leg spination typical for genus. Retrolateral tibial apophysis expanded at about half its length (fig. 507); tegulum greatly expanded at base of embolus; median apophysis vestigial; tegular apophysis small; embolus recurved apically (fig. 506).

FEMALE: Total length 6.1. Coloration as in 
male. Leg spination: femur III p0-0-1; tibia IV r1-1-1. Epigynum long, triangular, with narrow, arched hood (fig. 508); ducts restricted to posterior portion of epigynum (fig. 509).

Other Material Examined: New South Wales: Bruxner Park, Orara East State Forest, Coffs Harbour, $30^{\circ} 18^{\prime} \mathrm{S}, 153^{\circ} 08^{\prime} \mathrm{E}$, May 25-26, 1986 (D. Hirst, SAM N1997/80), 10 . Queensland: Binna Burra, Lamington National Park, $28^{\circ} 12^{\prime} \mathrm{S}, 153^{\circ} 11^{\prime} \mathrm{E}$, May 17 , 1983, on tree trunk at night (V. Davies, J. Gallon, D. Court, QMB S26508), 1 \%, Mar. 27-30, 1976, night (R. Raven, V. Davies, QMB S26513), 20, Apr. 5, 1995, rainforest, night (R. Raven, QMB S27528), $10^{\dagger}$; Conondale Range, $26^{\circ} 45^{\prime} \mathrm{S}, 152^{\circ} 37^{\prime} \mathrm{E}$, May $1-3$, 1976 (R. Raven, QMB S29347), 1 đ; Sunday Creek Road, junction with Gigher Creek Road, Kenilworth State Forest, $26^{\circ} 42^{\prime} \mathrm{S}$, $152^{\circ} 33^{\prime} \mathrm{E}$, May 7, 1998, wet sclerophyll forest (G. Milledge, AMS KS52281), 10 , 3 ; ; Mount Glorious, $27^{\circ} 20^{\prime} \mathrm{S}, 152^{\circ} 46^{\prime} \mathrm{E}$, Apr. 13-May 26, 1983, rainforest, malaise trap (A. Hiller, QMB S26477), 10, May 26-Aug. 10, 1983, rainforest, malaise trap (A. Hiller, QMB S26490), 20; O’Reillys, Lamington National Park, $28^{\circ} 14^{\prime} \mathrm{S}, 153^{\circ} 08^{\prime} \mathrm{E}$, Sept. $25-$ 26, 1986, rainforest (R. Raven, J. Gallon, QMB S26861), 1 ㅇ.

Distribution: Southeastern Queensland and northeastern New South Wales (map 56).

\section{Queenvic, new genus}

TyPe SPECIES: Queenvic mackay, new species.

ETYMOLOGY: The generic name is an arbitrary combination of letters, considered feminine in gender.

DiAgnOSIS: Males are best recognized by the prolaterally arched terminal apophysis (figs. 510, 514, 518, 522), females by the epigynum having a pair of lateral pockets and medially situated, recurved ducts (figs. 512, 516, 520, 524).

DESCRIPTION: Small spiders, total length of males 2.5-2.9, of females 2.6-3.2. Carapace brownish orange, almost entirely coated with large seta-bearing tubercles, pars cephalica elevated, pars thoracica elevated to region behind thoracic groove, steeply sloping posteriorly; thoracic groove short, deep. Eight eyes in two rows, anterior medians smallest, circular, dark, laterals subequal in size, oval, light, posterior medians largest, irregularly oval, flattened, light; from above, anterior eye row slightly recurved, posterior row slightly procurved, from front, both rows slightly procurved; anterior medians separated by more than their diameter, closer to anterior laterals; posterior medians separated by roughly their diameter,by about as much from posterior laterals; anterior and posterior laterals separated by about their diameter; median ocular quadrangle wider in back than in front, wider in back than long. Chelicerae, sternum, and mouthparts light brownish orange; chilum narrow, triangular, reflexed under medially elongated clypeus, accompanied by second, longitudinal posterior chilum (extremely narrow sclerite separating bases of chelicerae posteriorly); chelicerae with distinct lateral boss, anterior surface with large, sharply pointed, seta-bearing tubercles, with diffuse, relatively unsclerotized area near promargin; promargin with row of long setae originating in line along base of fang, seta closest to fang bent near base at almost $90^{\circ}$ angle, extending to near midline; promargin with three teeth, median one largest, proximal one smallest; retromargin with two large, narrowly separated teeth. Labium elongate, triangular, gradually narrowed anteriorly, posterior margin truncate, anterior margin slightly invaginated at middle, surface distinctly depressed medially. Endites obliquely depressed, with sharply demarcated, deep groove along margin near labium; serrula long, with single row of teeth. Sternum slightly elevated, with gradually sloping lateral margins, not expanded anteriorly, with triangular extensions to and between coxae; surface smooth medially, with large tubercles around margins. One epimeric sclerite on each side, above each coxa, reaching but not fused to sternal triangles, not fused to carapace or pedicel. Pedicel consisting of large, pentagonal sclerite bearing wide anterior margin meeting posterior tip of sternum at transverse line.

Anterior edge of abdomen of male with complete sclerotic ring formed by strong epigastric scutum surrounding pedicel; males with strong dorsal abdominal scutum covering entire front edge of abdomen, reaching 
entire length of abdomen, females with dorsal scutum represented only by small transverse plate above pedicel, restricted to lower one-third of anterior surface of abdomen; cuticle with long, recumbent setae, with white scale-like setae over pair of anterior white spots and pair of transverse white stripes at about one-third of abdominal length; epigastric scutum accompanied posterolaterally by pair of oval, deeply invaginated sclerites bearing clearly elevated anterior rim; anterior rim of sclerites fitting under epigastric scutum; colulus represented only by pair of long setae; posterior spiracle transverse, on small, triangular plate. Anterior lateral spinnerets tubular, separated by about their diameter, cuticle representing distal, second spinneret segment restricted to semicircle surrounding major ampullate gland spigots (piriform gland spigots surrounded only by soft cuticle); posterior median spinnerets of males large, tubular, of females with anteriorly expanded tips, bases occupied by three enormously widened cylindrical gland spigots; posterior lateral spinnerets two-segmented, those of females with greatly widened cylindrical gland spigots.

Leg spination greatly reduced; typical leg spination pattern (only surfaces with spines listed): tibiae IV v0-0-1p. Most leg surfaces with long setae; both sexes with all coxae dorsally tuberculate; anterior coxae with slightly protuberant posterolateral corners, slightly widened at about half their length; trochanters unnotched; tarsi without distinct scopulae; posterior metatarsi with thick, distal preening brushes; tarsi with two finely dentate claws, claw tufts composed of lateral pads of short, closely appressed setae; trichobothria present in double row on tarsi, single row on metatarsi and tibiae. Female palpal patella, tibia, and tarsus with long, thin spines; female palpal tarsus with long, basally dentate claw accompanied by triad of short spines.

Male palp with long retrolateral tibial apophysis; cymbial surface slightly excavated opposite tibial apophysis; tegulum wide, with long, distally hooked median apophysis; large terminal apophysis excavated laterally, functioning as conductor; embolus originating posteriorly, long, looping through terminal apophysis to actual conductor recessed at retrolateral side of terminal apophysis, with complex lobes; embolar base without apophysis. Epigynum with anterior hood, pair of median openings, and pair of posterolateral pockets; spermathecae accompanied by looping ducts.

\section{Key to Species of QueENVIC}

1. Retrolateral tibial apophysis long, narrow throughout its length (figs. 511, 515); anterior portion of epigynal ducts oriented transversely (figs. 512, 516) . . . . . 2

- Retrolateral tibial apophysis shorter, widened at figs. 519, 523); anterior portion of epigynal ducts oriented obliquely (figs. 520, 524) ................. 3

2. Ventral edge of retrolateral tibial apophysis convex (fig. 511); anterior portion of epigynal ducts far from epigynal hood (fig. 512) ................ mackay

- Ventral edge of retrolateral tibial apophysis concave (fig. 515); anterior portion of epigynal ducts near epigynal hood (fig. 516) .................. kelty

3. Retrolateral tibial apophysis widened, flattened near tip (fig. 523); epigynal pockets relatively small (fig. 524) ... piccadilly

- Retrolateral tibial apophysis narrowed at tip (fig. 519); epigynal pockets relatively large (fig. 520) ................ goanna

\section{Queenvic mackay, new species}

Figures 510-513; Map 57

TyPES: Female holotype and male allotype taken in Berlese sample of Acacia harpophylla litter $90 \mathrm{mi} \mathrm{S}$ Mackay, $21^{\circ} 09^{\prime} \mathrm{S}$, $149^{\circ} 12^{\prime} \mathrm{E}$, Queensland (July 18, 1968; L. Mound), deposited in QMB.

ETYMOLOGY: The specific name is a noun in apposition taken from the type locality.

DiAGNOSIS: Males can be recognized by the long, narrow, ventrally convex retrolateral tibial apophysis (fig. 511), females by the transverse anterior loops of the epigynal ducts situated far from the epigynal hood (figs. 512).

MALE: Total length 2.8. Abdominal dorsum with pair of white spots at anterolateral corners and pair of transverse white stripes at about one-third of length; venter gray with two paramedian longitudinal rows of orange sclerites; anterior femora, posterior femora through metatarsi darkened laterally. Leg spination typical for genus. Retrolateral tibial 

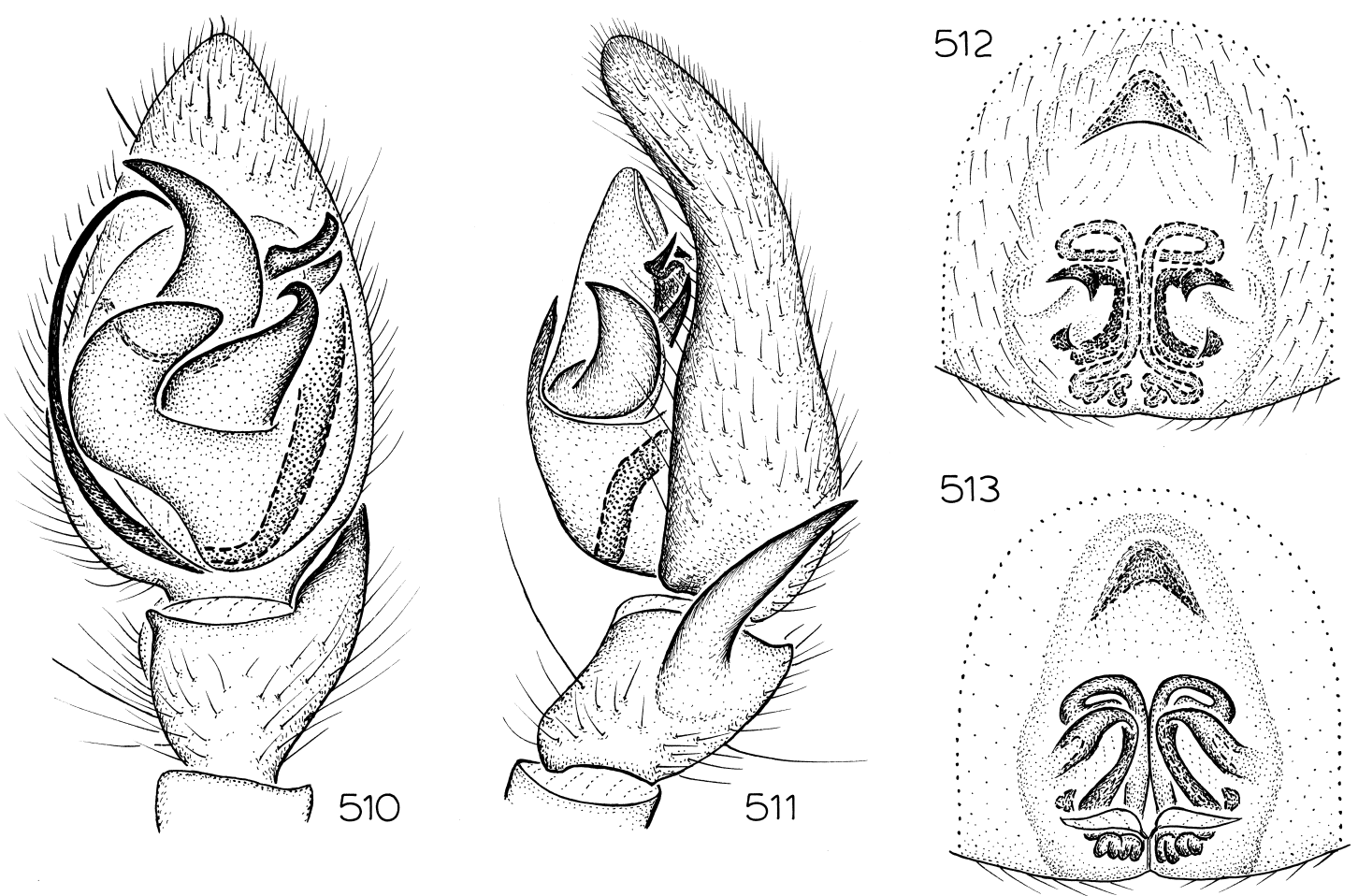

Figs. 510-513. Queenvic mackay, new species. 510. Left male palp, ventral view. 511. Same, retrolateral view. 512. Epigynum, ventral view. 513. Same, dorsal view.

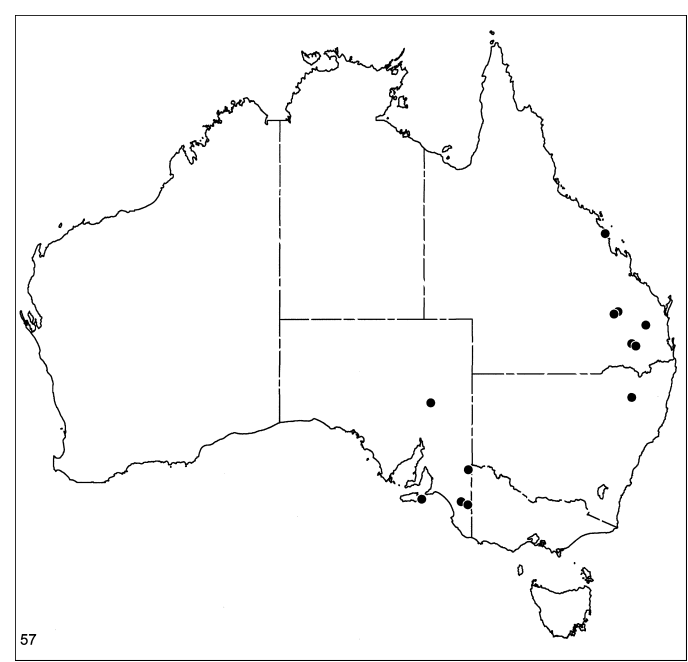

Map 57. Records of Queenvic mackay, new species. apophysis long, narrow (fig. 511); conductor with two retrolaterally directed processes, anterior one heavily sclerotized (fig. 510).

Female: Total length 3.0. Coloration as in male. Leg spination typical for genus. Epigynal hood situated far anterior of ducts (fig. 512 ); anterior portion of epigynal ducts oriented transversely (fig. 513).

Other Material Examined: New South Wales: Nandewar Range, $40 \mathrm{~km}$ E Narrabri on Bingara-Narrabi Road, $30^{\circ} 10^{\prime} \mathrm{S}$, $150^{\circ} 51^{\prime} \mathrm{E}$, Oct. 1,1990 , litter, open forest 3 $\mathrm{km}$ from road (J. Thompson, AMS K29915), 1 ․ Queensland: Boggomoss, Taroom District, $25^{\circ} 28^{\prime} \mathrm{S}, 150^{\circ} 01^{\prime} \mathrm{E}$, Sept. 10-Nov. 11, 1996, pitfall, field (P. Lawless, QMB S34306), 1 \% ; Braemar State Forest, $27^{\circ} 13^{\prime} \mathrm{S}$, $150^{\circ} 50^{\prime} \mathrm{E}$, Feb. 4-8, 1980 (R. Raven, QMB S26494), 1o, 1 웅 Keysland, $26^{\circ} 12^{\prime} \mathrm{S}$, $151^{\circ} 44^{\prime}$ E, Sept. 29-Dec. 5, 1994, intercept trap, open forest (G. Monteith, QMB S34311), 10; Lake Broadwater, via Dalby, $27^{\circ} 21^{\prime} \mathrm{S}, 151^{\circ} 06^{\prime} \mathrm{E}$, Jan. 26-Feb. 18, 1985, pitfall (QMB S28611), 1 ㅇ, Feb. 19-Mar. 26, 

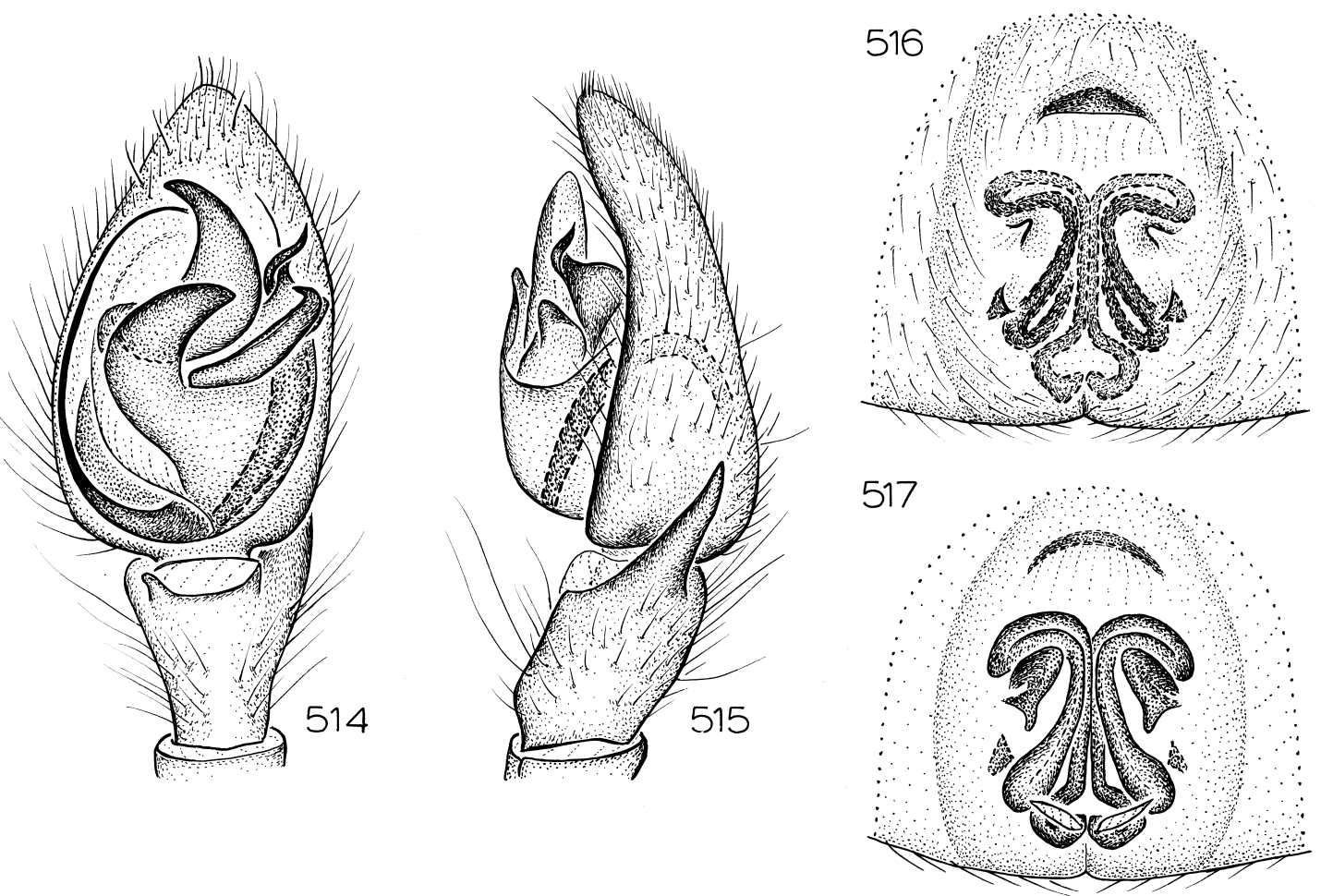

Figs. 514-517. Queenvic kelty, new species. 514. Left male palp, ventral view. 515. Same, retrolateral view. 516. Epigynum, ventral view. 517. Same, dorsal view.

1985, pitfall (M. Bennie, QMB S34308), 1 ㅇ, Mar. 25-May 16, 1985, pitfalls (QMB S30486), 20, 1 9 , May 16-Nov. 23, 1985, pitfalls (QMB S28591), 80ิ, 9오, Nov. 24, 1985-Jan. 3, 1986, pitfalls (M. Bennie, QMB

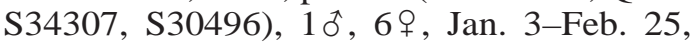
1986, pitfalls (M. Bennie, QMB S30495), 5ô, 9 ㅇ, Feb. 25-Apr. 22, 1986, pitfalls (M. Bennie, QMB S30497), 50ิ, 3 ㅇ, Apr. 22June 12, 1986, pitfalls (M. Bennie, QMB S28598), 30 , 1 9 ; 7 km N Ooline Scrub, Taroom District, $25^{\circ} 36^{\prime} \mathrm{S}, 149^{\circ} 46^{\prime} \mathrm{E}$, Sept. 11Nov. 12, 1996, pitfall, roadside (P. Lawless, QMB S34309), 10, Nov. 12, 1996-Jan. 13, 1997, pitfall, roadside (P. Lawless, QMB S36686), 40ิ, 4 ㅇ. South Australia: between Bucks Camp Well and Rabbit Island, Mount Rescue Conservation Park, $35^{\circ} 56^{\prime} \mathrm{S}$, $140^{\circ} 19^{\prime}$ E, Mar. 18, 1992, vibration (D. Hirst, SAM N1997/84), 19 ; Dudley Conservation Park, Kangaroo Island, $35^{\circ} 48^{\prime} \mathrm{S}, 137^{\circ} 51^{\prime} \mathrm{E}$, Nov. 5-11, 1990 (E. Matthews, J. Forrest, SAM N1997/81), 10ิ; Dudley Conservation Park, Kangaroo Island, $35^{\circ} 48^{\prime} \mathrm{S}, 137^{\circ} 52^{\prime} \mathrm{E}$,
Nov. 11-12, 1987 (D. Hirst, SAM N1997/ 126, 127), 2 \% 2.4 km SE Graythwaite High School, $36^{\circ} 07^{\prime} \mathrm{S}, 140^{\circ} 44^{\prime} \mathrm{E}$, Dec. 12-15, 1995, pitfall (SAM N1997/82), 1 \% ; Renmark, $34^{\circ} 11^{\prime} \mathrm{S}, 140^{\circ} 45^{\prime} \mathrm{E}$ (QMB), $1 \%$; Telford Scrub Conservation Park, $30^{\circ} 28^{\prime} \mathrm{S}$, $138^{\circ} 25^{\prime} \mathrm{E}$, Feb. 12, 1995 (T. Collins, SAM N1997/83), 1 오.

Distribution: Mid-eastern Queensland south to eastern South Australia (map 57).

\section{Queenvic kelty, new species}

Figures 514-517; Map 58

TYPE: Female holotype taken under eucalypt bark at Separation Creek, 38 38'S, $143^{\circ} 54^{\prime} \mathrm{E}$, Victoria (Sept. 19-21, 1989; M. Harvey, M. Blosfelds), deposited in WAM (96/204).

ETYMOLOGY: The specific name is an arbitrary combination of letters.

Diagnosis: This seems to be the sister species of $Q$. mackay; males can be distinguished by having the longer, thinner, and 
more distally directed distal prong of the palpal conductor situated farther from the subdistal prong (fig. 514), females by the longer anterior epigynal ducts situated near the epigynal hood (fig. 516).

MALE: Total length 2.5. Coloration as in Q. mackay except abdominal dorsum with anterior pair of spots weak, posterior stripes situated at about half of abdominal length; anterior femora darkened on all sides. Leg spination typical for genus. Retrolateral tibial apophysis shorter, basally wider than in $Q$. mackay (fig. 515); distal prong of conductor narrow, long, directed distally, well separated from retrolaterally directed subdistal prong (fig. 514).

Female: Total length 3.2. Coloration as in male. Leg spination typical for genus. Epigynal hood near anterior ducts (fig. 516); anterior ducts extending to sides of epigynum (fig. 517).

Other Material Examined: South Australia: Grassdale, Kelly Hill Conservation Park, $36^{\circ} 00^{\prime} \mathrm{S}, 136^{\circ} 52^{\prime} \mathrm{E}$, Nov. 9, 1987, pitfall (D. Hirst, SAM N1997/85), $1 \delta^{\star}$. Victoria: Separation Creek, $38^{\circ} 38^{\prime} \mathrm{S}, 143^{\circ} 54^{\prime} \mathrm{E}$, Aug. 28, 1988, Berlese, rotting bark (M. Harvey, M. Blosfelds, WAM 96/203), 1 ơ, Sept. 19-21, 1989, under eucalypt bark (M. Harvey, M. Blosfelds, WAM 96/205), 1 ㅇ.

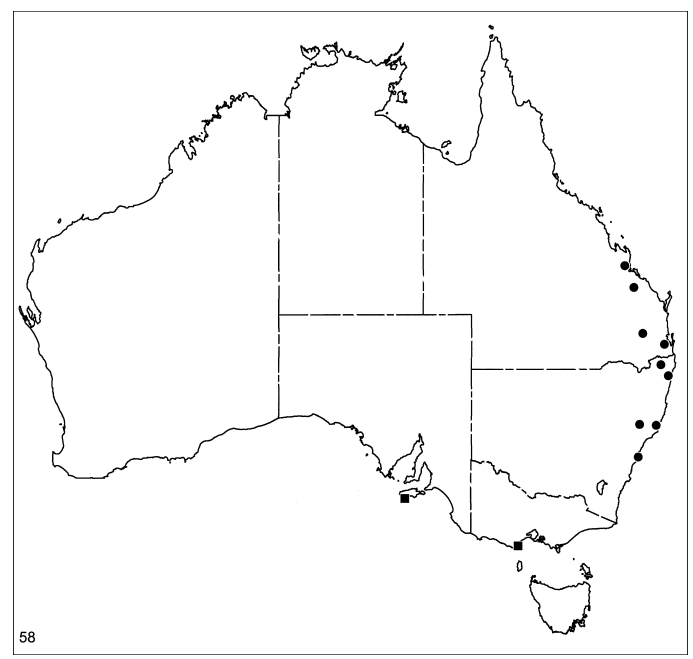

Map 58. Records of Queenvic kelty, new species (squares) and $Q$. goanna, new species (circles).
Distribution: South Australia and Victoria (map 58).

\section{Queenvic goanna, new species}

Figures 518-521; Map 58

TYPE: Female holotype taken in pitfall trap at an elevation of $545 \mathrm{~m}$ on Goanna Creek Road, Richmond Range State Forest, $28^{\circ} 36^{\prime} \mathrm{S}, 152^{\circ} 41^{\prime} \mathrm{E}$, New South Wales (Feb. 4-Apr. 9, 1993; M. Gray, G. Cassis), deposited in AMS (KS36011).

ETYMOLOGY: The specific name is a noun in apposition taken from the type locality.

DiAgnosis: This seems to be the sister species of $Q$. piccadilly, sharing with it a similar, transversely oriented distal prong on the male palpal conductor and longitudinally oriented anterior epigynal ducts; males can easily be distinguished by the shorter, wider retrolateral tibial apophysis (fig. 519), females by the short, wider epigynal hood and larger epigynal pockets (fig. 520).

MALE: Total length 2.9. Coloration as in Q. mackay except carapace with patches of white scales laterally on pars cephalica and on intercoxal areas of pars thoracica. Leg spination typical for genus. Retrolateral tibial apophysis relatively short, wide, sharply pointed (fig. 519); distal lobe of conductor oriented transversely, subdistal lobe large, rounded (fig. 518).

Female: Total length 2.9. Coloration as in male. Leg spines absent. Epigynal hood wide, short, lateral pockets large (fig. 520); anterior ducts oriented longitudinally, expanded anteriorly (fig. 521).

Other Material Examined: New South

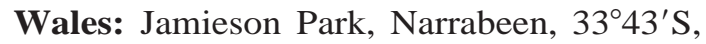
$151^{\circ} 18^{\prime}$ E, Nov. 6-20, 1995, pitfall, angophora woodland, ridge top, elev. $20 \mathrm{~m}$ (M. Gray, H. Smith, AMS KS49606), 10; Kiwarrak State Forest, near Taree, $31^{\circ} 58^{\prime} \mathrm{S}, 152^{\circ} 26^{\prime} \mathrm{E}$, Aug.6-Sept. 19, 1979, pitfall, litter (W. King, AMS KS3954), 10̊; Mount Brown Road, 0.1 km N Y intersection at Mount Brown, Richmond Range State Forest, $28^{\circ} 37^{\prime} \mathrm{S}, 152^{\circ} 43^{\prime} \mathrm{E}$, Feb. 4-Apr. 9, 1993, pitfall, elev. 480 m (M. Gray, G. Cassis, AMS KS36021), $10^{\star}$; junction, Range and Lockleys Roads, Doubleduke State Forest, $29^{\circ} 14^{\prime} \mathrm{S}, 153^{\circ} 11^{\prime} \mathrm{E}, \mathrm{Feb}$. 4-Apr. 9, 1993, pitfall, elev. 30 m (M. Gray, G. Cassis, AMS KS39598), $1 \%$; junction, 

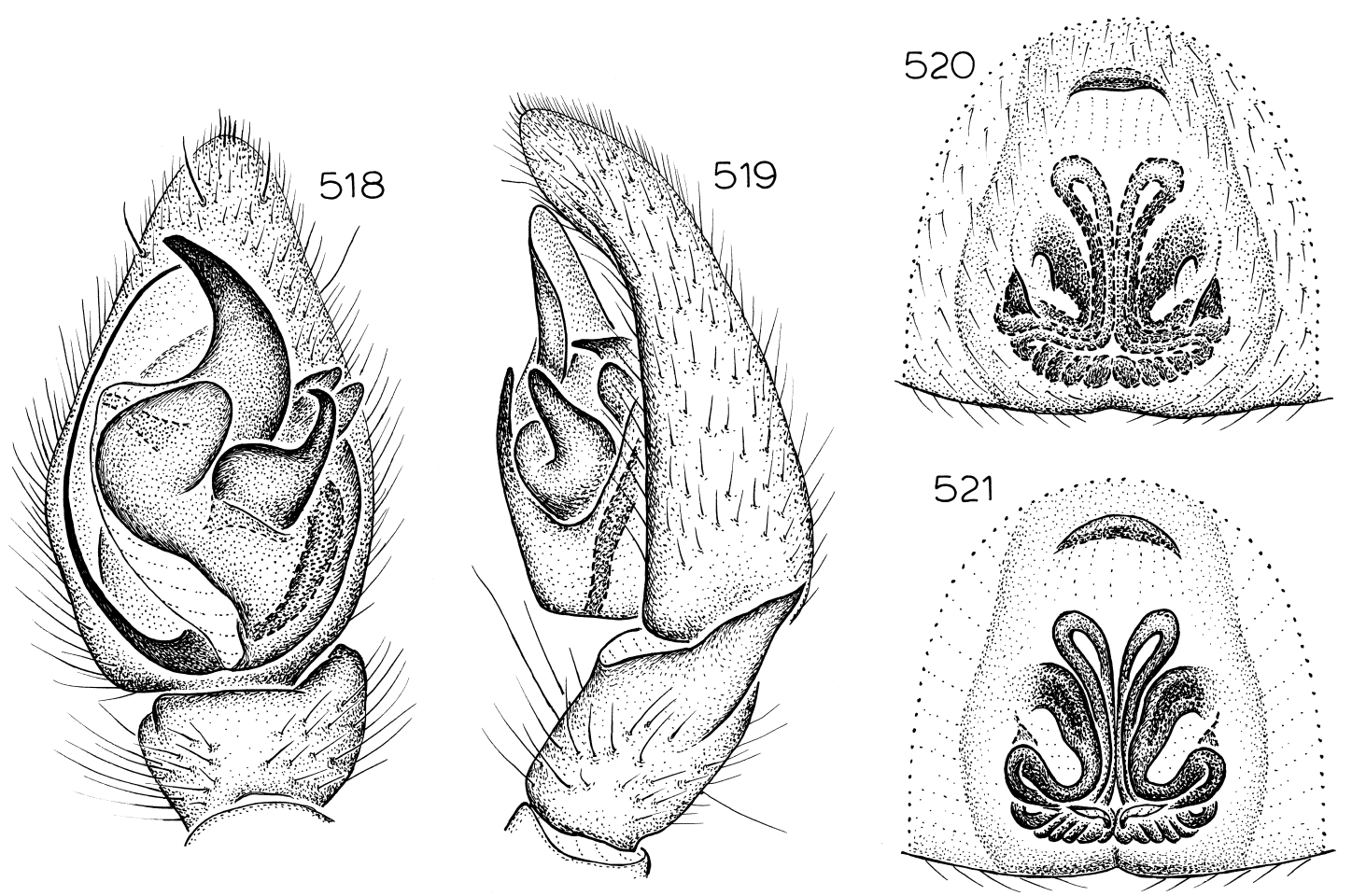

Figs. 518-521. Queenvic goanna, new species. 518. Left male palp, ventral view. 519. Same, retrolateral view. 520. Epigynum, ventral view. 521. Same, dorsal view.

Tubrabucca and Omadale Brook Roads, Stewarts Brook State Forest, $31^{\circ} 55^{\prime} \mathrm{S}, 151^{\circ} 24^{\prime} \mathrm{E}$, Nov. 22-23, 1996, elev. 1360 m (N. Platnick, R. Raven, V. Ovtsharenko, K. Catley, AMNH), 19 . Queensland: Kroombit Tops, $24^{\circ} 22^{\prime} \mathrm{S}, 151^{\circ} 01^{\prime} \mathrm{E}, \mathrm{Feb} .25,1982$, open forest, under logs (R. Raven, QMB S28283), 10; Marlaybrook, Bunya Mountains, $26^{\circ} 54^{\prime} \mathrm{S}, 151^{\circ} 35^{\prime} \mathrm{E}$, Mar. 2, 1976, litter (R. Raven, V. Davies, QMB S28117), 20; Olsens Caverns, The Caves, $23^{\circ} 10^{\prime} \mathrm{S}, 150^{\circ} 28^{\prime} \mathrm{E}$, Apr. 21-July 18, 1990, open forest, pitfall (D. Wallace, R. Raven, QMB S30407), 10; Upper Brookfield, $27^{\circ} 30^{\prime} \mathrm{S}, 152^{\circ} 55^{\prime} \mathrm{E}$, Oct.Nov. 1980, araucaria litter (R. Raven, V. Davies, QMB S28121), 1 .

DisTRIBUTION: Queensland and New South Wales (map 58).

\section{Queenvic piccadilly, new species}

Figures 522-525; Map 59

TYPE: Female holotype taken at an elevation of $800 \mathrm{~m}$ at Piccadilly Circus, $35^{\circ} 19^{\prime} \mathrm{S}$, $148^{\circ} 51^{\prime} \mathrm{E}$, Australian Capital Territory (Jan. 16, 1970; R. Taylor), deposited in QMB.

ETYMOLOGY: The specific name is a noun in apposition taken from the type locality.

DIAGNOSIS: This seems to be the sister species of $Q$. goanna (see above); males can easily be distinguished by the flattened, distally angled retrolateral tibial apophysis (fig. 523), females by the more triangular epigynal hood and smaller epigynal pockets (fig. 524).

MALE: Total length 2.7. Coloration as in Q. mackay except carapace with patches of white scales laterally on pars cephalica and on intercoxal areas of pars thoracica; abdominal dorsum with posterior stripes situated at about half of abdominal length; femora slightly darkened but legs otherwise unmarked. Leg spination typical for genus. Retrolateral tibial apophysis relatively long, uniformly wide, with distal portion flattened, angled (fig. 523); distal prong of conductor oriented transversely, close to relatively small subdistal prong (fig. 522). 

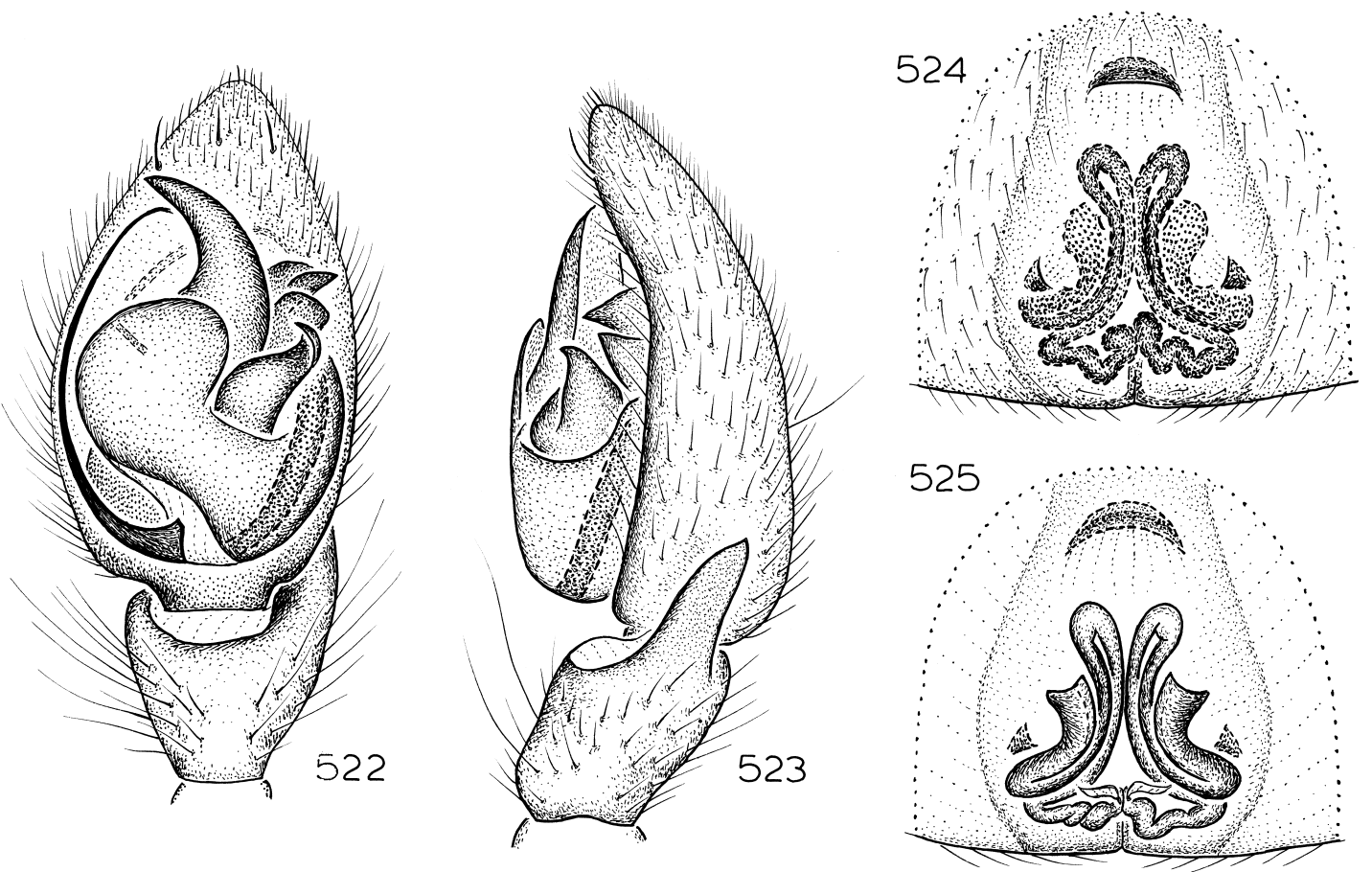

Figs. 522-525. Queenvic piccadilly, new species. 522. Left male palp, ventral view. 523. Same, retrolateral view. 524. Epigynum, ventral view. 525. Same, dorsal view.

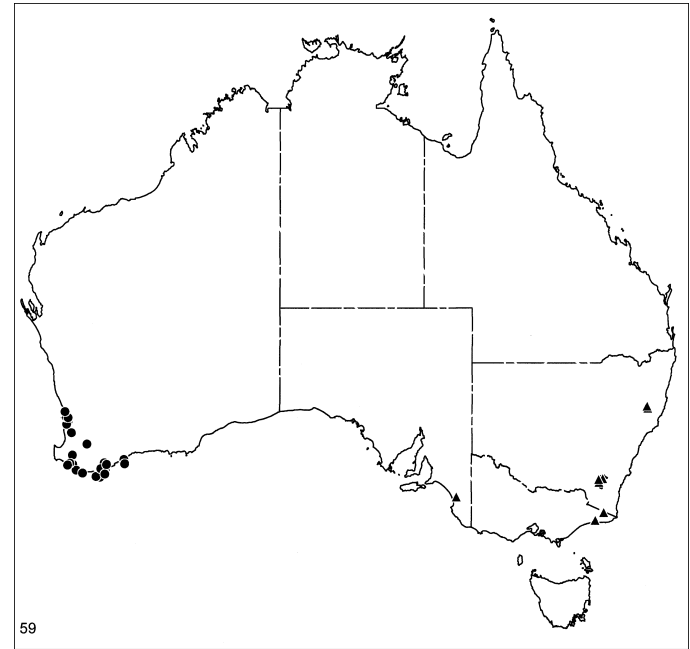

Map 59. Records of Queenvic piccadilly, new species (triangles) and Prionosternum scutatum Dunn (circles).
Female: Total length 2.6. Coloration as in male. Leg spination typical for genus. Epigynal hood triangular, lateral pockets relatively small (fig. 524); anterior ducts oriented longitudinally, expanded anteriorly (fig. 525).

Other Material Examined: Australian Capital Territory: Black Mountain, $35^{\circ} 16^{\prime} \mathrm{S}, 1^{\circ} 9^{\circ} 06^{\prime} \mathrm{E}$, Aug. 7, 1970, dry sclerophyll, elev. 620 m (J. Summers, QMB), 3 đै, 2 ㅇ, July 10, 1976, dry sclerophyll (T. Weir, QMB), 1 ㅇ, Dec. 1983-Jan. 1984, pitfalls (R. Moran, QMB), 10, 1\%; $0.8 \mathrm{~km} \mathrm{~N}$ Black Mountain peak, $35^{\circ} 16^{\prime} \mathrm{S}, 149^{\circ} 06^{\prime} \mathrm{E}$, June 17 , 1970, Berlese, dry sclerophyll, elev. $620 \mathrm{~m}$ (J. Simmons, QMB), 20; Tidbinbilla Nature Reserve, $35^{\circ} 28^{\prime} \mathrm{S}, 148^{\circ} 52^{\prime} \mathrm{E}$, Mar. 9, 1978, pitfall, litter (P. Ormay, AMS KS13949), 10; Wombat Creek, $6 \mathrm{~km}$ NE Piccadilly Circus, $35^{\circ} 19^{\prime} \mathrm{S}, 148^{\circ} 51^{\prime} \mathrm{E}$, Feb. 1984, elev. $750 \mathrm{~m}$ (T. Weir, J. Lawrence, Johnson, QMB), $30^{\star}$. New South Wales: Bondi State Forest, S Bombala, $37^{\circ} 08^{\prime} \mathrm{S}, 149^{\circ} 09^{\prime} \mathrm{E}$, Oct. $15-$ Nov. 14, 1980, eucalypt litter (G. Gowing, AMS KS11168, 11911), 10, $1 \%$; Dodds Fire Trail, 
$1 \mathrm{~km}$ from Enfield Road, Enfield State Forest, $31^{\circ} 23^{\prime} \mathrm{S}, 151^{\circ} 53^{\prime} \mathrm{E}$, Feb. 4-Apr. 9, 1993, pitfall, elev. $1050 \mathrm{~m}$ (M. Gray, G. Cassis, AMS KS37375), 1 đo; Mummel Forest Road, $8.8 \mathrm{~km} \mathrm{~N}$ junction with Enfield Forest Road, Enfield State Forest, $31^{\circ} 16^{\prime} \mathrm{S}, 151^{\circ} 50^{\prime} \mathrm{E}$, Feb. 4-Apr. 9, 1993, pitfall, elev. $1350 \mathrm{~m}$ (M. Gray, G. Cassis, AMS KS37364), 30 . South Australia: lagoon edge, Gum Lagoon Conservation Park, $36^{\circ} 18^{\prime} \mathrm{S}, 140^{\circ} 02^{\prime} \mathrm{E}$, Mar. 26, 1992, vibration (D. Hirst, SAM N1997/86), $1 \delta$; northern lagoon, Gum Lagoon Conservation Park, $36^{\circ} 17^{\prime} \mathrm{S}, 140^{\circ} 01^{\prime} \mathrm{E}$, Mar. 25, 1992, vibration (D. Hirst, J. Forrest, SAM N1997/87), 1․ Victoria: Rich-Murrungowar Coupe, $37^{\circ} 34^{\prime} \mathrm{S}, 148^{\circ} 38^{\prime} \mathrm{E}$, Oct. $29-$ Nov. 6, 1991, pitfall, gully (R. Coy, NMV K3537), 1 + , Mar. 18-25, 1992, pitfall, midslope (R. Coy, NMV K3527), 1 t.

Distribution: Southeastern Australia, except Tasmania (map 59).

\section{Bigenditia, new genus}

TyPe SPeCIES: Bigenditia zuytdorp, new species.

ETYMOLOGY: The generic name is an arbitrary combination of letters, considered feminine in gender.

DiAGNOSIS: Males can easily be recognized by the highly modified chelicerae, which have a projection on each side of the base of the fang, and endites, which have greatly expanded anterolateral corners; females are easily recognized by their massive, deeply excavated and rebordered epigyna with longitudinal median ridges (figs. 528, 533). These are the only known Australian centrothelines in which the sternum has fused with the ventral part of the pedicel (that character occurs also in the New Caledonian genus Centrocalia).

DESCRIPTION: Medium to large spiders, total length of males 6.1-6.5, of females 6.97.3. Carapace dark red, surface coated with large tubercles, sparsely set with long, dark setae; thoracic groove long, longitudinal, deep. Eight eyes in two rows, anterior medians circular, dark, larger than subequal, oval, light laterals, posterior medians largest, irregularly oval, flattened, light; from above, both eye rows almost straight, from front, both rows strongly procurved; anterior me- dians separated by more than their radius, closer to anterior laterals; posterior medians separated by much less than their radius, much farther from posterior laterals; anterior and posterior laterals separated by more than their diameter; median ocular quadrangle wider in back than in front, wider in back than long. Chelicerae, sternum, and mouthparts dark orangish red; chilum wide, triangular, tuberculate, accompanied by second, Ishaped, posterior chilum (extremely narrow sclerite separating bases of chelicerae posteriorly); chelicerae tuberculate except on distinct lateral boss, sparsely set with long, dark setae, anterior surface with depressed, relatively unsclerotized oval area near promargin; promargin with row of long setae originating in line along base of fang, seta closest to fang bent near base at almost $90^{\circ}$ angle, extending behind other promarginal setae to near midline; promargin with three teeth, median one largest, proximal one smallest; retromargin with two large, widely separated teeth; cheliceral gland openings presumably in excavated, reticulate area medial of proximal retromarginal tooth. Chelicerae of males highly modified, with large, dark projections both anterior and posterior of fang base. Labium elongate, base narrowed at about onethird of labial length, posterior margin procurved, anterior margin medially invaginated, surface depressed medially. Endites obliquely depressed, with sharply demarcated, wide, deep groove along margin near labium, groove wider anteriorly than posteriorly; serrula long, strong with single row of teeth. Endites of males highly modified, with large, dark projections at anterolateral corners. Sternum slightly elevated, with slightly sloping lateral margins, not expanded anteriorly, with triangular extensions to and between coxae; surface tuberculate, with distinct elevations opposite, and depressions between, coxae. One epimeric sclerite on each side, above each coxa, entirely fused to sternal extensions, including extensions between coxae I and endites, and between coxae IV, fused area constituting base of pedicel, reaching around sides of pedicel, but not fused above pedicel, not fused to carapace. Dorsal elements of pedicel not fused with sternum.

Anterior edge of abdomen of male with 
complete sclerotic ring formed by strong epigastric scutum; additional strong dorsal scutum reaches to about two-thirds of abdominal length, females with dorsal scutum reduced to small strip above pedicel, not fused to epigastric scutum; cuticle with short, recumbent setae; epigastric scutum accompanied posterolaterally by pair of oval, deeply invaginated sclerites bearing clearly elevated anterior rim; sclerites separated by membranous lobe, anterior rim of sclerites fitting under epigastric scutum; colulus represented only by setae; tiny transverse sclerite, well removed from spinnerets, marking position of small posterior spiracle. Anterior lateral spinnerets tubular, separated by more than their diameter, cuticle representing distal, second spinneret segment restricted to semicircle surrounding major ampullate gland spigots (piriform gland spigots surrounded only by soft cuticle); posterior median spinnerets of males large, tubular, of females with anteriorly expanded tips, bases occupied by three enormously widened cylindrical gland spigots; posterior lateral spinnerets two-segmented, those of females with two greatly widened cylindrical gland spigots.

Leg spination reduced only on anterior legs; typical leg spination pattern (only surfaces with spines listed): tibiae III, IV v1p2-2; metatarsi III, IV p0-0-1, v2-2-2, r0-0-1. Most leg surfaces with short setae originating from large, tuberculate bases; anterior coxae with slightly protuberant posterolateral corners; trochanter very slightly notched; anterior metatarsi and tarsi with divided scopulae, composed of laterally directed setae; posterior metatarsi with thick, dark, distal preening brushes; tarsi III, IV with sparse, divided scopulae; tarsi with two dentate claws, claw tufts composed of lateral pads of closely appressed setae. Female palpal tibia, and tarsus with long, thin spines; female palpal tarsus with long, basally dentate claw.

Male palp with strong retrolateral tibial apophysis; cymbial surface excavated opposite tibial apophysis, not produced into distinct lobe on ventral side of tibial apophysis; tegulum largely restricted to retrolateral side of bulb, bearing large, laterally expanded median apophysis opposing large, elongated terminal apophysis; embolus originating posteriorly, long, occupying prolateral side of bulb, accompanied distally by long, heavily sclerotized conductor; embolar base without apophysis. Epigynum with anterior hood fused to elevated median, longitudinal ridge; atrium on either side of ridge deep, frequently filled with hard plug; spermathecae extended anteriorly beyond epigynal hood.

\section{Key to Species of Bigenditia}

1. Median apophysis relatively wide (fig. 526); posterior margin of epigynum transverse (fig. 528) .............. zuytdorp

- Median apophysis relatively narrow (fig. 530); posterior margin of epigynum oblique (fig. 532) .............. millawa

Bigenditia zuytdorp, new species

Figures 526-529; Map 60

TyPES: Female holotype from taken in dry pitfall trap at Zuytdorp, $27^{\circ} 16^{\prime} \mathrm{S}, 114^{\circ} 04^{\prime} \mathrm{E}$, Western Australia (May 16-21, 1995; M. Harvey), deposited in WAM (96/1396).

ETYMOLOGY: The specific name is a noun in apposition taken from the type locality.

DiAGNOSIS: This western species can be distinguished from its eastern congener by the much wider median apophysis of males (fig. 526) and the posteriorly widened epigynum of females (fig. 528).

MALE: Total length 6.5. Abdominal scutum dark brownish orange, dorsum dark gray except for large, almost triangular white spot above spinnerets; venter almost white except posteriorly, where dark gray markings extend from dorsum around spinnerets; femora olive brown, other leg segments yellow. Leg spination typical for genus. Retrolateral tibial apophysis triangular (fig. 527), median apophysis relatively wide (fig. 526).

Female: Total length 6.9. Coloration as in male except abdominal dorsum with pair of paler patches anteriorly. Leg spination typical for genus. Epigynal atrium widened posteriorly (fig. 528); spermathecae extending only slightly anterior of epigynal hood (fig. 529).

Other Material Examined: South Australia: $10.2 \mathrm{~km}$ E Ampeinna Hills, $27^{\circ} 05^{\prime} \mathrm{S}$, $131^{\circ} 14^{\prime} \mathrm{E}$, Mar. 19-23, 1995, pitfall (SAM N1996/439), 1 ơ; 10.5 km E Ampeinna Hills, $27^{\circ} 05^{\prime} \mathrm{S}, 131^{\circ} 13^{\prime} \mathrm{E}$, Mar. 12-23, 1995, pitfall (SAM N1996/438), 10; 28 km SW Illintjitja, $26^{\circ} 19^{\prime} \mathrm{S}, 130^{\circ} 09^{\prime} \mathrm{E}$, May 8-12, 1993, pitfall 

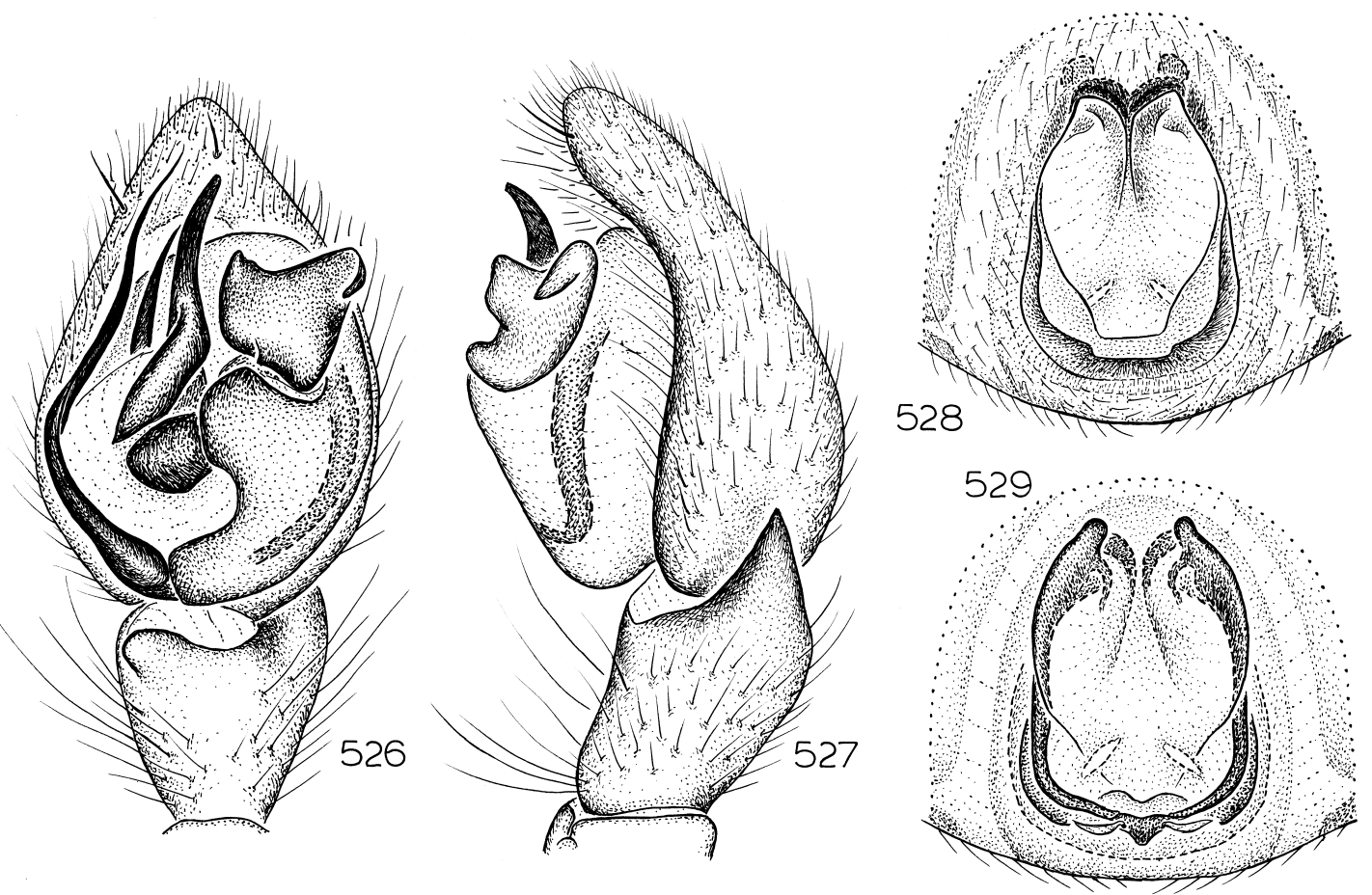

Figs. 526-529. Bigenditia zuytdorp, new species. 526. Left male palp, ventral view. 527. Same, retrolateral view. 528. Epigynum, ventral view. 529. Same, dorsal view.

(SAM N1996/435, 436), 2 ; $9.4 \mathrm{~km} \mathrm{SE}$ Maryinna Hill, $27^{\circ} 01^{\prime} \mathrm{S}, 131^{\circ} 17^{\prime} \mathrm{E}$, Mar. $14-$ 18, 1995, pitfall (SAM N1996/437), 10 . Western Australia: Bidgemia Station, Gasgoyne Junction, $25^{\circ} 03^{\prime} \mathrm{S}, 115^{\circ} 18^{\prime} \mathrm{E}$, June 6Aug. 20, 1995, pitfall (N. Hall, WAM 96/ 1402), 1 \% ; Bidgemia Station, Gasgoyne Junction, $25^{\circ} 13^{\prime} \mathrm{S}, 115^{\circ} 31^{\prime} \mathrm{E}$, Jan. 13-June 5, 1995, pitfalls (J. Waldock, WAM 96/1399, 1400), $20^{\top}$; Boolathana Station, $24^{\circ} 25^{\prime} \mathrm{S}$, $113^{\circ} 40^{\prime} \mathrm{E}$, Jan. 15-May 30, 1995, pitfall (J. Waldock, WAM 96/1407), 10 ; Boolathana Station, $24^{\circ} 25^{\prime} \mathrm{S}, 113^{\circ} 46^{\prime} \mathrm{E}$, Jan. 15-May 31, 1995, pitfall (J. Waldock, WAM 96/1391), $1 \delta^{\circ}$; Bush Bay, $25^{\circ} 07^{\prime} \mathrm{S}, 113^{\circ} 44^{\prime} \mathrm{E}$, May $23-$ Aug. 23, 1994, pitfalls (N. Hall, WAM 96/ 210-212), 30े, Aug. 16-Sept. 28, 1994, pitfalls (P. West, WAM 96/1387-1389), 30ิ, Apr. 4-May 23, 1995, pitfalls (P. West, WAM 96/1393), 10, May 21-26, 1995, dry pitfall (P. West, WAM 96/1403), 1 \% ; Bush Bay, $25^{\circ} 08^{\prime} \mathrm{S}, 113^{\circ} 46^{\prime} \mathrm{E}$, Jan. 16-May 23, 1995, pitfalls (P. West, WAM 96/13791386), 7 ๙, 1 오, May 23-Aug. 23, 1995, pitfalls (N. Hall, WAM 96/1397, 1398), 2 웅

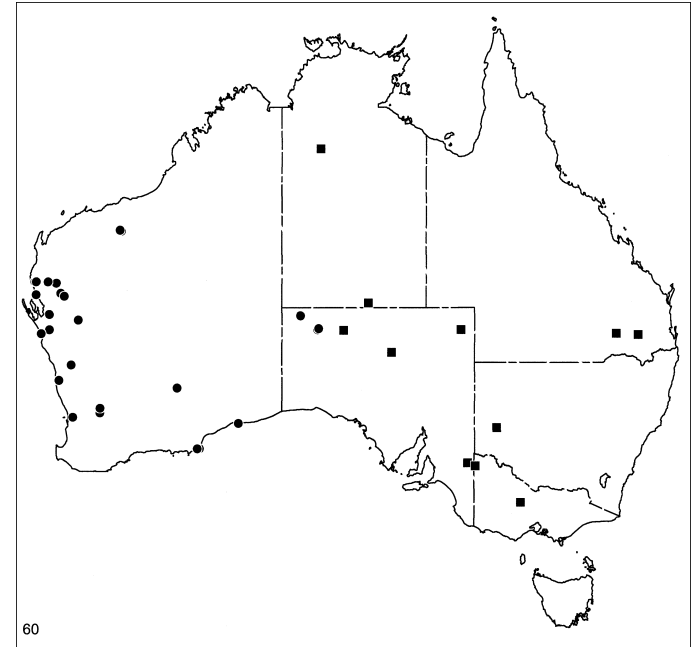

Map 60. Records of Bigenditia zuytdorp, new species (circles) and B. millawa, new species (squares). 
Eneabba, $29^{\circ} 50^{\prime} \mathrm{S}, 115^{\circ} 11^{\prime} \mathrm{E}$, Mar. 22, 1988 (R. McMillan, WAM 96/220), 1 \%; 0.3 km N Eyre Bird Observatory, $32^{\circ} 14^{\prime} \mathrm{S}, 126^{\circ} 18^{\prime} \mathrm{E}$, Mar. 1984 (W. Humphries, WAM 96/207, 208), $20^{\circ}$; Gutha, $29^{\circ} 00^{\prime} \mathrm{S}, 115^{\circ} 56^{\prime} \mathrm{E}$, May 23-Sept. 17, 1996, pitfall (M. Harvey, J. Waldock, WAM 96/1533), 1 \% ; Israelite Bay, $33^{\circ} 37^{\prime} \mathrm{S}, 123^{\circ} 52^{\prime} \mathrm{E}$, Mar. 1996 (SAM N1997/ 88), 10; Kennedy Range National Park, $24^{\circ} 30^{\prime} \mathrm{S}, 115^{\circ} 01^{\prime} \mathrm{E}$, Oct. 3-8, 1994, dry pitfall (M. Harvey, WAM 96/1404), 19 ; Mardathuna Station, $24^{\circ} 24^{\prime} \mathrm{S}, 114^{\circ} 28^{\prime}$ E, Jan. $14-$ May 24, 1995, pitfall (A. Sampey, WAM 96/ 1401), 10, May 23-28, 1995, dry pitfall (A. Sampey, WAM 96/1390), 1 \%, May 24-Aug. 26, 1995, pitfall (N. Hall, WAM 96/1406), 1 \%; Mardathuna Station, $24^{\circ} 26^{\prime} \mathrm{S}, 114^{\circ} 30^{\prime} \mathrm{E}$, Jan. 14-May 24, 1995, pitfall (A. Sampey, WAM 96/1408), 10 ; McLelland's property, $10 \mathrm{~km}$ NW Kellerberrin, $31^{\circ} 38^{\prime} \mathrm{S}, 117^{\circ} 43^{\prime} \mathrm{E}$,

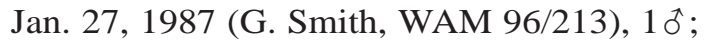
Mount Narryer Station, $26^{\circ} 32^{\prime} \mathrm{S}, 116^{\circ} 22^{\prime} \mathrm{E}$, Apr. 15, 1979 (A., M. Douglas, WAM 84/ 1170), $1 \delta^{\star}$; Nerren Nerren Station, $27^{\circ} 03^{\prime} \mathrm{S}$, $114^{\circ} 35^{\prime} \mathrm{E}$, May 9-14, 1995, dry pitfall (P. West, WAM 96/1405), 1 \% ; $11 \mathrm{~km}$ W Point Dempster, 333' S, $123^{\circ} 44^{\prime} \mathrm{E}$, Mar. 11, 1984 (R. How, WAM 96/215), 1 \% ; Ryan's property, c. $8 \mathrm{~km}$ E Durokoppin Field Station, $31^{\circ} 23^{\prime} \mathrm{S}, 117^{\circ} 44^{\prime} \mathrm{E}$, Mar. 12 , 1987, pitfall (G. Smith, WAM 96/214), 1 ơ, May 6, 1987 (G.

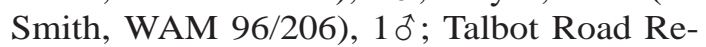
serve, $31^{\circ} 52^{\prime} \mathrm{S}, 116^{\circ} 03^{\prime} \mathrm{E}$, Jan. 6-Mar. 18, 1994, pitfall (M. Harvey, J. Waldock, WAM 96/209), 1 §, Mar. 18-May 19, 1994, pitfall (M. Harvey, J. Waldock, WAM 96/216), 1 ; Woodleigh Station, $26^{\circ} 13^{\prime} \mathrm{S}, 114^{\circ} 35^{\prime} \mathrm{E}$, May 17-Aug. 21, 1995, pitfall (N. Hall, WAM 96/ 1378), 1 \%; Woodstock Station, $21^{\circ} 37^{\prime} \mathrm{S}$, $118^{\circ} 58^{\prime}$ E, May 3-9, 1988, fence line (J. Dell, WAM 96/218, 221), 2ð, Apr. 21, 1989 (M.

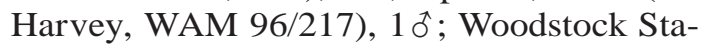
tion, $21^{\circ} 40^{\prime} \mathrm{S}, 119^{\circ} 03^{\prime} \mathrm{E}$, May 3-9, 1988, drift pitfall (J. Waldock, WAM 96/219), 1 \% ; $15 \mathrm{~km} \mathrm{~N}$ Yindi Station, $30^{\circ} 16^{\prime} \mathrm{S}, 122^{\circ} 29^{\prime} \mathrm{E}$, Mar. 11, 1996, in burrow on salt lake (P. Hudson, SAM N1996/457), 1 ‘; Zuytdorp, $27^{\circ} 16^{\prime} \mathrm{S}, 114^{\circ} 04^{\prime} \mathrm{E}$, May 18-Aug. 16, 1995, pitfall (N. Hall, WAM 96/1392), 1 ㅇ.

DistRIBUTION: Western sister species of $B$. millawa (map 60).
Bigenditia millawa, new species Figures 530-533; Map 60

TYPE: Male holotype taken in drift fence pitfall trap $20.4 \mathrm{~km} \mathrm{~N}$ Millawa South Bore, $34^{\circ} 35^{\prime} \mathrm{S}, 141^{\circ} 03^{\prime} \mathrm{E}$, Victoria (Feb. 1987; A. Yen), deposited in NMVS.

ETYMOLOGY: The specific name is a noun in apposition taken from the type locality.

DIAGNOSIS: This eastern sister species of $B$. zuytdorp can easily be distinguished by the much narrower median apophysis of males (fig. 530) and the posteriorly narrower epigynum of females (fig. 532).

MALE: Total length 6.1. Coloration as in B. zuytdorp except femora not darkened, all legs orange. Leg spination: tibiae IV v1p-1p2; metatarsi III v2-0-2. Retrolateral tibial apophysis angular (fig. 531), median apophysis relatively narrow (fig. 530).

Female: Total length 7.3. Coloration as in male except abdominal dorsum with pair of paler patches anteriorly. Leg spination: tibiae IV v1p-1p-2; metatarsi III v2-0-2. Epigynal atrium not widened posteriorly (fig. 532); spermathecae extending far anterior of epigynal hood (fig. 533).

Other MAterial ExAmined: New South Wales: Kinchega National Park, $32^{\circ} 28^{\prime} \mathrm{S}$, $142^{\circ} 22^{\prime} \mathrm{E}$, Mar. 29-30, 1984, pitfall (J. Smith, QMB), 1 0 . Northern Territory: Bullock Creek, Camfield Homestead, $17^{\circ} 10^{\prime} \mathrm{S}, 131^{\circ} 25^{\prime} \mathrm{E}$, Aug. 19-20, 1982 (I. Archibald, MNT), 19 ; near Lambert Centre, Australian Centre marker, $25^{\circ} 37^{\prime} \mathrm{S}, 134^{\circ} 24^{\prime} \mathrm{E}$, Mar. 29, 1993 (D. Hirst, SAM N1996/440), 10 . Queensland: Lake Broadwater, site 5, $27^{\circ} 21^{\prime} \mathrm{S}, 151^{\circ} 06^{\prime} \mathrm{E}$, Feb. 25-Apr. 22, 1986, pitfall (M. Bennie, QMB S34353), 10 ; Windermere, near Glenmorgan, $27^{\circ} 17^{\prime} \mathrm{S}$, $149^{\circ} 45^{\prime}$ E, July-Sept. 1991 (R. Raven, D. Smyth, QMB S30361), 20. South Australia: $1 \mathrm{~km}$ W Blue Hills Bore, $27^{\circ} 07^{\prime} \mathrm{S}$, $132^{\circ} 51^{\prime} \mathrm{E}$, Mar. 17, 1993, pitfall (SAM N1996/434), 1ơ; $7.89 \mathrm{~km} \mathrm{~N}$ Coongie, $27^{\circ} 06^{\prime} \mathrm{S}, 140^{\circ} 09^{\prime} \mathrm{E}$, May 7, 1987, pitfall (J. Reid, SAM N1996/431), 10; 9.42 km NNE Coongie, $27^{\circ} 06^{\prime} \mathrm{S}, 140^{\circ} 09^{\prime} \mathrm{E}$, Mar. 14, 1987 (J. Reid, SAM N1996/341), 10, May 7, 1987, pitfall (J. Reid, SAM N1996/433), 1 우 $10.9 \mathrm{~km}$ NNE Coongie, $27^{\circ} 05^{\prime} \mathrm{S}, 140^{\circ} 09^{\prime} \mathrm{E}$, May 7, 1987, pitfall (J. Reid, SAM N1996/ 432), 1\%; Edwards Creek, $28^{\circ} 20^{\prime} \mathrm{S}$, 

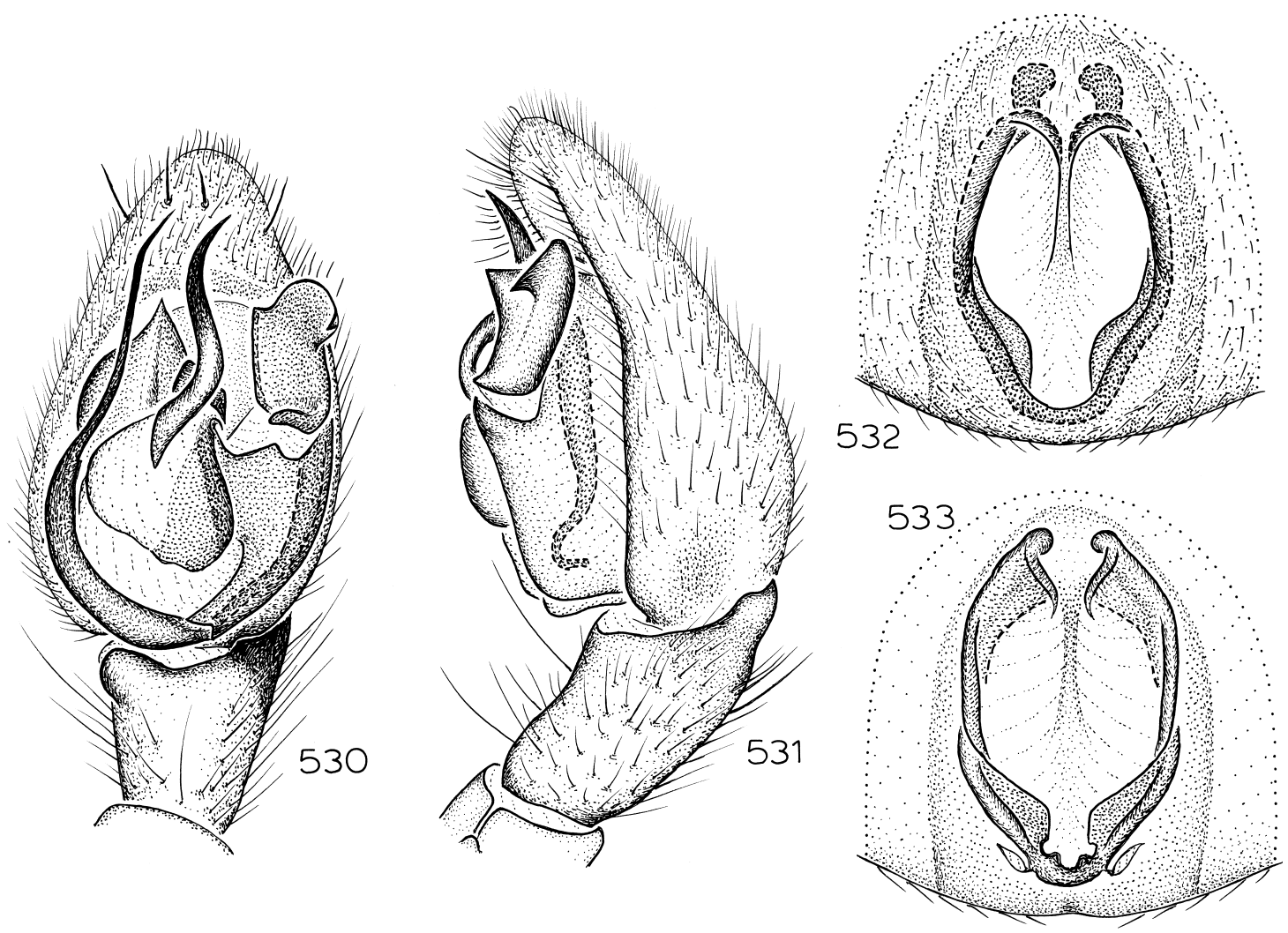

Figs. 530-533. Bigenditia millawa, new species. 530. Left male palp, ventral view. 531. Same, retrolateral view. 532. Epigynum, ventral view. 533. Same, dorsal view.

135 51'E, Mar. 16, 1993 (D. Hirst, SAM N1996/429, 430), 20; Katarapko Game Reserve, $34^{\circ} 25^{\prime} \mathrm{S}, 140^{\circ} 34^{\prime} \mathrm{E}$, May 28, 1991, pitfall (A. McArthur, SAM N1996/428), 19 . Victoria: $7.5 \mathrm{~km}$ ENE Kingower, $36^{\circ} 36^{\prime} \mathrm{S}$, $143^{\circ} 50^{\prime} \mathrm{E}$, Mar. 26-29, 1990, mallee (A. Coventry, NMV K4653), 2 우.

DisTRIBUTION: Eastern sister species of $B$. zuytdorp (map 60).

\section{Prionosternum Dunn}

Prionosternum Dunn, 1951: 13 (type species by original designation Prionosternum scutatum Dunn).

DiAGNOSIS: Males can be recognized by their porrect chelicerae and small, simple retrolateral tibial apophysis (figs. 534, 538), females by their epigynal conformation, with paired anterior epigynal hoods and bipartite spermathecae (figs. 536, 537).

DESCRIPTION: Medium size spiders, total length of males 3.5-4.2, of females 4.0-4.4. Carapace reddish brown, pars thoracica coated with anastomosing rows of large tubercles, pars cephalica with long, dark, thin setae but fewer tubercles; thoracic groove long, longitudinal, deep. Eight eyes in two rows, anterior medians smallest, circular, dark, laterals subequal in size, oval, light, posterior medians largest, irregularly oval, flattened, light; from above, both eye rows slightly procurved, from front, both rows procurved; anterior medians separated by more than their radius, closer to anterior laterals; posterior medians separated by less than their radius, much farther from posterior laterals; anterior and posterior laterals separated by almost their diameter; median ocular quadrangle wider in back than in front, longer than wide in front. Chelicerae, sternum, and mouthparts reddish brown; chilum wide, short, triangular, accompanied by second, longitudinal 
posterior chilum (extremely narrow sclerite separating bases of chelicerae posteriorly); chelicerae with distinct lateral boss, anterior surface with depressed, relatively unsclerotized oval area near promargin; promargin with two rows of long setae originating in line along base of fang, seta closest to fang not bent near base, not extending behind other promarginal setae to near midline; promargin with three teeth, median one largest, proximal one smallest; retromargin with two large, narrowly separated teeth; male chelicerae porrect, with deep median excavation, borders of excavation with strong tubercles. Labium elongate, triangular, only gradually narrowed anteriorly, posterior margin truncate, anterior margin very slightly invaginated at middle, surface slightly depressed medially. Endites obliquely depressed, with sharply demarcated, deep groove along margin near labium, groove wider anteriorly than posteriorly; serrula long, with single row of teeth; anterior surface with distinct sieve plate of maxillary gland openings set in oval of unsclerotized cuticle. Sternum slightly elevated, with gently sloping lateral margins, not expanded anteriorly, with triangular extensions to and between coxae; surface with few punctations. One epimeric sclerite on each side, above each coxa, not reaching sternal triangles, not fused to carapace or pedicel. Pedicel consisting of large, diamond-shaped sclerite bearing wide anterior margin meeting posterior tip of sternum at slightly procurved line.

Anterior edge of abdomen of male with complete sclerotic ring formed by strong epigastric scutum plus strong dorsal abdominal scutum covering entire front edge of abdomen, reaching to more than three-fourths of abdominal length, females with dorsal scutum represented only by small transverse plate above pedicel, restricted to lower half of anterior surface of abdomen; cuticle with long, recumbent setae; epigastric scutum accompanied posterolaterally by pair of oval, deeply invaginated sclerites bearing clearly elevated anterior rim; sclerites separated by membranous lobe, anterior rim of sclerites fitting under epigastric scutum; colulus represented only by pair of long setae; posterior spiracle procurved, inconspicuous. Anterior lateral spinnerets tubular, separated at base by about their diameter, cuticle representing distal, second spinneret segment restricted to semicircle surrounding major ampullate gland spigots (piriform gland spigots surrounded only by soft cuticle); posterior median spinnerets of males large, conical, of females with anteriorly expanded tips, bases occupied by three enormously widened cylindrical gland spigots; posterior lateral spinnerets two-segmented, those of females with at least one greatly widened cylindrical gland spigot.

Leg spination reduced; typical leg spination pattern (only surfaces with spines listed): femur IV d1-0-0; tibiae: III v1p-0-2; IV v1p-1p-2, r0-0-1; metatarsus IV v1p-1p-0. Most leg surfaces with long setae; both sexes with all coxae dorsally tuberculate; anterior coxae with slightly protuberant posterolateral corners, widened at about half their length; trochanters unnotched; anterior tarsi with weak, divided scopulae, composed of laterally directed setae; posterior metatarsi with thick, distal preening brushes; posterior tarsi with weak scopulae; tarsi with two dentate claws, claw tufts composed of lateral pads of short, closely appressed setae, restricted to ventral portion of claw tip; trichobothria present on distal leg segments, in two rows. Female palpal tibia and tarsus with long, thin spines; female palpal tarsus with long, basally dentate claw.

Male palp with short retrolateral tibial apophysis; cymbial surface not excavated opposite tibial apophysis; tegulum bulbous, with slight median projection; embolus short, originating at middle of prolateral side of bulb, extending behind hooked median apophysis, accompanied behind median apophysis by short, angular conductor; embolar base without apophysis. Epigynum with paired anterior hoods; spermathecae bipartite, accompanied by anteromedian ducts.

\section{Key to Species of Prionosternum}

1. Males .................... 2

- Females (those of $P$. porongorup unknown) $\ldots \ldots \ldots \ldots \ldots \ldots \ldots \ldots$

2. Palpal tibia with distinct dorsal apophysis at about one-third its length (fig. 535) ....

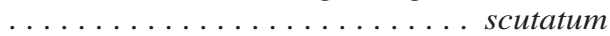

- Palpal tibia with only small dorsal expansion (figs. 471, 539) ............. 3 
3. Retrolateral tibial apophysis relatively short, stubby (figs. 538, 539) . . . . . nitidiceps

- Retrolateral tibial apophysis longer, on distinct base (figs. 470, 471) . . . . . porongurup

4. Anterior epigynal hoods relatively long (fig. 536) ................ scutatum

- Anterior epigynal hoods relatively short (fig. 540) .............. nitidiceps

\section{Prionosternum scutatum Dunn}

Figures 534-537; Map 59

Asadipus nitidiceps (misidentification): Simon, 1909: 178 (male paralectotype, presumably from East Fremantle, Western Australia, in MNHN, examined; male paralectotype from same locality, in $\mathrm{ZMB}$, examined; not female lectotype).

Prionosternum scutatum Dunn, 1951: 14, figs. 57 (male holotype from "Pimlea," actually Pimelea, Western Australia, in NMV, examined).

DiAgNosis: Males can easily be recognized by the presence of a second, retrodorsal tibial apophysis at about half the tibial length (figs. 534, 535), females by the long epigynal hoods (fig. 536) and transversely oriented anterior epigynal ducts (fig. 537).

MALE: Total length 3.7. Carapace reddish orange, chelicerae darkest, sternum lighter than mouthparts; abdominal scutum shiny brownish orange; legs unmarked, yellowish orange, anterior femora darkened. Leg spination typical for genus. Second, hookshaped tibial apophysis present at about half length of segment (fig. 535); palpal bulb globose (fig. 534).

FEMALE: Total length 4.4. Abdominal dorsum mottled gray with hairline light chevrons posteriorly; venter pale white with central gray markings. Leg spination typical for genus. Anterior epigynal hoods opening almost directly toward posterior (fig. 536); anterior epigynal ducts transverse (fig. 537).

Material ExAmined: Western Australia: Arthur River, $33^{\circ} 21^{\prime} \mathrm{S}, 117^{\circ} 02^{\prime} \mathrm{E}$, Aug. 15, 1992, under eucalypt bark (C. Car, WAM 96/ 222), $10^{\star} ; 13 \mathrm{mi} \mathrm{W}$ Bridgetown, $33^{\circ} 57^{\prime} \mathrm{S}$, $116^{\circ} 08^{\prime} \mathrm{E}$, Sept. 25, 1962, elev. $320 \mathrm{~m}$ (E. Ross, D. Cavagnaro, CAS), $10^{\hat{0}}$; Chester Pass, Stirling Range, $34^{\circ} 23^{\prime} \mathrm{S}, 118^{\circ} 06^{\prime} \mathrm{E}$, Sept. 24, 1962, elev. $200 \mathrm{~m}$ (E. Ross, D. Cavagnaro, CAS), $1 \%$; Crowea, $34^{\circ} 28^{\prime} \mathrm{S}$, $116^{\circ} 10^{\prime} \mathrm{E}$, Oct. 22, 197, pitfall, open (cleared) forest (S. Curry, WAM 96/225),
10, 6 Dec. 1, 1978, pitfall, closed (uncleared) forest (S. Curry, WAM 96/231), 1 o, Oct. 31, 1980, pitfall, open forest (S. Curry, WAM 96/226), 1 ô, Nov. 13, 1980, pitfall (S. Curry, WAM 96/227-229, 236), 40े, Nov. 21, 1980, pitfall, open forest (S. Curry, WAM 96/223), 10 , pitfall, closed forest (S. Curry, WAM 96/ 230), 10; SW (above) Dingo Beach, West Cape Howe, $35^{\circ} 08^{\prime} \mathrm{S}, 117^{\circ} 36^{\prime} \mathrm{E}$, July $11-26$, 1986, pitfall (B. Main, CBM), 1 우 Dog Pool on Shannon River, $20 \mathrm{~km}$ S Shannon,

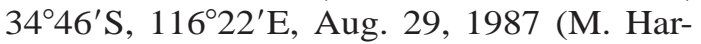
vey, J. Blyth, WAM 96/238), $10^{\star}$; Dwellingup, $32^{\circ} 43^{\prime} \mathrm{S}, 116^{\circ} 04^{\prime} \mathrm{E}$, Oct. 1, 1976 (J. Majer, WAM 96/224), 10, Sept. 29, 1977, pitfall (S. Curry, WAM 96/225), 10 , Nov. 11, 1977, pitfall (S. Curry, WAM 96/233), 10; East Fremantle, $32^{\circ} 03^{\prime} \mathrm{S}, 115^{\circ} 46^{\prime} \mathrm{E}$ (Michaelsen and Hartmeyer, MNHN), $10^{\star}$ (paralectotype), (same, ZMB 27147), 10 (paralectotype); Fitzgerald River National Park, Mount Maxwell, $34^{\circ} 12^{\prime} \mathrm{S}, 119^{\circ} 19^{\prime} \mathrm{E}$, May 25, 1994, under rock (M. Harvey, J. Waldock, WAM 96/292), 1 \% ; Hilltop Road, Walpole-Nornalup National Park, $34^{\circ} 56^{\prime} \mathrm{S}, 116^{\circ} 46^{\prime} \mathrm{E}$, Oct. 10-Nov. 3, 1984, pitfall (J. Lawrence, QMB), 20; Jandakot Airport, $32^{\circ} 06^{\prime} \mathrm{S}$, $115^{\circ} 52^{\prime} \mathrm{E}$, July $16-$ Sept. 1, 1994, pitfall (J. Waldock, A. Longbottom, WAM 96/243), 10; Landsdale School, $31^{\circ} 49^{\prime} \mathrm{S}, 115^{\circ} 51^{\prime} \mathrm{E}$, July 13-Sept. 25, 1995, pitfall (M. Harvey, J. Waldock, WAM 96/240), 1 o; Marangaroo Reserve, $31^{\circ} 50^{\prime} \mathrm{S}, 115^{\circ} 50^{\prime} \mathrm{E}$, July 13-Sept. 25, 1995, pitfall (M. Harvey, J. Waldock, WAM 96/239), 1ð; S end, Millinup Pass, Porongurup National Park, $34^{\circ} 42^{\prime} \mathrm{S}$, $117^{\circ} 54^{\prime} \mathrm{E}$, Mar. 30, 1993, in rotting $\log (\mathrm{M}$. Harvey, J. Waldock, WAM 96/1538), Apr. 28-Sept. 2, 1996, pitfalls (M. Harvey, WAM 96/1539-1548), 10우, Sept. 2-Dec. 16, 1996, pitfall (M. Harvey, J. Waldock, B. Main, WAM 96/1537), 1 ㅇ ; Mount Hawthorn, 31 $55^{\prime} \mathrm{S}, 115^{\circ} 50^{\prime} \mathrm{E}$, Aug. 14, 1993 (M. Harvey, WAM 93/1759), 1 đ。; Nedlands, $31^{\circ} 59^{\prime} \mathrm{S}, 115^{\circ} 48^{\prime} \mathrm{E}$, June 2, 1990 (A. Baynes, WAM 96/232), $10^{\top}$; Parmelia, $32^{\circ} 15^{\prime} \mathrm{S}$, $115^{\circ} 47^{\prime} \mathrm{E}$, June 22, 1990 (A. de Jong, WAM 96/237), 10; Pimelea, $34^{\circ} 24^{\prime} \mathrm{S}, 115^{\circ} 58^{\prime} \mathrm{E}$, Sept. 7, 1947 (R. Pescott, NMV K1028), $10^{\text {đ }}$ (holotype); Tooreburrup Hill, $34^{\circ} 26^{\prime} \mathrm{S}$, $119^{\circ} 22^{\prime} \mathrm{E}$, May 30, 1994, under rock (M. Harvey, J. Waldock, N. Brown, WAM 96/ 284), 1 ; ; Torndirrup National Park, $35^{\circ} 10^{\prime} \mathrm{S}$, 

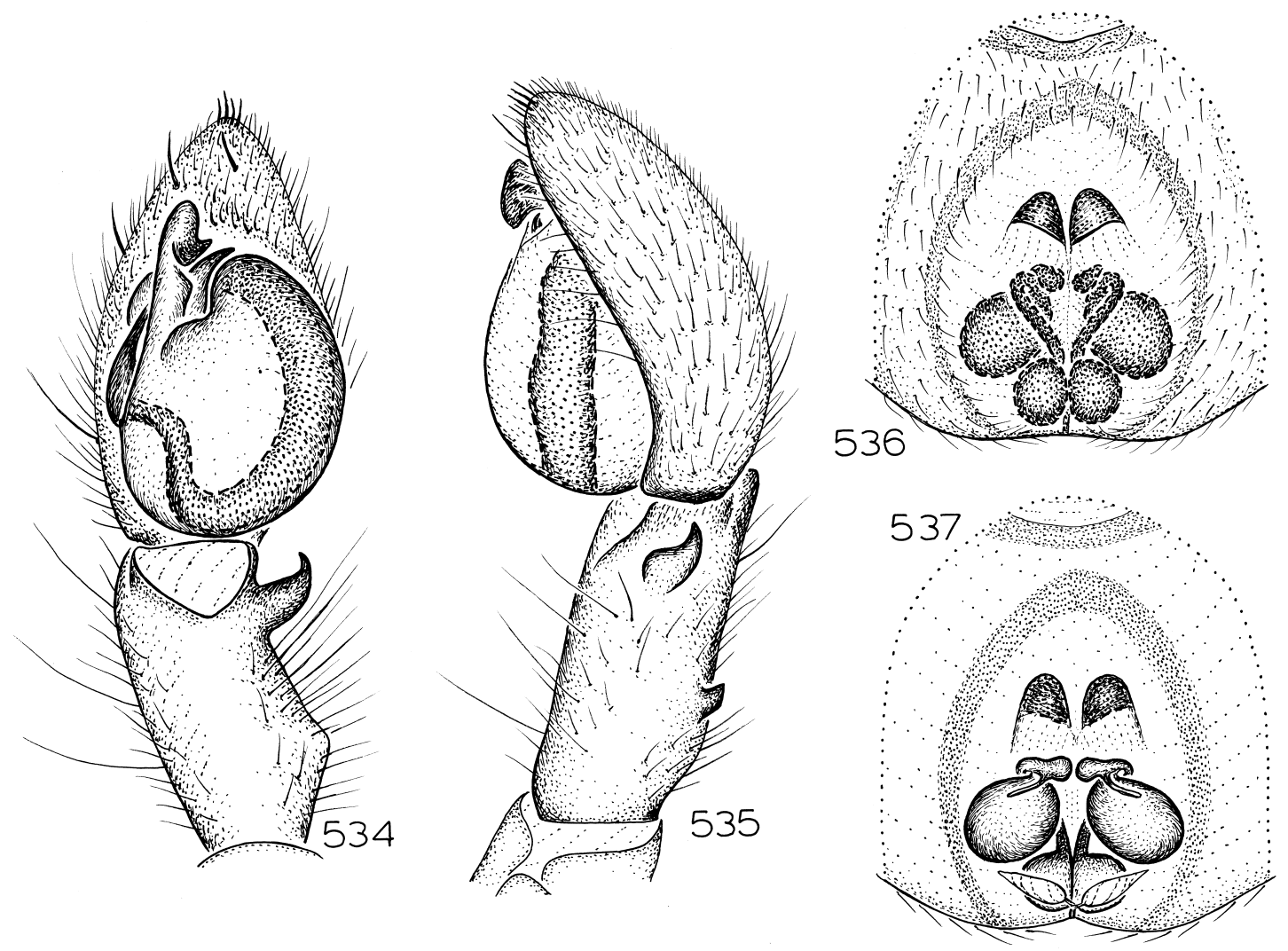

Figs. 534-537. Prionosternum scutatum Dunn. 534. Left male palp, ventral view. 535. Same, retrolateral view. 536. Epigynum, ventral view. 537. Same, dorsal view.

$117^{\circ} 50^{\prime}$ E, July 6-13, 1983, pitfall (P. Dyer, J. Lyon, WAM 96/293), 19 , July 13-20, 1983, pitfall (P. Dyer, J. Lyon, WAM 96/285, 286), 2 q , July 20-27, 1983, pitfall (P. Dyer, J. Lyon, WAM 96/290), 19, Aug. 24-31, 1983, pitfall (P. Dyer, J. Lyon, WAM 96/ 291), 1 \% , Sept. 7-14, 1983, pitfalls (P. Dyer, J. Lyon, WAM 96/287-289), 20, 1 क ; Trigg Dune Bush, $31^{\circ} 52^{\prime} \mathrm{S}, 115^{\circ} 46^{\prime} \mathrm{E}$, July $13-$ Sept. 25, 1995, pitfall (M. Harvey, J. Waldock, WAM 96/242), 10; Tuart Hill, $31^{\circ} 53^{\prime} \mathrm{S}, 115^{\circ} 52^{\prime} \mathrm{E}$, July 20-Sept. 23, 1993, pitfall (J. Waldock, A. Sampey, A. Thorpe, WAM 96/241), 10; NW Two Mile Lake, Stirling Range National Park, $34^{\circ} 28^{\prime} \mathrm{S}$, $118^{\circ} 15^{\prime} \mathrm{E}$, Sept. 20-30, 1990, pitfall (G. Friend, WAM 96/1536), 10; Two Peoples Bay, Sinker Reef Road, 34 $59^{\prime} \mathrm{S}, 118^{\circ} 09^{\prime} \mathrm{E}$, Nov. 14, 1998 (V. Ovtsharenko, AMNH), 1\%; Warren National Park, $34^{\circ} 30^{\prime} \mathrm{S}$,

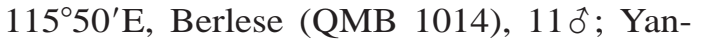

chep, $31^{\circ} 33^{\prime} \mathrm{S}, 115^{\circ} 41^{\prime} \mathrm{E}$, Aug. 24, 1978, tree trap (S. Curry, WAM 96/234), 10 .

Distribution: Southwestern Australia (map 59).

\section{Prionosternum nitidiceps (Simon), new combination \\ Figures 538-541; Map 61}

Asadipus nitidiceps Simon, 1909: 178 (female lectotype, here designated, from Busselton, Western Australia, in ZMH, examined; not male paralectotypes, $=P$. scutatum) .

Diagnosis: Males resemble those of $P$. porongurup but can be distinguished by the shorter retrolateral tibial apophysis and less pronounced tegular projection (fig. 539); females can be recognized by the obliquely oriented anterior epigynal hoods (fig. 540).

MALE: Total length 3.5. Coloration as in $P$. scutatum. Leg spination typical for genus. 

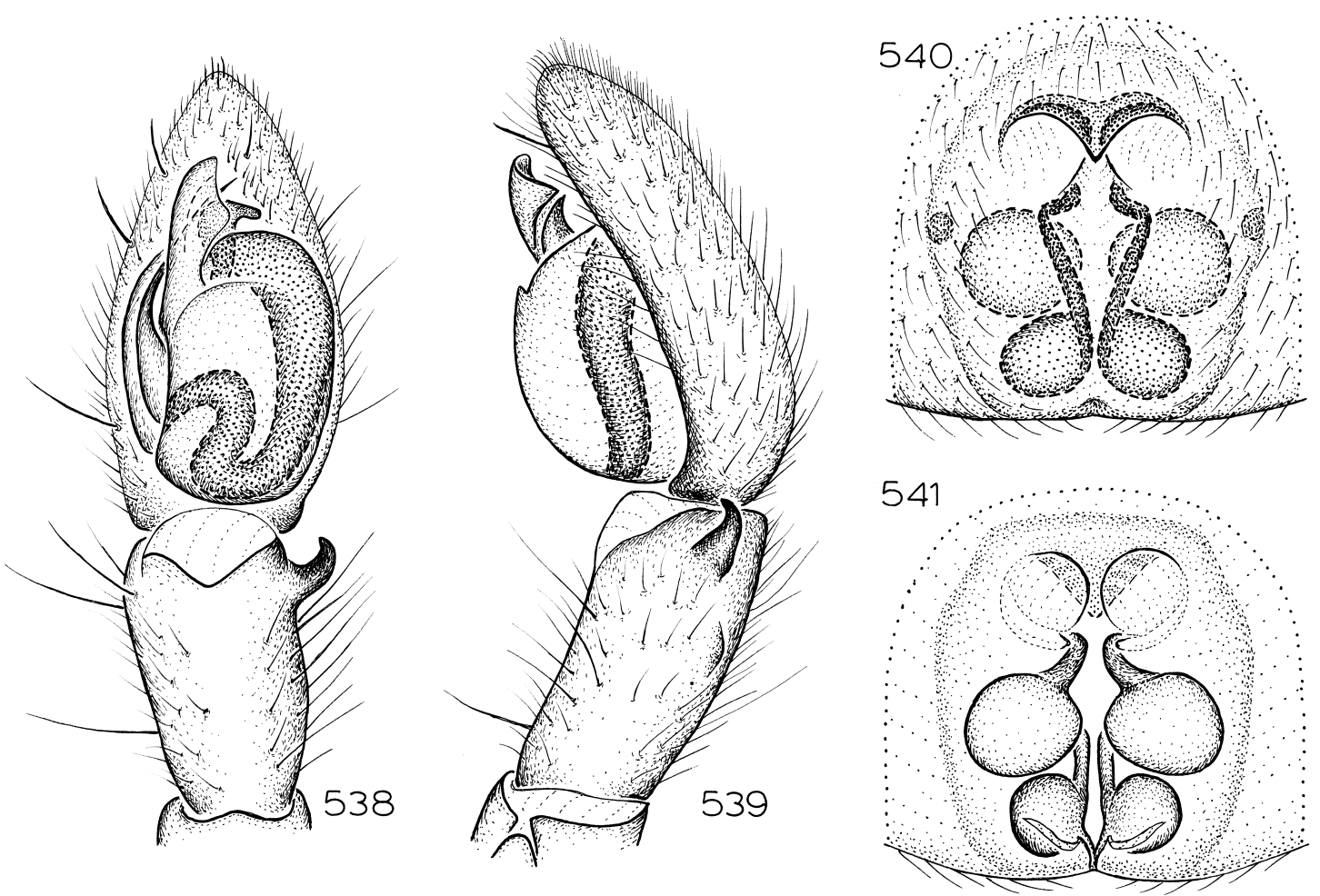

Figs. 538-541. Prionosternum nitidiceps (Simon). 538. Left male palp, ventral view. 539. Same, retrolateral view. 540. Epigynum, ventral view. 541. Same, dorsal view.

Single, subterminal retrolateral tibial apophysis small, with short base, originating at middle of segment height (fig. 539); tegulum with small anterior projection (fig. 540).

FEMALE: Total length 4.0. Coloration as in $P$. scutatum. Leg spination: tibia IV $1 \mathrm{p}-0-0$. Anterior epigynal hoods obliquely oriented (fig. 541); spermathecae bipartite, with anteriorly extended ducts (fig. 542).

Material Examined: Australian Capital Territory: Wombat Creek, 6 km NE Piccadilly Circus, $35^{\circ} 19^{\prime} \mathrm{S}, 148^{\circ} 51^{\prime} \mathrm{E}$, Feb. 1984, elev. $750 \mathrm{~m}$ (Weir, Lawrence, Johnson, QMB), 1 ․ New South Wales: Bonnie Vale Campground, Bundeena, Royal National Park, $34^{\circ} 06^{\prime} \mathrm{S}, 151^{\circ} 07^{\prime} \mathrm{E}$, Aug. 11-13, 1990, open coastal sclerophyll (C. Griswold, T. Meikle, USNM), $10^{\top}$; Leura, $33^{\circ} 43^{\prime} \mathrm{S}$, $150^{\circ} 20^{\prime} \mathrm{E}$ (Mann, MCZ), 1\%; $2.35 \mathrm{~km} \mathrm{~N}$ Moggs Swamp Creek, Moggs Swamp Fire Trail, Warra State Forest, $2^{\circ} 57^{\prime} \mathrm{S}, 151^{\circ} 58^{\prime} \mathrm{E}$, Feb. 4-Apr. 9, 1993, pitfall, elev. $1170 \mathrm{~m}$ (M. Gray, G. Cassis, AMS KS36364), 1 ㅇ.
South Australia: Aldinga Scrub, $35^{\circ} 17^{\prime} \mathrm{S}$, $138^{\circ} 29^{\prime} \mathrm{E}$, June 26,1987 , in casuarina litter (D. Hirst, SAM N1996/376), 10, Sept. 1522, 1987, pitfall (E. Matthews, J. Forrest, SAM N1996/377-379), 10 , 1 \%; Breakneck Creek National Park, Kangaroo Island, 35 $56^{\prime} \mathrm{S}, 136^{\circ} 37^{\prime} \mathrm{E}$, Nov. 7, 1987 (D. Hirst, SAM N1996/381), 1 $q$; Cape du Couedic, 36언 $\mathrm{S}, 136^{\circ} 42^{\prime} \mathrm{E}$, Dec. 2-7, 1982 (B. Guerin, J. Thurmer, D. Lacis, SAM N1996/382), 19 ; Dudley Conservation Park, Kangaroo Island, $35^{\circ} 48^{\prime} \mathrm{S}, 137^{\circ} 51^{\prime} \mathrm{E}$, vibration, Nov. $11-$ 12, 1987 (D. Hirst, SAM N1996/390-392), 39 ; Forestville, Adelaide, $34^{\circ} 56^{\prime} \mathrm{S}$, $138^{\circ} 36^{\prime}$ E, Aug. 1983-1984 (B. Guerin, SAM N1996/373-375), 30; Kelly Hill Caves camping area, Kangaroo Island, $36^{\circ} 01^{\prime} \mathrm{S}$, $136^{\circ} 55^{\prime} \mathrm{E}$, Nov. 9-10, 1987, pitfall (D. Hirst, SAM 1996/342), 10; Langhorne Creek,

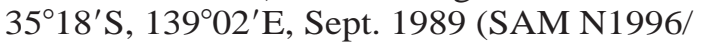
393, 394), 20; Largs North, Adelaide, $34^{\circ} 56^{\prime} \mathrm{S}, 138^{\circ} 36^{\prime} \mathrm{E}$, Aug. 21, 1975, on mallee roots (R. Cook, SAM N1996/372), 1 đ; Mit- 
cham, Adelaide, $34^{\circ} 56^{\prime} \mathrm{S}, 138^{\circ} 36^{\prime} \mathrm{E}$, Oct. 131, 1976, indoors (R. Southcott, SAM

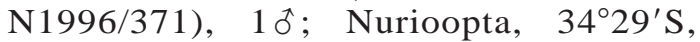
$139^{\circ} 00^{\prime} \mathrm{E}$, Sept. 24, 1992, in shed (A. Longbottom, WAM 96/248), 10َ; 7 km N Ravine des Casoars, Flinders Chase, Kangaroo Island, $35^{\circ} 45^{\prime} \mathrm{S}, 136^{\circ} 36^{\prime} \mathrm{E}$, Nov. 2-7, 1990 , pitfall (E. Matthews, J. Forest, SAM N1996/ 380), 1 đo; Rocky River crossing, W Ranger's Station, Flinders Chase, Kangaroo Island, $35^{\circ} 57^{\prime} \mathrm{S}, 136^{\circ} 44^{\prime} \mathrm{E}$, Nov. 7-9, 1987, pitfall (D. Hirst, SAM N1996/383, 384), 20; 4 km W Rocky River, $35^{\circ} 57^{\prime} \mathrm{S}, 136^{\circ} 42^{\prime}$ E, Nov. 17, 1990 (E. Matthews, J. Forrest, SAM N1997/89), 1 \%; Waterfall Creek, Western River Conservation Park, Kangaroo Island, $35^{\circ} 42^{\prime}$ S, $136^{\circ} 54^{\prime} \mathrm{E}$, Nov. 3-4, 1987, pitfall (D. Hirst, SAM N1996/385-389), 40, 1 오.

Tasmania: Eddystone Point, $41^{\circ} 00^{\prime} \mathrm{S}$, $148^{\circ} 19^{\prime}$ E, Oct. 1986-1987, coastal heath (T. Churchill, QVM 3604), 20, Nov.-Dec. 1986-1987, coastal heath (T. Churchill, QVM 1078, 4093), 2 \% , Nov. 1993, coastal heath (P. Cranston, J. Trueman, QMB S34290), $10^{\circ}$; Trevallyn, $41^{\circ} 27^{\prime} \mathrm{S}, 147^{\circ} 10^{\prime} \mathrm{E}$, Nov. 12, 1952, grass tussocks (V. Hickman, AMS KS29291), 2q; Waterhouse Point, $40^{\circ} 51^{\prime} \mathrm{S}, 147^{\circ} 38^{\prime} \mathrm{E}$, Sept.-Nov. 1986-1987, coastal heath (T. Churchill, QVM), 550, Oct.-Jan. 1986-1988, coastal heath (T. Churchill, QVM), 45 \% . Victoria: Balwyn, $37^{\circ} 49^{\prime} \mathrm{S}, 145^{\circ} 04^{\prime} \mathrm{E}$, Jan. 1 , 1983 , compost

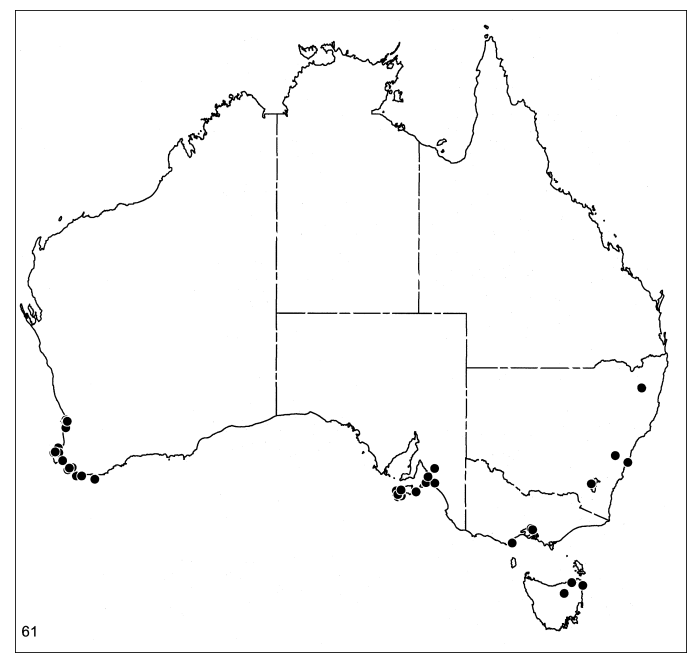

Map 61. Records of Prionosternum nitidiceps (Simon). heap (M. Harvey, QMB), 1 \% ; Glen Waverley, $37^{\circ} 53^{\prime} \mathrm{S}, 145^{\circ} 10^{\prime} \mathrm{E}$, Oct. 10, 1974 (V. Salinitri, QMB), $1 \delta^{\star}$; Separation Creek, $38^{\circ} 38^{\prime} \mathrm{S}, 143^{\circ} 54^{\prime} \mathrm{E}$, Sept. 19-21, 1989, under eucalypt bark (M. Harvey, M. Blosfelds, WAM 96/244-247), 40. Western Australia: Brockman National Park, 16 km S Pemberton, $34^{\circ} 28^{\prime} \mathrm{S}, 116^{\circ} 01^{\prime} \mathrm{E}$, Dec. 8, 1976, Berlese, karri litter and mold (J. Kethley, FMNH), 1 ; ; $3 \mathrm{~km}$ NE Broke Inlet, 345' ${ }^{\prime} \mathrm{S}$, $116^{\circ} 27^{\prime} \mathrm{E}$, Feb. 12, 1987 (B., M. Baehr, CBB), 19 ; Busselton, $33^{\circ} 39^{\prime} \mathrm{S}, 115^{\circ} 20^{\prime} \mathrm{E}$, Aug. 3-4, 1905 (W. Michaelsen, ZMH), 1 우

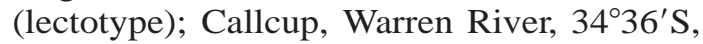
$115^{\circ} 54^{\prime} \mathrm{E}$, Nov. 2-7, 1997, pitfall on sand dune (C. Deeleman, CCD), 1 o , 1 으, Oct. 30Nov. 5, 1997, bush, elev. 100 m (J. Murphy, JAM 22926), 10, $4 \%$; Crowea, $34^{\circ} 28^{\prime} \mathrm{S}$, $116^{\circ} 10^{\prime}$ E, Nov. 15-Dec. 20, 1979 (S. Curry, WAM 84/687, 718, 719), 39 ; 3 km W Darradup, $34^{\circ} 05^{\prime} \mathrm{S}, 115^{\circ} 34^{\prime} \mathrm{E}$, Nov. 10 , 1991, litter at base of red gum (J. Bannister, WAM 96/302), 1 \% ; Dingo Beach, West Cape Howe, $35^{\circ} 08^{\prime} \mathrm{S}, 117^{\circ} 36^{\prime} \mathrm{E}$, Sept. 19-Nov. 8, 1985, pitfalls (B. Main, CBM), 4o, Dec. 26, 1984-Jan. 6, 1985, pitfall (B. Main, CBM), 2\%; Dombalup State Forest, Marri Road, $34^{\circ} 30^{\prime} \mathrm{S}, 116^{\circ} 00^{\prime} \mathrm{E}$, Jan. 27-Mar. 5, 1979. pitfall in litter (M. Gray, AMS KS15247), 1 웅 Dunsborough, $33^{\circ} 36^{\prime} \mathrm{S}, 115^{\circ} 06^{\prime} \mathrm{E}$, Nov. 13, 1976, on beach at night (A. Wilson, M. Shaud, J. Kethley, FMNH), 19 ; Hepburn Heights, $31^{\circ} 49^{\prime} \mathrm{S}, 115^{\circ} 47^{\prime} \mathrm{E}$, July $13-$ Sept. 25, 1995, pitfall (M. Harvey, J. Waldock, WAM 96/298), 1 \% ; Margaret River at Great North Road, $33^{\circ} 23^{\prime} \mathrm{S}, 115^{\circ} 18^{\prime} \mathrm{E}$, Aug. 27, 1987, under bark (M. Harvey, J. Blyth, WAM 96/294), 10; Nedlands, $31^{\circ} 59^{\prime} \mathrm{S}$, $115^{\circ} 48^{\prime} \mathrm{E}$, July 29, 1990 (A. Baynes, WAM 295, 296) 2\%, Aug. 6, 1990, garden (A. Baynes, WAM 297), 1 \%, Sept. 25, 1991 (A. Baynes, WAM 96/301), $1 \%$; Parmelia, $32^{\circ} 15^{\prime} \mathrm{S}, 115^{\circ} 47^{\prime} \mathrm{E}$, Aug. 28-30, 1989 (A. De Jong, WAM 96/299), 1 \% ; Torbay Head, West Cape Howe, $35^{\circ} 08^{\prime} \mathrm{S}, 117^{\circ} 39^{\prime} \mathrm{E}$, Oct. 16-30, 1982, pitfall (B. Main, CBM), 1 đ; Tuart Hill, $31^{\circ} 53^{\prime} \mathrm{S}, 115^{\circ} 52^{\prime} \mathrm{E}$, July 20-Sept. 23, 1993 , pitfall (J. Waldock, A. Sampey, A. Thorpe, WAM 96/300), 1 \% ; Walpole-Nornalup National Park, 34 $56^{\prime} \mathrm{S}, 116^{\circ} 46^{\prime} \mathrm{E}$ (QMB), 1 \% ; West Cape Howe, $35^{\circ} 08^{\prime} \mathrm{S}, 117^{\circ} 36^{\prime} \mathrm{E}$, Sept. 16-Oct. 8, 1983, pitfall (B. Main, CBM), 
$10^{\wedge}$; Yallingup, $33^{\circ} 39^{\prime} \mathrm{S}, 115^{\circ} 01^{\prime} \mathrm{E}$ (E. Waite, SAM N1996/395), 1 ô.

Distribution: Southern Australia, including Tasmania (map 61).

\section{Prionosternum porongurup, new species}

Figures 470, 471; Map 62

TYPE: Male holotype taken in a pitfall trap at an elevation of $470 \mathrm{~m}$ on Hayward Peak, Porongurup National Park, Western Australia, 344ㄴ ${ }^{\prime} \mathrm{S}, 117^{\circ} 52^{\prime} \mathrm{E}$ (Mar. 22, 1995; S. Barrett), deposited in WAM (96/303).

ETYMOLOGY: The specific name is a noun in apposition taken from the type locality.

DIAGNOSIS: Males can be recognized by the relatively long tibial apophysis, which originates near the ventral surface of the segment and has a sinuous base, and by the pronounced anterior tegular projection (figs. 470, 471); females are unknown.

MALE: Total length 4.2. Coloration as in $P$. scutatum. Leg spination typical for genus. Palpal tibia relatively long, with sinuous distal apophysis originating near ventral surface of segment, wrapping around retrolateral surface (fig. 471); tegulum with distinct anterior projection (fig. 470).

FEMALE: Unknown.

Other Material Examined: Western Australia: S end, Millinup Pass, Porongurup National Park, 34 $42^{\prime}$ S, $117^{\circ} 54^{\prime}$ E, Mar. 30, 1993 (M. Harvey, J. Waldock, WAM 96/ 304), 1 ๙ , Apr. 28-Sept. 2, 1996, pitfall (M. Harvey, WAM 96/1549), 1 đ; Stirling Range Caravan Park, $34^{\circ} 19^{\prime} \mathrm{S}, 118^{\circ} 11^{\prime} \mathrm{E}$, Apr. 22May 3, 1996 (M. Harvey, J. Waldock, B. Main, WAM 96/1535), 10; Torbay Head, $35^{\circ} 08^{\prime} \mathrm{S}, 117^{\circ} 39^{\prime} \mathrm{E}$, Mar. 20-Apr. 3, 1983, pitfalls (B. Main, CBM), 40 .

Distribution: Southwestern Australia (map 62).

\section{Graycassis, new genus}

TYPE SPECIES: Graycassis marengo, new species.

ETYMOLOGY: The generic name is in honor of the two collectors who are responsible for obtaining most of the known specimens, Drs. M. Gray and G. Cassis of the Australian Museum, and is considered masculine in gender.

DiAGNOSIS: Males are easy to recognize because of their greatly elaborated median apophysis (as in figs. 542, 574) and lobate retrolateral tibial apophyses (as in figs. 543, 575). Females have varied epigyna, but the posterior ducts are typically y-shaped, with each duct having a straight base leading to an oblique continuation (as in figs. 545, 577).

DESCRIPTION: Medium size spiders, total length of males 3.4-8.8, of females 4.8-9.9. Carapace reddish brown, surface with scattered low tubercles, pars cephalica with long, dark, thin setae; thoracic groove very long, longitudinal, shallow. Eight subequal eyes in two rows, anterior medians circular, dark, laterals oval, light, posterior medians irregularly oval, flattened, light; from above, both eye rows nearly straight, from front, anterior row nearly straight, posterior row strongly procurved; anterior medians separated by their diameter, slightly closer to anterior laterals; posterior medians separated by more than their diameter, farther from posterior laterals; anterior and posterior laterals separated by more than their diameter; median ocular quadrangle wider in back than in front and than long. Chelicerae, sternum, and mouthparts reddish brown; chilum wide, triangular, accompanied by second, longitudinal posterior chilum (extremely narrow sclerite separating bases of chelicerae posteriorly); chelicerae with distinct lateral boss, anterior sur-

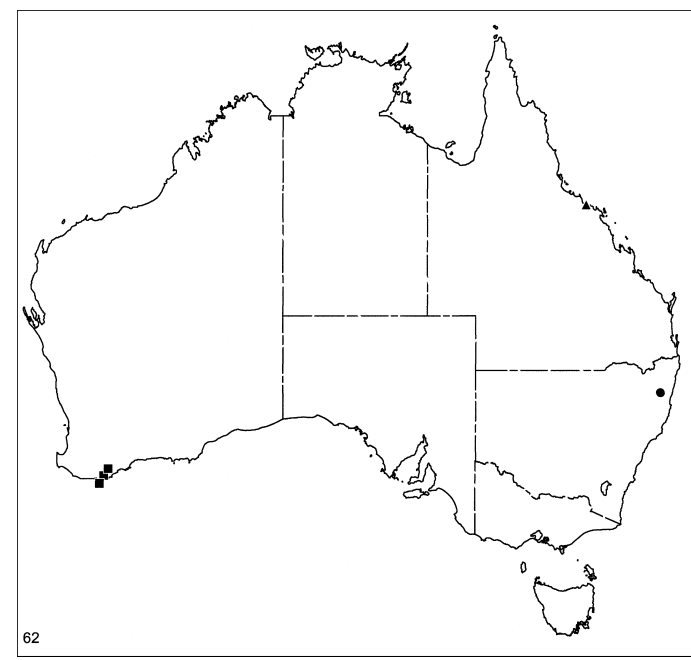

Map 62. Records of Prionosternum porongurup, new species (squares), Graycassis marengo, new species (circle), and Asadipus uphill, new species (triangle). 
face with depressed, relatively unsclerotized oval area near promargin; promargin with row of long setae originating in line along base of fang, seta closest to fang bent near base, extending behind other promarginal setae to near midline; promargin with three teeth, median one largest, proximal one smallest; retromargin with two large, widely separated teeth. Labium elongate, rectangular, only gradually narrowed anteriorly, posterior margin truncate, anterior margin distinctly invaginated at middle, surface not depressed medially. Endites obliquely depressed, with deep groove along margin near labium not sharply demarcated, groove wider anteriorly than posteriorly; serrula long, with single row of teeth; anterior surface with distinct sieve plate of maxillary gland openings set in oval of unsclerotized cuticle. Sternum slightly elevated, with gently sloping lateral margins, not expanded anteriorly, with triangular extensions to and between coxae; surface with few low tubercles. One epimeric sclerite on each side, above each coxa, not reaching sternal triangles, not fused to carapace or pedicel. Pedicel consisting of large, diamond-shaped sclerite bearing narrow anterior margin meeting posterior tip of sternum at slightly procurved line.

Anterior edge of abdomen of male with complete sclerotic ring formed by strong epigastric scutum plus strong dorsal abdominal scutum covering about one-half of length of dorsum, females with dorsal scutum represented only by small rectangular plate above pedicel, restricted to lower half of anterior surface of abdomen; cuticle with long, recumbent setae; epigastric scutum accompanied posterolaterally by pair of oval, deeply invaginated sclerites bearing clearly elevated anterior rim; sclerites separated by membranous lobe, anterior rim of sclerites fitting under epigastric scutum; colulus represented by setae on slightly sclerotized cuticle; posterior spiracle procurved, inconspicuous. Anterior lateral spinnerets tubular, separated at base by about their diameter, with about three piriform gland spigots, cuticle representing distal, second spinneret segment restricted to semicircle surrounding major ampullate gland spigots (piriform gland spigots surrounded only by soft cuticle); posterior median spinnerets of males large, tubular, of fe- males with anteriorly expanded tips, bases occupied by three enormously widened cylindrical gland spigots; posterior lateral spinnerets two-segmented, those of females with two greatly widened cylindrical gland spigots.

Leg spination reduced; typical leg spination pattern (only surfaces with spines listed): femora I-IV d1-0-0; tibiae: III p0-1-1, v1p-2-2, r0-1-1; IV p0-0-1, v2-2-2, r1-0-1; metatarsi: III p0-1-0, v2-1p-0, r0-1-0; IV p01-1, v2-2-0, r0-1-0. Most leg surfaces with long setae; coxae with very slight tubercles; anterior coxae with slightly protuberant posterolateral corners, widened at about half their length; trochanters with shallow but distinct notch; anterior tarsi with weak, divided scopulae, composed of laterally directed setae; posterior metatarsi with weak distal preening brushes; posterior tarsi with very weak scopulae; tarsi with two dentate claws, claw tufts reduced to few long setae, restricted to ventral portion of claw tip; trichobothria present on distal leg segments, in two rows. Female palpal tibia and tarsus with long, thin spines; female palpal tarsus with long, basally dentate claw.

Male palp with lobe-shaped, often bipartite retrolateral tibial apophysis; cymbial surface excavated opposite tibial apophysis; tegulum bulbous, with slight median projection; embolus relatively short, originating at middle of prolateral side of bulb, accompanied by membranous conductor; median apophysis enormously elaborated, often with spurs; embolar base with at most a slight translucent flange, without distinct apophysis. Epigynum with anterior ridge; spermathecae heavily sclerotized, often with accessory lobes.

\section{Key to Species of GRAYCASSIS}

1. Males .................. 2

- Females (those of G. enfield and G. scrub unknown) ............... 11

2. Median apophysis distally bifid, with long prolateral prong directed distally (figs. 542, $546,550) \ldots \ldots \ldots \ldots \ldots$

- Median apophysis otherwise ........ 5

3. Embolus long, narrow, unmodified (fig. 550) ............. dorrigo

- Embolus short, wide, twisted (figs. 542, 546) 
4. Median apophysis relatively wide (fig. 542) $\ldots \ldots \ldots \ldots \ldots$ marengo

- Median apophysis relatively narrow (fig. 546)

5. Median apophysis with elongated, prolaterally directed tip (figs. 570, 574, 578) . . 6

- Median apophysis with short tip (figs. 554, $558,562,566) \ldots \ldots \ldots$

6. Median apophysis distally convex (fig. 578) $\ldots \ldots \ldots \ldots \ldots \ldots$ scrub

- Median apophysis distally concave (figs. 570, 574)

7. Tip of median apophysis relatively narrow (fig. 570) ................. boss

- Tip of median apophysis relatively wide (fig. 574) ............... bulga

8. Tip of median apophysis twisted, sinuous (figs. 558, 562) ............. . 9

- Tip of median apophysis not twisted (figs. $554,566) \ldots \ldots \ldots \ldots \ldots$

9. Tip of median apophysis relatively long (fig.

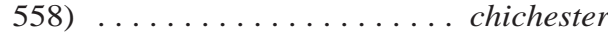

- Tip of median apophysis relatively short (fig. 562) ............. barrington

10. Median apophysis relatively short (fig. 566) ............... enfield

- Median apophysis relatively long (fig. 554) .................. bruxner

11. Epigynum with distinct anteromedian septum (figs. 544, 548, 552, 564, 572, 576) . 13

- Epigynum without anteromedian septum (figs. 556, 560) . . . . . . . . . 12

12. Epigynal ducts relatively short (figs. 556, 557) ............... bruxner

- Epigynal ducts relatively long (figs. 560, 561)

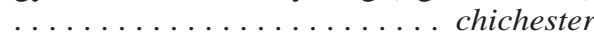

13. Epigynal septum relatively wide, separating anterior epigynal margins (figs. 572, 576)

- Epigynal septum narrow, not separating terior epigynal margins (figs. 544, 548, $552,564) \ldots \ldots \ldots \ldots \ldots \ldots$

14. Epigynum relatively narrow (fig. 572) .... $\ldots \ldots \ldots \ldots \ldots \ldots$ boss

- Epigynum relatively wide (fig. 576) . . bulga

15. Epigynal septum relatively long (fig. 564) .. $\ldots \ldots \ldots \ldots$ barrington

- Epigynal septum relatively short (figs. 544, $548,552) \ldots \ldots \ldots \ldots \ldots$

16. Anterior portion of epigynum relatively long (fig. 544) ............. marengo

- Anterior portion of epigynum shorter (figs.

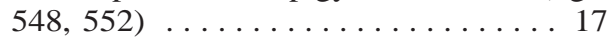

17. Median epigynal ducts oblique (figs. 548, 549) . . . . . . . . . . . . styx

- Median epigynal ducts recurved (figs. 552, 553) ............... dorrigo
Graycassis marengo, new species Figures 542-545; Map 62

TYPES: Female holotype and male allotype taken in pitfall traps at an elevation of 1120 m $2.2 \mathrm{~km}$ NE along Chimney Road from Chaelundi Road, Marengo State Forest, $30^{\circ} 06^{\prime} \mathrm{S}, 152^{\circ} 24^{\prime} \mathrm{E}$, New South Wales (Feb. 4-Apr. 9, 1993; M. Gray, G. Cassis), deposited in AMS (KS35332).

ETYMOLOGY: The specific name is a noun in apposition taken from the type locality.

DiAGNOSIS: The bifid median apophysis, bearing a long and distally incised prolateral prong (fig. 542) of males, and the narrow anteromedian epigynal septum and shape of the anterior portion of the epigynum (fig. 544) are diagnostic.

MALE: Total length 5.3. Carapace dark reddish-brown, abdominal scutum brown, soft portions of abdomen gray, venter with longitudinal pale markings; legs brownish orange, femora darkest. Leg spination typical for genus. Retrolateral tibial apophysis bifid, with ventral prong hook-shaped (fig. 543); median apophysis with long medial prong (fig. 542).

Female: Total length 5.6. Coloration as in male. Leg spination typical for genus. Epigynum wide, anterior half divided by septum (fig. 544); spermathecae each with anterior accessory lobe (fig. 545).

Other Material Examined: New South Wales: Big Bull Creek, $2.7 \mathrm{~km}$ NE along Foamy Creek Road from Chaelundi Road, Marengo State Forest, $30^{\circ} 07^{\prime} \mathrm{S}, 152^{\circ} 25^{\prime} \mathrm{E}$, Feb. 4-Apr. 9, 1993, pitfall, elev. 920 m (M. Gray, G. Cassis, AMS KS35331), 13 क ; 0.9 km S along Chaelundi Road from Big Bull Creek Road, Mount Hyland Nature Reserve, $30^{\circ} 09^{\prime} \mathrm{S}, 152^{\circ} 27^{\prime} \mathrm{E}$, Feb. 4-Apr. 9, 1993, pitfall, elev. 1080 m (M. Gray, G. Cassis, AMS KS35320), 160, $1 \%$; $1.9 \mathrm{~km} \mathrm{~N}$ along Chaelundi Road from Big Bull Creek Road, Mount Hyland Nature Reserve, $30^{\circ} 08^{\prime} \mathrm{S}$, $152^{\circ} 26^{\prime}$ E, Feb. 4-Apr. 9, 1993, pitfall, elev. $1160 \mathrm{~m}$ (M. Gray, G. Cassis, AMS KS35323), 180, $1 \% ; 0.4 \mathrm{~km}$ SW along Chimney Road from junction of Buckboard and Foamy Creek Roads, Marengo State Forest, $30^{\circ} 06^{\prime} \mathrm{S}, 152^{\circ} 25^{\prime} \mathrm{E}$, Feb. 4-Apr. 9, 1993, pitfall, elev. $1090 \mathrm{~m}$ (M. Gray, G. Cassis, AMS KS35340), 10َ; $2.2 \mathrm{~km} \mathrm{NE}$ along 

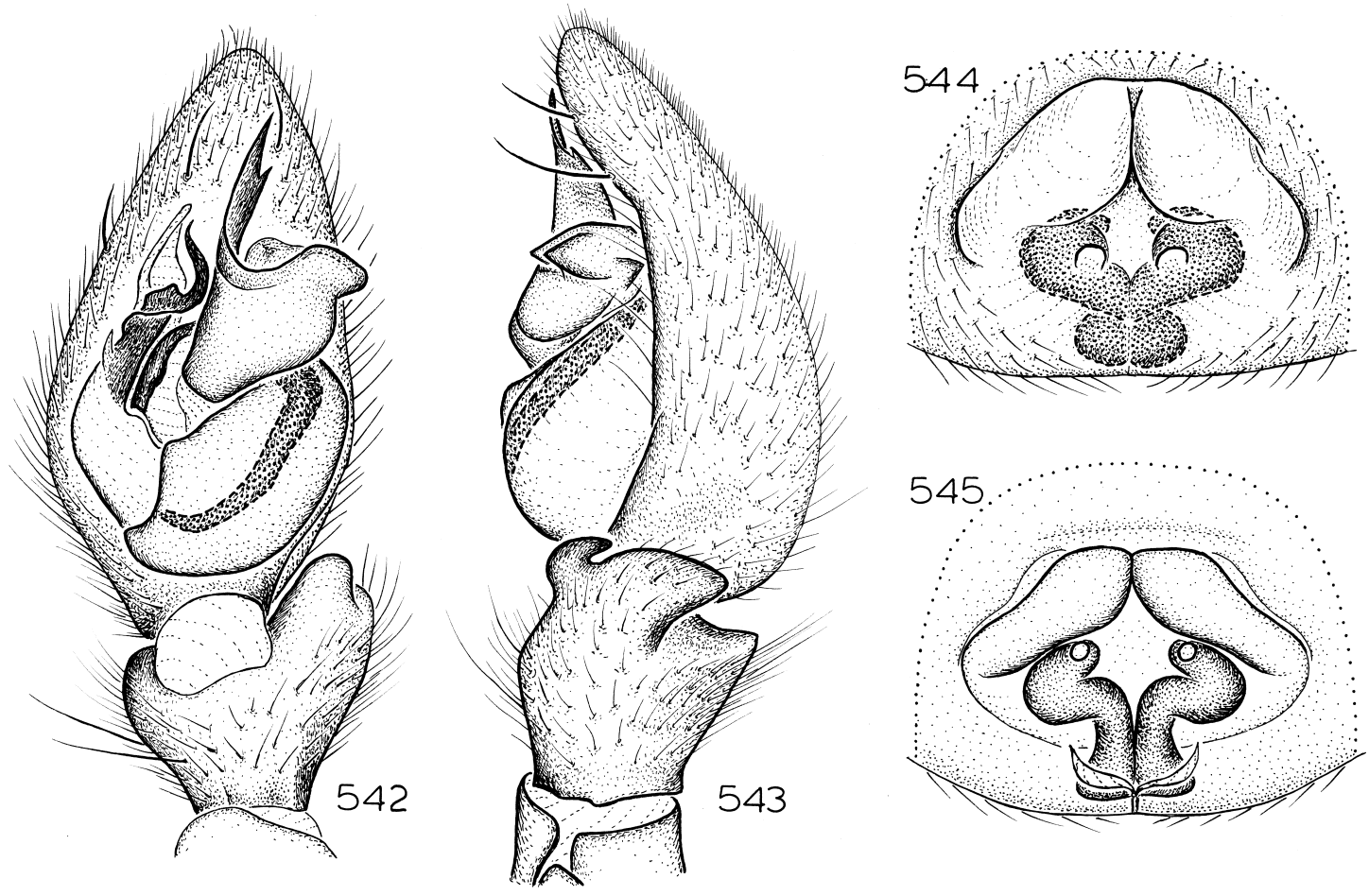

Figs. 542-545. Graycassis marengo, new species. 542. Left male palp, ventral view. 543. Same, retrolateral view. 544. Epigynum, ventral view. 545. Same, dorsal view.

Chimney Road from Chaelundi Road, Marengo State Forest, $30^{\circ} 06^{\prime} \mathrm{S}, 152^{\circ} 24^{\prime} \mathrm{E}, \mathrm{Feb}$. 4-Apr. 9, 1993, pitfall, elev. $1120 \mathrm{~m}$ (M. Gray, G. Cassis, AMS KS35332), 20; 0.4 $\mathrm{km}$ ENE junction of Hardens and Chaelundi Roads, Marengo State Forest, $30^{\circ} 08^{\prime} \mathrm{S}$, $152^{\circ} 25^{\prime}$ E, Feb. 4-Apr. 9, 1993, pitfall, elev. 1290 m (M. Gray, G. Cassis, AMS KS35328), 10َ; Obeloe Creek, 2.0 km SW along Obeloe Road from Chaelundi Road, Mount Hyland Nature Reserve, $30^{\circ} 09^{\prime} \mathrm{S}$, $152^{\circ} 27^{\prime} \mathrm{E}$, Feb. 4-Apr. 9, 1993, pitfall, elev. 910 m (M. Gray, G. Cassis, AMS KS35326),

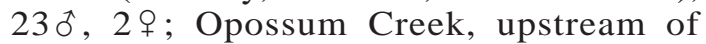
Foamy Creek Road, Marengo State Forest, $30^{\circ} 06^{\prime} \mathrm{S}, 152^{\circ} 25^{\prime} \mathrm{E}$, Feb. 4-Apr. 9, 1993, pitfall, elev. $830 \mathrm{~m}$ (M. Gray, G. Cassis, AMS KS35343), 18 ô, 3 ㅇ․

Distribution: New South Wales (map 62).

\section{Graycassis styx, new species}

Figures 546-549; Map 63

TyPES: Female holotype and male allotype taken in pitfall traps at an elevation of 1070 m off Cunnawarra Trail, c. 2 km N Cunnawarra Creek, Styx River State Forest, $30^{\circ} 32^{\prime} \mathrm{S}, 152^{\circ} 20^{\prime} \mathrm{E}$, New South Wales (Feb. 4-Apr. 9, 1993; M. Gray, G. Cassis), deposited in AMS (KS35371).

ETYMOLOGY: The specific name is a noun in apposition taken from the type locality.

Diagnosis: This species seems closest to $G$. marengo but can be distinguished by the basally enlarged median prong on the median apophysis (fig. 546) of males, and the longer epigynal ducts (fig. 549) of females.

MALE: Total length 5.8. Coloration as in G. marengo. Leg spination: tibiae III v2-2-2. Retrolateral tibial apophysis bifid, with ventral prong hook-shaped (fig. 547); median apophysis with long, basally widened medial prong (fig. 546).

FEMALE: Total length 6.5. Coloration as in G. marengo. Leg spination typical for genus. Epigynum wide, anterior one-third divided by septum (fig. 548); spermathecae each with anterior accessory lobe situated posterior of their tip (fig. 549). 

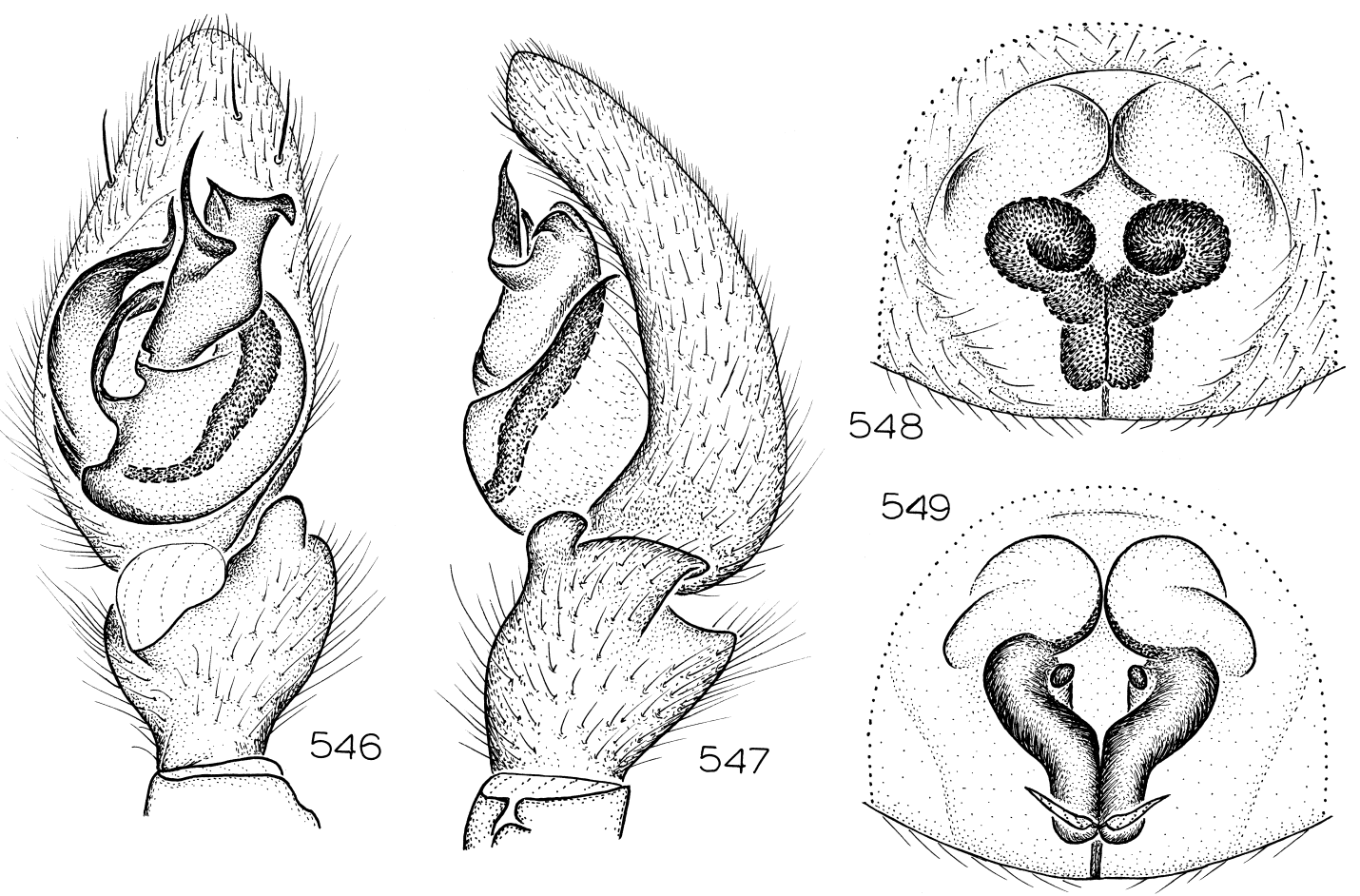

Figs. 546-549. Graycassis styx, new species. 546. Left male palp, ventral view. 547. Same, retrolateral view. 548. Epigynum, ventral view. 549. Same, dorsal view.

Other Material Examined: New South

Wales: bottom end of Cliffs Trail, c. $1.3 \mathrm{~km}$ from Oxley Road, Styx River State Forest, $30^{\circ} 33^{\prime}$ S, $152^{\circ} 20^{\prime}$ E, Feb. 4-Apr. 9, 1993, pitfall, elev. $1080 \mathrm{~m}$ (M. Gray, G. Cassis, AMS KS35362), 7ðิ, 2우 ; off Cunnawarra Trail, Styx River State Forest, $30^{\circ} 32^{\prime} \mathrm{S}, 152^{\circ} 20^{\prime} \mathrm{E}$, Feb. 4-Apr. 9, 1993, pitfall, elev. $1130 \mathrm{~m}$ (M. Gray, G. Cassis, AMS KS35367), 80, 3 9; off Cunnawarra Trail, $800 \mathrm{~m}$ N Cunnawarra Creek in gully running SE, Styx River State Forest, $30^{\circ} 33^{\prime} \mathrm{S}, 152^{\circ} 19^{\prime} \mathrm{E}$, Feb. 4-Apr. 9, 1993, pitfall, elev. $950 \mathrm{~m}$ (M. Gray, G. Cassis, AMS KS35375), 3 ơ; off Cunnawarra Trail, c. 2 km N Cunnawarra Creek, Styx River State Forest, $30^{\circ} 32^{\prime} \mathrm{S}, 152^{\circ} 20^{\prime} \mathrm{E}$, Feb. 4-Apr. 9, 1993, pitfall, elev. 1070 m (M. Gray, G. Cassis, AMS KS35371), $20^{\star}$.

Distribution: New South Wales (map 63).

Graycassis dorrigo, new species

Figures 550-553; Map 64

TYPES: Female holotype and male allotype taken in pitfall traps in rainforest at an ele-

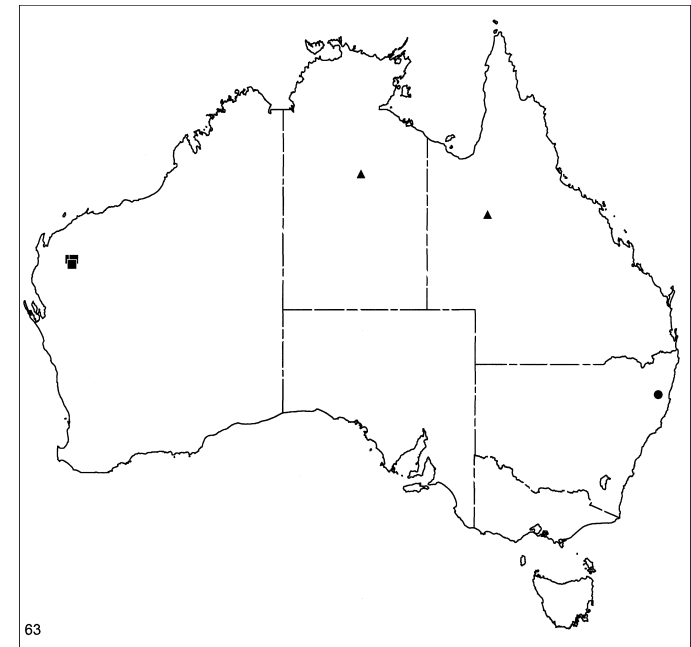

Map 63. Records of Graycassis styx, new species (circle), Asadipus barlee, new species (squares), and A. julia, new species (triangles). 

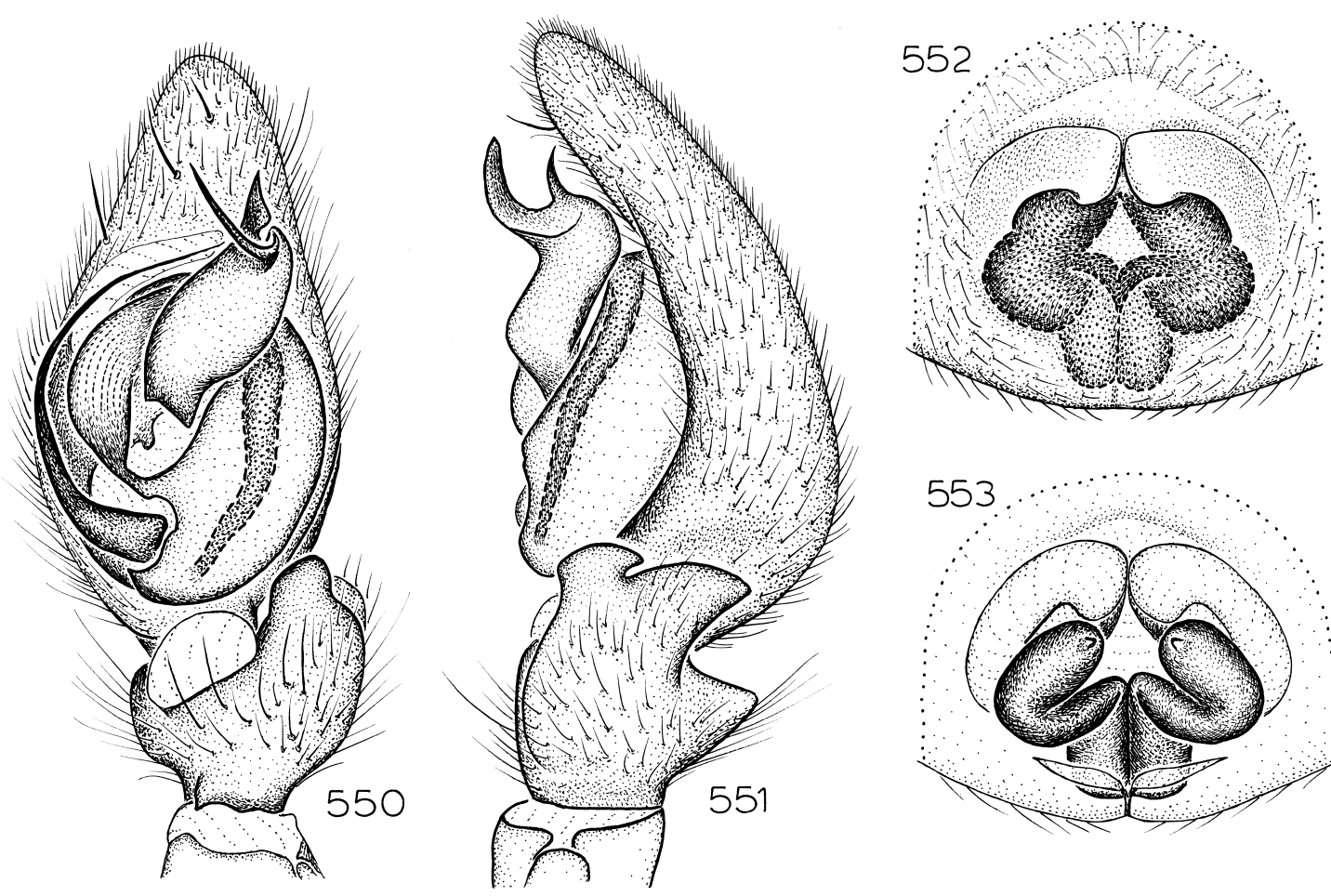

Figs. 550-553. Graycassis dorrigo, new species. 550. Left male palp, ventral view. 551. Same, retrolateral view. 552. Epigynum, ventral view. 553. Same, dorsal view.

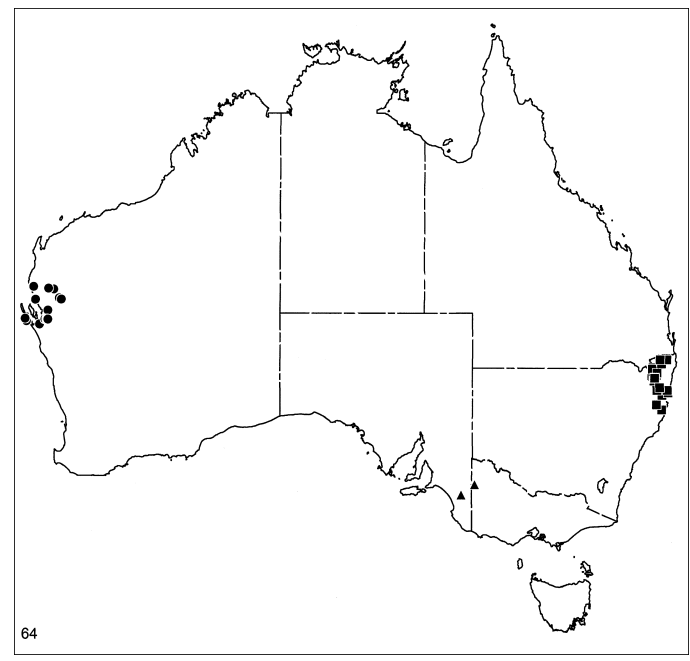

Map 64. Records of Graycassis dorrigo, new species (squares), Asadipus woodleigh, new species (circles), and A. bucks, new species (triangles). vation of $700 \mathrm{~m}$ at Never Never, Dorrigo National Park, $30^{\circ} 22^{\prime} \mathrm{S}, 152^{\circ} 45^{\prime} \mathrm{E}$, New South Wales (Nov. 12, 1980-Mar. 16, 1981; G., S. Monteith), deposited in QMB (S26848).

ETYMOLOGY: The specific name is a noun in apposition taken from the type locality.

DiAgnOSIS: This species resembles $G$. $m a$ rengo and $G$. styx in having a long median prong on the median apophysis of males, but differs in having a much smaller tip on the median apophysis (figs. 550, 551) of males and longer spermathecae (fig. 553) in females.

MALE: Total length 4.9. Coloration as in G. marengo. Leg spination: tibiae III v1p-1p2. Retrolateral tibial apophysis bifid, with ventral prong hook-shaped (fig. 551); median apophysis with long medial prong and narrow tip (fig. 550).

Female: Total length 6.1. Coloration as in G. marengo. Leg spination typical for genus. Epigynal openings directed anteriorly (fig. 552); spermathecae relatively long, folded (fig. 553). 
Other Material Examined: New South Wales: Boonoo Forest Drive, $1.8 \mathrm{~km}$ from Mount Lindsay Highway, Boonoo State Forest, 28 $56^{\prime} \mathrm{S}, 152^{\circ} 06^{\prime} \mathrm{E}$, Feb. 4-Apr. 9, 1993, pitfall, elev. $1060 \mathrm{~m}$ (M. Gray, G. Cassis, AMS KS36331), 10; Branch Creek tributary, $250 \mathrm{~m}$ downslope from Fife Fire Trail at point about $2.5 \mathrm{~km}$ NE Fife's Knob Road, $30^{\circ} 54^{\prime} \mathrm{S}, 152^{\circ} 23^{\prime} \mathrm{E}$, Feb. 4-Apr. 9, 1993, pitfall, elev. 350 m (M. Gray, G. Cassis, AMS KS39575), 5 क ; Bruxner Park, via Coffs Harbour, $30^{\circ} 14^{\prime} \mathrm{S}, 153^{\circ} 06^{\prime} \mathrm{E}$, Mar. 22-Nov. 13, 1980, rainforest pitfall, elev. $150 \mathrm{~m}$ (G., S. Monteith, QMB S26847), 30; 2.2 km NE along Chimney Road from Chaelundi Road, Marengo State Forest, $30^{\circ} 06^{\prime} \mathrm{S}, 152^{\circ} 24^{\prime} \mathrm{E}$, Feb. 4-Apr. 9, 1993, pitfall, elev. $1120 \mathrm{~m}$ (M. Gray, G. Cassis, AMS KS35333), 20; main N/S ridgeline, along Colongon Road, 1 km NW Boorook Creek junction, Boorook State Forest, $28^{\circ} 50^{\prime} \mathrm{S}, 152^{\circ} 10^{\prime} \mathrm{E}$, Feb. 4-Apr. 9, 1993, pitfall, elev. 970 m (M. Gray, G. Cassis, AMS KS36325), 40َ; Cooperabung Range Road, c. 4.5 km N Narang Road, Ballengarra State Forest, $31^{\circ} 11^{\prime} \mathrm{S}, 152^{\circ} 42^{\prime} \mathrm{E}$, Feb. 4-Apr. 9, 1993, pitfall, elev. 90 m (M. Gray, G. Cassis, AMS KS39596), 1 đ; Copper Creek Road, $1.7 \mathrm{~km}$ down from Carrai Road, then downslope $200 \mathrm{~m}$ to gully, Carrai State Forest, $30^{\circ} 57^{\prime} \mathrm{S}, 152^{\circ} 23^{\prime} \mathrm{E}$, Feb. 4-Apr. 9, 1993, pitfall, elev. 330 m (M. Gray, G. Cassis, AMS KS39574), 10; Dome Road, c. $1 \mathrm{~km}$ W Never Never Picnic Area, Dorrigo National Park, $30^{\circ} 21^{\prime} \mathrm{S}, 152^{\circ} 47^{\prime} \mathrm{E}$, Feb. 4Apr. 9, 1993, pitfall, elev. 690 m (M. Gray, G. Cassis, AMS KS35350), 12ð; Dome Road, c. $2 \mathrm{~km}$ W Never Never Picnic Area, Dorrigo National Park, $30^{\circ} 21^{\prime} \mathrm{S}, 152^{\circ} 47^{\prime} \mathrm{E}$, Feb. 4-Apr. 9, 1993, pitfall, elev. 710 m (M. Gray, G. Cassis, AMS KS35350), 100; c. $150 \mathrm{~m}$ up small track S Dome Road, c. 2.5 km W Never Never Picnic Area, Dorrigo National Park, $30^{\circ} 21^{\prime} \mathrm{S}, 152^{\circ} 47^{\prime} \mathrm{E}$, Feb. 4-Apr. 9, 1993, pitfall, elev. $700 \mathrm{~m}$ (M. Gray, G. Cassis, AMS KS35347), 50; down steep slope off Dorrigo-Bellingen Road, c. 19.5 km NW Bellingen, $30^{\circ} 24^{\prime} \mathrm{S}, 152^{\circ} 44^{\prime} \mathrm{E}$, Feb. 4-Apr. 9, 1993, pitfall, elev. $380 \mathrm{~m}$ (M. Gray, G. Cassis, AMS KS35360), 10; upper reaches, Eagle Hawk Creek, Washpool National Park, $29^{\circ} 24^{\prime} \mathrm{S}, 152^{\circ} 16^{\prime} \mathrm{E}$, Feb. 4-Apr. 9, 1993, cage traps walked in down gulley, elev. 980 m (M. Gray, G. Cassis, AMS
KS36409), 7ð; N Ewingar Road, Ewingar State Forest, $29^{\circ} 08^{\prime} \mathrm{S}, 152^{\circ} 25^{\prime} \mathrm{E}$, Feb. 4-Apr. 9, 1993, pitfall, elev. 760 m (M. Gray, G. Cassis, AMS KS40462), 10; Farnell Fire Trail, c. $1.2 \mathrm{~km} \mathrm{~N}$ junction with Upper Rocky River Fire Trail, Spirabo State Forest, $29^{\circ} 20^{\prime} \mathrm{S}, 152^{\circ} 09^{\prime} \mathrm{E}$, Feb. 4-Apr. 9, 1993, pitfall, elev. 1110 m (M. Gray, G. Cassis, AMS KS36447), 20; Fifes Knob Road, c. 3 km from Fifes Fire Trail, Carrai State Forest, $30^{\circ} 54^{\prime} \mathrm{S}, 152^{\circ} 22^{\prime} \mathrm{E}$, Feb. 4-Apr. 9, 1993, pitfall, elev. 670 m (M. Gray, G. Cassis, AMS KS39573), 20; Gibraltar Range, lower, $29^{\circ} 35^{\prime} \mathrm{S}, 152^{\circ} 13^{\prime} \mathrm{E}$, Mar. 30-Nov. 10, 1980, rainforest pitfall, elev. $450 \mathrm{~m}$ (G., S. Monteith, QMB S26476, 26483), 20, Mar. 15, 1981, elev. 450 m (G., S. Monteith, QMB S26492), 10 , 19 ; The Glade, Dorrigo National Park, $30^{\circ} 24^{\prime} \mathrm{S}, 152^{\circ} 44^{\prime} \mathrm{E}$, Mar. 22Nov. 12, 1980, rainforest pitfall, elev. $800 \mathrm{~m}$ (G., S. Monteith, QMB S26845), 20, Nov. 12, 1980-Mar. 16, 1981, rainforest pitfall, elev. 800 m (G., S. Monteith, QMB S26488, 26846), 20 , 3 \% ; Goanna Creek Road, 0.4 $\mathrm{km}$ from junction with Sandy Creek Road, Richmond Range State Forest, $28^{\circ} 37^{\prime} \mathrm{S}$, $152^{\circ} 42^{\prime} \mathrm{E}$, Feb. 4-Apr. 9, 1993, pitfall, elev. 575 m (M. Gray, G. Cassis, AMS KS35998), 40̊; Goanna Creek Road, $1.8 \mathrm{~km}$ from junction with Sandy Creek Road, Richmond Range State Forest, $28^{\circ} 36^{\prime} \mathrm{S}, 152^{\circ} 42^{\prime} \mathrm{E}, \mathrm{Feb}$. 4-Apr. 9, 1993, pitfall, elev. $580 \mathrm{~m}$ (M. Gray, G. Cassis, AMS KS40464), 40خ; Lionsville Road, Ewingar State Forest, $29^{\circ} 11^{\prime} \mathrm{S}$, $152^{\circ} 26^{\prime}$ E, Feb. 4-Apr. 9, 1993, pitfall, elev. 520 m (M. Gray, G. Cassis, AMS KS35994), 40ิ; Never Never, Dorrigo National Park, $30^{\circ} 22^{\prime} \mathrm{S}, 152^{\circ} 45^{\prime} \mathrm{E}$, Mar. 22-Nov. 12, 1980, rainforest pitfall, elev. $700 \mathrm{~m}$ (G., S. Monteith, QMB S26481, 26812), 40; North West Fire Trail, Washpool National Park, $29^{\circ} 27^{\prime} \mathrm{S}$, $152^{\circ} 16^{\prime}$ E, Feb. 4-Apr. 9, 1993, pitfall, elev. 950 m (M. Gray, G. Cassis, AMS KS36414), 19 ; North West Fire Trail, Washpool National Park, $29^{\circ} 27^{\prime} \mathrm{S}, 152^{\circ} 17^{\prime} \mathrm{E}$, Feb. 4-Apr. 9, 1993, pitfall, elev. 950 m (M. Gray, G. Cassis, AMS KS36404), 10, 1\%; Nothofagus Mountain, via Woodenbong, $28^{\circ} 24^{\prime} \mathrm{S}$, $152^{\circ} 36^{\prime} \mathrm{E}, 1981-1982$, rainforest pitfall, elev. 950 m (G. Monteith, QMB S7019), 10َ; Old Growth Road, $2.85 \mathrm{~km}$ from E end, $1.15 \mathrm{~km}$ from W end, off Sherwood Road, Conglomerate State Forest, $30^{\circ} 06^{\prime} \mathrm{S}, 153^{\circ} 03^{\prime} \mathrm{E}$, Feb. 
4-Apr. 9, 1993, pitfall, elev. 320 m (M. Gray, G. Cassis, AMS KS39571), 20; Richmond Gap, $28^{\circ} 20^{\prime} \mathrm{S}, 152^{\circ} 58^{\prime} \mathrm{E}$, 1979, elev. $520 \mathrm{~m}$ (G. Monteith, QMB S28197), 1 \%; Shannon Creek, Shannon Creek Road, Boundary Creek State Forest, $29^{\circ} 57^{\prime} \mathrm{S}$, $152^{\circ} 35^{\prime} \mathrm{E}$, Feb. 4-Apr. 9, 1993, pitfall, elev. 300 m (M. Gray, G. Cassis, AMS KS39572), 10; Solferino Creek, track off Lionsville Road, Ewingar State Forest, $29^{\circ} 10^{\prime} \mathrm{S}$, $152^{\circ} 26^{\prime} \mathrm{E}$, Feb. 4-Apr. 9, 1993, pitfall, elev. $520 \mathrm{~m}$ (M. Gray, G. Cassis, AMS KS40463), $2{ }^{\star}$; Spirabo Fire Trail, $0.5 \mathrm{~km} \mathrm{~N}$ three-way intersection near Five Bull Creek, Spirabo State Forest, $29^{\circ} 18^{\prime} \mathrm{S}, 152^{\circ} 06^{\prime} \mathrm{E}$, Feb. 4-Apr. 9, 1993, pitfall, elev. 1070 m (M. Gray, G. Cassis, AMS KS36454), 1 đ; $1.3 \mathrm{~km} \mathrm{~N}$ gate near intersection on Spirabo Fire Trail, Spirabo State Forest, $29^{\circ} 18^{\prime} \mathrm{S}, 152^{\circ} 06^{\prime} \mathrm{E}$, Feb. 4Apr. 9, 1993, pitfall, elev. 1160 m (M. Gray, G. Cassis, AMS KS36450), 80; $2.3 \mathrm{~km} \mathrm{~N}$ gate near intersection on Spirabo Fire Trail, Spirabo State Forest, $29^{\circ} 17^{\prime} \mathrm{S}, 152^{\circ} 05^{\prime} \mathrm{E}$, Feb. 4-Apr. 9, 1993, pitfall, elev. $1180 \mathrm{~m}$

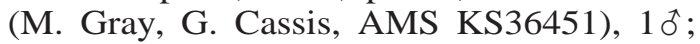
Tributary Grasstree Creek, Nogrigar Road, Ewingar State Forest, $29^{\circ} 08^{\prime} \mathrm{S}, 152^{\circ} 25^{\prime} \mathrm{E}$, Feb. 4-Apr. 9, 1993, pitfall, elev. 720 m (M. Gray, G. Cassis, AMS KS40461), $20^{\star}$; Tunners Road, Richmond Range State Forest, $28^{\circ} 37^{\prime} \mathrm{S}, 152^{\circ} 42^{\prime} \mathrm{E}$, Feb. 4-Apr. 9, 1993, pitfall, elev. 560 m (M. Gray, G. Cassis, AMS KS36035), 10; Tunners Road, junction with Peacock Creek, Richmond Range State Forest, $28^{\circ} 37^{\prime} \mathrm{S}, 152^{\circ} 42^{\prime} \mathrm{E}$, Feb. 4-Apr. 9, 1993, pitfall, elev. $470 \mathrm{~m}$ (M. Gray, G. Cassis, AMS KS36009), 50; Tweed Range Road, $500 \mathrm{~m}$ N Sheepstation Creek Track, Border Ranges National Park, $28^{\circ} 24^{\prime} \mathrm{S}, 153^{\circ} 01^{\prime} \mathrm{E}$, Feb. 4-Apr. 9, 1993, pitfall, elev. 470 m (M. Gray, G. Cassis, AMS KS35939), 1 đ; Tweed Range Road, $1.2 \mathrm{~km}$ N Sheepstation Creek turnoff, Border Ranges National Park, $28^{\circ} 24^{\prime} \mathrm{S}, 1^{\circ} 3^{\circ} 01^{\prime} \mathrm{E}$, Feb. 4-Apr. 9, 1993, pitfall, elev. $530 \mathrm{~m}$ (M. Gray, G. Cassis, AMS KS35964), 1 đo; Washpool Forest Way, Washpool National Park, $29^{\circ} 25^{\prime} \mathrm{S}, 152^{\circ} 16^{\prime} \mathrm{E}$, Feb. 4-Apr. 9, 1993, pitfall, elev. $890 \mathrm{~m}$ (M. Gray, G. Cassis, AMS KS36424), 20; Washpool State Forest, $29^{\circ} 16^{\prime} \mathrm{S}, 152^{\circ} 22^{\prime} \mathrm{E}$, Feb. 22-Mar. 20, 1992, pitfall (M. Gray, P. Croft, AMS KS38710, 38712, 38713), 30; $1.4 \mathrm{~km}$ $\mathrm{S}$ intersection with Wattle Ridge Road on
Farnell Fire Trail, Spirabo State Forest, $29^{\circ} 19^{\prime} \mathrm{S}, 152^{\circ} 09^{\prime} \mathrm{E}$, Feb. 4-Apr. 9, 1993, pitfall, elev. $1130 \mathrm{~m}$ (M. Gray, G. Cassis, AMS KS36446), 20; Wonga Walk, c. 200 m SW Hardwood Lookout, Dorrigo National Park, $30^{\circ} 22^{\prime} \mathrm{S}, 152^{\circ} 44^{\prime} \mathrm{E}$, Feb. 4-Apr. 9, 1993, pitfall, elev. $630 \mathrm{~m}$ (M. Gray, G. Cassis, AMS KS35352), 5 o; Wonga Walk, on small stream below Tristania Falls, Dorrigo National Park, $30^{\circ} 22^{\prime} \mathrm{S}, 152^{\circ} 43^{\prime} \mathrm{E}$, Feb. 4-Apr. 9, 1993, pitfall, elev. $630 \mathrm{~m}$ (M. Gray, G. Cassis, AMS KS35355), 10̋; Wonga Walk, c. $600 \mathrm{~m} \mathrm{~N}$ Tristania Falls, Dorrigo National Park, $30^{\circ} 22^{\prime} \mathrm{S}, 152^{\circ} 43^{\prime} \mathrm{E}$, Feb. 4-Apr. 9, 1993, pitfall, elev. 730 m (M. Gray, G. Cassis, AMS KS35354), 30 , 1 \% ; junction Woolool Wooloolni and Basket Swamp Road, Boonoo State Forest, $28^{\circ} 55^{\prime} \mathrm{S}, 152^{\circ} 08^{\prime} \mathrm{E}$, Feb. 4-Apr. 9, 1993, pitfall, elev. $1070 \mathrm{~m}$ (M. Gray, G. Cassis, AMS KS36334), 10; Yabbra State Forest, $28^{\circ} 30^{\prime} \mathrm{S}, 152^{\circ} 40^{\prime} \mathrm{E}$, Feb. 7-Mar. 15, 1992, pitfall (M. Gray, P. Croft, AMS KS38441, 38444, 38445, 38447, 38450, 38792, 38795, 38797, 38800), 15 ๙, Mar. 15-Apr. 2, 1992, pitfall (M. Gray, P. Croft, AMS KS38454, 38456-38462), 180, 1 ㅇ.

Distribution: New South Wales (map 64).

\section{Graycassis bruxner, new species \\ Figures 554-557; Map 65}

TyPES: Female holotype and male allotype taken in pitfall traps in rainforest at an elevation of $150 \mathrm{~m}$ in Bruxner Park, near Coffs Harbour, $30^{\circ} 14^{\prime} \mathrm{S}, 153^{\circ} 06^{\prime} \mathrm{E}$, New South Wales (Mar. 22-Nov. 13, 1980; B. Monteith), deposited in QMB (S26487).

ETYMOLOGY: The specific name is a noun in apposition taken from the type locality.

DiAGNOSIS: This species seems closest to G. chichester; males can be distinguished by the shape of the tip of the median apophysis (figs. 554, 555), females by the shorter spermathecae and more widely separated epigynal openings (figs. 556, 557).

MALE: Total length 3.6. Abdominal dorsum with pair of transverse white stripes at about half of length, coloration otherwise as in G. marengo. Leg spination: tibiae III v01p-2, r0-0-1; IV v2-1p-2; metatarsi IV p0-10 . Retrolateral tibial apophysis bifid, with ventral prong ledge-shaped (fig. 555); medi- 

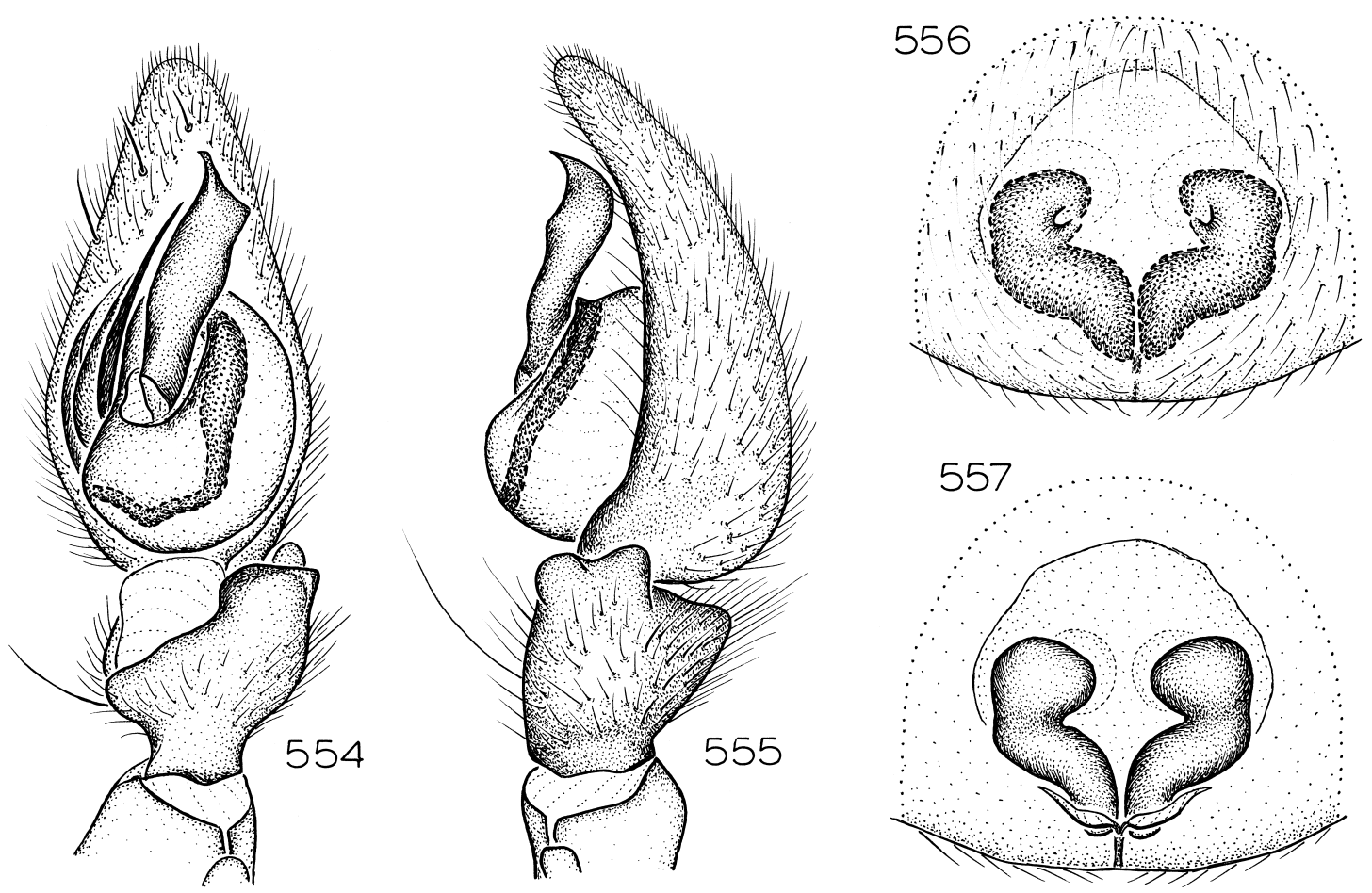

Figs. 554-557. Graycassis bruxner, new species. 554. Left male palp, ventral view. 555. Same, retrolateral view. 556. Epigynum, ventral view. 557. Same, dorsal view.

an apophysis with twisted tip, ending in narrow, median point (fig. 554).

Female: Total length 4.8. Coloration as in male. Leg spination: tibiae III v0-1p-2, r0-01; IV v1p-1p-2; metatarsi IV p0-1-0. Epigynal openings small, widely separated (fig. 556); spermathecae short, curled around epigynal openings (fig. 557).

Other Material ExAmined: New South Wales: Bennetts Road, c. 10 km NW Urbenville, Beaury State Forest, $28^{\circ} 25^{\prime} \mathrm{S}$, $152^{\circ} 27^{\prime} \mathrm{E}$, Feb. 4-Apr. 9, 1993, pitfall, elev. 890 m (M. Gray, G. Cassis, AMS KS36081), 20; Block and Tackle Ridge, $500 \mathrm{~m}$ from Carrai Road, Carrai State Forest, 30 $57^{\prime} \mathrm{S}$, $152^{\circ} 23^{\prime} \mathrm{E}$, Feb. 4-Apr. 9, 1993, pitfall, elev. 540 m (M. Gray, G. Cassis, AMS KS39620), $30 ; 1 \mathrm{~km}$ E main road, Boorook State Forest, $28^{\circ} 49^{\prime} \mathrm{S}, 152^{\circ} 11^{\prime} \mathrm{E}$, Feb. 4-Apr. 9, 1993, pitfall, elev. 900 m (M. Gray, G. Cassis, AMS KS36303), 1 ơ; $^{\dagger} 0.45 \mathrm{~km} \mathrm{SW}$ along Boundary Creek Road from junction of Dungel and Shannon Creek Roads, Boundary Creek State Forest, $29^{\circ} 56^{\prime} \mathrm{S}, 152^{\circ} 33^{\prime} \mathrm{E}$, Feb. 4-Apr.
9, 1993, pitfall, elev. 600 m (M. Gray, G. Cassis, AMS KS39612), 10; Branch Creek tributary, $250 \mathrm{~m}$ downslope from Fife Fire Trail, $30^{\circ} 54^{\prime} \mathrm{S}, 152^{\circ} 23^{\prime} \mathrm{E}$, Feb. 4-Apr. 9, 1993, pitfall, elev. 350 m (M. Gray, G. Cassis, AMS KS39616), 20; Bruxner Park, near Coffs Harbour, $30^{\circ} 14^{\prime} \mathrm{S}, 153^{\circ} 06^{\prime} \mathrm{E}$, May 14 , 1969, rainforest Berlese (R. Taylor, QMB), 10, 1 ㅇ, Mar. 22-Nov. 13, 1980, rainforest pitfall, elev. $150 \mathrm{~m}$ (B. Monteith, QMB S26487), 1 oิ, 2 q , Nov. 13, 1980-Mar. 17, 1981, rainforest pitfall, elevation $150 \mathrm{~m}$ (B. Monteith, QMB S26816), 1\%; $1.9 \mathrm{~km} \mathrm{~N}$ along Chaelundi Road from Big Bull Creek Road, Mount Hyland Nature Reserve, $30^{\circ} 08^{\prime} \mathrm{S}, 152^{\circ} 26^{\prime} \mathrm{E}$, Feb. 4-Apr. 9, 1993, pitfall, elev. 1160 m (M. Gray, G. Cassis, AMS KS35324), 20; Chandler Creek at confluence with Stockyard Creek, Boundary Creek State Forest, $29^{\circ} 57^{\prime} \mathrm{S}, 152^{\circ} 32^{\prime} \mathrm{E}$, Feb. 4-Apr. 9, 1993, pitfall, elev. 240 m (M. Gray, G. Cassis, AMS KS39611), 50̈; 2.2 km NE along Chimney Road from Chaelundi Road, Marengo State Forest, $30^{\circ} 06^{\prime} \mathrm{S}, 152^{\circ} 24^{\prime} \mathrm{E}$, 
Feb. 4-Apr. 9, 1993, pitfall, elev. $1120 \mathrm{~m}$ (M. Gray, G. Cassis, AMS KS35334), 20 ; main N/S ridgeline along Colongon Road, 1 km NW Boorook Creek junction, Boorook State Forest, $28^{\circ} 50^{\prime} \mathrm{S}, 152^{\circ} 10^{\prime} \mathrm{E}$, Feb. 4-Apr. 9, 1993, pitfall, elev. 970 m (M. Gray, G. Cassis, AMS KS36328), 20; Copper Creek Road, Carrai State Forest, $30^{\circ} 57^{\prime} \mathrm{S}, 152^{\circ} 23^{\prime} \mathrm{E}$, Feb. 4-Apr. 9, 1993, pitfall, elev. 330 m (M. Gray, G. Cassis, AMS KS39619), 1 đ̊; Daisy Patch Road, 3.7 km S Enfield Road, Daisy Patch Flora Reserve, $31^{\circ} 21^{\prime} \mathrm{S}, 151^{\circ} 55^{\prime} \mathrm{E}$, Feb. 4-Apr. 9, 1993, pitfall, elev. $1130 \mathrm{~m}$ (M. Gray, G. Cassis, AMS KS37370), 50, 2 웅 $100 \mathrm{~m} \mathrm{~S}$ junction of Dilli and Boundary Creek Roads, Boundary Creek State Forest, $29^{\circ} 58^{\prime}$ S, $152^{\circ} 36^{\prime}$ E, Feb. 4-Apr. 9, 1993, pitfall, elev. 540 m (M. Gray, G. Cassis, AMS

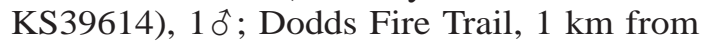
Enfield Road, Enfield State Forest, $31^{\circ} 23^{\prime} \mathrm{S}$, $151^{\circ} 53^{\prime} \mathrm{E}$, Feb. 4-Apr. 9, 1993, pitfall, elev. $1050 \mathrm{~m}$ (M. Gray, G. Cassis, AMS KS37377), 5 ơ; Dodds Fire Trail, 2 km from Enfield Road, Enfield State Forest, $31^{\circ} 23^{\prime} \mathrm{S}$, $151^{\circ} 52^{\prime}$ E, Feb. 4-Apr. 9, 1993, pitfall, elev. 930 m (M. Gray, G. Cassis, AMS KS37371), 30 , 1 웅 Dodds Fire Trail, c. 3 km from Enfield Road, Enfield State Forest, $31^{\circ} 24^{\prime} \mathrm{S}$, $151^{\circ} 52^{\prime}$ E, Feb. 4-Apr. 9, 1993, pitfall, elev. 910 m (M. Gray, G. Cassis, AMS KS37372),

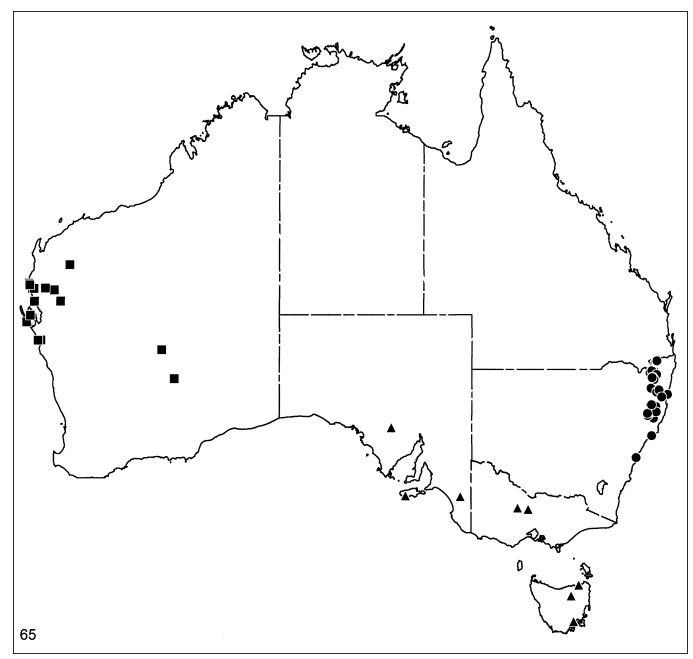

Map 65. Records of Graycassis bruxner, new species (circles), Asadipus banjiwarn, new species (squares), and A. longforest, new species (triangles).
11 ô, 1 ㅇ ; upper reaches, Eagle Hawk Creek, Washpool National Park, $29^{\circ} 24^{\prime} \mathrm{S}, 152^{\circ} 16^{\prime} \mathrm{E}$, Feb. 4-Apr. 9, 1993, cage traps walked in down gulley, elev. 980 m (M. Gray, G. Cassis, AMS KS36409), 20; East Kunderang Trail, $1.35 \mathrm{~km}$ E West Kunderang Trail, $30^{\circ} 48^{\prime} \mathrm{S}, 152^{\circ} 02^{\prime} \mathrm{E}$, Feb. 4-Apr. 9, 1993, pitfall, elev. $890 \mathrm{~m}$ (M. Gray, G. Cassis, AMS

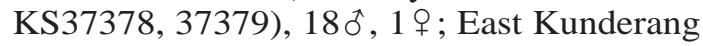
Trail, $2.1 \mathrm{~km} \mathrm{E} \mathrm{West} \mathrm{Kunderang} \mathrm{Trail,}$ $30^{\circ} 49^{\prime} \mathrm{S}, 152^{\circ} 03^{\prime} \mathrm{E}$, Feb. 4-Apr. 9, 1993, pitfall, elev. 845 m (M. Gray, G. Cassis, AMS KS37380), 40; Elkhorn Road, Ewingar State Forest, $29^{\circ} 06^{\prime} \mathrm{S}, 152^{\circ} 26^{\prime} \mathrm{E}$, Feb. 4-Apr. 9, 1993, pitfall, elev. 710 m (M. Gray, G. Cassis, AMS KS40467), 4o, pitfall, elev. $670 \mathrm{~m}$ (M. Gray, G. Cassis, AMS KS40466), 5 đ̊; Ewingar Creek, Elkhorn Road, Ewingar State Forest, $29^{\circ} 05^{\prime} \mathrm{S}, 152^{\circ} 26^{\prime} \mathrm{E}$, Feb. 4-Apr. 9, 1993, pitfall, elev. 640 m (M. Gray, G. Cassis, AMS KS40465), 5 ơ $^{\dagger}$ Fife Fire Trail, $500 \mathrm{~m}$ NE Fifes Knob Road, Carrai State Forest, $30^{\circ} 55^{\prime} \mathrm{S}, 152^{\circ} 23^{\prime} \mathrm{E}$, Feb. 4-Apr. 9, 1993, pitfall, elev. 650 m (M. Gray, G. Cassis, AMS KS396187, 40; Fife Fire Trail, 1.6 km NE Fifes Knob Road, Carrai State Forest, $30^{\circ} 55^{\prime} \mathrm{S}, 152^{\circ} 23^{\prime} \mathrm{E}$, Feb. 4-Apr. 9, 1993, pitfall, elev. 670 m (M. Gray, G. Cassis, AMS KS39618), 1 ơ; Fifes Knob Road, 2 km from Fifes Fire Trail, Carrai State Forest, $30^{\circ} 54^{\prime} \mathrm{S}$, $152^{\circ} 22^{\prime}$ E, Feb. 4-Apr. 9, 1993, pitfall, elev. 800 m (M. Gray, G. Cassis, AMS KS39622), 7ð; Fifes Knob Road, c. $3 \mathrm{~km}$ from Fifes Fire Trail, Carrai State Forest, $30^{\circ} 54^{\prime} \mathrm{S}$, $152^{\circ} 22^{\prime}$ E, Feb. 4-Apr. 9, 1993, pitfall, elev. 670 m (M. Gray, G. Cassis, AMS KS39623), $8{ }^{\dagger}$; Fifes Knob Road, c. $4 \mathrm{~km}$ from Fifes Fire Trail, Carrai State Forest, $30^{\circ} 54^{\prime} \mathrm{S}$, $152^{\circ} 22^{\prime}$ E, Feb. 4-Apr. 9, 1993, pitfall, elev. 740 m (M. Gray, G. Cassis, AMS KS39621), $11 \delta^{\star}$; Gordon, $33^{\circ} 44^{\prime} \mathrm{S}, 151^{\circ} 09^{\prime} \mathrm{E}$, Mar. 24Apr. 4, 1983, pitfall in litter (C. Horseman, AMS KS10884), 4ð, Feb. 16-Mar. 15, 1984, pitfall in litter (C. Horseman, AMS KS14040), 10, Mar. 15-Apr. 10, 1984, pitfalls in litter (C. Horseman, AMS KS14245), 50; Apr. 10-May 9, 1984, pitfalls in litter (C. Horseman, AMS KS14391), 60; Grahams Gully, downstream of Boundary Creek Road, Boundary Creek State Forest, $29^{\circ} 58^{\prime} \mathrm{S}$, $152^{\circ} 34^{\prime}$ E, Feb. 4-Apr. 9, 1993, pitfall, elev. 430 m (M. Gray, G. Cassis, AMS KS39613), 10; Grey Gums Forest Road, $1.1 \mathrm{~km}$ from 
Doyles River Road, Bulga State Forest, $31^{\circ} 33^{\prime} \mathrm{S}, 152^{\circ} 14^{\prime} \mathrm{E}$, Feb. 4-Apr. 9, 1993, pitfall, elev. $620 \mathrm{~m}$ (M. Gray, G. Cassis, AMS KS39580), 40; Grey Gums Forest Road, 2.1 $\mathrm{km}$ from Doyles River Road, Bulga State Forest, $31^{\circ} 33^{\prime} \mathrm{S}, 152^{\circ} 14^{\prime} \mathrm{E}$, Feb. 4-Apr. 9, 1993, pitfall, elev. 560 m (M. Gray, G. Cassis, AMS KS39580), 20 ; 0.5 km ENE junction of Hardens and Chaelundi Roads, Marengo State Forest, $30^{\circ} 08^{\prime} \mathrm{S}, 152^{\circ} 25^{\prime} \mathrm{E}$, Feb. 4-Apr. 9, 1993, pitfall, elev. $1290 \mathrm{~m}$ (M. Gray, G. Cassis, AMS KS35329), 1 후 0.95 km W Henry Road on London Bridge Road, London Bridge State Forest, $29^{\circ} 50^{\prime} \mathrm{S}$, $152^{\circ} 12^{\prime} \mathrm{E}$, Feb. 4-Apr. 9, 1993, pitfall, elev. 990 m (M. Gray, G. Cassis, AMS KS36388), 10 ; $240 \mathrm{~m}$ E junction Kunderang East and Kunderang West Road, $30^{\circ} 48^{\prime} \mathrm{S}, 152^{\circ} 02^{\prime} \mathrm{E}$, Feb. 4-Apr. 9, 1993, pitfall, elev. 900 m (M. Gray, G. Cassis, AMS KS37381), 50; NE facing slope above Kunderang Station Creek, $30^{\circ} 48^{\prime} \mathrm{S}, 152^{\circ} 06^{\prime} \mathrm{E}$, Feb. 4-Apr. 9, 1993, pitfall, elev. 410 m (M. Gray, G. Cassis, AMS KS37389), 60; $250 \mathrm{~m}$ upstream of London Bridge Fire Trail, House Creek, Oakwood State Forest, $29^{\circ} 54^{\prime} \mathrm{S}, 152^{\circ} 05^{\prime} \mathrm{E}$, Feb. 4-Apr. 9, 1993, pitfall, elev. 1000 m (M. Gray, G. Cassis, AMS KS36373), 80; Mount Boss State Forest, $31^{\circ} 12^{\prime} \mathrm{S}, 152^{\circ} 24^{\prime} \mathrm{E}$, Oct. 1980 (G. Webb, AMS KS43557), 1; Mummel Forest Road, $7.6 \mathrm{~km} \mathrm{~N}$ junction with Enfield Forest Road, Enfield State Forest, $31^{\circ} 17^{\prime} \mathrm{S}$, $151^{\circ} 51^{\prime} \mathrm{E}$, Feb. 4-Apr. 9, 1993, pitfall, elev. $1340 \mathrm{~m}$ (M. Gray, G. Cassis, AMS KS37359), 1 ơ; Nogrigar Road, Ewingar State Forest, $29^{\circ} 09^{\prime} \mathrm{S}, 152^{\circ} 26^{\prime} \mathrm{E}$, Feb. 4-Apr. 9, 1993, pitfall, elev. $800 \mathrm{~m}$ (M. Gray, G. Cassis, AMS KS40468), 4ð゚; Opossum Creek, upstream of Foamy Creek Road, Marengo State Forest, $30^{\circ} 06^{\prime} \mathrm{S}, 152^{\circ} 25^{\prime} \mathrm{E}$, Feb. 4-Apr. 9, 1993, pitfall, elev. 830 m (M. Gray, G. Cassis, AMS KS35344), $10^{\text {t }}$; Poverty Point, SE Tenterfield, $29^{\circ} 03^{\prime} \mathrm{S}$, $152^{\circ} 01^{\prime} \mathrm{E}$, Oct. 12 , 1977-Feb. 8, 1978, rainforest pitfall, elev. $945 \mathrm{~m}$ (G. Monteith, QMB S26403), 1 \% ; Rivertree Fire Trail, Gilgurry State Forest, $28^{\circ} 45^{\prime} \mathrm{S}, 152^{\circ} 15^{\prime} \mathrm{E}$, Feb. 4-Apr. 9, 1993, pitfall, elev. 770 m (M. Gray, G. Cassis, AMS KS36436), 10; SW end, Rocky Waterhole Road, Beaury State Forest, $28^{\circ} 33^{\prime} \mathrm{S}, 152^{\circ} 19^{\prime} \mathrm{E}$, Feb. 4-Apr. 9, 1993, pitfall, elev. 530 m (M. Gray, G. Cassis, AMS KS36129), 10; Sharpers Creek,
Boundary Road, N Sharpers Road, Nerong State Forest, $32^{\circ} 31^{\prime} \mathrm{S}, 152^{\circ} 07^{\prime} \mathrm{E}$, Feb. 4-Apr. 9, 1993, pitfall, elev. 30 m (M. Gray, G. Cassis, AMS KS39597), 3 đ; $1.9 \mathrm{~km} \mathrm{~W}$ along Sheas Nob Road from Boundary Creek Road, Boundary Creek State Forest, 2959'S, $152^{\circ} 34^{\prime}$ E, Feb. 4-Apr. 9, 1993, pitfall, elev. 550 m (M. Gray, G. Cassis, AMS KS39615), 3 o ; saddle along ridge, Spirabo State Forest, $29^{\circ} 18^{\prime} \mathrm{S}, 152^{\circ} 11^{\prime} \mathrm{E}$, Feb. 4-Apr. 9, 1993, pitfall, elev. 920 m (M. Gray, G. Cassis, AMS KS36402), 20; Timbarra Trig, Boonoo State Forest, $28^{\circ} 56^{\prime} \mathrm{S}, 152^{\circ} 08^{\prime} \mathrm{E}$, Feb. 4-Apr. 9, 1993, pitfall, elev. 1130 m (M. Gray, G. Cassis, AMS KS36345), 30; 1.9 km along Tucker Box Road from Tooloom Rest Area, Beaury State Forest, $28^{\circ} 28^{\prime} \mathrm{S}, 152^{\circ} 24^{\prime} \mathrm{E}$, Feb. 4Apr. 9, 1993, pitfall, elev. 760 m (M. Gray, G. Cassis, AMS KS36107), 30े; UrbenvilleLegume Road, gully in middle of Toolom Scrub Flora, Toolom Scrub Flora Reserve, $28^{\circ} 28^{\prime} \mathrm{S}, 152^{\circ} 23^{\prime} \mathrm{E}$, Feb. 4-Apr. 9, 1993, pitfall, elev. 715 m (M. Gray, G. Cassis, AMS KS36126), 20; $\mathrm{N}$ along Wallaby Creek, Beaury State Forest, $28^{\circ} 26^{\prime} \mathrm{S}, 152^{\circ} 27^{\prime} \mathrm{E}$, Feb. 4-Apr. 9, 1993, pitfall, elev. 830 m (M. Gray, G. Cassis, AMS KS36090), 10; N along Wallaby Creek Road, Beaury State Forest, $28^{\circ} 24^{\prime} \mathrm{S}, 152^{\circ} 27^{\prime} \mathrm{E}$, Feb. 4-Apr. 9, 1993, pitfall, elev. 910 m (M. Gray, G. Cassis, AMS KS36147), 50; Washpole Forest Way, Washpole National Park, $29^{\circ} 25^{\prime} \mathrm{S}$, $152^{\circ} 16^{\prime}$ E, Feb. 4-Apr. 9, 1993, pitfall, elev. 900 m (M. Gray, G. Cassis, AMS KS36423), 70; Wattle Creek Road, Spirabo State Forest, $29^{\circ} 18^{\prime} \mathrm{S}, 152^{\circ} 10^{\prime} \mathrm{E}$, Feb. 4-Apr. 9, 1993, pitfall, elev. 930 m (M. Gray, G. Cassis, AMS KS36400), 10; $1.4 \mathrm{~km} \mathrm{~S}$ intersection with Wattle Ridge Road on Farnell Fire Trail, Spirabo State Forest, $29^{\circ} 19^{\prime} \mathrm{S}, 152^{\circ} 09^{\prime} \mathrm{E}$, Feb. 4-Apr. 9, 1993, pitfall, elev. $1130 \mathrm{~m}$ (M. Gray, G. Cassis, AMS KS36445, 36446), 60, $1 \%$; Westerly branch, Kunderang Station Creek, $30^{\circ} 48^{\prime} \mathrm{S}, 152^{\circ} 06^{\prime} \mathrm{E}$, Feb. 4-Apr. 9, 1993, pitfall, elev. 310 m (M. Gray, G. Cassis, AMS KS37385), 25 đ, 6 ; Wonga Walk, c. 200 m SW Hardwood Lookout, Dorrigo National Park, $30^{\circ} 22^{\prime} \mathrm{S}$, $152^{\circ} 44^{\prime}$ E, Feb. 4-Apr. 9, 1993, pitfall, elev. 630 m (M. Gray, G. Cassis, AMS KS35353), 30 .

Distribution: New South Wales (map 65). 

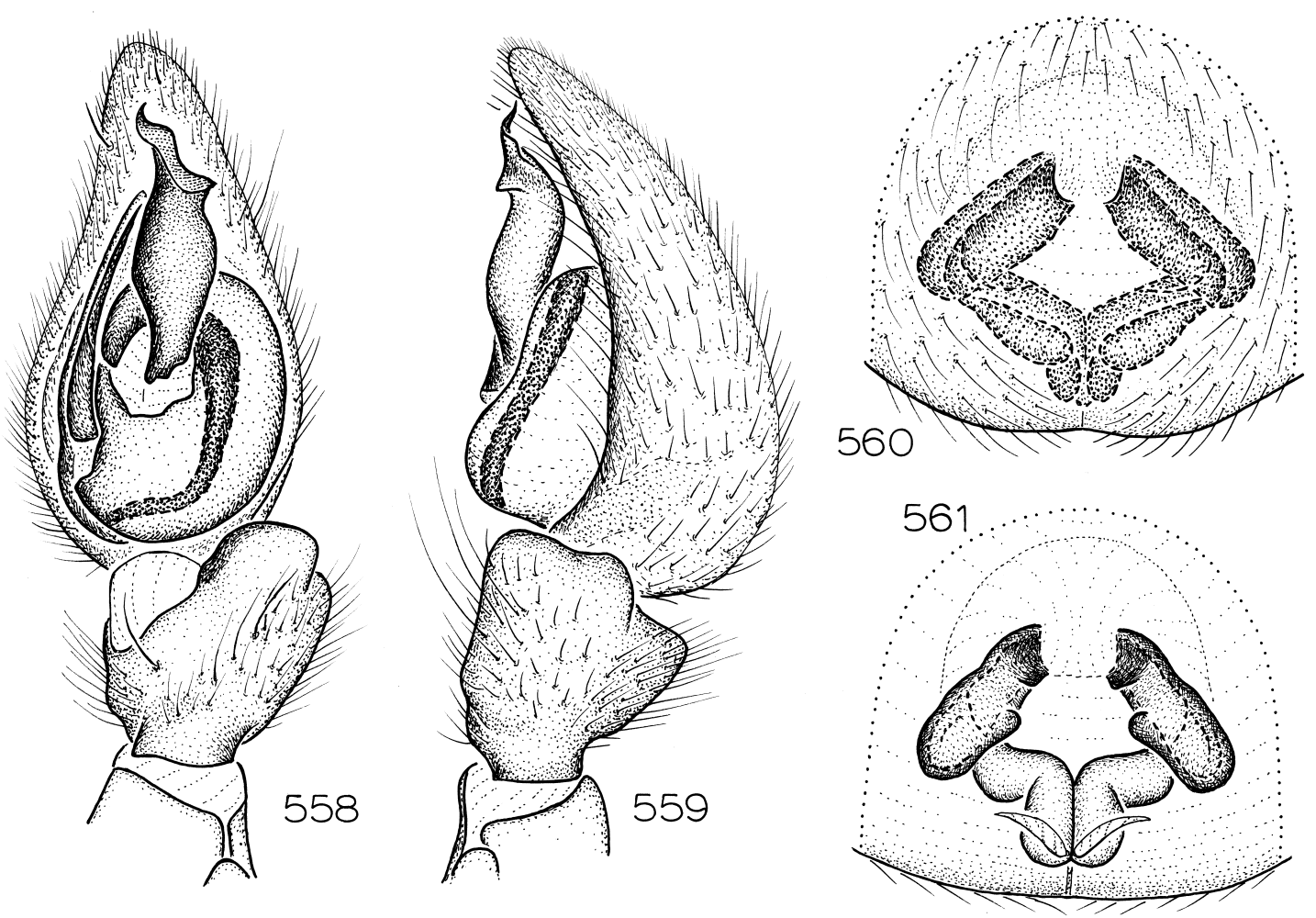

Figs. 558-561. Graycassis chichester, new species. 558. Left male palp, ventral view. 559. Same, retrolateral view. 560. Epigynum, ventral view. 561. Same, dorsal view.

\section{Graycassis chichester, new species} Figures 558-561; Map 66

TyPES: Female holotype and male allotype taken in pitfall traps at an elevation of 385 $\mathrm{m}$ on Allyn River Forest Road, $500 \mathrm{~m} \mathrm{~S}$ Mount Allyn Road, Chichester State Forest, $32^{\circ} 09^{\prime} \mathrm{S}, 151^{\circ} 28^{\prime} \mathrm{E}$, New South Wales (Feb. 4-Apr. 9, 1993; M. Gray, G. Cassis), deposited in AMS (KS37356).

ETYMOLOGY: The specific name is a noun in apposition taken from the type locality.

Diagnosis: This species resembles $G$. bruxner but can be distinguished by the sharp point on the retrolateral side of the median apophysis in males (fig. 558) and the closely spaced, anteriorly situated epigynal openings of females (fig. 560).

MALE: Total length 5.1. Coloration as in G. bruxner. Leg spination: tibiae: III v0-1p2, r0-0-0; IV v2-1p-2; metatarsi IV p0-1-0. Retrolateral tibial apophysis not bifid, with ventral edge ledge-shaped (fig. 559); median apophysis with twisted tip originating from sharp point on retrolateral side (fig. 558).

FEMALE: Total length 4.9. Coloration as in male. Leg spination: femur I d0-0-0; tibiae: III v0-1p-2, r0-0-1; IV v1p-1p-2; metatarsi IV p0-1-0. Epigynal openings situated anteriorly, closely spaced (fig. 560); spermathecae sharply angled (fig. 561).

Other Material Examined: New South Wales: Allyn River Forest Road, $1.1 \mathrm{~km} \mathrm{~N}$ Mount Allyn Road, Chichester State Forest, $32^{\circ} 08^{\prime} \mathrm{S}, 151^{\circ} 28^{\prime} \mathrm{E}$, Feb. 4-Apr. 9, 1993, pitfall, elev. 378 m (M. Gray, G. Cassis, AMS

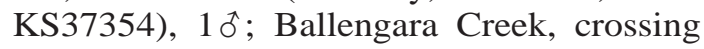
on Gregs Road, Ballengara State Forest, $31^{\circ} 13^{\prime} \mathrm{S}, 152^{\circ} 45^{\prime} \mathrm{E}$, Feb. 4-Apr. 9, 1993, pitfall, elev. $40 \mathrm{~m}$ (M. Gray, G. Cassis, AMS KS42079), 20; Bobbin Head, Kuring-gai Chase National Park, $33^{\circ} 38^{\prime} \mathrm{S}, 151^{\circ} 12^{\prime} \mathrm{E}$, June 22, 1991, sieved eucalypt litter (D. Black, WAM 96/1531, 1532), 2 q ; Broken Head, $28^{\circ} 43^{\prime} \mathrm{S}, 153^{\circ} 37^{\prime} \mathrm{E}$, Mar. 6-Nov. 7, 
1976, rainforest pitfall, elev. $30 \mathrm{~m}$ (G., S. Monteith, QMB S26703, 28111, 28112), 30; Gloucester-Bowendoc Road, beside Barnard River, $31^{\circ} 47^{\prime} \mathrm{S}, 151^{\circ} 54^{\prime} \mathrm{E}$, July 9, 1992, Berlese, rainforest litter (G. Hunt, AMS KS49465), 1 \% ; Gordon, $33^{\circ} 44^{\prime} \mathrm{S}, 151^{\circ} 09^{\prime} \mathrm{E}$, Apr. 14-June 9, 1983, pitfall in litter (C. Horseman, AMS KS12371, 12399), 2 , Nov. 24-Dec. 22, 1983, pitfall in litter (C. Horseman, AMS KS13562), 1 옹 Aug. 4Sept. 9, 1983, pitfall in litter (C. Horseman, AMS KS13293), 1 \%, Mar. 3-May 9, 1984, pitfalls in litter (C. Horseman, AMS KS14245, 14391), 2 $;$; $1.2 \mathrm{~km}$ up track $\mathrm{N}$ Grey Gums Forest Road, $2.1 \mathrm{~km}$ from Doyles River Road, Bulga State Forest, $31^{\circ} 32^{\prime}$ S, $152^{\circ} 14^{\prime}$ E, Feb. 4-Apr. 9, 1993, pitfall, elev. $620 \mathrm{~m}$ (M. Gray, G. Cassis, AMS KS39521), 8 $0^{\star}, 1$; ; 3-5 km NW Harrington, $31^{\circ} 52^{\prime} \mathrm{S}, 152^{\circ} 42^{\prime} \mathrm{E}$, litter on sandy soil, littoral rainforest (G. Williams, AMS KS23424), 3 \% ; Homewoods Road, 2.8 km W Knodingbul Road, Bulga State Forest, $31^{\circ} 37^{\prime} \mathrm{S}, 152^{\circ} 07^{\prime} \mathrm{E}$, Feb. 4-Apr. 9, 1993, pitfall, elev. 690 m (M. Gray, G. Cassis, AMS KS39526), 250 ; $600 \mathrm{~m}$ N Homewoods Road, 2.8 km W Knodingbul Road, Bulga State Forest, $31^{\circ} 37^{\prime} \mathrm{S}, 152^{\circ} 07^{\prime} \mathrm{E}$, Feb. 4-Apr. 9, 1993, pitfall, elev. 650 m (M. Gray, G. Cassis, AMS KS39525), 9ð̊, 2q; $1.8 \mathrm{~km} \mathrm{~N}$ ford of Karuah River on Karuah River Road, Chichester State Forest, $32^{\circ} 05^{\prime} \mathrm{S}, 151^{\circ} 43^{\prime} \mathrm{E}$, Feb. 4-Apr. 9, 1993, pitfall (M. Gray, G. Cassis, AMS KS37335), 350; 2.3 km N Karuah River on Karuah River Road, Chichester State Forest, $32^{\circ} 05^{\prime} \mathrm{S}, 151^{\circ} 43^{\prime} \mathrm{E}$, Feb. 4 Apr. 9, 1993, pitfall, elev. 520 m (M. Gray, G. Cassis, AMS KS3732), 29 $0,1 \%$; Kerewong State Forest, near Lorne, $31^{\circ} 36^{\prime} \mathrm{S}$, $152^{\circ} 34^{\prime} \mathrm{E}$, Nov. 7-Dec. 10, 1978, pitfall in litter (D. Milledge, AMS KS16197), 1\%; Middle Brother State Forest, near Lorne, $31^{\circ} 40^{\prime} \mathrm{S}, 152^{\circ} 41^{\prime} \mathrm{E}$, Sept. 24-Dec. 20, 1978, pitfall in litter (D. Milledge, AMS KS16108), 19 ; junction, Mount Allyn Road and Allyn River Forest Road, Chichester State Forest, $32^{\circ} 08^{\prime} \mathrm{S}, 151^{\circ} 28^{\prime} \mathrm{E}$, Feb. 4-Apr. 9, 1993, pitfall, elev. 355 m (M. Gray, G. Cassis, AMS KS37353), 50; Mount Allyn Road, 300 m E Shellbrook Forest Road, Chichester State Forest, $32^{\circ} 09^{\prime} \mathrm{S}, 151^{\circ} 27^{\prime} \mathrm{E}$, Feb. 4-Apr. 9, 1993, pitfall, elev. 580 m (M. Gray, G. Cassis, AMS KS37347), 80; Mount Allyn Road,
800 m N Shellbrook Forest Road, Chichester State Forest, $32^{\circ} 09^{\prime} \mathrm{S}, 151^{\circ} 27^{\prime} \mathrm{E}$, Feb. 4-Apr. 9, 1993, pitfall, elev. 636 m (M. Gray, G. Cassis, AMS KS37349), 10; Mount Allyn Road, 2.3 km N Shellbrook Forest Road, Chichester State Forest, $32^{\circ} 08^{\prime} \mathrm{S}, 151^{\circ} 27^{\prime} \mathrm{E}$, Feb. 4-Apr. 9, 1993, pitfall, elev. 770 m (M. Gray, G. Cassis, AMS KS37350), 50, 1 $q$; Mountain Road, $0.2 \mathrm{~km} \mathrm{~S}$ junction with $\mathrm{Ku}-$ nungra Road, $32^{\circ} 08^{\prime} \mathrm{S}, 151^{\circ} 44^{\prime} \mathrm{E}$, Feb. 4Apr. 9, 1993, pitfall (M. Gray, G. Cassis, AMS KS37345), 230, 1 ; ; Mountain Trail, $0.8 \mathrm{~km}$ from junction with Kunungra Road, $32^{\circ} 08^{\prime} \mathrm{S}, 151^{\circ} 45^{\prime} \mathrm{E}$, Feb. 4-Apr. 9, 1993, pitfall, elev. 300 m (M. Gray, G. Cassis, AMS KS37341), 11 ô, 1 ; ; Mountain Trail, $2.1 \mathrm{~km}$ from junction with Kunungra Road on SE side, ford over Kunungra River, $32^{\circ} 08^{\prime} \mathrm{S}$, $151^{\circ} 45^{\prime}$ E, Feb. 4-Apr. 9, 1993, pitfall, elev. 180 m (M. Gray, G. Cassis, AMS KS37341), $7 \delta^{\prime}$; Pacific Palms, $32^{\circ} 21^{\prime} \mathrm{S}, 152^{\circ} 31^{\prime} \mathrm{E}$, May 11-25, 1992, pitfalls (C. Peterson, AMS KS55278), 20 , June 1-23, 1992, pitfall (C. Peterson, AMS KS55279), 10; Padmans Road, near junction of Pole Dump Road, Bulga State Forest, $31^{\circ} 36^{\prime} \mathrm{S}, 152^{\circ} 10^{\prime} \mathrm{E}$, Feb. 4-Apr. 9, 1993, pitfall, elev. $730 \mathrm{~m}$ (M. Gray, G. Cassis, AMS KS39523), 60; Running Road, 100 m E Rocky Creek crossing, Whian Whian State Forest, $28^{\circ} 36^{\prime} \mathrm{S}$,

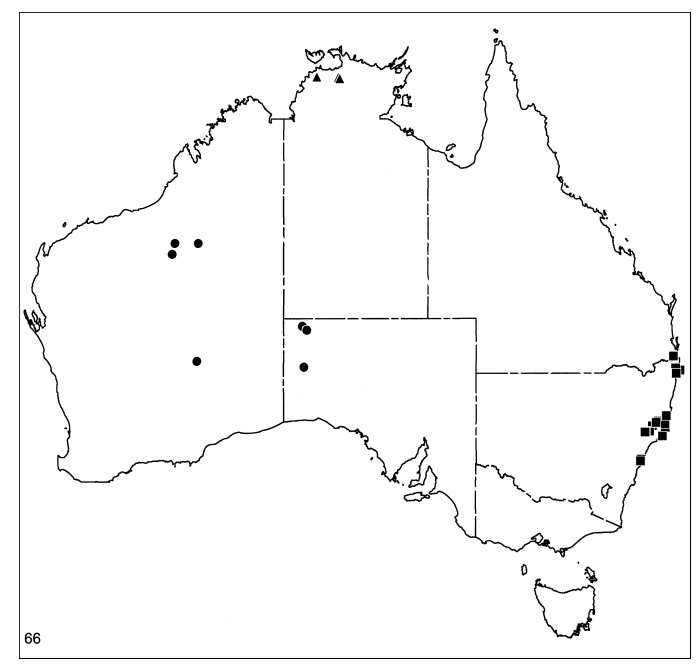

Map 66. Records of Graycassis chichester, new species (squares), Asadipus auld, new species (circles), and A. humptydoo, new species (triangles). 

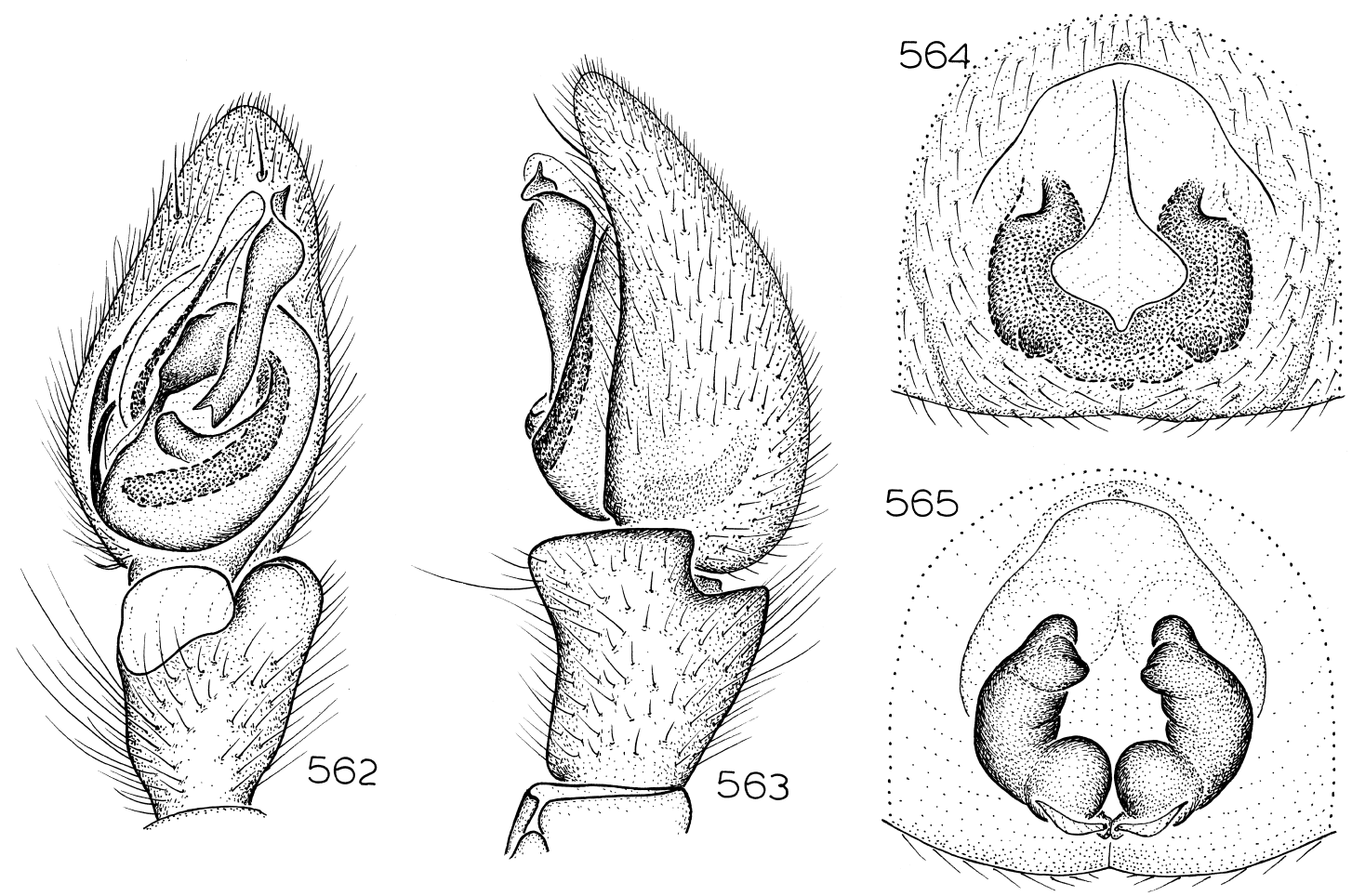

Figs. 562-565. Graycassis barrington, new species. 562. Left male palp, ventral view. 563. Same, retrolateral view. 564. Epigynum, ventral view. 565. Same, dorsal view.

$153^{\circ} 21^{\prime}$ E, Feb. 4-Apr. 9, 1993, pitfall, elev. 250 m (M. Gray, G. Cassis, AMS KS35916), $20^{\top}$; Victoria Park, via Alstonville, $28^{\circ} 53^{\prime} \mathrm{S}$, $153^{\circ} 23^{\prime} \mathrm{E}$, Mar. 23-Aug. 3, 1975, rainforest pitfall (G., S. Monteith, QMB S28329), 1 ô, Aug. 3-Nov. 16, 1975, rainforest pitfall, elev. 213 m (G., S. Monteith, QMB S12206), 10, Dec. 16-26, 1974, pitfall (G., S. Monteith, QMB S31319), 1 ; Wild Cattle Creek, $400 \mathrm{~m}$ NE Spur Track, Bulga State Forest, $31^{\circ} 36^{\prime} \mathrm{S}, 152^{\circ} 07^{\prime} \mathrm{E}$, Feb. 4-Apr. 9, 1993, pitfall, elev. 400 m (M. Gray, G. Cassis, AMS KS39524), 5 $0^{\star}$. Queensland: Mount Tambourine, $27^{\circ} 58^{\prime} \mathrm{S}, 153^{\circ} 12^{\prime} \mathrm{E}$, Sept. 14-Oct. 12, 1978, pitfall (J. Grimshaw, QMB S30192), 3 ; ; Mount Tambourine, Joalah National Park, $27^{\circ} 56^{\prime} \mathrm{S}, 153^{\circ} 12^{\prime} \mathrm{E}$, Oct. 18-21, 1978, rainforest litter (J. Lawrence, T. Weir, QMB), 19 ; Palm Grove, Mount Tambourine, $27^{\circ} 56^{\prime} \mathrm{S}, 153^{\circ} 12^{\prime} \mathrm{E}$, Mar. 22-May 25, 1975, rainforest pitfall, elev. $670 \mathrm{~m}$ (G., S. Monteith, QMB S28350), 1 t.

Distribution: New South Wales and adjacent Queensland (map 66).
Graycassis barrington, new species Figures 562-565; Map 67

TyPES: Female holotype and male allotype taken in pitfall traps at an elevation of 1460 $\mathrm{m} 0.4 \mathrm{~km} \mathrm{~S}$ along Barrington Trail from southern Green Gap turnoff, Stewarts Brook State Forest, $31^{\circ} 56^{\prime} \mathrm{S}, 151^{\circ} 26^{\prime} \mathrm{E}$, New South Wales (Feb. 4-Apr. 9, 1993; M. Gray, G. Cassis), deposited in AMS (KS39670).

ETYMology: The specific name is a noun in apposition taken from the type locality.

DIAGNOSIS: Males can easily be recognized by the greatly elongated and basally narrowed median apophysis (figs. 562, 563), females by the long anterior epigynal margin and widely separated epigynal openings (figs. 564, 565).

MALE: Total length 8.8. Coloration as in G. marengo. Leg spination: tibiae III v2-2-2; metatarsi IV p0-1-0. Retrolateral tibial apophysis entire, ledge-shaped (fig. 563); median apophysis long, basally narrowed, distally twisted (fig. 562). 
Female: Total length 9.9. Coloration as in $G$. marengo except abdominal venter pale white, with four longitudinal rows of tiny orange sclerites. Leg spination: tibiae: III v21p-2; IV p0-0-1, v2-1p-2; metatarsi IV p0-10 . Anterior epigynal margin long, extending down sides of epigynum, openings widely separated (fig. 564); spermathecae long, bulbous posteriorly (fig. 565).

Other Material Examined: New South Wales: Barrington Tops, $32^{\circ} 00^{\prime} \mathrm{S}, 151^{\circ} 28^{\prime} \mathrm{E}$, Jan. 1932, elev. $5000 \mathrm{ft}$ (P. Darlington, MCZ), 1 \% $0.4 \mathrm{~km} \mathrm{~S}$ along Barrington Trail from southern Green Gap turnoff, Stewarts Brook State Forest, 31 ${ }^{\circ} 56^{\prime} \mathrm{S}, 151^{\circ} 26^{\prime} \mathrm{E}$, Feb. 4-Apr. 9, 1993, pitfall, elev. $1460 \mathrm{~m}$ (M. Gray, G. Cassis, AMS KS39670), 17ð; Beech Walk Trail, Barrington Tops National Park, $50 \mathrm{~km}$ W Gloucester, 32 $05^{\prime} \mathrm{S}$, $151^{\circ} 37^{\prime} \mathrm{E}$, Aug. 14, 1990, Nothofagus moorei forest, elev. $1000 \mathrm{~m}$ (C. Griswold, T. Meikle, USNM), 19 ; junction, Bull Ridge and Barrington Forest Roads, Stewarts Brook State Forest, $31^{\circ} 57^{\prime} \mathrm{S}, 151^{\circ} 23^{\prime} \mathrm{E}$, Feb. 4-Apr. 9, 1993, pitfall, elev. 1440 m (M. Gray, G. Cas-

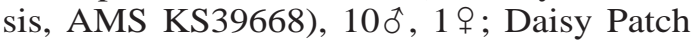
Fire Trail, $1.9 \mathrm{~km}$ S Enfield Road, Enfield State Forest, $31^{\circ} 20^{\prime} \mathrm{S}, 151^{\circ} 54^{\prime} \mathrm{E}$, Feb. 4-Apr. 9, 1993, pitfall, elev. 1130 m (M. Gray, G. Cassis, AMS KS37368), 10; Daisy Patch Road, $3.7 \mathrm{~km}$ S Enfield Road, Enfield State Forest, $31^{\circ} 21^{\prime} \mathrm{S}, 151^{\circ} 55^{\prime} \mathrm{E}$, Feb. 4-Apr. 9, 1993, pitfall, elev. 1130 m (M. Gray, G. Cassis, AMS KS37369), 10; Double Dumps Fire Trail. $700 \mathrm{~m}$ off Daisy Patch Road, Enfield State Forest, $31^{\circ} 20^{\prime} \mathrm{S}, 151^{\circ} 54^{\prime} \mathrm{E}$, Feb. $4-$ Apr. 9, 1993, pitfall, elev. 1080 m (M. Gray, G. Cassis, AMS KS37366), 20; $1.8 \mathrm{~km} \mathrm{~N}$ ford of Karuah River on Karuah River Road, Chichester State Forest, $32^{\circ} 05^{\prime} \mathrm{S}, 151^{\circ} 43^{\prime} \mathrm{E}$, Feb. 4-Apr. 9, 1993, pitfall (M. Gray, G. Cassis, AMS KS37336), 10ㅊ, 2 우 NE facing slope above Kunderang Station Creek, $30^{\circ} 48^{\prime} \mathrm{S}, 152^{\circ} 06^{\prime} \mathrm{E}$, Feb. 4-Apr. 9, 1993, pitfall, elev. 410 m (M. Gray, G. Cassis, AMS KS37387), 40; junction Kunungra and Berrico Roads, Chichester State Forest, $32^{\circ} 06^{\prime} \mathrm{S}$, $151^{\circ} 46^{\prime} \mathrm{E}$, Feb. 4-Apr. 9, 1993, pitfall, elev. $1000 \mathrm{~m}$ (M. Gray, G. Cassis, AMS KS37339), 20; Lumeah Road, $1.7 \mathrm{~km}$ from Mount Allyn Road, Chichester State Forest, $32^{\circ} 06^{\prime} \mathrm{S}, 1^{\circ} 1^{\circ} 26^{\prime} \mathrm{E}$, Feb. 4-Apr. 9, 1993, pitfall, elev. 970 m (M. Gray, G. Cassis, AMS
KS37351), 130ే; Mountain Trail, 2. 1 km S intersection with Kunungra Road on SE side of ford over Kunungra River, $32^{\circ} 08^{\prime} \mathrm{S}$, $151^{\circ} 45^{\prime} \mathrm{E}$, Feb. 4-Apr. 9, 1993, pitfall, elev. 180 m (M. Gray, G. Cassis, AMS KS37342), 20 ; Mount Allyn Road, adjacent to Mount Allyn Forest Park, $900 \mathrm{~m}$ from junction of Mount Allyn Lookout Road, Chichester State Forest, $32^{\circ} 07^{\prime} \mathrm{S}, 151^{\circ} 25^{\prime} \mathrm{E}$, Feb. 4-Apr. 9, 1993, pitfall, elev. 970 m (M. Gray, G. Cassis, AMS KS37352), 18 $\hat{0}, 1 \%$; Mummel Forest Road, $7.6 \mathrm{~km} \mathrm{~N}$ junction with Enfield Forest Road, Enfield State Forest, $31^{\circ} 17^{\prime} \mathrm{S}$, $151^{\circ} 51^{\prime} \mathrm{E}$, Feb. 4-Apr. 9, 1993, pitfall, elev. $1340 \mathrm{~m}$ (M. Gray, G. Cassis, AMS KS37357), 20; Pol Blue Creek, above Tubrabucca Road, Stewarts Brook State Forest, $31^{\circ} 55^{\prime} \mathrm{S}, 151^{\circ} 23^{\prime} \mathrm{E}$, Feb. 4-Apr. 9, 1993, pitfall, elev. $1240 \mathrm{~m}$ (M. Gray, G. Cassis, AMS KS37392), 20; W junction of Thunderbolts Track and Devils Hole Track, Barrington Tops State Forest, $31^{\circ} 54^{\prime} \mathrm{S}, 151^{\circ} 28^{\prime} \mathrm{E}$, Feb. 4-Apr. 9, 1993, pitfall, elev. 1420 m (M. Gray, G. Cassis, AMS KS39669), 10; 100 m N Trig Tower, Berrico Road, Chichester State Forest, $32^{\circ} 06^{\prime} \mathrm{S}, 151^{\circ} 45^{\prime} \mathrm{E}$, Feb. 4-Apr. 9, 1993, pitfall, elev. 940 m (M. Gray, G. Cassis, AMS KS37340), 10َ; 0.25 km S junction Tubrabucca and Omadale Brook Roads, Stewarts Brook State Forest, 31 ${ }^{\circ} 54^{\prime}$ S,

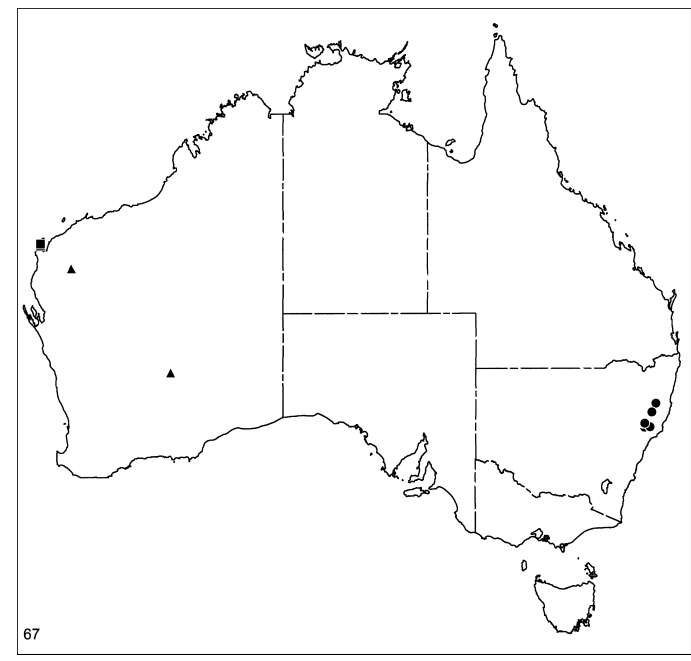

Map 67. Records of Graycassis barrington, new species (circles), Asadipus cape, new species (squares), and A. yundamindra, new species (triangles). 

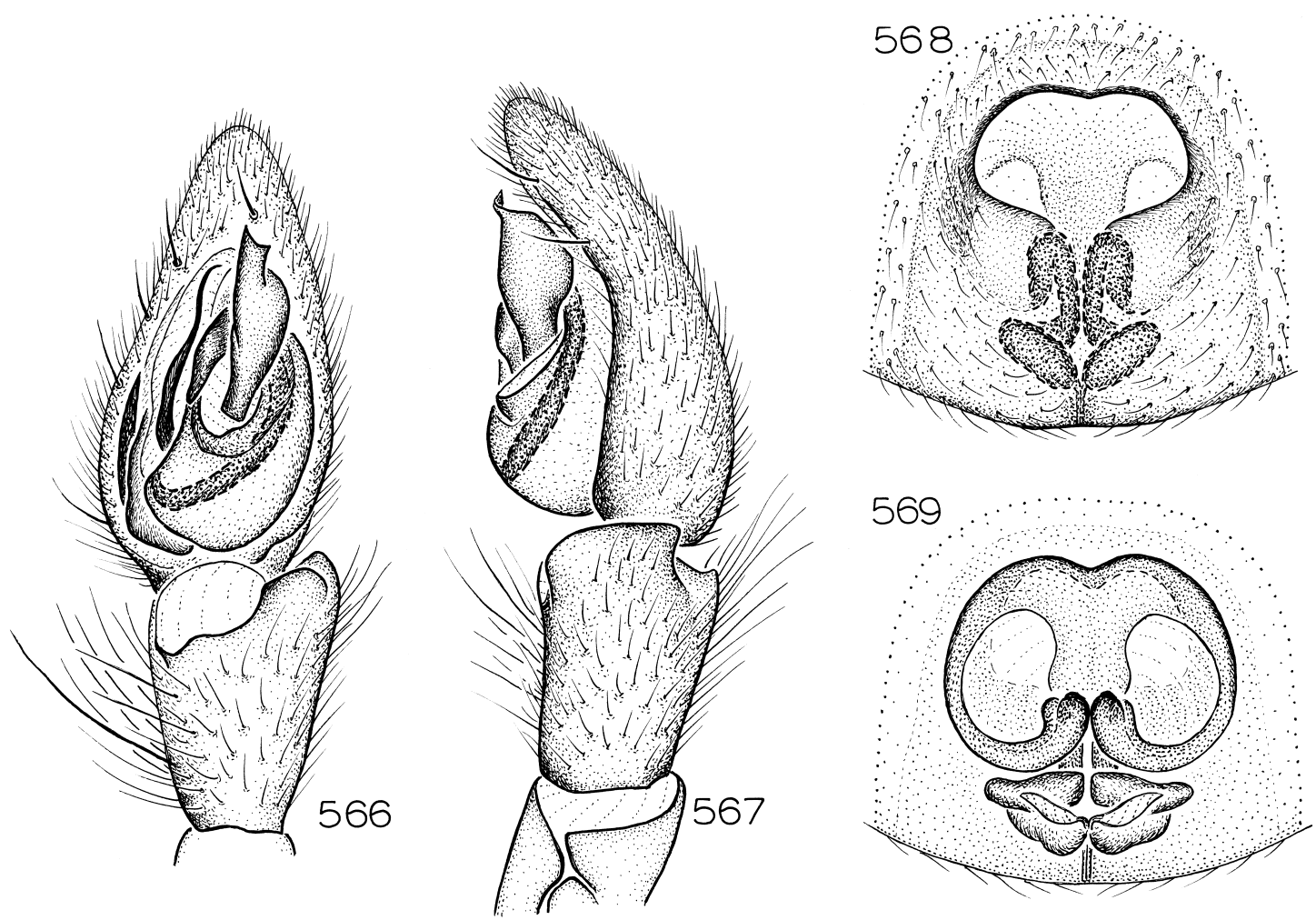

Figs. 566-569. 566, 567. Graycassis enfield, new species. 568, 569. Asadipus barant, new species. 566. Left male palp, ventral view. 567. Same, retrolateral view. 568. Epigynum, ventral view. 569. Same, dorsal view.

$151^{\circ} 23^{\prime}$ E, Feb. 4-Apr. 9, 1993, pitfall, elev. 1290 m (M. Gray, G. Cassis, AMS KS37396), 3 đ; Tugalow Creek, E side Barrington Trail, Barrington Tops State Forest, $31^{\circ} 54^{\prime} \mathrm{S}, 151^{\circ} 26^{\prime} \mathrm{E}$, Feb. 4-Apr. 9, 1993, pitfall, elev. $1370 \mathrm{~m}$ (M. Gray, G. Cassis, AMS KS39667), 7ð, 1 오.

Distribution: New South Wales (map 67).

Graycassis enfield, new species Figures 566, 567; Map 68

TYPE: Male holotype taken in a pitfall trap at an elevation of $1130 \mathrm{~m}$ on Daisy Patch Fire Trail, $1.9 \mathrm{~km} \mathrm{~S}$ Enfield Road, Enfield State Forest, $31^{\circ} 20^{\prime} \mathrm{S}, 151^{\circ} 54^{\prime} \mathrm{E}$, New South Wales (Feb. 4-Apr. 9, 1993; M. Gray, G. Cassis), deposited in AMS (KS37368).

ETYMOLOGY: The specific name is a noun in apposition taken from the type locality.

DiAGNOSIS: Males resemble those of $G$. barrington in the general shape of the me- dian apophysis, but that apophysis is both shorter and wider posteriorly (fig. 566) than in $G$. barrington.

MALE: Total length 7.1. Coloration as in G. marengo. Leg spination: tibiae: III v2-22; IV r1-1-1; metatarsi IV p0-1-0. Retrolateral tibial apophysis entire, ledge-shaped (fig. 567); median apophysis long, gradually widened from base, distally produced (fig. 566).

Female: Unknown.

VARIATION: The single male taken at Mummel Forest Road has the median apophysis distally larger, and bearing a relatively larger distally produced lobe. It could conceivably represent a closely related species, but much larger samples would be needed to test that hypothesis.

Other Material Examined: New South Wales: $0.9 \mathrm{~km} \mathrm{~S}$ along Chaelundi Road from Big Bull Road, Mount Hyland Nature Reserve, $30^{\circ} 09^{\prime} \mathrm{S}, 152^{\circ} 27^{\prime} \mathrm{E}$, Feb. 4-Apr. 9, 
1993, pitfall, elev. 1080 m (M. Gray, G. Cassis, AMS KS35321), 10\%; Double Dumps Fire Trail. $700 \mathrm{~m}$ off Daisy Patch Road, Enfield State Forest, $31^{\circ} 20^{\prime} \mathrm{S}, 151^{\circ} 54^{\prime} \mathrm{E}$, Feb. 4Apr. 9, 1993, pitfall, elev. 1080 m (M. Gray, G. Cassis, AMS KS37366), 10; Mummel Forest Road, $6.1 \mathrm{~km} \mathrm{~N}$ junction with Enfield Road, Enfield State Forest, $31^{\circ} 17^{\prime} \mathrm{S}$, $151^{\circ} 51^{\prime} \mathrm{E}$, Feb. 4-Apr. 9, 1993, pitfall, elev. $1330 \mathrm{~m}$ (M. Gray, G. Cassis, AMS KS37365), 10; Obeloe Creek, 2.0 km SW along Oboleo Road from Chaelundi Road, Mount Hyland Nature Reserve, $30^{\circ} 09^{\prime} \mathrm{S}$, $152^{\circ} 27^{\prime} \mathrm{E}$, Feb. 4-Apr. 9, 1993, pitfall, elev. 910 m (M. Gray, G. Cassis, AMS KS36327), 5 ô.

Distribution: Known only from Mount Hyland Nature Reserve and Enfield State Forest in New South Wales (map 68).

\section{Graycassis boss, new species} Figures 570-573; Map 69

TyPES: Female holotype and male allotype taken in pitfall traps at an elevation of 690 $\mathrm{m}$ on Rimau Road, c. $11.2 \mathrm{~km}$ E Cockerawombeeba Road, Mount Boss State Forest, $31^{\circ} 11^{\prime} \mathrm{S}, 152^{\circ} 21^{\prime} \mathrm{E}$, New South Wales (Feb. 4-Apr. 9, 1993; M. Gray, G. Cassis), deposited in AMS (KS39564).

ETYMOLOGY: The specific name is a noun in apposition taken from the type locality.

DIAGNOSIS: This species is very close to $G$. bulga, but males can be distinguished by the longer, narrower median extension on the median apophysis (figs. 570, 571), females by the posteriorly narrower and anteriorly wider epigynal septum (figs. 572, 573).

MALE: Total length 6.4. Coloration as in G. marengo. Leg spination: metatarsi III v22-0. Retrolateral tibial apophysis bifid, with ventral prong hook-shaped (fig. 571); median apophysis with long, narrow, prolaterally directed extension, retrolateral edges of apophysis rounded (fig. 570).

Female: Total length 7.2. Coloration as in G. marengo. Leg spination: tibiae III v0-2-2. Epigynum with median septum, posterior portion narrow, diamond-shaped, anterior portion almost half width of posterior portion (fig. 572); spermathecae with large anterior extensions (fig. 573).

Other Material Examined: New South
Wales: Rimau Road, c. $10 \mathrm{~km}$ by track E Cockerawombeeba Road, Mount Boss State Forest, $31^{\circ} 11^{\prime} \mathrm{S}, 152^{\circ} 21^{\prime} \mathrm{E}$, Feb. 4-Apr. 9, 1993, pitfall, elev. 700 m (M. Gray, G. Cassis, AMS KS39563), 240ึ; Rimau Road, c. $11,2 \mathrm{~km}$ E Cockerawombeeba Road, Mount Boss State Forest, $31^{\circ} 11^{\prime} \mathrm{S}, 152^{\circ} 21^{\prime} \mathrm{E}$, Feb. 4-Apr. 9, 1993, pitfall, elev. 690 m (M. Gray, G. Cassis, AMS KS39564), 460; Rimau Road, c. 13.8 km E Cockerawombeeba Road, Mount Boss State Forest, $31^{\circ} 11^{\prime} \mathrm{S}$, $152^{\circ} 22^{\prime}$ E, Feb. 4-Apr. 9, 1993, pitfall, elev. 760 m (M. Gray, G. Cassis, AMS KS39563),

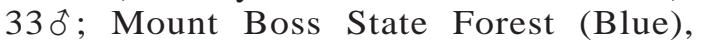
$31^{\circ} 12^{\prime} \mathrm{S}, 152^{\circ} 24^{\prime} \mathrm{E}$, Oct. 1980 (G. Webb, AMS KS42848), 19 ; Mount Boss State Forest (South Plateau), $31^{\circ} 12^{\prime} \mathrm{S}, 152^{\circ} 24^{\prime} \mathrm{E}$, Oct. 1980 (G. Webb, AMS KS42889), 1 \% ; Mount Boss State Forest (Valley), $31^{\circ} 12^{\prime} \mathrm{S}$, $152^{\circ} 24^{\prime} \mathrm{E}$, Oct. 1980 (G. Webb, AMS KS42855), 2 ; Mount Tindal Road, Ramornie State Forest, $29^{\circ} 41^{\prime} \mathrm{S}, 152^{\circ} 35^{\prime} \mathrm{E}$, Feb. 4Apr. 9, 1993, pitfall, elev. 490 m (M. Gray, G. Cassis, AMS KS39562), 13ㅊ, 1우 ; Mount Tindal Road, Ramornie State Forest, $29^{\circ} 42^{\prime} \mathrm{S}, 152^{\circ} 35^{\prime} \mathrm{E}$, Feb. 4-Apr. 9, 1993, pitfall, elev. 380 m (M. Gray, G. Cassis, AMS

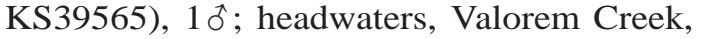
track off Mount Tindal Road, Ramornie State Forest, $29^{\circ} 42^{\prime} \mathrm{S}, 152^{\circ} 35^{\prime} \mathrm{E}$, Feb. 4-Apr. 9,

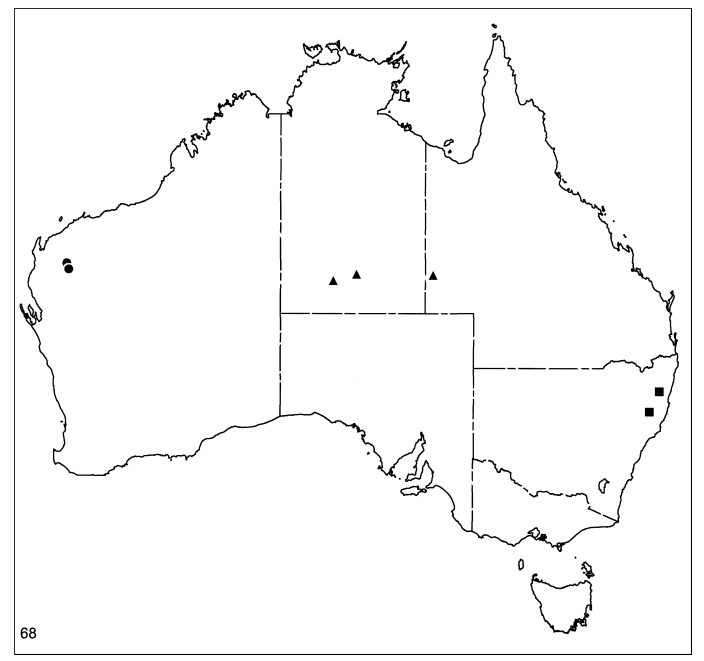

Map 68. Records of Graycassis enfield, new species (squares), Asadipus baranar, new species (circles), and $A$. areyonga, new species (triangles). 

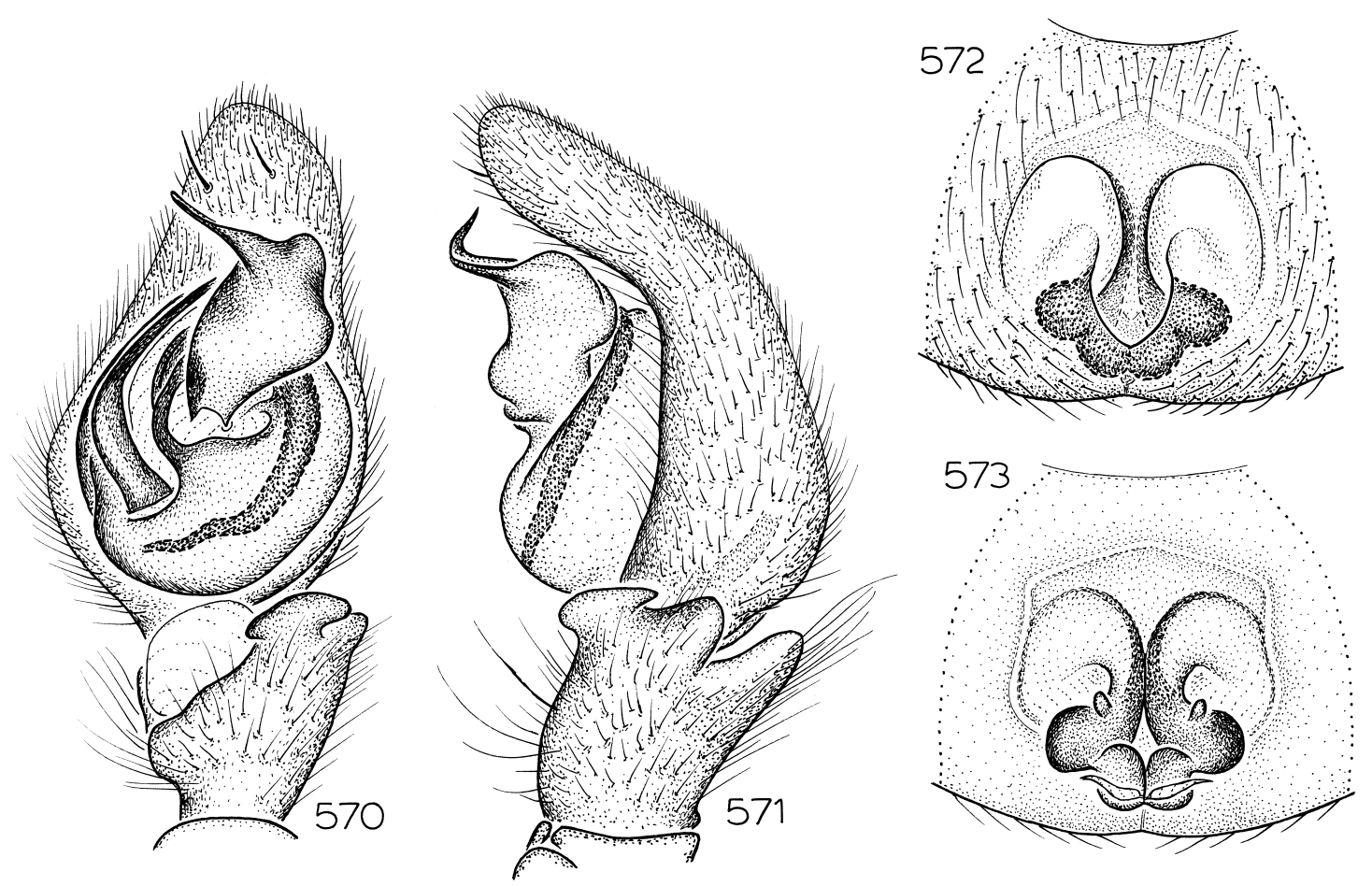

Figs. 570-573. Graycassis boss, new species. 570. Left male palp, ventral view. 571. Same, retrolateral view. 572. Epigynum, ventral view. 573. Same, dorsal view.

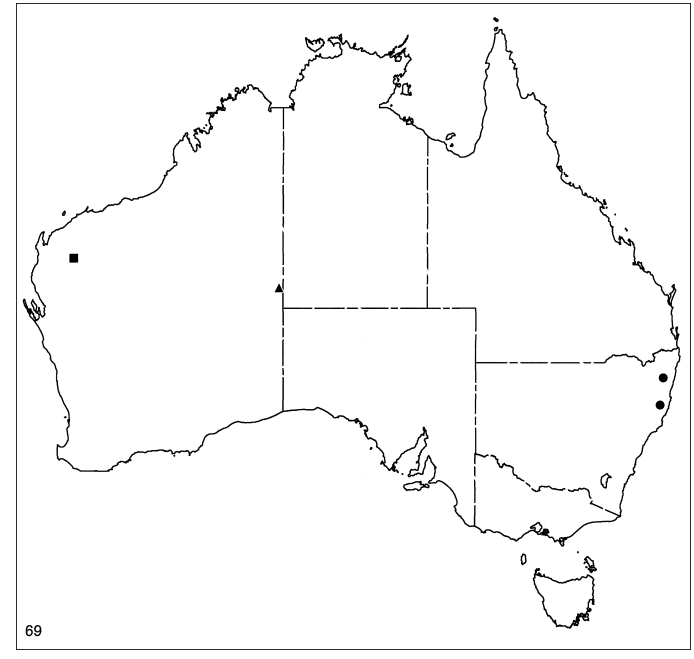

Map 69. Records of Graycassis boss, new species (circles), Asadipus barant, new species (square), and A. mountant, new species (triangle).
1993, pitfall, elev. 380 m (M. Gray, G. Cassis, AMS KS39566), 1 to.

DistRIBUTION: Known only from Mount Boss and Ramornie State Forests, New South Wales (map 69).

\section{Graycassis bulga, new species \\ Figures 574-577; Map 70}

TYPE: Male holotype taken in a pitfall trap at an elevation of $730 \mathrm{~m}$ on Bobbin Fire Trail, $100 \mathrm{~m}$ from Padmans Road, Bulga State Forest, $31^{\circ} 37^{\prime} \mathrm{S}, 152^{\circ} 10^{\prime} \mathrm{E}$, New South Wales (Feb. 4-Apr. 9, 1993; M. Gray, G. Cassis), deposited in AMS (KS39579).

ETYMOLOGY: The specific name is a noun in apposition taken from the type locality.

DiAGNOSIS: Males and females have not yet been collected at the same sites, but are matched here on the basis of their respective great similarities to those of $G$. boss; males have a shorter, narrower median extension on the median apophysis (figs. 574, 575); females have a posteriorly wider and anteriorly narrower epigynal septum (figs. 576, 577). 

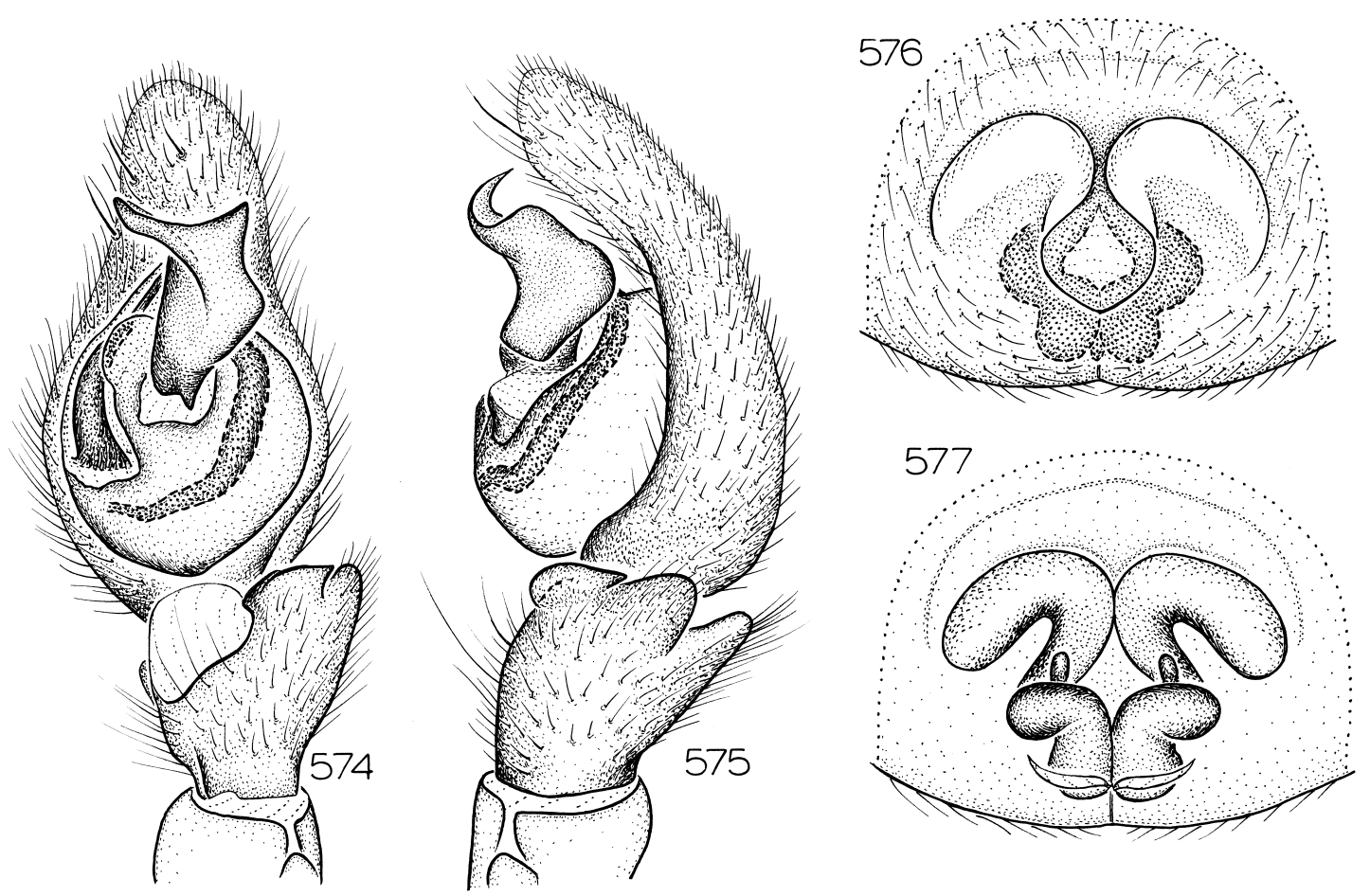

Figs. 574-577. Graycassis bulga, new species. 574. Left male palp, ventral view. 575. Same, retrolateral view. 576. Epigynum, ventral view. 577. Same, dorsal view.

MALE: Total length 5.7. Coloration as in G. marengo. Leg spination: tibiae III v0-1p2. Retrolateral tibial apophysis bifid, with ventral prong hook-shaped (fig. 575); median apophysis with relatively short, wide, prolaterally directed extension, retrolateral edges of apophysis angular (fig. 574).

FEMALE: Total length 8.3. Coloration as in G. marengo. Leg spination: tibiae III v0-2-2. Epigynum with median septum, posterior portion relatively wide, diamond-shaped, anterior portion much less than half width of posterior portion (fig. 576); spermathecae with large anterior extensions (fig. 577).

Other Material Examined: New South Wales: Bellangary State Forest, Wilson River Flora Reserve, $31^{\circ} 13^{\prime} \mathrm{S}, 152^{\circ} 29^{\prime} \mathrm{E}$, Mar. 10, 1981, rainforest pitfall (M. Gray, AMS KS9588), $20^{\star}$; Bobbin Fire Trail, $100 \mathrm{~m}$ from Padmans Road, Bulga State Forest, $31^{\circ} 37^{\prime} \mathrm{S}$, $152^{\circ} 10^{\prime}$ E, Feb. 4-Apr. 9, 1993, pitfall, elev. 730 m (M. Gray, G. Cassis, AMS KS39579), $660^{\top}$; E slope, below Breakneck Lookout, Kiwarrak State Forest, S Taree, $31^{\circ} 58^{\prime} \mathrm{S}$, $152^{\circ} 26^{\prime} \mathrm{E}$, Feb. 25, 1991, dry rainforest (G.
Williams, AMS KS31980), 19 ; Dodds Fire Trail, c. $3 \mathrm{~km}$ from Enfield Road, Enfield State Forest, $31^{\circ} 24^{\prime} \mathrm{S}, 151^{\circ} 52^{\prime} \mathrm{E}$, Feb. 4-Apr. 9, 1993, pitfall, elev. 910 m (M. Gray, G. Cassis, AMS KS37372), 1ð; Grey Gums Forest Road, $2.1 \mathrm{~km}$ from Doyles River Road, Bulga State Forest, $31^{\circ} 33^{\prime} \mathrm{S}, 152^{\circ} 14^{\prime} \mathrm{E}$, Feb. 4-Apr. 9, 1993, pitfall, elev. 560 m (M. Gray, G. Cassis, AMS KS39580), 5 ơ; 1.2 $\mathrm{km}$ up track N Grey Gums Forest Road, 2.1 $\mathrm{km}$ from Doyles River Road, Bulga State Forest, $31^{\circ} 32^{\prime} \mathrm{S}, 152^{\circ} 14^{\prime} \mathrm{E}$, Feb. 4-Apr. 9, 1993, pitfall, elev. 620 m (M. Gray, G. Cassis, AMS KS39576), 240; Homewoods Road, 2.8 km W Knodingbul Road, Bulga State Forest, $31^{\circ} 37^{\prime} \mathrm{S}, 152^{\circ} 07^{\prime} \mathrm{E}$, Feb. 4-Apr. 9, 1993, pitfall, elev. 690 m (M. Gray, G. Cassis, AMS KS39526, 39577), 240ే; Kerewong State Forest, near Lorne, $31^{\circ} 36^{\prime} \mathrm{S}$, $152^{\circ} 34^{\prime}$ E, Sept. 26-Dec. 10, 1978, pitfall in litter (D. Milledge, AMS KS16127, 16159), 2 \%; Padmans Road, near junction Pole Dump Road, $31^{\circ} 36^{\prime} \mathrm{S}, 152^{\circ} 10^{\prime} \mathrm{E}$, Feb. 4-Apr. 9, 1993, pitfall, elev. 730 m (M. Gray, G. Cassis, AMS KS39578), 40. 
Distribution: New South Wales (map 70).

Graycassis scrub, new species Figures 578, 579; Map 70

TYPE: Male holotype taken in a pitfall trap at a site NW of Rocky Creek crossing in Big Scrub Flora Reserve, $28^{\circ} 38^{\prime} \mathrm{S}, 153^{\circ} 19^{\prime} \mathrm{E}$, New South Wales (Feb. 4-Apr. 9, 1993; M. Gray, G. Cassis), deposited in AMS (KS35930).

ETYMOLOGY: The specific name is a noun in apposition taken from the type locality.

DIAGNOSIS: Males can easily be recognized by the enormously widened distal tip of the median apophysis (figs. 578, 579).

MALE: Total length 3.4. Coloration as in G. bruxner. Leg spination: tibiae: III v0-1p2 ; IV v1p-1p-2; metatarsi III v1p-1r-0. Retrolateral tibial apophysis bifid, ventral portion separated from dorsal portion by distinct notch (fig. 579); median apophysis enormously widened distally (fig. 578).

FEMALE: Unknown.

Other Material ExAmined: None.

DisTRIBUTION: Known only from the type locality in New South Wales (map 70).

\section{Asadipus Simon}

Asadipus Simon, 1897a: 186 (type species by original designation Trachelas insolens Simon).

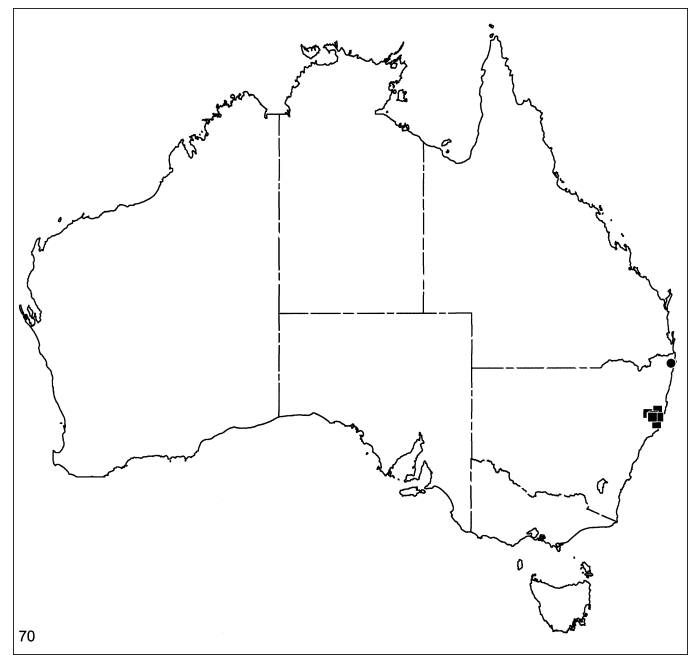

Map 70. Records of Graycassis bulga, new species (squares) and G. scrub, new species (circle).
Aristerus Simon, 1909: 179 (type species by monotypy Aristerus phaleratus Simon). NEW SYNONYMY.

DiAGNOSIS: Males are easily recognized by their ventrally incised retrolateral tibial apophysis (figs. 583, 627); females have a characteristically flattened epigynum, with heavily curled ducts visible through the cuticle (figs. 584, 588).

DESCRIPTION: Medium to large spiders, total length of males 3.8-7.5, of females 4.89.0. Carapace dark red, coated with large tubercles bearing long, white setae, margins with long, dark setae; thoracic groove long, longitudinal, deep. Eight eyes in two rows, posterior medians largest, other subequal, anterior medians circular, dark, laterals almost circular, light, posterior medians irregularly oval, flattened, light; from above, anterior eye row slightly recurved, posterior row slightly procurved, from front, both rows strongly procurved; anterior medians separated by roughly their radius, about as far from anterior laterals; posterior medians separated by than radius, much farther from posterior laterals; anterior and posterior laterals separated by about their diameter; median ocular quadrangle slightly wider in back than in front, about as long as wide in back. Chelicerae, sternum, and mouthparts dark orangish red; chilum wide, triangular, short, accompanied by second, I-shaped, posterior chilum (extremely narrow sclerite separating bases of chelicerae posteriorly); chelicerae with distinct lateral boss outlined by rows of tubercles bearing short, stiff setae, remainder of anterior and lateral surfaces with strong tubercles, anterior surface with depressed, relatively unsclerotized oval area near promargin; promargin with two rows of long setae originating in line along base of fang, seta closest to fang bent near base at almost $90^{\circ}$ angle, extending behind other promarginal setae toward midline; promargin with three teeth, median one largest, proximal one smallest; retromargin with four large teeth originating from common base. Labium elongate, base narrowed at about one-fifth of labial length, posterior margin procurved, anterior margin with slight medial invagination, surface not depressed medially. Endites obliquely depressed, with sharply demarcated, deep groove along margin near labium, 

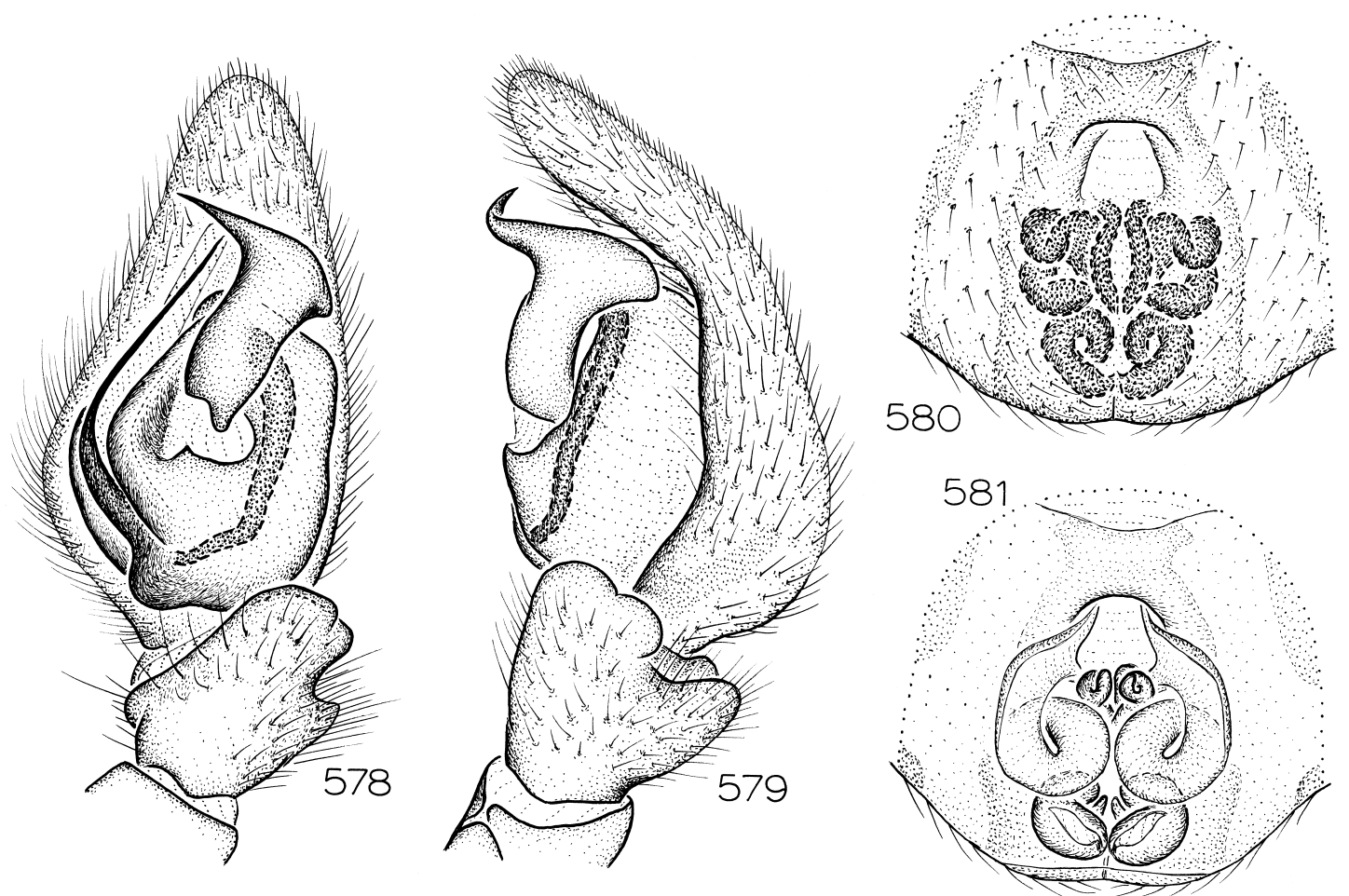

Figs. 578-581. 578, 579. Graycassis scrub, new species. 580, 581. Asadipus mountant, new species. 578. Left male palp, ventral view. 579. Same, retrolateral view. 580. Epigynum, ventral view. 581. Same, dorsal view.

groove wider anteriorly than posteriorly; serrula long, with single row of teeth; anterior surface with distinct sieve plate of maxillary gland openings set in oval of unsclerotized cuticle. Sternum slightly elevated, with inclined lateral margins, not expanded anteriorly, with triangular extensions to and between coxae; surface strongly tuberculate, with distinct elevations opposite, and depressions between, coxae. One epimeric sclerite on each side, above each coxa, reaching sternal triangles only by narrow strips of weakly sclerotized cuticle (strongest strip between coxae II and III), not fused to carapace but partially fused to sclerite surrounding pedicel. Pedicel consisting of large, diamondshaped sclerite narrow anterior margin meeting posterior tip of sternum at slightly procurved line.

Anterior edge of abdomen of male with complete sclerotic ring formed by strong epigastric scutum separated from strong dorsal abdominal scutum covering almost entire dorsal surface of abdomen, females with dorsal scutum represented only by small rectangular plate above pedicel, restricted to lower one-third of anterior surface of abdomen; cuticle with large tubercles bearing long, recumbent setae; epigastric scutum accompanied posterolaterally by pair of oval, deeply invaginated sclerites bearing clearly elevated anterior rim; sclerites separated by membranous lobe, anterior rim of sclerites fitting under epigastric scutum; colulus represented only by setae; tiny transverse sclerite, well removed from spinnerets, marking position of small posterior spiracle. Anterior lateral spinnerets tubular, separated by more than their diameter, cuticle representing distal, second spinneret segment restricted to semicircle surrounding major ampullate gland spigots (piriform gland spigots surrounded only by soft cuticle); posterior median spinnerets of males large, tubular, of females with anteriorly expanded tips, bases occupied by three enormously widened cy- 
lindrical gland spigots; posterior lateral spinnerets two-segmented, those of females with two greatly widened cylindrical gland spigots.

Leg spination reduced only on anterior legs; typical leg spination pattern (only surfaces with spines listed): tibiae: III p0-1-1, v2-2-2, r0-1-1; IV p0-1-1, v1p-2-2, r0-1-1; metatarsi III v2-0-0; IV v2-2-0. Most leg surfaces coated with short setae; both sexes with all coxae and trochanters dorsally tuberculate; anterior coxae with slightly protuberant posterolateral corners; trochanters unnotched; anterior metatarsi and tarsi with divided scopulae, composed of laterally directed setae; posterior metatarsi with thick, dark, distal preening brushes; tarsi III, IV with divided scopulae; tarsi with two dentate claws, claw tufts composed of lateral pads of closely appressed setae; trichobothria present on tibiae, metatarsi, and tarsi. Female palpal tibia and tarsus with long, thin spines; female palpal tarsus with long, basally dentate claw.

Male palp with strong, excavated retrolateral tibial apophysis; cymbial surface excavated opposite tibial apophysis; tegulum occupying retrolateral surface of bulb, bearing largely membranous (sometimes reduced) median apophysis opposing large, bifid terminal apophysis; embolus originating ventrally, long, looping around prolateral and distal sides of bulb, accompanied distally by long, membranous conductor. Epigynum flattened, usually with inconspicuous lateral margins; spermathecae accompanied medially and anteriorly by highly convoluted ducts.

SYNONYMY: In most collections, the generic name Aristerus has been used for these spiders, and the name Asadipus has commonly been applied instead to the species treated below under the name Notsodipus. Although the type species of Asadipus, which is the older name, has never been recollected, the male holotype has the incised retrolateral tibial apophysis characteristic of Aristerus, and that younger name must unfortunately therefore fall into synonymy, despite its substantial (but only informal) usage.

Misplaced SPecies: Asadipus lifoui Berland was transferred above to Centrocalia;
Asadipus nitidiceps Simon was transferred above to Prionosternum.

\section{Key to SPECIES of AsAdipus}

1. Males (those of A. barant, A. mountant, and A. areyonga unknown) ......... 2

- Females (those of A. insolens and A. palmerston unknown) ............. 18

2. Retrolateral tibial apophysis relatively long, extending at least half length of cymbium (as in figs. 595, 647) ............ 3

- Retrolateral tibial apophysis relatively short, extending only about one-third length of cymbium (as in figs. 583, 643) ..... 11

3. Retrolateral tibial apophysis extending most of cymbial length (figs. 607, 627) ... . 4 4

- Retrolateral tibial apophysis extending only about half of cymbial length (as in figs. $595,647) \ldots \ldots \ldots \ldots \ldots \ldots$

4. Tegulum and palpal bulb relatively wide (fig. 606) .............. kunderang

- Tegulum and palpal bulb relatively narrow (fig. 626) .......... yundamindra

5. Embolar base relatively narrow (figs. 594, 598) . . . . . . . . . . . . . 6 6

- Embolar base relatively wide (figs. 610, 618, $622,634,646) \ldots \ldots \ldots \ldots \ldots \ldots 7$

6. Basal portion of embolus near tegulum (fig. 594) .............. phaleratus

- Basal portion of embolus removed from tegulum (fig. 598) . . . . . . . . . . barlee

7. Proximal prolateral edge of terminal apophysis overlapping embolus in ventral view (figs. 610, 622, 646) $\ldots \ldots \ldots \ldots .8$

- Proximal prolateral edge of terminal apophysis not overlapping embolus (figs. 618, 634) .................. 10

8. Prongs of terminal apophysis forming vshaped structure (fig. 622) .... baranar

- Prongs of terminal apophysis otherwise (figs. 610, 646) ................. 9

9. Prolateral edge of terminal apophysis greatly widened (fig. 610) ........ . banjiwarn

- Prolateral edge of terminal apophysis moderately widened (fig. 646) ....... uphill

10. Tegulum relatively small (fig. 618) .. cape

- Tegulum relatively large (fig. 634) ..... $\ldots \ldots \ldots \ldots$ longforest

11. Tip of retrolateral apophysis invaginated (figs. 583, 587, 591) . . . . . . 12

- Tip of retrolateral tibial apophysis smoothly rounded (as in figs. 603, 643) ...... 14

12. Median apophysis relatively small (fig. 586, 590) . . . . . . . . . . . . . 13

- Median apophysis relatively large (fig. 582) $\ldots \ldots \ldots \ldots$ insolens 
13. Median apophysis with distal hook (fig. 586)

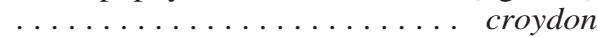

- Median apophysis rounded (fig. 590) .... ............... palmerston

14. Dorsal prong of terminal apophysis short, rounded, heavily sclerotized, visible behind membranous median apophysis (fig. 602) $\ldots \ldots \ldots \ldots \ldots$ woodleigh

- Dorsal prong of terminal apophysis long, protruding (figs. 614, 630, 638, 642) .... 15

15. Dorsal prong of terminal apophysis relatively wide, directed distally (figs. 630, 638) ... $\ldots \ldots \ldots \ldots \ldots \ldots \ldots$

- Dorsal prong of terminal apophysis relatively narrow, directed retrolaterally (figs. 614, 642) . . . . . . . . . . . . . . . 17

16. Ventral prong of terminal apophysis relatively narrow (fig. 630) .......... humptydoo

- Ventral prong of terminal apophysis relatively wide, extending under embolus (fig. 638) ................ bucks

17. Tegulum relatively long, sperm duct sinuous (fig. 614) ............... auld

- Tegulum relatively short, sperm duct evenly looping (fig. 642) ............. julia

18. Epigynum with pair of medially directed projections (fig. 604) . . . . . . . woodleigh

- Epigynum without pair of projections . . 19

19. Epigynum with tiny, anteromedian hood (figs. 640, 644, 648) ............ 20

- Epigynum without tiny, anteromedian hood

20. Epigynal ducts relatively short, wide (figs $644,645) \ldots \ldots \ldots \ldots \ldots \ldots$ julia

- Epigynal ducts relatively long, narrow (figs. $640,648) \ldots \ldots \ldots \ldots \ldots 21$

21. Epigynal ducts extending anteriorly of openings (fig. 648) ............. uphill

- Epigynal ducts extending only to openings (fig. 640) ................ bucks

22. Epigynum with anterior atrium (figs. 568, $580,616,624) \ldots \ldots \ldots 23$

- Epigynum without anterior atrium ... . . 26

23. Epigynal atrium relatively large (figs. 568, 624) . . . . . . . . . . . . 24

- Epigynal atrium relatively small (figs. 580,

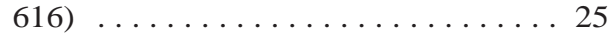

24. Epigynal atrium relatively wide (fig. 568) . .

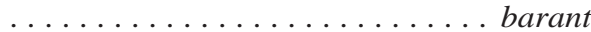

- Epigynal atrium relatively narrow (fig. 624) ............... baranar

25. Epigynal atrium with anteromedian septum (fig. 616) ................ auld

- Epigynal atrium without septum (fig. 580) .. $\ldots \ldots \ldots \ldots \ldots$ mountant

26. Epigynum with pair of anterior, anteromedially directed, triangular or rectangular openings (figs. 584, 600, 620, 628) . . 27
- Epigynum without such openings .... . 30

27. Epigynum with distinct anterior margin (figs. $584,620) \ldots \ldots \ldots \ldots \ldots 28$

- Epigynum without distinct anterior margin (figs. 600, 628) . . . . . . . . . . . 29

28. Epigynum relatively long (fig. 584) ..... $\ldots \ldots \ldots \ldots \ldots$ areyonga

- Epigynum relatively short (fig. 620) . . cape

29. Anterior epigynal ducts making two transverse loops (figs. 628, 629) ......... $\ldots \ldots \ldots \ldots$ yundamindra

- Anterior epigynal ducts making single transverse loop (figs. 600, 601) ..... barlee

30. Epigynum with pair of posterolateral margins (fig. 632) ............ humptydoo

- Epigynum without posterolateral margins .. $\ldots \ldots \ldots \ldots \ldots \ldots \ldots \ldots$

31. Epigynum with arched anterior margin (figs. $588,608) \ldots \ldots \ldots \ldots \ldots \ldots \ldots \ldots \ldots \ldots$

- Epigynum without arched anterior margin .. .................. 33

32. Median epigynal ducts extending as far posteriorly as lateral ducts (figs. 608, 609) ..

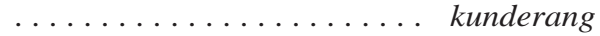

- Lateral epigynal ducts extending posteriorly of median ducts (figs. 588, 589) ..... $\ldots \ldots \ldots \ldots \ldots$ croydon

33. Anterior epigynal ducts coiled (figs. 613, 637) ................ . 34

- Anterior epigynal ducts not coiled (figs. 596, 597) .............. phaleratus

34. Anterior epigynal ducts relatively short (fig. 612) ............... banjiwarn

- Anterior epigynal ducts relatively long (fig. 636) ............. longforest

\section{Asadipus insolens (Simon)}

Figures 582, 583; Map 71

Trachelas insolens Simon, 1896: 413 (male holotype from Cooktown, Queensland, in MNHN, examined).

Asadipus insolens: Simon, 1897a: 186, fig. 181.

DiAGNOSIS: Males can easily be recognized by the extremely short retrolateral tibial apophysis, which is less deeply excavated than in other members of the genus (figs. 582,583 ).

MALE: Total length 6.1. Coloration as in A. phaleratus (see below), except venter of abdomen with orange median sclerotization immediately behind epigastric furrow, with most of venter coated with setae originating from long tubercles. Leg spination: tibiae III, IV p0-0-0, v0-0-2, r0-0-0; metatarsi III, IV v0-0-0. Retrolateral tibial apophysis short, 

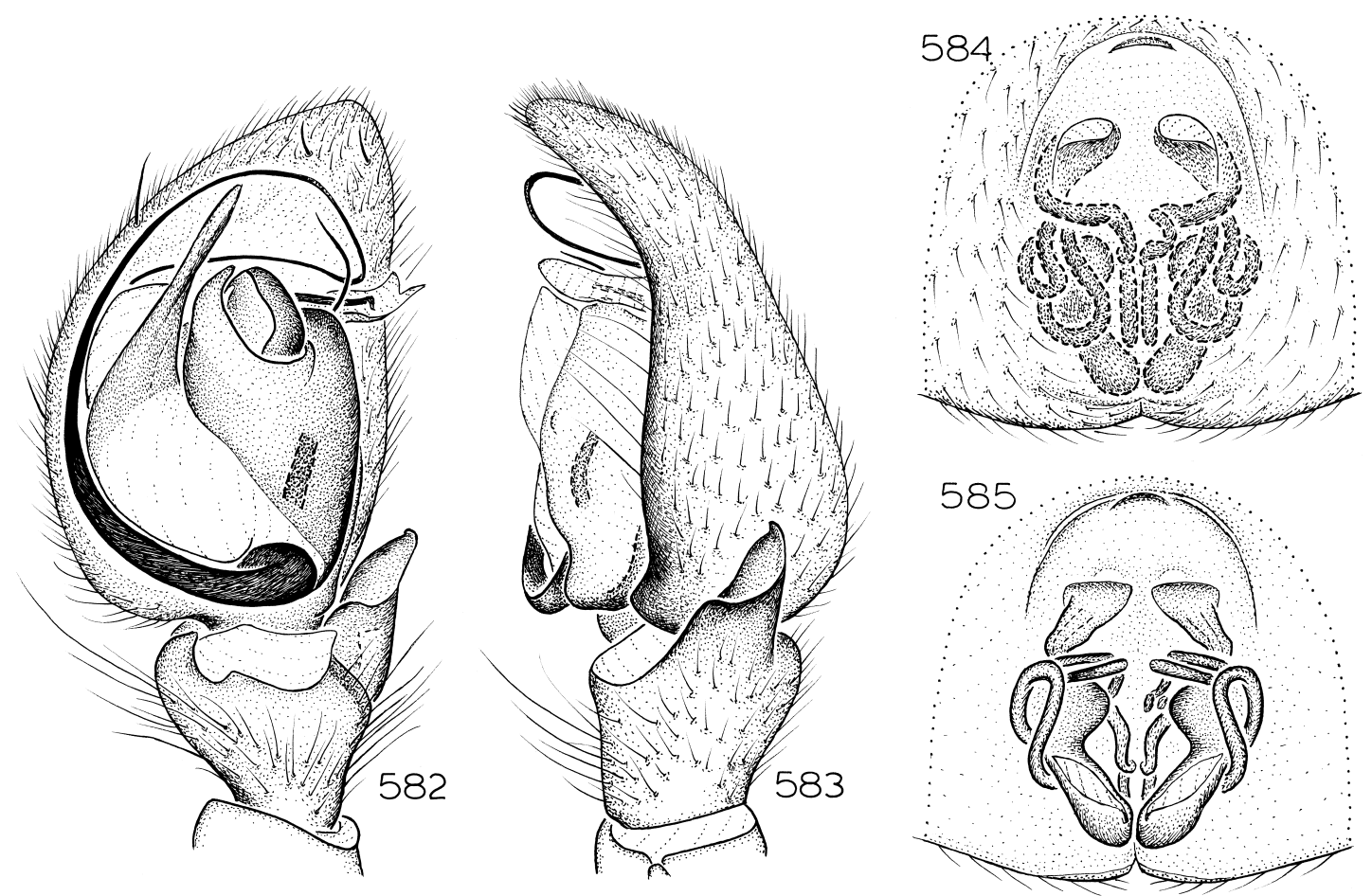

Figs. 582-585. 582, 583. Asadipus insolens (Simon). 584, 585. A. areyonga, new species. 582. Left male palp, ventral view. 583. Same, retrolateral view. 584. Epigynum, ventral view. 585. Same, dorsal view.

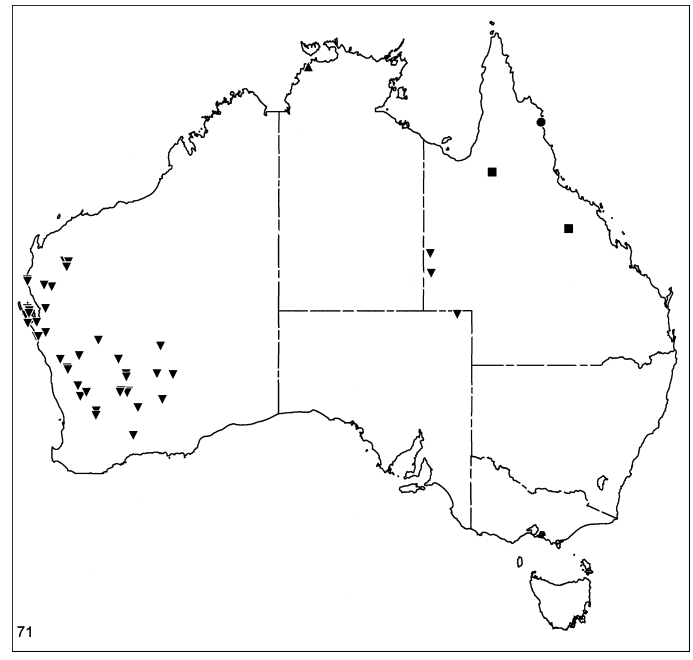

Map 71. Records of Asadipus insolens (Simon) (circle), A. croydon, new species (squares), A. palmerston, new species (triangle), and $A$. phaleratus (Simon) (inverted triangles). reaching only to about one-sixth of cymbial length (fig. 583); ventral prong of terminal apophysis relatively long, distally narrowed, dorsal prong extremely long, narrow, extending around back of alveolus (fig. 582).

Female: Unknown.

MATERIAL EXAMINED: Queensland: Cooktown, $15^{\circ} 28^{\prime} \mathrm{S}, 145^{\circ} 15^{\prime} \mathrm{E}$ (MNHN 18271), 1 के (holotype)

DisTRIBUTION: Known only from northern Queensland (map 71).

\section{Asadipus croydon, new species Figures 586-589; Map 71}

TYPES: Female holotype and male allotype taken in pitfall traps at Marsupial Creek, via Croydon, $18^{\circ} 13^{\prime} \mathrm{S}, 142^{\circ} 14^{\prime} \mathrm{E}$, Queensland (Mar. 1996-Mar. 1997; J. and P. Hasenpusch), deposited in QMB (S43919).

ETYMology: The specific name is a noun in apposition taken from the type locality.

DiAGNOSIS: Males can easily be recognized by the short, narrow, apically incised retro- 

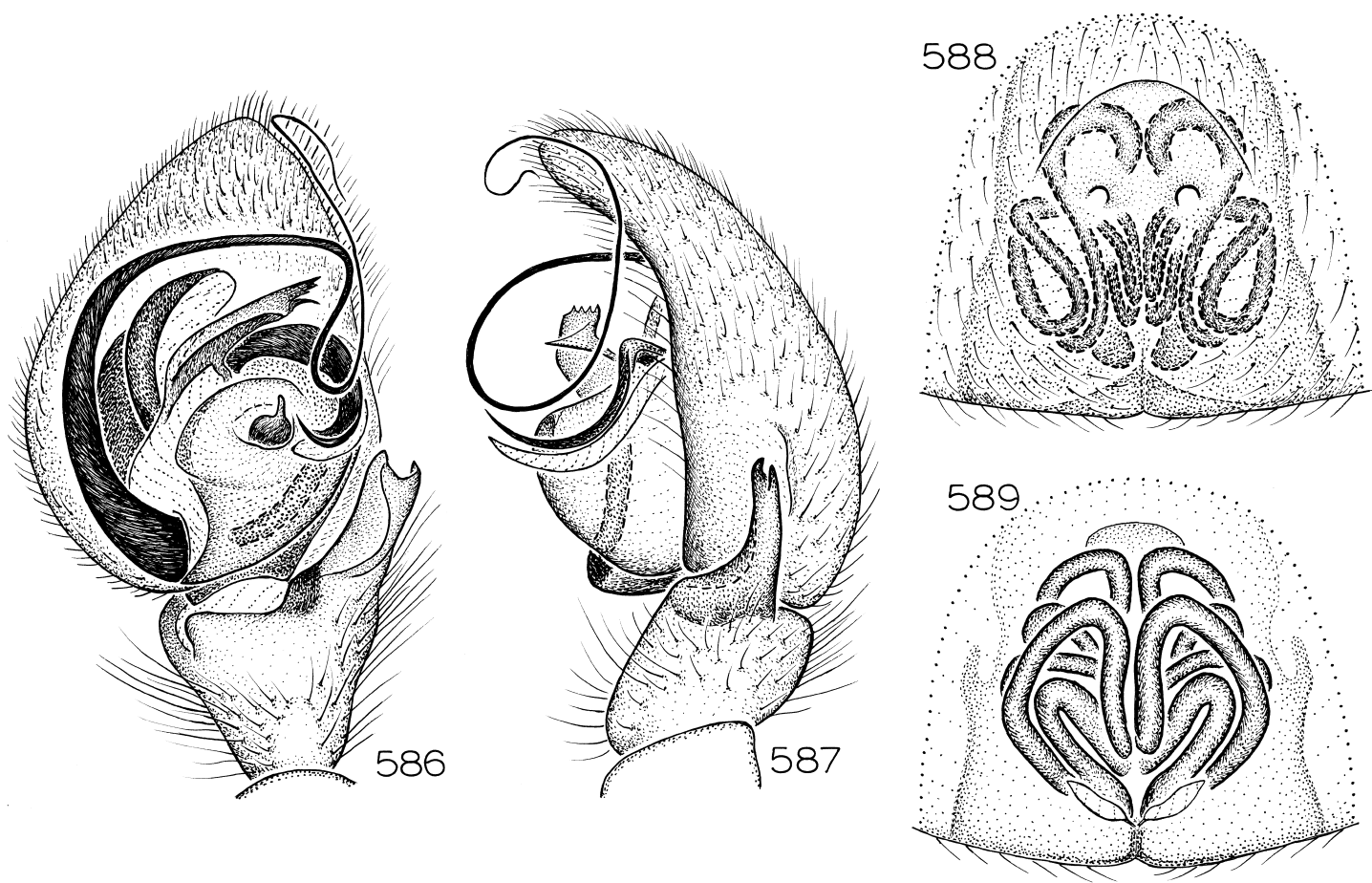

Figs. 586-589. Asadipus croydon, new species. 586. Left male palp, ventral view. 587. Same, retrolateral view. 588. Epigynum, ventral view. 589. Same, dorsal view.

lateral tibial apophysis (figs. 586, 587), females by the short posteromedian epigynal ducts (fig. 588).

MALE: Total length 6.6. Abdominal venter pale white with four longitudinal rows of small, circular sclerites, most anterior sclerite of two median rows enlarged; legs red. Leg spination: tibiae III, IV p0-0-0, v0-0-2, r0-00 ; metatarsi III, IV v0-0-0. Retrolateral tibial apophysis short, narrow, distally bifid (fig. 587); median apophysis small, hooked, heavily sclerotized (fig. 586).

FEMALE: Total length 7.1. Coloration as in A. uphill (see below). Leg spination: tibiae III, IV p0-0-0, v0-0-2, r0-0-0; metatarsi: III v0-0-0, IV v0-1p-0. Epigynum with distinct anterior margin (fig. 588); most posteromedian portion of ducts short, folded (fig. 589).

Other Material Examined: Queensland: Marsupial Creek, via Croydon, $18^{\circ} 13^{\prime} \mathrm{S}$, $142^{\circ} 14^{\prime} \mathrm{E}$, Mar. 1996-Mar. 1997, pitfall (J., P. Hasenpusch, QMB S35383), 19 ; Rosetta Station, $50 \mathrm{~km}$ from Mount Coolon, $21^{\circ} 20^{\prime} \mathrm{S}$, 1465'ㄹ, Apr. 23-May 1, 1991 (K. Plowman, QMB S24125), 1 .
DisTRIBUTION: Known only from northern Queensland (map 71).

\section{Asadipus palmerston, new species Figures 590, 591; Map 71}

TyPE: Male holotype taken in a house at Palmerston, $12^{\circ} 27^{\prime} \mathrm{S}, 130^{\circ} 50^{\prime} \mathrm{E}$, Northern Territory (Feb. 19, 1986; Turner), deposited in AMS (KS50314).

ETYMOLOGY: The specific name is a noun in apposition taken from the type locality.

DIAGNOSIS: This species is closely related to A. croydon, with a similarly bifid tip on the retrolateral tibial apophysis, but males can be distinguished by the much wider terminal apophysis (fig. 590).

MALE: Total length 7.5. Abdominal venter pale orange with four longitudinal rows of small, circular sclerites, sclerites of two median rows much larger than those of lateral rows; legs blackish red. Leg spination: tibiae III, IV p0-0-0, v0-0-2, r0-0-0; metatarsi III, IV v0-0-0. Retrolateral tibial apophysis short, narrow, distally bifid (fig. 591); median 

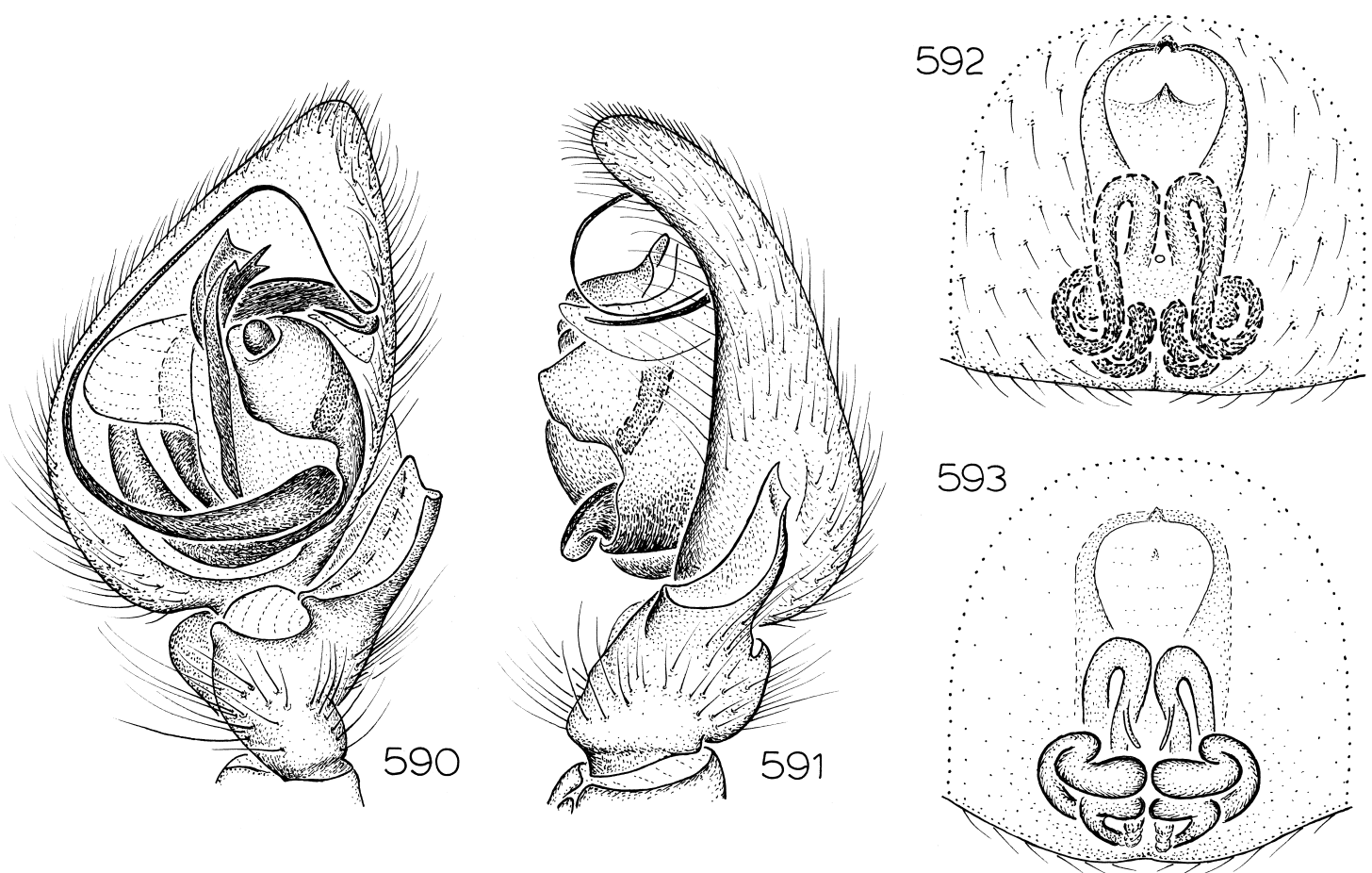

Figs. 590-593. 590, 591. Asadipus palmerston, new species. 592, 593. Notsodipus blackall, new species. 590. Left male palp, ventral view. 591. Same, retrolateral view. 592. Epigynum, ventral view. 593. Same, dorsal view.

apophysis small, folded, heavily sclerotized, terminal apophysis with wide, translucent, prolateral flange (fig. 590).

FEMALE: Unknown.

Other Material ExAmined: None.

Distribution: Known only from the Northern Territory (map 71).

\section{Asadipus phaleratus (Simon), new combination \\ Figures 594-597; Map 71}

Aristerus phaleratus Simon, 1909: 179 (male and female syntypes presumably from Day Dawn, Western Australia, in MNHN, examined; male syntype from Yalgoo, Western Australia, in WAM, examined; male and two female syntypes from Boorabbin, Western Australia, in ZMH, examined).

Diagnosis: Males resemble those of the sometimes sympatric $A$. barlee but have a longer embolus that almost reaches the retrolateral side of the cymbium (figs. 594, 595); females have relatively indistinct, v-shaped epigynal margins and obliquely oriented anterior ducts (figs. 596, 597).

MALE: Total length 7.0. Soft portions of abdominal cuticle gray, legs orange, femora darkest, unmarked. Leg spination: tibiae: III r0-0-1; IV v1p-1p-2; metatarsi IV v2-1p-0. Retrolateral tibial apophysis reaching to more than half of cymbial length (fig. 595); ventral prong of terminal apophysis distally expanded, membranous, dorsal prong terminating in long, sclerotized spur accompanying membranous conductor (fig. 594).

Female: Total length 7.0. Coloration as in male. Leg spination: metatarsi IV r0-1-0. Epigynum with inconspicuous lateral margins outlining v-shaped atrium (fig. 596); spermathecae variable, bean-shaped to dumbbell-shaped, surrounded by arched posterior and oblique anterior ducts (fig. 597).

MATERIAL EXAMINED: Queensland: Ethabuka Station Simpson Desert, 234' $\mathrm{S}$, $138^{\circ} 28^{\prime} \mathrm{E}$, July 9-29, 1992, pitfall, dune base (J. Henschel, QMB S34295), 1ð; 1 m. NE 

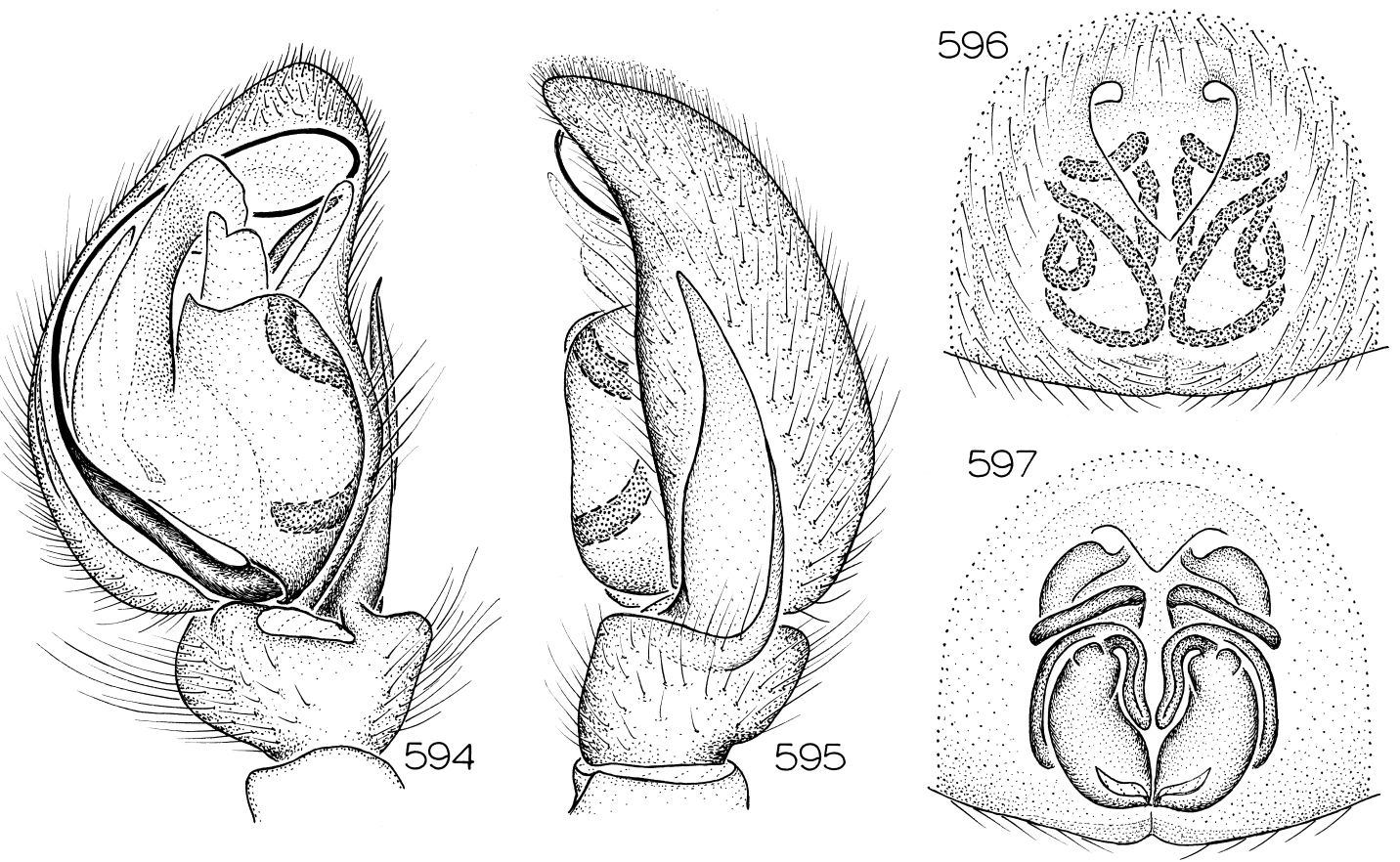

Figs. 594-597. Asadipus phaleratus (Simon). 594. Left male palp, ventral view. 595. Same, retrolateral view. 596. Epigynum, ventral view. 597. Same, dorsal view.

Linda Creek, Glenormiston Station, $22^{\circ} 41^{\prime} \mathrm{S}$, $138^{\circ} 26^{\prime} \mathrm{E}$, June 13, 1974, night collecting (A. Elliot, QMB S29377), 1 o. South Australia: 0.7 N Frew Well, $26^{\circ} 03^{\prime} \mathrm{S}, 140^{\circ} 06^{\prime} \mathrm{E}$, May 5, 1995, pitfall (R. Brandle, SAM N1996/420), $10^{\top}$. Western Australia: Ballidu, $30^{\circ} 35^{\prime} \mathrm{S}$, $116^{\circ} 45^{\prime} \mathrm{E}$, May 24-Sept. 16, 1996, pitfall (M. Harvey, J. Waldock, WAM 96/1567), $10^{\top}$; Banjiwarn, $27^{\circ} 48^{\prime} \mathrm{S}, 121^{\circ} 41^{\prime} \mathrm{E}$, May $26-$ 31, 1974, mulga, eremophila, bunchgrass (R. How, WAM 96/405), $1 \delta^{\dagger}$; Barlee Range Nature Reserve, $23^{\circ} 03^{\prime} \mathrm{S}, 115^{\circ} 49^{\prime} \mathrm{E}$, June $15-$ 18, 1994, dry pitfall (P., G. Kendrick, WAM 96/318), 10; Barlee Range Nature Reserve, $23^{\circ} 04^{\prime} \mathrm{S}$, $115^{\circ} 47^{\prime} \mathrm{E}$, June 15-18, 1994, dry pitfall (P., G. Kendrick, WAM 96/312), $10^{\top}$; Barlee Range Nature Reserve, $23^{\circ} 05^{\prime} \mathrm{S}$, $115^{\circ} 47^{\prime} \mathrm{E}$, June $15-18,1994$, dry pitfalls (P.,

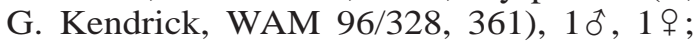
Barlee Range Nature Reserve, $23^{\circ} 06^{\prime} \mathrm{S}$, $116^{\circ} 00^{\prime} \mathrm{E}$, June $19-22$, 1994, dry pitfall (P., G. Kendrick, WAM 96/313, 334), $10,1 \%$; Barlee Range Nature Reserve, $23^{\circ} 07^{\prime} \mathrm{S}$, $116^{\circ} 01^{\prime} \mathrm{E}$, June 19-22, 1994, dry pitfall (P., G. Kendrick, WAM 96/305), 10; Barlee Range Nature Reserve, $23^{\circ} 23^{\prime} \mathrm{S}, 115^{\circ} 53^{\prime} \mathrm{E}$,
June 11-14, 1994, dry pitfalls (P., G. Kendrick, WAM 96/589-593), 40; Barlee Range Nature Reserve, $23^{\circ} 25^{\prime} \mathrm{S}, 115^{\circ} 54^{\prime} \mathrm{E}$, June $11-$ 14, 1994, dry pitfalls (P., G. Kendrick, WAM 96/325-327), 30, Sept. 1995, pitfall (S. van Leeuwen, B. Bromilow, WAM 96/306), 1 ; Big Lagoon, N Peron Peninsula, $25^{\circ} 43^{\prime} \mathrm{S}$, $113^{\circ} 28^{\prime} \mathrm{E}$, Aug. 4, 1989, pitfall (G. Harold,

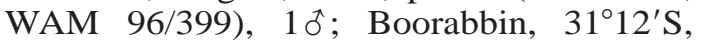
$120^{\circ} 18^{\prime} \mathrm{E}$, July 3, 1905 (ZMH), $10^{\Uparrow}, 2$ 우 (syntypes); Bulong, $30^{\circ} 45^{\prime} \mathrm{S}, 121^{\circ} 48^{\prime} \mathrm{E}$, June 22 , 1969 (L. Koch, WAM 96/331), 1 ठ̊; Bungalbin Hill, $30^{\circ} 17^{\prime} \mathrm{S}, 119^{\circ} 43^{\prime} \mathrm{E}$, Sept. 1979 , shrubland (R. How, WAM 96/407, 408), 20; Bungalbin Hill, $30^{\circ} 18^{\prime} \mathrm{S}, 119^{\circ} 43^{\prime} \mathrm{E}$, Sept. 1979, woodland (R. How, WAM 96/406), $10^{\top}$; Buntine Rocks Nature Reserve, 2959'S, $116^{\circ} 36^{\prime} \mathrm{E}$, May 22-Sept. 17, 1996, pitfall (M. Harvey, J. Waldock, WAM 96/1568), 10; Cape Cuvier, Quobba Station, $24^{\circ} 08^{\prime} \mathrm{S}$, $113^{\circ} 27^{\prime} \mathrm{E}$, May $28-J u n e ~ 3,1995$, dry pitfalls (A. Sampey, WAM 96/353, 354), 1 tै, 1 우 May 31-Aug. 25, 1995, pitfalls (N. Hall,

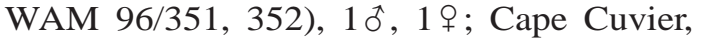
Quobba Station, $24^{\circ} 12^{\prime}$ S, $113^{\circ} 27^{\prime}$ E, Jan. 15May 29, 1995, pitfall (A. Sampey, WAM 96/ 
315), 1 đ, May 29-June 3, 1995, dry pitfalls (A. Sampey, WAM 96/316, 317), $10^{\top}$; May 29-Aug. 25, 1995, pitfall (N. Hall, WAM 96/ 319), 1 ; ; Cape Cuvier, Quobba Station, $24^{\circ} 13^{\prime} \mathrm{S}, 113^{\circ} 28^{\prime} \mathrm{E}$, May 28-June 2, 1995, dry pitfalls (A. Sampey, WAM 96/387-390), 4ô, May 30-Aug. 24, 1995, pitfall (N. Hall, WAM 96/314), 10; Cape Cuvier, Quobba Station, $24^{\circ} 13^{\prime} \mathrm{S}, 113^{\circ} 30^{\prime} \mathrm{E}$, May $28-\mathrm{June} 2$, 1995, dry pitfalls (A. Sampey, WAM 96/

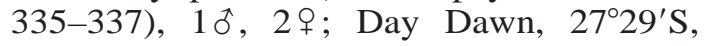
$117^{\circ} 51^{\prime} \mathrm{E}$ (Michaelsen, MNHN 6975), $1 \widehat{o}^{\widehat{t}}$, 1 우 (syntypes; locality data per original description); c. $8 \mathrm{~km}$ E Durokoppin Field Station, $31^{\circ} 23^{\prime} \mathrm{S}, 117^{\circ} 44^{\prime} \mathrm{E}$, May 7, 1987, pitfall (G. Smith, WAM 96/386), 1 đ; Edel Land, $26^{\circ} 04^{\prime} \mathrm{S}, 113^{\circ} 23^{\prime} \mathrm{E}$, Sept. 15-20, 1989 (G.

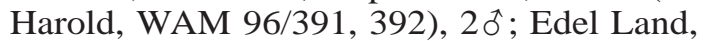
$26^{\circ} 32^{\prime} \mathrm{S}, 113^{\circ} 30^{\prime} \mathrm{E}$, May 9-Aug. 30, 1995, pitfall (N. Hall, WAM 96/393), $10^{\text {to; }}$ Francois Peron National Park, $25^{\circ} 49^{\prime} \mathrm{S}, 113^{\circ} 32^{\prime} \mathrm{E}$, Jan. 18-May 24, 1995, pitfall (M. Harvey, WAM 96/398), 10; May 22-27, 1995, dry pitfalls (M. Harvey, WAM 96/402-404), 30, May 24-Aug. 30, 1995, pitfall (N. Hall, WAM 96/ 360), 1 \% ; Francois Peron National Park, $25^{\circ} 50^{\prime} \mathrm{S}, 113^{\circ} 36^{\prime} \mathrm{E}$, May 22-27, 1995, dry pitfall (M. Harvey, WAM 96/401), 1 ơ ; Francois Peron National Park, $25^{\circ} 53^{\prime} \mathrm{S}, 113^{\circ} 33^{\prime} \mathrm{E}$, May 22-27, 1995, dry pitfalls (M. Harvey, WAM 96/394, 397), 20 , May 25-Aug. 30, 1995, pitfall (N. Hall, WAM 96/395), 10;; Francois Peron National Park, $25^{\circ} 59^{\prime} \mathrm{S}$, $113^{\circ} 34^{\prime} \mathrm{E}$, May 22-27, 1995, dry pitfall (M. Harvey, WAM 96/396), 1 ô, May 26-Aug. 30, 1995, pitfall (N. Hall, WAM 96/395),

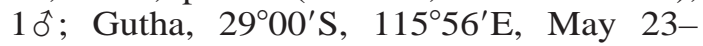
Sept. 17, 1996, pitfall (M. Harvey, J. Waldock, WAM 96/1570), 1 o; Helena-Aurora Ranges, 30 23'S, $119^{\circ} 38^{\prime} \mathrm{E}$, Sept. 24, 1995 , pitfall (R. McMillan, WAM 96/400), 10َ; Kalannie, $30^{\circ} 21^{\prime} \mathrm{S}, 117^{\circ} 07^{\prime} \mathrm{E}$, June 19,1979 (R. McMillan, WAM 96/339-345), 4o, 3 \% Kellerberrin, $31^{\circ} 38^{\prime} \mathrm{S}, 117^{\circ} 43^{\prime} \mathrm{E}$ (G. Smith, WAM 96/356. 357), 2 \% ; 10 km NW Kellerberrin, $31^{\circ} 38^{\prime} \mathrm{S}, 117^{\circ} 43^{\prime} \mathrm{E}$, May 6, 1987 (G. Smith, WAM 96/382), 10; Kennedy Range National Park, $24^{\circ} 30^{\prime} \mathrm{S}, 115^{\circ} 01^{\prime} \mathrm{E}$, May $27-$ June 1, 1995, dry pitfalls (P. West, WAM 96/ 323, 324), 1 ô, 1 \% ; Kennedy Range National Park, $24^{\circ} 30^{\prime} \mathrm{S}, 115^{\circ} 02^{\prime} \mathrm{E}$, May 27-June 1, 1995, dry pitfall (P. West, WAM 96/381), 1 o, May 29-Aug. 28, 1995, pitfall (N. Hall,
WAM 96/384), $10^{\Uparrow}$; Kennedy Range National Park, $24^{\circ} 31^{\prime} \mathrm{S}, 114^{\circ} 58^{\prime} \mathrm{E}$, May 27-June 1, 1995, dry pitfall (P. West, WAM 96/383), $10^{\circ}$; Kookynie, $29^{\circ} 20^{\prime} \mathrm{S}, 121^{\circ} 29^{\prime} \mathrm{E}$, July 21 , 1986 (R. McMillan, WAM 96/338), 1 우 Mardathuna Station, $24^{\circ} 24^{\prime}$ S, $114^{\circ} 28^{\prime}$ E, May 24-Aug. 26, 1995, pitfall (N. Hall, WAM 96/ 374), $1 \delta^{\top}$; Mardathuna Station, $24^{\circ} 26^{\prime} \mathrm{S}$, $114^{\circ} 30^{\prime} \mathrm{E}$, Jan. 14-May 24, 1995, pitfalls (A. Sampey, WAM 96/320-322), 20, 1 으, May 24-Aug. 26, 1995, pitfall (N. Hall, WAM 96/ 375), $10^{\text {oे }}$ McDermid Rock, $32^{\circ} 45^{\prime} \mathrm{S}$, $120^{\circ} 02^{\prime} \mathrm{E}$, July 1979 , woodland (R. How, WAM 96/378), $10^{\top}$; Meedo Station, $25^{\circ} 43^{\prime} \mathrm{S}$, $114^{\circ} 36^{\prime} \mathrm{E}$, Oct. 11, 1994-Jan. 12, 1995, pitfall (N. McKenzie, J. Rolfe, WAM 96/346), 1 \& , May 19-Aug. 22, 1995, pitfalls (N. Hall, WAM 96/332, 333), 1 ๙ , 19 ; Mount Elvire Station, $29^{\circ} 20^{\prime} \mathrm{S}, 119^{\circ} 35^{\prime} \mathrm{E}$, Sept. 13-17, 1994, dry pitfalls (A. Burbidge, WAM 96/ 329, 330), 10, 1\%; Mount Elvire Station, $29^{\circ} 30^{\prime} \mathrm{S}, 119^{\circ} 36^{\prime} \mathrm{E}$, Sept. 13-17, 1994, dry pitfalls (A. Burbidge, WAM 96/376, 377), $20^{\star}$; Mount Jackson, $30^{\circ} 16^{\prime} \mathrm{S}, 119^{\circ} 16^{\prime} \mathrm{E}$, Sept. 1979, tree mallee (R. How, WAM 96/ 380), 10; Mount Jackson, $30^{\circ} 21^{\prime} \mathrm{S}$, $119^{\circ} 12^{\prime} \mathrm{E}$, May 19, 1969 (M. de Graaf, WAM 96/379), 10, Sept. 6-11, 1979, heath (R. How, WAM 88/180), 19 ; Mullewa, $28^{\circ} 32^{\prime} \mathrm{S}, 115^{\circ} 31^{\prime} \mathrm{E}$, Sept. 11, 1931 (P. Darlington, MCZ), 1옹 Sept. 18, 1931 (W. Wheeler, MCZ), 1 \% ; Nanga Station, $26^{\circ} 29^{\prime} \mathrm{S}$, $114^{\circ} 03^{\prime} \mathrm{E}$, Aug. 23-Oct. 16, 1994, pitfall (P. West, WAM 96/350), 1 \%, Oct. 16, 1994-Jan. 19, 1995, pitfall (N. McKenzie, J. Rolfe, WAM 96/355), 1 \% , May 11-Aug. 30, 1995, pitfalls (N. Hall, WAM 96/373-373), $3 \sigma^{\text {; }}$; Nerren Nerren Station, $27^{\circ} 00^{\prime} \mathrm{S}, 114^{\circ} 32^{\prime} \mathrm{E}$, Aug. 25-Oct. 15, 1994, pitfall (J. Waldock, WAM 96/358), 10; Nerren Nerren Station, $27^{\circ} 03^{\prime} \mathrm{S}, 114^{\circ} 35^{\prime} \mathrm{E}$, Oct. 16, 1994-Jan. 11, 1995, pitfall (N. McKenzie, J. Rolfe, WAM 96/359), 1 ㅇ, May 11-Aug. 18, 1995, pitfall (N. Hall, WAM 96/369, 370), 20 ; Nerren Nerren Station, $27^{\circ} 03^{\prime} \mathrm{S}, 114^{\circ} 36^{\prime} \mathrm{E}$, May $11-$ Aug. 18, 1995, pitfall (N. Hall, WAM 96/

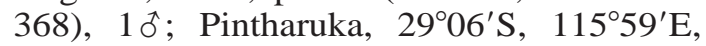
May 23-Sept. 17, 1996, pitfalls (M. Harvey, J. Waldock, WAM 96/1565, 1566), 20; Yalgoo, $28^{\circ} 20^{\prime} \mathrm{S}, 116^{\circ} 41^{\prime} \mathrm{E}$, July 11,1905 (WAM 4312), $10^{\star}$ (syntype); Yuinmery, $28^{\circ} 32^{\prime} \mathrm{S}, 119^{\circ} 06^{\prime} \mathrm{E}$, May 1979 , mallee/triodia (R. How, WAM 96/367), 1 §; Yundamindra, 

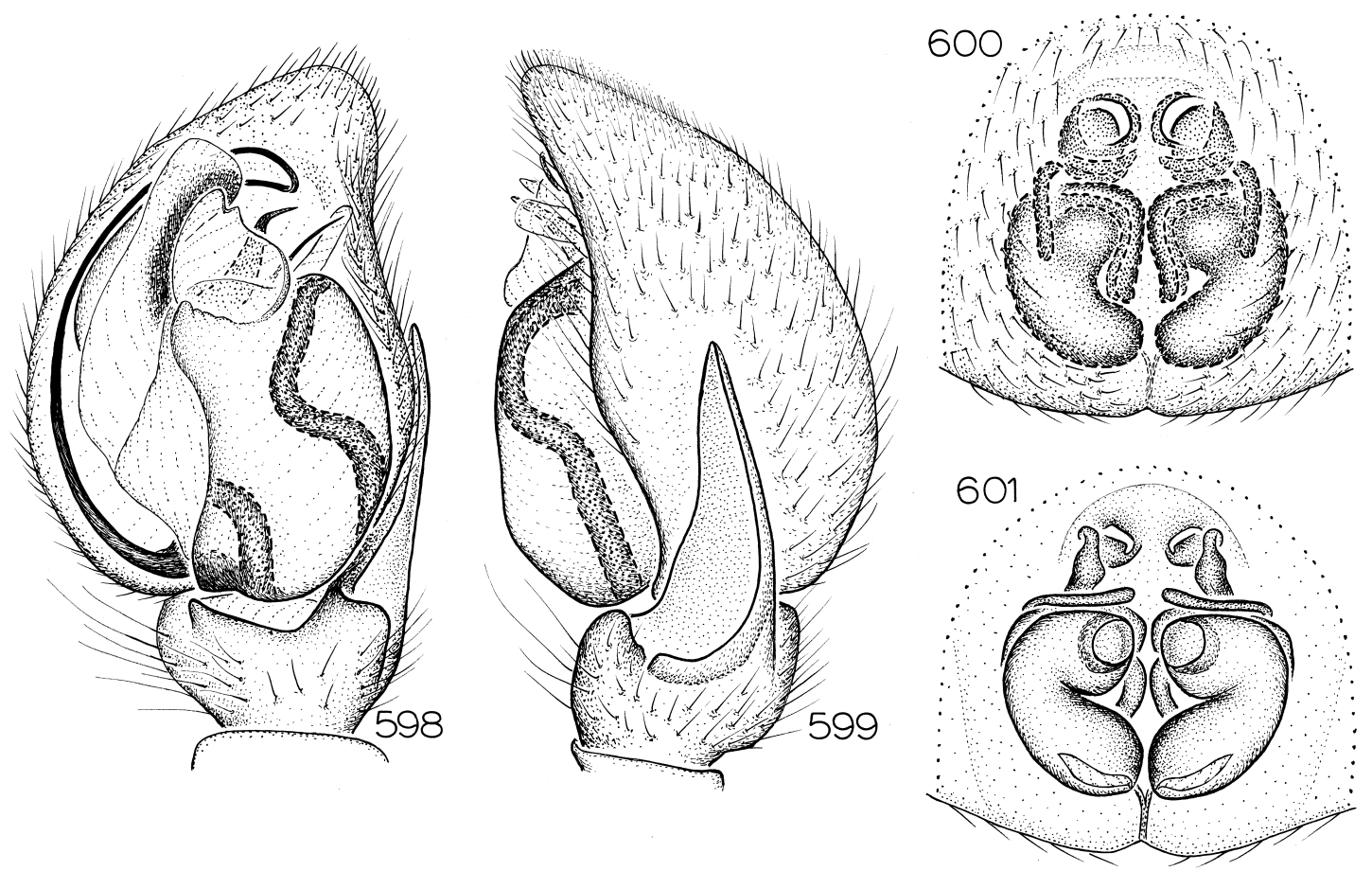

Figs. 598-601. Asadipus barlee, new species. 598. Left male palp, ventral view. 599. Same, retrolateral view. 600. Epigynum, ventral view. 601. Same, dorsal view.

$29^{\circ} 23^{\prime} \mathrm{S}, 122^{\circ} 28^{\prime} \mathrm{E}$, July 1981 , mulga/shrubs (W. Humphreys, WAM 96/366), 10; Zuytdorp, $27^{\circ} 16^{\prime} \mathrm{S}, 114^{\circ} 01^{\prime} \mathrm{E}$, May $16-21,1995$, dry pitfalls (M. Harvey, WAM 96/362, 363),

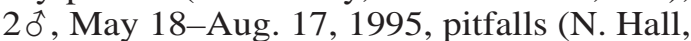
WAM 96/365), 10; Zuytdorp, $27^{\circ} 16^{\prime} \mathrm{S}$, $114^{\circ} 09^{\prime} \mathrm{E}$, May $16-21,1995$, dry pitfall (M. Harvey, WAM 96/364), 10, May 18-Aug. 16, 1995, pitfalls (N. Hall, WAM 96/347349), 2 ㅊ, 1 ㅇ․

Distribution: Western Australia to western Queensland (map 71).

\section{Asadipus barlee, new species}

Figures 598-601; Map 63

TYPE: Female holotype and male allotype taken in dry pitfall traps in Barlee Range Nature Reserve, $23^{\circ} 05^{\prime} \mathrm{S}, 115^{\circ} 47^{\prime} \mathrm{E}$, Western Australia (June 15-18, 1994; P., G. Kendrick), deposited in WAM $(96 / 593,594)$.

ETYMOLOGY: The specific name is a noun in apposition taken from the type locality.

Diagnosis: The species resembles A. phaleratus, and occurs together with that species; males can be distinguished by the shorter embolus, which is restricted to the prolateral side of the bulb (fig 598), females by the longer epigynum with larger anterior openings (fig. 600).

MALE: Total length 6.6. Coloration as in A. phaleratus except legs uniformly dark brownish red. Leg spination: tibiae III, IV p0-0-0, v1p-1p-2, r0-0-0; metatarsi III, IV v1p-0-0. Retrolateral tibial apophysis reaching to about half of cymbial length (fig. 599); embolus originating on prolateral side of bulb, ventral portion of terminal apophysis distally expanded, membranous, dorsal portion terminating in long, sclerotized spur accompanying membranous conductor (fig. 598).

Female: Total length 7.3. Coloration as in A. phaleratus. Leg spination: tibiae III, IV p0-0-0, v1p-1p-2, r0-0-0; metatarsi: III v0-00 ; IV v1p-0-0. Epigynum anteriorly elongated, with conspicuous, oval openings (fig. 600); ducts elongated, enlarged anteriorly (fig. 601). 
Other Material Examined: Western Australia: Barlee Range Nature Reserve, $23^{\circ} 05^{\prime} \mathrm{S}, 115^{\circ} 47^{\prime} \mathrm{E}$, June 15-18, 1994, dry pitfalls (P., G. Kendrick, WAM 96/595-597, 599, 614, 616), 4ô, 2 ; Barlee Range Nature Reserve, $23^{\circ} 06^{\prime} \mathrm{S}, 115^{\circ} 45^{\prime} \mathrm{E}$, June 1994 , pitfall (S. van Leeuwen, B. Bromilow, WAM 96/605-608), 30, 1 ; ; Barlee Range Nature Reserve, $23^{\circ} 06^{\prime} \mathrm{S}, 116^{\circ} 00^{\prime} \mathrm{E}$, June 1994 , pitfalls (S. van Leeuwen, B. Bromilow, WAM 96/609-611), 20, 1, June 19-22, 1994, dry pitfall (P., G. Kendrick, WAM 96/598), 10; Barlee Range Nature Reserve, $23^{\circ} 23^{\prime} \mathrm{S}$, $115^{\circ} 53^{\prime} \mathrm{E}$, June 1994 , pitfall (S. van Leeuwen, B. Bromilow, WAM 96/600), 1 ô, June 11-14, 1994, dry pitfalls (P., G. Kendrick, WAM 96/307-311, 601-604, 612, 613), 60ิ, 5 ㅇ.

DisTRIBUTION: Known only from the Barlee Range Nature Reserve, Western Australia (map 63).

\section{Asadipus woodleigh, new species}

Figures 602-605; Map 64

TYPES: Female holotype and male allotype taken in pitfall traps at Woodleigh Station, $26^{\circ} 12^{\prime} \mathrm{S}, 114^{\circ} 25^{\prime} \mathrm{E}$, Western Australia (May 17-Aug. 21, 1995; N. Hall), deposited in WAM $(96 / 566,567)$.

ETYMOLOGY: The specific name is a noun in apposition taken from the type locality.

DiAGNOSIS: Males can be recognized by the very short retrolateral tibial apophysis and the short terminal apophysis having its dorsal prong short, curved, very heavily sclerotized, and easily visible through the translucent median apophysis (figs. 602, 603 ), females by the pair of medially directed projections on the lateral epigynal margins (fig. 604).

MALE: Total length 6.6. Coloration as in A. phaleratus. Leg spination: tibiae: III v1p2-2; IV v1p-1p-2; metatarsi III, IV v1p-0-0. Retrolateral tibial apophysis very short, reaching to less than one-fourth of cymbial length (fig. 603); dorsal prong of terminal apophysis heavily sclerotized, rounded, situated medially of ventral portion, easily visible through translucent median apophysis (fig. 602).
Female: Total length 7.7. Coloration as in A. phaleratus. Leg spination: tibiae: III p00-1, v1p-1p-2, r0-0-0; IV p0-0-0, v1p-1p-2; metatarsi: III v0-0-0; IV v0-1p-0. Epigynum with distinctive pair of medially directed projections on lateral margins (fig. 604); ducts extending anterolaterally (fig. 605).

Other Material Examined: Western Australia: Bidgemia Station, Gasgoyne Junction, $25^{\circ} 03^{\prime} \mathrm{S}, 115^{\circ} 18^{\prime} \mathrm{E}$, Aug. 17-Oct. 5, 1994, pitfall (A. Sampey, WAM 96/413), 1 \% , Jan. 6-June 6, 1995, pitfalls (J. Waldock, WAM 96/421-437), 13 ऽ, 4 , June 6-Aug. 20, 1995, pitfall (N. Hall, WAM 96/412), 1 \% B Bidgemia Station, Gasgoyne Junction, $25^{\circ} 07^{\prime} \mathrm{S}, 115^{\circ} 26^{\prime} \mathrm{E}$, Jan. 13-June 6, 1995, pitfalls (J. Waldock, WAM 96/417-420), 40ิ, June 6-Aug. 20, 1995, pitfalls (N. Hall, WAM 96/414-416), 30; Boolathana Station, $24^{\circ} 25^{\prime} \mathrm{S}, 113^{\circ} 40^{\prime} \mathrm{E}$, Jan. 15-May 29, 1995, pitfalls (J. Waldock, WAM 96/472-483), 120; May 29-Aug. 25, 1995, pitfall (N. Hall, WAM 96/467), 10; Boolathana Station, $24^{\circ} 25^{\prime} \mathrm{S}, 113^{\circ} 41^{\prime} \mathrm{E}$, Aug. 20-Sept. 30, 1994, pitfall (A. Sampey, WAM 96/468), 1 , Jan. 15-May 30, 1995, pitfalls (J. Waldock, WAM 96/491-518), 28ð, May 28June 2, 1995, dry pitfalls (J. Waldock, WAM 96/484, 485), 2 ơ, May 30-Aug. 25, 1995, pitfalls (N. Hall, WAM 96/486-490), $5 \sigma^{\dagger}$; Boolathana Station, $24^{\circ} 25^{\prime} \mathrm{S}, 113^{\circ} 42^{\prime} \mathrm{E}$, Jan. 15-May 29, 1995, pitfalls (J. Waldock, WAM 96/469-471), 30̊; Bush Bay, 2505'S, $113^{\circ} 43^{\prime} \mathrm{E}$, Jan. 16-May 23, 1995, pitfalls (P. West, WAM 96/456-460), 50, May 21-26, 1995, dry pitfalls (P. West, WAM 96/462466), 5 o, May 23-Aug. 23, 1995, pitfalls (N. Hall, WAM 96/452-455), 4ô; Bush Bay, $25^{\circ} 07^{\prime} \mathrm{S}, 113^{\circ} 44^{\prime} \mathrm{E}$, Apr. 4-May 23, 1995 , pitfalls (P. West, WAM 96/1394, 1395), 20 , May 21-26, 1995, dry pitfall (P. West, WAM

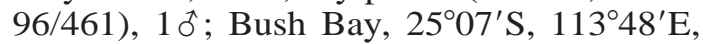
Aug. 16-Sept. 29, 1994, pitfall (M. Harvey, WAM 96/440), 19, Jan. 16-May 23, 1995, pitfalls (P. West, WAM 96/441-445, 451), 60., May 21-26, 1995, dry pitfalls (P. West, WAM 96/446, 448, 449), 30; Bush Bay, $25^{\circ} 08^{\prime} \mathrm{S}, 113^{\circ} 46^{\prime} \mathrm{E}$, Jan. 16-May 23, 1995, pitfalls (P. West, M. Harvey, WAM 96/438, 439, 450), $30^{\top}$; Bush Bay, $25^{\circ} 08^{\prime} \mathrm{S}, 113^{\circ} 49^{\prime} \mathrm{E}$, Jan. 16-May 23, 1995, pitfall (P. West, WAM 96/447), $10^{\top}$; Edel Land, $26^{\circ} 18^{\prime} \mathrm{S}, 113^{\circ} 16^{\prime} \mathrm{E}$, Aug. 26, 1989 (G. Harold, WAM 96/411), 

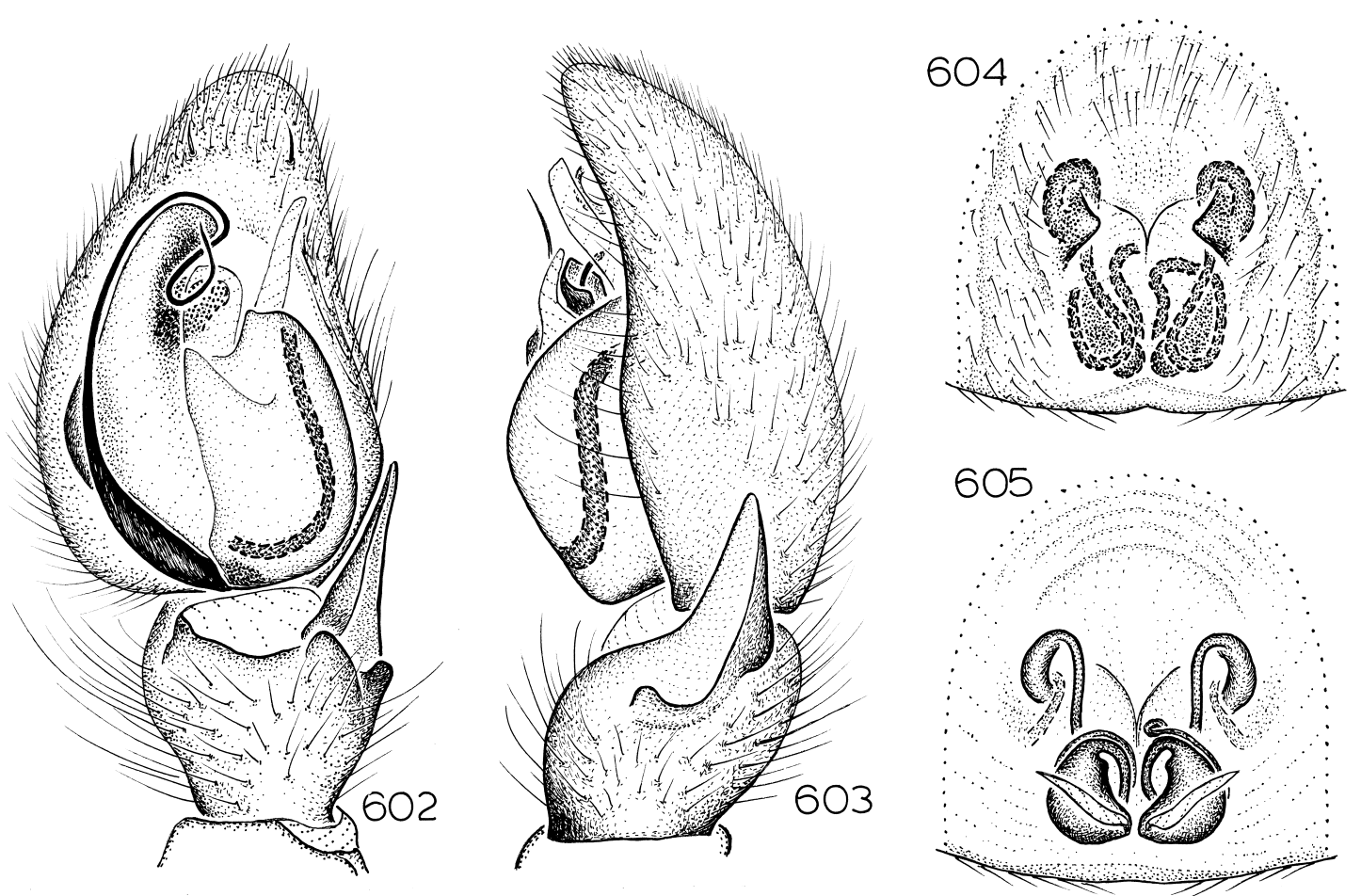

Figs. 602-605. Asadipus woodleigh, new species. 602. Left male palp, ventral view. 603. Same, retrolateral view. 604. Epigynum, ventral view. 605. Same, dorsal view.

1 ; Kennedy Range National Park, 243' $\mathrm{S}$, $114^{\circ} 58^{\prime} \mathrm{E}$, Jan. 14-May 29, 1995, pitfalls (P. West, WAM 96/530-539), 7o, 3 o, May 29Aug. 28, 1995, pitfalls (N. Hall, WAM 96/ 519-522), 4o; Kennedy Range National Park, $24^{\circ} 34^{\prime}$ S, $114^{\circ} 57^{\prime}$ E, Jan. 14-May 29, 1995, pitfalls (P. West, WAM 96/526-529), 4ô, May 29-Aug. 28, 1995, pitfalls (N. Hall, WAM 96/523-525), 20, 1 ; ; Mardathuna Station, $24^{\circ} 31^{\prime} \mathrm{S}, 114^{\circ} 38^{\prime} \mathrm{E}$, Apr. 7-May 26, 1995, pitfalls (A. Sampey, WAM 96/546551), 60ิ, May 23-28, 1995, dry pitfalls (A. Sampey, WAM 96/544, 545), 20 , May 26Aug. 26, 1995, pitfall (N. Hall, WAM 96/ 543), 1 \% ; Meedo Station, $25^{\circ} 41^{\prime} \mathrm{S}, 114^{\circ} 37^{\prime} \mathrm{E}$, Jan. 12-May 18, 1995, pitfall (A. Sampey, WAM 96/540), $10^{\dagger}$; Meedo Station, 254' S, $114^{\circ} 36^{\prime} \mathrm{E}$, May 16-21, 1995, dry pitfall (A. Sampey, WAM 96/541), 10, May 19-Aug. 22, 1995, pitfall (N. Hall, WAM 96/542), $10^{\prime}$; Nanga Station, $26^{\circ} 29^{\prime} \mathrm{S}, 114^{\circ} 05^{\prime} \mathrm{E}$, Jan. 19-May 11, 1995, pitfalls (A. Sampey, WAM 96/554-560), 7ð, May 11-15, 1995, dry pitfall (A. Sampey, WAM 96/552), 10 ,
May 11-Aug. 30, 1995, pitfall (N. Hall, WAM 96/553), 10; Shark Bay, Edel Land, $26^{\circ} 10^{\prime} \mathrm{S}, 113^{\circ} 11^{\prime} \mathrm{E}$, Apr. 18, 1979, under ground litter (N. Sarti, WAM 96/409, 410), $20^{\star}$; Woodleigh Station, $26^{\circ} 12^{\prime} \mathrm{S}, 114^{\circ} 25^{\prime} \mathrm{E}$, Jan. 12-May 17, 1995, pitfalls (P. West, WAM 96/568, 582, 583), 20, 1 \% ; Woodleigh Station, $26^{\circ} 12^{\prime} \mathrm{S}, 114^{\circ} 31^{\prime} \mathrm{E}$, Jan. 12May 17, 1995, pitfalls (P. West, WAM 96/ 586-588), 30; Woodleigh Station, $26^{\circ} 12^{\prime} \mathrm{S}$, $114^{\circ} 32^{\prime} \mathrm{E}$, Jan. 12-May 17, 1995, pitfalls (P. West, WAM 96/577-580), 40, May 14-20, 1995, dry pitfalls (P. West, WAM 96/584, 585), 20; Woodleigh Station, $26^{\circ} 13^{\prime} \mathrm{S}$, $114^{\circ} 35^{\prime} \mathrm{E}$, Aug. 22-Oct. 12, 1994, pitfall (M. Harvey, WAM 96/565), 19 , Jan. 12-May 17, 1995, pitfalls (P. West, WAM 96/569574, 581), $70^{\star}$; Woodleigh Station, $26^{\circ} 13^{\prime} \mathrm{S}$, $114^{\circ} 36^{\prime}$ E, Jan. 12-May 17, 1995, pitfalls (P. West, WAM 96/575, 576), 20, May 15-21, 1995, dry pitfalls (P. West, WAM 96/562564), 20, 1 \% , May 17-Aug. 21, 1995, pitfall (N. Hall, WAM 96/561), 1 \% .

DisTRIBUTION: Western Australia (map 64). 

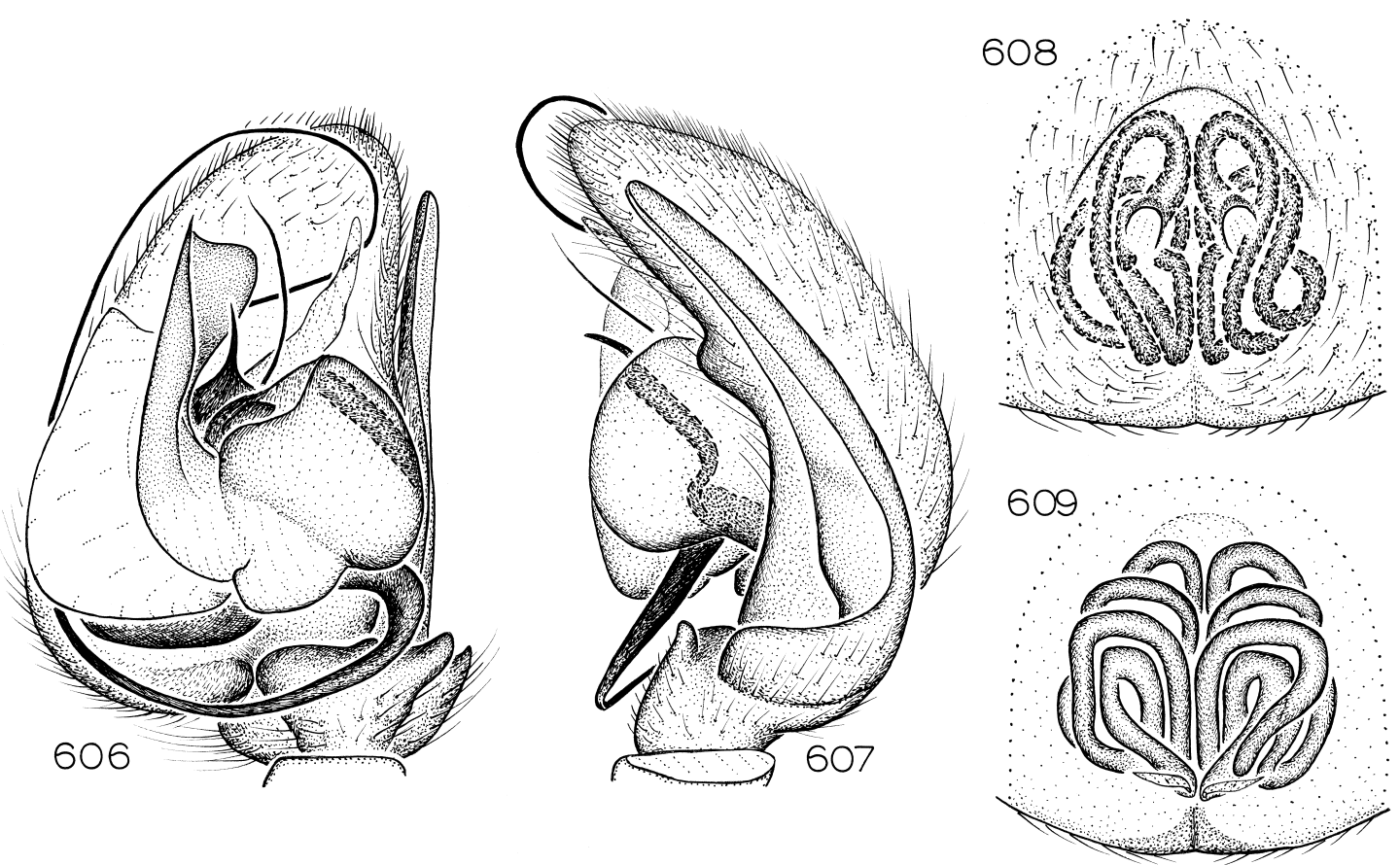

Figs. 606-609. Asadipus kunderang, new species. 606. Left male palp, ventral view. 607. Same, retrolateral view. 608. Epigynum, ventral view. 609. Same, dorsal view.

\section{Asadipus kunderang, new species} Figures 606-609; Map 72

TYPES: Female holotype and male allotype taken in pitfall traps at an elevation of 890 $\mathrm{m}$ on East Kunderang Trail, $1.35 \mathrm{~km}$ E West Kunderang Trail, $30^{\circ} 48^{\prime} \mathrm{S}, 152^{\circ} 02^{\prime} \mathrm{E}$, New South Wales (Feb. 4-Apr. 9, 1993; M. Gray, G. Cassis), deposited in AMS (KS40287).

ETYMOLOGY: The specific name is a noun in apposition taken from the type locality.

DiaGNOSIS: Males of this distinctive species can be recognized easily by the enormously elongated retrolateral tibial apophysis, which reaches almost to the distal tip of the cymbium, and the relatively wide palpal bulb and tegulum (figs. 606, 607), females by the anteromedially directed epigynal openings and ducts (fig. 608).

MALE: Total length 6.4. Carapace and abdominal scutum extremely dark brownish red, especially near anteriorly produced and setose clypeal area; legs dark reddish brown, femora darkest. Leg spination: tibiae: III p00-0, v0-0-1p, r0-0-0; IV p0-0-0, v0-1p-2, r00-0; metatarsi III, IV v0-0-0. Retrolateral tib- ial apophysis extremely long, reaching almost to distal tip of cymbium (fig. 607); embolus extremely long, ventral portion of terminal apophysis expanded prolaterally (fig. 606).

Female: Total length 7.5. Coloration as in A. phaleratus. Leg spination: tibiae: III p00-0, v0-0-1p, r0-0-0; IV p0-0-0, v0-1p-2, r00-0; metatarsi III, IV v0-0-0. Epigynal openings directed anteromedially (fig. 608), leading to looping ducts making at least three oval circuits around epigynal sides (fig. 609).

Other Material Examined: Australian Capital Territory: Piccadilly Circus, $35^{\circ} 22^{\prime} \mathrm{S}, 148^{\circ} 48^{\prime} \mathrm{E}$, Mar. 1984, elev. $1240 \mathrm{~m}$ (J. Lawrence, T. Weir, M. Johnson, QMB), 10; Wombat Creek, 6 km NE Piccadilly Circus, $35^{\circ} 19^{\prime} \mathrm{S}, 148^{\circ} 51^{\prime} \mathrm{E}$, Apr. 1984 , elev. 750 m (T. Weir, J. Lawrence, M. Johnson, QMB), 1 \%, Mar. 1985, elev. $750 \mathrm{~m}$ (T. Weir, J. Lawrence, M. Johnson, QMB), 1 , May 1985, elev. 750 m (T. Weir, J. Lawrence, M. Johnson, QMB), 2o. New South Wales: hilltop on ridge between Big Marsh and Esk River, Bundjalung National Park, 29 $17^{\prime} \mathrm{S}$, 
$153^{\circ} 16^{\prime} \mathrm{E}$, Feb. 4-Apr. 9, 1993, pitfall (M. Gray, G. Cassis, AMS KS40282), $10^{\text {to }}$; midway between Boorook Creek and Gilcurry Creek on Conlongan Road, Boorook State Forest, 28 $51^{\prime} \mathrm{S}, 152^{\circ} 11^{\prime} \mathrm{E}$, Feb. 4-Apr. 9, 1993, pitfall, elev. 980 m (M. Gray, G. Cassis, AMS KS40284), $10^{\Uparrow}$; Boorook State Forest, $1 \mathrm{~km}$ E main road, $28^{\circ} 49^{\prime} \mathrm{S}, 152^{\circ} 11^{\prime} \mathrm{E}$, Feb. 4-Apr. 9, 1993, pitfall, elev. 900 m (M. Gray, G. Cassis, AMS KS40283), 10 ; Booti Booti National Park, $32^{\circ} 16^{\prime} \mathrm{S}, 152^{\circ} 31^{\prime} \mathrm{E}$, July 15, 1996, pitfall, sand dune (L. Wilkie, AMS KS50522), 19 ; Grey Gums Forest Road, 1.1 $\mathrm{km}$ from Doyles River Road, Bulga State Forest, $31^{\circ} 33^{\prime} \mathrm{S}, 152^{\circ} 14^{\prime} \mathrm{E}$, Feb. 4-Apr. 9, 1993, pitfall, elev. 620 m (M. Gray, G. Cassis, AMS KS40290), 1 ; ; Grey Gums Forest Road, $2.1 \mathrm{~km}$ from Doyles River Road, Bulga State Forest, $31^{\circ} 33^{\prime} \mathrm{S}, 152^{\circ} 15^{\prime} \mathrm{E}$, Feb. 4Apr. 9, 1993, pitfall, elev. 560 m (M. Gray, G. Cassis, AMS KS40289), 10; East Kunderang Trail, $1.35 \mathrm{~km}$ E West Kunderang Trail, $30^{\circ} 48^{\prime} \mathrm{S}, 152^{\circ} 02^{\prime} \mathrm{E}$, Feb. 4-Apr. 9, 1993, pitfall, elev. 890 m (M. Gray, G. Cassis, AMS KS40287), 20, 1 ; ; East Kunderang Trail, $2.1 \mathrm{~km}$ E West Kunderang Trail, $30^{\circ} 49^{\prime} \mathrm{S}, 152^{\circ} 03^{\prime} \mathrm{E}$, Feb. 4-Apr. 9, 1993, pitfall, elev. 845 m (M. Gray, G. Cassis, AMS KS40286), 1 đ; $^{\text {; }} 240$ m E junction Kunderang East and Kunderang West Trails, $30^{\circ} 48^{\prime} \mathrm{S}$, $152^{\circ} 02^{\prime} \mathrm{E}$, Feb. 4-Apr. 9, 1993, pitfall, elev. 900 m (M. Gray, G. Cassis, AMS KS40288), 10ิ, 1; Mummel Forest Road, 7.6 km N junction Enfield Forest Road, Enfield State Forest, $31^{\circ} 17^{\prime} \mathrm{S}, 152^{\circ} 51^{\prime} \mathrm{E}$, Feb. 4-Apr. 9, 1993, pitfall, elev. 1340 m (M. Gray, G. Cassis, AMS KS40285), 10 ; Padmans Road, near intersection of Pole Dump Road, Bulga State Forest, $31^{\circ} 36^{\prime} \mathrm{S}, 152^{\circ} 10^{\prime} \mathrm{E}, \mathrm{Feb}$. 4-Apr. 9, 1993, pitfall, elev. $730 \mathrm{~m}$ (M. Gray, G. Cassis, AMS KS40291), 10; saddle along ridge, Spirabo State Forest, $29^{\circ} 18^{\prime} \mathrm{S}$, $152^{\circ} 11^{\prime} \mathrm{E}$, Feb. 4-Apr. 9, 1993, pitfall, elev. 920 m (M. Gray, G. Cassis, AMS KS40281), $10^{\star}$; N Wee Jasper at Carey's Cave, $35^{\circ} 04^{\prime} \mathrm{S}$, $148^{\circ} 40^{\prime} \mathrm{E}$, Oct. 7 , 1992, semi-evergreen vine thicket (J. Stanisic, G. Ingram, QMB S21967), 1 ㅇ. Northern Territory: top end, Sherwin Creek, $14^{\circ} 39^{\prime} \mathrm{S}, 134^{\circ} 21^{\prime} \mathrm{E}$, May 1996 (A. Hertog, CSID A0263), $1 \delta^{\dagger}, 4$ ㅇ. Queensland: Bedourie, $24^{\circ} 22^{\prime} \mathrm{S}, 139^{\circ} 28^{\prime} \mathrm{E}$, Aug. 25-28, 1978, under stone, under bark (M. Jahnke, QMB S26525), 2ð; The Bluff,
Keysland, $26^{\circ} 15^{\prime} \mathrm{S}, 151^{\circ} 43^{\prime} \mathrm{E}$, July 29-Oct. 23, 1995, pitfall in vine scrub, elev. $530 \mathrm{~m}$ (G. Monteith, QMB S34291), 20 ; Koy Property, Brigooda, $26^{\circ} 16^{\prime} \mathrm{S}, 151^{\circ} 25^{\prime} \mathrm{E}$, Jan. 26Apr. 20, 1995, pitfall in vine scrub (G. Monteith, QMB S34293), 10; Lake Broadwater, via Dalby, $27^{\circ} 11^{\prime} \mathrm{S}, 151^{\circ} 16^{\prime} \mathrm{E}$, Feb. $25-$ Apr. 22, 1986, pitfall (M. Bennie, QMB S34294), 1\%; Lake Moondarra, via Mount Isa, $20^{\circ} 41^{\prime} \mathrm{S}, 139^{\circ} 30^{\prime} \mathrm{E}$, Apr. 17-July 25, 1996, pitfall (R. Raven, R. McKay, QMB S31204), $10^{\top}$; Mount Archer, $23^{\circ} 20^{\prime} \mathrm{S}, 150^{\circ} 35^{\prime} \mathrm{E}$, July 1-Oct. 22, 1990, pitfall in open forest (D. Wallace, R. Raven, QMB S35355), 10; Mount Cameron Station, Winton, $22^{\circ} 59^{\prime} \mathrm{S}$, $142^{\circ} 34^{\prime}$ E, May 15-18, 1977 (A. Rozenfelds,

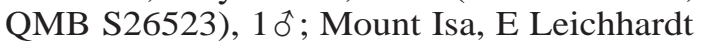
River, $1 \mathrm{~km}$ downstream from Dam Wall, 204' S, 139²9'E, 1988 (K. Mitchell, B., W. Barton, AMS KS30269), 10; Mount Rose, via Taroom, $25^{\circ} 25^{\prime} \mathrm{S}, 149^{\circ} 58^{\prime} \mathrm{E}$, July $12-$ Sept. 23, 1997, pitfall, softwood scrub (D. Cook, QMB S43925), 3 ; ; Rochedale State Forest, Brisbane, $27^{\circ} 37^{\prime} \mathrm{S}, 153^{\circ} 09^{\prime} \mathrm{E}$, May 28-June 12, 1980, pitfall (V. Davies, R. Raven, QMB S26547), 10; Thylungra, $26^{\circ} 04^{\prime} \mathrm{S}, 143^{\circ} 28^{\prime} \mathrm{E}$, Oct. 1995 , pitfall (T. Churchill, CSID A0248), 10 . South Australia: Alligator Gorge, Mount Remarkable National Park, $32^{\circ} 45^{\prime} \mathrm{S}, 138^{\circ} 03^{\prime} \mathrm{E}$, Apr. 13, 1993, on ground (D. Hirst, SAM N1996/ 425), 1 ; ; Burnside, $34^{\circ} 56^{\prime} \mathrm{S}, 138^{\circ} 38^{\prime} \mathrm{E}$ (O.

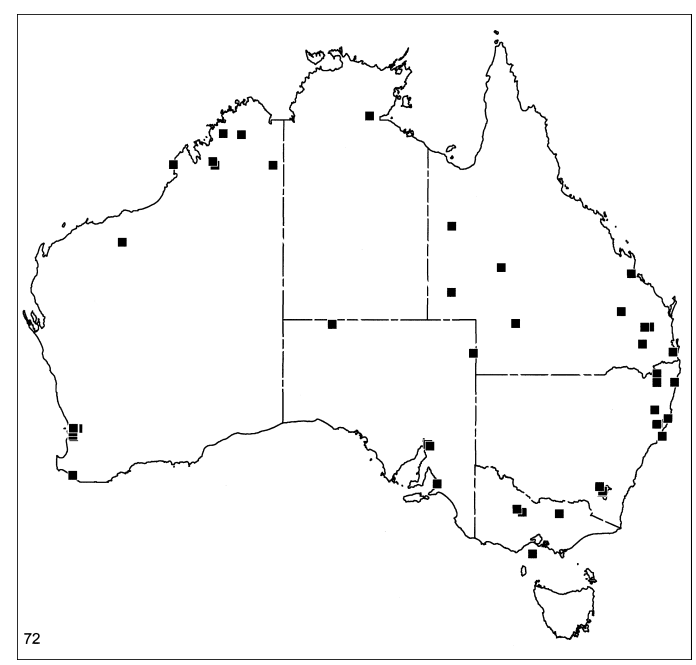

Map 72. Records of Asadipus kunderang, new species. 
Tepper, SAM N1996/424), 1 ; Cullyamurra Water Hole, Coopers Creek, $27^{\circ} 42^{\prime} \mathrm{S}$, $140^{\circ} 50^{\prime} E$, Sept. 21-24, 1990 (J. Shaw, SAM N1997/90), 1 iq; Melrose, $32^{\circ} 50^{\prime} \mathrm{S}, 138^{\circ} 11^{\prime} \mathrm{E}$ (A. Lea, SAM N1996/426), $19 ; 4$ km SSW Mount Cuthbert, $26^{\circ} 08^{\prime} \mathrm{S}, 132^{\circ} 04^{\prime} \mathrm{E}$, Oct. 21-22, 1994 (J. Forrest, SAM N1996/421, 422), 1 o , 1 ㅇ․ Victoria: Eyensbury, $38^{\circ} 48^{\prime} \mathrm{S}$, $144^{\circ} 33^{\prime} \mathrm{E}$, Nov. 13-20, 1991, pitfall, grey box woodland (B. Van Praagh, R. Kay, A. Kobelt, NMVS), $1 \%$; 7.5 km NNW Inglewood, $36^{\circ} 30^{\prime} \mathrm{S}, 143^{\circ} 53^{\prime} \mathrm{E}$, Oct. 15-20, 1989, mallee (A. Coventry, NMV K4650), 19 ; Upper Lurg, $36^{\circ} 35^{\prime} \mathrm{S}, 146^{\circ} 11^{\prime} \mathrm{E}$, Apr. 12, 1996, house at night (J. Strudwick, NMV), 1 t, Apr. 22, 1997, house (J. Strudwick, NMV), $1 \delta^{\top} ; 7.5 \mathrm{~km}$ SSW Wychitella, $36^{\circ} 20^{\prime} \mathrm{S}$, $143^{\circ} 35^{\prime} \mathrm{E}$, Mar. 26-29, 1990, mallee (A. Coventry, NMV K4652), 1 o. Western Australia: ALCOA minesite and forests, $\mathrm{N}$ and NW of Jarrahdale, $32^{\circ} 21^{\prime} \mathrm{S}, 116^{\circ} 04^{\prime} \mathrm{E}$, Mar.Aug. 1993 (S. Simmonds, WAM 96/928), 10; Bushmead, Ridge Hill Road, $31^{\circ} 56^{\prime} \mathrm{S}$, $116^{\circ} 02^{\prime} \mathrm{E}$, Apr. 16-June 17, 1996, pitfall (J. Waldock, P. West, A. Longbottom, WAM 96/ 622), 10; Cardup Reserve, $32^{\circ} 15^{\prime} \mathrm{S}$, $115^{\circ} 59^{\prime} \mathrm{E}$, Apr. 16-June 17, 1996, pitfalls (J. Waldock, P. West, A. Longbottom, WAM 96/

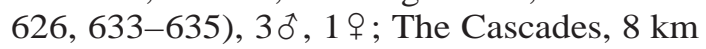
SSW Pemberton, $34^{\circ} 30^{\prime} \mathrm{S}, 116^{\circ} 00^{\prime} \mathrm{E}$, May 3, 1990 (M. Harvey, J. Waldock, WAM 96/ 624), $1 \delta^{\star}$; Drysdale River Station, $15^{\circ} 42^{\prime} \mathrm{S}$, $126^{\circ} 23^{\prime} \mathrm{E}$, June 18, 1993, litter near shed (A. Longbotton, WAM 96/631), 1 \%, Feb. 25, 1994, under drum on airfield (A. Longbottom, WAM 96/623), 10; Great Eastern Highway, York turnoff, $31^{\circ} 53^{\prime} \mathrm{S}, 116^{\circ} 19^{\prime} \mathrm{E}$, with eggsac on leaf on ground (B. Main, WAM 96/632), 1 \% ; Hovea, $31^{\circ} 53^{\prime} \mathrm{S}$, $116^{\circ} 06^{\prime} \mathrm{E}$, June 6,1993 , indoors at night (A. Sampey, WAM 96/620), 10; Kimberley, Lennard River Crossing, Gibb River Road, $17^{\circ} 23^{\prime} \mathrm{S}, 124^{\circ} 44^{\prime} \mathrm{E}$, July $14-28,1988$, pitfall in riverine thicket (T. Houston, WAM 96/ 617-619, 630, 638-640), 40, 3; $4 \mathrm{~km} \mathrm{~W}$ King Cascade, $15^{\circ} 38^{\prime} \mathrm{S}, 125^{\circ} 15^{\prime} \mathrm{E}$, June $12-$ 16, 1988, Berlese, closed forest litter ( $\mathrm{T}$. Weir, QMB), 1 \% ; W Kurrajong Camp, Purnululu National Park, $17^{\circ} 23^{\prime} \mathrm{S}, 128^{\circ} 20^{\prime} \mathrm{E}$, May 5, 1994, under limestone (A. Longbottom, WAM 96/1569), 1 ㅇ (penultimate but with fully formed epigynum); Norman Road, Cardup, $32^{\circ} 16^{\prime} \mathrm{S}, 116^{\circ} 01^{\prime} \mathrm{E}$, Apr, 16-June 17,
1996, pitfall (J. Waldock, P. West, A. Longbottom, WAM 96/625), 1 ơ; $^{\text {}}$ toward Old Napier Downs, $30 \mathrm{mi}$. E Derby, $17^{\circ} 11^{\prime} \mathrm{S}$, $124^{\circ} 36^{\prime} \mathrm{E}$, June 8, 1970 (Lemley, WAM 96/ 621), 1ठ; Point Coulomb, c. $45 \mathrm{mi} \mathrm{N}$ Broome, $17^{\circ} 21^{\prime} \mathrm{S}, 122^{\circ} 09^{\prime} \mathrm{E}$, June-July, 1971 (W. Butler, WAM 84/1123), 1 \% ; Rushton Road, Martin, $32^{\circ} 04^{\prime} \mathrm{S}, 116^{\circ} 01^{\prime} \mathrm{E}$, Apr, 16June 17, 1996, pitfall (J. Waldock, P. West, A. Longbottom, WAM 96/627), $1 \delta^{\Uparrow}$; Talbot Road Reserve, $31^{\circ} 52^{\prime} \mathrm{S}, 116^{\circ} 03^{\prime} \mathrm{E}$, May $10-$ June 24, 1993 (M. Harvey, J. Waldock, WAM 96/629), 1 오․ Aug. 24-29, 1993, dry pitfall (J. Dell, WAM 96/637), 1 \% ; Woodstock Station, $21^{\circ} 37^{\prime} \mathrm{S}, 119^{\circ} 01^{\prime} \mathrm{E}$, Feb. 1017, 1989, dry pitfall, acacia/spinifex (J. Dell, R. How, J. Waldock, WAM 96/636), 1 .

Distribution: Widespread in Australia, except Tasmania (map 72).

\section{Asadipus banjiwarn, new species}

Figures 610-613; Map 65

TYPES: Female holotype and male allotype from mallee, mulga, and triodia assemblage at Banjiwarn, $27^{\circ} 48^{\prime} \mathrm{S}, 121^{\circ} 40^{\prime} \mathrm{E}$, Western Australia (May 1979; R. How), deposited in WAM $(96 / 644,645)$.

ETYMOLOGY: The specific name is a noun in apposition taken from the type locality.

DiAGNOSIS: Males of this distinctive, small species can be recognized easily by the widened palpal bulb, with the terminal apophysis widely overlapping the embolus (fig. 610), females by the large epigynal openings and medially coiled epigynal ducts (figs. 612, 613).

MALE: Total length 3.8. Coloration as in A. phaleratus, with clypeus area anteriorly produced. Leg spination: tibiae: III p0-0-0, v0-0-1p, r0-0-0; IV p0-0-0, v1p-1p-1p, r0-00 ; metatarsi: III v0-0-0, IV v0-1p-0. Retrolateral tibial apophysis extending about onehalf of cymbial length (fig. 611); ventral portion of terminal apophysis overlapping embolus in ventral view, dorsal portion almost sickle-shaped, median apophysis originating on recessed ledge (fig. 610).

Female: Total length 5.0. Coloration as in A. phaleratus. Leg spination: tibiae III, IV p0-0-0, v0-0-1p, r0-0-0; metatarsi III, IV v00-0. Epigynum with large, anteriorly directed openings (fig. 612); ducts irregularly coiling 


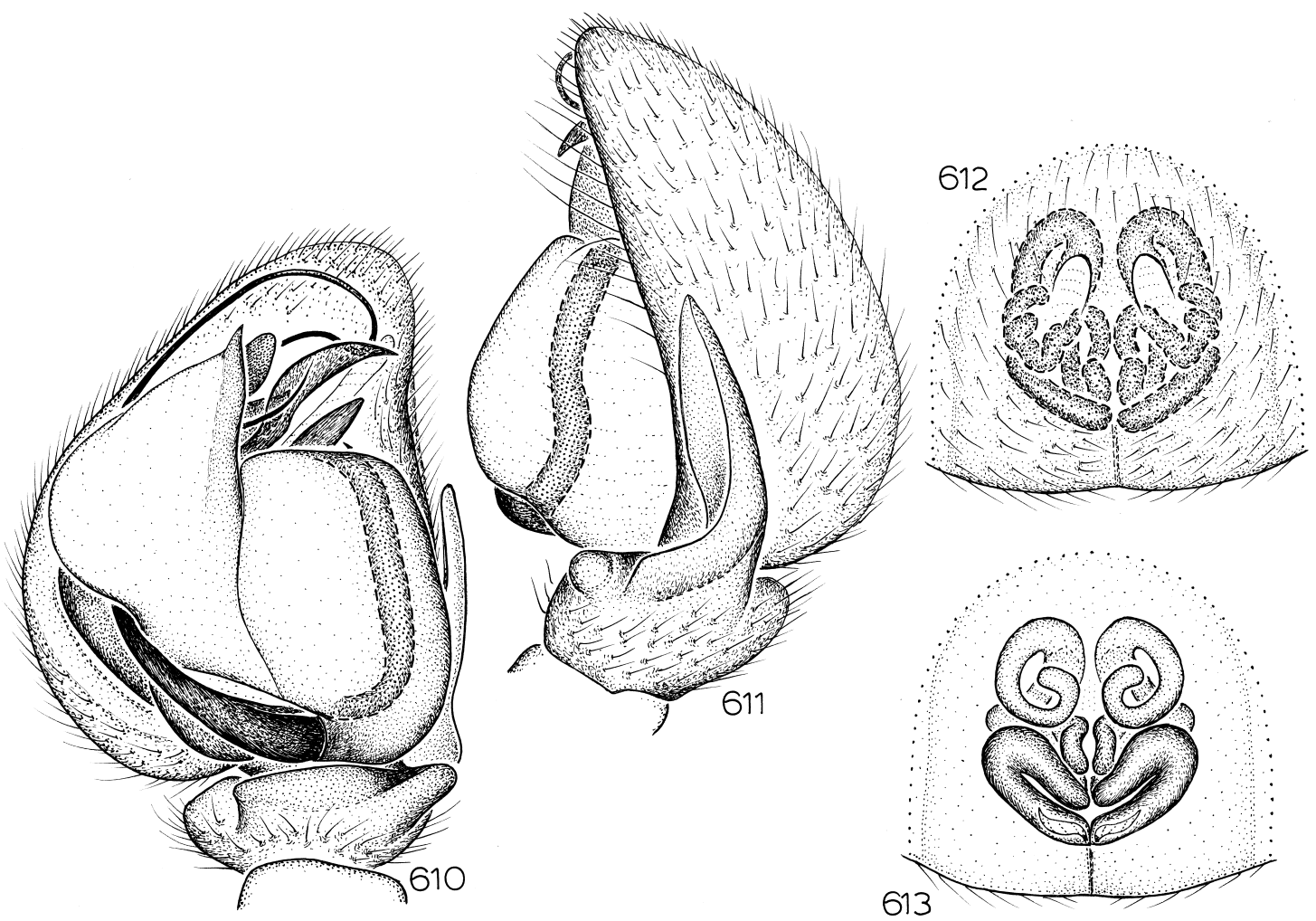

Figs. 610-613. Asadipus banjiwarn, new species. 610. Left male palp, ventral view. 611. Same, retrolateral view. 612. Epigynum, ventral view. 613. Same, dorsal view.

between and anterior of spermathecae (fig. 613).

Other Material ExAmined: Western Australia: Banjiwarn, $27^{\circ} 48^{\prime} \mathrm{S}, 121^{\circ} 41^{\prime} \mathrm{E}$, May 26-31, 1974, mulga, eremophila, bunchgrass (R. How, WAM 96/643), $10^{\text {t; }}$ Barlee Range Nature Reserve, $23^{\circ} 06^{\prime} \mathrm{S}$, $116^{\circ} 00^{\prime} \mathrm{E}$, June 19-22, 1994, dry pitfall (P., G. Kendrick, WAM 96/646), $1 \delta^{\dagger}$; Bidgemia

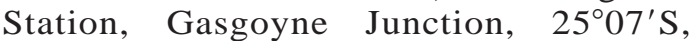
$115^{\circ} 26^{\prime} \mathrm{E}$, June 6-Aug. 20, 1995, pitfalls (N. Hall, WAM 96/642), 1\%; Boolathana Station, $24^{\circ} 25^{\prime} \mathrm{S}, 113^{\circ} 40^{\prime} \mathrm{E}$, May 28-June 2, 1995, dry pitfall (J. Waldock, WAM 96/649), 10; Boolathana Station, $24^{\circ} 25^{\prime} \mathrm{S}, 113^{\circ} 46^{\prime} \mathrm{E}$, May 31-Aug. 25, 1995, pitfall (N. Hall, WAM 96/650), 1 ơ, Aug. 20-Sept. 30, 1994, pitfall (A. Sampey, WAM 96/648), 1 \% ; Bush Bay, $25^{\circ} 07^{\prime} \mathrm{S}, 113^{\circ} 48^{\prime} \mathrm{E}$, May 23-Aug. 23, 1995, pitfall (H. Hall, WAM 96/647), 10;; Cape Cuvier, Quobba Station, $24^{\circ} 08^{\prime} \mathrm{S}$, $113^{\circ} 27^{\prime} \mathrm{E}$, May 31-Aug. 25, 1995, pitfall (N. Hall, WAM 96/652), 10; Cape Cuvier,
Quobba Station, $24^{\circ} 12^{\prime} \mathrm{S}, 113^{\circ} 27^{\prime} \mathrm{E}$, Aug. 21-Sept. 29, 1995, pitfall (P. West, WAM 96/ 653), 10; Cape Cuvier, Quobba Station, $24^{\circ} 13^{\prime} \mathrm{S}, 113^{\circ} 30^{\prime} \mathrm{E}$, May 30-Aug. 24, 1995, pitfall (N. Hall, WAM 96/651), $10^{\text {t }}$; Edel Land, $26^{\circ} 15^{\prime}$ S, $113^{\circ} 19^{\prime}$ E, Sept. 9-14, 1989 (G. Harold, WAM 96/641), 10; Francois Peron National Park, 25 $53^{\prime} \mathrm{S}, 113^{\circ} 33^{\prime} \mathrm{E}$, May 22-27, 1995, dry pitfall (M. Harvey,

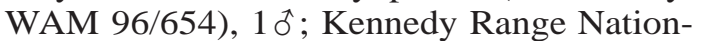
al Park, $24^{\circ} 30^{\prime} \mathrm{S}, 115^{\circ} 01^{\prime} \mathrm{E}$, May 27-June 1, 1995, dry pitfall (P. West, WAM 96/656), $10^{\top}$; Kennedy Range National Park, $24^{\circ} 30^{\prime} \mathrm{S}$, $115^{\circ} 02^{\prime} \mathrm{E}$, May $27-\mathrm{June} 1,1995$, dry pitfall (P. West, WAM 96/655), 10; Mardathuna Station, $24^{\circ} 24^{\prime} \mathrm{S}, 114^{\circ} 28^{\prime} \mathrm{E}$, May 23-May 28, 1995, dry pitfall (A. Sampey, WAM 96/658), 10, May 24-Aug. 26, 1995, pitfall (N. Hall, WAM 96/657), $10^{\top}$; Yundamindra, 29 $23^{\prime} \mathrm{S}$, $122^{\circ} 28^{\prime} \mathrm{E}$, July 1981 , mulga/shrubs (W. Humphreys, WAM 96/659), 1 đ; Y Yundamindra, $29^{\circ} 24^{\prime} \mathrm{S}, 122^{\circ} 28^{\prime} \mathrm{E}$, July 1981 , pitfall, mallee, mulga, triodia (W. Humphreys, 

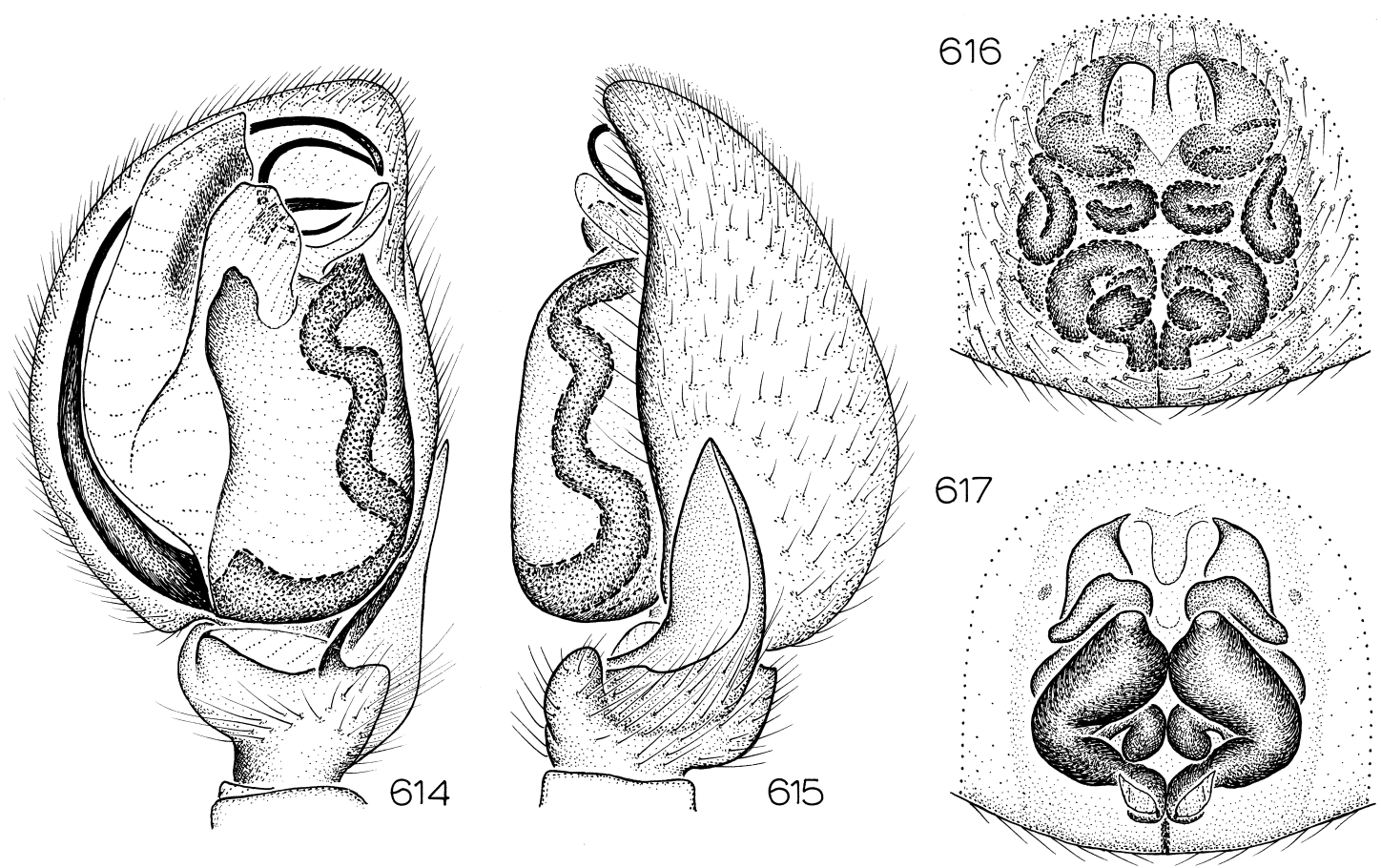

Figs. 614-617. Asadipus auld, new species. 614. Left male palp, ventral view. 615. Same, retrolateral view. 616. Epigynum, ventral view. 617. Same, dorsal view.

WAM 96/684), 1 \%; Zuytdorp, $27^{\circ} 15^{\prime} \mathrm{S}$, $114^{\circ} 12^{\prime} \mathrm{E}$, May 17-Aug. 16, 1995, pitfall (N. Hall, WAM 96/660), 1 \% ; Zuytdorp, $27^{\circ} 16^{\prime} \mathrm{S}$, $114^{\circ} 02^{\prime} \mathrm{E}$, May $18-\mathrm{Aug} .17,1995$, pitfall (N. Hall, WAM 96/661), 1 \% .

Distribution: Western Australia (map 65).

Asadipus auld, new species

Figures 614-617; Map 66

TYPES: Male holotype taken in a fence pitfall trap at Lake Auld, $21^{\circ} 44^{\prime} \mathrm{S}, 123^{\circ} 40^{\prime} \mathrm{E}$, Western Australia (June 13, 1986; L. Charlton), deposited in WAM (87/1142), and female allotype with the same data but captured one day earlier, deposited in WAM (87/ 1143).

ETYMOLOGY: The specific name is a noun in apposition taken from the type locality.

DiAGNOSIS: Males can be recognized by the thickened embolus and sinuous sperm duct (figs. 614, 615), females by the rectangular anterior epigynal openings (fig. 616) and anteriorly massive spermathecae (fig. 617).

MALE: Total length 7.0. Coloration as in
A. phaleratus. Leg spination: tibiae: III r0-01; IV v2-2-2; metatarsi IV v1p-2-0. Retrolateral tibial apophysis reaching to about one-third of cymbial length (fig. 615); embolus originating on prolateral side of bulb, thickened throughout its length, ventral portion of terminal apophysis distally expanded, membranous, dorsal portion terminating in long, sharply bent, sclerotized spur accompanying membranous conductor (fig. 614).

Female: Total length 7.9. Coloration as in A. phaleratus. Leg spination: tibiae: III v1p2-2, r0-0-1; IV p0-0-1. Epigynal openings situated anteriorly, rectangular (fig. 616); spermathecae anteriorly inflated (fig. 617).

Other Material Examined: South Australia: $23 \mathrm{~km}$ WSW Illintjitja, $26^{\circ} 17^{\prime} \mathrm{S}$, $130^{\circ} 10^{\prime} \mathrm{E}$, May 9-13, 1993, pitfall (SAM N1996/419), 1 đ; $^{\text {7 }} 7.5$ km NNW Mount Kintore, $26^{\circ} 30^{\prime} \mathrm{S}, 130^{\circ} 27^{\prime} \mathrm{E}$, May 9, 1994 (SAM 1997/91, 92), $20^{\star} ; 41 \mathrm{~km}$ W Vokes Hill corner, $28^{\circ} 32^{\prime} \mathrm{S}, 130^{\circ} 16^{\prime} \mathrm{E}$, Apr. 14, 1994, pitfall (D. Hirst, SAM 1996/418), 10. Western Australia: edge, Great Sandy Desert, windmill $30 \mathrm{~km}$ on Telfer Road, $21^{\circ} 43^{\prime} \mathrm{S}$, 

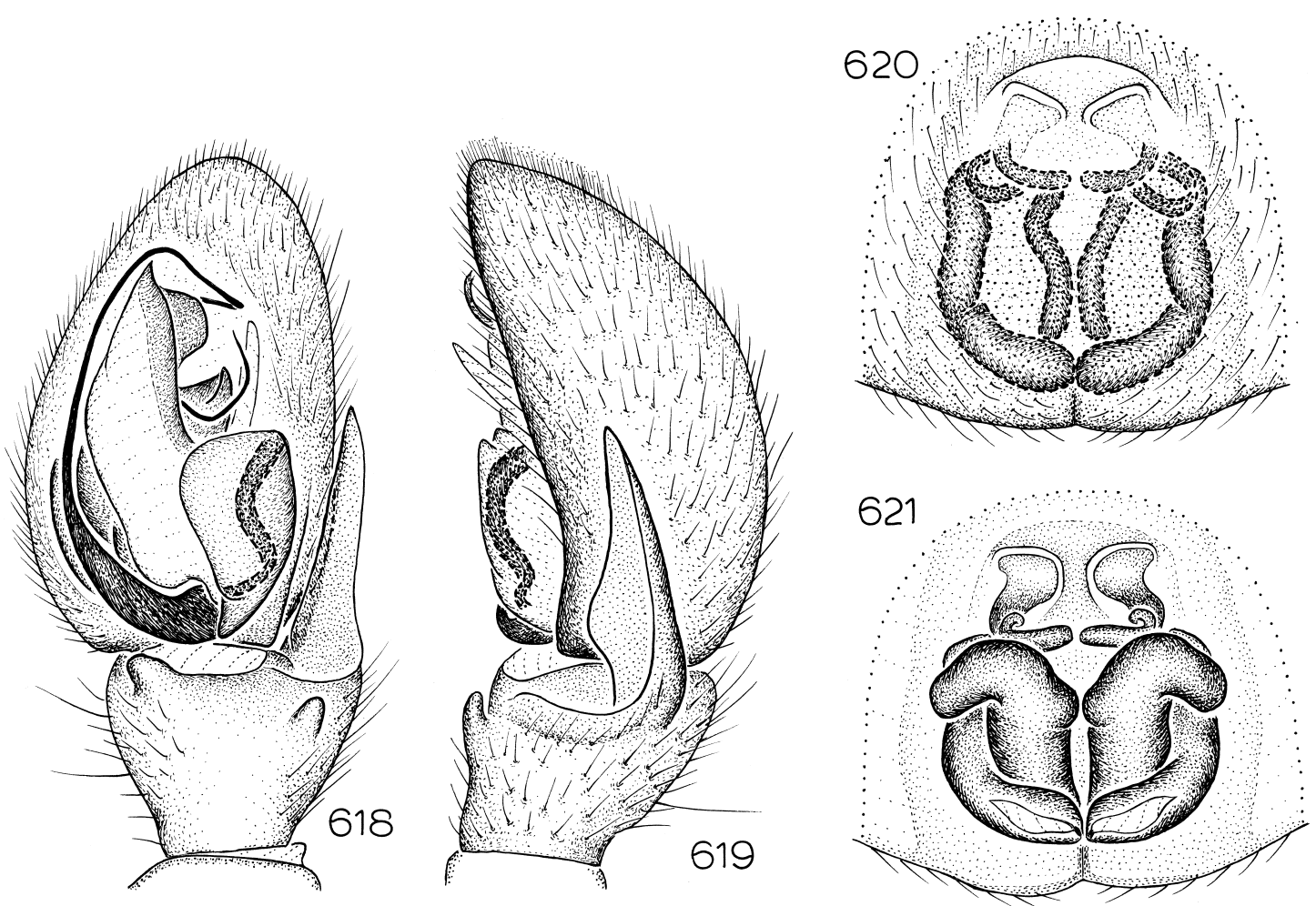

Figs. 618-621. Asadipus cape, new species. 618. Left male palp, ventral view. 619. Same, retrolateral view. 620. Epigynum, ventral view. 621. Same, dorsal view.

$122^{\circ} 14^{\prime}$ E, June 28-29, 1988 (D. South, L. McKenna, WAM 96/661), 1 ơ; Kintyre, $22^{\circ} 19^{\prime} \mathrm{S}, 122^{\circ} 04^{\prime} \mathrm{E}$, Nov. 1988 (S. Harold, WAM 96/669), 19; 7-8 km WNW Point Salvation, $28^{\circ} 12^{\prime} \mathrm{S}, 123^{\circ} 36^{\prime} \mathrm{E}$, Oct. 11-13, 1990, pitfall (E. Pianka, WAM 96/668), 1 ㅇ․

Distribution: Western and South Australia (map 66).

\section{Asadipus cape, new species \\ Figures 618-621; Map 67}

TYPE: Female holotype from Cape Range area, $21^{\circ} 59^{\prime} \mathrm{S}, 113^{\circ} 59^{\prime} \mathrm{E}$, Western Australia (June-Sept. 1989; W. Humphreys), deposited in WAM (96/663).

ETYMOLOGY: The specific name is a noun in apposition taken from the type locality.

DiAGNOSIS: Males of this distinctive species can easily be recognized by the relatively small palpal bulb (fig. 618), females by the massively inflated posterior epigynal ducts (fig. 621).
MaLE: Total length 5.7. Coloration as in A. phaleratus. Leg spination: tibiae: III p00-0, v0-0-1p, r0-0-0; IV p0-0-0, v1p-1p-2, r0-0-0; metatarsi: III v0-0-0; IV v1p-0-0. Retrolateral tibial apophysis reaching to about half of cymbial length (fig. 619); ventral portion of terminal apophysis narrow, dorsal portion folded (fig. 618).

Female: Total length 7.0. Coloration as in A. phaleratus, except medial portion of abdominal dorsum paler than sides. Leg spination: tibiae III, IV p0-0-0, v1p-1p-2, r0-00; metatarsi: III v1p-0-0; IV 2-0-0. Epigynum elongated, anterior portion rectangular (fig. 620); spermathecal and posterior epigynal ducts inflated (fig. 621).

Other MAterial ExAmined: Western Australia: Cape Range, near Cape Range No. 2 Well, $22^{\circ} 06^{\prime} \mathrm{S}, 114^{\circ} 00^{\prime} \mathrm{E}$, May 1965 (W. Kendrick, G. Hitchin, WAM 96/662), 10 .

Distribution: Known only from the Cape Range, Western Australia (map 67). 

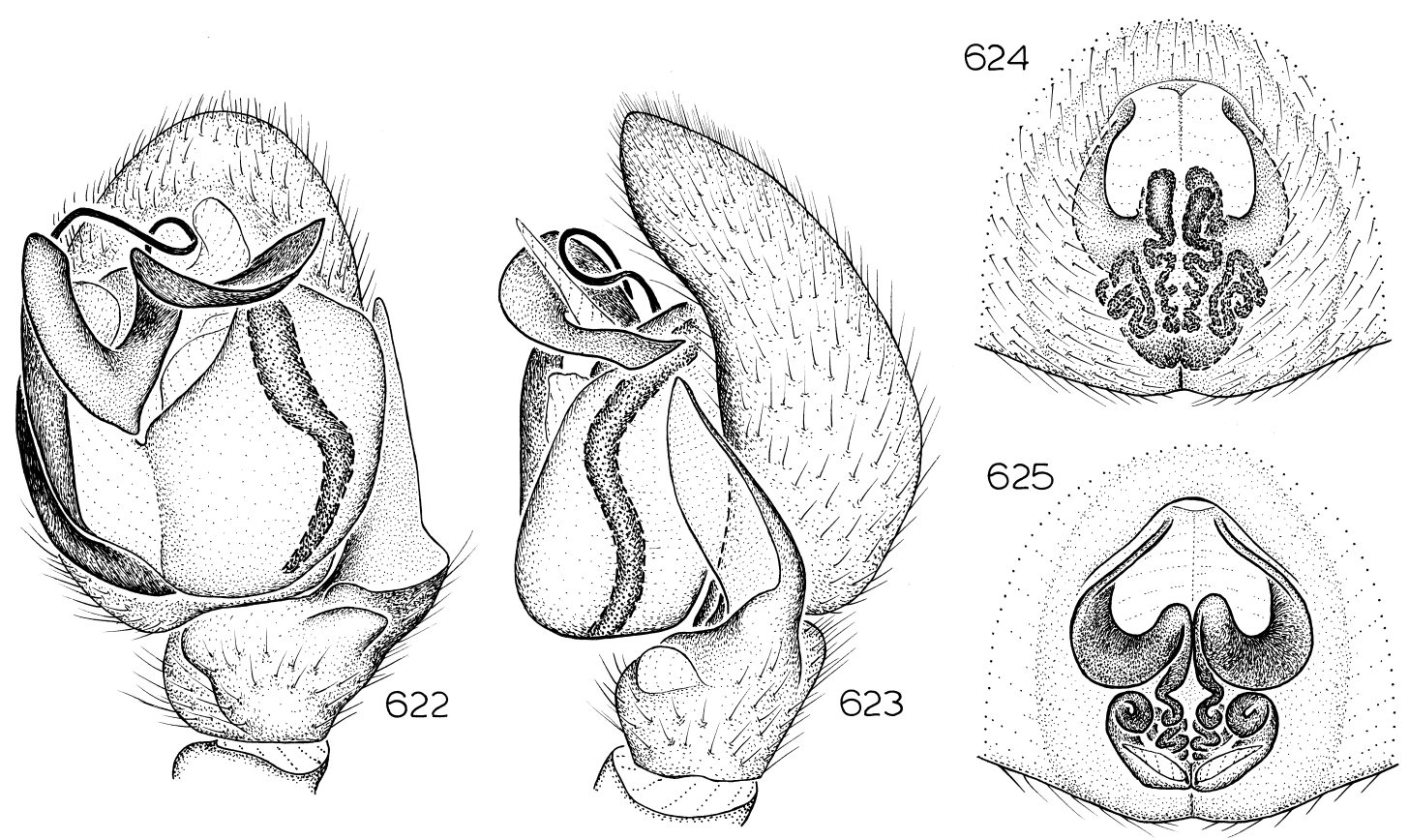

Figs. 622-625. Asadipus baranar, new species. 622. Left male palp, ventral view. 623. Same, retrolateral view. 624. Epigynum, ventral view. 625. Same, dorsal view.

\section{Asadipus baranar, new species} Figures 622-625; Map 68

TYPE: Female holotype taken in dry pitfall trap in Barlee Range Nature Reserve, $23^{\circ} 23^{\prime} \mathrm{S}, 115^{\circ} 53^{\prime} \mathrm{E}$, Western Australia (June 11-14, 1994; P., G. Kendrick), deposited in WAM (96/665).

ETYMOLOGY: The specific name is an arbitrary combination of letters.

Diagnosis: Males of this distinctive species can easily be recognized by the greatly enlarged dorsal prong of the terminal apophysis (fig. 622), females by the inverted heartshaped epigynal atrium (fig. 623) and enlarged anterior epigynal ducts (fig. 624).

MALE: Total length 4.0. Coloration as in A. phaleratus. Leg spination: tibiae: III p0$0-0$, v0-0-0, r0-0-0; IV p0-0-0, v1p-1p-1p, r0-0-0; metatarsi: III v0-0-0; IV v0-1p-0. Palpal femur shortened, thickened distally, with small, rounded knob at about two-thirds of length of retrolateral surface; retrolateral tibial apophysis reaching to about half of cymbial length (fig. 623); dorsal portion of terminal apophysis greatly enlarged, forming ushaped functional conductor (fig. 622).
Female: Total length 7.1. Coloration as in A. phaleratus. Leg spination: tibiae: III p00-0, v0-0-1p, r0-0-0; IV p0-0-0, v1p-1p-2, r0-0-0; metatarsi: III v0-0-0; IV v0-1p-0. Epigynum with almost completely unsclerotized, inverted heart-shaped anterior atrium (fig. 624); anterior epigynal ducts enlarged (fig. 625).

Other Material Examined: Western Australia: Barlee Range Nature Reserve, $23^{\circ} 03^{\prime} \mathrm{S}, 115^{\circ} 49^{\prime} \mathrm{E}$, Aug. 1993, pitfall (S. van Leeuwen, B. Bromilow, WAM 96/666), 1 ơ;

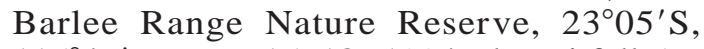
$115^{\circ} 47^{\prime} \mathrm{E}$, June $15-18,1994$, dry pitfall (P., G. Kendrick, WAM 96/1564), $10^{\lambda}$; Barlee Range Nature Reserve, $23^{\circ} 25^{\prime} \mathrm{S}, 115^{\circ} 54^{\prime} \mathrm{E}$, Aug. 1993, pitfall (S. van Leeuwen, B. Bromilow, WAM 96/664), 1 ô.

DisTRIBUTION: Known only from the Barlee Range Nature Reserve, Western Australia (map 68).

\section{Asadipus barant, new species Figures 568, 569; Map 69}

TYPE: Female holotype taken in a dry pitfall trap in the Barlee Range Nature Reserve, 
$23^{\circ} 07^{\prime} \mathrm{S}, 116^{\circ} 01^{\prime} \mathrm{E}$, Western Australia (June 19-22, 1994; P., G. Kendrick), deposited in WAM (96/710).

ETYMOLOGY: The specific name is an arbitrary combination of letters.

Diagnosis: Females of this species resemble those of $A$. baranar but have more highly developed anterior epigynal ducts (fig. 569).

MALE: Unknown.

Female: Total length 4.8. Abdominal dorsum gray with posterior chevrons and oblique lines composed of small pale patches. Leg spination: tibiae: III p0-0-0, v0-0-0, r0-0-0; IV p0-0-0, v1p-0-1p, r0-0-0; metatarsi: III v0-0-0; IV v1p-0-0. Epigynum with pronounced anterior atrium and thickened anterior margins (fig. 568); anterior ducts occupying about two-thirds of epigynal length (fig. 569).

Other Material Examined: None.

DisTRIBUTION: Known only from the Barlee Range Nature Reserve, Western Australia (map 69).

\section{Asadipus mountant, new species}

Figures 580, 581; Map 69

TYPE: Female holotype from Mount Ant, $24^{\circ} 46^{\prime} \mathrm{S}, 128^{\circ} 45^{\prime} \mathrm{E}$, Western Australia (July 11, 1969; D. Giuliani), deposited in WAM (90/336).

ETYMOLOGY: The specific name is a noun in apposition taken from the type locality.

DIAGNOSIS: Females of this species can easily be recognized by the oblique anterolateral epigynal margins (fig. 580).

MALE: Unknown.

Female: Total length 5.7. Abdominal dorsum gray with posterior chevrons and oblique lines composed of small pale patches. Leg spination: tibiae: III p0-0-0, v0-0-1p, r0-0-0; IV p0-0-0, v1p-2-1p; metatarsi: III v0-0-0; IV v2-0-0. Epigynum with transverse anterior and oblique anterolateral margins (fig. 580); median ducts enlarged, translucent (fig. 581).

Other Material Examined: None.

DisTRIBUTION: Known only from Mount Ant, Western Australia (map 69).

\section{Asadipus yundamindra, new species Figures 626-629; Map 67}

TYPE: Female holotype from Yundamindra area, $29^{\circ} 07^{\prime} \mathrm{S}, 122^{\circ} 02^{\prime} \mathrm{E}$, Western Australia (early June 1989; S. Gilligan), deposited in WAM (96/615).

ETYMOLOGY: The specific name is a noun in apposition taken from the type locality.

Diagnosis: The male of this distinctive species can easily be recognized by the long retrolateral tibial apophysis and the extremely long embolus, which originates on the retrolateral side of the palpal bulb (fig. 627); the female here associated with that male is from a different area, but has extremely long and narrow epigynal ducts (figs. 628, 629) that may be correlated with the male embolus length.

MALE: Total length 6.2. Coloration as in A. phaleratus. Leg spination: tibiae: III p00-0, v1p-2-2, r0-0-0; IV p0-0-0, v1p-1p-2; metatarsi: III v0-0-0; IV v1p-0-0. Retrolateral tibial apophysis reaching three-fourths length of cymbium (fig. 627); embolus originating on retrolateral side of bulb, greatly extended beyond proximal edge of bulb, dorsal prong of terminal apophysis elongate (fig. 626).

FEMALE: Total length 7.6. Coloration as in A. phaleratus. Leg spination: tibiae: III p00-1, v0-1p-2, r0-0-0; IV p0-0-0, v1p-1p-2; metatarsi: III v1p-0-0; IV v2-1p-0. Epigynum anteriorly elongated, with conspicuous, oval openings (fig. 628); ducts elongated, highly twisted anteriorly (fig. 629).

Other Material Examined: Western Australia: Barlee Range Nature Reserve, $23^{\circ} 23^{\prime} \mathrm{S}, 115^{\circ} 53^{\prime} \mathrm{E}$, June 1994 , pitfall (S. van Leeuwen, B. Bromilow, WAM 96/667), 1 ઈ. Distribution: Western Australia (map 67).

\section{Asadipus areyonga, new species}

Figures 584, 585; Map 68

TYPE: Female holotype from $6 \mathrm{mi} \mathrm{N}$ Areyonga Aboriginal Settlement, $24^{\circ} 04^{\prime} \mathrm{S}$, $132^{\circ} 16^{\prime} \mathrm{E}$, Northern Territory (May 16, 1976; I. Kowanko), deposited in SAM (1996/423).

ETYMOLOGY: The specific name is a noun in apposition taken from the type locality.

DiAgNOSIS: Females of this species resemble those of A. yundamindra in having relatively long and narrow epigynal ducts, but differ in having a longer epigynum with a relatively elongated anterior portion (fig. 584) and spermathecae that are not greatly enlarged anteriorly (fig. 585). 

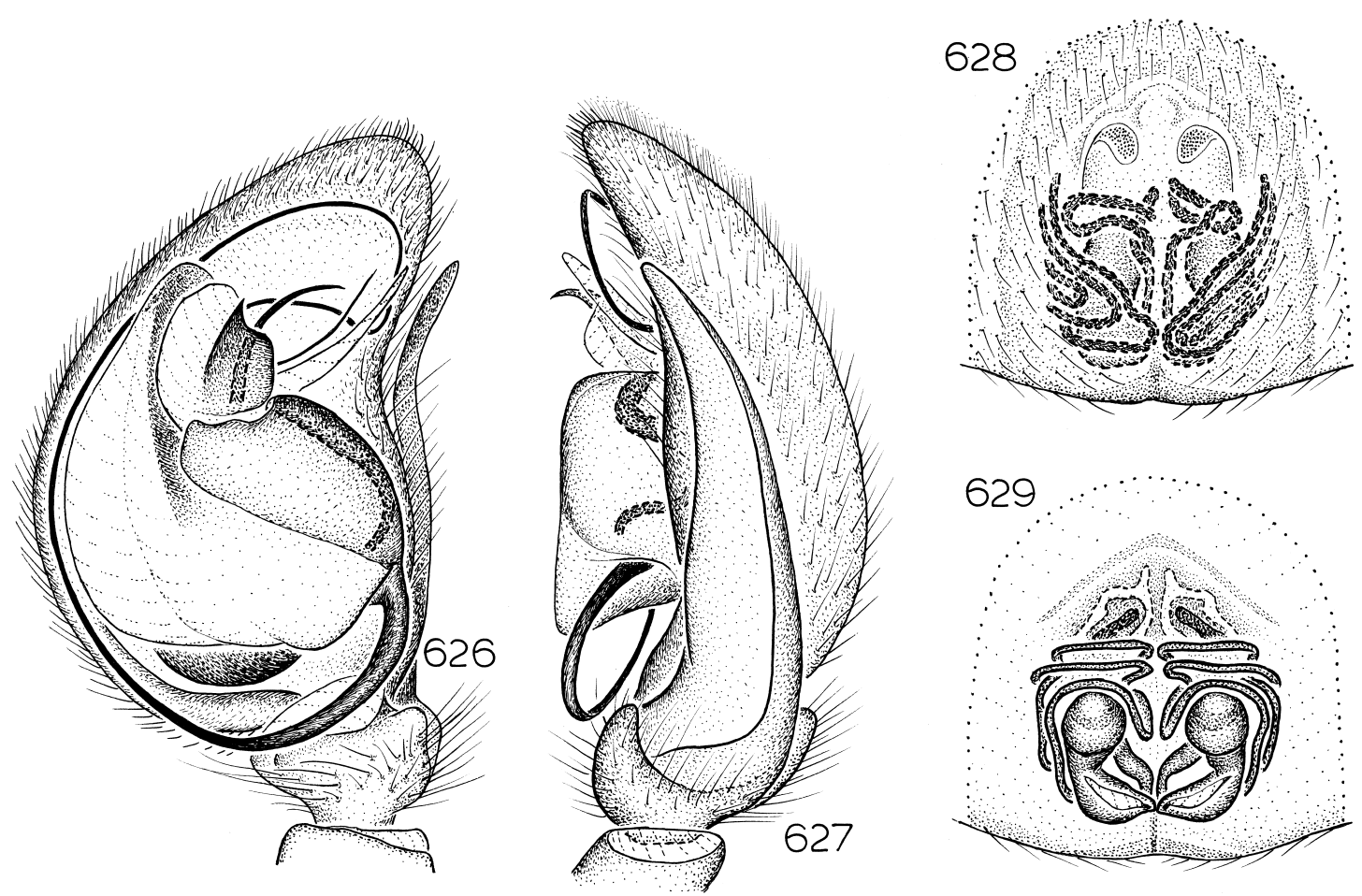

Figs. 626-629. Asadipus yundamindra, new species. 626. Left male palp, ventral view. 627. Same, retrolateral view. 628. Epigynum, ventral view. 629. Same, dorsal view.

MALE: Unknown.

Female: Total length 9.0. Abdominal dorsum gray with posterior chevrons and oblique lines composed of small pale patches. Leg spination: tibiae III, IV p0-0-0, v1p1p-2, r0-0-0; metatarsi: III v1p-0-0; IV v1r$1 \mathrm{p}-0$. Epigynum elongated, especially anterior of most ducts (fig. 584); spermathecae relatively uniform in width throughout their length, ducts long, narrow (fig. 585).

OTHER MATERIAL EXAMINED: Northern Territory: Cassia Hill Track, Simpson Gap, $23^{\circ} 43^{\prime} \mathrm{S}, 133^{\circ} 43^{\prime} \mathrm{E}$, May 1986 , under stone (B. Scott, NMV K3416), 1우. Queensland: Ethabuka Station, Simpson Desert, $23^{\circ} 46^{\prime} \mathrm{S}$, $138^{\circ} 28^{\prime} \mathrm{E}$, July 29 , 1992 , desert, dune swale floodplain (J. Henschel, QMB S34292), 1 ㅇ․

DisTRIBUTION: Northern Territory and western Queensland (map 68).

\section{Asadipus humptydoo, new species} Figures 630-633; Map 66

TyPE: Female holotype from Trepang Road, Humpty Doo, $12^{\circ} 35^{\prime} \mathrm{S}, 131^{\circ} 05^{\prime} \mathrm{E}$,
Northern Territory (Mar. 21, 1993; J. Webber), deposited in MNT.

ETYMOLOGY: The specific name is a noun in apposition taken from the type locality.

DiAGNOSIS: Males of this distinctive species can be recognized by the longitudinal dorsal branch of the terminal apophysis (fig. 630), females by the anterior epigynal hood and long epigynal ducts (figs. 632, 633).

MALE: Total length 5.0. Coloration as in A. phaleratus. Leg spination: tibiae: III v1p1p-2, r0-0-0; IV v1p-1p-2; metatarsi: III v00-0; IV p0-1-0, v1p-0-0, r0-1-0. Retrolateral tibial apophysis very short, reaching only about one-fifth length of cymbium (fig. 631); ventral prong of terminal apophysis relatively short, wide, dorsal prong oriented longitudinally (fig. 630).

Female: Total length 6.8. Coloration as in A. phaleratus. Leg spination: tibiae III, IV p0-0-0, v0-0-2, r0-0-0; metatarsi III, IV v0$0-0$. Epigynum with small, triangular anterior hood, anteromedian portion of atrial surface heavily sclerotized (fig. 632); ducts long, anteriorly looped (fig. 633). 

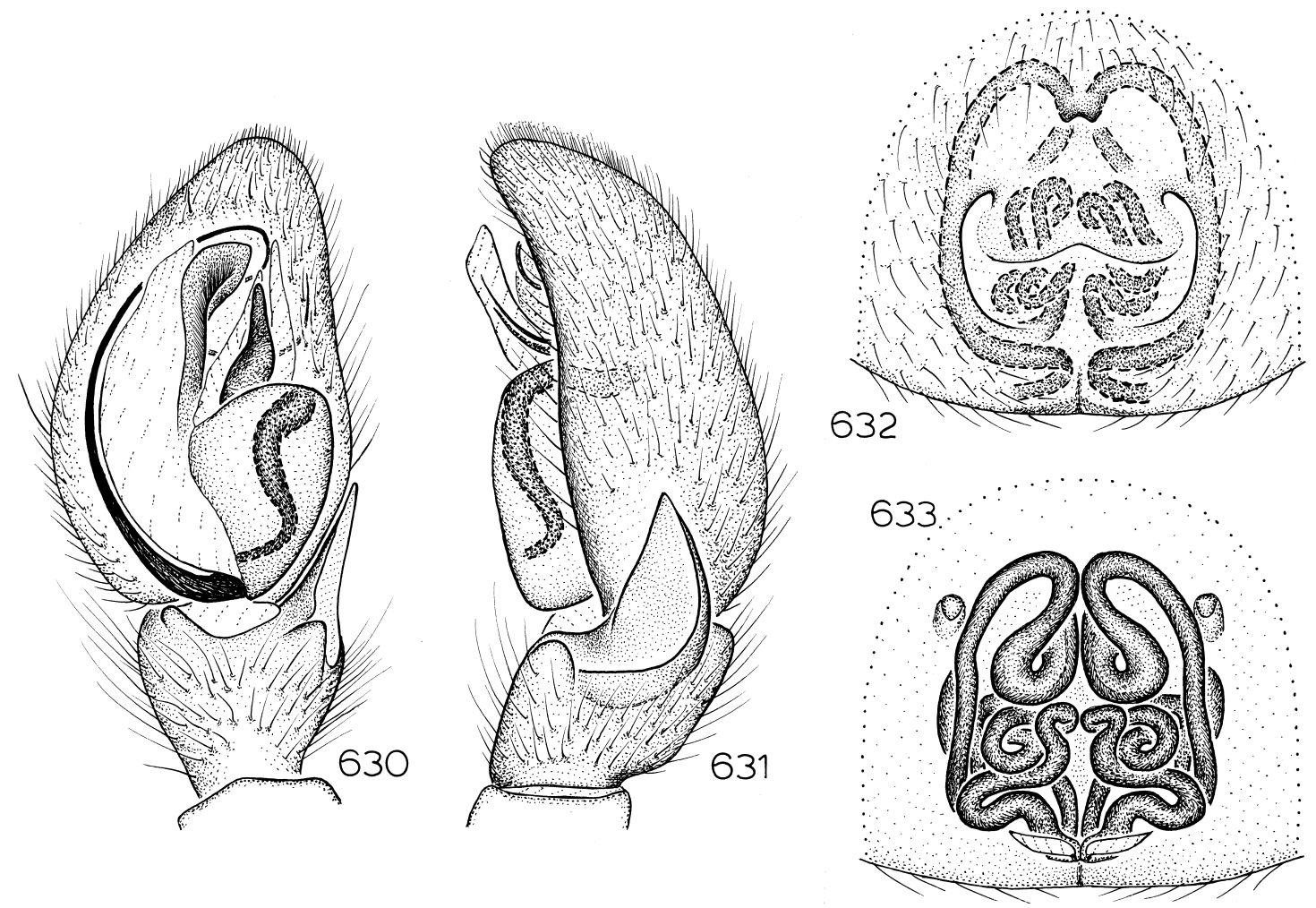

Figs. 630-633. Asadipus humptydoo, new species. 630. Left male palp, ventral view. 631. Same, retrolateral view. 632. Epigynum, ventral view. 633. Same, dorsal view.

Other MATERIAl ExAmined: Northern Territory: Kapalga, $12^{\circ} 36^{\prime} \mathrm{S}, 132^{\circ} 25^{\prime} \mathrm{E}$, Apr. 28, 1992 (A. Andersen, WAM 96/670), $10^{\text {; }}$; South Alligator River, $12^{\circ} 40^{\prime} \mathrm{S}, 132^{\circ} 30^{\prime} \mathrm{E}$, Apr. 13, 1977 (R. Pengilley, MNT), 1 ㅇ.

DistRibution: Known only from far northern Australia (map 66).

\section{Asadipus longforest, new species Figures 634-637; Map 65}

TyPES: Female holotype and male allotype taken in pitfall traps in bull mallee and grey box woodland at Longforest, $36^{\circ} 39^{\prime} \mathrm{S}$, $144^{\circ} 30^{\prime} \mathrm{E}$, Victoria (Mar. 13-19, 1992; B. Van Praagh, P. Lillywhite), deposited in NMVS.

ETYMOLOGY: The specific name is a noun in apposition taken from the type locality.

DiAGNOSIS: Males can be recognized by the arched dorsal prong of the terminal apophysis (fig. 634), females by the anterior epigynal ducts making a full circle (fig. 637).
MALE: Total length 4.3. Coloration as in A. phaleratus. Leg spination: tibiae: III p00-0, v0-0-1p, r0-0-0; IV p0-0-0, v1p-1p-2, r0-0-0; metatarsi: III v0-0-0; IV v1p-0-0. Retrolateral tibial apophysis reaching to about half of cymbial length (fig. 635); ventral prong of terminal apophysis relatively narrow, dorsal prong arched, distally twisted (fig. 634).

FEMALE: Total length 5.4. Coloration as in A. phaleratus. Leg spination: tibiae: III p00-0, v1p-0-2, r0-0-0; IV p0-0-0, v1p-1p-2, r0-0-0; metatarsi: III v0-0-0; IV v1p-0-0. Anterior epigynal ducts forming full circle around epigynal openings (fig. 636); spermathecae situated medially, semicircular (fig. 637).

Other Material Examined: South Australia: $4 \mathrm{~km} \mathrm{~N}$ Bucks Camp, Mount Rescue Conservation Park, $35^{\circ} 56^{\prime} \mathrm{S}, 140^{\circ} 18^{\prime} \mathrm{E}$, Mar. 18, 1992, vibration (D. Hirst, SAM N1997/ 93), 1 ; $0.1 \mathrm{~km}$ N Grainger Lagoon, Kan- 

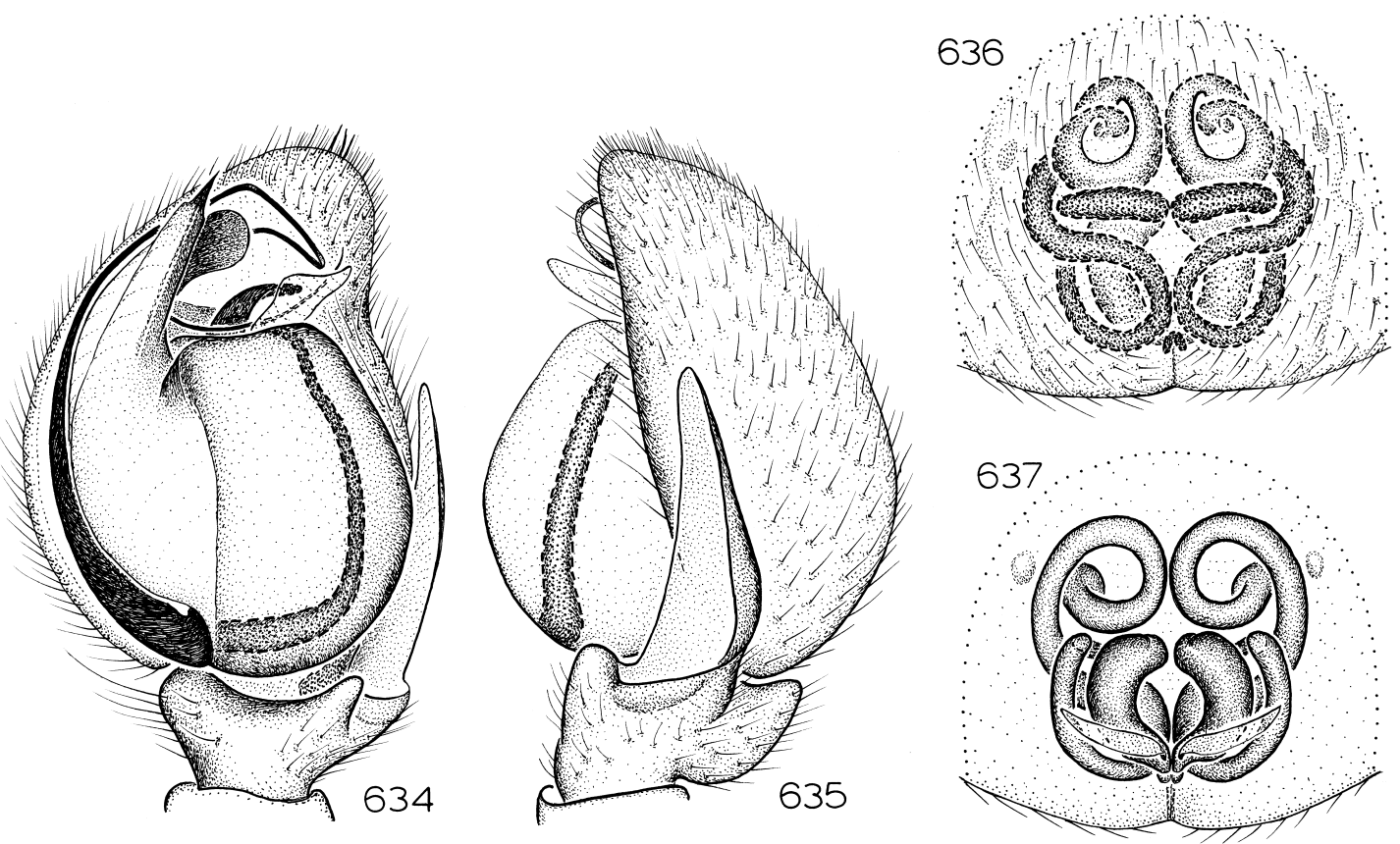

Figs. 634-637. Asadipus longforest, new species. 634. Left male palp, ventral view. 635. Same, retrolateral view. 636. Epigynum, ventral view. 637. Same, dorsal view.

garoo Island, $35^{\circ} 54^{\prime} \mathrm{S}, 136^{\circ} 53^{\prime} \mathrm{E}$, Mar. 9-15, 1995, pitfall (T. Herbert, SAM N1996/427), 10; Pinkawillinie Conservation Park, $32^{\circ} 07^{\prime} \mathrm{S}, 136^{\circ} 00^{\prime} \mathrm{E}$, May 21,1990 , pitfall (D. Hirst, SAM N1997/94), 10 . Tasmania: Queens Domain, 42 $52^{\prime}$ S, $147^{\circ} 19^{\prime}$ E, Aug.Sept. 1942, in cranberry bushes (V. Hickman, AMS KS29241), $10^{\text {to }} 1$ \% ; Trevallyn, $41^{\circ} 27^{\prime} \mathrm{S}, 147^{\circ} 10^{\prime} \mathrm{E}$, Mar. 17, 1928, under stone (V. Hickman, AMS KS29289), 19; Waterhouse Point, $40^{\circ} 51^{\prime} \mathrm{S}, 147^{\circ} 38^{\prime} \mathrm{E}$, Apr. 1987, coastal heath (T. Churchill, QVM 13: 1981), $1 \delta^{\star}$. Victoria: $7 \mathrm{~km}$ ENE Kingower, $36^{\circ} 35^{\prime} \mathrm{S}, 143^{\circ} 50^{\prime} \mathrm{E}$, Mar. 26-29, 1990, mallee (A. Coventry, NMV K4651), 10; Longforest, $36^{\circ} 39^{\prime} \mathrm{S}, 144^{\circ} 30^{\prime} \mathrm{E}$, Mar. 13-19, 1992 , pitfalls, bull mallee, grey box woodland (B. Van Praagh, P. Lillywhite, NMVS), $2 \hat{0}, 1$ ㅇ․

Distribution: South Australia, Victoria, and Tasmania (map 65).

\section{Asadipus bucks, new species} Figures 638-641; Map 64

TYPE: Female holotype taken by vibration at a site between Bucks Camp Well and Rabbit Island, Mount Rescue Conservation Park, $35^{\circ} 56^{\prime} \mathrm{S}, 140^{\circ} 19^{\prime} \mathrm{E}$, South Australia (Mar. 18, 1992; D. Hirst), deposited in SAM (N1997/ 95).

ETYMOLOGY: The specific name is a noun in apposition taken from the type locality.

DiAGNOSIS: Males and females have not been collected together but are paired on the basis of their respective similarities to those of A. humptydoo and A. julia, which share a relatively unexpanded male palpal bulb and short retrolateral tibial apophysis, and on the shared presence of unusually long leg spines; males can be distinguished by the relatively short embolus (fig. 638), females by the relatively short epigynal ducts (fig. 640).

MALE: Total length 4.1. Coloration as in A. phaleratus. Leg spination: tibiae: III p00-0, v1p-1p-2, r0-0-0; IV p0-0-0, v1p-1p-2, r0-0-1; metatarsi: III v1p-0-0; IV v2-1p-0. Retrolateral tibial apophysis reaching to about one-fourth of cymbial length (fig. 639); ventral prong of terminal apophysis relatively short, dorsal prong basally widened, long (fig. 638).

Female: Total length 5.1. Coloration as in A. phaleratus. Leg spination: tibiae: III p0- 

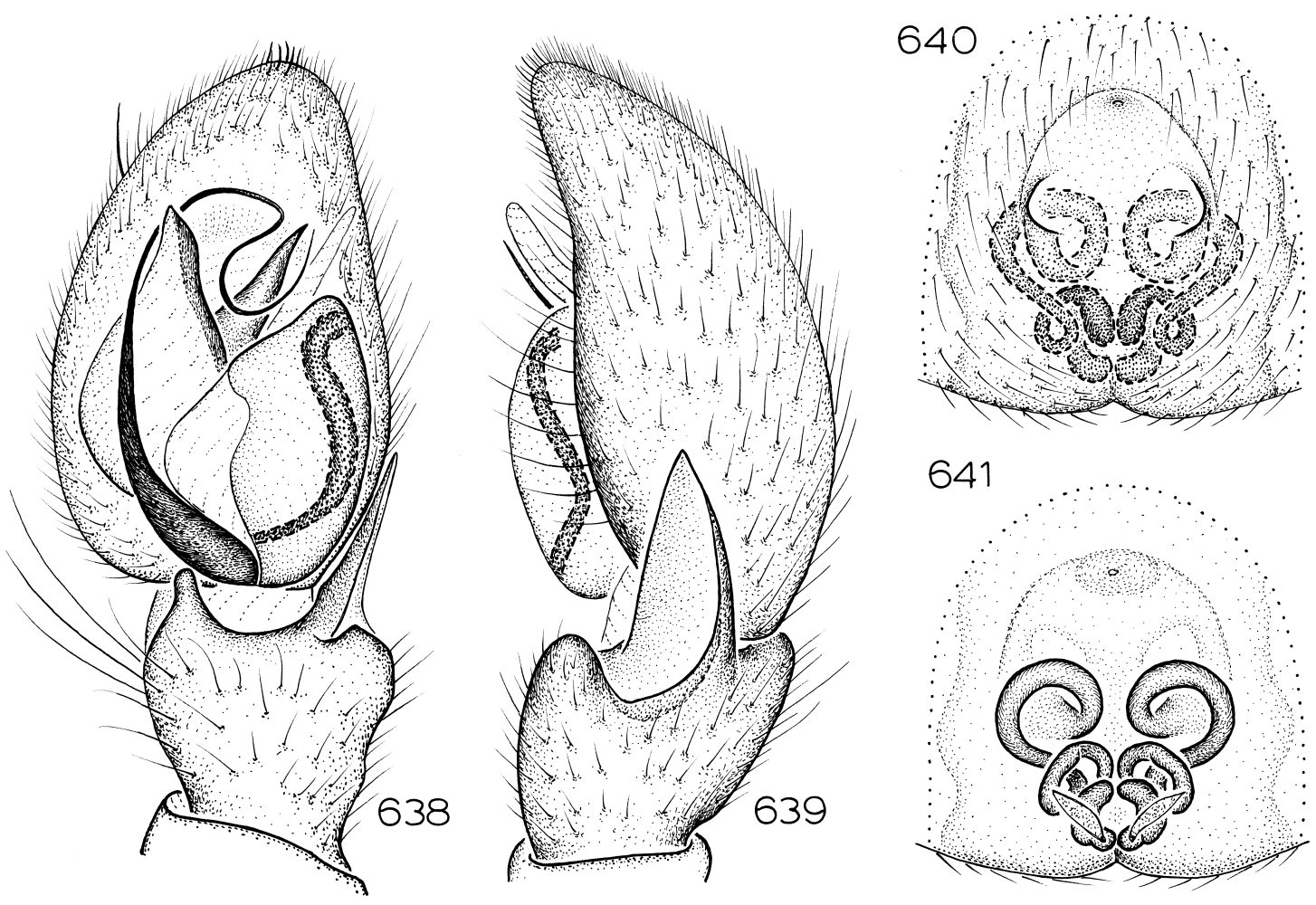

Figs. 638-641. Asadipus bucks, new species. 638. Left male palp, ventral view. 639. Same, retrolateral view. 640. Epigynum, ventral view. 641. Same, dorsal view.

0-0, v1p-1p-2, r0-0-0; IV p0-0-0, v1p-2-2, r0-0-1; metatarsi: III v1p-0-0; IV v2-0-0. Epigynal openings situated at about half length of epigynum (fig. 640); ducts enlarged anteriorly (fig. 641).

Other MAterial Examined: Victoria: $12.3 \mathrm{~km}$ SSW Murrayville Post Office, $35^{\circ} 22^{\prime} \mathrm{S}, 141^{\circ} 09^{\prime} \mathrm{E}$, Feb. 1986 , drift fence pitfall (A. Yen, NMVS), 10 .

Distribution: Eastern South Australia and western Victoria (map 64).

Asadipus julia, new species Figures 642-645; Map 63

TYPE: Female holotype taken in sheep shed at Julia Creek, $20^{\circ} 40^{\prime} \mathrm{S}, 141^{\circ} 45^{\prime} \mathrm{E}$, Queensland (May 1985; P. Chapman), deposited in QMB (S26519).

EтYMOLOGY: The specific name is a noun in apposition taken from the type locality.

Diagnosis: This species resembles $A$. humptydoo and A. bucks in having a rela- tively unexpanded male palpal bulb and short retrolateral tibial apophysis; males can be distinguished by the more rounded ventral prong of the terminal apophysis (fig. 642), females by anteriorly enlarged and folded epigynal ducts (fig. 645).

MALE: Total length 4.7. Coloration as in A. phaleratus. Leg spination: tibiae III, IV p0-0-0, v0-1p-1p, r0-0-0; metatarsi: III v0-00 ; IV v1p-0-0. Retrolateral tibial apophysis reaching to about one-fourth of cymbial length (fig. 643); ventral prong of terminal apophysis relatively short, rounded, dorsal prong long, not basally widened (fig. 642).

FEMALE: Total length 7.3. Coloration as in A. phaleratus. Leg spination: tibiae III, IV p0-0-0, v0-0-2, r0-0-0; metatarsi III, IV v00-0. Epigynum resembling that of A. humptydoo in having triangular anterior hood and anteromedian portion of atrial surface heavily sclerotized, but ducts much shorter (figs. 644, 645). 

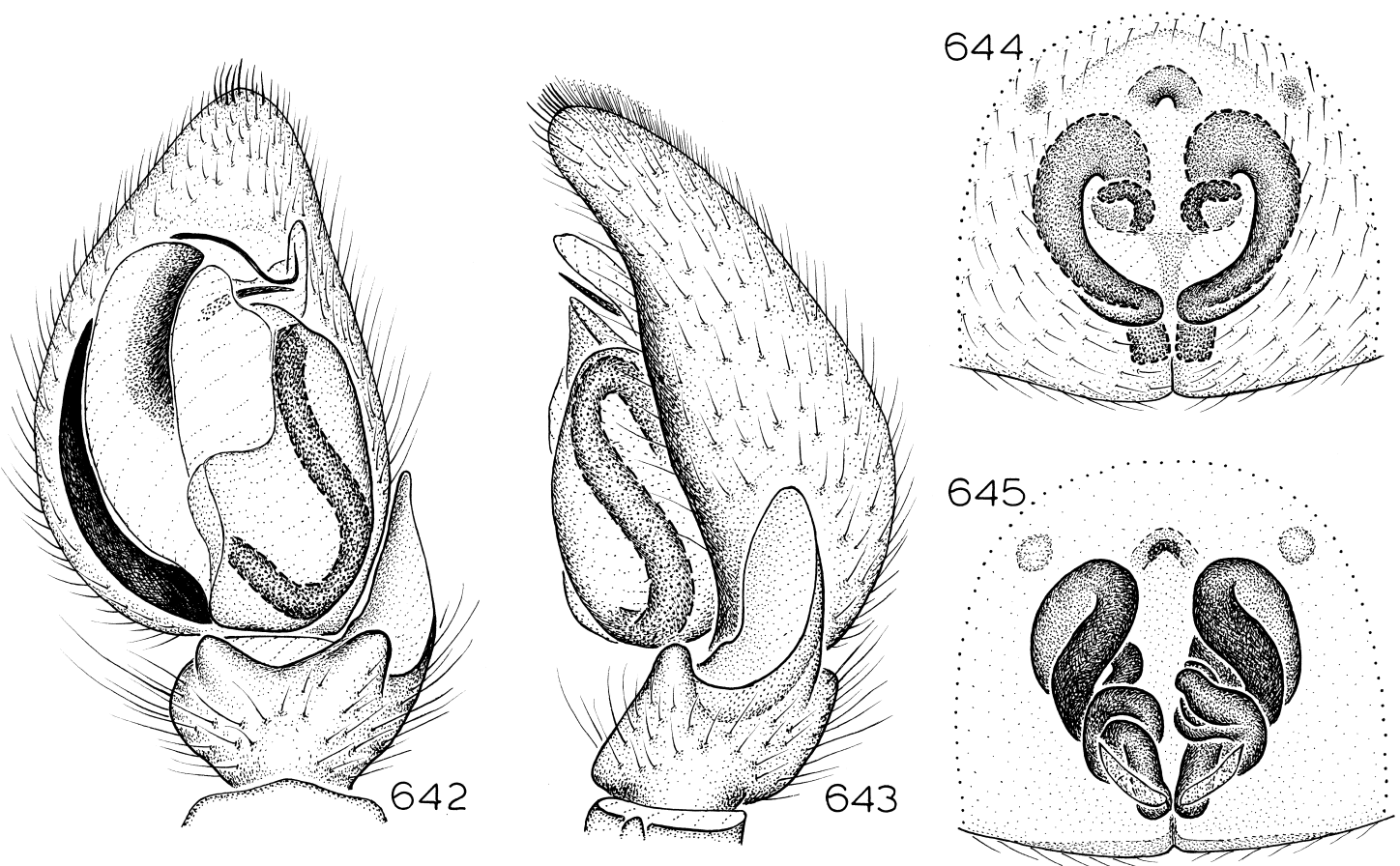

Figs. 642-645. Asadipus julia, new species. 642. Left male palp, ventral view. 643. Same, retrolateral view. 644. Epigynum, ventral view. 645. Same, dorsal view.

Other Material ExAmined: Northern Territory: Helen Springs Station, Barkly Tablelands, $18^{\circ} 26^{\prime} \mathrm{S}, 133^{\circ} 52^{\prime} \mathrm{E}$, Mar. 1996, pitfall (T. Churchill, CSID A0433), 10 .

Distribution: Northern Territory and western Queensland (map 63).

\section{Asadipus uphill, new species}

Figures 646-649; Map 62

TYPE: Male holotype taken in a pitfall trap at Cape Upstart, E of Station Hill, $19^{\circ} 47^{\prime} \mathrm{S}$, $147^{\circ} 50^{\prime} \mathrm{E}$, Queensland (Oct. 25, 1991-July 27, 1992; P. Lawless, R. Raven, M. Shaw), deposited in QMB (S21799), and female allotype taken in open forest at an elevation of $50 \mathrm{~m}$ at Cape Upstart, $2.5 \mathrm{~km}$ SE of Station Hill, 19 $47^{\prime} \mathrm{S}, 147^{\circ} 49^{\prime} \mathrm{E}$, Queensland (Apr. 20, 1998; G. Monteith), deposited in QMB (S42453).

ETYMOLOGY: The specific name is an arbitrary combination of letters.

DIAGNOSIS: Males can easily be recognized by the distinctively short and ventrally directed dorsal prong of the terminal apophysis (fig. 646); females resemble those of A. kunderang but can be distinguished by the presence of a tiny anteromedian epigynal hood (figs. 648, 649).

MALE: Carapace 2.2 long (abdomen missing). Coloration as in A. phaleratus except legs light brown. Leg spination: tibiae: III p0-0-0, v0-0-0, r0-0-0; IV p0-0-0, v0-0-1p, rO-0-0; metatarsi III, IV v0-0-0. Retrolateral tibial apophysis reaching to about half of cymbial length (fig. 647); ventral prong of terminal apophysis relatively short, sharply pointed distally, dorsal prong short, directed ventrally rather than retrolaterally (fig. 646).

Female: Total length 8.9. Abdominal dorsum dark gray with posterior white chevrons and oblique rows of small white spots; legs dark brown. Leg spination: tibiae: III p0-00 , v0-0-2, r0-0-0; IV p0-0-0, v0-1p-2, r0-00 ; metatarsi: III v0-0-0; IV v0-1p-0. Epigynum with tiny anteromedian hood (fig. 648); ducts asymmetrical (fig. 649).

Other Material EXAMined: None

DisTRIBUTION: Known only from mid-eastern Queensland (map 62). 

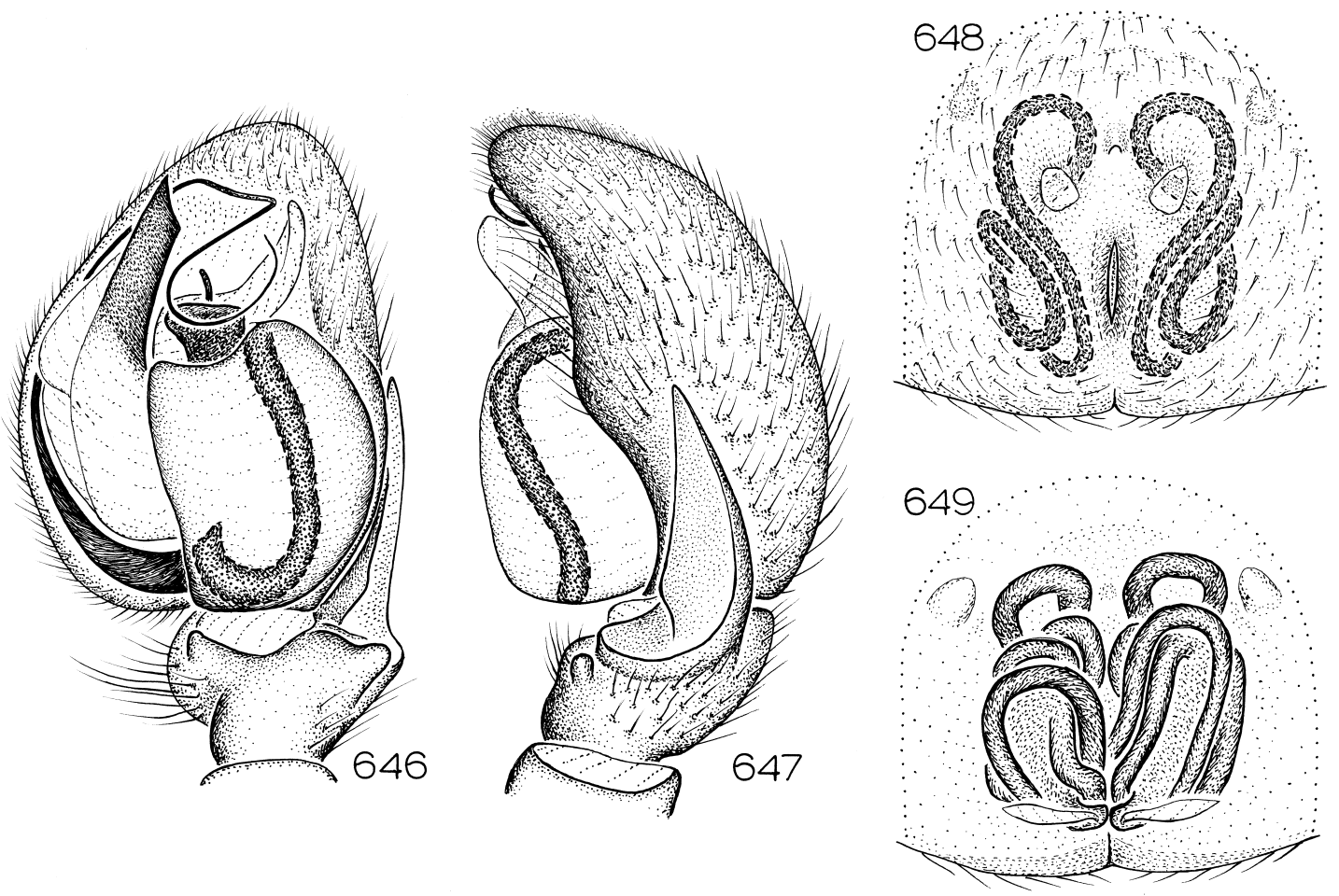

Figs. 646-649. Asadipus uphill, new species. 646. Left male palp, ventral view. 647. Same, retrolateral view. 648. Epigynum, ventral view. 649. Same, dorsal view.

\section{Notsodipus, new genus}

TyPe SPECIES: Notsodipus dalby, new species.

ETYMOLOGY: The generic name is an arbitrary combination of letters, considered masculine in gender.

Diagnosis: Members of this genus resemble species of Asadipus in having a relatively long and sinuous embolus, and equally long and sinuous epigynal ducts, but can be distinguished by the unexcavated retrolateral tibial apophysis of males (figs. 651, 663) and the deep anterior epigynal atrium and highly coiled posterior ducts of females (figs. 652, 653).

DESCRIPTION: Small to medium-sized spiders, total length of males 1.8-5.0, of females 1.9-5.7. Carapace dark orange, coated with large tubercles bearing long, erect setae, margins with long, dark setae; thoracic groove short, almost circular, deep. Eight eyes in two rows, posterior medians largest, other subequal, anterior medians circular, dark, laterals almost circular, light, posterior medians irregularly oval, flattened, light; from above, anterior eye row slightly recurved, posterior row slightly procurved, from front, both rows procurved; anterior medians separated by more than their radius, closer to anterior laterals; posterior medians separated by less than their radius, much farther from posterior laterals; anterior and posterior laterals separated by about their radius; median ocular quadrangle slightly wider in back than in front, about as long as wide in back. Chelicerae, sternum, and mouthparts orangish red; chilum wide, triangular, short, accompanied by second, I-shaped, posterior chilum (extremely narrow sclerite separating bases of chelicerae posteriorly); chelicerae with distinct lateral boss and scattered tubercles bearing short, stiff setae, anterior surface with depressed, relatively unsclerotized oval area near promargin; promargin with row of long setae originating in line along base of fang, seta closest to fang bent near base at 
almost $90^{\circ}$ angle, extending behind other promarginal setae toward midline; promargin with three teeth, median one largest, proximal one smallest; retromargin with two well separated teeth. Labium elongate, base narrowed at about one-fifth of labial length, posterior margin procurved, anterior margin with slight medial invagination, surface not depressed medially. Endites obliquely depressed, with sharply demarcated, deep groove along margin near labium, groove wider anteriorly than posteriorly; serrula long, with single row of teeth; anterior surface with distinct sieve plate of maxillary gland openings set in oval of unsclerotized cuticle. Sternum slightly elevated, with inclined lateral margins, not expanded anteriorly, with slight triangular extensions to and longer extensions between coxae; surface not tuberculate, with only slight elevations opposite, and depressions between, coxae. One epimeric sclerite on each side, above each coxa, extending toward but not fused with sternal triangles, not fused to carapace or to sclerite surrounding pedicel. Pedicel consisting of large, diamond-shaped sclerite with narrow anterior margin meeting posterior tip of sternum at slightly procurved line.

Anterior edge of abdomen of male with complete sclerotic ring formed by strong epigastric scutum separated from strong dorsal abdominal scutum covering almost entire dorsal surface of abdomen, females with dorsal scutum represented only by small, almost circular plate above pedicel, extending slightly on to abdominal dorsum (except in $N$. keilira, where extending over length of abdominal dorsum); cuticle with small tubercles bearing long, erect setae; epigastric scutum accompanied posterolaterally by pair of oval, deeply invaginated sclerites bearing clearly elevated anterior rim; sclerites separated by membranous lobe, anterior rim of sclerites fitting under epigastric scutum; colulus represented only by pair of setae; tiny transverse sclerite, well removed from spinnerets, marking position of small posterior spiracle. Anterior lateral spinnerets tubular, separated by less than their diameter, cuticle representing distal, second spinneret segment restricted to semicircle surrounding major ampullate gland spigots (piriform gland spigots surrounded only by soft cuticle); poste- rior median spinnerets of males large, tubular, of females with anteriorly expanded tips, bases occupied by three enormously widened cylindrical gland spigots; posterior lateral spinnerets two-segmented, those of females with two greatly widened cylindrical gland spigots.

Leg spination greatly reduced; typical leg spination pattern (only surfaces with spines listed): tibiae: III v0-0-1p; IV v0-0-2. Most leg surfaces coated with long setae; males with all coxae and trochanters dorsally tuberculate; anterior coxae with slightly protuberant posterolateral corners; trochanters unnotched; anterior metatarsi and tarsi without scopulae; posterior metatarsi with thick, distal preening brushes; tarsi III, IV without scopulae; tarsi with two dentate claws, claw tufts composed of lateral pads of closely appressed setae; trichobothria present on tibiae, metatarsi, and tarsi. Female palpal tibia and tarsus with long, thin, prolateral spines; female palpal tarsus with long, basally dentate claw.

Male palp with long, unexcavated retrolateral tibial apophysis; cymbial surface flattened opposite tibial apophysis; tegulum occupying retrolateral surface of bulb, produced into lobe medially, bearing large, elaborate terminal apophysis; embolus originating prolaterally, long, looping around prolateral, distal, and proximal sides of bulb; median apophysis absent. Epigynum flattened, with conspicuously excavated anterior atrium; spermathecal ducts long, sinuous.

\section{Key to SPECIES OF NotSODIPUS}

1. Males (those of N. blackall unknown) ... 2

- Females (those of N. broadwater unknown)

2. Ventral portion of terminal apophysis terminating in narrow, sharp point (as in figs. $650,654) \ldots \ldots \ldots \ldots \ldots \ldots \ldots$

- Ventral portion of terminal apophysis not terminating in narrow, sharp point (figs. 666, $670,678,706,710) \ldots \ldots \ldots \ldots \ldots 3$

3. Ventral portion of terminal apophysis relatively broad at tip (figs. 666, 670) . . . 4

- Ventral portion of terminal apophysis relatively narrow at tip (figs. 678, 706, 710)

4. Tip of terminal apophysis with three points (fig. 670) ............. magdala 
- Tip of terminal apophysis with single point (fig. 666) ............... marun

5. Embolus relatively long, looping to proximal half of tegulum (fig. 678) .... renmark

- Embolus relatively short, restricted to distal half of tegulum (figs. 706, 710) . . . 6 6

6. Embolus relatively wide at base (fig. 706) . . ................... keilira

- Embolus relatively narrow at base (fig. 710) ................... domain

7. Tegulum with large proximal apophysis (figs. $702,703) \ldots \ldots \ldots \ldots \ldots \ldots$ visio

- Tegulum without proximal apophysis . . . 8

8. Tip of terminal apophysis bifid (fig. 674) . . ................. broadwater

- Tip of terminal apophysis not bifid .... 9

9. Embolus very long, making extra loop proximally (fig. 662) .......... muckera

- Embolus shorter, not making proximal loop ................ 10

10. Dorsal prong of terminal apophysis forming tube-shaped structure behind ventral prong (figs. 650, 654, 658) .......... 11

- Dorsal prong of terminal apophysis otherwise $\ldots \ldots \ldots \ldots \ldots \ldots \ldots \ldots$

11. Retrolateral tibial apophysis abruptly narrowed at tip (figs. 655, 659) $\ldots \ldots .12$

- Retrolateral tibial apophysis gradually narrowed toward tip (fig. 651) ..... dalby

12. Tegular bulge situated near anterior edge of tegulum (fig. 654) . . . . . . . . innot

- Tegular bulge situated farther posteriorly (fig. 658) ............. upstart

13. Dorsal prong of terminal apophysis with prolaterally directed spur near base (fig. 686) ................... barlee

- Dorsal prong of terminal apophysis without prolaterally directed spur ......... 14

14. Retrolateral tibial apophysis relatively wide at base (figs. 683, 691) ........... 15

- Retrolateral tibial apophysis relatively narrow at base (figs. 695, 699) . . . . . . . 16

15. Tegulum relatively narrow, embolus relatively short (fig. 682) . . . . . . . capensis

- Tegulum relatively wide, embolus relatively long (fig. 690) . . . . . . . . . . meedo

16. Retrolateral tibial apophysis relatively short (fig. 695) .............. quobba

- Retrolateral tibial apophysis relatively long (fig. 699) . . . . . . . . . bidgemia

17. Epigynum with pair of lateral pockets and anteromedian septum (fig. 712) ... domain

- Epigynum without lateral pockets .... 18

18. Epigynum relatively long, narrow (figs. 592, $704,708) \ldots \ldots \ldots \ldots \ldots \ldots$

- Epigynum not narrow and elongated (as in figs. 652,692$) \ldots \ldots \ldots \ldots \ldots \ldots 21$
19. Epigynal atrium situated medially (fig. 708) .................. keilira

- Epigynal atrium situated anteriorly (figs. 591, 704) ................. 20

20. Epigynal ducts extending to anterior half of epigynum (figs. 592, 593) . . . . blackall

- Epigynal ducts restricted to posterior half of epigynum (figs. 704, 705) ...... visio

21. Epigynal atrium relatively small (figs. 664,

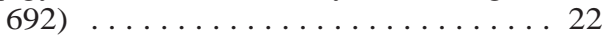

- Epigynal atrium relatively large (as in figs. $652,660) \ldots \ldots \ldots \ldots \ldots \ldots 23$

22. Epigynal atrium very short (fig. 692) ..... ................... meedo

- Epigynal atrium longer (fig. 664) .. . muckera

23. Epigynal atrium relatively short (figs. 652, $660) \ldots \ldots \ldots \ldots \ldots \ldots \ldots 24$

- Epigynal atrium longer (as in figs. 680, 700) .................. 25

24. Epigynal atrium wider than long (fig. 660) .............. upstart

- Epigynal atrium longer than wide (fig. 652) ................. dalby

25. Epigynal atrium widest anteriorly or medially ................... 27

- Epigynal atrium widest posteriorly (figs. 668, $688) \ldots \ldots \ldots \ldots \ldots \ldots \ldots 26$

26. Lateral epigynal ducts relatively long (figs. $668,669) \ldots \ldots \ldots \ldots \ldots$ marun

- Lateral epigynal ducts relatively short (figs. $688,689) \ldots \ldots \ldots \ldots \ldots$ barlee

27. Epigynal atrium widest anteriorly (figs. 680, $696,700) \ldots \ldots \ldots \ldots \ldots \ldots 28$

- Epigynal atrium widest at middle (figs. 656, $672,684) \ldots \ldots \ldots \ldots \ldots \ldots 30$

28. Epigynal atrium relatively long (fig. 696) .. ................ quobba

- Epigynal atrium relatively short (figs. 680, 700) . . . . . . . . . . . . . 29

29. Epigynal atrium relatively small (fig. 680) .. ............... renmark

- Epigynal atrium relatively large (fig. 700) .. .................... bidgemia

30. Epigynal atrium relatively short (fig. 656) ..

................. innot

- Epigynal atrium relatively long (figs. 672, 684) ................. 31

31. Lateral epigynal ducts relatively long (figs. $672,673) \ldots \ldots \ldots \ldots \ldots$ magdala

- Lateral epigynal ducts relatively short (figs. $684,685 \ldots \ldots \ldots \ldots \ldots \ldots$ capensis

\section{Notsodipus dalby, new species Figures 650-653; Map 73}

TYPES: Female holotype and male allotype taken in pitfall traps at Lake Broadwater, via Dalby, Queensland, $27^{\circ} 21^{\prime} \mathrm{S}, 151^{\circ} 06^{\prime} \mathrm{E}$, (May 

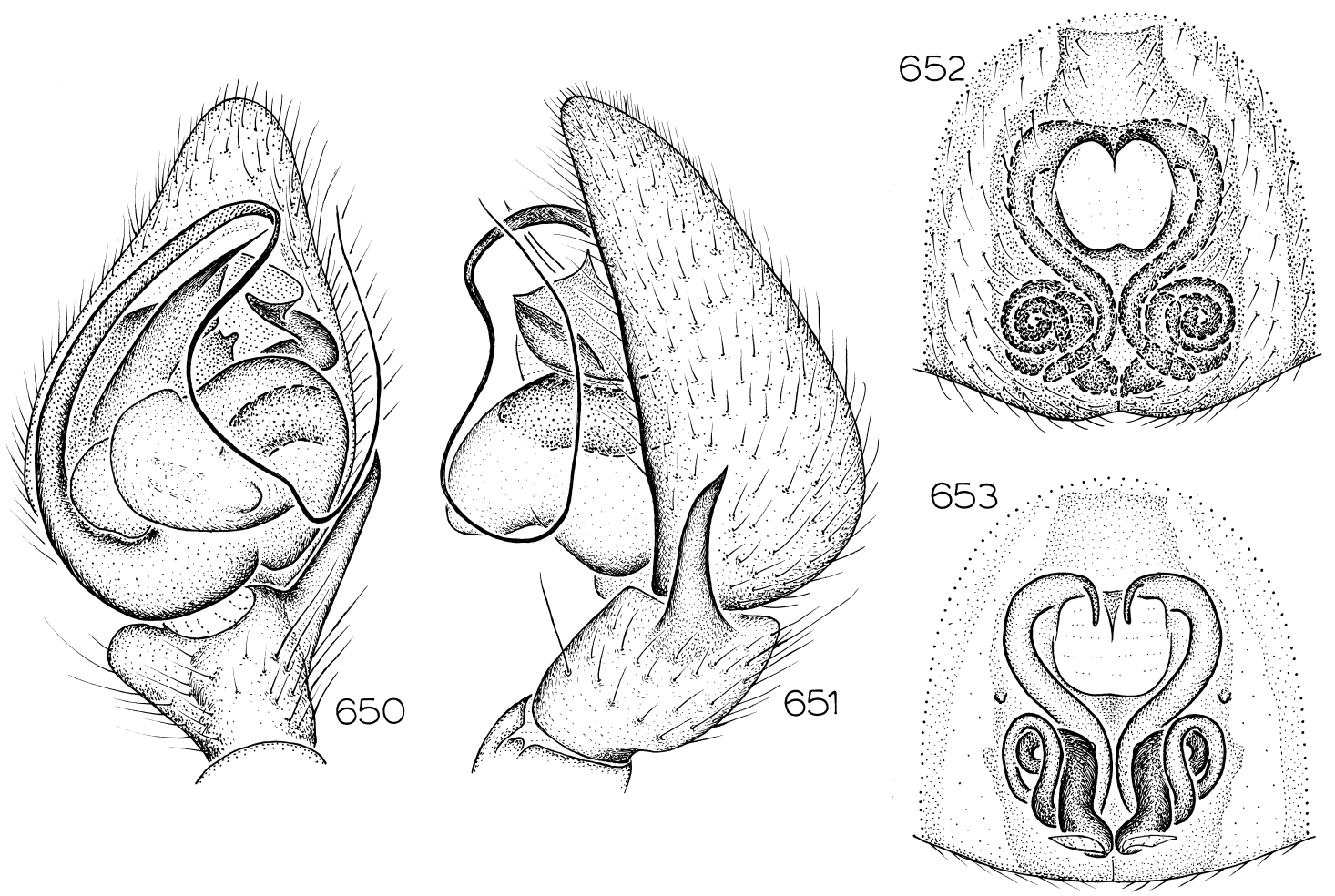

Figs. 650-653. Notsodipus dalby, new species. 650. Left male palp, ventral view. 651. Same, retrolateral view. 652. Epigynum, ventral view. 653. Same, dorsal view.

16-Nov. 23, 1985), deposited in QMB (S28523).

ETYMOLOGY: The specific name is a noun in apposition taken from the type locality.

DiAGNOSIS: This species closely resembles $N$. innot (see below), but can be distinguished by the shorter embolus and smaller terminal apophysis (fig. 650) of males and by having the posteromedian epigynal ducts largely overlain by the posterolateral ducts (fig. 653) in females.

MALE: Total length 3.5. Abdominal dorsum with two pairs of lighter muscle impressions visible under dark scutum; venter gray, with anterior pair of circular orange sclerites and large, rectangular, median, orange sclerite. Leg spination: tibia III v0-0-0. Retrolateral tibial apophysis long, smoothly tapered (fig. 651); terminal apophysis with distally narrow median prong, distal elements relatively short, narrow, embolus relatively short, not reaching to ventral edge of bulb (fig. 650).
Female: Total length 2.6. Abdominal dorsum gray, with two pairs of lighter muscles impressions and several thin, narrow, posterior pale chevrons; venter without sclerites, with two oblique rows of pale spots. Leg spination typical for genus. Epigynal atrium almost rectangular (fig. 652); spermathecae ductlike, widely separated anteriorly, with lateral ducts fully overlying their lateral ends (fig. 653).

Other Material Examined: New South Wales: Yanga Creek, $7 \mathrm{~km}$ SSE Balranald, $34^{\circ} 42^{\prime} \mathrm{S}, 143^{\circ} 35^{\prime} \mathrm{E}$, Oct. 24, 1983, under bark of Eucalyptus camaldulensis (D. Rentz, M. Harvey, QMB), $1 \delta^{\star}$. Queensland: Lake Broadwater, via Dalby, $27^{\circ} 21^{\prime} \mathrm{S}, 151^{\circ} 06^{\prime} \mathrm{E}$, May 16-Nov. 23, 1985 (QMB S28523), 17ô, Nov. 24, 1985-Jan. 3, 1986, pitfalls (M. Bennie, QMB S28633), 20, 2 \% . South Australia: $7 \mathrm{~km}$ SE Lake Werta Wert, Chowilla, $33^{\circ} 56^{\prime} \mathrm{S}, 140^{\circ} 52^{\prime} \mathrm{E}$, Oct. 10,1988 (S. Lewer, SAM N1988/404), 10; $52 \mathrm{~km}$ ENE Wolseley, $36^{\circ} 20^{\prime} \mathrm{S}, 140^{\circ} 57^{\prime} \mathrm{E}$, Dec. $13-$ 
15, 1995 (SAM 1997/96, 97), 10, 1 ㅇ. Victoria: Booths Road, $0.2 \mathrm{~km} \mathrm{~S}$ Murray Valley Highway, $36^{\circ} 08^{\prime} \mathrm{S}, 145^{\circ} 11^{\prime} \mathrm{E}$, Dec. 2-7, 1994, pitfall (J. Evans, M. Griffiths, S. Hinkley, NMVS), $10^{\text {; }}$; Brooms Road, $7.5 \mathrm{~km} \mathrm{NE}$ Yambuna, $36^{\circ} 06^{\prime} \mathrm{S}, 145^{\circ} 02^{\prime} \mathrm{E}$, Dec. 2-7, 1994, pitfall (J. Evans, M. Griffiths, S. Hinkley, NMVS), 10; Brooms Road, $9 \mathrm{~km} \mathrm{SW}$ Nathalia, $36^{\circ} 06^{\prime} \mathrm{S}, 145^{\circ} 07^{\prime} \mathrm{E}$, Dec. 2-7, 1994, pitfall (J. Evans, M. Griffiths, S. Hinkley, NMVS), $1 \%$; Cohuna, $35^{\circ} 50^{\prime} \mathrm{S}, 144^{\circ} 11^{\prime} \mathrm{E}$, Nov. 16-21, 1996, pitfall, shelterbelt (J. Shield, J. Hooper, CVIC), 1 ô, Dec. 1996Jan. 1997, pitfalls (J. Hooper, CVIC), $20^{\star}$; Deep Creek, $7 \mathrm{~km}$ SSE Barmah, $36^{\circ} 05^{\prime} \mathrm{S}$, $144^{\circ} 59^{\prime}$ E, Dec. 2-7, 1994, pitfall (J. Evans, M. Griffiths, S. Hinkley, NMVS), $1 \delta^{\text {t }}$; Goulburn River, $12 \mathrm{~km}$ SSE Nathalia, $36^{\circ} 10^{\prime} \mathrm{S}$, $145^{\circ} 14^{\prime} \mathrm{E}$, Dec. $2-7,1994$, pitfall (J. Evans, M. Griffiths, S. Hinkley, NMVS), $1 \delta^{\dagger} ; 5 \mathrm{~km}$ ESE Kaarimba, $36^{\circ} 10^{\prime} \mathrm{S}, 145^{\circ} 14^{\prime} \mathrm{E}$, Dec. 2 7, 1994, pitfall (J. Evans, M. Griffiths, S. Hinkley, NMVS), 90; Kotupna-Barmah Road at Ellingtons Bridge, $36^{\circ} 05^{\prime} \mathrm{S}$, $145^{\circ} 03^{\prime} \mathrm{E}$, Dec. 2-7, 1994, pitfall (J. Evans, M. Griffiths, S. Hinkley, NMVS), 20 , 1 ; ; 5 $\mathrm{km}$ ESE Lower Moira, 36 $05^{\prime} \mathrm{S}, 145^{\circ} 03^{\prime} \mathrm{E}$, Jan. 17-22, 1994, pitfall, hand collecting (G. Milledge, P. Lillywhite, NMVS), 29 , Dec. 2-7, 1994, pitfall (J. Evans, M. Griffiths, S. Hinkley, NMVS), 40, 1 ; ; McDonalds Road, 1.8 km S Shepparton-Barmah Road, $36^{\circ} 04^{\prime} \mathrm{S}, 145^{\circ} 02^{\prime} \mathrm{E}$, Dec. 2-7, 1994, pitfall (J. Evans, M. Griffiths, S. Hinkley, NMVS), 5 ô, $4 \%$; Murray Valley Highway, Deep Creek Crossing, $36^{\circ} 08^{\prime} \mathrm{S}, 145^{\circ} 11^{\prime} \mathrm{E}$, Dec. 2-7, 1994, pitfall (J. Evans, M. Griffiths, S. Hinkley, NMVS), 20, 2 ; ; Murray Valley Highway, Skeleton Creek Crossing, $36^{\circ} 07^{\prime} \mathrm{S}$, $145^{\circ} 11^{\prime} \mathrm{E}$, Jan. 26-30, 1995, pitfall (J. Evans, S. Hinkley, J. Wainer, NMVS), $1 \%$; Murray Valley Highway, Skeleton Creek Crossing, $36^{\circ} 00^{\prime} \mathrm{S}, 145^{\circ} 14^{\prime} \mathrm{E}$, Dec. $2-7$, 1994, pitfall (J. Evans, M. Griffiths, S. Hinkley, NMVS), $1{ }^{\star}$; Murray Valley Highway, $0.3 \mathrm{~km}$ NNW Walshs Bridge Road, $36^{\circ} 06^{\prime} \mathrm{S}, 145^{\circ} 12^{\prime} \mathrm{E}$, Jan. 17-22, 1994, pitfall (G. Milledge, NMVS), 1 \%, Dec. 2-7, 1994, pitfall (J. Evans, M. Griffiths, S. Hinkley, NMVS), $10^{\star}$; Pomfrets Road, $0.6 \mathrm{~km}$ S Picola-Katunga Road, $36^{\circ} 07^{\prime} \mathrm{S}, 145^{\circ} 11^{\prime} \mathrm{E}$, Dec. $2-7$, 1994, pitfall (J. Evans, M. Griffiths, S. Hinkley, NMVS), 40; Rathbones Road, 3 km E Booths Road, $36^{\circ} 09^{\prime} \mathrm{S}, 145^{\circ} 13^{\prime} \mathrm{E}$, Dec. $2-7$, 1994, pitfall (J. Evans, M. Griffiths, S. Hinkley, NMVS), 3 के, 1 웅 Schwenkes Road, Cohuna, $35^{\circ} 46^{\prime} \mathrm{S}$, $144^{\circ} 05^{\prime} \mathrm{E}$, Nov. 16-21, 1996, pitfall (J. Shield, J. Hooper, CVIC), $10^{\text {t }}$; State Forest. $3.5 \mathrm{~km}$ NE Yambuna, $36^{\circ} 06^{\prime} \mathrm{S}, 145^{\circ} 02^{\prime} \mathrm{E}$, Dec. 2-7, 1994, pitfall (J. Evans, M. Griffiths, S. Hinkley, NMVS), $20^{\top}$; State Forest. $11 \mathrm{~km}$ SE Yambuna, $36^{\circ} 09^{\prime} \mathrm{S}, 145^{\circ} 01^{\prime} \mathrm{E}$, Dec. 2-7, 1994, pitfall (J. Evans, M. Griffiths, S. Hinkley, NMVS), 1 t.

DisTRIBUTION: Southern Queensland south to Victoria and eastern South Australia (map 73).

\section{Notsodipus innot, new species} Figures 654-657; Map 73

TyPES: Female holotype and male allotype taken in pitfall traps in dry woodland near Running Creek, Innot Springs, Queensland, $17^{\circ} 19^{\prime} \mathrm{S}, 145^{\circ} 18^{\prime} \mathrm{E}$ (Nov. 5, 1991-July 25, 1992; P. Lawless, R. Raven, M. Shaw), deposited in QMB (S24113).

ETYMOLOGY: The specific name is a noun in apposition taken from the type locality.

DiAgNosis: This species closely resembles $N$. dalby but can be distinguished by the longer embolus and larger terminal apophysis (fig. 654) of males and by having the posteromedian epigynal ducts only slightly over-

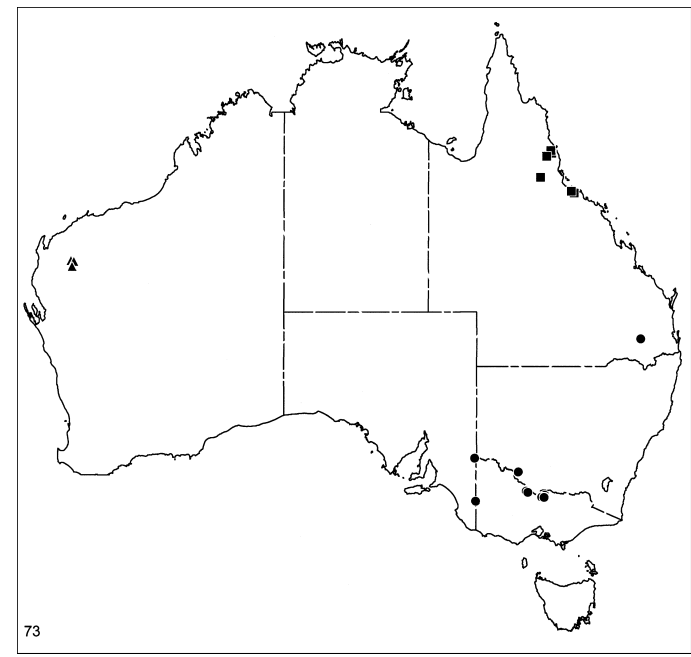

Map 73. Records of Notsodipus dalby, new species (circles), N. innot, new species (squares), and $N$. barlee, new species (triangles). 

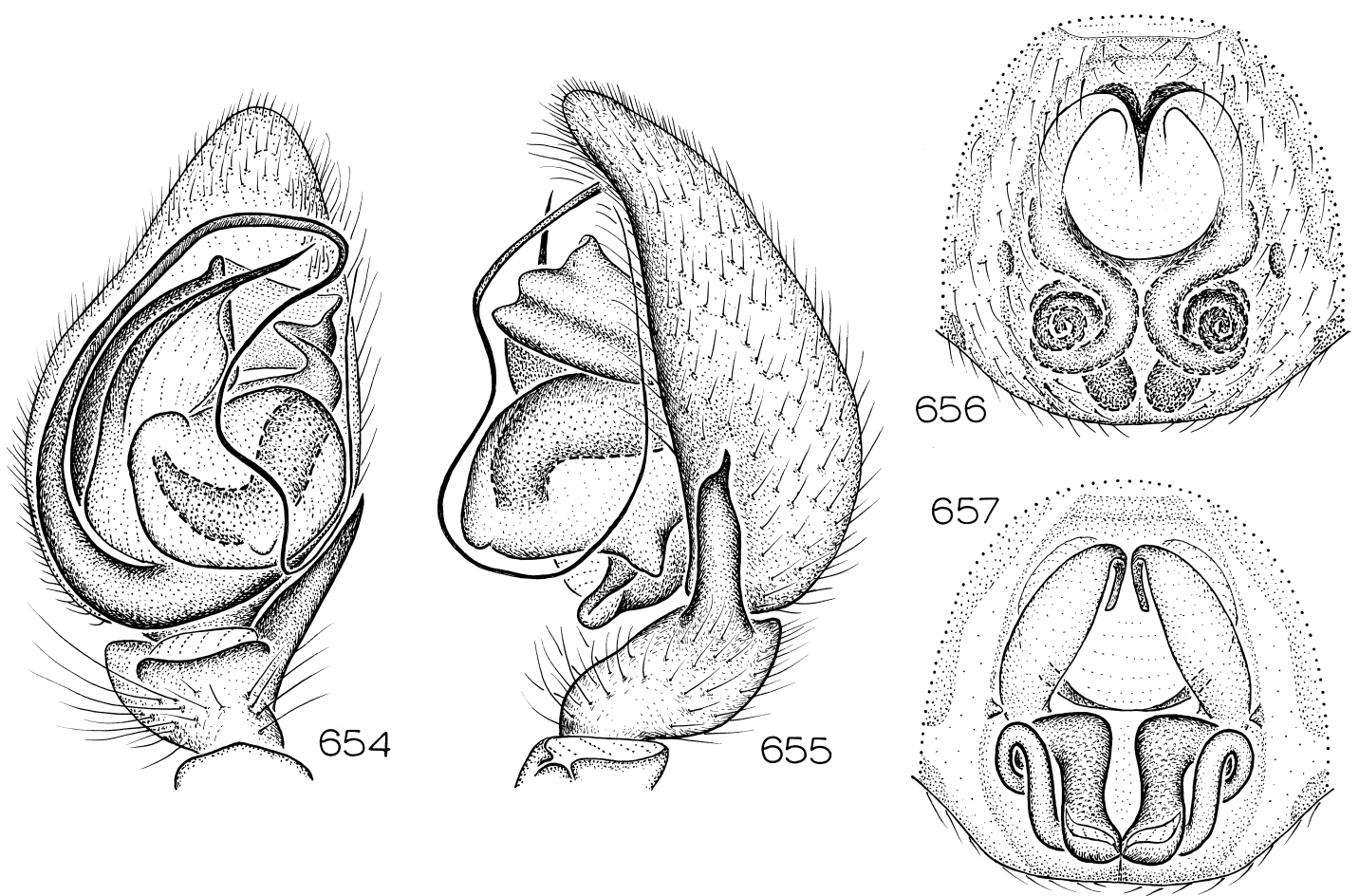

Figs. 654-657. Notsodipus innot, new species. 654. Left male palp, ventral view. 655. Same, retrolateral view. 656. Epigynum, ventral view. 657. Same, dorsal view.

lain by the posterolateral ducts (fig. 657) in females.

MALE: Total length 3.7. Abdominal dorsum with posterior chevrons visible under scutum; venter gray, with four longitudinal rows of small, circular, orange sclerites. Leg spination typical for genus. Retrolateral tibial apophysis long, subdistally widened, distally sinuous (fig. 655); terminal apophysis with distally narrow median prong, distal elements relatively long, wide, heavily sclerotized retrolaterally, embolus relatively long, extending past ventral edge of bulb (fig. 654).

FEMALE: Total length 3.5. Abdominal dorsum gray, with posterior chevrons; venter without sclerites or pale spots. Leg spination typical for genus. Epigynal atrium relatively large, almost circular (fig. 656); spermathecae ductlike, not widely separated anteriorly, with lateral ducts overlying only the tips of their lateral ends (fig. 657).

Other MAterial ExAmined: Queensland: Cape Cleveland Road, $19^{\circ} 21^{\prime} \mathrm{S}, 147^{\circ} 01^{\prime} \mathrm{E}$,
July 27-Dec. 2, 1992, pitfall (R. Raven, P., E. Lawless, M. Shaw, QMB S19877), 1\%; Danbulla State Forest, $17^{\circ} 09^{\prime} \mathrm{S}, 145^{\circ} 37^{\prime} \mathrm{E}$, Aug. 6, 1992 (J. Murphy, JAM 21339), 1 \%; Davies Creek National Park, $17^{\circ} 00^{\prime} \mathrm{S}$, $145^{\circ} 34^{\prime}$ E, Oct. 29, 1991-July 23, 1992, pitfall (P. Lawless, R. Raven, M. Shaw, QMB S25154), 1 ㅇ, July 23-Nov. 26, 1992, pitfall (R. Raven, P., E. Lawless, M. Shaw, QMB S19856), 1\%; near Running Creek, Innot Springs, $17^{\circ} 19^{\prime} \mathrm{S}, 145^{\circ} 18^{\prime} \mathrm{E}$, Nov. 5, 1991July 25, 1992, pitfall, dry woodland (P. Lawless, R. Raven, M. Shaw, QMB S24113, 24137), $20^{\text {\%, }} 2$ 우, July 25-Nov. 30, 1992, pitfall, dry woodland (R. Raven, P., E. Lawless, M. Shaw, QMB S24309, 24312), 2q; 2 mi W Saunders Beach, Townsville, $19^{\circ} 15^{\prime} \mathrm{S}$, $146^{\circ} 50^{\prime} \mathrm{E}$, July 21 , 1968, Berlese (L. Mound, QMB), 10, 1 ; ; Wyandotte Creek, $18^{\circ} 29^{\prime} \mathrm{S}$, $144^{\circ} 55^{\prime} \mathrm{E}$, Nov. 6, 1991-July 26, 1992, pitfall, dry eucalypt woodland (P. Lawless, R. Raven, M. Shaw, QMB S21784), 10.

DISTRIBUTION: Known only from northand mid-eastern Queensland (map 73). 

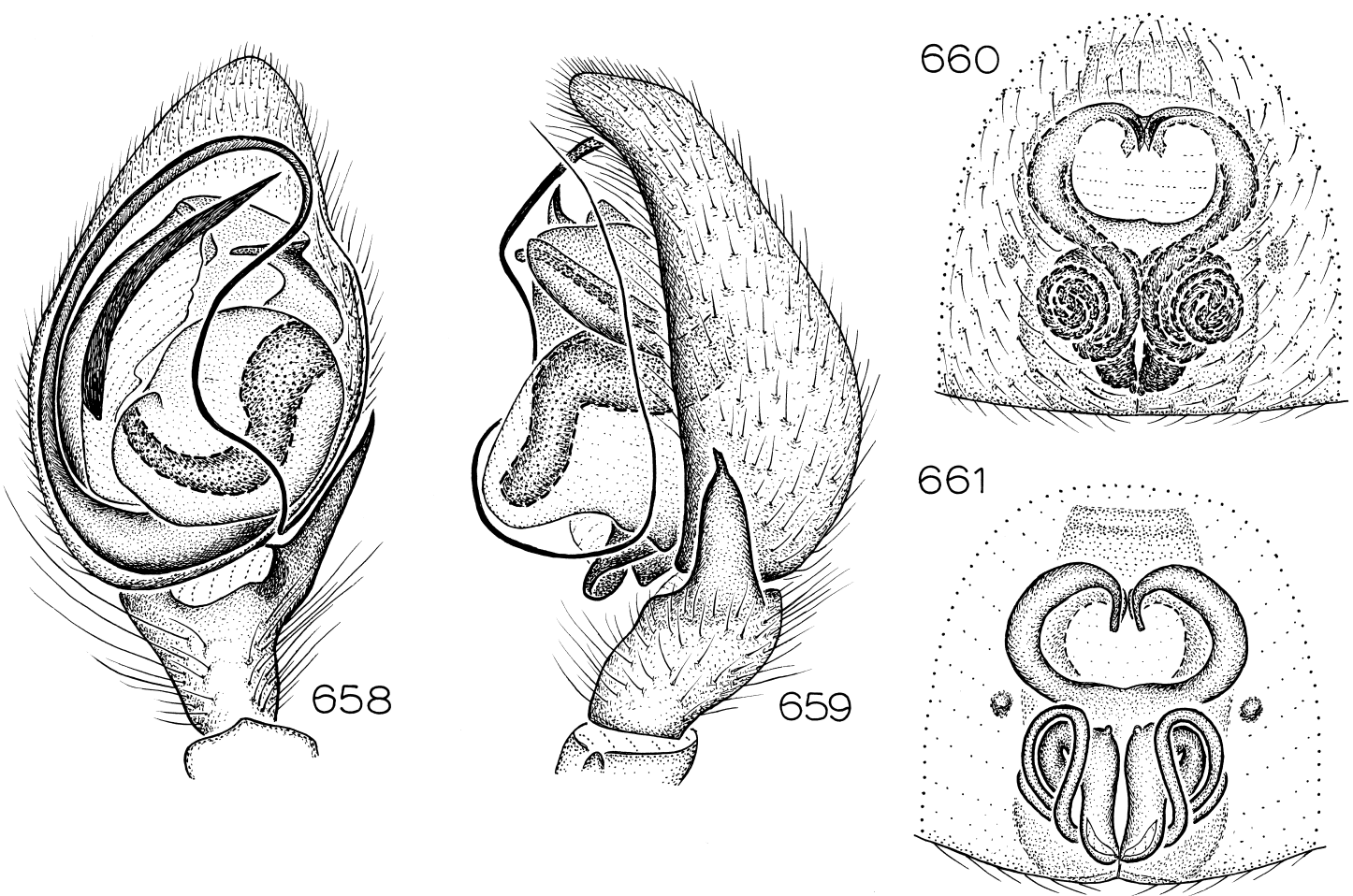

Figs. 658-661. Notsodipus upstart, new species. 658. Left male palp, ventral view. 659. Same, retrolateral view. 660. Epigynum, ventral view. 661. Same, dorsal view.

\section{Notsodipus upstart, new species}

Figures 658-661; Map 74

TYPES: Female holotype and male allotype taken in pitfall traps at Cape Upstart, E of Station Hill, $19^{\circ} 47^{\prime} \mathrm{S}, 147^{\circ} 50^{\prime} \mathrm{E}$, Queensland (July 27-Nov. 25, 1992; R. Raven, P., E. Lawless, M. Shaw), deposited in QMB (S21807, S21818).

EтYMOLOGY: The specific name is a noun in apposition taken from the type locality.

DiAgNosis: This species is closely related to $N$. dalby and $N$. innot but can be distinguished by the beak-shaped tip of the retrolateral tibial apophysis (fig. 659) of males and the much shorter epigynal atrium (fig. 660) of females.

MALE: Total length 3.3. Abdominal dorsum with posterior chevrons visible under scutum; venter gray, with four longitudinal rows of small, circular, orange sclerites. Leg spination presumed typical for genus (leg III missing). Retrolateral tibial apophysis abruptly narrowed, beak-shaped distally (fig.

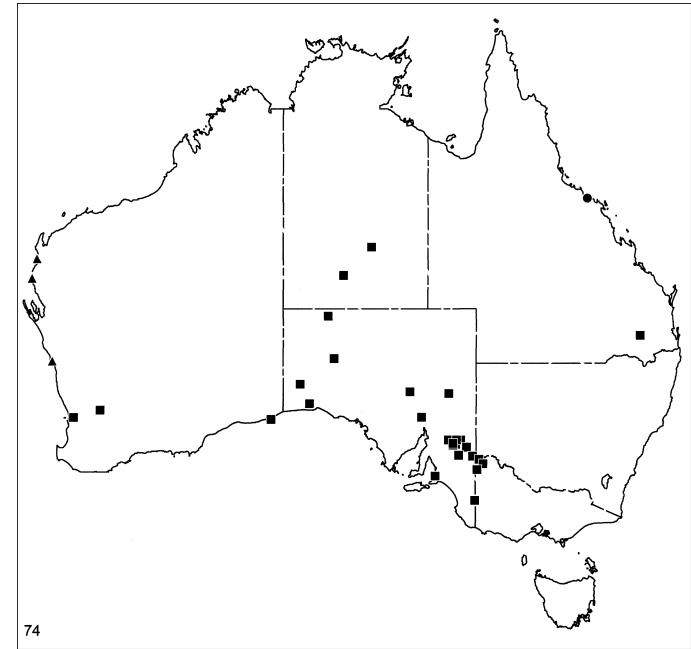

Map 74. Records of Notsodipus upstart, new species (circle), N. muckera, new species (squares), and N. quobba, new species (triangles). 

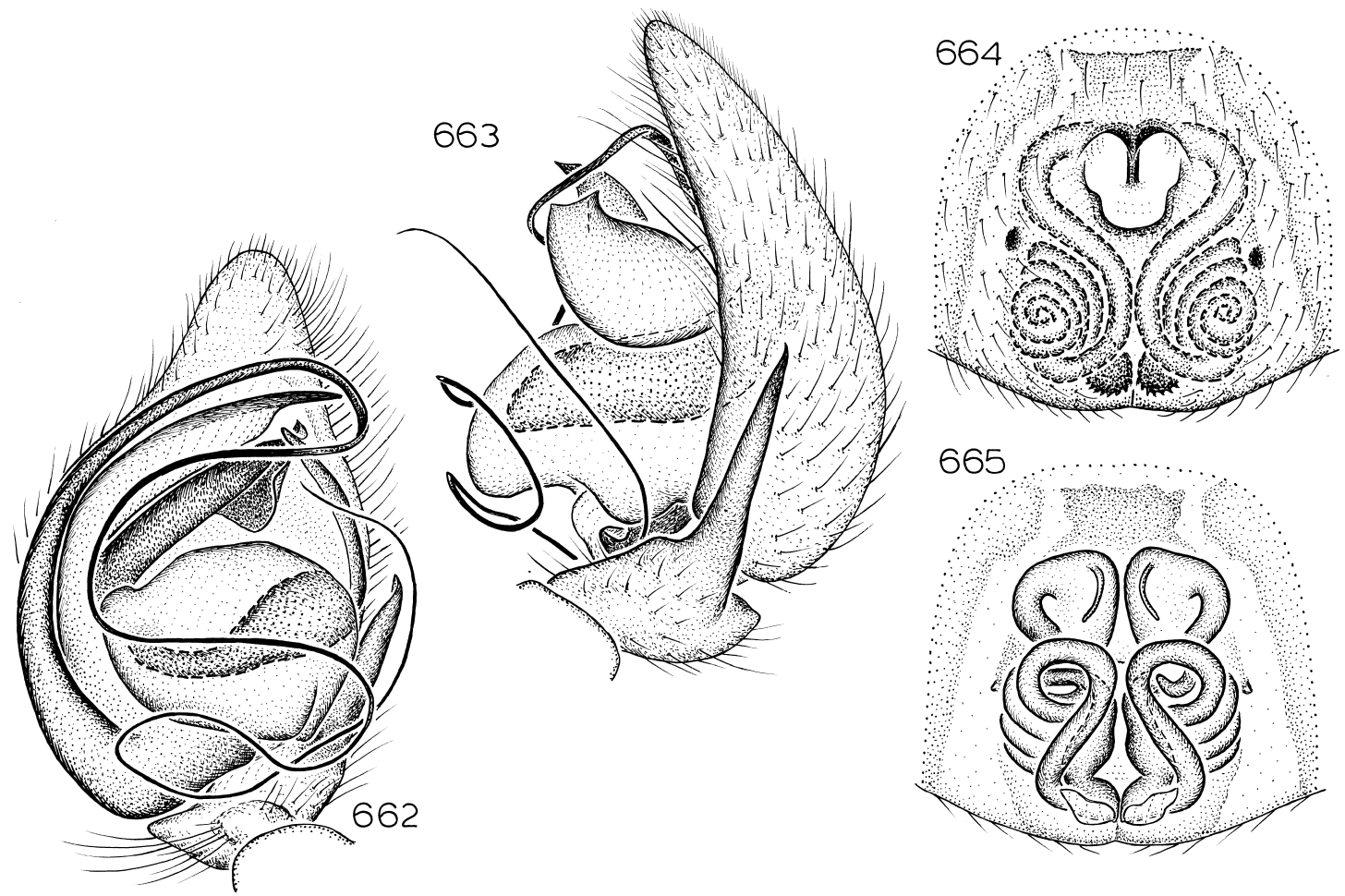

Figs. 662-665. Notsodipus muckera, new species. 662. Left male palp, ventral view. 663. Same, retrolateral view. 664. Epigynum, ventral view. 665. Same, dorsal view.

659); terminal apophysis with distally narrow median prong, distal elements relatively short, wide, heavily sclerotized retrolaterally, embolus relatively long, extending to ventral edge of bulb (fig. 658).

FEMALE: Total length 3.3. Abdominal dorsum gray, with posterior chevrons; venter with two longitudinal rows of small, circular, orange sclerites. Leg spination typical for genus. Epigynal atrium short, wide (fig. 660); spermathecae ductlike, almost touching anteriorly, with lateral ducts overlying most of their lateral ends (fig. 661).

Other Material Examined: One female taken with the types (QMB S24861).

DisTRIBUTION: Known only from mid-eastern Queensland (map 74).

\section{Notsodipus muckera, new species} Figures 662-665; Map 74

TYPES: Female holotype and male allotype taken in pitfall traps at Muckera, $30^{\circ} 02^{\prime} \mathrm{S}$, $130^{\circ} 03^{\prime} \mathrm{E}$, South Australia (Sept. 1984; B.
Guerin), deposited in SAM (N1996/349, 350).

ETYMology: The specific name is a noun in apposition taken from the type locality.

DiAGNOSIS: Males can be recognized by the long, arched median prong of the terminal apophysis, which is abruptly narrowed near its tip (fig. 662), females by the relatively long median and highly coiled lateral epigynal ducts (fig. 665).

MALE: Total length 3.8. Abdominal dorsum apparently uniformly gray under scutum, venter with four longitudinal rows of small, circular, orange sclerites largely overlain by large, rectangular orange sclerite. Leg spination typical for genus. Retrolateral tibial apophysis very long, narrow (fig. 663); median prong of terminal apophysis long, accompanied for most of its length by membranous lobe, distal elements very large, smoothly arched (fig. 662).

Female: Total length 4.6. Abdominal dorsum mottled gray, venter with two parame- 
dian longitudinal rows of small, brownish sclerites. Leg spination typical for genus. Epigynal atrium relatively shallow, divided for most of its length (fig. 664); median epigynal ducts very long, accompanied by highly coiled lateral ducts (fig. 665).

Other MATERIAL EXAMINED: Northern Territory: Finke Gorge National Park, E bank Finke River, 11.3 km S Hermannsburg, $24^{\circ} 03^{\prime} \mathrm{S}, 132^{\circ} 45^{\prime} \mathrm{E}$, Oct. 1994, pitfall (NMV K4654), 1 \% ; Waite Creek, Alcoota, $22^{\circ} 29^{\prime} \mathrm{S}$, $134^{\circ} 30^{\prime} \mathrm{E}$, Oct. 21, 1982 (I. Archibald, MNT), 10 . Queensland: Lake Broadwater, via Dalby, $27^{\circ} 21^{\prime} \mathrm{S}, 151^{\circ} 06^{\prime} \mathrm{E}$, May $17-$ Nov. 24, 1985 (M. Bennie, QMB S34338. 34339), 10, 19 . South Australia: near Balcoona High School, $30^{\circ} 33^{\prime} \mathrm{S}, 139^{\circ} 18^{\prime} \mathrm{E}$, Sept. 20, 1993, pitfall (SAM N1996/353), 10; $5.2 \mathrm{~km}$ NNE Bullock Dam, $33^{\circ} 23^{\prime} \mathrm{S}, 139^{\circ} 43^{\prime} \mathrm{E}$, Oct. 22, 1922, pitfall (SAM N1996/364), 10; 7.6

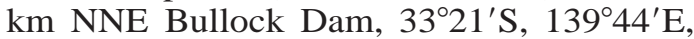
Oct. 23, 1922, pitfall (SAM N1996/365), 1 \%; $2 \mathrm{~km}$ ENE The Coffins Dam, 33⒉' $139^{\circ} 34^{\prime} \mathrm{E}$, Oct. 21,1992 , pitfall (SAM N1996/366, 367), 10, 1 \% ; 1.2 km S Custon, $36^{\circ} 27^{\prime} \mathrm{S}, 140^{\circ} 55^{\prime} \mathrm{E}$, Dec, 4-8, 1995, pitfall (SAM 1997/99), 10; $1.1 \mathrm{~km}$ SE Elmore Dam, $33^{\circ} 07^{\prime} \mathrm{S}, 140^{\circ} 03^{\prime} \mathrm{E}$, Oct. 16 , 1992, pitfall (SAM N1996/362), 10; $4.5 \mathrm{~km} \mathrm{NW}$ Emu, $28^{\circ} 38^{\prime} \mathrm{S}, 132^{\circ} 10^{\prime} \mathrm{E}$, Oct. 11,1976 , among Eragrostis eriopoda roots (D. Lee, SAM N1996/348), 1 ; 2.75 km NE Faraway Hill Station, $33^{\circ} 07^{\prime} \mathrm{S}$, $139^{\circ} 47^{\prime} \mathrm{E}$, Oct. 16, 1992 (SAM N1996/354-357), 30 0 , 1 \% ; Kudna Rockhole, $31^{\circ} 07^{\prime} \mathrm{S}, 130^{\circ} 38^{\prime} \mathrm{E}$, Sept. 30, 1988 (D. Hirst, SAM N1996/351), 1 \%; edge of Lake Torrens, near Beda Hill, $31^{\circ} 52^{\prime} \mathrm{S}$, $137^{\circ} 37^{\prime} \mathrm{E}$, Dec. 5, 1989 (D. Hirst, SAM N1996/352), 19 ; 7 km NE Mount Woodroffe, $26^{\circ} 17^{\prime} \mathrm{S}, 131^{\circ} 48^{\prime} \mathrm{E}$, Oct. 16 , 1994, under bark of dead hakea (D. Hirst, SAM N1996/347), 1 \%; Olympic Dam Site, Roxby Downs, $30^{\circ} 27^{\prime} \mathrm{S}, 136^{\circ} 53^{\prime} \mathrm{E}$, Mar.-June 1987 , pitfall (A. Smith, SAM N1997/102), 10; Port Stanvac, N of coastal cliff, $35^{\circ} 06^{\prime} \mathrm{S}$, $138^{\circ} 28^{\prime} \mathrm{E}$, Dec. 6, 1966, in Goodenia amplexans and litter (H. Cooper, SAM N1996/ 343), 1 \% ; $19 \mathrm{~km} \mathrm{~N}$ Renmark, $34^{\circ} 00^{\prime} \mathrm{S}$, $140^{\circ} 47^{\prime} \mathrm{E}$, Sept. 7-Oct. 12, 1995, flight intercept pitfall (K. Pullen, QMB S34342), 30 , Oct. 10-26, 1995, pitfall, saltbush (K. Pullen, A. Lambie, QMB), 1 \% , Nov. 8-Dec. 14, 1995, flight intercept pitfall (K. Pullen, QMB
S34298), 2 đ̃, Nov. 8-22, 1995, pitfalls, saltbush (A. Lambie, K. Pullen, QMB S34344), 20; $79 \mathrm{~km}$ NNW Renmark, 3331's, $140^{\circ} 24^{\prime} \mathrm{E}$, Sept. 6-Oct. 12, 1995, flight intercept pitfall (K. Pullen, QMB), 20; $0.4 \mathrm{~km}$ WSW Teatrick, $36^{\circ} 24^{\prime} \mathrm{S}, 140^{\circ} 53^{\prime} \mathrm{E}$, Dec. 48, 1995, pitfall (SAM N1997/100, 101), 10ิ, $1 \%$; $1 \mathrm{~km}$ NW Tractor Dam, $33^{\circ} 18^{\prime} \mathrm{S}$, $139^{\circ} 34^{\prime} \mathrm{E}$, Oct. 5, 1992, pitfall (SAM N1996/

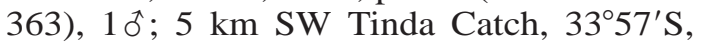
$139^{\circ} 55^{\prime} \mathrm{E}$, Oct. 2, 1992, pitfall (SAM N1996/ 368), $10^{\top} ; 2.3 \mathrm{~km}$ SW Wingoona, $33^{\circ} 07^{\prime} \mathrm{S}$, $139^{\circ} 17^{\prime}$ E, Oct. 19-24, 1992, pitfall (SAM N1996/358-361), 3o, 1 ㅇ․ Victoria: $8.5 \mathrm{~km}$ SE confluence of Lindsay River and Mullaroo Creek, $34^{\circ} 11^{\prime} \mathrm{S}, 141^{\circ} 11^{\prime} \mathrm{E}$, Nov. 1985 , drift fence pitfall (A. Yen, NMVS), 1 \% 9.4 $\mathrm{km}$ ESE Meringur, $34^{\circ} 25^{\prime} \mathrm{S}, 141^{\circ} 26^{\prime} \mathrm{E}$, Oct. 1986, drift fence pitfall (A. Yen, NMVS), 1 ; $3.7 \mathrm{~km}$ N Millewa South Bore, $34^{\circ} 45^{\prime} \mathrm{S}$, $141^{\circ} 04^{\prime} \mathrm{E}$, Nov. 1985, drift fence pitfall (A. Yen, NMVS), 1 o. Western Australia: Durokoppin Nature Reserve, $31^{\circ} 30^{\prime} \mathrm{S}$, $117^{\circ} 44^{\prime} \mathrm{E}$, Nov. 1-3, 1987 (D. Mitchell, WAM 96/682), 1q, Nov. 3-14, 1988 (D. Mitchell, WAM 96/679), 1 đ̃; East Yorkrakine Reserve, $31^{\circ} 28^{\prime} \mathrm{S}, 117^{\circ} 41^{\prime} \mathrm{E}$, Nov. 3-14, 1988 (D. Mitchell, WAM 96/680), 1 \% ; Eucla, 31 $59^{\prime} \mathrm{S}, 128^{\circ} 15^{\prime} \mathrm{E}$, Sept. 23, 1966 (J. Lowry, WAM 96/95), $10^{\star}$; Talbot Road Reserve, $31^{\circ} 52^{\prime} \mathrm{S}, 116^{\circ} 03^{\prime} \mathrm{E}$, Nov. $18-$ Dec. 10 , 1993, pitfall (J. Waldock, WAM 96/681), 1 오.

Distribution: Southern Australia, except Tasmania (map 74).

\section{Notsodipus marun, new species Figures 666-669; Map 75}

TYPES: Female holotype and male allotype taken in Berlese sample of litter from closed forest at Marun, Prince Frederick Harbour, $15^{\circ} 00^{\prime} \mathrm{S}, 125^{\circ} 21^{\prime} \mathrm{E}$, Western Australia (June 6-11, 1988; I. Naumann), deposited in QMB.

ETYMOLOGY: The specific name is a noun in apposition taken from the type locality.

DiAGNOSIS: Males can be recognized by the folded tip of the median prong of the terminal apophysis (fig. 666), females by the very large epigynal atrium (fig. 668).

MALE: Total length 3.5. Abdominal dorsum with large pair of pale, anterior spots and few pale posterior chevrons showing 

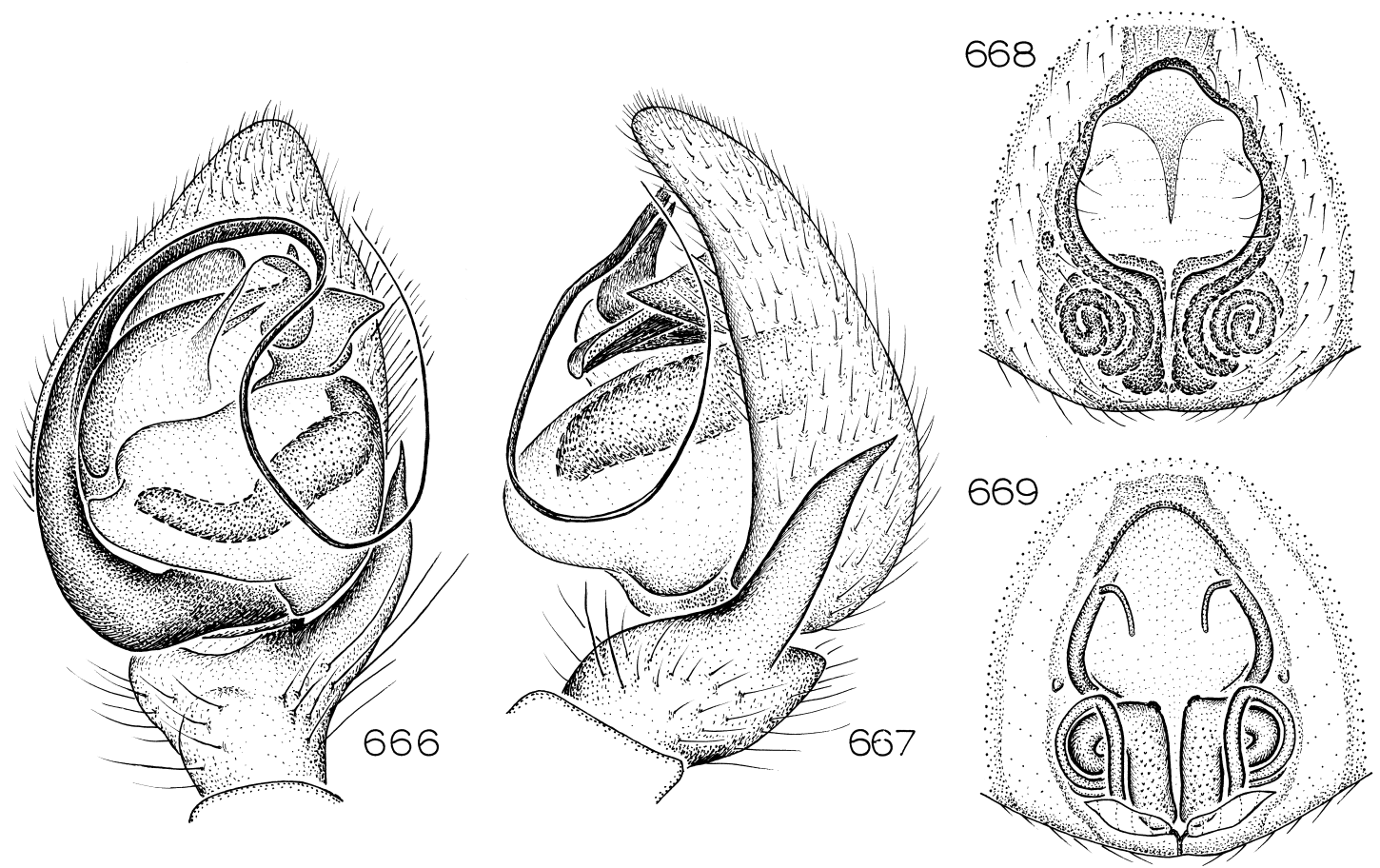

Figs. 666-669. Notsodipus marun, new species. 666. Left male palp, ventral view. 667. Same, retrolateral view. 668. Epigynum, ventral view. 669. Same, dorsal view.

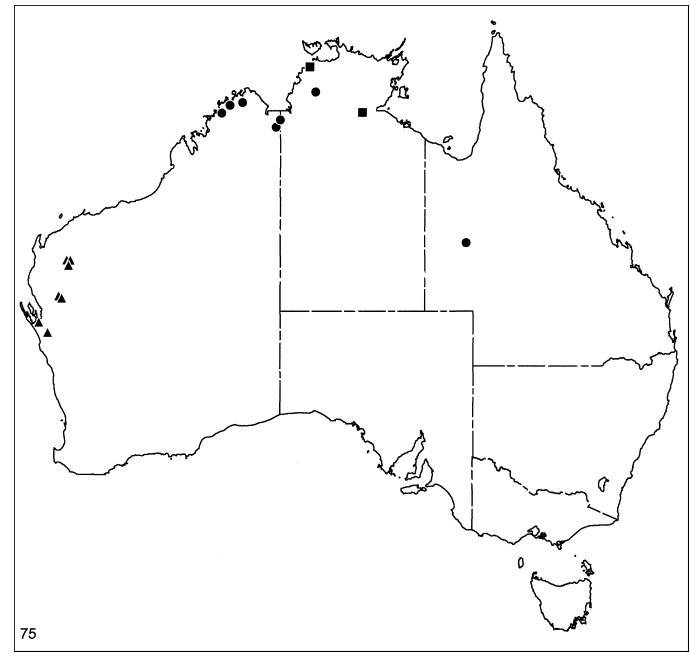

Map 75. Records of Notsodipus marun, new species (circles), N. magdala, new species (squares), and N. bidgemia, new species (triangles). through scutum, venter with large, rectangular orange sclerite. Leg spination typical for genus. Retrolateral tibial apophysis long (fig. 667); median prong of terminal apophysis sharply curved near base, distally folded (fig. 666).

Female: Total length 3.5. Coloration as in male except venter without sclerite, with median rectangular patch of gray. Leg spination typical for genus. Epigynal atrium large, occupying over half of epigynal length (fig. 668); median spermathecal ducts approximate throughout their length (fig. 669).

Other MATERIAl ExAmined: Northern Territory: Douglas Daly, $13^{\circ} 50^{\prime} \mathrm{S}, 131^{\circ} 11^{\prime} \mathrm{E}$, July 3-9, 1996, pitfall, loam site (T. Churchill, CSID), 1 . Queensland: Osbourne Mines, $22^{\circ} 08^{\prime} \mathrm{S}, 140^{\circ} 34^{\prime} \mathrm{E}$, Apr. 19-July 2, 1996, pitfall (R. Raven, A. Nicholson, QMB S31232, 31249), 10, 1\%; Osbourne Mine site, SSE Mount Isa, $22^{\circ} 07^{\prime} \mathrm{S}, 140^{\circ} 34^{\prime} \mathrm{E}$, Dec. 1996 (A. Nicholson, QMB S34340, 34341), 3 ㅇ. Western Australia: $12 \mathrm{~km} \mathrm{~S}$ Kalumburu Mission, $14^{\circ} 25^{\prime} \mathrm{S}, 126^{\circ} 38^{\prime} \mathrm{E}$, June 7-11, 1988, Malaise trap with trough, closed forest 

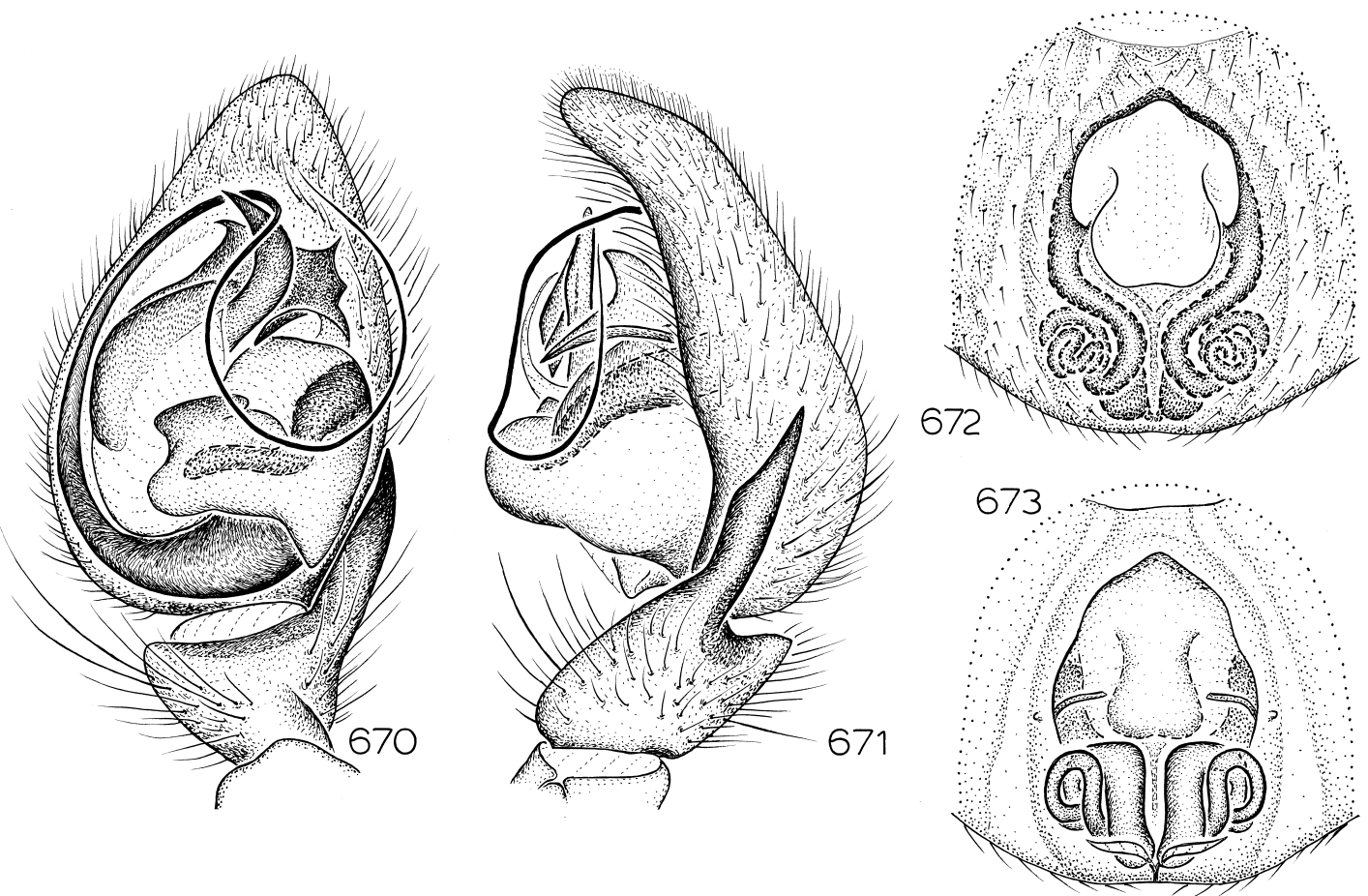

Figs. 670-673. Notsodipus magdala, new species. 670. Left male palp, ventral view. 671. Same, retrolateral view. 672. Epigynum, ventral view. 673. Same, dorsal view.

(T. Weir, QMB), 10, Berlese, closed forest litter (T. Weir, WAM 96/1409, 1410) 2 \% ; Kununurra, $15^{\circ} 47^{\prime} \mathrm{S}, 128^{\circ} 44^{\prime} \mathrm{E}$, Mar. 19,1970 , Berlese, dry scrub, grassland (R. Bartell, QMB), 10; Marun, Prince Frederick Harbour, $15^{\circ} 00^{\prime} \mathrm{S}, 125^{\circ} 21^{\prime} \mathrm{E}$, June 6-11, 1988, Berlese, litter, closed forest (I. Naumann, QMB), 10, 2q; Walsh Point, Admiralty Gulf, $14^{\circ} 34^{\prime} \mathrm{S}, 125^{\circ} 51^{\prime} \mathrm{E}$, May 16, 1983 (J. Balderson, QMB), 1\%; Weaber Range, 20 $\mathrm{km}$ NNE Kununurra, $15^{\circ} 23^{\prime} \mathrm{S}, 128^{\circ} 59^{\prime} \mathrm{E}$, June 7, 1992, litter (M. Harvey, J. Waldock, WAM 96/683), 1 ㅇ․

DisTRIBUTION: Northern parts of Western Australia east to western Queensland (map 75).

Notsodipus magdala, new species Figures 670-673; Map 75

TyPE: Male holotype from East Magdala Woodland, Jabiru, $14^{\circ} 58^{\prime} \mathrm{S}, 134^{\circ} 07^{\prime} \mathrm{E}$, Northern Territory (Apr. 2, 1982; Morton), deposited in SAM (N1997/103).
ETYMOLOGY: The specific name is a noun in apposition taken from the type locality.

Diagnosis: This species resembles $N$. $m a$ run in having a folded median prong of the terminal apophysis of the male palp and a relatively large epigynal atrium. Males can be distinguished by the shape of the lateral prong of the terminal apophysis (fig. 670), females by the wide epigynal septum (fig. 672).

MALE: Total length 4.0. Abdominal dorsum with large pair of pale, anterior spots and few pale posterior chevrons showing through scutum, venter with weak, narrow, rectangular orange sclerite overlaying two longitudinal rows of small, orange, circular sclerites. Leg spination typical for genus. Retrolateral tibial apophysis long, narrowed distally and subbasally (fig. 671); median prong of terminal apophysis sharply curved near base, distally folded, lateral prong with three cusps (fig. 670).

FEMALE: Total length 3.6. Coloration as in male except venter without rectangular scler- 


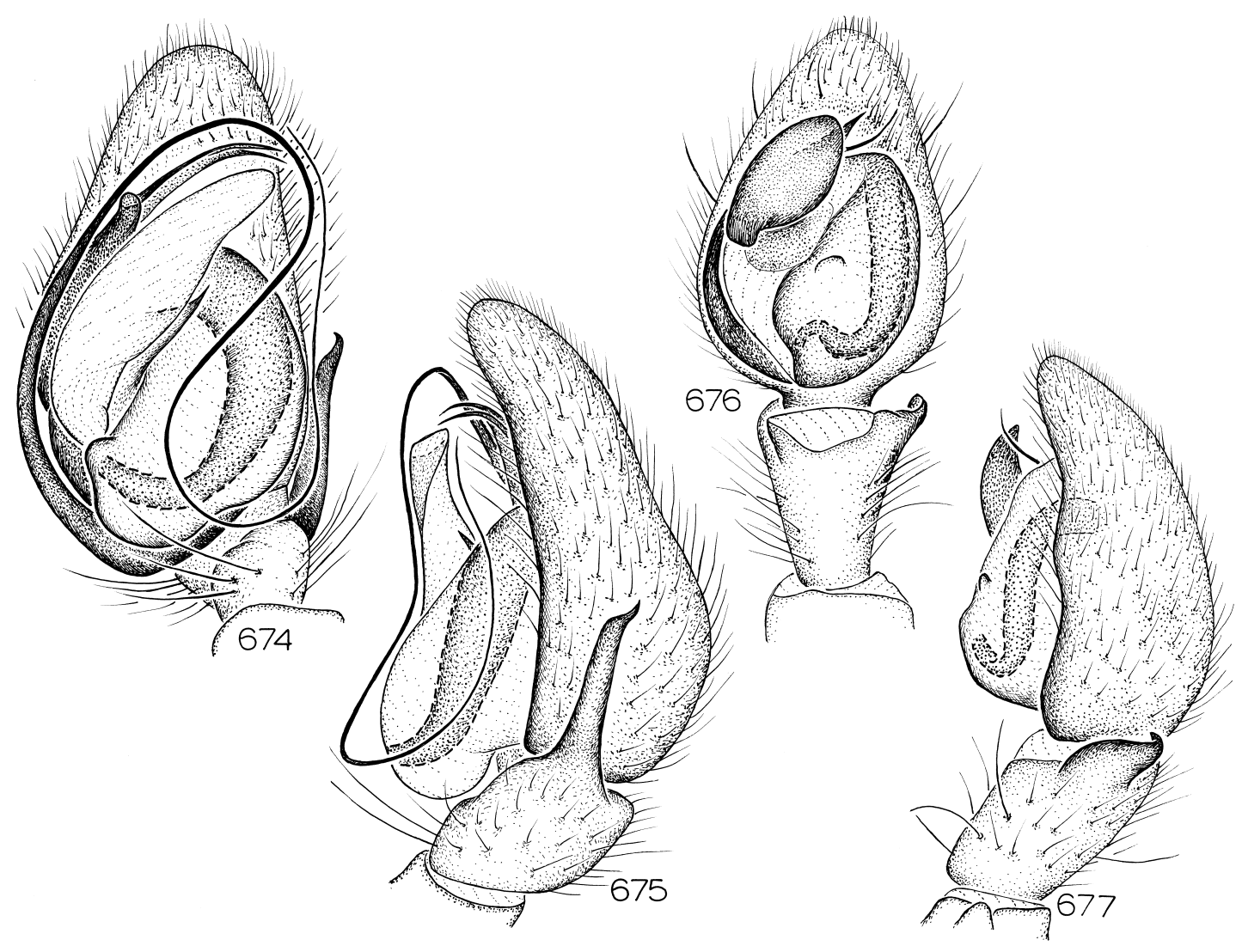

Figs. 674-677. 674, 675. Notsodipus broadwater, new species. 676, 677. Longepi durin, new species. 674, 676. Left male palp, ventral view. 675, 677. Same, retrolateral view.

ite. Leg spination typical for genus. Epigynal atrium large, occupying about half of epigynal length, with wide median septum (fig. 672); median spermathecal ducts well separated throughout most of their length (fig. 673).

Other MATERIAL EXAMined: Northern Territory: East Point, $12^{\circ} 25^{\prime} \mathrm{S}, 130^{\circ} 49^{\prime} \mathrm{E}$, May 1-31, 1975 (R. Pengilley, MNT T023), $1 \%$; Thorak, Darwin, $12^{\circ} 28^{\prime} \mathrm{S}, 130^{\circ} 50^{\prime} \mathrm{E}$, Apr. 1996, pitfall, savanna (T. Churchill, CSID), 20 .

Distribution: Known only from the Northern Territory (map 75).

Notsodipus broadwater, new species Figures 674, 675; Map 76

TYPE: Male holotype taken in pitfall trap at Lake Broadwater, via Dalby, $27^{\circ} 21^{\prime} \mathrm{S}$, $151^{\circ} 06^{\prime} \mathrm{E}$, Queensland (May 17-Nov. 24,
1985; M. Bennie), deposited in QMB (S34338).

ETYMology: The specific name is a noun in apposition taken from the type locality.

DiAGNOSIS: Males of this distinctive species can easily be recognized by the large, rounded, membranous ventral prong of the terminal apophysis (fig. 674).

Male: Total length 3.2. Abdominal dorsum with three pairs of small, pale, anterior spots and few pale posterior chevrons showing through scutum, venter with two longitudinal rows of small, orange, circular sclerites. Leg spination typical for genus. Retrolateral tibial apophysis long, narrow (fig. 675); median prong of terminal apophysis long, membranous except along dorsal edge, tegulum with prolaterally situated lobe (fig. 674).

FeMAlE: Unknown. 
Other Material Examined: One male taken with the type (QMB S34339).

DisTRIBUTION: Known only from southeastern Queensland (map 76).

\section{Notsodipus renmark, new species}

Figures 678-681; Map 76

TYPE: Female holotype taken in flight intercept trap $31 \mathrm{~km}$ NW Renmark, 33⒌ $59^{\prime} \mathrm{S}$, $140^{\circ} 30^{\prime}$ E, South Australia (Sept. 5-Oct. 12, 1995; K. Pullen), deposited in QMB (S34343).

ETYMOLOGY: The specific name is a noun in apposition taken from the type locality.

DiAgnosis: This species resembles $N$. broadwater closely in the shape of the median prong of the terminal apophysis; males can be distinguished by the differently shaped tegulum (fig. 678); females (unknown in $N$. broadwater) have a triangular epigynal atrium (fig. 680).

MALE: Total length 3.0. Abdominal dorsum without distinct patterning visible under scutum, venter with four longitudinal rows of small, orange, circular sclerites. Leg spination typical for genus. Retrolateral tibial apophysis long, narrow (fig. 679); median prong of terminal apophysis long, membranous except along dorsal edge, tegulum with medially situated lobe (fig. 678).

FEMALE: Total length 2.6. Coloration as in male, except abdominal venter without longitudinal rows sclerites. Leg spination typical for genus. Epigynal atrium triangular (fig. 680), median ducts widely separated (fig. 681).

Other Material Examined: South Australia: $14 \mathrm{~km}$ WNW Renmark, $34^{\circ} 07^{\prime} \mathrm{S}$, $140^{\circ} 37^{\prime} \mathrm{E}$, May 2-June 7, 1995, pitfall, mallee on dune (K. Pullen, QMB S32520), 1 万ิ. Victoria: $9 \mathrm{~km}$ ESE Hattah, $34^{\circ} 48^{\prime} \mathrm{S}$, $142^{\circ} 22^{\prime} \mathrm{E}$, Apr. 12, 1989, live Triodia irritans foliage (J. Ludeke, NMV), 10 .

Distribution: South Australia and Victoria (map 76).

Notsodipus capensis, new species Figures 682-685; Map 77

TYPE: Female holotype and male allotype from Cave C.64, Cape Range, $22^{\circ} 03^{\prime} \mathrm{S}$, $114^{\circ} 01^{\prime} \mathrm{E}$, Western Australia (June 27, 1989; B. Vine), deposited in WAM $(96 / 685,686)$.
ETYMOLOGY: The specific name refers to the type locality.

Diagnosis: This species seems closest to $N$. barlee; males of both have long, narrow tips on both the median and lateral prongs of the terminal apophysis (fig. 682), females of both have long, posteriorly directed extensions of the anterior epigynal ducts (fig. 685). Males of $N$. capensis have the base of the lateral prong of the terminal apophysis rounded, without a prolaterally directed tubercle (fig. 682); females have a narrower epigynal atrium (fig. 684).

MALE: Total length 5.0. Abdominal dorsum and venter pale white, venter with two longitudinal rows of small, circular, orange sclerites. Leg spination typical for genus. Retrolateral tibial apophysis with distinct dorsal protuberance on base (fig. 683); median prong of terminal apophysis with narrow, anteriorly directed tip, lateral prong with narrow, prolaterally directed tip (fig. 682).

Female: Total length 3.9. Abdomen brownish gray, venter with sclerites as in male. Leg spination typical for genus. Epigynal atrium relatively large, with narrow anteromedian ridge (fig. 684); anterior ducts with posteriorly directed extensions (fig. 685).

Other Material Examined: Western Australia: Cave C.64, Cape Range, $22^{\circ} 03^{\prime} \mathrm{S}$,

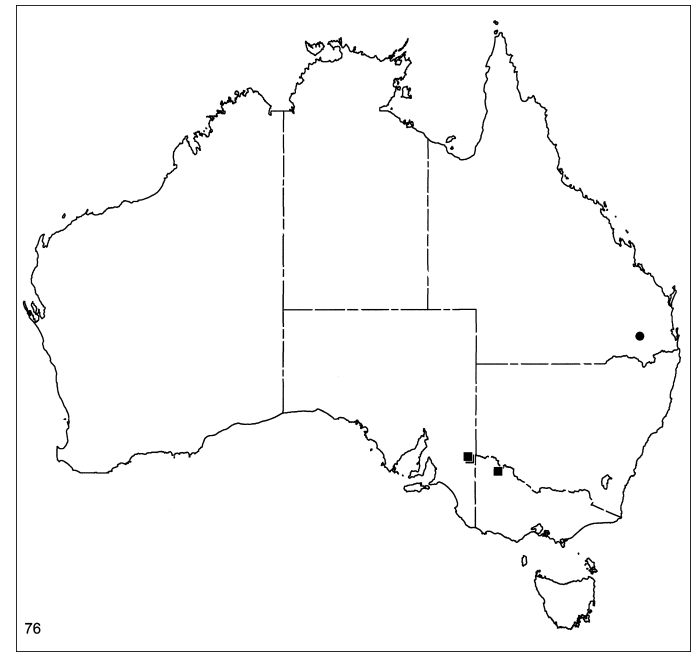

Map 76. Records of Notsodipus broadwater, new species (circle), and $N$. renmark, new species (squares). 

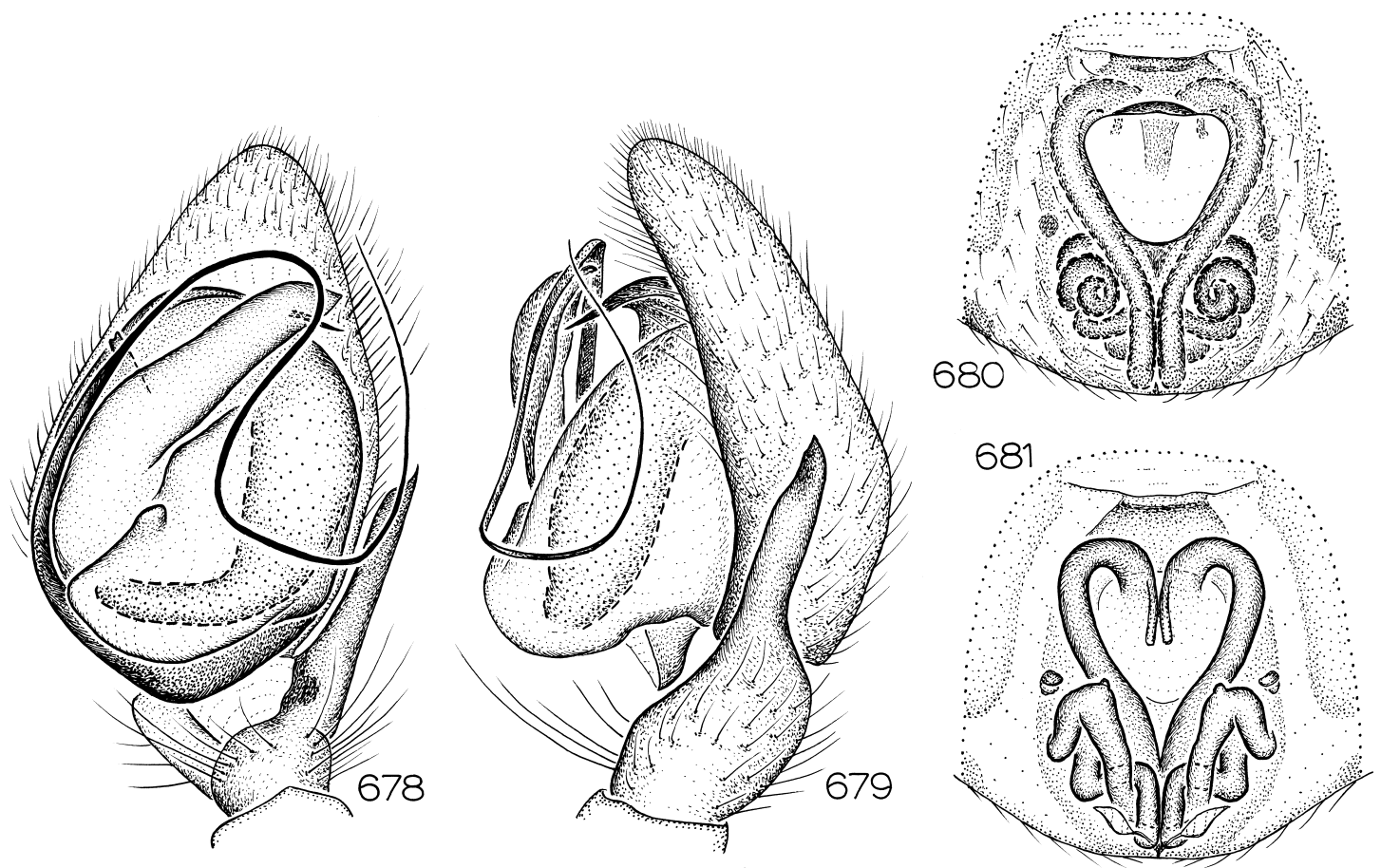

Figs. 678-681. Notsodipus renmark, new species. 678. Left male palp, ventral view. 679. Same, retrolateral view. 680. Epigynum, ventral view. 681. Same, dorsal view.

$114^{\circ} 01^{\prime} \mathrm{E}$, June 27, 1989 (B. Vine, WAM 96/ 687), 1 penultimate + (with full epigynum); Cave C.118, Cape Range, $22^{\circ} 09^{\prime} \mathrm{S}, 113^{\circ} 59^{\prime} \mathrm{E}$, July 23, 1989, pitfall $4 \mathrm{~m}$ outside cave (E. Pryor, WAM 96/690), 1 ô, July 27, 1989, pitfall $8 \mathrm{~m}$ outside Cave (E. Pryor, WAM 96/ 689), 1 ơ $^{\text {; }}$ Cave C.224, Cape Range, $21^{\circ} 56^{\prime} \mathrm{S}$, $114^{\circ} 06^{\prime} \mathrm{E}$, June 30, 1989 (M. East, WAM 96/ 688), 1 穴.

Distribution: Known only from the Cape Range, Western Australia (map 77).

Notsodipus barlee, new species Figures 686-689; Map 73

TYPE: Female holotype taken in pitfall trap at Barlee Range Nature Reserve, $23^{\circ} 06^{\prime} \mathrm{S}$, $116^{\circ} 00^{\prime} \mathrm{E}$, Western Australia (Aug. 1993; S. van Leeuwen, B. Bromilow), deposited in WAM (96/693).

ETYMOLOGY: The specific name is a noun in apposition taken from the type locality.

DiAGNOSIS: This species seems closest to $N$. capensis (see above); males have a distinctive prolaterally directed tubercle on the base of the lateral prong of the terminal apophysis (fig. 686), and females have a wider epigynal atrium (fig. 688).

MALE: Total length 3.9. Abdomen light gray, venter with four longitudinal rows of small, circular, orange sclerites. Leg spination typical for genus. Retrolateral tibial apophysis with distinct dorsal protuberance on base (fig. 687); median prong of terminal apophysis with narrow, anteriorly directed tip, lateral prong with narrow, prolaterally directed tip, base of lateral prong with prolaterally directed tubercle (fig. 686).

Female: Total length 4.6. Coloration as in male. Leg spination typical for genus. Epigynal atrium relatively wide, with narrow anteromedian ridge (fig. 688); anterior ducts with posteriorly directed extensions, posterior ducts with anteromedian tubercle (fig. 689).

Other Material Examined: Western Australia: Barlee Range Nature Reserve, $23^{\circ} 03^{\prime} \mathrm{S}, 115^{\circ} 49^{\prime} \mathrm{E}$, June $15-18$, 1994, dry pitfalls (P., G. Kendrick, WAM 96/1559, 1560), 20; Barlee Range Nature Reserve, $23^{\circ} 06^{\prime} \mathrm{S}, 116^{\circ} 00^{\prime} \mathrm{E}$, June 1994 , pitfall (S. van 

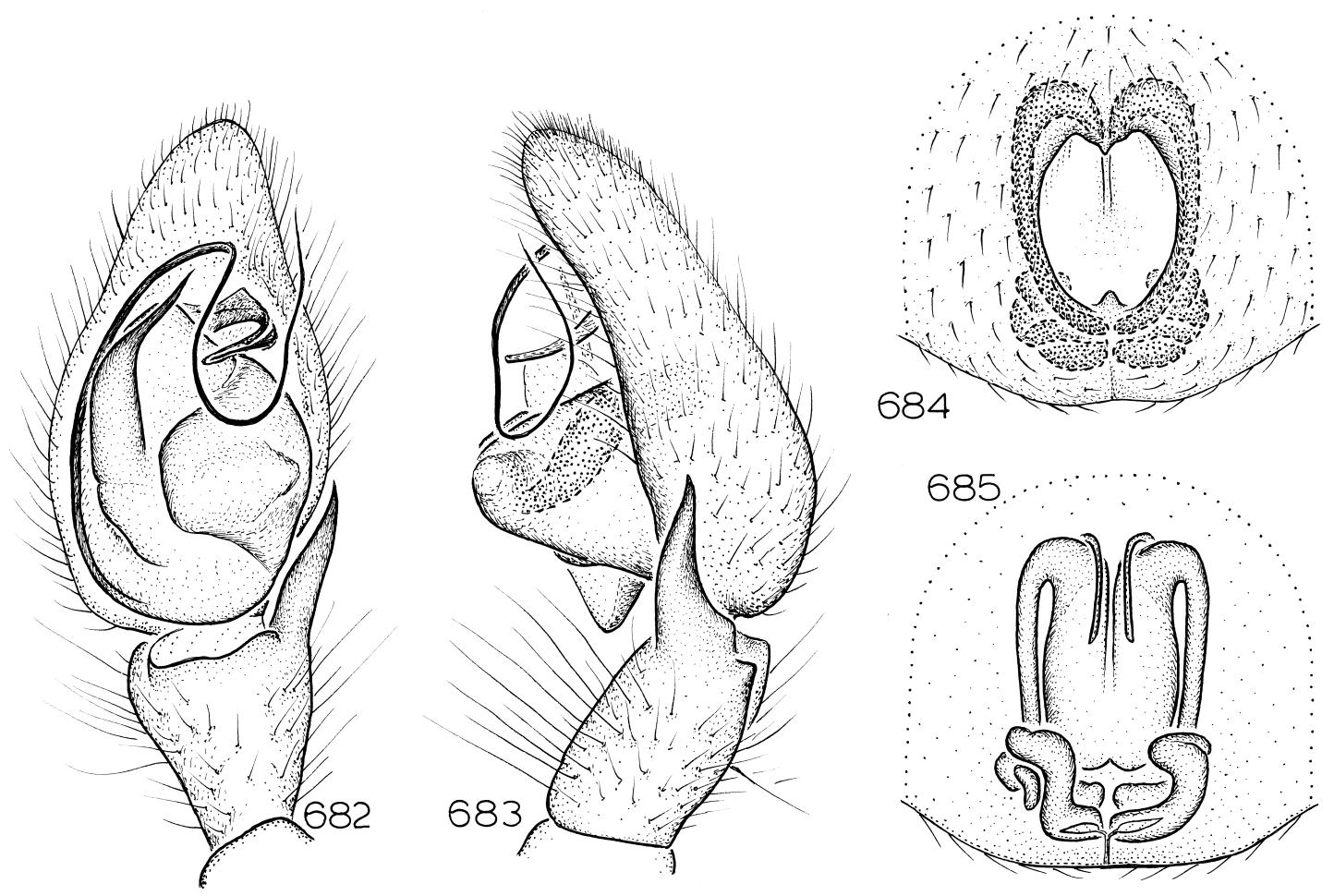

Figs. 682-685. Notsodipus capensis, new species. 682. Left male palp, ventral view. 683. Same, retrolateral view. 684. Epigynum, ventral view. 685. Same, dorsal view.

Leeuwen, B. Bromilow, WAM 96/691), 1ð, June 19-22, 1994, dry pitfall (P., G. Kendrick, WAM 96/1557), 10; Barlee Range Nature Reserve, $23^{\circ} 23^{\prime} \mathrm{S}, 115^{\circ} 53^{\prime} \mathrm{E}$, June 1994, pitfall (S. van Leeuwen, B. Bromilow, WAM 96/692), 10, June 11-14, 1994, dry

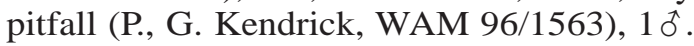

DisTRIBUTION: Known only from the Barlee Range Nature Reserve, Western Australia (map 73).

\section{Notsodipus meedo, new species} Figures 690-693; Map 77

TYPE: Female holotype taken in pitfall trap at Meedo Station, $25^{\circ} 39^{\prime} \mathrm{S}, 114^{\circ} 38^{\prime} \mathrm{E}$, Western Australia (Aug. 22-Oct. 11, 1994; P. West), deposited in WAM (96/706).

ETYMOLOGY: The specific name is a noun in apposition taken from the type locality.

DIAGNOSIS: Males have the embolar tip relatively short and sharply bent (fig. 690), females have a relatively small epigynal atrium (fig. 692).

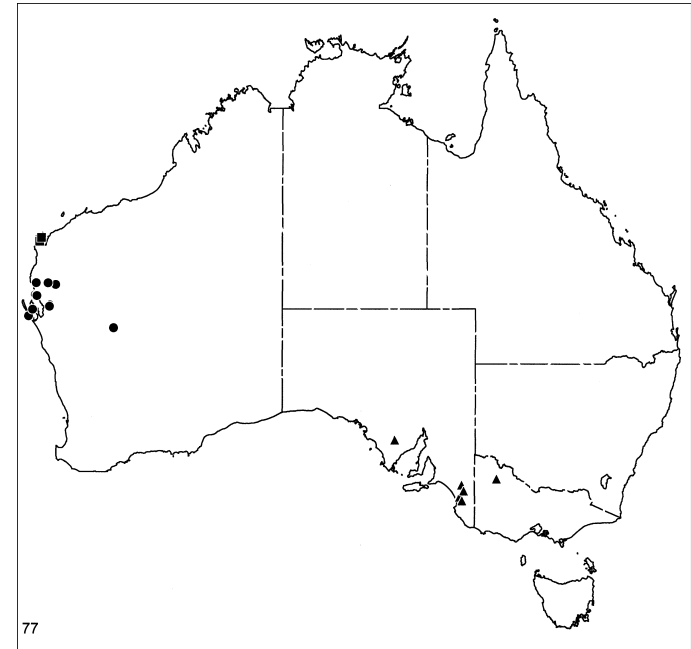

Map 77. Records of Notsodipus capensis, new species (squares), N. meedo, new species (circles), and N. keilira, new species (triangles). 

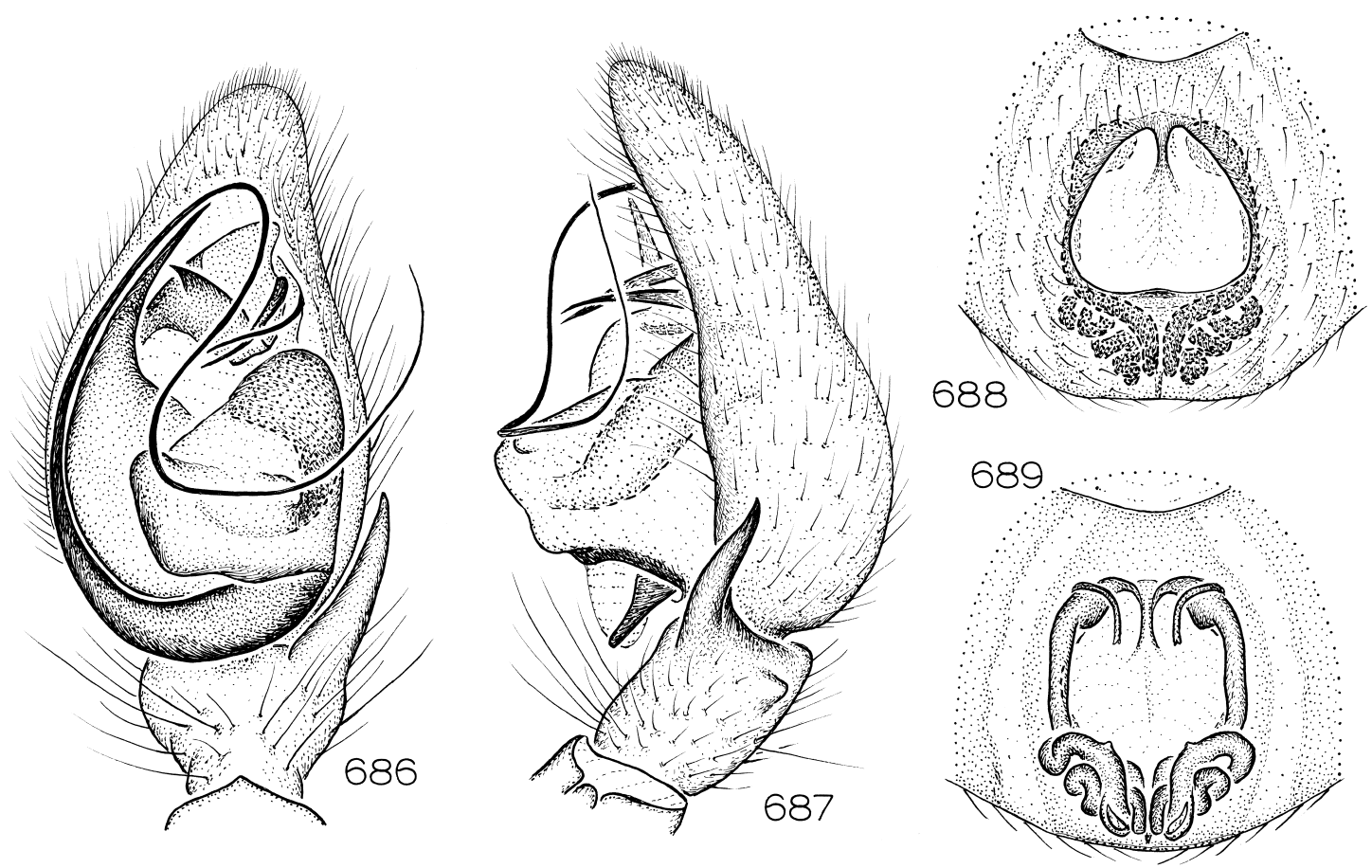

Figs. 686-689. Notsodipus barlee, new species. 686. Left male palp, ventral view. 687. Same, retrolateral view. 688. Epigynum, ventral view. 689. Same, dorsal view.

MALE: Total length 3.3. Abdominal dorsum with anterior light spot, venter with two longitudinal rows of small, circular, orange sclerites. Leg spination typical for genus. Retrolateral tibial apophysis with distinct dorsal protuberance on base (fig. 691); median prong of terminal apophysis very long, with narrow tip, lateral prong with narrow, prolaterally directed tip, base of lateral prong with small, prolaterally directed tubercle, distal portion of embolus bent twice at sharp angles (fig. 690).

FEMALE: Total length 3.5. Abdominal dorsum gray with scattered white maculations, venter as in male. Leg spination typical for genus. Epigynal atrium relatively small, deep, with narrow anteromedian ridge (fig. 692); anterior ducts heart-shaped, with posteriorly directed extensions, posterior ducts with anteromedian tubercle on enlarged median portion (fig. 693).

Other Material Examined: Western Australia: Boolathana Station, $24^{\circ} 25^{\prime} \mathrm{S}$, $113^{\circ} 46^{\prime} \mathrm{E}$, May 31-Aug. 25, 1995, pitfall (N. Hall, WAM 96/701), $10^{\dagger}$; Bush Bay, $25^{\circ} 05^{\prime} \mathrm{S}$, $113^{\circ} 43^{\prime}$ E, Aug. 16-Sept. 30, 1994, pitfall (M. Harvey, WAM 96/705), 1 \%; Bush Bay, $25^{\circ} 07^{\prime} \mathrm{S}, 113^{\circ} 48^{\prime} \mathrm{E}$, May 21-26, 1995, dry pitfalls (P. West, WAM 96/697, 698), $20^{\circ}$; Bush Bay, $25^{\circ} 08^{\prime} \mathrm{S}, 113^{\circ} 49^{\prime} \mathrm{E}$, May 23-Aug. 23, 1995, pitfall (N. Hall, WAM 96/700), $10^{\circ}$; Edel Land, $26^{\circ} 15^{\prime} \mathrm{S}, 113^{\circ} 16^{\prime} \mathrm{E}$, Aug. 21, 1989 (G. Harold, WAM 96/704), 1 \%; Edel Land, $26^{\circ} 15^{\prime} \mathrm{S}, 113^{\circ} 18^{\prime} \mathrm{E}$, Sept. 9-14, 1989 (G. Harold, WAM 96/702), 19 ; Francois Peron National Park, 25 $53^{\prime} \mathrm{S}, 113^{\circ} 33^{\prime} \mathrm{E}$, Aug. 24-Oct. 10, 1994, pitfall (A. Sampey, WAM 96/703), 19 ; Kennedy Range National Park, $24^{\circ} 31^{\prime} \mathrm{S}, 114^{\circ} 58^{\prime} \mathrm{E}$, May 27-June 1, 1995, dry pitfall (P. West, WAM 96/696), 10; Mardathuna Station, $24^{\circ} 24^{\prime} \mathrm{S}, 114^{\circ} 28^{\prime} \mathrm{E}$, May 23-28, 1995, dry pitfall (A. Sampey, WAM 96/695), 10; Mardathuna Station, $24^{\circ} 26^{\prime} \mathrm{S}, 114^{\circ} 30^{\prime} \mathrm{E}$, May 23-28, 1995, dry pitfall (A. Sampey, WAM 96/699), 1 ô; Meedo Station, $25^{\circ} 43^{\prime} \mathrm{S}, 114^{\circ} 36^{\prime} \mathrm{E}$, May 19-Aug. 22, 1995, pitfall (N. Hall, WAM 96/694), $1{ }^{\top}$; Polelle Station, $26^{\circ} 55^{\prime} \mathrm{S}, 118^{\circ} 33^{\prime} \mathrm{E}$, Aug. 5, 1982 (B. Main, WAM 96/1552), 1 ㅇ.

Distribution: Western Australia (map 77). 

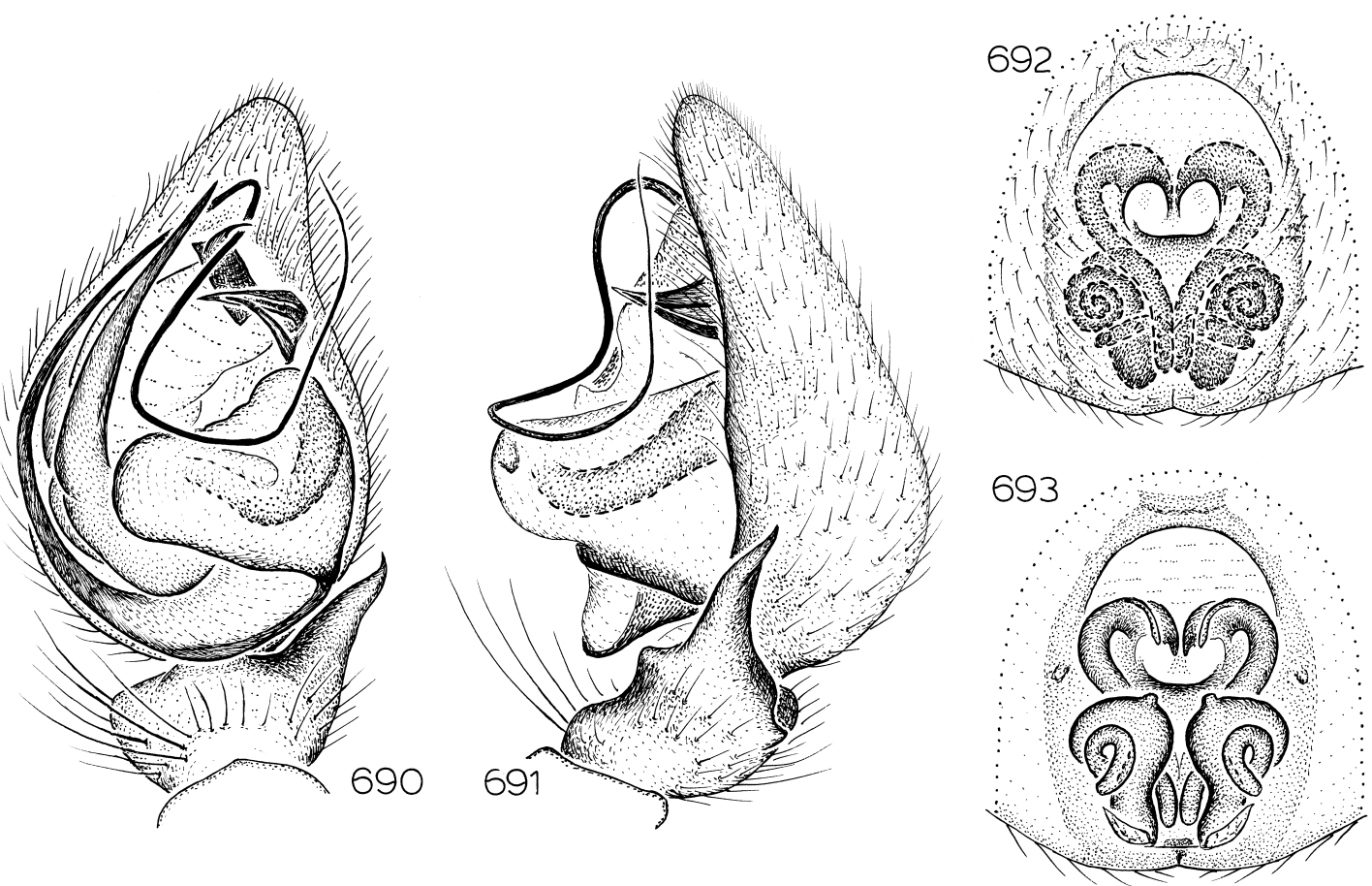

Figs. 690-693. Notsodipus meedo, new species. 690. Left male palp, ventral view. 691. Same, retrolateral view. 692. Epigynum, ventral view. 693. Same, dorsal view.

Notsodipus quobba, new species

Figures 694-697; Map 74

TYPE: Male holotype taken in dry pitfall trap at Quobba Station, Cape Cuvier, $24^{\circ} 08^{\prime} \mathrm{S}, 113^{\circ} 27^{\prime} \mathrm{E}$, Western Australia (May 28-June 3, 1995; A. Sampey), deposited in WAM 96/708).

ETYMOLOGY: The specific name is a noun in apposition taken from the type locality.

DiAGNOSIS: Males have a relatively small lateral prong of the terminal apophysis and a relatively short embolus (fig. 694); females (which have not been collected with males) have a distinctively rectangular epigynum (fig. 696).

MALE: Total length 3.2. Abdominal dorsum gray with scattered tiny white spots, venter unmarked. Leg spination: tibia IV v1p-0-2. Retrolateral tibial apophysis without distinct dorsal protuberance on base (fig. 695); median prong of terminal apophysis very narrow, lateral prong with distal ele- ments relatively small, embolus relatively short (fig. 694).

Female: Total length 2.4. Coloration as in male. Leg spination: tibia IV r0-0-1. Epigynum rectangular, with v-shaped anterior septum (fig. 696); spermathecae situated posteriorly, coiled (fig. 697).

Other Material Examined: Western Australia: Coral Bay, $23^{\circ} 08^{\prime} \mathrm{S}, 113^{\circ} 46^{\prime} \mathrm{E}$, May 12, 1990, leaf litter (A. Longbottom, WAM 96/709), 10; Moonyoonooka, near Geraldton, $28^{\circ} 46^{\prime} \mathrm{S}, 114^{\circ} 43^{\prime} \mathrm{E}$, Nov. 5, 1998, pitfall (V. Ovtsharenko, J. Waldock, AMNH), 1 \%; Quobba Station, Cape Cuvier, $24^{\circ} 12^{\prime} \mathrm{S}$, $113^{\circ} 27^{\prime} \mathrm{E}$, May $29-J u n e$ 3, 1995, dry pitfall (A. Sampey, WAM 96/707), 1 ô.

Distribution: Western Australia (map 74).

Notsodipus bidgemia, new species Figures 698-701; Map 75

TYPE: Male holotype taken in dry pitfall trap at Bidgemia Station, Gasgoyne Junction, 

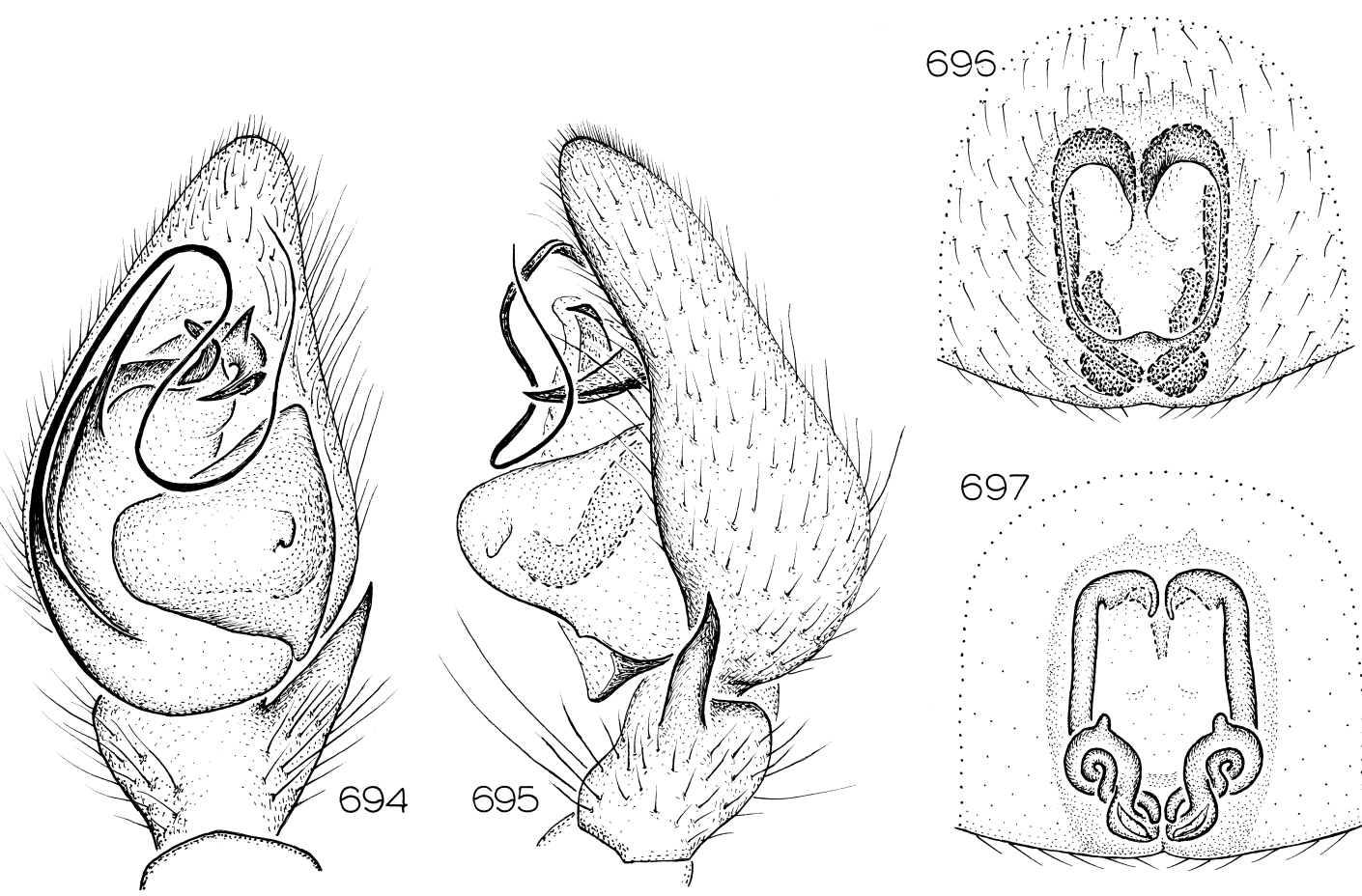

Figs. 694-697. Notsodipus quobba, new species. 694. Left male palp, ventral view. 695. Same, retrolateral view. 696. Epigynum, ventral view. 697. Same, dorsal view.

$25^{\circ} 03^{\prime} \mathrm{S}, 115^{\circ} 18^{\prime} \mathrm{E}$, Western Australia (June 3-8, 1995; J. Waldock), deposited in WAM (96/712).

ETYMOLOGY: The specific name is a noun in apposition taken from the type locality.

DiAGNOSIS: Males can be recognized by the dorsally excavated retrolateral tibial apophysis and the wide lateral prong of the terminal apophysis (figs. 698, 699), females (which have not been collected together with males, but which match them in size and color pattern) by the heart-shaped epigynal atrium (fig. 700).

MALE: Total length 4.5. Abdominal dorsum with large posteromedian white spot above spinnerets, venter with four longitudinal rows of small, circular, orange sclerites. Leg spination typical for genus. Retrolateral tibial apophysis dorsally excavated (fig. 699); median prong of terminal apophysis narrow for most of its length, lateral prong wide, embolus relatively short (fig. 698).

FEMALE: Total length 4.7. Abdominal dorsum as in male, venter with two longitudinal rows of small, circular, orange sclerites. Leg spination typical for genus. Epigynal atrium large, heart-shaped (fig. 700); median epigynal ducts widely separated (fig. 701).

Other Material Examined: Western Australia: Barlee Range Nature Reserve, $23^{\circ} 05^{\prime} \mathrm{S}, 115^{\circ} 47^{\prime} \mathrm{E}$, June 15-18, 1994, dry pitfalls (P., G. Kendrick, WAM 96/15531555, 1561), 40; Barlee Range Nature Reserve, $23^{\circ} 06^{\prime} \mathrm{S}, 116^{\circ} 00^{\prime} \mathrm{E}$, June $19-22,1994$, dry pitfalls (P., G. Kendrick, WAM 96/1556), 10; Barlee Range Nature Reserve, $23^{\circ} 23^{\prime} \mathrm{S}$, $115^{\circ} 53^{\prime} \mathrm{E}$, June 11-14, 1994, dry pitfalls (P., G. Kendrick, WAM 96/1558, 1562), 2 бే; Bidgemia Station, Gasgoyne Junction, $25^{\circ} 11^{\prime} \mathrm{S}, 115^{\circ} 29^{\prime} \mathrm{E}$, June 3-8, 1995, dry pit-

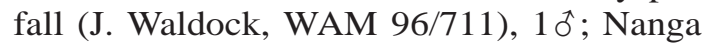
Station, $26^{\circ} 29^{\prime} \mathrm{S}, 114^{\circ} 03^{\prime} \mathrm{E}$, Aug. 23-Oct. 16, 1994, pitfall (P. West, WAM 96/715), 19; Nerren Nerren Station, $27^{\circ} 03^{\prime} \mathrm{S}, 114^{\circ} 36^{\prime} \mathrm{E}$, May 11-Aug. 18, 1995, pitfalls (N. Hall, WAM 96/713, 714), 2 ㅇ.

Distribution: Western Australia (map 75). 

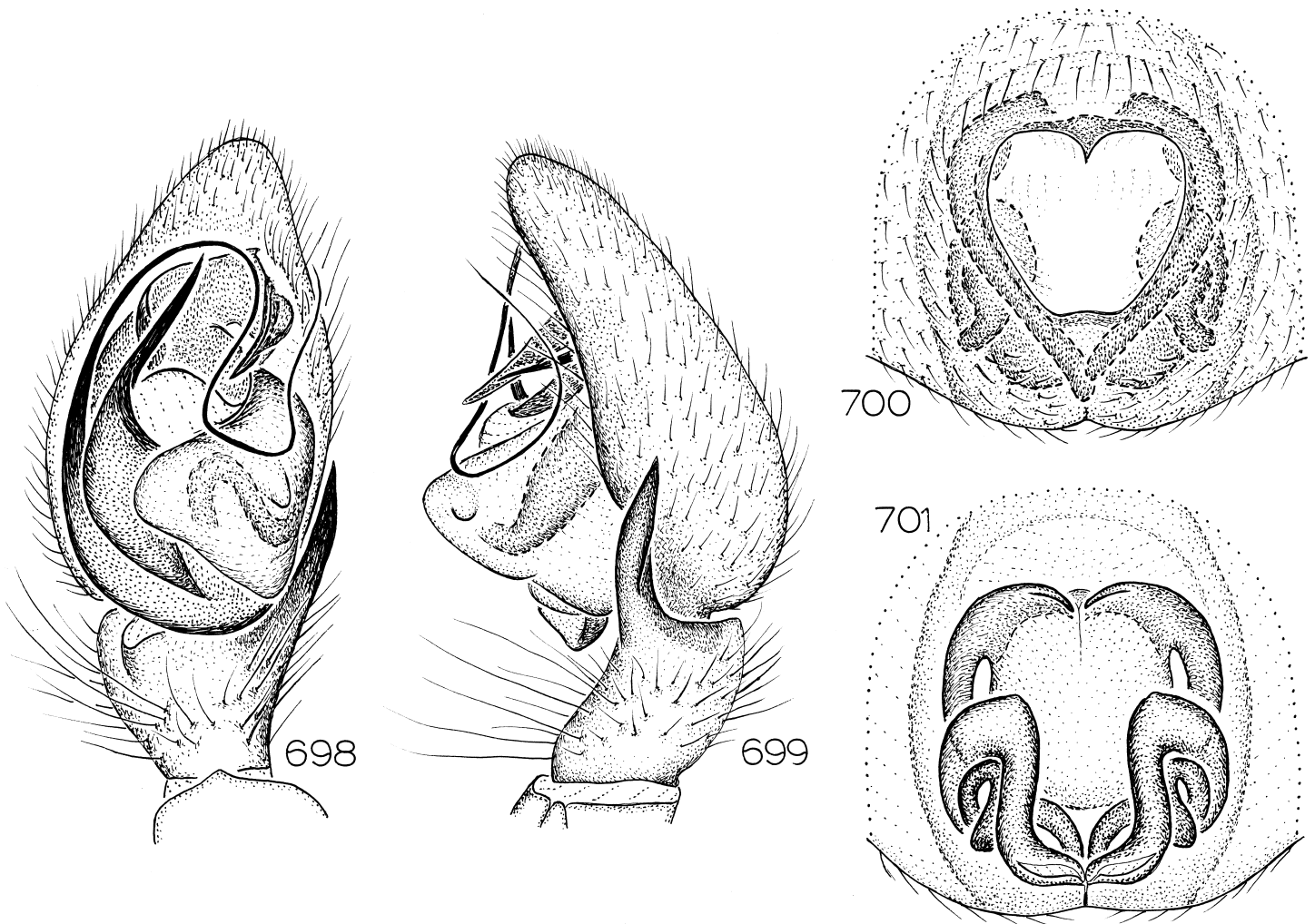

Figs. 698-701. Notsodipus bidgemia, new species. 698. Left male palp, ventral view. 699. Same, retrolateral view. 700. Epigynum, ventral view. 701. Same, dorsal view.

\section{Notsodipus visio, new species} Figures 702-705; Map 78

TyPES: Male holotype and female allotype taken in Eucalyptus wandoo litter at Mount Observation, $31^{\circ} 54^{\prime} \mathrm{S}, 116^{\circ} 33^{\prime} \mathrm{E}$, Western Australia (Aug. 13, 1994; M. Harvey, M. Blosfelds), deposited in WAM (96/1550, 1551).

ETYMOLOGY: The specific name is an arbitrary combination of letters.

DiAGNOSIS: This aberrant species (and its hypothesized sister taxon, $N$. blackall, new species) are easily distinguished from all other Notsodipus by the very small size and very narrow abdomen. Males have a uniquely developed tegular lobe (figs. 702, 703); females of both have a distinctively elongated epigynum (figs. 592, 704) that nevertheless has the posteriorly directed duct extensions found in other species of the genus; in $N$. visio, the median ducts are much shorter than in N. blackall (fig. 705).
MALE: Total length 1.8. Abdominal dorsum long, narrow, dark gray with scattered white hairlines under full scutum, venter pale white with large rectangular orange scutum covering most of surface behind epigastric furrow. Legs without spines. Retrolateral tibial apophysis incised at tip (fig. 703); tegular knob enormously enlarged into distinctive apophysis (fig. 702).

FEMALE: Total length 1.9. Coloration as in male except dorsal and ventral abdominal scuta absent. Legs without spines. Epigynum long, atrium situated anteriorly, with two anterior hoods (fig. 704); median ducts relatively short, with short, posteriorly directed extensions (fig. 705).

Other Material Examined: South Australia: $79 \mathrm{~km}$ NNW Renmark, $31^{\circ} 31^{\prime} \mathrm{S}$, $140^{\circ} 24^{\prime} \mathrm{E}$, Aug. 9-Sept. 7, 1995, intercept trap (K. Pullen, QMB), 10 . Western Australia: Trigg Dune Bush, $31^{\circ} 53^{\prime} \mathrm{S}, 116^{\circ} 46^{\prime} \mathrm{E}$, Sept. 25-Nov. 28, 1995, pitfall (M. Harvey, 

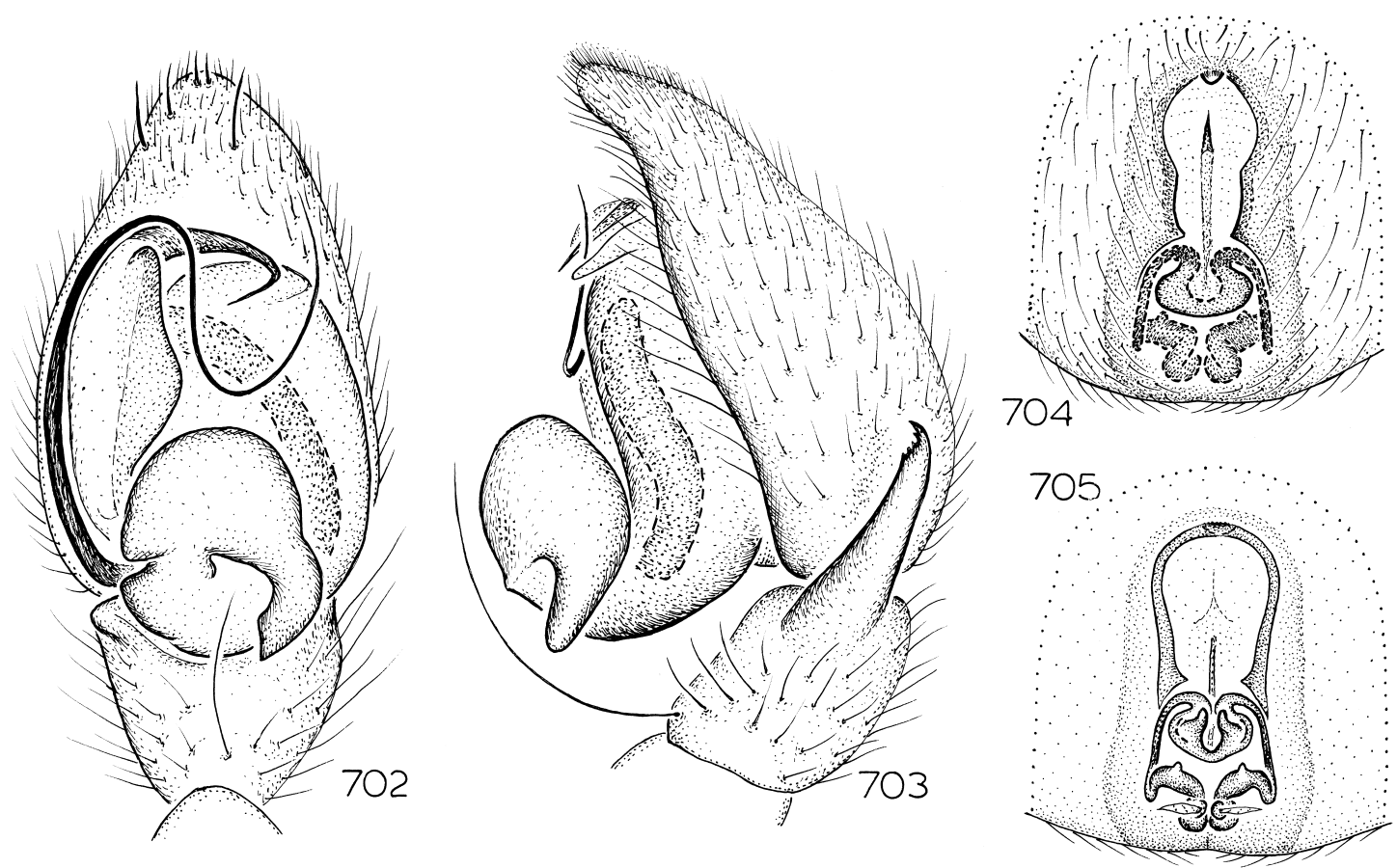

Figs. 702-705. Notsodipus visio, new species. 702. Left male palp, ventral view. 703. Same, retrolateral view. 704. Epigynum, ventral view. 705. Same, dorsal view.

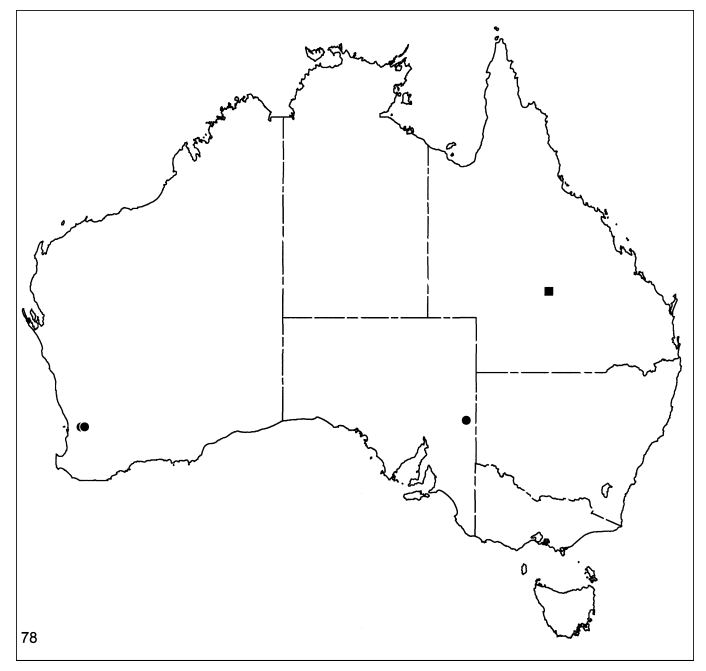

Map 78. Records of Notsodipus visio, new species (circles), and N. blackall, new species (square).
J. Waldock, WAM 96/717), 1 đ; near York, $31^{\circ} 53^{\prime} \mathrm{S}, 116^{\circ} 46^{\prime} \mathrm{E}$, Nov. 16, 1991, powderbark leaf litter (J. Bannister, WAM 96/716), 10 .

Distribution: Despite the large geographic gap between them, I've found no characters by which to distinguish the South Australian male from those known from southwestern Australia (map 78).

\section{Notsodipus blackall, new species}

Figures 592, 593; Map 78

TYPE: Female holotype taken in dry leaf litter (brigalow, wilga) from 33 mi. S Blackall, $24^{\circ} 25^{\prime} \mathrm{S}, 145^{\circ} 28^{\prime} \mathrm{E}$, Queensland (May 31, 1956; F. Perkins), deposited in QMB.

ETYMOLOGY: The specific name is a noun in apposition taken from the type locality.

DiAGNOSIS: Females can be distinguished from those of its hypothesized sister species, $N$. visio, by the much longer median epigynal ducts (figs. 592, 593).

MALE: Unknown.

Female: Total length 2.4. Coloration as in male of $N$. visio except dorsal and ventral 

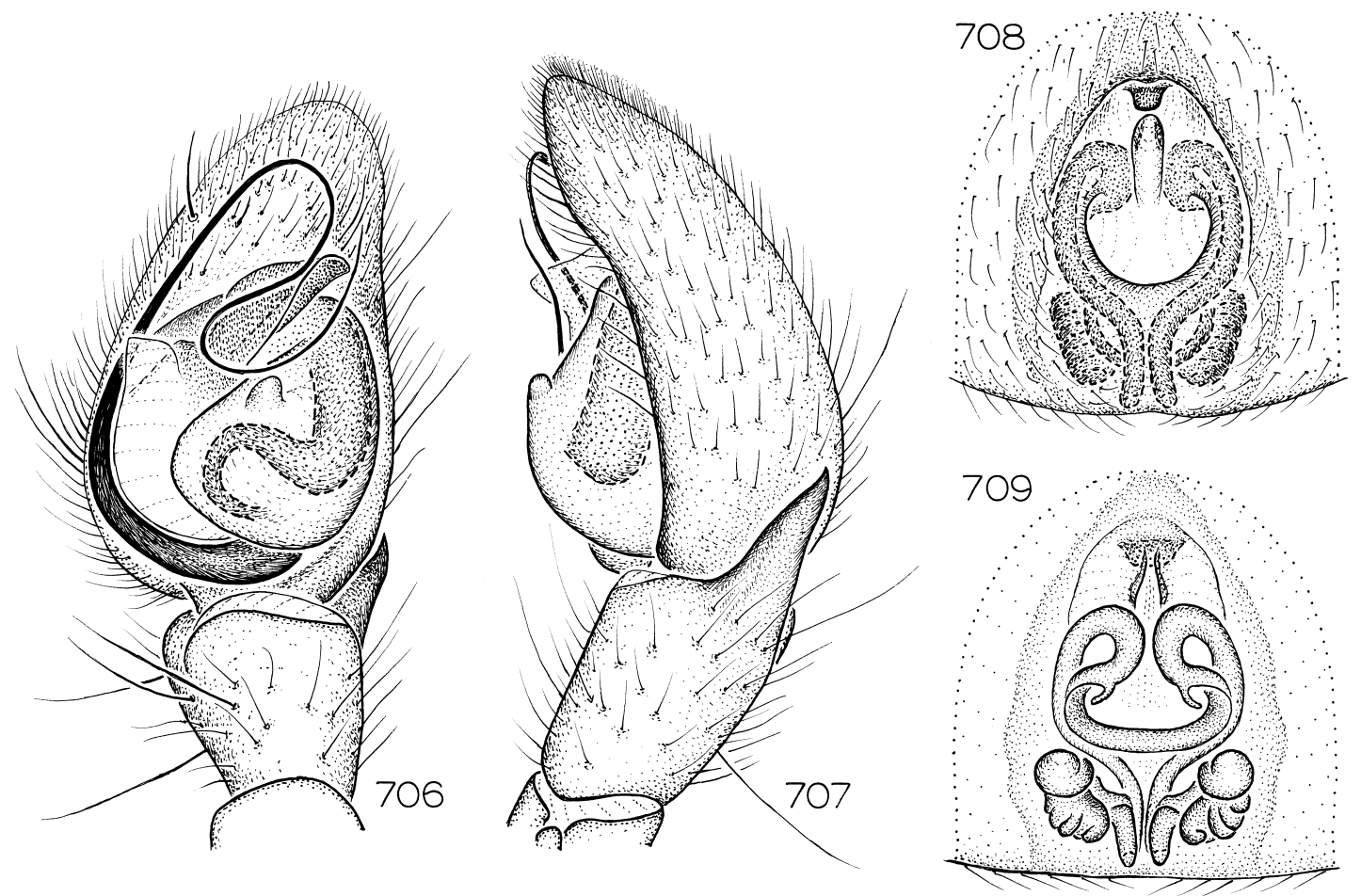

Figs. 706-709. Notsodipus keilira, new species. 706. Left male palp, ventral view. 707. Same, retrolateral view. 708. Epigynum, ventral view. 709. Same, dorsal view.

abdominal scuta absent. Legs without spines. Epigynal atrium large, situated far anteriorly (fig. 592); median ducts elongated, with posteriorly directed extensions (fig. 593).

Other MATERial EXAmined: None.

Distribution: Known only from central Queensland (map 78).

\section{Notsodipus keilira, new species Figures 706-709; Map 77}

TYPE: Female holotype taken by vibration $13 \mathrm{~km}$ N Keilira Station, $35^{\circ} 37^{\prime} \mathrm{S}, 140^{\circ} 10^{\prime} \mathrm{E}$, South Australia (Mar. 22, 1992; D. Hirst), deposited in SAM (N1997/105).

ETYMOLOGY: The specific name is a noun in apposition taken from the type locality.

DiAgNosis: Males of this distinctive species can easily be recognized by the short, translucent medial prong of the terminal apophysis (fig. 706), females by the narrow epigynal hood (fig. 708). This species is odd in both somatic and genitalic features, including the elongated dorsal abdominal scu- tum of females, the short, translucent median lobe of the male terminal apophysis, and the presence of a narrow anterior epigynal hood and septum in females. It could conceivably represent a highly autapomorphic species of Longepi, or even the sister group of Longepi plus Notsodipus. However, the presence of the characteristic embolar conformation suggests that it is an aberrant member of Notsodipus instead.

MALE: Total length 4.6. Abdominal venter with two paramedian longitudinal rows of small, circular, orange sclerites. Leg spination: metatarsus IV v1p-0-0. Retrolateral tibial apophysis simple, triangular (fig. 707); median prong of terminal apophysis reduced to small translucent lobe, embolus elongate (fig. 706).

FEMALE: Total length 5.7. Abdominal scutum enlarged extending more than half length of abdominal dorsum. Leg spination: tibiae: III v1p-0-2; IV v1p-1p-2; metatarsus IV v1p-0-0. Epigynum with narrow anterior 
hood and narrow anterior septum (fig. 708); ducts widely separated medially (fig. 709).

Other Material Examined: South Australia: SE Big Desolation, Gum Lagoon Conservation Park, $36^{\circ} 16^{\prime} \mathrm{S}, 139^{\circ} 59^{\prime} \mathrm{E}$, Mar. 25 , 1992, vibration on stony ground (D. Hirst, SAM N1996/401), $1 \%$; Gum Lagoon Conservation Park, $36^{\circ} 17^{\prime} \mathrm{S}, 140^{\circ} 02^{\prime} \mathrm{E}$, Mar. 26, 1992, vibration, lagoon edge (D. Hirst, SAM N1996/402, 403), 2 ; Jimmys Well, Mount Rescue Conservation Park, $35^{\circ} 51^{\prime} \mathrm{S}$, $140^{\circ} 18^{\prime} \mathrm{E}$, Mar. 16-21, 1992, pitfall (D.

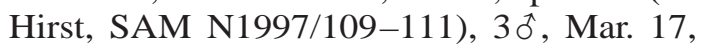
1992, vibration (D. Hirst, SAM N1997/108), 1 , Mar. 21, 1992, pitfall (D. Hirst, SAM N1997/104), 10َ; 1 km N Jimmys Well, Mount Rescue Conservation Park, $35^{\circ} 51^{\prime} \mathrm{S}$, $140^{\circ} 18^{\prime} \mathrm{E}$, Mar. 19, 1992, vibration (D. Hirst, SAM N1997/107), 10̋; N Jimmys Well, Mount Rescue Conservation Park, 35 51' S, $140^{\circ} 18^{\prime} \mathrm{E}$, Mar. 18, 1992, vibration (D. Hirst, SAM N1996/408), 1 đో; NE Jimmys Well, Mount Rescue Conservation Park, 35 51' S, $140^{\circ} 19^{\prime} \mathrm{E}$, Mar. 20, 1992, vibration (D. Hirst, SAM N1996/409-416), 20, 6웅 Jip Jip Conservation Park, $36^{\circ} 29^{\prime} \mathrm{S}, 140^{\circ} 11^{\prime} \mathrm{E}$, Mar. 23, 1992, vibration (J. Forrest, D. Hirst, SAM N1996/407), 19 ; 13 km N Keilira Station, $35^{\circ} 37^{\prime} \mathrm{S}, 140^{\circ} 10^{\prime} \mathrm{E}$, Mar. 22, 1992, vibration (D. Hirst, SAM N1997/10), 1 ㅇ, Mar. 23, 1992, vibration (D. Hirst, SAM N1996/ 404-406), 3 ㅇ ; Museum Hill, N Bucks Camp Well, Mount Rescue Conservation Park, $35^{\circ} 56^{\prime} \mathrm{S}, 140^{\circ} 18^{\prime} \mathrm{E}$, Mar. 18, 1992, vibration (D. Hirst, SAM N1997/112), 1; Pinkawillinie Conservation Park, $33^{\circ} 07^{\prime} \mathrm{S}, 136^{\circ} 00^{\prime} \mathrm{E}$, Mar. 20, 1996, vibration (D. Hirst, SAM N1996/396-400), $5 q$. Victoria: Calder Highway, $25 \mathrm{~km} \mathrm{~S}$ Ouyen, $35^{\circ} 17^{\prime} \mathrm{S}$, $142^{\circ} 20^{\prime} \mathrm{E}$, July 10,1994 , under bark of roadside mallee (P. Hudson, SAM N1996/417), 1 ㅇ.

Distribution: South Australia and Victoria (map 77).

Notsodipus domain, new species Figures 710-713; Map 79

TyPES: Female holotype and male allotype taken in grass tussocks at Queens Domain, $42^{\circ} 52^{\prime} \mathrm{S}, 147^{\circ} 19^{\prime} \mathrm{E}$, Tasmania (Feb. 23, 1968; V. Hickman), deposited in AMS (KS29336).
ETYMology: The specific name is a noun in apposition taken from the type locality.

DIAGNOSIS: Like the preceding species, this taxon is very odd and might be misplaced. Males can easily be recognized by the sharply pointed median projection on the palpal bulb and the small median apophysis (fig. 710), females by the divided anterior epigynal atrium (fig. 712). The lateral pockets on the epigynum suggest a possible relationship to Queenvic, but the palpal conformation suggests that the species is better placed here.

MALE: Total length 2.7. Abdominal dorsum with two pairs of lighter muscle impressions visible anteriorly under dark scutum, transverse white stripe visible medially under scutum; venter gray, with large, rectangular, median, dark orange sclerite. Leg spination: tibia IV v0-0-1p. Retrolateral tibial apophysis long, triangular (fig. 711); terminal apophysis with longitudinally oriented narrow median prong, distal elements short but still with typical, recurved retrolateral elements, embolus relatively short, confined to distal portion of bulb, which bears sharply pointed median projection (fig. 710).

Female: Total length 3.3. Coloration as in male except abdominal dorsum dark gray, without rectangular sclerite. Leg spination: tibiae: III v0-0-0, IV v0-0-1p. Epigynal atrium deep, but with lateral edges poorly demarcated, divided (fig. 712); posterior epigynal ducts coiled (fig. 713).

Other Material ExAmined: New South Wales: Bondi State Forest, S Bombala, $37^{\circ} 08^{\prime} \mathrm{S}, 149^{\circ} 09^{\prime} \mathrm{E}$, May 6, 1980 , pitfall, open forest, litter (G. Gowing, AMS KS18040), 1 đ. South Australia: Pinkawillinie Conservation Park, $32^{\circ} 07^{\prime} \mathrm{S}, 136^{\circ} 00^{\prime} \mathrm{E}$, May 21, 1990, pitfall (D. Hirst, SAM N1997/ 98), 1 \% ; $31 \mathrm{~km}$ NW Renmark, 3359'S, $140^{\circ} 30^{\prime} \mathrm{E}$, May 2-June 8, 1995, flight intercept pitfall (K. Pullen, QMB), 1 \%, Aug. 8Sept. 7, 1995, flight intercept pitfall (K. Pullen, QMB S34310), 10; 79 km NNW Renmark, 333' $\mathrm{S}, 140^{\circ} 24^{\prime} \mathrm{E}$, May 3-June 6, 1995, flight intercept pitfall (K. Pullen, QMB), 19 . Tasmania: Mount Rumney, $42^{\circ} 52^{\prime} \mathrm{S}, 147^{\circ} 27^{\prime} \mathrm{E}$, June 8,1963 , in grass tussock (J., V. Hickman, AMS KS29300), 1; Queens Domain, $42^{\circ} 52^{\prime} \mathrm{S}, 147^{\circ} 19^{\prime} \mathrm{E}$, Feb. 23, 1968, in grass tussocks (V. Hickman, AMS KS29336), 10, 1 ㅇ. Western 

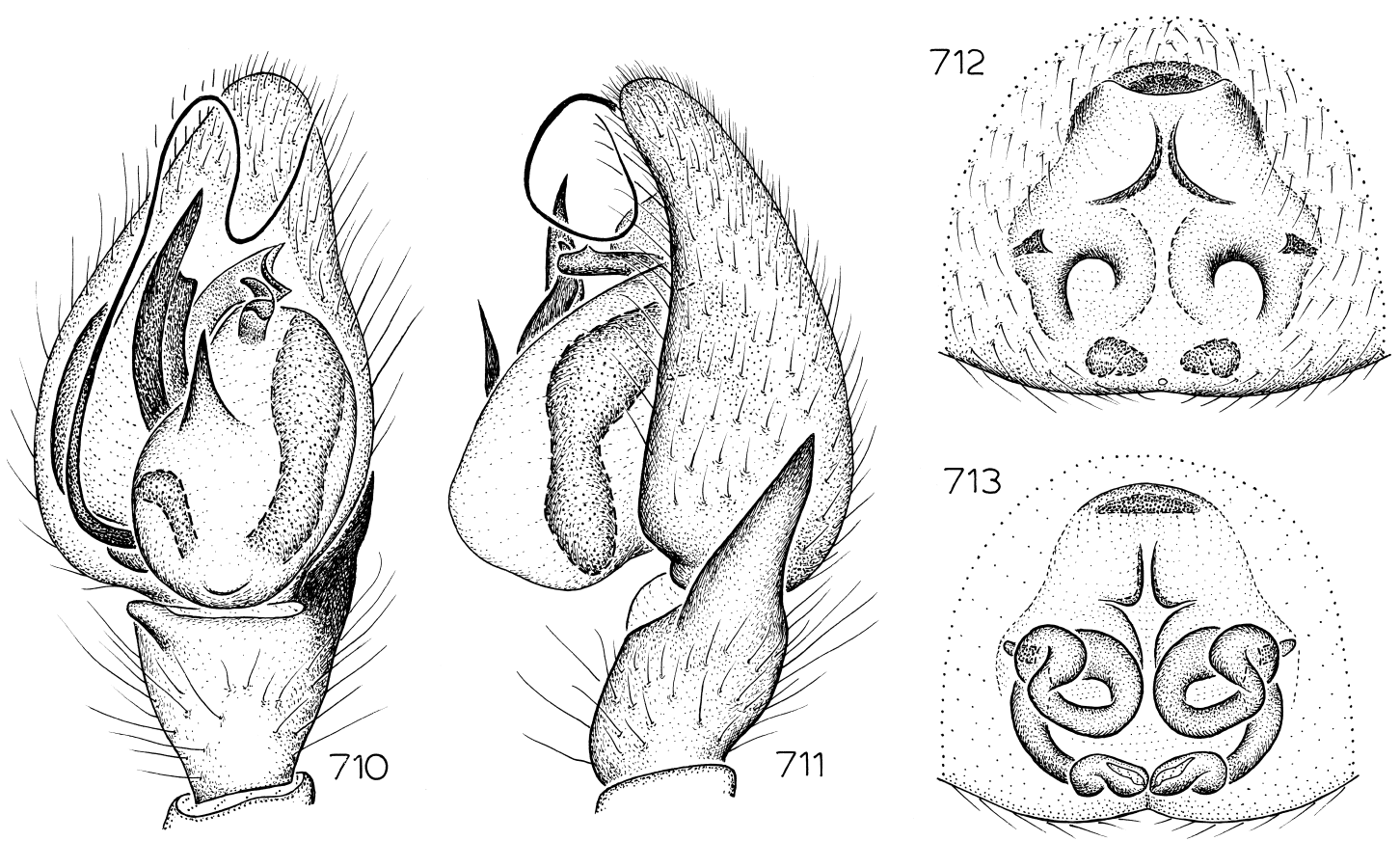

Figs. 710-713. Notsodipus domain, new species. 710. Left male palp, ventral view. 711. Same, retrolateral view. 712. Epigynum, ventral view. 713. Same, dorsal view.

Australia: Heitmans Scrub, $31^{\circ} 31^{\prime} \mathrm{S}$, $117^{\circ} 37^{\prime} \mathrm{E}$, Nov. 3-14, 1988 (D. Mitchell, WAM 96/671-678), 60, 2; Woodline, Goldfields, $31^{\circ} 55^{\prime} \mathrm{S}, 122^{\circ} 24^{\prime} \mathrm{E}$, Aug. 1980, pitfall (W. Humphreys, WAM 90/421), 1 \% .

Distribution: Southern Australia, including Tasmania (map 79).

\section{Longepi, new genus}

TyPe Species: Longepi boyd, new species.

ETYMOLOGY: The generic name is an arbitrary combination of letters, considered masculine in gender.

DiAGNOSIS: Males are most easily recognized by the enlarged terminal apophysis situated anteriorly on the prolateral edge of the palpal bulb and obscuring, in ventral view, the distal portion of the embolus (as in figs. 714,738 ). Females have a long epigynum, reaching almost to the pedicel, with a complex anterior hood (as in figs. 716, 740) and (like those of Notsodipus) a pair of blind ducts (as in figs. 717, 741).

DESCRIPTION: Medium size spiders, total length of males 2.8-4.1, of females 3.3-5.3. Carapace brownish orange, pars thoracica coated with anastomosing rows of large tubercles, pars cephalica with long, dark, thin setae; thoracic groove long, longitudinal, deep. Eight eyes in two rows, anterior medians smallest, circular, dark, laterals sub-

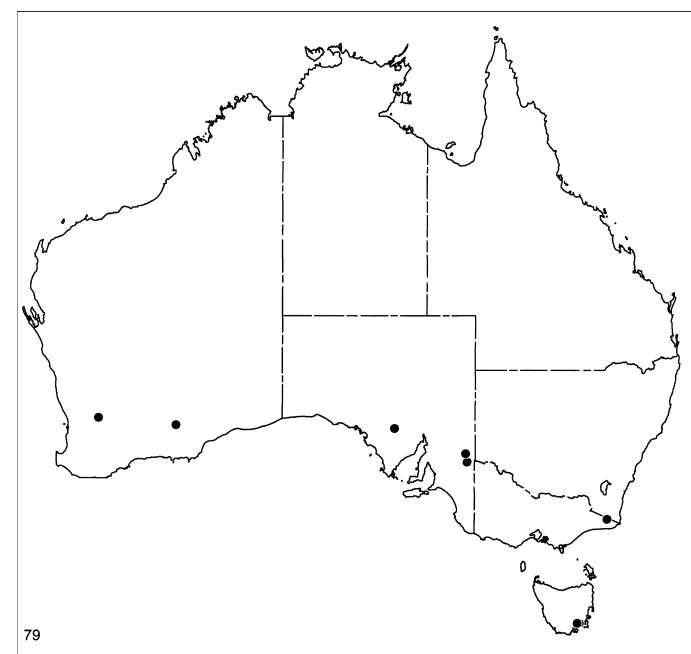

Map 79. Records of Notsodipus domain, new species. 
equal in size, oval, light, posterior medians largest, irregularly oval, flattened, light; from above, anterior eye row slightly recurved, posterior row straight, from front, both rows slightly procurved; anterior medians separated by more than their radius, slightly closer to anterior laterals; posterior medians separated by less than their radius, much farther from posterior laterals; anterior and posterior laterals separated by almost their diameter; median ocular quadrangle wider in back than in front, longer than wide in front. Chelicerae, sternum, and mouthparts light brownish orange; chilum wide, triangular (sometimes reduced in size or even fused to anterior edge of carapace, resulting in median prolongation), accompanied by second, longitudinal posterior chilum (extremely narrow sclerite separating bases of chelicerae posteriorly); chelicerae with distinct lateral boss, anterior surface with depressed, relatively unsclerotized oval area near promargin; promargin with two rows of long setae originating in line along base of fang, seta closest to fang bent near base at almost $90^{\circ}$ angle, extending behind other promarginal setae to near midline; promargin with three teeth, median one largest, proximal one smallest; retromargin with two large, narrowly separated teeth. Labium elongate, triangular, only gradually narrowed anteriorly, posterior margin truncate, anterior margin medially invaginated, surface slightly depressed medially. Endites obliquely depressed, with sharply demarcated, deep groove along margin near labium, groove wider anteriorly than posteriorly; serrula long, with single row of teeth; anterior surface with distinct sieve plate of maxillary gland openings set in oval of unsclerotized cuticle. Sternum not elevated, with flat lateral margins, not expanded anteriorly, with triangular extensions to and between coxae; surface smooth, without distinct elevations or tubercles. One epimeric sclerite on each side, above each coxa, not reaching sternal triangles, not fused to carapace but fused to pedicel. Pedicel consisting of large, diamondshaped sclerite bearing wide anterior margin meeting posterior tip of sternum at transverse line.

Anterior edge of abdomen of male with complete sclerotic ring formed by strong epigastric scutum plus strong dorsal abdominal scutum covering entire front edge of abdomen, reaching to about three-fourths of abdominal length, females with dorsal scutum represented only by small transverse plate above pedicel, restricted to lower half of anterior surface of abdomen; cuticle with long, recumbent setae; epigastric scutum accompanied posterolaterally by pair of oval, deeply invaginated sclerites bearing clearly elevated anterior rim; sclerites separated by membranous lobe, anterior rim of sclerites fitting under epigastric scutum; colulus represented only by pair of long setae; posterior spiracle transverse, inconspicuous. Anterior lateral spinnerets tubular, separated by about their diameter, cuticle representing distal, second spinneret segment restricted to semicircle surrounding major ampullate gland spigots (piriform gland spigots surrounded only by soft cuticle); posterior median spinnerets of males large, tubular, of females with anteriorly expanded tips, bases occupied by three enormously widened cylindrical gland spigots; posterior lateral spinnerets two-segmented, those of females with two greatly widened cylindrical gland spigots.

Leg spination reduced; typical leg spination pattern (only surfaces with spines listed): tibiae: III v0-0-2; IV v1p-1p-2, r0-0-1. Most leg surfaces with long setae; males with all coxae dorsally tuberculate; anterior coxae with slightly protuberant posterolateral corners, widened at about half their length; trochanters unnotched; anterior tarsi with divided scopulae, composed of laterally directed setae; posterior metatarsi with thick, distal preening brushes; posterior tarsi with weak scopulae; tarsi with two dentate claws, claw tufts composed of lateral pads of short, closely appressed setae; trichobothria present on distal segments. Female palpal tibia and tarsus with long, thin spines; female palpal tarsus with long, basally dentate claw.

Male palp with elaborate, often bifid retrolateral tibial apophysis; cymbial surface excavated opposite tibial apophysis; tegulum often almost obscured, in ventral view, by large terminal apophysis, but with distinct excavation on retrolateral surface; median apophysis large, usually completely hidden (in ventral view) behind terminal apophysis; embolus originating posteriorly, long, looping behind terminal apophysis to prolateral 
side of bulb, accompanied distally by recessed conductor; embolar base without apophysis. Epigynum very long, reaching almost to pedicel, with T-shaped anterior hood and posterior atrium; spermathecae accompanied by anteromedian and anterolateral ducts.

\section{KEY TO SPECIES OF LONGEPI}

1. Males .................... 2

- Females (those of $L$. durin unknown) . . 9

2. Retrolateral tibial apophysis relatively small (figs. 677, 759) .............. 3

- Retrolateral tibial apophysis larger (as in figs. $715,735) \ldots \ldots \ldots \ldots \ldots \ldots 4$

3. Terminal apophysis relatively small (fig. 676) ................... durin

- Terminal apophysis relatively large (fig. 738) ................. canungra

4. Retrolateral tibial apophysis with small, basal hook (figs. 715, 719) . . . . . . . . . 5

- Retrolateral tibial apophysis bifid (figs. 723, 727, 731, 735) . . . . . . . .6

5. Retrolateral tibial apophysis very long (fig. 715) ................. boyd

- Retrolateral tibial apophysis shorter (fig. 719) ..................... tarra

6. Dorsal prong of retrolateral tibial apophysis longer than ventral prong (fig. 727) .....

cobon

- Dorsal prong of retrolateral tibial apophysis shorter than ventral prong (figs. 723, 731, 735) ................. 7

7. Terminal apophysis relatively large (fig. 730) $\ldots \ldots \ldots \ldots$.................... bondi

- Terminal apophysis relatively small (figs.

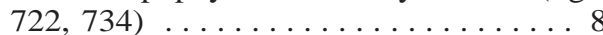

8. Tip of terminal apophysis long, sharp (fig. 722) ............... barmah

- Tip of terminal apophysis shorter, more rounded (fig. 734) ........ woodman

9. Epigynum with distinct atrium (figs. 716, $720,724) \ldots \ldots \ldots \ldots \ldots$

- Epigynum without distinct atrium (figs. 728, $732,736,740) \ldots \ldots \ldots 12$

10. Anterior epigynal hood very wide (fig. 716) .................... boyd

- Anterior epigynal hood narrower (figs. 720, 724) . . . . . . . . . . . . . 11

11. Epigynal hood widest anteriorly (fig. 720) ..

- Epigynal hood widest at middle (fig. 724) . . ................ barmah

12. Epigynal hood with pair of anterolaterally directed lobes (figs. 728, 732) ....... 13

- Epigynal hood otherwise (figs. 736, 740) ..
13. Epigynal hood relatively wide posteriorly (fig. 728) ............... cobon

- Epigynal hood relatively narrow posteriorly (fig. 732) ............. bondi

14. Epigynal hood relatively wide (fig. 736) ... .................. woodman

- Epigynal hood relatively narrow (fig. 740) $\ldots \ldots \ldots \ldots \ldots$ canungra

\section{Longepi boyd, new species}

Figures 714-717; Map 80

TYPE: Female holotype taken in a Berlese sample of mallee scrub litter at Boyd Plateau, Kanangra area, $33^{\circ} 59^{\prime} \mathrm{S}, 150^{\circ} 08^{\prime} \mathrm{E}$, New South Wales (Sept. 28, 1973; M. Gray), deposited in AMS (KS17658).

ETYMOLOGY: The specific name is a noun in apposition taken from the type locality.

DIAGNOSIS: Males can easily be recognized by the extremely long retrolateral tibial apophysis, which reaches three-quarters of the cymbial length (fig. 715), females by the very wide epigynal hood (fig. 716).

MALE: Total length 3.9. Abdominal scutum brownish orange, similar in color to carapace; sides of abdomen gray, venter white with large median square of gray; legs unmarked. Leg spination: tibia III v0-0-1p. Retrolateral tibial apophysis greatly elongated, with basal hook on dorsal side (fig. 715); terminal apophysis greatly enlarged, covering over half of ventral bulb surface (fig. 714).

FEMALE: Total length 4.9. Abdominal dorsum mottled gray, coloration otherwise as in male. Leg spination: tibia III v1p-0-2. Epigynal hood wide, with distinct lateral pockets (fig. 716); median ducts bean-shaped, lateral ducts long (fig. 717).

Other Material Examined: Australian Capital Territory: Blundells Creek, $3 \mathrm{~km} \mathrm{E}$ Piccadilly Circus, $35^{\circ} 22^{\prime} \mathrm{S}, 148^{\circ} 50^{\prime} \mathrm{E}$, Jan.Mar. 1985, elev. $850 \mathrm{~m}$ (Weir, Lawrence, Johnson, QMB), 60, 23 ; ; Blundells Creek Road, $3.5 \mathrm{~km}$ E Piccadilly Circus, $35^{\circ} 22^{\prime} \mathrm{S}$, $148^{\circ} 50^{\prime} \mathrm{E}$, Jan. 27, 1983, elev. $850 \mathrm{~m}$ (J. Lawrence, QMB), 19 ; Lees and Blundells Creek, Brindabella Ranges, $35^{\circ} 22^{\prime} \mathrm{S}$, $148^{\circ} 50^{\prime} \mathrm{E}, 1979-1980$, pitfalls (C. Dickman, QMB), 20 , Oct. 1981, pitfall (C. Dickman, QMB), 10; $1 \mathrm{~km} \mathrm{~N}$ Mount Gingera, 35 33'S, $148^{\circ} 47^{\prime} \mathrm{E}$, Feb. 18, 1981, Berlese, leaf litter (A. Calder, QMB), 19 ; Tidbinbilla Nature Reserve, $35^{\circ} 28^{\prime} \mathrm{S}, 148^{\circ} 52^{\prime} \mathrm{E}$, Mar. 9, 1978, 

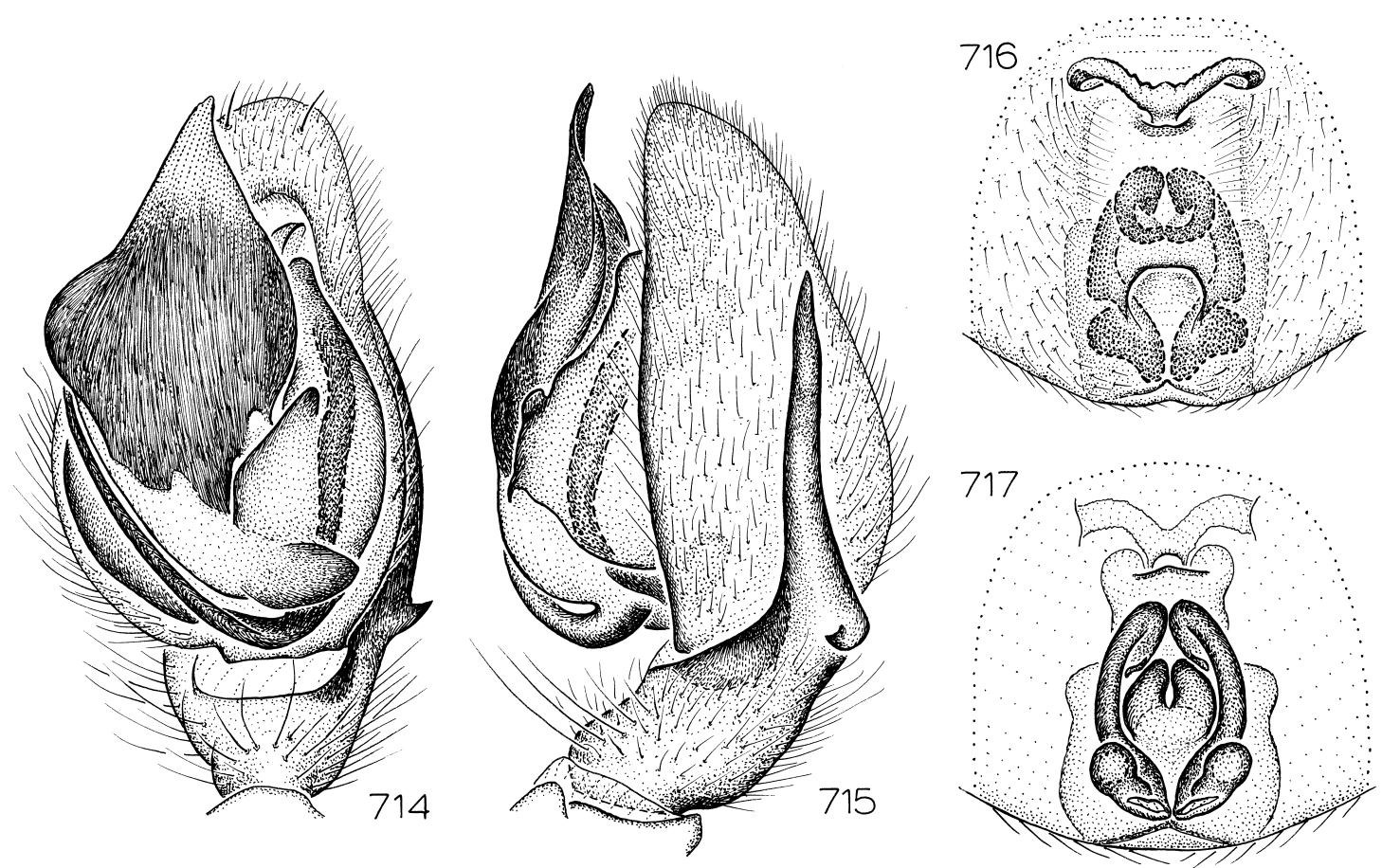

Figs. 714-717. Longepi boyd, new species. 714. Left male palp, ventral view. 715. Same, retrolateral view. 716. Epigynum, ventral view. 717. Same, dorsal view.

pitfall (P. Ormay, AMS KS13829), 2 ㅇ. New South Wales: Bondi State Forest, S Bombala, $37^{\circ} 08^{\prime} \mathrm{S}, 149^{\circ} 09^{\prime} \mathrm{E}$, Feb. 3, 1980, pitfall, litter, open forest (G. Gowing, AMS

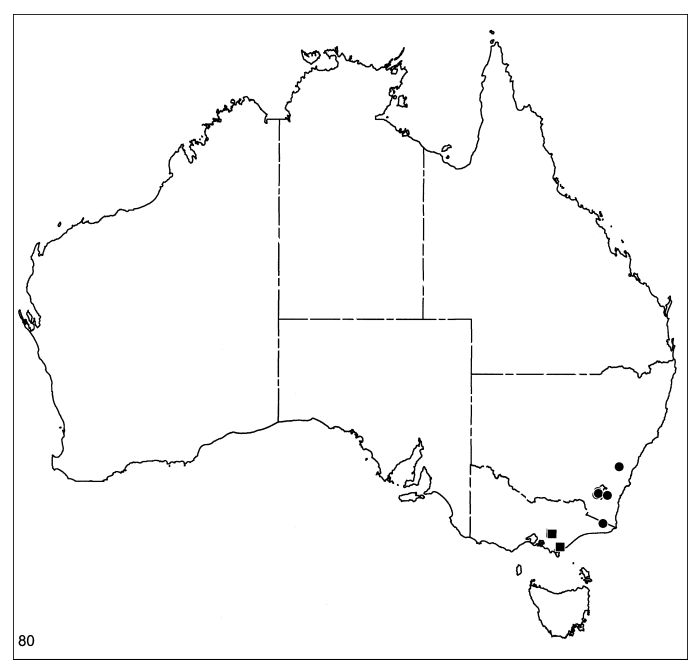

Map 80. Records of Longepi boyd, new species (circles) and L. tarra, new species (squares).
KS18039), $19 ; 8.8$ km ESE Captain's Flat, $35^{\circ} 35^{\prime} \mathrm{S}, 149^{\circ} 26^{\prime} \mathrm{E}$, Jan. 10 , 1970 , dry sclerophyll (R. Taylor, QMB), 1 \%; South Ramshead, Feb. 1983, pitfall, elev. $2000 \mathrm{~m}$ (K. Green, QMB), 2 \% .

Distribution: New South Wales and the Australian Capital Territory (map 80).

\section{Longepi tarra, new species \\ Figures 718-721; Map 80}

TYPE: Female holotype taken in pitfall trap in Nothofagus cunninghamii forest $0.2 \mathrm{~km} \mathrm{~W}$ Tarra Valley Picnic Area, Tarra-Bulga National Park, Strzlecki Ranges, $38^{\circ} 27^{\prime} \mathrm{S}$, $146^{\circ} 32^{\prime} \mathrm{E}$, Victoria (Nov. 14, 1995-Jan. 10, 1996; G. Milledge), deposited in NMV (K4658).

ETYMOLOGY: The specific name is a noun in apposition taken from the type locality.

DiAGNOSIS: Males and females have not been collected together, but are matched here on the basis of their respective similarities to those of L. boyd; males have a shorter retrolateral tibial apophysis (fig. 719) and a longer 

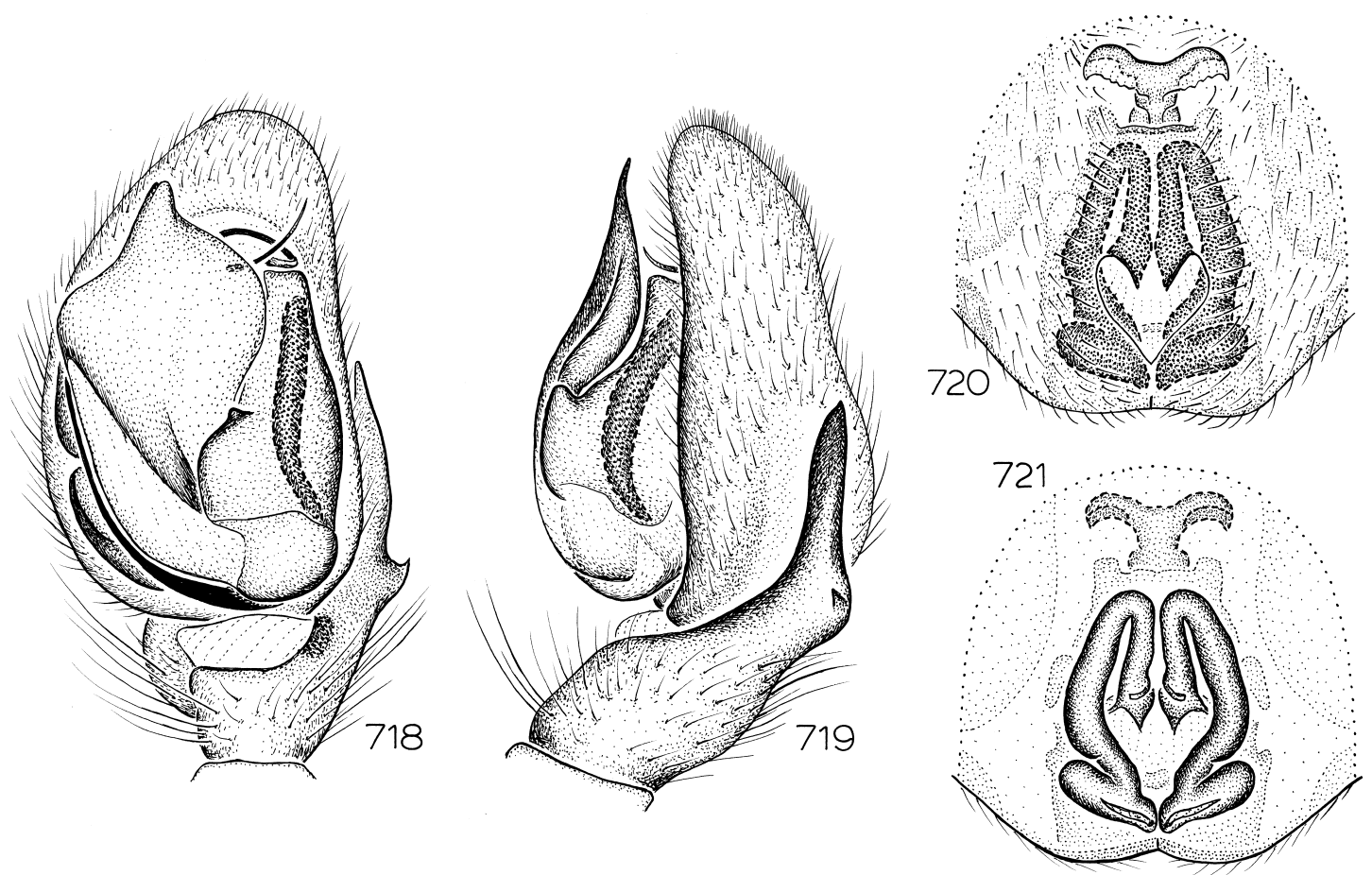

Figs. 718-721. Longepi tarra, new species. 718. Left male palp, ventral view. 719. Same, retrolateral view. 720. Epigynum, ventral view. 721. Same, dorsal view.

narrowed portion of the terminal apophysis (fig. 718); females have a wider, heart-shaped epigynal atrium (fig. 720).

MALE: Total length 3.7. Abdominal scutum dark reddish brown; coloration otherwise as in L. boyd. Leg spination: tibia IV v0-0-2. Retrolateral tibial apophysis with basal hook, extending only about half of cymbial length, sharply bent at about onethird its length (fig. 719); terminal apophysis wide but narrowed apical portion relatively long (fig. 718).

FEMALE: Total length 4.5. Abdominal dorsum mottled gray, coloration otherwise as in male. Leg spination typical for genus. Epigynal atrium wide, heart-shaped, with anterolateral openings (fig. 720); median ducts long, longitudinal (fig. 721).

Other MATERIAl ExAmined: Victoria: $11.4 \mathrm{~km}$ ENE McMahons Creek, 37²1's, $145^{\circ} 57^{\prime} \mathrm{E}$, Nov. 14-24, 1988 (L. Lumsden, NMV K3632), 10; $9.2 \mathrm{~km} \mathrm{NW} \mathrm{Toorongo,}$ 374ㄴ' S, $146^{\circ} 02^{\prime} \mathrm{E}$, Nov. 14-24, 1988 (L. Lumsden, NMV K3630), 10.

DisTRIBUTION: Victoria (map 80).
Longepi barmah, new species Figures 722-725; Map 81

TYPE: Female holotype from taken in pitfall trap on McDonalds Road, $1.8 \mathrm{~km}$ S Shepparton-Barmah Road, $36^{\circ} 04^{\prime} \mathrm{S}, 145^{\circ} 02^{\prime} \mathrm{E}$, Victoria (Jan. 17-22, 1994; G. Milledge), deposited in NMV.

ETYMOLOGY: The specific name is a noun in apposition taken from the type locality.

DiaGNOSIS: Males of this distinctive species can easily be recognized by the short, bifurcated retrolateral tibial apophysis (fig. 723) and narrow terminal apophysis (fig. 722), females by the lateral pockets of the epigynal hood (fig. 724) and posteriorly enlarged lateral ducts (fig. 725).

MaLE: Total length 3.6. Coloration as in $L$. boyd. Leg spination: tibiae: III v1p-0-2; IV r0-1-1. Retrolateral tibial apophysis bifurcate, sinuous (fig. 723); terminal apophysis narrow, distal portion directed retrolaterally (fig. 722).

FEMALE: Total length 3.7. Coloration as in L. boyd. Leg spination as in male. Epigynal 

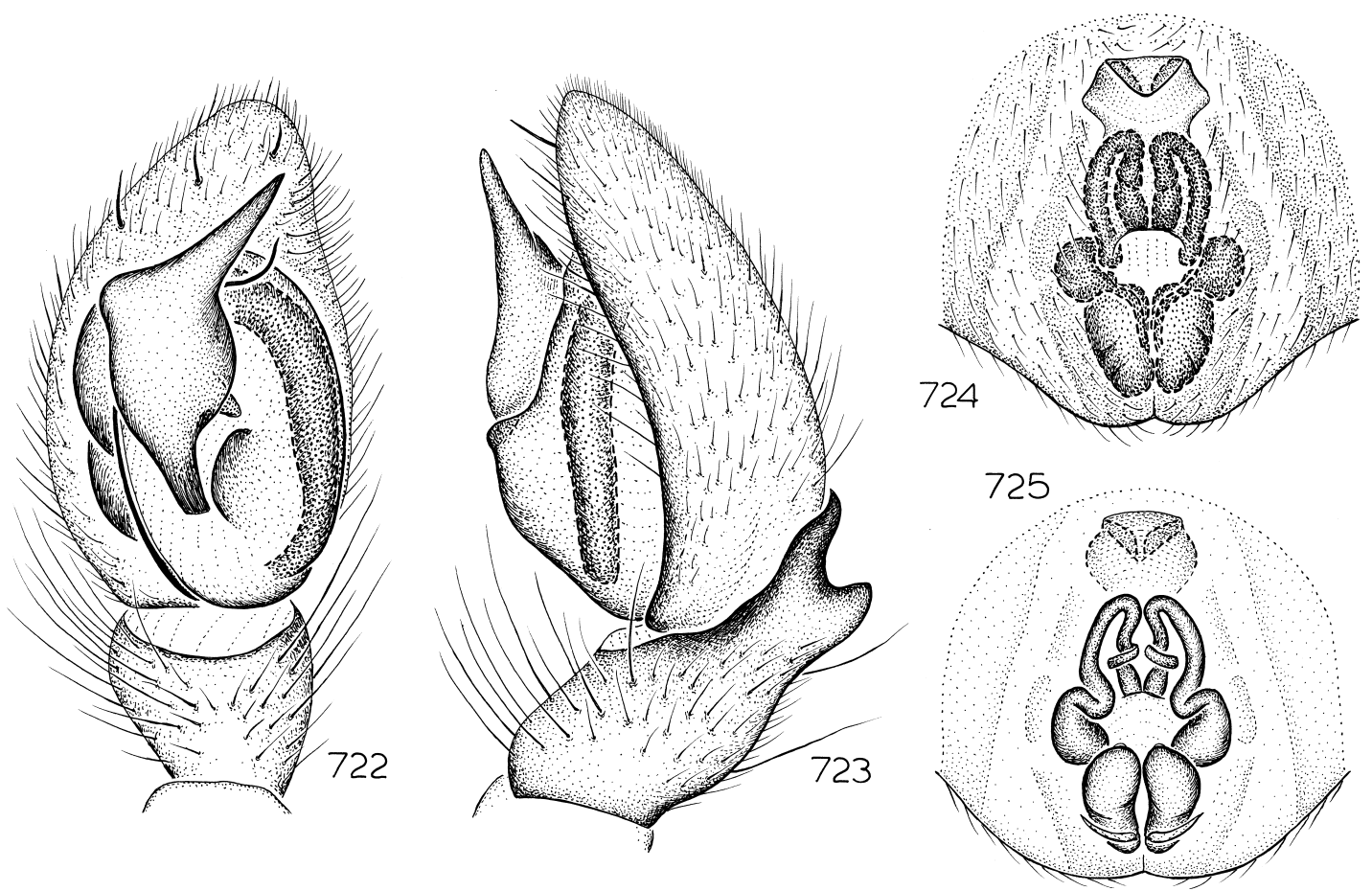

Figs. 722-725. Longepi barmah, new species. 722. Left male palp, ventral view. 723. Same, retrolateral view. 724. Epigynum, ventral view. 725. Same, dorsal view.

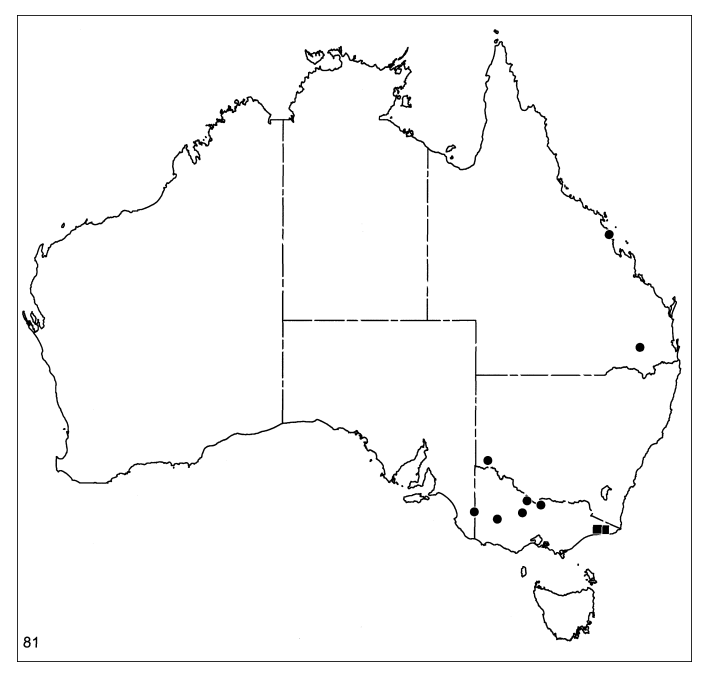

Map 81. Records of Longepi barmah, new species (circles) and L. cobon, new species (squares). hood beak-shaped, with lateral pockets (fig. 724); lateral ducts enlarged, sinuous posteriorly (fig. 725).

Other Material Examined: New South Wales: $5 \mathrm{~km} \mathrm{~N}$ Bunningeree, at crossing of Ana branch of Darling River, at Silver City Highway, $33^{\circ} 36^{\prime} \mathrm{S}, 141^{\circ} 45^{\prime} \mathrm{E}$, Aug. 22, 1981, eucalypt litter (M. Kotzman, QMB), 1 ㅇ․ Queensland: Lake Broadwater, via Dalby, $27^{\circ} 21^{\prime} \mathrm{S}, 151^{\circ} 06^{\prime} \mathrm{E}$, May 6-Nov. 24, 1985, pitfalls (M. Bennie, QMB S28596, S28635), 35 ๙ , 9 오, Nov. 24, 1985-Jan. 3, 1986, pitfalls (M. Bennie, QMB S28667), 4어, Jan. 3Feb. 25, 1986, pitfalls (M. Bennie, QMB S28617), 2\%; $90 \mathrm{mi} \mathrm{S}$ Mackay, $21^{\circ} 09^{\prime} \mathrm{S}$, $149^{\circ} 12^{\prime} \mathrm{E}$, July 18 , 1968 , Berlese, Acacia harpophylla litter (L. Mound, QMB), 1 ㅇ. South Australia: $3.7 \mathrm{~km}$ NE Pooginagoric, $36^{\circ} 27^{\prime} \mathrm{S}, 140^{\circ} 55^{\prime} \mathrm{E}$, Dec. 5-8, 1995, pitfall (SAM N1997/80), 1 ㅇ․ Victoria: Cohuna, $35^{\circ} 50^{\prime} \mathrm{S}, 144^{\circ} 11^{\prime} \mathrm{E}$, Nov. 16-21, 1996, shelterbelt pitfall (J. Shield, J. Hooper, CVIC), 19 , Dec. 1996-Jan. 1997, pitfall (J. Hooper, CVIC, 2 ; $1 \mathrm{~km}$ NNW Inglewood, 36 34'S, $143^{\circ} 51^{\prime} \mathrm{E}$, Oct. 15-20, 1989 (A. Coventry, 


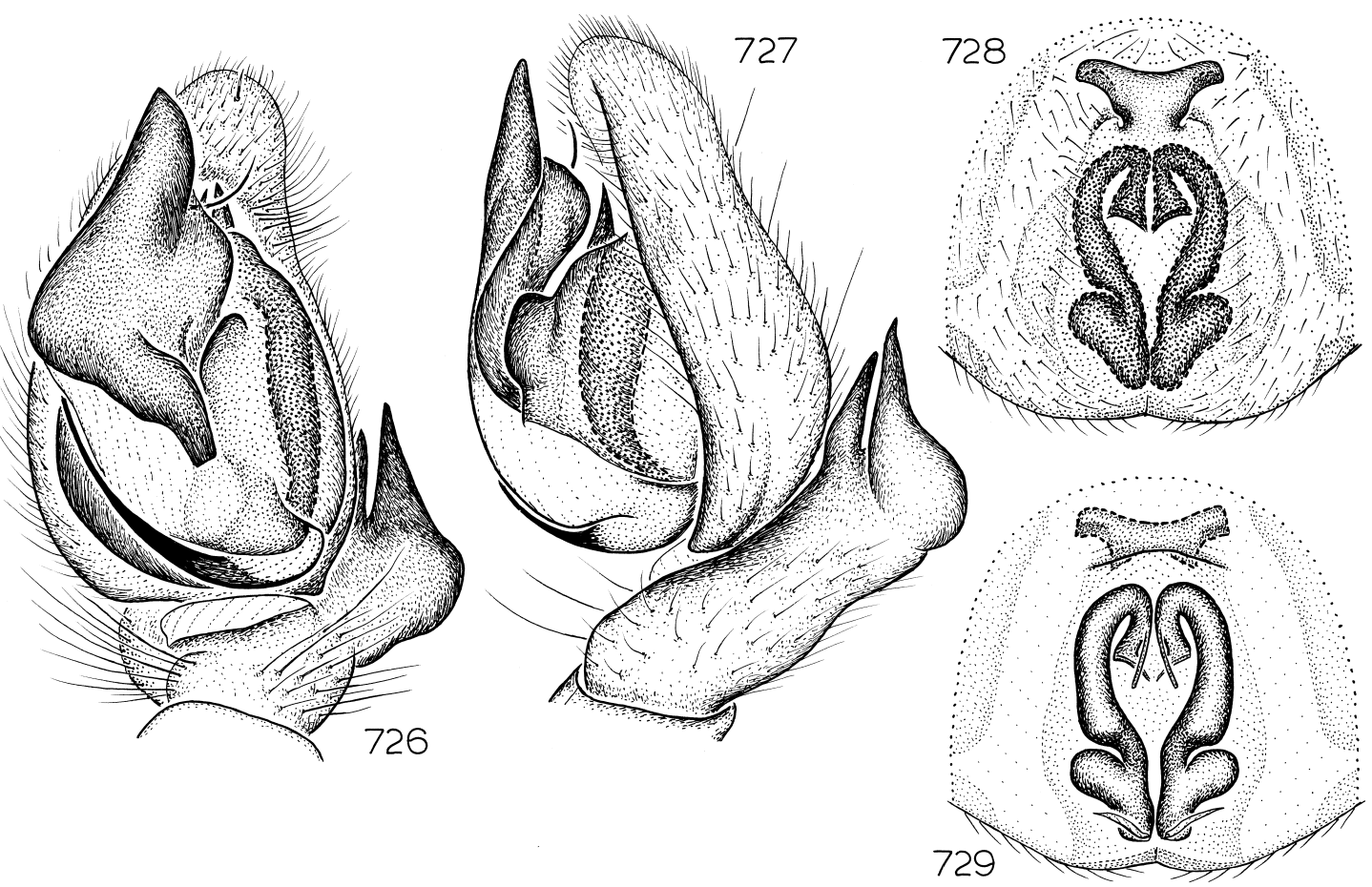

Figs. 726-729. Longepi cobon, new species. 726. Left male palp, ventral view. 727. Same, retrolateral view. 728. Epigynum, ventral view. 729. Same, dorsal view.

NMV K4640), 2ð; 2 km NNW Inglewood, $36^{\circ} 33^{\prime} \mathrm{S}, 143^{\circ} 51^{\prime} \mathrm{E}$, Oct. 15-20, 1989 (A. Coventry, NMV K4640), 10; 7.5 km NNW Inglewood, $36^{\circ} 30^{\prime} \mathrm{S}, 143^{\circ} 53^{\prime} \mathrm{E}$, Oct. 15-20, 1989 (A. Coventry, NMV K4643), 3 đ; Potter Creek, $1.7 \mathrm{~km} \mathrm{~S}$ Western Highway, $36^{\circ} 50^{\prime} \mathrm{S}, 142^{\circ} 21^{\prime} \mathrm{E}$, Nov. 6, 1991, pitfall (S. Larwill, NMV K3644), 10.

Distribution: Eastern Australia, except Tasmania (map 81).

\section{Longepi cobon, new species Figures 726-729; Map 81}

TYPE: Female holotype from midslope pitfall trap at Cobon North Coupe, $37^{\circ} 22^{\prime} \mathrm{S}$, $148^{\circ} 56^{\prime} \mathrm{E}$, Victoria (Dec, 12-20, 1991; R. Coy), deposited in NMV (K3621).

ETYMOLOGY: The specific name is a noun in apposition taken from the type locality.

DiAGNOSIS: Males can easily be recognized by the expanded knob on the dorsal prong of the retrolateral tibial apophysis (fig. 727), females by the Y-shaped epigynal hood (fig. 728).
MALE: Total length 3.5. Coloration as in $L$. tarra except abdominal venter uniformly gray. Leg spination: tibia IV v0-0-2. Retrolateral tibial apophysis with two prongs, subequal in length, dorsal prong with expanded knob (fig. 727); terminal apophysis only partially obscuring median apophysis in ventral view (fig. 726).

FEMALE: Total length 5.3. Coloration as in L. tarra except abdominal venter uniformly gray. Leg spination typical for genus. Epigynal hood Y-shaped, with posterolateral pockets, atrium reduced to $\mathrm{V}$-shaped ridge anterior of openings (fig. 728); lateral ducts very long (fig. 729).

OTHER MATERIAL EXAMINED: Victoria: Cobon South Coupe, $37^{\circ} 25^{\prime} \mathrm{S}, 148^{\circ} 58^{\prime} \mathrm{E}$, Nov. 10-17, 1991, midslope pitfall (R. Coy, NMV K3606), 1 đ, Dec. 9-15, 1991, upslope pitfalls (R. Coy, NMV K3602), 20 ; Sardine Coupe, $37^{\circ} 24^{\prime} \mathrm{S}, 148^{\circ} 31^{\prime} \mathrm{E}$, Jan. 23-30, 1992, pitfall, gully (R. Coy, NMV K3557), 10 .

DistRIBUTION: Eastern Victoria (map 81). 

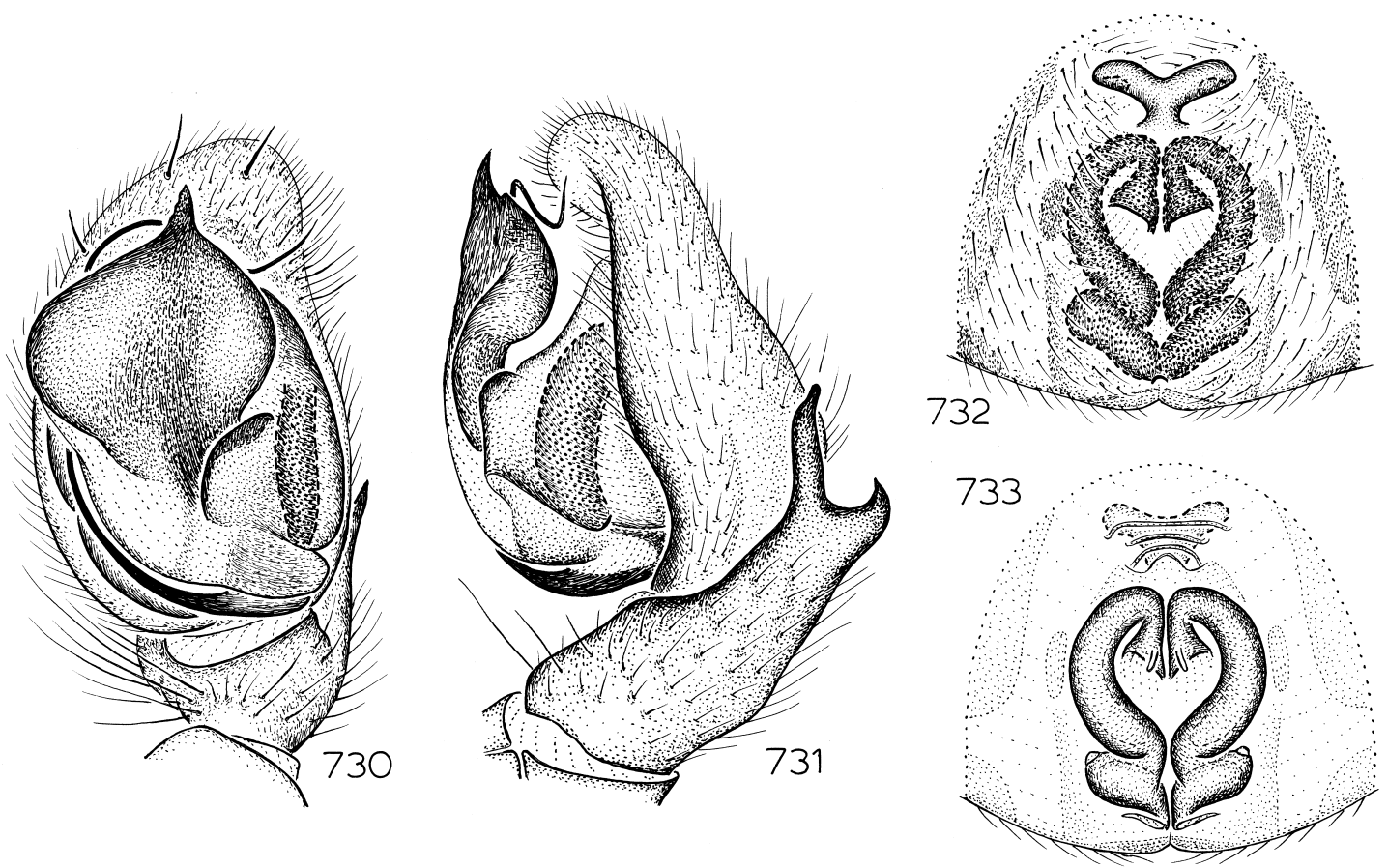

Figs. 730-733. Longepi bondi, new species. 730. Left male palp, ventral view. 731. Same, retrolateral view. 732. Epigynum, ventral view. 733. Same, dorsal view.

\section{Longepi bondi, new species} Figures 730-733; Map 82

TYPE: Female holotype taken in eucalypt litter in Bondi State Forest, S Bombala, $37^{\circ} 08^{\prime} \mathrm{S}, 149^{\circ} 09^{\prime} \mathrm{E}$, New South Wales (Dec. 13, 1980; G. Gowing), deposited in AMS (KS11889).

ETYMOLOGY: The specific name is a noun in apposition taken from the type locality.

DIAGNOSIS: Males and females have not been collected together, but are matched here on the basis of their respective similarities to those of $L$. cobon; males can be recognized by the relatively short dorsal prong on the retrolateral tibial apophysis (fig. 731), females by the v-shaped epigynal hood (fig. 732).

MALE: Total length 3.8. Coloration as in $L$. tarra. Leg spination: tibiae: III missing; IV v1p-0-2. Retrolateral tibial apophysis with long, rectangular base, short dorsal fork, and sinuous ventral fork (fig. 731); terminal apophysis very wide (fig. 730).

FEMALE: Total length 4.9. Coloration as in L. tarra. Leg spination as in male. Epigynal hood narrow posteriorly, with posterolateral pockets, atrium reduced to $\mathrm{v}$-shaped ridge anterior of openings (fig. 732); lateral ducts almost semicircular (fig. 733).

OTHER MATERIAL EXAMINED: Victoria: Jeeralang West Road, $0.1 \mathrm{~km} \mathrm{~N}$ Binns Hill Junction, Strzelecki Ranges, $38^{\circ} 27^{\prime} \mathrm{S}$, $146^{\circ} 29^{\prime}$ E, Nov. 14, 1995-Jan. 10, 1996, pitfall, eucalypt forest (G. Milledge, NMV

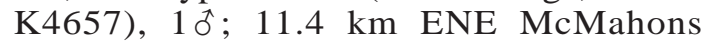
Creek, $37^{\circ} 41^{\prime} \mathrm{S}, 145^{\circ} 57^{\prime} \mathrm{E}$, Nov. 14-24, 1988 (L. Lumsden, ex NMV K3632), 10 .

DistRIBUTION: Eastern Victoria and adjacent New South Wales (map 82).

\section{Longepi woodman, new species} Figures 734-737; Map 82

TyPES: Female holotype and male allotype taken in pitfall traps at Woodman Point, $32^{\circ} 08^{\prime} \mathrm{S}, 115^{\circ} 45^{\prime} \mathrm{E}$, Western Australia (June 28-Sept. 1, 1994; J. Waldock, A. Longbottom), deposited in WAM $(96 / 265,266)$.

ETYMOLOGY: The specific name is a noun in apposition taken from the type locality.

DiAGNOSIS: Males can be recognized by 

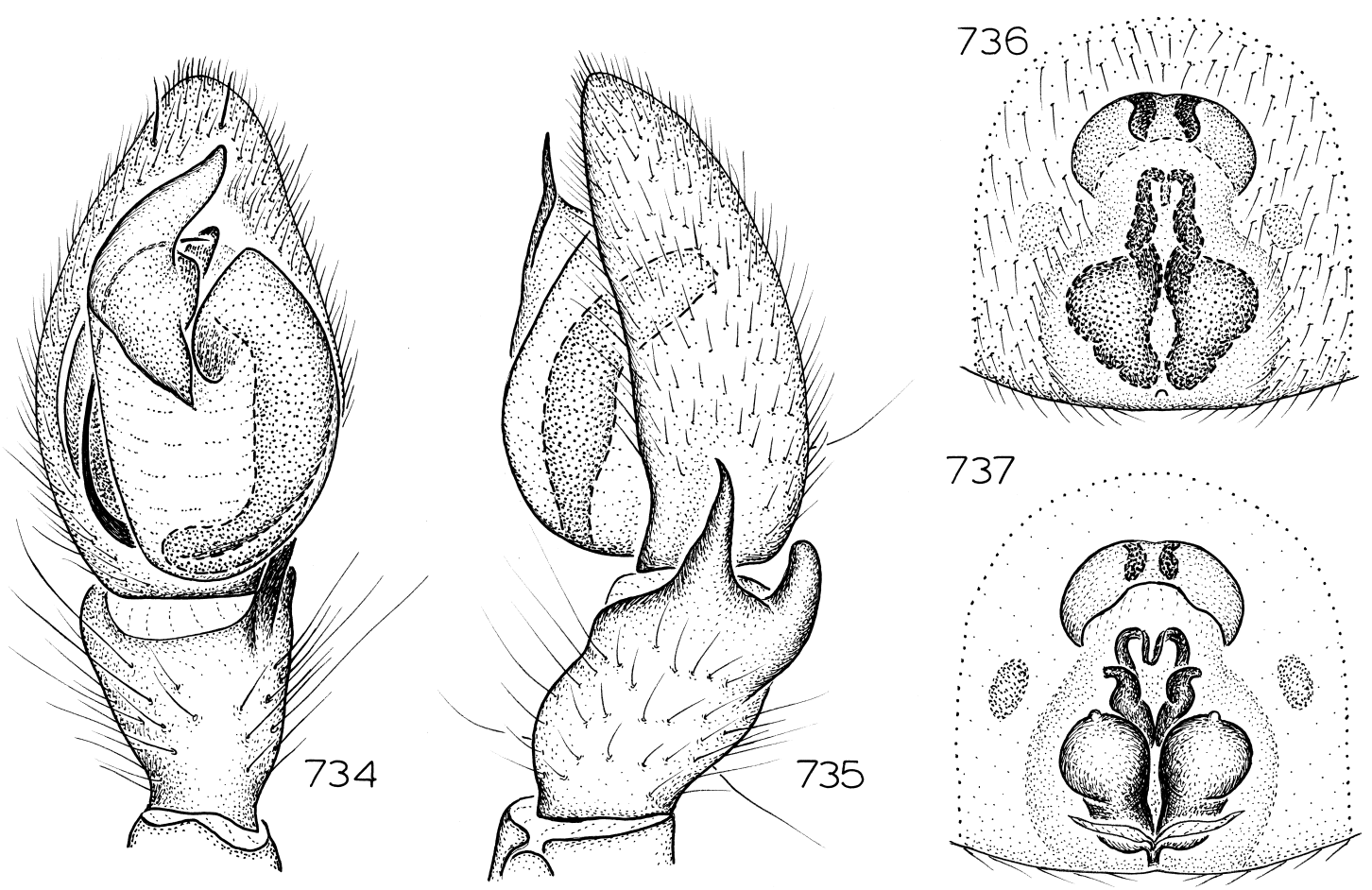

Figs. 734-737. Longepi woodman, new species. 734. Left male palp, ventral view. 735. Same, retrolateral view. 736. Epigynum, ventral view. 737. Same, dorsal view.

the widely separated arms of the bifid retrolateral tibial apophysis (fig. 735) and the relatively small terminal apophysis (fig. 734), females by the rounded epigynal hood (fig. 736) and small anterior epigynal ducts (fig. 737).

MALE: Total length 2.8. Dorsal abdominal scutum darker than carapace, coloration otherwise as in L. boyd. Leg spination: femur IV d1-0-0; tibiae: III v1p-0-2; IV r1-0-1; metatarsus IV v1p-0-0. Retrolateral tibial apophysis bifid, arms widely separated (fig. 735); terminal apophysis relatively small (fig. 734).

FEMALE: Total length 3.3. Coloration as in L. boyd. Leg spination: femur IV d1-0-0; tibiae: III v1p-0-2; IV r1-0-1; metatarsus IV v1p-0-0. Epigynal hood relatively large, rounded (fig. 736); anterior epigynal ducts relatively small (fig. 737).

Other Material Examined: Australian Capital Territory: Black Mountain, $35^{\circ} 16^{\prime} \mathrm{S}, 149^{\circ} 06^{\prime} \mathrm{E}$, July 10 , 1976 , dry sclerophyll (T. Weir, QMB), 20, Oct.-Nov. 1983,

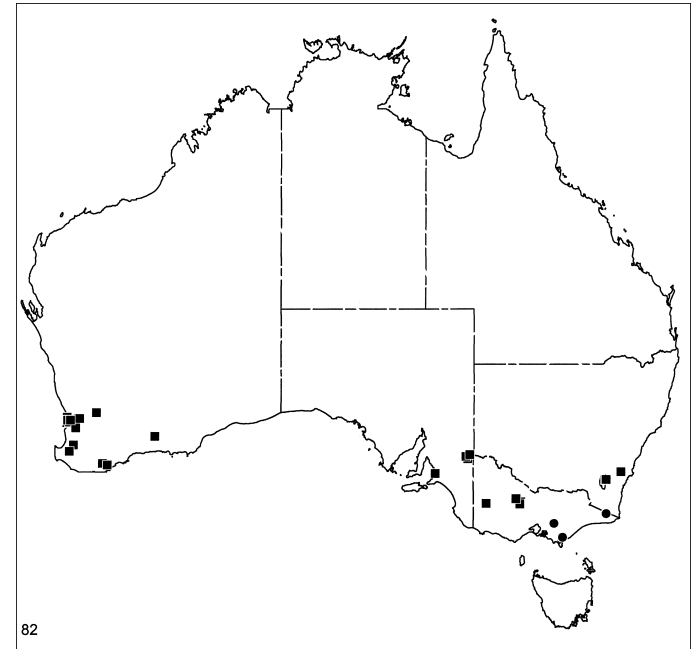

Map 82. Records of Longepi bondi, new species (circles) and L. woodman, new species (squares). 
pitfalls (QMB), 7ð; $\mathrm{N}$ face, Black Mountain, $35^{\circ} 16^{\prime} \mathrm{S}$, $149^{\circ} 06^{\prime} \mathrm{E}$, Dec. 5, 1969, Berlese, dry sclerophyll, elev. $640 \mathrm{~m}$ (C. Brooks, QMB), 19 ; Canberra, $35^{\circ} 17^{\prime} \mathrm{S}, 149^{\circ} 13^{\prime} \mathrm{E}$, Oct. 17, 1991, on wall in garden (A. Longbottom, QMB), 1ơ; Cook, Sept. 30, 1983, on floor (M. Harvey, QMB), $1 \delta^{\text {* }}$, Oct. 3, 1983, under clothes (M. Harvey, QMB), 30 , Oct. 9, 1983, in grass (M. Harvey, QMB), $1 \hat{0}$, Oct. 16, 1983, in house (M. Harvey, QMB), $10^{\star}$; E face, Mount Ainslie, $35^{\circ} 16^{\prime} \mathrm{S}$, $149^{\circ} 10^{\prime} \mathrm{E}$, Nov. 25 , 1969, Berlese, dry sclerophyll litter, elev. 840 m (C. Brooks, QMB), $1 \%$; Mount Pleasant, Dec. 13, 1969, Berlese, dry sclerophyll (C. Brooks, QMB), 1 ㅇ. New South Wales: Bungonia Caves, near Information Center, $34^{\circ} 50^{\prime} \mathrm{S}, 150^{\circ} 04^{\prime} \mathrm{E}$, mid Oct.mid Nov. 1989, pitfall (G. Hunt, AMS KS22561), $5 \sigma^{\star}$. South Australia: Mitcham, Adelaide, $34^{\circ} 56^{\prime} \mathrm{S}, 138^{\circ} 36^{\prime} \mathrm{E}$, Sept. 1-30, 1975 (R. Southcott, SAM N1996/370), 10; $14 \mathrm{~km}$ WNW Renmark, 34 $07^{\prime} \mathrm{S}, 140^{\circ} 37^{\prime} \mathrm{E}$, Aug. 8-21, 1995, pitfall, mallee on dune (K. Lambie, QMB), 10; $31 \mathrm{~km}$ NW Renmark, $33^{\circ} 59^{\prime} \mathrm{S}, 140^{\circ} 30^{\prime} \mathrm{E}$, July 6-Aug. 10, 1995, flight intercept (K. Pullen, QMB), $10 ; 32 \mathrm{~km}$ N Renmark, $33^{\circ} 53^{\prime} \mathrm{S}, 140^{\circ} 44^{\prime} \mathrm{E}$, July 6-Aug. 10, 1995, flight intercept (K. Pullen, QMB), $10^{\circ}$; Windsor Gardens, Adelaide, $34^{\circ} 56^{\prime} \mathrm{S}$, $138^{\circ} 36^{\prime}$ E, Sept.-Oct. 1983 (D. Hirst, SAM

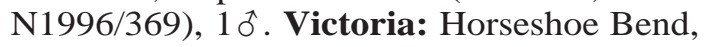
Little Desert National Park, 36 $35^{\prime} \mathrm{S}$, $141^{\circ} 45^{\prime} \mathrm{E}$, July 6, 1982, Eucalyptus camaldulensis litter (M. Harvey, B. Roberts, NMV), $10^{\star} ; 2 \mathrm{~km}$ NNW Inglewood, $36^{\circ} 33^{\prime} \mathrm{S}$, $143^{\circ} 51^{\prime} \mathrm{E}$, Oct. $15-20,1989$, mallee (A. Cov-

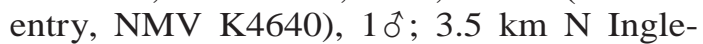
wood, $36^{\circ} 32^{\prime} \mathrm{S}, 143^{\circ} 51^{\prime} \mathrm{E}$, Oct. 15-20, 1989, mallee (A. Coventry, NMV K4641), $10^{\text {ț }} 5$ $\mathrm{km} \mathrm{N}$ Inglewood, $36^{\circ} 31^{\prime} \mathrm{S}, 143^{\circ} 52^{\prime} \mathrm{E}$, Oct. 15-20, 1989, mallee (A. Coventry, NMV K4642), $10^{\star} ; 7.5 \mathrm{~km}$ NNW Inglewood, $36^{\circ} 30^{\prime} \mathrm{S}, 143^{\circ} 53^{\prime} \mathrm{E}$, Oct. 15-20, 1989, mallee (A. Coventry, NMV K4643), 10; 7 km SSE Kingower, $36^{\circ} 37^{\prime} \mathrm{S}, 143^{\circ} 50^{\prime} \mathrm{E}$, Oct. 15-20, 1989, mallee (A. Coventry, NMV K4646, 4664), $20^{\text {` }} 77.5 \mathrm{~km}$ ENE Kingower, $36^{\circ} 36^{\prime} \mathrm{S}$, $143^{\circ} 50^{\prime} \mathrm{E}$, Oct. $15-20,1989$, mallee (A. Coventry, NMV K4644, 4645), 20; 4 km S Wychitella, $36^{\circ} 19^{\prime} \mathrm{S}, 143^{\circ} 36^{\prime} \mathrm{E}$, Oct. 15-20, 1989, mallee (A. Coventry, NMV K4647), $10^{\top} ; 9 \mathrm{~km} \mathrm{~S}$ Wychitella, $36^{\circ} 21^{\prime} \mathrm{S}, 143^{\circ} 35^{\prime} \mathrm{E}$, Oct. 15-20, 1989, mallee (A. Coventry,
NMV K4648), 10 . Western Australia: Bold Park, $31^{\circ} 57^{\prime}$ S, $115^{\circ} 46^{\prime}$ E, July $20-$ Sept. 24, 1993, pitfalls (J. Waldock, WAM 96/253, 275-277), 30ิ, 1오. Aug. 24-29, 1993, dry pitfall (R. How, WAM 96/261), 1 đ̊; Collie, $33^{\circ} 22^{\prime} \mathrm{S}, 116^{\circ} 09^{\prime} \mathrm{E}$, Oct. 4 , 1977 , tree pitfall (S. Curry, WAM 96/254), $1 \delta^{\dagger}$; East Victoria Park, $31^{\circ} 59^{\prime} \mathrm{S}, 115^{\circ} 54^{\prime} \mathrm{E}$, Sept. 22, 1994, found dead (J. Waldock, WAM 96/258), 1 đ。; junction, Great Southern Highway and Yarra Road, $31^{\circ} 54^{\prime} \mathrm{S}, 116^{\circ} 28^{\prime} \mathrm{E}$, Aug. 13, 1994, under bark of log (M. Harvey, M. Blosfelds, WAM 96/260), 10; Hepburn Heights, $31^{\circ} 49^{\prime} \mathrm{S}, 115^{\circ} 46^{\prime} \mathrm{E}$, July 13-Sept. 25, 1995, pitfall (M. Harvey, J. Waldock, WAM 96/ 252, 257), $20^{\circ}$; Jandakot Airport, $32^{\circ} 06^{\prime} \mathrm{S}$, $115^{\circ} 53^{\prime}$ E, July 16-Sept. 1, 1994, pitfall (J. Waldock, A. Longbottom, WAM 96/259), $10^{\dagger}$; Kirup, $33^{\circ} 43^{\prime} \mathrm{S}, 115^{\circ} 54^{\prime} \mathrm{E}$, Nov. 18, 1977, pitfall (S. Curry, WAM 96/283), 10; Mount Claremont, $31^{\circ} 58^{\prime} \mathrm{S}, 115^{\circ} 46^{\prime} \mathrm{E}$, June 24-Sept. 1, 1994, pitfalls (J. Waldock, A. Longbottom, WAM 96/249, 250), 20 , Sept. 1-Nov. 4, 1994, pitfall (J. Waldock, A. Longbottom, WAM 96/274), 1 \%; Mount Cooke, $32^{\circ} 25^{\prime} \mathrm{S}, 116^{\circ} 18^{\prime} \mathrm{E}$, Sept. 19-Nov. 28, 1991, pitfall (J. Waldock, C. Car, WAM 96/251, 262), 2o; Mount Observation, $31^{\circ} 54^{\prime} \mathrm{S}, 116^{\circ} 33^{\prime} \mathrm{E}$, Aug. 13, 1994, under rock (M. Harvey, M. Blosfelds, WAM 96/ 256), 1 ò $^{\dagger}$ Nedlands, $31^{\circ} 59^{\prime} \mathrm{S}, 115^{\circ} 48^{\prime} \mathrm{E}$, Sept. 29, 1993 (A. Baynes, WAM 96/278, 279), 2 \%; North Bungalla, $31^{\circ} 33^{\prime} \mathrm{S}$, $117^{\circ} 35^{\prime}$ E, June 23-Aug. 4, 1987, pitfall (B. Main, WAM 96/282), $10^{\text {t; }}$ North Bungalla, $31^{\circ} 35^{\prime} \mathrm{S}, 117^{\circ} 35^{\prime} \mathrm{E}$, June 23-Aug. 4, 1987, pitfall (B. Main, WAM 96/280), $1 \delta^{\top}$; Peak Charles, 32 $53^{\prime} \mathrm{S}, 121^{\circ} 10^{\prime} \mathrm{E}$, Aug. 2, 1992 , salmon gum litter (M. Cowan, WAM 96/ 281), $10^{\circ}$; Perth Airport, $31^{\circ} 59^{\prime} \mathrm{S}, 115^{\circ} 58^{\prime} \mathrm{E}$, Sept. 24-Nov. 18, 1993, pitfall (J. Waldock, WAM 96/272), 1 \% ; Talyuberlup Picnic Area, Stirling Range National Park, $34^{\circ} 24^{\prime} \mathrm{S}$, $117^{\circ} 57^{\prime}$ E, Oct. 29-Nov. 4, 1984 (QMB), 30'; W Two Mile Lake, Stirling Range National Park, $34^{\circ} 29^{\prime}$ S, $118^{\circ} 15^{\prime}$ E, Sept. 10-20, 1990, pitfall (G. Friend, WAM 96/1534), $1 \delta^{\text {; }}$; Warwick Open Space, $31^{\circ} 51^{\prime} \mathrm{S}, 115^{\circ} 49^{\prime} \mathrm{E}$, July 13-Sept. 25, 1995, pitfall (M. Harvey, J. Waldock, WAM 96/255), 10; Woodman Point, $32^{\circ} 08^{\prime} \mathrm{S}, 115^{\circ} 45^{\prime} \mathrm{E}$, June $28-$ Sept. 1 , 1994, pitfalls (J. Waldock, A. Longbottom, WAM 96/267-269), 3 o, Sept. 1-Nov. 4, 

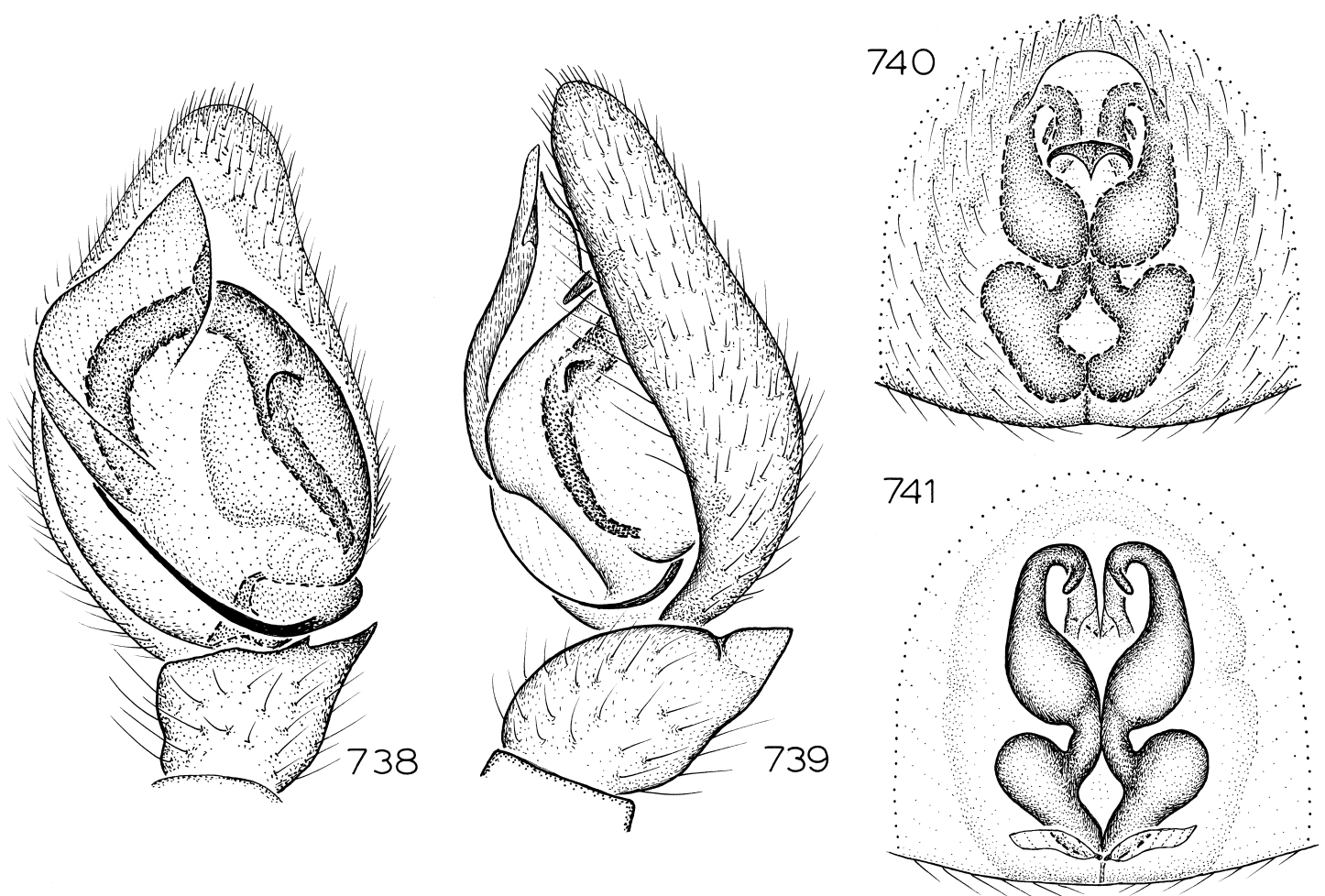

Figs. 738-741. Longepi canungra, new species. 738. Left male palp, ventral view. 739. Same, retrolateral view. 740. Epigynum, ventral view. 741. Same, dorsal view.

1994, pitfalls (J. Waldock, A. Longbottom, WAM 96/263, 264, 270, 271, 273), 1 ㅊ, 4 ㅇ․

Distribution: Southern Australia, except Tasmania (map 83).

\section{Longepi canungra, new species}

Figures 738-741; Map 83

TYPE: Female holotype taken in Berlese sample of stick brushings at Curtis Farm, Canungra, $28^{\circ} 04^{\prime} \mathrm{S}, 153^{\circ} 07^{\prime} \mathrm{E}$, Queensland (Sept. 9, 1979; G. Monteith), deposited in QMB (S26512).

ETYMOLOGY: The specific name is a noun in apposition taken from the type locality.

DiAGNOSIS: Males of this distinctive species can easily be recognized by the very short retrolateral tibial apophysis (fig. 739), females by the very small anterior epigynal hood (fig. 740).

MALE: Only known specimen badly faded, in poor condition. Total length 3.3. Coloration was apparently as in L. boyd. Leg spination apparently: tibiae: III v0-2-2; IV v0-

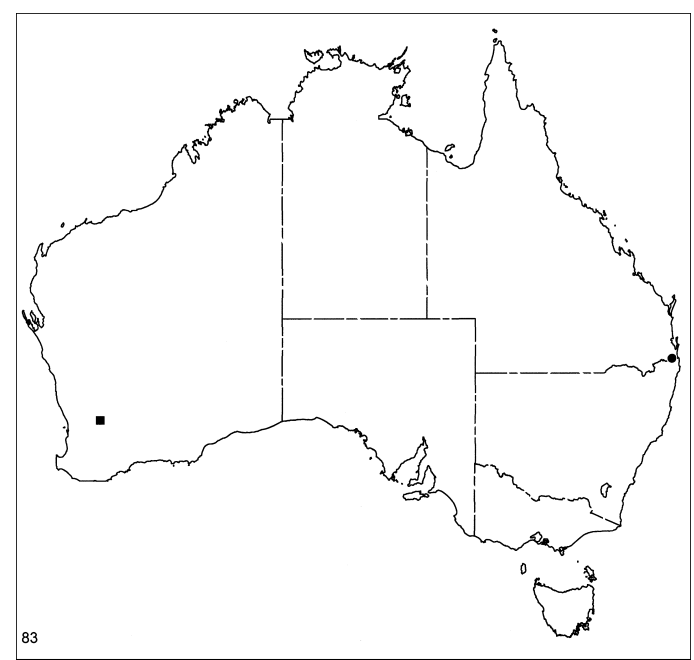

Map 83. Records of Longepi canungra, new species (circle) and L. durin, new species (square). 
0-2, r0-0-0. Palp faded, sclerites difficult to see; retrolateral tibial apophysis short, bifid (fig. 739); terminal apophysis occupying anteroprolateral quarter of bulb (fig. 738).

FEMALE: Total length 4.6. Unsclerotized portions of abdomen uniformly dirty white, coloration otherwise as in L. boyd. Leg spination: tibiae: III v0-0-0; IV v0-0-2. Epigynal openings situated anteriorly on either side of short septum (fig. 740), ducts with ventral diverticula anterior of openings (fig. 741).

Other MATERIAl ExAMINED: Queensland: Curtis Farm, Canungra, $28^{\circ} 04^{\prime} \mathrm{S}, 153^{\circ} 07^{\prime} \mathrm{E}$, Aug. 13-Dec. 2, 1977, scrub, elev. $120 \mathrm{~m}$, pitfall (G., S. Monteith, QMB S28357), 1 t.

DisTRIBUTION: Known only from southeastern Queensland (map 83).

\section{Longepi durin, new species} Figures 676, 677; Map 83

TYPE: Male holotype from Durokoppin Nature Reserve, $31^{\circ} 30^{\prime} \mathrm{S}, 117^{\circ} 44^{\prime} \mathrm{E}$, Western Australia (Nov. 3-14, 1989; D. Mitchell), deposited in WAM (96/718).
ETYMOLOGY: The specific name is a noun in apposition, formed by contraction from the type locality, in honor of W. D. Platnick.

DiAGNOSIS: Males can easily be recognized by the small, obliquely oriented, and distally hooked retrolateral tibial apophysis (fig. 677). Discovery of the female could show that this enigmatic species is misplaced in Longepi; it could conceivably represent the sister group of the known Prionosternum species instead.

MALE: Total length 4.1. Abdominal venter gray, with two longitudinal paramedian rows of small, orange, circular sclerites. Leg spination: tibia IV r0-0-0; metatarsus IV v1p-0o0. Retrolateral tibial apophysis relatively small, obliquely oriented, hooked at tip (fig. 677); terminal apophysis moderately enlarged, covering prodistal quarter of bulb area (fig. 676).

FEMALE: Unknown.

Other MAterial ExAmined: None.

Distribution: Known only from Western Australia (map 83).

\section{PSEUDOLAMPONINAE, NEW SUBFAMILY}

Type Genus: Pseudolampona, new genus.

DiaGNOSIS: Members of this subfamily are easily distinguished from both lamponines and centrothelines by the absence of a deep groove on the median surface of the palpal endites, as well as by the tiny, inconspicuous postepigastric sclerites. Because of their very small size and elevated carapace, they look at first glance more like textricellines than lamponids, but their typically gnaphosoid posterior median eyes easily separate them from textricellines.

Distribution: Australia, including Tasmania (but not yet recorded from the Northern Territory).

InCLUded Genera: Pseudolampona and Paralampona, new genera.

\section{Key to Genera of Pseudolamponinae}

1. Abdomen usually with dark chevrons; males with patch of extremely thick, scalelike setae on posterior half of sternum and large median apophysis on palp; epigynum with lateral pockets ....... Pseudolampona

- Abdomen usually unpatterned; males without scalelike sternal setae and without median apophysis on palp; epigynum without lateral pockets .......... Paralampona

\section{Pseudolampona, new genus}

Type SPECIES: Pseudolampona warrandyte, new species.

ETYMOLOGY: The generic name refers to the falsely lamponine appearance of these spiders.

DiAGNOSIS: The presence of a chevroned abdomen usually separates members of this genus from those of Paralampona, as do the presence in males of thick setae on the sternum and a median apophysis on the palp, and in females of lateral pockets on the epigynum.

DESCRIPTION: Small spiders, total length of males 1.9-3.0, of females 2.3-3.8. Carapace dark orange, pars thoracica coated with long setae originating from low tubercles, pars cephalica elevated, lightest (especially posteriorly), with few setae; thoracic groove short, longitudinal. Eight eyes in two rows, poste- 
rior medians largest, others subequal in size; anterior medians circular, dark, other eyes light, laterals oval, posterior medians irregularly oval, flattened; from above, anterior eye row slightly recurved, posterior row procurved, from front, both rows strongly procurved; anterior medians separated by about their radius, by about their radius from anterior laterals; posterior medians almost contiguous, separated by almost their diameter from posterior laterals; anterior and posterior laterals separated by less than their diameter; median ocular quadrangle wider in front than in back, longer than wide. Chelicerae dark orange, sternum and mouthparts orange; chilum small, triangular, accompanied by second, posterior chilum (extremely narrow sclerite separating bases of chelicerae posteriorly); chelicerae with distinct lateral boss, promargin with series of short setae originating in line along base of fang plus one long, thick seta originating closest to fang, immediately bent at 90 degree angle, extending to median line; promargin with single large tooth and one or two denticles, retromargin without teeth. Labium elongate, rounded distally, truncated posteriorly, distinctly depressed medially. Endites obliquely depressed, with sharply demarcated, deep groove along margin near labium. Sternum slightly elevated, with steep, rebordered lateral margins, not expanded anteriorly, with extensions to and between coxae; surface smooth, males with patch of short, thick, dark setae posteriorly. Two epimeric sclerites on each side, one opposite palpal endites, one surrounding all coxae, not reaching sternal triangles, not fused to carapace. Pedicel composed of two small, flat dorsal sclerites and triangular sclerite covering venter and sides.

Abdomen without dorsal or anterior scutum in either sex; epigastric scutum accompanied posterolaterally by tiny, inconspicuous pair of oval, deeply invaginated sclerites bearing clearly elevated anterior rim; sclerites separated by membranous lobe; anterior edge of oval sclerites often fitting under epigastric scutum; colulus represented by pair of strong setae; posterior spiracle not on distinct sclerite. Anterior lateral spinnerets tubular, separated by less than their diameter, cuticle representing distal, second spinneret segment restricted to semicircle surrounding major ampullate gland spigots (piriform gland spigots surrounded only by soft cuticle); posterior median spinnerets small, tubular, without anteriorly expanded tips; posterior lateral spinnerets two-segmented.

Legs spineless; in males, all coxae and posterior trochanters dorsally tuberculate; anterior coxae with protuberant posterolateral corners; trochanters unnotched; anterior metatarsi and tarsi without scopulae; posterior metatarsi with distal preening brushes; posterior tarsi not scopulae; tarsi with two elongate claws, claw tufts reduced to just few setae; trichobothria present on distal segments. Female palpal tibia and tarsus without spines; female palpal tarsus with small claw.

Male palp with retrolateral tibial apophysis; cymbium unmodified; tegulum not expanded; embolus prolaterally situated, elongated, accompanied by membranous conductor; median apophysis present, enlarged. Epigynum simple, usually with small anterior hood, lateral pockets, and pair of globose spermathecae.

\section{Key to Species of PSEUdolampona}

1. Males ................... 1

- Females (those of P. wyandotte unknown) .. .................... 13

2. Median apophysis situated at retrolateral edge of bulb (fig. 754), retrolateral tibial apophysis very long (fig. 755) .... woodman

- Median apophysis situated more medially, retrolateral tibial apophysis shorter .... 3

3. Median apophysis expanded at or below tip (figs. 742, 746, 750, 784) ...... 4

- Median apophysis gradually narrowed toward tip (as in fig. 758) . . . . . . . . . 7

4. Embolus relatively wide (figs. 742, 784) . . 5

- Embolus relatively narrow (figs. 746, 750) . . $\ldots \ldots \ldots \ldots \ldots \ldots \ldots \ldots$

5. Median apophysis widest at half its length (fig. 742) ............ warrandyte

- Median apophysis widest distally (fig. 784) ............... glenmore

6. Expansion on median apophysis directed obliquely and distally (fig. 750) .. emmett

- Expansion of median apophysis directed retrolaterally (fig. 746) .......... boree

7. Retrolateral tibial apophysis relatively long (figs. 759, 767) ............8

- Retrolateral tibial apophysis shorter ... . .9

8. Median apophysis relatively long (fig. 758) $\ldots \ldots \ldots \ldots$ jarrahdale 
- Median apophysis relatively short (fig. 766) ................... taroom

9. Median apophysis relatively short (figs. 770, 778) ............... 10

- Median apophysis relatively long (figs. 762, $774,782) \ldots \ldots \ldots \ldots 11$

10. Embolus extending far retrolaterally (fig. 770) $\ldots \ldots \ldots \ldots \ldots \ldots$ spurgeon

- Embolus not extending retrolaterally (fig. 778) .............. binnowee

11. Tegulum relatively wide (fig. 774) ..... ..................kroombit

- Tegulum relatively narrow (figs. 762, 782) ................... 12

12. Tibia with proximal heel (fig. 783) ....

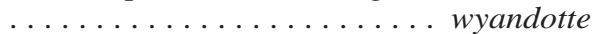

- Tibia without proximal heel (fig. 763) . . . .

13. Epigynum with weak but distinct lateral margins (as in figs. 744, 748) . . . . . 15

- Epigynum without distinct lateral margins (figs. 768, 780) . . . . . . . . 14

14. Spermathecae extending anterior of epigynal pockets (fig. 768) . . . . . . . . taroom

- Spermathecae not extending anterior of epigynal pockets (fig. 780) ..... binnowee

15. Epigynal pockets situated anterior of spermathecae (figs. 756, 760, 786) . . . . 16

- Epigynal pockets more posteriorly situated (as in figs. 744, 748) . . . . . . . . 18

16. Epigynal pockets relatively widely separated

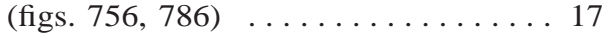

- Epigynal pockets closer to each other (fig. 760) .............. jarrahdale

17. Lateral epigynal margins relatively short (fig. 756) .............. woodman

- Lateral epigynal margins relatively long (fig. 786) ............... glenmore

18. Spermathecae very short (figs. 772, 776) ...

- Spermathecae longer (figs. 744, 748, 752, 764) ............... 20

19. Anterior epigynal hood far from spermathecae (fig. 772) ............. spurgeon

- Anterior epigynal hood closer to spermathecae (fig. 776) . . . . . . . . . kroombit

20. Epigynal pockets situated at anterior edge of spermathecae (figs. 744, 752) ...... 21

- Epigynal pockets situated more posteriorly (figs. 748, 764) . . . . . . . . . 22

21. Spermathecae relatively large (fig. 744) ....

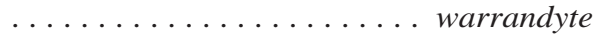

- Spermathecae relatively small (fig. 752) ... $\ldots \ldots \ldots \ldots \ldots$ emmett

22. Epigynal pockets situated at about half of spermathecal length (fig. 764) .... marun

- Epigynal pockets farther anterior (fig. 748) boree
Pseudolampona warrandyte, new species Figures 742-745; Map 84

TYPES: Female holotype and male allotype from Warrandyte, $37^{\circ} 45^{\prime} \mathrm{S}, 145^{\circ} 13^{\prime} \mathrm{E}$, Victoria (July 12, 1980; R. Raven), deposited in QMB (S25988).

ETYMOLOGY: The specific name is a noun in apposition taken from the type locality.

DiAGNOSIS: Males can be recognized by the very long and medially expanded median apophysis (fig. 742), females by the long and widely separated lateral epigynal margins (fig. 744).

MALE: Total length 3.0. Abdominal dorsum and sides with six dark chevrons, last three fused at sides; venter with three dark, longitudinal stripes (anterior along midline, pair of more posterior paramedians); legs unmarked. Retrolateral tibial apophysis greatly widened at base (fig. 743); median apophysis long, widest near midpoint (fig. 742).

FEMALE: Total length 3.4. Coloration as in male. Lateral epigynal margins reaching as far anterior as anterior tip of spermathecae, widely separated anteriorly (fig. 744); spermathecae relatively long, thick (fig. 745).

Other Material Examined: Australian Capital Territory: Black Mountain, $35^{\circ} 16^{\prime} \mathrm{S}, 149^{\circ} 06^{\prime} \mathrm{E}$ (NMV K3625), 1 ㅇ. New South Wales: Bungonia, $34^{\circ} 52^{\prime} \mathrm{S}, 149^{\circ} 57^{\prime} \mathrm{E}$, mid Dec. 1989-mid Feb. 1990, pitfall (G. Hunt, AMS KS22731), 19 ; Bungonia Caves, near Information Center, $34^{\circ} 50^{\prime} \mathrm{S}, 150^{\circ} 04^{\prime} \mathrm{E}$, mid Oct.-mid Nov. 1989, pitfall (G. Hunt, AMS KS22561), 1 ; ; Mount Flora, near Mittagong, $34^{\circ} 22^{\prime} \mathrm{S}, 150^{\circ} 26^{\prime} \mathrm{E}$, Apr. 15, 1976, Berlese, dry sclerophyll (R. Sadler, R. Taylor, QMB), 20, 1 ㅇ. Victoria: Coranderrk, Sept. 2, 1976 (V. Salanitri, QMB S26776), 10; Rich-Murrungowar Coupe, $37^{\circ} 34^{\prime} \mathrm{S}, 148^{\circ} 38^{\prime} \mathrm{E}$, May 7-13, 1992, upslope pitfall (R. Coy, NMV K3531, 3539), 20; Warrandyte, $37^{\circ} 45^{\prime} \mathrm{S}, 145^{\circ} 13^{\prime} \mathrm{E}$, July 12,1980 (R. Raven, QMB S25988), $10^{\star}$.

DisTRIBUTION: Southern New South Wales, the Australian Capital Territory, and eastern Victoria (map 84).

\section{Pseudolampona boree, new species Figures 746-749; Map 84}

TYPES: Female holotype and male allotype taken in a Berlese sample from a dry scler- 

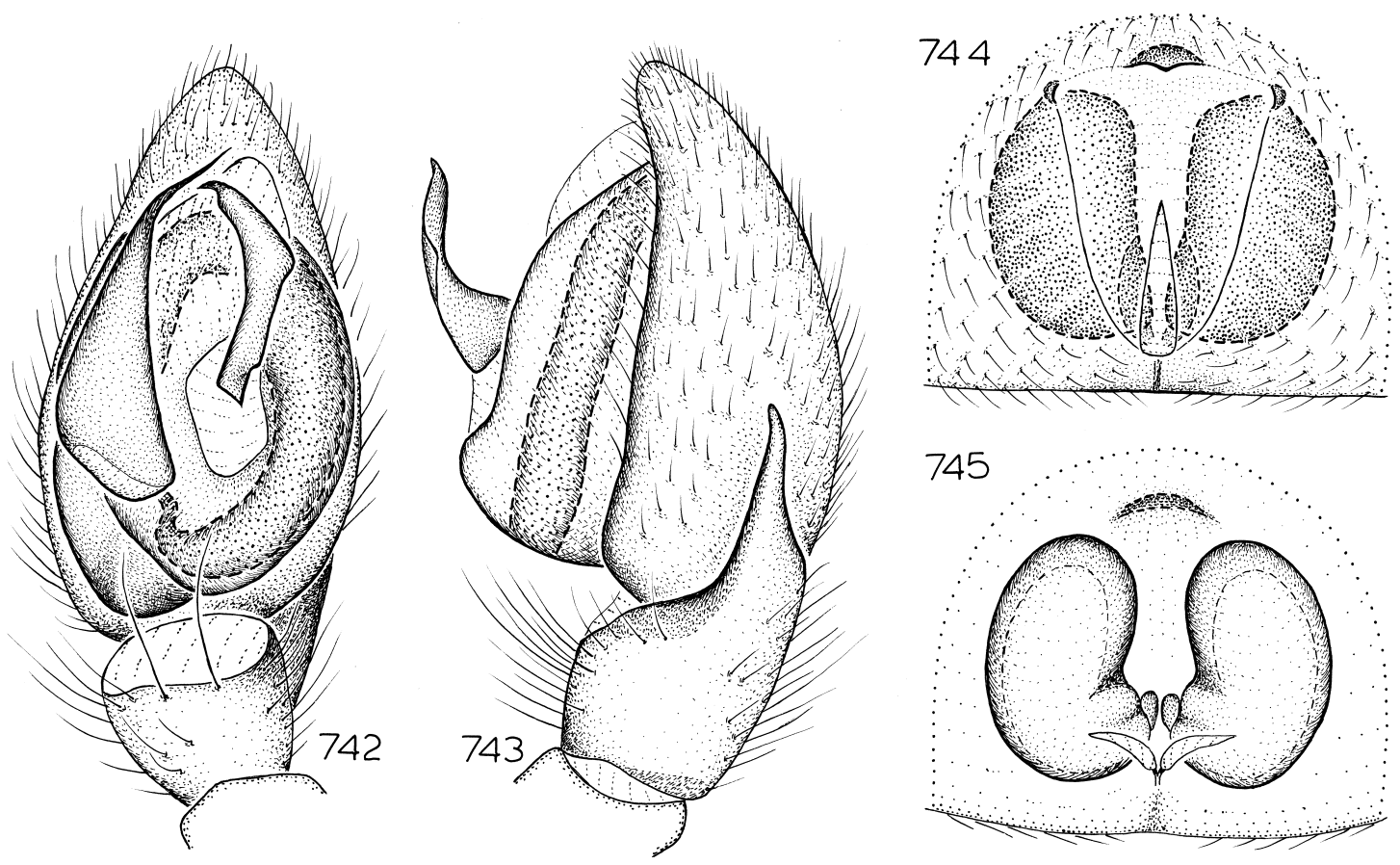

Figs. 742-745. Pseudolampona warrandyte, new species. 742. Left male palp, ventral view. 743. Same, retrolateral view. 744. Epigynum, ventral view. 745. Same, dorsal view.

ophyll forest at Boree Creek, $35^{\circ} 07^{\prime} \mathrm{S}$, $146^{\circ} 37^{\prime} \mathrm{E}$, New South Wales (June 10, 1984; R. Moran), deposited in QMB.

ETYMOLOGY: The specific name is a noun in apposition taken from the type locality.

DIAGNOSIS: Males can be recognized by the subdistally expanded median apophysis (fig. 746), females by the relatively short lateral epigynal margins (fig. 748).

MALE: Total length 2.9. Coloration as in $P$. warrandyte. Retrolateral tibial apophysis gradually narrowed toward tip (fig. 747); median apophysis expanded subdistally (fig. 746).

Female: Total length 3.2. Coloration as in $P$. warrandyte. Lateral epigynal margins relatively short, not reaching anterior tip of spermathecae (fig. 748); spermathecae large, approximate (fig. 749).

Other Material Examined: New South Wales: Boree Creek, $35^{\circ} 07^{\prime} \mathrm{S}, 146^{\circ} 37^{\prime} \mathrm{E}$, June 10, 1984, Berlese, dry sclerophyll (R. Moran, QMB), $2 q$. South Australia: $1 \mathrm{~km}$ $S$ Box Flat, Ngarkat Conservation Park, 35 36' S, $140^{\circ} 23^{\prime} \mathrm{E}$, Mar. 19, 1992 (D. Hirst, SAM N1997/115), 1 ; ; Kolay Hut, Paney
Station, $32^{\circ} 33^{\prime} \mathrm{S}, 135^{\circ} 36^{\prime} \mathrm{E}$, Dec. 8-11, 1992, pitfall (D. Hirst, SAM N1997/113), $1 \% ; 0.5$ $\mathrm{km}$ W Ngarutjara Homeland, $26^{\circ} 14^{\prime} \mathrm{S}$, $131^{\circ} 48^{\prime} \mathrm{E}$, Oct. $12-17,1994$, pitfall (J. Forrest, SAM N1997/128), $1 \%$; $1 \mathrm{~km} \mathrm{~N}$ Point Tinline, Cape Gantheaume. Kangaroo Island, $35^{\circ} 59^{\prime} \mathrm{S}, 137^{\circ} 37^{\prime} \mathrm{E}$, Nov. $10-11$, 1987, pitfall (D. Hirst, SAM N1997/130), $10^{\text {ta }} 5.9 \mathrm{~km} \mathrm{~W}$ Pooginagoric, $36^{\circ} 26^{\prime} \mathrm{S}, 140^{\circ} 46^{\prime} \mathrm{E}$, Dec. $12-$ 15, 1995, pitfall (SAM N1997/114), 10; 14 km WNW Renmark, 34 $07^{\prime} \mathrm{S}, 140^{\circ} 37^{\prime} \mathrm{E}$, July 4-Aug. 10, 1995, intercept trap (K. Pullen, QMB), 20; $31 \mathrm{~km}$ NW Renmark, 3359'S, $140^{\circ} 30^{\prime} \mathrm{E}$, May 2-June 8, 1995, intercept trap (K. Pullen, QMB), 20; 32 km N Renmark, $33^{\circ} 53^{\prime} \mathrm{S}, 140^{\circ} 44^{\prime} \mathrm{E}$, Aug. 9-Sept. 7, 1995, intercept trap (K. Pullen, QMB), 20; $79 \mathrm{~km}$ NNW Renmark, $33^{\circ} 31^{\prime} \mathrm{S}, 140^{\circ} 24^{\prime} \mathrm{E}$, Aug. 9Sept. 7, 1995, intercept trap (K. Pullen, QMB), 20; South Gap Station, Beda Hill, $31^{\circ} 51^{\prime} \mathrm{S}, 137^{\circ} 37^{\prime} \mathrm{E}$, Dec. 4-6, 1989, pitfall (D. Hirst, SAM N1996/319), 10 . Victoria: $4 \mathrm{~km} \mathrm{~S}$ Nowingi, $34^{\circ} 36^{\prime} \mathrm{S}, 142^{\circ} 14^{\prime} \mathrm{E}$, Sept. 22, 1985, under rock (M. Harvey, B. Scott, L. Hoare, WAM 96/719, 720), 2 \%. Western Australia: Francois Peron National Park, 

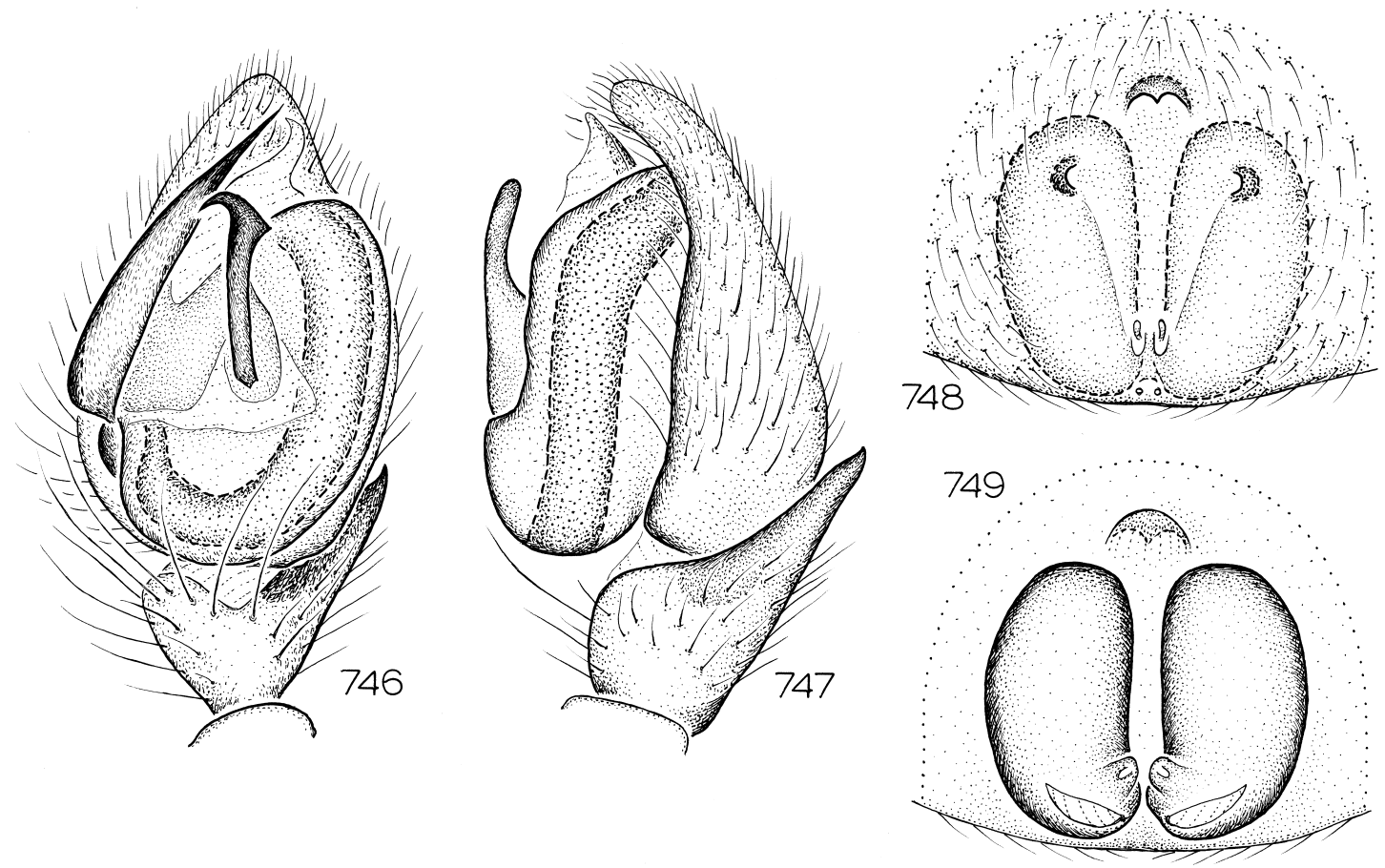

Figs. 746-749. Pseudolampona boree, new species. 746. Left male palp, ventral view. 747. Same, retrolateral view. 748. Epigynum, ventral view. 749. Same, dorsal view.

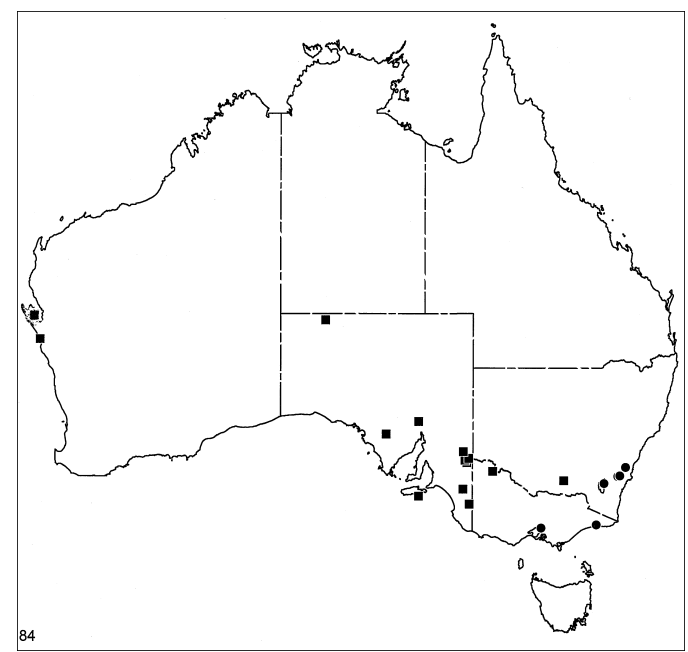

Map 84. Records of Pseudolampona warrandyte, new species (circles) and $P$. boree, new species (squares). $25^{\circ} 59^{\prime} \mathrm{S}, 113^{\circ} 34^{\prime} \mathrm{E}$, Jan. 18-May 26, 1995, pitfall (M. Harvey, WAM 96/721), 1 oे $^{\text {; Zu- }}$ ytdorp, $27^{\circ} 16^{\prime} \mathrm{S}, 114^{\circ} 04^{\prime} \mathrm{E}$, Oct. $17,1994-$ Jan. 11, 1995, pitfall (N. McKenzie, J. Rolfe, WAM 96/1512), 1 ઈ.

Distribution: Southern Australia, except Tasmania (map 84).

\section{Pseudolampona emmett, new species} Figures 750-753; Map 85

TYPES: Female holotype from pitfall trap at Emmett Creek, S of Townsville, $19^{\circ} 27^{\prime} \mathrm{S}$, $147^{\circ} 03^{\prime} \mathrm{E}$, Queensland (Oct. 26, 1991-July 27, 1992; P. Lawless, M. Shaw, R. Raven), deposited in QMB (S21954), and male allotype (same data except pitfall set Dec. 2, 1992-Apr. 12, 1993), deposited in QMB (S34331).

ETYMOLOGY: The specific name is a noun in apposition taken from the type locality.

DiAGNOSIS: Males of this small species can be recognized by the distally bifid median apophysis (fig. 750), females by the long lateral epigynal margins and widely separated spermathecae (fig. 752). 

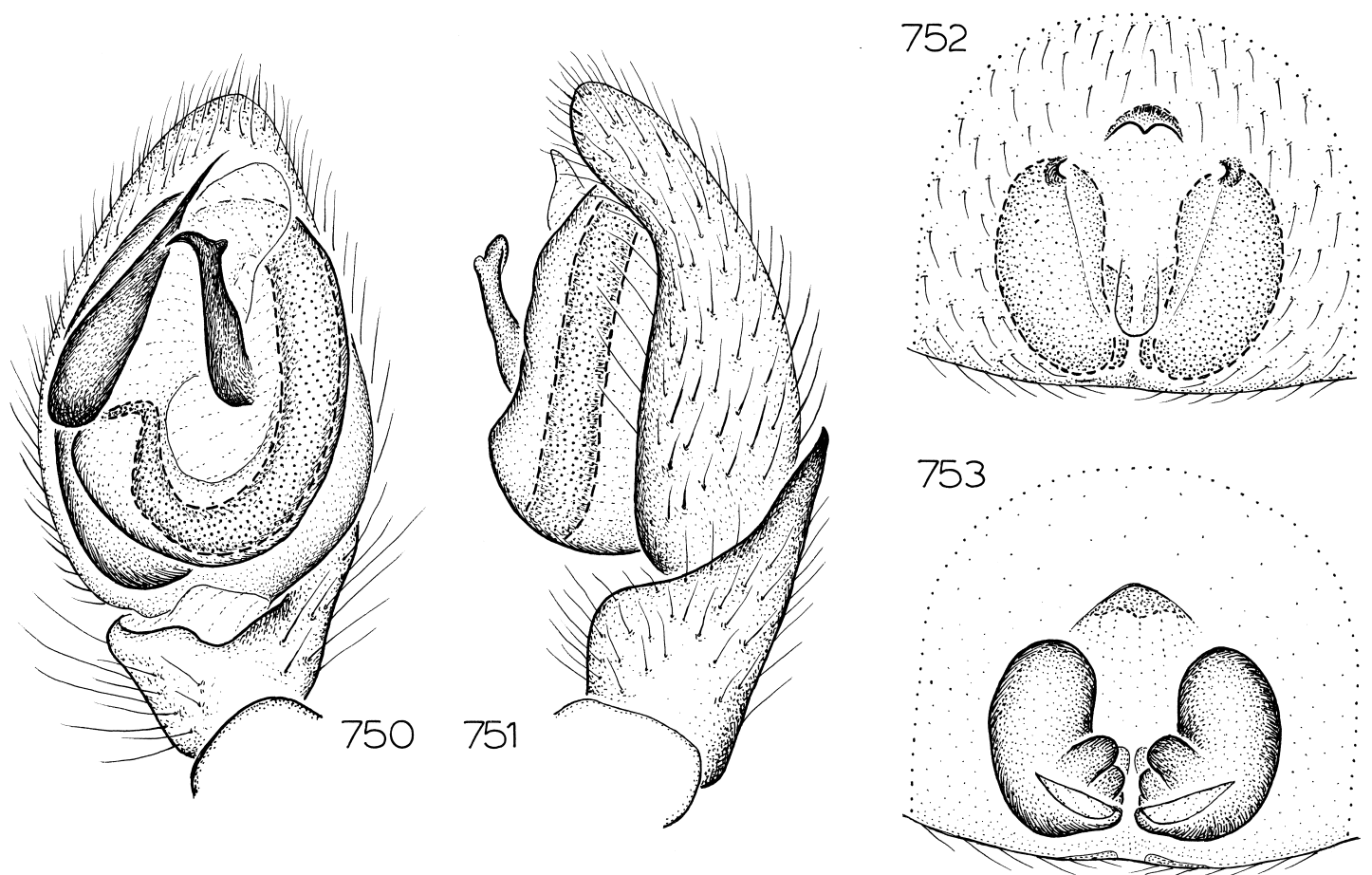

Figs. 750-753. Pseudolampona emmett, new species. 750. Left male palp, ventral view. 751. Same, retrolateral view. 752. Epigynum, ventral view. 753. Same, dorsal view.

MALE: Total length 1.9. Coloration as in $P$. warrandyte except abdominal dorsum with only four dark chevrons (posterior stripe interrupted medially), venter with only a pair of small, posterior, dark spots. Retrolateral tibial apophysis small, triangular (fig. 751); median apophysis distally bifid (fig. 750).

FEMALE: Total length 3.3. Coloration as in male. Lateral epigynal margins relatively long, reaching full length of spermathecae (fig. 752); spermathecae widely separated anteriorly (fig. 753).

OTHER MATERIAl ExAmined: Queensland: Emmett Creek, S Townsville, $19^{\circ} 27^{\prime} \mathrm{S}$, $147^{\circ} 03^{\prime} \mathrm{E}$, Oct. 26, 1991-July 27, 1992, pitfall (P. Lawless, R. Raven, M. Shaw, QMB S24755), 10, July 27-Dec. 2, 1992, pitfall (R. Raven, P., E. Lawless, M. Shaw, QMB S24748), 1 \% , Dec. 2, 1992-Apr. 12, 1993, pitfall (P. Lawless, M. Shaw, R. Raven, ex QMB S34331), 10; Killymoon Creek, S Townsville, $19^{\circ} 24^{\prime} \mathrm{S}, 147^{\circ} 01^{\prime} \mathrm{E}$, Oct. 26, 1991-July 27, 1992, pitfall (P. Lawless, R. Raven, M. Shaw, QMB S19955), 10, 1옹

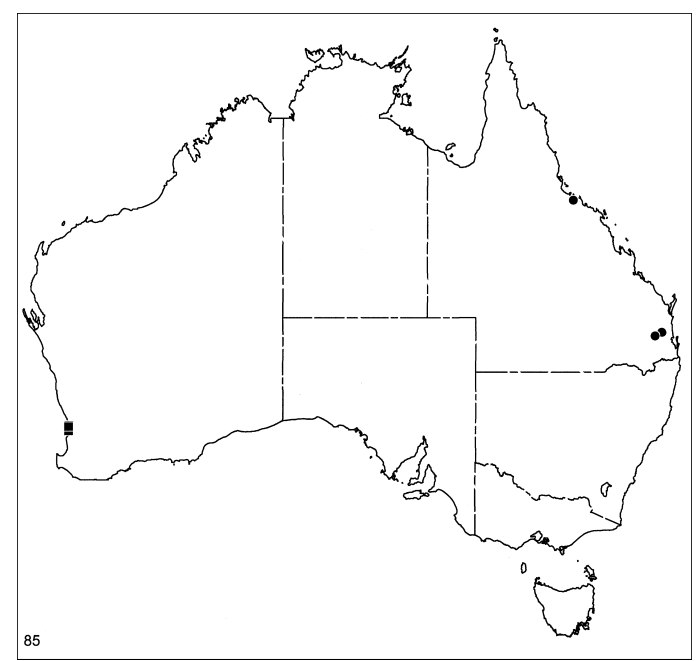

Map 85. Records of Pseudolampona emmett, new species (circles) and $P$. woodman, new species (squares). 

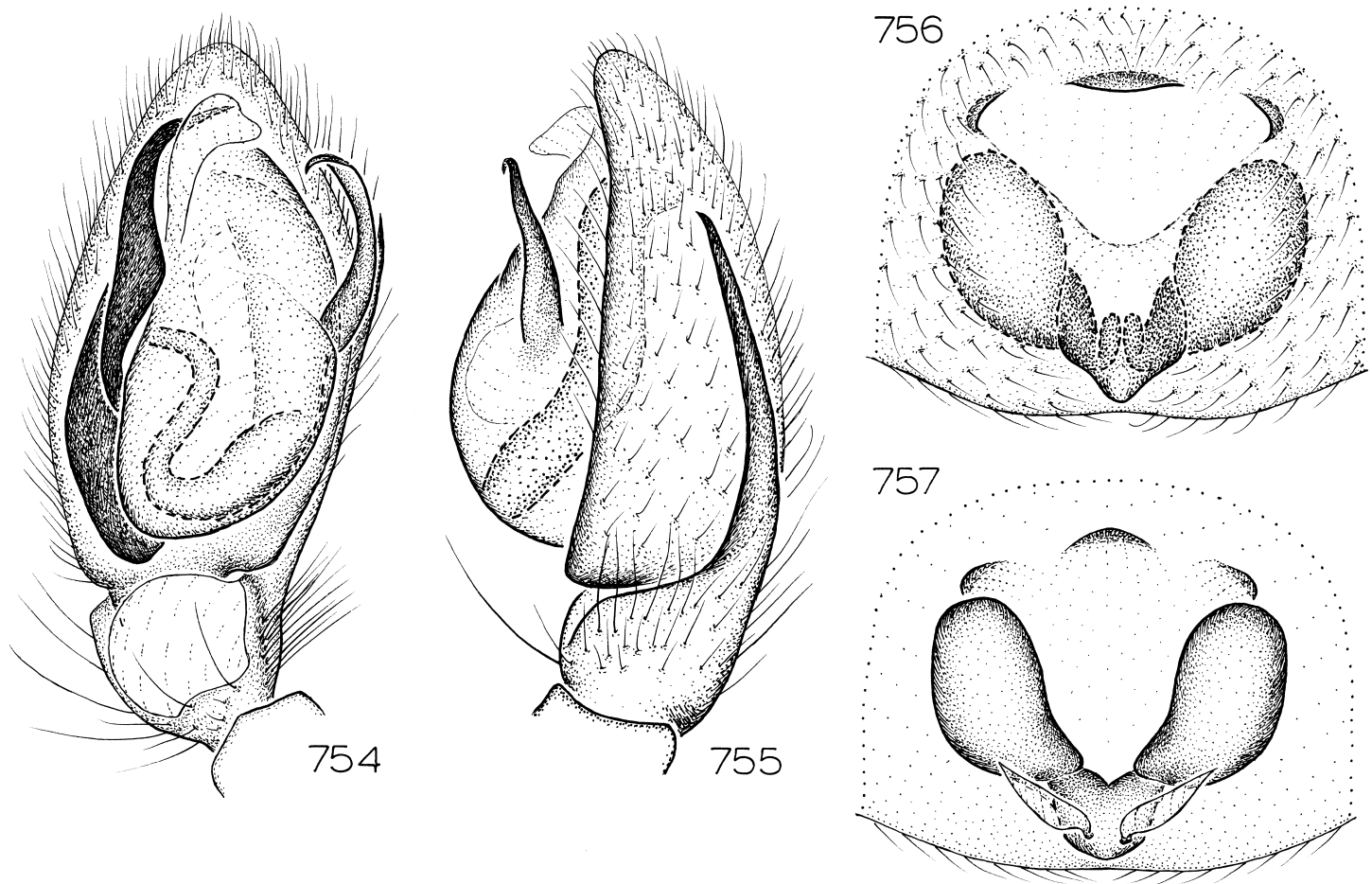

Figs. 754-757. Pseudolampona woodman, new species. 754. Left male palp, ventral view. 755. Same, retrolateral view. 756. Epigynum, ventral view. 757. Same, dorsal view.

July 27-Dec. 2, 1992, pitfall (R. Raven, P., E. Lawless, M. Shaw, QMB S22979), 10; Tungi Creek, Jimna, $26^{\circ} 40^{\prime} \mathrm{S}, 152^{\circ} 28^{\prime} \mathrm{E}$, Dec. 18, 1996-Jan. 20, 1997, rainforest pitfalls (G. Monteith, QMB S35377), 30; 5 km E Yarraman, $26^{\circ} 52^{\prime} \mathrm{S}, 152^{\circ} 03^{\prime} \mathrm{E}$, June 19,1982 , Berlese, closed forest turkey mound litter (L. Hill, QMB), 1 \% .

DISTRIBUTION: Known only from southand mid-eastern Queensland (map 85).

\section{Pseudolampona woodman, new species} Figures 754-757; Map 85

TYPES: Female holotype and male allotype taken in pitfall traps at Woodman Point, $32^{\circ} 08^{\prime} \mathrm{S}, 115^{\circ} 45^{\prime} \mathrm{E}$, Western Australia (June 28-Sept. 1, 1994; J. Waldock, A. Longbottom), deposited in WAM $(96 / 722,723)$.

ETYMOLOGY: The specific name is a noun in apposition taken from the type locality.

DIAGNOSIS: Males can easily be recognized by the greatly elongated retrolateral tibial apophysis (fig. 755), females by the relatively wide epigynum (fig. 756).
MALE: Total length 2.1. Coloration as in $P$. warrandyte except abdominal dorsum with five dark chevrons. Retrolateral tibial apophysis greatly elongated, reaching almost to distal tip of bulb (fig. 755); median apophysis long, originating on retrolateral side of bulb (fig. 754).

Female: Total length 3.1. Coloration as in male. Lateral epigynal pockets widely separated (fig. 756); spermathecae widely separated (fig. 757).

Other Material Examined: Western Australia: Hepburn Heights, $31^{\circ} 49^{\prime} \mathrm{S}$, $115^{\circ} 46^{\prime} \mathrm{E}$, Nov. 28, 1995-Jan. 29, 1996, pitfall (M. Harvey, J. Waldock, WAM 96/728), 1; Trigg Dune Bush, 31 ${ }^{\circ} 53^{\prime} \mathrm{S}, 115^{\circ} 45^{\prime} \mathrm{E}$, Nov. 28, 1995-Jan. 29, 1996, pitfall (M. Harvey, J. Waldock, WAM 96/727), 1 \% ; Woodman Point, $32^{\circ} 08^{\prime} \mathrm{S}, 115^{\circ} 45^{\prime} \mathrm{E}$, June $28-$ Sept. 1, 1994, pitfall (J. Waldock, A. Longbottom, WAM 96/724), 1 \%, Sept. 1-Nov. 4, 1994 (J. Waldock, A. Longbottom, WAM 96/1522), 1 ô, Nov. 4, 1994-Jan. 19, 1995, pitfalls (J. Waldock, M. Harvey, WAM 96/725, 726, 729-735), 6ð, 3 ㅇ․ 

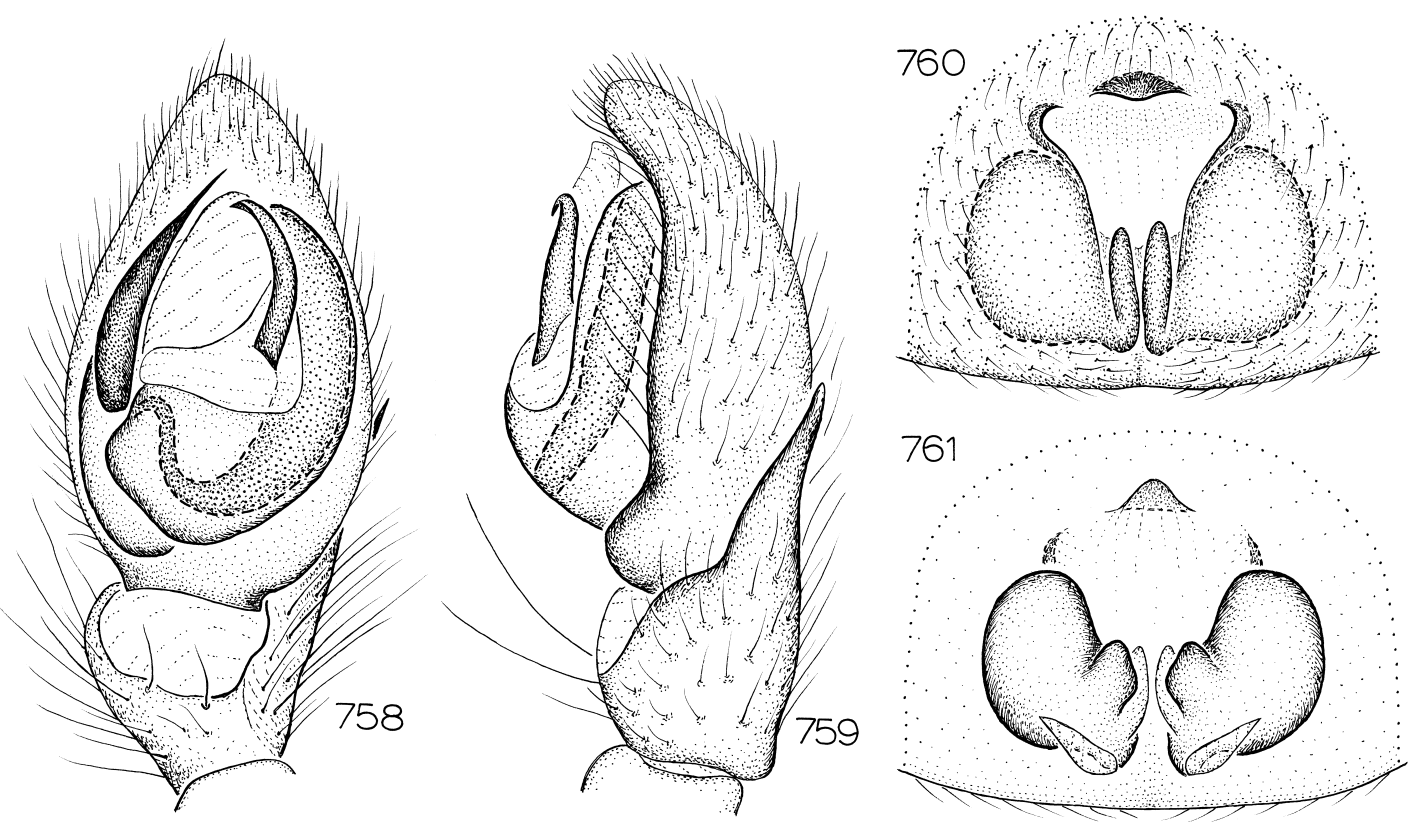

Figs. 758-761. Pseudolampona jarrahdale, new species. 758. Left male palp, ventral view. 759. Same, retrolateral view. 760. Epigynum, ventral view. 761. Same, dorsal view.

DisTRIBUTION: Known only from Western Australia (map 85).

\section{Pseudolampona jarrahdale, new species Figures 758-761; Map 86}

TYPE: Male holotype from Alcoa minesite and forest, $\mathrm{N}$ and NW of Jarrahdale, $32^{\circ} 20^{\prime} \mathrm{S}$, $116^{\circ} 04^{\prime} \mathrm{E}$, Western Australia (Mar.-Aug. 1993; S. Simmonds), deposited in WAM (96/ 737).

ETYMOLOGY: The specific name is a noun in apposition taken from the type locality.

DIAGNOSIS: Males can easily be recognized by the strongly arched median apophysis (fig. 758), females by the almost u-shaped spermathecae (fig. 761).

MALE: Total length 2.9. Coloration as in $P$. woodman except abdominal venter with dark markings only around spinnerets. Retrolateral tibial apophysis straight (fig. 759); median apophysis highly arched (fig. 758).

Female: Total length 3.1. Coloration as in male. Epigynum showing pair of darkened posteromedian ducts (fig. 760), spermathecae rotund, almost u-shaped because of large, median projections (fig. 761).

Other Material Examined: Western
Australia: Dwellingup, $32^{\circ} 43^{\prime} \mathrm{S}, 116^{\circ} 04^{\prime} \mathrm{E}$, Jan. 12, 1978, pitfall (S. Curry, WAM 96/ 1530), 1 \% , Feb. 28, 1979 (S. Curry, WAM 95/736), $19 ; 16 \mathrm{~km}$ E Dwellingup on Williams Road, $32^{\circ} 40^{\prime} \mathrm{S}, 115^{\circ} 55^{\prime} \mathrm{E}$, Feb. 15, 1979 (M. Gray, AMS KS14848), 1 ㅇ․

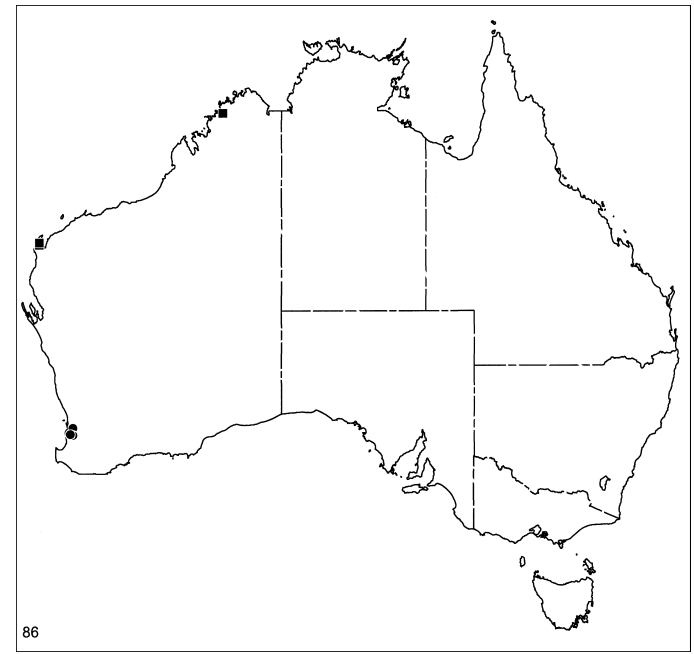

Map 86. Records of Pseudolampona jarrahdale, new species (circles) and $P$. marun, new species (squares). 

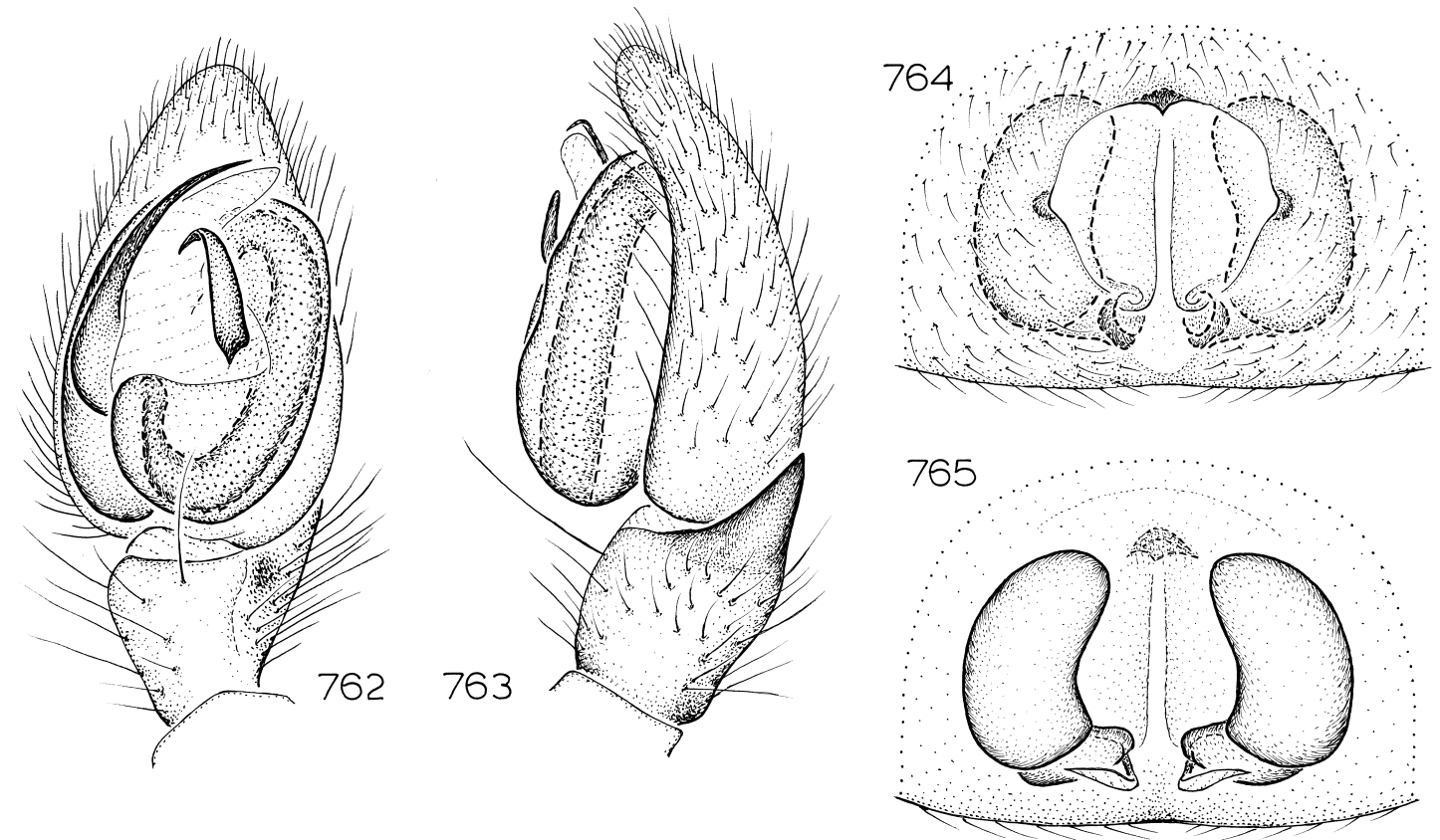

Figs. 762-765. Pseudolampona marun, new species. 762. Left male palp, ventral view. 763. Same, retrolateral view. 764. Epigynum, ventral view. 765. Same, dorsal view.

Distribution: Known only from Western Australia (map 86).

\section{Pseudolampona marun, new species} Figures 762-765; Map 86

TyPE: Male holotype taken in Berlese sample of closed forest litter at Marun, Prince Frederick Harbour, $15^{\circ} 00^{\prime} \mathrm{S}, 125^{\circ} 21^{\prime} \mathrm{E}$, Western Australia (June 6-11, 1988; I. Naumann), deposited in QMB.

ETYMOLOGY: The specific name is a noun in apposition taken from the type locality.

DiAGNOSIS: Males and females have not been taken together, and are from distant localities, but are matched here until addition specimens indicate that more than one species of this genus inhabits the northern half of Western Australia. Males can be recognized by the relatively narrow embolus and short retrolateral tibial apophysis (figs. 762, 763), females by the almost circular epigynal margins (fig. 764).

MALE: Total length 2.3. Abdominal dorsum with four dark chevrons, venter unmarked. Retrolateral tibial apophysis very short, triangular (fig. 763); embolus relatively narrow, especially in distal half (fig. 762).

FEMALE: Total length 3.5. Coloration as in male. Lateral epigynal margins anchored by lateral pockets at about half of epigynal length, forming almost circular rim (fig. 764), spermathecae with short posterior lobes (fig. 765).

Other Material Examined: Western Australia: cave C.15, Cape Range, $22^{\circ} 13^{\prime}$ S, $113^{\circ} 59^{\prime} \mathrm{E}$, June 28, 1989 (M. Harvey, WAM 96/738), 1 \% ; site TL-5, NW Cape Peninsula, $22^{\circ} 06^{\prime} \mathrm{S}, 114^{\circ} 00^{\prime} \mathrm{E}$, June 3, 1990, litter (J. Waldock, WAM 96/739), 1 ㅇ.

DisTRIBUTION: Known only from the northern half of Western Australia (map 86).

Pseudolampona taroom, new species Figures 766-769; Map 87

TYPE: Male holotype taken in a pitfall trap at Boggomoss, Taroom District, $25^{\circ} 25^{\prime} \mathrm{S}$, $150^{\circ} 01^{\prime} \mathrm{E}$, Queensland (Sept. 9-Nov. 11, 1996; P. Lawless), deposited in QMB (S34317). 

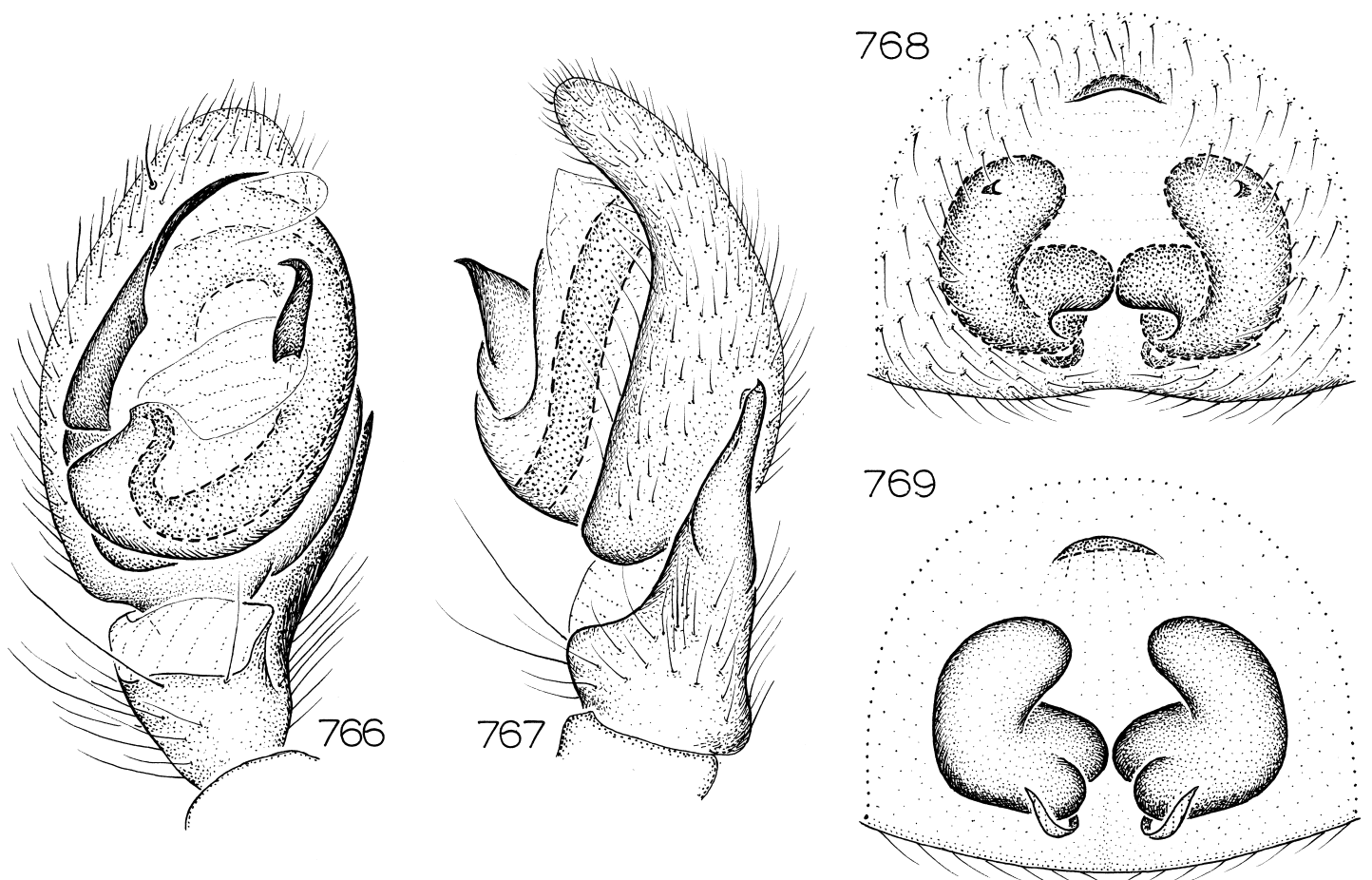

Figs. 766-769. Pseudolampona taroom, new species. 766. Left male palp, ventral view. 767. Same, retrolateral view. 768. Epigynum, ventral view. 769. Same, dorsal view.

ETYMOLOGY: The specific name is a noun in apposition taken from the type locality.

DiAGNOSIS: Males can be recognized by the distally folded embolus (fig. 766), females by the absence of lateral epigynal margins and the enlarged lobes that separate the spermathecae (fig. 768).

MALE: Total length 2.5. Coloration as in P. warrandyte. Retrolateral tibial apophysis relatively long, slightly expanded at about half its length (fig. 767); embolus narrow, folded at about half its length (fig. 766).

FEMALE: Total length 3.1. Coloration as in $P$. warrandyte. Lateral epigynal margins absent, lateral pockets small, often difficult to see (fig. 768); spermathecae separated by enlarged, medially directed lobes (fig. 769).

VARIATION: The two females from New South Wales have relatively short spermathecae, and it is possible that they could prove to represent a different species.

Other Material Examined: New South Wales: $700 \mathrm{~m}$ E Arrinya Road on logging road 900 m from Burns Road junction, Kan-

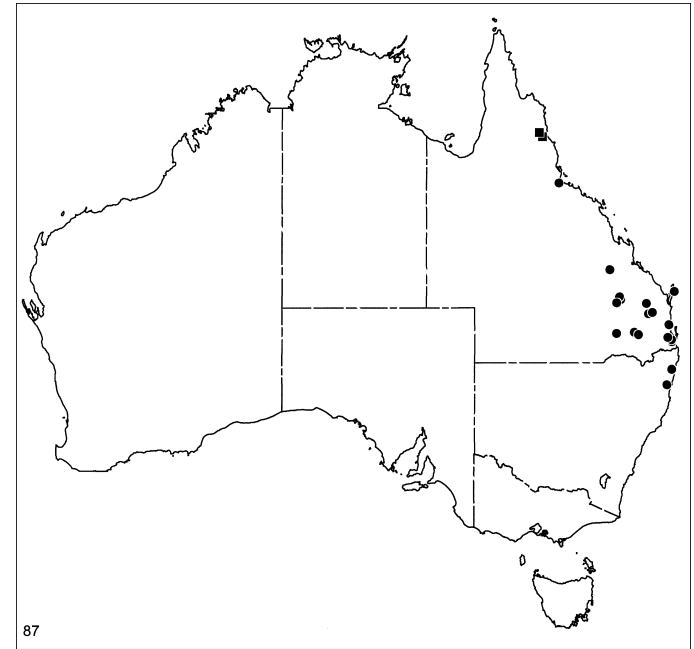

Map 87. Records of Pseudolampona taroom, new species (circles) and $P$. spurgeon, new species (squares). 

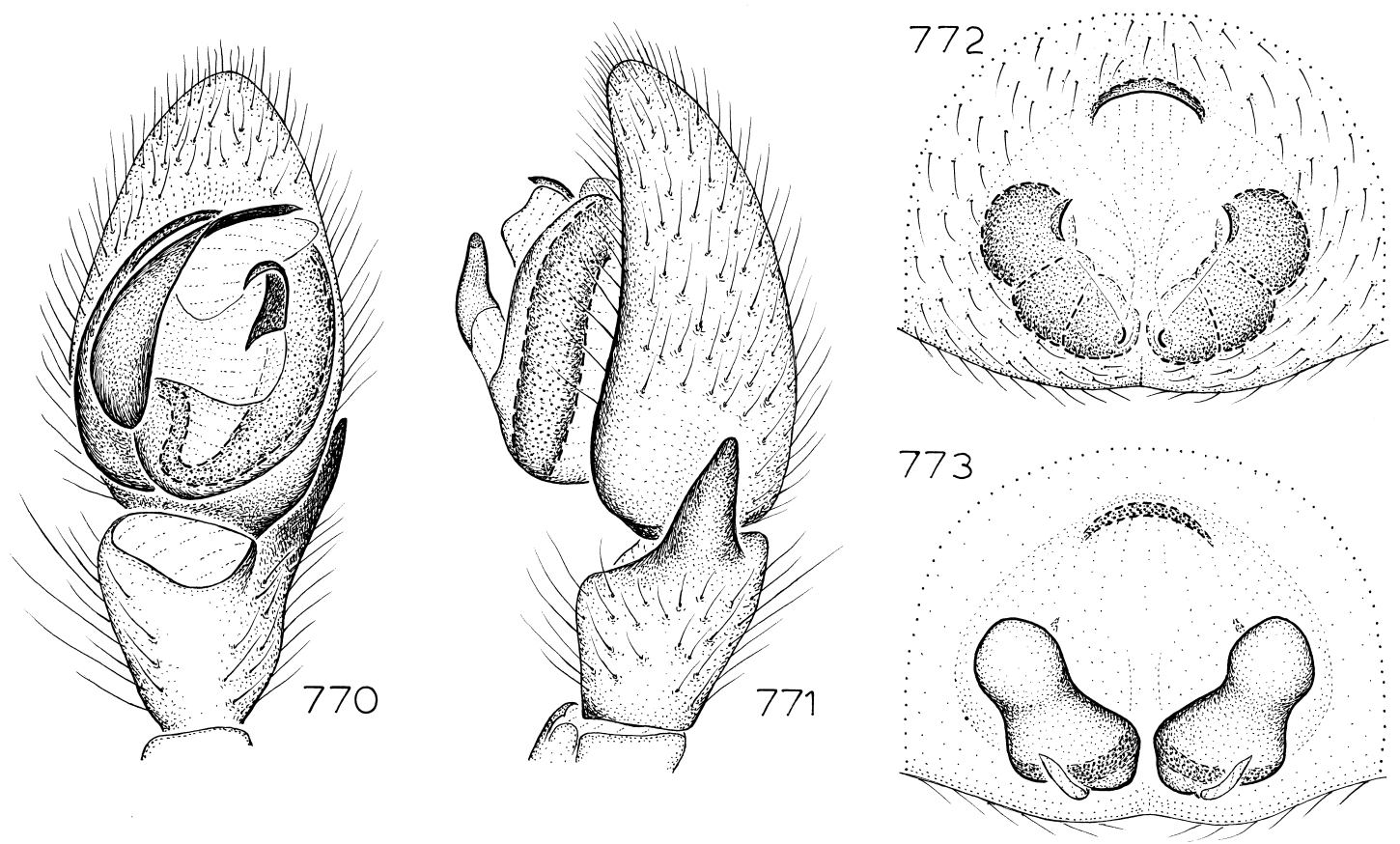

Figs. 770-773. Pseudolampona spurgeon, new species. 770. Left male palp, ventral view. 771. Same, retrolateral view. 772. Epigynum, ventral view. 773. Same, dorsal view.

garoo River State Forest, $30^{\circ} 04^{\prime} \mathrm{S}, 152^{\circ} 52^{\prime} \mathrm{E}$, Feb. 4-Apr. 9, 1993, pitfall (M. Gray, G. Cassis, AMS KS39568), 1\%; junction of Range Road and Lockleys Road, Doubleduke State Forest, $29^{\circ} 14^{\prime} \mathrm{S}, 153^{\circ} 11^{\prime} \mathrm{E}$, Feb. 4-Apr. 9, 1993, pitfall (M. Gray, G. Cassis, AMS KS39567), 1․ Queensland: Bahr's Scrub, $27^{\circ} 44^{\prime} \mathrm{S}, 153^{\circ} 10^{\prime} \mathrm{E}$, Apr. 30, 1980, dry litter (R. Raven, QMB S34312), 1 \% B Beerwah Forestry Reserve, $26^{\circ} 51^{\prime} \mathrm{S}, 152^{\circ} 57^{\prime} \mathrm{E}$, Nov. 21, 1990, pitfall, heath (M. Glover, QMB S32336), 10, Aug. 25, 1991, pitfall, heath (M. Glover, QMB S32328, 32329), $10^{\dagger}, 1 \%$; Braemar State Forest, $27^{\circ} 13^{\prime} \mathrm{S}$, $150^{\circ} 50^{\prime} \mathrm{E}$, Oct. 15-19, 1979, cypress, brigalow litter (R. Raven, QMB S26911, 28115), 10, 19 ; Ewan Maddock Dam, 26 48'S, $152^{\circ} 59^{\prime} \mathrm{E}$, Oct. 27, 1992-Jan. 30, 1993, pitfalls, open forest (M. Glover, QMB S32397), 30., Aug. 22, 1993-Mar. 22, 1994, pitfalls, open forest (M. Glover, QMB S28702, 28715, 30389), 7o; 3 mi SE Gayndah, $25^{\circ} 38^{\prime} \mathrm{S}, 151^{\circ} 36^{\prime} \mathrm{E}$, July 17 , 1968, Berlese, eucalypt litter (L. Mound, QMB), $1 \%$; Isla Gorge, $8.5 \mathrm{~km}$ SSW lookout, $25^{\circ} 16^{\prime} \mathrm{S}$, $149^{\circ} 56^{\prime} \mathrm{E}$, Dec. 19, 1997-Mar. 3, 1998, pit- fall, vine scrub, elev. $360 \mathrm{~m}$ (G. Monteith, D. Cook, QMB S44205), 10; Keysland, $26^{\circ} 12^{\prime} \mathrm{S}, 151^{\circ} 44^{\prime} \mathrm{E}$, Sept. 29-Dec. 5, 1994, intercept trap, open forest (G. Monteith, QMB S32466), 2q; Lake Broadwater, via Dalby, $27^{\circ} 21^{\prime} \mathrm{S}, 151^{\circ} 06^{\prime} \mathrm{E}$, May 16-Nov. 23, 1985, pitfall (QMB S28589), 1 o , Jan. 3-Feb. 25, 1986, pitfall (M. Bennie, QMB S28585), 10 , Feb. 22, 1986, Berlese, brigalow, sieved litter, (G. Monteith, V. Davies, D. Thompson, QMB S34324), 19; Nangur State Forest, $26^{\circ} 08^{\prime} \mathrm{S}, 151^{\circ} 58^{\prime} \mathrm{E}$, Oct. 24-Nov. 24, 1995, pitfall, elevation $320 \mathrm{~m}$ (G. Monteith, QMB S34315), 1 \% ; North Mimosa Creek, Blackdown Tableland National Park, $23^{\circ} 46^{\prime} \mathrm{S}$, $149^{\circ} 20^{\prime} \mathrm{E}$, July 8, 1991, sieved litter, open forest (D. Black, WAM 96/1529), 1 \% ; Orchid Beach, Fraser Island, $24^{\circ} 58^{\prime} \mathrm{S}$, $153^{\circ} 19^{\prime}$ E, Mar. 7-Oct. 1, 1996, pitfall, heath, banksia, eucalypt (R. Raven, QMB S31269), 20, 2 \% $5 \mathrm{~km}$ W Paluma, $19^{\circ} 00^{\prime} \mathrm{S}, 146^{\circ} 12^{\prime} \mathrm{E}$, July 12, 1971, Berlese, rainforest (Taylor, Feehan, QMB), 10; Rochedale State Forest, Brisbane, $27^{\circ} 37^{\prime} \mathrm{S}, 153^{\circ} 09^{\prime} \mathrm{E}$, June $12-30$, 1980, litter (V. Davies, R. Raven, QMB S34314, 34322), 2q; $7 \mathrm{~km} \mathrm{~N}$ Taroom, 

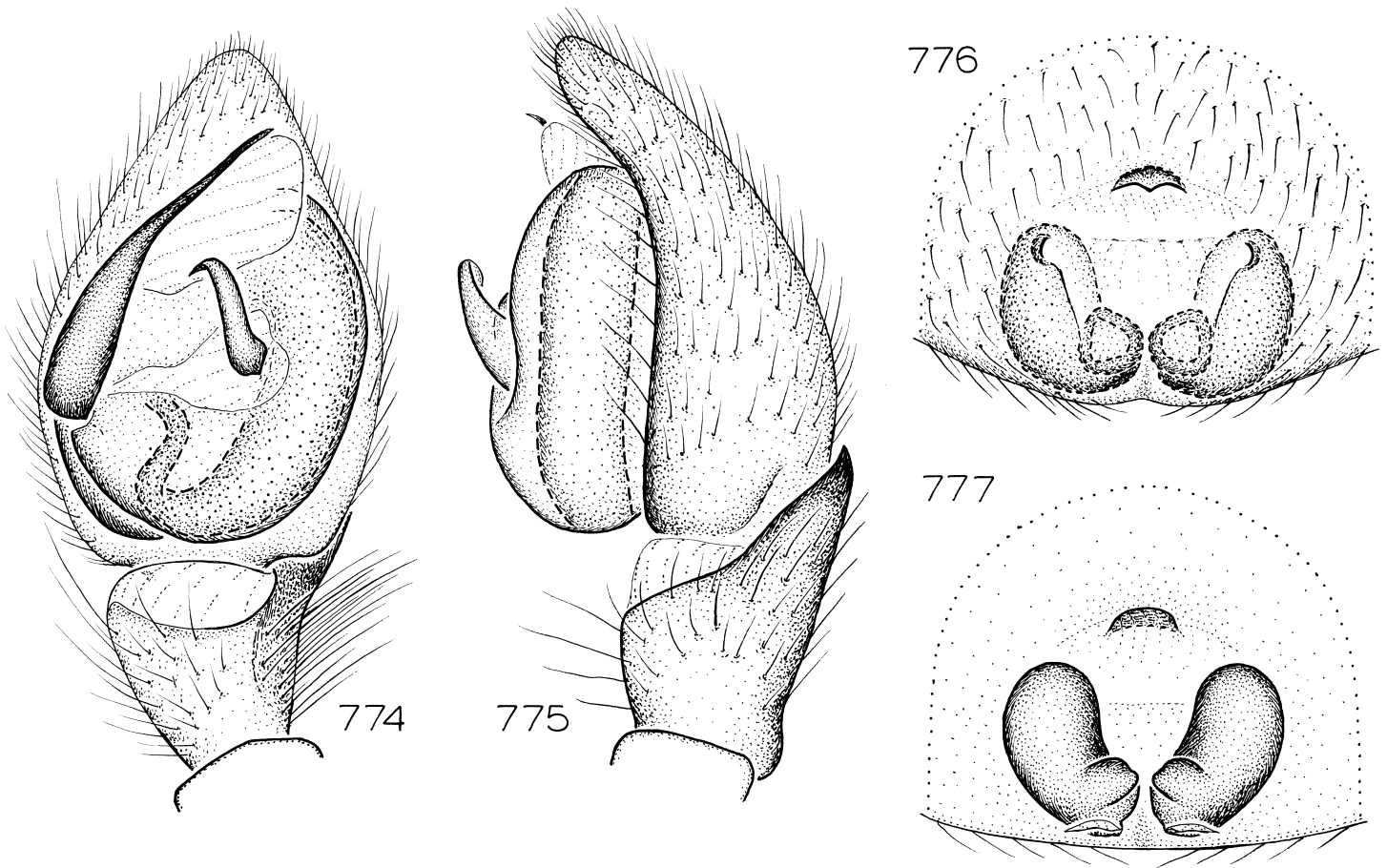

Figs. 774-777. Pseudolampona kroombit, new species. 774. Left male palp, ventral view. 775. Same, retrolateral view. 776. Epigynum, ventral view. 777. Same, dorsal view.

$25^{\circ} 36^{\prime} \mathrm{S}, 149^{\circ} 46^{\prime} \mathrm{E}$, Nov. 12, 1996-Jan. 31, 1997, pitfall, roadside, ooline scrub (P. Lawless, QMB S36681), 20 ; Upper Brookfield, $27^{\circ} 30^{\prime} \mathrm{S}, 152^{\circ} 55^{\prime} \mathrm{E}$, Oct. 30, 1980 , araucarianotophyll vineforest, litter (R. Raven, V. Davies, QMB S34330), 1 \%; Windermere, near Glenmorgan, $27^{\circ} 17^{\prime} \mathrm{S}, 149^{\circ} 45^{\prime} \mathrm{E}$, July-Sept. 1991, open forest pitfall (R. Raven, D. Smyth, ex QMB S30361), 1 ㅇ.

DistRIBUTION: Known only from Queensland and New South Wales (map 87).

\section{Pseudolampona spurgeon, new species} Figures 770-773; Map 87

TYPE: Male holotype taken in a pitfall trap at an elevation of $1100 \mathrm{~m}$ on Mount Spurgeon, $16^{\circ} 27^{\prime} \mathrm{S}, 145^{\circ} 11^{\prime} \mathrm{E}$, Queensland (Nov. 19, 1997-Feb. 8, 1998; G. Monteith, D. Cook), deposited in QMB (S43938).

ETYMOLOGY: The specific name is a noun in apposition taken from the type locality.

Diagnosis: Males resemble those of $P$. $t a-$ room in having a distally folded embolus, but can be recognized by the distinctly beaked median apophysis (fig. 770); females differ from those of $P$. taroom in having the anterior spermathecal lobes distinctly demarcated (figs. 772, 773).

MALE: Total length 2.2. Abdominal dorsum with four dark chevrons, two anterior ones large, fused at sides; venter with dark markings only around spinnerets. Retrolateral tibial apophysis small, spike-shaped (fig. 771); embolus strong, folded at midlength, median apophysis short, beaked (fig. 770).

Female: Total length 2.3. Coloration as in male. Epigynal openings at about half of epigynal length (fig. 772), spermathecae with distinctly demarcated anterior lobes (fig. 773).

Other MAterial Examined: Queensland: Mount Windsor Tableland, Whypalla, $16^{\circ} 13^{\prime} \mathrm{S}, 144^{\circ} 59^{\prime} \mathrm{E}$, summer $1992-1993$, pitfalls, elev. $1120 \mathrm{~m} \mathrm{(S.} \mathrm{Burnett,} \mathrm{QMB}$ S22660, 24403, 30450, 33168, 33205), 9 ㅊ, 1 ㅇ.

Distribution: Known only from northeastern Queensland. 
Pseudolampona kroombit, new species Figures 774-777; Map 88

TYPES: Male holotype from rainforest pitfall at Three Moon Creek, Kroombit Tops, $45 \mathrm{~km}$ SSW Calliope, $24^{\circ} 25^{\prime} \mathrm{S}, 151^{\circ} 03^{\prime} \mathrm{E}$, Queensland (Dec. 9-19, 1983; V. Davies, J. Gallon), deposited in QMB (S34332), and female allotype taken in a Berlese sample sieved from rainforest litter at an elevation of $940 \mathrm{~m}$ at the same locality (Dec. 15, 1983; G. Monteith, V. Davies, J. Gallon, D. Thompson), deposited in QMB (S25981).

ETYMOLOGY: The specific name is a noun in apposition taken from the type locality.

DiAGNOSIS: Males can be recognized by the relatively short retrolateral tibial apophysis (fig. 775), relatively long embolus, and simple median apophysis (fig. 774), females by the angular lateral epigynal margins and weak transverse anterior epigynal ridge situated posterior of the epigynal hood (fig. 776) and the rounded median spermathecal lobes (fig. 777).

MALE: Total length 2.4. Abdominal dorsum with four dark, transverse stripes, anterior stripe extended posteriorly on sides of abdomen, posterior two or three stripes broken at middle, producing spotted appearance; venter as $P$. warrandyte. Retrolateral tibial apophysis short, triangular (fig. 775); embolus elongate, reaching almost to retrolateral

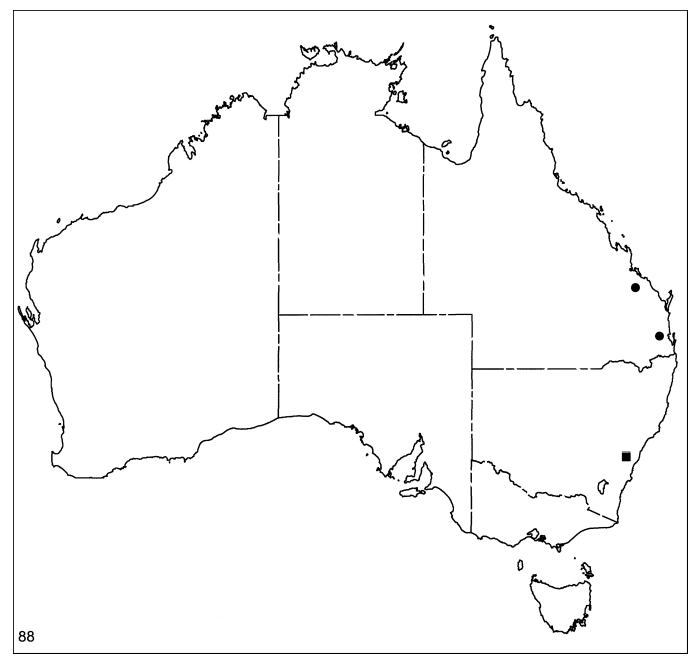

Map 88. Records of Pseudolampona kroombit, new species (circles) and $P$. binnowee, new species (squares). edge of bulb, median apophysis simple, hooked (fig. 774).

Female: Total length 3.1. Coloration as in male. Epigynum with weak but distinct anterior ridge situated behind anterior hood (fig. 776); spermathecae widely separated by rounded median lobes (fig. 777).

OTHER MATERIAL EXAMINED: Queensland: Mount Brisbane, $27^{\circ} 05^{\prime} \mathrm{S}, 152^{\circ} 32^{\prime} \mathrm{E}$, Oct. 6, 1979 (R. Raven, QMB S34337), 1 ô; Three Moon Creek, Kroombit Tops, $45 \mathrm{~km}$ SSW Calliope, $24^{\circ} 25^{\prime} \mathrm{S}, 151^{\circ} 03^{\prime} \mathrm{E}$, Dec. 9-19, 1983 (V. Davies, J. Gallon, QMB ex S34332), 3 ô.

Distribution: Known only from southeastern Queensland (map 88).

\section{Pseudolampona binnowee, new species} Figures 778-781; Map 88

TYPE: Male holotype taken in pitfall trap on Binnowee Drive, Blue Mountains National Park, $33^{\circ} 40^{\prime} \mathrm{S}, 150^{\circ} 28^{\prime} \mathrm{E}$, New South Wales (Aug. 15, 1996), deposited in AMS (KS55292).

ETYMOLOGY: The specific name is a noun in apposition taken from the type locality.

DiAgnosis: This species seems closest to $P$. kroombit, but males have a shorter embolus, shorter median apophysis, and narrower retrolateral tibial apophysis (figs. 778, 779) and females have more rounded spermathecae (figs. 780, 781).

MALE: Total length 2.5. Abdominal dorsum with four dark, transverse stripes, each extended posteriorly on sides of abdomen, middle two stripes extended anteriorly at middle; venter as $P$. warrandyte. Retrolateral tibial apophysis short, narrow (fig. 779); embolus short, median apophysis short, hooked (fig. 778).

Female: Total length 3.8. Coloration as in male. Epigynum short, with small lateral pockets, hood narrow (fig. 780); spermathecae rounded, widely separated, with long, thumb-shaped median lobes (fig. 781).

Other Material Examined: New South Wales: Ridge Street, Woodford, $33^{\circ} 44^{\prime} \mathrm{S}$, $150^{\circ} 29^{\prime}$ E, Sept. 30-Oct. 4, 1996, pitfalls (AMS KS51713, 55307), 2 ㅇ․

Distribution: Known only from the Blue Mountains region of New South Wales (map 88). 

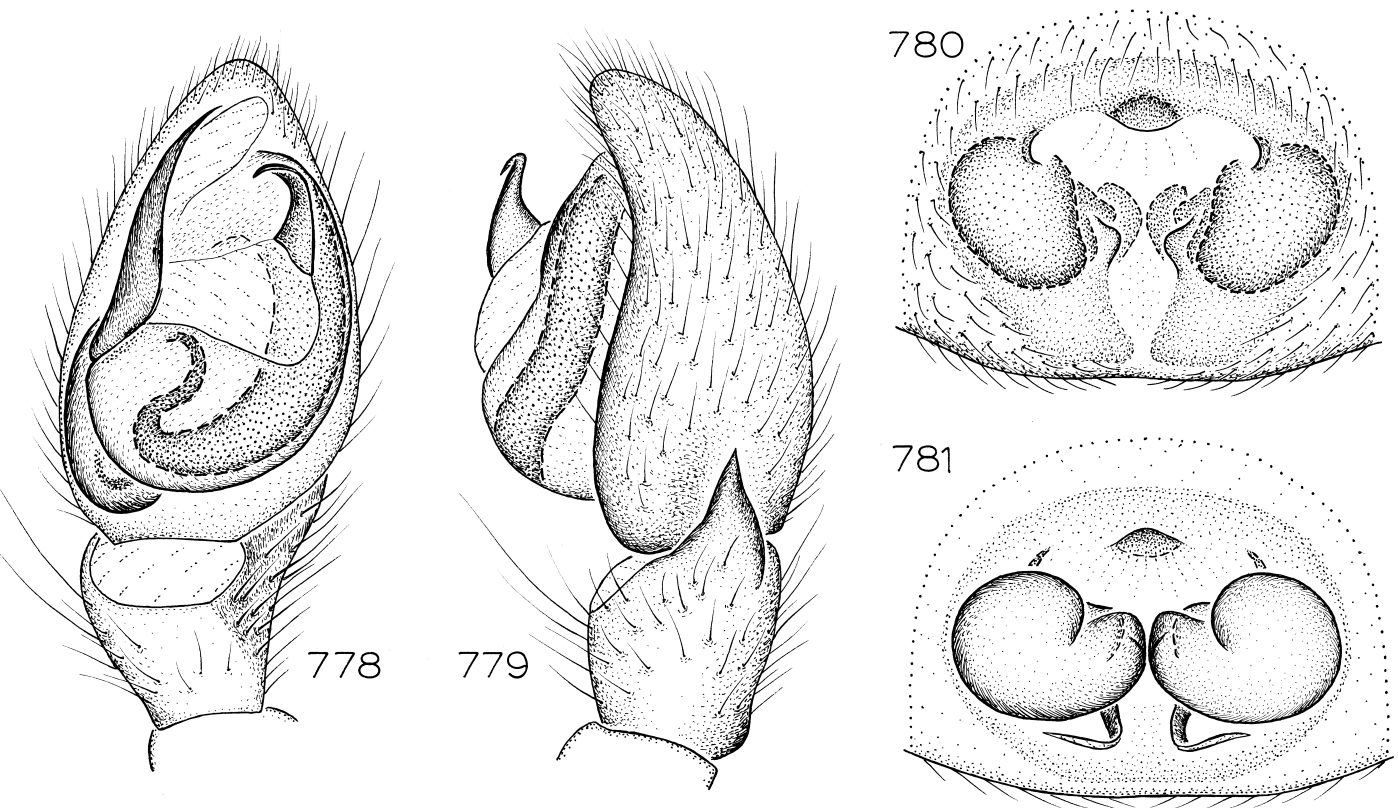

Figs. 778-781. Pseudolampona binnowee, new species. 778. Left male palp, ventral view. 779. Same, retrolateral view. 780. Epigynum, ventral view. 781. Same, dorsal view.

Pseudolampona wyandotte, new species Figures 782, 783; Map 89

TYPE: Male holotype taken in a pitfall trap in dry eucalypt woodland at Wyandotte Creek, $18^{\circ} 29^{\prime} \mathrm{S}, 144^{\circ} 55^{\prime} \mathrm{E}$, Queensland (July 26-Dec. 1, 1992; R. Raven, P., E. Lawless, M. Shaw), deposited in QMB (S24340).

ETYMOLOGY: The specific name is a noun in apposition taken from the type locality.

DiAgNosis: Males can easily be recognized by the elongated palpal bulb and excavated median apophysis (fig. 782).

MALE: Total length 2.5. Abdominal dorsum with five dark chevrons; venter unmarked. Retrolateral tibial apophysis distinctly narrowed at about half its length (fig. 783); palpal bulb elongated, median apophysis excavated (fig. 782).

FeMale: Unknown.

Other Material Examined: None.

DisTRIBUTION: Known only from northeastern Queensland (map 89).

\section{Pseudolampona glenmore, new species} Figures 784-787; Map 89

TYPES: Male holotype and female allotype taken in open forest pitfalls at Glenmore, $23^{\circ} 20^{\prime} \mathrm{S}, 150^{\circ} 30^{\prime} \mathrm{E}$, Queensland (Mar. 21Sept. 3, 1991; D. Wallace, R. Raven), deposited in QMB (S34320).

ETYMOLOGY: The specific name is a noun in apposition taken from the type locality.

DiAGNOSIS: Males can easily be recognized by the enlarged tip of the median apophysis (fig. 784), females by the anteriorly advanced lateral epigynal margins (fig. 786).

MALE: Total length 2.0. Abdominal dorsum with five narrow dark chevrons, venter with dark markings only around spinnerets. Retrolateral tibial apophysis long, narrow (fig. 785); median apophysis greatly expanded distally (fig. 784).

FEMALE: Total length 2.4. Coloration as in male. Epigynum with anteriorly advanced lateral margins outlining triangular atrium (fig. 786), spermathecae bean-shaped (fig. 787).

Other MATERIAl ExAMINED: Queensland: Glenmore, $23^{\circ} 20^{\prime} \mathrm{S}, 150^{\circ} 30^{\prime} \mathrm{E}$, Mar. 21-Sept. 3, 1990, open forest pitfall (D. Wallace, R. Raven, QMB S32527), 10; Kabra, 2328'S, $150^{\circ} 24^{\prime} \mathrm{E}$, July $19-$ Oct. 22,1990 , open forest pitfall (D. Wallace, R. Raven, K. Williams, QMB S26321), 10, 1q, Mar. 21-Sept. 3, 

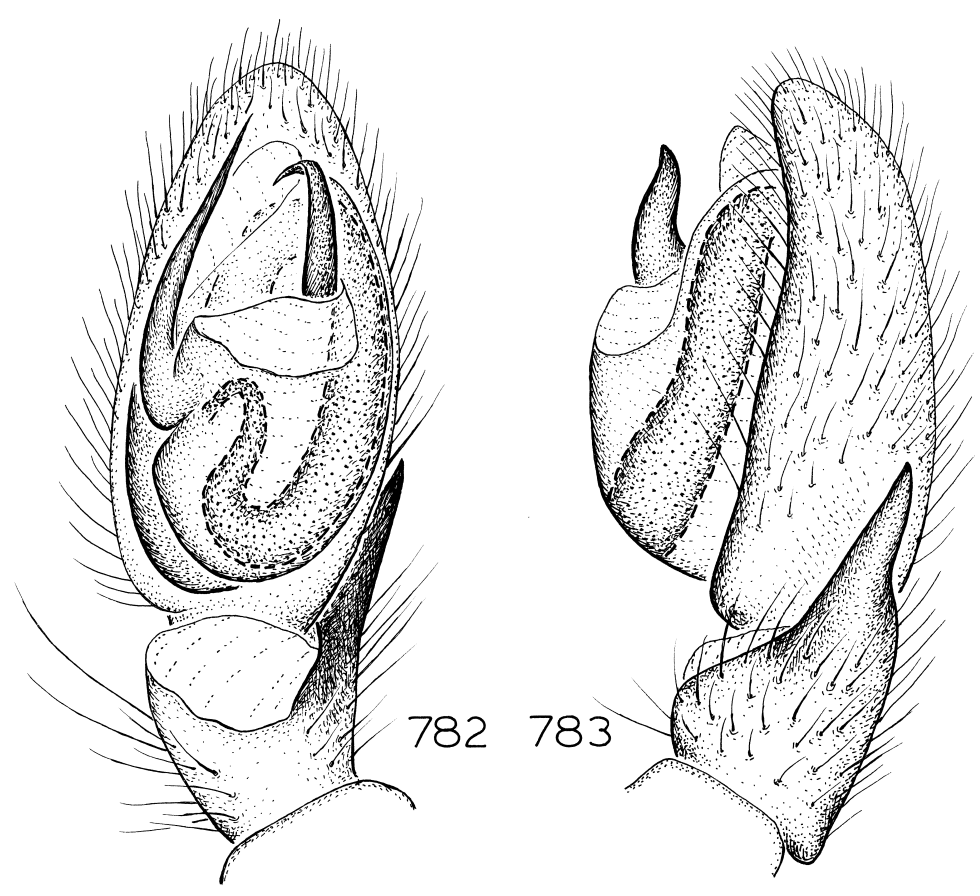

Figs. 782, 783. Pseudolampona wyandotte, new species. 782. Left male palp, ventral view. 783. Same, retrolateral view.

1991, open forest pitfall (D. Wallace, R. Raven, K. Williams, QMB S32390, 32533), $20^{\top}$; Mount Archer, $23^{\circ} 20^{\prime} \mathrm{S}, 150^{\circ} 35^{\prime} \mathrm{E}$, July 18-Oct. 22, 1990, open forest pitfall (D.

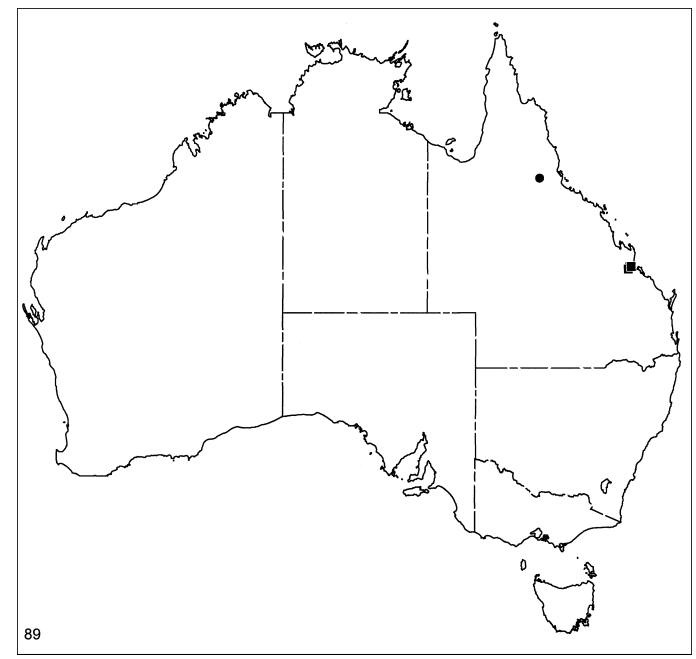

Map 89. Records of Pseudolampona wyandotte, new species (circle) and $P$. glenmore, new species (squares).
Wallace, R. Raven, K. Williams, QMB S19596), 10 .

Distribution: Known only from mid-eastern Queensland (map 89).

\section{Paralampona, new genus}

TyPE SPECIES: Paralampona domain, new species.

ETYMOLOGY: The generic name refers to the falsely lamponine appearance of these spiders.

DiAGNOSIS: The absence of a chevroned abdomen usually separates members of this genus from those of Pseudolampona, as do the absence in males of thick setae on the sternum and a median apophysis on the palp, and in females of lateral pockets on the epigynum.

DESCRIPTION: Small spiders, total length of males 1.3-2.1, of females 1.7-2.5. Carapace light orange, pars thoracica coated with long setae originating from anastomosing rows of low tubercles, pars cephalica elevated, elongated, lightest (especially posteriorly), with few setae; thoracic groove short, longitudi- 

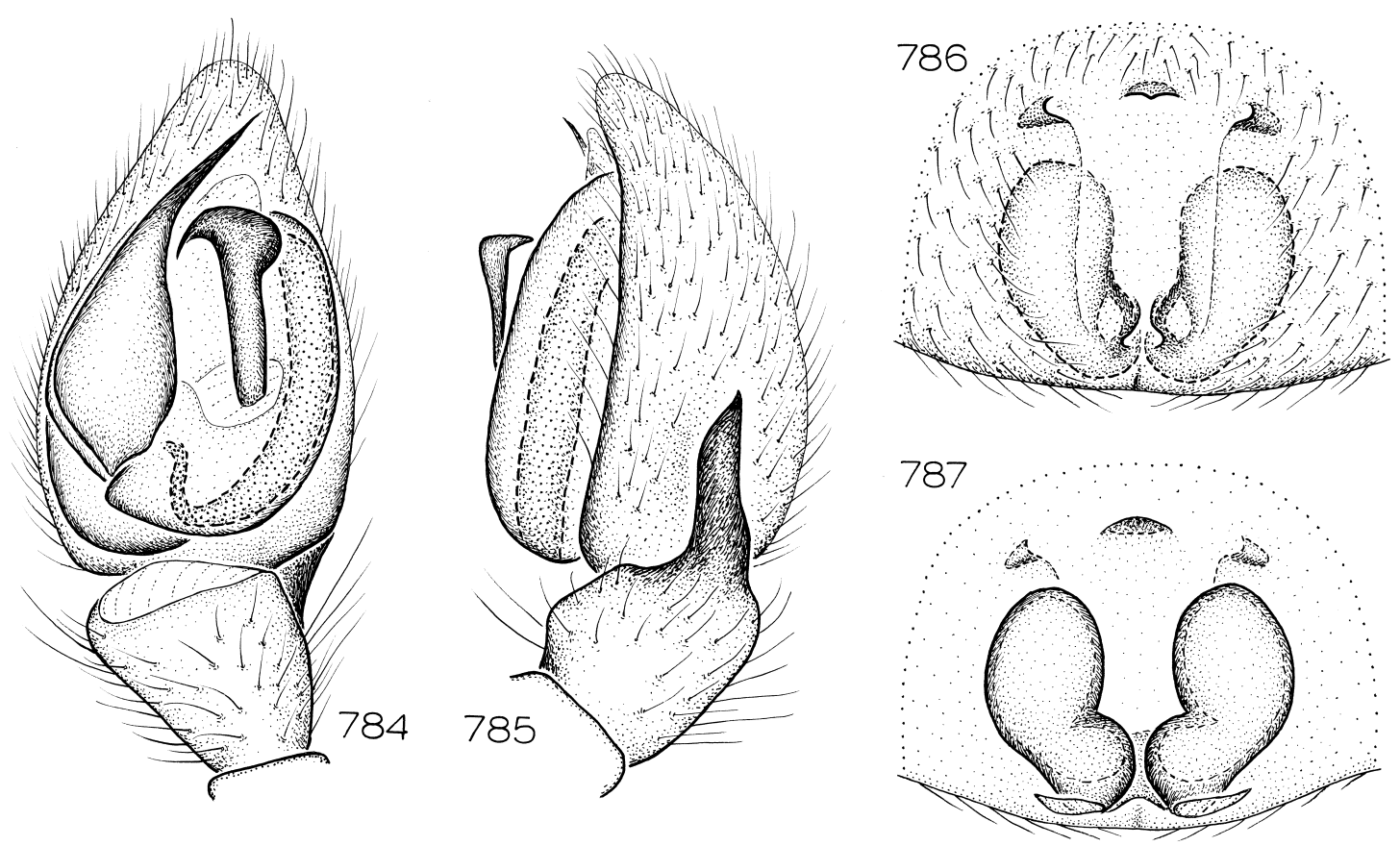

Figs. 784-787. Pseudolampona glenmore, new species. 784. Left male palp, ventral view. 785. Same, retrolateral view. 786. Epigynum, ventral view. 787. Same, dorsal view.

nal, situated back at about four-fifths of carapace length. Eight eyes in two rows, posterior medians largest, others subequal in size; anterior medians circular, dark, other eyes light, laterals oval, posterior medians irregularly oval, flattened; from above, anterior eye row slightly recurved, posterior row slightly procurved, from front, both rows strongly procurved; anterior medians separated by almost their diameter, by about their radius from anterior laterals; posterior medians separated by almost their radius, by almost their diameter from posterior laterals; anterior and posterior laterals separated by less than their diameter; median ocular quadrangle wider in front than in back, about as long as wide in back. Chelicerae, sternum and mouthparts light orange; chilum small, triangular, accompanied by second, small, oval posterior chilum; chelicerae with distinct lateral boss, promargin with series of short setae originating in line along base of fang plus one long, thick seta originating closest to fang, immediately bent at 90 degree angle, extending to median line; promargin with single large tooth and one or two denticles, retromargin with two tiny denticles near base of fang. Labium elongate, triangular, with broad distal tip, truncated posteriorly, distinctly depressed medially. Endites obliquely depressed, without sharply demarcated, deep groove along margin near labium; serrula present, teeth in single row. Sternum very slightly elevated, with rebordered lateral margins, not expanded anteriorly, with extensions to and between coxae; surface smooth, males without patch of short, thick, dark setae posteriorly. Two epimeric sclerites on each side, one opposite palpal endites, one surrounding all coxae, not reaching sternal triangles, not fused to carapace. Pedicel composed of two small, flat dorsal sclerites and almost rectangular sclerite covering venter and sides.

Abdomen without dorsal or anterior scutum in either sex; cuticle with thick setae; epigastric scutum accompanied posterolaterally by tiny, inconspicuous pair of narrow, transverse, invaginated sclerites; sclerites separated by membranous lobe, sometimes continued as slight sclerotization around lateral edge of epigastric scutum; colulus rep- 
resented by pair of strong setae; posterior spiracle not on distinct sclerite. Anterior lateral spinnerets tubular, separated by almost their diameter, cuticle representing distal, second spinneret segment restricted to semicircle surrounding major ampullate gland spigots (piriform gland spigots surrounded only by soft cuticle); posterior median spinnerets small, tubular, without anteriorly expanded tips; posterior lateral spinnerets twosegmented.

Legs spineless; most surfaces with thin setae; coxae not notably tuberculate; anterior coxae with protuberant posterolateral corners; trochanters unnotched; anterior metatarsi and tarsi without scopulae; posterior metatarsi without distal preening brushes; posterior tarsi not scopulae; tarsi with two short claws, claw tufts reduced to just few setae; trichobothria present on distal segments. Female palpal tibia and tarsus without spines; female palpal tarsus with small claw.

Male palp with retrolateral tibial apophysis, sometimes with modified femur; cymbium unmodified; tegulum not expanded; embolus prolaterally or medially situated, elongated, accompanied by membranous conductor; median apophysis absent. Epigynum simple, usually with small anterior hood and pair of globose spermathecae.

\section{Key to Species of PARALAMPONA}

1. Males .................. 2

- Females ................... 9

2. Embolus relatively straight throughout its length (figs. 792, 804, 808, 816) ..... 3

- Distal part of embolus hook-shaped (figs. 788, 796, 800, 812) ........6 6

3. Retrolateral tibial apophysis relatively long (figs. 793, 809) . ...........4 4

- Retrolateral tibial apophysis relatively short (figs. 805, 817) ............ 5

4. Tegulum greatly widened posteriorly (fig. 792) . . . . . . . . . marangaroo

- Tegulum not widened posteriorly (fig. 808) ........................ kiola

5. Retrolateral tibial apophysis straight (fig. 805) ................ aurumagua

- Retrolateral tibial apophysis sinuous (fig. 817) ............... renmark

6. Embolus relatively long (figs. 788, 800) . . 7

- Embolus relatively short (figs. 796, 812) . . 8

7. Tip of embolus relatively short (fig. 788) ..

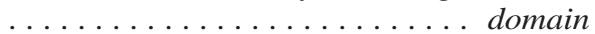

- Tip of embolus relatively long (fig. 800) ... .........................

8. Retrolateral tibial apophysis greatly widened, sinuous (figs. 796, 797) ...... sherlock

- Retrolateral tibial apophysis narrow, straight (figs. 812, 813) . . . . . . . . wogwog

9. Anterior epigynal hood forming concave arch (figs. 790, 794, 798) . . . . . . . 10

- Anterior epigynal hood straight or convex (as in figs. 802, 806, 818) . . . . . . 12

10. Spermathecae elongate (figs. 795, 799) . . 11

- Spermathecae rotund (fig. 791) ... . domain

11. Spermathecae relatively wide (fig. 795) ....

............... marangaroo

- Spermathecae relatively narrow (fig. 799) .. ................ sherlock

12. Epigynum with strong posterior margin (figs.

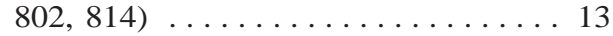

- Epigynum without distinct posterior margin (figs. 806, 810, 818) . . . . . . . 14

13. Spermathecae with lateral lobes (fig. 803) ..

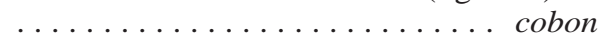

- Spermathecae with medial lobes (fig. 815) $\ldots \ldots \ldots \ldots \ldots \ldots$ wogwog

14. Posterior portion of spermathecae bilobed (figs. 818, 819) ......... renmark

- Posterior portion of spermathecae not bilobed (figs. 807, 811) ............ 15

15. Epigynal septum relatively narrow (fig. 806) .............. aurumagua

- Epigynal septum relatively wide (fig. 810) .. ........................ kiola

Paralampona domain, new species Figures 788-791; Map 90

TyPES: Female holotype and male allotype taken in grass tussocks at Queens Domain, $42^{\circ} 52^{\prime} \mathrm{S}, 147^{\circ} 19^{\prime} \mathrm{E}$, Tasmania (Sept. 1, 1934; V. Hickman), deposited in AMS (KS29340).

ETYMOLOGY: The specific name is a noun in apposition taken from the type locality.

DIAGNOSIS: Males can be recognized by the distally narrowed and hooked embolus (figs. 788, 789), females by the medially directed spermathecal lobes (figs. 790, 791).

MALE: Total length 1.5. Abdomen grayish brown, legs light orange. Palpal femur unmodified, retrolateral tibial apophysis long, narrow (fig. 789); embolus basally wide, distally narrow, hooked (fig. 790).

FEMALE: Total length 2.5. Abdomen pale tan. Epigynum with posterior ducts forming semicircle (fig. 791), spermathecae with medially directed terminal lobes (fig. 792).

Other Material Examined: Australian 

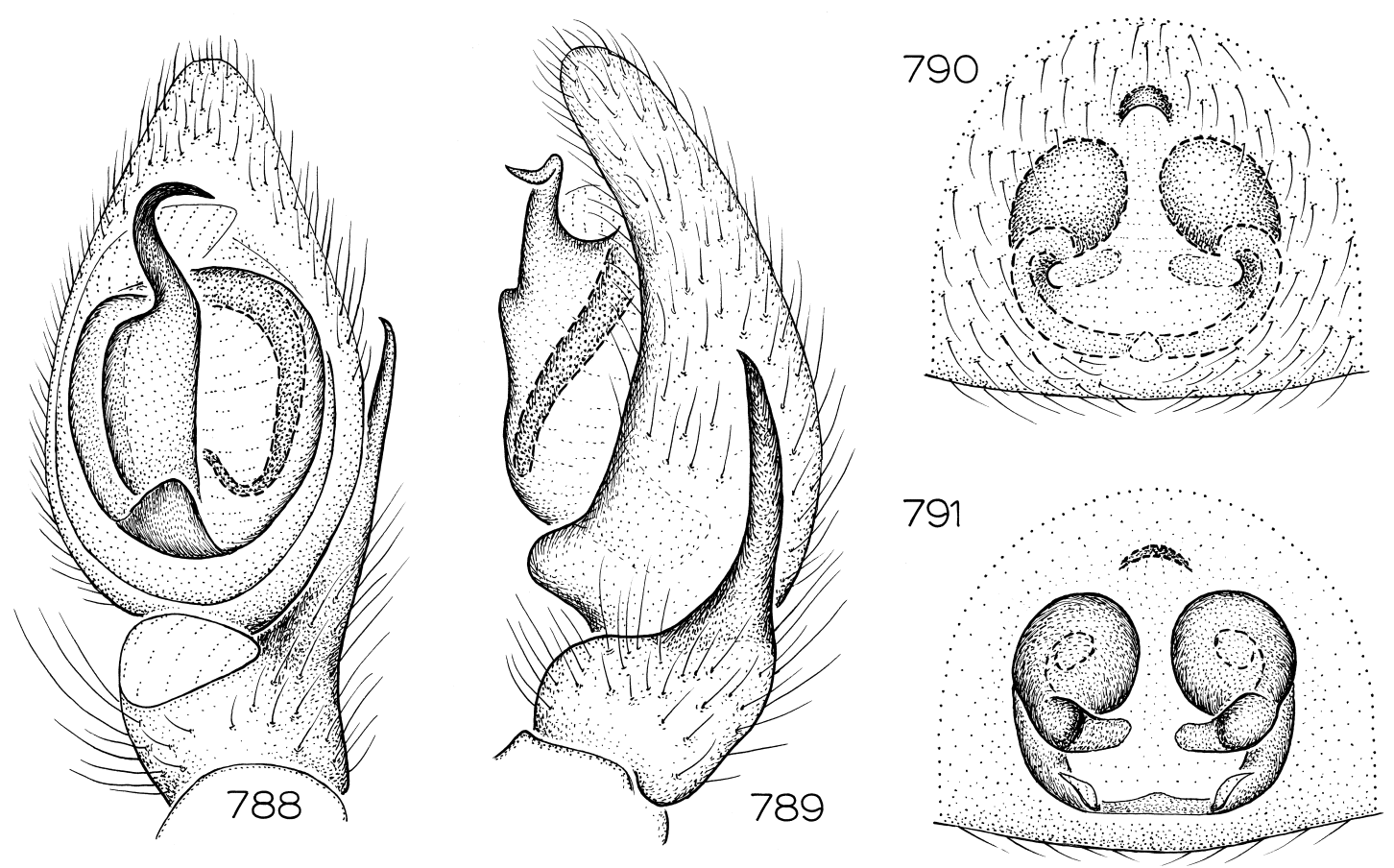

Figs. 788-791. Paralampona domain, new species. 788. Left male palp, ventral view. 789. Same, retrolateral view. 790. Epigynum, ventral view. 791. Same, dorsal view.

Capital Territory: Black Mountain, $35^{\circ} 16^{\prime} \mathrm{S}, 149^{\circ} 06^{\prime} \mathrm{E}$, Oct. 7,1976 , Berlese, dry sclerophyll (T. Weir, QMB), 1 , , Oct.-Nov. 1983, pitfall (R. Moran, QMB), 1 ㅇ. New South Wales: Booti Booti National Park, $32^{\circ} 15^{\prime} \mathrm{S}, 152^{\circ} 32^{\prime} \mathrm{E}$, July 20,1996 , litter, bitou bush (L. Wilkie, AMS KS55296), 2 \% ; Jerrabomberra Hill, near Queanbeyan, 35 $23^{\prime} \mathrm{S}$, $149^{\circ} 13^{\prime}$ E, Sept. 30 , 1976, Berlese, dry sclerophyll, elev. $783 \mathrm{~m}$ (R. Taylor, T. Weir, QMB), $1 \%$; Murrumbidgee River, Narranderra, $34^{\circ} 45^{\prime} \mathrm{S}, 146^{\circ} 33^{\prime} \mathrm{E}$, July 5, 1968, Berlese, eucalypt litter (J. Watson, QMB), 1 ㅇ. Queensland: Lake Broadwater, via Dalby, $27^{\circ} 21^{\prime} \mathrm{S}, 151^{\circ} 06^{\prime} \mathrm{E}$, Feb. 25-Apr. 22, 1986, pitfalls (M. Bennie, QMB S34316, 34325), 2 . South Australia: Aldinga, $35^{\circ} 16^{\prime} \mathrm{S}$, $138^{\circ} 29^{\prime} \mathrm{E}$, Berlese (QMB), 1 \% ; Breakneck River, Flinders Chase, Kangaroo Island, $35^{\circ} 56^{\prime} \mathrm{S}, 136^{\circ} 37^{\prime} \mathrm{E}$, Nov. 8, 1990, litter (E. Matthews, SAM N1997/119), 19 ; Kelly Hill Caves camp area, Kangaroo Island, $35^{\circ} 59^{\prime} \mathrm{S}$, $136^{\circ} 54^{\prime} \mathrm{E}$, Nov. 10, 1987, litter under thomasia (D. HIrst, SAM N1997/116, 117), 2 ; Loftia Recreation Park, Mount Lofty Ranges,

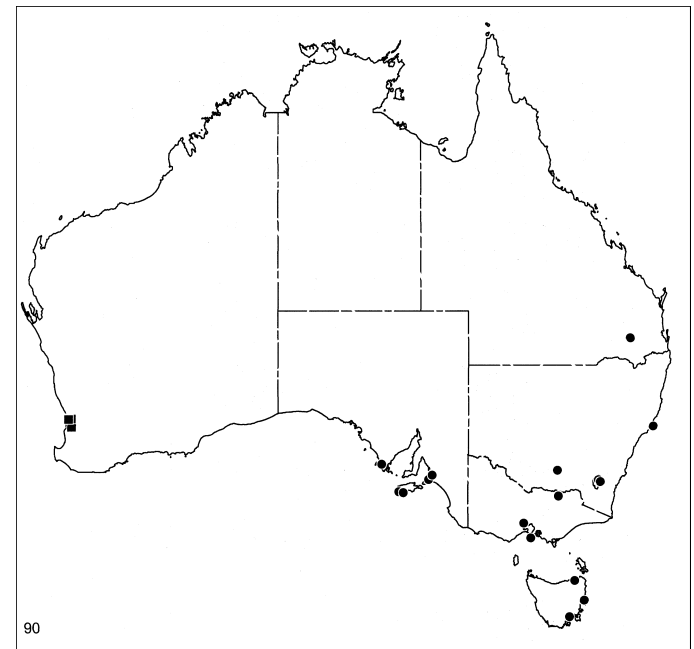

Map 90. Records of Paralampona domain, new species (circles) and $P$. marangaroo, new species (squares). 

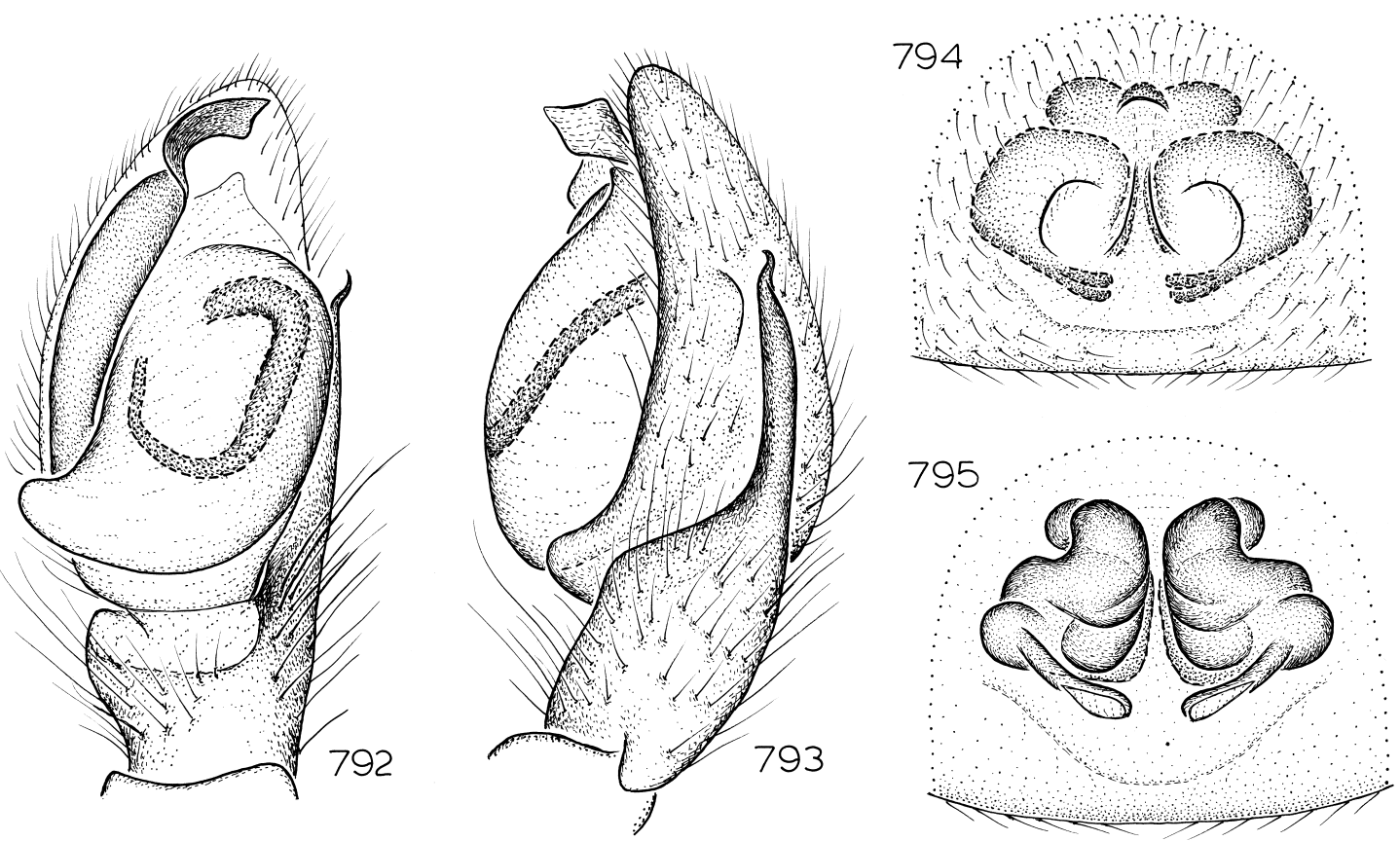

Figs. 792-795. Paralampona marangaroo, new species. 792. Left male palp, ventral view. 793. Same, retrolateral view. 794. Epigynum, ventral view. 795. Same, dorsal view.

$35^{\circ} 02^{\prime} \mathrm{S}, 138^{\circ} 42^{\prime} \mathrm{E}$, Sept. 14 , 1989, litter (D. Hirst, SAM N1997/120), 19 ; foot, Marble Range, near homestead, $34^{\circ} 25^{\prime} \mathrm{S}, 135^{\circ} 31^{\prime} \mathrm{E}$, Mar. 29, 1987, litter near creek (D. Lee, D. Hirst, SAM N1997/118), 1 ㅇ. Tasmania: 15 km ENE Cranbrook, $41^{\circ} 57^{\prime} \mathrm{S}, 148^{\circ} 14^{\prime} \mathrm{E}$, Jan. 28, 1983, Berlese, leaf litter (I. Naumann, J. Cardale, QMB), 19 ; Maggs Mountain, Sept. 23, 1981 (R. Green, QVM 13:42041), 1\%; Northeast Tasmania, Feb. 1993 (QVM 13: 20837, 20871), 10, 2q; Queens Domain, $42^{\circ} 52^{\prime} \mathrm{S}, 147^{\circ} 19^{\prime} \mathrm{E}$, Sept. 1, 1934 , grass tussocks (V. Hickman, AMS KS29340), 10 , $2 \%$, Aug.-Sept. 1942, grass tussocks (V. Hickman, AMS KS29238), 60ิ, 5ㅇ, May 1948, grass tussocks (V. Hickman, AMS KS32533), 10, June 5, 1948 (V. Hickman, AMS KS32534), 1 \% , Mar. 1968, grass tussocks (V. Hickman, AMS KS29348), 1 ; Waterhouse Point, $40^{\circ} 51^{\prime} \mathrm{S}, 147^{\circ} 38^{\prime} \mathrm{E}$, Nov. 1986, coastal heath (T. Churchill, QVM 13: 1152), 1 क, Oct. 1987, coastal heath (T. Churchill, QVM 13:3567), 19, 1987, coastal heath (T. Churchill, QVM 13:3930), 1 ㅇ. Victoria: Cape Schanck, $38^{\circ} 30^{\prime} \mathrm{S}, 144^{\circ} 53^{\prime} \mathrm{E}$, May 28, 1978 (V. Salanitri, QMB S26735),
1ิึ, 1 ㅇ, July 8, 1978 (V. Salanitri, QMB S26714, 26746), 2q; Chiltern Forest, $36^{\circ} 10^{\prime} \mathrm{S}, 146^{\circ} 37^{\prime} \mathrm{E}$, Feb. 1967, Berlese, leaf litter (R. McInnes, QMB), 19 ; Lederderg Gorge, Aug. 12, 1981, under rock (M. Harvey, NMV K3624), 2 \% ; Longforest Road, $6.5 \mathrm{~km}$ NE Bacchus Marsh, $37^{\circ} 41^{\prime} \mathrm{S}$, $144^{\circ} 27^{\prime} \mathrm{E}$, Aug. 25, 1981, eucalypt litter (M. Harvey, B. Roberts, NMV K3623), 4 ㅇ ; Rotamah Island, The Lakes National Park, June 11, 1990, sieved Banksia litter (D. Black, WAM 96/1527, 1528), 2 ․

DistRIBUTION: Southeastern Australia, including Tasmania (map 90).

Paralampona marangaroo, new species Figures 792-795; Map 90

TYPES: Female holotype and male allotype taken in pitfall traps in Marangaroo Reserve, $31^{\circ} 50^{\prime} \mathrm{S}, 115^{\circ} 50^{\prime} \mathrm{E}$, Western Australia (July 13-Sept. 25, 1995; M. Harvey, J. Waldock), deposited in WAM $(96 / 740,741)$.

ETYMology: The specific name is a noun in apposition taken from the type locality.

DIAGNOSIS: Males can easily be recognized 

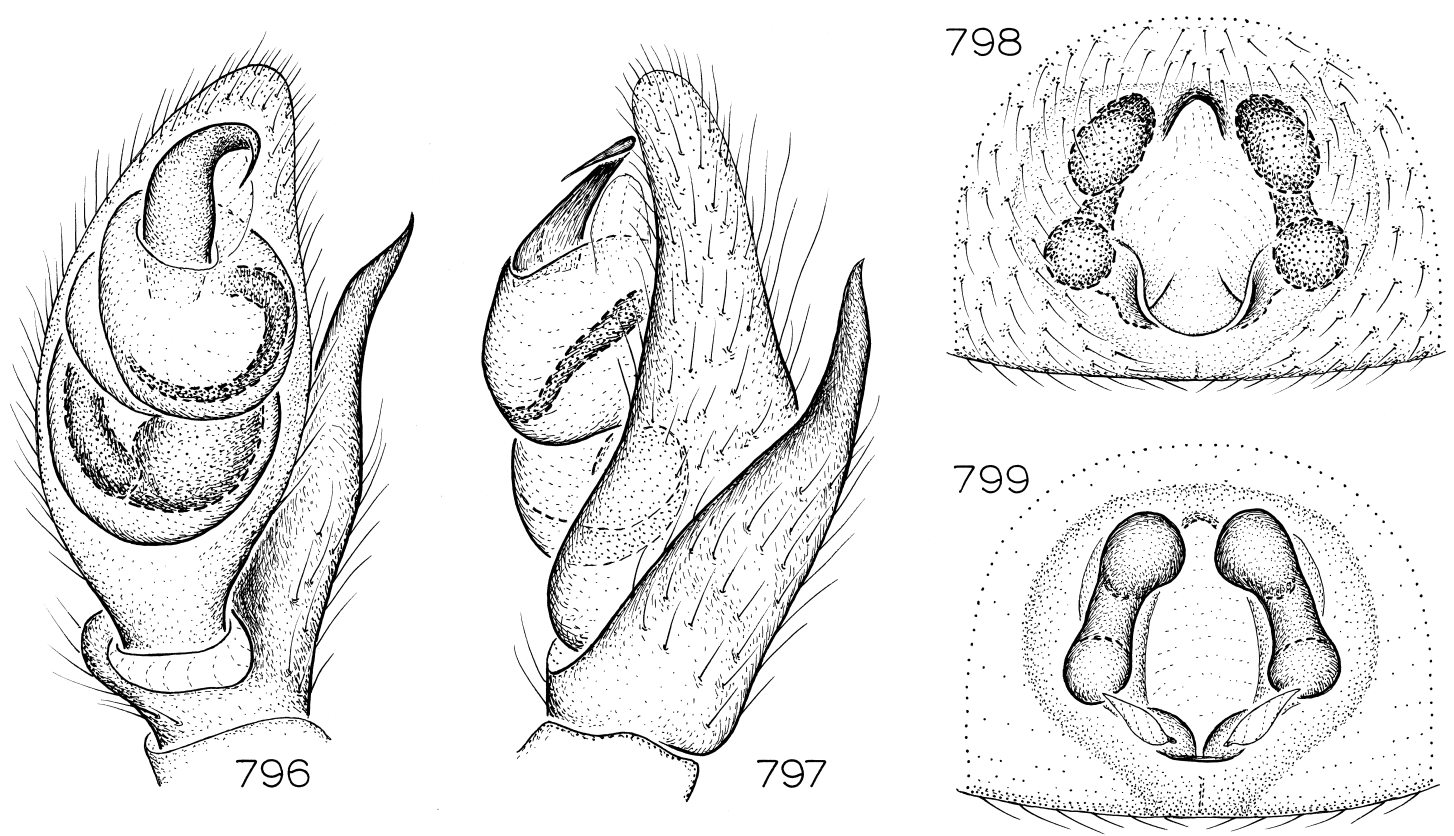

Figs. 796-799. Paralampona sherlock, new species. 796. Left male palp, ventral view. 797. Same, retrolateral view. 798. Epigynum, ventral view. 799. Same, dorsal view.

by the greatly widened palpal femur (fig. 793) and the wide, prolaterally situated embolus (fig. 392), females by the long, narrow, median epigynal septum (fig. 794).

MALE: Total length 2.1. Abdomen dirty white, legs pale orange. Palpal femur greatly enlarged, with heavy setae distodorsally, retrolateral tibial apophysis long (fig. 793); embolus prolaterally situated, wide, distally twisted (fig. 792).

FEMALE: Total length 2.4. Coloration as in male. Epigynum with median septum (fig. 793); spermathecae elaborate (fig. 795).

Other Material Examined: Western Australia: Jarrahdale Mine, $32^{\circ} 20^{\prime} \mathrm{S}$, $116^{\circ} 04^{\prime} \mathrm{E}$, Sept. 12-19, 1997 (K. Brennan, WAM 96/745), 1 \%; Talbot Road Reserve, $31^{\circ} 52^{\prime} \mathrm{S}, 116^{\circ} 03^{\prime} \mathrm{E}$, Nov. 18-Dec. 10, 1993, pitfall (J. Waldock, WAM 96/742), 1 q ; Tuart Hill, $31^{\circ} 53^{\prime} \mathrm{S}, 115^{\circ} 52^{\prime} \mathrm{E}$, May $10-J u l y ~ 20$, 1993, pitfall (M. Harvey, J. Waldock, WAM 96/744), 1 9 , July 20-Sept. 23, 1993, pitfall (J. Waldock, A. Sampey, A. Thorpe, WAM 96/743), 1 ô.

Distribution: Western Australia (map 90).
Paralampona sherlock, new species Figures 796-799; Map 91

TYPE: Female holotype from a Berlese sample of mallee litter taken $3.2 \mathrm{~km} \mathrm{~W}$ of

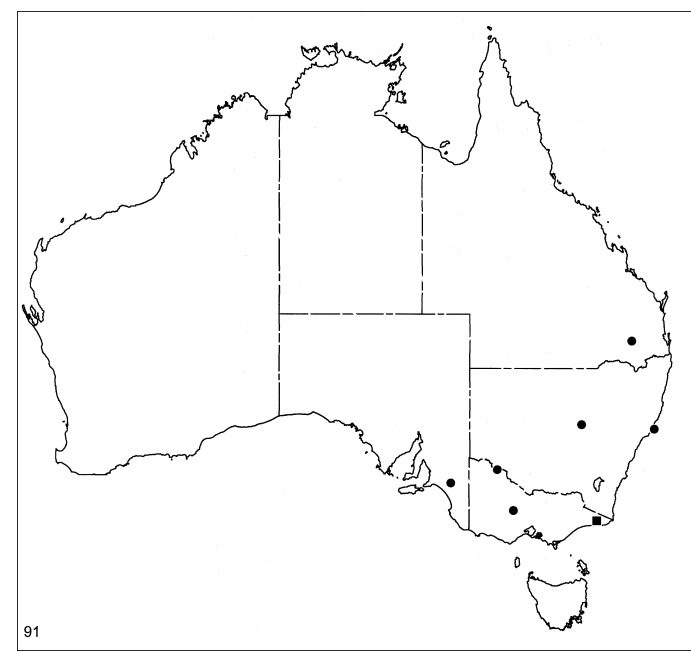

Map 91. Records of Paralampona sherlock, new species (circles) and $P$. cobon, new species (square). 

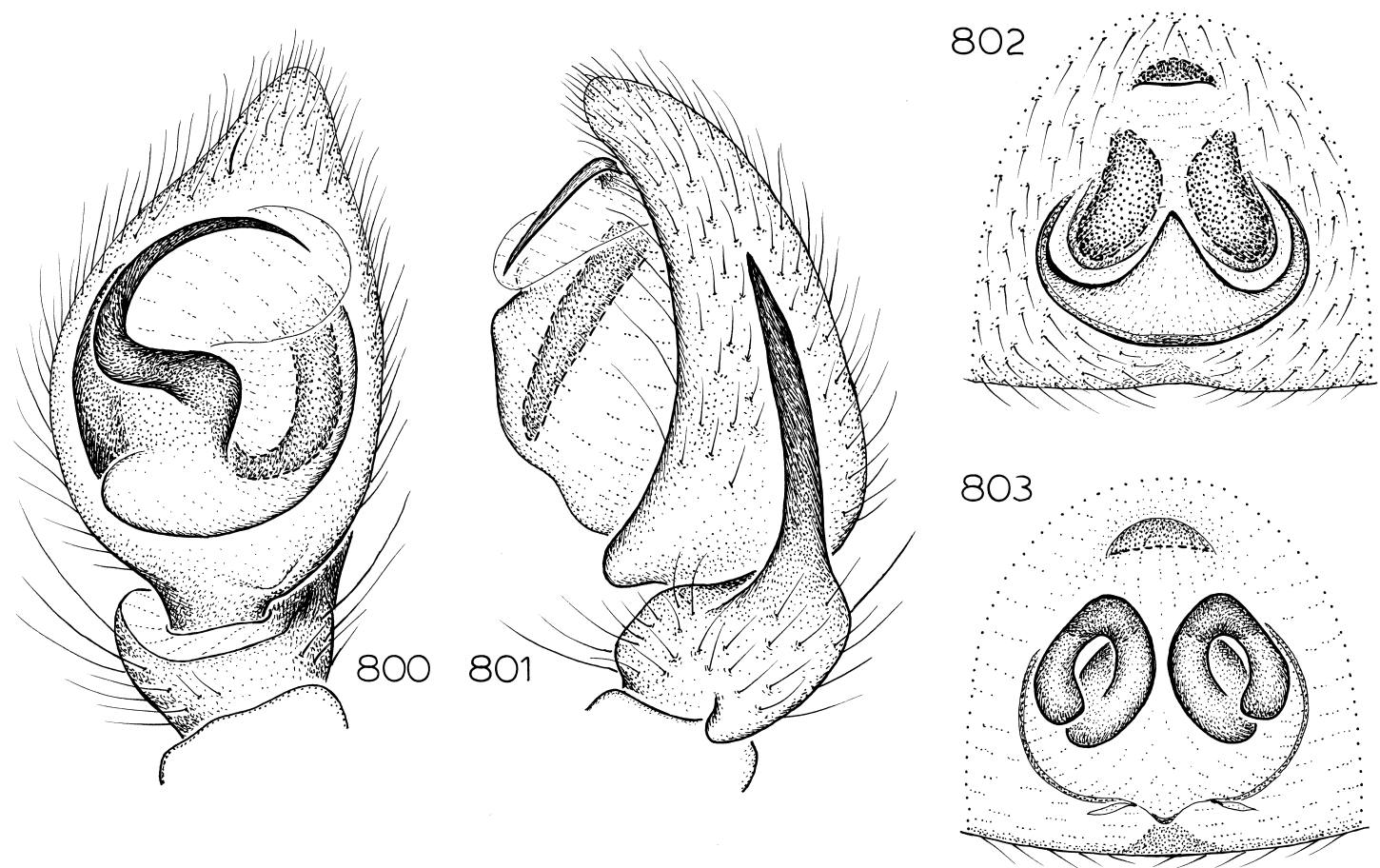

Figs. 800-803. Paralampona cobon, new species. 800. Left male palp, ventral view. 801. Same, retrolateral view. 802. Epigynum, ventral view. 803. Same, dorsal view.

Sherlock, $35^{\circ} 19^{\prime} \mathrm{S}, 139^{\circ} 49^{\prime} \mathrm{E}$, South Australia (Jan. 12, 1970; R. Taylor), deposited in QMB.

ETYMOLOGY: The specific name is a noun in apposition taken from the type locality, in honor of N. S. Platnick.

DiAGNOSIS: Males and females have not been collected together but are matched here on the basis of their bizarre genitalia and their similar distributions in mallee habitats. Males can easily be recognized by the massively inflated retrolateral tibial apophysis (figs. 796, 797), females by the dumbbellshaped spermathecae (figs. 798, 799).

MALE: Total length 1.3. Abdominal dorsum gray, darkest posteriorly. Palpal femur unmodified; retrolateral tibial apophysis inflated (fig. 797); embolus situated distally, curved (fig. 796).

FEMALE: Total length 2.3. Abdomen white, legs pale orange. Epigynum with short, posteriorly situated median septum (fig. 798); spermathecae dumbbell-shaped (fig. 799).

Other Material Examined: New South Wales: Booti Booti National Park, $32^{\circ} 16^{\prime} \mathrm{S}$, $152^{\circ} 32^{\prime} \mathrm{E}$, July $12-16,1996$, pitfalls, sand dunes (L. Wilkie, AMS KS50517-50521), $50^{\circ} ; 26 \mathrm{~km}$ E Euston, $34^{\circ} 34^{\prime} \mathrm{S}, 142^{\circ} 44^{\prime} \mathrm{E}$, Feb. 6, 1970, Berlese, open mallee (C. Brooks, QMB), 19 ; Trangie, $32^{\circ} 02^{\prime} \mathrm{S}$, $147^{\circ} 59^{\prime}$ E, Mar. 8, 1968, dry sclerophyll leaf litter (L. Mound, QMB), 10 . Queensland: Lake Broadwater, via Dalby, $27^{\circ} 21^{\prime} \mathrm{S}$, $151^{\circ} 06^{\prime}$ E, May 17-Nov. 24, 1995, pitfall (M. Bennie, QMB S34321), 10ิ, Apr. 22-June 12, 1986, pitfall (M. Bennie, QMB S34313), 10. Victoria: $5 \mathrm{~km}$ E Dunolly, $36^{\circ} 51^{\prime} \mathrm{S}$, $143^{\circ} 44^{\prime} \mathrm{E}$, Aug. 4, 1983, leaf litter (R. Moran, QMB), $1 \hat{\sigma}$.

DistRIBUTION: Apparently widespread in drier areas of southeastern Australia, except Tasmania (map 91).

\section{Paralampona cobon, new species} Figures 800-803; Map 91

TyPES: Female holotype and male allotype from midslope pitfall traps at Cobon South Coupe, $37^{\circ} 25^{\prime} \mathrm{S}, 148^{\circ} 56^{\prime} \mathrm{E}$, Victoria (Apr. 6- 

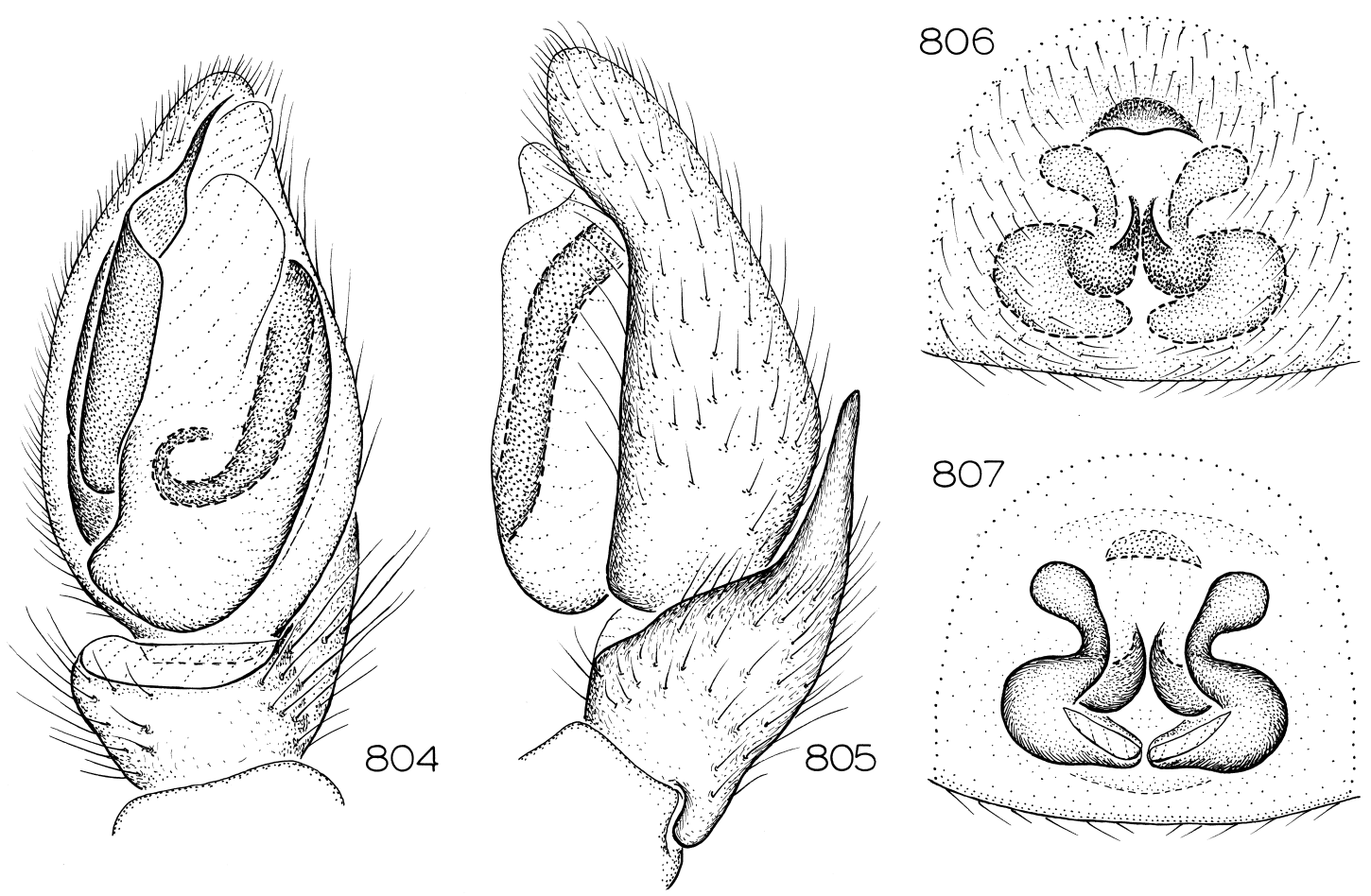

Figs. 804-807. Paralampona aurumagua, new species. 804. Left male palp, ventral view. 805. Same, retrolateral view. 806. Epigynum, ventral view. 807. Same, dorsal view.

14, 1992; R. Coy), deposited in NMV (K3601).

ETYMOLOGY: The specific name is a noun in apposition taken from the type locality.

DiAGNOSIS: Males can be recognized by the long, arched embolus (fig. 800), females by the anterior spermathecal lobes (fig. 802).

MALE: Total length 1.9. Abdomen light gray dorsally, white ventrally, legs light orange. Palpal femur unmodified; retrolateral tibial apophysis extending from base of tibia almost to tip of palpal bulb (fig. 801), embolus long, arched (fig. 800).

FEMALE: Total length 2.5. Coloration as in male. Epigynum with rounded posterior margin (fig. 802); spermathecae with posteriorly directed anterior lobes (fig. 803).

Other MATERIAl ExAMined: Victoria: Cobon South Coupe, $37^{\circ} 25^{\prime} \mathrm{S}, 148^{\circ} 56^{\prime} \mathrm{E}$, Apr. 6-14, 1992, midslope pitfalls (R. Coy, NMV K3601), 10, upslope pitfall (R. Coy, NMV 3549), 1 ठิ.
DisTRIBUTION: Known only from eastern Victoria (map 91).

Paralampona aurumagua, new species Figures 804-807; Map 92

TYPES: Female holotype and male allotype taken in litter at Gold Creek Reservoir, Brookfield, $27^{\circ} 30^{\prime} \mathrm{S}, 152^{\circ} 55^{\prime} \mathrm{E}$, Queensland (June 2, 1981; R. Raven, V. Davies), deposited in QMB (S34327).

ETYMOLOGY: The specific name is am arbitrary combination of letters.

Diagnosis: This species seems closest to $P$. kiola (see below), but males have a shorter retrolateral tibial apophysis that is situated more dorsally (figs. 804, 805), and females have the anterior spermathecal lobes more widely separated (figs. 806, 807).

MALE: Total length 1.5. Abdominal dorsum variable (uniformly gray, with gray chevrons, or uniformly pale); anterior legs light orange, posterior legs paler. Palpal fe- 

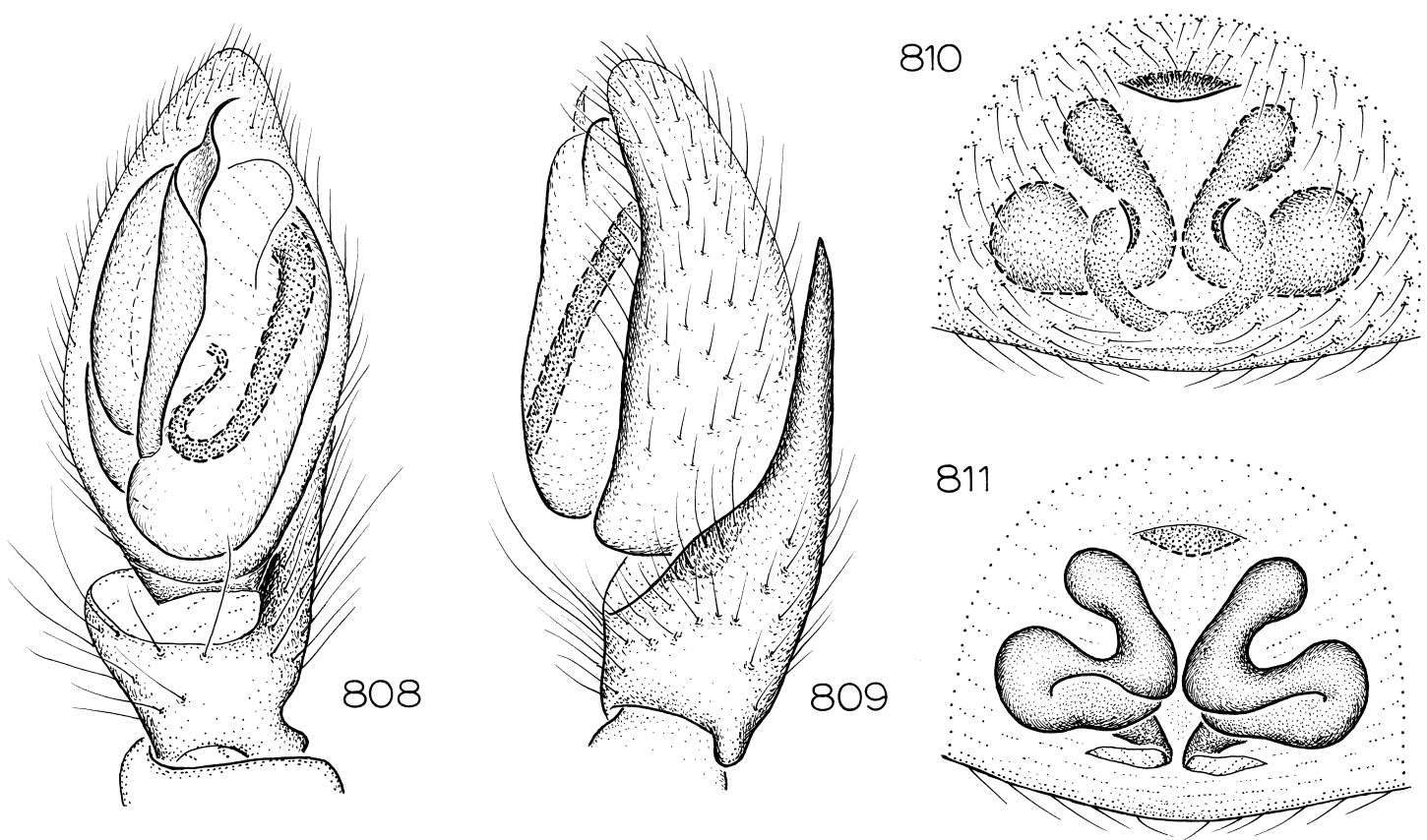

Figs. 808-811. Paralampona kiola, new species. 808. Left male palp, ventral view. 809. Same, retrolateral view. 810. Epigynum, ventral view. 811. Same, dorsal view.

mur distally incrassate; retrolateral tibial apophysis relatively short, situated almost dorsally (fig. 805); embolus long (fig. 804).

FEMALE: Total length 2.3. Coloration as in male. Epigynum with narrow median septum (fig. 806); anterior spermathecal lobes widely separated (fig. 807).

Other MATERIAl ExAmined: Queensland: Frenchville, $23^{\circ} 20^{\prime} \mathrm{S}, 150^{\circ} 34^{\prime} \mathrm{E}$, Apr. 21-July 18, 1990, pitfall, open forest (D. Wallace, R. Raven, QMB S34318), 10; Gold Creek Reservoir, Brookfield, $27^{\circ} 30^{\prime} \mathrm{S}, 152^{\circ} 55^{\prime} \mathrm{E}$, June 23, 1980, litter (R. Raven, V. Davies, QMB S34319), 10, 3 \% , Aug. 7, 1980, litter (R. Raven, QMB S34333), 20, $3+$, Nov. 28Dec. 16, 1980, litter, closed forest (QMB S34326), 2 q , Mar. 18, 1981, litter, closed forest (R. Raven, V. Davies, QMB S34336), 1ô, 2 \%, Mar. 23-Apr. 8, 1981, litter, closed forest (R. Raven, V. Davies, QMB S34328), 20, 2 ㅇ, Apr. 23-May 13, 1981, litter (R. Raven, V. Davies, QMB S34329), 10, 2 운, June 17-July 3, 1981, litter (R. Raven, V. Davies, QMB S34335), 10, 2 \%, Aug. 14, 1881, litter (R. Raven, V. Davies, QMB S34323), 1\%; Mount Coot-tha, Brisbane, $27^{\circ} 28^{\prime} \mathrm{S}, 152^{\circ} 58^{\prime} \mathrm{E}$, Dec. 1-22, 1979, pitfall (R. Raven, QMB S34334), 3 , Nov. 4, 1996-July 4, 1997, pitfalls, eucalypt forest, south slope (R. Raven, QMB S31370), 20.

DistRIBUTION: Known only from eastern Queensland (map 92).

\section{Paralampona kiola, new species} Figures 808-811; Map 92

TYPES: Female holotype and male allotype taken in pitfall traps in litter on Forest Drive, Kiola State Forest, $15 \mathrm{~km} \mathrm{~N}$ Batemans Bay, $35^{\circ} 37^{\prime} \mathrm{S}, 150^{\circ} 16^{\prime} \mathrm{E}$, New South Wales (Jan. 30-Feb. 22, 1979; C. Horseman), deposited in AMS (KS2596).

ETYMOLOGY: The specific name is a noun in apposition taken from the type locality.

Diagnosis: This species seems closest to $P$. aurumaqua, but males have a longer retrolateral tibial apophysis (fig. 809) and females have a wider epigynal septum (fig. 810).

MALE: Total length 1.7. Abdominal dorsum gray, lightest in cardiac area, venter white, legs pale yellow. Palpal femur slightly 

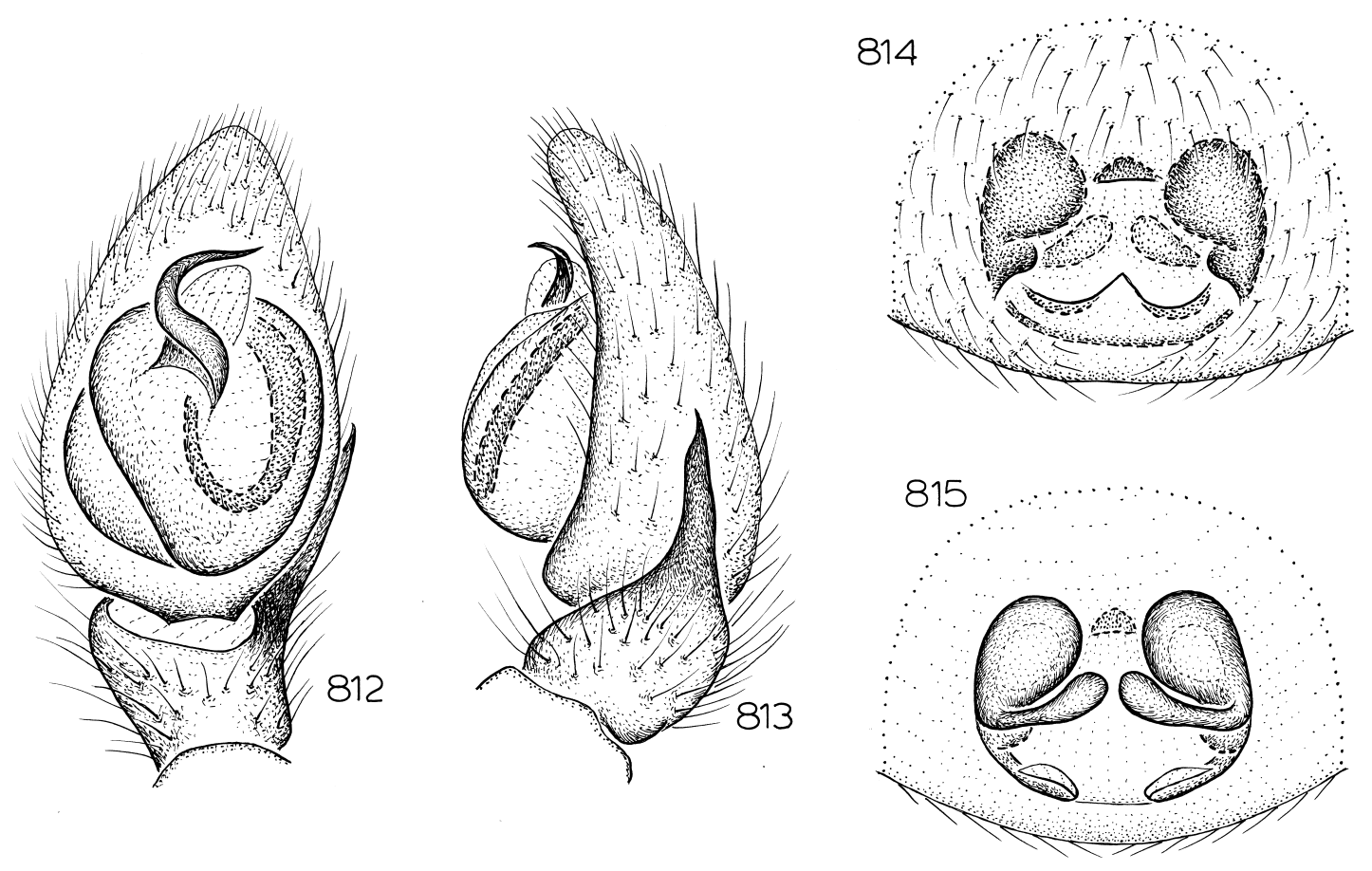

Figs. 812-815. Paralampona wogwog, new species. 812. Left male palp, ventral view. 813. Same, retrolateral view. 814. Epigynum, ventral view. 815. Same, dorsal view.

enlarged distally; retrolateral tibial apophysis long, not shifted dorsally (fig. 809); embolus long (fig. 808).

FEMALE: Total length 2.1. Coloration as in male. Epigynal septum relatively wide (fig. 810), spermathecae with recurved anterior lobes (fig. 811).

Other Material Examined: Australian Capital Territory: Urriara, Berlesate 228 (QMB), 1 \% ; Wombat Creek, $8 \mathrm{~km} \mathrm{~N}$ Piccadilly Circus, $35^{\circ} 19^{\prime} \mathrm{S}, 148^{\circ} 51^{\prime} \mathrm{E}$, elev. $750 \mathrm{~m}$ (Weir, Lawrence, Johnson, QMB), 7 $0^{\star}$. New South Wales: Batemans Bay, $35^{\circ} 42^{\prime} \mathrm{S}$, $150^{\circ} 11^{\prime} \mathrm{E}$, May 27, 1978, Berlese, wet log and stump, elev. $10 \mathrm{~m}$ (S., J. Peck, AMNH), $1 \% ; 3 \mathrm{~km} \mathrm{~N}$ Batemans Bay, $35^{\circ} 43^{\prime} \mathrm{S}$, $150^{\circ} 11^{\prime} \mathrm{E}$, June 9-Aug. 30, 1982, intercept trap, elev. 50 m (S., J. Peck, AMNH), 1 đ; Benandarah State Forest, $8 \mathrm{~km}$ N Batemans Bay, $35^{\circ} 40^{\prime} \mathrm{S}, 150^{\circ} 14^{\prime} \mathrm{E}$, Jan. 30-Feb. 22, 1979, litter pitfalls (C. Horseman, AMS KS2609), 50, 1 ㅇ, Sept. 21-Oct. 18, 1979, pitfalls (C. Horseman, AMS KS3927, 5549), 2\%; Blue Mountains, road to Ingar Picnic Area, $33^{\circ} 46^{\prime} \mathrm{S}, 150^{\circ} 29^{\prime} \mathrm{E}$, Oct. 3, 1996, pitfall

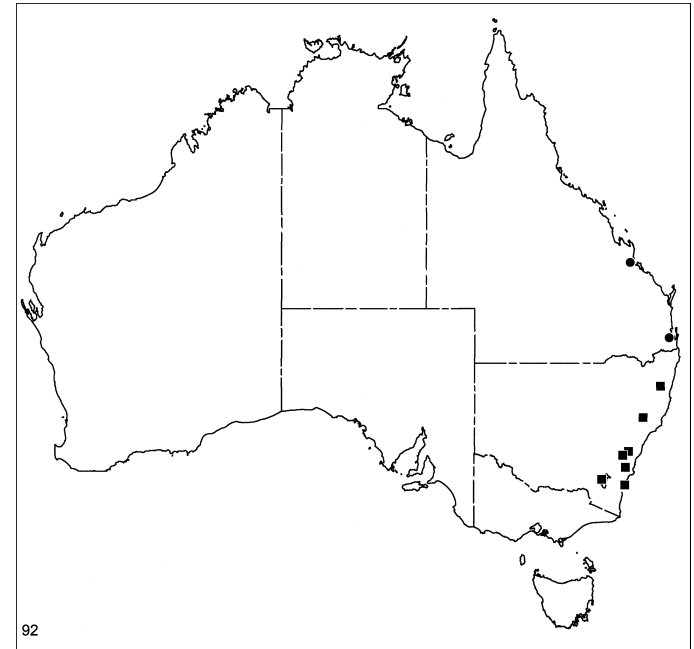

Map 92. Records of Paralampona aurumagua, new species (circles) and $P$. kiola, new species (squares). 

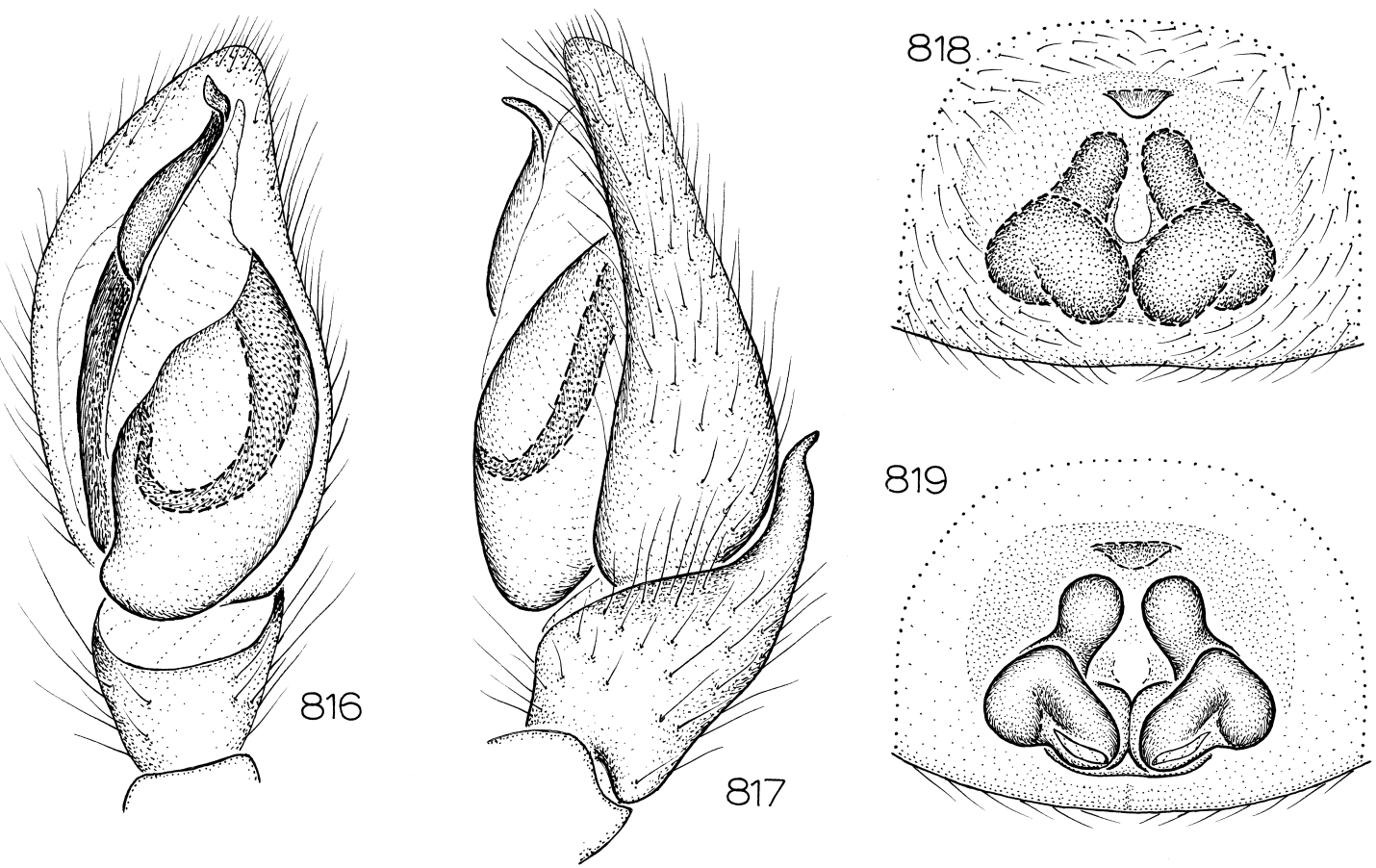

Figs. 816-819. Paralampona renmark, new species. 816. Left male palp, ventral view. 817. Same, retrolateral view. 818. Epigynum, ventral view. 819. Same, dorsal view.

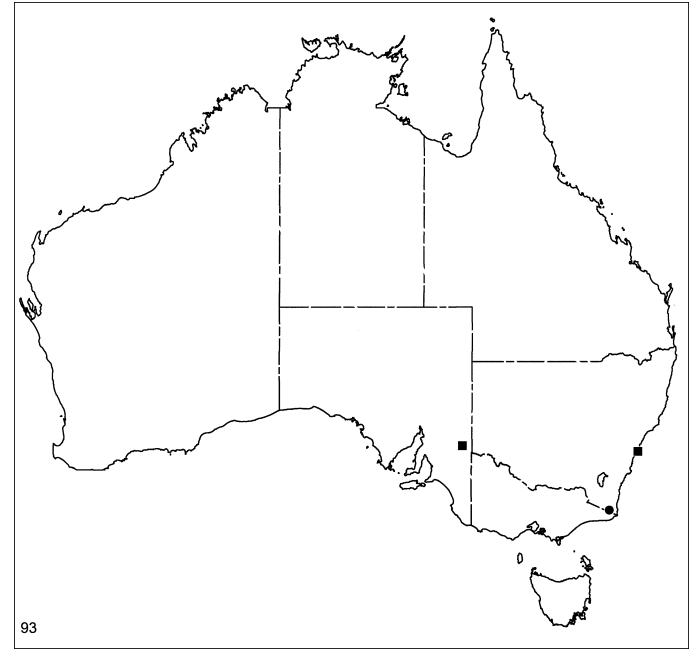

Map 93. Records of Paralampona wogwog, new species (circle) and $P$. renmark, new species (squares).
(AMS KS51708), 1 ; $0.9 \mathrm{~km} \mathrm{~S}$ along Chaelundi Road from Big Bull Road, Mount Hyland Nature Reserve, $30^{\circ} 09^{\prime} \mathrm{S}, 152^{\circ} 27^{\prime} \mathrm{E}$, Feb. 4-Apr. 9, 1993, pitfall, elev. $1080 \mathrm{~m}$ (M. Gray, G. Cassis, AMS KS35377), 1 ; 2 $\mathrm{km}$ SSE Bundanoon, $34^{\circ} 39^{\prime} \mathrm{S}, 150^{\circ} 18^{\prime} \mathrm{E}$, Apr. 3, 1982, Berlese, wet sclerophyll, ferns, bracken, dicksonia, elev. $550 \mathrm{~m}$ (L. Hill, QMB), 10; Forest Drive, Kiola State Forest, $15 \mathrm{~km} \mathrm{~N}$ Batemans Bay, $35^{\circ} 37^{\prime} \mathrm{S}, 150^{\circ} 16^{\prime} \mathrm{E}$, Jan. 30-Feb. 22, 1979, litter pitfalls (C. Horseman, AMS KS2596), 30 , 1 \% , Sept. 7Oct. 4, 1979, litter pitfalls (C. Horseman, AMS KS3906), 50, 4 우 ; Kanangra-Boyd National Park, $33^{\circ} 59^{\prime} \mathrm{S}, 150^{\circ} 08^{\prime} \mathrm{E}$, Mar. 20, 1982, Berlese, closed forest litter and moss (L. Hill, QMB), 1 ; $0.7 \mathrm{~km}$ along logging trail from Omadale Brook Road, Stewarts State Forest, $31^{\circ} 54^{\prime} \mathrm{S}, 151^{\circ} 23^{\prime} \mathrm{E}$, Feb. 4-Apr. 9, 1993, pitfall, elev. 1250 m (M. Gray, G. Cassis, AMS KS37395), 10 .

Distribution: Know only from New South Wales and the Australian Capital Territory (map 92). 
Paralampona wogwog, new species

Figures 812-815; Map 93

TYPES: Female holotype and male allotype taken in pitfall traps $4 \mathrm{~km}$ NE Mount Wog Wog, $17 \mathrm{~km}$ SE Bombala, $37^{\circ} 05^{\prime} \mathrm{S}$, $149^{\circ} 28^{\prime} \mathrm{E}$, New South Wales (May 1985; C. Margules), deposited in QMB.

ETYMology: The specific name is a noun in apposition taken from the type locality.

DiAgnosis: Males of this distinctive species can be recognized by the relatively short embolus (fig. 812) and retrolateral tibial apophysis (fig. 813), females by the strong posterior epigynal margin (fig. 814) and long, anteromedially directed spermathecal lobes (fig. 815).

MALE: Total length 1.3. Abdominal dorsum dark gray, venter white, legs pale yellow. Palpal femur unmodified, retrolateral tibial apophysis relatively short (fig. 813), embolus relatively short, strongly hooked (fig. 812).

Female: Total length 1.7. Coloration as in male. Epigynum with pronounced posterior margin (fig. 814); spermathecal lobes long, anteromedially directed, widened at tip (fig. 815).

Other Material Examined: New South Wales: $4 \mathrm{~km}$ NE Mount Wog Wog, $17 \mathrm{~km}$ SE Bombala, $37^{\circ} 05^{\prime} \mathrm{S}, 149^{\circ} 28^{\prime} \mathrm{E}$, Feb. 1985 , pitfall (C. Margules, QMB), 1ð, 1 , , May 1985, pitfall (C. Margules, QMB), 2 ㅇ.

Distribution: Known only from the southeastern New South Wales (map 93).
Paralampona renmark, new species Figures 816-819; Map 93

TYPE: Male holotype taken in flight intercept trap at a site $79 \mathrm{~km}$ NNW Renmark, $33^{\circ} 31^{\prime} \mathrm{S}, 140^{\circ} 24^{\prime} \mathrm{E}$, South Australia (Aug. 9Sept. 7, 1995; K. Pullen), deposited in QMB.

ETYMOLOGY: The specific name is a noun in apposition taken from the type locality.

DiAGNOSIS: Males and females have not been taken together. Males of this distinctive species can be recognized by the distally sinuous retrolateral tibial apophysis (fig. 817) and the long, doubly twisted embolus (fig. 816 ), females by the posterolateral spermathecal lobes (fig. 818, 819).

MALE: Total length 2.1. Abdominal dorsum dark gray except near anterolateral corners, where pale, venter white, legs pale yellow. Palpal femur expanded, hirsute distally, retrolateral tibial apophysis sinuous, abruptly narrowed at tip (fig. 817); embolus long, twisted twice (fig. 816).

FEMALE: Total length 2.4. Abdomen light gray, legs pale yellow. Epigynal hood triangular, prominent (fig. 818); spermathecae with posterolaterally directed lobes (fig. 819).

Other Material Examined: New South

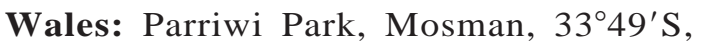
$151^{\circ} 14^{\prime} \mathrm{E}$, June 6, 1992, litter (G. Hunt, AMS $\mathrm{KS} 49488), 1$ ㅇ․

DisTRIBUTION: Known only from eastern South Australia and New South Wales (map 93).

\section{REFERENCES}

Atkinson, R. K., and L. G. Wright

1991. Studies of the necrotic actions of the venoms of several Australian spiders. Comp. Biochem. Physiol. 98C: 441444.

Berland, L.

1929. Araignées recueillies par Madame Pruvot aux Iles Loyalty. Bull. Soc. Zool. Fr. 54: 388-399.

Bonnet, $\mathrm{P}$.

1957. Bibliographia araneorum. Toulouse, 2(3): 1927-3026.

Dalmas, R. de.

1917. Araignées de Nouvelle Zélande. Ann. Soc. Entomol. Fr. 86: 317-430.
Dunn, R. A.

1951. Spiders of the Russell Grimwade expedition. Mem. Natl. Mus. Melb. 17: 9-18.

Farris, J. S.

1969. A successive approximations approach to character weighting. Syst. Zool. 18: 374-385.

1988. Hennig86, version 1.5. Computer program distributed by its author.

Forster, R. R.

1979. The spiders of New Zealand. Part V. Cycloctenidae, Gnaphosidae, Clubionidae. Otago Mus. Zool. Bull. 5: 1-95. 
Goloboff, P.

1993. Estimating character weights during tree search. Cladistics 9: 83-91.

1997. Pee-Wee, version 2.6. Computer program distributed by its author.

Gray, M.

1989. A significant illness that was produced by the white-tailed spider, Lampona cylindrata. Med. J. Aust. 151: 114-116.

Harvey, M. S., and R. J. Raven

1991. Necrotising arachnidism in Australia: a simple case of misidentification. Med. J. Aust. 154: 856.

Hickman, V. V.

1950. Araneae from Reevesby Island, South Australia. Proc. R. Soc. Vict., n. ser., 60: $1-16$.

1967. Some common spiders of Tasmania. Tasman. Mus. Art Gallery, 112 pp.

Koch, L.

1866. Die Arachniden-Familie der Drassiden. Nürnberg, 304 pp.

1872. Die Arachniden Australiens. Nürnberg, 1: 105-368.

1873. Die Arachniden Australiens. Nürnberg, 1: 369-472.

Main, B. Y.

1964. Spiders of Australia: a guide to their identification with brief notes on the natural history of common forms. Brisbane: Jacaranda Press, 124 pp.

Petrunkevitch, A.

1928. Systema aranearum. Trans. Conn. Acad. Arts Sci. 29: 1-270.

Platnick, N. I.

1990. Spinneret morphology and the phylogeny of ground spiders (Araneae, Gnaphosoidea). Am. Mus. Novitates 2978: $42 \mathrm{pp}$.

Rash, L. D., E. A. Davis, R. G. King, and W. C. Hodgson

1998. A comparison of the pharmacological activity of venom from the male and female white-tailed spider. Toxicon 36: $1311-1312$.

Raven, R. J., and M. S. Harvey

1991. Necrotising arachnidism in Australia [Reply]. Med. J. Aust. 155: 208.
Roewer, C. F.

1955. Katalog der Araneae von 1758 bis

Simon, E. 1940, bzw. 1954. Bruxelles, 2: 1-1751.

1893. Histoire naturelle des araignées. Paris, 1: 257-488.

1896. Descriptions d'arachnides nouveaux de la famille des Clubionidae. Ann. Soc. Entomol. Belg. 40: 400-422.

1897a. Histoire naturelle des araignées. Paris, 2: 1-192.

1897b. Description d'arachnides nouveaux. Ann. Soc. Entomol. Belg. 41: 8-17.

1898. Histoire naturelle des araignées. Paris, 2: 193-380.

1908. Araneae. 1re partie. In W. Michaelsen and R. Hartmeyer (eds.), Die Fauna Sudwest-Australiens. Jena, 1(12): 359446.

1909. Araneae. 2e partie. Ibid. 2(13): 152212.

Strand, E.

1913. Über einige australische Spinnen des senckenbergischen Museums. Zool. Jahrb. Syst. 35: 599-624.

1922. Über einige indoaustralische Spinnen. Arch. Naturg. 88(A4): 143-149.

Sutherland, S. K.

1987. Watch out, Miss Muffet! Med. J. Aust. 147: 531 .

1991. Necrotising arachnidism in Australia. Ibid. 155: 136.

Thorell, T.

1869. On European spiders. Part I. Review of the European genera of spiders, preceded by some observations on zoological nomenclature. Nova Acta R. Soc. Sci. Upsaliae, ser. 3, 7: 1-108.

Urquhart, A. T.

1887. On new species of Araneida. Trans. N. Z. Inst. 19: 72-118.

1892. Catalogue of the described species of New Zealand Araneidae. Ibid. 24: 220230.

1893. On new species of Tasmanian Araneae. Pap. Proc. R. Soc. Tasman. 1892: 94130.

White, J., D. Hirst, and E. Hender

1989. 36 cases of bites by spiders, including the white-tailed spider, Lampona cylindrata. Med. J. Aust. 150: 401-403. 